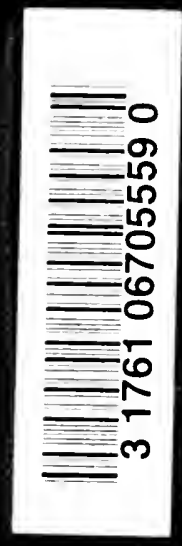




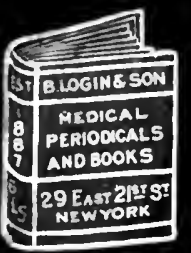


. 
Digitized by the Internet Archive in 2007 with funding from Microsoft Corporation 



\title{
MODERN TREATMENT
}

\section{THE MANAGEMENT OF DISEASE WITH MEDICINAL AND NON-MEDICINAL REMEDIES}

IN CONTRIBUTIONS BY AMERICAN AND

FOREIGN AUTHORI'TIES

\author{
EDITED BY
}

\section{HOBART AMORY HARE, M.D.}

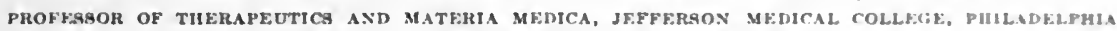
PHYSICIAN TO TIF JRFFHLON COLLEG: HOSHTAL

\section{ASSISTED BY}

\section{H. R. M. LANDIS, M.D.}

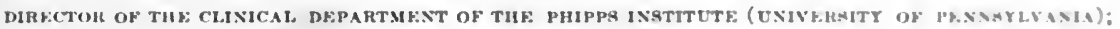
VISITING IIYSICIAN TO TIL: WIITE IAVEN AANATORIUM

\section{INT THO YOLUMES TOI,UME I}

ILLUSTRATED

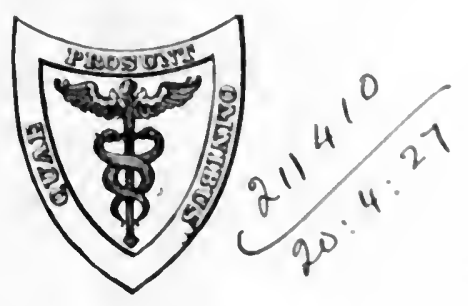

LEA \& FEBIGER PHILADELPHIA AND NEW YORK 
Entered according to Act of Congress, in the year 1910, by LEA \& FEBIGER, in the Office of the Librarian of Congress. All rights reserved.

Authority to use for comment the Pharmacopœia of the United States of America (Eighth Decennial Revision), in this volume, has been granted by the Board of Trustees of the United States Pharmacopœial Convention; which Board of Trustees is in no way responsible for the accuracy of any translations of the Official Weights and Measures, or for any statement as to strength of Official Preparations. 


\section{INTRODUCTION.}

EvEn practitioner of medicine and surgery is deeply interested in the treatment of disease by medicines and by remedial measures which do not depend upon drugs for their effects. At no time in the history of the medieal art has this interest been keener than it is today. Still face to face with many problems that called forth the best efforts of our predecessors, we are also called upon to meet additional conditions laid bare by the advances in medical science, all departments of which, even if remote from bedside practice, nevertheless have as their chice reason for existence the hope that our measures for the cure or alleviation of disease may be improved. Heretofore the means in our hands to this end consisted solely in drugs, in climate, in hydrotherapy and electrotherapy, but within the last fifteen or twenty years the wide and fruitful fields of therapentics covered by serum therapy, and so-called vaccine therapy, have been developed with a far greater approach to specific treatment than we ever possessed before. During the time that these advances have been made the pharmacologist, the chemist, and the pharmacist have enriched the materia medica list, with the result that remedies are better understood, new ones of inestimable value have been evolved, and all of them placed in our hands in a form in which they can be readily employed.

The appearance of a work which places in the hands of the physician a competent summary of all these advances is therefore timely, and the specialist and general surgeon may well scan its pages not only for facts which are directly connected with their particular lines of work, but also because he who treats his patients skilfully, in so far as their general condition is concerned, is he who wins success.

As one who has devoted many years to the study of therapentics, it may not be out of place for the editor of these volumes to say a few words about the subject of therapentics as it exists today, because it would seem that we are passing through a period in which, notwithstanding our wonderful advances, there still exist those who for lack of knowledge not rarely endeavor to ridicule the procedures which are often resorted to by competent and experienced men. 'Their tendency' is to throw doubt upon any method which fails to stand before what they call known scientific facts, meaning by scientific facts laboratory results, and to ignore the fact that even if the explanation given as to the means by which a remedy does good is wrong, this in no way impairs the value of the statement that it does good. Such critics do good in that they push to the front the need of rational processes in treatment, and because they cause the practitioner to hesitate and think before he proceeds to the use of a remedy on hearsay or the advice of some 
one else. 'They often do harm in that they discourage those who are doing their best with the knowledge that scientific investigation and clinical experience has given them, and like the religious agnostic, they take away faith and give nothing in its place, although this faith is often founded upon years of careful bedside experience and hundreds of tests made moler conditions the most severe.

Brilliant as the advances have been, the well-balanced physician recognizes that the fields yet untilled are still filled with clinical conditions which are not susceptible as yet of scientific study. Yet there are men with laboratory training, but little or no bedside experience, who would have us refuse to trespass upon these fields because the means we have at hand are not based upon so-called scientific laboratory facts. As Sir Clifford Allbutt has well said, where would England be if her expansion lyad waited until cartographers mapped out new areas before pioneers were allowed to travel abroad. 'The whole progress of human knowledge is characterized by the advances of the pioneer and explorer, and after him has come the master scientist to place the results where they should be. As long as the realm of Medicine remains in part nnexplored there will remain grave conditions which must be met by empiricism, and as long as human beings in health and sickness vary individually as to their symptoms and reactive powers to disease and drugs there will be fields which must be dealt with without the accuracy given us by the laboratory worker. In other words, to quote Sir Clifford's simile again, in part, the physician at the bedside must ever be the leader or prime factor in whose path must follow the scientific laboratory investigator who will discover the reasons for the procedures which his practical colleague has found useful. Rare exceptions to this rule do not negative it, but emphasize its general truth. If, therefore, the knowledge of the laboratory investigator leads him to the belief that a given therapentic procedure is irrational, it is for him, if he finds that the procedure has welhigh universal approval, not to marshal what facts he has to prove the clinician is wrong, but to bend his energies to discover the facts, as yet unknown to all of us, whereby the results are produced. In other words, it is for him to prove how it does good, and not his function to try to prove that his colleague is in error. 'The cry today is for the conservative therapeutist, who, like Ehrlich, will build up our resources, and not for the iconoclast, who would take from us that which we have obtained by years of careful observation.

'The skill of the individual physician in adjusting his doses to the needs of a particular case, in choosing one drug instead of another of the same type for a particular action, is still the skill of the empiric and not the skill of the scientific laboratory investigator. If a given dose of a given remedy were suitable for a given ailment, there would be little need for the physician except as a diagnostician. It is the never-ceasing changes in all living persons and the different effects of disease in different individuals that gives the man with bedside experience his great value. 'The pharmacologist may lay down the fundamental processes, but the clinician must make the distinctions in their use which develop results 
which we desire. 'The variation from the normal in the Iroly atferent by disease may completely reverse the known effect of a drug upon at healthy organism. A host of illustrations might be cited, but a few mulfin. Nearly all of our knowledge of the so-called physiologrical effects of alcohol is that obtained by investigations of its effects on fiealthy aninals or men. In the healthy organism the alcohol must te burnt up in addition to the ordinary processes of oxidation; whereas, in the folbrile patient it is not only more readily burnt up, but hy its oxiclation it protects the tissues of the patient. Another instance is the extraordinary impaiment of the value of digritalis by the presence of fever. Still another is the immunity to chloroform possessed by parturient women. Other instances are the tolerance of iodides by syphilitics and of opinm by those in pain.

There can be no doubt that of tentimes remedies gain a reputation that they do not deserve chiefly because of coincidence of improvement with their administration, when in reality there is no relationship of cause and effect, or because the imagination of the physician, or of the patient, plays a role which in the ease of the former should be controlled, but which in the patient is certainly advantageous. In either event a remedy may seem beneficial when really inert. But these errors are rarely harmful, and a great number of remedies have stood the test and have proved themselves of positive value, even if their effects are inexplicable. In many instances the advance of scientific knowedge has discovered how they do good many years after thousands of lives have been saved hy their use, as with iquinine, for example, or with digitalis, the so-called physiological action of which was at first thought to be quite the reverse of what we know it to be today. It is the duty of the physician, therefore, to resort to all measures proved useful by long experience unless it can be proved that they possess power for harm. At the same time that it is the duty of the pharmacologist to be a constunctive agent, so is it also the duty of the bedside physician to be continually on the alert to improve his methods, to analyze his practice, to improve his mental processes, and to avoid griving a remedy by rule of thumb or hecause someone else has done so. He should be constantly on the lookout to discover why a remedy does good, and not be content with the result alone, since by this means be acts by reason and not by slavish example. 'T'o put it briefly, the attitude of the competent medical man of today must be that of clinical investigator, who welcomes every gain in knowledge as an additional aid in his eflorts to do good. He must hold fast to that which is good, being careful that what he holds is worthy, and he must ever be controlled in his practice hy the fact that he is treating a patient, not a disegse, a patient whose powers of dealing with a malady are marvellous, with an organism that is to be directed, not driven or forced into the right path. Iatst of all, let him he so careful of what he does that the maxim, "do no harm," is never disregarded.

H. A. H.

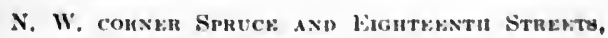
I'HLIDELPHA, 1910. 



\section{CON'TRIBU'TORS TO VOLUME I}

ARISTIDES AGRAMONTE, M.D.,

President of the Board of Infectious Diseases, and Member of the National Boand of Health; Professor of Hacteriology and Experimental Pathology, National University, Havana, C'uba.

OSWALD T. AVERY, M.D.,

Department of Bacteriology, Iloagland Laboratory, Brooklyn, X. Y.

W. JARVIS BARLOW, M.D.,

Dean and Professor of Clinical Medicine in the Los Angeles Department, College of Medicine, University of California; Member of the American Climatological Association.

SIMON BARUCH, M.D.,

Professor of Hydrotherapy in Columbia University (College of Physicians and Surgeons), New York; Consulting Physician to the J. Hood Wright Memorial Hospital and Montefiore Hospital for Chronic Invalids and Consumptives.

S. P. BEEBF, M.D., Pi.D.,

Professor of Experimental Therapeutics, Cornell University Medical School, New York.

THOMAS A. CLAYTOR, M.D.,

Clinical Professor of Medieine, George Washington University; Physician to the Garfield Memorial Hospital, and to the Tubereulosis Hospital, Washington, D. C.

F. X. DERCUM, M.D.,

Professor of Nervous and Mental Diseases in the Jefferson Medical College of Philadelphia; Neurologist to the Philadelphia General Hospital; Membre Cormespondant Etrauger de la Société de Neurologie de Paris.

CHARLES HUNTER DUNN, M.D.,

Clinical Instructor in Pediatrics, Harvanl Melical School; Assistant Plyysician at the Children's Hospital, Boston; Assistant Physician and Pathologist at the Infants' Hospital, Boston.

JULIUS FRIEDENWAI.D, M.D.,

Professor of Diseases of the Stomach, ('ollege of Plyysicians and Sungeons, Baltimore; Visiting Gastro-enterologist to the Mercy Hospital, Bay View Hospital, St. Agnes Hospital, Union Protestant Infirmary, and the Cluceh Home, Baltimore.

GUY HINSDALE, A.M., M.D.,

Secretary of the American Climatological Association: Fellow of the Royal Society of Medicine of Great Britain; Fellow of the College of Physicians of Philadelphia, etc. 
S. DANA HUBBARD, M.D.,

Attending Plyysician, Department of Dermatology, Vanderbilt Clinic, Columbia University, N. Y.; Attending Dermatologist, New York City Children's Ilospitals; formerly Diagnostieian, Department of Health, New York; and Chief Medieal Inspeetor, Borough of Brooklyn, Department of Health, New York.

AMIBROSE HUNSBERGLR, P'H.(i.,

Ieeturer on Commercial I'harmacy, Medieo-('hirurgical College, Department of Plarmacy, Philadelphia.

THOMAS WRIGITT JACKSON, M.D.,

Fort Hunt, Va., Melieal Reserve Corps, U. S. Army; Member of The American Society of Tropical Nedicine; Nember of the Manila Medical Society; Lately Captain and Assistant Surgeon, U. S. Volunteers; Lately Leeturer on Tropical Medieine, Jefferson Medical College, Philadelphia.

J. W. KERR, M.I).,

United States Public ILealtlı and Marine-Hospital Service.

H. R. M. LANDIS, M.D.,

Director of the (linical I Jepartment of the Phipps Institute (University of P'enn.yliania); l'isiting Physician to the White Haven Sanatorium.

FRANK SHLRMAN IIEARA, M.D.,

Professor of Therapeuties in the Comell Lniversity Medical Sehool, New York.

W. H. PARK, M.D.,

Professor of Bacteriology and Hygiene in the Cniversity and Bellevue Hospital Medieal College; Direetor of the Researeh Laboratory of the Department of Health of New York ('ity.

GEORGE E. PFAHLER, M.D.,

Professor of Roentgenology in the Medico-Chirurgical College, and Direetor of the X'rity Lathoratory of the Medico-Chirurgical Hospital, Philadelphia.

F. M. POTTLAGER, M.D.,

Nerlical Director of the Pottenger Sanatorium, Monrovia, Cal.

NATIANIEI, BOWDITCII l'OTTER, M.D.,

Visiting Physician to the New York ('ity Hospital, to the Freneh Hospital, and to the Hospital for Rup tured and ('rippledl; ('onsulting Physician to the Manhattan State Hospital: Assoeiate in ('linieal Medieine in the Columbia University, New lork.

GEORGE E. I'RIC'E, M.D.,

Assistant Professor of Norvous and Mental Diseases in the Jefferson Medical College; Assistant Neurologist to the Infferon ('ollege Hospital, Pliiladelphia.

D.MHI) RIESIA. M.D.,

Professor of ('linical Mfedicine, Philadelphia Polyelinic, and Assistant Professor of Medicine in the Iniversity of Pennsylrania.

LEONARD ROGERS, M.D., F.R.C.P'.,

Professor of Pathology and I'lyysician-in-Charge of the Cholera Wards in the Nerlical college of Calcutta.

B. F. ROYER, M.I).,

Chief Medieal Inspector in the Department of Health of Pennsylvania; formerly ('hief Resident in the Municipal Hospital for Contagious Diseases of Pliiladelphia. 
ROBERT DAWSON RUDOLF, M.D. (EDIN.), F.R.C.P. (IOND.),

Professor of Therapeutics in the University of Toronto; Assintant Physician to the Toronto General Hospital and the Sick Children's Hospital.

JOHN RUHRÄH, M.D.,

Professor of Diseases of Children and Therapeutics, College of Physicians and Surgeons; Visiting Physician to the Robert Garrett Hospital for Children, Nursery and Child's Hospital, Merey Hospital, and (onsulting l'hysician to the Church Home and Infirmary, Baltimore.

ARTHUR M. SHRADY, M.1).,

Chief of Clinic of Hydrotherapy in the Columbia Iniversity, New York: (onsulting Physician to the Seton Iospital; Assistant Attending Physician (") the Harlem Hospital, New York.

Thomas A. STOREY, A.M., M.D., Ph.D.,

Professor of Physical Instruction and Hygiene, and Director of that Department in the College of the City of New York.

OSCAR H. WILSON, M.D.,

Instruetor in Clinical Medieine in the Jefferson Medieal College of Philadelphia.

HORATIO C. WOOD, JR., M.D.,

Second Vice-Clıairman of the Committee of Revision of the U.S. Pharmacopoeia; Formerly Associate Professor of Pharmacology in the University of Pennsylvania: Member of the American Society for Pharmacology and Experimental Therapeutics. 



\section{CONTENTS OF VOLUME I}

INTRODUCTION

\section{PAR'T I \\ GENERAL, CONSIDERA'TIONS}

MODERN PHARMACOLOGY AND ITS BEARING ON PRACTICAI,

THERAPEUTICS................................ 17

Br horatjo C. Wood, Jr., M.D.

THE CONBINATION OF DRUGS. PRESCRIPTION WRITING....... 65

By Ambiose Hunsberger, Ph.G.

THE UNTOWARD EFFECTS OF DRUGS.................. 109

By Robert Dawson Rudolf, M.D. (Edin.), F.R.C.P. (Lond.)

\section{PART II}

THE TREATMENT OF DISEASE BY NON-MEDICINAI, MEASURES

DEFINITION OF CLIMATIC THERAPEUTICS.

By W. Jarvis Barlow, M.I).

GENERAL EXERCISE.............................. 163

Bу Thомas A. Stokey, M.D., PH.D.

MINERAL SPRINGS

BY GuY HiNsDale, M.D.

HYDROTHERAPY.

By Simon Baruch, M.D., and Arthul M. Shrady, M.D.

ELECTROTHERAPEUTICS, INCLUDING HIGH-FREQUENCY CURRENTS.

BY Gronge E. Prick, M.D.

THE TREATMENT OF DISEASE BY THE $X$-RAYS AND RADIOACTIVE SUIBSTANCES

By George E. Prahlem, M.D.

THE REST CURE

BY F. X. DerCum, M.D.

NUTRITION AND FOODS.

By Juluus Friedenwald, M.D., AND Johx RUHRÄl, M.D. 

HYGIENIC MEASURES: THE MANAGEMENT OF EPIDEMICS AND

DISINFECTION ............................ 435 BY J. W. KerR, M.D.

SERUM THERAPY

BY W. H. PARK, M.I.

OPSONINS AND VACCINE THERAPY 515

By Nathaniel Bowmitch Potter, M.D., and Oswald T. Avery, M.D.

GLANDUIAR THERAPY.. 551 By S. P. IBeebe, M.D., P'H.D.

TUBERCULIN AS A THERAPEUTIC AND IIAGNOSTIC AGENT.... 573 By F. M. Potthager, M.D.

\section{PART III}

\section{THE TREATMENT OF 'THE INFEC'TIOUS DISEASES}

TYPHOID FEVER

By Datid Riesmax, M.D.

SCARLATINA, MEASLES, RUBELIA, VARICELLA, AND VARIOLA. 639 By S. Dana Hubrard, M.D.

CEREBrospinal MENINGitis. 685

By Charles Hunter Dunn, M.D.

PNEUMONIA AND PIEURISY ..................... 707

Iy Frank Sherman Meara, M.D.

TUBERCULOSIS.

BY H. R. M. LaNins, M.D.

DIPITTHERIA

By B. Frankuin Roter, M.D.

ACUTE ARTICILAR RIIEUMATISM

By Thomas A. Claytor, M.D.

CHOLERA

Br Leonard Rogkrs, M.D., F.R.C.P'

PLAGUE.

By Thomas Wright Jackson, M.D.

INFLUENZA 875

by Oscar Wilson, M.D.

YELLOW FEVER 889

By Aristides Agranonte, M.D.

INDEX 


\title{
PAR'T I \\ GENERAL CONSIDERATIONS
}

\section{MODERN PHARMACOLOGY AND IT'S BEARING ON PRACI'ICAL THERAPEU'TICS}

\author{
By HORATIO C. WOOD, Jr., M. D.
}

\section{THE VALUE AND LIMITATIONS OF PHARMACODYNAMICS}

RAtronal efforts at the relief of diseased conditions may be based upon their etiology, pathology, or symptomatology. The most logical and the most successful treatment is one which will eliminate the cause of the disease. Quinine, by destroying the plasmodium in the blood, renders any further medication unnecessary in a large proportion of cases of malarial fever; or the removal, by surgical measures, of an inflamed appendix abruptly sets aside the pain and the fever which were evidences of an inflammatory process in the body. 'The therapeutist, therefore, should always endeavor to find the underlying cause of the symptoms which are presented in the ease before him.

'To give a name to the symptom complex presented is no diagnosis, at least from the viewpoint of the therapeutist. It is necessary that the clinician bear in mind the pathological changes in the organ which give rise to the symptoms, and then seareh for the cause which gave rise to the lesion. For instance, let us imagine a patient with a paralytic condition of the extensor muscles of the foot. As possible causes of this symptom the physician considers lesions in the brain, the spinal cord. the peripheral nerves, or the muscles themselves. One after another of these causes are excluded, until the pathologic condition is diagnosed, let us say, to be a neuritis. For successful trentment of this condition, however, it is requisite that the cause of the changes in the nerve he discovered. 'The treatment will evidently be very different according to whether this neuritis is the result of ehronic lead-poisoning, chronic arsenical poisoning, trammatism, or any of the other causes which may bring about inflammation in a nerve-trunk.

Unfortunately, however, in many cases the origin of the disease may baffle the most careful and skilful diagnosticians, and in other instances 
the cause, even although discoverable, may be beyond the power of chemical or mechanical agents to effect. 'Thus, we may know that dyspnea and edema are due to lesions of the valves of the heart, but we know of no way to repair the torn valve. In such cases we may rationally attempt to antagonize the results of the pathological lesions; for instance, in endocarditis we have for our object to increase the functional power of the heart muscle, so that, despite the disadvantages under which it labors, it may still be able to fulfil the necessities of the circulation. 'To take another example, suppose the kidneys are diseased, and not properly eliminating the waste-products of the body, we may try either to increase the activity of the remaining healthy kidney structure to such an extent that it may carry on the whole excretory function of the organ, or to relieve the kidney by stimulating the other channels of elimination through the use of sudorifics or eathartics.

At times, however, either owing to our ignorance of the real pathological conditions or to the profoundness of the changes, we are unable to do anything beyond merely assuaging the symptoms, conserving the patient's strength, and preventing needless suffering, until such time as the unaided, but unhindered, forces of the body itself shall have destroyed the cause of the disease.

'The diseases in which the etiological factor may be reached with chemical agents - that is, with drugs - are, after all, comparatively few, and it is doubtful if their number is likely to be materially increased for many years. In a general sense, therefore, the scientific therapeutist is more frequently called on to combat the results of pathological lesions than their causes. If there is circulating in the system a poison, whether of bacterial origin or inorganic in its nature, he may strive to eliminate the toxic agent as rapidly as possible from the blood, but also he seeks some drug whose action is physiologically antagonistic to the poison, so that life may last until the patient recovers. It is evident that, for these efforts to be successful, it is necessary that the physician understands the effects of the drugs which he employs upon the various organs of the body.

Although a large number of facts, many of them of prime importance in the development of the science of pharmacology, have come from observations made in the sick-room, yet the greater part of our knowledge, as well as all hope for the systematizing of it has come and must continue to come through experimentation upon the lower animals. The proof of this statement is seen, in comparing the number of valuable remedies which have been introduced into medicine from the laboratory, in the half century of its existence, with the few drugs of real value which a thousand years of bedside experience have given us. Omitting references to mere pharmaceutical progress, such as is shown in the advantages the alkaloids offer over the crude drugs from which they are derived, and considering only those drugs alssolutely unknown, either in crude or pure form, until their value had been discovered through animal experimentation, the list includes such agents as nux vomica, nitroglycerine, strophanthus, hyoscine, physostigma, chloral hydrate, and practically all of the 
other somnifacients, salicylic acid and its salts, acetanilid and the other coal-tar antipyretics, theobromine, theophylline, suprarenal capsules, practically all the antiseptics, the antitoxins, eucaine and the other local anestheties, etc. Besides these substances, the first suggestion for the use of which in elinical therapeusis we owe to the laboratory investigator, there are a number of other substances whose range of utility has been so greatly modified, or extended throngh animal experimentation, that they might well be included in this list. For instance, digitalis sixty years ago was ranked as a cardiac sedative, and used in conditions of exeited circulation; that elinicians have come to regard it as a stimulant to the heart is due solely to the pharmacological researches of such men as 'Traube and Brunton; likewise an entirely new field of usefulness for ergot, although its utility as a stimulant of the uterus had long been known through elinical experience, was opened through the experiments of Holmes, who first demonstrated its power to contract blood-vessels.

In view of the increasing reliance which is being placed upon laboratory experimentation, as the doorway through which drugs must enter clinical medicine, it becomes a matter of vital moment to the practitioner to know how far conclusions concerning the action of chemieal agents, drawn from studies upon healthy lower animals, hold true in diseased conditions of the human being. 'There are evidently two distinct divisions to this question: first, is the action of drugs the same upon man as upon the lower animals? and, second, is the action of drugs the same in disease as in health?

While it is, of course, indisputable that drugs do not always cause the same symptoms in animals of different species, yet, after all, the difference in symptoms does not prove that physiological effects of the drug in the two animals are not identical. Thus atropine causes a great increase in the pulse-rate in the dog, but has comparatively little influence on the pulserate in the rabbit; but in each instance the pneumogastric nerve has been paralyzed. In the rabbit, paralysis of the ragus does not affect the pulserate; in the dog, it produces a great inerease in the pulse-rate; hence, although the action of the drug in both animals is the same, the symptoms which it causes are different. Similarly, we find that those remedies which affect the higher intellectual centres, such as morphine, act relatively more violently upon man, because of the superior development of his cerebrum, than they do upon the lower animals. But studies in comparative physiology have enlightened us concerning the differences in most of the vital functions between man and various members of the animal kingdom, so we can make proper allowances for these variations, and the careful pharmacologist is able to draw conclusions, from laboratory experimentation, which will hold true almost without exception for the human animal.

On the other hand, diseased conditions may so affect the functional capacity of the body that the response to cheinical agents may depart widely from that of health. 'This difference in the susceptibility of diseased and normal organs is well illustrated in the case of the antipyretie group. These drugs in moderate doses produce practically no change in 
the bodily temperature in healthy individuals, and yet, in febrile conditions, at times produce so profound a disturbance of the thermogenic centre as to lead to serious collapse. A number of similar examples of various morbid conditions, which experience has shown us modify the action of therapeutic agents might be cited, but as yet we have not acquired a sufficient amount of data to permit any systematization of this knowledge, so that new conclusions concerning the therapeutic value of drugs generally need to be confirmed by clinical experience before they can be finally accepted.

\section{RELATIONS OF CHEMICAL STRUCTURE TO BIOLOGICAL EFFECTS}

Science consists not merely in the collection of facts, but essentially in the correlation of these facts. While the effects of certain drugs on various organs of the body have long been known, it is only within comparatively recent years that any serious effort has been made to systematize known facts of drug force, and the science of pharmacodynamics may, therefore, be regarded as still being in its infancy. Two distinct tendencies may be noted in the development of this science, which we may call the chemical and the physiological. These, while distinct, are closely bound together, and the progress of each mutually dependent on that of the other.

The connection between chemical composition and physiological action might, at first thought, seem a matter of interest only to the scientist. If, however, the facts of pharmacology are of any importance to the practising physician,- - and I am convinced that successful clinical therapeutics is well-nigh impossible without them,-an understanding of the basic principles of the science is of great assistance. To attempt to remember all the clinically important facts of pharmacology, as isolated from each other, is a feat of mental gymnasties worthy of a disciple of Confucius. It is not merely that the practical application of a science is so much more successful if the basic principles are apprehended, but the number of facts which it is necessary to remember are greatly reduced. The degree to which a knowledge of some of the fundamental laws of pharmacology simplifies the subject may be illustrated by a reference to the inorganic salts. Of these, there are about 150 recognized by the Pharmacopoia, but if we classify them according to ionic theory as given below, we have to remember the properties of only about 40 substances as opposed to the 150. Again, there are being introduced to-day by synthetic chemists an immense number of new organic compounds, many of which are brought to the attention of the medical profession accompanied with statements which one who knows aught of the laws of relation of structure to action can brand as impossible; a knowledge of this branch of pharmacology on the part of the physician would have saved many a patient from experiments which were foredoomed to failure.

'The great fundamental question, which must be answered before we can hope to be able to completely systematize the laws of the relation between structure and action, is how do drugs produce their effects? What 
are the nature of the changes in the body produced by chomical angents which lead to the alterations in functions? A few years ago this qurestion would have been almost unhesitatingly answered by saving that thes. wero. chemical. 'The common concept of the action of a drug was that it formerl a chemical union with the protoplasm of the cell, and that this new form of protoplasm was endowed with properties more or less at varianee with those of the original cell. But to-day this theory is being strongly disputed, at least as being universally applicable. For instance, Meycr has shown that the narcotic drugs have in common the physical property that they are more soluble in fatty menstrua than in watery, and that, therefore, they will be readily taken up by the lipoids of the nervous svistem from the blood. Curci conceives of the action of the narcotics ass not chemieal, but physical. His theory is that, after one of these substances enters into the cell-contents, it does not form a chemical union with the protoplasm, but "infiltrates itself into the protoplasm amongit the gramules, the organelles, and the mucleus, in such a manner that the diflerent parts of the cell are surrounded by the hydrocarbon, and, becoming isolated, lose their contact with each other." 1

Although at present we are uable to state positively what are the nature of the changes in the cells eaused by drugs, it is probable that they are not all of one kind-some may be physical and some chemical. Many of the olsserved phenomena, especially as concerns the organic compounds, can be most easily explained by a theory similar to that put forward by Ehrlich to illuminate the neutralization of bacterial toxius.

All substances may be divided into two groups-electrolytes and nonelectrolytes. An electrolyte is a substance which is eapable of being decomposed by the electric current. 'The most convenient examples of this class of compounds are the inorganic salts, as sodium bromide, magnesium sulphate, potassium citrate, and similar salts. The current theory is that such substances, in dilute solution, undergo partial decomposition into their constituent elements or radicals, which are called ions. 'Thus, sodium chloride, if dissolved in water, exists, at least in part, as commingled, but not chemically bound, sodium kations and chlorine anions. 'This dissociation of the ions is, however, never complete; some of the sult appears to exist in the solution as a salt, not as its constituent ions. The proportion of ionization which occurs depends on the specific dissociability of the substance, the character of the solvent, the concentration of the solution, etc.

A non-electrolyte is a substance which eannot be further decomposed without losing its chemical identity. A simple distinction between ionic dissociation and ehemical decomposition is that, in the former. when the solvent is evaporated, there is obtained the salt in the same state that it was before solution, but, after chemieal decomposition, evaporation will not reunite the separated ingredients. 'The non-electrolytes, from a medical standpoint, are found chiefly among the organic drugs.

When an electrolyte is introduced into the blood, it has a threefold effect on the functions of the body-that which is due to the influence of its

1 XV. Cong. Internat. de Med., 1906, Section 4, p. 49. 
kation, that due to the influence of its anion, and that which is due to its effect as a salt. In many cases the activity of one or the other ion is so great as to overwhelm the influence of the two other factors. For instance, in the case of potassium cyanide the cyanogen ion is so poisonous that the influence of the potassium is imperceptible, and it is impossible to introduce a sufficient amount of the salt into the body to exercise the salt action. Sometimes it is the basic and sometimes it is the acid ion which exercises the preponderant power, and in other compounds neither ion may be extremely poisonous, in which case we get the typical salt effect. When the two ions are of approximately the same toxicity, the physiological action of the compound will be the sum of the effects of both ions. In such instances, where these salts are being used for therapeutic purposes, we can well modify the action of the important constituent by changing the unimportant ion; thus, while all the inorganic bromides are very similar in their physiological action, yet there is a distinct difference between the effects of potassium and ammonium bromide. The bromine ion, while somewhat more active than most of the alkaline earths, is nevertheless given frequently in doses which are so large that the effects of the kation may become evident. We may get, therefore, from the free use of potassium bromide, evidences of the depressant influence of the potassium on the circulation and central nervous system, and with the ammonium bromide, the effects of ammonium in stimulation of these functions.

Various efforts have been made to discover some constant relation between physiological effects of inorganic ions and various chemical or physical properties, as, for instance, atomic weight. None of these have proved successful, and it is doubtful whether any such connection will ever be shown, so that our knowledge of the action of the constituents which make up a salt must always be determined experimentally. While it is impossible to foretell what the effects of an ion, whose action has never been expcrimentally investigated, will be, yet wherever we have a compound made up of several ions, each of whose action is known, we can generally predict with accuracy what the effect of the compound will be. Two facts, however, prevent us from making absolutely certain predictions as to the effect of an untested salt. The first of these is that, by introducing new elements, we may alter the plan of dissociation; thus, by adding iron to potassium cyanide in certain proportion we destroy its toxicity, because a salt is formed which no longer yields up a cyanogen ion, but a ferrocyanide, which is comparatively non-poisonous. 'The other influence, which may disprove our prophecy as to the action of the compound, may be that, owing to some alteration in physical properties, the substance may become unabsorbable, and, therefore, cannot act at all when introduced into the alinentary tract.

When an aqueous solution of a crystalline substance is separated from pure water by an animal membrane, there is a tendency for the crystalline body to pass into the water, and the water to pass into the solution, until the fluids on the two sides of the animal membrane become of equal density. 'This, which is known as the process of osmosis, is 
the basis of the so-called salt action. If we introduce a large quantity of some non-toxic salt into the circulation, fluid will be drawn into the vessels from the surrounding tissues, until the concentration of the salt in the blood-stream approximates that outside the vessels, or, to use the physiological expression, until the blool becomes isotonic with the surrounding fluid. 'The increased volume of fluid in the vessels under these conditions will have the same effect as if we had increaserl the volume of fluid in the vessels by the intravenous injection of an isotonic salt solution; there will be a more rapid elimination of water through the various excretory organs, especially throngh the kidneys. 'This is the basis of the diuretic action of the neutral salts. As pointed out above, however, when either of the constituent ions of the salt are especially poisonous, we cannot introduce a large enough quantity of the substance into the circulation to produce any perceptible salt action, and the increased secretion is therefore abseni.

'The division of chemicals into organie and inorganic compounds is not a universally available one for pharmacologic purposes, because, while most inorganic eompounds act ionically, a number of organic ones also do so. 'Thus, the salicylic ester of quinine is certainly an organic compound, yet it acts ionically, showing the effects of both quinine and salieylic acid. Since, however, a large number of organic compounds do not ionize, the term organic is sometimes used in a loose way to refer to the non-electrolytic organic drugs.

That our knowledge of the laws which govern the relation of chemical structure to biological action is so imperfect is not surprising when we consider, on the one hand, the complexity of the subject, and, on the other, the short space of time which has elapsed since pharmacology became a true science. Even the chemistry of many of the most important vegetable drugs is only a matter of conjecture, and with some not even that. Limited, on one side, by ignorance of the biological laws, and, on the other, by unsolved ehemical problems, it is no discredit to the young science that it has not found the answer to the riddle.

Being as yet uncertain concerning both the fundamental chemical and biological problems involved, our search for guiding principles is, to a large extent, groping in the dark. Here and there we come across, more or less accidentally, certain rules which harmonize a large number of observed facts, and which, therefore, we accept, provisionally, at least, as some of the laws for which we are searching. It would be aside from the purpose of a treatise like the present to attempt to consider in detail the various theories which have been suggested to explain the physiological relations of chemical compounds, since these theories are at the best at least unproved, if not improbable. I shall, therefore, give only those principles which at present seem to be generally applicable, and are of practical importance to clinical modicine.

It is impossible to discuss intelligently the pharmacology of organic drugs unless one is familiar with the terms employed in organic chemistry. As it may be that some of the readers of this article have not followed closely the recent developments in this ficld, I have thought it advisable, 
even at the risk of repeating facts already known, to give a brief résumé of some of the points in organic ehemistry which are especially important for our present purpose. It is, of course, out of the question to give even the barest outline of so vast a subject within the limits of an article of the character of this one, and I shall, therefore, only attempt to refresh the mind of the reader concerning the use of certain terms which are indispensable in the consideration of the relation of chemical structure to physiological action.

'The derivatives of carbon, which constitute the substances generally ineluded under the term of organic chemicals, may be divided into two great classes-the "open-chain" and the "closed-chain" compounds.

The open-chain series is of ten spoken of as the "fatty acid" series, because the fats belong to it, or as the "methane homologues," from the lowest member of the series. The series is built up as follows: Carbon being tetravalent, four atoms of hydrogen are required to satisfy one of (arbon, and the lowest member is $\dot{\mathrm{C}} \mathrm{H}_{4}$ (methane), which may be graphically represented:<smiles>C</smiles>

When two such molecules unite, there is an elision of two hydrogen atoms, the two carbon atoms mutually satisfying one valence of each other, and we have $\mathrm{C}_{2} \mathrm{H}_{5}$ (ethane)-<smiles>CC</smiles>

The series of compounds built up in this manner are known as high as pentatriacontane, $\mathrm{C}_{35} \mathrm{H}_{72}$.

When one of these homologues is oxidized, we have a series of stages or degrees of oxidation proceeding as follows: First, one hydrogen atom is replaced by a hydroxyl (OH) group, forming an alcohol; then an atom of oxygen, which is bivalent, is substituted for the hydroxyl and one hydrogen, and we have an aldehydc; finally, another atom of hydrogen is displaced by a hydroxyl, the resulting substance being an organic acid.<smiles>C</smiles>

Methane.<smiles>CO</smiles>

Methyl alcohol.<smiles>C=O</smiles>

Formaldehyde.<smiles>O=CO</smiles>

Formic acid.

In the higher members of the series more than one of the hydrogen atoms may be replaced by hydroxyl, forming what is known as a polyatomic alcohol. 'The most familiar of this class of compounds is glycerin. Derived from the polyatomie alcohols are the sugars: 
<smiles>OCC(O)CO</smiles><smiles>O=CC(O)C(O)(I)C(O)CO</smiles>

An ether is an oxide of a hydrocarbon in which two radicals, cach yielding up an atom of hydrogen, are bound together by the oxygen. 'Thus we have ethyl ether-<smiles>CCOC</smiles>

the anesthetic commonly known simply as "ether."

One or more of the hydrogen atoms may be substituted by various other elements or radicals, as in ethyl chloride $\left(\mathrm{C}_{2} \mathrm{H}_{5} \mathrm{Cl}\right)$, iodoform $\left(\mathrm{CHI}_{3}\right)$, etc. When the radical introduced is an acid, organic or inorganic, the compound is an ester, as, for instance, the salicylic ester of methyl or amyl nitrite $\left(\mathrm{C}_{5} \mathrm{H}_{11} \mathrm{NO}_{2}\right)$. The esters act in the bodly like salts, that is, they are broken up, liberating the acid, which acts ionically.

Of the closed chain compounds, the most important, from a medical standpoint, are those built around the benzene ring, sometimes known as the aromatic hydrocarbons. 'The benzene ring consists of six atoms of carbon, each of which has three valences satisfied by neighboring carbon atoms and one by a hydrogen. The structure of this ring is usually represented by a hexagonal figure, the number of lines between the carbon atoms signifying the number of valences. For convenience, the ring is sometimes indicated merely by the hexagon, the letters signifying the elements being omitted.<smiles>c1ccccc1</smiles>

Benzene.

Any or all of the hydrogen atoms in this ring may he replaced by another element or radical, in which may be built up compounds of extriordinary complexity. If one $\mathrm{H}$ is replaced by an $\mathrm{OH}$, there is formed a phenol. When an alcohol of the open-chain series is introduced into the benzene ring, the resultant compound is capable of undergoing a series of oxidative changes similar to those of the alcohol itself. Thus we have:<smiles>OCc1ccccc1</smiles>

Benzyl alcohol.<smiles>O=C(O)c1ccccc1</smiles>

Benzaldehyde.<smiles>O=C(O)c1ccccc1</smiles>

Benzoic acid. 
If a carbon atom from the benzene ring is displaced by nitrogen, we have a body known as pyridine. If a similar displacement occurs in naphthalene - which may be regarded as two benzene rings fused together-the resultant substance is called quinoline. 'These two bodies, pyridine and quinoline, are of great interest from the fact that nearly all the alkaloids are derivatives of one or the other of them:<smiles>c1ccncc1</smiles>

Pyridine.<smiles>c1ccc2ncccc2c1</smiles>

Quinoline.

Another series of compounds of pharmacologic interest may be regarded as derivatives of ammonia, in which one or more atoms of hydrogen have been replaced by an organic radical of either the open-chain or closed-chain series. If this radical is basic, the compound is known as an amine; when the radical is acid, as an amide. Familiar examples are formamide and aniline:

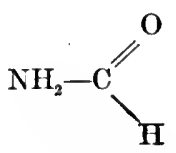

Formamide.<smiles>Nc1ccccc1</smiles>

Phenylamine (aniline).

In studying the relation of the molecular structure of an organic compound to the alterations produced in the functions of the body by it we have to consider the effects, not only of the substance itself, but of all the various derivatives which may be formed within the body as the result of the vital chemical activities. Compounds which are inert of themselves may, as the result of being split up in the system, become highly toxic, and, on the other hand, many substances which are poisonous to protoplasm become oxidized, or otherwise changed, in the body to innocuous substances.

(1) 'The action of an organic radical, no matter with what it is combined, is always essentially the same. We may increase or diminish its potency by so eombining it that the new compound penetrates more rapidly or less rapidly the cell protoplasm, but we cannot change the type of the action of a radieal without destroying its chemical identity. 'This law is open to apparent, but not real, exceptions, as noted under (3).

(2) Almost as a corollary of this law is one of great clinical importance, that is, the introduction of inert radicals into the molecule will not alter the kind, although it may modify the degree, of action.

It must be noted, however, that at times the addition to the molecule of a radical, practically impotent in itself, may bring out some latent 
property not previously manifested by the original nucleoss. In these instances it is probable that the inert radical acts as an "anchoring" gromp, analogous to the toxophore of Ehrlich's side-chain therory. As an instance of this may be mentioned the local ancsthetics. Cixane is methyl-benzoyl-ecgonine. 'The ecgonine is evidently not the active radical, we can substitute for it a simple amide, as in the syuthetic known as orthoform which is an amido-oxybenzoic methyl ester $\left(\mathrm{C}_{6} \mathrm{H}_{3} \mathrm{COO}\left(\mathrm{CH}_{3}-\right.\right.$ $\mathrm{NH}_{2} \mathrm{OH}$ ). Nor is the effect on the sensory nerves due to the methyl group, for it can be substituted by ethyl, as in anesthesin $\left(\mathrm{C}_{6} \mathrm{H}_{4} \mathrm{CO}\right) \mathrm{CC}_{2}^{\circ}$ $\mathrm{H}_{5} \mathrm{NH}_{2}$ ). It would seem, therefore, that the active nucleus in these compounds is the amido-benzoic acid, but that the ethyl or methyl radical is necessary to permit union with the peripheral nerve-endings.

(3) Where two radicals of dissimilar action are joined together, the effect of the resultant compound will be the sum of the actions of the two radicals. In this way we may overcome the unpleasant by-effects of certain substances by introducing into the molecule radicals which oppose the undesired action. For instance, the depressant influence upon the circulation of the ethyl radical in the narcotics may be overcome by the addition of a stimulating ammonia derivative, hence the relative safety of ethyl carbamate as a hypnotic.

This rule does not contradict, as at first thought it might seem, Rule 1. In the example quoted, the depressant tendency remains; it is merely antagonized by a simultaneous stimulating effect

(4) Of two compounds whose activity depends upon their decomposition with the liberation of the same nucleus, the one which decomposes more easily, that is, liberates the active nucleus more rapidly, will, generally speaking, be the more toxic, and is also usually the more efficacious. 'This is well illustrated by the coal-tar antipyretics. Acetanilid and acetphenetidin both owe their activity to the formation within the body of paramidophenol, but as the oxidation processes which produce this substance occur more slowly with the phenetidin group, acetanilid is more toxic than acetphenetidin:<smiles>Nc1ccc(O)cc1</smiles>

Paramidophenol.

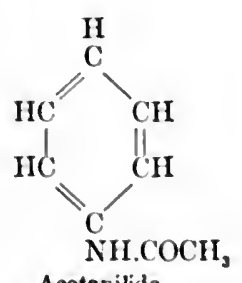

Acetanilide.

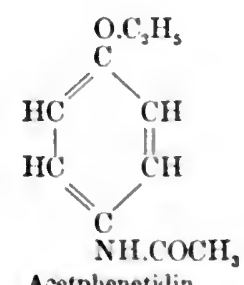

Asetphenetidin.

(5) Dervatives of the fatty (open-chain) series, as far as they are absorbable, all seem to exercise a narcotic action, except when in the condition of organic acids, in which case they act ionically and are relatively inert. There are some exceptions to this rule in the case of certain aldehydes which are rapidly oxidized in the boly into acids.

The narcotic power of the fatty series increases as we ascend the series; that is, as the molecule grows larger. For instance, ethyl alcohol 
is more narcotic than methyl alcohol and propyl alcohol is more narcotic than ethyl. 'Thus, we may arrange the alcohols in ascending scale of activity as follows: methyl alcohol $\left(\mathrm{CH}_{3} \mathrm{OH}\right)$, ethyl $\left(\mathrm{C}_{2} \mathrm{H}_{5} \mathrm{OH}\right)$, propyl $\left(\mathrm{C}_{3} \mathrm{H}_{7} \mathrm{OH}\right)$, butyl $\left(\mathrm{C}_{4} \mathrm{H}_{9} \mathrm{OH}\right)$, etc. 'This law holds true as long as the compounds are absorbable, but, as we ascend higher into the series, the physical character of the derivatives gradually change, and they are inert, as regards the system, because they cannot be taken up by the blood.

'The presence of more than one hydroxyl in the molecule tends to weaken or destroy the narcotic power, so that polyatomic alcohols, as glycerin, $\mathrm{C}_{3} \mathrm{H}_{5}(\mathrm{OH})_{3}$, and their derivatives, the carbohydrates, are relatively inert.

(6) Ammonia derivatives are stimulating in their effects. The alkaloids being ammonia derivatives are, therefore, generally speaking, excitants to nerve centres. The apparent exceptions to this generalization as concerning alkaloids can mostly be explained on one of two grounds. First, they may have some peripheral effect which hides the central stimulation; thus paralysis of the peripheral motor nerves, produced by curare, entirely hides the strychnin-like stimulation of the spinal cord. In other instances, the stimulant effect of the ammonia may be overwhelmed by the depressant power of some other radical. 'Thus urethane, $\mathrm{CO}\left(\mathrm{OC}_{2} \mathrm{H}_{5}\right) \mathrm{NH}_{2}$ is depressant rather than excitant, because the ethyl overcomes the action of the amide radical. Nevertheless, as pointed out above, this compound is less depressant than others of the series because of the presence of the $\mathrm{NH}_{2}$ group.

\section{CIRCUMSTANCES MODIFYING THE EFFECTS OF DRUGS}

The conditions produced by disease which call for the use of dynamically acting drugs, that is, those agents which directly influence the physiological processes of the body, may be divided into three groups: Conditions in which an organ is-(1) overactive or underactive, owing to the presence of some poison in the system; (2) exhausted from too great strain; (3) where there has been a functional destruction of part of the organ, so that an abnormal amount of labor is thrown upon the remaining tissue, and (4) where, owing to changes in other parts of the body, it becomes necessary to excite a healthy structure to abnormal activity.

When two poisons which act upon the same tissue in opposite ways are introduced into the blood, they tend to more or less neutralize the effects of each other; the lowering of funetion, which is brought about by a depressant poison, may be overcome by the administration of a stimulant, and vice versa, the effects of an exciting poison may be abolished by the use of a sedative. 'This antagonism, however, remains true only within certain limits, because, if given in sufficient dose, the depressant poison will always overpower the stimulant. Thus, we can always rclax the convulsions in a case of tetanus, at least temporarily, with spinal depressants, but we cannot overcome in all instances the depressant effects of a pneumotoxin upon the vasomotor system. In like manner a periph- 
erally acting drug will always overpower a centrally acting one; it is evident that, no matter how much we may stimulate the spinal cord, if the motor nerves are paralyzed, we can get no response in the muscles. However, when the peripherally acting drug is not present in too large amounts, the nerve centres may still exercise at least partial control over the function of an organ. For instance, if we give a dose of atropine sufficient to lower the conductivity, but not completely paralyze the pueumogastric nerve, irritation of the nerve by mild electrical currents may have no effect upon the pulse-rate, but we may still slow the heart by increasing the strength of the stimulus.

A sharp distinction must be made between a depression of function, brought about by the restraining influences of a poison, and the exhaustion of an organ the result of overwork. In the former instance, the rational treatment is to give an exciting drug which shall antagonize the depressant poison. But when an organ is really exhausted, while stimulants may, perhaps, temporarily increase functional activity, they do so at the expense of the reserve power of the organ; to employ stimulation under these circumstances is to aggravate the disease. Only two agents can restore power to an exhausted organ-rest and food.

There are some circumstances, however, in which the exciting drugs may be indicated in conditions of exhaustion. We cannot stop the heart and allow it to rest, because the activity of the whole body depends upon a constant supply of oxygen and food, and if the kidneys excrete not at all, there soon accumulates in the system a sufficient amount of poisonous metabolites to stop the living machine. It may be necessary, therefore, to spur the exhausted heart or the degenerated kidney with a stimulant poison, in the hope that something will happen to turn the tide of the disease if we can only keep the patient alive.

Where there has been an anatomical destruction of the tissue, and the amount of functionally capable substance remaining in an organ is diminished, the indications for treatment are very similar to those in states of exhaustion, with this difference, however, that there is more chance for a compensatory hypertrophy of the remaining healthy tissue if we maintain life by gentle stimulation.

In conditions of exhaustion or of structural diminution much may frequently be done to relieve the diseased organ by increasing the activity of a vicarious one. Thus we guard against the exhaustion of kidney function in cases of nephritis by increasing excretion through the bowels and skin. A modern example of this is found in the use of the hacterial vaccines; the bodies of dead bacteria are injected into the svistem for the purpose of stimulating the cells of the organism to an abnormal production of the antitoxic substances.

From what has been said above, it is manifest that, in the presence of antagonistic poisons produced by disease, larger doses of pharmacal agents will be born than in health. The converse is equally true, that the presence of synergists heightens the susceptibility of the tissues to drugs. Hence the folly of using, as I have seen suggested, strychnine as a vasomotor stimulant in tetanus; the tetanotoxin has made the spinal cord 
so excitable that the drugs which act on nerve centres will first show their effects in increased convulsiens.

Organs which are irritated or inflamed, even if not physiologically excited, respond more delicately to stimulant remedies. Stimulation is, after all, closely akin to irritation, and doses, which to normal organs are stimulant, may prove violently irritant to inflamed ones; in acute nephritis a stimulating diuretic may check instead of increase secretion.

Other things being equal, the more concentrated the solution of a drug in the blood, the nore violent will be its effect. Now, as in human medicine we rarely inject drugs directly into the blood-stream, but introduce them into various structures from which they are absorbed more or less rapidly, and as the system begins to eliminate poison from the blood a!most the instant it enters it, it is evident that the amount which is in the blood-stream at any one moment will depend not only upon the size of the dose which has been placed in the stomach, but upon the rapidity of absorption, as compared to that of elimination. As the rate of absorption differs widely according to the methods of administration, it is evident that, when we come to consider those factors which influence dosage, we have to have regard for three conditions-the amount of blood in the patient, the size of the dose, and the channel of administration.

The amount of blood in any species of animal bears, under ordinary circumstances, a more or less constant relation to the body-weight, and the only way we can guess at the amount of blood in a given individual is from his weight. But while the physician should, in considering a question of dosage, differentiate between a patient markedly under size and one greatly over the average, the fact that most very heavy persons owe their weight to an excess of adipose prevents the dose being scaled accurately to size. Fatty tissues are poorly supplied with blood, and, therefore, corpulent persons require in proportion to their weight relatively smaller doses than thin persons. Speaking generally, women are smaller than men, and, therefore, should be ordered smaller amounts of drug, but the greatest variation in size is found in children at different ages. The difference in dosage required at different periods of life depends, however, not only upon variations in size, but also upon the unequaled rate of development of the vegetative and animal functions. 'Ihus, we find that immature individuals demand relatively' smaller doses of those drugs which act upon the central nervous system, and relatively larger doses of those which act upon the intestinal tract, than they should aceording to a ealeulation based strietly upon weight relations. Nevertheless, the various methods for calculating doses for children, Young's and Cowling's rule, are merely means of estimating the weights of children. In passing, I may express a preference for Cowling's rule, as being not only more simple, but also more accurate, than Young's. According to Cowling's rule, the dose for a child is determined by making a fraction, of which the age of the child at the next birthday is taken as the numerator and the figure 24 as the denommator; thus, a child in his sixth year would require $\frac{f^{n}}{24}$, or $\frac{1}{4}$ of the adult dose. 
Unless there is some reason to do otherwise, drugs should always be given by the mouth. Nature, intending the stomach and small intestines to be used as absorbing surfaces, has made ample provision for lissolving and alsorbing in this portion of the body. "The circumstances under which we may prefer other channels of administration are: (1) when we waint great promptness of action; (2) where we fear the result of local action upon the mucous membrane of the upper alimentary tract; (3) when we are using a drug likely to have its therapeutic value destroyed by the chemical aetion of the digestive juices; and ( $t)$ when the pattent cannot, or will not, swallow.

In the first of these conditions, that is, where quickness is a great desideratum, we have recourse to the hypodermic injection or to inhalation, or, more rarely, to intravenous injection. 'The last two methorls of administering remedies are so restricted by absolute circumstanees that they are to be thought of only under special conditions-for instance, the use of the pulmonary tissue for the absorption of volatile anesthetics. Where time is not a matter of importance, but it is necessary to avoid the possibility of irritating the stomach, there are two modes of administration-the endermic and the rectal. Concerning the latter, there is a popular superstition that drugs administered by the rectum have a more marked action upon the urinary organs than if given by the month. For this belief there is not the slightest support. Outside of the slowness and uncertainty of their absorption, and the possible local effect upon the mucous membrane of the lower bowel, the action of drugs, when administered in the form of suppositories, is exactly the same as when given by the mouth, and, when we are employing such centrally acting remedies as opium, I can conceive of no just reason for preferring this form of administration. Because absorption is so much slower through the reetum or through the skin, it requires approximately about double the dose to produce the same degree of effect as when the remedy is administered by the mouth.

The ehief channels through which drugs eseape from the system are the kidneys and the bowels. Besides these, however, certain ilrugs are eliminated, at least in part, through the lungs, the skin, or various glands of the body. The rate of elimination varies greatly with individual subrstances. In a broad general way the same factors which increase the rapidity of absorption also render escape comparatively prompt. There are, however, several important exceptions to this rule; for instance, the inorganic bromides are alsorbed comparatively readily but eliminated slowly, and, therefore, tend, if given in repeated dose, to accumulate in the system.

Sulstances which in relatively coneentrated solution are locally irritant, and prone, therefore, to give rise to digestive disturhances whein exhibited by the mouth, nlso show a tendeney to irritate at the point from which they escape from the system. 'This is illustrated in the effect of such substanees as eantharides and turpentine upon the kidneys, and in the violent purging eaused by such poisons as arsenic, the diarrhoa 
being due to the irritant action of the drug upon the intestinal glands during the process of elimination.

A number of drugs, especially those of the organic kingdom, are more or less completely destroyed during their sojourn in the system. Thus it has been determined that approximately 75 per cent. of morphine is oxidized in the body to a comparatively harmless substance known as oxymorphine; alcohol is so completely burnt up in the body that it escapes almost entirely in the form of water and carbonic acid. These chemical changes are carried on largely in the liver, and we find, therefore, that some irritant poisons are likely to produce inflammation of this organ. 'The toxic power of other agents is destroyed, through combinations with various metabolites, and form comparatively inert substances. In this manner phenol is changed into a non-toxic phenolsulphonic acid, and a number of substances unite with glycuronic acid.

In many instances the therapeutic activity of a drug depends upon the decomposition taking place in some portion of the system. Common examples of this are salol, which is broken up in the intestinal tract into phenol and salicylic acid, acetphenetidin, oxidized in the body to paramidophenol, and hexamethylenamin, decomposed after it has passed through the kidney, liberating formaldehyd.

\section{RELATION OF PHYSIOLOGICAL ACTION TO THERAPEUTIC USES}

In studying the changes in function produced by drugs upon the system, whereby they become of service in the treatment of disease, much advantage is derived from considering them in groups. Our knowledge of remedial agents has now advanced sufficiently far to permit of a pharmacological elassitication with a comparative degree of accuracy. Such a grouping is, however, of little value for the purpose of the clinical therapeutist, for it necessitates the association of substances of such widely varying therapeutic properties as nicotine, physostigmine, and adrenaline, and the separation of such therapeutic kin as the diuretic caffeine and potassium salts, or the analgesic morphine and acetphenetidin, or the diaphoretic nitre and pilocarpine.

For the purposes of such a treatise as the present I believe a classification of remedies, acording to their clinical uses, is more serviceable. I shall, therefore, divide the drugs which I wish to discuss in the following groups:

Nervines.-Drugs used to promote sleep; drugs used to relieve pain; drugs used to check convulsions.

Cardiants.-Drugs used to slow the pulse; drugs used to elevate bloodpressure; drugs used to lower blood-pressure.

Eliminants.-Drugs used to inerease urine; drugs used to cause catharsis; drugs used to increase the sweat.

Pectorants.-Drugs used to excite the respiratory centre; drugs used to soothe the respiratory centre; drugs used to affect bronchial mucous membrane. 
Somnifacients. - As stated above, the derivatives of the methanc homologues are narcotic. 'The rapidity of their action is largely governed by their physical condition. 'Those which are very volatile at ordinary' temperatures, such as ethyl oxide (ether), act so rapidly that they are useful only where profound narcosis of short duration is desired, as in the production of surgical anesthesia. For the purpose of producing ordinary sleep, the fugaciousness of their effects renders them unsuitable. In like manner, among those which are used as somnifacients, the promptness of their effects is still determined by their physical rather than their chemical constitution. 'Thus chloral hydrate, slightly volatile and extremely soluble in water, is very prompt in its action, but not at all permanent; urethane is somewhat less soluble and slower; and trional, sparingly soluble in water, is very slow, but persistent in its effects.

Of this series of narcotics, those which have proved themselves clinically useful may be divided into the following group: (1) the simple derivatives; (2) those containing a halogen; (3) the carbamic derivatives; (4) the sulphone derivatives.

The chemical relations of the narcotics can be readily seen by referring to their structural formula:<smiles>CC=O</smiles>

Aldeliyde.<smiles>[14CH3]C(N)=O</smiles><smiles>O=CC(Cl)(Cl)Cl</smiles><smiles>CCO[Se]C(C)(C)OCC</smiles><smiles>CC(O)C(Cl)(Cl)Cl</smiles><smiles>CCCC(C)(OCC)C(=O)OCC</smiles>

Of the first group, the only one which has been used to any considerable extent in the treatment of "insomnia is the polymer of acetaldehyde, known as paraldehyde. This is a liquid, soluble in eight parts or water, and miscible with alcohol and fixed oils in all proportions. Its action is rapid, becoming manifest generally in fifteen to twenty minutes after its administration, and lasting from one to two hours. It is but feebly depressant to the circulation, or to the lower nerve centres in the medulla and spinal cord, and is, therefore, comparatively safe.

'The introduction of a halogen, especially of chloride, into a molecule of an ethyl, or homologous derivative greatly increases the depressant action upon the brain, so that these drugs are more powerful as hypnotics, but, at the same time, it also decreases the depressint action upon the circulation and the vital centres, so that they are likewise more dangerous. 'This is illustrated in the case of chloroform, which is about eight times as powerful as ether, but is also more depressant to the circulation. Of this group I shall mention two-chloral hydrate and isopral. 'The former, which chemically is the hydrate of ethyl trichloraldehyde $\left(\mathrm{CHCl}_{3} \mathrm{CO}_{7}\right)$, is very freely soluble in water and alcohol. 
and acts with great promptness, but with little persistency. Isopral differs from chloral essentially in the substitution of a propyl for an ethyl radical. As pointed out previously, the propyl alcohols, having a larger molecule, are more active than the ethyl derivatives, and clinical experience has shown this deduction to hold true in this instance. But while isopral is more powerfully narcotic than chloral, the experiments of Sollmann and of Hatcher indicate that it is also more powerfully depressant to the vital centres, so that the relative safety of isopral is no greater than of the older remedy.

Various efforts have been made to overcome the depressant action of chloral by combining it with other radicals. Of the productions of these efforts only one deserves mention, and that is chloralformamide. The a mide radical in this substance has, at least in part, overcome the depressant action of the chlorine, but, as chloralformamide is broken up in the system into its two component constituents gradually, the substance, while less toxic than chloral hydrate, is also less efficient as a hypnotic. (See Rule 4, p. 27.)

By combining with the depressant ethyl the stimulating ammonia derivative, amide compounds have been obtained which are as nearly absolutely safe as one could readily hope. 'The series of carbamates, or urea derivatives, include a large number of the more recent sleep-producing drugs which have been brought forward by synthetic chemists. Of this group, one is official, the ethyl carbamate, commonly known as urethane. Urethane occurs as a crystalline solid, freely soluble in both water and alcohol. It is relatively prompt in its action, and, although generally free from dangerous by-effects, it is somewhat uncertain in its hypnotic effects in the more obstinate cases of insomnia. Another member of this group which has been widely employed is veronal (diethyl malonylurea). As this contains two ethyl radicals instead of one, as in urethane, it is more actively hypnotic, but we should expect it also to be more depressant. Although scientific data as to its relative safety is, as far as my reading goes, lacking, the general clinical evidence, I think, confirms this conclusion. The sodium salt of this compound has also been introduced under the name of medinal, but has not achieved great popularity. As this group of lypnotics are eliminated from the system as urea, they frequently give rise to diuresis, and may prove irritant to inflamed kidneys.

Of the sulphone group, only two are employed to any extent in this country. 'These are sulphonal (diethylsulphonedimethylmethane) and trional (diethylsulphonemethylethylmethane). Both of these drugs are very sparingly soluble, and, therefore, act with great slowness, but much persistency. When used over long periods of time, they tend to accumulate in the system, and their halitual employment has in many cases led to serious and fatal intoxication. As trional is less insoluble than sulphonal, its action is somewhat more powerful, and it is more readily eliminated, and, therefore, less likely to give rise to chronic intoxication. For these reasons, sulphonal is to-day comparatively infrequently prescribed. 
All the narcotic drugs, if given in sufficient dose, destroy, at least in part, the sense of pain, but their action upon the intellectual centres, by virtue of which they produce sleep, occurs before the anodyne effect, and, in order to destroy the perception of pain, comparatively enormous doses are required-quantities large enough to cause a condition of coma. In the case of the volatile members of this series, on account of the rapidity of elimination, this degree of narcosis can be produced with comparative safety, so that such drugs as ether, chloroform, and ethyl chloride are used largely for the purpose of producing surgical anesthesia. But with those agents which are eliminated more slowly it is impossible to accurately control the depth of nareosis, so that, as pain-relieving drugs, such sul,stances as paraldehyde and the other somnifacients are almost useless. When given in small dose, their effect upon pain is practically nil, and in those conditions of sleeplessness which are due to suffering the narcotics of the open-chain series are of little value. In these cases we should aim rather to alleviate the pain than to depress the intellectual centres in the brain, and the hypnotic for such conditions is to be looked for among the analgesies rather than the true somnifacients.

Among those remedies which are employed in insomnia due to nervous excitement or exhaustion three factors influence our choice. 'These are the rapidity of action, the certainty of effect, and the relative safety of the drug.

Two general types of insomnia may be distinguished-one in which the patient is restless and wakeful from the moment of retiring, but, if once asleep, shows little tendency to awake cluring the night, and the second, in which the patient may drop to sleep naturally, but, even if put to sleep by drugs, wakes in a few hours, or as soon as the drug has been eliminated from the system. It is evident that these two cases demand hypnotics of very different properties. In the first instance we require a remedy which shall act powerfully and promptly, but not necessarily persistently. In the second case we need a drug whose action is well sustained, that is, one which is slowly eliminated from the sistem; whether its effects appear tardily or promptly is a matter of indifference, because this ean be overcome by regulating the time at which the sleeping potion is administered. The same factors which make for the quickness with which the effect comes on, influence also the quickness with which it disappears, so that the promptly acting hypuotics are fugacious in proportion to their promptness. On this basis, the narcotic drugs may be divided into three groups - the rapidly acting, those of moderate rapidity, and the slow, persistent ones. In the first class fall especially chloral hydrate and paraldehyde; in the second group come the carbamic derivatives, urethane and veronal; and in the third, the ethyl-sulphonic group, of which, as we have mentioned above, sulphonal is the slower, but trional the preferable. 'The action of sulphonal, indeed, is so persistent that the patient frequently complains of drowsiness the next morning.

In comparing the power or certainty of effect of the somnifacient, we consider the probability of sleep being produced by the drug when 
given in doses which are not large enough to produe serious effects on the vital organs. Any one of these remedies is almost certain to produce sleep if given in large enough quantity, but it is not permissible to push them to this extreme, for while enormous doses of various members of this group of remedies have been taken on occasions with no permanent ill effects, nevertheless, when given in such quantities, they are likely at times to prove unexpectedly toxic. 'The potency of a somnifacient remedy is most readily gauged in the more obstinate types of insomnia, such as those which occur in the course of some forms of insanity. The chlorine derivatives, chloral hydrate and isopral, separate themselves from the rest of the somnifacients as being by far the most reliable of the group. As mentioned above, however, their power is gained at the expense of safety. Of the two, the border-line between therapeutic activity and danger is a little broader in the case of chloral hydrate, so that, where a very powerful hypnotic action is wanted, preference should be given to this drug. The other members of this series may be arranged about in the following order, as regards the certainty of their effect-paraldehyde, trional, chloralformamide, veronal, and urethane.

The relative safety of the hypnotics is to be judged from two standpoints-the likelihood of the remedy acting treacherously and the probability of dangerous symptoms from an overdose. It is characteristic of the chlorine derivatives of the methane homologues that at times, for some unknown reason, they aet with unforeseeable violence, and dangerous depression may follow from even relatively small doses. This is seen in the case of the sudden deaths which have occurred from the inhalation of but one or two whiffs of chloroform, and from the occasional instances of serious cardiac depression which have been reported from chloral hydrate. For this reason it is rarely advisable to give more than 15 to 20 grains of chloral hydrate at a dose. Although, in ordinary quantities, neither paraldehyde nor veronal is likely to give unhappy results, the claim of being the safest hypnotic probably rests between ethyl carbamate and trional.

A word may be said concerning the administration of these agents. All those which are soluble in water, including chloral hydrate, chloralformamide, paraldehyde, and ethyl carbamate, are preferably administered dissolved in aromatic water, in doses ranging around 15 grains, except paraldehyde, which is generally given in from 30 to 60 minims. The insoluble remedies, and especially the sulphone derivatives, should not be exhibited in the form of pills or tablets. 'The best means of administering sulphonal or trional is to stir them up in a glass of hot milk or hot water, and administer one-half to one hour before the time of desired effect.

Drugs Used to Relieve Pain.-An ordinary pain sensation begins with an irritation of the peripheral ending of an afferent nerve, passes up the nerve-trunk to the sensory traets of the spinal cord, and through this is conveyed to the perceptive centres of the brain. It is evident that we may abolish pain by acting upon any one of these three tracts; that is, upon the peripheral nerve, upon the spinal cord, or upon the brain, and 
the analgesic drugs may, therefore, be classed into three corresponding divisions.

Peripheral Anodynes. - The local anestheties, those which paralyze the peripheral endings of the sensory nerves, mostly belong either to what we may call the coeaine series or to the phenols. Besides these, however, we have a few other substances which are oecasionally serviceable. The most active of the group are those which are allied to cocaine.

The cocaine series may be defined as derivatives of amido-benzoic acid, containing a methyl or an ethyl side group. Cocaine itself is a methylbenzoyl-cegonine. Its analgesic properties are due to the methyl-benzoyl nucleus, while many of its systemic effects are to be attributed to the ecgonine. By substituting for the exgonine various other ammonia derivatives, substances may be prepared possessed of the local anesthetic action of cocaine, but free from its systemic effects; this is the basis of the numerous synthetic alkaloids which have been recently brought forward as substitutes for cocaine. 'The most important of these are cucaine, novocaine, orthoform, and stovaine. All of these substances differ from cocaine physiologically, in that they do not produce the violent spasm of the arterioles which follows the local application of the natural alkaloid, nor do they dilate the pupil nor stimulate the higher nerve centres when absorbed. While all the substitutes mentioned are less toxic than cocaine, eucaine and novocaine are the least poisonous of the whole series. None of them, however, appears to equal cocaine in local anesthesic power, so that they must be used in slightly more concentrated solution, but even making allowances for the relative potency, novocaine and eucaine are only about one-quarter as dangerous as cocaine.

In order to act upon the nerve-endings, a drug must come into contaet with them, but the skin offers a practically impenetrable barrier to the passage of the cocaine group, and, therefore, applied over the skin surfaces they have almost no effect. On the other hand, mucous membranes offer comparatively little resistance to penetration. 'The ease with which the cocaine group pass through mueous membranes-that is, their degree of power on these surfaces-depends upon the thickness of the mucous membrane and the mode of application. 'The effect of local anestheties is more marked when applied in watery solution than in the form of an ointment.

Orthoform, probably on account of its slight solubility. penetrates even unucous membranes with difficulty, and finds its chief field of usefulness as a dusting-powder to raw surfices, as in open uleers or laryngeal tuberculosis.

The phenol series of local anesthetics include a large number of compounds, among which may be mentioned earbolic acid, menthol, and certain of the volatile oils, especially oil of cloves. 'They are, in distinction from the cocaine series, capable of passing through unbroken skin, and eanse, therefore, a certain degree of anesthesia when applied externally. It is impossible, however, to produce the same completeness of anesthesia with these remedies, beciuse, if applied in sufliciently concentrated solu- 
tion, they become very irritant. They are, therefore, used chiefly to partially benumb sensation in various irritative conditions of the skin.

Among the miscellaneous substances which are occasionally useful as local anesthetics may be mentioned aconite, antipyrine, atropine, and iodoform. Aconite is a very powerful paralyzant of sensory nerveendings, but is so poisonous that its use is rarely justifiable. Atropine is comparatively feeble in its effects upon peripheral nerves, but is occasionally employed in the form of an ointment or a powder, especially in painful spasm, in which case its action upon the motor nerves, as well as those of sensation, makes it of service. Iodoform has a very considerable degree of local ancsthetic effect, but its strong odor interferes with its employment in a large number of conditions in which it might otherwise be useful. In cases of painful hemorrhoids, however, a suppository of 5 grains of iodoform will often give more relief than any other drug. Antipyrine, in its local effects, is very similar to cocaine, although distinctly inferior in power.

Spinal Analgesics.-Practically the only drugs which are clinically useful for their action upon the sensory cord are the bromides. In man, however, the effect of the bromides upon the brain is so powerful that we are rarely justified in giving large enough doses to greatly depress the spinal cord. For this reason the bromides by themselves are of little service in the treatment of painful conditions. They appear in some way to greatly enhance the analgesic properties of those drugs which act chiefly upon the brain.

Central Anodynes.-'The cerebrally acting anodynes include morphine, closely allicd alkaloids, and the so-called coal-tar derivatives.

The effects of morphine upon the lower animals may be briefly summarized as being a depression of the higher cerebral centres, depression of the respiratory centres, stimulation of motor tracts of the spinal cord, and certain effects upon peripheral organs, as the intestines, which are, for the present purpose, of little interest. When given in fatal dose to any of the higher mammals, death is caused by an arrest of respiration. The three important alkaloids of this group-morphine, codeine, and heroine- possess all the properties mentioned, but in varying degree. Morphine is relatively much more powerful in its action upon the brain than any of the other opiates, and, while both codeine and heroine possess a certain degree of anodyne property, in doses which are justifiable for human beings they are vastly inferior to morphine. The chief value of the last-named alkaloids is their effect upon the respiratory centre. ${ }^{1}$

The scientific study of the effects of drugs upon the higher cerebral functions, such as the perception of pain, is, for obvious reasons, almost impossible, and consequently we have no very accurate conception of the mode of action of this group of remedies, and, although we believe that the pain-relieving powers of both morphine and acetanilide are due to their action upon the brain, clinical experience has shown that their effects are not the same. While morphine will relieve any form of pain,

1 The effects of this group must not be confounded with the anesthesia produced by injecting cocaine, or one of its congeners, into the spinal canal. 
exeept that which is due to diseases of the hrain or its membranes, the coal-tar derivatives are valuable only when the seat of the pain is within the nervous system itself. 'Thus, for instance, the pain of a broken leg will be relieved by morphine, but not by antipyrine, but the prain of a neuralgia, or even of locomotor ataxia, yiclds at least partially to both remedies. 'The coal-tar analgesics a re of great value in practical medicine because in many cases, by their use, we can avoid the danger of the morphine habit and the disturbances of digestion which are produced by this drug. Moreover, even in those cases where morphine is imperative, by combining the coal-tar with an opiate, we may be able to alleviate the suffering with smaller doses of morphine, and to postpone the time when the patient shall have heeome habituated to the use of the drug so that no further analgesic effect can be derived.

The number of these coal-tar anodynes is legion, and there has been an extraordinary amount of exaggeration of statement concerning the qualities, both desirable and undesirable, of the whole group and of each individual member. By venders of nostrums they are lauded as veritable gifts from heaven, but by certain clinicians they have been uniformly condemned as devices of the evil one. 'That many cases of serious and even fatal collapse have been caused by single doses of these substances and that many a heart muscle has been weakened by their repeated use seem hardly open to doubt. But the evil effect from prolonged selfmedication which has resulted from these remedies is hardly a cogent argument against their employment as anodynes under professional supervision. The cases of collapse which have followed the ingestion of a single dose have nearly all occurred under one of two circumstances either enormously large quantities have been administered or they have been used as antipyretics.

The superstition that the coal-tar derivatives are depressant to the heart has not been experimentally confirmed. 'The cardiac failure, which has occurred following their administration in febrile conditions, is probably secondary to the sudden reduction of temperature. It is a well-known fact that a moderate degree of heat acts upon the heart as a stimulant, and it is readily conceivable how a heart which has been accustomed for days to working at a temperature of $103^{\circ}$ or $104^{\circ} \mathrm{F}$., if suddenly bathed with a blood at a temperature of $97^{\circ} \mathrm{F}$. , or perhaps even $96^{\circ} \mathrm{F}$., is greatly depressed by the withdrawal of heat. When given to healthy animals, enormous quantities can be administered without perceptible alteration in the circulatory system. 'Thus, I have injected into the vein of a $\operatorname{dog} 0.5 \mathrm{gram}$ per kilo of acetphenetidin (which would be equivalent to a dose of about one ounce for an ordinary man), with no distinct effect upon the circulation. 'The cases of chronic heart weakness which have followed the prolonged use of these drugs in all probability depend upon secondary degenerations in the heart muscle, brought about by the destruction of hemoglobin, which is one of the characteristic toxic effects of this series.

Except antipyrine, all the coal-tar antipyretics which are employed clinically to any considerable extent owe their activity to the liberation 
or paramidophenol in the system. Antipyrine differs from the other coal-tar anodynes not only in its chemical nature, but also in the fact that it is soluble in water, and that it appears to exercise more or less stimulating effect upon the medulla and spinal cord. The toxic effects of the paramidophenol compounds, as pointed out in a previous chapter, appear to vary according to the rapidity with which they liberate the active nucleus in the body. Aniline requires but the introduction of one atom of oxygen to become paramidophenol, and, while it produces the same effects as the elinically useful members of this series, has proved itself to be so toxic as to forbid its employment. In acetanilide, the molecule being more complex, the oxidation takes place more gradually, and there is less danger of undesirable action. In the phenetidin compounds the molecule is still larger, and these drugs are the least likely of any of the series to act dangerously. There is, however, no apparent reason to believe that there is any ground for preference among the various individuals of the phenetidin group. Phenetidin can be combined with any one of a large number of organic acid radicals, but that the citrylphenetidin has any advantage over the acetyl-phenetidin (the official representative of the group) is highly improbable.

One other centrally acting analgesic should be mentioned, and that is cannabis indica. 'This drug is remarkable for the narrowness with which its effects are limited to the brain. Upon any of the vital organs its action is so extremely feeble as to be almost negligible. Theoretically, it ought to be a valuable addition to our armamentarium. Practically, however, the variability in the potency of its preparations and our ignorance of its pharmacy almost annihilate its serviceability.

Drugs Used to Check Convulsions.-Convulsions are of two types, cerebral and spinal. The spinal, or reflex, convulsion is caused by hyperexcitability of either the sensory or motor ganglia of the spinal cord. The impulse for the movement begins with an irritation of a sensory nerve, passes up to the sensory ganglia, thence is transferred to the motor centres of the cord, from which the impulse passes out through the nerve to the muscle. Theoretically, interruption of the reflex arc at any point will prevent the convulsion, but, practically, the drugs which are useful in controlling spinal convulsions act upon either the sensory or motor ganglia of the spinal cord. Drugs which paralyze the peripheral motor nerves, as conium and curare, paralyze, along with the other motor nerves, those which supply the diaphragm and thoracic muscles, preventing respiration, and are, therefore, not available in clinical medicine. As far as the paralyzants of sensory nerves are concerned, the practical fact is that we have none which do not act more powerfully on the vital organs than upon nerves of sensation.

The cerebral convulsion originates in the motor area of the brain, passing downward, thence passes through the motor tracts of the cord, and thence out through the nerve to the muscle. It is evident that this type of convulsion may be checked either at its fountain-head, the psychomotor area, or by acting upon the motor side of the cord.

It is evident that those drugs that affect the motor area in the spinal 
cord are available for the relief of both spinal (tetanic) and cerebral (epileptiform) convulsions, while those acting upon sensation are useful, at least theoretically, only in the reflex convulsions.

In man the effect of the bromine ion is felt first upon the brain, although in the lower animals the spinal cord is more sensitive to its action. 'The effect of a bromide upon the brain is very different from that of the narcotic group, for whereas the latter act preeminently upon the intellectual centres, the bromine affects first the motor area of the hrain, and, only when given in large quantities, the higher cerebral functions. This differentiation is of importance, as it bears strongly upon the clinical use of the bromides as somnifacients. I am convinced that, for the purpose of directly producing sleep, they are very inferior remedies. 'Their value in insomnia depends probably upon their power of benumbing the perceptive apparatus, so that the various disturbances, as noise and physical discomfort, which tend to wakefulness, have less influence.

If given in somewhat larger doses, the bromine ion depresses the sensory side of the spinal cord; whether or not there is an effect upon the perceptive contres in the cerebrum is uncertain. It is only if given in very large or toxic doses that they exercise an influence upon the lower motor neuron.

The therapeutic value of these salts depends entirely on the bromine, but the effects of the basic ion are sufficiently powerful to influence their availability in certain types of eases; and as I shall not have another oceasion to consider the comparative effects of the different earths, they may be described here, in order to point out how far they may modify the action of the bromine.

Potassium weakens the vigor of the cardiac contraction and also lessens the reflex activity; in other words, it is depressant to both the circulation and the central nervous system. 'The potassium bromide, therefore, is the most powerful anticonvulsant of this group, but is also somewhat depressant to the cireulation, and incidentally is the most liable to upset the stomach. Lithium is similar in its effects to potassium, although its action is not so well marked. The ammonium ion is stimulating to the central nervous system and to the circulation. On account of the first action, the ammonium bromide should not be employed when we are using the drug to impress the spinal cord, as the depressant action of the bromine would be more or less neutralized by the ammonium. When, however, we desire a cerebral action, as, for instance, in epilepsy, the ammonium bromide is eminently available, since some of the undesirable effects of the bromide are more or less overcome by the basic ion. 'The metal sodium is, so to speak, neutral; it has neither marked exciting nor marked depressing properties on the circulation or nervous system, and sodium bromide might be desimated as the salt of preference for routine usage. Although both calcium and strontium have a tonic effect upon the heart, and probably also cause constriction of the blood-vessels, yet they are both so poorly alsorber from the intestinal truet that, in the use of these salts of hydrohromic acid, the effect of the kation hardly comes into consideration. 'They 
represent, perhaps, the purest bromine action it is possible to obtain from the inorganic salts with the least disturbance of the stomach.

'The action of the anesthetics, ether and chloroform, involves the whole of the central nervous system, the order in which is it affected being about as follows: first, the brain, then the sensory cord, and then the motor cord. In deep anesthesia all these portions of the nervous system are completely paralyzed. Chloroform is so much more powerful, and so much more prompt, than ether that the latter is rarely used as an antieonvulsant. The remainder of the clinically useful anticonvulsants-namely, chloral hydrate, the nitrites, and physostigmine-all act upon the motor side of the cord.

'The comparative utility of the anticonvulsants depends chiefly upon the rapidity of their action. Chloroform acts very promptly, but its effects disappear almost immediately on its withdrawal, so that, if it is desired to keep the convulsion suppressed with the use of chloroform, it is necessary to continue its administration. It is, however, manifestly impossible to keep the patient under the influence of chloroform any great length of time, and, therefore, chloroform is useful only in very violent convulsions, where life is immediately threatened, as in strychninepoisoning or eclampsia. Still more rapid in its effects than chloroform is amyl nitrite, but, on account of its powerful influence upon the circulation, it can rarely be pushed to the same degree of paralysis that can chloroform. It is, however, a very valuable anticonvulsant in certain types of epilepsy and in strychnine poisoning. The other nitrites, although slower in their action than amyl nitrite, are too fleeting in their effects to be useful as continuous sedatives, and lack the extraordinary promptness of action which makes amyl nitrite especially valuable.

Practically the most useful remedies we have for the relief of convulsions are the bromides and chloral hydrate. In convulsions of the cerebral type, ehloral hydrate will generally act more certainly than the bromides. It must be remembered that in human beings the action of both of these remedies upon the brain occurs earlier than that upon the spinal cord, and that, therefore, larger doses are needed when they are used as anticonvulsants than when they are employed as cerebral sedatives.

Drugs Used to Slow the Pulse.-It may be a new concept to some to regard the slowing of the pulse itself as an end to be strived for, but I believe its correctness is easily demonstrable. In chronic heart disease our object should be, not merely to temporarily increase the work done by the heart, nor to raise the blool-pressure so that certain symptoms may be set aside, but to permanently increase the potential strength of the heart muscle, so that it shall be sufficient for the added labor which is thrown upon it, and a brief study of the physiological conditions of the circulation will, I think, show how the reduction in the pulse-rate must spare carliac energy and increase those anabolic processes which tend toward hypertrophy.

'The changes in the rate at which the heart beats, whether brought on 
by chemical agents or by physiologieal ones, are due almost solely to differences in the length of the diastolic period; for while it is true that certain drugs do prolong also the systole, the amount of time which is consumed in this longer contraction is insignificant in comparison with that which is spent in the lengthened diastole. Now, during the period of cardiac relaxation, the blood is flowing constantly from the great veins into the auricle, and through the auricle into the ventricle-the auricular contraction is but the sharp completion of the diastolic flow which fills the ventricle. It is evident, therefore, that cateris paribus, if the diastole is prolonged, a larger amount of blood will be in the ventricle at the beginning of its contraction, and the pulse volume be correspondingly increased. 'This conclusion has been abundantly confirmed by experimentation, and Elving and Wendt have shown that the increased size of the pulsewaves almost compensate for the reduction in their number, so that a heart which is beating anywhere between 60 and 100 times a minute pumps almost the same quantity of blood at all rates. As the systolic period is the same in both instances, if the heart which contracts but 60 times in a minute drives forward as much blood as if beating one-half again as rapidly, it follows that the slowly acting heart has more time to rest and exerts also much smaller total of muscular power. 'The lost muscular power in the rapidly acting heart is explained by the fact that, if the ventricular cavity is incompletely filled at the beginning of the systole, no useful work can begin until there has been sufficient shortening of the chamber to correspond to the amount of blood it contains.

The slowing of the pulse, however, not only diminishes the catabolic changes which take place during functional activity, but also favors the reconstructive metabolism, for it is a fundamental physiological principle that the reparative processes go on during the time of functional rest, which, for the heart, is during diastole.

Moreover, a large pulse-wave is favorable for a better filling of the coronary artery, and hence tends to better nutrition of the heart inuscle. Finally, as clinical evidence of the correctness of my logic, I may call attention to the fact, which has caused much surprise to some clinicians, that even in cases of heart disease, with abnormally high blood-pressure, digitalis may prove beneficial.

The drugs which slow the pulse may be divided into two groups-those which increase the tone of the heart muscle and tend to raise blood-pressure and those which do not. In the first class, the most important are digitalis, strophanthus, apocynum, convallaria, adonidins, and perhaps sparteine; in the second class, aconite and veratrum are the only ones still employed to any large extent.

Digitalis Group. - Stimulation of the cardio-inhilitory mechanism produces an increase not only in the duration but also in the completeness of the diastole; that is, a lessening of the muscle tone of the heart. It is evident that in cases where there is already dilatation of the heart, and especially where the peripheral resistance is high, this loss of tone, brought about through stimulation of the pars vagum, may easily prove harmful as tending toward further dilatation. 'The digitalis group, how- 
ever, have, besides their action upon inhibition, a powerful effect in increasing the tone of the heart muscle. This is seen, not only in the more vigorous contraction of the ventricle during systole, but also in the less complete relaxation during diastole. This effect upon the heart muscle in normal conditions will entirely overcome the diminished tone, brought about through their effeets upon the pneumogastric nerve, and renders this group of drugs peculiarly valuable in those cases of chronic heart weakness in which there is a loss of muscle tone.

A third effeet of the digitalis group must be borne in mind as influencing the clinical use of these drugs, and that is their effect upon vasomotor mechanism. All of the digitalis group, as far as they have been studied, are more or less stimulating to vasomotion, but some of them are more active in this regard than others. Strophanthus is one of the feeblest vasoconstrictors of the group, but the statement which is sometimes made that it has no effect upon the vasomotor system is very far from the truth. Adonidine differs from the other digitalis drugs, according to the experiments of Kakowski, in that it is the only one of the series which does not constrict the coronary vessels. What bearing this coronary constriction has upon the therapeutic use of this group of drugs is as yet not clear.

While upon this subject of the effeets of digitalis upon the vasomotor mechanism, I wish to call attention to the fact that this action is not always the unmixed evil which some have believed. On account of the anatomical relations of the coronary artery to the aorta, a considerable degree of pressure is required in the aorta to afford the proper bloodsupply to the heart muscle. If the peripheral resistance is low and the heart muscle feeble, there will be a reduction in the proportion of blood which passes to the heart and a consequent impoverishment of its nutrition. 'Tigerstedt has found that dilatation of the peripheral blood-vessels diminishes the output of the heart; despite the lessened resistance in front of it, and the consequent greater ease with which its contents can be expelled, the heart is unable to pump as much blood when the peripheral resistance is low. Tigerstedt attributes this, probably correctly, to the fact that under the low peripheral resistance there is a diminution in the blood-supply to the heart, and consequent weakening of the muscle is the result of insufficient food and oxygen. It is manifest, therefore, that, if the blood-pressure is low, the vascular constriction produced by digitalis favors better nutrition of the heart muscle, so that the increased obstruction to the passage of blood is more than balanced. But, on the other hand, if the blood-pressure is very high, as is not infrequently the case in certain types of heart disease, there is nothing gained by further contracting the peripheral blood-vessels, and the vasomotor action of digitalis may prove harmful by placing a resistance before a heart whose enfeebled museulature, unable to cope with the added burden, gives way in the form of further dilatation.

By common clinical experience, both strophanthus and adonidine have been deemed less trustworthy than digitalis in severe cases of heart weakness. In the pharmacological laboratory this relation has not been 
observed. A plansible explanation of this unreliability may be found in the fact that the absorption of strophanthus from the intestinal tract is extremely irregular.

While on the subject of digitalis, I wish to express my belief that there is no single principle of digitalis which ean clinically replace the drug itself. 'The one most widely employed, digitoxin, has been shown by Fränkel to be the most dangerously cumulative of all this group of drugs, and, while the various glueosides of digitalis are similar in their action, it is too much to claim that they are identical, and the value of the drug rests in the blending of the effects of its different principles.

The diuretic action of the digitalis group, which is partly the result of better circulation, but partly also of a direct action on the kidneys, will be considered in connection with the drugs used to increase the flow of urine. Apocynum and squill, which belong, pharmacologically, to the same group are also considered among the diuretics.

Aconite Group.-There are only two drugs at all widely used, that belong to the second class of substances which slow the pulse, and they are aconite and veratrum. These remedies were formerly classed as cardiac depressants, but, as neither one of them lowers the bloodpressure when given in therapeutic dose after section of the pneumogastric nerve, it is evident that they have little if any real depressing influence upon the heart muscle. Indeed, there is reason to believe that in small quantities their influence upon the heart is stimulating rather than weakening. The pronounced fall of blood-pressure which is produced by them is due solely to the great slowing of the pulse, the result of stimulation of the cardio-inhibitory centre. As far as the circulation is concerned, these remedies are almost purely inhibitory stimulants. As pointed out above, however, this stimulation of the pneumogastric nerve leads to a diminution of the muscular tone of the heart, not as a result of the direct influence of the drug, but simply as one of the evidences of excessive inhibition.

'They are used in medicine in those cases in which the muscular tone of the heart muscle is not low, as, for instance, in the so-called "irritable heart" of athletes, and in which it is desired to reduce the pulse-rate in order to prevent the heart exhausting its energy in uncontrolled action.

It has been taught in the past that there was an essential difference between aconite and veratrum, in that the latter produced dilatation of the blood-vessels and that aconite did not. More recent investigation, however, seems to have demonstrated that veratrum does not dilate the blood-vessels if given in anything approaching therapeutic quantities, so that, as far as the effects of small quantities are concerned, the action of the two drugs upon the circulation is practically identical. When, however, larger quantities are given, aconite produces a marked disturbance of the cardiac rhythm, so that the auricle and ventricle no longer act synchronously, whereas, in the case of veratrum, under toxic dose, the heart simply becomes slower and weaker, but maintains its normal rhythm. Veratrum may be pushed to full physiological action with more freedon than aconite, for the reasons, first, that excessive quantities will generally 
promote emesis before serious poisoning, and, for the second, because it is less likely to disturb the irritability of the heart muscle. On the other hand, for ordinary conditions aconite is preferable, as it is less prone to upset the stomach.

Drugs which are Used to Raise the Blood-pressure.-We may increase the blood-pressure either by increasing the force of the heart or by contracting the blood-vessels. Consequently, we may divide the circulatory stimulants into two groups - those whose action is chiefly upon the heart and those whose action is chiefly upon the vasomotor system. Some of the circulatory stimulants belong to both groups, that is, they possess a power over the blood-vessels as well as the heart, but I shall consider these in the class in which, in my opinion, they show the predominating effect.

Cardiac Stimulants.-The cardiac stimulants include alcohol, ammonia, caffeine, camphor, and the digitalis group.

It may not be amiss to emphasize the distinction between what we may call the alcohol-camphor group and the drugs allied to digitalis, for, while in a sense both sets may be called cardiac stimulants, their effects on the heart are quite unlike. The most striking dissimilarity, from a pharmacological standpoint, is seen in the effeets of poisonous doses, whereas, under digitalis, the contraction of the heart musele becomes constantly more vigorous and prolonged, even until death; that is, there is a constant stimulation from all doses. With the other group, as soon as a certain maximum dose is surpassed, the stimulation passes into depression. The practical application of this fact is that the alcohol-camphor group must not be given too freely; if these drugs fail to better the circulation in ordinary doses, it is useless to give more, for one would but add to the danger of the disease that of the secondary depressant influence of the remedy.

Another important practical distinction lies in the fact that, as they do not stimulate inhibition, the alcohol-camphor group lack the tonic properties of digitalis. 'Their influence is purely that of an excitant or temporary stimulant, and the increased action is at the expense of the reserve energy of the heart. For this reason they are not suitable drugs for the treatment of chronic heart disease, save, of course, for emergency use, where immediate death is threatened.

There has been a great deal of dispute as to whether alcohol stimulates the heart or not. It is universally agreed that the large dose of alcohol has a depressant influence upon this organ, but, concerning what we might call the therapeutic dose, corresponding to about from one-half to one ounce of whisky, there is decided difference of opinion. The evidence of a stimulant influence upon the heart up until within the last four or five years was far from convincing, and, we, therefore, find that, among the older pharmacologists, the opinion is generally held that any dose of alcohol sufficient to affect the circulation acts as a depressant. Recent investigations, however, by Kochman, Brandini, Wood and Hoyt, and others seem to pretty clearly demonstrate that, with proper doses, there is a distinct if not great increase in the functional activity of the heart. 
Since, however, the blood-vessels of the skin are dilated by this dose, the pressure within the arteries generally is not greatly altered. In conditions of exhaustion the influence of alcohol upon the heart seems to he more marked than it is in the normal animal. While I believe that alcohol is capable of acting as a stimulant to the circulation in certain conditions of exhaustion or depression, as occur, for instance, during the infectious fevers, I am convinced that it has been too much relied upon in these conditions, and too frecuently employed in conditions for which it is in no wise suitable. Its value in such states as surgical shock is extremely questionable, because, althongh it has more or less stimulant influence on the heart, it has a depressant action upon the vasomotor mechanism which is usually already below par.

Ammonia is a rapidly aeting but fugacious stimulant to the circulation, affecting both the heart and the blood-vessels. It is so rapidly destroved however, and so slowly absorbed through the mucous membranes, that it is wellnigh impossible to obtain any effect from it through oral administration. If an action is desired from this substance, it should be administered hyporlermically.

Concerning the effects of caffeine, our information is not as yet enough to permit of positive statement. It is definitely determined that it accelerates the heart-beat independent of any aetion on inhibition, and probably also slightly augments the size of the contraction, so that we properly class it as a cardiac stimulant. It also causes some constriction of the vessels, but whether by a central or peripheral action is uncertain. Clinicians sometimes employ caffeine as a heart stimulant in cases of chronic valvular disease, but it is, in my opinion, bad therapeutics. While caffeine may be of service in these conditions, it is in those cases in which there is marked dropsy that it becomes of value through its influence on the kidneys.

While camphor has apparently little effect on the normal heart, it seems to counteract in some way the influence of depressing poisons. Thus, Heubner and others have found that camphor will excite the heart to renewed activity after muscarine, and Böhme asserts that the same is true of the heart arrested by chloral. Clinical evidence is abundant that camphor exercises a powerful stimulating effect, when given hypodermically, which lasts from one to two hours.

Sufficient has been said of the action of digitalis in the section on drugs which slow the pulse, except to point out that its action on inhibition to a certain extent antagonizes its blood-pressure-raising properties. It might, therefore, be advisable, when using it, to combat acute circulatory failure, to combine it with atropine as an inhihitory paralyzant.

Vasomotor Stimulants.--The arterial pressure is the result of the pumping force of the heart, driving fluid against the resistance offered by the blood-vessels. The vaseular resistance varies chicfly with the calibre of the ressels. For the maintenance of proper degree of blookpressure a condition of semicontraction of the blood-ressels is as necessary as is sufficient muscle power in the heart. General dilatition of the blood-vessels, vasomotor paralysis, will so greatly diminish the 
resistance that, despite a perfectly healthy heart, the blood-pressure may fall to zero and death occur. Cases of sudden death due to vasomotor paresis have been observed, for instance, after violent blows on the abdomen, which paralyze the splanchnic area. It is only within comparatively recent times that the inportance of vasomotor tone for the maintenance of proper circulation has been realized. Many of those conditions of low blood-pressure, such as are seen in surgical shock and in the later stages of infectious fevers and the like, which the profession were wont at one time to speak of as heart failure, are now regarded as vasomotor failure. Speaking generally, where our object is to simply elevate the blood-pressure, more striking results can be obtained from the vasomotor stimulants than from heart stimulants. Among the drugs which are useful for increasing vasomotor tone may be mentioned digitalis, strychnine, atropine, ergot, and adrenaline.

The suprarenal capsules contain a principle chemically allied to the alkaloids, which is an extraordinarily powerful stimulant to the sympathetic nerves, including the vasomotor nerves. There is no substance employed in medicine which is capable of causing a greater rise in the blood-pressure than this principle. There are a number of conditions, however, which greatly limit its field of usefulness as a general vasomotor stimulant. In the first place, its action is so extremely fugacious that, when given by the mouth, it exercises practically no influence upon the circulation, because it escapes from the blood-stream faster than the mucous membrane can absorb it, and, even when injected hypodermically, its influence is comparatively feeble. To develop the full pharmacological effect of this drug it is necessary to administer it intravenously. The operation of preparing for an intravenous injection consumes a considerable amount of time, and, as the great value of the drug is the promptness of its action, maintaining circulation until slower and more persistent remedies can be introduced, it is evident that this feature greatly detracts from its clinical utility as a general stimulant. The repeated use of adrenaline in the rabbit has been shown unequivocally to produce degeneration of the arterial walls, probably through a direct irritant action upon the endothelial lining, and, while it has not yet been demonstrated that similar changes are caused in human arteries, there is no reason to doubt that the irritant action of the drug would be the same in the human being as in the rabbit. In other instances violent pulmonary edema has resulted from the experimental use of this drug, even from a single dose. This latter condition arises from the fact that the principle exercises comparatively little effect upon the pulmonary vessels, and the violent spasm of the systemic arteries must, therefore, cause acute congestion in the lungs and consequent escape of fluid from the vessels. This accident is not likely to happen unless the blood-pressure is elevated considerably above normal, and is hardly to be feared in cases of very low bloodpressure. On the other hand, it should make the physician very cautious in the use of adrenaline in cases in which the blood-pressure is only moderately low, and in cases in which there is reason to bclicve that the heart is in any degree weakened. 'Therefore, I do not believe that this agent, 
as powerful as it is physiologically, has a very wide range of utility as a general stimulant in clinical medicine. As a local vascular stimulant, for instance, in local hemorrhages or congestions, it may be used with great benefit.

Atropine is a vasomotor stimulant of moderate power, whose usefulness, however, is sometimes greatly lessened by its cffects upon other organs. As its action upon the glands is more powerful than upon the circulation, it is necessary, in order to obtain any great rise in the bloodpressure, to give doses which almost completely dry up secretions. As this condition is one of great discomfort to the patient, its use as a vasomotor stimulant is practically limited to cases in which there is immediate danger of death. In these conditions, however,-that is, where collapse is severe enough to threaten the patient's life at once, - the action of the drug in checking secretion is usually beneficial, as the drying of the skin tends to maintain the bodily temperature. Moreover, the paralytic action upon the cardio-inhibitory meehanism increases the rate of the pulse, and, therefore, augments the work done by the heart. While atropine is in no true sense a stimulant to the heart muscle, yet the increase in cardiac rate which follows its administration must tend to an elevation of the blood-pressure. 'The drug, therefore, in cases of surgical shoek or in cases of poisoning, is of service as a circulatory stimulant.

Strychnine does not possess the action of atropine upon the glands, and is probably even more powerful as a vasomotor stimulant. It is probably the most commonly employed drug where vasomotor stimulation is desired. Ordinarily, however, it is given in too small dose to exercise its full effects upon the circulation. 'To obtain stimulation of the vasomotor centre it must be pushed to the full degree of tolerance-that is, until the beginning of exaggerated reflexes shows the action upon the spinal cord.

Digitalis is frequently forgotten in considering the vasomotor stimulants. It is, however, one of the most powerful drugs for this purpose that we possess, the vasomotor effects frequently surpassing the heart action.

Ergot is a very powerful vasomotor stimulant, but is absorbed comparatively slowly, either through the intestinal tract or from subcutaneous tissues. Moreover, the preparations of ergot which are commercially obtainable are generally almost inert, so that, while theoretically it should be a very valuable drug, practically, in conditions of acute circulatory failure, it is of little utility. On the other hand, in conditions of chronic congestions, as in certain types of diarrhea, it has proved itself clinically of much value.

Drugs which are Used to Lower the Blood-pressure.- Three types of drugs have been used to reduce abnormally high arterial pressure those which act by slowing the pulse (aconite group), those which weaken the contractile power of the heart (antimony), and those which dilate the blood-vessels (nitrites). Besides these direet agents, efforts are sometimes made to lower the blood-pressure by extreme depletion, as with the cathartics.

VoL. I. $\rightarrow$ 
The use of antimony as a means of reducing the force of the circulation has almost entirely disappeared and justly so. I cannot imagine any justification for lowering the functional capacity of the heart when we can produce an equal degree of diminution of cardiac action by such drugs as aconite or veratrum, whose depressant influence does not essentially weaken the heart and can be immediately abolished by an inhibitory paralyzant, as atropine. Most commonly, however, the indication to lower the pressure is met by the use of the vasodilators.

Unfortunately, the agents at our command for producing arterial relaxations are extremely limited. They include only the drugs which liberate the nitrite ion. The belief, held by some clinicians, that the iodides have the power of dilating blood-vessels is without any scientific basis.

Among the nitrites, five have been used to a considerable extent in practical medicine. These are amyl nitrite, ethyl nitrite, sodium nitrite, glyceryl nitrate (nitroglycerine), and erythrol tetranitrate, but practically, the only difference between these remedies is in the rapidity of their effects. The extraordinary rapidity and fugaciousness of these substances are not appreciated by the majority of clinicians. The following table is taken from a paper by Wallace and Ringer, ${ }^{1}$ showing the quickness of their effect on human beings when given by the mouth:

Average Blood-pressure Results from Administration of Nitroglycerine, Sodium Nitrite, and Erythrol Tetranitrate to Normal Persons

\begin{tabular}{|c|c|c|c|}
\hline Drug. & $\begin{array}{c}\text { Beginning } \\
\text { of action. } \\
\text { Min. }\end{array}$ & $\begin{array}{l}\text { Time of } \\
\text { maximum } \\
\text { effect. } \\
\text { Min. }\end{array}$ & $\begin{array}{c}\text { Duration of } \\
\text { action. } \\
\text { Min. }\end{array}$ \\
\hline
\end{tabular}

Amyl nit rite $(3$ minims) ......... 1

Nitroglycerine ( $1 \frac{1}{2}$ minims 1 per cent.

solution). . . . . . . . . . . 2

Sodium njtrite $(1$ gr. $) \ldots \ldots \ldots \ldots \ldots \ldots 10$

Erythrol tetranitrate ( $\frac{1}{2}$ gr.) $\ldots \ldots \ldots 15$

3

7

830

$25 \quad 60$

$32 \quad 120-240$

The custom of giving 1 to 2 minims of spirits of nitroglycerine three or four times a day, in the light of this research is a manifest absurdity. Attention should be called to the fact that this table represents not experiments upon dogs or eats, but observations under clinical conditions on human beings.

The physiological action of the nitrites may be summed up about as follows: In therapeutic doses they cause a widening of the blood-vessels and an increase in the pulse-rate through a paralyzing action on the cardioinhibitory centres, and exercise a depressant influence over the motor centres in the spinal cord; if given in toxic dose, they interfere with the oxygen-carrying power of the blood by causing chemical changes in the hemoglobin. Some believe that the nitrites increase the power of the heart muscle, but in my opinion the evidence in this direction is far from convincing. The increased fulness of the pulse which sometimes follows the exhibition of nitroglycerine in conditions of high arterial tension does not mean that the heart is contracting more forcibly, but merely that the

1 Jour. Amer. Med. Assoc., vol. liii, p. 1629. 
disappearance of the excessive resistance allows the ventricle to empty itself more completely. While, through the acceleration of the heart-rate, these agents may, perhaps, slightly augment the output of blexd, I do not believe that any of the nitrites have any true stimulant effect on the heart miscle, and $\mathrm{I}$ am convinced that the use of the nitrites in conditions of shock and collapse has no rational foundation and is capable only of harm.

'The action of the nitrites in relaxing blood-ressels is due to an effect either upon the arterial muscles or on the nervous mechanism controlling these muscles. It is manifest that where, as a result of pathologic processes, the muscular coat of the artery has been largely replaced by fibrous tissue, the nitrites are ineapable of dilating the blood-vessels, and, for this reason, very frequently they fail when their effect is most needed, as in cases of advanced arteriosclerosis with high blood-pressure.

Cathartics.-'The older writers divided the cathartics, according to the power of their action, into the laxatives, the purgatives, and the drastics. The difference between these groups, however, is, after all, largely a question of dosage, for a drug which in full dose may be a powerful drastic, in smaller quantity may aet as a mild aperient, and the pharmacological division is not only more scientific, but more useful praetically. 'The catharties, according to their physiological action, may be divided as follows: the salines, the anthraquinone group, the cathartic oils, and the resinous group.

Salines.-When two solutions, containing an inorganie salt in different concentrations, are separated by an animal membrane, there is a tendency for the interchange of fluid and solid constituents through the membrane until the fluids on the two sides become of equal density. If, therefore, sodium chloride be introduced into the intestinal tract, it should theoretically determine the passage of fluid into the bowel; but practically the sodium chloride itself passes so rapidly through the wall of the gut that enormous quantities would have to be introduced into the bowel to perceptibly disturb the osmotic balance. The salts of certain acids, however, appear to be unabsorbable, or at least difficultly absorbable, by the intestines. The experiments of Pauli indicate that this property is in some way connected with their power of precipitating albumins. For whatever reason the salts are not absorbed, however, the fact that they remain in the intestines leads to a determination of liquids from the surrounding tissues into the lumen of the bowel, as well as prevents the absorption of any fluid which may already be present. When enough fluid has aceumulated in the intestines to cause moderate distention, the internal pressure reflexly augments the peristalsis and provokes eatharsis. Since the eflects on peristalsis do not occur until there is sufficient pressure within the intestines, it follow's that the more water which is ingested with the cathartic salt, the more promptly it will aet. 'There is some evidence, however, that these saline cathartics exercise a direct stimulating aetion upon the glands of the intestinal mucosa, and, while this effect may in part explain their laxative action, that it is not the sole, nor even the chief, cause of the catharsis is shown by the 
comparative slowness of action when taken in concentrated solution. 'The saline cathartics are the neutral salts of sulphuric, phosphoric, tartaric, and citric acids.

Among the salines, the choice is to be made, I believe, largely on the ground of palatability. 'The sulphates, especially magnesium sulphate, are perhaps somewhat more active than the phosphates or the tartrates, but the latter are generally efficient in ordinary conditions. Magnesium sulphate has an umpleasant bitter flaror, which may, however, be partially disguised by exhibing it in effervescent form, as in the official efferrescent magnesium sulphate; the double tartrate of sodium and potassium, commonly known as Rochelle salts, is much less unpleasant, and if given in effervescent form, is one of the most elegant saline laxatives. It may be mentioned in passing that the natural laxative waters, as well as the various proprietary salines upon the market, are simply mixtures in varying proportions of official salines with various other salts, as sodium chloride and bicarbonate of soda.

Anthraquinone Series.-A number of vegetable cathartics owe their action to the presence of principles which are chemical derivatives of anthraquinone. 'The drugs which come under this class are aloes, cascara sagrada, rhubarb, and senna.

The action of these drugs is due to a local irritant influence upon the walls of the bowcl, increasing peristalsis, and perhaps the secretion of the intestinal glands. The effect upon peristalsis seems to be chiefly limited to the large intestines. Magnus found that, when senna was added to the food, the passage along the intestines of the cat was no more than usual in the normal animal until the mixture reached the colon, when there occurred a marked increase in the peristaltic activity of the lower bowel, and consequent hurrying forward of its contents. Most of these drugs also contain bitter principles, which may exercise more or less tonic influence upon the bowel, and make them, therefore, useful, especially in chronic constipation; there is a wide-spread clinical opinion that this is especially true of cascara sagrada. Rhubarb contains, besides its cathartic principles, notable proportions of tannin, and, therefore, exercises a marked astringent action upon the stomach and upper intestines. For this reason it is sometimes employed to clean out the bowel in eases of diarrhœea, but, for reasons given below, I deem it inferior to other drugs for this purpose.

Cathartic Oils.-Oleic acid is slightly stimulant to the intestinal peristalsis, and olive oil is, therefore, sometimes of service in the milder degrees of constipation. Some of the near relatives of oleic acid, however, are actively cathartic; among these may be mentioned especially ricinoleic acid, from castor oil, and crotonoleic acid, from croton oil. Although widely different in their therapentic uses, the mode of action of these two drugs is probably similar in kind, although very different in degrec.

The purgative effect of the cathartic oils depends upon their decomposition in the intestinal tract, with the liberation of an irritant fatty acid. In the case of croton oil, a certain amount of free crotonoleic acid 
is found in the oil, and this drug, therefore, being irritant to all mucous membranes, is liable to produce emesis as well as catharsis, and acts promptly. On the other hand, while old specimens of castor oil may contain the free acid, fresh specimens lo not, and it is necessary for the oil to be saponified before it can act. As this decomposition takes place generally in the duodenum, it acts in the stomach simply as any other bland oil and is in no way irritating, and, as the liberation of the ricinoleice acid takes place gradually, its action is not manifest for several hours after the administration of a dose of castor oil.

The purgation caused by ricinoleic acid appears to be chiefly due to an increase in the peristaltic movements of the bowel, the result of a local irritation. 'This stimulating effect upon peristalsis diflers notably from that of the anthraquinone series in that it affects the upper bowel as well as the lower. 'There is a popular belief that the purgation by castor oil is more than ordinarily likely to be followed by constipation. 'The constipation, however, is probably due to the fact that the whole of the alimentary tract has been so thoroughly emptied that it requires a considerable amount of time before a normal mucous membrane is able to secrete enough fluid into the bowel to stimulate its movements, rather than to any direct influence of the drug.

Resinous Group.-'The old group of "drastic" cathartics includes a number of drugs which contain as their active prineiples resinous bodies, mostly either acidic or glucosidal. While the subject has not been thoroughly worked out, the active principles of this group of cathartics appear to be closely allied chemically, and, at least from a therapeutic standpoint, their effects are practically identical, except in so far as they may differ in degree. The group includes colocynth, gamboge, elaterin, podophyllum, jalap, and scammony.

'These cathartics are more or less irritant to mucous membranes and likely to give rise to nausea, yet, as the presence of bile scems essential for the full development of their power, the effect upon the stomach is less marked than that upon the bowel. As the result of the irritation of the mucous membranes of the intestines, we have not only an increase in the peristalsis, but a large secretion by the glands; in other words, a condition similar to that in an intestinal catarrh. The amount of fluid which is poured into the bowd under these circumstances is sufficient to influence the total liquids of the body, so that these agents are useful for the purpose of evacuating dropsical effusions. Some of the older writers have believed that elaterin has a special property of enusing the elimination of urea through the intestinal glands, and is, therefore, of peculiar value in kidney diseases, but the scientific evidence in favor of this view is, to say the least, questionable.

Mercury.-Certain salts of mercury, probahly by virtue of a local irritant action, excite the intestines to abnormal peristalsis. 'The question of greatest interest in connection with the mercurials is their influence upon the liver. While clinically they are universally cmployed as cholagogues, pharmacologically their effect upon hepatic secretion is practically nil. While it may be accepted as definitely proved that calo- 
mel and the other mercurials do not increase the secretion of bile, there is strong evidence that they do increase the amount of bile in the feces. Exactly how this is brought about is still a matter of conjecture. It is in my opinion, however, probably the result of two causes: first, by virtue of an antiseptic action, the mercurial prugatives diminish intestinal putrefaction and thereby lessen the fermentative decomposition of the bowel, and, second, they may increase in some manner the outpouring of storedup lile from the gall-bladder and hepatic ducts.

Therapeutic Uses.-'The importance of a knowledge of the physiological effects of the catharties for a proper appreciation of their relative values can be most easily shown by taking up the various types of conditions in which a purgative is required. 'These are: (1) To empty the bowel of irritating or poisonous substances, as in cases of acute enteritis or in excessive intestinal putrefaction with the formation of ptomaines. (2) 'I'o aid in the elimination of toxic materials from the blood, as in the treatment of gout or uremia. (3) To evacuate fluid in various forms of dropsy. (4) To maintain the action of the bowels in chronic constipation.

When used to empty the bowels of toxic materials, the properties of the ideal purgative would be-that it affect the whole intestines, that it shall act promptly, and that it shall not be too highly irritant. These requisites are most nearly fulfilled by the so-called saline laxatives. If given well cliluted, they usually act within an hour, and their effect is due rather to purely physical causes than any form of irritation. The salines are, for these reasons, of frequent service in the beginning of an enteritis and similar states to evacuate the offending material. But, when the trouble is clue to bacterial infection, the antiseptic influence of mercury makes it of great value as a cathartic, and, although less prompt than the salines, ealomel may frequently be substitued for them with great advantage in the preliminary treatment of acute eatarrh of the intestines. Castor oil has also been widely used as a laxative in inflammatory conditions of the bowel, with the idea that, on account of its oleaginous nature, it will exercise more or less soothing influence upon the inflamed mucous membranes, but, as it is broken up with the splitting off of irritant ricinoleic acid, this soothing influence is probably slight at the very best; however, it is an efficient cathartic, affecting the whole of the i: testines, and, since the prime object is to get rid of the source of an infammation, it has frequently proved of service. The vegetable catharties, loth of the anthraquinone series and the resinous group, should never l.e used for this indication. As pointed out above, the effect of the anthraguinone derivatives is chiefly upon the colon, and generally the offending material is in the upper bowel. Moreover, both classes of drugs are highly irritant to mucous membranes and may easily add to the inflammation.

In cases of edema it is evident that the cathartie of choice should be one which causes the greatest amount of water to pour into the intestinal tract. For this purpose, either the salines or "drastics" may be employed. The latter cause very profuse secretion in the intestinal tract, 
but are harsher in their action than the purgative salts. At times the salines and the drasties may be combined with gorsl results. Clinical experience has given the preference among the drastics to either claterin or jalap. When the salines are being used to evaluate fluid from the body or to deplete, the mode of use differs from that where we are using them to simply empty the intestinal tract, for, while the more dilute the solution of the saline cathartic is, the more rapid will he its effect, yet it is evident that if the osmotic factor plays a part in the effects of these remedies, the amount of fluid which is drawn from the surrounding tissues into the bowel will be greater if the salt is given concentrated.

In cases of chronic constipation it is generally the muscular action of the intestines which is at fault, and for this reason we should give preference to a laxative which increases peristalsis. 'These are the vegetable cathartics. On account of the highly irritant effect of the resinoid group, they are used only in those eases in which the anthraguinone derivatives are not sufficient by themselves. Much benefit is sometimes obtained in these cases by combining with the cathartic those remedies which act directly upon unstriped muscle. 'The most important of these agents, from a clinical standpoint, are atropine and physostigmine, although stryehnine is widely used. The exhaustive studies of Magnus have shown that, in not too large doses, atropine increases both the tone and the activity of the rhythmic contraction of the intestinal muscle, probably through an action upon Auerbach's plexus, but the most marked effect is a regulation of the peristaltic movements, so that the contraction waves became of nearly equal height and oceurred at rhythmic intervals. The same author also confirmed the previous finding of Fraser and others that physostigmine increased the activity of the intestinal muscles through an action probably upon the musele substance. It would seem probable that to the peculiar regulating action of atropine upon peristalsis is due its value to prevent griping when combined with the vegetable cathartics.

Drugs Used to Increase Quantity of Urine.-Drugs augment the volume of urine in one of two ways, either by causing an increased amount of blood to pass through the kidney, or by direct stimulation of the secreting structure of this organ. 'The increase in the volume of blood passing through the kidney may be the result either of a greater activity of the circulation, as through stimulation of the heart, or of a larger volume of blood; it is through the latter effect that the injection of normal salt solution or the copious drinking of water increases the quantity of urine. We may, therefore, divide the diureties into three classes-those which act by stimulating the circulation, those which increase the volume of blood, and those which have a direct effect upon the secreting structure of the kidney.

Circulatory Stimulants.-'The volume of blood which passes through the kidney is evidently not dependent solely upon the height of the general arterial pressure, but upon the state of the renal blood-vessels in relation to those of the rest of the body. If the arteries all over the body are uniformly contracted, although the hlood-pressure may be greatly clevated, it 
is evident that the proportion of blood in the kidney will be just the same as in normal conditions; the ordinary vasomotor stimulants, therefore, do not increase the output of urine. On the other hand, if the bloodvessels of the kidneys remain at their normal size, while those of other parts of the body become contracted, the kidney will receive more than its just share of blood, and, therefore, its secretion will be encouraged. 'This is the condition of affairs under the influence of caffeine, although it is probable in this instance that the dilatation of the renal vessels is secondary to the demand for more blood, due to the increased activity of the kidney.

When, however, the kidneys are the seat of a local fibroid change, the resistance to the passage of blood may be so much higher in these organs than in the rest of the body that they receive less than their normal quota of blood. In this instance general vasomotor stimulation, by tending to equalize the resistance in the rest of the body, may enforce a proper circulation through the kidneys, and it is for this reason that the high bloodpressure in cases of nephritis is so frequently a conservative effort on the part of nature.

In the case of digitalis, while there is a marked constriction of the vessels the increased heart action may more than counterbalance the vasomotor effect, and more blood be forced through the kidney, so that the effects on the circulation will lead to an increased secretion of urine. Moreover, most, if not all, of this group of remedies exercise more or less stimulating action directly upon the secretory epithelium of the kidney, although some of them are comparatively feeble in this regard. Digitalis has but slight direct action upon the kidney, and, if in any individual case the vasomotor system happens to be more sensitive than usual to the effects of the drug, the constriction of the renal blood-vessels may entirely prevent the occurrence of diuresis. Because strophanthus is less powerful as a vasomotor stimulant than digitalis, it is generally more efficient as a renal stimulant. Two drugs of the digitalis group stand out prominent for the fact that their stimulating action upon the kidney is so marked that it almost completely overshadows their effects upon the circulation in general. These are squill and apocynum. Apocynum, on account of the intensity of its local irritant influence upon the mucous membranes of the stomach and bowel, is greatly limited in its range of usefulness, but, in those cases where the alimentary tract can bear it, it is a remedy of value for the evacuation of dropsical effusions. Squill, although liable to upset the stomach, is less so than apocynum, and is more generally useful as a diuretic. It must be remembered, however, that both of these drugs, in their effects upon the kidney, may pass beyond the limits of stimulation into the realm of irritation, especially when the kidney is the seat of an inflammatory process. 'Therefore, ncither of them should be cmployed as diuretics in the case of acute Bright's disease, and only with great caution where there is any lesion of the kidney more active than a simple cirrhotic process.

It is not only with digitalis itsclf, but with all its congeners, which are used as diureties, that the vasomotor effect hinders the action upon 
the kidney, even if in many euses it does not prevent. Therefore, very: frequently beneficial results may be obtained in the use of this group of remedies as cliureties by combining them with a nitrite in order to prevent excessive vascular contraction.

Saline Diuretics. - As has already been pointed out, if we introduce a saline substance into the blood-stream, the higher concentration of the saline constituents of the blood leads to a flow of water from the surrounding tissues into the blood-vessels, so that the total volume of blood becomes increased. Any non-toxic crystalloid is, therefore, capable of acting as a diuretic hy inereasing the amount of the blood. Some salts, being easily diffusible themselves, escape from the vessels so rapidly that there is a relatively small attraction for water into the circulation. For this reason we find the salts of potassium are more diuretic than the salts of sodium, and the acetates and carbonates more diuretic than the chlorides. For practical purposes the saline diuretics include several of the salts of potassium, sodium acetate, and ammonium acetate. 'There is, however, a room for choice among the salts of potassium. Formerly the nitrate was used to a considerable extent as a diuretic, but the nitric ion is so irritant to the kidney that this salt should not be employed where there is any tendency toward renal inflammation; indeed, it is hardly safe to give it in large enough quantities to obtain the salt action even when the organ is healthy. Potassium chlorate acts in the body very much as does the nitrate, and is not available as a saline diuretic. 'The chloride, being comparatively readily diffusible, for reasons just pointer] out, is less efficient as a diuretic. 'The salts of potassium which are practically useful as diuretics are the acetate, citrate, bitartrate, and the bicarbonate. 'The acetate and citrate undergo in the system a process of oxidation and appear in the urine in the form of carbonates, so that they not only increase the volume of urine, but tend to render it alkaline. The bitartrate does not seem to be changed in the system, but is less readily absorbed from the intestines, and, therefore, frequently acts as a laxative rather than a diuretic. The older writers considered that this salt had a direct stimulating action upon the kidney, but this is probably erroneous.

'Theoretically, the saline diuretics should increase only the watery constituents of the urine, but practical experience has shown that they will also increase, although to a much less degree, the solid matters, so that they are useful not only to evacuate dropsical effusions, but to increase elimination of waste poisons in conditions of failing kidney function. They are generally less efficient than the direct stimulants to the organ, but, on the other hand, are not so likely to irritate an inflamed kidney, and are preferable where it is desired to increase the volume of urine in eases suffering from nephritis.

Stimulating Diuretics. - Almost any irritant substance which is capable of entering the blood-stream, if given in the right dose, will exercise more or less diuretic action, and we find that among the older therapeutists many of the volatile oils were used clinically to inerease the secretion of urine, such as oil of turpentine, oil of juniper, ete. If the dose 
of such an irritant be too large, however, one may easily pass beyond the stage of stimulation into that of irritation or inflammation, in which case there will be a diminution instead of an increase in the secretion of the kidney. It must be remembered, moreover, that in an organ which is the site of an inflammatory process, even if not very active, what in normal conditions would be stimulating may prove irritant, and it has been clinically found that the border line between stimulation and irritation with the drugs mentioned above is comparatively narrow, so that they are only occasionally available. The stimulating diuretics which are largely employed to-day are, first, certain members of the digitalis series (already considered), second, the xanthin or purin derivatives, and, third, the salts of mercury.

The most widely used of the stimulating diuretics are without doubt the xanthin derivatives, of which three are to-day employed in clinical medicine. 'These are caffeine, theobromine, and theophylline (theocine). Although caffeine constricts most of the blood-vessels of the body, the vessels of the kidney are dilated. This dilatation is probably the result of the greater functional activity of the organ demanding more blood, and is in opposition to the general effect of caffeine upon the circulation. It is evident, however, that the tendency toward constriction of the bloodvessels will more or less antagonize the action of caffeine upon the kidneys, and we do not get the full degree of vaseular relaxation, which is most favorable for the action of this organ. Caffeine is, therefore, in healthy kidneys less powerful as a diuretic than those members of the xanthin series which do not affect the circulation. On the other hand, when the blood-pressure is very low, the effect of caffeine upon the circulation may make it especially desirable as a kidney stimulant, but even under these conditions, on account of its effect upon the central nervous system, it is frequently impossible to give large enough doses to get the full stimulant action upon the kidney. For these reasons, the use of caffeine as a diuretic has been to a large extent given up in favor of the closely allied bodies mentioned above. 'Theobromine, an alkaloid found in the chocolate bean, has practically no effect upon the circulation, except to slightly increase the pulse-rate, and is much fecbler in its action upon the nerve centres than is eaffeine. It is generally administered either in the form of the double acetate or the double salicylate of theobromine and sodium. 'The alkaloid theophyllin, although known for many years as a constituent of tea-leaves, is present in such extremely small quantities as not to be clinically available until a process for manufacturing it synthetically was discovered; this synthetic theophyllin occurs in the market under the name of theocine. Although a potent stimulant to the kidney, when used over a considerable period of time for some reason it loses its diuretic power. Albanese believes that this is due to the accumulation of the crystals of trimethylxanthine in the renal canaliculi, and, therefore, recommends the ingestion of large quantities of water with the alkaloid. If the administration of this substance is interrupted every two or three days for twenty-four hours, diuretic action from it may be obtained over a long period of time. 
Drugs Used to Increase the Sweat.- The sudorifics are divisible into those which are directly stimulant to secretion, and those which act indirectly through changes in the circulation in the skin.

The indirect sudorifies include those which aet-(1) By producing nausea (ipecacuanha); (2) by dilating the skin-vessels (opium and alcohol); (3) reflexly, through irritation of the mucous membrane of the stomach (camphor and the volatile oils); (4) by lowering the general blookl-presure (aconite and the nitrites). 'The coal-tar derivatives, as antipyrine and the salicylates, produce at times violent perspiration, but, as this effect seems to be due to their action on the thermogenic centre, they are not generally useful unless fever is present, and then must be used with cauiion, on account of the danger of excessive effect.

'The indirect sudorifics are comparatively feeble, and, therefore, are of service chiefly in conjunction with other diaphoretic measures to increase elimination in febrile and semifebrile complaints.

While there are a number of substances which act upon some portion of the nervous mechanism governing secretion, the only one which is used clinically is pilocarpine. This alkaloid stimulates the peripheral endings of the secretory nerves-probably of all glandular structures. As a result of this action, there is an increase in the flow of saliva, in the secretion of the various glands of the digestive tract, but especially in the secretion of sweat.

Two drawbacks to the practical usefulness of pilocarpine are its liability to produce nausea and even vomiting, and the supposed danger of pulmonary edema. 'The first of these dangers is not likely to occur unless the dose has been large and may generally be avoided, in what we may call the milder conditions in which sweating is indicated, as in the abortion of certain febrile complaints, by combining it with other sudorifics, as the nitrites or ipecac, and encouraging the sweating by the external application of heat.

Where, however, it is necessary to produce a profound perspiration, as for the purpose of eliminating toxic materials from the blood or to aid in the evacuation of dropsical fluids, while we should encourage the sweating with the use of heat, it is frequently necessary to give comparatively large doses-from $\frac{1}{8}$ to $\frac{1}{4}$ grain-to produce the desired results.

In such instances the remedy should be given hypodermically.

'The popularity of pilocarpine has been greatly restricted by the fear of pulmonary oedema, an accident which has undoubtedly followed its use. 'This effeet is probably the result of an increased secretion by the bronchial glands, with a simultaneous constriction of the bronchioles-due to an action on the pulmonary terminals of the pneumogastric nerve-which hinders the easy evacuation of the lungs. It is manifest that, if a large amount of fluid is eliminated from the system through the skin, the secretion of other glands will be diminished; hence, if the action of pilocarpine is diverted toward the sweat-glands, the danger of the accumulation of fluid in the lungs will be markedly dininished. For this reason it is of great advantage, not only on account of more powerful effects, but also because of greater safety, to encourage the action of the drug 
upon the skin by the use of hot-packs and similar diaphoretic measures. Atropine antagonizes both of the above-mentioned actions of pilocarpine on the respiratory tract, and is likely to prove of great service if pulmonary odema occur; but, on the other hand, it overcomes the action of pilocarpine also on the sweat-glands, and will, therefore, also stop the diaphoretic effect.

Drugs Used to Increase Respiration.-'The central nervous system acts more or less as a unit in its relation to chemical agents-that is, those drugs which act upon one part of the central nervous system are likely to have a similar influence upon other portions; thus, we find that such substances as chloral hydrate, ether, etc., which depress the cerebral function, also lessen the activity of the spinal cord and many of the medullary centres. Although anatomically the medulla is contained within the cranial cavity, physiologically it is more nearly related to the spinal cord than to the brain, and those drugs which have a marked action upon the spinal ganglia almost without exception affect to a greater or lesser degree the centres in the medulla. From the standpoint of the pharmacologist, the most important of these centres are those which govern the circulatory and the respiratory muscles. It is, therefore, no cause for surprise to find the respiratory stimulants to be, almost without exception, excitants of the spinal cord. The respiratory stimulants include strychnine, cocaine, atropine, caffeine, and ammonia.

'The rhythmical contractions of the diaphragm and thoracic muscles, which constitute breathing, are caused by periodic impulses discharged from the respiratory centre. The cause of the rhythmical discharge of the respiratory centre has been the subject of some dispute among physiologists, but the generally accepted opinion to-day is that it is the result of three factors-afferent nervous impulses, reaching the centre through the vagus, the influence of certain materials in the blood, notably carbonic acid, and metabolic processes within the centre itself.

A knowledge of the physiology of the respiratory movement is important, because it affords us the clue to a pharmacological fact which is also of much importance to clinical therapeutics, and that is that, by the administration of several respiratory stimulants, we are able to produce a greater exaltation of function than by giving large doses of any one. While in a certain sense atropine may be regarded as a spinal stimulant, yet its action upon the cord is entirely distinct from that of strychnine. Just wherein lies the difference between the action of atropine and strychnine upon nerve centres is as yet merely a matter of conjecture, but it is at least reasonable to suppose that if these drugs affect, physiologically, different structures in the spinal cord, they will also affect different portions or functions of the respiratory centre. For example, it is conceivable that strychnine might act upon the respiratory centre by increasing the sensitiveness toward the irritating influence of carbonic acid, while atropine might hasten that internal metabolism which provokes the centre to periodie discharge of motor impulses. I wish to emphasize, however, that this conception is a purely thcoretical one, and, while it explains certain pharmacological faets may perhaps, be shown, by future 
investigation, to be untenable. At least, however, it affords a working hypothesis which is practically servicealle.

There is another element which increases the advantage of mixed stimulants as against the single drug, and that is, what certain writers have spoken of as the "crossed action" of drugs. 'The theory of the "crossed action" is that, by using two drugs which synergize on some one organ of the body, we get the maximum effeet upon that organ, with the minimum effect upon other functions of the body. Let us suppose, for example, that we desire to increase the activity of the respiratory centre. We give our patient first a dose of atropine. 'This drug increases not only the functional activity of the respiratory centre, but it also stimulates the vasomotor mechanism, paralyzes the peripheral endings of the pneumogastric and of the secretory nerves, and effects various other organs in the body to a greater or less degree; if we attempt to push atropine, not only do we run the risk of carrying the action upon the respiratory centre beyond the stage of stimulation into that of secondary depression, but we are also liable to produce an unpleasant degree of action upon the circulation, secretion, ete. Caffeine is without influence upon secretion or cardiac inhibition, so that, by adding this drug to our atropine, while we do not increase the undesirable effects, we get the synergistic action upon the respiratory centre. This agent has in its turn, however, certain effects which are in no way advantageous in the present situation; for instance, excitation of the brain, the kidneys, and heart muscle. These latter effects are not, however, produced by strychnine, and we may, therefore, in cases where we wish to produce a profound influence upon the respirafory centre, add a third drug to our stimulants. This principle of "crossed action," however, cannot be carried out indefinitely, because, as we have seen above, all these substances affect the vasomotor as well as the respiratory centre, and have also more or less influence upon the spinal cord.

Auother factor of practical moment is that most, if not all, respiratory stimulants are secondarily depressant; that is, if given in toxic dose, instead of increasing the activity of the respiration, they lessen it. If, therefore, in an indiviclual case, the depression of the respiratory centre is so great that it does not yield to a therapentic dose of one of these drugs, it is inadvisable to attempt to arouse it by further administration of the remedy, and we must have recourse to some synergist.

Drugs Used to Soothe the Respiratory Centre.-Excessive breathing of itself is rarely harmful, but there are two groups of cases in which it may be desirable to reduce the sensibility of the respiratory centre. For instance, in eases of cardiac or renal disease, so far advanced that sufficient aëration of the blood is impossible, the violent dyspnoa serves little if any useful purpose, and the relief of the distressing feeling of airhunger with a benumbing drug, as morphine, is not only an act of mercy, but may, by permitting a much-needed rest, actually encourage the reestablishment of circulatory compensation.

In another group of cases the respiratory centre is abnormally sensitive to afferent nerve impulses, and a cough reflex is brought about by 
ordinarily inadequate peripheral irritations. Cough is generally a conservative, or even a necessary, symptom; when the bronehial mueous membrane is seereting freely there is no other means by which the lungs ean rid themselves of accumulating fluids. But at times the cough may be out of all proportion to the amount of secretion; indeed, may be most distressing when the membrane is quite dry. 'This condition arises, either as the result of a local inflammation and consequent irritation of the peripheral nerves in the bronehial mucous membrane, or of hypersensitiveness of the respiratory centre. The drugs useful to relieve peripheral irritations will be considered in the next section. Here I wish simply to consider those which are useful to quiet the respiratory centre. The most important of these are morphine and allied drugs, although at times the narcotics of the methane series are useful.

Morphine itself is a very powerful sedative to the respiratory centre, and frequently of much value for the relief of excessive cough. It has, however, the drawbacks, not only of the danger of the formation of a habit, a danger which must be an ever-present spectre with the physician, and of its marked effeet upon the digestive organs, but also that it checks the bronehial secretions. The dryness of the mucous membrane caused by it may so irritate the sensory nerves that at times the drug will increase instead of relieve the cough.

The morphine substitutes, as has been pointed out, being relatively feeble in their effects upon cerebral functions, are less liable to give rise to narcomania. 'They are likewise less prone to produce digestive disturbance, and are generally to be preferred to morphine as cough sedatives. They differ among themselves more in the degree than in the quality of their action. The acetyl morphine, commonly known as heroine, appears to be the most powerful respiratory sedative of the group, exceeding even morphine itself in this direetion.

'The asthmatic paroxysm consists essentially of an obstruetion of air in and out of the lungs, commonly due to a spasm of the bronchial museles, brought about through abnormal impulses from the respiratory centre. It is erident that we ean orereome this spasm, not only by lessening the functional activity of the respiratory eentre, but also by diminishing the conductivity of the peripheral pathway to the bronehial museles. 'The most valuable agents for this latter effect are atropine and hyoscyamine. By paralyzing the pulmonic terminals of the vagus, which is the motor nerve of the bronchial museles, these drugs are able to relax the spasm. As their action is peripheral, better results ean be obtained, with less disturbances of other functions of the body, if they are introduced loeally by inhalation than if administered systemically. Lobelia appears to have a similar effect on the bronehial nerve supply, but tends rather to increase than diminish secretion.

Drugs Used to Affect Bronchial Secretion.-The proper application for an acutely inflamed mucous membrane is a demulcent. In the case of the bronchi, however, because of the anatomical condition the difficulties of applying directly soothing drugs, practically the only efficient means of soothing an inflammation is to increase the demulcent secre- 
tion of these tubes. 'The sedative expectorants are those drugs which increase the bronchial secretions.

In the general relaxation and increased glandular activity which occurs during nausea the bronchial mucous membrane shares, and the central emetics, therefore, all tend to increase the bronchial secretions Of the nauseating expectorants, ipecacuanha is the most generally useful. Antimony or tartar emetic, while sometimes of service in sthenic cases, has so powerful an influence upon the circulation that it should be employed only in robust individuals. Apomorphine, which is theoretically: valuable, is so unstable a body, especially in solution, that it is difficult to be sure how much active drug the patient is recciving. There is some evidence that ipecac exercises not only a direct, but also an indirect, influence upon the lungs, for Pecholier has shown that toxic doses of emetine produced marked pathologic changes in the lungs. When using this class of remedies as expectorants, it is not necessary that they be given in doses sufficient to vomit, but only to preduce a mild degree of nausea.

Potassium citrate tends to increase the quantity and fluidity of the bronchial secretions. 'This effect is probably the result of two factors. Its salt action increases the volume of the secretion of the bronchial as well as the other glands of the body, and, being converted in the system into a carbonate, it maintains the alkalinity of the secretions, hence favoring their fluidity.

The effects of the ammonia salts, especially the chloride and the carbonate, which are so popularly employed in the treatment of acute bronchitis, are probably due almost entirely to a salt action, although it is possible that minute quantities are eliminated through the bronchial mucous membranes and exercise a direct influence.

The so-called stimulating expectorants inchude a large number of irritant substances, which are elininated in greater or lesser part through the lungs, and exercise, therefore, upon the bronchial mucous membranes a direct local irritant action which tends to restore tone to passively dilated blood-vessels. 'They are to be avoided in conditions of active inflammation, as in the early stages of acute bronchitis, and find their field of utility in the later stages of bronchitis, after the acute irritation has subsided, or in those forms of bronchitis which are chronic from the beginning. They do not affect directly bronchial secretion, but when this is overabundant from congestion, their local influence on the blood-ressels will lead toward the reesstablishment of normal secretion.

'This group includes such remedies as eucalyptol, creosote, the various turpentine derivatives (terebene, terpin hydrate, etc.), tar, oil of sandalwood, balsam of Tolu, and a host of other aromatic and balsamic drugs. Precisely what the relative clinical value of these substances is it is hard to say, but if creosote, guaiacol, eucalyptol, and tcrebene are left, the others might all be dispensed with in the treatment of bronchitis and no one be the loser. 



\title{
THE COMBINATION OF DRUGS AND PRESCRIP'IION-WRITING
}

\author{
By A.MBRose HUNSBERGER, Ри.D.
}

IT is not the purpose of this article to consider the purely fundamental parts of prescription construction, these being available in several wellwritten works, and are thoroughly taught in the progressive medical colleges of to-day, but rather to refer to the practical results of this training in some medical schools, as indicated by a more or less close observation of a large number of prescriptions. Such suggestions or comments as are ventured by the writer are offered in good faith, with the one thought in mind, that here and there the reader may note some fact which heretofore seemed somewhat troublesome to understand.

It should be borne in mind that all these observations are made from the viewpoint of the apothecary, and therapentic considerations and questions of practice generally are excluded.

'The availability and efficiency of drug therapeutics are to be measured to some extent by the degree of proficiency in the art of formulation developed by the physician. Lack of precise knowledge of the action to be anticipated from a drug or combination of drugs; selection of improperly prepared drugs; under- or overdosage; improper combinations; pharmaceutical incompatibilities; neglect to observe the saturation point in the patient; infrequent or overfrequent repetition of doses, not to mention the psychic effect of unsightly, nauseating, and vile-smelling potions, are credited with being potent factors in creating a certain feeling of distrust in the minds of many medical practitioners as to the benefits to be derived from the application of drug therapy. 'These factors have had the further effect of leading some physicians to lose confidence in their ability to construct a preseription that would he therapentically effective, and at the same time acceptable to the senses of sight, taste, and smell; and they, therefore, receive with open arms the ready-made product of the manufacturer-the elegant pharmaceuticals, which sometimes are found to be more elegant than eflective.

It is generally coneeded, however, that drug therapy of this type is both unseientific and unnecessary-unseientific, because it allows no latitude for the application of the physician's fundamental scientific training, and unnecessary, because any ohservant medical practitioner should be able to acquire a proficiency in prescription construction, which, as far as therapeutic efficiency goes, will meet the requirements of any emergency that may arise, and possess a degree of elegance that will compare favorably with its efficiency. 
It must be borne in mind, of course, that the point to which palatability in an extemporaneous prescription can be carried is limited by the general character of the drug, and the necessity at times of using a certain method of administration. Strychnine, for instance, is said to be discernible to the organs of taste when diluted in 20,000 parts of water. It is obvious, therefore, that if it be expedient to administer this substance in distilled water a patient with a strong aversion to bitter substances would find this dose quite objectionable. When the physician is not limited in his selection of the formula, however, the matter of masking the taste of such a substance becomes very simple indeed.

It may be said then that-(1) correct prescription writing, (2) selection of the proper dosage, (3) precise wording, and (4) legible writing constitute four essentials in the construction of an order, uttered by a medical practitioner, calling for the preparation and administration of a medicinal substance, or combination of substances, such an order being termed a prescription.

The necessity for legible writing and precise wording is, however, not limited to the order for ready-made or extemporaneous preparations; it is equally important to exercise the same care when uttering a written order for any of the numerous auxiliary agents and appliances considered necessary for the prompt alleviation of suffering and the ultimate restoration of the patient.

\section{THE PRESCRIPTION BLANK}

The first topic properly taken up in a discussion of prescription-writing is the prescription blank. The use of a convenient and neat form upon which to transcribe the order for medicine indicates a methodical interest in the patient's welfare, and the practising physician should at no time during active practice find himself without a pad of blanks in his pocket. A general observation of this caution would preclude the uncertainty inrolved in deciphering a prescription hastily scrawled upon the margin of a newspaper, a piece of a paper bag, a diminutive visiting card, or a frayed fragment of coarse wrapping-paper-a practice that is happily infrequent and might well be unknown.

In selecting a form of prescription blank, those of diminutive or colossal size, bizarre coloring, and awkward type should be studiously avoided. 'The form of preseription blank finding general favor consists of a sheet of strong, smooth finish linen or bond paper of medium weight, measuring $3 \frac{1}{2} \times 5 \frac{1}{2}$ inches. The color is usually white, although a pale blue shade is occasionally used. The color of ink used in printing the heading is preferably black or blue-black and not glossy, and the type used, while not necessarily severely plain, should be readily legible.

'The printed heading should include the name of the practitioner, address or addresses, consultation hours, telephone number, and, if a limited or partly limited prescription, it must also include a printed statement which indicates its limited character.

The use of an ornamental letter or monogram alone as a heading for a prescription blank is not to be encouraged, since it is difficult at times 
to establish the identity of the author of a prescription writton upon such a blank, especially when the signature is not readily deciplucralle. ()ecasions arise reguiring that the preseriber le communicated with, and it should not be necessary to interrogate the patient as to the mance and address of his medical adviser.

A prescription written on a blank such as the above is usually recognized as being unlimited; that is to say, the patient uses his own discretion as regards continued use of the medicine by hiuself (or his fricnds) without consulting the preseriber. A simple method of placing a limitation upon a prescription written upon this form consists in writing above the preseriber's signature an appropriate phrase, such as "Not to be Renewed or Copied," or "This Preseription must not be Renewed without Written Assent of the Prescriber," or "Ne Repetatur."

The attention of the patient should be direeted to this limitation at the time of consultation, since limiting the use of a prescription is designed solely for his benefit and protection. It furthermore afiords an opportunity for the preseriber to impress upon his patient the propriety of giving the medical adviser full sway in the applieation of necessary remedial measures. The use of such injunctions as "ne repetatur," which might seem to the patient to be lacking in frankness, is rather to be advised against from the druggists' standpoint. When confronted with a notice of limitation at the time of applying for renewal of the prescription the patient usually resents what he terms an abridgement of his rights, and, because he was not made aware of the limitation by the author of the prescription at the time of its issuance, he is prone to criticize it as a surreptitious act borne of an ulterior motive. Such an attitude of mind need not be engendered, however, if a free and frank understanding exists between the two directly interested parties, the doctor and the patient.

'The utter futility of attempting to limit a patient's use of a medicine, when he is carrying a copy of the preseription about with him is obvious. While the original compounder would, in all probability, refuse to renew the preseription, there is little doubt that some one could be found willing to disregard the limitation of a copy and aid the patient in circumventing the first compounder as well as the preseriber.

When the worls, "Copy formula on label" are in the prescription, it bears an injunction, the propriety of which has been seriously questionerl, since indiscriminate circulation is given in this way to such preseriptions.

The copy of a preseription thus brought constantly before the eyes of a patient furnishes an incentive to self-medieation. There appears to be a certain fascination about studying prescription formulas that appeals strongly to many patients, some of whom derive a peculiar pleasure from airing their somewhat limited knowledge of drug therapy, and applying the same in a more or less indiscriminate fashion to their ailing associates and themselves.

In some cases formulas acquired in this fashion are filed by the patient. and these files are consulted in quite the same fashion as the "Family" Doctor Book." Again, they are used for purposes of comparison with 
any subsequent prescription that may be written for the family. This self-inspired confidence often reaches the point of passing judgment upon the value and applicability of such remedial agents as the medical attendant may see fit to prescribe, even to the point of requesting the compounder to omit or add other ingredients, a request never to be acceded to.

'This little and dangerous knowledge of names of drugs is no doubt largely responsible for the ever-increasing, indiscriminate, and reckless use by the laity of such substances as acetanilide, antipyrine, strychnine, arsenic trioxide, mercurial preparations, organotherapy products, and hypnoties of all types, the catchy trade-names of the coal-tar chemicals rendering this class of products particularly susceptible to misuse.

The profound respect once universally entertained for the dangerous qualities of such substances has been supplanted by a contempt bred by familiarity with the prescription formula. The pharmacist who has the temerity to warn such laymen of the risks involved in the haphazard use of potent drugs is usually discomfited by the argument that a doctor ordered it originally, and the medicine is therefore perfectly safe and harmless.

Arguments in favor of printing the injunction directing a copy of the prescription to be placed upon the label consist in the facility with which precise information is supplied to the physician as to the dosage, combination, etc., of medicine preseribed upon a previous oceasion, perhaps quite remote. Such information is, of course, at all times to be had from the custodian of the prescription, or from the file of carbon copies kept by many physicians, but of these resources the first is time-consuming and the second might fail in the case of a visit to the patient's abode. Under such conditions the advantage of having the formula placed on the label is obvious.

The practice of requiring such copies should invariably be followed when one physician assists another in his practice, and particularly when a physician is contemplating a prolonged absence from practice and the work is to be given in charge of another practitioner.

If it is deemed desirable to keep a complete record of prescriptions written, this may be accomplished by making use of a duplicate prescription-blank pad, having each blank interleaved with a thin sheet of paper. By inserting carbon paper between the two sheets, exact copies of the prescriptions written remain in pad form after the originals are torn off. 'These copies, if numbered consecutively, dated and filed in proper order, greatly simplify the keeping of case records, in that, as the case progresses, instead of copying each individual preseription upon the record card, reference may be made to the number of the carbon copy of the prescription. By this method effort, time, and card space are economized.

The plan of using ruled spaces for the several divisions of weights and measures is a landable effort in the direction of simplifying the art of prescription-writing, but it raises the question whether it is not as simple to distinetly write the proper symbol as it is to locate the column set aside for it. 'The likelihood of selecting the wrong column seems 
greater than that of writing the wrong symbol. "l'here would not be the slightest difficulty in fixing the responsibility when this form is used for an error made by inadvertently using the wrong column.

Some physicians use a blank ruled in the following manner:

Orvicr: Hours:

JoHn Jonfs, M.D.,

21 North Fourteenth Street, Philadelphia.

Telephone.

IF.

Oz. Dr. Gr.

An innovation, known as the "Square Deal" blank, was originated by a prominent Philadelphia pharmacist. 'The limit of use to which a prescription may be restricted, when written on this blank, becomes absolute if it is properly signed.

This prescription is written for the person whose name appears thereon, for the present indications only; henee it is NOT TO BE RENEWED without my written consent, and NO COPY OF SAME IS TO BE GIVEN. The pharmacist compounding it will kindly preserve this sheet on his preseription file.

If, for reasons of his own, the patient is disinelined to accept treatment under the conditions imposed, he will properly terminate the relationship at that point. If he accepts the conditions as outlined, and pays the required fee, he enters into tacit, if not actual, contract relations with the prescriber, and is in honor bound to observe rigidly such injunctions as his medical adviser has issued.

Requests for the renewal of limited prescriptions are very rare, indicating that practically all patients are quite willing to have the physician control their use of medicines, as well as restrict their diet, curtail their amusements, and rearrange their mode of living, provided it is made clear to them that it is a necessary part of the treatment. 'They will resent, however, having any one but the physician demand rigid observance of the restriction.

When desirous of placing an absolute limitation upon the use of medicine ordered on this blank, it is imperntive for the physician to attach his 
signature to the conditions on the reverse side. Failure to do this would be understood as indicating that the prescription was not intended to be a limited one, and a request for renewal of such a prescription would be honored by the original compounder. It will be observed, therefore, that this form of blank may be used for both limited and unlinited prescriptions. In the first case the conditions must be signed, and in the second they are ignored, the prescriber simply attaching his signature beneath the body of the prescription in the usual manner.

In summarizing the discussion of prescription-blank forms it may be said that it is generally recognized that the physician, being the originator of the prescription, is, therefore, responsible for its proper application, and, having assumed that responsibility, it is quite equitable that full control of the prescription must be afforded him, to the extent of stating the conditions under which it is ordered, the use (brief or extended) to which it shall be put, by whom it is to be used, and what final disposition shall be made of it.

In this connection it is suggested that the practitioner familiarize himself with the laws as to narcotics of his district, many States having enacted statutes which specifieally forbid reissuing copies of prescriptions containing certain potent drugs. A request for a copy proscribed by law would, of course, be refused, and embarrassing explanations might be necessary to justify the refusal.

If the physieian decides that his obligations to himself, his patients, and to the community demand that he limit the indiscriminate use of all or some of his prescriptions, he will derive considerable aid in carrying out his determination by adopting the prohibitive form of prescription blank.

\section{PRESCRIPTION-WRITING}

Prescriptions should preferably be written with pen and ink, and the excess ink be carefully absorbed with a blotter before handing the prescription to a patient. In emergencies, a lead pencil may be used, which should be of a medium degree of hardness and well pointed. Blunt or soft-lead pencils and indelible pencils are to be advised against, as it is difficult to form the characters properly with them, and with the latter the prescription becomes an undecipherable blotch if a drop or two of water is splashed upon the written surface.

'The character of the prescription to be written, whether for a single ingredient or a combination, should be thoroughly evolved before the act of writing begins. Patients or their families are quick to note and comment upon any apparent indecision. Furthermore, erasures, changing of quantities by writing one figure upon the other, and tearing up and rewriting the prescription are procedures to be guarded against.

'The hand-writing should be firm, the lines straight, and characters properly formed, and alsogether free from flourishing displays of chirographic dexterity. "I's" should be dotted, 't's" crossed, and recognized rules of punctuation followed.

Whenever possible, the name and sex of the patient, which may be 
indicated by the prefixes Mr., Mrs., or Miss, or the given names, "John" or "Elizabeth," should be stated. 'The address is properly added also, and frequently serves a good purpose in differentiating between two patients of the same name who are under treatment at the same time. The date upon which the prescription was given to the patient should also be stated.

The ingredients of the prescription should be stated so fully and clearly that there cannot be the slightest grounds for conjecture. While it is not necessary, in most instances, to write the final " $i$ " or "ium" in a fiveor six-syllable name in order to fully convey the prescriber's intention, in should not be assumed that the same lopping-off process may be applied with impunity to a two- or three-syllable word. Abbreviations like the following examples are involved, indefinite, and likely to prove dangerous:

"Sodium chlor." may indicate chloride or chlorate of sodium.

"Sodium nitr." may indicate nitrite or nitrate of sodium.

"Sodium sulph." may indieate sulphite or sulphate of sodium.

"Hydr. chlor." may indicate calomel, corrosive sublimate, or hydrated chloral.

"Quinin. hyd." may indicate hydrochloride or hydrobromate of quinine.

These instances might be multiplied indefinitely, but the examples given will serve to indicate the nature of ambignous abbreviations. When a substance is prescribed that is infrequently used in the practice of medicine, yet having a name similar to one often prescribed, a distinct mark should indicate that the infrequently used drug is the one to be dispensed, e. g., Acidi Sulphurosi.

A similar plan is well followed when large doses of potent substances are to be dispensed:

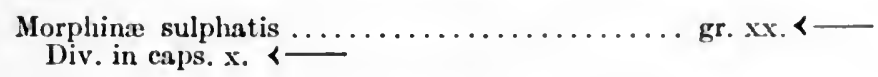

The two symbols, 5 and 5 , indicating respectively the ounce and dram, are sometimes difficult of identification, because of blurred writing or careless forming of the additional curve on the ounce symbol. 'This should receive especial attention, plenty of space being used on the blank to give it prominence. Equal catution must be observed in forming the dram mark, so that there may not be the slightest suspicion of the extra eurve which designates the ounce mark. A hair-line unwittingly placed in proximity might mean a dose increased eight times. 'The scruple mark is little used, most prescribers preferring to employ its equivalent in grains.

When measures of volume are preseribed, the corresponding symbols should be employed, thus, $f z$ or $f 5$.

As a rule, the names of such official substances as are preseribed by volume indicate their fluid character, but there are exceptions to the rule which require the use of the symbol denoting measure by volume; therefore the habit of differentiating between gravimetric and volumetric quantities should be formed $-\overline{3}-\bar{z}$ for solids, $\mathrm{f} \overline{\mathrm{z}}-\mathrm{f} \mathbf{3}$ for liquids.

When stating the ingredients of a prescription, it is well to platee the 
quantity desired after each ingredient at once. The habit of stating all the ingredients before any quantities are specified frequently results in the latter being forgotten.

When quantities are once stated, no attempt should be made to change by writing on top of the figures first placed. The original figures should be obliterated by erasure or cross-marking, and the final figures be placed upon a clean surface. 'The method of stating directions to the patient should be neither brief nor pedantic. 'The nature of the dosage for should receive consideration in its relationhip to the directions, as, for instance:

"'Take or swallow one capsule, with water, after each meal."

"Place one powder on the tongue, take a sip of water, and swallow."

"Immerse one konseal in water several seconds, take up with spoon, and swallow."

"Dissolve one on the tongue every hour."

"Insert one suppository at night."

"Inject three times a day."

The following examples clearly imply the degree of action:

"Apply to scalp with gentle (or vigorous) massage."

"Apply to part with gentle (or vigorous) friction."

"Anoint face with gentle rubbing."

These clearly outline important details of application:

"Saturate a 4-inch square (or circle) of lint with lotion, apply, and cover with waxed paper (or oiled silk)."

"Dissolve one teaspoonful (or one tablet) in one-half glass of water and drink during effervescence."

"Dissolve one tablespoonful in a quart of boiled water and use lukewarm for injection."

Such brief statements as the following should be avoided as a general rule:

"One pill," “Apply,” “Lotion,” “Wash (Salve, Dusting-powder, Eye Drops)," "Rub in," “Headache Powders," “Inhale," “T. i. d.," "As directed."

While, no doubt, supplementary verbal directions will be given in most cases, it should be remembered that these are fixed in the patient's mind only in the degree permitted by his mental state, which may vary frequently from being a trifle off the normal to a point approaching actual liysteria.

When medicine is to be administered three times a day, the thoughts of the ambulant patient clrift to the meal hours. If these are the hours in the mind of the prescriber, the directions should state whether the medicine is to be taken before, during, or after meals. If meal hours can be disregarded, a clear way to state directions is as follows: "Take one teaspoonful (pill, powder, or capsule) at 9 A. м., 3 P. м., and 9 Р. м." Meaningless directions may indirectly contribute toward fatal errors in prescription compounding, as is well illustrated in several instances in which "lysol," a poison, was dispensed when "laxol," an innocuous castor-oil preparation, was prescribed. It is conceivable that an unhappy 
combination of careless writing, and still more careless reading, of a preseription might transform laxol into lysol, and that the latter would be dispensed under an "As Directed" label without a thought of impending tragedy, but it incomprehensible that the error could occur if the directions were properly stated on the prescription.

Involved directions should also be avoided. 'The following is a specimen which presumes a nice sense of discrimination in the patient for whom the medicine is intended: "Take one, two, or three teaspoonfuls every two, three, or four hours." A better form to state this would be "l'ake one teaspoonful and repeat as needed, every two, three, or four hours."

The next is a specimen in repetition that has little dignity of expression: "For Charles Jones." "Give Charles one small teaspoonful in about a cuarter of a tumbler of cold water, fifteen minutes after breakfast, after lunch, and after dinner, three times a day as directed." 'The same meaning is conveyed by stating: "For Charles Jones: Give a teaspoonful in one-fourth tumbler of water three times a day fifteen minutes after meals."

Summarized directions on preseriptions should define the dose, frequency, and time of repetition, method of administration, and, in a degree, imply the character of the dosage form. Supplementary and extended directions, which cannot conveniently be placed upon a prescription label, should be written out on a separate preseription-blank and given to the patient or attendant.

Iists of supplementary appliances required in the treatment should also be written on a prescription blank, and the style, size, capacity, construction, ete., stated. 'The reason for this is the fact that most of the sick-room adjuncts exist in infinite variety, and the patient or his messenger is perhaps less qualified to select, than the plyysician is to indicate, the proper article to be procured. As an illustration of this, attention may be directed to a simple verbal order calling for a syringe. 'This might mean an ear, nose, rectal, urethral, male or female, hard or soft rubber, glass, tin, bulb, fountain, or piston, and of a capacity ranging from one dram to four quarts. Orders describing explicitly that which is desired may be stated as follows:

"Hard-rubber urethral syringe, 2 dram, blunt point."

"Soft-rubber ear syringe, one ounce, long flexible point."

"Glass ear syringe, curved bulbous point, one-half ounce."

"Fountain syringe, one quart, attach soft-rubber catheter No. 23 Fr." Fr."

"Fountain syringe, two quart, attach colon (or rectal) tube, No. 30

"Invalid cushion, very large (large, medium, or small)."

"Ice-bag (child or adult) for neck (or head, spine, knee, etc.)."

"Hot-water bag (size should be stated; varying from one-half pint to four cuarts)."

"Cotton, one ounce (two, four, eight, or sixteen ounces)."

"Adhesive plaster (deseribe width, length, and whether plain or medicated). 
"Oiled silk" (approximate size required should be stated).

"Atomizer (hard rubber, metal, oil, or water: for nose or throat)."

"Soft-rubber syringe attachments for giving enemas" (state which is desired, eatheter, length 14 inches, size 9 to 30 Fr.; rectal tube, size 22 to 35 Fr., length 20 inches, or colon tube, size 22 to 35 Fr., length 30 inches.

"Salts, Epsom (or Rochelle)," "Sodium phosphate: plain, granular, granular effervescent, or liquid)."

“Bandages," cotton (or gauze, woven elastie, flannel, linen, rubber, ete.), state width, length, and number desired.

"Douche pans, bed pans, etc."; size should be stated.

"Disinfectants" - character of same should be stated and method of using deseribed.

\section{EXTEMPORANEOUS DOSAGE FORMS}

The form in which the medicinal substance is to be administered to the patient merits the consideration of the prescriber. 'This in no sense implies that he needs to pander to every drug antipathy or idiosyncrasy of the whimsical or querulous individual, but rather to elicit in a diplomatie manner his likes and dislikes as related to drug administration. A patient with a strong dislike for a sweet, cloying liquid substance will often be appreciative of a combination that to another would seem extremely bitter and nauseating, and vice versâ, and there is the individual who, under no stress of eireumstances, can swallow a pill, but who will swallow a capsule three times as large with gusto. Others again prefer to take the dose in powder or liquid form, regardless of taste, rather than swallow either pill or capsule.

With small children the administration of drugs assumes a more difficult aspeet, the ingenuity of the experienced practitioner being sometimes taxed to the utmost in the effort to produce an attraetive and palatable dose. 'The only compensating factor lies in the fact that with children a materially reduced quantity of the active substance is necessary to produce the desired effeet, allowing a somewhat wider latitude for dilution and flavoring. Pills, tablets, capsules, and konseals should not be preseribed for young children.

The general rule of exhibiting the drug in the form preferable to the patient may be conveniently followed with a large number of substances, particularly with those which are given in small quantities and oceur in dry form. Very pungent, odoriferous, and deliqueseent substances, and those occurring as lirpuids, being less susceptible of adaptation to the available methods of administration.

'The liquid form is usually recognized as possessing the greater number of advantages, for the reason that soluble substances can be administered in their most readily assimilable form-inerease or diminution of the dose is easily secured, proper dilution is readily accomplished, and the addition of correctives is simplified. The composition of a vehicle for solutions usually includes water, glycerine, and alcohol, in varying proportions and combinations, or used singly, and colored, 
flavored, or medicated. 'The facility with which most extemporanerous liquid preparations of soluble substances may be supplied is a factor to be remembered when urgent necessity for innediate remedial moalsures of a medicinal characier arise. I) insoluble, in the vehicle indicated, may be given in this wat by increasing the density of the earrier through the addition of proper quantities of syrup plain, flavored, or medicated, glyeerine, acacia, tragacanth, I rish moss, gelatine, etc. Such liquid forms are termed suspensions, and they are of an aqueous character. Insoluble substances exhibited in this manner must, however, be susceptible of being reduced to a state of minute subdivision, the finer powders being capable of more prolongerd suspension. All insoluble powders, of a greater or lesser specific gravity than the vehicle, will collect in the top or bottom of the vessel, and the vehicle should, therefore, be of a degree of limpidity that will facilitate complete disintegration of the lumped powder, and insure thorough distribution of the medicinal substance throughout the vessel. 'The patient should be direeted to shake the vessel vigorously until the appearance of the contents indicates thorough admixture, then pour out the dose immediately. It is not advisable to use the above method for insoluble substances of high potency, for the reason that the patient would find it extremely diflicult to determine the degree of sliaking necessary because of the uncertainty of the ocular test (his only one) as applied to the minute quantity of the drug necessarily present. Among the substances to be excluded as not fitted for exhibition in the form of aqueous suspensions are alkaloids, insufficiently soluble salts thereof, elaterin, phosphorus, the insoluble salts of mercury, antimony, etc.

Insoluble drugs, to be used in the form of injections, should, as a rule, be exhibited in the form of suspensions, the vehicle being made sufficiently dense to maintain proper distribution of the drug during the time required to fill and empty the syringe, the substances best adapted for this purpose being bland and non-irritating mucilages of acacia, chondrus, eydonium, starch or tragacanth jelly, properly diluted, In some cases liquid white petrolatum answers admirahly.

Another type of suspension is the emulsion. 'This consists of a homogeneous admixture of substances, such as oils, oleoresins, gum-resins, balsams, camphor, ete., which are ordinarily immiscible with, or insoluble in, desirable vehicles. 'Through the addition of gummy or gelatinous substances, and by aid of proper manipulation, what is known as emulsification is brought about, whereupon the addition of adjuvants, correctives, flavors, and a desirable vehicle is reatily accomplisherl. Other liquid forms include collyria, lotions, liniments, washes, types of which will be indieated later.

Next in desirability to the liquid form of drug administration and application is the dry or powder form. 'This method is limited to substances that are reasonably permanent under exposure to atmospheric conditions, and which are, comparatively speaking, tasteless, olorless, and free from overly pungent qualities. Doses of very large or extremely minute bulk may be given in this manner, by having the patient mix the 
former with a proper quantity of water, milk, or wine, to produce a semiliquid consisteney, and the latter may be directed to be made up to an appreciable bulk by the addition of milk-sugar, cane-sugar, or other comparatively inert substances.

As suggested before, only such substances as are reasonably free from disagreeable odor and taste should be administered in powder form. Obscrvation of this precaution alone, however, will oceasionally fail to overcome aversion to drugs displayed by some patients, particularly ehildren. This may invariably be remedied, and the dose made attractive instead of repugnant, if the prescriber will direct the addition of a few drops of oil of peppermint, anise, cinnamon, or such other flavor as the patient may express a preference for.

It is quite necessary to add a sufficient quantity of sugar in the presence of these flavoring agents to impart a distinetly sweet taste to the powder, the attractiveness of the essential oils existing only in their agreeable taste - as brought out by the sweetening agent.

If the use of sugar is precluded, the same degree of palatability may be seeured by mixing the powder in a spoon with an aromatic water containing 25 per cent. of glycerine.

Next in favor to the powder as a dosage form is a gelatine capsule. These are supplied in two forms, commonly termed hard and soft. 'The hard capsule consists of two eaps of very thin gelatine, the larger fitting closely over the open end of the smaller, making a compact, tight, and readily soluble covering for powders, solids, masses, semisolids, and oily liquids having a disagreeable odor and taste. The hard eapsules are available in a variety of sizes, the smallest being capable of holding about one grain of milk-sigar, and from this size they gradually increase to a size limited only by the swallowing capacity of the human being.

Very deliquescent substances should not be direeted to be dispensed in capsules, as these, beeause of their watery character, are prone to dissolve the gelatine.

When directing a patient to use capsules, he should be directed to hold the capsule in the mouth for a fraction of a minute before swallowing. This softens the gelatine and facilitates its disintegration in the stomach.

Soft capsules consist of a somewhat thicker shell of gelatine, of a soft flexible consisteney, maintained by the presence of glycerine in the gelatine, oval in shape, and open at onc end to allow for the introduction of the liquids which they are designed to carry into the stomach. After filling, the open ends are sealed with hot gelatine and the capsule is ready to be swallowed.

Although the soft capsule has a bulk considerably larger than the hard type, swallowing is no more difficult because of its flexible, yielding character. Soft capsules are employed for the exhibition of oils, oleoresins, ete., and but rarely for dry sulstances. Watery or fluid preparations, made with diluted alcohol or glycerine, because of their solvent action upon the gelatine, cannot be dispensed in soft capsules.

Preseriptions can be dispensed in hard eapsules more expeditiously 
than when soft eapsules are directed, a factor to be borne in mind when emergency remedies are to be supplied. Quantities varying from five minims to eighty minims, may conveniently be administered in soft capsules.

Konseals are bowl-shaped disks, made of dried flour paste, and are splendidly adapted for administering powders that are given in more or less bulky doses, such as combinations of rhubarb, magnesia, charcoal, ete.

Quantities up to 20 grains may be given by this methorl with less discomfort to the patient than would be experienced by swallowing a eapsule containing an equal weight of poweler.

'The patient should be directed to immerse the konseal in water for several seconds before taking it. 'This softens the flonr-paste shell and renders swallowing exceedingly easy. 'The konseal should be taken up, floated upon a portion of the water in a spoon.

Pills are round, oval, or double-convex forms of suitable size for swallowing, and containing in each form a proper dose of medicinal substance. Pills are very well adapted for the administration of solid extracts, resinous and gummy substances, and other solid drugs, whose single-dose bulk does not preclude swallowing without discomfort.

A pill presents the dose of the drug in its most compact form, a distinct advantage when there is difficulty in swallowing. When freshly made, with the proper excipient, disintegration is prompt and invariable.

Occasional reports by medical men have indicated the passige of pills through the body intact. The reason for occurrences of this sort can, no doubt, be traeed to ready-made pills of uncertain age or to the mistaken zeal of a dispenser, who has the erroneous notion that a perfect pill must needs be a hard pill.

A pill-mass should not be stiffened beyond the point necessary to retain a reasonable semblance of shape.

When a pill has been well covered with the proper dusting-powder and the external surface has dried, the taste of the drng is scarcely perceptible to the patient swallowing it. If it be expedient, however, and time allows, pills can readily be supplied with a tasteless coating. 'The esthetic sense of the patient may be appealed to by applying a gold or silver coating, or the pills may be coated with gelatine, sugar, chocolate, tolu, or salol, the latter when drug action is sought in the intestines.

If it is desirable to cover the taste of the pill-mass and time is limited, the pill may be placed in a gelatine capsule, bearing in mind that the bulk of the dose will be somewhat increased thereby.

Tablet triturates furnish an ideal method for administering drugs given in small doses, such as atropine, strychnine, hyoseine, calomel, nitroglycerine, ete., which have the quality of comparative tastelessness. 'These tablets may be prepared extemporaneously with expedition, are of small bulk (from one-half to one grain), and disintegrate immerliately upon contact with water.

Tablet triturates usually consist of the medieament, cane-sugar, milk-sugar, or both, moistened to a firm paste with alcohol, and presied 
into suitable molds, from which they are removed after a few moment's "setting" and dried.

'This form of dosage can be made particularly attractive to petulant children, since the flavor of a favorite confection can be imitated by the addition of a trace of one or more of the essential oils.

When prescribing tablet triturates, the error of referring to the form desired as "triturates" should be avoided. The term "triturate" can only imply what the word means-namely, a drug or combination of drugs rubled to a fine powder-and can in no sense be taken to indicate that a form of tablet is clesired. It is obvious, therefore, that confusion can only be avoided if the full name of the dosage form be employed, $i . e$, "tablet triturates."

Hypodermic tablets, as the name indicates, are primarily intended to supp'y a ready means for preparing solutions of medicinal substances to be used subcutaneously. 'These tablets are but rarely prepared extemporaneously, partly for the reason that their almost invariable use is of an emergency character, and partly because most medicaments used in this form are reasonably stable, and can, therefore, be carried in stock by the dispenser without risk of marked deterioration in strength.

Should the stock form of hypodermic tablets be undesirable or lacking in the needed combination, they may be prepared extemporaneously, provided the necessary time may be had. The method of preparation is similar to that of tablet triturates, except that the diluent used must be freely soluble, non-irritating, and sterile.

Hypodermic tablets may be, of course, given by the mouth to bridge over an emergency, when time and distance preclude the prompt availability of other dosage forms.

Compressed tablets are small, round disks, having convex or flat upper and lower surfaces, of sizes which vary with the bulk of the dose of the medicinal ingredient exhibited, but usually of a size that can be swallowed comfortably and eonveniently by the adult patient.

The particles of substance in this form are held together as a result of heavy pressure exerted upon the powdered or granulated drug confined in a strong metal cylinder in dose portions. The pressure is exerted by appropriate machinery, operating a plunger with a stroke, which is atljusted to produce the proper degree of compression necessary to form the loose particles into a solid disk.

'This dosage form has achieved wide-spread popularity, probably because of the extreme economy of labor which may be practised in the production on a large seale, and the consequent low price at which large inanufacturers have exploited ready-made compressed tablets. Some idea may be gained of the facilities with which stock tablets are produced when one considers that a single machine will compress from 150 to 400 tablets per minute. 'The term "stock tablet" refers to compressed tablets, prepared from stereotyped formulas and kept in stock by the dispenser.

'The wisdom of the somewhat general use made of this form has been seriously questioned by medieal practitioners at different times. While 
granting the admissibility of administering in this form readily soluble substances, such as the bromides, iodides, etc., other less soluble or practically insoluble substances, such as salol, quinine, terpin hydrate, ett., have been thought to be entirely precluded from being exhibited in form of compressed tablets. 'There is a strong probability of such tablets failing to disintegrate because of the insoluble character of the drug substance. Cases have been reported describing the passage of compresied tablets through the body and recovery from the feces in practically intact form.

While it is possible that failure of compressed tablets to disintegrate may be due to drug insolubility, it is quite probable that the devices employed to produce a stock tablet that will not crumble may also be a factor. This applies particularly to tablets designed for shipment and frequent handling.

Such tablets are frequently found to be of a brick-like degree of hardness, which may be the result of compression under tremendous force, or may be due to the presence of adhesive substances, such as acacia, tragacanth, dextrine, and glucose, which are added in most cases to promote cohesion of the particles of drug substance when granulating the powder for compression.

The author of a work on compressed tablet manufacture remarks, "Substances requiring granulation comprise a large majority of medicaments entering into compressed tablets. . . . In order to produce tablets which will possess the proper qualities ... medicaments entering into their composition must be mixed with certain excipients adapted to the needs of the particular substance or combination." Further on, in this same work on compressed tablets, it may be found that among the various foreign substances that enter into the manufacture of compressed tablets, in addition to the adhesives above mentioned, are flour, cherry gum, cane-sugar, milk-sugar, ammonium chloride, sodium chloride, starch, magnesium carbonate and oxide, tale, boric acid, and petroleum oil, these added excipients and adhesives constituting from 15 to 75 per cent. of the weight of some tablets. A list of permissible artificial coloring agents is also given.

This would seem to indicate that the innocent-looking little disk of pure drug substance is a rather more formidable proposition than would appear to the cursory observer.

While some manufacturers have honestly striven to extend the list of remedies that may be made in this form, by experimentation with various methods of combination, and prompt elimination of those found to be lacking in the qualities necessary to produce a tablet that would be true to the formula, and could be depended upon to produce the desired result, it is also unfortunately true that other manufacturers have sadly lacked this spirit of discretion, and have offered tablets in combinations that are practically useless.

The published results of investigations, made by the Council of Pharmacy of the American Medical Association, indicate that the day has not yet arrived when implicit trust and confidence may be placed in com- 
pressed tablets as a desirable dosage form for any very extended list of remedies.

Extemporaneous preparation of compressed tablets, as may be noted, is limited to such drugs as are available in, or may be readily reduced to, a coarse granular powder. Given such a substance, one that is not affected by unfavorable atmospheric conditions, and compressed tablets can be prepared with the same facility as are capsules or konseals.

Considerable difficulty would be experienced by the average pharmacist, however, in manipulating a preseription calling for hygroscopic substances, such as iodides, powdered extracts, ete., on a humid day, or in preparing for prompt administration a combination requiring trituration, moistening, granulating, and drying, previous to eompression.

Since their only raison d'etre is cheapness, and that only applying to stock tablets, it would seem as if the compressed tablet, lacking the equal advantage of other solid dosage forms, might well be eliminated from the list of desirable extemporaneous dosage forms.

If it be expedient to make use of stock tablets, the prescriber should assure himself of the reliability of their source, the proper admixture of the medicament, the accuracy of dose, proper disintegrating or soluble qualities, and that their cost is not lower than the actual cost of the ingredients they are supposed to contain.

'Troches or lozenges are hard, flat, solid disks of a consisteney that insures their slow solubility in the mouth. They are used to exhibit medieinal substances whose prolonged contact with the mucous surfaces of the mouth and throat is desired, this result being aecomplished by having the patient dissolve them on the tongue.

The component parts of the lozenge mass usually consist of the medicinal ingredient, flaroring agent, cane-sugar, tragacanth, acacia, or elm bark, proper cohesion being seeured by the addition of water, syrup, or mucilage, and the whole kneaded into a tenacious mass.

The mass is rolled out to the necessary thickness, the lozenges are cut to the proper shape and size, and dried-spontaneously, if the medicinal ingredients are of a volatile character, and with slow heat if no volatile substances are present. Palatable flavoring agents, available for use in lozenges, include oils of anise, peppermint, wintergreen, acacia, etc. Extract of glycyrrhiza finds favor with many patients.

When lozenges requiring extemporaneous preparation are preseribed the patient should be informed that from one to four hours is necessary for their preparation.

Suppositories are solid forms of various shapes, used for the purpose of conveying medicinal substances into the rectum, urethra, ragina, nose, and ear. Cacao-butter is the general vehicle used in making suppositories, its melting-point being just below the body temperature, and yet sufficiently high to allow necessary manipulation at ordinary temperature.

There are three processes used in the preparation of suppositories made from eacao-butter, namely, fusion, compression, and mass method. The last method is to be preferred, for the reason that it facilitates thorough admixture of the medicament with the vehicle, eliminates the danger 
slowly poured upon the exposed wool, where it is gradually absorbed, filling the body of the capsule with a medicinally saturated wool. The cap of the capsule is then replaced and the tampon is ready for insertion, after being dipped in warm water to secure necessary lubrication. The patient should always be directed to medicate the tampon just previous to use.

'The action of the glycerine within, and the body warmth and moisture enveloping the tampon, dissolve the gelatine capsule, allowing ready contact of the medicinal substance with the parts. An attached thread accomplishes removal of the pledget of wool when its medicinal property is exhausted.

Substances, such as iodine, boroglyceride, alum, tannin, opium, ichthyol, and many others may be dissolved or suspended in glycerine in the proper proportion and applied by this method.

Prescriptions for medicaments intended for use in tampons should be so adjusted that one fluiddram or less will constitute the dose to be applied. Quantities greater than this exceed the absorbent capacity of the wool. Tampons intended for average adult use have a diameter of about one inch and are two and a half inches long. Smaller sizes may be specificd for use by patients of very diminutive stature, tampons of a diameter of only one-half inch being available.

Ointments are prepared by thoroughly mixing medicinal ingredients with fatty substances, such as petrolatum, benzoinated lard, etc., and are usually of such consistency as to retain a solid form at the ordinary temperature, yet of a sufficiently low melting-point to assure liquefaction and ready distribution when applied to the body by inunction.

Ointments are prepared by several methods, the two generally employed in their extemporaneous preparation being those of fusion, when the ingredients are readily miscible, and mixing on a porcelain slab, when ingredients enter into the combination which are immiscible with or insoluble in a desirable vehicle. In selecting a vehicle for an ointment, consideration should be given to its qualities of absorption by the skin, its protective claracter, its miscibility with aqueous solution of medicaments, and to its freedom from irritating properties.

Yellow petrolatum is an ideal substance for use in conditions where a purely loeal action is indicated, or when it is desirable to apply a bland protective coating to a cutaneous surface. The better grades of yellow petrolatum have the desirable qualities of body, cohesiveness, freedom from irritative and odoriferous characteristics, and possess a further advantage of moderate cost.

White petrolatum is less desirable as a vehicle than yellow, because of the absence of the cohesive and homogeneous quality which characterizes the latter. 'This is particularly true of the snow-white variety, which is frequently found to be of a consistency which utterly lacks homogeneity, separating into a liquid and granular body at ordinary room temperature.

With such a vehicle it is difficult to produce an ointment of the requisite smoothness and physical stability. 
The variety known ats pearl white is somewhat more eligible, and may be used when the esthetic sense of the patient is not reconcilable to the yellow product.

'The prescriber should bear in mind that, in an ointment prepares with the petrolatum vehicle, absorption of the medicinal ingredient is reduced to the minimum.

Petrolatum has been exploited extensively under a variety of trademarked names. 'The use of such titles in prescription-writing is not to be commended. 'The official title should invariably be employed.

Benzoinated lard, when properly rendered, and impregnated with gum benzoin, provides an efficient vehicle for ointments, ranking second to petrolatum only in price, its cost being about five times that of the best petrolatum.

Benzoinated lard is white in color, possesses good body and consistency, a fragrance of its own, and is absorbed by the skin quite readily, Watery substances, up to about 10 per cent. of the weight of the ointment, may be added when this vehicle is employed.

When prescribing lard, the qualifying term "benzoinated" should always be used.

Hydrous wool-fat, as a vehicle for ointments, lends itself well to the addition of from 20 to 30 per cent. watery solutions, possessing in this feature a distinet advantage over other fatty substances. It furthermore is said to provide the best means of exhibiting medicaments designed to be absorbed by the skin. It is not to be recommended as a general ointment vehicle, however, as it possesses a disagreeable "sheepv" odor; it is not readily manipulated with dry powders because of its sticky nature; it does not readily mix with appreciable quantities of essential oils, camphor, etc.; nor does it answer well for inunction because of its tough consistence. The consistence of hydrous wool-fat can be altered somewhat through a "shortening" brought about by the addition of 25 per cent. of petrolatum. The facility of absorption and miscibility with water is, of course, correspondingly reduced by this means.

Solid saponated petrolatum is an ointment vehicle of good penetrating quality. It is a solid saponaceous combination of petrolatum, oleic acid, and spirit of ammonia, and is well adapted for substances such as methyl salieylate, salicylic acid, iodine, ete.

A liquid vehicle for external application having the same qualities for penetration is prepared by substituting liquid petrolatum for the solid variety. This is known as Liquid Saponated Petrolatmm, and it provides a splendid medium for exhibiting in solution substances lihe menthol, chloral, iodine, guaiacol, methyl salicylate, salicylic acid, ete.

Glycero-gelatin is a semisolid substance composed of a mixture of gelatine, glycerine, and water. 'Ihis mass melts at the body temperature and is used chiefly in dermatological practice as a vehicle for such substances as zinc oxide, ichthyol, iodoform, resorein. 'This preparation is entirely free from grease, and may, therefore, be remored by washing with water.

Dermatologic paste consists of a medicament such as precipitated 
sulphur, zinc oxide, starch, etc., worked to a stiff paste by the addition of an excipient such as soft soap, petrolatum, lard, glycerine, linseed oil, or dextrinated paste.

Paste pencils are hard cylindrical masses composed of a mixture of starch, tragacanth, dextrine, and sugar. These are also used in dermatological practice, chiefly for medicaments that are to be used frequently and applied directly to the skin.

Salve mulls are medicated ointments of a sufficiently high meltingpoint to allow handling after they have Been spread upon gauze by the compounder. 'The velicle usually consists of benzoinated lard hardened by the addition of suet or yellow wax. Salve mulls somewhat resemble medicated plasters, except that plaster vehicles usually contain rubber as part of the mass.

Cerates are prepared similarly to salve mulls, except that the patient spreads them just previous to use.

Ointments.-Unless there is a special reason for ordering a very soft ointment, the prescriber should avoid doing so. A reasonably firm ointment presents a very much more attractive appearance than one of the "sloppy" sort.

If the medicament or vehicle is of a disagreeable odor a few drops of oil of rose geranium, lavender, wintergreen, or other suitable substance that is calculated to mask the repugnant odor, may be added. Care must be exercised, however, that the quantity of oils added will not be sufficiently large to produce irritation of the parts for which the ointment is designed.

Some ointments undergo marked color changes while being used by the patient. This is particularly noticeable in light-colored combinations containing resorcin.

To overcome this tendency to change in appearance it has been suggested that coloring matter in sufficient quantity to produce a fairly dark ointment be added when the prescription is originally compounded. If this is thought desirable, a small quantity of powdered charcoal may be added.

Applying ointments with the finger is distasteful to many patients, and may be avoided if a glass or hard-rubber applicator is directed to be used. 'The convex side of a small spoon will answer in many cases, but the use of a metallic instrument is precluded when iodine, the mercurials, etc., are to be applied.

Most ointments are prone to turn rancid, and, therefore, should be ordered in quantities not to exceed a week's supply. On the other hand, if the condition of the patient warrants frequent and liberal applications, the quantity directed should be a proportionate one. Four repetitions of a one-ounce ointment will cost the patient considerably more than a single compounding of a four-ounce prescription.

Ointments are usually dispensed in porcelain jars or collapsible tubes of soft metal. While the latter is a cleaner method and adaptable to most combinations, long-established custom dictates the invariable use of the porcelain jar, unless tubes are specified. 
Ointments to be used in divided doses are usually dispensed in dose portions, these being wrapped in wax paper. 'This method is not a neat one, however. A mueh better impression will be made upon the patient if the divided doses are directed to be dispensed in individual tin boxes. Mercurial ointment may be exhibited in this manner, provided the inner surface of the tin is properly coated to prevent contact with the mercury. Dose portions of ointments prepared by the fusion method may also be directed to be dispensed in soft capsules of an appropriate size. The ends of these dose capsules are to be punctured by the patient when ready for an inunetion and the contents ejected upon the area by pressure. The preparation of sueh a prescription is less expeditious and more expensive than one dispensed in individual tin boxes.

The two methods above referred to are of particular advantage to the ambulant patient, in that he may conveniently carry one or more doses with him for midday use while following his usual vocation, thus insuring regular and methodical compliance with the instructions given by the medical adviser. 'The convenience of this same type of patient is also subserved when liquids are prescribed for internal use if the physician will direct that the preseription be dispensed in two portions, one to be used at home and one at his place of business.

\section{DOSE MEASURES}

The selection of appropriate dose measures for use by the patient usually receives little consideration from the prescriber. Probably the major portion of liquid medicines consumed is taken in doses which appreciably lack, or exceed, the quantity directed by the physician. Commendable progress has been made in the direction of improvement in pharmaceutical preparations. Precise elemical or physiological standards have been established for many drugs and many more are to follow. Standardization must go further than this, however, to be effective. If all the good is to be derived therefrom, the patient must be instructed to use a measuring medium of standard capacity.

The dose measures for liquids invariably indicated on prescriptions written in the apothecary's system are the dropper, teaspoon, dessertspoon, and tablespoon. Doses thus directed are calculated from the following equivalents:

$$
\begin{aligned}
& \text { One drop } \\
& \text { One teaspoonful }=\text { one minim. } \\
& \text { One dessertspoonful }=\text { two fluiddram. } \\
& \text { One tablespoonfut }=\text { four fluiddrams. }
\end{aligned}
$$

These equivalents are unreliable and dangerously variable. The average spoon is not made with any idea of accurate capacity in mind, investigation having indieated a variation of 10 to 35 per cent. from the assumed apothecary's equivalent, the variation being usually in excess of the supposed capacity.

The volume of a drop is still more variable, its size being influenced by the size of the orifice from which it is delivered, and by the physical properties of the substance used. 
A reference to the drug bromoform, a liquid that is frequently prescribed in drop doses, will illustrate the extreme uncertainty involved in calculating drop doses from the standpoint of an accurate relationship between drops and minims.

Sixty minims of bromoform weigh 150 grains in round figures. The same volume of this substance vietds 300 drops when delivered from a dropper with an orifice so constructed as to deliver a drop of distilled water of exactly one minim volume.

The official average dose of bromoform is stated in the U.S. Pharmacopœia at 3 minims. In order to give this dose by the drop method it is necessary to direct the patient to take 15 drops, provided a dropper is used that will deliver exactly 60 drops of distilled water to the fluiddram. Each deviation in the shape of the dropping end of the medicine-dropper will upset the proportionate number of drops to be directed.

These instruments are notoriously irregular, and an effort was made to correct the condition through the following recommendation, which was submitted to the U. S. P. Convention in 1900: "It is recommended that an official medicine-dropper have its delivery end $3 \mathrm{~mm}$. in external diameter, and adapted to deliver 20 drops of distilled water to a gram at $15^{\circ}$ C." 'This was referred to the Committee on Revision without recommendation.

The adoption of a standard dropper as suggested in the above recommendation would clarify the situation to the extent of eliminating those that fail to conform to the regulation as to size of delivery end. It would further have the effect of encouraging a schedule of drop doses arbitrarily based upon the activity of a portion of drug preparation as represented in a drop delivered from a standard dropper.

The activity of liquid preparations of drug substances is usually based on the stated number of strength units per fluiddram or cubic centimeters, neither of which bear a systematic relationship to a drop, standard or not; therefore the practitioner will find it more simple and precise to calculate his smaller doses in subdivisions of the quantities stated, than to attempt to remember the drop doses of the various preparations. Digitalis, for instance, is administered in the form of the powdered leaf, and it is, therefore, necessary to know the dose of the substance. It is also available in the form of fluidextract, tincture, and infusion, each preparation being of a percentage strength that differs from the other.

Members of the same classes of pharmaceutical preparations usually carry the same percentage of drug content, therefore it is necessary to remember only as many percentages of strength as there are classes.

The practitioner having acquired this knowledge, need but to know the dose of digitalis leaf, and the measured dose of the preparation thereof is readily calculated.

The arbitrary drop dose of each preparation would ha ve to be memorized as a separate entity, since the drops in each bear absolutely no sistematically calculable relation to the strength of the preparation nor to each other-the drop of the fluidextract being less than one-half, and the tincture a little over one-half, the size of a drop of infusion. 
In order to secure accurate administration of medicines, the patient should be directed to procure a dose glass about two inches wide at the top and tapering to an inside diameter of not over three-eighths of an inch at the bottom. The markings should be etched upon the glass and are properly graduated in fluiddrams with the exception of the first division, which should also indicate the half dram. 'The markings must, of course, be based upon accurate measurements.

Quantities of from 5 to 20 minims may be measured in pipettes graduated to 5, 10, 15, and 20 minims. Doses of less than 5 minims should be adjusted to that figure by adding a sufficient quantity of diluent of a suitable character.

If the metric system is used, small doses may be adjusted to quantities of $\frac{1}{4}, \frac{1}{2}, \frac{3}{4}$, and 1 c.c., and the patient be directed to use a pipette with the corresponding graduations. Doses from 1 c.e. up may be measured in a dose glass graduated in 1, 5, 10, 15, 20, and 30 c.c. measurements.

Dose measures of accurate adjustment, and of the style above described, are readily obtained at a low cost. 'The very common glass of wide diameter at the bottom having graduations pressed into the side is but little better than a spoon as a convenient and accurate measure, and is not to be recommended.

\section{PRESCRIPTION TYPES}

The following list of preseriptions covers most of the dosage forms: They are presented in the knowledge that no type forms are possible that would meet the requirements of all eases. Intelligent modification of the various forms, and a study of efficient vehicles available, will provide an armamentarium that will supply the needs of the average medical practitioner.

When prolonged use of one drug is indieated, it sometimes becomes necessary to alter the color, taste, or odor of a combination, or even change the dosage form, to avoid discouragement in the patient.

'This may be achieved with substances such as the iodides, bromides, etc., by using at first a vehicle consisting of an aromatic water sweetened with plain syrup; then changing to one eonsisting of distilled water with 20 per cent. each of glycerine and compound tincture of gentian; then change to equal parts of an aromatic water (not used previously), syrup, and elixir of cinchona; again a change to equal parts of water and compound syrup of sarsaparilla; then change to equal parts of compound tineture of cardamom, glycerine, and water. 'The essence of pepsin of the N. F. has found much favor as a general vehicle and may be used as such during a long period without presenting the same color, taste, or odor twice, if the plan outlined above is followed carefully.

Substances not adapted to liquid forms of administration may be exhibited alternately in the form of mass pills, round and flat; tablet triturates, capsules, konseals, and in the form of powders to which have been added various flavoring ingredients. 
The following formulæ illustrate the best manner of prescribing a large number of drugs commonly used.

\section{LIQUID FORMS FOR INTERNAL USE \\ Extemporaneous Solutions}

R. Sodii iodidi......................

Aqua destillat...................... ad f f $\mathrm{j} .-\mathrm{M}$.

Ft. sol.

Sig.-Ten minims in one-fourth tumbler of milk three times a day before meals.

This indicates a type of prescription requiring the least time for extemporaneous preparation and also an accurate method of administration.

P. Ammonii bromidi.............. 5iv;

Syrup. aurant. cort................ f f 5 j;

Aq. cinnamomi..................... ad f fij.-M.

Ft. sol.

Sig.-One dram in a half-glass of water before meals and at bedtime.

This indicates a type of flavored vehicle which is susceptible of numberless variations.

P. Ammonii chloridi ................ 3 ij;

Codeinæ.................... gr. iij;

Elixir cinchonæ...................... ad fón.

Ft. sol.

Sig. - $5 \mathrm{j}$ in water every three hours.

This type is adapted to patients who dislike very sweet, cloying vehicles.

\section{Liquors: Extemporaneous Dilutions.}

R. Liquor. potassii arsenitis............ f $\mathbf{z} \mathrm{j}$;

Tinct. cardamomi comp................ f f

Sig.-Five minims in water during meals.

P. Liquor. iodi composit.............. f $\mathbf{3}$ iij;

Sig.-Five minims in water after each meal.

These types indicate methods of increasing the dose portion to the capacity of the dose measure.

\section{Simple Suspensions or Mixtures}

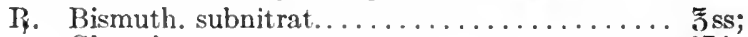

Glycerin.................... f $\mathrm{f}_{\mathrm{z}} \mathrm{j}$

Tinct. cardamomi comp.............. f f $\mathrm{s} s$;

Aq. menth. piperit................... ad f fiij.-M.

Sig. - $f$ jj after meals and at bedtime. Shake.

The addition of glycerine in this prescription indicates a method of increasing the density which promotes thorough admixture during the time required to measure the dose. 'The glycerine incidentally acts as a sweetening agent.

R. Terpin. hydrat................... gr. lxxij;

Heroin. hydrochlor................. gr. j;

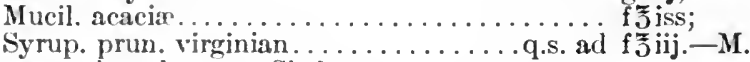

Sig.-f5j every three hours. Shake.

To this vehicle may be added numerous substances, such as eucalyptol, terebene, creosote, etc. 
Suspensions by Emulsification

Ol. ricini..................... $f \mathrm{f}^{\mathrm{j}} \mathrm{j}$

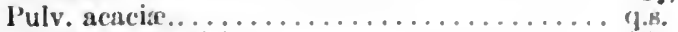

Spirit. vini gallici................ f ij;

Syrup........................ $\mathrm{f}_{3} \mathrm{j}$

Aq. cinnamomi. ........................ ad fon.

Sig.-Tablespoonful at bedtime.

R. Ol. gossyp. sem................ f fiv;

Ol. amygd. amar...................... q.

Syrup....................

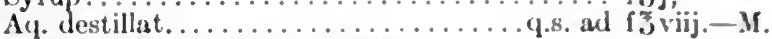

Sig. - $\mathrm{f}$ ss three times a day after meals.

'These two preseriptions indicate elegant methods for administering substances which otherwise might have a tendency to produce nausea. The flavors may be modified to suit the taste of the patient.

LIQUID FORMS FOR EXTERNAL USE

Collyrium

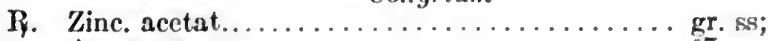

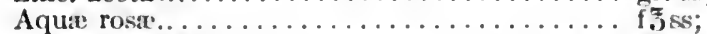

Aqua destillat....................... ad $\mathrm{f}_{\mathrm{Jj}} \mathrm{ij}$.-M.

Ft. sol.

Sig.-Gtt. $j$ in right eye every two hours.

R. Acid. boric................. $5 \ldots \ldots$

Aq. camphor..................... foss;

Aq. destil...................... ad fỗ $v j .-M$.

Ft. sol.

Sig.-Bathe left cye every four hours, using eye-cup.

'These two preseriptions indicate the importance of explicitly stating directions.

Lotion

R. Resorcin..................... 5 j;

Aquæ cologn.................. f

Ol. ricini.................... f

Alcoholis................................ ad fo.

Ft. sol. Dispense in sprinkler-top bottle.

Sig.- Sprinkle on sealp and massage vigorously at night.

R. Hydrarg. chlor. corros................ gr. ij;

Phenolis................... 5 ss;

Spir. violæ odorat................ f

Glyecrin.................... f $5 \mathrm{ss}$

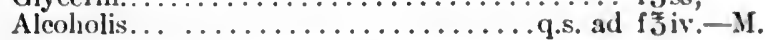

Ft. sol.

Sig.-Apply to sealp with dropper and massage gently once daily.

These two preseriptions indicate methods whereby the use of remedial agents of this type may be made agreeable to the patient.

\section{Douche}

R. Liquor antisep. alkalin.............. f

Sig.-Mix f $5 \mathrm{j}$ with 5 iij of warm water and use as nasal douche twice daily.

Mouth-urash

R. Liquor. formaldehyd................ mx;

Liquor, antisepticl...............

Glycerin...........................

Sig.-Use full strength as mouth-wash twice daily.

f.3j,-M.

This indicates an agreeable method of exhibiting the valuable but somewhat disagreeable solution of formaldehyde. 
P. Menthol................... gr. $\mathbf{x}$; Ft. sol.

Aqua cologn......................s. ad f $\tilde{z}^{\mathrm{j}} \mathrm{ij} .-\mathrm{M}$.

Sig.-Apply gently to forehead and temples with tips of fingers. Avoid contact with eyes.

The directions here indicate a proper warning to the patient.

P. Sat. sol. magnes. sulph.............. f f xvj.

Sig.-Saturate compress and apply, covering with waxed paper.

Indicates clearly proper method of use by the patient.

R. Potass. sulphid.,

Zinc. sulphat.................. āā ōiss;

Aquæ rosæ............................ ad f f

Sig.-Apply to face at night, using soft cloth. Shake.

'The odor of the vehicle in this prescription admirably covers the disagreeable odor of the medicinal ingredients.

\section{Gargle}

IF. Potass. ehlorat............... $\mathbf{3} \mathbf{j} ;$

Tinet. ferri ehlor.................. f fiv;

Glyeerin................... f

Aquæ destillata...................... ad f fiv.-M. Ft. sol.

Sig.-Use full strength as a gargle.

Nasal Spray

R. Menthol..................... gr. ij;

Camphor........................ gij;

Ol. eucalypt................. f $5 \mathrm{ss}$;

Ol. gaultheriæ..................... nxv;

Petrolat. liquid. alb.................s. ad f fo Fit. sol.

Sig.-Use in oil atomizer to spray nasal passages.

\section{Throat Spray}

R. Iodi.......................... gr. iv;

Potass. iodid.................. gr. viij;

Glycerin..................... f 5 iss;

Aquæ menth. piper.................s. ad f f $^{\mathrm{j}} \mathrm{j} . \mathrm{-M}$.

Ft. sol.

Sig.-Use in hard-rubber atomizer for spraying throat.

These two preseriptions indieate the proper use of modifying ingredients; while the directions specifically imply the type of instruments to be used.

\section{Alcoholic Liniment}

R. Ol. sassafras...................... f5iv;

Ol. origan...............

Tinct. capsiei..................... f $5 \mathrm{ij}$

Alcoholis........................ ad fo.

Sig.-Apply externally with gentle frietion.

\section{Oily Liniment}

R. Camphorx. $5 \mathrm{ij}$;

Ol. terebenth..................... f Jiv;

Ol. gossyp. semin.................. ad f $\mathrm{f}_{\mathrm{Z}} \mathrm{ij}$.-M.

Ft. sol.

Sig.-Apply externally with vigorous friction. 
F. Tinct. opii,

Soapy Liniment

Tinct. aconit.

Chloroformi,

Methyl salicyl................

Liniment. saponis..................... ad f f $^{3}$ ij. -M.

Sig.-Apply to affected part with gentle rubbing.

IF. Menthol.................

Chloral. hydrat.................

Methyl salicylat.................. f

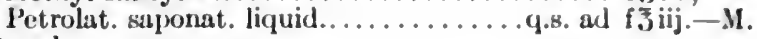

Ft. sol.

Sig.-Apply with gentle massage.

li. Iodi........................... gr

Petrolat. saponat. liquid................... ad $\mathrm{f}^{\mathrm{J}} \mathrm{ij}$.

Ft. sol.

Sig.-Apply with prolonged rubbing.

The foregoing types give a somewhat faint idea of the many admirable vehicles available for use with liniments.

Rectal Injection

R. Bismuth. subcarb............... $\boldsymbol{3}_{\mathrm{j} \text {; }}$

Tinct. opii....................... f

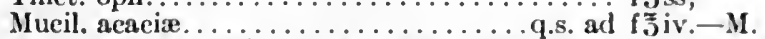

Sig.-Mix one tablespoonful with a quart of warm starch water and use for rectal injeetion. Shake.

This prescription indicates an effectual suspension method.

\section{Urethral Injection}

R. Argent. nitrat...................... gr. ij;

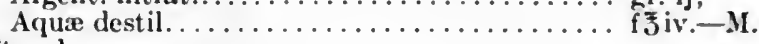

Ft. sol.

Sig.-Inject $f \tilde{3} \mathrm{j}$ twice daily.

The directions here preclude accidental wrong application.

R. Zinc. sulphat.,

\section{Urethral Injection-Suspension}

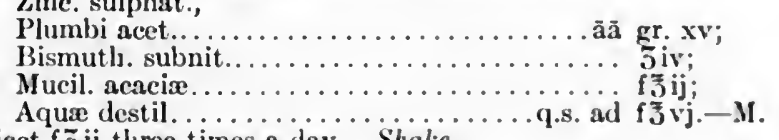

Sig.-Injeet f 5 ij three times a day. Shake.

The addition of a substance such as mucilage of acacia gives the proper density to the vehicle. Withont such addition it is quite impossible to maintain a proper admixture of the medicinal ingredients while the dose is being drawn into the syringe.

\section{Paints}

R. Phenolis........................... $\mathrm{gr}$;

Aleoholis............................

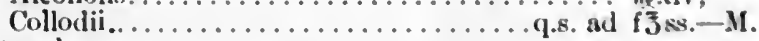

Ft. sol.

Sig.-A pply with a small glass rod.

While camel's-hair brushes are usually directed for use with a prescription of this type, they become practically useless after the first few applications, and the glass rod will be found to be a decided improvement. 
R. Chloral hydrat.,

Camphore....................

Sig.-Apply to painful parts with camel's-hair brush.

This prescription indicates method of securing full local effect of drug action.

\section{SOLID FORMS FOR INTERNAL USE}

\section{Powders}

P. Hydrarg. ehlor. mit................. gr. j;

Pulv. ipecac....................... gr.

Sacchar. lac.................

Ol. anisi..........................

Div. chart. no. $x$.

Sig.-Give one powder on tongue followed by sip of water; or mix one powder with water in a spoon.

The flavor of a prescription such as this may be altered in many ways by the substitution of differently flavored essential oils, etc.

R. Pulv. carbon lign.,

Pulv. rhei,

Magnes. oxid.,

Ext. glycyrrh. pulv............... āã $\bar{\jmath} s s .-M$.

Sig.- Take an even teaspoonful in four tablespoonfuls of water twice daily.

The addition of powdered extract of licorice to this combination renders palatable a dose that would otherwise be nauseating to many patients.

R. Sodii sulph. exsic.,

Magnes. sulph. exsic.,

Sodii phos. exsic................ āā $\overline{5} \mathbf{j}$;

Sodii chloridi..................

Sodii bicarbonat.............

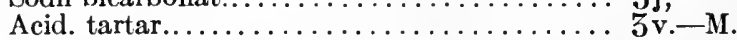

Sig.-Dissolve $5 \mathrm{j}$ in one-half glassful of water each morning and drink during effervescence.

This method of prescribing effervescent powders may be followed in cases where the official forms do not answer the requirements.

\section{Solid Substances in Hard Capsules}

R. Strychnin. sulph.................... gr. $\frac{1}{4}$;

Quininæ valcrian.................... gr. xx;

Ferri valerian.............

Div. in caps. no. $\mathrm{xx}$.

Sig.-Take one capsule before cach meal.

R. Aloin,

Ext. belladonna.................āā gr. ij;

Strychninæ sulph................... gr. $\frac{1}{3}$

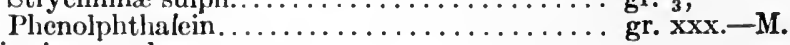

Div. in capsul. no. $x x$.

Sig.-One morning and evening.

Method of masking bitter and odoriferous substances. Quantities of the various ingredients may be adjusted to fit the individual case. 


\section{Hard Capsule. Combination of Solid and Liquid}

R. Heroin. hydrochlor.................. gr. j;

Terpin. hydrat................. 5 ji

Eucalyptol..................... n w

Pulv. glycyrrhizii radicis............ q.8.-S.

Div. in capsul. no. xx.

Sig.-One capsule cvery three hours.

Other substances such as terebene, creosote, in equal volume may be substituted for the eucalyptol.

\section{Hard Capsules. Semi-solid}

R. Ichthyol................... 5 j;

Pulv. glycyrrh. rad.................... (1.8. -M.

Div. in eapsul. no. xx.

Sig.-Take one capsule before each meal.

Substances such as strychnine, arsenic trioxide, aloin, ete., may be added in combination.

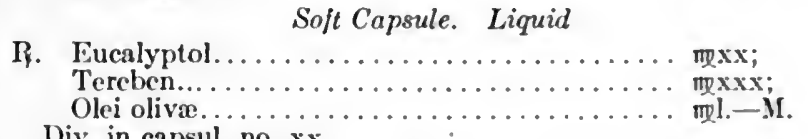

Div. in capsul. no. $\mathrm{xx}$.

Sig.-Take one capsule after meals.

'This combination fills the smallest size of soft capsule. If desirable, cod-liver oil may be substituted for olive oil and quantity increased to 40 minims per dose.

Soft Capsule. Solid Dissolved in Liquid

R. Salolis........................ gr.
Ol. santal.................... f 5 ij.

Ft. sol. Pone in capsul. no. xx.

Sig.-Take one capsule cvery thrce hours.

To the above combination may be added such substances as balsam copaiba, oil of cubebs, ete. The addition of $1 \mathrm{minim}$ of oil of cassia to the capsule overcomes disagreeable eructations.

\section{Mass Pills}

R. Strychnina sulphat is............... gr. f;

Aloin,

Ext. belladonna.................. åa gr. ij.-MI.

Div. in pilula no. $x x$.

Sig.-One night and morning.

Type of very small pill.

R. Elaterin....................... gr. j;

Ext. lyoscyani.................... gr. xx;

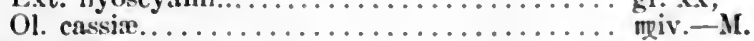

Div, in pil. no. $\mathrm{xx}$.

Sig.-One pill night and moming.

14. Ext. nucis vomica................ gr. iv;

Ext. cascare sagrade,

Ext. sumbul..................... a ga gr. xx;

Mass. ferri carb...................... gr. c. $-M$.

Div. in pil. no. $\mathrm{xx}$ (double convex).

Sig.-Take one after each meal.

When it is desirable to give a large-sized pill, the double convex shape should be directed, as it is more readily swallowed. 
R. Stryehnin. sulph.................. gr. $\frac{1}{3}$;

Fel. bovis inspis............. gr. $\mathbf{x x x}$

Panereatin......................... gr. xl.-M.

Div. in pil. no. $\mathrm{xx}$ (salol coated).

Sig.-One pill before meals.

Mass dosage forms requiring a coating to disguise taste or odor should be dispensed in hard capsules if needed for emergency use, since some time is required to apply and dry the usual pill coating.

Konseals

R. Aloin......................... j;

Quininæ sulphatis................

Acetphenetidina................... gr. c;

Caffeinæ citrat........................... gr. $\mathrm{xl}$

Sodii bicarb...................

Div. in konseals no. $x x$.

Sig.-Immerse one in water, take out with a spoon and swallow, as directed.

Indicates best method for administering large doses of bitter powders.

\section{Tablet Triturates}

R. Hydrarg. chlor. mit............... gr. ij;

Pulv. ipecac..................... gr. ss;

Sacchar. lactis..................... q.s.

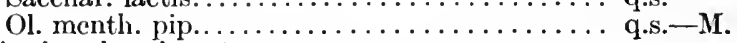

Div. in tab. triturat. no. xx.

Sig.-Take one tablet every thirty minutes.

Any favored flavor may be substituted for the oil of peppermint.

P. Strychninæ nitratis.................. gr. j;

Spirit. glycerylis nitratis.............. ml;

Sacchar. lac............................

Div, tab. triturat. no. 1 .

Sig.-Take one every four hours.

This illustrates the combination of a liquid with a solid.

\section{Lozenges}

P. Orthoform..................... gr. xx;

Mentholis........................ gr. $\mathrm{j}$;

Phenolis............................... gr. $x$

Sacehar. et pulv. tragacanth...........āā q.S. -M.

Div, in troch. no. $\mathrm{xx}$.

Sig.-Allow one lozenge to dissolve on the tongue every hour.

\section{SEMI-SOLIDS FOR LOCAL APPLICATION Futty}

R. Ichthyol.................... $3 \mathrm{ij}$

Adipis benzoinatis.........

Sig.-Apply every four hours, rubbing gently.

R. Mentholis...................... gr. ij;

Camphora..................... gr. $\mathbf{x}$;

Methyl. salicylatis....................

Ol. oliva $\ldots \ldots \ldots \ldots \ldots \ldots \ldots \ldots \ldots \ldots$ f 5 ss;

Sig.-Apply with gentle friction.

Adipis lani hydros.................

'The two above represent absorptive ointments. 
R. Acidi borici.

Bismuth. subnitrat. ...........

Phenolis........................... $x$;

Petrolati....................... כ vj.-MI.

Sig.-Apply to affected part twice daily.

This type of vehicle gives a purely local effect.

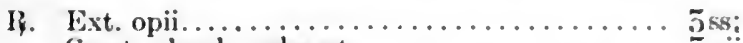

Cerat. plumb. subacet................

Sig. - Spread upon muslin and apply. Renew twice daily.

R. Ext. belladonna,

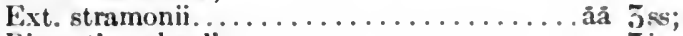

Bismuth. subgall...................

Adip. lani. hydros...................

Petrolati.......................

Sig.-Use in pile pipe within rectum morning and evening.

R. Ung. hydrarg..................

Adipis lani hydros..................

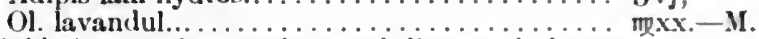

Divide into twelve portions and dispense in boxes.

Sig.-Use one box daily by inunction.

The above prescriptions indicate different methods of dispensing and applying ointments.

R. Ichthyol.,

Zinc. oxidi,

Acidi borici ................

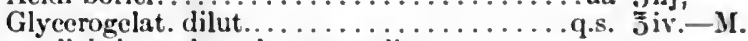

Sig.-Warm slightly and apply on muslin. water.

Local applications of this type may be removed by washing with

Suppositories

R. Extract. hamamelid............... 5 ss;

Extract. opii.................... gr. xviij;

Acid. tannic...................... gr. xviij;

Ol. theobroma...............

Div. in suppos. no, xii (adult rectal).

Sig.-Insert one morning and evening.

If. Ichthyolis. gr. vi;

Extract. opii...................... gr. iij;

Ol. theobroma q.s.-.I.

Div. suppos. no. vi (adult nasal).

Sig.-Insert into affected nostril at night.

R. Cocaina hydrochlor.................. gr. j:

Extract. opii........................

Ol. theobroma............... q.s.-M.

Div. suppos. no. vi (adult aural).

Sig.-Insert into the ear once daily.

R. Ichthyol................... .

Glycerit. boroglycerin.............. faiv;

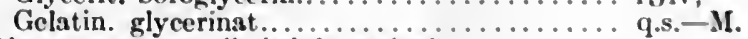

Div. suppos. no. xii. (adult vaginal).

Sig.-Insert one at night. 
R. Protargol................... gr. vj;

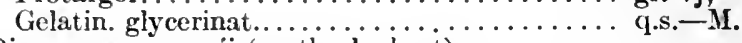

Sig.-Inscrt one at bedtime.

The above types clearly indicate the character of vehicles, size, style, and method of use.

Tampon
P. Glyeerit. acidi tannic ............. f5ij;

Glycerini...................

Sig.-Pour one teaspoonful upon tampon and insert as directed.

Wool tampons, large, no. xii.

For use with liquid as directed.

This indicates method of prescribing tampons and medication.

P. Pulv. alumen,

\section{Powders for Local Use}

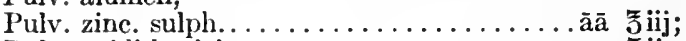

Pulv. acidi borici.................

Phenolis................... f3 j;

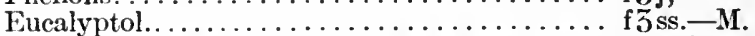
亏ij measure.

Sig. - $5 \mathrm{ij}$ dissolved in two quarts of hot water and use as douche. Dispense in

I\%. Bismuth. subgall.,

Thymol. iodidi,

Zinci stearat.,

Acidi boriei...................āa $\bar{z} s s ;$

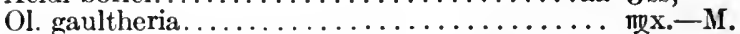

Dispense in shaker-top box.

Sig.--Sprinkle upon affected surface twice daily.

This indicates method of application and masking drug odor.

If. Saceharin.,

Mentholis................... āa gr. j;

Camphoræ.................... gr.

Cocainæ hydroehlcridi................ gr. v;

Talc. purificat.,

Acidi borici...................āā $\mathbf{5} \mathbf{i j} .-M$.

Sig.-Apply to throat with powder blower twice daily.

A method of overcoming disagreeable taste in a throat application.

While the foregoing list will give a general idea of the variety of methods available for administering and applying medicinal agents, many variations of these methors will suggest themselves to the practitioner. Excellent results in the production of palatable and elegant prescription forms are achieved frequently through consultation of the physician with the pharmacist. While it is probably true that some pharmacists fail to measure up to their responsibilities, it is equally true that there are scattered throughout the country many capable and conseientious workers in the pharmaceutical field whose constant ambition is the advancement and uplift of their profession.

The difficulty in the matter, of course, lies in selecting the one who is a capable pharmaceutical consultant. A fair description of such a pharmacist probably is that given in a booklet distributed by the Phila- 
delphia Branch of the American Pharmaceutical Association at the Convention of the American Medical Association held at Atlantic City, New Jersey, in 1907. Under the caption, "Ilow to 'Tell a Competent Pharmacist" this booklet goes on to say: " . . Not an inconsiderable number of retail druggists have given their attention largely to the attention of other business than drugs. . . . There are, however, many apotheearies who are anxious to clivoree themselves from the traffic in nostrums and notions and devote their energy to the practice of pharmacy along strietly professional lines. . . The corle of ethies of the American Medical Association advises that its members shall recommend to their patients competent apothecaries, but the differentiation between the reliable and untrustworthy is not always easy. There are three readily applied tests which are of great importance in judging between the real pharmacist and the mere shop-keeper. 'The first is, Does he show or take an active interest in the deliberations of societies that are devoted to the advancement of the science of pharmacy? If a pharmacist is at all worthy of the name, he must be interested, and keep himself informed of the advances that are made from day to day. 'To do this he must become conversant with the current pharmaceutical literature and be willing to contribute his observations to the sum total of knowledge in this particular line. . . . The second test concerns the text-book or works of reference that are to be found in his shop. Any pharmacist to fully comply with the existing laws designed to regulate the practice of pharmacy, should have and use the latest editions of the Pharmacopoeia of the United States and of the National Formulary. . . 'The third test is to be found in the facilities and appliances that the pharmacist has to make the official preparations and to test the drugs and chemicals that he may be compelled to buy. . . . Any physician who insists that these three comparatively simple tests be complied with by the apothecary whom he recommends to his patients, may rest assured that his prescriptions will be accurately compounded, and that he and his patients will receive the best service that the resources of modern pharmacy ean offer."

Further evidence of this proper interest in the advancement of the allied interests of medicine and pharmacy is displayed in the adoption by the Philadelphia County Medical Society and the Philadelphia Braneh of the American Pharmaceutical Association of a "Declaration of the Status of the Preseription," which declares that:

"First: The preseription is an utterance of the preseriber, who alone should direct and control its employment. It should, whenever practicable, be dated, and the name of the patient with his (or her) age, if a minor, should be specified.

"Second: Prescriptions are either of limited or unlimited character. A limited prescription is one on which restrictions have been placed by the preseriber, viz., to control the ownership, to limit the privilege of renewal, to state if a copy shall be given by the pharmacist compounding it, to direct the disposition of the prescription, and thus control all of the afore-mentioned rights. 'These restrictions should be specified over the 
signature of the prescriber. Limited prescriptions should be employed by the prescriber when writing for a medicinal substance commonly called a narcotic or habit-forming drug. The physician, when delivering a restricted prescription, should impress upon the patient that it is an order for medicine required only for the time stated and present needs; and the attention of the patient should be directed to the character of the restrictions thereon.

"An unlimited prescription is one which has no restrictions placed thereon by the prescriber, all rights being waived by the author. An unlimited prescription should be used when the copy of the prescription is directed to be placed upon the label of the container of the finished preparation.

"Third: The pharmacist who prepares the medicine should retain the prescription for reference as a record of his services and for a certain period of time-not less than five years-for the protection of the prescriber, pharmacist, and patient; and all restrictions placed upon the prescriptions must be respected by the compounder thereof. The attention of the patient should be directed to the nature of the restriction in a prescription by affixing an appropriate notice on the container of the medicine.

"Fourth: A copy of a restricted prescription should be written upon a special blank upon which is clearly stated the nature of the limitations, and if such direct that no copy of the original shall be given, none should be issued except upon the written request of the author. error."

"All copies of prescriptions are made without recourse for possible

\section{A LIST OF SUBSTANCES WITH REFERENCE TO DOSAGE, FORMS, VEHICLES, ETC.}

In addition to the sample prescriptions already given, the following list of commonly used drugs will prove valuable for ready reference.

The official average doses are stated in the figures immediately following the name of the article. The suggested methods of administration are based upon the doses stated. Solubilities are approximated to the quantity usually exhibited in one fluiddram of vehicle, and are stated as "A.," alcohol; "W.," water; "G.," glycerine.

Acetanilidum (4 grs.). Sol. A. Give as powder, or in capsules and konseals; also in aromatic elixir.

Acetphenetidinum ( $7 \frac{1}{2}$ grs.). Sol. A. Give as acetanilide.

Acid. benzoic. ( $7 \frac{1}{2}$ grs.). Sol. A. Give in capsules and konseals; also solution in compound tinct. lavender.

Acid. citric. ( $7 \frac{1}{2}$ grs.). Sol. W. A. Give in syrupy vehicle, well diluted.

Acid. gallic. (15 grs.). Give in konseals or capsule.

Acid. hydriodic. dil. ( 8 min.). Give in syrupy vehicle, well diluted.

Acid. hydrochloric. dil. (15 min.). Give in lemon flavor syrupy vehicle through glass tube. 
Acid. hydrocyanic. dil. (1 $\frac{1}{2}$ min.). Give in equal parts syrup and water.

Acid. hypophosphorous dil. (8 min.).

Acid. nitric. dil. (30 min.).

Acid. nitrohydrochloric. dil. (15 min.).

Acirl. phosphorie. dil. (30 min.).

Acid. salicylic. ( $7 \frac{1}{2}$ grs.). Sol. A. Give in capsules or konseals.

Acid. sulphuric. aromat. (15 min.). Give with aromatic elixir.

Acid. sulphuric. dil. (30 min.).

Acid. tannic. ( $7 \frac{1}{2}$ grs.). Give in capsules or konseals, or as glycerite acid. tannic.

Acid. trichloracetic. Apply externally with glass rod. elixir.

Aconitina $\left(\frac{1}{0} \frac{1}{6} \mathrm{gr}.\right)$. Sol. A. Give in pill, capsule, or in aromatic

Ether $(15 \mathrm{~min}$.$) . Give in equal parts of alcohol and aromatic$ elixir.

Ether acetic. (15 min.).

Ethylis carbamas (15 grs.). Give as powder, or in konseals or capsules.

Alcohol. When ordering, specify kind desired and percentage.

Aloe purif. (4 grs.). Give in capsules.

Aloinum ( 1 gr.). Give in pill or capsule.

Alumen ( $7 \frac{1}{2}$ grs.). Sol. W. or G. Give in capsule or konseal.

Ammon. benzoas (15 grs.). Capsule, konseal, or suspension in syrupy liquid.

Ammon. bromidi (15 grs.). Sol. W. Give in aromatic water with 25 per cent. syrup or glycerine.

Ammon. carbonas (4 grs.). Sol. W. Equal parts aromatic water and demulcent syrup. Reacts with syrup squills and ipecac, clanging in part to ammonium acetate.

Ammon. chloridum ( $7 \frac{1}{2} \mathrm{grs}$.). Sol. W. Equal parts water or brown mixture, and syrup.

Ammon. iodidum (4 grs.). Sol. W. Give in 25 per cent. water and 75 per cent. syrup.

Ammon. salicylas. (4 grs.). Give in aromatic elixir with 10 per cent. comp. tinct. cardamom.

Ammon. valeras ( $7 \frac{1}{2}$ grs.). Given preferably as elixir.

Amyl. nitris. (3 min.). Direct in crushable glass bulbs or glassstoppered bottle.

Antimon. et potass. tart. ( $\frac{1}{10}$ gr.). Sol. W. Give as wine or in powder.

Antipyrine (4 grs.). Sol. W. Give in capsule, konseal, or in aromatic elixir.

Apomorphin. hydrochlor. ( $\frac{1}{3} \mathrm{gr}$.). Give in pill or capsule. Solution undergoes color change.

Aqua (medicated waters). Those having aromatic qualities which permit of their use as flavoring agents, as well as solvent vehicles, are as follows: 
Bitter almond, anise, orange flower, cinnamon, fennel, peppermint, spearmint, and rose waters.

Aqua hydrogen. dioxid. Instruct patients to avoid use of metallic instruments in contact with this liquid.

Argenti nitras. ( $\frac{1}{5}$ gr.). Readily decomposed. Give dry in capsule with diluent of bismuth subnitrate. Distilled water must be used for solutions.

Argenti oxid. (1 gr.). Give in capsule.

Arsenii iodidum ( $\left.\frac{1}{10} \mathrm{gr}.\right)$. Give in capsule.

Arsenii trioxid. ( $\frac{1}{30} \mathrm{gr}$.). Give in capsule, pill, or aqueous solution. Asafotida (4 grs.). Give in capsule.

Aspidium. Give oleoresin in soft capsule.

Atropinæe sulphas $\left(\frac{1}{160} \mathrm{gr}.\right)$. Give in tablet triturate, pill, capsule, or in watery solution.

Aurii et sodii chlor. ( $\frac{1}{10} \mathrm{gr}$.). Sol. W. Give in capsule or pill.

Balsam. peruvian. (15 grs.). Give in soft capsule.

Bismuth salts. Give as powder or in capsules and konseals, and in suspensions with syrup.

Bromoform (3 min.). Give as emulsion with acaciæ flavored with wintergreen.

Caffeine (1 gr.). Give in capsule or konseal, or in solution with sodium benzoate.

Caffeine citrate, effervescent (60 grs.). Palatable form for administering caffeine.

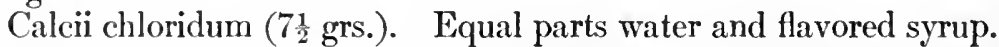

Calcii hypophosphis ( $7 \frac{1}{2} \mathrm{grs}$.). Give with water and lemon syrup.

Camphora. (2 grs.). Sol. A. Give in capsule or emulsion with 25 per cent. syrup tolu.

Calx sulphurata $(1 \mathrm{gr}$.). Give dry in capsules. Unstable salt.

Camphor. monobrom. (2 grs.). Give in capsules.

Carbo ligni (15 grs.). Give as powder or in konseal.

Chloralformamid. (15 grs.). Capsule or solution in aromatic elixir.

Chloral. hydras. (15 grs.). Sol. W. Give in aromatic water and syrup. sion.

Chloroform $(5 \mathrm{~min}$.$) . Sol. A. Give in alcoholic solution or enul-$

Cinchonidine and cinchonine salts should be given in capsules or konseals when the official liquid preparations do not answer.

Codeina and its salts ( $\frac{1}{2} \mathrm{gr}$.) may be given in hydroalcoholic solution, tablet triturate, pill, capsule, or powder.

Colchicini $\left(\frac{1}{100}\right.$ gr.). Sol. W. Give alone or in combination with salicylates in watery solutions, capsules, or konseals.

Copaila (15 min.). Give in soft capsules or as emulsion.

Creosotum (3 min.). Give in soft capsules, or syrupy cmulsion.

Cresol. Available for local application as liquor cresol comp.

Digitalis. Available as fluidextract, tincture, solid extract, and infusion. 'The liquid forms may be combined with any flavored hydroalcoholic vehicle; and the solid forms in pill or capsule. 
Elaterin ( $\left.\gamma^{\frac{1}{\gamma}} \mathrm{gr}.\right)$. Give in pill or capsule.

Elixir adjuvans. Good general velicle composed of fluidextract of glyeyrrhiza and aromatic elixir.

Elixir aromaticum. Good general vehicle composed of aromatic oils, syrup, water, and 25 per cent. alcohol.

Elixir ferri, quinina et strychnina phosphatum (1 fl. dr.). Give as such.

Emulsum olei morrhuae (2 fl. drs.). Is a 50 per cent. emulsion of cod-liver oil flavored with wintergreen.

Ergota. Used chiefly in form of fluidextract (1 drachm), which may be combined with aromatic elixir. sion.

Eucalyptol (5 min.). Sol. A. Give in soft capsule or syrupy emul-

Extractx. Used chiefly in solid dosage forms.

Fel bovis purificatum ( $7 \frac{1}{2}$ grs.). Give in pill or capsule.

Ferri carbonas saccharat. (4 grs.). Give as powder or in capsules and konseals.

Iron salts and combinations occurring as scales are usually given in aromatic elixir, most of them being available in official preparations.

Ferri sulphas exsiceat. (2 grs.). Give in capsules.

Fluidextracts, as a rule, are susceptible of admixture with flavored hydroalcoholic vehicles, the several striking exceptions requiring nearly full-strength alcohol vehicles being those of cannabis indica, cubeb, eriodictyon, eucalyptus, ginger, grindelia, lupulin, and veratrum.

In many instances the addition of glycerine to the vehicle is advisable to prevent precipitation upon standing.

Galla. Combined with benzoated lard for external use.

Gelatinum glycerinatum. Combination of gelatine, glycerine, and water, used as a vehicle for suppositories and substances for local application.

Glandula supraren. sicca. (4 grs.). Give in capsules.

Glandula thyroid. sicca. (4 grs.). Give in capsules.

Glandular extracts should, as a rule, be administered in unchanged form because of the delicate nature of the active principle.

Graiacol (S min.). Sol. A. Give in soft capsule.

Guaiacol carbonas (15 grs.). Give as powder or in capsule and konseal.

Hexamethylenamina (5 grs.). Sol. W. A. Give in capsules or aromatic elixir.

Hydrarg. chlor. corrosiv. ( $\frac{1}{26}$ gr.). Give in pill or capsule.

Hydrarg. chlor. mit. (1 gr.). Powder, capsule, or tablet triturate.

Hydrarg. iodid. flav. ( $\frac{1}{5} \mathrm{gr}$.). 'Tablet triturate, pill, or capsule.

Hydrarg. iodid. rub. ( $\frac{1}{20} \mathrm{gr}$.). Give as above.

Hydrarg. cum creta. (4 grs.). Give in capsule.

Hydrastina ( $\frac{1}{5} \mathrm{gr}$.). Gire in pill or capsule.

Hyoscine hydrobromide ( $\frac{1}{1} 0 \mathrm{gr}$.). Tablet triturate, pill, capsule, or in aromatic watery solution.

Hyoseyamine hydrobromide ( $\left.\frac{1}{2} \sigma \mathrm{gr}.\right)$. Give as above.

Infusum digitalis (2 fl. drs.). May be flavored by addition of syrup. 
Iodoform (4 grs.). Give in capsule. Externally in collodion, or in suspension with glycerinated gelatine.

Iodine $(10 \mathrm{gr}$.$) . Give in watery solution with potassium iodide,$ flavored with aromatic elixir.

Ipecac. ( $1 \mathrm{gr}$.). Give as powder, pill, or capsule; or in form of syrup.

Jalap (15 grs.). Combine powder with equal quantity of flavored sugar; or give in capsule or konseal.

Liquor antisepticus ( $1 \mathrm{fl} . \mathrm{dr}$.). This is a combination of boric and benzoic acids, thymol, eucalyptol, oils of peppermint, gaultheria, and thyme, in a vehicle of 25 per cent. alcohol and 75 per cent. water. Used internally and externally.

Liquor ferri et ammonii acetatis ( $4 \mathrm{fl}$. drs.). Combination of tincture of ferric chloride, with solution of ammonium acetate, aromatic elixir, and water. Excellent form for iron administration.

Liquor formaldehydi. An effective combination for mouth-wash is a 1 per cent. solution of this in a vehicle composed of 10 per cent. glycerine, 10 per cent. tinct. card. comp. in peppermint water.

Liquor iodi compositus ( $3 \mathrm{~min}$.). 5 per cent. solution of iodine in water. May be given in 25 per cent. solution of glycerine in cinnamon water.

Liquor potassii arsenitis ( $3 \mathrm{~min}$.). Give in this form or with an aromatic water.

Liquor sodii phosphatis comp. (2 fl. drs.). This dose represents 120 grs. sodium phosphate crystals.

Lithii benzoas ( 15 grs.). Sol. W. Give as powder or in capsules and cachets; also syrupy, aromatic water, vehicle.

Lithii bromidum (15 grs.). Sol. W. Very deliquescent. Give only in solution in syrup and aromatic water.

Lithii carbonat. ( $7 \frac{1}{2} \mathrm{grs}$.). Give as powder, capsules, or konseals.

Lithii citras ( $7 \frac{1}{2}$ grs.). See Lithii bromid.

Lithii citras effervescens ( 2 drs.).

Lithii salicylat. (15 grs.). Give as capsules or konseals.

Magnesii sulphas (240 grs.). Give in sweetened water flavored with lemon.

Magnesii sulphas effervescens (240 grs.). Give in water.

Mangani dioxid. praecip. (4 grs.). Give in capsules.

Menthol (1 gr.). Sol. W. Give in capsules. Externally in fatty or oily vehicle.

Methylis salicylas (15 min.). Give in soft capsules or syrupy emulsion.

Methylene-blue (4 grs.). Give in capsules.

Mistura creta ( $4 \mathrm{fl}$. drs.). Mixture of chalk powder with cinnamon and distilled water. Palatable vehicle for insoluble bismuth salts.

Mistura ferri comp. (4 fl. drs.). Combination of iron sulphate and potassium carbonate in rose water.

Mistura glycyrrhiza comp. (2 fl. drs.). A palatable combination of extract glycyrrhiza, syrup, tinct. opium camph., wine of antimony, 
and spirit of nitrous ether in water, largely used as a vehicle for ammonium salts, expectorant syrups, etc.

Mistura rhei et sodae (1 fl. dr.). Combination of sodium bicarbonate, fluidextracts of rhubarb and ipecac, glycerine, spirit of peppermin:, and water.

Morphine salts are adjustable to any of the dosage forms.

Myrrha ( $7 \frac{1}{2}$ grs.). Give in capsules.

Naphthalinum (2 grs.). Give in capsules or konseals.

Nux vomica. Usually given in form of extract in the usual dosage forms, or as tincture in hydroalcoholic aromatic vehicle.

Oleates include those of atropine ( 2 per cent.), cocaine ( 5 per cent.), mercury (25 per cent.), quinine (25 per cent.), veratrine (2 per cent.), in oleic acid, and oleic acid and olive oil.

Oils. Volatile oils whose aromatic qualities may be of use in disguising odor or taste of drug substances include bitter almond, anise, orange-peel, betula, cajuput, caraway, cloves, cinnamon, coriander, eucalyptus, fennel, wintergreen, lavender, lemon, peppermint, spearmint, nutmeg, allspice, rose, rosemary, santal, sassafras, and thyme. Oils lacking the agreeably odoriferous qualities of those included in the above list, but which are used frequently in medical practice are: Ethereal oil, oils of sweet almond, cade, worm-seed, copaiba, cubeb, erigeron, cotton-seed, juniper, cod-liver, olive, tar, castor, turpentine, mustard, theobroma, and eroton. A few of these are rarely if ever used internally. 'Those that are so used may be given in soft capsules or syrupy emulsions.

Opium. 'This substance is available in several official liquid forms. Give the solid substance in pill or capsule.

Pancreatin. ( $7 \frac{1}{2}$ grs.). Largely prescribed in capsules, but recommended by some to be given in salol-coated pills.

Paraldehydum (30 min.). Available as elixir.

Pelletierin. tannas (4 grs.). Give in capsule.

Pepo (pumpkin seed) (1 oz.). Give as emulsion.

Pepsinum (4 grs.). Give in capsule or as essence of pepsin, N. F.

Petrolatum. A yellowish colored vehicle for ointments. 'Takes up little water and is not well absorbed by the skin.

Petrolatum album. White ointment vehicle; less desirable than the above.

Petrolatum liquidum. Useful liquid vehicle for local application of oily substances; absolutely bland and non-irritating. water.

Phenol (1 gr.). Give in vehicle of syrup of ginger and cinnamon

Phenylis salicylas ( $7 \frac{1}{2}$ grs.). Give in capsule, konseal, or as powder.

Phosphorus ( $\frac{1}{2} \sigma$ gr.). Give in pill or capsule or as elixir.

Physostigmine salts ( $\frac{1}{60} \mathrm{gr}$.). Give in hydroalcoholic aromatic vehicle or in capsules.

Pilocarpine salts $\left(\frac{1}{5} \mathrm{gr}\right.$.). Give in syrupy aromatic water vehiele or capsule.

Pills. There are fourteen official formulas for pills, including a 
number of combinations that have been used largely. With the exception of pills of ferrous iodide, most of these combinations are better adjusted by extemporaneous prescriptions, as the individual requirements of different patients can be better met in this fashion.

Piper ( $7 \frac{1}{2}$ grs.). Give in capsules.

Piperina (3 grs.). Give in capsules.

Pix liquida ( $7 \frac{1}{2}$ grs.). Give in capsule or as syrup.

Plumbi acetas (1 gr.). Give in pill or capsule.

Podophyllum. Use resin ( $\frac{1}{10} \mathrm{gr}$.) in pill or capsule.

Potassii acetas (30 grs.). Sol. W. Give with lemon syrup and water.

Potassii bicarbonas (30 grs.). Scl. W. Give with syrup of tolu and aromatic water.

Potassii bitartras (30 grs.). Give in sweetened water and lemonjuice.

Potassii bromidum (15 grs.). Sol. W. Give with syrup and aromatic water.

Potassii carbonas (15 grs.). Sol. W. Give like bromide.

Potassii chloras (4 grs.). Sol. W. Use chiefly as gargle in combination with tinct. ferri chlor., glycerine, and water. Should not be prescribed dry in combination with sugar because of danger of explosion.

Potassii citras (15 grs.). Give in vehicle of lemon syrup and water.

Potassii citras effervescens. A palatable effervescent preparation of the above.

Potassii cyanidum ( $\frac{1}{5}$ gr.). Sol. W. Give in aromatic water or capsule.

Potassii dichromas ( $\frac{1}{5}$ gr.). Give in capsule.

Potassii et sodii tartras (120 grs.). Sol. W. Give in sweetened water with lemon-juice or carbonated water.

Potassii iodidum ( $7 \frac{1}{2}$ grs.). Sol. W. Give in aromatic water and syrup vehicle, or in essence pepsin, N. F., or as concentrated solution administered in milk.

Potassii nitras ( $7 \frac{1}{2}$ grs.). Sol. W. Give in aromatic water with syrup or in capsules.

Potassii permanganas ( $1 \mathrm{gr}$.$) . Give in capsule.$

Pulvis acetanilid. comp. (7 grs.). Composed of acetanilide, caffeine, and sodium bicarbonate. Give as powder or in capsule and konseal.

Pulvis aromaticus (15 grs.). Composed of cinnamon, ginger, cardamom, and nutmeg. Used as carminative, also to contribute flavor to substances administered as dry powders.

Pulvis glycyrrhiza comp. ( $60 \mathrm{grs}$.). Combination of senna, licorice, sulphur, flavored with oil of fenmel and sweetened with sugar. Give as such mixed with water.

Pulvis ipecac. et opii ( $7 \frac{1}{2}$ grs.). Composed of ipecac 1, opium 1, and sugar of milk 8 . Give as powder or in capsule.

Pulvis jalap comp. (30 grs.). Consists of jalap 35 and potassium bitartrate 65. Give as such, mixed with water or in konseals. 
Pulvis rhei comp. (30 grs.). Composed of rhubarb, magnesium oxide, and ginger. Give mixed with water or in konseals, or combined with powdered extract of licorice.

Quinine salts, all of which have distinetly bitter tastes, are best administered in capsules or konseals. If these forms are precluderl, the salts may be suspended in chocolate syrup or aromatic syrup of yerba Santa.

Resorcinol (2 grs.). Sol. W., A., or G. Best administered in capsules. Solutions undergo color change.

Rhamnus purshiana. Give extract in pill or capsule form; or use aromatic fluidextract, or plain fluidextract, combined with glycerine and aromatic elixir.

Rheum ( 15 grs.). Give as powder with powdered extract of licorice or in capsules and konseals.

Salicinum (15 grs.). Give in capsules or konseals.

Santoninum (1 gr.). Give as powder with flavored sugar milk.

Scilla (2 grs.). Give in eapsules or as syrup, tincture, and vinegar.

Senega. Use syrup senega.

Senna ( 60 grs.). Give as powder in compound licorice powder or in form of syrup.

Sodii benzoas ( 15 grs.). Sol. W. Give in aromatic water and syrup vehicle.

Sodii bicarbonas (15 grs.). Give mixed with water, and in capsules or konseals.

Sodii bromidum (15 grs.). Give like preceding bromides.

Sodii citras (15 grs.). Give with water and lemon-syrup.

Sodii iodidum ( $7 \frac{1}{2}$ grs.). Give like potassii iodid.

Sodii nitras (15 grs.). Give like potassii nitras.

Sodii nitris (1 gr.). Give in solution with distilled water; or dilute well with inert powder and give in capsule.

Sodii phenolsulphonas ( $4 \mathrm{grs}$.). Give in capsule.

Sodii phosphas (30 grs.). Sol. W. Give in copious quantity of very hot water, or as liq. sod. phos. comp., or the effervescent salt.

Sodii salicylas (15 grs.). Sol. W. Give in aromatie water and syrup or essence of pepsin, N. F., or in capsules and konseals.

Sodii sulphas (240 grs.). Give dissolved in water, sweetened, and flavored with lemon-juice.

Spartein sulphas $\left(\frac{1}{6} \mathrm{gr}.\right)$. Sol. W. Give in pills, capsules, or aromatic elixir.

Spirit. xtheris comp. $(1 \mathrm{fl}$. dr.). Combined with comp. tincture lavender, tinct. card. comp., and give in sweetened water.

Spirit. ather. nitros. (30 min.). Combine with aromatic elixir.

Spirit. ammonii aromaticus (30 min.). Give as such in carbonated water.

Spirit. glyceryl. nitrat. (1 min.). Combine with aromatic elixir, or give in form of tablet triturate and capsule.

Strontii bromidum (15 grs.). Give in aromatic water and syrup. Incompatible with regetable substances. 
Strontii iodidum ( $\left.7 \frac{1}{2} \mathrm{grs}.\right)$. Give like potassium iodide.

Strontii salicylas (15 grs.). Give in capsules or konseals.

Strophanthinum $\left(\frac{1}{200}\right.$ gr.). Give in tablet triturate, pill, or capsule, or aromatic water.

Strychnine salts are extremely bitter and should be given in tablet triturate, pills, or capsules, unless these forms are precluded.

Sulphonethylmethanum (15 grs.). Give in capsule or konseal.

Sulphonmethanum (15 grs.). Give in capsules or konseals.

Sulphur lotum (60 grs.). Give as powder mixed with jelly or thick syrupy substances.

Syrups. The syrups available as flavoring, sweetening, and suspending agents are: Plain syrup, syrup acacia, citric acid, bitter almond, orange, orange flower, wild cherry, rose, rubus, sarsaparilla comp., tolu, and ginger. Those serving as dosage forms in themselves are syrups of hydriodic acid, calcium lactophosphate, lime, ferrous iodide, phosphates of iron, quinine, and strychnine, hypophosphites, hypophosphites comp., ipecac, krameria, lactucarium, tar, rhubarb, rhubarb aromatic, squill, squill comp., senega, and senna. Most of the syrups are susceptible of combination with each other or with other aqueous liquids.

Talcum purificatum. A useful substance in itself as a local application to inflamed surfaces, or as vehicle for medicinal agents used for a similar purpose.

Taraxacum. Available in an agreeable form in compound elixir taraxacum.

Terebenum ( 8 min.). Give in soft capsules or syrupy emulsion.

Terpini hydras (2 grs.). Sol. A. Give in capsules or as elixir terpin hydras.

Thymol (2 grs.). Give in capsules or syrupy emulsion.

Tinct. aconiti $(10 \mathrm{~min}$.). Give in aromatic elixir or other flavored hydroalcoholic combinations.

'Tinct. aloes (30 min.). Combine with tinctura cardamomi compositus.

Tinct. asafœtidæ (15 min.). Use equal parts of this, tinct. card. comp., and spirit lavender comp.

'I'inct. belladonna. fol. ( 8 min.). Give with aromatic elixir or any similar vehicle.

Tinct. benzoin. comp. (30 min.). Miscible only with strongly alcoholic vehicles.

'Tinet. calumbæ (1 fl. dr.). Combine with hydroalcoholic vehicle.

Tinct. cannabis indica (10 min.). Give with comp. spirit of lavender, or elixir fortified with alcohol.

Tinet. cantharides (5 min.). Give as above.

Tinct. capsici ( $8 \mathrm{~min}$.). Give with tinct. of cinnamon and aromatic elixir well diluted.

'Tinct. cimicifugæ (1 fl. dr.). Give in any hydroalcoholic aromatic vehicle.

'Tinct. cinchona comp. ( $1 \mathrm{fl}$. dr.). Give with tinct. card. comp. or alone as bitter tonic. 
Tinet. cinnamon (30 min.). Give in aromatic elixir.

'Tinct. colchici sem. (30 min.). Give in aromatic hydroalcoholic vehicle. elixir.

'Tinct. digitalis (15 min.). Give with tinct. card. comp. or aromatic

'Tinct. ferri chlor. (8 min.). Give in glycerine and water vehicle through glass tube.

Tinct. galla (1 fl. dr.). Give in glycerine and aromatic elixir vehicle.

Tinct. gambir comp. (1 fl. dr.). Give in aromatic elixir combined with spirit lavender comp.

Tinet. gelsemii ( $8 \mathrm{~min}$.). Give in hydroalcoholic aromatic vehicle.

'Tinct. gentian comp. ( $1 \mathrm{fl}$. dr.). Give along as bitter stomachic, or with glycerine and sherry wine.

'Tinct. guaiaci $(1 \mathrm{fl} . \mathrm{dr}$.$) . Give in glycerine and syrup of acacia$ vehicle.

Tinct. guaiaci ammoniat. (30 min.). Give with comp. spirit lavender.

'Tinct. hydrastis $(1 \mathrm{fl}$. dr.). Give in aromatic hydroalcoholic combination.

Tinct. hyoscyami (30 min.). Give in aromatic elixir.

Tinct. iodi ( 1 min.). Give in glycerine and aromatic elixir combination.

Tinct. ipecac et opii ( $8 \mathrm{~min}$.$) . Give in aromatic elixir.$

'Tinct. kino (1 fl. dr.). Give in glycerine and comp. tinct. cardamom.

Tinct. krameria $(1 \mathrm{fl}$. dr.). Give with comp. spirit lavender and glycerine.

'Tinct. lavandula comp. (30 min.). Carminative and flavoring agent. Frequently referred to as spirit lavender comp.

'Tinct. lobelix (15 min.). Combined with aromatic hydroalcoholic vehicles.

Tinct. musk ( $1 \mathrm{fl} . \mathrm{dr}$.$) . Give with comp. tinct. of lavender.$

'Tinct. nucis vomic. (10 min.). Combine with tinct. gentian comp., or tinct. card. comp., and aromatic elixir vehicle.

Tinct. opii ( $8 \mathrm{~min}$.$) . Give with tinct. lavender comp.$

Tinct. opii camphorat. (2 fl. drs.). Use in this form.

'Tinct. opii deodorati $(8 \mathrm{~min}$.). Give like tinct. opium.

'Tinct. plysostigmatis (15 min.). Give in tinct. lavender comp. or in strong alcoholic vehicle.

Tinct. quassix (30 min.). Combine with comp. tincture of gentian.

Tinct. rhei aromatica $(30 \mathrm{~min}$.). Give in this form.

'Tinct. sanguinaria $(15 \mathrm{~min}$.). Give in aromatic elixir fortified with alcohol.

'Tinct. scilla $(15 \mathrm{~min}$.). Give with comp. tinct. lavender.

Tinct. serpentarixe (1 fl. dr.). Give with comp. tinct. cardamom and glycerine.

'Tinct. stramonii ( $8 \mathrm{~min}$.$) . Give in aromatic elixir.$

'Tinct. strophanthi ( 8 min.). Give in comp. tinct. of cardamom and aromatic elixir.

Tinct. valerian $(1 \mathrm{fl}$. dr.). Give with comp. tinct. lavender. 
Tinct. valerian ammoniata (30 min.). Give in this form well diluted. Tinct. veratri $(15 \mathrm{~min}$.). Give with comp. tinct. lavender.

Tinct. vanilla. Useful flavoring agent for powders or liquids.

Tinct. zingiberis $(30 \mathrm{~min}$.). Give in this form well diluted with sweetened water.

Troches. Official formulas for troches include those of tannic acid, ammonium chloride, cubeb, gambir, licorice and opium, krameria, chlorate of potash, santonin, and sodium bicarbonate.

Unguentre. An excellent official ointment vehicle is ointment of rose water. 'This is composed of spermaceti, white wax, oil of sweet almond, borax, and strong rose water. It possesses an agreeable odor and splendid emollient qualities. It may be used as a diluent for a number of the official ointments or as vehicle for such substances as boric acid, zinc stearate, etc.

Vinum antimonii $(15 \mathrm{~min}$.). Give with any aromatic water.

Vinum cocæ (4 fl. drs.). Give in this form.

Vinum colchici seminis (30 min.). Combine with any hydroalcoholic tincture, or give with aromatic elixir.

Vinum ergotæ (2 fl. drs.). Give in this form.

Vinum ferri (2 fl. drs.). Give in this form.

Vinum ferri amarum (2 fl. drs.). Give in this form.

Vinum ipecacuanhæ (15 min.). Give in any aromatic water.

Vinum opii ( $8 \mathrm{~min}$.$) . Give in aromatic elixir.$

Zinci acetas ( 2 grs.). Give in capsules.

Zinci iodidum (1 gr.). Give in aromatic elixir.

Zinci oxidum (4 grs.). Give in capsules.

Zinci phenolsulphonas ( 2 grs.). Give in capsules.

Zinci sulphas (15 grs.). Give in capsules. Externally in watery solution.

Zinci valeras (2 grs.). Give in capsules. 


\section{THE UNTOWARD EFFECTS OF DRUGS}

\section{BY ROBERT DAWSON RUDOLF, M.D. (EDin.), F.R.C.I. (IAND.)}

WheN an unusual and unexpected series of symptoms follow the administration of some substance, we may assume that this result is the effect of some law. Nothing happens without a cause, and often by a careful investigation into the facts of the case the reason for the unexpected result will become clear. "In physiological studies we must always carefully note any fact which does not aceord with received ideas. It is always from the examination and the discussion of this exceptional fact that a discovery will be made, if there is one to make" (Claude Beruard). It is quite certain that the cause of such an unusual result must lie either-(a) in the remedy or its administration, or $(b)$ in the individual to whom it has been given.

I. Between the writing of a prescription by the physician and the actual taking of the medicine by the patient many possible causes of unexpected results may occur. 'The prescription itself may he at fault, either as regards the dosage of the drugs or in the using of some incompatibility which may quite neutralize or completely alter the action of the principal ingredients. Or the wrong dispensing of a correct preseription may be the cause of the trouble, and many fatal mistakes have occurred from this source. 'The risk of a wrong prescription being a source of trouble is lessened by the fact that it must pass under the eye of the dispenser, unless, indeed, the doctor does his own dispensing; but the dispenser is not thus checked, as a rule, although nowadays many of the best druggists do check all dispensing.

But, granted that the drugs have been correctly prescribed and correctly dispensed, still untoward effects may occur, and be due to the drugs, rather than to the patient himself. 'The pharmacopoias lay down certain rules as regards the strength and composition of their preparations, but many sources of error crecp in, and more often than is generally supposed abnormal effects are due to the drugs themselves. "This is specially true as regards vegetable preparations, which are very prone, with time, to alter in strength and composition. Ergot becomes useless in time, and in the same way hyoscyamus depreciates very much with keeping. In a recent investigation, carried out by the Canadian In:land Revenue Department it was found that many samples of sweet spirit of nitre, gathered at random throughout the Dominion, contained little or no nitrite of ethyl, to which principle the preparation owes its vasodilating and diuretic action; forty-nine out of the seventy-seven examples examined ( 63 per cent.) contained less than 1.75 of this borly, and 
fifteen contained less than 0.5 per cent., while four contained none at all. From long keeping the preparation becomes inert through oxidation. Morphine, when kept long, is found to be more apt to produce emesis, and this is put down by most writers to the partial transformation of the drug by slow oxidation into apomorphine. Chloroform again depreciates with keeping, especially in the presence of light. Some preparations, especially alcoholic solutions, tend to become stronger by keeping, the alcohol by evaporating leaving a more concentrated solution.

The activity of drugs depends upon many things, which sources of uncertainty are largely guarded against by the pharmacopoias, but still more or less exist. Digitalis grown wild on the hills is much more active than that which is grown in valleys or artificially cultivated. Socotrine aloes is several times more active than the Arabian drug, and Indian opium is very different in composition from the Turkish.

The season of the year in which the plant is gathered makes a difference in the activity of many. 'Thus, the squirting cucumber (elaterium) gathered in July is some six times as strong as the same gathered in August, while that collected in September has hardly any purgative action (Lewin).

It will thus be seen how all-important it is for the practitioner to make sure that his drugs are reliable in every way, as otherwise his treatment, however well thought out, may fail. The best way to be sure of his weapons is to use only the preparations of the best firms, who have a reputation to maintain, and, in case of his doing his own dispensing, to see that his stock does not remain too long without renewal.

Givell, then, that the practitioner is employing only preparations which are in every way reliable, untoward effects may still be met with, due to causes such as the following: (a) Form in which the drug is administered; (b) time of administration; (c) relation to meals; and $(d)$ idiosyncrasy.

Drugs are very frequently administered in capsule form, and, as a rule, this is a very convenient way of doing so, but occasionally, when so given, they may give rise to nausea and vomiting, while if taken in a mixture, no such effects may occur. Tannin, for example, if given in powder or capsule form, may cause considerable irritation of the stomach, when, if diluted, no such result is seen. The proper amount of dilution is thus very important, and may make the difference between success and failure in the use of some preparation.

The use of tablets and pills is frequently very convenient, and these are largely employed, and yet often, owing to the solidity of such, the patient may fail to react to the medicine, and may appear to be exhibiting an abnormal tolerance to the drug, when, as a matter of fact, the medicine has passed through the alimentary tract unchanged. This is specially seen in the case of iron and of quinine preparations, and again and again it has been the writer's experience to see malarial cases fail to react to quinine, and on inquiry and investigation it has been found that solid preparations of the drug have been userl, which would not dissolve and hence might as well not have been given. If there be any 
doubt about the solubility of tablets or pills, they should be broken or cut up before being taken. Another objection to such solid preparations is seen when a powerful drug, such as opium, is administered in pill form, and at first no effect is produced and then suddenly toxic symptoms set in. What has happened is that the patient's alinentary tract has, for some reason, not been albsorbing for some time, and has then suddenly begun to do so. Such an accident could scarcely occur if the drug had been in fluid form, as it would not have accumulated in the same way in the digestive canal.

'The route by which a drug is introduced into the system has often the greatest possible influence in determining the kind and amount of its action. l'erhaps the best example of this is seen in the case of adrenalin, the active principle of the suprarenal glands. 'This boly acts powerfully upon the vessels, probahly by stimulating the nerve-endings, and produces vasoconstriction. If the drug be given by the mouth, or even hypodermically, it produces very little effect upon the general bloodpressure, the reason being that it has produced so much local constriction of the vessels that no absorption, or little absorption, takes place, and it is soon destroyed by the tissues in situ. But if the drug he given directly into a vein at once a marked rise in blood-pressure occurs, the sphygmomanometer perhaps registering a rise of 100 millimeters of mercury or more in a few seconds. 'The marked effect in this case, and the slight one in the former, is due to the fact that only in the latter has the drug really entered the circulation.

'The time of day at which a drug is given may make much difference in its action; for example, when soporifies are given in the morning they will not produce sleep nearly so easily as when given at the end of the day, when the tired condition of the individuil predisposes him toward sleep.

'The question of whether a medicine is to be given before or after food is one to which the laity are fully alive, and it is an important one, as many drugs, such as iron and digitalis, are apt to produce irritation when given while the stomach is empty, but can be taken without inconvenience when given after meals.

II. The Patient Himself-I ord Byron, in his reported conversations with the Countess of Blessington, remarked to her, that "medical men do not sufficiently attend to the idiosyncrasies on which so much depends, and often hurry to the grave one patient by a treatment that has succeded in another. 'The moment they ascertain the disease to be the same as one they have known, they conclude that the same remelies that enred the first must remove the symptoms of the second, not making allowance for the peculiarities of temperament, habits, and disposition, which last has a great influence in maladies." Dr. G. D. Pollock, to whom I am indebted for this quotation, says that "these remarks are simply an exaggeration of feeling and fact. Byron was not enamored with the medical profession more than he was with the profession of Divinity, but he was an neute observer, and it was interesting to read his views respecting idlosyncrasies. He judged rightlp- 
very rightly-of their importance in connection with the practice of medicine." That one man's meat is another man's poison is an old and true saying.

Very many people find that certain articles of food do not suit them, and, without being able to give any further reason, they learn to avoid such things. Certain foods are specially apt to affect a minority in a special way, and every one has met individuals who could not take strawberries or shell-fish withont being more or less, in some cases very seriously, disturbed. In the same way certain drugs are prone in certain individuals to produce untoward effects, which follow an abnormally small dose, or, on the other hand, they may produce symptoms in susceptible people which apparently have no resemblance to the ordinary toxic effects of such drugs.

As an example of the first class of cases one may cite the case of an individual who, as the result of a small amount of alcohol, becomes intoxicated. Here the toxic effects of the drug are produced by what in most individuals would be a medicinal dose.

FIG. 1.

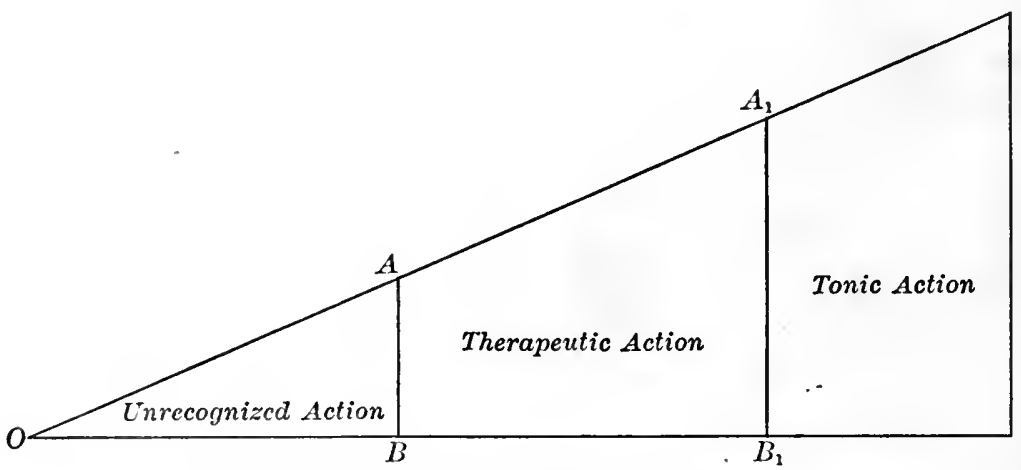

Diagram illustrating the effects of different quantities of a drug.

Harrington Sainsbury gives a good diagram (Fig. 1) illustrating the ignorable, therapeutic and toxic effects of drugs, and I take the liberty of reproducing it here in a slightly modified way. In an individual in whom there is an unusual tolerance toward a drug the lines $A B$ and $A_{1} B_{1}$ will be moved further than usual from $O$; while, where an unusual susceptibility exists, these lines will be nearer to $O$, and an unusually small dose will produce the therapeutic effects of the drug, and even an unusually small dose may take him into the region of toxic action.

As an example of the class where a drug produces in certain people symptoms that are in no way like those produced by a toxic dose, one may mention iodide of potassium, which may cause a petechial eruption in certain individuals, and yet in most people the drug will produce no such effect, even when given in enormous and poisonous amounts.

Occasionally the unusual results of a drug are manifested in the dircetion of want of action, so that large or even toxic doses of it may 
produce no symptoms of any kind. Il. A. Hare published some years ago a marvellous case of this nature. 'The patient, a hysterical fernale, was suffering from headache and fever, and was given the following drugs in surprising amounts without any toxie or other effects:

Three-fourths grain of pilocarpine in three doses hyjoxlermicallyno effect. 'Then 20 minims of tincture of cannabis indical cvery four hours. 'I 'hen $\frac{1}{2}$-grain pills of solid cannabis indica every hour until 15 grains were taken-all without effect.

Then 15 grains of bisulphate of quinine-no effect. She also was given 40 to 50 grains of this every day for three days, with the same negative result. Finally she was given 60 grains of antipyrine in sixtcen hours without effect.

Such a case is, of course, extremely exceptional, but it is very common to find people in whom the lines $A B$ and $A_{1} B_{1}$ are moved away from (). 'The great difference in the amount of anesthetics, especially chloroform, that is required to produce anesthesia, is another example of the great difference in susceptibility that exists in different individuals.

Most drugs when introduced into the system enter into chemical combination with the protoplasm of the cells, and by so doing alter the functions of these cells and thus produce their pharmacological effects. When one considers how complicated such combinations may be, and how slight a difference in organie combinations it takes to make a marked difference in the chemical bodies resulting, it is no wonder, but rather the reverse, that people do not always react similarly to drugs or, indeed, to foods.

The effect of age is marked in varying the actions of drugs, and it might be said that infancy possesses an idiosyncrasy toward morphine in the direction of a much-increased susceptibility to the influence of the drug, while, on the other hand, young children stand atropine so well that they will take nearly the same dose as adults without harm.

In old age, when the vital powers are on the ebb, drugs may produce untoward effects, and must, as a rule, be used in smaller doses than in robust middle life. Iodoform is specially apt to give trouble in old people.

'The influence of sex is not marked, although certain drugs, for example antipyrine, seem to be more prone to produce toxie effects in women than in men, especially during the menstrual period.

So far we have dealt with idiosynerasy in the apparently healthy, but many drugs may have most untoward effects when administered during disease, which are free from such when the recipients are healthy. Many of these effects may be explained upon physiological grounds, while others are hard to understand, and are merely known from clinical experience. It is easy to understand why it is dangerous to use opium preparations freely in eases of bronchorrhea. 'The patient is kept alive by his frequent cough, which prevents the bronchial tubes from filling up with secretion. Opium dulls the bronchial reflex and depresses the respiratory center, and thus doubly hampers Nature's efforts to keep the respiratory passages free. In the same way one can see how in nephritis Vol. $I-8$ 
opium is apt to produce untoward effects, as it is only got rid of with difficulty by the crippled renal epithelium.

People who suffer from vasomotor ataxia are specially apt to show idiosyncrasy to drugs, and S. Solis Cohen illustrates this fact by the case of a young man with blue extremities from such cause, who gave up his position as a druggist because he could not tolerate the dispensing of ipecacuanha; and the writer has recorded the case of a woman who had great vasomotor instability, in whom a marked hypersusceptibility existed toward morphine and also atropine, each drug on several occasions producing toxic effects from minimum doses.

As already remarked, in every case an untoward effect is the result of some law, which, if only understood, would make clear the unexpected action. As a rule, opium produces constipation, the probable reason for this being that it stimulates the inhibitory nerves of the bowel. But occasionally we find its administration followed by diarrhœa. Here apparently a most unlikely event has occurred, and yet it might be explained as follows-depression is usually the result of overstimulation, and, if in an individual the inhibitory apparatus of the bowel be unusually irritable, this stage will be soon reached, and the result will be increased peristalsis with consequent diarrhœa. This is the normal reaction of many animals to the drug, and opium usually produces purging in dogs.

Again, opium has a stimulating action upon the motor cells of the cord, and in frogs and many other animals this effect is so marked that the drug throws them into spasms and convulsions, nuch like that occurring in strychnine poisoning. In human beings this stimulation of the cord is slighit, and so masked by the sedative effect of the drug upon the higher centres that it is not evident at all, but occasionally one meets with an individual in whom the spasms may be a marked feature, as in a woman recently seen, in whom a small dose of morphine hypodermically given produced so much spasm and twitching as to suggest strychnine. Under appropriate treatment these untoward symptoms disappeared, and then the patient remembered that on more than one other occasion when she had been given morphine she had reacted in the same way. No doubt very many cases of idiosyncrasy can be explained in the same manner.

In 1902, Richet invented the term anaphylaxis, to denote the curious property possessed by certain poisons of increasing instead of diminishing the sensitiveness of the organism to their action. The property may be described as an inverted immunity. Using a highly toxic congestin got from mussels, he found that a second injection of it into dogs might find them five to twenty-five times more sensitive than on the first occasion. It is not our province here to discuss the nature of anaphylaxis, but, suffice it to say, that many have used it to explain cases of idiostnerasy, and no doubt it does explain certain untoward results arising from some therapeutic agents, such as antitoxin and tuberculin, where a second dose occasionally produces symptoms which were absent after the first injection. In a recent leader in the British Medical 
Journal this matter is discussed, and the advice is there given that a second injection of any serum should be massive mather than small, as. strange to say, the phenomenon of anaphylaxis is more apt to follow small than large doses. Some would go so far as to try to explain all calses of hypersusceptibility to drugs, etc., as due to anaphylaxis, but when individuals react in an unusual way to a first dose of any substance, it is hard to accept such an explanation of the event.

When physiologists are working with the organs of lower animals, especially the heart, they constantly find great differences between the susceptibility of tissues of the same species of animal towart varions drugs. This difference in susceptibility is innate, and no doubt rexists equally in human tissues, and accounts, if one may accept such an coxplanation, for much of the idlosyncrasy that we come across. 'The different temperaments, of which the older physicians made so much, really meant the same thing, and probably nowadays we do not, at loast as teachers, put enough weight upon the essential difference between people.

Drugs.-Within the limits of this chapter it would be impossihle to even mention all the untoward effects that have been described as occurring from the use of various drugs, much less to completely describe such, and all that will be attempted will be a short account of the more common and important variations from the average.

Aconite.-Occasionally, after a single pharmacopocial dose of aconite, symptoms of poisoning occur. 'Tingling of the mouth, throat, and extremities is complained of, and the pulse falls in frequency and strength. 'The patient feels weak and looks anxious, and the respirations may become shallow and irregular. Nausea and vomiting may occur. No doubt such symptoms after a therapeutic dose suggest an idiosyncrasy, but the maximum dose of the U. S. P. and B. P. will verv often produce more or less of such results, and, in the writer's opinion, it is too large a one. 'The safest way to use aconite is to give one or two minim doses frequently repeated, the patient being closely watched for any untoward symptoms the while. 'Ten minims of the tincture should be the maximum single dose, instead of the 15 allowed by the pharmacopocias.

Aconitine is officinal, but different samples vary so much in strength and purity that it is better avoided in practice.

The Antipyretics (Acetanilide and Antipyrine Series). Since 1SA4, when the first of this series was introduced, these drugs have been enormously used both by the profession and the laity, and many fatal cases of poisoning have oceurred. As a rule, such eases have been the result of excessive doses, but in some instances have oceurred from amounts which are ordinarily safe. H. A. Iare collected from medical literature a great number of cases in which untoward results had occurred, and since then many more have been published. 'The three most commonly used members of the group are antipurine, antifebrine, and phenacetine.

Antipyrine.-'This drug often produces a slight cynosis and duski- 
ness of the hands, nose, and lips, with coldness of the extremities and irritation of the skin. Erythematous patches may occur, and occasionally bullæ may be produced. Symptoms of cardiac weakness are not uncommon, and in the rare cases where death has supervened, heart failure has been the immediate cause. Women seem to be specially susceptible to the drug. The dose producing unpleasant symptoms may have been from 4 to 10 grains - a dose which in most instances would be perfectly safe. IV. G. A. Robertson published a case in which 5 grains always produced faintness and collapse, with marked urticaria.

Acetanilide.- This is the most powerful member of the series, but very rarely has it produced serious symptoms, unless in excessive doses. Cases of poisoning have occurred from its use as a dusting-powder for wounds, as in one mention ed by Beaumont Small, in which prostration and cyanosis followed the use of between 20 and 40 grains applied to a burnt surface. In another, an infant four days old died from its application to the umbilical cord. The symptoms produced resemble those of antipyrine poisoning.

Acetphenetidine (Phenacetin).-'This is the mildest of the series, and, as a rule, large amounts may be taken without poisonous results, but in rare instances such have occurred, as in a case reported by Hollopeter, quoted by Wood. Here $22 \frac{1}{2}$ grains in six hours produced collapse, with marked lividity, great dyspnea and restlessness, cold perspiration, and slightly dilated pupils; and Wood mentions a fatal case in a baby of one year from 10 grains taken in ten hours. When untoward symptoms occur, they resemble those of the rest of the group. The presence of impurities, especially paraphenetidin, is, according to Beaumont Small, the cause of many of the unlooked-for effects of the drug.

Usually it is considered that the addition of caffeine to these drugs lessens the tendency to collapse, but recently Worth Hale has seemed to show that caffeine increases the toxicity of at least acetanilide.

Arsenic.-Nore or less severe cases of poisoning from the therapeutic use of arsenic are far from uncommon, and every practitioner of any experience has seen examples of them. The symptoms may be grouped roughly into three classes, although these tend to overlap. 'The most common untoward effects of the drug are due to gastric irritation, the patient complaining of anorexia, nausea, and vomiting, and frequently of severe epigastric pain. Occasionally diarrhœa may occur, or there may be constipation. 'The tongue is thickly coated and the breath foul.

Probably the next most frequent group to appear are those symptoms due to multiple neuritis, this being ushered in by numbness and tingling of the extremities, and often by acute pain along the course of the nerves of the limbs. 'The lower limbs are most frequently affected, and the knee-jerk is early lost. Very soon the muscles atrophy, and their reaction to faradic, and next even to the galvanic, current nay disappear.

In the third place, the resentment of the sustem to arsenic may show itself in skin disturbances, either as an increase in pigment, suggestive of Addison's disease, or more commonly in the form of various erup- 
tions. These may be papular, vesicular, or erythematous in type, and herpes is not uncommon. But of all the skin manifestations, the one most commonly seen is slight oedema around the eyes, accompanied by redness of the conjunctive and occasionally by catarrh of the upper air-passages. Very often this is the first sign that hints to the physician thant he has reached this special patient's physiologieal limit for arsenic.

Skin symptoms are specially apt to arise where already there exists some eruption, and it is quite common to see a slight, perhaps localized, skin lesion lighted up and made to rapidly spread by even small doses of arsenic. Henre the drug should he used with caution, if at all, in all but the most chronic types of skin disease; an exception to this rule is probably penphigus, where arsenic is often very efficacious.

'That a marked idiosyncrasy exists toward arsenic is well shown by the accounts of instances where large communitics have been poisoned from some common source, and yet only a small percentage of the individuals exposed have suffered. The historical cases of arsenical beer in Manchester, and of milk diluted with arsenical water in London, illustrate this point well.

Many people cannot stand a dose of 3 minims of Fowler's solution thrice daily for any length of time without showing symptoms of poisoning, while others may take several times this amount without any untoward effects. Children usually bear arsenic extremely well.

Belladonna.- When used externally, belladonna, in the form of liniment or plaster, may produce irritation, with a resulting erythematous rash, and in rare cases a vesicular one with much itching. 'This last form occurred twice in a patient of mine from the use of a plaster. When atropine is instilled into the conjunctival sac, besides the expected dilatation of the pupil, a conjunctivitis may be set up. Symptoms of general poisoning have followed the use of collyria of the drug, probably due to the poison having reached the alimentary tract by way of the tearduets.

When untoward effects occur from the internal use of belladonna or atropine, they usually take the form of dilatation of the pupil, dryness of the mouth and throat, flushing of the skin, and quickening of the heart's action, and occasionally by the produetion of a scarlatiniform eruption. These unlooked-for symptoms rapidly disappear upon the discontinuance of the drug, but the dilatation of the pupil may persist for days.

Children, like rabbits, are very tolerant of belladonna, while adults resemble carnivora in being more susceptible to the drug. It would be interesting to note the relative activity of belladonna in flesh-eaters and vegeturians.

Bismuth.-When soluble salts of bismuth, such as the bismuth and ammonium citrate, are injected into the veins or subcutameous tissues of animals, acute poisoning ensues. 'The symptoms are chiefly due to a primary stimulation, or rather irritation, of the nervous system, soon followed by fatal depression. The blool-pressure falls, due partly to weakening of the heart muscle and partly to depression of the vasomotor centers. If injected in smaller quantities, symptoms occur, largely refer- 
able to the alimentary tract, such as stomatitis, vomiting, and diarrhœa, and, later, tetanic convulsions and renal irritation. "In these chronic poisonings the organs affected are those by which the drug is excretedthe mouth, hidneys, large intestine, and cæcum" (Cushny). It is evident that in most cases bismuth is not absorbed to any extent, even when given in enormous doses, but simply passes through the alimentary tract, exerting a local influence there. When it is applied to a wound. however, absorption may take place, when symptoms similar to those produced in animals by injection of the drug take place; viz., salivation, swelling of the gums, tongue, and throat, and even gangrene of the soft tissues of the mouth, vomiting, diarrhoea, and albuminuria. Occasionally such symptoms occur after the administration by the mouth and are due to an unusual degree of absorption. Bennecke reports a case of poisoning ending fatally in a feeble infant, to whom between 3 and 4 grams had been given for skiagraphic purposes. In most cases, however, when such untoward symptoms occur, they are believed to be referable to impurities in the drug, ehiefly arsenic and lead. The so-called bismuth breath is due to the presence of tellurium. Constipation following the administration is almost a constant event, and is, in fact, one of the chief reasons for the use of the drug, but it may occur with unusual persistence and require to be corrected, or the drug to be discontinued. The ammonio citrate of bismuth is a stimulant, astringent, and probably an antiseptic, but in large amounts is irritating. Preparations of bismuth cause the stools to assume a grayish color, probably from the presence. of sulphide of bismuth.

Boric Acid.-'This drug seldom produces untoward effects, but large quantities, when taken by the mouth, cause some gastric and intestinal irritation, and in some cases it is said to act as an aperient, even in moderate doses.

When boric acid is used as a surgical dressing, it is not entirely without risk, and many cases of serious and even fatal poisoning are on record. It produces, then, chiefly alimentary symptoms, such as romiting and diarrhœa, with collapse, and a number of skin eruptions have been described as due to its continuous use, either as a dressing or internally. Lewin cites two cases where fatal effects followed the injection of a 5 per cent. solution into the pleural cavity in the one instance, and into a large abscess cavity, in the other. In both cases vomiting occurred, followed by an erythematous rash, collapse, and death. He salys that in such cases the drug is slowly and continuously absorbed, and that thus a continuous poisoning is kept up, while in the case of a large single dose the drug is, as we know, quickly eliminated by the kidneys.

Borax acts in much the same way as does boric acid, and may in rare cases produce such untoward effects as nausea, vomiting, and diarrhoea, and the formation of scarlatiniform, eczematous, or papular eruptions. A peculiarity of these last eruptions is that they tend to be distributed round the joints (Hare).

A bluish line on the gums after the long and toxic use of boric acid or 
its sodium salt (borax) has been described, resembling that produced by lead. It is possible that the amount of boric acid used in the preserving of various foods may in a few predisposed people give rise to gastrointestinal irritation, but the evidence is very contradictory. H. C. Wood, Jr., has made a useful summary of this subject.

Bromides.-Bromides are very frequently used in medicine, and every practitioner is familiar with the most common untoward effects that they may produce in the form of an acne eruption. Such acnes may become pustular and may even lead to abscess formation. Occasionally the eruption is of an erythematous type and it may be very irritable. 'The digestion is commonly disturbed, and the patient has a foul breath, anorexia, and may even have nausea and vomiting. 'The nervous system is dulled and cxcessive sleep may occur, but such is not of a pleasant nature, as a rule. While awake the patient may have headache and feel dull and stupid. There may be irritation of the respiratory tract, but this is not nearly so common or as marked a feature as in the case of the iodides. The circulation is sometimes depressed and the general vitality lowered, but this is specially and almost only seen in the case of the potassium salt, and is probably due rather to the base than to the bromide ion. 'The most commonly used bromides are the strontium, ammonium, sodium and potassium salts, and they may be put in that order as regards their tendency to depress and produce unpleasant effects, beginning with the mildest.

The effect of arsenic in lessening the tendency to bromide eruptions is generally admitted to be marked, and it is no uncommon experience to see a bromide rash disappear upon the addition to the bromide mixture of 2 or 3 minims of the liquor arsenicalis. It is a good plan to combine small doses of arsenie with bromides, in order to lessen the tendency of the rash to appear.

Cantharides.-Cantharides is seldom used internally nowadays, but when so used is apt, in all but very small doses, to produce gastro-intestinal irritation, with intense thirst. After absorption its action is chiefly on the urinary tract, where it produces a sense of heat in the bladder and urethra, priapism, strangury, and even acute nephritis.

When used in the ordinary way-i.e., externally-sufficient cantharidine may be absorbed to produce the urinary symptoms mentioned, ard Huchard and others, according to Hare, have reported cases in which, without previous disease of the kidneys, a blister has caused violent acute nephritis, with resulting uremia. This is no doubt an extremely unlikely thing to happen when the kidneys are healthy, but very apt to occur when they are acutely diseased. Hence, one should use blisters with great caution in such conditions.

Phenol.-Since the time when carbolic acid was introduced so largely as an antiseptic in surgery, very many cases of poisoning following its therapeutic use have occurred, and there is no doubt that idiosyncrasy has played a powerful rôle in its production. When the carbolic spray was used in operations, such cases were specially common, and Röhrig showed in 1878 that finely atomized watery solutions 
of the drug, as of other substances, were absorbed by the skin more easily than when the solution was simply applied. Now that the spray is no longer used, poisoning is not so common, but still may occur from dressings of wounds, from solutions applied to extensive areas of normal skin, and especially from cavities, such as the bladder, rectum, and uterus.

Poisoning by therapeutic doses of the drug, given by the mouth, is seldom seen. 'The earliest symptoms which are produced by the absorption from any of these channels of an amount of the drug, which for a given individual is toxic, are usually a darkened hue of the urine, with pain in the lumbar region, restlessness, or cerebral disturbance, then symptoms of collapse, with weak frequent pulse, and often marked dyspnoea. There may be an early rise in temperature.

If the case go on to a fatal termination, such a result is usually due to respiratory failure. 'The dark color of the urine becomes more marked on standing, and is probably due to an educt of phenol, which has not yet been isolated, perhaps hydrochinon. Occasionally hematuria may occur, and even suppression of urine. In animals convulsions are almost constant in phenol poisoning, but in man they rarely occur. Headache, nausea, and vomiting are common. The hypersensibility of some individuals to the local action of phenol is extreme, and many cannot wet their hands with a 1 in 60 solution without suffering from well-marked local anesthesia, which may last for hours. In others, its frequent use may produce an eczema of the hands, which resists all ordinary treatment as long as the source of the trouble persists. Many cases occur of local gangrene of the fingers and toes, produced by the continuous application of a 3 per cent., or stronger, solution. In the Medical Review of 1900 , p. 449 , is given a list of 132 case of gangrene due to weak solutions of the acid. Such a result usually calls for amputation of the affected digit.

Chloral Hydrate.-Chloral hydrate is not characterized by many untoward effects short of actual poisoning, but occasionally produces engorgement of the conjunctiva and eruptions of the skin. 'These latter may be erythematous, papular, urticarial, vesicular, and even petechial. Occasionally, instead of the quiet sleep-which is the normal effect of chloral-occurring, an excited condition supervenes, resembling alcoholic intoxication.

Cases are on record where moderate doses, 20 and 30 grains, have proved fatal, and many where closes ranging from these up to 4 grams have caused death. The symptoms in such cases are deep narcosis, with flushed, swollen face and blood-shot eyes. The pupils are usually dilated, and, even if contracted, dilate upon the subject being roused. Such serious symptoms occurring from such doses can only be explained by idiosyncrasy.

Cocaine.--Very many cases of poisoning from cocaine have occurred since it was introduced in the latter part of the last centiry. 'The drug is chiefly used for producing local anesthesia of the skin, conjunctiva, and mucous membranes, and the susceptibility toward the drug being very variable, cases of poisoning have followed comparatively small 
doses. Thus, Wood quotes a case of death occurring forty seconds after the hypodermic injection of twelve drops of a 4 per cent. solution in a girl of eleven years, and another, where death is said to have followerl the application of a 5 per cent. solution to the gum; and he wisely concludes that not more than $\frac{3}{4}$ grain shonld be used locally, either hyperlermically or upon the mucous membranes or the conjunctiva.

'The early symptoms of poisoning are great restlessness and nervous excitement, with occasionally a sense of well-being, but more often than not this is absent. 'The pulse-rate is raised, as is also the respiration, and frequently muscular twitchings occur and some numbness over the whole body is felt. 'There may be nausea and vomiting. 'The pupils are dilated. Such are the results of slight poisoning, such as may, in susceptible individuals, follow very small doses of the drug.

'The cocaine habit is perhaps the most easily acquired of any, and it is well, if the drug is prescribed for use in hay asthma and the like, to append $\mathrm{Ne}$ repetatur to the prescription.

Digitalis.-Foxglove is chiefly used therapeutically when a fairly healthy heart muscle is unable, from some handicap, to perform its work properly, and as a result threatens to fail. It is the most powerful weapon at our command in dealing with heart troubles, and, when wisely used, often saves life. 'There are, however, many contraindications to its use, and when these are ignored, the drug may do great harmwith this aspect of the subject, however, we are not here concerned. Digitalis is irritating locally, and hence tends in many instances to cause nausea and vomiting, especially when given on an empty stomach. On this account it has often to be dispensed with, or liquids given only hypodermically. The drug is cumulative, and in a case of, say, mitral regurgitation, where at first the failing compensation has been successfully met by its use, a stage may be reached when the improved pulse becomes suddenly fast and perhaps irregular, the increased urine gives place to a scanty flow, and vomiting may set in. Here the patient has entered the region of toxic action of the drug. Sometimes such poisoning sets in suddenly, more especially after ascites has been relieved by tapping, and is probably due to the sudden absorption into the circulation of a quantity of the drug which has been accumulating in more neutral parts of the system.

When a patient is taking digitalis in full doses, he should be kept horizontal, as syncope is apt to occur upon his suddenly assuming the erect posture.

'The strength of digitalis preparations varies greatly, and this fact may account for many cases where we fail to get the expected good effects of the drug.

The digitaline preparations of commerce vary much in composition, some being almost inert, while others are very toxic. Very often considerable pain is produced locally by their use hypodermically, and even abscess formation has occurred.

Children do not stand digitalis well, as a rule, and are apt to show an irregular pulse under its use. 
Ergot.-Many eases of serious and even fatal poisoning have occurred from the use of ergot. 'The symptoms of a toxic dose are mainly apparent as gangrene of the extremities and disturbances of the central nervous system. Usually it takes an enormous dose to produce such results, but occasionally small ones will so act in persons possessing an idiosyncrasy in regard to the drug.

R. B. Faulkner mentions a case in which a dram of the fluid extract eaused drowsiness, swelling of the feet, and violent pricking of the extremities; and gangrene of small areas, such as the toes, may oceasionally oecur from small single doses.

When taken continuously for some time, as used to be recommended in cases of fibroid of the uterus, symptoms of chronic poisoning may ensue, similar to those that used to oecur in Europe when great numbers would suffer from ergot poisoning, due to the contamination of bread by the ergot fungus. Gangrene of the extremities, generally superficial and almost limited to the skin, would oceur. Occasionally it involved a whole limb, and even deep structures, such as the eye, uterus, or stomach. At other times, or along with the gangrene, nervous symptoms predominate, such as drowsiness, headache, cramp of the limbs, and even convulsions. Such epidemies are now a thing of the past, except in Russia.

Although it is rare nowadays to have toxic symptoms from the use of ergot, it is well to remember that very frequently a supposed unusual resistance to the action of the drug occurs, and that, at a time, perhaps, when it is most required, as when postpartum hemorrhage is threatening. Such an unusual tolerance, of eourse, may exist, but most frequently the trouble lies in the drug itself, which has become inert from some cause, such as long keeping.

Iodine and the Iodides. - When iodine is applied externally, usually in the form of the tincture, it produces discoloration of the skin and more or less irritation, and, on account of this irritating action, is greatly used in medicine as a counterirritant. The susceptibility, however, of different individuals to the drug is very various, many not being able to stand the local applieation of the tincture for more than a single occasion, while others may permit of the frequent painting on of the tincture without complaining. An eruption frequently oceurs from the cxternal use of iodine, generally of an erythematous or vesicular type, and, as showing that this is at least partially due to the absorption and subsequent effect of the drug on the skin is the fact that the eruption is often not limited to the area of application, but may be wide-spread, and of the same nature as that often produced when iodine or the iodides are given nternally. IThen the iodides are applied externally, they are not absorbed. There is a marked and very common idiosyncrasy to the iodides taken internally, some people being able to take them in large amounts without trouble, while others are affected by very small doses. 'Three gr ins, repeated a few times, is a sufficient amount in some individuals to set up symptoms of iodism. There is much difference of opinion as to whether iodide symptoms are more apt to be set up by large or by small doses, 
but, in the writer's experience, the amount of iodism bears little or no relation to the amount of iodides given. It is very rare to see one dram of iodide of potassium taken thrice daily give rise to symptoms, unless it be some disturbance of the stomach. Such large doses of the drug, of course, are practically only given in tertiary syphilis, and many believe that absence of symptoms in large doses is a therapeutic sign of this disease.

Symptoms. - The first thing usually noticed by a person who is heginning to feel the effects of iodism is a metallic taste in the mouth, especially in the mornings, followed soon by coryza, with great congestion of the eyes, and occasionally by bronchitis. 'The stomach is apt to be disturbed, with loss of appetite, nausea, and even vomiting, and of ten there is considerable epigastric pain.

Eruptions of the skin are frequent, and may take the most diverse forms,-pustular or papulo-pustular, acneiform spots being the most common; or erythema, or urticaria, with considerable odema. Rarely one sees petechiae, and these may be very wide-spread. Such a case eame under my notice some time ago when $2 \frac{1}{2}$ grains of potassium iodide given three times daily soon gave rise to a most marked petechial eruption. Other symptoms may occur, such as a fast heart, with tremor, suggestive of thyroidism (and possibly due to stimulation of the thyroid gland), and such are specially apt to be seen when the patient is suffering from an already overactive thyroid gland. Edema of the glottis has occurred, and probably has been ingrafted upon an inflammatory condition there. In one case, where this proved fatal, small uleerations were found in the larynx, as well as the fatal oedema. Some have attributed the occurrence of iodism to the presence of iodates in the iodides, but it has been abundantly proved that it occurs from the pure iodides. Probably the effects are chiefly due to the setting free of iodine, but something more than this would seem to be necessary when marked local symptoms occur, such as petechix, from minute doses.

Iodoform. - When this drug was extensively used in surgical practice, many eases of poisoning occurred. In wounds some of the iodoform decomposes, and the free iodine unites with the alkali of the fluids to form iodides, which may in turn produce symptoms of iodism, but most of the troubles which have arisen through the use of iodoform have been due to the unchanged drug. In the first place, the smell of the material is most objectionable to many people, both for itself and on account of its suggestion of disease. 'The characteristic symptoms that may' occur from the use of iodoform are nervous ones, the patient becoming anxious and depressed, and soon delirious and even maniacal. Giddliness and headache are common. The pulse is hastened and there may be fever, and in fatal cases collapse sets in. There may be a marked erythema. Such toxic symptoms have occurred much more commonly in adults than in children, and the aged seem specially prone to suffer. I.ewin states that females are especially prone to show sumptoms of toxemiat. Nearly all toxic cases have occurred from the use of the drug in wounds, abscess cavities, etc., but a few examples have been recorded from the 
internal use of the remedy. One grain has produced pronounced symptoms, as in a case reported by Dr. 'Tiffany, of Kansas City.

Iron.-This is one of the most commonly used of tonics, and when an examination of the blood shows that iron is present to too small an extent, especially when this want is shown by a low color-index, it is one of the most valuable of drugs. But the use of iron is frequently abused, the drug being given when not required, and in such instances is specially apt to produce untoward effects.

The digestion may be disturbed by iron, and want of appetite, nausea, and even vomiting may be produced, and this is specially the case where it is given upon an empty stomach. Constipation is apt to be induced, and the stools are blackened from the formation of the sulphide of iron. As a rule, the ferrous salts are better borne by the digestive tract than are the ferric oncs. The teeth tend to be blackened by iron, from the formation of the sulphide. It is necessary for this that there be a formation of sulphuretted hydrogen, either from caries or from decomposition of food between the teeth. Headaches follow the administration of iron sometimes, and there are often seen people who say that they cannot take the drug in any form. Probably these headaches arise from digestive disturbances, and they are not nearly so apt to occur if the bowels are kept freely open during the course of treatment. It is a popular belief that iron produces an abnormally free menstrual flow, and hence should be stopped when the period is due; such a result, if it occur at all, is merely an jdiosyncrasy, and by no means a constant thing.

Lead.-Chronic poisoning from lead is extremely common, and usually results from long-continued introduction of the poison into the system from food, or in the course of various trades in which lead is used. Susceptibility to the poison undoubtedly exists in certain people, as when all in a trade are equally exposed to the danger, but only a few suffer. Women and children are specially prone to be affected, and a lowered condition of the general health seems to greatly predispose to the poison. When an individual has once been affected, he is very apt to suffer again.

Only rarely is poisoning produced from the therapeutic use of lead, but it has occurred from the very free use of the acetate. Baker, quoted by Lewin, noted it in the case of a man who had taken only 1 grain of the acetate night and morning for four days; there occurred loss of appetite, pain in the stomach, constipation, and paralysis of three weeks' duration.

There seems to be much evidence that the drug may be absorbed through the skin, or from wounds, with poisonous results. Edward Curtis calls attention to the risks of using lead compounds about the eye; for, if by any chance there be a lesion of the conjunctiva, one may produce quickly an indelible, opaque, white streak over the area of exposed corneal tissue.

When poisoning occurs, the usual symptoms are loss of appetite, with nausea and even vomiting, marked constipation, and severe colic, with rigidity and constriction of the abdominal walls. A blue line on the gums, where they join the tecth, is common in chronic cases. Marked anemia is an early symptom, and is chiefly due to breaking down 
of the red blood-cells, which may even lead to jaundice. 'I'hese colls often show characteristic granules, staining with hasophilie dres. Paralysis, due probably to neuritis, is a common simptom, and is chicefly limited to the extensor muscles of the band-licnce the characteristic wrist-drop. It may be accompanied by mumbness and tingling and violent neuralgic pains. 'The paralysis, however, may he much more widlely extended than this, and J. J. Putnam points ont that in children the loges and feet are commonly affected. Various cereloral symptoms may (xour, such as severe headache, epilepsy, and even delirim, and such brain involvements are spccially apt to occur in alcoholics.

'The effects of lead on the circulation are marked, and take the form symptomatically of high blood-pressure. Arterioselerosis is produced and frequently gramular contracted kidney. 'There is a close rolation between lead-poisoning and gout, the former seeming to strongly predispose to the other.

'The diagnosis of chronic lead-poisoning is, as a rule, easy, but the condition may occur in such a variety of forms that sometimes it is very difficult to recognize and is often overlooked. H. A. Hare makes the good practical suggestion that the occurrence of rare and anomalous symptoms in a given case should at once bring to the physician's mind the possibility of syphilis or lead-poisoning.

Trcatment. - The treatment of these cases mav come under three healings: (1) To prevent the further ingestion of the poison; (2) the getting rid of the poison that has already been taken; (3) the treatment of symptoms.

It is important to tell the sufferers from lead-poisoning that, having once had the trouble, they are much more prone to be again affected, and hence should take more than ordinary care in their uccupations that they may avoid such a possibility. Great cleanliness and the aroidance of dust containing the poison are important. A drink containing sulphuric acid is often used, and is no doubt useful, as it will tend to make any lead oceur as the sulphate instead of the more poisonous carbonate.

As regards the getting rid of the poison in the system, iodides are usually given, and seem to have the support of much empirical experience, but experiment; would appear to show that they are no more valuable than are the bromides or chlorides of potassium.

Purgatives, such as castor oil, guarded with opium, are useful in relieving the colic and possibly getting rid of any of the poison that may still be in the alimentary tract.

In paralysis, massage and stimulation of the affecterl muscles with the faradic, or, in its failure, with galvanism, is most useful. Internally strychnine is usually-and with good reason-given. Into the detailed treatment of chronic lead-poisoning, however, it is beyond our province to enter.

Mercury.-Mercury is a powerful poison, to which there is much idiosynerasy, and, as it is used very freely, both in merlical and surgieal practice, it is not surprising that many cases occur which show more or less degrees of poisoning under its use. 
When used externally, as the blue ointment, it is quite common to get considerable irritation of the skin, resulting in an erythema or eczema, and this may spread far beyond the area of inunction. In some people it is only the prolonged use of this preparation that produces these undesired results, but in others they soon appear, and may be accompanied by considerable fever, headache, and gastric disturbance. The eruption may be limited to the part treated or may spread widely. If eczematous, there may be pus-formation, with much crusting and subsequent desquamation. Many believe that mercury, taken internally, can produce similar eruptions, and only recently I watched the case of a woman who, on several occasions, had suffered from an acute eczema of the face and axillax, following and apparently due on each occasion, to her taking 2 grains of gray powder thrice daily for a few days.

When mercury is taken internally in medicinal doses, it sometimes produces symptoms of general poisoning. The first untoward effect noticed is usually salivation, with tenderness and sponginess of the gums and a fetid breath. In the old days, when mercury was deliberately pushed, in the treatment of syphilis, to the production of salivation, necrosis of the jaw, with falling out of the teeth, sometimes occurred. Loss of appetite, vomiting, and diarrhœa are frequent, and not rarely albuminuria and even rephritis occur.

Tremors, suggestive of paralysis agitans, often are seen, and "the occurrence of choreic movements in an adult should cause inquiry as to any exposure to mercury. In other cases a brownish discoloration of the skin, resembling that of Addison's disease, may appear. Blindness, deafness, sensory disturbances, such as hyperesthesia and anesthesia, may develop, and localized wasting of muscles, or of groups of muscles, may assert itself" (H. A. Hare).

All such general symptoms may follow the use of the drug, internally, externally, or by injection through the skin, or, indeed, by inhalation.

The earliest srmptoms of mercurialism should at once suggest the suspension of the drug, the use of mouth-washes, such as one of chlorate of potash, and probably the administration of iodide of potassium, which drug is believed by many to assist in the elimination of the poison. The romiting, cczema, and other untoward symptoms that may have occurred will call for appropriate treatment, and when the condition is early recognized and the cause removed, such care is quickly successful. With the more profound cases of mercurial poisoning we are not here concerned.

It is commonly stated that children are more tolerant of mercury than. are adults, and this is true as regards salivation. It is very doubtful, however, whether they do not suffer as easily from blood-poisoning from the drugg, and one must not be led into a position of false security by the absence of symptoms of stomatitis.

The Nitrites. - The most evident action of the various nitrites is vasodilatation, but this varies in individuals, depending upon the stability or instability of the vasomotors apparatus and the preparations ingested. In some people the inhalation of 3 minims of the nitrite of 
amyl will produce very marked flushing, with a throbbing headache, vertigo, and embarrassment of the respiration and threatened collapse. On the other hand, many persons can stand such a dose with very little effect beyond some flushing of the face and hastening of the pulse. Visual effeets are not uneommon, subjective sensations of light, usually yellow light, being noticed.

All the nitrites act much in the same way, the effects being only different in their speed of appearance and in their duration. Woxd mentions a case where one minim of the spirit of glyceryl nitrate (nitroglycerin) eaused insensibility, and another where this was complete unconscionsness, with absence of the pulse at the wrist, after 2 minims of the same preparation. Such effeets are entirely unusual, as generally such doses may be given without untoward effects.

It is important to remember that the nitrites are very prone to depreciate, becoming oxidized, and thus tend to become inert. As already mentioned, the sweet spirit of nitre on the market is not uncommonly quite inert, and sodium nitrite is very apt to become so too.

Opium and Morphia.-This extremely important drug has probably yielded more examples of idiosyncrasy than has any other, and medical literature is permeated with cases in which marvellously small doses had produced toxic effects. So much is this the case that one is tempted, while fully admitting that marked eases of hypersusceptibility do oceur, to doubt inany of the cases published; for example, Lewin quotes different cases where fatal effects followed $\frac{1}{4}$ minim of laudanum and $\frac{1}{20}$ of a grain of opium.

The susceptibility to opium varies with the age and sex of the individuals to whom it is administered, children and females being more easily affected by it than are adult males, and it is very prone to eause trouble in certain diseases. On the other hand, the contimued use soon leads to a marked tolerance, and in the East whole peoples are opiumeaters, taking enough to kill the average individual, without showing the least intoxication.

Symptoms. - When one considers the markedly different way in which different lower animals react to opium, it seems to at least partially explain why the human subject shows so much variation in the way it behaves toward the drug. In dogs, for example, opium produces, as a rule, purging; in cats it is apt to cause convulsions; in most animals, as in man, the pupil contracts under its action, but in birds it remains unaffeeted, and in animals in which morphine eauses movements and excitement, the pupil widely dilates.

One frequently sees srmptoms of slight poisoning produced by doses which, in most individuals, would be simply therapeutic. Anorexia, nausea, vomiting, and constipation are very common results of opium. Mental excitement and headache, followed by stupor, may occur, and deep sleep may set in, from which the patient is aroused only with difficulty. The pupil is contracted. All these symptoms have, in my experience, arisen from $\frac{1}{8}$ grain of morphia given hypodermieally. 
In certain cases abnormal symptoms oecur. For example, skin eruptions appear, chiefly of the roseola type, and there may be profuse perspiration. Occasionally the nausea and vomiting may be out of all proportion to the other symptoms, the drug acting as a powerful emetic. This, as already mentioned, may possibly be due to the partial changing of morphine into apomorphine, but may probably occur apart from this. One now and then sees diarrhøea follow the administration of opium, here the drug acting as it generally does in dogs. In rare cases marked mental exeitement sets in, just as it usually does in eats, and in some the spinal stimulation is so marked that the patient may appear to suffer from strychnine poisoning. Such effects occur normally in frogs.

As regards the use of opium and morphine in young infants, it is important to remember that, while they are extremely susceptible to the drug, yet often small doses are very valuable indeed in acute conditions, like cholera infantum. The same remark applies as regards nephritis, where one must, of course, aet warily.

Phosphorus.-Cases of poisoning from therapeutic doses of phosphorus are not at all common, but some have been recorded in which as small a single dose as $\frac{1}{30}$ grain has been followed by very unpleasant results, such as vomiting, diarrhœe with tenesmus, and in rare cases jaundice. The jaundice, when it occurs, is very persistent. Chamot mentions a case where a child had died from sucking the heads of two matches. It would seem, according to Lewin, that symptoms are specially apt to oceur when the drug is given in pill form or in oil, as in such cases it remains longer unabsorbed in contact with the mucous membrane, and has thus more time to produce irritation.

Pilocarpine.-This powerful drug is often very valuable, but it must be used with caution, as it is apt to produce serious and depressing symptoms if used in too large doses, and occasionally even when administered in therapeutic amounts. Profuse sweating is what is expected, but this may be excessive in amount. Occasionally the stomach is disturbed, even when the drug is given hypodermically, and the cireulation may be depressed, with low blood-pressure and symptoms of collapse. But what chiefly constitutes a danger is the tendency to the occurrence of pulmonary edema--so much so that fatal asphyxia may result. Such untoward effects are extremely rare or unknown if the drug be used in small therapeutic doses, say $\frac{1}{10}$ grain of the nitrate by the mouth, or half that amount hypodermically. Such an amount will produce nothing more than a gentle action of the skin, and it may be repeated two or three times in the day. Samuel West says that it is a most valuable drug in chronic renal disease, and with this I would fully agree.

Children, and oecasionally adults, according to Ringer, are very tolerant of the drug.

Atropine is an antidote to piloearpine, and hence should be used promptly when any untoward effects oecur.

Potassium Chlorate.-Chlorate of potash has been very largely used internally in practice, chiefly in diphtheria and other inflammations of the throat and mouth, and alṣo in conditions where it was hoped that 
the drug would help to oxidize the blood, but such an effect has been most conclusively shown not to be possible.

For many years the drug was supposed to be innocuons, until, indeed, Jacobi, in 1879, published a long list of eases in which serious or fatal results had followed its use. Since then it has been fully recogni\%ed as an edged tool. There is much idiosyncrasy toward the drug, some people being able to stand enormous doses without showing any symptoms, while in others, especially where the kidneys are discused, serious symptoms have been set up by so-called therapeutic doses, and so small an amount as 15 grains has proved fatal in a child (Cushny). 'The same idiosyncrasy is seen in animals - certain species, as the rabbit, being able to stand almost any dose, while others, such as the dog, are easily poisoned, and that, too, with the same symptoms as oceur in man. 'The brunt of the attack seems to be borne by the blood, which becomes markedly changed, both by hemolysis and also by the changing of the hemoglobin into methemoglobin and also hematin. These decomposition products appear in the urine, and cause this to become dark and even black in color. 'The urinary flow may become very scanty and even suppressed, chiefly due to the mechanical plugging of the uriniferous ducts with the decomposition debris. Vomiting and diarrhoea are common, and jaundice may appear, and the patient suffers from uremia, and also from the direct action of the drug upon the central nervous system. An erythematous rash may appear. Normally nearly a 100 per cent. of the drug appears in the urine, only traces being discoverable in the saliva and other secretions. In the light of this fact, it would seem far from wise to give potassium chlorate freely by the stomach in order that it may be secreted in the saliva. Far better and free from risk is it to use the drug either as a gargle or mouth-wash for its local effect.

Quinine.-Many people show a peculiar susceptibility to quinine, either in the direction of more easily exhibiting its toxic effects, or else in showing some quite abnormal symptoms. This susceptibility has been said to be hereditary, and to run in families. Of this there seems to be much doubt. When quinine is used in large doses it is usually for malaria, and, when given in this disease, it may not produce any toxic symptoms, although, if given in the same amount and to the same individual at a time when he is not under the malarial infection, it may art very markedly. It would seem as if the malarial poison and the drug tended to neutralize one another. Similar observations have been made in the case of mercury and syphilis. Cases of serious poisoning from large doses of quinine are exceedingly rare, but a few have been published. Roberts mentions the ease of a girl who recovered after taking 5 draims of the drug. She suffered from deep coma, shallow breathing, slow pulse, and absolute deafness and blindness. It has been stated that enough quinine may be eliminated in the milk to produce death of the infant, but this is very doubtful.

In people who have an idiosynerasy for quinine small doses may produce marked ringing in the ears and deafness, which may be very persistent. Total blindness is not uncommon, and is accorimpanied by a Vor. I -9 
very marked contraction and even obliteration of the retinal vessels, and sometimes by degenerative changes in the retinal nerve-cells, and even by atrophy of the optic nerve (Cushny).

Very commonly irritation of the skin occurs, and various eruptions are set up. These are usually erythematous, but eczematous ones are not uncommon, and purpuric spots occasionally occur. I know of a woman who always suffers from an irritable eruption, chiefly on the face, even after as small a dose as 1 grain of quinine. Some people who work with cinchona bark suffer from great irritation of the skin, according to Lauder Brunton, and I. Burney Yeo reports that 2 grains repeated once produces in himself an itchy erythema.

Gastro-intestinal catarrh is not very rare from moderate doses of quinine, and may produce vomiting and diarrhœa. Irritation of the urinary tract is a frequent untoward effect, albuminuria may be induced, and after considerable doses a marked hematuria may occur. Quinine seems to have a stimulating effect upon the uterine muscle, and many physicians use it to increase the pains of labor. Some hold that it only very occasionally has any effect here, and believe that when it has it is in the nature of an idiosyncrasy. In any case, it is well to be cautious with the use of the drug during pregnancy, as there seems to be some risk of its producing abortion.

The untoward effects of quinine quickly disappear upon the suspension of the drug. The unreliability of solid preparations of it has already been referred to. The drug had best be administered either in the form of powder or solution.

Salicylic Acid and the Salicylates.-The use of salicylic acid and its salts very frequently produces untoward effects, as much difference in the susceptibility of individuals to the drug exists. In sufficiently large doses the drug will produce in any person toxic effects, which chiefly consist in gastric disturbance, depression of the circulation, with delirium and hallucinations of sight and hearing. Excessive perspiration, and frequently irritation of the renal epithelium, may occur, with resulting albumin, casts, and even blood in the urine, but with these normal toxic effects we are not here concerned. In some individuals more or less of these symptoms, however, may occur from medicinal doses. The most common complaint is one of singing in the ears and deafness, and, next to that, disturbance of vision, which may take the form of flashes of light, usually yellow or green in color, or of more definite hallucinations as to vision. Salicylic acid itself very frequently produces nausea and vomiting; next to that, the synthetic salicylate of soda; then aspirin (acetylsalicylic acid), then the natural sodium salt, and, least likely, salicin. 'The unpleasant effects of the salicylates may be avoided or greatly lessened, according to Lees, by administering them with double the quantity of bicarbonate of soda, and the writer fully agrees with this.

Santonin. - Santonin is rarely employed for anything nowadays but as a means of ridding a patient of round worms. Slight unpleasant poisoning symptoms are very common from its use in therapeutic doses. The urine is nearly always, after ordinary doses of, say, 1 or 2 grains 
in the adult, colored yellow, and tends to turn red upon the addition of an alkali. Very frecpently disturbances of vision occur, objecets at first appearing of a blue color, changing to yellow very soon. ()casionally hallucinations of vision occur, in which the subjective ohjects are of the corresponding colors. In rare instances disturbances of titste and smell are complained of. As a rule, most of the drug passes through the allimentary tract unabsorbed, but under certain little understoul ionditions more than usual of it is absorbed, and it is then that the nntowarel effects occur. Retention of urine has been noted after the taking of the drug. When it is experimentally introduced directly into the filood, santonin is a violent poison, killing by its action on the central nervous system, with the production of convulsions and final asphyxia, and, in rare instances, such a result has followed the use of it by the mouth, and has evidently been due to an unusual degree of absorption. In one case, reported by Grimm, 2 grains produced convulsions and sudden death. 'The administration of santonin should always be succeederl by a purgative, in order to remove both the round worms and the drug.

Silver.-The only untoward effect of silver that need be mentioned is the production of argyria. 'This is due to a deposit of the metal, or more probably an organic salt, in the tissues, and it occurs after the prolonged use of the drug. When this distressing condition occurs, it is practically permanent, although the administration of iodide of potassium is said to slightly lessen it.

Argyria is rarely seen nowadays. In order to avoid the possibility of its occurrence, silver should not be given continuously for more than two months, and the total quantity in that time must not exceed one ounce.

Strychnine.-Cases of idiosyncrasy to strychnine are not common, and this in spite of the fact that it is so largely used. Cases, however, have been reported where a scarlatiform rash has occurred from its use. Children seem to be specially susceptible to the influence of the drug, and Christison cited the case of a child of three who had been killed in four hours by $\frac{1}{16}$ of a grain. It has often seemed to me that the hypotonic condition that occurs in some cases of profound typhoid toxemia has been made worse by the therapentic use of the drug.

Sulphones.- The two members of this group most used in practice are sulphonal and trional. 'They act much in the same way-as hypnoties. Sulphonal is most often followed by undesired effects. A single dose of either has proved fatal in people predisposed to them; thus, Petitt records the death of a woman from one dose of 2 grams of sulphonal, but, on the other hand, enormous amounts have been taken without serious symptoms resulting. 'The most common untoward results that follow occur from the long use of therapentic doses. After an-individual has been taking a sulphone, especially sulphonal, for some time, quite suddenly, perhaps, he may suffer from poisoning. 'The first thing noticerl is probably a pink diseoloration of the urine, due to the production of hematoporphyrinuria, with lassitude, gastro-intestinal symptoms, weakness of the extremities, collapse, and perhaps deatli. Various skin 
eruptions occur. Where such symptoms set in, death occurs in more than half of them, and hence the importance of stopping the drug upon the first suggestion of such poisoning; or, of course, better still never giving sulphones to such an extent. The sulphonal habit is far from uncommon.

Turpentine.-It is comparatively common to see skin eruptions follow the internal use of turpentine. 'This is most commonly noticed where the drug has been used in an enema. The eruption is usually of a scarlatiniform type, and tends to be very irritable. Turpentine is occasionally used as an anthelmintic, and here there is some danger of the production of albuminuria, with blood and casts in the urine. A frequently quoted clinical rule is to use the drug only in very small or very large doses. In the former case there is not enough used to produce harm, while in the latter the drug acts as an intestinal irritant, and is swept out of the bowel so quickly that little is absorbed.

Externally, the effects of turpentine in different individuals vary greatly, the liniment producing, in some people, an erythematous or vesicular eruption, while in others it has no such effect. When the skin shows an unusual tolerance toward turpentine, so that its expected rubefacient effect is not obtained, it is well to increase its stimulating action by the addition of tincture of iodine, in the proportion of say 1 dram of this to the ounce of the liniment. 


\section{PART II \\ THE 'TREA'TMEN'T OF IDISEASE BY NON-MEDICINAL MEASURES}

\section{DEFINITION OF CLIMATIC THERAPEUTICS}

BY W. JARVIS BARLOW, A.B., M.D.

Definition of Climatic Therapeutics.-In its restricted and perhaps more accurate sense, climatic therapeutics is the utilization or application of climatic factors in the treatment of the sick and in the prevention of disease. In the following pages, however, on climatotherapy I shall not take this view of the definition, because it is well recognized that many of the factors, other than those dependent on meteorological conditions, must be considered if one seeks advantages plus climatic treatment. These other elements, to be necessarily considered and entering into a suecessful use of climate, may be termed non-climatic factors.

Climatic Factors.-These are the conditions of the atmosphere of a place that affect any climate, as follows:

1. Temperature.

2. Humidity.

3. Atmospheric pressure.

4. Movements of winds.

5. Purity of air.

The amount of sunshine, so important in the consideration of any elimate, is governed largely by the humidity; therefore, in contemplating a change, or giving a patient climatic treatment, the two most important factors of the place for your determination are temperature and humidity. These conditions of the atmosphere depend on the following influences:

(1) Distance from the equator (latitude).

(3) Elevation above sea-level (altitude).

(3) Distribution of lands and water over its surface.

(4) Prevailing winds.

(5) Purely local influences-nearness to mountains; nature of soil; vegetation; cultivation; population; smoke, etc.

Non-climatic Factors.-Before a change of climate is advised, irrespective of the diagnosis made, non-climatic factors must be considered as follows: 
(1) The Stage of the Disease.-Fortunately, the time has passed when physicians send hopeless invalids to a health resort, the only exception being the case that realizes the outcome, has plenty of resources, and decides to make a change that he may be more comfortable in his last days.

(2) Financial Resources. - Without sufficient means to command comfort and proper treatment under a local physician the case should remain at home.

(3) Mental Attitude of the Patient.-The consideration of the patient's wishes are important.

(4) Separation From or Breaking Up a Home.-If the patient is married and too many sacrifices must be made, or the future home jeopardized, the case will usually do better without the advantages of climate.

(5) Individual Peculiarities.-Certain cases are easily depressed or become lonely, subject to notalgia, or without resources within themselves. Such factors often play a most important part, and here individualization must be made. Each case is a law unto itself. If the patient is easily depressed, or becomes lonely in new or isolated localities, it is better to advise against the additional treatment by climate.

Recognizing these non-climatic factors, I propose to give the practical side of climatic treatment as I see it, limiting the space allotted me to those types of climate and resorts in the United States which seem more or less adaptable to our use in various pathological conditions. It is unwise to rely entirely upon climatic conditions and exclude other factors. To summarize this treatment, climatotherapy is the close study of all conditions that arise in relation to the resort selected, viz., atmospheric, topographic, sociologic, psychic, and economic.

Principal Types of Climates and Health Resorts in the United States.-Climatologists have always found it difficult to present a clear and definite classification of climates. The different types cannot be sharply defined. I will use here the classification made in a previous publication on climatic therapeutics, written by me and published in 1909:
(1) Ocean: $\left\{\begin{array}{l}(a) \text { Sea voyage. } \\ (b) \text { Island. } \\ (c) \text { Coast. }\end{array}\right.$
(a) Low altitude (up to 1000 feet).
(2) Inland:
(b) Medium altitude (1000 to 3000 feet).
(c) High altitude (3000 to 7000 feet).
(d) Deserts.

Since the characteristics of each type depend on the temperature, the humidity, atmospheric pressure, movements of winds, and the purity of the air, and since any or all of these elements may be modified by local factors or atmospheric conditions, it is readily understood that the elements of any one of these groups may be so changed as to approach closely the next in line. 'The character of soil, nearness or distance from lakes or large bodies of water, forests, vegetation, density of population, dust and smoke, will each have its effect in changing the conditions. 
A coast climate which has close proximity to high and desert mountainous regions may lose much of its ocean factors and take on inland climatic conditions, so that such a coast climate, in its pure meteorological condition known as enervating, may be so changed as to become sedative or mildly stimulating. It might prove most helpful to the clinician to make another classification:

(a) Enervating (Florida).

(b) Sedative or relaxing (coast of California).

(c) Stimulating (mountainous regions).

In what follows-a discussion of these types, resorts, or the several diseases-I will endeavor to include these terms, but it must at the same time be remembered that a climate which is tonic and stimulating to one person may be relaxing and sedative to another, and what is enervating to one may be tonic to the other.

I cannot do better in describing the main classification than by repeating in part the words published a year ago:

I. Ocean Climates.-Here temperature, according to latitude, varies less than with inland climates. The general characteristics and important properties of ocean climates are pure air and freedom from dust and pathogenic organisms; in general, they are moist and equable. The evenness of the temperature is characteristic, the difference of the temperature between day and night, for both summer and winter, is less than the inland climate, and in southern latitudes the temperature is equable the year round, modified more or less by the Gulf Stream and other strong ocean currents.

The physiologic properties of the ocean climate, according to Schroeder and Blumenfeld (1904), show that the heart action is strengthened and that the pulse is slowed, for the moist air and air-currents cool the skin and lead to the contraction of the bloodvessels. 'This in turn reflexly leads to increased heart action and dilatation of the bloodvessels of the skin, and, finally, to a cutaneous hyperemia. 'The ocean climates, in general, have a sedative and relaxing effect on the nervous system. The mucous membrane and skin are more active. 'The increased pressure leads to increased depth and slowing of respiration. 'The metabolism is considerably augmented, and, on account of the respiration, more carbon dioxid is thrown out. With this change of metabolism comes an increase of weight and an increase of blood-cells and hemoglobin.

This important effect on metabolism is a matter of individuality. Many persons, sick or well, who have made a change to an ocean climate, with whom such a climate agrees, may show just such results, while others will not improve at all in the same climate. Based on this adaptability of the individual to particular climates, Huggard (1906), in discussing types of elimate, states that "the tonic or relaxing character of a climate turns ehiefly on the ability of the organism to adapt itself to the requirements. Other things being equal, that climate is most tonic which demands the greatest amount of tissue change that a given organism ean permanently yield." He shows that a climate that 
is tonic and stimulating to one person may be relaxing and sedative to another, and vice versâ.

(a) Sea-voyages.-On a voyage with favorable weather conditions one may, for a long period, experience the general characteristics of the occan climate, receiving the benefit of outdoor life in the purest of air. On the other hand, the weather conditions may be unfavorable, with rain and high winds. In general, this type of climatic treatment is only applicable in the earliest stages of tuberculosis when there are no constitutional symptoms, or for arrested cases. In both instances the patient should be proof against seasickness. The objections to ocean trips are that a sufficiently long royage, under unfavorable weather conditions, may cause much confinement, and this, with the poor food too often found on the ships that go to semitropical and tropical countries, may cause disastrous effects. When a sea voyage can be made in a sanatorium ship, types of which have been constructed on the continent, then such a sea voyage may be free from many of the drawbacks which ordinarily are a part of these voyages.

(b) Island Climates.-The climates of small islands possess practically the same characteristics as the ocean climate, and, besides, persons can be made more comfortable on an island and not suffer from seasickness. The smaller islands are not recommended for tuberculous persons, because of the disadvantage of poor accommodations and poor food and isolation of the patient, which too often leads to nostalgia. One of the advantages of living on an island or on the coast is that sea-bathing is possible, but this only applies to patients in the arrested stages of disease, for those with active trouble should not bathe in the open sea. Sea-bathing may be stimulating if not indulged in excessively. The first bath should be short-a mere dip of two or three minutes; longer bathing may cause much depression. If the limit of endurance has been exceeded, it will be marked by a chilly feeling, vertigo, or nausea.

The larger island, with mountainous districts, adds much to the scenic effects and beauty, by presenting an elevated region in close proximity to the ocean, but the climate no longer partakes of the island character, but resembles more the coast type.

(c) Coast Climates. - The coast region gives equable moist conditions, and practically offers the only ocean climate for selected, individual cases of tuberculosis. The atmosphere, most of the time, is cool and damp, often foggy, especially along the western coast of the United States and North Sea, Germany. Places with coast climate have the advantage of being accessible, and are often provided with suitable resorts in which it is agrecable to live, and where excellent accommodations may be found, with occasionally a sanatorium for the treatment of tuberculosis.

Many coast climates may be warm and moist during the summer months, depending on prevailing winds-the land and sea breezeswhich make the climate equable. During the day the breeze blows from the sea to the land, and during the night from the cool land to the sea. The physiological propertics of the ocean climates, given above, apply 
especially to this type. 'The cases to be sent to such places should be individualized, just as for all other climates.

II. Inland Climates.-'The temperature varies gencrally, according to the season, the distance from the ocean, and the proximity to the mountains, as well as the distance from the equator. As the altitude increases the atmospheric pressure decreases, the humidity lessens, the changes of temperature between day and night increase, and the sun's rays become more intense.

(a) Low Altitude.-Generally speaking, the climate in a low altitude is moist and cold in winter, moist and hot in summer, the mean temperature diminishing with the distance from the equator, being more equable according to the proximity of the ocean climate and drier according to the relative position of the mountains.

(b) Medium Altitude.-The climate here is warm, moderately moist in summer, cool and moderately dry in winter. In places where the rainfall occurs only in winter the climate is warm and moderately dry in summer, these factors being modified by local conditions, such as forests, winds, soil, and regetation.

(c) High Altitude--Here purity of air is the dominant feature. The temperature is cool and dry in summer, espceially in altitudes over 4000 feet; cold and dry in winter, but modificd by local conditions. 'The climate is characterized by the changes incident to diminished airpressure, by abundance of sunshine, snow, rain in showers, and electric storms in summer time. In former times ozone was considered an important factor. There seems now, however, no scientific evidence that ozone is liberated anywhere in such quantities as to be of special service.

'The physiological effects of the high altitude consist of an increase in the respiratory and cardiac functions, and an increase in the appetite and general metabolism. Muscular power and the seeretions of the mucous membranes are diminished. The nervous system is stimulated.

'The effeet on the blood has long been a matter of dispute. 'The blood-pressure is held to be decreased slightly (Huggard, 1906). 'The amount of hemoglobin is increased. The white cells do not increase. That there is an inerease in the number of red blood-cells is acknowledged, but the question at issue is, How much is apparent and how much is real? 'The theories that have been brought forward to account for this increase are based either on changes in the peripheral circulation (Camplell and Hoagland, 1902), by changes in the density of the blood (Grawit\%, 1895), by error in instruments used (Brunings, 1593), and by changes due to the improved general health, either from a prolonged life of the red cells (Fick, 1595), or by formation of new blood-elements (Schaumann and Rosenquist, 1897). 'There is, undoubtedly, some increase in the number of new blood elements formed, but hardly as great as the blood counts would indicate. It is an interesting fact that the increase in the number of red cells is most marked in the first fow days, reaching its maximum in the first month, and then the number rapidly: diminishes on descending to lower levels. Weinzirl (1903) has shown 
that cold is an important factor in the production of the blood changes at high altitudes. It should be remembered, also, that stimulating climates other than those of altitude, through their effects on appetite and metabolism, lead to an increase in blood formation (Schroeder and Blumenfeld, 1904).

The stimulating effects of altitude may, however, be dangerous, in that the demand made on the respiratory, circulatory, and muscular apparatus produce a definite strain on the system, and if the patient is not sufficiently robust, or if he is injudicious in exercising before being acclimated, serious results may follow. In the selection of an altitude for cases of tuberculosis local conditions are as important as is the consideration of the constitutional symptoms.

(d) Desert Climates.-To this type belong the inland climates of low and medium altitudes. A few desert places of value in the United States are below sea-level. These are characterized by an abundance of sunshine, purity of air, low humidity, frequent winds, and dust storms. The disadvantages of this type are the unpleasant winds, at times accompanied by much dust and sand, unsuitable accommodations, poor food, which often lead to nostalgia and mental depression.

Health Resorts in the United States.-An enumeration of these must of necessity be limited in a brief article, but those in my experience which seemed most important will be given, and the cases recommended for each stated definitely on a later page.

In general the open resorts are here intended, and whenever they have the advantage of containing sanatoria for the tuberculous or other diseases, this fact will be mentioned. For the recommendations and prices of these institutions, a useful book is the "Directory of Institutions and Societies dealing with Tuberculosis in the United States and Canada," published by the National Society for the Study of Pulmonary 'Tuberculosis.

The vast extent and topography of the United States, with the latitude, varying altitudes of the several mountain ranges, east and west, the lakes, forests, deserts, and long coasts on two oceans and gulf, make it possible to find any type of climate for health or disease, not surpassed in climatic factors by any European or Continental country. Coast climates may be found in the regions along the Atlantic and Pacific and their islands; inland climates of all altitudes in the east, west, north, and south. 'The east offers proximity to the Appalachians, the chain of mountains extending from the New England States to Georgia, and including the White mountains of New Hampshire, Adirondacks, and Catskills of New York and Vermont, the Blue Ridge of Pennsylvania, and Alleghenies farther south. 'The west offers the Rocky Mountains and the Pacific Coast Range. 'The north offers the Canadian Rockies. Desert climates may be found in portions of Arizona, 'Texas, and southern California.

The Coast Climate Resorts.-The coast climate resorts may be separated for the two seasons, summer and winter, the two exceptions being the resorts of southern New Jersey and California, which are 
available the whole year; in fact, the resorts of somthern Californiat are preferable, more equable, and comfortable in summer that in winter.

Summer Coast Resorts.-Cases suitably selected, which need the relaxing conditions of coast climates, may be sent to the following places: The coast of Maine and its islands, that of Massachusetts and Connecticut, Nantucket and Martha's Vineyard, I ong Island, the northern part of the coast of New Jersey, which has many resorts. 'The facilities offered at these places are chicfly boating, bathing, and fishing. The southern coast of New Jersey, represented by Atlantic City and Cape May, and southern (alifornia, represented by Santa Barbara and the beaches near I os Augeles (as I ong Beach, Santa Monica, Ocean Park, Redondo, Hermosa, and many smaller places), Catalina Islands, and, farther south, I a Jolla; ('oronado Beach and San Diego are both cool in summer and mild in winter, California, of course, having the greater advantage climatically. Similar conditions prevail further north, between Point Conception (which separates the north and south) and San Francisco, and give cooler and more tonic resorts, as Monterey, Pacific Grove, and Carmel by the sea. About San Francisco are many coast suburbs, protected from the severe winds and fogs of that bay, and each give all-year-around comforts. 'The air is more stimulating, has more moisture and less equability than the southern resorts.

The southern California coast has the advantage of being the driest marine climate, and during the summer months has no storms or rains and always cool nights. Health-seekers agree that the summer climate of these places is absolutely perfect-even the hot part of the few warm days is not perceptible on account of the dryness of the atmosphere. 'This dryness is due to the nearness of the desert, distance from storm centres, and that the rain appears only in the winter months, from November to March, so that the summer climate is drier than the winter, and makes the California coast climate, for at least one-half of the year, an exception to the usual type of ocean climate.

I am equally enthusiastic over its equability, and believe no other coast approaches it for an all-year comfort and peace. On this point, read what the former professor in Kings College, London (Burney Yeo), says:

"This coast, with the portion of the Pacific slope lying directly" hehind it, possess one of the most genial and equable climates in the world. It is under the influence of the warm ocean eurrent from Japan and ranges of high mountains form its northern boundary, the land sloping gradually down from the east to the ocean. It has mild winters and relatively cool summers, without extremes either of heat or cold. In the northern regions of this const the utmosphere is humid, and rainfall is considerable, but this diminishes as we pass southward, and there is a great difference between the climate of the town on the northern part of the coast and that of the resorts to the south."

'The chief objection is fog, which is present for part of the day on half 
the days of early summer, but proves a benefit to inland regions by cooling the air for many miles.

Winter Coast Resorts.-Atlantic City and Cape May, for their situations, have exceptionally mild winters, and afford an escape from the changeable spring months of other places. They are popular with people returning early from Florida and other southern points.

Old Point Comfort is an early winter resort; Savannah, Ga., and Charlestown, S. C., formerly popular winter resorts, have the disadvantages of large and commercial cities. Many of the suburbs have the climatic advantages without the disadvantages.

'The islands off the South Atlantic coast have warm, moist, and sedative conditions.

Bermuda is probably the most fashionable, and its season is from December to March. It has excellent commodious hotels.

'The Bahamas (January, February, and March), represented by Nassau, closely resemble the climate of Madeira, which has always been heralded as an excellent relaxing resort. Each has summer weather in midwinter, with plenty of sunshine and pure air, and is soothing and invigorating.

Puerto Rico and the Coast of Cuba, from December to March, have the same climatic factors as Bermuda and Nassau, and, when the accommodations are provided, will become even more popular.

Finally, the most important winter resorts of this type are to be found in Florida and southern California, both of which will bear comparison, each having advantages for certain classes of disease.

\section{WINTER MONTHS.}

FLORIDA.

Short season (December to April).

More rain and humidity.

Moist and enervating.

Warm winter months.

less average hours of sunsline.

Slight diurnal elianges.

Accessibility to eastern States.

Evenings like summer.

Balmy air-eurrents.

Iess wind and dust.

Rich tropical vegetation (in abundance).

\section{Southern California}

Whole year (summer even better than winter).

Less rain and humidity.

Less moist, relaxing, and often tonic.

Colder winter months.

Greater average of hours sunshine.

Great diurnal changes.

Inaccessibility to eastern States.

Cold evenings and nights.

Chilly air-currents.

More wind and dust.

Semi-tropical vegetation by irrigation. Much dry barren country.

By examining these columns, one can see that, to make a change for disease, California is far preferable, excepting its distance from the temperate eastern States, while Florida is advantageous to the tourist, or those secking a change and rest for a short period of time.

In general the southern California coast is cool, equable, moist, and bracing; Florida is warm, equable, moist, and enervating. The winter resorts of Florida are Jacksonville, St. Augustine, and small places along the St. John's River. 'Toward the south are Orlando and Winter Park; in the south are Palm Beach and Miami, with Tampa on the Gulf at the terminus of the railway. Palm Beach and Miami are favorite 
resorts, with excellent commodious hotels and a short season (January, February, and March). 'The winter resorts of the southern California coast are the same as those given for the summer months.

Inland Climatic Resorts.-Low Altitude.-For summer months are the places along Lake Champlain, Lake George, the 'Thousand Islands, and regions along the St. Lawrence River, all of which are cool and mildly stimulating, offering most attractive facilities for boating and sailing. These locations, as well as numerous places near the northeastern States and near the Great Lakes, are most excellent sections for a limited number of health-seekers who are sufficiently strong to enjoy the sports, or convalescents from acute diseases; otherwise, more suitable regions should be chosen. 'The central plateau of Massachusetts, elevation 500 to 1200 feet, partakes of this low inland type, as the country about Worcester and Rutland, where is situated the State Sanatorium. The western part of the State offers still more attractive summer places, among the Berkshire hills, part of which belong to the medium altitude group, but are best included here: Lenox, Stockbridge, Pittsfield, Great Barrington, Adams, and Williamstown; the air is cool and mildly stimulating, especially in the early autumn and winter months. Saratoga, New York, has always been a most famous mineral-water resort from June to September. It has some very hot, moist days, which may be a benefit to the class of invalids recommended, but September is usually uncomfortable. It has excellent accommodation for all classes and a sanatorium.

Northern New Jersey, called often the Pine region, represented by Lakewood, Brown's Mills, and Lake Hopatcong, is indicated for the winter, spring, and fall months rather than midsummer.

Morristown and Summit, altitude 500 feet, the Oranges and Bernardsville, are quiet, attractive, residential districts, well suited for outdoor life to people who cannot go far from New York.

For the Winter Months.-Most resorts of low altitude are chiefly adapted for the winter time. Lakewood, New Jersey, altitude 60 feetseason October 1 to June 1 - has sandy soil, is well protected by pine forests, and, on account of its proximity to the ocean, is more equable than other inland New Jersey places. It has excellent hotel accommodations and medical men.

Delaware Water Gap, Pa., 600 feet, is comparatively dry; has good porous soil and adequate accommodations.

Southern Pines, North Carolina, altitude 700 feet, ahout 100 miles from the coast, is a favorable stopping-place for travellers to and from the more southern regions. Its nearness to the ocean moderates the cold weather of the higher regions of this State. It is well protected, and has a sanatorium for lung cases. I'inehurst is three miles distant.

Aiken, S. C., 565 feet, season, December to May, is well protected by forests, and has many attractions. It has the driest climate in low altitude of any place east of the Rocky Mountains, with an abundant sunshine and relatively few variations of temperature. Its mean winter 
temperature is about $50^{\circ} \mathrm{F}$. It is a popular resort for catarmal and pulmonary cases, having good aecommodations and a sanatorium.

Augusta, Ga., altitude 139 feet, and North Augusta, S. C., altitude 600 feet, are separated by the Savannah River and climatically similar to Aiken. Both have equable mild winters, and are suitable for a large class of invalids, as well as others who wish to avoid the rigorous and changeable conditions of the northern regions. Both are delightful places for the winter months, where many amusements may be had. Recently better accommodations and small sanatoria have been provided.

Atlanta, Ga, altitude 1059 feet, is placed in this group, as it cannot compete with the resorts of medium altitude. It has the disadvantages of population, of its situation, colder in winter and warmer in summer, than other resorts of the State. Its mean winter temperature is $44^{\circ} \mathrm{F}$; mean summer, $77^{\circ} \mathrm{F}$; the best season is during March, April, and May.

'Thomasville, Ga., altitude 330 feet, has had for many years the reputation of being a good winter resort. It has the advantages of being well situated, in a pine forest on sandy soil in the southern part of the State, and being warmer in winter; the mean temperature is $53^{\circ} \mathrm{F}$. It has a mild relaxing climate, and is suitable for cases which do not bear cold weather. The country about is beautiful and attractive for riding and driving.

Some of the inland places of Florida might be included in this group, but they are all so similar to coast climates that I prefer to keep them under that heading.

San Antonio, Texas, altitude 680 feet, is a fairly dry, equable, and relaxing climate. It should be recommended only for winter months, December to April. The temperature occasionally goes to and below freezing point. Its disadvantages are a short season, poor accommodations and facilities.

Yuma, Arizona, altitude 140 feet, at the line of southern California, near the Mexican border, is our best type of desert climate, and nearest the climate of Egypt. It is characterized by extreme dryness, pure air, and abundance of sunshine. The section about Yuma has the lowest average rainfall during all four seasons of any region in the United States. The climate is comfortable and suitable from December to April; the average temperature is $60^{\circ} \mathrm{F}$; ; average humidity, $40^{\circ} \mathrm{F}$. Unfortunately, this town and the surrounding country are not properly equipped in any way for health-seekers or invalids.

A valley, recently made prosperous by the leashing of a part of the Colorado River, and situated in southern California, has the same desert climate, where invalids ean get limited accommodations. I refer to the Imperial Valley, which has wonderfully dry, equable conditions. Banning and P'alm Springs, California, are similar climatic resorts of a desert trpe, situated on the west side of the Pacific coast, and cut off from its humidity and rain-storms. Each has limited accommodations for pulmonary cases, and Banning has a small sanatorium. 
'The chief objection to all these desert places is the difficulty in obtaining good food and the great heat of summer.

Additional low types of southern California partake more or less of the coast climate, modificd by nearness to this Colorado Desert, and depending upon this modification are the places suitable for all-year-round resorts or as only suitable for winter. Places up to 1000 feet elevation, and within 40 miles of the coast, are comfortable the whole year; the nearer the ocean, the cooler in summer and the warmer in winter, and vice versa. Fogs are numerous in the spring and summer months for a part of many days, extending many miles over the inland places,- usually 30 miles, and often 50 miles,-and disappear earlier the greater the distance from the coast. 'The days of summer without these fogs are often hot ones for these inland resorts. Sections beyond forty miles from the coast, or from 1000 to 3500 feet, are suitable only during the winter and spring months, November to June, as, according to the distance, they take on more of the desert and less of the coast factors. 'The separation of towns in southern California, and the lack of accommodations and comforts, which were former drawbacks, are gradually disappearing, and the counties of San Bernardino, Riverside, Los Angeles, Ventura, Santa Barbara, and San Diego, which contain many health resorts, are gradually being populated in a most astounding manner.

Redlands, elevation 1200 feet, and Riverside, about 900 feet, 70 miles from the coast, are the two most attractive winter resorts to be found, and are at one end of the San Gabriel Valley, which extends to Pasadena. Along this valley are several small towns, each of which is a delightful residential place, containing ranch homes and boarding-houses, inadequately supplied for caring for invalids, but where no better climate can be found for the group of cardio, renal, and circulatory diseases, or where the outdoor life is more agreeable and attractive.

People, to have the right care in southern California outside of a sanatorium, must be in a position to keep house or live with friends. Most of the numerous boarding-houses are not suitable for any except healthy people or those who can lead normal lives.

From Redlands down may be mentioned San Bernardino, Riverside, Ontario, Pomona, Claremont (college town), Azusa, Duarte, Monrovia, Sierra Madre, Altadena, and Pasadena. Winter climates for low altitude of this group are Redlands, San Bernardino, Riverside, and Ontario. 'The others are suitable for all-year-round residence, with some hot days in summer, when the temperature will reach $9 S^{\circ}$ and $100^{\circ} \mathrm{F}$., but the relative dryness always saves any great discomfort.

Monrovia, Sierra Madre, and Altadena are at the base of the coast range, with altitudes of 1000 to 1400 feet. Many invalids reside in these places, especially tubereulous cases, that need low altitude treatment under equable conditions. Sanatorium treatment may be had at Monrovia.

Pasadena, altitude 800 feet, nine miles from Los Angeles and 25 miles from the coast, is an excellent all-year-round place. It is, however, more suitable for winter sensons, and, on account of the local 
topography, has less fog than many sections equally distant from the ocean, is one of the most charming residential districts on the American continent, with beautiful homes and grounds, and, together with Santa Barbara, the most popular for tourists. It is rapidly growing out of the resort class, but its outlying districts are still well adapted for invalids. 'Three miles to the east, at Lamanda Park, is an excellent sanatorium for nervous cases of the neurasthenic type, convalescents, and patients broken down from physical or nervous strain.

Los Angeles is fifteen miles from the coast, and in consequence has cooler summers than Pasadena. Having grown to be cities of the larger class, with over 300,000 population, these towns have, therefore, all the disadvantages of a modern city, which have lessened their desirability as health resorts. Their climate, however, suits many chronic cases who become permanent residents, and its outlying districts or suburbs are filled with desirable and grateful patients. Farther north lies the Ojai Valley, a few miles from Santa Barbaxa, which telongs to this low altitude type. This valley is an excellent place for winter, spring, and fall; hot in summer, but with climatic advantages for an ideal outdoor life, and with the disadvantages of inadequate accommodations and difficulty of obtaining food.

Nearer Santa Barbara are other valleys (Montecito) which are ideal the year round, and other places in the foot-hill regions which have strong admirers. An excellent sanatorium for nervous troubles and convalescents can be had at Santa Barbara. Near San Diego are the El Cajon and Lakeside Valleys, with ideal climates the greater part of the year. In the northern part of the State, near San Francisco, are protected inland places that serve many people. Santa Clara Valley is represented by San José, Los Gatos, and Palo Alto; near the bay are Berkeley and Alameda. The conditions are comfortable and suitable the whole year.

Residence in a climate may make one too enthusiastic in its coloring, and it is pleasant to have my experience indorsed by the words of a European authority. Huggard says:

"Owing to the mildness of its climate, the moderate degree of its humidity, and the absence of extremes of heat and cold, southern California is suitable for a greater variety of invalids than is any country of similar size in the world. The heat is seldom oppressive, and the cold is rarely so great as to be trying even to delicate invalids. The days, even when warm, are succeeded by cool evenings, which, to most people, form a refreshing contrast. . . There is no climate in the world without drawbacks, but fewer climatic disadvantages for permanent residence are probably to be found in southern California than in any other country under the sun."

Medium Altitude (1000 to 3000 feet).-Whole Year.-Resorts of this group are available and suitable the whole year, except those of Arizona (Phoenix and Tucson), which are only winter resorts.

Phoenix, Arizona (altitude 1087 feet), is favorably located, and well known for its mild winter weather, dryness, and great amount of sunshine. 
It is suitable in the late antumn, winter, and spring months (November to May) for all cases that need ontdoor life in dry air, and who, on account of some complication of cardiac, renal, or circulatory system, are unsuitable for high altitude. It has, in common with all Arizona, the advantages of pure air, a great amount of sunshine, dryness, mild winters, and few bad storms. 'The greatest rainfall is in July and August; a little again during the winter months, but not much at any time, the anmual mean being six or seven inches. The mean temperature for the three seasons is $53^{\circ} \mathrm{F}$. to $70^{\circ} \mathrm{F}$. 'The summers are entirely too hot for comfort in health or otherwise. Suitable and comfortable aceommodations may be had, with exeellent medical treament. 'T'empe, a small town sonth of Phoenix, has the same climate without the other advantages.

Tueson, Arizona (altitude 2400 feet), in the southwestern part of the State, shares the excellent climatic conditions of this State for winter months. On account of the altitude, it has the advantage of a more stimulating climate, for many cases, over l'hoenix, and many who have spent the winter in both places speak more favorably of 'T'ueson. It has the disadvantages of the shorter season, December to April, but my experience has been that pulmonary cases, under as good care, during those montlis will do better in 'Tueson than Phoenix. Better accommodations are being yearly provided at 'Tueson, which was formerly its greatest drawback. Redlands, Beaumont, and Banning, California, might well be mentioned in this class as well as among those of the low inland types.

The White Mountain resort region of New Hampshire, elevation from 1200 to 1500 feet, is well suited for certain cases during summer and autumn months, and may be represented by the town of Bethlehem (1500 feet). 'The air is cool, moist, and mildly tonic in spring and autumn, and often dusty and warm in summer. The Adirondack Mountains of New York (altitude 1500 to 2500 feet) are represented by the many lake resorts of this region, which are all available for summer and autumn. Saranac Lake, Lake Placid, Paul Smith's, Loon Lake, Chateaugay, etc., where excellent aecommodations may be hao.

Saranac Lake, for the whole year, is the principal resort, near which is the town of 'Trudeau, made famous by Dr. 'Trudeau, who stands responsible for the great reputation of this section in the successful outdoor treatment of pulmonary tuberculosis. At 'Trudeau is situated The Adirondack Cottage Sanatorium. 'This region is studded by small lakes, forests, and evergreens, and has abundance of pure air and water. 'The air is cool, moist, and mildly stimulating in spring, summer, and autumn, cold and stimulating in winter.

The Catskill Momntains (elevation 1000 to 2000 feet), represented by 'Tannersville and Kaaterskill and other attrative stations, are used chiefly as summer reorts and have no special provision for invalids. The climate is similar to the Adirondacks, but the air is not as bracing or stimulating. 'To the sonth are the hills of Sullivan County, which have become popular and available for many chronic casces, esperially. Vor. I-10 
of the pulmonary type, and represented by Liberty (altitude 2300 feet), near which stands the Loomis Sanitarium for tuberculosis.

The Blue Ridge Mountain region of Pennsylvania has been more or less heralded for its pure dry air, which is true of this section of the State. It has good porous soil, and may here be represented by Glen Summit, 2000 feet, near which is situated the White Haven Sanitarium for Poor Consumptives. 'This section is more agreeable during the winter and spring months; in summer it is warm.

In Virginia there are several places with natural hot and cold springs, but the best known, best equipped, and most popular is Hot Springs, in Bath County, altitude 2300 feet. It is an excellent example of the mountainous regions of both Virginia and West Virginia, having the advantages of climate and baths. The air is cool, moderately dry, and stimulating. The best season and more agreeable weather is found in the autumn and the early winter and spring months. The midwinter is cold and the midsummer hot. It is well equipped for invalids, and has a bathing establishment of modern type, with every luxury and comfort which are necessary to compete with European spring resorts. Most of our spring resorts are wanting in equipment, amusements, and luxurious comforts, although their natural opportunities are often equal to the best. The value of Hot Springs has been described by Hinsdale in his article on Mineral Springs.

Asheville (altitude 2255 feet) is the principal resort of North Carolina, and is an excellent all-year-round climate. Its best season is from October to June, though the summer is comparatively cool and dry. The country is hilly and well wooded, with higher mountains a few miles distant. Those who have known this section say it is one of the most delightful east of the Rocky Mountains. Asheville has gained an enviable reputation for the treatment of pulmonary disease. Certainly, I know of no better place for all-year-round residence for cases that need altitude, and for definite reasons cannot be placed in high elevations. The summers are cool, with mean of the maximum temperature $82^{\circ} \mathrm{F}$. The winters are cold, with mean of the maximum temperature $29^{\circ} \mathrm{F}$. The frost and snow are light and disappear quickly. The air is tonic and stimulating. A patient who should remain in one place a whole year, and needing a medium type of altitude, should choose Asheville, especially if he can exercise a little. Accommodations in sanatoria and wellregulated boarding-places may be had. Hot Springs, N. C., is only 35 miles distant from Asheville, is an all-year-round resort for those who need the water treatment, and is another good example of the mountainous region of the western part of the State.

High Altitude (3000 to 7000 feet).-The chief resorts for this treatment are to be found in the Rocky Mountain region of Colorado and New Mexico. All share similar conditions and characteristics, and are especially advantageous in the treatment of pulmonary diseases, Colorado leading in accommodations, care, and medical attention. New Mexico is a little superior in climatic advantages. This whole region is a suitable, natural resort for pulmonary tuberculosis; the winters are cold 
and stimulating, the summers cool and bracing. ()ther churacteristices are pure air, dryness, elevation, abundance of sunshine, diurnal changes, or wide difference of temperature between day and night, and between sumshine und shade. 'The disadvantages seem to lee ronfined to the oceasional wind and dust storms and the isolation felt at the most of these places. 'The latter has been the greatest oljeection on the part of patients, in my experience.

Colorado has elevations varying from $4(x)(0) 10,(x)$ feet, with mountain-peaks over 14,000 feet. ('haracteristic features of the State are: Altitude, great distance from the ocean, dryness, marked diurnal changes, abundance of sunshine, and pure air. "The climate of the eastern and southeastern parts is best. 'The mean temperature for winter is about $30^{\circ} \mathrm{F}$; mean of the maximum, $40^{\circ}$ to $50^{\circ} \mathrm{F}$; mean of the minimum, $12^{\circ}$ to $18^{\circ} \mathrm{F}$; the mean for summer, $70^{\circ}$ to $76^{\circ} \mathrm{F}$. 'The average relative humidity is from $4 S$ to 50 per cent., which is lowered as a higher temperature prevails. 'The winters are cold and the summers cool and dry. Many summer health resorts are to be found. The two chice resorts of the State are Denver and Colorado Springs, with their ontlying districts. For much information as to the advantages and atmospheric conditions of this State we shall always be indebted to Solly, whose book is referred to for further information. 'The indications for these resorts are those given for high altitude, and there are no better places for the treatment and eare of pulmonary tuberculosis, or a climate that will agree with as many patients. It can be compared most favorably with the famous resorts of the mountains in Switzerland. Objections may be found to any climate under the sun. 'The objections to Colorado are the oceasional wind and dust storms, and, to a few patients, the sharp diurnal changes and the cold of winter.

Denver (altitude 5183 to $5600 \mathrm{fect}$ ) is an active, busy city, beautifully constructed and located. It has all the advantages of a modern city, with few of the disadvantages. If one is well enough, he may enter a business life and find congenial companionship, which is never to be underestimated; the climate is at all times stimulating. One can find good care, splendid accommodations, and excellent medical attention. Several sanatoria are available, among them "The Home" and the "Agnes Memorial Sanatorium."

Colorado Springs (altitude $6098 \mathrm{feet}$ ) is as beautifully situated as any resort in the country, surrounded by higher mountains, and with Pike's Peak in the distance. 'The porous soil, drainage, and water-supply' are all that a resort should have, and the construction of the town, with its wide streets and comfortable simple homes, impresses every observer that outdoor life is here easily maintained. 'The advantages of this resort are largely due to the help of the late General Palmer. It has all the elimatic advantages of Denver, without the disadvantages of a large city. Good accommodations, care, and medical attention may be had, and there is a sanatorium for tuberculosis. 'The objections to the place are those of the region, especially the wind and dust storms, which prevail in spring and fall, and against which invalids must be protecter. 
The climate is at all times stimulating, and throughout the year is cooler than Denver.

\begin{tabular}{|c|c|}
\hline n & Colorado Springs. \\
\hline & $\ldots 7^{\circ} \mathrm{F}$ \\
\hline Mean of the minimum. & $\ldots 34^{\circ} \mathrm{F}$ \\
\hline
\end{tabular}

Though these differences do not secm great, they are sufficient to make the summers better and more agreeable at Colorado Springs than 1)enver, and to make Denver warmer in winter. As a fact, many people who live in Denver in the summer go to higher places where it is cooler and more bracing. Colorado Springs has ideal summer weather. Both places, however, are all-year-round resorts.

Manitou (altitude 6300 feet) is five miles west of the Springs, at the foot of Pike's Peak; it has no suitable accommodations for invalids, and is sheltered from the cool breezes in summer. Glenwood Springs (5£00 feet) is recommended for summer only, It has a comfortable hotel.

Estes Park (altitude 7000 feet), 75 miles from Denver, is used as a summer resort, and has excellent accommodations and pure mountain air. Many cases make this change for the summer season.

The New Mexico resorts have similar elimatic conditions to Colorado, characterized by altitude, dryness, abundance of sunshine, diurnal changes, and pure air. 'The winters and summers are both slightly warmer, which may be more suitable, depending upon the individual. Owing to the dryness, however, the heat of summer at any of the resorts is never uncomfortable or oppressive, and the nights always delightful. I have been in most of the places at different times of the year, and feel that, when more accommodations and comforts are provided, the resorts of New Mexico will offer for the whole year residence advantages excelled by none.

The State possesses an excellent climate for outdoor life the whole year. The annual mean temperature is $54^{\circ} \mathrm{F}$; winter average, $36^{\circ} \mathrm{F}$.; summer average, $72^{\circ} \mathrm{F}$; average annual precipitation, 13 inches; and the mean relative humidity, 40 per cent. 'The objections to some of the resorts may be stated in the frequency of the severe winds during the spring months, the lack of accommodations, and difficulty of getting comforts. The principal resorts can now supply good medical stimulation as trained men are yearly becoming residents of this State. In the southern part of the State, Silver City (altitude 6000 feet) is approached in two hours from the main railroad at Deming. Its situation is proteeted from the winds, and has become well known for its climatic advantages during the winter and spring for pulmonary cases, and can surely be safely recommended to possess advantages during the whole year. 'The summers are not too hot nor the winters too cold. Bullock, in his article, gives the mean anmual temperature $54^{\circ} \mathrm{F}$, relative humidity, 46 per cent., rainfall 12 inches, and only 37 eloudy days in the year. Climatically, there is no hetter region in the Cnited States, but the section is isolated, and still lacks the accommodations, comforts, and 
amusements of modern settled resorts. One must le prepared to rent a house or enter one of the sanatoria, three of which offer good accommodations and medical attention, the largest and best expuipped being the "New Mexico Cottage Sanatorium."

Fort Bayard (altitude 6200 feet) is nine miles from Silver (ity. 'The large Government Sanatorium for the officers and men of the U.S. army was placed here as offering the best climatic conditions of our States.

Ieming ( 4315 feet) is an unattractive small town, with a good winter climate, but cannot be recommended as a resort in any way. lt has no accommodations, and its location, without sheltering hills, exposes it to the severe wind and dust storms.

Santa Fé (altitude 7013 feet), Las Vegas (altitude $6500 \mathrm{fect}$ ), and Albuquerque (altitude 5200 feet) are three cities in the northern part of New Mexico, on the line of the Santa Fé Railroad, and are the bestknown resorts. The climate of these places is very similar to that of Denver. 'Ten years' record of the weather bureau shows that the winter temperature in the above resorts does not fall so low as that of Denver, nor is the summer temperature as high. 'The humidity is less, and in Santa Fé there is less wind. An exception might be made of I as Vegas, the summer temperature of which is higher, though we have no proved record of the fact. Santa Fé, in a ten years' record, shows a winter mean temperature of $31^{\circ} \mathrm{F}$; s summer, $67^{\circ} \mathrm{F}$; the lowest for winter is $13^{\circ} \mathrm{F}$. below zero; the highest during summer is $97^{\circ} \mathrm{F}$. The relative anmual mean humidity is 55 per cent. at $8 \mathrm{~A}$. $\mathrm{M}$. and 36 per cent. at $S \mathrm{r}$. . with an average of 279 hours of total sunshine for the month. 'The prevailing wind is southeast, and is less intense during any part of the year than in either of the other places.

Las Vegas is warmer and dustier in summer than Santa Fé, has higher winds in spring, with much the same fine winter climate.

Albuquerque is warmer, both in winter and summer, than Santa Fé, has less precipitation, but has severer and more frequent winds. The mean temperature for winter is $35^{\circ} \mathrm{F}$; for summer it is $76^{\circ} \mathrm{F}$. The lowest temperature is $10^{\circ} \mathrm{F}$. below zero; the highest is $104^{\circ} \mathrm{F}$. 'The prevailing wind is south. The anmual mean rainfall is 7.2 inches, while Santa Fé has 14.2 inches. Albucyuerque is a most thriving and progressive city, and better accommodations and food may be ohtained there than in Santa Fe, but the climatic conditions are not so favorable. On account of the lower humidity and the greater amount of sunshine. these resorts would be better than either Denver or Colorado Springs if the elimate alone were to be considered, but the resorts of Colorado possess the other factors so necessary in climatotherapv. 'There are sanntoria and limited accommodations at each of these three places.

The high altitude resorts of Arizona are limited, and, of the two mentioned, Preseott is by far the best and most important. 'These elevated regions have all the characteristic climatic advantages of the Rocky Mountain region, except that the winters are milder and warmer-altitude, dryness, great amount of sunshine, marked diurnal changes, mild winter, cool summers, and pure air. Mean temperature, $60^{\circ}$ to $65^{\circ} \mathrm{F}$; 
relative humidity, 30 to 50 per cent. Prescott (altitude 5260 feet) is practically the only all-year-round resort of high altitude in the State. It is a prosperous, growing town, but still retains much of the old mining ways. It has limited accommodations and a good sanatorium for tuberculous cases. Both the winter and summer climate are excellent, and many health-seekers from lower altitudes go to Prescott for the summer months. Its location is protected from severe winds and dust storms.

Flagstaff, Arizona (altitude 7000 fcet), has an excellent all-year-round climate, cold and snow in winter, as occurs at Prescott, and cool days all summer, but, as yet, there is no provision made for invalids; it is beautifully situated, with forests of pine, and the country is interesting for those who like the wonderful scenery and coloring of this land.

El Paso, 'Texas (altitude 3700 feet), is similar climatically to the southern part of New Mexico, but its situation and lower elevation make the summers impossible for health-seekers. From November to May the climate is good for invalids; the air is cool, dry, and stimulating, with a relative humidity of about 50 per cent.; there are limited accommodations, and a hospital and sanatorium for the tuberculous.

Southern California, in the mountainous regions, possesses the climatic conditions of high altitude without the disadvantages of wind or dust storms-San Jacinto and San Bernardino, Idyllwild in the Strawberry Valley on San Jacinto, and Bear Valley on San Bernardino, elevation 5200 feet. The climate is excellent the whole year; mild winters, with snow and ice, and cool summers; the air is tonic and stimulating. Idyllwild, has been closed as a resort for several years, but good accommodations may be had here during the summer for those who are not invalids, and a limited number of people can be taken in Bear Valley.

In the northern part of the State is Lake Tahoe (altitude 6000 feet). It is well equipped for summer tourists.

Advantages and Disadvantages of Climatic Treatment.-We have come to a more rational view of elimatic treatment, and, through experience and results of many years, realize that the larger number of chronic diseases will be benefited (other things being equal) by adding to other means a suitable climate. Our science teaches us to properly use and control nature's resources, which, rightly directed in disease, brings anticipated good results. We use numerous physical agents to effect a cure-massage, application of cold and heat, electric therapy, radiotherapy, and here, in climatotherapy, we give all such agents at the same time. It is nature's way of bringing benefit or cure, and, with the exception of tuberculosis, it has been grossly and inexcusably neglected in our text-books under the treatment of other chronic diseases. With this one exception it seems, in company with psychotherapy, to have been put aside and left in the hands of the untrained and ignorant. Specific directions are scareely ever given to the student or physician.

One is left without a suggestion of the proper resort, or a word of direction as to the place, time, and dosage; not even what to avoid in preseribing a climatic change. On the other hand, where a drug that has scemingly benefited some cases is recommended, speeifie dircetions 
for time and dosage are given. If I can read correctly the present "writing on the wall," there is a decided change in vogue. All fresh and open-air treatment is part of climatic treatment, and it can be most effectually carried out in places or resorts with the greatest atmospheric advantages, or what we eall best climate. 'The best climate is that place which will allow the patient to be in the fresh air the whole twenty-four hours or lead an ideal outdoor life. For a decade climate or open-air treatment has been insisted upon in the care of the tuberculous, and we see it now urged for other diseases. I believe the time is near at hand when every hospital ward will be ineomplete without its open-air balcony, porch, or pavilion adjacent, which will be as necessary as a trained nurse and attendants, and which will prove of such benefit that the entire ward will be utilized merely as a working or dressing room. Children and adults are now treated in the open air for most of the acute and chronic diseases. 'They should be put out-of-doors in every elimate in all but the worst weather. Statistics show they will improve. Pneumonia has lost some of its terrors through this treatment, and eastern physicians, who strongly advocate it, say that they have never seen anything like it in the treatment of any disease, except antitoxin in diphtheria, many cases having been saved that formerly would have seemed hopeless.

That atmosphere has a decided influence on body metabolism is a well-recognized fact. Of the influenee of elimatic conditions on metabolism, Nichols (1901) says:

"The main avenues by which heat is dissipated are three: (1) Radiation, conduction; (2) evaporation of water in perspiration and expired air; (3) external work. The atmospheric conditions affecting the escape of heat from the body are temperature, humidity, and the wind. 'The greater the difference between the temperature of the body and that of the surrounding objects and air, the greater is the radiation and conduction of heat from the warmer to the cooler. Hence the body gives off more heat in a cold than a warm environment, and to make up for the loss, oxidation within the body is increased by the heat-regulating mechanism. As the surrounding temperature rises, the heat lost diminishes, and oxidation within the body also falls." Such circumstances prove, what experience has taught those of us who have studied cases in different elimates, that more food in cold climates is needed to make up for this heat loss and less food in warmer climates. If all persons responded in sim ilar manner to the same influences, the best results might be found in: cold dry climate, but here, as elsewhere, individual peculiarities exist and people, handicapped by certain diseases, find more benefit in warm, equable elimates, where less demand is made.

Bullock and Sands give results and comparisons of elimatic treatment as exereising a definite, favoring influence on pulmonary cases.

Any of the ehronic diseases mentioned in subsequent pages will be benefited by a change to more favorable climatic conditions; when long travel is impossible, removal for short distances, where more time may be spent outdoors and the air is purer, may suffice.

Other advantages than changed meteorological conditions are free- 
dom from lusiness and home cares, mental and physical stimulation from changed surroundings, and the stimulus that often comes from the different physician who seems to the patient at the resort to understand his particular trouble. Very often the separation from other members of the family acts adrantageously, and, with some chronic troubles, will prove the one thing needed, as many physicians can testify. The cases that especially call for climatic treatment are the pulmonary, cardiac, and renal diseases, which will receive specific directions under their several classes.

'The disadvantages of this treatment have keen touched upon, chief among which are the expense involved, the sefaration from home and friends, the relinquishing of business affairs, the distance, and the journey. In not a few resorts I must add the temptations created by too much amusement, dissipation, or exercise. Iocal disadvantages attached to the resort may be considered as poor or unsuitable accommedations, food, hygiene, and care, lack of comforts and amusement. and, most of all, good medical attention.

Grecly gocs so far as to say that "the best climatic results are largely due to good hygiene and care."

Again, to emphasize the importance of hygienic conditions, I would mention the experience of Gorgas in Panama and Cuba. Gorgas lays less and less stress upon the climatic conditions of those places, and more and more upon hygienic requirements, as being responsible for the healthy conditions and low death-rate. When these results can be gained in the poor climatic conditions of such countries, how much better must results be with the same hygienic surroundings under good climatic conditions?

'The right medical attention at health resorts must be emphasized, because it secms folly to send any case that needs attention to a climatic resort withont the home physician making sure that his patient will have good medical care. Most resorts are like big sanatoria, where all kinds of chronic invalids and hypochondriacs are treated by as many kinds of cranks, and where quacks and charlatans eke out a good living. 'Too often patients fall into the hands of quacks because they have received inadequate instructions before leaving home.

Indications and Contraindications for Climatic Treatment.Under this head will be given the diseases and class of cases which indicate or contraindicate climatic treatment, using the preceding classifieation of the types of climate. After indieating what diseases may best be treated in a coast climate, low altitudes or high altitudes, I will then give, under the several diseases, such resorts as may fill these indications.

It must be remembered that the early stages of some chronic diseases may get well in any climate under right cireumstances, and, therefore, such cases will be indicated in more than one group, for example: Ineipient pulmonary tuberculosis, under right management, may get well in any of these climates, and will be found in each group of diseases indieated. It must le left to the individual peculiarities, or knowledge of a choice, which place scems best for each case. Having had fifteen 
vears' experience in an open-air resort for health-seckers, I have learned that the physician who individualizes most carcfully will experience the best results. In order not to repeat too often, I will first inention the diseases that are benefited by and suitable for a change to any of the types of elimate:

Incipient pulmonary tuberculosis, fibroid phthisis, early cardiae disease with good compensation, early interstitial nephritis, without cardiac complications or marked rise of blood-pressure, early forms of insanity, the anemias, digestive disturbanees, certain cases of asthma and chronie rheumatism, neurasthenia, and functional neuroses.

Indications for Coast Climate.-(1) Pulmonary eases: incipient or early eases of tuberculosis without fever, and with few or no constitutional symptoms. (A history of early hemorrhage is no contraindication.)

Chronic fibroid cases, with or without marked bronchitis and $\mathrm{cm}-$ physema (the accompanying bronchitis should be a more or less dry type). Tuberculosis of the upper air-passages. Elderly people with long-standing inactive trouble. Cases complicated with eardiorenal diseases. Chronic bronchitis and emphysema (especially old and very young people). Marked emphysema (any age).

(2) Kidney cases. Chronic parenchymatous and interstitial nephritis, especially with tendency to high blood-pressure.

(3) Heart cases. In valvular trouble, with or without loss of compensation. Myocarditis, arterioselerosis, and angina.

(4) Diseases of the nervous system, with marked neurotic manifestations.

(5) Acute or chronic bone lesions of childhood.

Contraindications.- The great mass of pulmonary tuberculous cases, especially the active type. Acute bronchitis.

Indications for Inland Climates. - It is difficult to draw any sharp lines for eases that should be sent to either low or medium altitude. Certain general principles and classes of patients are a distinct guide, but individualization must be used here as well as decision between medium and high altitudes. Besides the pressure, much depends upon the other atmospheric conditions, and the nature of the place at the time of year. 'There are very distinct indieations for and against high altitude, which are a guide for inland types.

Indications for High Altitude Treatment (3000 to 7000 Fcet).-(1) Pulmonary cases: the great mass of tubereulous eases (hemoptysis no contraindication); early tuberculosis, with or withont fever and constitutional symptoms; early or mild latent tubcreulosis-cases with infiltration or beginning destruction, with pleurisy, or old pleuritie adhesions; tuberculosis with compensated cardiac disease.

(2) Forms of asthma that have no great degree of emphysema.

(3) Secondary anemias and chronic digestive disturbances without cardiac or renal involvements.

(4) Convalescents from acute diseases that have not involved the heart.

Contraindications for High Altitude.-(1) Pulmonary. Acute pneu- 
monic cases of tuberculosis with high fever, rapid pulse, and respiration (high fever alone is no contraindication), advanced cases, with extensive involvement of both lungs, or cavity formation of both lungs, with extensive adhesions and dyspnea on exertion, all of which will do better at low or medium altitude; chronic fibroid cases, with accompanying bronchitis and emphysema; late laryngeal cases; young children and infants, with any pulmonary condition, which will do better in low or medium altitudes; emphysema, chronic bronchitis of the advanced type, bronchiectasis, empyema, and, in general, any pulmonary case that has cardiac, renal, hepatic, or severe gastro-intestinal complications.

(2) Kidney cases in general.

(3) Heart cases, except those of moderate involvement, with good compensation; patients whose heart muscle is handicapped by chronic disease; arteriosclerosis.

(4) Diseases of the nervous system and those with marked neurotic history.

Indications for Medium Altitude (1000 to $3000 \mathrm{Feet}$ ).-By individualizing cases, one may now readily perceive that any one of the cases indicated for high altitude may improve and do well at a medium altitude, especially when the resort most conveniently located or better equipped for the individual is more available. Always keep in mind, however, that the more such cases can approach the atmospheric conditions of high altitudes, the more satisfactory will be the result. I often choose a medium altitude during the winter months, November to May, and a high altitude for the summer, May to November, on account of the severe cold and changeable weather that will occur in most resorts of high altitude. Remember, on the other hand, that some patients feel better in cold weather, so that the decision must be made for each individual case.

Indications for low altitudes (up to 1000 feet) are much the same as for coast climates, with similar contraindications. Here I manage to have patients make two changes in the year, oftener than with cases sent to higher elevations. I find most of the cases indicated for coast climates will do better during the winter months a short distance from the sea; the slight elevation and dryness add to their comfort and improve metabolism. When spring or summer weather approaches, it seems best to send them nearer or directly to the coast.

After fifteen years in southern California I feel that this is a rule that can be safely followed along the Pacific Coast, and should work similarly in the temperate climates of the cast.

Cases that have been mentioned as contraindicated for high altitude will, I believe, be handled best in such a manner, and they may even be sent to a medium altitude for the winter. Certainly the pulmonary and renal cases of that group have, in my hands, been benefited by this procedure.

Indications for Desert Climate.-Since the main features are due to abundance of sunshine, extreme dryness, warmth, purity of air, and no sudden changes, the cases indicated are: 
Advanced pulmonary tuberculous eases, with or without laryngale involvement, chronic bronchitis or emphysema, with pronounced expectoration; many cases of nephritis; simple albuminuria; rheumatism and gout; arthritis deformans, especially carly cases; neuritis; arteriosclerosis.

It is surprising how oceasionally an advanced pulmonary case will do well in deserts without altitude, after having tried in vain other cliinatic resorts.

Diseases Benefited by Climatic Treatment and the Places Indicated.-Tuberculosis. - As a general rule, it is well to remember that another region or climate than the one in which the disease is contracted should be selected. All climatic resorts may have the disease originate in them, but certain regions have distinct advantages for treatment. All pulmonary cases except those contraindicated under high altitude should be sent to the Colorado or New Mexico resorts for the whole year.

For less altitude choose Asheville, N. C., for the whole year. When a warmer climate is desired for the winter months, the resorts of Arizona may be selected.

The lower altitudes, which are to be preferred, are the Adirondacks, New York, and, for the winter, the resorts of South Carolina, Georgia, and southern California.

My plan for patients who do not respond well to cold winter weather, and who cannot go far to the mountain regions, or who can make two annual changes, is to advise 'Tueson, Phonix, or the foot-hills of southern California, preferably Redlands, during the winter months, then Colorudo Springs, Denver, Santa Fé, or Silver City, summer and autumn. Remember that an incipient case, without constitutional symptoms, may go to any resort and get well, but I write here as definitely as possible for the great number.

In six months the disease is often arrested, but the residence under climatic treatment should continue for one year. It is unsafe for patients to return to former climatic conditions within less time. Those pulmonary cases contraindicated for high altitude, or such as must go to a lower altitude, I should send for the winter to 'Tucson, Phonix, and the foot-hills of southern Califormia, preferably Redlands, Banning, or Palm Springs, or the resorts of South Carolina and Georgia; for the summer, the Adirondacks, White, and Catskill Mountains. Other cases may do well in the clevated parts of Long Island and northern New Jersey. For the whole year, the foot-hills of southern California, near the coast, may be selected.

Babcock says, incipient cases, in fairly good physical state, may go to almost any resort where they can live ontdoors day and night. "He prefers Colorado, New Mexico, or resorts of the Engadine. "They may get on well, however, in California and those elevated parts of Arizona, Mexico, and southern 'lexas, where conditions of living, already designated, can be secured; many of them do well also in parts of North Carolina, Georgia, and even liorida." 
'The rule for laryngeal tulerculosis does not differ materially from that for pulmonary except that it is more essential that these cases be in resorts freest from dust, and in small regions away from the irritating influences of a city.

The tuberculous diseases of childhood are best treated at or near the seashore by an ocean climate. A few cases will improve more rapidly in a dry, low altitude or moderately elevated regions. The tuberculous bones and joints are best treated in sanatoria at the seashore, under skilled orthopedic care, and such may be had at Sea Breeze, near New York, Atlantic City, and Cape May. The coast of southern California, preferably San Diego, is ideal climatically for such cases, but unfortunately there is no special provision made. Suitable regions are Sea Breeze, Atlantic City, Cape May, the California coast, southern shore of Long Island, and in winter, Florida, Bermuda, and Nassau. Ely and Whitlock give interesting details and results of surgical tuberculosis in children at Sea Breeze.

Chronic Bronchitis and "Chronic Winter Cough."-When the sputum is scanty and the cough dry, it is best to choose warm, moist climates, such as the resorts of Florida and the Pacific coast, Bermuda, Nassau, Cuba, and Puerto Rico, or, when the sputum is abundant and cough loose, the drier resorts should be chosen-Aiken, Augusta, and the inland places of southern California, where exposure to cold and dampness are minimized.

Southern California has many inland places that can be safely recommended for this condition. In addition, there are the resorts in Arizona, Colorado, and Asheville, North Carolina, for cases feeling better in high altitudes.

Southern Pines and Aiken, South Carolina, are the best places in the south when a low altitude is desired. A. McPhedran says:

"There is no disease in which it is more necessary that the patient, and not the disease, should receive first consideration than in chronic bronchitis. The therapeutic indications are much less definite than in the acute affection, and therefore a variety of methods may le tried in the effort to suit the treatment to the individual. It is of first importance to avoid a cold, wet, and foggy climate; but it is in just such climates that the affection is most frequent, and unfortunately most sufferers are unable to seek a better one. Those who have the means should live in the most favorable climate. A mild sea air is usually most beneficial in the winter, such as Nassau, Bermuda, Jamaica, Cuba, Florida, and southern California in America, in Europe the Mediterranean coast, Sicily, Madeira, the Canary Islands, and the Isle of Wight.

Some patients, especially those with tuberculous tendencies, do better in a dry, warm climate, such as Mexico, New Mexico, Arizona, and Colorado, and, in the east, the higher parts of Georgia and the Carolinas. Not rarely it will be found best to try the moist and dry climates alternately.

In the summer months a more bracing climate will usually be found most beneficial if a continuous outdoor life is led, especially in the 
forest districts or on the plains. In Canada, Muskoka, the forests of northern Ontario and Quebee, the plains of the northwest, the foot-hills of the Rocky Mountains, and many parts of British Columbia, offer admirable opportunities for such a life. In the Lnited States the Xew Eingland and the Pacific coast States are best. Experience has proved that all these parts afford excellent resorts for tuberculous paticnts, and they should be equally favorable for chronic bronchitis, but as ret little consideration has been given to the subject. In time it will probably be shown that in chronie l,ronchitis, as in tuberculosis, the all-important matter in the treatment is not the mild climate, but the ontdoor life, care being taken to keep the boly and limbs warm so as to prevent congestion of the bronchial mucous membranes. Even in the lukon, long-standing cases of cluronic bronchitis have quickly recovered."

Emphysema.- For this trouble a low altitude or a coast climate should he chosen, for this, as well as bronchitis, outdoor life, hygiene, care of the diet, and attention to elimination are more important than the place chosen.

Southern California offers many advantages to those who wish permanent residence. Other suitable resorts are those of South Carolina and Georgia. It is best to keep these cases under 2000 feet elevation; a few only do well above 1000 feet altitude.

Asthma.- There is no rule for this class that can be safely followed. Each case is a study in itself. When nervous symptoms are present, any change may relieve or prevent an attack. A change to any high altitude resort is most beneficial in cases without emphysema. 'The patient must help by his own experience. I have seen most excellent results in eastern visitors from a residence in southern California, and others doubtless experienced equal or better results at their own resorts.

Convalescents from acute respiratory diseases are benefited by any change where the case may be longer in the open air, and this is usually sufficient. If the patient lives near the coast, the best location is inland, to a higher elevation, and some such change is of ten necessary to effect a cure. Hay-fever, or rose cold, is often avoided br any change to regions with less or a different vegetation-from the country to the city is often sufficient. Places most suitable are the White Mountains, Adirondacks, Catskills, and Berkshire Hills, Glen Summit in the Blute Ridge, Pacific Coast, 'Thousand lslands, Long Island shore, and Staten Island.

Diseases of the Kidney. - Next to pulmonary cases chronic Bright's disease, and convalescents from acute Bright's disease, are most benefited through elimatic treatment. If these cases arc under careful management early, and in the right climate, there is no reason why a eure should not result in quite the same mamer as with pulmonary tuberculosis. Changeable cold weather and high altitudes, which increase blood-pressure, act injuriously, so temperate climates and mountainous regions are out of the question. We know that nephritis occurs infrequently in warm equable, climates, and that these cases improre on 
removal from changeable irregular weather to an equable region. It is important that residence in such a climate should extend over a period of one to two years under proper medical supervision. Attention to the food, underclothing, outdoor life, and proper elimination through the skin, kidneys, and bowels are essential. I have no apologies to make when I urge for these cases southern California, Santa Barbara, Los Angeles, San Diego, with their suburbs, outlying valleys and foot-hill regions, which offer coast and low altitude climates, with their varying accommodations, to suit individual comforts throughout the whole year. There is no other class of disease, especially the interstitial type of nephritis, that will do equally well in southern California. The average eastern physician and layman does not realize that, while the winter climate of southern California is much warmer and more equable than that of the east, its summer climate is proportionately cooler and drier, a fact scarcely believable to those who have not experienced the difference. There are regions in California which more nearly approach the climatic conditions of Egypt than can be obtained elsewhere in the United States. Besides the necessary comforts for the care of nephritic cases, it has the additional advantage of affording a year's residence; for children and young infants it has no superior. Osler says: "A patient in good circumstances may be urged to go away during the winter months, or, if necessary, to move altogether to a warm, equable climate, like that of southern California. There is no doubt of the value in these cases of removal from the changeable, irregular weather which prevails in the temperate regions from November to April." Again: "In the convalescence from acute Bright's disease, care should be taken to guard the patient against cold. A change of air is often beneficial, particularly a residence in a warm, equable climate."

Other places of value for winter months are Southern Pines, North Carolina, the resorts of South Carolina, Georgia, and Florida, Bermuda, Nassau, and Cuba. Those of South Carolina may be utilized for the entire year, though the patient might be wise to make a summer change; when drier conditions are wanted choose low altitude resorts of Arizona.

Diseases of the Circulatory System.-Heart Disease.-As a general rulc, unless there are other reasons and the heart lesion is compensated, high altitude resorts are contraindicated; on account of the decrease of pressure in elcvated regions, the heart must be able to meet the increased demands put upon it, or acute dilatation on overexertion after arrival in high altitude regions may occur. Whether a case should go to a colder or warmer equable climate must largely depend upon the individual. Many feel better at a slight elevation, and can with safety be sent. Advanced cases of heart disease should be kept at low elevations. They are usually better off at home than seeking a climatic change. For winter the resorts of South Carolina, Georgia, Florida, and southern California may be selected. For summer, Berkshire Hills, northern New Jersey, Catskills, Adirondacks, Long Island, coast of Maine, and California are preferable. 
Resorts with proper bathing facilities in low elevations may be best suited in individual cases.

'To give the views in part of a high-altitude physician, Hall, of I)enver, says: "Without statisties to prove it, I am equally impressed with the belief that atheromatous patients with increased blood-pressure are more liable to disaster than those without such increase. The failure of even a small margin to maintain the required pressure disturbs the circulatory conditions profoundly. Patients with hearts greatly exhausted by chronic disease - as phthisis, pernicious anemia, and similar troublesare occasionally seriously embarrassed by the increased demands of the altitude, even though no physical exertion be made." 'This seems quite true, and if heart cases, on going to a reasonable altitude, show dyspnea from loss of compensation, it is best, even after one failure of compensation, to go to lower altitude.

Arteriosclerosis is benefited by residence in warm, equable climates, or where the patients with this trouble can escape changeable cold and damp weather. 'The treatment of this disease is certainly helped by climate. Much of the benefit is due to the outdoor life afforded and the avoidance of any acute catarrhal conditions. High and medium altitudes should be excluded, for reasons already given. 'The low altitude and coast region resorts are practically all suitable, and the choice should be made according to the circumstances and preference of the patient. I prefer the low altitude regions near the coast of southern California, but not directly on the coast, on account of the increased dampness; for winter alone, Florida and South Carolina and Georgia. Boardman Reed recently wrote on the treatment of this disease, urging climatotherapy along these lines: "Moderate warmth and dryness promote the action of the skin, and, joined with equability, afford the arterioselerotic the most favorable external conditions for attaining an old age in spite of his disease. Many places in the southwest suit well, especially for the winter months. When the patient can live all the year round in such a comparatively dry and equable climate as that of southern California, at nearly the sea-level, his chances for improvement will be greatly increased. 'This is especially true for the more favored localities of that region near the coast, though, as a rule, not directly on the seashore. The blood-pressure is not disturbed in this region, either by altitude or by violent storms, cold waves, or extreme changes of temperature, such as prevail so much of the time in many parts of our country."

For extreme cases it is necessary to add to climate rest and sanatorium treatment.

Chronic Rheumatic Affections and Gout.-Freelom from changeable, damp, and cold weather should be urged. As a rule, warm and dry resorts are indicated, rather than eold and damp regions. 'The presence of natural or artificial baths is an advantage. I would prefer a moist resort, with the proper facilities for baths, than a dry resort without them. 'The nature of the soil at any chosen region is important. A moderate altitude with dry conditions probably benefits the greatest number. Virginia Hot Springs may be well recommended for all these affections, 
and for any time of year, although autumn, winter, and spring are the best seasons. It can boast of modern equipment and accommodations for treatment and care. Hot Springs, North Carolina, has the advantage of both elimate and baths. The low altitude region of southern California has the climate, but the natural waters existing here are not yet made available, or the places sufficiently comfortable for the average health-seeker. Some adults are benefited by residence here and others not, while children always improve, due to outdoor life. Other resorts recommended are Saratoga, New York, summer and fall, Aiken, South Carolina, Augusta and Thomasville, Georgia, and Florida, for winter and early spring. Of Florida, Fremont Smith says: "Gouty and rheumatic subjeets, with ineident neuralgias or neuritis, have frequently and rapidly improved under my observation in this greatly eliminative atmosphere."

Arthritis Deformans.-For this condition, the resorts of Arizona, 'Texas, the desert and inland places of southern California, are suitable. On elimatie treatment of arthritis deformans, MeCrae says: "There is no doubt of a benefit of a change of climate, especially from the north to the south in winter. Whether this is due to anything more than the possibility of being out-of-doors, and leading a life more in the open air, is difficult to say. The more equable climate undoubtedly has an influence. Certain patients are more apt to have recurrence in colder weather, and improve immediately with a change to a warmer climate. As a rule, a dry equable climate suits the patients best."

Diabetes.-Contentment and diet are most important to the diabetic, and ean usually be found at home. If to these, without jeopardizing either, climate can be added, some benefit may be gained. As the nervous system is so easily irritated in this disease, do not advise any high altitude resort. Low altitudes are better than the coast, where there is equability and the patient can live outdoors. I have seen many improve on coming to southern California from the east, the percentage of sugar diminishing markedly without making any change in diet. I attribute this to the outdoor life and freedom from responsibilities. Resorts of South Carolina and Georgia may be tried-a few do well in Florida.

Hare advises a medium altitude, such as is found at Asheville, North Carolina, or the low altitude at 'Thomasville, Georgia, saying: "High altitudes are nearly always harmful, as they inerease the nervous irritability of the patient, are apt to be cold, and to produce congestions by chilling him. Similarly, seashore resorts are not advantageous, as diabetics do not withstand a damp atmosphere, as a rule."

'To this I make an exception of the seashore resorts of southern California in the summer months, especially San Diego and Santa Barbara.

Diseases of the Digestive System.-For the serions organic diseases no ehange should be contemplated, except it be to make the patient more comfortable, or to remove him from a cold, danp, or a hot malarions region. 
For functional diseases of the digestive system any change will doVirginia Hot Springs, Saratoga, New York, and, in general, any of the medium low altitude health resorts.

Diseases of the Nervous System.-'The organic lesions do best in low and medium altitudes, which offer dryness and equability. Arizona, parts of southern California, and Asheville, North Carolina, are suitable.

If there is much irritability, select a coast climate. If anemia is present the high altitudes of New Mexico and Colorado are best. 'The functional diseases of the nervous system are, like the digestive, benefited by any change; the choice must necessarily be individual, after consulting the patient's wishes. A relaxing climate is usually best, on account of the nervous irritability.

Florida, Bermuda, and Nassau are excellent for cases of neurasthenia and insomnia, convalescents, and those who need rest from business strain. 'The resorts of South Carolina and Georgia will suit many. The low altitude places of southern California seem to increase nervous irritability, while the coast often decreases it. If anemia is present in these cases as the probable cause, or for anemia itself, choose the high altitude resorts, as Colorado, New Mexico, and Asheville, North Carolina.

VoL. I-11 



\title{
GENERAL EXERCISE
}

\author{
By 'THOMAS A. STORFY, А.M., M.I., Рн.D.
}

For obvious logical reasons, I have arranged the topieal discussion of General Exercise in this chipter as follows: First, the Physiology of Exercise; second, 'The Prophylactic and 'Therapentic Action of Fxercise; third, 'The Untoward Effects of Exercise; fourth, Indications for Exercise; fifth, Contraindications; sixth, The Administration of Exercise; seventh, 'The Varieties of Exercise at the Disposal of the Practitioner; eighth, 'The General Application of Exercise.

Physiology of Exercise. - The rational prescription of exercise must rest upon an intelligent knowledge of the more important physiological activities that are directly and indirectly affected by the contraction of the skeletal muscles. 'The following outline of these more important physiological activities is presented in order to bring ont the rationale of the later discussion. 'The scope of this chapter elininates any consideration of minor physiological details and limits the presentation to a brief outline of the main relevant facts.

The contracting muscle has a mechanieal squeering, pumping influence upon the vascular tissues with which it comes into pressure contact. 'This influence serves to accelerate the lymph and venous rirculation during exercise. 'The pressure of the contrating muscles also stimulates the local afferent nerve endings initiating vasomotor reflexes which lead to local vascular dilatation. In these ways the contracting muscle is given an increased blood supply and an increased venous and lymphatic drainage. In addition, the contraction of voluntary muscle is accomplished at the expense of some or all of the varions chemical constituents of the neuromuseular mechanisms involved, i.e., the neuron and the striated muscle fiber. In the muscle fiber these chemical reactions result in the diminution of glycogen and in the production of such wastes as carbon dioxide, sarcolactic acid, acid potassium phosphate, and creatin. In the nerve cell the products of activity are less well known. but it has been established that the physiological activity of nerve centres is nccompanied by the production of acid wastes. Linder the extreme conditions of experimental fatigue in the laboratory it has been shown by the microscope that muscle fiber and nerve cells undergo extratordinary change in their staining reactions. Even the nerve fiber under tests for fatigue displays an altered reaction to histological stains.

It is evident, then, that voluntary muscular contraction is accompanied by chemical losses in the nerve cell and muscle fiber. 'The waste products of these chemical reactions are emptied into the lymph and blood streams surrounding these tissues, and must be reckoned with 
qualitatively and quantitatively in estimating the probable effects of exercise in any given case. The chemical losses sustained by the muscle fiber and the neuron are replaced by supplies furnished these organs from the blood and lymph. These supplies are the food substances absorbed from the intestinal canal and from the alveolar spaces of the lungs. The ultimate source of these supplies (tissue foods) are the proteids, fats, carbohydrates, salts, and water taken in by the way of the alimentary canal, and the oxygen taken in by the way of the respiratory tract. It is evident, then, that exercise involves a heightened metabolism in muscle and neuron, producing an increase in katabolic waste and anabolic demand. 'This necessarily increases the excretory activity of the tissue cells filling the lymph and the blood with waste products and bringing those products into intimate association through the blood stream with all the cells of the body, giving opportunity for hormone action, and finally throwing a greater burden upon the excretory organs, chiefly the kidneys, the lungs, and the skin.

It is apparent, then, that for our purposes we must consider the physiology of exercise from the standpoint of the local mechanical effects of muscular contraction and from the standpoint of katabolism, hormone action, excretion, anabolism, anatomical structure, and organic function.

1. The Local Mechanical Effects of Muscular Contraction.-The alternation of contraction and relaxation of muscle during exercise serves to press the fluid contents out of the smaller lymph spaces in the muscle tissue and to squeeze the lymphatic and venous streams in the direction of least resistance. Because of the valvular arrangements in these systems this pressure is afferent, and results in accelerating the lymphatic and blood circulation within and about the muscle mass. This assists in the circulatory removal of the chemical products of tissue activity.

In addition the local intra- and extramuscular movements excite the sensory nerve endings within the muscle substance and in the adjacent tissues, so that various nervous reflexes are established. These reflexes probably have much to do with the remarkable refinements of neuromuscular coördination which are so commonplace to us. For our purpose the important fact in this connection is that these reflexes are also largely vasomotor in character and have much to do with the increase in blood supply to the contracting muscles and with the general increase in peripheral circulation present during and for a short time following general physical exercise.

2. The Influence of Exercise upon Katabolism.-All organic activity is accomplished at the chemical expense of the tissues involved. Physical exercise is fundamentally a function of the motor neuron and the striated muscle fiber, but it is necessarily inseparably associated with the normal functional activity of various connective-tissue sheaths, aponeuroses, tendons, ligaments, bones, joints, lymphatic spaces, blood capillaries, lymphatic and blood-vascular systems, the heart and lungs, the hemapoietic organs, various afferent and efferent nerve tracts and systems, the spinal and cranial nerve centres, the special senses, and the various glandular structures of the alimentary tract. In fact, practically 
all the organs of the body may, under the inftnence of physical exercise, be thrown into greater functional activity, and therefore be called upon for an increased expenditure of chemical resource. Even though the increased loss in any given tissue cell may be infinitesinal, the summated increase from some millions of tissue cells, dependent upon the extent of the physical exercise, may amount to a great deal compared to the normal loss.

The chemical reactions which take place during the functional activity of the tissue cell, and on which the nature of that activity depends, are chemical reactions within the substance of the cell. 'T'hese reactions form products which are eliminated from the cell as wastes. As a result of this action the cell loses a part of the material which it has constructerl, and, in addition, suffers more or less structural wear and tear. 'This destruetive process-katabolism-throws various waste products into the lymph and blood streams.

'The contraction of the voluntary muscle fiber is accompanied by an increased evolution of heat and the elimination from the fiber of carbon dioxide, sarcolactic acid, acid potassium phosphate. and creatin. 'These substances are called "fatigue substances." Without going farther into the details, it may be stated that each functionating tissue contributes its so-called wastes as a result of katabolic action, and that these wastes are carried in one form or another by the blood all over the body. Each tissue is bathed by a solution of these waste products in blood. Some of these products enter into chemical nssociations in the blood, which may alter their possible toxicity; carbon dioxide is largely eliminated by the lungs before it reaches the arterial stream. But the fact remains that the chemical wastes or fatigue products of voluntary muscular contraction and the increase of chemical waste from other tissues affected by physical exercise must have a more or less stimulating, irritating, or depressing influence, as the case may be, upon the general tissues and organs of the body, particularly upon the cells of the central nervous system, before they are finally excreted from the body.

3. The Hormone Action of the Katabolic Products of Physical Exercise.These fatigue or waste products come into contact first with tissues of the voluntary muscles themselves.

Lee's investigations show that "the physiological action on skeletal muscle of each of the commonly recognized fatigue substances, namely. carbon dioxide, paralactic acid, and monopotassium phosphate, is of two opposite modes, the appearance of the one or the other mode being dependent upon the quality of the substance that is present and the duration of its activity. If present in small quantity, or moderate quantity, for a brief time each substance causes an angmentation of activity of the muscle, which is characterized by an increase in irritability and working power. If present in moderate or large quantity, or in smaller quantity, for a longer time, each substance causes a depression of activity or fatigue of the muscle, which is churacterized by a decrease in irritability and working power." 
In my own investigations I have shown that "human voluntary muscle is made more irritable by successive excitations (electrical); that the irritability of human voluntary muscle is immediately very greatly increased by a moderate amount of work (Fig. 2); and that it is very greatly decreased by a fatiguing amount of work $^{1}$ (Fig. 3).

FIG. 2

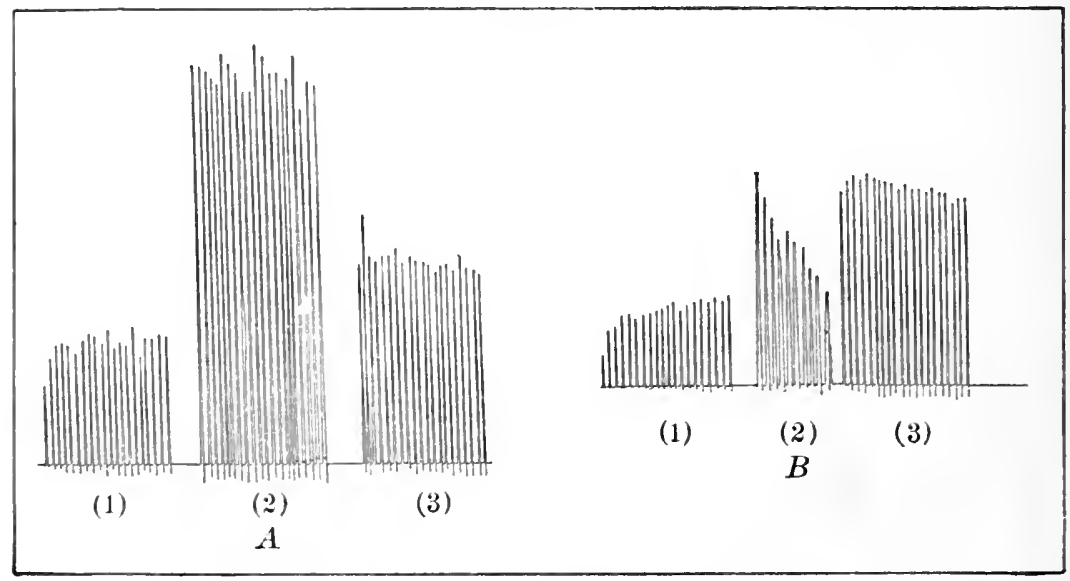

The curves read from left to right. A. (1) Contractions of the human abductor indicis muscle upon electrical stimulation, against very slight resistance; (2) voluntary contractions against considerably increased resistance: (3) conditions as in (1). B. Conditions as in Series $A$, except that the resistance to voluntary contraction was very much greater

FIG. 3

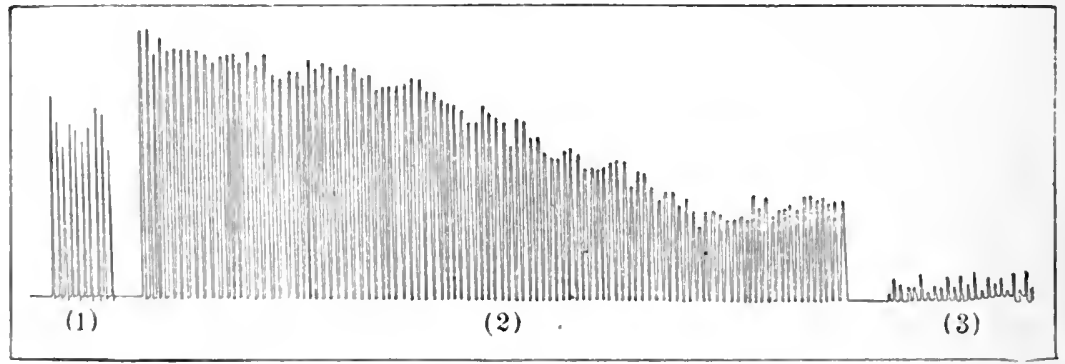

(1) Electrical stimulation, very slight resistance; (2) voluntary contraction against oonsiderably incrensed resistance: (3) conditions as in (1).

These waste products have a further local influence upon the arterioles of the contracting muscle. 'The local vasodilatation is largely a vasomotor reflex, but it is also probably brought about through the medium of a vasodilator hormone given off by the working muscle. 'This influence assists in securing an increase in the blood supply of the contracting muscles. 
'The lymph and blood streams carry these waste products in solution, or in some form of chemical association, away from their places of origin and distribute them in the blood stream to all the tissues. Onr present knowledge of the susceptibility and response of the fixed and circulating tissue cells of the body to the stimulating action of circulating irritants of various sorts leads us to expect that these various circulating acid wastes of voluntary muscular contraction must have a more or less inportant influence upon the functional activity of all the organs of the borly: 'This is the simplest explanation of the various more remote influences of exercise. 'This is particularly true in the relation between muscular work and the functional activity of the central nervous system. A number of central and spinal centres are stimulated to greater activity through the products of muscular work. As soon as the tension of carbon dioxide in the pulmonary alveoli and in the pulmonary veins exceeds the normal, this chemical waste becomes a stimulant of the respiratory centre, causing a more rapid respiratory rhythm, and therefore a more rapid removal of carbon dioxide from the alveoli of the lungs. When these katabolic products are carried to the central nervous system in sufficient amount they apparently stimulate the cardiac centres and thus assist in accelerating the heart beat.

In the same general way the nerve centres controlling the action of the sweat glands are stimulated to greater activity because of the action of these centres of chemical wastes given off by the working muscle and brought in contact with the nerve centres by the blood.

The increase in blood pressure accompanying exercise is another phenomenon which is at least partially due to the action of these products on the vasomotor centres.

It is possible, too, that the general growth stimulus that follows systematic exercise in younger persons may be due to an anabolic stimulus from some hormone given off during muscular contraction.

Experiments which I performed in the physiological laboratory of Stanford University, in 1899, show that moderate exercise of the larger musele groups is accompanied by an increase in the irritability of the motor centres of the brain. After such exercise smaller muscle groups -in this case the deep flexors of the forearm-previously voluntarily fatigued, could be voluntarily stimulated to do a normal quantity of work in spite of their diminished irritability. 'This effect was in all probability a result of the stimulating influence of the chemical products of muscular activity carried to the cerebral cortex and there brought in contact with the nerve cells of the motor centres.

So far we have been considering the general stimulating influences of these waste products. We noted in considering the influence of these chemicals upon the muscle fiber that a depressing influence followed their excessive or prolonged production. 'The same law operates in the general influence of these products. A depressing or fatiguing influence accompanies the rapid excessive production of these chemical wastes. And, too, it may be stated that any prolonged production in excess of the ability of the organism to neutralize or eliminate those toxic products 
will eventuate in respiratory, cardiac, and general fatigue, functional depression, febrile reaction, and, in extreme cases, in organic injury.

All of our various theories, offered in explanation of sleep, include prominently the depressing effects on the higher nerve centres by the acid wastes of tissue metabolism attendant upon the day's work.

4. The Influence of Exercise upon Excretion.-The elimination of these various waste products from the blood is the function of the several excretory organs, chiefly the lungs, skin, and kidneys.

The lungs and skin excrete the carbon dioxide produced by the low metabolism of the tissues at rest or by the high katabolism of the tissues in activity. In each case the increase in the amount of the excretion, and therefore the increase in the amount of work performed by the excretory organs, is directly proportional to the quantity of carbon dioxide produced by the working tissues.

The elimination of water by way of the lungs and skin, like the elimination of carbon dioxide by those organs, is relatively proportional to the amount of physical exercise taken by the individual. There is, concomitantly, a reduction in the amount of water eliminated by the kidneys and the mucous membrane of the alimentary tract. Under these circumstances we find a drier mouth, drier fecal discharges, and a more concentrated urine, unless the increased elimination of water is compensated by a proportional increase in the ingestion of liquids.

Most of the remaining wastes are excreted by the kidneys. The solid wastes eliminated by the skin may be neglected for our purposes. In general we find that the tissues waste, and particularly the wastes from the muscular tissues, eliminated by the kidneys, are ammonia, the endogenous purin bases, and creatin. The ammonia formed by hydrolytic cleavage of the protein molecule in the tissues is converted into urea by the liver and excreted by the kidney as urea. The uric acid and other endogenous purin bases excreted in the urine are derived mainly from the dissimilation or katabolism of the voluntary muscles. The amount of uric acid in the urine is increased within a few hours after exercise.

Creatin is a normal product of the metabolism of skeletal muscle. It appears in the urine as creatinin. Some authorities state that the elimination of creatin is increased by muscular exercise. Others state that there is no such relationship.

In addition to these eliminations by way of the kidney, I have found uniformly that the urine contains albumin after severe physical exercise. This has been the finding in all the investigations that have come under my observation. In some cases the investigation has included a microscopic exmination of the urinary sediment, and it has not been infrequently found that severe physical exercise has caused the appearance of acetone, blood cells, and casts in urine which ordinarily contained neither acetone, albumin, nor abnormal sediment.

5. The Influence of Exercise upon Anabolism.-The loss of chemical material in the tissue cells, whatever and wherever those cells may be, is replaced from the food content-the chemical supply-of the blood. 
These cells rebuild themselves and restore their peculiar losses from this common source-the blood. 'The muscle cells not only repair themselves structurally, but they also store up glycogen for future contractile activity. 'The neuron not only repairs any losses from wear and tear in its own structure, but also stores up its chromophile substance for further functional activity in the elaboration of its nerve impulse. And so each tissue cell that has participated more or less directly and more or less extensively in the functional activity of the motor neurons and the striaterl fibers concerned in physical exercise, or that has been brought directly or indirectly into chemical coördination through the influence of the circullating chemical products of that activity repairs its structural losses and its functional losses from the same source; that is, the blood, and the blood, of course, derives its food supply through the digestive and respiratory tracts. 'The extent of this building-up and repairing process, and the extent of the demands upon the foor supply of the blood, depends upon the quantity and quality of the physical exercise taken.

It is evident, then, that anabolism, the reconstruction and repair of cellular structure and the manufacture of the various special tissue substances, is more or less profoundly affected by physical exercise.

A wise prescription of exercise, in the absence of such incompatibilities as bad habits of eating, insufficient nourishment, bad habits of excretion, nervous strain, and insufficient rest, and in the absence of pathological contraindications, may, therefore, in the young person promote growth, and in the older person secure an approximately normal weight.

6. The Influence of Exercise on Structure. - It is a well-established fact in physiology that an unused organ does not develop properly; that a period of long disuse leads to atrophy; that regular exercise of an organ leads to its normal development and growth; and that organs that are exercised a great deal are, in most cases, hypertrophied. 'These structural changes are associated with the anabolic effects of exercise, and are most apparent in the nervous and muscular tissues, in the heart, and in the tendons, ligaments, connective-tissue sheaths, bones, and joints associated with the voluntary musculature.

It seems to be true also that in certain tissues at least exercise not only increases the size of the individual element (muscle fiber, for instance), but also increases the number of the tissue elements present. So that there are more muscle fibers in the regularly exercised muscle and more nerve cells in the regularly exercised motor centre than in those muscles and centres that are not regularly exercised.

The voluntary muscles have another characteristic reaction to exercise. A muscle that is exercised a great deal tends to shorten permanently. 'This accounts for some of the minor asymmetries that are found in the trained athlete and "strong man," such as uneven height of shoulders, excessive flexion of the elbow at rest, certain types of "round shoulders," and so on. 'The orthopedic surgeon takes advantage of this characteristic in treating faulty attitudes, chest deformities, scoliosis, flat foot, and other similar conditions. 
7. The Influence of Exercise upon Function.-Exercise has a local and a general effect in its rclation to the functional activity of the human organism. Locally the exercised muscle, with its increased size and number of individual fibers, develops a greater contractile power. And the governing motor centre sends out stronger and more effective nerve impulses. Depending upon the nature of the exercise, the reaction time, the speed of motion, and the endurance of the neuromuscular mechanism are increased, normal coördination becomes more perfect, and new coördinations may be established. 'The functional efficiency of the muscle tendon, connective-tissue sheaths, ligaments, bones, and joints is, within physiological limitations, directly increased in proportion to the extent of their participation in exercise.

Associated with its beneficial influence upon general metabolism, physical exercise causes a general increase in the functional efficiency of the organ. 'The heart develops a greater strength, regularity, and endurance. Circulatory activities are improved. 'The depth of inspiration is increased. 'The rhythm of respiration is slowed. 'The strength, endurance, and coördination of the neuromuscular elements controlling the movements of respiration are improved. 'The necessary and very' intimate coördination between the complex respiratory machine, the complex circulatory machine, and the complex vasomotor machine is brought into more perfect adjustment and efficiency. 'The heat regulation of the body is improved. Digestion, metabolism, and excretion are improved.

In fact, under the influence of wise habits of physical exercise, other conditions being normal, the human organism approximates its limit of structural and functional efficiency.

The Prophylactic and Therapeutic Effects of Exercise.-'The facts presenter in the preceding discussion indicate the prophylactic and therapentic values of physical exercise.

One of the most important factors in preventive medicine is the resistance or immunity of the individual to disease.

"The production of active or potential immunity is a function of some of the fixed and circulating cells of the body. When pathogenic organisms within certain linits of virulence gain access to the tissues they are destroyed or rendered innocuous by one or more of several processes. 'Ther may be devoured by phagocytes; they may be killed and dissolved by agents in solution in the tissue juices; they may be imprisoned and walled off locally from the rest of the tissues; and their toxic products may be neutralized or destroyed by soluble antibodies in the tissue fluids. These several defensive activities are carried on directly by the tissue cells or indirectly by the products of the vital activities of those cells. On the cells of the body depends the exhibition of the phenomena of immunity.

"'The degree of immunity produced is related directly to the health of the cell. An impoverished, poorly nourished, unhealthy cell will not react to the same extent and with the same success as will the normal, healthy, well-nourished cell.

"'The health, and therefore the immunity producing power, of the cell 
depends upon its nourishment, including food, water. andel oxiencon; upon its relief from the toxic influence of its own waste probluts: upon its exereise; upon its opportunities for rest and repair. and upon at reasonable freedom from the direct and indirect influences of pathogenic organisms. 'lhese facts have everywhere been forced upon the attention of men who are experimenting with the immmity reactions of the blowl. 'Ihey find healthy blood is neressary for good reactions. Bactericiclal phenomena, phagocytosis, bacteriolysis, agglutination, the profuctions of antitoxins, and the other phenomena of immunity are all more marked in bloxl taken from healthy animals. It has been found in laboratories everywhere that a reduction in mortality and a more successful experimentittion with the vital phenomena of cellular structures in experimental animals accompanies the provision of good and sufficient food, exercise, and careful sanitation. 'The health and, therefore, the immunity of the whole body depends upon the health of all its constituent parts- on the health of its cells. If the cells are all well nourished, active, and protected from extremes of pathogenic influcnces, their summated health will be the health of the individual whose borly they in combination make. 'That such a healthy individual is possessed of a certain degree. of immunity has been proved empirically and experimontally, and it is equally well established that the possession and conservation of the healthy boly depend upon the observance of several simple hygienic procedures. 'These hygienic procedures are the same as those which 1 have already stated were essential to the boly cells. Furthermore, these procedures must be the fundamental procedures in any wise and wellordered policy of personal health control.

"'The well-planned policy of personal health control teaches men to eat properly, to drink properly, to breathe properly, to take proper eare of the excretions and wastes, to exercise wisely, to rest wisely, and to keep reasonably clean with reference to disease-breeding organisms. Such governing principles lead to and conserve human health. Failure to respect any one of these principles will jeopardize the success of the others. No rational scheme should rely upon exercise alone for health. nor upon any other one procedure or habit. One must respect all of these several requisites in order to work under a wise poliey of personal health control. "The man who regulates his habits of cating and takes no exercise camnot expect health. 'The man who exercises properly and eats wisely will fail to secure health if his habits of slece are bad. On the other hand, a reasonable observance of these several simple hygienic laws cannot fail to secure and conserve health for the average individual.

"Such an individual will be possessed of millions of active, healthy" cellular structures working for his protection, constructing for him a defensive armamentarimm, and ready on demand to respond with supreme reaction against the invasion of disease."

'Therapeutically, we mar consider that the general physiological con-

'Storey, Proceedings of Section V. International ('ongress on 'Tuherrulusis Warehington, D. C., $190 \mathrm{~S}$. 
dition of the actively healthy body makes its recovery from infection more easily possible. 'This is an overlapping of the prophylactic and therapeutic effects of exercise. It is the only way in which it may be said that exercise has a therapeutic mission in the acute stages of febrile diseases. Health of cell and health of body depend, among other things, upon physical exercise. Exercise of the body exercises the cells of the body. Without bodily exercise, the tissue cells cannot be actively healthy. In a well-ordered policy of personal health control, wise habits of exercise do their full and important share in improving the physiological efficiency of the tissue cells of the organism, developing their powers of resistance to disease. In one case this resistance may enable the tissues to destroy the invading bacteria of disease immediately on entry into the tissues. In another case it may enable the tissue to develop its physiological defences successfully but slowly, so that there is an acute disease attack followed by recovery. 'This is the common experience of the organism in the course of infections from which it recovers. It is only in such a way that physical exercise may be used "therapeutically" in the treatment of infections during their acute stages. This treatment is prophylactic in point of time.

I know of no diseased condition in which exercise is indicated during the acute febrile stages, and there are very few non-febrile diseases in which it is indicated during the acute attack or exacerbation. Our additional therapeutic uses of exercise, then, are limited to applications during recuperation, to treatment of certain chronic non-febrile conditions, and to special prescriptions arranged for general health effects in the presence of more or less seriously disqualifying chronic lesion.

In estimating the therapeutic, prophylactic, and general health effects of exercise, one must take into consideration the following physiological effects of exercise:

(a) An immediate, transient, local increase in the blood supply of the muscle.

(b) An immediate, transient, local increase in the lymphatic and venous drainage of the muscle.

(c) An immediate and transient increase in the heart rate, blood pressure, and rate of respiration.

(d) An immediate and transient increase in the superficial circulation.

(e) A later, more lasting increase in the size, strength, and functional endurance of the exercised muscle.

( $f$ ) A later, more lasting decrease in the length of the exercised muscle.

(g) A later, more lasting increase in the size and strength of the tendons, ligaments, sheaths, bones, and joints directly associated in exercise.

(h) A later, more lasting increase in the size and efficiency of the nerve cells in the exercised motor centres.

(i) An improvement and perfection of old neuromuscular coördinations.

(j) The establishment of new neuromuscular coördination with the possibility of developing new paths for the afferent and efferent nerve impulses. 
(k) An increase in the size, strength, functional endurance, and! efficiency of the heart.

(l) An improvement in the regulation of the vasomotor balance.

(m) An improvement in the blood and lymph circulation.

(n) An increase in the strength, endurance, and coordination of the musculature of respiration.

(o) An improvement in the depth and rhythm of the thoracic and diaphragmatic movements governing the ventilation of the pulmonary alveoli, and augmenting the speed of the general lymphatic and venons circulation.

(p) An improvement in the operation of the heat regulating mechanisms.

(q) A perfection of the mutual neuromuscular coördinations associating cardiac, circulatory, pulmonary, and vasomotor activities.

(r) An increase in general katabolic activity.

(s) An increased circulation of the products of the chemical activities of all the tissues and organs associated directly and indirectly in physical exercise.

( $t$ ) A larger hormone effect of these products (among them carbon dioxide, acid potassium phosphate, sarcolactic acid, ammonia, creatin, and the endogenous purin bases).

(u) An increase in the elimination of carbon dioxide by the lungs; of water and carbon dioxide by the skin; of uric acid and creatin by the urine.

(v) A desire for rest after exercise, and a desire for sleep at the end of the day.

(w) An improved appetite, better digestion, more food in the blood for the tissues, a greater demand for food by the tissue cells (a benignant (ircle).

(x) An increase in the anabolic activities of all the tissues concerned directly and indirectly in exercise, with, therefore, a general structural and functional benefit.

(y) A gain in weight or an increased growth, or both, continuing until the inetabolic equilibrium is reëstablished.

(z) A perfection of the physiological efliciency of the whole organism, with consequently

(a) A better development of the powers of potential and active inmmunity.

The Untoward Effects of Exercise. - The neuromuseular mechanism becomes adjusted to meet the daily demands male upon it. Is long as those daily demands are reasonable and are not greatly varied or increased this mechanism performs its work with ease and comfort. When any new coördination is brought into active play, or when a relatively excessive demand is made on the old coomdinations, there result several effects which are more or less disagreable.

'The muscles that have been thus unusually exercised become tender to the touch and painful on active motion. 'This disconfort may be considerable. It may be temporarily incapacitating. It is probably 
due to the local and general toxic influence of tissue wastes and to local mechanical traumatisms.

A well-muscled man who returns to physical exercise after a period of inactivity may enter too actively and abruptly into the performance of his old exercises. Under such circumstances muscle tendons, and even bones, have been broken by the power of the muscular contraction acting on these structures weakened through disuse. The local soreness is usually greater in these cases, because there is a likelihood of more extensive tissue tramma than there is in individuals with weaker muscles, and special care should be taken in these cases to begin with mild exercise and increase the activity gradually.

Physical exercise employed for health purposes need never have any injurious effects. If the prescription is made with intelligent concessions to the previous training, present condition, strength, and organic limitations of the individual, there will be no injurious effects. Physical exercise employed for athletic purposes may very easily have injurious effects. But even then the excessive demands of physical exercise may be without apparent injury to the picked men that undergo the ordeal of athletic training. The scope of this chapter does not include a consideration of exercise in athletics, but a knowledge of the common injuries and undesirable effects of the excessive exercise inseparably associated with modern athletics is of importance. Many praetitioners find their younger patients entering into public school and collegiate athletics without reference to their organic capacity for such work. 'There is a fortunate tendency on the part of these educational institutions, as well as the better class of athletic clubs, to exercise some sort of a medical supervision over the admission of the candidate to athletic training and over his continuance in training. But for obvious financial reasons it is only rare that this institutional supervision is adequate. The amount of time which the examiner can spend on each individual and the number of times he can see any given case under these systems is entirely too small. As a result, we find too frequently that the health of the individual is sacrificed in the interest of athletic success.

The more common injuries of excessive exercise in athletics are: Muscular hypertrophy and shortening, with consequent anatomical asymmetries, such as uneven height of shoulders, forward protrusion of the shoulders, with rounding of upper back, semiflexion of elbows at rest, semiflexed hand, and large chest, with small hips and legs, as in the case of the expert gymnast; excessive cardiac hypertrophy, with dilatation; arteriosclerosis in adults; and inguinal and femoral hernia.

In case of organic weakness the effect of the strain in excessive exercise, particularly in competitive athletics, is often made manifest at the point of weakness. In this way excessive exercise has an untoward effect upon such conditions as chronic endocarditis, chronic myocarditis, chronic pericarditis, established arteriosclerosis, aneurysm, varicose veins, incipient, quiescent, and arrested pulmonary tuberculosis, emphysema, chronic bronchitis, asthma, chronic pleurisy, hernia, chronic or recurrent appendicitis, chronic nephritis, g!necological displacement, spinal Pott's, scoliosis, sacro-iliac discise, hip disease, and flat foot. 


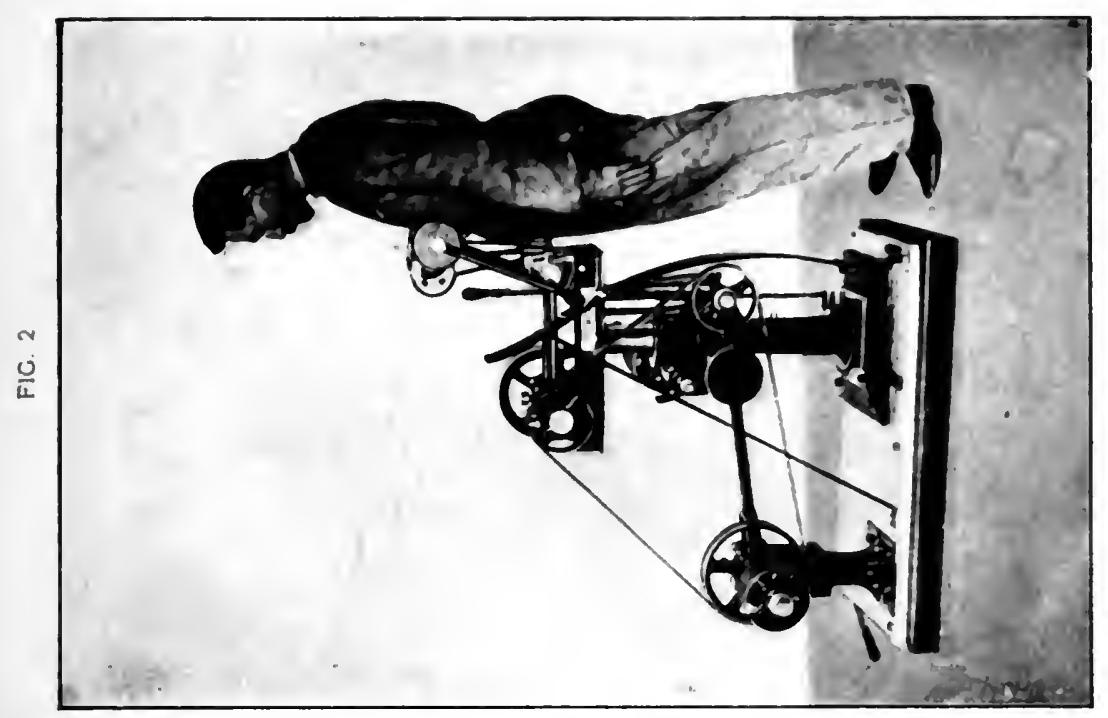

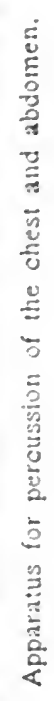

感

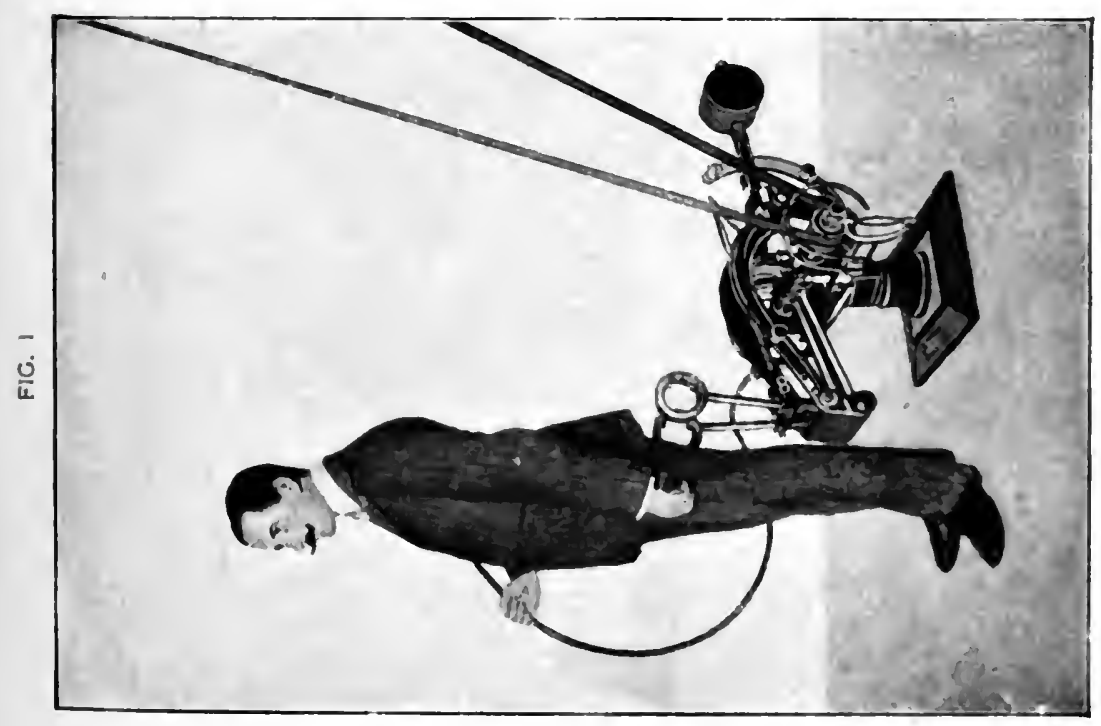

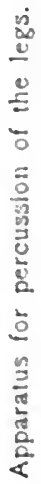




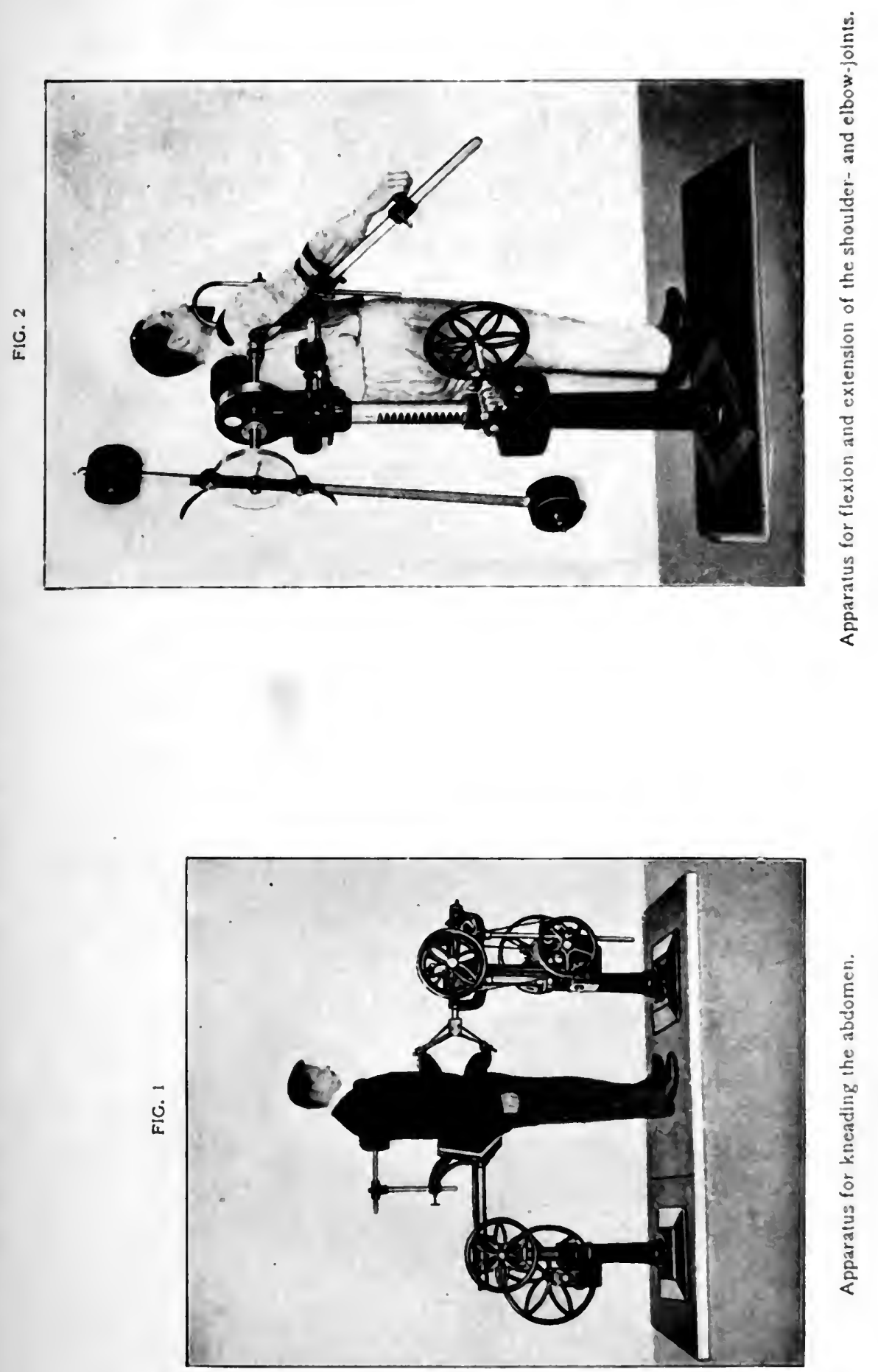

है 



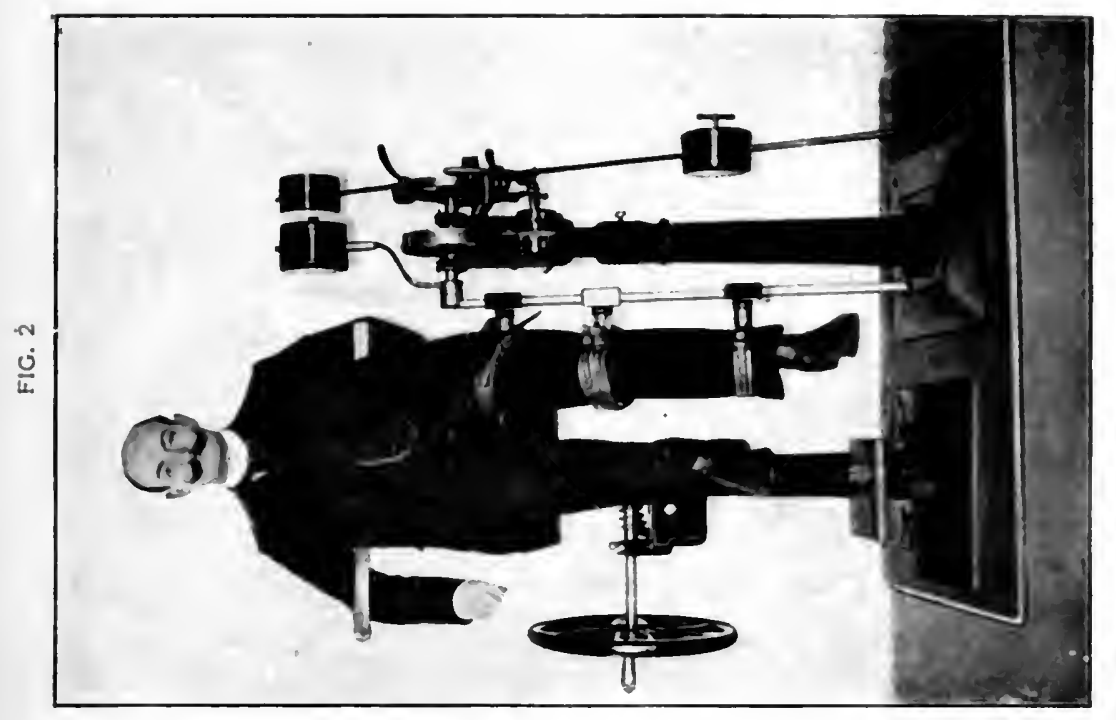

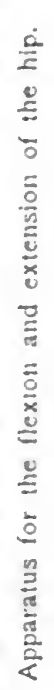

$\Xi$
5
5

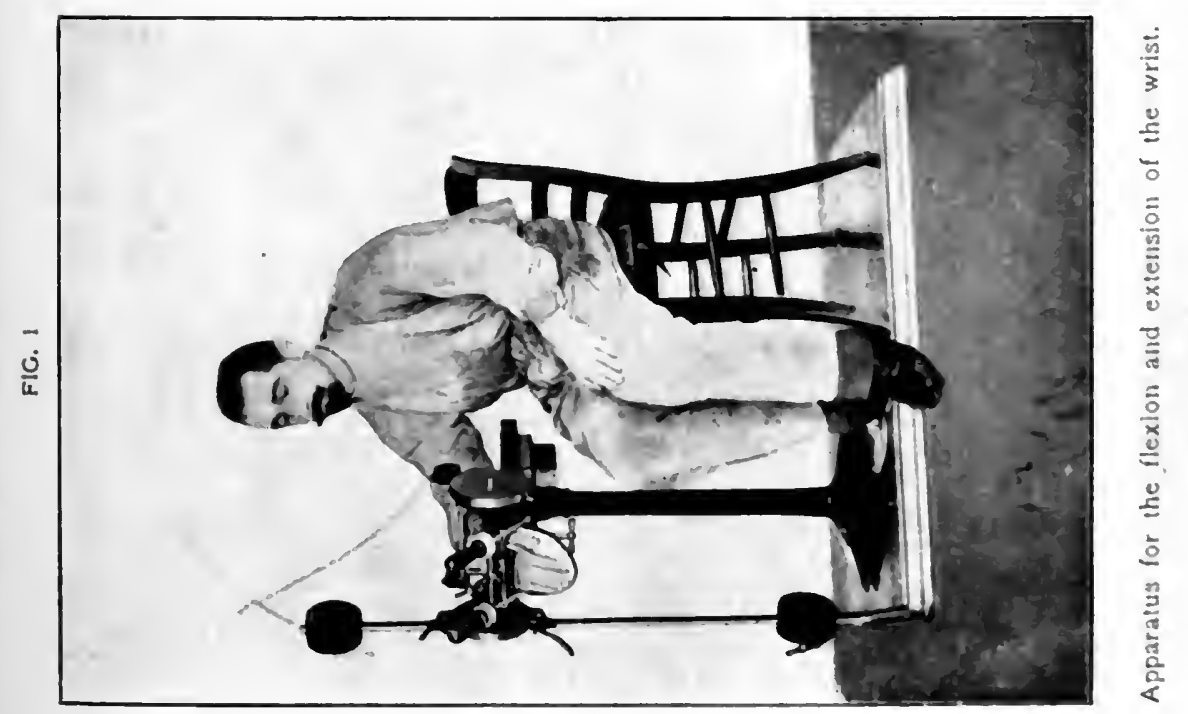





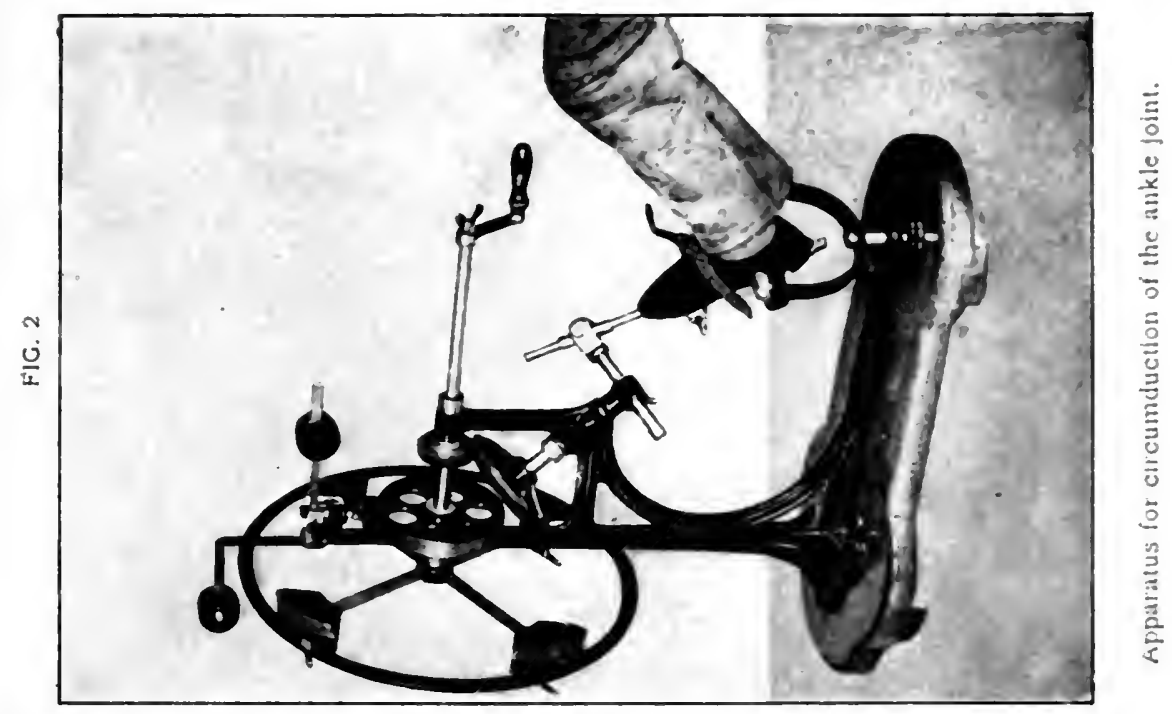

$\geq$

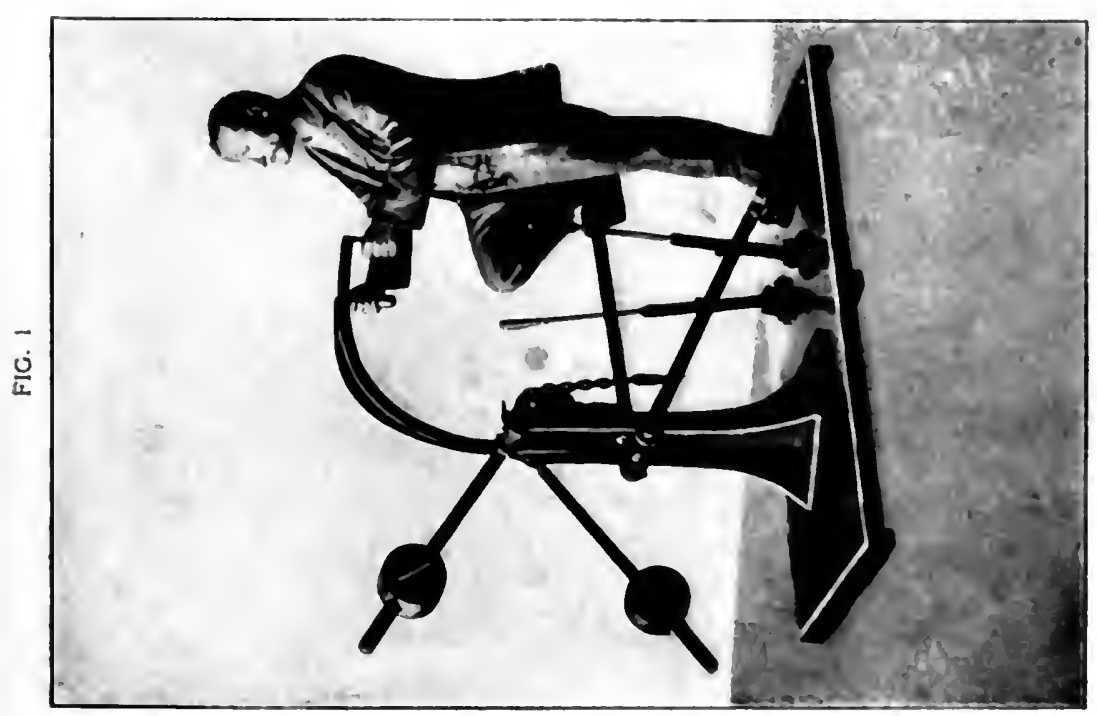

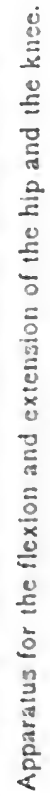





\section{PLATE V}

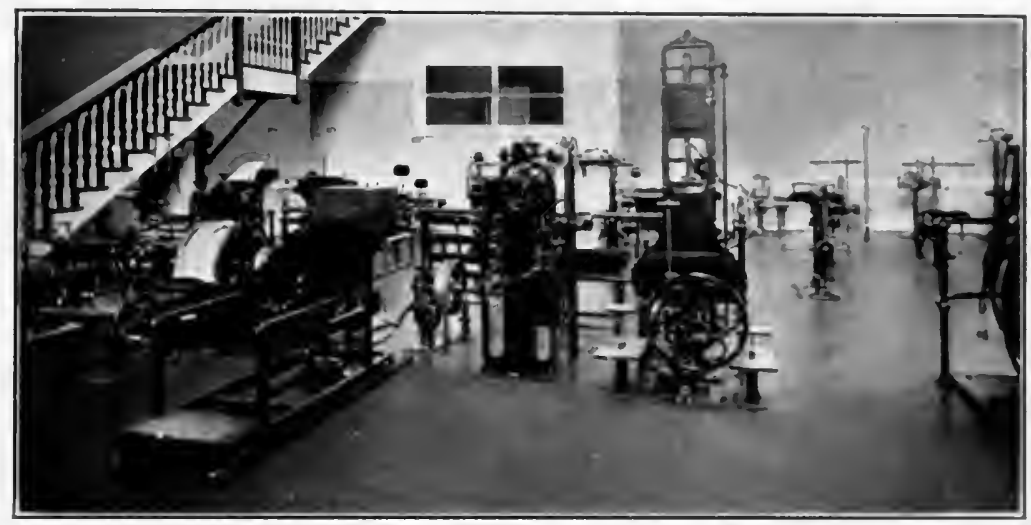

The Zander Room of the Massachusetts General Hospital, Boston.

Nore.-Plates I to $\mathrm{V}$, inclusive, have been secured through the courtesy of the kny-Scheerer Company, New York City. 

Indications for the Administration of Excercise. - Some form of exercise is necessary to individual health at all anges and in all stages of development and under all conditions save those present during the acute attacks, exacerbations, or terminal stages of disease.

Many people secure enough physical exercise in the performance of their day's work to satisfy their physiological needs. Exercise that is accomplished under pleasant, interesting, and recreative conditions is more valuable than that which comes from the routine of daily housework or from the routine of office work. But, though less desirable, such exercise has its physiological influence, and in many cases serves to maintain some degree of health for the individual. 'There is no such thing as physiological efficiency in the absence of all exercise. Under such circumstances the individual is necessarily bedridden, and suffers structural and functional deterioration which must eventuate in death.

In health the variety and quantity of exercise indicated depends upon the age, sex, habits, physique, and condition of the individual. 'The babe must have freedom for the kicking, squirming, grasping, and twisting movements that develop his musculature, incite and perfect his larger coörlinations, and stimulate his whole organism to normal functional activity. 'The growing child continues these absolutely essential influences through his play, games, and sports, and secures these physiological benefits more or less completely in spite of the restrictions of the home, the school, and urban life. 'The mature individual either deliberately plans for exercise or secures it incidentally in the pursuit of his pleasures, his day's work, or both.

If the babe is bound fast, he does not grow. If the child is forced to lead an absolutely sedentary or bedridden life, he does not develop. If the mature human being reduces his exercise to a critical minimum, he is soon without strength or endurance, and is without resistance to disease. Such a babe rarely reaches childhood, and the days of the child and the man are numbered.

In the normal individual some form and amount of exercise is indicated at all ages. 'The following abnormal conditions are types which indicate special prescriptions of exercise for local and generil effects:

1. 'The poor appetite, indigestion, constipation, dull headache, and bad breath of sedentary life.

2. Weak, flably muscles.

3. Overweight and underweight.

4. Cardiac and circulatory weakness.

5. Chronic cardiac and circulatory lesions.

6. Undeveloped lung capacity.

7. Chronic pulmonary lesions.

8. Chronic kidney lesions.

9. Gout and rheumatism.

10. Nervous excitement, fatigue, and exhaustion, and the various consequent neurological diseases.

11. Sexual perversion and excesses.

12. Mental diseases. 
13. Convalescence from acute disease.

14. Neurological diseases, with approximate or complete loss of function.

15. Chronic joint diseases.

16. Ventral, inguinal, and femoral hernia, bubonocele, and weak abdominal walls.

17. Unhealed wounds, fractures, sprains, etc.

The Contraindications of Exercise--Reasonable exercise is never absolutely contraindicated in all its forms save during the acute attacks, exacerbation, or terminal stages of disease. Excessive exercise is always contraindicated, particularly in the presence of any of the various organic conditions and lesions that would invite injury through its influence. The term excessive is relative. Exercise that is mild for one man would be excessive for some other. Exercise that would produce breathlessness, and consequent injury, in one case of pulmonary disease, might be without effect upon the respiratory rhythm of another better trained pulmonary case. Exercise that will send one individual to bed the next day with exquisitely tender muscles, a bad headache, a slight fever, and a concentrated urine will have no apparent effect upon some other better conditioned individual. Exercise which is moderate for the normal individual is usually excessive for patients suffering with various cardiac, circulatory, pulmonary, and nervous diseases.

Excessive exercise which produces a great increase in lieart rate and blood pressure is contraindicated in the presence of such conditions as chronic endocarditis, myocarditis, tachycardia, bradycardia, arteriosclerosis and its various complications, thrombosis, aneurysm, varicose veins, Addison's disease, and exophthalmic goitre.

Exercise which is accompanied by a great increase in pulmonary respiratory movement is contraindicated in chronic pulmonary diseases in which there is danger of rupture and pulmonary hemorrhage, or pneumothorax.

The general nervous stimulation and excitement accompanying excessive exercise is injurious to patients suffering with fatigue, chorea, hysteria, neurasthenia, epilepsy, nervous indigestion, exophthalmic goitre, etc.

The burden of the resulting increase in excretory activity contraindicates excessive exercise in such conditions as chronic nephritis, chronic cystitis, asthma, emphysema, chronic bronchitis, and chronic moist skin lesions.

The Administration of Exercise.-The amount and sort of exercise that may be given wisely varies with the age, sex, previous habits of exercise, and the present strength and organic condition of the individual. In any case, the first exercise should be mild. 'That is to say, there should, as a rule, be none of the muscular soreness, stiffness, and other disagrecable effects that are consequent upon taking too much exercise after a period of inactivity, or that are consequent upon changing abruptly from one line of exercise to another. If the amount of exercise is gradually increased, the capacity of the individual will increase in 
proportion, so that the desired physiological effects maty be seconred without diseomfort or accident.

Exercise is better given in the middle of the morning or fairly late in the morning, or in the midalle or liate part of the afternoon. Exercise immediately after meals interferes with normal digestion. ("anmon found that gastric and peristaltic movenents ceased when the lalworatory animal strugerled. I have found, as did I ombarl and oflecs, that the power of voluntary muscoular contraction is less in the early morning and immediately after meals than at other times of the day. "The variation which I secured indicated a period of maximum power roughly between 11 A.s. and 1 p.u., and between 4 and 6 i..., and a period of minimum power eartier in the morning and ealy in the afternoon.

Exercise should be taken under counfortable and pleasing surronndings. 'This is particularly desirable in nervous and impressionable cases. Interest is a sine qua nem in younger immature persons and a valuable aljuvant in any case. Comfortable clothing, good ventilation, goul illumination, and satisfactory heating are necessary for indoor exercise.

Outcloor exercise is better than indoor exercise, other factors being equal.

Exercise should be regular. It should be taken daily. It should in most eases be followed by a bath, preferably a cool bath. 'The temperature of the bath must, however, be regulated to suit the reaction of the individual case. (ieneral massage after exereise is a valuable adjurant where the nature of the case will permit.

\section{The Varieties of Exercise at the Disposal of the Practitioner.} Exercise may be either passive or active. Under the term "passive exercise" we include those procedures by means of which we secure" exercise effects from the manipulation of the muscles without making any demands upon the nervous energy of the individual. 'These proeedures are massage, mechanical vibration, passive motion, and electric excitation. 'They are of particular value where general nervous, respiratory, vasomotor, or cardiac excitement is contraindicated, and where the condition of the patient makes it unwise or impossible for him to stimulate his muscles voluntarily.

Massage assists in the removal of wastes from the tissues treated by increasing the lymphatic and venous flow. 'This is a mechanical inflience like the squeezing and pmoping process described above with reference to the local influence of muscoular contraction. 'This secures a moderate or minimum increase in hormone action and subsepuent exeretory activity. The mechanieal effect of the massage also initiates some degree of vasomotor action, with consequent local vasorlilatation and increased blood supply. 'The final influence of massage, then, is better tissue nourishment, with a minimm effect upon the nerve centres, respiration, cardinc action, circulation, perspiration, and cxcretion. In addition, massage may be applied without disturbing woumds or other injuries which make ordinary exercise impossible.

Mechanical percussion and vibration have the same general efferets as massage, but in certain cases they cannot be administered as intellivol. $1-12$ 
gently, nor are they as capable of the same degree of adjustment as is massage. On the other hand, mechanical devices like some of the Zander machines have very important special applications.

Pure passive motion has a minimum effect upon conditions in the muscles. 'These effects are like those of massage, though less in degree. In addition, passive motion has a direct and important effect on joint surfaces, tendon and tendon sheath surfaces, aponeuroses and ligaments, restoring in some chronic joint cases almost the normal amplitude and facility of movement.

Electric excitation, inducing muscular contraction, lies somewhere between massage and active exercise in its physiological influence. Under careful and intelligent supervision, the electric stimulation of voluntary muscles appears to secure the same results in lesser degree and extent that are common to active physical exercise, with this exception-the voluntary nerve centres are not brought into activity. 'This form of exercise is applicable to those cases in which it is undesirable or impossible for the individual to exercise his motor centres.

Active exercise may include any and every form of movement accomplished by the normal activity of the neuromuscular mechanism. We have formal exercise without apparatus; exercise in which the contraction of one muscle group is resisted by the contraction of its antagonistic group; and exercise in which one individual, the operator, resists formal movements performed by another individual, the patient.

We have exercise with light apparatus (see Plates VI to X, inclusive), exercise with heavy apparatus, exercise in which the individual moves the apparatus about in a formal way, exercise in which the apparatus is fixed and the individual moves himself, with the apparatus as his support. These are varieties of exercise characteristic of the gymnasium.

And then we have exercises through games, sports, and play.

Formal exercise without apparatus or with light apparatus is of value, because the exercise may, within certain limits, be localized; and the severity of the exercise may be regulated to a minimum. Untoward respiratory, cardiac, vasomotor, nervous, and excretory influences may be reduced or practically eliminated. 'The amount of exercise here, as elsewhere, depends upon the rhythm of movement and the resistance to be overcome.

Formal exercise with heavy apparatus and exercise in which the body is moved about a fixed piece of apparatus is more likely to be interesting to the capable individual than are the formal lighter exercises. These heavy exercises are likely to produce anatomical asymmetries. They give large opportunity for the strain of weak organs, because of the increased blood pressure and the increased intra-abdominal and intrathoracic pressures that arise with the great effort common to such work. Such exercises very greatly increase the blood pressure and are contraindicated in all cases of cardiac, pulmonary, and vascular weakness, and in the presence of hernia, incipient, present, or probable (as in the presence of a separation of the recti abdomini, weak abdominal scar, and large inguinal ring). 


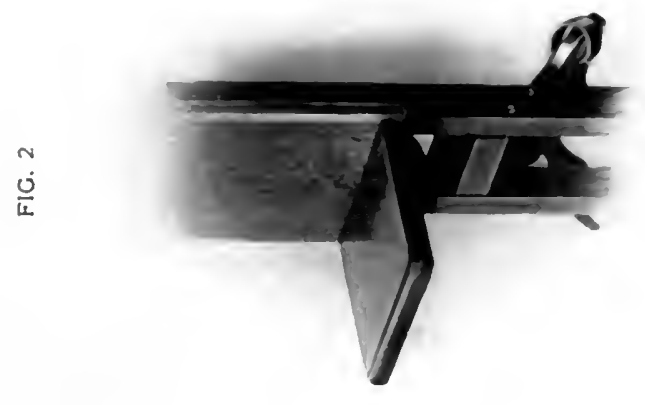

5
5
5
5

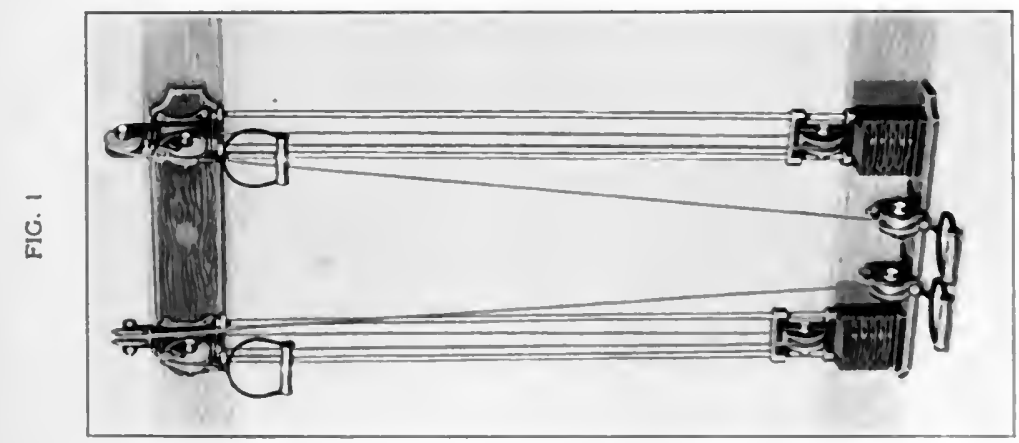

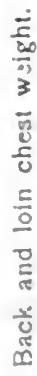



PLATE VII

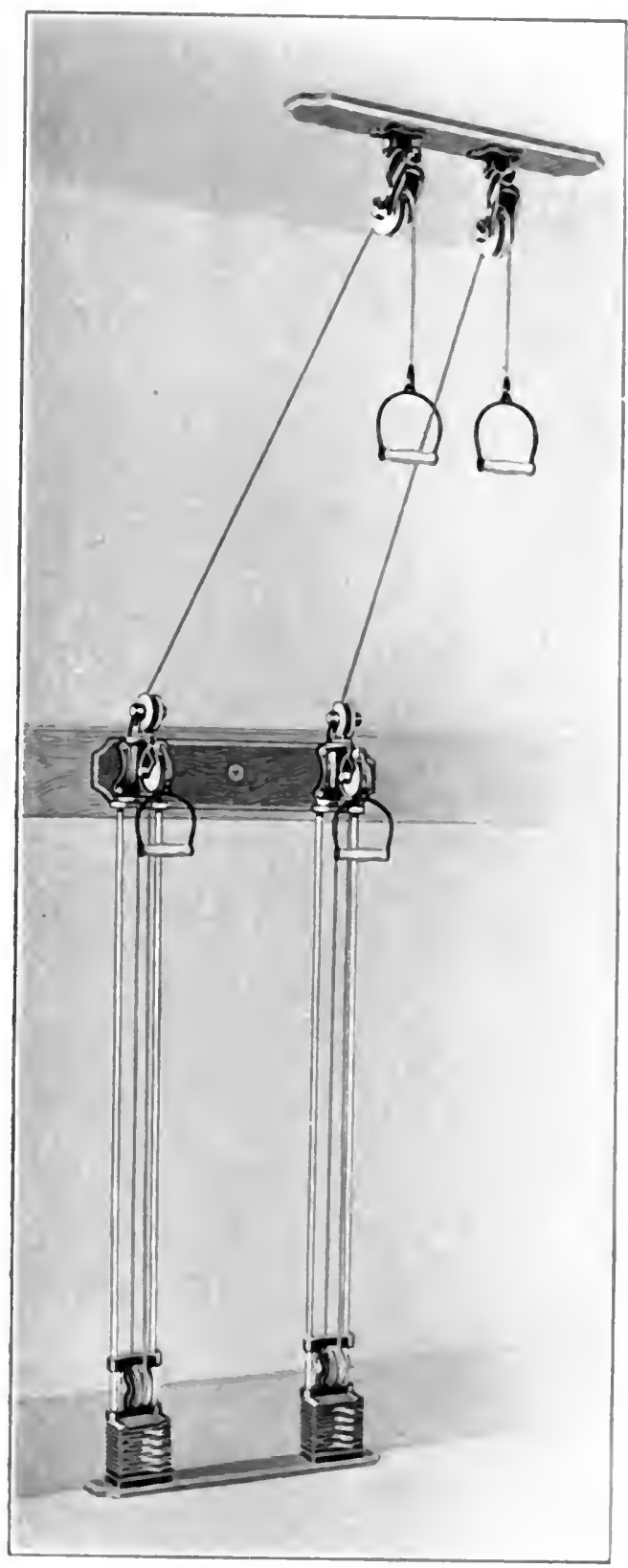

Duplex intercostal chest weight. 



\section{PLATE VI:I}

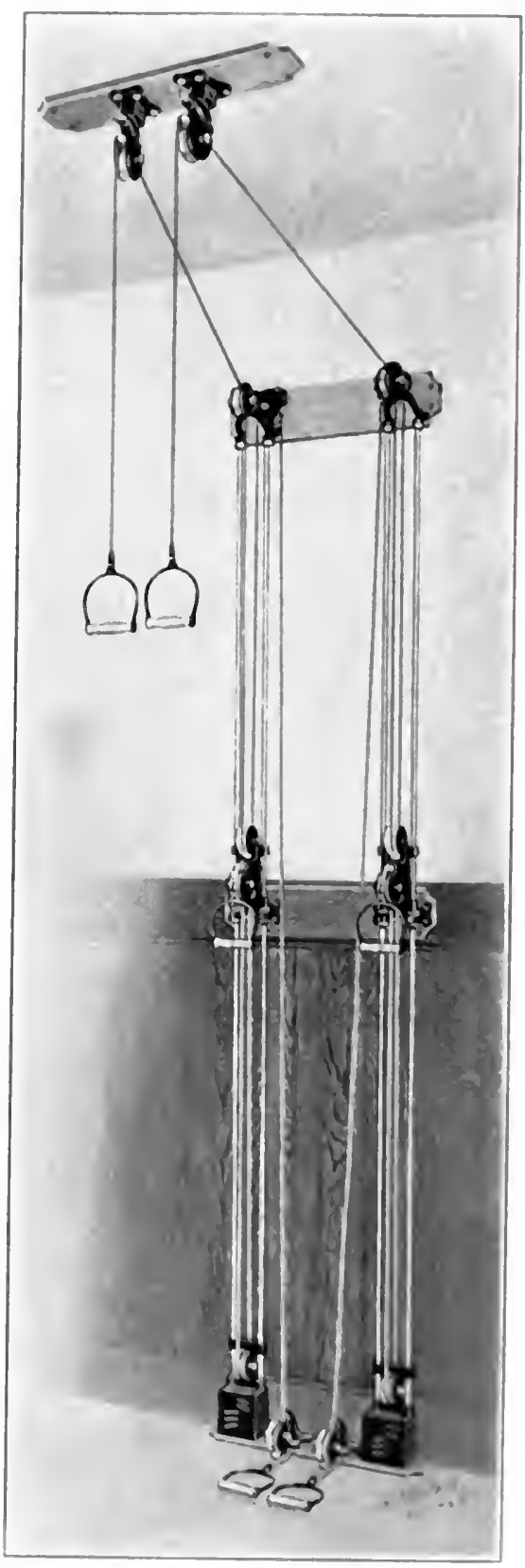

Triplex under-lift pulley weigh. 



\section{PLATE IX}

FIC. 1

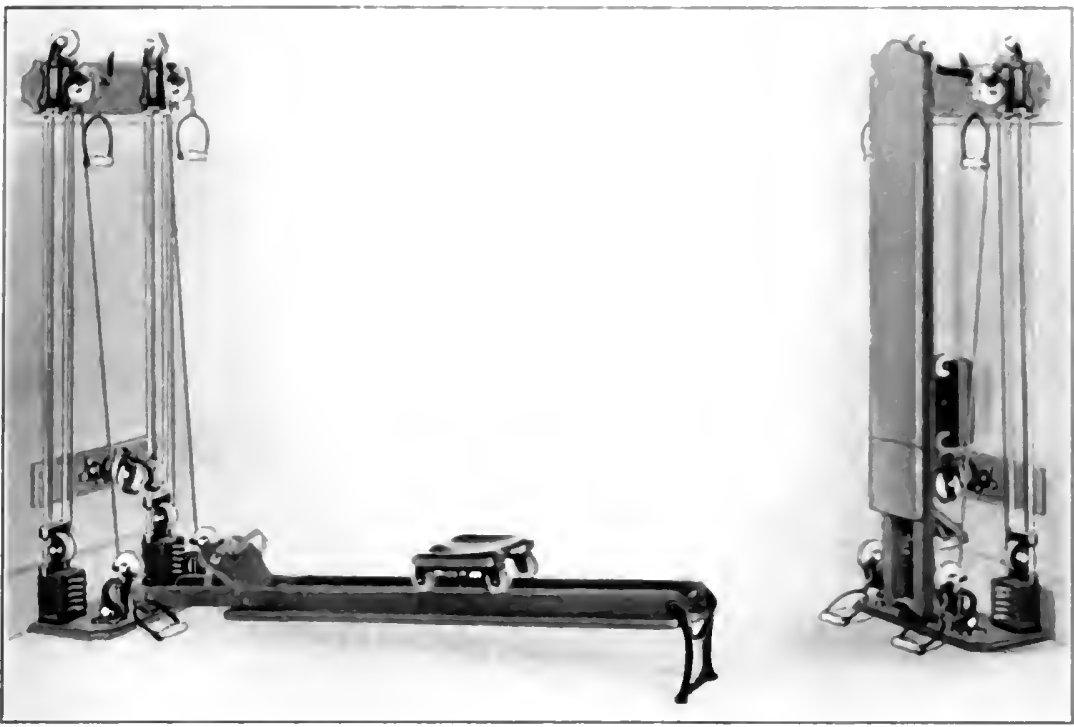

Rowing pulley weight.

FIG. 2

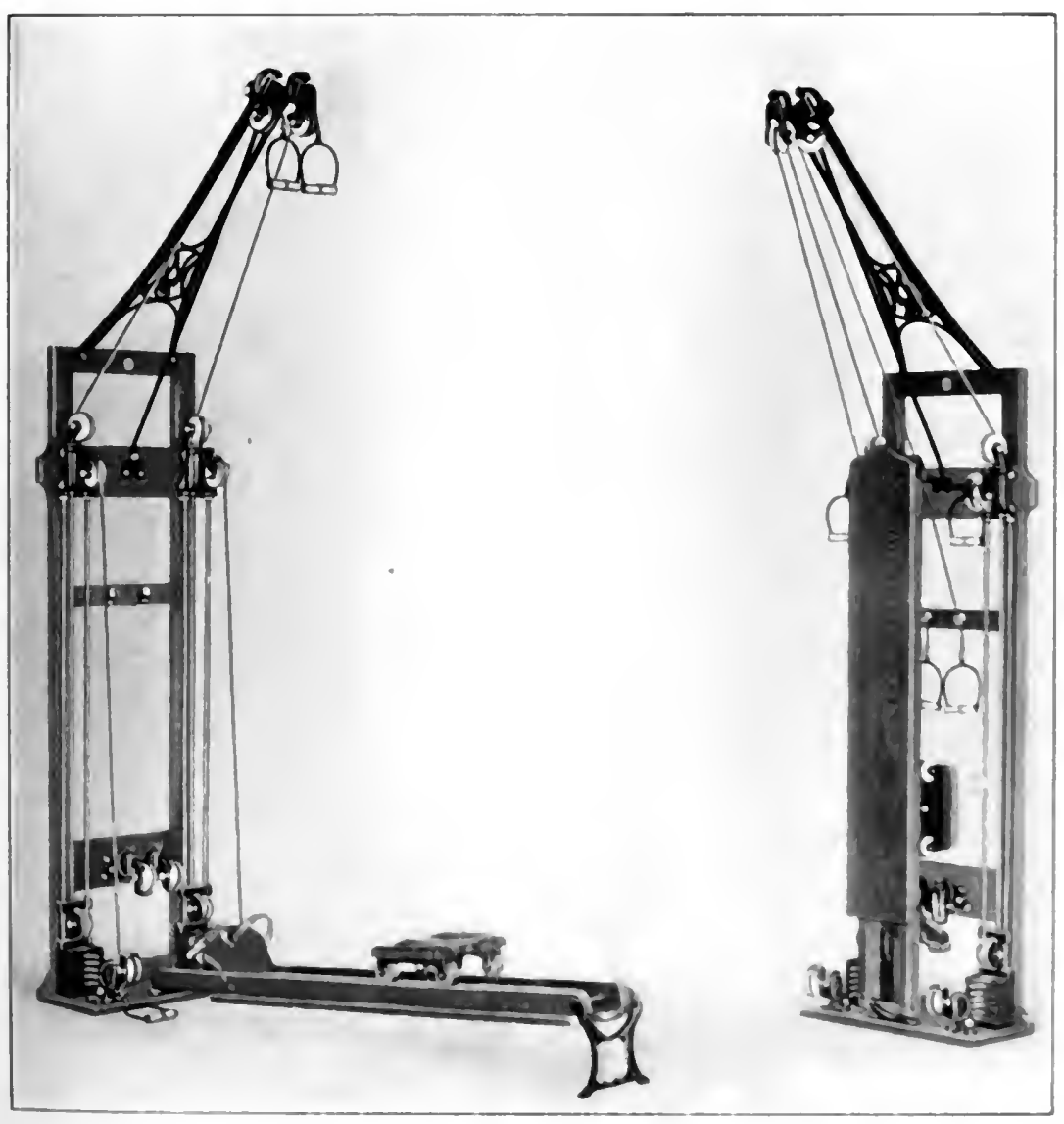





\section{PLATE $X$}

FIC. 1

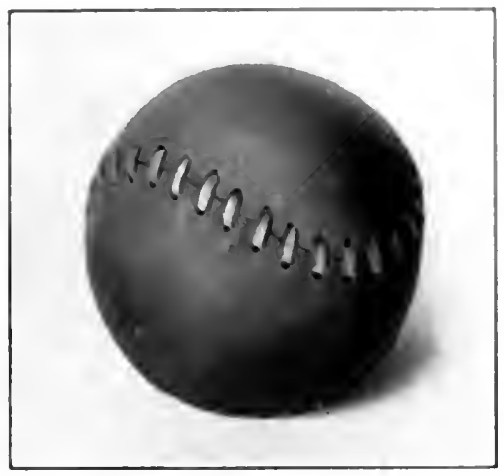

"Medicine ball."

FIG. 2

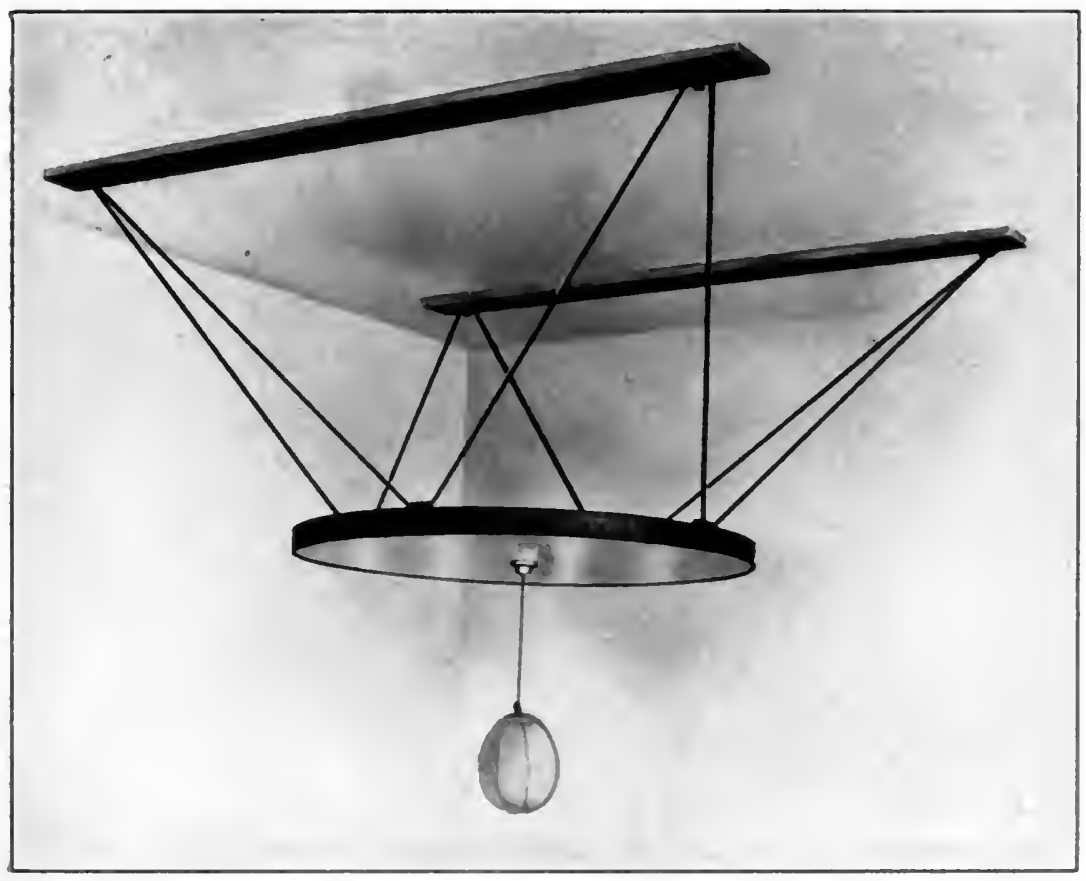

Siriking bag and drum.

Nore.-Plntes VI to $\boldsymbol{X}$, inclusive, have been secured through the courtesy of the Narmgansett Machine Company, Provillence, R. I. 

Games, sports, and platy are the most satisfactory forms of physical exereise for the normal younger individual. Silch atetivities usmally take place in the open and are often at tratcive and interesting. 'Iho demands which such forms of exercise make upon the structural and functional status of the individual vary within large linits. ("ropucet goes well with arteriosclerosis, but hand-hall would be langerous. (On the other hand, the reaction of the individual to the physiological demands of any one of these forms of exercise depends upon his physigue, lis training, and his condition. As I have stated elscwhere, exercise that is mild for one patient may be severe for another.

In advising any of the above forms of exercise, passive or active, the practitioner will be guided by the object for which he is preseribing; the structural and functional effects of the sort and quality of exercise which he prescribes; and the age, sex, previons experience, phosique and present condition of his patient.

The following calisthenic and chest-weight exercises are offered as types of drills which ean be utilized for light, moderate, or heav work. If the rhythm of movement is very slow, if no hand apparatus, or if lighter weights are used, if the heavier movements are eliminated, and if periods of rest are entered at regular intervals, the physician may secure a very mild type of exercise. If, on the other hand, the rhythm is made rapid, if dumb-bells are used for some of the movements, and if the number of counts to each movement is increased, the patient may be given a severe exereise.

Calisthenic Exercises.-Directions for Form and Execution.-Pusition: Heels alout a foot apart, toes direetly forward, arms in side hang. chest up, chin drawn down and back.

lixecution: Contime each movement for four, eight, sixteen, or more counts. Rhythm from one to three movements per second. Slower rhythm, one to three seconds hetween each movement. with frepuent periods of rest when it is desired to reduce the excitatory and excretory eflects to a minimum.

Movements. - 1. Inhale, raising arms through sile horizontal to vertical position, then arms to side hang; exhale. Repeat.

2. Inhale, raising arms to vertical through front horizontal; arms to side hang through side horizontal; exhale.

3. Inhale, raising arms to front horizontal, then to side horizontal; arms to side hang; exhale.

4. Open and close hands.

(a) Repeat, arms in side horizontal.

(b) In front horizontal.

(c) In vertical.

(d) In side horizontal.

(e) Position.

5. Arms in sille hang. (a) Flex forearms, bringing fists to shoulders. (b) Repeat in the four positions, i.e., arms in side horizontal, front horizontal, side horizontal, and vertical. 
6. From position with arms vertical.

(a) Arm circles inward, crossing arms in front of body (full arm circumduction).

(b) Repeat, making the movements in the opposite direction.

7. (a) Rotate full arm.

(b) Repeat in the four-arm positions.

Position: Legs well apart; hands on hips, thumbs pointing backward.

8 . Bend trunk to the right (knees and hips firm; chest, neek, and head in good position); then left.

9. 'Trunk bend forward and return to erect position (position as in No. S, bending at hips). Repeat.

10. Alternate No. 8 and No. 9.

11. Rotate trunk to the right; then left.

12. Hands between ankles; with body bend forward (slight bend at knees); arms through front horizontal to vertical; body erect.

13. Same; arms through front horizontal to side horizontal.

14. Same, to front horizontal; half turn of body to the right; right knee bent: arms reaching far to the right; alternate with same movement to the left. Position: heels and toes about twelve inches apart; hands on hips.

15. Squat and return (flex and extend thighs on legs).

16. Spring from toes (knees and hips straight).

17. Spring from toes, alternating position of thighs from abduction to adduction.

18. Standing run, raise knees high in front.

Position: Body extended on floor with the weight sustained by the toes and arms; knees, hips, back, and neek straight.

19. Spread legs and return.

20. Flex and extend arms (lowering and raising the body).

21. Flex hips and knees, bringing feet between hands; rise to erect position (in good form). return through same position to "front rest on Hoor."

Position: Supine, arms at side or clasped back of head.

22. Flex right knee, bringing heel to buttock.

(a) Repeat left.

(b) Alternate.

(c) Combine.

23. Flex right thigh on trunk. knee and ankle straight.

(a) Repeat left.

(b) Alternate.

(c) Combine.

24. Spreat legs and return (abduct and adduct thighs).

25. Raise head from floor.

26. Raise thorax from floor, resting weight on oceiput and buttocks.

27. Raise hearl and chest from floor; neek straight.

28. Raise trunk to sitting position and return.

29. Raise body, resting weight on heels and occiput. 
30. Arms at side, inhale while bringing arms through latteral extconderl position to cephalad extended position (remenuler the body is supine). Return arms to sides; exhale.

31. Inhale, bringing arms thromgh front extended position to (eephalad extended position; return arms through lateral extended position to side; extiale.

32. Inhale, bringing arms to front extended position and to lateral extended position. Return directly to side position; exhate.

Type Exercises for Chest Weights.-Position: Far'e machine, feet parallel and about twelve inches apart, toes directly forward, arms hanging at sides, chest up, chin drawn back and down.

Fixecution: Rhythm, one or two movements a second, and larger weights for greater physiological effects. One or two seconds to a movement, and lighter weights for minimum effects. Four, eight, sixteen, or more movements to each exercise.

Movements.-1. Arms to front horizontal and return.

2. Arms to front horizontal, to side horizontal, to front horizontal, to side hang.

3. Arms to front horizontal, side horizontal, front horizontal, vertical, and return; through same series.

4. Right arm vertical; alternate left and right to vertical.

5. Arms to vertical and return.

6. Arms front horizontal, flex to shoulders, front horizontals, and down. I eges well apart.

7. Hands between ankles; swing arms to front horizontal; half turn of trunk to right; slight bend of right knee.

8. Same, left.

9. Alternate 7 and $S$. Feet as before.

10. Arms vertical; step right leg far to the right; hands to floor in front cf right foot; return to stand, with arms in vertical. arms to side.

11. Repeat on left.

12. Alternate right and left. Face away from machine.

13. Arms to front horizontals, side horizontals, front horizontals, and down.

14. Arms to front horizontals, side horizontals, front horizontals, vertical; return through same series to side hang.

15. Alternate right and left, arms to vertical.

16. Arms in front horizontal, flex to shoulders. 
CHEST WEIGH'T EXIRCISES.

Frg. 4

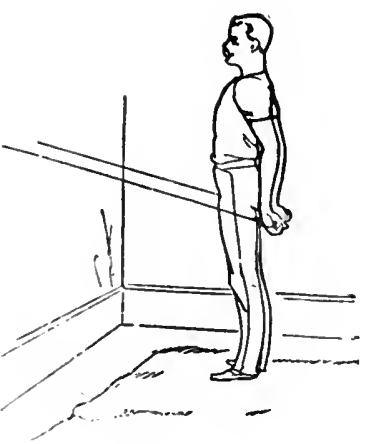

FIg. 7

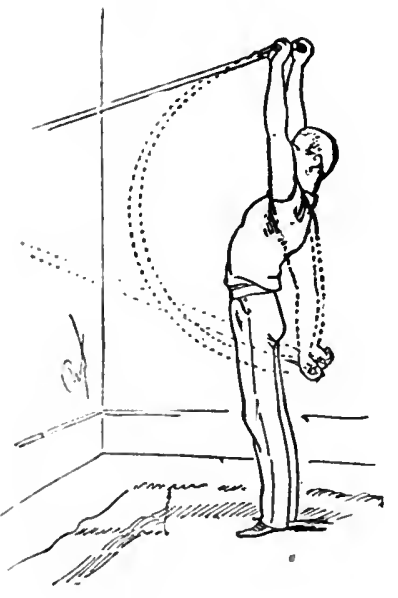

FIG, 10

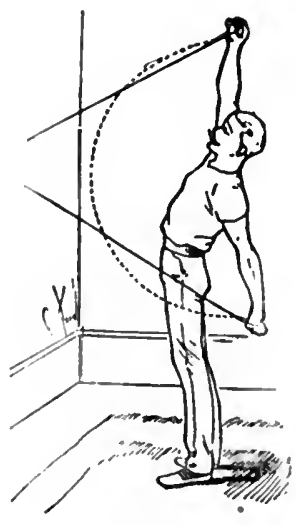

Fig. 5

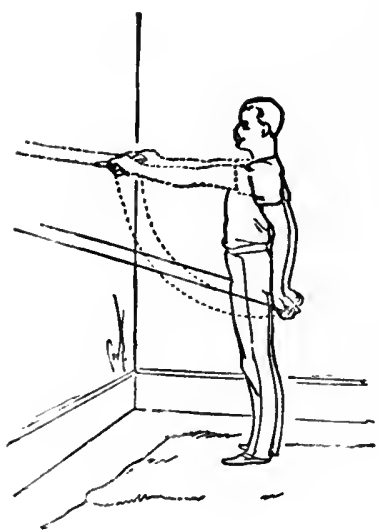

F10. 8

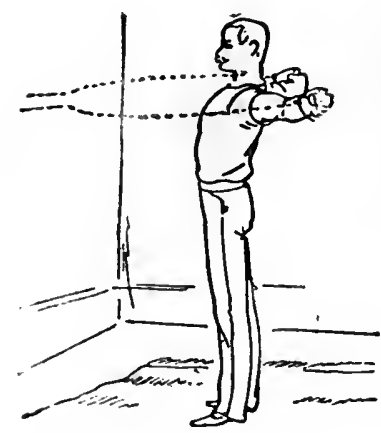

FIg, 11

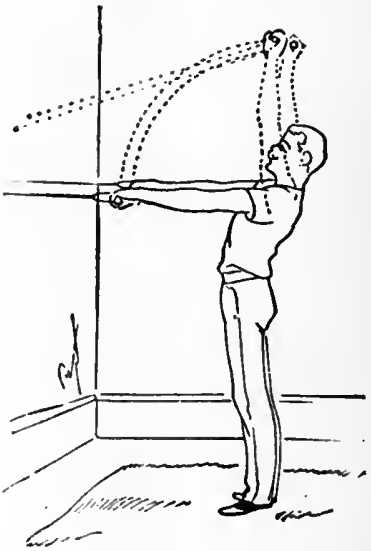

Fre, 9

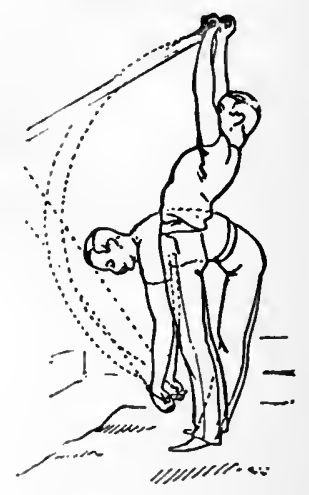

FIG 12
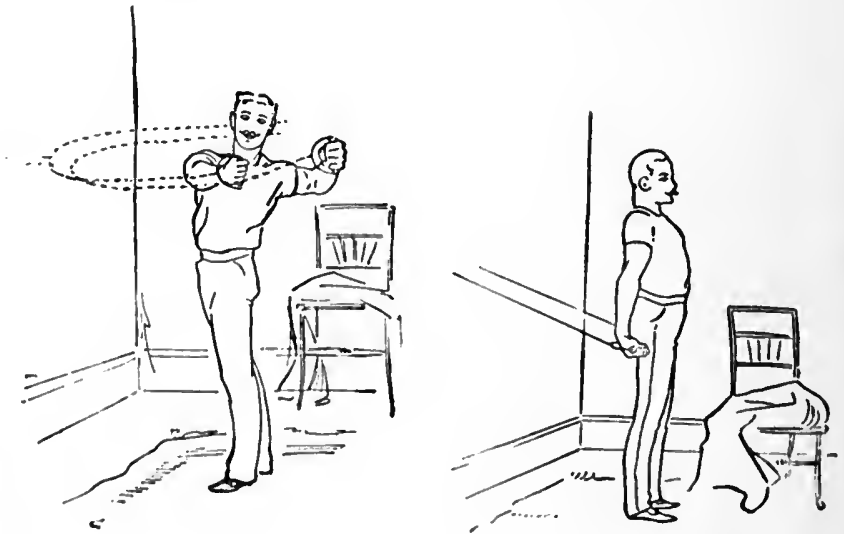
FI\%, 13
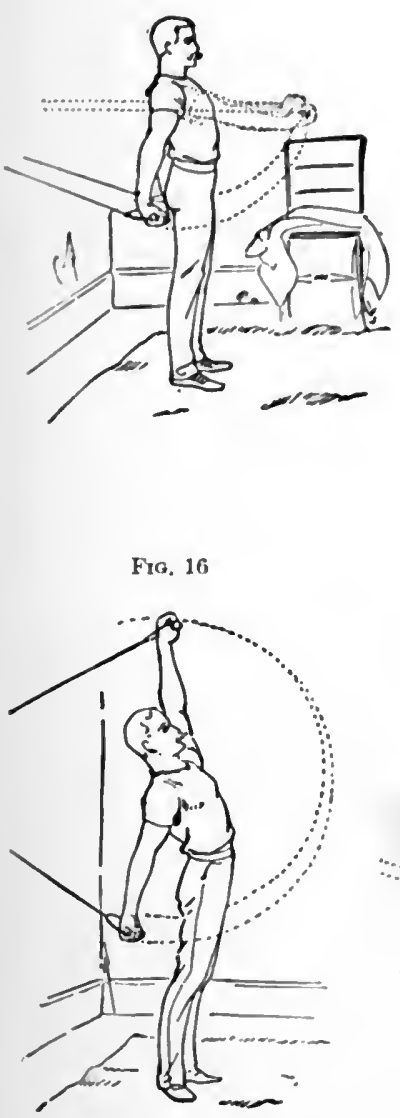

FIo. 19

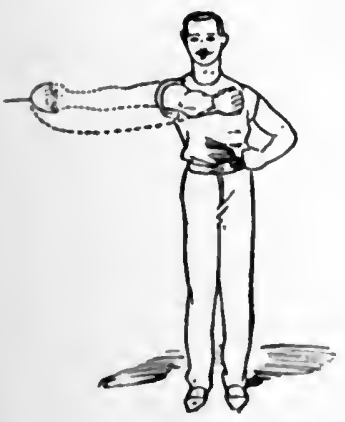

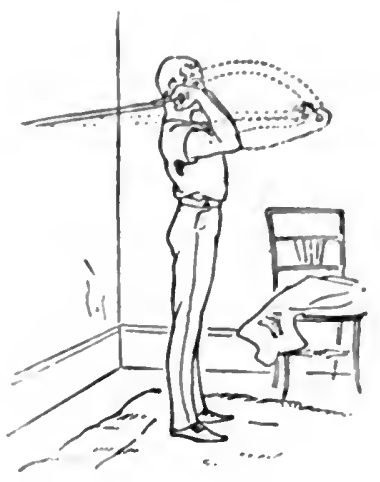

Fro. 17

Fir. It

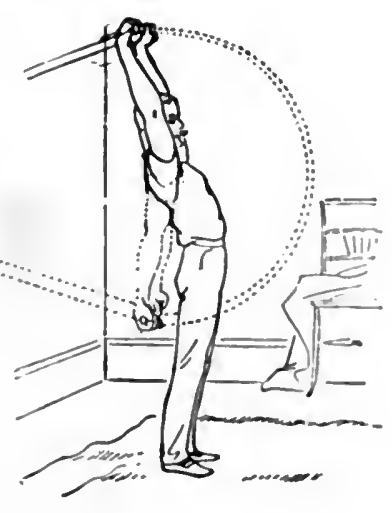

Fic. 20

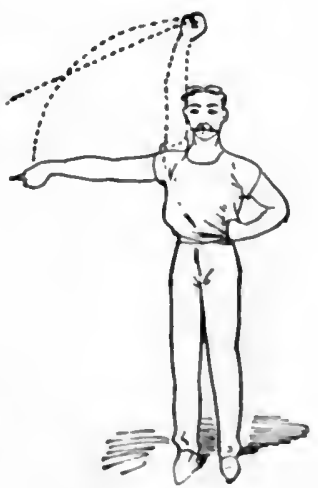

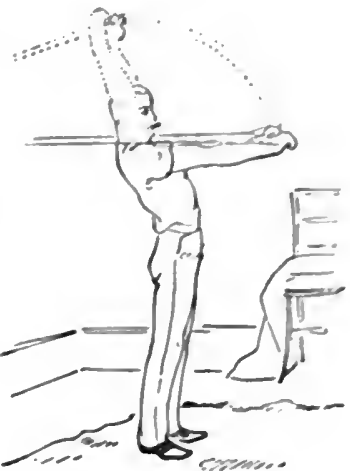

FIG. 18

FIs. 1:

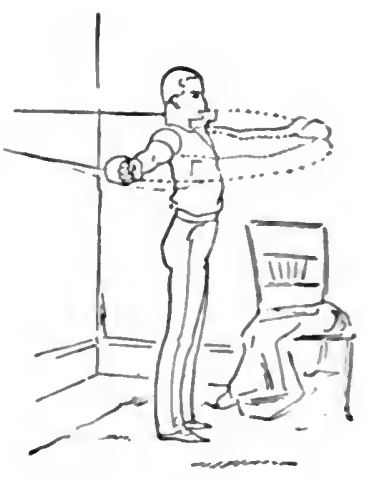

FIG. 21

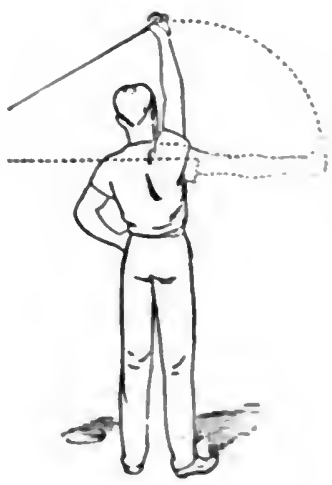


184

FIG, 2:

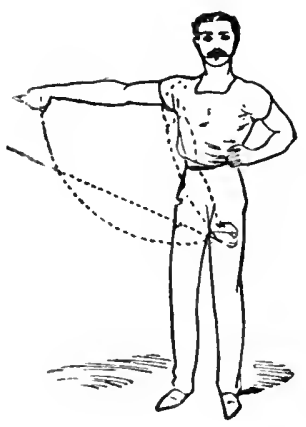

Frr. 25

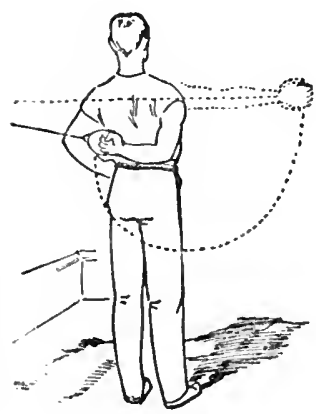

Fra. 24

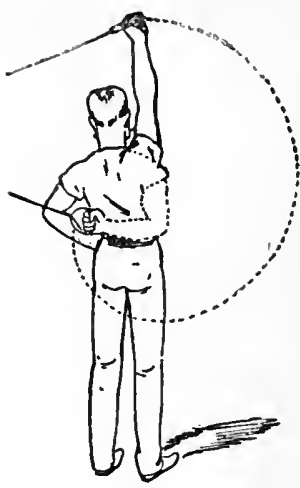

Frg. 27

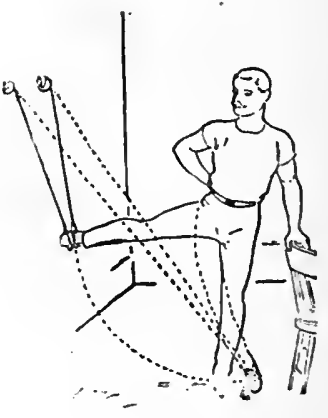

FIG. 29

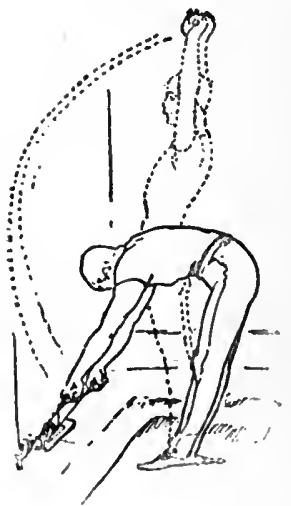


Fin. :3)

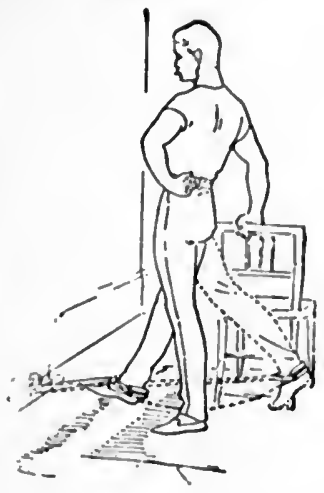

Fro. 32

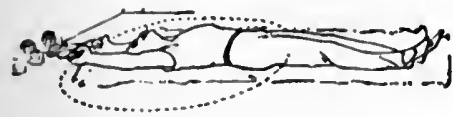

Fto. 34

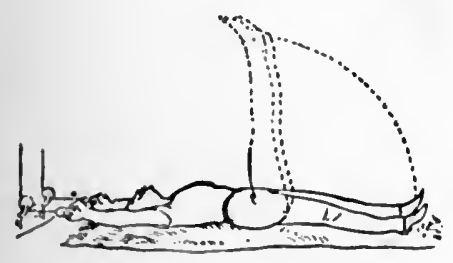

Fเo. 36

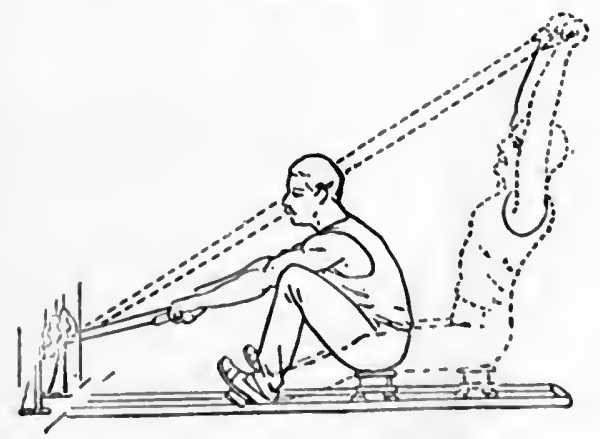

Rowing machine exereises.

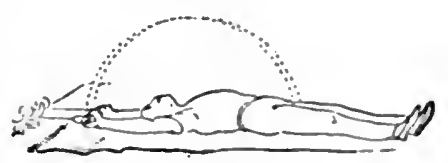

Fro. 35

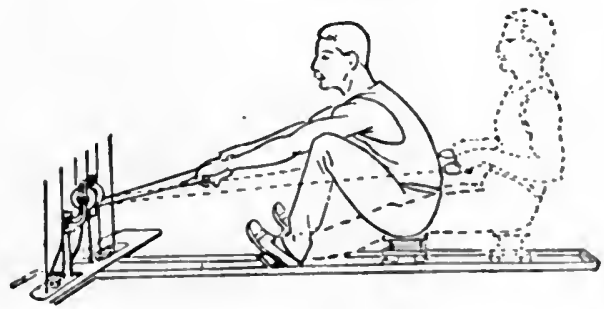




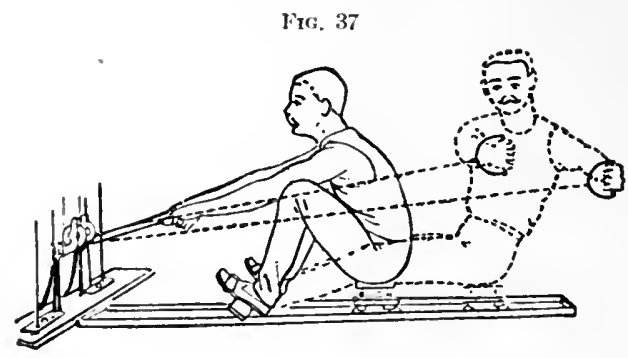

Fig. 38

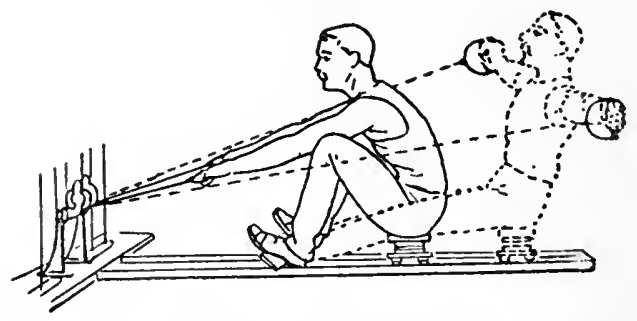

Rowing machine exercises.

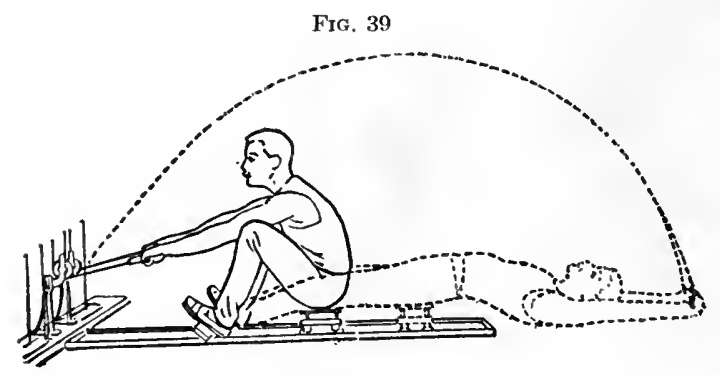

lowing machine exercises.

Note.-I am indebted to the Narragansett Machine Company for illustrations Nos. 4 to 39 , inclusive.

The General Application of Exercise.-In discussing the general application of exercise I will not attempt to go into the details of treatment in any given disease. Such a discussion belongs to the therapeutics of that disease. In addition, I will limit myself to a discussion of the general principles involved. The special details belong to the special practitioner, as in the reëstablishment of coördinations in locomotor ataxia, or in the treatment of structural scoliosis through exercise. The limitations of this chapter and its subject preclude the exploitation of such details.

General Exercise for the Normal Individual.-Every well-ordered individual policy of personal health control must include regular habits of 


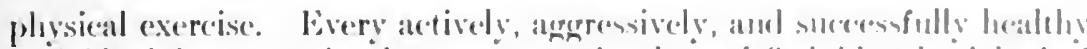
individual hats conscionsty or nuronscionsty satisfical his physiological demand for exercise. But no persomal health policy is complete or successful which does not include several other essential health hathits. We have seen that exercise is intimately and inseparably assoriated with rest, nourishment, excretion, and phesiological protection. 'These considerations must inevitably enter into every intelligent plan for the utilization of exercise as a health procedure. And, on the aterage, every normal man that exercises wisely, rests wisely, eats, drinks, and breathes intelligently, takes care of his exeretions, and reasonably avoids the agents and carriers of disease, will develop and maintain a high degree of tissue health and consequent physiological efficiency. Such an individual will be less easily infected by our common communicable diseases, and, once infected, he will more eaisily recover. IIis active and potential immunity will be more highly developed.

Exercise, for the normal individual, shoudd be taken daily. Gireater benefit will be secured through exercise that cause's moderate respiratory, cardiac, and circulatory excitement with sweating, but very satisfactory results follow exercise which falls short of such effects. Exercise should be followed by a bath, preferably cool, and a rub-down. A very satisfactory effect may be obtained by following the bath with a brisk rub with a coarse towel.

The normal individual may select his exercise from a variety of sources with special reference to his individual needs and limitations. He may use formal gymmastics, with or without apparatus, chest weights, heavy gymuatic apparatus, recreative gardening, woodworking, walking, billiards, croquet, quoits, mountain climbing, hunting, fishing, rowing, swimming, bieydling, horseback riding, tennis, golf, hand-ball, the punching bags, boxing, fencing, skating, skiing, and other games and sports.

The Application of Exercise in Abrormal Cases. - 1. Sedentary Habits. - The accompaniments of sedentary habits are commonly indigestion, constipation, acne, lack of appetite, headache, bad breath, and mental dulness, with often obesity. The nervous type presents varied degrees of indigestion, constipation, insomnia, headache, irritability, and more or less underweight. 'The two types are not neressarily. distinct.

In such cases the sedentary habit and its undesirable and more or less dangerous accompaniments ind sefuela may be "cured" by instituting regular habits of exercise and establishing wise habits of rest, nomrishment, excretion, and bathing. Such patients should undertake mild exereise at first. Otherwise they will suffer from muscle soreness. They may begin with light, formal gymnastics, casy walking, chest weights, croquet, quoits, billiards, and golf. Massage will be of benefit at all stages. As the strength and condition of the patient improve, he should increase the amount of exercise taken, either by increasing the vigor with which he performs those already undertaken or by adopting new and more vigorous exercises. Within certain limitations it maly be 
truly said that one gets out of exercise what he puts into it. Bowling, tennis, fencing, boxing, bag-punching, horseback-riding, hand-ball, squash, and other heavier games and exercises may be prescribed when the individual becomes physically fit to meet the demands which they make upon his muscles, heart, lungs, and circulation.

2. Convalescence.-Convalescence may be hastened by the wise administration of exercise. During the period in which the patient is too weak to undertake roluntarily even the mildest form of physical exercise he may be treated by manipulation (passive movements) and massage. This treatment may accompany the routine sponging usually prescribed at this time. The transition from the recumbent to the sitting position in bed may be accomplished gradually by adding pillows for the higher support of the head and trunk. As soon as the sitting position may be sustained for several hours without untoward nervous, respiratory, cardiac, or circulatory disturbance the patient may be carried to a chair and possibly some attempt made to stand. Later, a walk from the chair to the bed may be added, and the physical demands gradually increased from day to day as the strength and functional reaction of the patient indicates its practicality. 'The success of such treatment will depend absolutely upon the success with which the rest, nourishment, excretion, and physiological protection of the patient is handled. Outdoor exercise should be taken as soon as practicable. The patient may at first sit on the porch, in the yard, or on the roof. Later, the vibratory effects of the carriage, trolley car, or automobile may be of benefit. 'The patient gradually gains strength and is able to walk increasing distances with firmer, more normal, and more active step. After a time, when his strength permits, he may undertake mild forms of gymmasties; then such games as croquet and quoits, and, later, golf. Each period of exercise should be followed by a period of rest, and the practitioner should carefully note the immediate effect of the exercise taken upon the heart, circulation, and respiration. This attention in the beginning must be constant and the functional indications faithfully respected. Overdoses of exercise will produce disproportionate and persistent cardiac, circulatory, and respiratory excitement. Such mistakes may lead to general fatigue, collapse, organic complications, and prolonged convalescence.

3. Conditions in which Rapid Pulse or Increased Blood Pressure are Contraindicated (e.g., Tachycardia, Bradycardia, Chronic Endocarditis, Chronic Myocarditis, Chronic Pericarditis, Quiescent Aneurysm, Varices, Arteriosclerosis, Chromic Nephritis, Addison's Disease).-'The various pathological conditions in which increased heart rate and blood pressure are likely to be injurious indicate habits of exercise in which there is no effort and in which the rhythm of movement is comparatively slow and the mechanical work done by any given single movement comparatively small. In all these pathological conditions the aim is to secure as much general and local physiological benefit as is possible without injury to weak organs. 'The physician will take into consideration the degree of local or general organic weakness, and the age, sex, and nervous 
irritability of the individual; his previous training, physique, and present condition, and gauge his prescription according to its effect upon heart rate, blood pressure, and respiratory rhythm. During the last fifteen years I have had opportunity to observe a number of these cases in which it was ultimately possible to secure a half or three-quarters of an hour of systematic exercise with no general increase in blood pressure and with a cardiac acceleration of only three or four beats to the minute. In these cases I have employed free-hand exercises, light dumb-bells, chest weights, and walking. 'The rhythm must be very slow, and in many' cases the mechanical work done insignificant. 'The patient must exercise without effort, always breathing freely, naturally, and guietly. Frequent short periods of rest interpolated at intervals during the exercise may be necessary in severe eases. Stronger patients may do well with croquet, quoits, and golf. Massage is always valuable. 'There is a general tendency to underestimate the amount of exercise which can be done by individuals suffering with these lesions. 'This is particularly true of valvular lesions of the heart. General physiological efficiency, health, and resistance to disease are sacrificed out of overrespert for these weaknesses. Each and all of these conditions are compatible with some degree of exercise. 'The amount and variety that can be borne with safety in any individual ease must be determined by the physician in charge, but in no case should excrcise be totally neglected unless contraindicated by acute exacerbations, complications, or sequels of the chronic affection. As the treatment progresses it is regularly possible, within limitations, to increase the amount of exercise without undesirable organic disturbances.

4. In Chlorosis and Anemia and Some Allied Pathological Conditions Characterizel by a Subnormal Amount of Hemoglobin or a Numerical Reduction in Erythrocytes. - The oxygen-carrying capacity of the blood is limited. For this reason the patient should not undertake any exercise that will increase the tissue respiration. Massage, passive motion, vibratory exercise, and very mild active exercise will assist in the removal of tissue wastes, favor metabolism, without respiratory excitation, and possibly effect desirable hormone action.

5. Conditions Contraindicating Great Increase in Carbon Dioxide Elimination (e. g., Chronic Bronchitis, Asthma, Emphysema, C'hronic Pneumonia, from Any Cause).-C'These diseased conditions are frequently associated with a considerable reduction in the alveolar surface areas for respiratory exchange. Under such conditions it is not advisable to prescribe exercise which will increase the need for carbon dioxide excretion beyond the capacity of the alveolar walls. 'The amount of exercise that may be borne may be increased by training. Exercises with slow rhythm and involving but little mechanical work, and exercises in which only a limited number of muscle groups participate, are aceompanied by slight respiratory excitement. 'These exercises are practically the same as those advised in cases where increased cardiac and circulator: excitement are contraindicated.

It has been my experience that these cases are able, after careful train- 
ing, to support a relatively large amount of exercise without undesirable physiological excitement.

6. Conditions Contraindicating Increased Intrathoracic Pressure (e.g., Pulmonary Tuberculosis and Other Chronic Diseases which Weaken the Bronchial and Alveolar Walls).-The presence of weakened alveolar or bronchial walls, with the consequent possibility of rupture and pulmonary hemorrhage or pneumothorax, contraindicates any exercise that may increase the intrathoracic pressure. This contraindication would include deep, labored breathing and exercises with effort. In the presence of such conditions all exercise should be accomplished with easy, free, effortless breathing and without increase in respiratory rate or blood pressure. Massage, passive movements, and mild active exercise, with slow rhythm and a minimum of mechanical work, are indicated. If the exercise is carefully undertaken there will be no untoward result, and the local pulmonary and the general physiological efficiency of the patient will be increased.

7. Conditions which Contraindicate Increased Intra-abdominal Pressure (e.g., Herniw, Bubonocele, Diastasis Recti, Scar Weakness in Abdominal Wall).- There is a variety of conditions in which increased intra-abdominal pressure is contraindicated. All exercise that is accomplished with an effort-that is, by holding the breath and simultaneous contraction of the abdominal musculature-will exert an internal pressure upon the abdominal parietes. Unless care is taken the simplest muscular acts may be accompanied by effort. These patients should be taught to exercise without holding the breath. All of their movements should be accomplished with easy, free, natural breathing. A considerable degree of respiratory activity may be present, with no undesirable effect upon the intra-abdominal pressure. With this precaution in mind, the physician may prescribe any exercise which the condition and circumstances of the patient indicates.

8. Conditions which Contraindicate Increased Demand upon the Excretory Functions of the Kidneys; Chronic Nephritis.-Exercise must be carefully regulated in the presence of an impairment of the functional activity of the kidneys. 'There is some reason for assuming that exercise excesses which are accompanied by the appearance in the urine of albumin, with or without blood and casts, are ultimately productive of serious pathological changes in the normal kidney. In the diseased kidney all exercise is contraindicated which throws an additional burden upon its excretory function. Under such conditions, mild exercise, with only slight cardiac, circulatory, and respiratory excitement, are indicated. 'The prescription of exercise may be checked up by urinary analysis. There should be no attendant consequential increase in pathological findings. Under some circumstances an increase in perspiratory activity with a consequent concentration of the urine, is the penalty for overexertion.

'The high blood pressure, cardiac and valvular changes, associated with chronic nephritis, are also contraindications for excessive, exercise. 
For obvious reasons the plan of exercise suggested for ehronic cardiac. conditions is equally applicable here.

9. Constitutional Discases which are Characterized by Intercals of Comparative Health (e.g., (iout, Diabetes, C'hronic Rheumatism).The various chronic constitutional affections which, in their intervals of quiescence, do not greatly limit the activity of the individual, derive an great deal of benefit from regular systematic exercise. Such cases are to be treated as normal individuals in so far as their general or special organic condition will permit.

10. Conditions in which Nervous Excitement is Contraindicated (e. g., Nerrous Fatigue, General Debility, Neurasthenia, IIysteria, Chorea, Epilepsy, Paralysis Agitans, and General Paresis).-Those conditions in which general nervous excitement is contraindicated! are best handled with passive exercise, and later with quiet, formal, moderately slow exercises, in which the effort, mental attention, and mechanical work is at a minimum. Such cases may be treated along the same lines as those suggested for general convalescence, the prescription of exercise being at all times adjusted to fit in with the other necessary lines of treatment indicated. On the other hand there are some of these cases in which it is desirable to engage the interest and attention of the patient, so that his mind may be relieved of the nervous excitement consequent upon his domestic, business, or social affinirs. In these cases, other things being equal, reereative games, sports, and plays are effective. Croquet, quoits, walking, golf, bowling, horsebackriding, bicycling, tennis, hand-ball, hunting, fishing, target-shooting, archery, and canoeing may be indicated in special instances.

11. Neurological Conditions Characterized by Approximate or Complete Loss of Function, Paralysis, Atrophy, or Contracture (e.g., Locomotor Ataxia, General Paresis, Progressive iluscular Atrophy, Spastic Paralysis, Anterior Poliomyelitis (Convalescent), Diffuse and Focal Lesions of the Brain and Cord, Diseases of the Peripheral Nerves (Nenritis), General and Functional Diseases).-Those neurological conditions that are characterized by loss of muscular function and impairment of museular nutrition are benefited by the passive exercise of the affected museles, and in appropriate cases by the educational exercise of nearly allied muscle groups. Passive exereise, through massage, passive motion, and electricity, may serve to delay muscular atrophy through its influence upon local metabolism. New coördinations approximating the utility of those lost may be established, to a limited extent, through the patient exercise of muscles that are still capable of functional activity. It wonld appear, too, that in some cases at least the careful exercise of the affected muscle group through the medium of a few nerve filaments not destroyed by disease or injury has led to the restoration of a consielerable degree of function throngh the new nerve patl.

12. Mental Diseases. - The health habits of mental cases need careful supervision. The same general principles operate in their interest as they do in the interest of the nornal individual. 'l'he same indications and contraindications for exercise present thenselves. The practitioner 
with a mental case on his hands will invariably find that his patient does better while submitting himself to an intelligent policy of personal health control in which regular systematic exercise figures conspicuously.

In institutional life these patients often do well in group games, sports, and play. Dancing is one of the commonest and most effective exercises in hospitals for the insane.

13. Bone and Convalescent and Chronic Joint Conditions Indicating Exercise for its Influence upon Local Nourishment, Anatomical Relationship, and Motility (e.g., Fractures, Dislocations, Subluxations, Anlyyloses, Chronic Arthritis, Faulty Attitudes, Scoliosis, Wcak Arches, Flat Feet). -Early massage and passive motion materially assist in the convalescence of fractures, dislocations, and subluxations, through their beneficial influence upon local nourishment and on arthritic mobility. Through such treatment the injured areas may be given a larger supply of arterial blood and therefore a greater amount of available tissue food. In addition, the improved venous and lymphatic drainage serves to remove more rapidly the products of local injury. Passive motion in these cases may prevent deposits in and about joint surfaces, tendons, and ligaments immobilized for purposes of treatment, which may prolong convalescence or eventuate in stiff joints or complete ankylosis.

Arthritic surfaces, ankylosed either as a result of prolonged immobilization during treatment or as a result of some form of arthritis, may in many cases be restored to a considerable degree of efficiency through forced, graded extension, passive motion, and mechanical vibration. The Zander apparatıs is particularly effective in some of these cases.

Anatomical asymmetries due to or accompanied by the stretching of weak muscles may be treated by exercise which will strengthen and therefore shorten the weak and stretched muscles. Such treatment is valueless unless conscientiously carried out for a considerable period of time. Furthermore, such exercise is without effect in removing asymmetries consequent upon bony abnormality or fixed bony asymmetry. Under such conditions, if these exercises can ever be of local corrective value, it can only be after removal of the fixed bony cause.

As a rule, in simple functional scoliosis the asymmetry may be corrected by general symmetrical exercises of the trunk. In such cases systematic exercise will secure a general muscular, ligamentous, and connective-tissue strengthening, with a corresponding improvement in functional activity. The supporting and locomotor tissues of the trunk will then perform their duties more evenly and successfully.

'The treatment of structural scoliosis is within the special province of the orthopedic surgeon.

Faulty attitudes are largely the result of habit, associated with a weak musculature. If the faulty attitude has not become fixed through muscular, connective tissue, and bony change, it can be corrected through persistent general exercise in correct position. 'Those ases in which the anatomical relationship has become rather rigidly and firmly fixed require special attention.

Weak arches are benefited by exercises in which the muscles that pro- 
duce plantar flexion and inversion of the feet are strengthened and shortened. Such cases should be taught to walk with the feet directly forward, or "toeing in." 'They should exercise more or less while supporting the weight of the body on the outer side of the plantar surface of the foot. Movements of plantar flexion and of ankle circunduction with the plantar surface of the foot facing the median line (pedal inversion) are useful. The muscular exercise secured through attempted plantar flexion while walking, and through pedal inversion while walking, is of value. In addition, general exercise with improved general condition will be of local benefit.

As in structural scoliosis and rigid faulty attitudes, flat foot comes under the province of the specialist.

VoL. I. -13 



\title{
MINERAL SPRINGS
}

\author{
BY GCY HINSDALE, A.M., M.D.
}

EvikY medical practitioner ought to be acquainted with at least the names and general qualities of the principal American and foreign mineral waters. 'Their use is remarkably large both in Europe and America, and the public are generally convinced of the benefits to be derived from them.

Natural mineral waters have been defined as all waters which, als obtained from nature, are distinguished from ordinary waters either by the salts or gases they contain in solution, or by their temperature being elevated; or by some therapeutic virtue being attributed to them. 'Thermal waters are, therefore, included, as has been the custom for ages, although many of them are of feeble mineralization. A water ought to have at least is grains per gallon of the commoner constituents to be classed as a mineral water; but in case of more potent chemicals, such as arsenic, bromine, lithia, or of salts of iron, smaller quantities contained in solution may fairly place it in this class. We do not recognize as lithia waters, however, those containing less than half a grain per gallon; or as sulphur waters those containing only the faintest trace of hydrogen sulphide. 'The United States Government, thromgh its geologieal survey, is trying to determine the proper classifieation and the real analysis of the waters of the United States which are of commercial importance. Wide discrepancies are commonly found between the advertised and historic analyses and those determined by the (iovernment chemists in their laboratories. In due time we may see American mineral waters properly labelled, just as we are begimning to see Anerican wines, drugs. and foods bearing their correct names and ingredients. France and Germany have adopted stringent laws as to labels of mineral waters, but in the United States manufacturers have, until very recently, put forth artificial waters under stich misleading names as "Reichsquellen," "Imperial spring," "Friedrichsquelle," although manufactured in the United States.

'The chemistry of mineral waters has undergone important changes in the last twenty or thirty years. We formerly understood that definite

1 The author has deseribed the use of mineral springs in his work on "Hydrotherupy," pp. 38s to 420. W. B. Saunders (0., Philadelphia, 1910. For the amaly ses of Ameriean mineral springs the reader is refernel to "Mlineral IIaters of the Inited States," hy J. K. Ihywood and B. H. Smith, Bulletin No. 91, Burean of Chemistry. U. S. Dept. of Agrieulture, Washington, 190;; also "Mineral springs of the l'nievl States and Their Therapentic l'ses," hy d. K. (rook, M.I).: l.ea of Fehiger, Isog, and

"A System of Physiolngieal "Therapeutics," vol. ix, Blakiston, Philadelphia, 192.

2F. Parkes Weber, "Climatotherap! und Balneotherapy," 1. 316. 
quantities of certain salts, like sodium bicarbonate, for example, could be determined, and that they existed as such in a given mineral water. We expected as much of the analysis of a water as we did of a sample of gold or silver ore. But this is evidently not possible and chemists nowadays state in their analyses what they term a "hypothetical combination" of bases and acids. Even this is now termed an antiquated statement of the chemistry of waters. As Haywood and Smith say in their report: "The authors have, much against their inclination, thought best to combine the acids and bases in a hypothetical combination to form salts, since the time does not seem ripe to abandon this antiquated method of reporting the results of water analyses. That such a combination has no basis in fact is doubtless true, since we have every reason to believe that in cases where various acid and basic ions are present in solution no base unites with any one acid to the exclusion of all other acids, or vice versa; but all possible combinations of the various basic and acid ions in solution are formed to some extent." The whole conception and appearance of graphic formulæ are now changed, with the introduction of what are termed "anions" and "kations," in chemical nomenclature.

It is impossible to give full analyses of the various mineral waters referred to in the text; for this the reader is referred to the special works mentioned on the previous page. I shall endeavor, at least, to state the general character of the best-known American and European waters.

Radioactivity of Mineral Waters.-Waters containing a comparatively small amount of mineral constituents have been accorded a therapeutic value which the ordinary chemical analysis fails, as a rule, to explain in any adequate manner. It is possible that the discovery of radioactive properties in some of these waters may afford some explanation of these qualities. A large number of the thermal springs of Europe, such as Baden, near Vienna; Bad Gastein, Joachimsthal, near Prague, and Buxton, England, have been tested in this respect and have been found to contain slightly varying quantities of argon and helium, with distinct radioactive properties; these elements showing a constant association with waters of this class. It is likely that this fact throws some light on one of the problems of the chemistry of mineral waters, namely, why a natural mineral water should have distinctly greater therapeutic power than a water to which is given artificially precisely similar chemical constituents according to a standard analysis.

Undoubtedly mineral waters are best taken at their source. They are more easily assimilated 'when used in their natural state with the gases which accompany them, and it is generally believed that after removal from the spring the salts held in solution undergo some unexplained change which diminishes their efficacy. This is especially true of waters containing iron, sulphur, and of all radioactive waters. Calcareous waters containing iron are also liable to form precipitates of oxide of iron after bottling. 'The alkaline, carbonated, and saline waters are probably best suited for transportation, and those calcareous waters

1 See "The Electrolytic Dissociation Theory," by Profs. H. P. Talbot and A. A. Blanchard, 'The Macmillan Co., 1907. 
devoid of iron salts, which are so abundant in Virginia, West Virginia, and Kentucky.

Another source of trouble is the use of bad corks and the consequent tendency of gases to escape.

Artificial Waters. - Whether artificial mineral waters can in any case equal natural waters is a debatable point. Vichy and Kissingen, artificially prepared from effervescent salts or compressed tablets, as manufactured by leading chemists, probably approach very closely natural waters; while the bottled artificial waters, prepared from distilled water and containing definite quantities of the requisite salts of sodium, potassium, lithium, or magnesium, as the case may be, and bearing the guarantee of reliable firms, are of undoubted therapentic value. ${ }^{2}$

There is a vast trade in artificial waters in siphons, but I do not have much confidence in their methods of manufacture or medicinal value. 'The terms by which they are known-Vichy, Seltzer, or plain soda-are more or less interchangeable. 'They are largely used at bars, in clubs, and to some extent at the table and in the sick room. As a diluent for milk, whisky, brandy, etc., they have some value. 'They are, however, often manufactured under very unhygienic conditions.

Water Drinking.-It seems to be pretty well determined that water is not absorbed by the mucous membrane of the stomach, but by the intestine. As far as the stomach is concerned, the ingestion of water may stimulate or restrict its secretions or may modify its physical state by either undue dilatation or by producing gastroptosis. If one drink a pint of water it is probable that none will be left in the stomach at the end of about three-quarters of an hour, and it has been found that hot water passes out more rapidly than cold; the motility of the stomach is more powerfully affected by hot water. Water actually hastens the digestion of some foods by favoring disintegration, but only when used in moderate quantities.

A healthy man of average size should take of water, independent of that contained in the food, about three to four pints daily. It is found that of this quantity about two-thirds will be exereted by the kidneys and the balance through the lungs and skin. But it is evident that the power behind excretion comes from the heart, which maintains the blood pressure, and hence the greater quantity ingested the greater are the demands on the circulation. If valvular defects are present and uncompensated, or if the arterial system or kidneys are diseased, it follows that great care must be exercised not to throw an unnecessary or dangeous load upon them. 'This is an abuse which is eommonly noted at wateringplaces, and especially among those who take the waters without proper medical advice.

People who travel five hundred or a thousand miles to drink certain waters are naturally disappointed at the restriction of fluid if such a plan

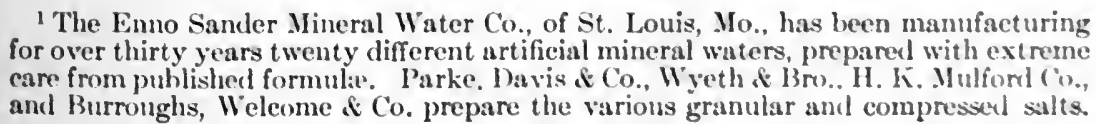


is instituted. As a rule, these patients more readily consent to a restricted diet, to the use of baths, massage, and other physical methorjs of treatment, and a little explanation is needed to convince such persons of the necessities of the case. Aside from diseases of the circulatory system, there are chronic gastric and intestinal affections, as well as undue fatness, in which restriction of fluid is required.

Von Noorden is positive, in reference to obesity, that fluids should be restricted to the point of almost continuous thirst.

P. B. Hawk has shown that copious water drinking causes an increased excretion of nitrogen and phosphorus by the urine. 'The increase in the amount of nitrogen eliminated is due, primarily, to washing out of the tissue, urea previously formed but which has not been removed in the normal processes, and secondarily, to a stimulation of proteid metabolism. 'The increased excretion of phosphorus is due to increased cellular activity and the accompanying catabolism of nucleins, lecithins, and other phosphorus-containing bodies. Experiments in which 4500 c.c of water were administered daily after nitrogen equilibrium had been established showed an increase of 12.8 per cent. in the excretion of nitrogen in the first day and an increase of 6.8 per cent. on the second day. 'The sulphate excretion was parallel with that of nitrogen. 'The phosphate excretion increased from 9.4 per cent. on the first day to 17.1 per cent. on the second day.

Hot Water Drinking.- - Hot water drinking is not so much in vogue as it was ten or fifteen years ago, and this is probably on account of the tendency to undue relaxation of the gastrointestinal tract when continued for long periods. In small quantities, hot water raises the pulse rate and lowers the blood pressure; after twenty minutes, the usual rate and pressure are restored. 'The principles applying to the use of hot or warm water draughts are equally applicable to the weaker natural thermal waters. 'These are indicated in hyperchlorhydria, gastrorrhea, and gastralgia; acute, suppurative, and interstitial nephritis. In the latter affection there are favorable reports from the use of a gallon a day in connection with hot applications to the back and abdomen. Hot water draughts are contraindicated in disease of the heart with failure of compensation. In this class of cases it is best to restrict the total quantity of water consumed to about 1200 c.c., independent of the water contained in the food. In administering water to promote diuresis, it must always be borne in mind that we have to look to the heart for the vis a tergo; while, if there is low pressure in the kidney there can never be satisfactory diuresis.

'Taken at their source the weaker natural thermal waters are usually more acceptable to the stomach, by reason of the quantity of carbonic acid gas commonly present. Artificial heat applied to any water drives off the contained gas, which will, in any event, escape unless very carefully bottled. Thermal springs usually contain carbonic acid gas, hydrogen sulphide, oxygen, nitrogen, argon, and helium, and these gases enter largely into the efficacy of mineral springs.

Thermal springs are found in Virginia, Arkansas, South Dakota, New 
Mexico, Colorado, Arizona, and ('alifornia, and a very large number are found in the Yellowstone National Park. 'l'he latter are not yet used therapeutically, but the day may cone when they will be utilizerl.

In Europe, those of Aix-la-Chapelle, Baden-Baden, Ems, Carlstad, Wiesbaden, Gastein, Aix-les-Bains, and Bath are the most famous.

Cold Water Draughts. - These are called for in febrile states, in hypochlorhydria, and in chronic constipation. 'They may be used morlerately. in chronic nephritis. 'The most favorable time is thirty or forty minutes before meals. Cool water should be used freely before and during the hot full bath, cabinet bath, and 'Turkish bath. Cold water is contraindicated in gastric dilatation, gastroptosis, colic, in all states of fatigue, and should be taken cautiously when there is profuse perspiration. Ice water is particularly dangerous at such times, although the temptation is very great to quench thirst.

The ingestion of cold water reduces the temperature of the borly; it lowers the pulse rate and raises arterial tension. A pint of water at $\dot{S}^{\circ} \mathrm{C}$. $\left(46.4^{\circ} \mathrm{F}\right.$.) taken into the stomach may lower the rectal temperature nearly $2^{\circ} \mathrm{F}$. and reduce the frequency of the pulse twenty to thirty beats within a few minutes. It is possible, on the other hand, to affect the temperature in the deep abdominal organs by rectal enemata of cold water.

In most cases the free use of water during meals is not arlvisable. It is believed to delay the digestion of starchy and other foods. Individuals, however, vary greatly in this respect, and many are accustomed to drink three or four glasses of water during meals with entire impunity. I believe that a moderate amount of cool water-one glassful-is not, as a rule, injurious. Patients who must avoid starchy foods, and who eat freely of meat, are no doubt benefited by the free use of pure water before and after meals. Most peopledrink too little, and this is particularly the case with women, leading to constipation, acid, dense urine, and occasionally to an irritability of the bladder accompanying urine of high specific gravity.

It is sometimes desirable to administer cold carbonated waters with as much of the gas present as possible. If they are artificially heated before drinking the gas inevitably escapes. 'To avoid chilling the stomach by the use of cold waters and yet retain the gas, they may be taken through a glass tube. At Spa, in Belgium, this plan is followed in using the Prince de Conde Spring. It is a chalybeate water, and the use of the glass tubes is not on account of the presence of iron, but to insure the slow absorption of the water without chilling the stomach. 'This practice might well be employed at some of our springs, where patients are inclinel to drain the glass in the shortest possible time.

It would seem almost unnecessary to warn against the use, o: misuse, of great quantities of water. 'Ihe owners of one Saratogra Spring, in their latest circular, publish the letter of a patron, who writes that on his first day, before retiring, he "had guzzled nineteen glasses, and felt first-class after its use. The next day I took twenty-one glasses and felt better. 'l'his was no attempt to see how much I could drink, but merely. to quench a raging thirst." I trust that when the State of New York 
assumes possession of the Saratoga Springs some rational use of these waters will be insured; and from present indications that day is not far distant.

Distilled Water.-'This is a chemically pure water made palatable by the addition of oxygen, with or without carbonic acid gas. Much discussion has arisen as to the propriety of using a water without any solid constituents, the claim having been made that its use tends to abstract valuable salts from the system. These objections are more theoretical than practical, the rather extensive use of distilled water on shipboard and elsewhere not having been attended by any bad results. Oxygenated and carbonated distilled water is widely sold under various trade names in siphons. It is much more palatable than the distilled water of the chemical laboratory, and more economical. Distilled water can be easily and cheaply produced for household use by means of a still, which is heated by gas or oil. A satisfactory apparatus is the Parmelee Automatic Aërating Water Still and Sterilizer. If oil is used to heat it, a blue flame Primus or Khotal oil stove should be employed.

Distilled water is no doubt an ideal drinking water, far preferable to the mineralized waters for constant use. In regions where limestone formations and alkaline deposits characterize the soil the use of distilled water would prevent the disturbances of the stomach and bowels commonly observed.

The purer the water the greater is the capacity for the solution and the elimination of the unnecessary salts produced in the process of digestion and assimilation. The food should provide all the necessary salts in abundance, and hence distilled water or the chemically indifferent waters are best for constant use. I do not wish to be construed as depreciating the value of the more highly mineralized waters; they are undoubtedly valuable for purgative and other purposes and favor elimination through the bowels or kidneys. They have their place for occasional use. Other individuals seem to require the constant use of an alkaline water, but the average man is better off on the purest water he can find.

The following are some of the American waters containing a minimum of salts:

Maine-Poland Springs; Highland Spring; Mount Hartford Spring; Indian Hermit Spring; Ogunquit; Pownal Spring.

Vermont-Equinox Spring.

Massachusetts-Commonwealth Mineral Spring; Massasoit Spring; Nobscot Mineral Spring; Ballardvale; Sheep Rock.

Connecticut-Stark Mineral Spring.

New York-Colonial Springs, L. I.; Great Bear Water; Sun Ray Spring.

Pennsylvania-Glen Summit Water, Roscommon Spring.

Maryland-Chattalanee Spring; Bentley Springs.

The Use of Water in Obesity.-Authorities differ greatly in this regard. Mr. Banting, who reduced himself in a year from 202 pounds to 156 , limited the total amount of fluid to 35 ounces. Oertel allows 8 to 16 ounces of water, 12 ounces of wine, and 2 cups of coffee, tea, or 
milk, daily. In hot weather the amount of fluid may be slightly increased and should be taken in small portions through the day. Schweninger suppresses all beverages at meals and allows no fluid until two hours have elapsed since food. Germain See, on the other hand, allow's large quantities of warm aromatic drinks. No doubt this is the best course in fat and gonty people, and such patients may be allowed half a pint of water an hour after each meal and at bedtime. Where physical exercise is recuired, and especially when depleting baths are used, water should be allowed. Even during meals some water should be taken, to aid in the solution and digestion of food.

In treating poorly nourished persons, water may be added to milk in the proportion of two tablespoonfuls of hot water to a glass of milk, to which is added 5 grains each of common salt and sodium bicarbonate. In this way the digestibility of the milk is favored.

Bronchitis.-In all cases of bronchitis with hard and ineffective cough, water generally gives relief. Warm drinks before retiring will frequently allay cough, and, as every mother knows, a drink of water will of ten stop a persistent cough in children after settling for the night. Cold water in quantities, from 4 to 6 ounces, should be given to children with measles or bronchopneumonia, and repeated every two or three hours.

Nephritis.-It is generally held that the free use of water internally is beneficial in nephritis. Certainly a glassful taken night and morning on an empty stomach will act as an eliminant. It does not follow, however, that where much is good more is better. It is quite possible to flood the heart and arteries with water and seriously overtax them. Ascites is a very evident contraindication. 'The underlying motive in giving water and milk extensively is to "flush the kidneys" and get rid of toxins or other irritants. I believe that unless there is also serious cardiac disease, with failure of compensation, it is a proper course. Von Noorden, however, in his monograph on nephritis, takes the ground that, as water is always very badly excreted by the kidneys when they are acutely diseased, and frequently also in subacute and subchronic forms of nephritis, the extensive use of milk and water is not logical. "A condition of hydremia must be regarded as a constant source of irritation of the kidneys, and the problem that confronts us is to prevent excessive stimulation of these organs as much as possible." Unless the heart is seriously at fault, I believe in giving water freely in nephritis, especially the lightly mineralized waters, skimmed milk, ginger ale, etc. People vary, but water drinking is certainly in most cases a physiological, hygienic, and remedial measure of value.

Typhoid Fever.-In typhoid fever, cool water should be administered in small quantities at frequent and definite intervals. It may be possible in this way to give as much as two or three quarts in twenty-four hours. 'The amount of urine is increased, the nerrous symptoms a re lessened, and patients are more comfortable. 'The intermal use of cold water is a good adjuvant to the cold bath, and it is believed to lessen the mortality from the disease. 'The method adopted by E. F. Cushing and 'T. II. Clarke 
in 100 cases treated by this method was to give not less than three pints daily. It was found that the amount of water which patients would take could be greatly increased. 'The quantity of ten reached a gallon or a gallon and a half. 'This was accomplished by giving 4 ounces of water every fifteen minutes during the waking hours. In addition, the patient received every two hours during the day and once or twice at night, alternately, 6 ounces of milk and 6 ounces of albumen water, representing some three pints of fluid. Besides all this, a bowlful of ice water was kept by the bedside, so that the patient could help himself. Occasionally a patient rebelled at first, but most of them took the water readily, and some greedily. 'The natural result was, first, a great increase in urine, which rose as high as eight to twelve pints, and was maintained at this standard during the febrile part of the disease. Fewer baths were required and headaches were not so troublesome; the mouth was noticeably clean and moist, and the nervous and toxenic symptoms less. 'There were few complications, and the mortality, as well as the severity of typhoid fever, seems to be lessened by this free use of water internally.

Alkaline Waters.-'These are classed according to their predominant bases, as sodic, sodic-magnesic, alkaline chalybeate, alkaline saline, calcic, or sodic calcic. With all their variations they embrace a very large number all over the United States and Europe. 'The milder waters, carbonated, are used largely for the table, for their antacid properties, and in the treatment of gout, rheumatism, diabetes, renal disease, bladder affections, and diseases of the liver and stomach. It would be impossible within the limits of this article to classify or even mention them here except in a general way. The names of the more prominent ones are household words-Apollinaris, Vichy, White Rock, Manitou, Perrier, Carlsbad, Giesshubler, Fachingen, Evian, Biliner, Allouez Magnesia, Bedford, Bethesda, Saratoga Vichy, Rhens, Vals, Selters, and Kaiser Brunnen.

Carbonated or Bicarbonated Alkaline Waters.-These depend for their action on carbonates or bicarbonates of sodium or potassium, or both together, with more or less carbonic acid gas. Other bases, such as calcium and magnesium, and small quantities of lithium or iron, are usually found in these waters. When large quantities of carbonic acid are present the salts retain their form of bicarbonates, and for this reason special care is taken to retain the original gas or add to it in the process of bottling. On testing at the spring with litmus these waters give an acid reaction, owing to the gas present, but the salts are alkaline and their action on the system conforms to that of alkalies. Next to pure water they constitute the most important and most useful class of waters used medicinally. 'Their chief use is to stimulate the gastric secretion, correct undue acidity of the stomach or urinary secretions, and promote diuresis. 'The milder alkaline waters are widely used at the table and have come into general use to mix with alcoholic drinks or as a substitute for them. 'They are of great value in catarrhal conditions of the mucous membranes, in rheumatism, gout, diabetes, and hyperchlorhydria. In cases of gastric ulcer, carbonated waters should be avoided, as there is 
too great a risk of hemorrlauge from any sudelen dilatation of the stomalds: nor should any very cold water be given in this condition.

It has been shown by Pawlow that sodium bicarbonate inhibits the secretions of the stomach and pancereas; its use tends to break the continuity of their secretion, and during the panses of secretory activity an opportunity is afforded for disease of these organs to abate. Hence it is that alkaline waters of the type of Carlsbad, Vichy, and Manitou afford relief in gastritis, in which the mucous nembrane is actively inflamed, or in hyperchlorhydria, in which the membrane is irritable. It is probable that the use of natural waters gives better results than the medicinal use of their principal salts as drugs, by reason of the dissociation of the positive and negative ions, now recognized in mineral waters. 'This capacity for dissociation, according to von Aldor, of Berlin, is a great factor in their pliysiologieal action. 'The more diluted a salt solution the higher the ionization; the more concentrated it is, the more the neutral molecules remain near the ions.

'The alkaline waters may be taken before meals or after them, or may be used with the meals, according to circumstances. 'Two glasses shomld be the maximum taken at any one time.

Lithia Waters. - 'This group of mineral waters came into use a little over forty years ago, acquiring popularity because of their asserted value in removing uric acid and urates from the system. 'They were strongly advocated by the late Dr. Garrod, who made experiments on the solubility of uric acid in solutions of lithium carbonate, and claimed that the administration of this substance would prevent the separation of uric acid and urates in the urine, since lithium urate is rather more soluble in water than the corresponding salts of potassium and sodium.' Iefinann, however, in a recent review of this subject points out that a simple association of lithium carbonate solution and uric acid in a test-tube is not at all comparable with the actual condition in which other salts, especially carbonates and phosphates, are present. Although lithium urate is the most soluble of the common urates, lithium carbonate and phosphate are decidedly less soluble than the corresponding sodium or potassium salts. 'lo charge the urine with much lithium is, therefore, to predispose to insoluble phosphatic deposits and the substitution of such a deposit for urate is no therapeutic gain. Accustomed as we have been to hold lithia waters in high estimation for all urinary and bladder diseases, the recent critieism of the use of these waters, and, what is of great importance, the recent analyses of the so-called lithia waters, onght to lead us to examine this subject more thoroughly. 'To begin with, the chemical estimation of lithium in a given water is a difficult problem; the spectroscopic test for the presence of this element is correspondingly easy and delicate, as it reveals an almost infinitesimal quantity.

The analyses of Waller and of I effimam were the first to open the

'See "Iithin Waters as Therapentic Agents," by Henry Leffmann, M.I)., 1'uthelogical Chemist to the Jefferson Medical College Hospital, Philadelphis, Monthly Cyclopedia of Practical Medicine, March. 1910. The anthor acknowinlges his indebtedness to 1)r. I.effmann for data on this suljjeet. 
eves of the medical profession to the fact that the popular lithia waters have been exploited under false statements as to their lithium content. These tests have been fully corroborated by the recent analyses in the United States Government laboratories by Haywood and Smith. Their report gives the analysis of: (1) Geneva Lithia Water, New York; (2) Jeffress Lithia Water, Virginia; (3) Otterburn Lithia Water, Virginia; (4) Buffalo Lithia Water, Virginia; (5) Golindo Lithia Water, Virginia; (6) Londonderry Lithia Water, New Hampshire; (7) White Rock Lithia Water, Wisconsin; (8) Crockett Arsenic Lithia Water, Virginia; (9) Augusta White Lithia Water, Virginia; (10) Bear Lithia Water, Virginia; (11) Rubino Healing Springs Water, Virginia. The exact method of determining the amount of lithium present is stated in the report, and is known as that of Gooch.

The Government analyses show enormous differences from those advertised, and the summaries contain the following: (1) "Although the water is advertised as containing a very large amount of lithium, 23.8 parts per million, it in reality contains only 0.1 part. It can, therefore, hardly be considered as a lithia water, as it would take about 300 liters of this water to give one full medicinal dose of lithium." (2) "It is, however, a misnomer to call this a lithia water." (3) "However, it is a misnomer to call this a lithia water, as it would take approximately 1000 liters to give a full medicinal dose of lithium." (4) "It is a misnomer to term such a water a lithia water." (5) "The spring hardly contains enough lithium to be called a lithia water." (6) "The advertised analysis giving 10.3 parts per million, while the Bureau of Chemistry shows only a spectroscopic trace." (7) "For this element the advertised analysis claims 38.1 parts per million, while the Bureau of Chemistry results show 12.6 parts. However, with the amount present as found by the Bureau of Chemistry, the water may be considered fairly rich in this element, as it amounts to 1.05 per cent. of the total salts present." (8) "It is a misnomer to term such a water as this a lithia water." (9 and 10) "It is a misnomer to call such a water as this a lithia water." (10) "The Bureau analysis shows only a spectroscopic trace of lithium, while the advertised analysis gives 0.91 part per million."

I believe that too much confidence has been reposed in the so-called lithia waters. We have been badly imposed upon, no doubt, and have attributed much greater value to lithia as a constituent than the facts warrant. We shall probably in the future hear less about lithia as a characteristic feature of mineral waters. Undoubtedly the formulæ of these waters have in many cases been constructed to suit the commercial demand.

The alkaline-calcareous or earthy mineral waters, containing calcium bicarbonate or sulphate and magnesium bicarbonate, are valuable for their antacid and diuretic properties. As a rule, they are well borne, counteracting hyperacidity of the stomach and urinary tract. For this reason they are largely used for the treatment of gout and rheumatism, and, on account of their astringent effect, aid in the treatment of diarrhea and an irritable mucous membrane. Most of these waters in the natural state contain considerable quantities of carbonic acid gas, and, therefore, are 
best used at their sonrce. Calcium salts are eagerly sought by the arcids arising from imperfect digestion. These acids readily combine with the bases furnished in mineral waters, and probably in this manner obviate union with similar bases found in bone, cartilage of joints, and in other natural tissues of the borly. A proper selection of food, such as vegetables rich in salts, accomplishes a similar purpose, and this diet goes hand-in-hand with the use of mineral waters in the treatment of these affections. Calcareous mineral waters favorably influence rheumatism of a subacute or chronic type, gout, gravel, pyelitis from kidney concretion, chronic gonorrhea, bronchial catarrh, with profuse secretion, and allied affections. 'They are credited with favorable results in scrofulosis, rachitis, and osteomalacia. Waters of this class are commonly termed "hard" waters, and by reason of the calcium sulphate or biearbonate present are not generally suited for constant use when more than 15 grains of calcium salts are present in a gallon. 'The calcium sulphate is the more harmful constituent, and dyspepsia, a feeling of heaviness or a tendency to dream or a derangement of the bowels may occur from their prolonged use by susceptible persons. Some of these waters are quite astringent and may cause constipation. 'They have also been credited with the development of goitre in localities where this affection is commonly met with.

In Europe, and especially at Wildungen, in Germany, these waters are believed to relieve affections of the urinary tract, such as chronic cystitis, pyelitis, enlarged prostate and its results, chronic gonorrhea, gleet, urethral stricture, and irritable bladder. The principal springs at Wildungen are the Helenenquelle and the Georg-V ictorquelle. 'The former, owing to its alkalinity, is preferable in cases of irritable bladder, with highly acid urine. It is better borne by the stomach and is less liable to constipate. 'The Georg-Victorquelle is used when there is much vesical catarrh, with alkalinity of the urine, or when there is phosphaturia without mucus or mucopus.

Contrexéville waters belong to the same class, but have a somewhat different effect in that they are more diuretic and have a slightly laxative action. 'They are used for uric-acid gravel, gouty oxaluria, and chronic cystitis; for gouty glycosuria or gouty conditions in weak subjects. 'These waters are claimed to correct nocturnal eneuresis in children, by removing some source of irritation. 'They are unsuited for cases in which there is extreme loss of vesical contractile power or great liability of prostatic congestion (Weber). Contrexéville water is widely sold in the United States.

'The best known American water of this class is that of Healing Springs, Virginia. It has enjoyed a favorable reputation for over one hundred years. It keeps well when bottled and is extensively userl at the Virginia Hot Springs. It contains 20.7 grains of calcium carbonate, 3 grains of magnesium carbonate, and 5.7 grains of magnesium sulphate per gallon. There is no sodium ehloride present. 'The analysis by 1)r. $\mathrm{H}$. Froehling, of Richmond, shows that it is apparently free from calcium sulphate, and in this respect it is different from the Bedford Chalybeate Spring, Pennsylvania, containing 107 grains of ealcium sulphate; the Greenbrier White Sulphur Spring, West Virginia, is grains of calcium 
sulphate; Clifton Springs, New York, 69 grains of calcium sulphate; and Richfield Springs, New York, 112 grains of calcium sulphate per gallon.

Other calcareous or earthy mineral springs in the United States are: Napa Soda Spring, California (10 gr. $\mathrm{CaCO}_{3}$ ), and Manitou Springs, Colorado ( 65 gr. calcium carbonate per gallon). 'The most extensively used thermal calcareous waters are at Hot Springs, Arkansas ( 7 gr. calcium carbonate).

In Europe cold earthy waters of wide reputation and popularity are found at Marienbad, in Bohemia; Contrexéville, in France; Wildungen, in Germany; Leukerbad, in Switzerland, and Bath, in England. Of this class the waters of Contrexéville are best known, and, as already stated, are extensively used in the United States. The waters of Kreuznach, Germany, are of this class, containing chlorine, iodine, and bromine in combination with the earthy bases, and are used internally for tertiary syphilides and strumous diseases.

Saline Waters.-These are characterized by predominance of sodium chloride, but a large number of other salts, such as calcium chloride, magnesium chloride, and sodium sulphate, or bicarbonate, may be associated. 'The waters are generally cold and well charged with carbonic acid gas, as at Saratoga Springs, Kreuznach, Homburg, and Kissingen, and SalsoMaggiore; or they may be hot, as at Wiesbaden, Baden-Baden, and Glenwood Springs, in Colorado.

Saline waters are classified in general as sulphated, muriated, or borated; and the two former again as chalybeate sulphated, sulphated and muriated, chalybeate muriated. 'To designate the predominate hases we have: sodic, magnesic, calcic, or sodic magnesic, calcic magnesic, calcic sodic, calcic chalybeate, or calcic-magnesic alumino-chalybeate, as the case may be. 'This form of basic and acid, or kationic and anionic, terminology is at once descriptive and rational. They are purgative, and exert, secondarily, a good effect on the liver and gastrointestinal functions. Their reputation having rested for years on a purely empiric foundation, it is highly gratifying to note the recent attempt to determine the true physiological action of these waters. We are indebted to von Noorden, of Frankfort, and Carl Dapper, of Kissingen, for the best exposition of the effects of saline waters on metabolism. 'Their observations were made on persons undergoing a drinking cure for various affections, comprising anacidity, subacidity, and hyperacidity of the gastric juice; chronic intestinal catarrh, gastric ulcer, obesity, diabetes mellitus, and the effects of alcohol. A curious observation was made, viz., that in numerous cases of gastric disorder. particularly in gastric catarrh, the use of saline mineral waters leads to an active and permanent increase in the production of $\mathrm{HCl}$. On the other hand, in cases accompanied by hyperacidity, particularly in nerrous dyspepsia, the moderate use of saline mineral waters learls to a decrease of the $\mathrm{HCl}$ production and a decrease of the subjective simptoms. That a given remedy should work a cure in cases of a diametrically opposite nature is one of the paradoxes of medicine. But the high standing of these climicians and the great care shown in minute examinations made of the gastric secretion 
while the patients were under observation leave no doubt that the (on)clusions ale correct.

Another important point brought out is that the adninistration of saline mineral waters does not call for any particular diet. Arlitrary and routine dietetic regulations are in vogne at many health resorts. 'There is a popular prejudice among many phrsicians, and it is shared by the general public, that each particular saline water calls for the exclusion of certain definite articles of fool from the diet, regardless of the disease. In other words, that each water calls for a definite diet. 'The governing prineiple should be that the diet should be adapted to the partieular needs of the patient and not be given in a routine way according to the water which is prescribed. Probably this antiquated method is more commonly employed in Europe than in America, although this error is not confined to European resorts. That the use of fats should be forbidden while taking saline waters is one of the fallacies which these anthors quoted have pointed out, and they make the valuable practical point that many patients need the fats of a normal diet to maintain their nutrition and strength. Undoubtedly there are patients with gastric and intestinal trouble, with diabetes or gout, many neurasthenies and convalescents from various diseases, whose needs call for fats and who are perfectly able to digest and assimilate them. That they are assimilated the observations and records of von Noorden plainly show.

'There need be no interdiction of raw fruit while using saline minoral waters unless the individual case demands it. 'The drinking of saline waters, or of any other water, per se, does not call for the restriction of any particular article of food.

Saline waters are generally taken, according to their density, in quantities of one to four glasses before breakfast, either hot or cold. When taken cold they generally act more promptly. 'They should be taken slow!y' or sipped, and, if possible, a morlerate amount of exercise should be taken between each glass, and a half hour should elapse between the last glass and breakfast, which should be a light one. 'The American tendency to haste usually results in taking the entire quantity at once and going immediately to breakfast; this, however, is not the best way to take laxative or any other waters.

'The use of saline waters does not affect the proteid metabolism, and hence they are suitable in eases of overfatness, in which we never want to diminish the proteid content of the bods. Several investigators have published the results of experiments on animals and in healthy persons, showing a slight increase in the excretion of urea after the ingestion of sodium chloride, but I believe that von Noorden's conclusion is correct, that proteid metabolism is not appreciably affected.

Regarding the excretion of uric aeid the conclusion is that in cases of gout the use of saline waters (c. g., Kissingen, Rakocy) causes an inerease, amounting occasionally to as much as 0.2 gram a day, although in exceptional eases no change ean be noted. I shall refer later on 10 the applicability of the saline waters to the treatment of gout.

The most famous saline springs of Europe ure those of Honsburg 
(Elizabeth-Brunnen and Kaiser-Brunnen), Kreuznach (Elizabethquelle), Nauheim (Kur-Brunnen and Karls-Brunnen), Kissingen (Rakoczy and Bitter Water), Friedrichshall, Baden-Baden, Wiesbaden (Koch-Brunnen), Soden (Warm-Brunnen), all in Germany. Hall and Salzburg, in Austria; Cheltenham and Leamington, in England.

In America the most noted saline springs are those of Saratoga; they have been in use for over one hundred years. The Congress Spring was discovered in 1792, and since then forty additional springs have been discovered. The Congress Spring contains over 600 grains of mineral salts per gallon, of which two-thirds is sodium chloride. The Hathorn Spring, near by, is of similar character. The waters from High Rock, Arondack, Star, Lincoln, Patterson, Champion, and other springs of Saratoga are all widely sold. The Saratoga Vichy is somewhat similar to the French water of that name. They are all highly carbonated. In recent years numerous borings have been made in Saratoga for the carbonic acid gas which exists in great quantity in the deeper strata. These borings have diminished greatly the pressure of gas in various springs, and much opposition has developed against the companies which compress this gas and ship it for commercial purposes. The State of New York has recently appropriated $\$ 1,000,000$ toward acquiring the Saratoga Springs, and, with further appropriations, it is hoped that these springs will be converted into the New York State Mineral Spring Reservation, and freed from the objectionable features of the past.

Other valuable saline springs in the United States are those of Ballston Spa and Glen Springs, New York; Mt. Clemens, Michigan; Waukesha, Wisconsin; Blue Lick, Kentucky; Eureka and Byron Springs, California; and numerous salt wells in Texas. In Canada there are: Caledonia Springs, St. Catherine's Well, and Sandwich Springs, all in Ontario, and all having rather more than local reputation.

Sodic-potassic-magnesic Sulphated Muriated Saline Waters.-These are commonly termed "bitter waters," and are valuable for their cathartic properties. 'The best examples are those found in Austria-Hungary, Bohemia, and Spain. The only well-known American water belonging to the sodic sulphated class is Abilena, from Kansas. This contains about 3200 grains of sodium sulphate per gallon.

Names of springs.

Abilena, Kansas

Apenta, Buda Pest, Hungary

Carabaña, Spain

Esculap, Hungary

Cheltenham, England .

Elster, (Salzquelle)

Franzensbad (Salzquelle).

Franz-Josef, Hungary

Pïllna, Bohemia

Rubinat, Condal, Spain

Villacabras, Spain

Hunjadi-Janos, Hungary .

Crab Orchard Spring, Kentucky

Vietoria, Buda Pest

Castalian Mineral Springs, California
Grains per gallon. Magnesium Sodium sulphate. $\begin{gathered}\text { Sulphate. } \\ \text { sulphats }\end{gathered}$

$\begin{array}{rr}3200 & \\ 1300 & 1420 \\ 7000 & 280 \\ 973 & 1204 \\ 154 & 49 \\ 364 & \\ 196 & \\ 1680 & 1722 \\ 665 & 756 \\ 3122 & 210 \\ 8540 & 63 \\ 1211 & 1169 \\ 70 & 245 \\ 2345 & 1693 \\ 779 & \end{array}$


'These waters stimulate the stomach and intestine, thereby promoting peristalsis and causing purgation. 'They promote the flow of bile, and are largely used in America and Europe for their cathartic qualitios. It is claimed that when the sulphates of sodium and magnesium are associated in nearly equal proportions the taste is not so objectionable as when one or the other preponderates; the advocates of a pure soxtium sulphate water claim, on the other hand, that it acts better as at cholagogne, increasing the production of sodium glycocholate and sodium taurocholate; and that magnesium sulphate has no cholagogue action whatever. Such statements, however, need confirmation. 'The dose of these waters may be regulated by observing the proportion of total saline contents and the needs and susceptibility of the individual.

'The strongest purgative water known is the Victoria, of Buda Pest. It contains a large proportion of magnesium sulphate and is a particularly disagreeable water. The linanz-Joseph Spring is a strong aperient, containing nearly equal parts of the sulphate of soda and magnesia. 'The dose of these waters is a small wineglassful, or more; they should he diluted for use with warm water, and taken in the morning on rising. Free purgation is obviotsly a great help in promoting a proper function of the stomach and the skin and aids the action of drugs.

Sulphuretted Waters.- These are characterized by the presence of hydrogen sulphide gas or the sulphide of sodium, potassium calcium, or magnesium, in conneetion with other salts, such as the chlorides of sodium and potassium, or the earthy salts. Unless waters eontain at least five cubie inches of hỵdrogen sulphide per gallon, or is grains of sulphides per gallon, we do not lay much stress on the sulphur as a potent factor. Waters of weaker composition are very numerous, and, though bearing the title of sulphur springs, are useful rather by reason of their thermal qualities or through other constituents, usually alkaline-calic or saline. 'The advantages of these salts have been discussed (see pp. 207 and 209 ).

When well eharged with sulphur they are useful in diseases of the liver and spleen, and probably also in gout, rheumatism, in skin diseases. such as acne, and in serofulous eruptions.

A mild antiseptie action may attend the internal use of the stronger sulphur waters in skin diseases; in diseases of the liver it is probable that improvement is due to some influence on the secretion of bile. Sulphur waters seem to increase this, and are believed to materially reduce an enlarged liver. On the other hand, there is reason to believe that the hemoglobin value of the red blood corpuscles is reduced, since a prolonged course of the internal use of sulphur water tends to induce an anemic condition, which, however, is of short duration. It is, of course, diflieult to determine the effect of the sulphuretted hydrogen apart from the sulphide or the saline or calcareous constituents of a given water, or to disassociate the infuence of diet, elimate, regime, and other features of mineral-water treatment at spas. 'These waters are generally tal en at spas for the reason that, as a rule, they do not keep well when bottlet. With great eare it is possible to bottle some of these waters. 'Those of Richfield Springs and Sharon Springs, New lork, and those of the Gieen-

Vor. 1. -11 
brier White Sulphur Springs, in West Virginia, are bottled and sold to some extent. 'The slightest exposure to the air sets up chemical changes which alter greatly the constituent gases and salts. As Peale says, the soluble sulphides are rather unstable and easily decomposed. They are rarely fully determined in mineral-water analyses, because a part of the necessary work of analysis must be done at the spring. 'The majority of water analyses are confessedly incomplete so far as the estimation of the gases is concerned. The chemistry of these waters before consumption and after ingestion is most complex and their physiological action is more or less in dispute, so that there is a good deal of skepticism as to whether there is any real value in their administration, as far as the sulphur content is concerned.

Iron or Chalybeate Waters; Steel Waters.-There are no absolutely pure chalybeate waters, but the iron exists in connection with carbonates, sulphates, chlorides, and with other bases, such as calcium, magnesium, and aluminum. They are thermal or non-thermal and are associated with carbonic acid and hydrogen sulphide. A water containing bicarbonate of iron is usually preferable, for continued use as a tonic, to one holding sulphate of iron, as the latter is more astringent. Free sulphuric acid and alum salts render some iron springs unfit for use; but where these constituents are in moderate quantity, and may be taken at their source, the effect is of ten beneficial. A relaxed mucous membrane with a tendency to diarrhea and anemia may be treated successfully with a water of this type. Good examples are Rockbridge Alum Water, Virginia, or the Oak Orchard Springs, New York.

For continued use in anemia and chlorosis we naturally choose a water with bicarbonate of iron from a cold spring with an abundance of carbonic acid gas, such as those of Spa, in Belgium; Schwalbach, in Germany; and St. Moritz, in Switzerland. These waters contain, respectively, in each gallon 6.4 grains, 5.6 grains, and 2.8 grains of bicarbonate of iron. They are agreeable to take, by reason of the large amount of free carbonic acid gas present.

English chalybeate spas include 'Tunbridge Wells, in Kent, and Flitwick Wells, in Bedfordshire. 'The Flitwick water contains 170 grains of sulphate of iron to the gallon, and is bottled for sale. The waters of Harrogate and Buxton also contain iron; the so-called "Kissingen" well, one of the eighty springs of Harrogate, contains about 10 grains of bicarbonate of iron per gallon; while the "Chloride of Iron Well" contains 13.3 grains of chloride of iron and 11.2 grains of carbonate of iron per gallon. The waters from these chalybeate springs of Harrogate are bottled after being artificially charged with carbonic acid gas. At Harrogate they are generally taken after being heated.

Waters containing bicarbonate of iron require great care in bottling, as most of the iron is liable to be deposited on the sides of the bottle in the form of an insoluble oxide. To prevent this the water must be sterilized, to prevent the growth of any microbes; they must be thoroughly charged with carbonic acid gas and be very carefully closed. Chalybeate waters always give the best results when taken directly from the spring. One 
reason why the iron waters do not act so well after bottling is that if much lime is present, as is frepuently the ase, the iron precopitates.

'The waters of Srhwalbach, on that account, keep better than those of Pyrmont, which contain lime in abundance. 'Those from the P'rince de Conde Spa keep best of all, and may be given in quantities from one to four or five tumblers a daỵ. 'They aid digestion and improve the qualities of the blood.

Iron waters are best taken after meals, in quantities varving from 4 to 8 ounces. 'The use of 8 ounces may produce headache in susceptible patients. It is best to order a saline water before breakfast when giving iron waters.

'The waters of Franzensbad, Marienbad, and Kissingen (Rakocyy) all contain some bicarbonate of iron, and are bottled for use. Iron springs are rather common in the United States; the principal ones are as follows:

('resson Springs, Pennsyl vania (iron spring)

Iron salts; crains in one gallon.

Cresson springs, Pennsylvania (alum spring)

28.5

44.6

11.5

14.2

liock Enon Springs, Virginia

3.1

Massanetta Springs, Virginia

7.9

28.0

'Topeka Mineral Well, Kansas .

1050.0

Chalybeate waters are useful in anemia, general debility, and scrofula. The more astringent waters may be chosen in cases of chronic diarrhea and in malarial cachexia. Iron waters are generally deemed unsuitable in any case of fever, in gastric irritability, and in pulmonary disease if hemorrhage is a feature. Like most mineral waters they are diuretic. If associated with a little arsenic or manganese their value is probably enhanced.

Arsenic Waters. - Waters of this class are found in the United States and Europe, the best known being as follows:

Grains of arsenic
per gallon.
0.07
0.6
7.140

Ia Bourboulc and Mont Dore, Puy de Döme, France, Thermal

I.evieo, Austrian Tyrol

Roncegno, Austrinn Tyrol

0.140

Arsenic oceurs in these waters as arsenic acid or arsenous salts. $\mathrm{Ib}$ sorption through the stomach is easy, but in the administration of the stronger waters, such as Ievico or Roncegno, dilution is necessary. 'The Ievico water is in two grades-strong and weak-and for export these are mixed and bottled. At the commencement of a course of this water a tablespoonful well diluted may be given two or three times a day, during or after meals. 'This quantity may be gives later three times a day or slightly increased.. 'The therapentic indications are those usual for arsenic, namely, in anemia, malarial cachexia, serofula, and skin affections. 
Iodobromic Waters.-Sodium bromide and sodium iodide are sometimes found in waters containing sodium chloride; but as those salts are subordinate to the great excess of the latter salt, waters of this class shonld not be used internally with a view to bromine or iodine medication. 'Their presence should not be ignored, however, when such waters as those of Saratoga, the Bowden Lithia, etc., are prescribed.

The chief European waters of this class are those of Kreuznach, Burton, and Woodhall; the last is the strongest known, containing nearly 5 grains of bromine and two-thirds of a grain of iodine to the gallon. 'The Saratoga Springs, such as Congress and Hathorn, Eureka, Excelsior, Champion, Geyser, and Lincoln, belong to this class; the Ypsilanti Mineral Well, the Port Huron Mineral Bath, of Michigan, and about sixty others in the United States.

'The bromo-iodine waters, with small saline content, are of value in the treatment of scrofula, syphilis, goitre, and diseases of the skin, but are contraindicated in chronic inflammatory states of the digestive organs.

Gout and Chronic Rheumatism.-The use of water internally in gout has many adrocates, and in England some rather distinguished opponents. I believe that pure water should be used freely, and better hot than cold. It certainly aids elimination of waste matter, and when taken hot stimulates the liver cells and favors biliary excretion. Undoubtedly the free and abundant use of water prevents gout, by temporarily diluting the blood, preventing precipitation of uratic salts about the joints and fibrous tissues.

It is well known that in many cases of gout the action of the kidneys is defective. 'The gonty kidney must be treated in much the same way as we would treat a nephritis of the interstitial type; and in choosing a water for this purpose we should avoid, as nearly as possible, those containing sodimm chloride.

We now know that renal elimination is impeded by the use of sodium chloride in the food and drink, and hence it is illogical to impose upon the kidneys any additional burden. Widal, in France, and Leonard Williams, in England, have presented this subject in a convincing manner, but the medical profession is still careless in applying these principles to the use of mineral waters in gouty and renal affections. Almost all mineral waters, irrespective of their contents, are advocated by their owners for use in gout, rheumatism, and Bright's disease, and physicians do not discriminate sufficiently in their choice, being content too often to follow the dictates of the label and the advertising matter. When saline waters are given to gouty patients in an active dose the hope and belief is that the excess of sodium chloride will be eliminated through the bowels. We shall see later on, in considering chronic constipation, that this opinion is not altogether tenable.

The suggestion has been made that salt in the food behaves in a manner different from that of salt contained in mineral waters; and that sodium chloride retention is much less liable to occur from the use of saline waters; these are rather beneficial than otherwise when of the hypotonic type with dissociation of the ions. We know that when a relatively 
minute quantity of sodium chloride is dissolved in a large quantity of water it undergoes dissociation into its $\mathrm{Na}$ and ( 1 ions; but when the anount is large this dissoriation is largcly wanting. If it is true, as has been claimed recently by Ackerley, that the weaker saline waters are beneficial in gout, notwithstandiug that the ingestion of salt-rontaining food is injurious, we may find a clue to the explanation of these apparently contradictory propositions in what I have said above about the principle of jonization.

'To take salt with food and to take the same amount of salt in mineral water, or in plain water, on an empty stomach involve ynite different phrsiological conditions. Ackerley has shown that even though there is a large excretion of sodium chloride after a meal containing it, the salt taken with food is retained longer in the body and excreted nore slowly than the same quantity of the single salt dissolved in water and taken on an empty stomach; also that 5 grams of sodium chloride taken on an empty stomach in from eight to twenty-four ounces of cold water will produce a rapid and watery salt-containing evacuation; while 5 grams and even 10 grams taken with food have no marked effect on the bowels.

'The intricacies of saline therapy are too great to be diseussed at length here, but the rational conclusion is undoubtedly to advise the gout to use pure waters or those having calcareous and alkaline bases and avoid saline waters altogether, unless of very slight mineralization.

'The best mineral waters for gouty subjects include nearly all the pure and alkaline waters on the list. It is said that the German Emperor, under presumahly the best advice, drinks largely of Fachingen water for this purpose. 'The waters of these springs contain 3.5 per mille of sodium bicarbonate and 6 per mille of calcium bicarbonate-a greater percentage of these salts than in any other water, as far as known.' 'This water is well horne, and is not particularly disanreeable.

Other excellent waters are Celestins Vichy, Evian, Vittel, Ems, the waters of Neuenahr, and Vals. American waters include the Bedford Magnesia, Gettysburg, and the Healing Springs Water. 'The baths of Virginia Hot Springs, Richfield Springs, and Mt. Clemens, in the United States, and those of Bath and Buxton, England, are serviceable. 'The systematic use of pure waters, like Poland Water, tends to the prevention of gout. In Europe, Giesshuebler and Fachingen are useyl largely for this purpose. They are well charged with carbonic acid gas. No bad results in uncomplicated cases should ensue from the use of 1 liter a day of these waters. A bottle of contrexeville Water every morning before breakfast is a favorite method of treating gouty patients, and this water enjoys a very good reputation in this disease.

Nephritis. In choosing a mineral water to be used in cases of nephritis we should avoid those containing sodium chloride. It has been very dearly shown by Widal and others that a diseased kidney does not eliminate this salt very well; indeed, the impermeability of the diseaser kidney to salines is a fair index of the extent of nephritis. When exeessive amounts are ingested water is retained in the boly. the increase of the osmotic pressure of the blood lessens perspiration and the anount 
of water lost by persp.ration. Edema may occur whether demonstrable or not; the albumin is increased by the ingestion of salt, and in patients with neplnitis without albuminuria it may then appear in the urine; headache, nausea, stupor may develop and favor the onset of uremia. Pure water, like Poland or carbonated distilled water, or the alkaline and alkaline calcic waters, or lithia, may be used.

Cholecystitis; Gallstones.-It is now recognized that cholecystitis begins as an infection, and that the presence of gallstones is a consecutive occurrence. A good deal of stress is laid on the etiology of the disease in carrying out treatment, and it is believed that the free use of alkaline water by the mouth and by the rectum aids in combating the bacterial infection that causes the intestinal catarrh present in these cases; hence the use of purges, of alkaline drinks, and irrigations, and the use of oil internally. 'The plan of treatment at Carlsbad by Dr. Ludwig r. Aldor at the Sanatorium for Diseases of the Stomach and Intestinal Tract is somewhat as follows:

Rest in bed is insisted on; from 7 A.M. until noon, and from 2 to 7 P.M., fomentations are applied over the region of the gall-bladder as hot as the patient can endure. From 150 to 200 c.c. (5 to 7 ounces) of Carlsbad Sprudel Water, at $50^{\circ}$ to $55^{\circ} \mathrm{C} .\left(122^{\circ}\right.$ to $130^{\circ} \mathrm{F}$.), are given every two hours, so that in twenty-four hours each patient must drink in the neighborhood of 700 to 1500 c.c. ( 25 to 50 ounces). The drinking hours begin at 7 A.M. and end at 7 P.M. Food is given freely, as much nourishment as the patient desires. This treatment is carried out not only until the patient ceases to have attacks of colic, but until all pain and tenderness have wholly disappeared from the gall-bladder region. This usually requires from four to five weeks; in rare cases as many as eight or ten weeks. In exceptional cases surgical operations are required. The most essential requisite for success is absolute rest during a course of treatment.

The alkaline waters of Carlsbad, Sprudel, Mühlbrunn, and Franz Josefsquelle have acquired a world-wide reputation in the treatment of cholelithiasis and diabetes. The favorable results are not alone due to the special skill of Carlsbad physicians in these cases, but to an inherent quality of these alkaline waters to correct morbid conditions.

Diabetes. - This disease in its milder or more benign form is often successfully treated with the alkaline mineral waters. 'There is, however, some tendency in the use of alkaline waters to increase tissue waste, and they should be given cautiously or altogether withheld in persons who are losing flesh. In gouty cases a trip to Carlsbad, Neuenahr, Vichy, or Brides-les-Bains may accomplish much good. 'These waters are alkaline sulphated, alkaline muriated, and sodic carbonated. The Vittel Springs, in France, to which diabetics are commonly sent, are calcic sulphated and carbonated; those of Royat are alkaline ferroarseniated, while those of Buxton, Gastein, and Evian are of very low mineralization. 'The change of climate and the dietetic and general hygienic régime contributes greatly to success in these cases. 'The use of mineral waters like those mentioned tends to check the inordinate desire for water drinking which is often a feature in diabetes. 
Diabetes Insipidus.-Chalybeate and arsenical waters may be advised.

Phosphaturia.-Saline (sodium chloride) waters may be very useful in connection with graduated exercises, sea bathing, or other forms of hydrotherapy.

Urinary Gravel; Oxaluria.-Inasmuch as oxalic acid can be produced from uric acid by oxidation, and as oxaluria is commonly associated with uric-acid gravel, we naturally choose the same mineral water for use in both these affections. Oxaluria sometimes seems to be connected with imperfect carbohydrate metabolism which, in a more arlvanced degree, may lead to the appearance of sugar in the urine as a form of alimentary glycosuria. In persistent oxaluria with dyspepsia, especially in arthritic subjects, the digestion and general metabolism which are at fault may often be successfully treated at mineral spas where a suitable regimen and diet are observed. 'The use of bottled waters for these affections is only a meagre substitute. The entire class of alkaline, alkaline saline, and alkaline-calcic waters may befound suitable; the most prominent examples are Vichy, Contrexéville, Wildungen, Apollinaris, White Rock, Healing Springs, and Buffalo, Virginia; Giesshuebler, Chatel-Guyon. When constipation is a complication we may use Saratoga Vichy, Congress, Hathorn, Bedford Magnesia, Pluto, Friedrichshall, or the Spanish bitter waters, such as Carabaña and Rubinat.

Chronic Constipation.- The saline, alkaline saline, sulphated or "bitter" waters are commonly used in this affection. 'They act best when taken cold on rising, in quantities graded according to individual requirements. One pint of Congress or Hathorn water or of any of the other waters of Saratoga, Crab Orchard, French Lick, answers the purpose quite as well as any imported water. 'The concentrated Pluto water of French Lick, Indiana, seems to enjoy at present the largest sale, and in its concentrated form needs dilution; so that 2 or 3 ounces diluted with twice that amount of pure water meets all ordinary requirements. The Abilena Water, of Kansas, containing a large quantity of sodium sulphate (Glauber's Salt); the Veronica Water, of California, and the popular "Red Raven" (sodium phosphate) are very satisfactory, and not unpleasant. Among the foreign waters, the bitter waters of Hungary, such as Hunjadi, Apenta; those of Spain, as, for example, Carabaña, Rubinat, and Condal, are equally efficacious.

'The treatment of chronic constipation by the use of salines and sulphates is not the simple matter that most of us imagine. 'To begin with, the causes of this affection are so diverse and often so obscure that mistakes in treatment commonly follow. Cases due to obstruction of the bowel by strictures, growths, and tumors are obviously surgical, and precious time is often lost in treating these with mineral waters. Others are due to faulty secretions from the liver and pancreas; others again to an inhibition of peristalsis through the nerve centres, due to chronic disease of the brain or spinal cord; or to diphtheritic paralysis or tabes. Cases such as these are for the neurologist. But in that 
large class due to some impaired physiological function or to some enterospasm or spasm of the sphincter, or to what is called alimentary constipation, the regulation of diet and drink and of the habits of the patients may accomplish a great deal of good.

'There is much confusion and there are wide differences of opinion as to the action of such common articles as sodium chloride, sodium sulphate, and magnesium sulphate on the bowel in the production of purgation.

Saline purgatives are believed to produce their effects in three ways: First, by preventing the absorption of fluid from the alimentary canal, thereby retaining liquid in the tube; secondly, if given in proper concentration, they cause a pouring out of fluid into the intestine, thereby adding to the amount that has been swallowed; and thirdly, it is generally supposed that they stimulate peristalsis and so hurry along the contents of the intestine toward the rectum. Hertz and his colaborers believe that when magnesium sulphate is given some of it is absorbed from the stomach and then acts through the blood, stimulating the neuromuscular mechanism of the colon. It is shown, in summarizing the evidence from various quarters, that if a solution of sodium chloride isotonic with the blood serum be administered by the mouth, little or none of it reaches a cecal fistula, since it is all absorbed in the stomach and small intestine. On the other hand, if the same quantity of an isotonic solution of sodium sulphate be administered, most of it escapes by the fistula, only some 10 or 20 per cent. having been absorbed by the stomach and smali bowel. In other words, the sodium sulphate prevents absorption which the sodium chloride does not prevent; and it is well known that sulphates, tartrates, citrates, lactates, and malates are comparatively unabsorbable; whereas the chlorides, iodides, bromides, and acetates are rapidly absorbed. 'The latter, therefore, excepting in the case of sodium chloride, do not produce purgation. There can be no doubt whatever that the use of a hypertonic solution draws fluid from the blood into the bowel, as will, also, a concentrated solution of common salt to a less degree. In other words, the sodium sulphate prevents the absorption of fluid, and the processes of osmosis cause an outpouring of fluid into the tube in order to make the fluids in the body and in the tube isotonic. 'The fact is cited also that when large quantities of fluid are present in the tissues, as in cases of dropsy, these fluids can be got rid of by the use of concentrated saline purges. 'The development of thirst after the use of salines is also an evidence that fluid has been lost and that its restoration is called for.

After a night's rest the stomach is in better condition to respond to the stimulus of a moderately saline, carbonated solution. 'The progress of the latter from the stomach to the bowel is rapid, cleansing and stimulating to the whole gastro-intestinal tract. "A further effect in the bowel of a solution of moderate strength is to produce an increase of glandular secretion and a slight excitation of peristalsis, but as the greater part is absorbed higher up, purgation need not follow. 'To realize the efficiency of saline solutions in chronic catarrh of any of the mucous 
membranes we have only to (all to mind their cleansing and stimulating action in nasal catarrh. In the early stages of a saline contrse there is an increased excretion of uric acid, together with a slight loss of weight, which is later succeded by a gain, dne to improved appetite and assimilation." Saline waters are, therefore, suitable for cases of chronic gastric catarrh with hypoacidity and for chronic constipation, or diarrlea, by local action on the mucosa. 'They are not at all suitable when the diarrhea is due to ulcerative lesions, or in any case of acute congestion or inflammation of mucous membranes.

Dr. Neville Wood makes a practical observation in comnection with sulphate waters, and that is that in (iermany and Austria it is betieved that the cold waters have a much stronger aperient action than the hot, and that the function of the hot Cartshad water is rather the treatment of diarrhea. We are informed that when a patient with constipation is sent to some of the Carlsbad physicians he is not unlikely to be told that the selection has been faulty, and sent on to Marienbad, where the waters are cold.

"There is too general a tendency to send these patients indiseriminately to the sodium and magnesium sulphate springs, due, apparently, to the fact that those salts are the only ones commonly used at lione which happen to be contained in mineral waters. Cases in which atony of the bowel is the chief eausative factor do badly at these spas and still worse on their return, for the muscular coat is weakened, and it is not easy to leave off the Glauber salt habit. For such cases muriated waters of medium strength are more suitable, the difficulty of discontinuance being less. It is only when constipation is associated with plethora that sulphated waters are indieated, and only when there is catarrh of the mucous membrane or insufficient glandular seeretion that muriated waters are of very great service. When the eatarrh is low down the methods of Chatei-Guyon and Plombières should be considered." The treatment of colitis at Plombières is by intestinal lavage; at ChatelGuyon they use, in addition, water by the mouth. Chatel is believed to be preferable in cases of atony of the colon, while the waters of I'lombières are to be preferred when there is spasm with irritability.

'To obtain mineral waters recently bottled, and without deterioration, is often difficult. Druggists and wholesale grocers keep the more popular brands in stock, and the freshest supply is usually found at the largest dealers. Stale water is always undesirable, and may be injurious. so that extreme care should be exereised in securing a supply. In the United States there are usually agencies for the sale of special waters, such as Vichy, Hunjadi, Fachingen, ete, but for those less frequently ealled for one must go to some importing house that makes a business of handling rapidly consignments as they come from the foreign springs. I have found satisfaction in dealing with the house of Morris \& Schrader. importers and dealers in foreign and domestic mineral waters, No. s Barclay Street, New York City. 


\section{American Waters.}

Abilena. Abilene, Kansas. Sodic sulphated; purgative.

Allouez Magnesia. Green Bay, Wiseonsin. Caleic bicarbonated; alkaline.

Arcadian. Waukesha, Wiseonsin. Alkaline.

Bear. Virginia. Caleie biearbonated; alkaline.

Bedford. Bedford, Pennsylvania. Calcic magnesic sulphated; saline.

Bethesda. Waukesha, Wiseonsin. Alkaline; ealeic.

Bowden Lithia. Lithia Springs, Georgia. Alkaline; caleie.

Buffalo. Virginia. Calcic sulphated; saline; diuretic.

Capon spring. Hampshire Co., West Virginia. Calcie bicarbonated; diuretic.

Castilian. California. Alkaline; saline; sulphated.

Crockett Arsenie Watcr. Shawsville, Virginia. Calcic bicarbonated; alkaline.

Deep Roek. Oswego, New York. Sodic muriated; saline.

Great Bear. Fulton, New York. Calcic bicarbonated; alkaline.

Geneva. Geneva, New York. Calcic sulphated; saline.

Healing Springs. Hot Springs, Virginia. Caleie biearbonated; alkaline.

Hndor Lithia. Pure water, with 40 grains lithium carbonate per gallon.

Londonderry. New IIampshire. Calcic bicarbonated; alkaline.

Manacea. Manganese, West Virginia. Caleareous.

Manitou. Manitou, Colorado. Sodic and ealeic bicarbonated; alkaline; $\mathrm{CO}_{2}$.

Mardela Springs. Maryland. Ferruginous and ealcic biearbonated; alkaline; $\mathrm{CO}_{2}$. Missesquoi. Sheldon, Vermont. Caleareous alkaline.

It. Clemens. Miehigan. Sodie muriated; saline; sulphuretted.

Oak Orchard. Genesee Co., New York. Calcie alumino-chalybeate sulphated; astringent.

Poland. Maine. Calcic bicarbonated; alkaline; very pure.

Pluto. French Lick, Indiana. Sodic sulphated; saline; purgative.

Red Raven. Harmarville, Pennsylvania. Sodium phosphate; purgative.

Red Sulphur. West Virginia. Calcic alkaline; sulphocarbonated.

Richfield Springs. New York. Calcic magnesic sulphated; alkaline; saline; sulphuretted.

Rockbridge Alum. Virginia. Iron; alum; sulphated; astringent.

Saratoga, New York. Arondack. Sodie and calcic muriated; alkaline; saline; laxative.

Saratoga, New York. Congress. Sodic and calcic muriated; alkaline; saline.

Saratoga, New York. Hathorn. Sodic muriated; alkaline; saline; laxative.

Saratoga, New York. Geyser (carbonated). Sodie biearbonated; alkaline;

saline; laxative.

Saratoga, New York. Carlsbad. Sodie biearbonated; alkaline; saline.

Saratoga, New York. Chief. Sodic biearbonated; alkaline; saline.

Saratoga, New York. Lincoln. Sodie and caleic muriated; alkaline; saline.

Saratoga, New York. Magnetic. Sodie and ealcic; muriated; alkaline; saline;

laxative.

Saratoga, New York. Peerless. Sodic and calcic muriated; alkaline; saline;

laxative.

Saratoga, New York. Star. Sodic muriated; saline.

Saratoga, New York. Victoria. Sodic muriated.

Saratoga, New York. Vichy. Sodic muriated; alkaline; saline.

Saratoga, New York. Patterson. Alkaline; saline; laxative.

Saratoga, New York. High Rock. Sodie and calcic muriated; alkaline; saline;

laxative.

Sheboygan. Wiseonsin. Sodie and calcic muriated; alkaline; saline; $\mathrm{CO}_{2}$.

'Thompson's Bromin. Arsenic Spring. Ashe Co., North Carolina. Tonic.

Veronica. Santa Barbara, California. Magnesic sulphated; saline; laxative.

White Sulphur. Greenbrier Co., West Virginia. Calcic sulphated and bicarbonated; sulphuretted.

West Baden Sprudel. Freneh Liek, Indiana. Chlorides; sulphates; aperient.

White Rock. Waukesha, Wisconsin. Sodic and calcic muriated; alkaline; saline; $\mathrm{CO}_{2}$.

Waukesha Water. Imperial Spring. Wisconsin. Calcareous magnesic; alkaline.

\section{Foreign Waters.}

Aix-la-Chapelle. Thermal sulphocarbonated; sodic muriated; saline.

A penta. Ilungary. Bitter water (sulphated); purgative. 
Apollinaris. Prussia. Carbonated sorlic: alkaline; $\mathrm{CO}_{2}$.

Armeniusquelle. Leppspringe, Germany. ('alcic and sodic sulphated; alkaline. Bilin. Bohemin. Sodic carbonated and sulphated; alkaline. Gastrie catarrh; gout.

Birresborner. I'russia. Alkaline; table water.

Birmenstorfer. Switzerland. Sulphated; aperient.

Bertriek. Bergquelle. Sodie sulphated; biearbonated. Dyspepsia, gont.

Carabaña. Sodie magnesie sulphated; purgative.

Carlshad. Muhlbrun. Thermal; carbonaterl; sorlic sulphated; alkaline; saline.

Carlsbad. Sprudel. Thermal; carbonated; sodic sulphated.

Carlsbad. Schlossbrunnen.

Contrexéville. Source du Pavilion. Caleic; alkaline; saline; sulphated.

Eger l'ranzbrunnen. See Franzensibad.

Ems Rock Spring. Vietoria. Thermal; sodic; alkaline; earbonated; muriated. Einser Kraenehen. Germany. Thermal; alkaline; saline; antieatarrhal.

Evian. Franee. Alkaline; table water.

Fachingen. Germany. Alkaline; sodie bicarbonated; antacid; table water. Gastric affections.

Franzensbad (Eger Franzbrunnen). Sodic; alkaline; saline; sulphated; aperient. Friedrichshall. Germany. (Bitter) magnesie sodie sulphated; $\mathrm{CO}_{2}$.

Franz Joseph. Hungary. Bitter water; sulphated.

Giesshuebler. Bohemia. Alkaline; antacid; table water.

Homburger Elisabeth. Germany. Sodie muriated; saline; carbonated.

Hungarian Kossuth. Hungary. Aperient.

Hunjadi Janos. Hungary. Bitter water; aperient.

Hunjadi Matyas. Hungary. Bitter water; aperient.

Kaiserbrunnen. Germany. Alkaline; table water.

Kïissingen (Rakoczy). Germany. Alkaline; saline; ferruginous.

Kireuznacher Elisabeth. Germany, Sodic caleic; saline.

Kronenquelle. Prussian Silesia. Alkaline.

Lippspringer. See Armeniusquelle

Levico. Austrian Tyrol. Arsenic; nemapoietic.

Marienbad Kireuzbrunnen. Sodic; ealcic; ferruginous; sulphated; carbonated, $\mathrm{CO}_{2}$

Neuenahrer Sprudel. Germany. Alkaline; carbonated; $\mathrm{CO}_{2}$.

Obersalzbrunnen. Prussian Silesia. Alkaline. Cystitis.

Puellna. Bohemia. Sodic magnesie sulphated.

Rhens. Germany. Alkaline; saline; table water.

Roneegno. Austrian Tyrol. Arsenic; antimalarial.

Rubinat Condal. Spain. Sulphated; purgative.

Rubinat Llorach. Spain. Sulphated; purgative.

Rubinat Serre. Spain. Sulphated; purgative.

St. Galmier. France. Alkaline; table water.

St. Leger. Franee. Calcic. Dyspepsia.

Salzschliefer Bonifacius. Germany. Gout.

Schlangenbad. Germany. Thermal; sodie; muriated; saline.

Sehwalbacher. Germany. Magnesic chalybeate; alkaline; carbonated.

Salzsehliefer Bonifacius. Germany. Gout.

Schwalbacher. Germany. Magnesic chalybeate; alkaline; carbonated.

Selters. Germany. Calcic sodie; saline; carbonated.

Tarasp, Luciusquelle. Switzerland. Alkaline; sulphated; aperient.

Vals, Source Desirée. France. Alkaline. Dyspepsia; gout.

Vichy, Celestins. France. Alkaline; $\mathrm{CO}_{2}$; antacil. Gout.

Vichy, Grand Grille. France. Alkaline; $\mathrm{CO}_{2}$; antacid. Gout.

Vichy, Hôpital. France. Alkaline; $\mathrm{CO}_{2}^{2}$ : antacid. Gout.

Vichy, Hauterive. France. Alkaline; $\mathrm{CO}_{2} ;$ antacid. Fout.

Victoria Brunnen. Buda Pest, Hungary. Bitter water; strong sodic magnesic; sulphated.

Villacabras. Spain. Sulphated: aperient.

Vittel. France. Calcie; antacid. Gout.

Wiesbaden Kochbrunnen. Germany. Thermal; saline.

Wiesbaden Gichtwasser. Germany. Thermal; saline.

Wildungen Helenenquelle. Calcic magnesic; bicarbonated.

Wildungen George Vietorquelle. Calcic magnesic bicarbonated; vesical catarrh.

Wilhelmsquelle. Germany. Alkaline; saline; table water. 



\section{HYDROTHERAPY}

BY SIMON BARUCH, M. D., A.ND ARTHUR M. SHRADY, A.M., M.I).

IT is the aim of this article to divest hydrotherapy of the confusing technicalities with which institutional hydrotherapists have invested it, and which have deterred the general practitioner from its use. 'The physician will find here an exposition of the rational and practical application of water in disease, which will enable him to utilize it at the bedside. 'The views expressed are the basis of our instruction in hydrotherapy from the chair and in the clinic.

The defection of a large number of patients from the clientele of the general practitioner to the various empirics, mental or Christian Science, osteopaths, and (in (iermany) Naturaerzte has led to the investigation of this remarkable phenomenon. 'To the authors it would seem clear that the main cause is to be sought in the inefficiency of drug treatment, upon which the modern doctor appears to depend as much as his predecessor, who has been criticized for bleeding and spoliative medication. 'The enormous production and sale of symptomatic remedies which flood our offices in samples presented by persuasive agents of the manufacturer, and the large amounts spent for advertising their virtues, would indicate that the average doctor prescribes these new drugs freely, and that we are now sadly emulating the polypharmacy of our predecessors. 'The result is the same as in the days of Hahnemann, when the sick deserted the "regulars" who filled their stomachs with nasty medicines and flocked to homeopathy. 'The chief difference between that not far-off period and the present appears to be that now the sick receive more palatable medicines, which are less destructive, but equally inefficient, except as palliatives. Since the chief agent in the removal of disease is vis medicatrix natura, the wisest men in the medical profession avoid polypharmacy, confining themselves to drugs of well-known action, and strive to aid nature in its effort to restore health.

In the furtherance of this object our knoviledge of the life-sustaining action of food, drink, air, exercise, rest, etc., has grouped these agencies under the head of physiological remedies. 'The latter have been introduced into the medieal curricula of the German schools more energetically. since a committee for the revision of medical instruction, headed by the veteran teacher, Kussmaul, pointed out that to their negleet in the universities may be attributed the rise of the Naturarat who had seriously encroached upon the regular physician's domain. We agree with this 
noted teacher that hydrotherapy is an important, if not the most active, of the physiological remedies, and that the average student receives too little information upon it. The average doctor is sadly ignorant of hydrotherapy, because books of reference rarely give information on this subject. The text-books on practice and therapentics have, until very recently, afforded the most meagre, and too often erroneous and contradictory, information, which has resulted in failure and abandonment, to the great detriment of patient and physician alike.

Hydrotherapy ( $\delta \dot{\delta} \omega \rho, \theta \varepsilon \rho a \pi \varepsilon(\alpha)$ means the treatment of disease by water. In one sense the term is misleading, since the water is only a means of administering the great remedial agents of heat and cold, and the term thermotherapy would be equally descriptive of this method of therapeutics. We use water as the most adaptable means for conveying heat and cold for various reasons. It is universally obtainable, cheap, and safe. It has the peculiar quality of absorbing a large amount of heat without a proportionate rise in temperature, thus affording an excellent container for heat without rapid changes in its thermometric readings. It also parts with its heat at a rate which is well adapted to its use as a therapeutic agent. In its flowing form its temperature, force of impact, amount, or bulk can be rapidly altered, and, therefore, is under absolute control. It may also be used as a solid or a vapor at more extreme temperatures.

The Dosage of Hydrotherapeutic Procedures.-The extreme flexibility of the water treatment may be seen if we recollect that at one extreme we have ice, so cold that it may cause necrosis, while at the other extreme we have boiling water, which can also cause death of tissue. Between these limits we have lessened degrees of activity, the more nearly we approach the temperature of the part with which it comes in contact; inasmuch as the skin is chiefly the region to which water is applied, we have our neutral point at about $92^{\circ}$ to $94^{\circ} \mathrm{F}$.

The hydrotherapeutic law of Baruch states that "the difference in intensity of effect is in proportion to the difference between the temperature of the water and the skin." Not only can we modify our treatment by thermometric measurement, but also by its duration. A certain procedure may last ten seconds, ten minutes, hours, or even days. Certainly this should be done by the watch, and not by indefinite adjectives, such as long, short, etc. It is this haphazard method of prescribing that has caused the use of water to be abandoned by many practitioners who have their patients report so many discouraging failures. A thermometer and a watch are both cheap instruments of precision which should accompany all baths, douches, etc., and which should be as accurately noted as are the administration and effects of drug treatment.

One important accompaniment which tends to enhance our successes when using cold is friction, without which cold water is rarely well borne; with the sick and delicate it is a sine qua non. The way in which it is used, whether light or severe, is also of moment. A sick patient is unable to administer this friction to himself, and then it is 
necessary to have the murse or attendant come to his assistance. 'These in turn are variable quantities, since sone nurses are strong and willing, and others are weak and la\%y, and it behooves us as phisicians to be present at the beginning of such treatments, to correct errors, to stimulate effort, or to change the assistant. Along with friction, al purely mechanical irritant, we might here consider such chemical irritants ats carbonic acid gas, salt, ete., which are often used as adjuvants to promote reatetion, for it is purely for overcoming the chilling effect of cold water that these chemical and mechanical means are added to the thermic shock.

Still another specification may be added to our prescription, $i$. $e$, the bulk of water employed. If we use a thimbleful of ice-water, it is by no means as great a demand upon the patient's reactive capacity as a tubful of water at $80^{\circ} \mathrm{F}$. So a sheet wet in water at a definite temperature is much colder to the patient if it is used dripping than if used wrung out. So a towel, containing less water than a sheet, becomes a milder agent. The area over which the application is to be made must also be carefully stated, else our prescription is indefinite. Certain parts of the body bear cold well, others bear it ill. A squitre foot of shin surface treated means a milder procedure than a square yard. If all these precautions are employed, we have a modus operandi which would give definite results were it not for the human element in our equation. Here the art, judgment, and experience of the physician comes into play. If, on the other hand, he employs common sense, understands the physiological acts which are taking place, he has one of the most easily modified and flexible methods of treatment at his command. If the patient does not fit his first prescription, he can make his subsequent prescriptions fit his patient. He can immediately modify the temperatures, the duration, or limit the area attacked, or change the amount of friction employed.

Physiological Action of Heat and Cold.-The main desideratum in all cold water procedures is reaction, for without this sequel our efforts may cause harm instead of good. 'The physiology of this phenomenon is interesting, and should be understood by all who employ hydrotherapy. 'The first effect of cold upon the periphery is to cause contraction of all the tissues of the skin, vascular, muscular, and fibrons; promptly the healthy afferent nerves signal for a greater supply of heat and the efferent nerves reply by causing a vasodilatation of the vessels in the chilled area. 'The ressels of the interior, viscera, muscles, etc., correspondingly contract and the heart responds to the new demand made upon it, and we have a tonic hyperemia in this particular area. Baruch has drawn attention to the role played by the muscular fibers of the skin, which, he claims, contract under the cold, and retaining more or less tonicity, serve as a check to a subsequent overdilatation of the bloodvessels, and thus maintain a higher blood-pressure. We, therefore, have a local effect on the circulation when a primary contraction is followed by dilatation of the ressels, provided that the application be brief enough. 'Too long an application causes a paralysis of the cutaneous muscles, with an ensuing cyanosis, a hyperemia, perhaps, but of the atonic variety, as illustrated under an iee-bag if it be prolonged. Here the stimulant 
effect of the cold is like that of other stimulants-if too little it is negative, if too much is.employed, we get results which are harmful. If properly measured and with proper adjuvants, we have an active, surging blood-current supplied to the part which nature supports whenever it is evident that it has cold to battle with, and there is a threatened destruction of a part.

Then we have the general effect on the circulation to consider. Let us consider the heart first. In Winternitz's experiments, confirmed by Baruch, he noted in sphygmographic tracings a primary acceleration of the pulse-rate, immediately followed by a lessening of the number of beats per minute when cold water was applied to the periphery. The reverse held true when high temperatures were emploved. The good results in fever when cold water is employed are due in part to this slowing of the pulse, and to an increase in blood-pressure which accompanies the lessened rate. Along with this cardiac change, and in large part causing it, we have the general vasomotor system aroused to perform its duties. In acute or chronic toxic states we have a vasomotor change which is decidedly detrimental to the patient's welfare, and if we can modify these abnormalities, an important advantage is gained. As an example, let us consider the typical dicrotic pulse of typhoid ferer and the influence of the cold bath upon the circulation. We here have paretic blood-vessels, into which the heart is making an effort to throw a sufficient amount of blood at a certain pressure, and often quite unsuccessfully. The heart in health is assisted by the blood-vessels in propelling the blood forward and in maintaining sufficient tension. In the typhoid state most clinicians fear the giving out of the vasomotor system more than that of the heart, and it is in cold that they place their main reliance to correct this weakness. As H. A. Hare has so well expressed it: "The vasomotor system is made up, on the one hand, of the vasomotor nervous apparatus, and, on the other, by the blood-ressels themselves. The resistance offered to the heart by the properly acting vasomotor nervous system, through its influence on the vessels, is identical with the friction offered to the driving wheels of a locomotive. The locomotive is intended to meet and stand any resistance, and if the resistance be removed by slippery rails, the wheels fly around ineffectually, racking the machinery and destroying its usefulness." A more graphic simile could hardly be offered us of a picture too common in infectious diseases, where we say the patient is overwhelmed by the toxemia. It is this toxic influence on the rasomotor system that we fear, although we often call it heart failure. Cold is our best supporting stimulant for such a condition, while drugs too often prove their inefficiency.

Along with this effect on the circulation, and in large part causing it, we have the effect on the nerrous system. This most important controller of all our vital functions is under the continued influence of its environment in the largest sense. Changes in weather, happiness, the presence of toxic irritants in the blood, etc., all react, and react powerfully, on this highly organized yet much abused srstem. In cold we have the most direct spur to make it perform its duties. When we wish to arouse 
ourselves from a state of lethargy, the sudden impact of cold water will often succed without further help. The morning cold plunge has a world-wide reputation for rapidly placing us in the condition where work seems a pleasure rather than a burden. In fevers, where the lethal poisons from bacteria are slowly undermining the patient's strength, where each successive benumbing of the nervous activity means the widening of a vicious circle, where the central nervous system fails to regulate the circulation, the secretions, etc., it becomes necessary for us to arouse this great regulation. 'Those who have used the cold friction bath in typhoid fever cannot fail to remark the return to a more nearly normal condition. 'The patient, when awake, is much brighter; when asleep, is in a more peaceful shmber, his secretions are more abumdant, his appreciation of his needs and his symptoms is more acute. 'This is accomplished in nearly every patient if we molify our treatment to suit the individual case. If the patient is first seen after the disease has made great inroads upon his strength, and where reaction from bolder procedures would be impossible, we should change the technique in accordance-in other words, lessen our dosage by using water less cold, for a shorter time, over limited areas, by the addition of chemical or mechanical irritation or awaiting a time when exhaustion is at its minimum, and the nervous system better able to respond to the fillip.

How different is the effect of heat on the circulation. If the temperature of the water be within the bounds of comfort, which is generally $104^{\circ} \mathrm{F}$. or under, we note no primary stimulation, and we have a gradually relaxing skin and a lowering of tone in the vasomotor system, giving a sluggish peripheral circulation without sufficient increase in cardiac energy to counteract this condition. 'There is a consequent fall of bloodpressure. If the temperature be over $104^{\circ} \mathrm{F}$., up to $110^{\circ}$ or $115^{\circ} \mathrm{F}$, we have a distinct primary stimulation of the peripheral vessels, which, however, soon ceases, and if the bath is continued, we have an entirely different train of events than if we were using cold. Here the skin relaxes, vasodilatation takes place, the heart hurries more and more, endeavoring to keep up a sufficient blood-pressure, but succeeding only in part. 'The general temperature of the interior rises, so that the effect is no longer confined to the skin, and the heart has to attempt to make up for this as well. If carried too far, the brain shows evidence of the lowered pressure, and feelings of faintness ensue.

Respiratory Effects,- The immediate effect of entering a bath which is cold or hot enough to stimulate the peripheral nerves in the skin is to cause a deep, sometimes irregular, gasping respiration, which if the submersion be mantained, soon becomes regular and more rapid. If the bath be cold, the breathing becomes deeper, and if hot, it becomes more shallow. Claude Bernard ascribes the sense of drspnea which is experienced in a hot bath to be the result of the heated blook circulating in the respirat ry centre.

Effect on Metabolism.-Inasmuch as we ure enalbled to regulate the distribution of the blood within certain bounds, so are we able to diminish or inerease the supply of blood to different viscera. In this way we can 
in part control the functions of these organs in so far as they depend upon the increase or decrease of their blood-supply. While the temperature of the interior is not materially affected, we find that cold and heat increase oxidation. When the temperature of the blood is raised or lowered, we have the additional direct effect of increased or decreased oxidation. Chemically, the cold procedures cause oxidation of the carbohydrates and hydrocarbons, while heat causes an oxidation or change in the nitrogenous elements. Of course, the duration of the various procedures have a direct bearing on the results both during and after the treatments. Judging from the clinical effects, we are forced to conclude that the results of these different hydrotherapeutic procedings are not merely chemical and thermic effects, but must be traced back to effect on living cells composing the various organs. It is their vital activity which is being altered by heat and cold, and in many ways. Each viscus of the body is under the influence of the other organs, as has been plainly shown in the experiments now being made on the internal secretions of the ductless and other glands. If we alter the chemical activity of one organ, this in turn has an effect on another, and such change may again affect the original or other tissues. The interdependence and multiplicity of these changes are so intricate that the laboratory can as yet not give us adequate explanations or measurement of all these phenomena, and we are forced in some instances to fall back upon our clinical observation of gross results.

Temperature Effect.- The temperature of the skin is, of course, cooler than that of the interior. Its source of supply is mainly from the blood flowing through it, which comes from the interior, and also by direct conduction, chiefly from underlying muscles. The venous blood, as it leaves the skin, is cooler than the arterial, while in the glandular and muscular tissues the reverse is true. It is fair to conclude that the skin is warmed chiefly by its blood, and not by chemical activity, as is the case with the viscera and muscles. The regulation of this blood-supply is by means of the nervous system, and that is particularly affected by the thermic irritation of the afferent nerves (and, according to Baruch, on the ganglionic nerve supply of the cutaneous muscles). It is a vital act, and certain vital aetivities are aroused which make the result quite different from that on inanimate bodies where an equalization of temperature tends to take place.

In health our control of the body-temperature is extremely limited so long as safe procedures are used. Our experiments in the Vanderbilt clinic, for instance, showed an increase of only one-tenth degree Fahrenheit in the rectum of a healthy man who had lain in a bath of $73^{\circ} \mathrm{F}$. for ten minutes. Liebermeister showed long ago that even after an extremely cold bath of brief duration the internal temperature was slightly raised. It would seem probable, therefore, that the effect of cold in health is shown chiefly upon the surface. The normal equilibrium is carefully guarded by the vital centres. In fevers the result is more noticeable, and we can lower the temperature several degrees. Even in such instances we notice great variations. If a typhoid patient be sub- 
jected to the Brand bath given at stated intervals, hy the same nurses, at the same temperature, and the same length of time, we find the results in the first week show little temperature reduction, and ats the discasc progresses, the drops in the fever-curve become greater and greater after each bath. If the heat abstraction were a purely physical process, this would not be so. Here again we must explain the result by the effect on the nervous system, which becomes less and less overwhelmed ly the bacterial poisons as nature learns to counteract them. At first the pattient is hut little helped in overcoming the fever, but as time goes on the same procedure has increasing effect, and toward the end of the disease he needs the baths less and less as a support to his nervous system. Of course, this refers to cases which show the tendency to recover ani suceessfully eombat the invading bacteria.

'The effeet of heat on healthy subjects is more striking than that of cold, for the organism is less able to comteract it and protect itself. For instance, if a patient is submerged to his neck in water above $105^{\circ} \mathrm{F}$., he is enabled to part with heat only by means of his lungs and the surface of his head. 'The consequence is, a heat stasis takes place, and the temperature rises 2 or 3 degrees in the mouth, and, of course, many more degrees in the skin. In consequence of the lowered blood-pressure due to the inereased amount of blood eirculating in the skin, cerehral anemia ensues, and a sense of faintness compels us to cease adding heat, and thereby confine our procedures within safe limits.

Blood-pressure. - In heat and cold we have agents which act in a surprising way on arterial tension. Cold applications, if of sufficiently low temperature and maintained for a proper length of time, will cause a rise of blood-pressure. 'Theodore Janeway has shown that there is a rise of several millimeters of $\mathrm{Hg}$ after a Brand bath.

'The effect of heat is different. At first there is a primary rise, which in turn is followed by a fall of pressure. 'This is easily understood when we note the relaxing effect of hot air or water upon the skin and its contained vessels. 'The heart hurries to make up for this subnormal pressure, but succeeds only in part.

Effect on Distribution of Blood-cells.-Careful blood counts made by reliable observers have shown a positive increase of both the red cells and leukocytes after cold baths, and a slight increase of red cells and a dimimution of leukocytes after warm haths in parts not exposed to the action of water (the lobe of the ear). 'This is not due to an actual increase in the number of cells in the blood, but to a stirring-up of cells which have been more or less dormant in the outlying vascular areas. Their more active participation in the blood-current must conduce to an enhancement of functional activity, and in infectious diseases promote phagocytosis. Although enthusiastic hydrotherapentists have misinterpreted this effect, the mathematical proof of a larger number of bloodcells in the peripheral vessels under good renction cannot he gainsaid.

Effect on Muscle Power.-Heat, when uncombined with friction or massage, and where it is acting upon a normal muscle, causes diminished power, while eold canses the reverse to a marked degree. 'The alterna- 
tions of heat and cold act in a way similar to cold when applied by douches, etc. Here the mechanical impact has a direct bearing on the result. This result probably is caused by the effect of heat and cold upon the nervous system, which is in control of the muscles.

Effect on the Nervous System.-Of course, many of the preceding effects of hydriatic treatment may be traced back to the nervous system, and in disease we rely upon arousing this great regulator to take command of its forces by means of peripheral irritation by heat and cold. The entire skin area affords us an immense number of nerve terminals upon which we apply our excitants, thus arousing innumerable reflex effects, which it is our object to control. Upon cold especially do we rely for a safe and active stimulant, and especially is this true when toxemia has so overwhelmed the nervous svstem that it shirks its duties and allows a chain of erents to occur which in turn threatens the life of the patient. Drugs like caffeine, strychnine, or other medicaments are not so powerful. Heat also acts as a stimulant, but it has the disadvantage of relaxing the skin and lowering the blood-pressure eventually, and, of course, may increase existing fever. If water at skin temperature be used, we have the reverse effect. Here the peripheral nerves are surrounded by an unirritating medium, causing a soothing effect, which is reflected upon the central nervous svstem, thus causing a state of quiet and often inducing sleep.

Technique of Hydrotherapeutic Procedures.-It will be the effort of the writers to here describe the technique of hydriatric procedures from the standpoint of the general practitioner. These methods have too long been in the hands of untrained men, who know little of physiology or disease, and whose rules are too rigid, with the natural consequence of a hit or miss result. Moreover, these methods are applicable to the acutely ill as well as to those afflicted with chronic ailments.

The Ablution.-The bed should be properly prepared with a rubber sheet, upon which are placed in order a blanket and a sheet, the latter preferably of linen. If the case be one of fever, the nurse should bathe the face with water at $50^{\circ}$ to $60^{\circ} \mathrm{F}$. 'These colder temperatures are well borne by this part of the skin, which is accustomed to the cold, and has an excellent reactive capacity. Then each part of the body is separately washed with water at $90^{\circ} \mathrm{F}$, reducing this temperature from $2^{\circ}$ to $5^{\circ} \mathrm{F}$. at each successive treatment until even $60^{\circ} \mathrm{F}$. is reached, provided prolonged chilliness does not occur. Care should be taken that active friction accompanies the application, and to this end the hand wash-cloth or a bath glove should be employed in preference to the sponge, which is too smooth, and moreover often contains too little water.

In the earlier trials of the patient's reactive power the water is to be used in small amounts at the higher temperatures, later more freely, and at lower temperatures. It is important to omit the extremities below the knees and elbows when they show a tendency to be cold, and if the patient has a normal temperature, they are also wisely omitted. The cold water is applied to the chest, abdomen, and back until they cease 
to warm up under the cold with friction, being careful to dip the glose, cloth, or hand into the cold water again and again, since it becomes warmed by the body's heat. If a reduction in the paticnt's temperature is particularly desired, no rubbing dry is permitted, since the evaporattion of the water has a cooling eflect which is wall colcrated hy the reacting skin. In asthenic individuals, where reatetion is poor, this is not true, and a thorough drying is advisable. Chilliness should be avoiderl, and too long procedures are contraindicated if the patient seens tired from their use. In the German "Abreilumg" a damp linen towel without fringes (dripping) is placed upon successive portions of the trunk, handfuls of water are dashed upon it, then brisk rubbing over the towel follows. This is more effective, but requires greater power of reaction on the part of the subject. 'The body may be thus treated twice or oftener and then dried. 'The parts of the body not being treated are properly protected by blankets.

In chronic cases, where the condition is such that little reactive power is present, we use the ablution in a somewhat different way. 'The patient should be previously warmed by a blanket pack, and then ordered to arise and stand in water 10 to 12 inches deep at a temperature of $104^{\circ} \mathrm{F}$. This foot-bath is employed to aid reaction, since this is poor if the foet are chilled by standing in the cold water which drops from him. A wash-cloth wrung out of water at $80^{\circ} \mathrm{F}$. is then rapilly passed over the trunk and upper parts of the limbs, using good friction for a definite time. 'The temperature is gradually reduced, perhaps to $50^{\circ} \mathrm{F}$., the application more and more prolonged, and the amount of water increased as the patient learns to react. Baruch has termed this educational method "neurovascular training," and in the ablution we have the primary conrse, which later shomld be followed by the more effective methods, like the cold rub, the drip sheet, etc. If, with all these precautions, cyanosis, chilling, exhaustion should ensue, we apply the water to only one part at a time, and then stop until the reactive power is aroused and allows us to proceed steadily although cautiously.

To properly understand the physiological processes here aroused ne must recollect that the warm skin is suddenly aroused by the impact of cold water, and the peripheral nerves appreciate a surprise or shock, which, if the patient is untrained, is disagreeable at the moment. 'This surprise should be suflicient to arouse the central nervous system and the reflex vascular effect if we are to obtain appreciable benefit. 'The skin-vessels are directed to maintain a hetter peripheral circulation, as is shown by the reddened epidermis and the hyperemia moreover, is active and not passive, as may be proved by the temporary pallor caused by pressing the hands on the rosy skin, which rapidly ceases on withdrawal of the pressure. 'This increased vascular tone helps the heart to propel the blood forward, the central nervous system adjusts properly the distribution of the blood, and the power of the heart and the deepened respiration add an effect in maintaining the flow of the blood thronghont the body in a more nearly normal manner. 'The effect on fevers is partieularly noteworthy, for here the toxemia is counteracted, particularly 
on the part of the circulation. There is a less important effect in the cooling of the blood, which is facilitated by its greater distribution to the surface as well as by direct heat abstraction by the cold environment and the effect of evaporation.

The ablution is, therefore, indicated in fevers, both for the comfort following it and as a means of sustaining the patient's strength and resistance. In the eruptive fevers spreading a wet towel and gently tapping it by the palm of the hand may be substituted, so as to avoid irritating the skin too much. In the milder cases of typhoid, in tonsillitis, la grippe, tuberculosis, the writers have seen distinct benefit ensue. In chronic diseases it is of use in depleted, run-down, neurasthenic individuals, in anemia, in fatigue, chronic catarrhs, etc. Of course, more heroic and, therefore, more efficient procedures should, if possible, be employed as the patient becomes educated by practice. In chronic cases the next step in Baruch's neurovascular training is the affusion after ablutions at $60^{\circ} \mathrm{F}$. are well borne, without chilling effect.

Affusion.-This method is more effective and more lasting in its results than the ablution. It is applied to a larger surface of the patient, and demands more response to the cold; therefore, in poor reaction cases we precede it by the milder method. The patient, if ambulant, stands in water at $102^{\circ} \mathrm{F}$., and has water poured upon his shoulders, back, and front alternately from a basin or pitcher at $90^{\circ} \mathrm{F}$., the temperature being reduced daily $2^{\circ}$ or $3^{\circ} \mathrm{F}$. until $50^{\circ}$ or $60^{\circ} \mathrm{F}$. are reached. 'The mechanical irritation of the periphery may be increased by allowing the water to be poured en masse and from a height, thus simulating a douche. The reason that this is a more effective and energetic method of applying cold than is the ablution is easily seen if one recollects that the amount of water is much greater, the area of the skin attacked is larger, and that due to the intermissions he has a series of active shocks, both thermic and mechanical. The number of applications may be increased, and the temperature lowered in accordance with the response of the patient. After its termination the patient is dried and sent into the open air to gently exercise. If, however, the subject is confined to bed, he is placed in a tub which is empty (or sometimes in 6 inches of water at about $102^{\circ}$ $\mathrm{F}$.), in the sitting or inclined position, and the application made in the same manner. The general practitioner will find this an excellent remedy in the toxic fevers, where an overwhelmed nervous system has allowed the various organs to perform their duties ineffectually. The patient is aroused from his lethargy, he becomes more nearly normal mentally, his circulation responds, as is evidenced by a shower and stronger heart's action; his respirations deepen, thus aiding the heart as well as overcoming a tendency toward hypostatic congestion and retention of bronchial mucus. 'The effect is often brilliant, for by breaking into the vicious circle, the result is not only local and immediate, but far reaching, and if repeated, long lasting. 'The timid practitioner need not fear this procedure, since it is distinctly stimulating if carefully done. Winternitz very truthfully portrays the truth in the following words: "Collapse is almost universally regarded as a contraindication to every application of 
cold. I would again express the opinion that I know no more powerful or effective agent for combating th reatening or existing collapse than the intense and intelligently applied excitation by cold. How often lave I seen, in advanced fever processes, in degenerated typhoids, a rapidly favorable change wrought in the corpse-like coldness of the extremities, in the most serious manifestations of nerve weakness, in the hypostatie. congestion of the lungs, by one dipping into a very cold bath or one cold application. I am firmly convinced that very often these manifestations are not due to heart feebleness, but to a collapse of the vessels, and here an evanescent, but energetic, excitation by cold is the only reliable remedy, as I have repeatedly proved."

Therapeutic Applications. - The conditions which call for the use of the affusions occur particularly in the acute infectious diseases. 'Their use seems to counteract the influence of many forms of toxemia, and inasmuch as it is a mild procedure, even a weak patient can react successfully and show increased power afterward. Scarlatina, with its rapid, low-tension pulse is a typical example in which we have a pure toxemia much benefited by cold. 'The poorly developed eruption may be mads to take on a ruddy hue by increasing the circulatory power in both the skin and the interior. Contrary to the general opinion, which has advocated warm baths, clinical experience shows that short cold water applications accomplish all the good of these and also afford additional benefits.

Hoffmann, in Nothnagel's encyclopedia, especially recommends the affusion, stating that this "is, therefore, the best method to use in the capillary bronchitis of children."

The Sheet Bath.- The bed is prepared as follows: Upon the mattress is spread a rubber sheet and over this a blanket. A coarse or fine linen sheet is then partly wrung out of water at $80^{\circ}, 70^{\circ}, 60^{\circ}$, or even $50^{\circ} \mathrm{F}$., the selection of the temperature being regulated by the effect desired and the patient's ability to react. 'The subject is then placed upon the sheet, his face is washed with water at $60^{\circ} \mathrm{F}$., and a wet turban placed about the forehead at the same temperature. 'The patient's arms are raised and the chest enveloped with one-half of the sheet; then the arms are placed at the side, and the remaining half is placed snugly ahout the shoulders, arms, and legs, tucking the loose sheet in between the thighs and feet. 'Thus the coaptation of two shin surfaces is prevented, and a large area of skin is surrounded by the cold wet sheet. If we fear a poor reaction in a feeble patient, the arms and legs may be omitted in the wrapping. 'The nurse then rubs the sheet with the flat hand, successively the posterior and afterward the anterior surfaces of the trunk, so as to have the aid of friction in establishing the reaction. So soon as each part becomes warmed, water is poured upon it from a pitcher in small quantities, and the part is again rubbed until warm. When each part fails to warm up, no further application of cold is made at that point; then another part is rubbed and cooled. After the entire trunk has been thus warmed and cooled, the patient is dried and made comfortable in his bed. If there be much fever, the drying may be omitted, the sheet 
being left in situ, but covered with one blanket for a half-hour, unless he sleeps, under which circumstances he is best let alone until awakening.

'The effect of this method of applying cold is a moderate one. At first the skin-muscles contract and the nervous system is aroused. Then reaction takes place, together with improved heart action. As the warm blood reaches the surface it is cooled again and again as the cold water is applied. In fever cases the heat is given up steadily and an approach to normal may be demonstrated by the thermometer. If the patient remain in the wet sheet and a single blanket, the gradual evaporation of the water and the increased supply of blood to the surface add to the antifebrile effect. A soothing hypnotic influence also is often noticeable. The respiratory act is particularly deepened by the successive applications of cold.

The Drip Sheet.-By this term is meant a linen sheet which is not wrung out, but which is applied dripping wet. One may realize how heroic a procedure this is by simply calculating the amount of water in the sheet. It must be remembered that the patient has to warm up the water, and the more there is in the sheet, the greater the effort demanded of him. If his reaction is poor, it cannot be used until he has become trained to cold-water applications by milder procedures. The subject is directed to have the room comfortably warm $\left(70^{\circ}\right.$ to $80^{\circ} \mathrm{F}$.), and to stand in water at $105^{\circ} \mathrm{F}$. so as to avoid chilling his feet by the drippings from the sheet, which would collect on the floor or tub. 'The chilling of these sensitive portions of the body interferes with the reactive capacity. The water to be used is carefully measured by the thermometer and should be about $85^{\circ} \mathrm{F}$. at first, and daily lowered a few degrees at subsequent applications until $60^{\circ} \mathrm{F}$. is reached.

The sheet is grasped by the attendant 6 to 9 inches below the upper hem, and is arranged in a roughly plaited manner, so that he can control it like one does a bandage. The patient then raises both his arms as he faces his nurse and the sheet is placed across his chest and in both axillæ (Plate XI, Fig. 1); he then lowers his arms and places them firmly against his sides, with the sheet intervening. 'This gives a strong hold on the first part of the sheet. He is then directed to revolve, thus turning his back to the operator, who sweeps the sheet across it and above the shoulder, across which it is pulled taut, and in a downward direction over its anterior aspect (Plate XI, Fig. 2). Here the operator's free hand holds it in place, while the hand which holds the sheet sweeps the latter upward over the opposite shoulder, thus making a fold which is kept in place by the tight overlapping edge. Finally, the tapering end of the sheet is tucked into the neck folds after the arms have been completely enveloped. The lower part of the sheet is held between the patient's knees, and, if possible, the elge of the sheet is grasped by the patient's hand to make it still more secure. 'The attendant then rapidly rubs different areas with the flat hands by rapid passes (Plate XII, Fig. 1).

Friction must be energetic, for we have to assist the patient to overcome a large dose of cold. 'This is obtained by active rubbing, slapping, etc., over the different areas, and never ceasing until the sheet warms 


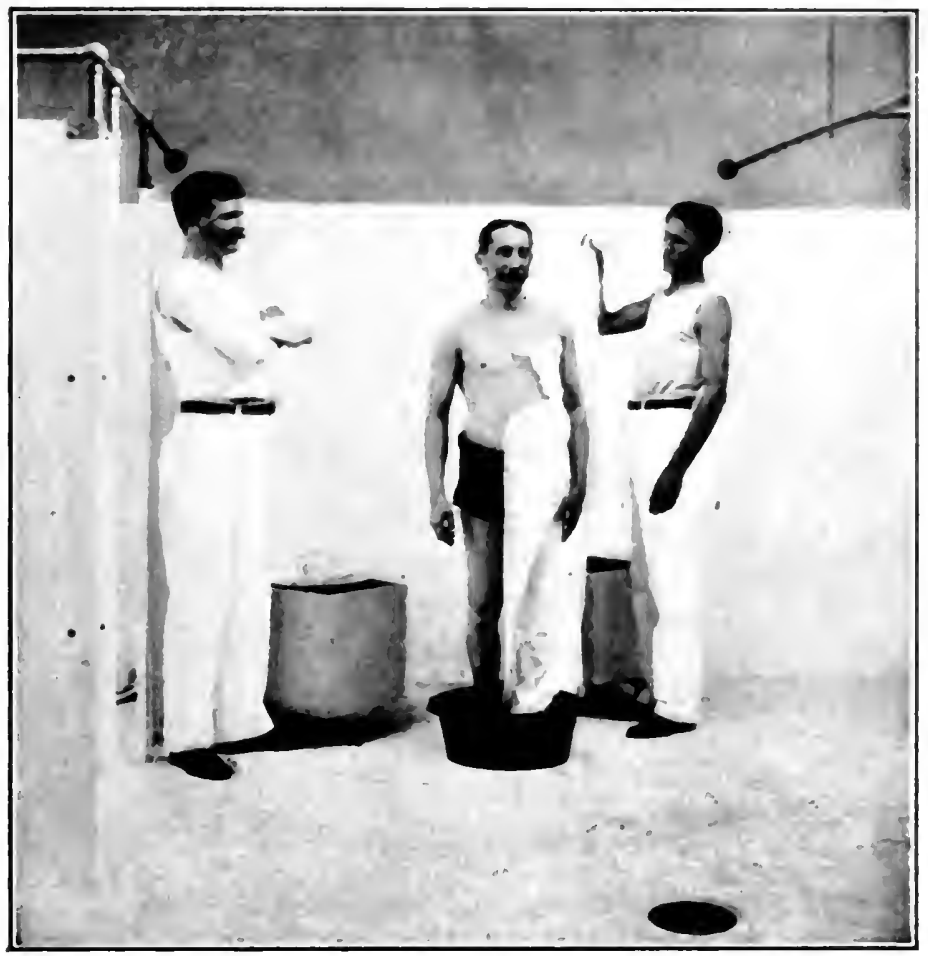

Fig. 1.-Drip sheet. First step.

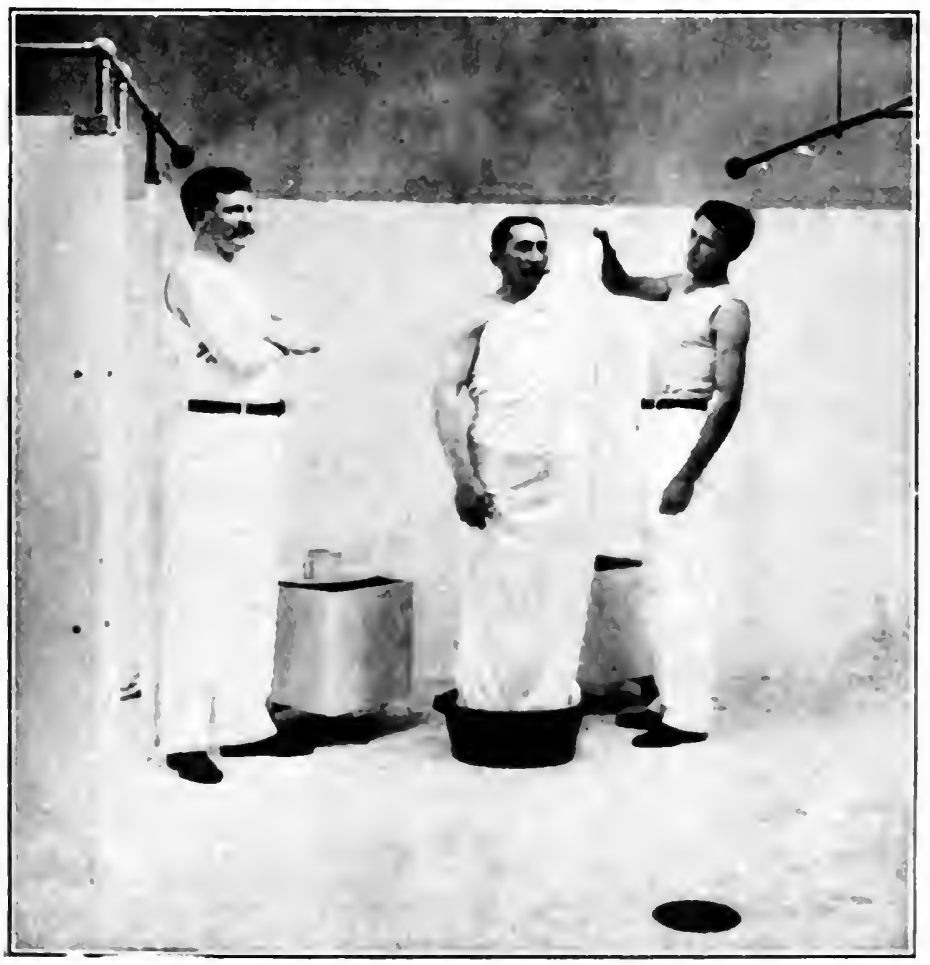

Fig. 2.-Drip sheet. Second step. Patient is directed to turn. 



\section{PLATE XII}

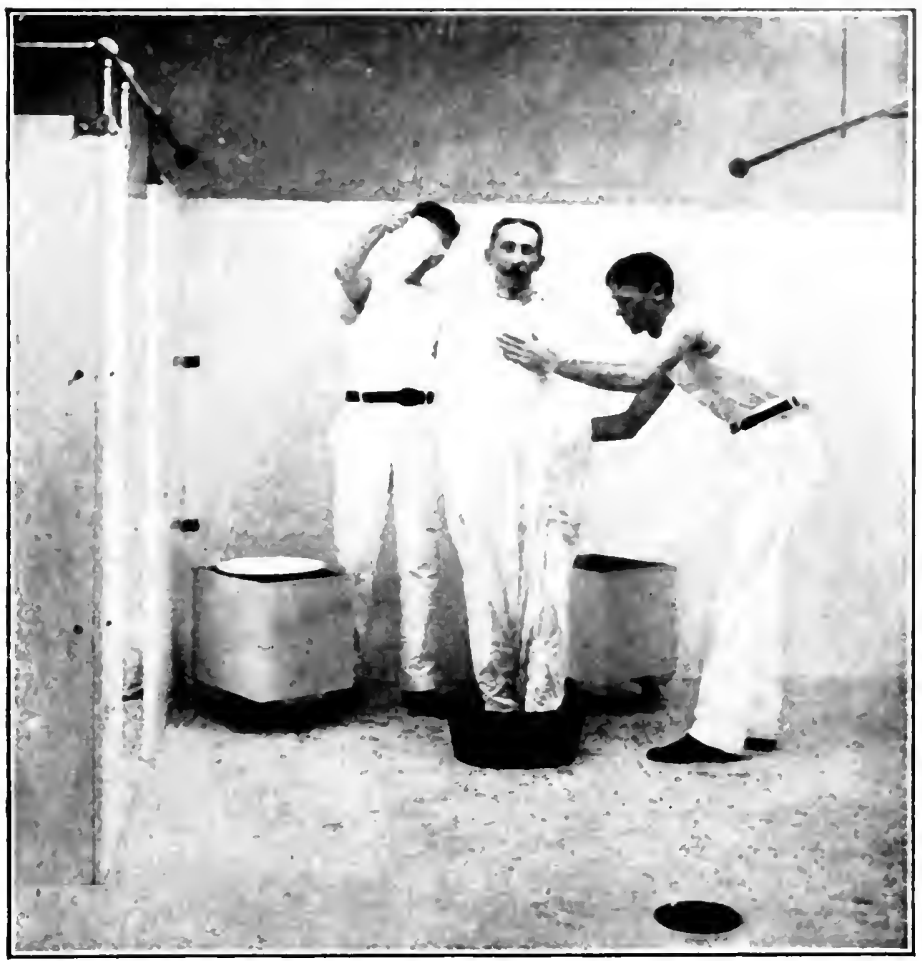

Fig. 1.-Drip sheet. Third step. Affusion and friction.

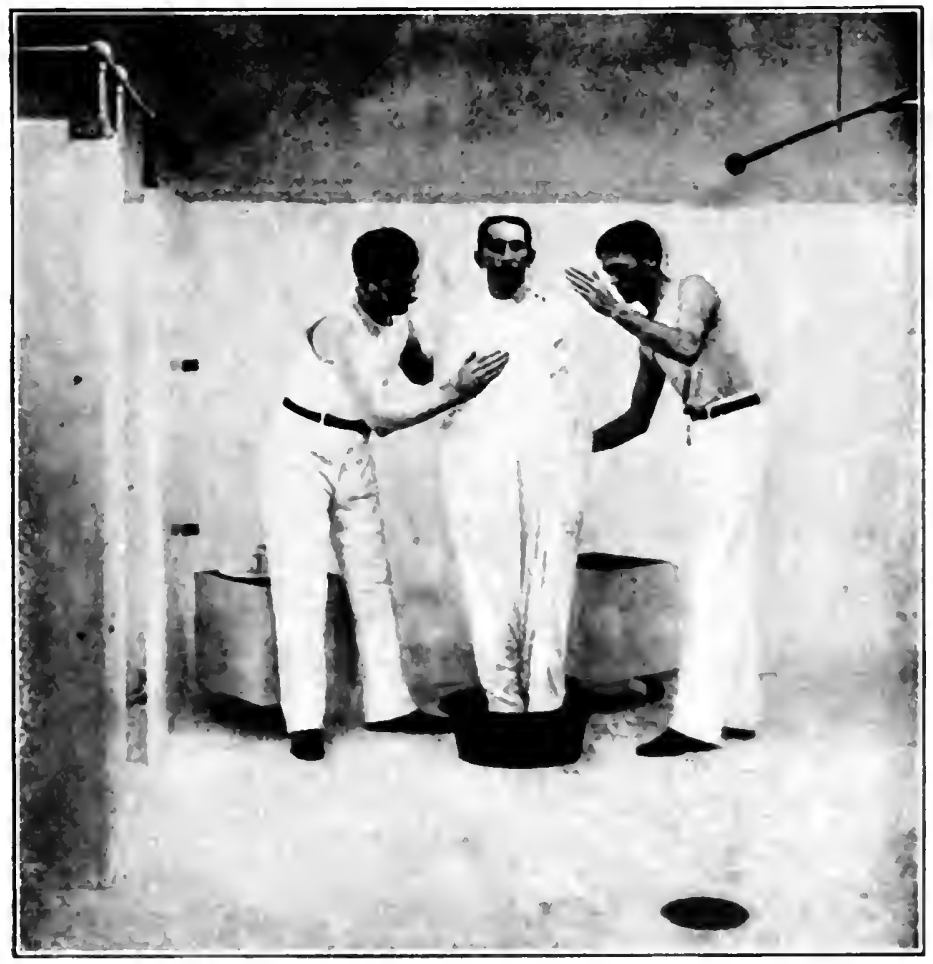

Fig. 2. Drip sheet, Fourth step. Mode of fricion. 



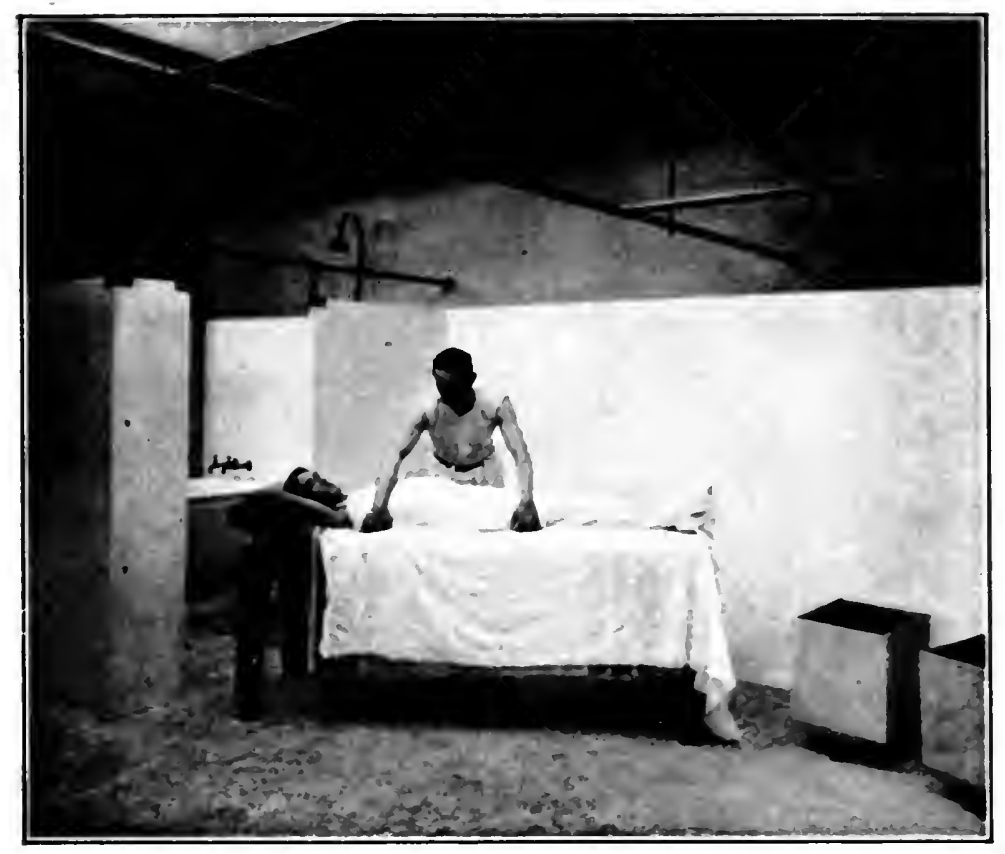

Fig. 1.-Wet-pack. First step. Applying wet sheet.

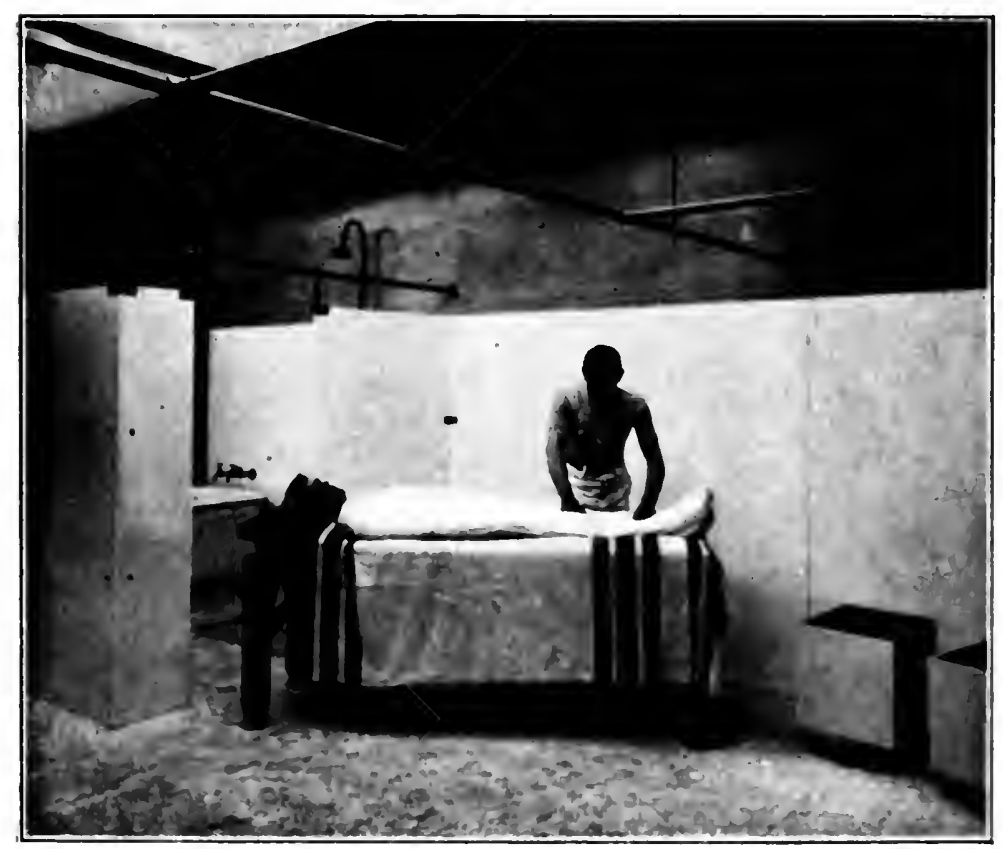

Fig. 2.-Wei-pack. Second step. Sheet wrap completed. 



\section{PLATE XIV}

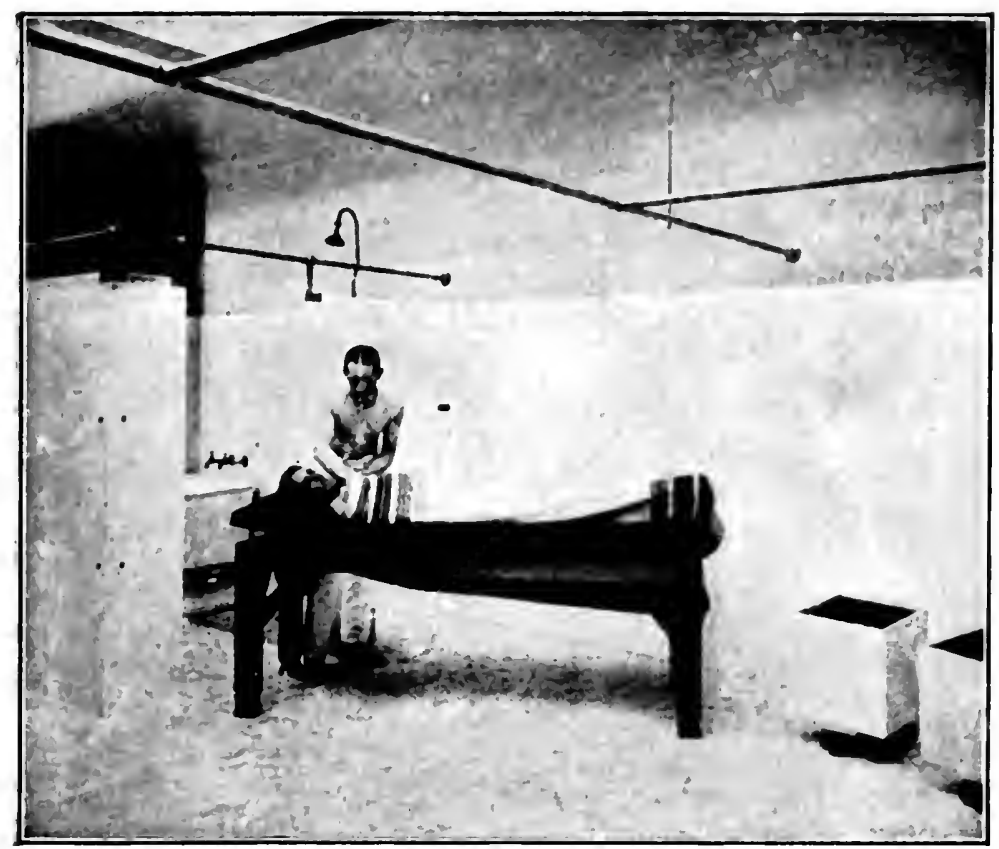

Fig. 1.-Wet-pack. Third step. Wrapping blanket, right to left.

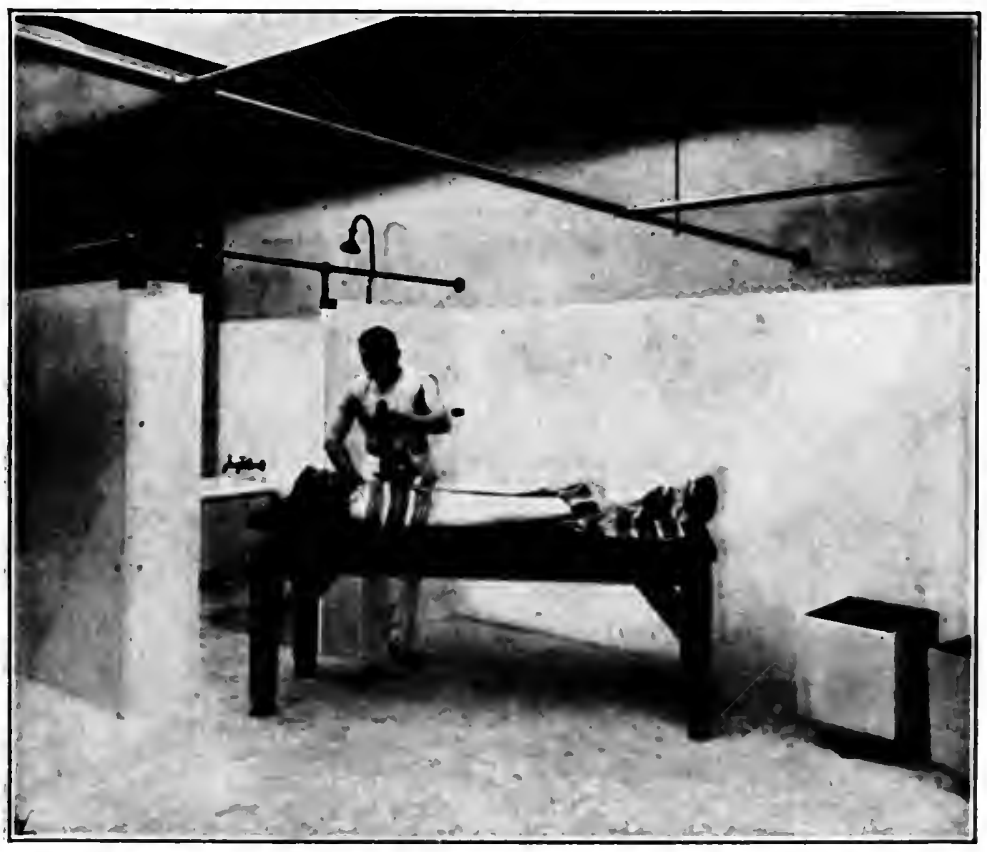

Fig. 2.-Wet-pack. Fourth step. Securing blanket, left to right. 

under these eflorts. 'The duration of this measure varies with the result and the conditions present. It may cease as soon as reaction is started, or it may be doubled or trebled in amount by pouring a basinful of water 5 or 10 degrees lower than that of the original application (Plate XII, Fig. 2), and following this by friction, and again cooling the sheet as many times as seens desirable.

Always cease if chilling is shown by shivering of any great degree, if the teeth chatter, or if the skin fails to warm the sheet. At the conclusion of the treatment the patient is actively dried, dressed, and, if able, is allowed to exercise in the open air. Judgment is necessary in measuring the patient's power to react; too great fatigue should not follow this method, and if it is jresent, indicates a modification in temperature or, better still, in duration of the treatment.

Therapeutic Application.- This is the acme in the course of neurovascular training, and as such is meant to follow the introductory methods, and is applied only to trained subjects or those with a naturally good reaction. It demands more of the subject and does more good than the milder methods previonsly deseribed, provided it is well borne. To obtain good results in neurasthenia or hysteria these bolder procedures seem necessary. Catarrhal diseases of the respiratory or alimentary systems are helped by the use of the drip sheet. A tendency to acute colds, ete., is also lessened by their systematic use. Anemic subjects are decidedly benefited, and show an increased hemoglobin content in their blood. 'The many concomitant symptoms of anemia, like headaches, neuralgias, etc., are consequently relieved.

In nephritis the sheet may be wrung out of water at $75^{\circ} \mathrm{F}$, and good reaction produced by rubling the patient, who, after being dried, is put to bed. Von Noorden particularly recommends this cold rubbing, saying that he knows no procedure which is of greater benefit if a good reaction follows it.

It may easily be seen how the drip sheet may be modified by applying cold water throngh thin towels over limited surfaces of the body, followed by active friction and drying.

The sheet bath, which is practically a recumbent drip sheet (see p. 231 ), is of use in all febrile diseases which show the eflect of toxic bacteria upon the nervous and circulatory system. Incidentally it lowers temperature, thereby lessening the strain on the patient. 'The pulse, if dicrotic, becomes less so; the mental condition is rendered more nearly normal, if there be need of sleep, the patient is soothed and often slumbers. All the eruptive fevers are greatly benefited by this procedure, and the exanthem becomes much more distinct, due to the better circulation in the skin. 'The widespread fear of a poorly developed rash is due to the fact that the toxie state often cause's a poor cutaneous circulation, allowing the skin to become mottled and cyanotic. 'The sheet bath corrects this condition and its eause, thus favoring not only an increased eruption, but also doing away with the cause behind it. 'The lethargy and malaise in influenza, tonsillitis, and other simpler infections are markedly lessened by this means of applying cold. Pneumonia seems to be more benefited by cold compresses, which 
will be described later. Delirium tremens, sunstroke, etc., also are helped by the sheet bath.

The Wet-pack.-'This procedure is quite different from the preceding ones, inasmuch as cold water is here used without friction. The gradations of temperature are not employed, since the only fillip employed is the thermic one, and to obtain reaction the water should be cold enough to cause a response on the part of the skin. Hence temperatures near that of the skin would afford us a nearly useless measure. Another distinction is that reaction depends upon the patient's own response; it is maintained by blankets surrounding the wet sheet. If the reaction is poor, two or even three blankets may be employed. It is inexpedient to employ the higher temperatures when reaction is poor. The technique of the wet-pack should be particularly accurate. A coarse linen sheet wrung out of water at $70^{\circ} \mathrm{F}$; later at $60^{\circ}$ or even $50^{\circ} \mathrm{F}$, as reaction indicates, is placed upon two or more blankets and a rubber sheet, previously prepared on a firm cot. The patient is placed thereon, with the centre of his occiput reaching the upper edge of the sheet and blanket and his feet nearly reaching the lower hem. He is then directed to raise his arms, and one side of the sheet is rapidly placed about his trunk and lower limbs (Plate XIII, Fig. 1). 'Then the arms are placed by the side, and the other part of the sheet is placed about them, and the thighs and legs are separated by tucked-in folds of the lower portion (Plate XIII, Fig. 2). 'Thus coaptation of two skin surfaces is avoided. This enveloping by the sheet need not be smoothly done, inasmuch as it should be done quickly to prevent chilliness; the blanket which is wrapped about it serves to make everything snug. 'The upper end of the blanket on one side is then firmly drawn about the neck and downward, with its upper edge crossing the clavicle at right angles and below the collar-bone for a distance of $\delta$ or 10 inches, where it is held by one hand while the other folds the blanket upward and over the opposite shoulder (Plate XIV, Fig. 1). The whole of one side of the blanket is then tucked snugly under the patient, surrounding trunk and limbs. The remaining half of the blanket is then treated in a similar manner, only reversing its direction (Plate $\mathrm{XIV}$, Fig. 2). The operator then uses considerable muscular force in tightening the woolen wrapping until he has forced out as much as possible the contained air. 'The less air in the pack, the less does the patient give up heat, and, therefore, the more easily does he react (Plate XV).

It is essential that the pack fit very closely about the neek, so that the entrance and egress of air shall be avoided, otherwise there would be a constant chilling and evaporation by the motion of the chest acting as a pump, and constantly propelling the air in and out of the pack. The feet should also be snugly wrapped, except in feeble patients, where the blanket alone surrounds them, the wet sheet stopping at the ankles. 'The duration of this procedure is from thirty to sixty minutes, and if the patient sleeps, he may remain undisturbed until he awakens. If it is used as an antipyretic measure, it may be repeated every ten minutes until four or five packs have been given. Liebermeister has estimated that five of these packs equal the full tub-bath of fifteen minutes' duration 


\section{PLATE XV}

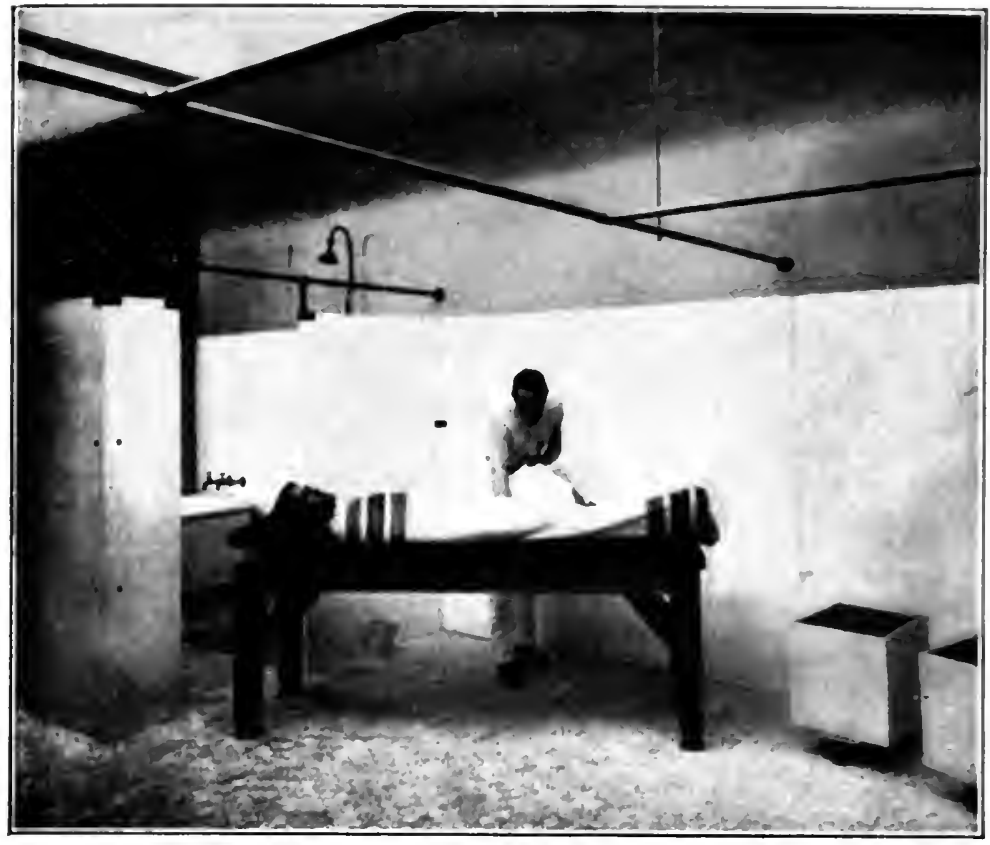

Fig. 1.-Wet-pack. Fifth step. Mode of tightening blanket.

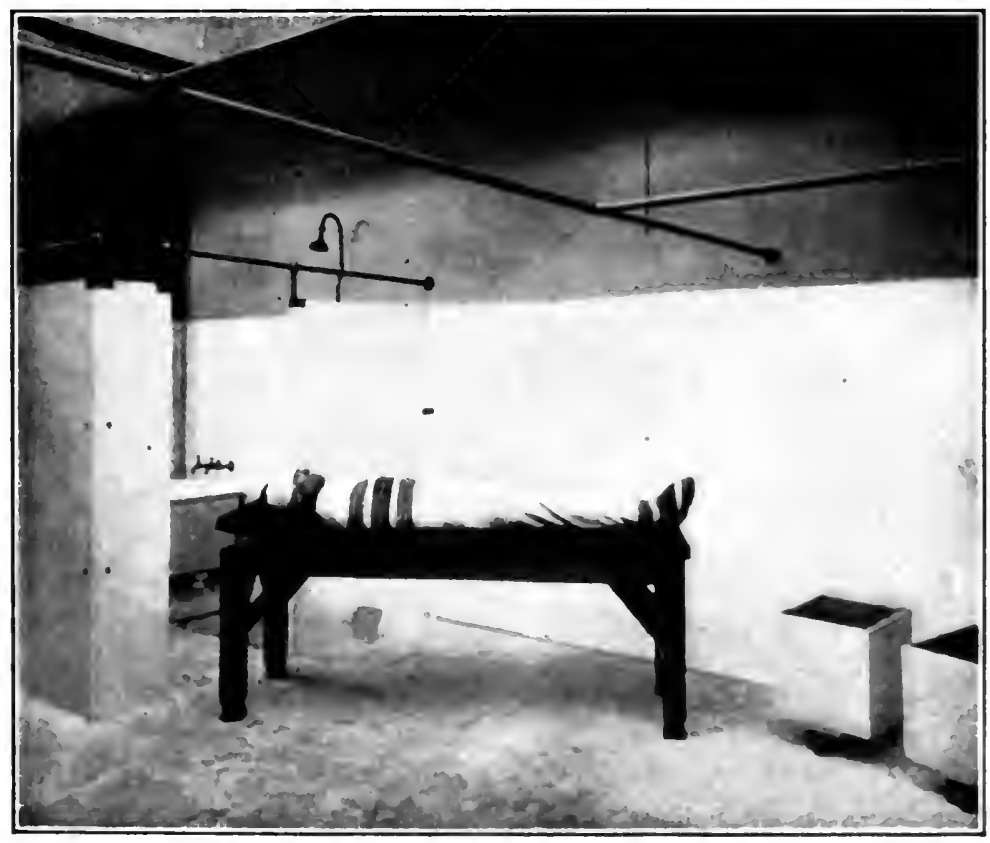

Fig. 2.-Wei-pack complese. 

in their ability to abstract heat. If reaction is poor, three blankets may be employed, removing the second and third ones at ten-minute intervals, but leaving the innermost blanket in place. At the termination of this measure the patient is rubbed with alcohol or cold water.

'The modus operandi of the wet-pact is as follows: 'I'he blood-vessels of the skin are suddenly brought under the stimulating effect of cold water about 30 or 40 degrees below that of the boly surface. 'lhere is a primary compression by contraction of the skin muscles and a temporary" increase of the blood content of the interior of the body. Along with this is a refreshing and stimulating effect on the whole nerions system, which encourages it to perform its work more normally and energetically. 'The patient perceives a sense of chilliness, which may last a few minutes, but soon ceases. Following this primary compression of the blood-vessels there is a reactive hyperemia of the skin, and blood is supplied to the surface in an active manner, which results in a lessening of internal congestions of the brain and other viscera. With this reactive tonic hyperemia heat is brought to the surface in a degree which is greater than if a pack were given with water at the shin temperature. Consequently the cold water ceases to be cool, and is warmed to about $95^{\circ}$ F., and the patient, after ten minutes' time, is enveloped in a vapor bath slightly cooler than the blood of the interior. If only one blanket is used, the vaporization of the water and the radiation of heat through the enveloping medium go on at a rate which is just sufficient to act as a soothing influence on the periphery and thus promote sleep. Should more blankets be used for any length of time, the patient would be in a warm pack, he would begin to perspire, and the hypnotic influence would be nullified. It would seem as if this increased blood-supply to the skin might act as an aid in the eliminative power of the sweat-glands, and the odor which often ensues in uremia and other toxic states is of some value as an evidence of this fact.

Therapeutic Application.-It is thus made evident that the manner in which the pack is given has a distinct bearing on its therapeutic uses. If it lasts but ten minutes or is repeated every ten minutes, the chief effects are nervous and circulatory stimulation. If it remains undisturbed for a longer period, there is a succeeding calmative effect, and often the subject sleeps. If it is surrounded by three blankets and the patient left in it for a longer period, it becomes a warm pack and induces perspiration, vasomotor relaxation, and increased metabolic activity. 'Therefore, it is wise to specify what method should be followed with an exactness that cannot be too strictly enforced. As an antifebrile agent, it may be used in all the acute infections and toxic states, such as scarlatina, delirium tremens, tonsillitis, etc. As a soothing hypnotic agent it is of use in insomnia, restlessness, the sleeplessness of fevers, autointoxication, and as an eliminant in nephritis, eclampsia, ete. When several blankets are used, this procedure has the advantage of producing a primary tonic hyperemia of the skin, whereas the hot blanket pack does not do so. 'The skin is thus stimulated to maintain the warmth of the pack, and requires less external aid from hot-water bottles, etc. 
In the various forms of insanity, paresis, mania, melancholia, it serves to induce sleep, improves the functions of the body, and often hastens a cure, if such be possible.

Wet Compresses. - This method of applying cold water is simply that of the wet-pack, but limited to smaller areas. 'The same general principles are to be followed and the same effects are to be expected, but in a minor degree. One or two layers of old linen cut to fit the part (chest, abdomen, joint, etc.) are wrung out of water at about $60^{\circ} \mathrm{F}$. If a towel is used, it should be without fringes, which promote dripping and an uneven distribution of the cold. The linen having been placed snugly over the area selected, is held in place by a single layer of flannel, which must extend beyond the areas covered by the wet compress. An inch margin is sufficient. 'The flannel is then secured by safety-pins, so that it confines the body or limb snugly, without constriction or interference with breathing if placed over the chest. No air should be allowed access to the wet linen, else it would evaporate the contained water too rapidly, and, therefore, chill the part and interfere with reaction.

The duration of each application varies according to the power to react, etc., this differing in each patient. It should never be removed until it is thoroughly warm, and the nurse should be well instructed on this point. Ordinarily, thirty to sixty minutes will be required, and a rough rule of one-hour intervals generally meets the condition. No harm results from a more prolonged period, since they dry and become ineffective. Sleep, of course, contraindicates their removal. One of the drawbacks to the continual application of wet compresses is the fact that they favor the infection of the skin, and pustular eruptions may follow. This may be obviated by keeping the linen scrupulously clean by boilin: it daily. A very common error on the part of the nurse is to cover nis application with oiled silk to prevent wetting the bedclothes. This, of course, converts the cooling, stimulating application into a poultice wi h heat retention, and the skin becomes cyanotic and its circulation sluggish. It thus defeats the purpose of the procedure. The same modifications may be employed as in the wet-pack. If reaction is poor, two layers of flannel may be employed. If stimulation to the circulation, respiration, etc., is desired, colder water may be substituted and more thorough wringing out. If stupor or delirium be present, the same modification is indicated.

The Chest Compress.- To obtain the full benefit of this measure the linen and flannel should be so fashioned that they fit the individual properly. For a man, an old waistcoat will serve as a pattern, making the overlapping parts somewhat longer. A pattern of this is diagrammatically represented in Fig. 40. It should be so fashioned that it reaches from the clavicles to the navel. Of course, the flannel binder extends about an inch beyond the linen at all places, so as to exclude air from the water-soaked linen. Since this measure is particularly applicable in pneumonia, it is important that it be applied carefully, and with a minimum of disturbance to the patient. 'Todo this, a large towel should be placed upon a table, and upon this in turn the dry flannel and the wet 
linen. One-half of this many-layered dressing is then rolled tightly, and the patient is carefully turned upon his side and this half inserterl close to him on the bed; he is then turned in the opposite dirertion, while the layers are unfolded on the rolled-up side. The patient is then replaced on his back and directed to raise his arms. 'The wet compress is rapidly' swept over the chest and shoulders, and in turn secured hy the flanne] binder and safety-pins. The towel is left flat below, to absorb moisture and protect the bed. When the linen is to be changed, the simc process should be followed, leaving the towel and binder flat on the bed, beneath the subject, unless too wet, when they should be changed. 'These changes should be made about once an hour.

It has been claimed that this frequent disturbance may be detrimental. If the patient is allowed to make no effort, all the work being done by the nurse, no harm results, and in a mild way the changes in position may serve to lessen hypostasis, which results from a long-continued recumbent position. No renewal should be

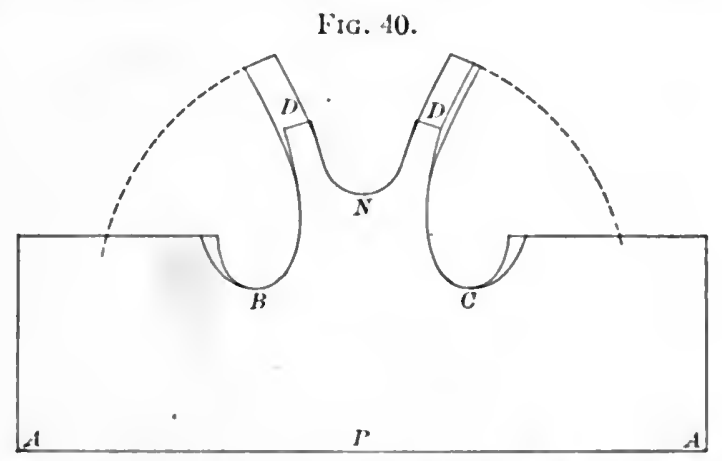

$\mathrm{N}=$ notch for back of neck. $\mathrm{B}$ and $\mathrm{C}$ for the axilla. $\mathrm{D}=$ shoulder straps. $\mathrm{A}=$ anterior. $\quad \mathbf{P}=$ losterior.

attempted unless a thorough warning of the compress has taken place. If at the end of an hour reaction is not evident, the wet compress may be removed, and only the dry binder left in place until the skin is again warm. When this takes place, the skin may be gently stimulated by friction and the compress reapplied, more thoroughly wrung out, or using thinner linen, thus using less water and demanding less of the patient's power to react.

Therapeutic Application.-'The chest compress is indieated in acute pulmonary troubles, pneumonia, acute bronchitis, phthisis, etc., where we wish stimulation and a revulsive action on the part of the circulation in the thorax. When first applied, there is a deep inspiration, which helps the heart to force along the bleod and helps the bronchi to rid themselves of mucus, as well as the minor advantage of increasing the oxygen supply to the system. It stimulates the nervous system to more normal action, particularly in preventing the overwhelining toxemia which is the eventual cause of vasomotor and even cardiac failure. 
The lessening of the congestion in the bronchial tubes favors the abatement of cough. Of course, it has no effect on the exudative inflammation, but simply serves to protect the patient from the toxic effects which are eventually the cause of death. 'The mechanical obstruction of the lobes in pneumonia can well be left to itself if the toxemia is successfully combated. The slight reduction of the temperature is of less importance, yet it scems to save the heart from beating so rapidly and to make the patient more confortable.

In chronic pulnonary conditions like tuberculosis and bronchitis, the compress may be applied only at night, and left in place until awakening occurs. Before removal, after arising the patient may have a cold ablution, sufficiently cold to produce a tonic reaction, so that they may not miss the warm covering after a night's wear.

The Abdominal Compress.- This procedure is employed in a similar manner to the foregoing, but is simpler in design. A towel is placed upon the bed, and in turn upon this is laid the flannel. The somewhat smaller layer or layers of wet linen are so fashioned as to reach from the xiphoid cartilage to the anterior superior spines, and to the postaxillary lines on the sides. Generally, the linen is not applied to the posterior aspect. The same rules for temperature, intervals of removal, and careful cleanliness are as necessary as in the chest compress.

Therapeutic Application.-'The abdominal compress is a valuable application in all febrile affections, and its hourly renewal serves to supply a gentle yet frequent stimulant to the whole nervous system, and a continuously acting antipyretic. Its local effect is also of value in maintaining the tone of the intestinal peristalsis and a lessening or prevention of tympanites. It may be used with or without the full bath. In cases of gastritis, ulcer of the stomach, chronic colic, enterocolitis, and in some cases of appendicitis it has proved useful. In neuroses of the gastro-intestinal canal it may prove of signal service. In ambulant cases it may be worn night and day, renewed only when dry, and maintained, if necessary, for weeks at a time. Cases of hyperemesis gravidarum may also be benefited by these applications, whether the patient is in bed or up and about.

Other Local Compresses.-Following the same technique, it will be found that the wet compress may be of use in such cases as acute tonsillitis, acute articular rhemmatism, acute gout, cellulitis, and other local inflammations. The more chronic inflammations show less benefit, but are improved after longer treatment. In subacute or chronic rheumatic or gouty synovitis a compress applied over the inflamed or tender joints is of special value in redueing hyperemic conditions.

Continuous Cold Applications. - These differ from the cold compress in that reaction is not songht. 'The skin and the underlying tissues for a short distance are chilled, and reflexly there is an apparent effect on the decper viscera. Care should be taken that the cold should not be maintained long enough to jeopardize the vitality of the area over which it is applied. A good rule would be to allow the cold to be active for forty-five minutes, and then removed for a quarter of an hour or more if 
the circulation is feeble. The ice-coil is the usual menus of maintaining cold. Several layers of damp linen are to be placed over the skin, and the rubber or metal coil is laid upon them. A bucket of ice water supplies a continual cold current, which is propelled by siphonage so regulated as to be not too rapid, and, therefore, not too cold. An ice-lang ma act in a similar manner, and should be removed at stated intervals for the same reasoll.

Therapeutic Application.-In pericarditis we adopt this method almost as a matter of routine. It strengthens and slows the heart's action, and seems to shorten the inflammatory process. I ess brilliant results are noted in endocardial troubles, and in myocarditis it is best omitted or used with great caution. In some of the neuroses it has proved beneficial. In paroxysmal tachycardia, however, it is of very doubtful value.

In appendicitis the use of continuous cold seems to add greatly to the paticnt's comfort, probably by lessening peristalsis in the inflamed area, and by its local anesthetic effect, which is superior to the morphine treatment, because it favors the employment of satisfactory palpation, and possibly may lessen the chance of peritonitis by localizing the disease. Surgery has reached such an efficient point to-day that local treatment is not regarded with favor, since the golden moments for interference may be frittered away.

The Cold Friction Bath.-This important therapeutic measure consists in a complete immersion of the body up to the neck in water below the temperature of the skin, and in the simultaneous employment of active friction to the skin surfaces. 'The term "friction bath" has been devised (Baruch), and this important feature should never be omitted. Without friction the bath is rarely a success, except in robust people in health.

The tub being filled with water at the prescribed temperature, the patient is lifted into it with care. During the bath, which may last from five to twenty minutes, the entire body should be rubbed with a large sponge or soft towel crumpled in the hand.

The temperature of this bath is adapted to the indications of each case. Repetition is also practised in accordance with the temperature, pulse, and general condition of the patient-usually from three to six hours. The graduated bath, beginning with $90^{\circ} \mathrm{F}$. and reduced rapidly by adding ice-water along the side of the tub to $80^{\circ}$ or $75^{\circ} \mathrm{F}$, is used by some physicians as more agreeable. The technique and rationale of the cold friction baths are more fully described on p. 250. For temperature reductions a prolonged graduated bath of moderate temperature is more effective than a shorter and colder bath. Hence the graduated bath is most useful as an antithermic agent, the sudden bath of $75^{\circ} \mathrm{F}$. or lower, for nerve and cardiac refreshment.

The Bed Bath.-Many methods have been suggested for making the bed into a bath-tub when, on account of crowding or expense, the tub could not be used. One of the simplest of these procedures is that of A. C. Haven, who suggested the tying of a clothes-line to the -four posts 
at a definite height above the mattress, and making this line very taut. Over this, and sinking onto the mattress, may be placed oil-cloth or rubber sheeting, and held in place by clothes-pins. This may be emptied by siphonage or by letting down one corner and draining into a bucket. Where there is insufficient nursing or the patient is unduly heavy, this substitute may well be employed.

Therapeutics of the Cold Friction Bath.-In all febrile diseases presenting a rectal temperature of $103^{\circ} \mathrm{F}$. or over, in septic cases, medical or surgical, the cold friction bath may be of advantage and often turn the tide in the course of the disease. It arouses the overwhelmed nervous, circulatory, and eliminating systems to energetic activity as no other agent can. Beginning with a bath of five minutes at $80^{\circ} \mathrm{F}$, repeating at $75^{\circ}$ or $70^{\circ} \mathrm{F}$, and then increasing the duration of the baths gradually, is the proper way to proceed, always aiming for the longest and coldest bath that is not followed by prolonged chilling. The practitioner will find the bath valuable and free from the cruelty with which it has been charged by those unacquainted with its correct technique. In the ephemeral fevers of children, the etiology not always being clear, the graduated cold friction bath of ten minutes is extremely useful, beginning with a bath at $95^{\circ} \mathrm{F}$. and rapidly reducing the temperature by the addition of cold water. Such a bath, administered with active friction with a large sponge, often reduces temperature and affords refreshing sleep.

Instead of ordering aconite, sweet spirit of nitre, ten-minute baths from $95^{\circ}$ to $80^{\circ} \mathrm{F}$. every four hours may prove of great value until the diagnosis is established. Not the least advantage of such cold friction baths is found in disabusing the minds of the patient and the parents of the apprehension so often existing and accustoming them to a procedure which may become absolutely essential to recovery.

Hammock Bath.-'This term is used with reference to the device employed in suspending the patient in a bath, so that he may lie there in quiet comfort without making any effort to balance himself, and without undue pressure on any part of his body. It is adapted for prolonged procedures, and generally is used with water at an indifferent temperature, $i$. $e$, about that of the blood.

Before the tub is filled with water at from $95^{\circ}$ to $100^{\circ} \mathrm{F}$., to a height sufficient to submerge the patient with the exception of the head and neck, a sheet is arranged so that when the patient lies upon it, it clears the bottom of the tub. If the edges of the tub protrude, two or three dozen clothes-pins (previously made wet, so that they may hold better) keep the borders of the sheet in position. Or if this method should prove impracticable, several handages may be sewn on the edges of the sheet and, by passing them below the tub and tying them, the sheet may be raised or lowered at will. Across the head of the tub a towel may be similarly placed, so as to serve as a head-rest. The main object in view is to make the patient thoroughly comfortable, and thus avoid all external irritations, which would serve to annoy or irritate. The patient is kept in this position for an hour or hours, and even for 
months, and it is essential that no strain should be put upon him. If the tub is built into the wall, it may be necessary to construct a worden frame to fit over its borders, and have the sheet suspended therefrom.

In non-febrile cases the bath is to be mantaind at $10(1)^{\circ} \mathrm{l}$., and never allowed to become cooler than $95^{\circ} \mathrm{F}$. Consequently frequent renewal of the water is necessary, and careful thermomotric observations must be made. Placing a blanket over the tub will aid in lessening the need of frequent additions of hot water. 'The patient must have constant attention to prevent accidents and to keep the water in the right condition. If possible, the patient is removed to answer the calls of nature, but if this is deemed unwise, the water may be removed and a fresh supply afforded. The feces and urine should, of course, be removed for asthetic and aseptic reasons.

'The effect of this bath is a negative one. Exterual changes of temperature are reduced to a minimum. 'The peripheral terminations of the nerves in the skin are, therefore, not irritated by heat or cold, and are possibly soothed by the succulent condition of the skin, and by the sedative effeet of equal pressure by the surrounding water. After the primary effect of submersion has passed off, the respiration and pulserate and temperature show a remarkably steady record, and the patient will become drowsy. The duration of this procedure must, of course, vary with the disease treated and the result obtained.

Therapeutic Application.-This procedure is particularly applic: able to cases of cerebral excitement, and as such has bccome popular in many insane asylums, and in the alcoholic wards of general hospitals. In acute mania or maniacal conditions, delirium tremens, extreme restlessness, a diminution of excitement follows the use of the indifferent temperature in the form of a hammock bath. In chronic organic diseases of the central nervous system, myelitis, locomotor ataxia, chronic meningitis, etc., good results are reported in the relief of lied-sores, contractures, and other sepuels. Riess, who used this procedure very often, claims to have noticed in over 100 cases, an improvement in over 50 per cent., not only in the symptoms, but in the disease itself. Cases of pemphigus, extensive burns, extensive suppurating wounds, crstitis, chronic diarrhoa, cancer of the urogenital tract, ete., maty be relieved. It has been used in typhoid fever at a temperature of $90^{\circ} \mathrm{F}$., and seems to shorten its course, according to the investigations of Riess. It has the advantage of being more easily borne, and of being more comfortable than the cold bath, and should be tried when the latter is not practicable. Barr, of Liverpool, treated 55 suecessive cases of trphoid with a bath at $93^{\circ} \mathrm{F}$., with but one death, maintaining the treatment while the temperature was above $100^{\circ} \mathrm{F}$. In acute nephritis there follows an increased excretion of urine, of nitrogen output, and chloride of sodium. The effect on the albumin was variable. In these cases one or two baths a day are to be administered, each lasting an hour and followed by rest in bed. As an antithermic agent, this bath of $95^{\circ}$ to $100^{\circ} \mathrm{F}$., if maintained for an hour or longer, gives excellent results. It is superior to the cold bath of shorter duration.

Vol., I. -16 
The Hot Bath Pack.-This name has been given to the most effective procedure which can be employed to cause sweating, oxidation of tissues, and the lowering of blood-pressure. The method to be employed is as follows: A bath-tub is filled about two-thirds full of water at $102^{\circ} \mathrm{F}$, and the patient is directed to enter it and keep his limbs and trunks completely submerged during the treatment. As he becomes accustomed to the heat, more hot water is rapidly added, and the temperature is gradually raised to $106^{\circ}, 108^{\circ}, 110^{\circ}$, or even $115^{\circ} \mathrm{F}$. The tolerance for heat varies in individuals, some bearing the higher temperatures for many minutes. This hot bath is maintained for five, ten, or fifteen minutes, provided the patient does not feel faint.

If observations be taken during the bath, the pulse will gradually become quicker and softer. A normal pulse of 70 will often be increased to 120 , and the blood-pressure, measured by Janeway's sphygmomanometer will show a decrease of 15 millimeters of $\mathrm{Hg}$. The mouth temperature will gradually rise, and often registers $102^{\circ} \mathrm{F}$. or over. The skin becomes filled with blood, and this portion of the body being the hottest, the temperature is steadily increased in the interior by the blood which has passed through the skin. It may readily be seen that the longer the bath, the greater the increase of temperature. 'The brain often shows the result of cerebral anemia, and the patient may complain of dizziness or faintness, which are signs for a cessation of the bath. This bath, when maintained, becomes depressing, and after the first few minutes the primary stimulation from heat administered to the skin passes away, and the patient will become exhausted if the procedure is prolonged. To prevent faintness the patient should rise slowly. So soon as he steps from the bath he is wrapped immediately in a hot sheet, which has been previously prepared in order to prevent the cooling effect of the surrounding air and the evaporation from the wet skin. He then lies upon one, two, or three blankets, which are wrapped snugly about him by the same method that has been described in the wet-pack. By following this order and technique, there is produced a heat accumulation sufficient to warm the whole body, periphery, and interior, and this heat stasis is maintained by obstructing all outlets by which heat may escape, except that of the lungs and head. There is a gradual escape through the blankets, but it is very little. The sweat-glands of the patient soon show the effect and act copiously. $\therefore$ 'The pack is kept intact for thirty to sixty minutes, and in order to refresh the patient and to promote perspiration a cold turban is wrapped about the forehead and small amounts of ice-water ( 2 ounces every ten minutes) are administered throughout. At the conclusion of the treatment the patient is rubbed with alcohol or given cold-water $\left(60^{\circ} \mathrm{F}\right.$.) affusions, and if the weather is cold, he should remain indoors for at least an hour.

It may readily be seen that there is a strong therapeutic agent in the hot-bath pack. Elimination by the sweat-glands reaches its maximum, lessening of internal congestions must necessarily follow such a filling by blood as is noticeable in the skin, for the skin can hold over one-half of the blood in the body. Oxidation of tissues must be enhanced by 
this high temperature of the body; therefore, metabolism is greatly stimulated, and the products are more thoroughly eliminated. 'There is such a readjustment of the supply of blood that a change must occur in every organ of the body, and the pressure at which it circulates is markedly lessened. It is a safer agent than pilocarpine, and if there is a danger-signal, may be immediately counteracted by removal and the dashing of cold water upon the patient.

Therapeutic Application.-In cases of gout, chronic or acute muscular rheumatism, some cases of autointoxication, lumbago, sciatica, neuralgias, etc., this method often meets with brilliant results. In stiffening of muscles or joints the patient should be manipulated while in the hot bath, for here the heat acts as an ansesthetic, and massage and passive or active motion is rendered both free and less painful. After fractures, long immobilization from bad treatment, or painful neuritis, the joint may be relieved from pain and its function restored. Sometimes, if the contracted tissues are tough and much stiffened, the improvement is very gradual, but none the less sure. In cases of obesity the oxidation of tissues causes a loss of weight, as well as the loss of moisture. In cases treated at the Vanderbilt Clinic a difference in weight of four pounds has been noted after a single hot-bath pack, so great was the sweating.

When using such a powerful agent, the physician should recognize the elements of danger in it. For instance, in weak, emaciated patients the method is too strenuous to be used effectively without gradually undermining the subject's strength. In cases neither robust nor weak, one or two treatments a week may be ordered, and some form of tonic cold treatment given on the intermediate days. Rarely should we use them oftener than once every other day. Sometimes the beneficial result is not immediate, but is more noticeable after the course of treatment has been discontinued.

In nephritis, acute or chronic, hot packs may be given more frequently. Here they act as eliminating agents, vasomotor relaxants, and serve to relieve internal congestions. 'They must be used cautiously so as not to overtax the patient's strength, and are contraindicated where there are low blood-pressure and weak heart action. At the same time the writers have noticed that they are better borne than the so-called hotpack, made from wringing out blankets in hot water. 'This latter procedure ceases to be hot after a few moments, and the skin does not receive the stimulating shock of the heat to a sufficient degree. Consequently an inspection of the cutaneous circulation after a hot bath will demonstrate an active hypersemia if it is not maintained over a few minutes, while after a hot blanket pack the skin appears mottled, showing a sluggish circulation, a condition most apt to appear in exhausted or poisoned patients.

In nephritic or gall-stone colic there is a distinet anusthetic effect, and probably an antispasmodic one. 'The writers know of patients who have recourse to such baths upon the onset of pain and before they obtain medical aid. 
In acute catarrhal diseases in the early stages this procedure seems to shorten and lessen the severity of the process, probably by lessening the internal congestions and subsequently restoring the natural resistarce of the tissues.

Hot Fomentations.- The technique of this procedure is simple, yet should be followed closely if accident is to be avoided. Four layers of woolen blanket are cut to the shape of the area to be treated and sewn together so as to form a pad. Two of these pads should be applied at one time after they have been saturated with boiling water and thoroughly wrung out by an effective wringer. A homely instrument for this purpose may be devised by a broom-handle sawed in halves and a stout piece of linen attached to them. By placing the pads in the linen and twisting the broom-sticks in opposite directions, nearly all the water may be expressed, leaving the pads impregnated with only a very hot vapor. 'The region over which they are to be applied is thoroughly anointed with lard or some other ointment, so as to prevent scalding. It is wise to allow the pads to be exposed for a few moments to the cold air, in order to test the sensitiveness of the skin of each individual, for this varies a great deal. Both pads arc then applied to the anointed surface and kept in position by a blanket upon which the patient lies. This adds a mild hot pack to the treatment, which may be of service as an eliminant. After five or ten minutes two or more steaming pads are applied, and the same procedure is repeated at the same interval until three or four applications have been made. If the patient's skin proves tolerant, the primary cooling of the pads is gradually lessened and finally omitted.

Therapeutic Application.-These local applications are of service in cases of lumbago, sciatica, stiffened muscles, etc., where the heat serves to dissipate the exudate causing the trouble, and also serves as an anæsthetic, such as may allow passive or active movements and massage, which otherwise would not be possible. The moist heat acts as an excellent conductor, and may produce pain at the moment of application. The old household remedy of several layers of flannel and a hot flat-iron serves a similar purpose, providing both heat and massage. The addition of massage is of prime inportance, since it may serve to complete the dissipation of exudates, change the circulation in the part treated, and enable the patient to start active motion, which may complete the cure. Immobilization of inflamed muscles seems to cause the trouble to persist.

The Hip Bath.-In using this bath, the so-called sitz-bath, the only things necessary are a tub, some oilcloth on which to place it, a blanket, and a thermometer with which to measure the temperature of the water. 'The patient is to be placed in a comfortable position, with the hips submerged in the bath and the knees, legs, and upper trunk outside, but properly protected by blankets if the room is cool. If this last precaution is not taken, a chilly sensation is engendered on account of the lack of friction over these parts. The submerged parts must be vigorously rubbed by the patient and the attendant. 'The rationale of a hot hip bath $\left(104^{\circ}\right.$ to $115^{\circ} \mathrm{F}$.) depends upon the relaxing effect on the arteries, with a 
subsequent fall of blood-pressure and acceleration of the pulse-rate. If a cold one is used, $-85^{\circ}$ to $75^{\circ} \mathrm{F}$,- - the reverse is true. Both heat and cold, however, have the common result of redistributing the blood-supply, and affect particularly the splanchnic circulation. 'There is a noticeatble increase of blood in the head with the lower temperatures, and a cold turban should be placed about the forehead to prevent the discomfort naturally resulting therefrom. The effect is chiefly reflex and partly hydrostatic.

Therapeutic Application.-In cases of unduly prolonged menstruation, where surgery cannot be of avail, this measure is often most useful. 'The cold sitz-bath at about $85^{\circ} \mathrm{F}$. is to be preferred in these instances and maintained for five to ten minutes with active friction. A general affusion of 10 to 15 degrees colder temperature will serve to complete the treatment, with added benefit. It is wise to begin these baths after the normal number of menstrual days has passed, but if this cannot be discovered, no harm is done if they are used when the discharge seens to be decreasing. By saving the patient's blood from excessive loss the anemia and exhaustion are lessened, and the nervous system has a chance to regulate itself.

In certain intestinal catarrhs, diarrhoa, when persistent, and abdominal neuroses, these cold hip baths act as a stimulant when of brief duration, but if prolonged, cause relaxation and a lessening of functional activity. Constipation is sometimes benefited, especially if accompanied by massage of the colon, arousing the activity of the latter by both thermic and mechanical means.

The indifferent temperatures are particularly indicated where there is a hypersensitiveness of the bladder, a neuralgic dysmenorrhoa, etc. Here the calmative effect predominates.

The hot hip bath gives the primary stimulating effect, acting therein like cold, but this is not maintained, and is soon succeeded by the antispasmodic and detergent effect, and consequently may be employed where these results are sought. 'Tenesmus, chordee, painful hemorrhoids, urethritis, ete., seem at times to be much relieved.

The Douche.-'This procedure consists of a nozzle of varying size connected to a flexible hose, which in turn is connected to a water-supply in such manner that considerable pressure may be obtained from it. 'This pressure will vary with the height of the supply and the size of the nozzle. In consequence of good pressure we have a percussing and massaging effect on the deeper structures, as well as the mechanical stimulation of the skin. Of course, the nearer the patient stands to the outlet, the stronger the impact. Hot or cold water and steam may be used singly or in combination, in accordance with the effect desired. By the use of the douche we, therefore, have the effect of heat and cold; of mechanical irritation of the skin, such as is provided by friction in other cold-water procedures, and the massage effect. The general usefulness of cold over the whole body, with its consequent nerve refreshment, is added to the local effect, such as is most marked in the alternating cold and warm (the so-called Scotch) douche. If merely the effect of the 
cold and mechanical. impact are desired, the so-called shower bath, if supplied with sufficient pressure, answers the purpose well. 'Technically, this is called a rain douche, or if the apertures are arranged so as to point to a common centre in which the patient is placed, it is called a circular douche. If the jet douche is caused to spread by placing the index-finger over the outlet, the term fan douche is generally used. This, of course, lessens the percussion effect.

The temperatures employed vary between $115^{\circ}$ and $40^{\circ} \mathrm{F}$. The apparatus should be such that these are definitely under control and can be delivered at any specified degree or pressure. 'The pressure usually employed is about 30 pounds to the square inch, sometimes less, but rarely more. A cold douche $-80^{\circ}$ to $40^{\circ} \mathrm{F}$.- acts as a distinct stimulant to the heart and peripheral circulation, accelerates the flow of lymph, energizes the muscles, brain, and the functions of the viscera. It is cumulative in its effect if given daily, and results in a marked increase of bodily strength and bienaise.

It is advisable to precede the cold douche by some warming procedure, such as the hot-air cabinet or warm dry pack. This introductory treatment makes the skin hyperæmic and more sensitive, and consequently demands less degrees of cold to beget a good reaction.

Therapeutic Indications.-In neurasthenia or hysteria the cold jet douche is particularly helpful. Here the temperatures are carefully graduated, beginning at $95^{\circ}$ to $90^{\circ} \mathrm{F}$., and lasting for one minute or less, and decreasing 2 or 3 degrees if well tolerated - to $70^{\circ}, 60^{\circ}$, or even $40^{\circ} \mathrm{F}$. These extremely low temperatures are not apt to be well borne, but are particularly useful in hysterical individuals if of a robust type. The duration may be gradually increased, sometimes to two minutes, and rarely to five. The patient is then dried, clothed, and directed to exercise in the fresh air immediately afterward.

In clumbago and sciatica, after a prolonged hot-air cabinet bath lasting from five to fifteen minutes, after perspiration begins the jet douche is to be recommended. If very sensitive to the impact of the water, lower pressures are to be employed; if this condition does not exist, fully thirty pounds will be indicated. Here the hot steam douche may be used or the hot-water douche at $110^{\circ}$ to $115^{\circ} \mathrm{F}$., lasting for a half minute, and alternated with a cold douche at about $80^{\circ} \mathrm{F}$. for fifteen seconds, and repeating these until two or three minutes' treatment has been administered. In the more chronic cases the full pressure cold douche serves to stimulate and massage the affected area. 'The douche is swept slowly over the painful localities, and may be employed as a general douche in addition, for such conditions are apt to occur in depreciated or poisoned individuals.

In chronic joint diseases-gout, rheumatism, arthritis, synovitisthis same procedure may be particularly helpful. It stimulates the absorption of fluid and other exudates, increases the mobility of the joint, and aids the patient to help himself. A preliminary hot-air cabinet treatment is to be advised in most of these cases if they are well nourished and strong. 
In autointoxications a general cold douche is most useful, again combining it with a cabinet bath. In anemia, chlorosis, headaches, the same cold douche is advisable, but the preceding hot-air bath should be short-just short of perspiration, in fact.

In enuresis of children the spinal douche or affusions of cold water $\left(80^{\circ}\right.$ to $60^{\circ} \mathrm{F}$ ) often are curative. It must be remembered that sinall children stand cold poorly, so that both duration and lower temperatures are to be avoided.

Irrigations.- The mechanical cleansing of the stomach or lower intestinal canal with water is termed irrigation, in contradistinction to the application of water as an emetic, when it is made irritating by the addition of salt or mustard, or as an enema, when it is made irritating by the addition of soap, etc.

Irrigation of the stomach has received the appellation lavage. In hydrotherapy it is used for diagnostic and therapentic purposes, as will be described later. 'The technique is as follows: A pitcher containing two or more quarts of water at $100{ }^{\circ} \mathrm{F}$. is made ready. A rubber stomach-tube of firm construction is moistened by dipping into water. The patient, whose person is protected by a rubber apron or sheet, is seated on a chair; the physician, standing at his right side, directs him to hold his head slightly forward, open his mouth, and not to protrude his tongue. 'The lower end of the tube, firmly' held between the thumb and index-fingers, is introduced just above the tongue, and when it impinges against the posterior pharynx, the patient is directed to swallow, while the tube is slipped into the canal. Reassuring the patient, who now gags, and directing him to hold his month open, the tube is steadily pushed through the pharynx until the circular mark on it reaches the teeth. 'The patient is now directed to hold the tube like a pen near the mouth, and not to close his teeth. He feels reassured by the knowledge of having control of the entire situation, but he must be warned against withdrawing the tube and thus necessitating a reintroduction. 'The funnel portion of the tube is now raised above the patient's head, and is filled with water from the pitcher several times; when a pint or more has flown into the stomach, the funnel is reversed while half full of unter and brought down over a basin previously provided on a chair in front of the patient. 'The water now flows out by siphon; if it does not, more water is poured into the stomach, and siphon action is again tried, while the patient is directed to make voluntary compression of the abdomen by contracting the abdominal muscles. The filling and siphoning are repeated until the stomach has been thoronghly irrigated.

It is important to calm the patient's apprehension before beginning lavage by telling him that it is not the length of the tube, but its diameter, that can possibly disturb him, and that he had doubtless often swallowed pieces of meat inuch larger than the thickness of the tube without choking. Impatience or anger on the part of the doctor is inexcusable and always leads to fuilure. It is far better to desist after the second attempt, 
and renew it on another day, than to persevere in the kindliest manner. 'The pharynx becomes irritable after repeated trials.

'The entire process is mechanical and cleansing.

Therapeutic Application.-Dyspepsia is the principal disease in which we have found lavage of great use. We group under this head all cases of difficult digestion, and divide them for practical purposes into catarrhal and nervous dyspepsia. Moreover, one of us has, for the past thirty years, adopted a course of diagnosis and treatment which has proved satisfactory. When a patient complains of positive stomach symptoms, he is directed to partake of a large meal containing especially such articles as he insists he cannot digest. When fear of executing this prescription is expressed, the patient is advised to seek other counsel. Five hours later the stomach is irrigated with warm water, as described. A rough but useful diagnosis is obtained by inspection of the contents of the basin. If the water is fairly clear, and contains little or no undigested food, a gastric neurosis is diagnosticated and a general tonic hydrotherapeutic régime is instituted. (See Neurovascular Training, p. 230.) The stomach is only washed at rare intervals, to remove gas or confirm the diagnosis. In these cases fear of food is common, and this is at once neutralized by the ocular demonstration of its fallacy. A diet is ordered consisting of hot milk and seltzer water, much butter and stale bread, soft and tender meats, absence of desserts and spices, abundance of egg yolks in soup, etc. If the water contains more or less undigested food with mucus of a tenacions character (the glairy mucus usually noted is due to faucial irritation), catarrhal dyspepsia is the diagnosis. In these cases too it has been possible sometimes to "point a moral" by showing the patient imperfectly chewed remnants of food. 'The diet is ordered accordingly; avoidance of hot breads, cakes, and condiments, addition of minced meat (Salisbury steak), no salads or fibrous vegetables, no cold milk, no desserts-thorough chewing, ete. 'The stomach is irrigated once a day before the best meal, to rid it of mucus and fermenting material.

In those not infrequent cases which have become catarrhal from long persistence, or in mixed cases, a middle course is indicated.

Irrigation of the intestine is known as enteroclysis. We are careful to distinguish the latter from an enema. In fact, the first step of an irrigation is the emptying of the bowel by an enema of soapsuds, if there has not been a natural evacuation quite recently. Formerly, one of us (Baruch) followed the accepted practice of introducing a long rubber catheter. The frequent difficulty of carrying the whole catheter into the bowel, by reason of its turning upon itself, has led him to use only sufficient tubing to pass the sigmoid flexure. 'The patient, if a child, is held upon the nurse's lap, which is protected by a doubled sheet and rubber cloth, the latter reaching, in the shape of a trough, into a large vessel or baby's bath-tub. 'Two quarts of boiled water, in which a level teaspoonful of salt is dissolved, are held in a clean fountain syringe. 'The tube is inserted and the water allowed to flow slowly under low pressure until it is expelled. 'The tube should be held firmly to prevent its heing 
washed out. 'The flow is continued until the water returus clear of mucus or fecal matter. 'That water entering the rectum through the customary hard-rubber rectal syringe tube may, under ordinary pressure, reach the ascending colon, has been demonstrated several times, with the Roentgen picture and in fistulas of the right iliac region. (Sce Baruch's Hydrotherapy, third edition, p. 286.)

Enteroclysis is a valuable procedure for the purpose of mechanically cleansing the large intestine.

Infantile diurrhar, when obstinate under other management, yields readily to daily irrigations of normal salt solution, which removes the products of decomposition, and leaves the mucous membrane clean. If a thorough purging with oleum ricini and proper feeding with pasteurized milk or starvation fail, the addition of one or more irrigations has, in my observation, saved life. When febrile conditions prevail, the graduated friction bath $-95^{\circ}$ to $85^{\circ} \mathrm{F}$.- for ten minutes refreshes the patient and calms the nervous system.

Dysentery of adults or children also yields to judiciously applied irrigations when other measures fail. In Shiga's dysentery the warm normal salt solution once or twice a day, just after a movement, removes irritating material, inhibits the bacillary growth, lessens toxemia, and relieves pain.

In amabic dyscntery, on the contrary, these irrigations are useless, while flushing with ice-water, not more than $40^{\circ} \mathrm{F}$., kills the amobre and destroys the spores which are imbedded in the mucous membrane and resist the strongest antiseptic solution that are safe. A glass funnel or large fountain syringe should be filled with cracked ice, and water at $40^{\circ} \mathrm{F}$. should be poured into it, and allowed to flush the intestine, $\frac{1}{2}$ pint at first, in an hour a pint, in two hours a larger quantity, al ways avoiding excessive pain and distention.

Catarrhal jaundice yields to enteroclysis of water at $75^{\circ} \mathrm{F}$. once in twenty-four hours. The water temperature should be reduced every day or two until $60^{\circ} \mathrm{F}$. is reached.

In uremia and renal insufficiency irrigation with water at $110^{\circ}$ to $120^{\circ} \mathrm{F}$. cautiously administered may reëstablish the failing secretion when other measures have been unsuccessful.

In pernicious anemia, which has been shown by Welch to be sometimes due to the hemolytic action of the Bacillus aerogenes capsulatus present in the colon, large normal salt solution irrigations administered once or twice a day are of undoubted value. In this intractable disease any measure that may add to our therapeutic resources must be appreciated and carefully used.

Lead colic yields frequently to the addition of large warm irrigations.

Irrigation of the bladder and ragina are so familiar to the practitioner that it would seem necessary to warn aguinst their too facile resort rather than to dwell upon their value. When the bladder is irritated in prostatic disense, irrigations should be used with great caution and infrequency.

Vaginal injections of hot water have become a routine practice against 
which we would raise a warning voice, especially in unmarried women, whom women doctors more often subject to needless examinations than do careful men. Actual disease of the uterus or adnexa is so rare in virgins that general tonic hydrotherapy often accomplishes satisfactory results without local treatment.

Internal Use of Water.-The practice of flushing the organism, as it is called, with water of indefinite temperature appears to be so popular that it should be mentioned that there are no positive experimental data to demonstrate its action. The authors believe that the diuretic action of water drinking, when the renal secretion is insufficient, may be obtained only in cases in which the heart and kidneys are fairly healthy, and that in these instances the diuretic action is due chiefly to the temperature effect upon the internal peripheral nerves and vessels, as in cold baths. The diuretic effect of the hot-water regime of Saulsbury and the enormous increase of urine in infectious diseases obtained by both ourselves and others from small doses of very cold water frequently administered, prove this statement. The danger of overtaxing the heart by large quantities of water must be clear to every clinician. Physiological experiments have demonstrated that very little water is absorbed from the stomach, and, therefore, its effect on the nerves and vessels of the stomach must be evanescent, and, therefore, stimulating, as is that of a cold dash upon the skin.

\section{CONDITIONS IN WHICH HYDROTHERAPY HAS PROVED EFFICACIOUS}

Typhoid Fever.-In the modern management of this disease the application of water has so completely changed the mortality that it is gratifying to have been in some measure instrumental in the diffusion of a better knowledge of its rational use in this country. There is still much lack of realization of the rationale or actual reasons for the remarkable effect of judicious hydrotherapy in this as in some other diseases.

That the cold friction bath has modified the aspect of typhoid ferer and made its prognosis more farorable is evident not only from our own observations, but also by the recorded reports of Gilman 'Thompson, who claims a reduction of mortality of 50 per cent. in his service of the Presbyterian and New York Hospitals; by those of the late H. P. Loomis, who, in a carefully collated report before the Practitioners Society, stated that the mortality in the principal New York hospitals has been reduced 50 per cent.; by Osler, 'Tyson, Wilson, Musser, Stockton, W. Hanna Thomson, and others in this country, and by many prominent French clinicians. 'The correctness of the statistics published by these authorities is borne out by those of Francis Hare, of Melbourne, Australia, who reported a mortality of 7.05 per cent. in 1923 cases treated in accordance with the Brand method. These are the only large statistics from civil hospitals, and they approximate those of Osler ( 7.5 per cent.), Tyson (7.25 per cent.), Gilman Thompson (7.75 per cent.), and Bouveret (6.9 per cent.). It is a singular and deplorable fact that English physicians still oppose the cold friction bath, despite its having origi- 
nated with Wright and Currie, and the recent convincing evidence of Australian physicians (Murtra reported 173 cases in private practice, with a mortality of 5.4 per cent.).

'The dread of shock has deterred many from this bath. As has been often stated, this is a chimera, for the proper "shock" acts as a thermic stimulant. It is an unpleasant suprise and not depressing when cautiously given. Some physicians oppose the strict Brand bath because it is heroic, inconvenient, and expensive. Does any one hesitate to adopt much more heroic procedures (laparotomy, amputation, ete.) in surgery when life may be saved by them? Why then should we deny the sick typhoid-fever patient this life-saving measure?

The present management of typhoid fever is the expectant method, and well does it deserve this appellation, for the doctor is in constant expectation of delirium, coma vigil, hypostatic pneumonia, heart failure, hemorrhage, perforation, tvmpanites, diarrhoa, bed-sores, etc. 'The mortuary statistics justify this apprehension, for these are the "complications" that kill. May they be prevented by the strict cold friction bath? This important query was first answered in the affirmative by Ernst Brand. By his convincing rationale, and by his irrefutable statistics, amply sustained by the largest statistics of others, one of the writers was convinced and converted from a scoffer at "heroic bathing" to an advocate and propagandist.

The procedure in all cases of undiagnosed fever is as follows: We advise against bathing altogether unless it can be done systematically and in an intelligent manner. When a patient presents a rectal temperature of $103^{\circ} \mathrm{F}$. or over without diagnosis, he is placed upon a small rocking chair, previously covered by a blanket and sheet, wrapped in the latter, and drawn backward into the bath-room. Here he is put into a previously prepared tub of water at $90^{\circ} \mathrm{F}$., bathed with friction for ten minutes, dried, and drawn back to bed. In half an hour his rectal temperature is recorded. Four hours after the bath, if his rectal temperature is still $103^{\circ}$ or over, he is again bathed with friction in water at $85^{\circ} \mathrm{F}$. This bath is repeated every four hours unless the patient is sleeping normally; each bath is reduced 5 degrees until $70^{\circ} \mathrm{F}$. are reached. If one of these baths is followed by a reduction of more than 2 degrees in the rectum, typhoid fever is improbable. The smaller the temperature reduction, the more probable is the diagnosis of typhoid fever. This is the diagnostic bath devised (by Baruch) fifteen years ago, and confirmed in reliability by frequent observation. 'This tentative method of bathing has the advantage, too, of accustoming both patient and family to the treatment.

'The diagnosis having been established in this or other manner, the systematic cold friction bath is given, the best type of which is that devised by Brand. Now the tub must be brought to the bedside, from which it is separated by a screen. In cities 6-foot tin tubs may be readily purchased; in smaller towns a discarded bath-tub may be obtained from a plumber. If this is impracticable, the bath-room must be used. The tub is placed securely upon some support to diminish the stooping 
involved during friction. 'The tub is partly filled before being placed in situ, and then filled two-thirds with water at $70^{\circ} \mathrm{F}$., either with buckets or by attaching a rubber tube or hose to the nearest faucet. Reassuring the patient, he is undressed, a light napkin is wrapped around the sexual parts, 4 ounces of hot coffee are administered, the face is bathed with ice-water, and then he is lifted into the tub. 'The sudden impact of water upon the entire cutaneous area produces more or less alarm, which must be counteracted by quiet suasion. Active friction diminishes the sense of cold and so-called shock. As the bath continues complaints of coldness become more urgent, but the patient must not be removed unless there be prolonged and decided shivering or chattering of teeth or facial cyanosis. Every part of the body should be rubbed with a large sponge. 'Iwice during the bath a basin of water at $50^{\circ} \mathrm{F}$. should be emptied over the head and shoulders. The bath is continued for fifteen minutes unless collapse threatens. The pulse is not reliable as an indication of collapse, because it may be smaller by reason of constriction of the radial artery; if it has diminished in frequency, its thready character may be disregarded. Premature removal from the bath must never be made on the patient's solicitation, but on demand of the patient's condition.

When the bath is completed, the patient is lifted upon a previously prepared sofa, covered with a blanket and sheet, or upon one-half of the bed similarly provided. If the temperature has been above $103.5^{\circ} \mathrm{F}$., he is wrapped in the sheet and blanket, and allowed to dry; this is an effective antipyretic. If he is shivering, he must be dried and replaced in bed. Iot bottles and artificial stimulation should be avoided if possible, but used if necessary to facilitate reaction. A better way is to avoid excessive chilling by shortening the duration of the next bath, and cmploy more friction during its use. The bath temperature must never be raised on this account; it is a hydrotherapeutic law that the more brief and intense the application, the better the reaction, provided it is not beyond the patient's capacity. Disregard of this rule has resulted often in abandonment of the cold friction bath. Half an hour after the bath the rectal temperature is recorded and the patient receives 4 ounces of ice-water. If the abdomen is warm, a compress consisting of two layers of coarse old linen or a small towel sufficiently large to cover the abdomen is wrung out of water at $60^{\circ} \mathrm{F}$. and secured with a thin flannel bandage slightly wider than the compress. This is renewed every hour if it is warm. 'The bath-water need not be renewed oftener than once after four baths unless it be soiled. The remoral is done through a faucet, or, in the absence of the latter, with buckets or by siphonage. 'The temperature for the next bath may be arranged by the addition of ice-water, not, however, in view of the patient.

'This is the ideal Brand bath, the statistics of which prove its superiority over all modifications. The latter are justifiable only when the exigencies of the case clemand it; $i . e$., when objections to it are insuperable, because every deviation is followed by less farorable results. It is true that some physicians have recently resorted to "less heroic" baths. Whether they are justified or not their statisties will surely show. 
In the mean time their patients will suffer and die if the muncrons observations quoted are correct. Recently, in a visit to two large hospitals, the physicians asserted that they conld get along with less severe baths, but in one instance a patient who was delirions and lying in his feces was receiving a sponge-bath, and in two other instances patients with muttering delirinm and serions hyperpyrexia were both receiving baths of $85^{\circ} \mathrm{F}$. for ten minutes. Such incidents are never observed when the strict Brand bath is practised, because the latter arouses the reflexes, and keeps the nervous system from fatally slumbering. With this knowledge carefully before one, the Brand bath may, but with great reluctance, be modified as follows:

1. The bath may be made of the same temperature- $70^{\circ} \mathrm{F}$, of only five minutes' duration, and repeated every three hours or oftener until the patient's reaction is established. 'Then a few minutes are added to each bath until fifteen minutes are again reached. Gradual reduction of the bath temperature is not approved, nor is raising the bath temperature.

2. If this cannot be done, the sheet bath may be given every three hours. This procedure, carefully given, so that each part of the trunk is successively cooled and warmed as follows, is an efficient substitute.

On an adjoining bed or cot, or on one side of a double bed, a rubber sheet or other protection is spread. 'This is covered by a blanket; a pillow is provided also. A small tub of water at $80^{\circ} \mathrm{F}$., and a large pitcher of water at $60^{\circ} \mathrm{F}$. and a sponge are made ready. A linen sheet or table-cloth is saturated with water at $80^{\circ} \mathrm{F}$., partly wrung out, and spread upon the blanket, a little above the line of the axills. 'The patient is placed upon the sheet, and requested to raise his arms; the sheet is quickly drawn across his chest from both sides and pressed between his knees, so as to be quite snugly adherent to the skin. 'The lower extremities are covered over the wet sheet with part of the blanket, while the trunk is treated as follows: From the pitcher water is gently poured upon the upper half of one side of the chest; this part is thoroughly rubbed by rapid passes of both flat hands until it feels warm; water is again poured upon this part and again rubbed warm. This process is repeated until the skin fails to warm up. Now another part of the chest is treated in like manner, and the whole anterior and posterior aspects of the trunk are thus gone over rapidly, avoiding severe chilling. Some chilliness is expected, but this soon passes off, when the excess of water is mopped up from the sides of the body and the patient is snugly wrapped in the blanket. If necessary, the lower extremities or the entire body may be rubbed and shapped with the ontstretched hands until chilliness ceases before the blanket is applied. So soon as the patient feels warm and eomfortable, the blanket may be loosened or removed. Many patients full asleep in the pack; they should remain in it until they awake; otherwise they may be returned to bed in half an hour after being dried and dressed. 'This sheet bath may be repeated every three hours if the rectal temperature and pulse demand it. 'This is the best substitute for the tub-bath. 
3. Affusions with water at $70^{\circ} \mathrm{F}$. may also be substituted. The water is squeezed from a large sponge, and at each application is reduced until $60^{\circ} \mathrm{F}$. are reached. 'The patient is placed on a rubber sheet covered with a cotton one, and so arranged that it forms a trough leading to a receptacle at the foot of the bed. This method of "slushing," if accompanied by friction with the sponge over the entire body, is a valuable substitute, because it affords the needed awakening of the central nervous system. A statistical record of this practice made by Cabot at the Massachusetts General Hospital is certainly encouraging, so far as comfort to the patient is concerned.

4. A still milder form of hydrotherapy is the ablution described on p. 228. Meigs, of Philadelphia, reports excellent results from the rubbing of the patient every two hours with pieces of gauze dipped in "cold" water. The comparative statements of these procedures are generally vitiated by want of precision in describing the technique of the procedures, the temperature of the water, duration of bath, etc., not always being stated.

5. Another substitute for the cold friction bath is the hammock bath described on p. 240. Large numbers of typhoid ferer cases have been recorded with a mortality not far above that quoted as resulting from the cold bath. This bath is more agreeable and meets with less opposition. The patient is placed in a tub containing water not less than $88^{\circ} \mathrm{F}$, and kept in it until his mouth temperature is reduced to $100^{\circ} \mathrm{F}$. He is then removed for two hours and returned when the temperature reaches $102.5^{\circ} \mathrm{F}$. This course may be continued throughout the entire case.

The hammock bath is often a useful expedient, when the patient is seen after the first week and is found with high temperature or delirious. By allowing the patient to lie in such a suspension bath, or even on the bottom of the tub protected by water cushions, in water in the neighborhood of $100^{\circ}$ to $95^{\circ} \mathrm{F}$. for an hour or longer, with a cold compress around his head, the temperature will be more surely reduced than by a colder bath; delirium and insomnia are almost invariably removed. The patient should again be placed in the bath whenever his rectal temperature registers $103^{\circ} \mathrm{F}$, and removed when it is reduced below $100^{\circ} \mathrm{F}$. In desperate cases also this bath may be of signal service; it does not refresh the central nervous system, but this deficiency may be remedied by affusions from basins of water at $50^{\circ} \mathrm{F}$. before removing the patient from the bath. The same bath water may be used for twenty-four hours unless it is soiled. bath.

A temperature of $107^{\circ} \mathrm{F}$. has been reduced to $101^{\circ} \mathrm{F}$. by such a

6. In some desperate cases the addition of $\mathrm{CO}_{2}$ to a bath of $80^{\circ} \mathrm{F}$. has proved of value in tiding the patient over a condition in which the reflexes failed to respond to the coldest water. When chilling is intense during the ordinary friction bath, the addition of the Cassebeer or 'Triton salts, or, better still, the Zeo preparation, stimulates the skin, warms it, and overcomes the patient's justifiable remonstrances. This 
practice should always be tried when objections are otherwise insurmountable. We obtain in this manner a combination of thermic, chemic, and mechanical excitation of the peripheral vessels and nerveendings.

'The efficiency of all hydrotherapeutic procedures depends upon a thorough understanding of their technique and rationale. 'This is not more difficult to master than is that of the action of medicinal agents, for the action of water in disease is entirely derived from the physiological action of cold and heat, as stated in the first page of this article. It is only necessary to bear this rationale in mind in order to attain the skill and judgment demanded for modification and adaptation of water procedures to the varying phases of disease. For many years it has been the aim of one of us to divest physicans of the idea that there is anything occult in hydrotherapy, as well as to wean them from an irrational or haphazard application of water.

'The immersion of a fever patient in water at $70^{\circ} \mathrm{F}$. arouses all the physiological activities of the organism for meeting this temperature invasion. There is a gasp and staceato breathing, due to the "surprise," which is not to be confounded with the misleading word shock. "The sensory terminals are stimulated by the thermic excitation, if it be not excessive, when it must become depressing according to physiological law. As is familiar even to the tyro in medicine, this stimulus is conveyed to the central nervous system, producing refreshment and relief of the greater or less depreciation incident to the attack from toxins. When the manifestations of typhoid fever are analyzed it is found that headache, adynamia, general apathy are but the forerunners of the more intense delirium, subsultus, etc., of later stages or of more severe types. 'The effect of this cold friction bath is positive in the majority of cases. The brightened countenance testifies to the refreshment is does the changed aspect of the entire case. The cold bath maintains this refreshment if begun in the first week, and, by reason of repetitions, does not permit the nervous system to relapse into apathy; it thus maintains the integrity of the latter as far as possible, under the deteriorating influences. In short, it is prophylactic, it prevents the deepening of apathy into coma or muttering delirium with all the depreciating effects upon the organs which depend upon it for force. I tet this point be constantly held in view, for the eurly and continued application of cold as a thermic stimulant is as important here as it and other irritants are in opium-poisoning. 'The Brand bath affords the most irtense action, the sheet bath an equally intense, but less enduring effect upon the sensorium, and the abhution or affusion a still milder stimulation. Here is ample opportunity for grading or dosage, and it is urged that the reader depend upon his own judgment just as soon as he has had some experience with the treatment here laid down.

Another effect of the cold immersion is upon the immense vascular area of the skin, and the resultant powerful action upon the heart and vasomotor system. For several years one of us (Baruch) has taught that the cold bath produced contraction of the cutaneous vessels, driving 
the blood from the surface, and upon withdrawal of the cold, the cutaneous vessels, dilated, producing a tonic hyperemia. This rationale, first suggested by Winternitz, the father of modern hydrotherapy, was controverted by Mathes, of Jena, now in Koeln, who held correctly that when the muscular walls of a vessel are stretched beyond their normal calibre, they are in an atonic condition, and that, therefore, the reaction after cold procedures could not be tonic. An investigation of this apparent contradiction between the clinically correct view of Winternitz and the physiologically correct view of Mathes, revealed that both based their deductions on the false premise that the cutaneous vessels which are contracted by a cold bath are provided with contractile muscular walls. The fact is that by far the larger vascular area in the skin consists of capillaries, that these are mere endothelial tubes filling the papillary spaces in enormous tortuous reduplication, and that the arterioles which are located in the subcutaneous tissue lie probably out of reach of any but the most intense cold procedures.

The skin is supplied with unstripped muscular fibres interwoven with white and yellow elastic tissue, varying in quantity between the superabundance in the scrotum and entire absence in the palmar and plantar surfaces. This anatomical peculiarity of the skin endows it with a function hitherto not recognized, as will presently be shown. These muscular fibres, called by Unna the oblique tensors of the skin, contract diagonally through the thickness of the skin in the same manner as the erectores pilorum, which also are intimately involved in the blood and lymph movement. Thomsa having confirmed this view, we feel justified in assuming that the cutaneous muscle-fibres replace the muscular coat which is absent in the papillary vessels, and that when constringed under cold they press the blood out of the latter. When these musele-fibres give way under the inpour of warm blood, after withdrawal of the cold water, the papillary vessels fill only to the extent which the elastieity of the skin admits. They cannot be overstretched like the arterial coats, nor are the latter capable of complete distention by reason of the firm obstacle presented by the skin muscles and fibrous tissues; thus is prevented atonic hyperemia. They also probably compress in their state of contraction the cutaneous arterioles. When the vessels are filled to repletion by the enhanced cardiac effort due to increased resistance at the periphery, the muscular and elastic structures hold them firmly; the heart, feeling the enhanced vasomotor impulse, sends more and better blood to the interior peripheral vessels also, thus improving the blood supply of all the organs.

The ruddy appearance of the skin under good reaction may be explained on Bier's "Blutgefühe" theory, an illustration of which is furnished by Ritter, who points out the curious faet that even when an arm is so constricted by an elastic cord that it turns cyanotic, a frozen spot will, on thawing, present a bright red surface, a phenomenon often observed, thus confirming Bier's idea that when compressed tissues are released they "select" arterial blood and not venous.

'The facts cited establish this theory (Baruch's) of reaction after cold 
procedures as highly probable. At any rate, the clinical result is an increase of the volume and cell capacity of the blood circulation in the skin, as has been ascertained by Breitenstein, 'Thayer, Baruch, and others. The combined enhancement of the functions of the central nervous sorstem and of the heart inures to the benefit of the poisoned organism. 'The resultant stimulus to organic activity differs vastly from that induced by alcohol, digitalis, or strychnine. Alcohol especially dilates the peripheral ressels, which are already in a semiparetic condition. It may drive the heart to increased exertion withont furnishing vasomotor foree, and may be likened to the spur which urges a jaded horse to increased effort only to drop exhausted. 'The cold frietion bath, on the contrary, is a physiologic stimulus. It may be aptly compared, as Hare has so well said, to sanding a slippery track for a locomotive whose wheels are revolving in vain, though ample power is supplied by the boiler.

Here, again, we would emphasize the fact that every organ feels the impetus of improved circulation. 'The sum total is as follows:

1. 'The entire nervous system is kept alert, preventing failure at the centres of vitality. A case under cold friction baths does not often die from exhaustion.

2. The heart is kept from the beginning in as nearly normal action as is possible under the toxemia. Heart failure is prevented. We do not wait as in the expectant plan until it occurs, and then "call upon Hercules."

3. The renal function is maintained, the quantity and toxieity of the urine are increased, as proved by actual analysis, and the quantity is frequently doubled. 'Tyson and others have shown the marked diminution of albumin in the urine. Nephritis is prevented.

4. 'The improved circulation is felt in Peyer's patches. Sloughing and consequent hemorrhage and perforation are to a great extent prevented, as is evident from the reliable comparative statisties of Hare, of Melbourne, Vogl, of Munich, and others. 'Thus another eause of death is eliminated.

5. The improved respiratory action and bettered pulmonary eirculation prevent death from hypostatic pneumonia, proved by Vogl, Brand, and others.

6. 'The soft tongue and month under this treatment indicate inproved cireulation in the gastro-intestinal canal, resulting in appetite and digestion being furthered and nutrition maintained. Vogl has proved this by weighing his soldier patients.

7. Sloughing bed-sores are almost unknown, as are involuntary movements or urination.

8. Hyperpyrexia, which cripples the entire organism, is prevented. 'The temperature is held in check by small but steady reduetions of the daily average. All these facts are cited to emphasize the prophylactic action of the cold friction bath and the fact that it is not a symptomatic (expectant) method.

Relapses have been regarded as more frequent after this treatment. 
'This is easily explained by the fact that there are a great many patients prevented from dying, and that among these a certain proportion relapse.

The first bath should be given in the presence of the physician, because it is not safe to trust any nurse, without special instruction, to overcome the objections of the patient and to follow the technique.

'The diet should consist of 4 to 6 ounces of milk or farinaceous broth, given alternately, every two hours. In the intervening hour 4 to 6 ounces of ice-water are given, and it should be insisted upon that the nurse record the quantity taken and retained. When the patient objects to the ice-water, it is given in smaller quantities, with the addition of 10 drops of dilute hydrochloric acid. The diuretic action of this "shock" to the nerves and vessels of the pharynx and stomach is important.

Other hydriatric procedures have been found worthless or out of proportion to the effects. The cold pack, consisting of a cold wet sheet wrapped around the patient, is a refrigerating procedure, but lacks the stimulating effect of active friction, and is not to be recommended. Sprinkling with ice-water may be cooling, but without accompanying friction the best effect is missed. The procedures mentioned in this article represent the best features of hydrotherapy as applied in typhoid fever, and will be found to suffice for all contingencies.

The Exanthemata.- In the management of eruptive fevers, hydrotherapy plays an important part. The mild types require only internal use of small quantities of ice-water, regularly administered for diuretic and refreshing effect.

In the prodrome of measles and scarlatina there occurs at times a sudden overwhelming of the organism, which manifests itself by imperfect eruption, cyanosis, and marked nervous symptoms. In these cases the stimulating action of a short bath in water from $105^{\circ}$ to $115^{\circ} \mathrm{F}$, lasting not over five minutes or less, in order to avoid the relaxing action of warm water, is of striking value. It arouses the central nervous system just as cold water does, but it is not refreshing. The hyperemia resulting from it flushes the skin and "brings out the eruption." Such a short bath may be repeated, if necessary, in an hour, and if not sufficient to arouse the system, it may be concluded with an affusion by pouring one or more basins of water at $50^{\circ} \mathrm{F}$. over the shoulders. Reaction must be encouraged by friction in the bath and wrapping in blankets after its completion.

When the eruption is established, and the temperature rises above $103^{\circ} \mathrm{F}$., the patient should be given a tub bath at $95^{\circ} \mathrm{F}$., reducing its temperature to $85^{\circ} \mathrm{F}$. for eight to ten minutes; complete submersion must be insisted on. Such a bath repeated every four hours promotes sleep, refreshes the patient, and reduces the temperature.

In the bronchopneumonia complicating measles, the wet chest compress at $60^{\circ} \mathrm{F}$., repeated whenever it has become warm, is of great service. 'The nephritis of scarlatina is prevented or modified favorably by baths of $95^{\circ} \mathrm{F}$. for half an hour or longer, followed by warm wrapping. A salt-free diet and careful hygienic management are necessary adjuncts. 
Pneumonia.-The similarity between preumonia and typhoid fever, both being infections diseases, and running a definite course, would indicate a similarity of management, modified by the difference in the effects of the micro-organisms involved. In the pneumonia (croupous) of adults, in whom more or less pleurisy is often present, the full bath has been abandoned becanse disturbance of the patient aggravates his distress, and milder procedures suffice to meet the indications. In the pneumonia of children (bronchopneumonia) the ten-minute graduated tub bath from $95^{\circ}$ to $85^{\circ} \mathrm{F}$., repeated every four hours, followed by the wet compress, is useful for sustaining the organism in the struggle.

In the management of adult pueumonia of all types the patient should be isolated in a room from which one or more windows have been removed, but not the blinds, thus providing for ample ventilation, which cannot be interfered with by officious friends or aërophobic nurses. If the room is very cold the patient's head is covered with a night-cap, and the nurses are instructed to wear sufficient clothes. A dose of ealomel (grs. $7 \frac{1}{2}$ ) is administered dry, and washed down after thoroughly rinsing the mouth, in order te destroy the pneumococci in the mouth, their favorite habitat. A concentrated solution of chlorate of potassium is used every hour to keep the mouth clean; 4 ounces of ice-water are administered regularly every two hours, alternating with 4 ounces of hot milk, into which cold seltzer water has been driven from a siphon. Active feeding is not neeessary, because the disease usually attacks the patient in the midst of health and lasts a comparatively short time; furthermore, undigested food may increase the intestinal distention, which appears to be a frequent result of pueumococeus infeetion.

The chief hydrotherapeutic measure is the chest compress. 'Two compresses of three layers each of linen are cut in the shape of a vest (see p. 237), so as to facilitate the wrapping of the entire thorax. 'The compress is wrung out of water at $60^{\circ} \mathrm{F}$. and sungly applied. A flannel cover of the same shape, but $\frac{1}{2}$ inch larger, is pimed over the compress to hold it in position. 'The compress requires removal every hour if it has become warm. For renewal, a fresh compress should be ready, in water at $60^{\circ} \mathrm{F}$, before removing the first. If temperature reduction is specially indicated, a larger quantity of water may remain in the compress; if there be stupor, colder water may be used. 'The effect on the cardiac, respiratory, and eliminative functions from this simple procedure is strikingly satisfactory. Stimulants are rarely needed, exeept in alcoholies.

Sunstroke.-In this disease cold water is used more or less by every physician, although it is generally applied in a faulty manner. A long time elapsed ere the antithermic bath in typhoid fever was abandoned, indeed, it is still found in some hospitals. Austin Flint, the elder, abandoned it in the Bellerue Hospital because it was fatal. Ball resumed it in the better cold-friction bath as a nerve and heart stimulating procedure with marked advantage. The constant agitation of this point has brought about a favorable change, and it is hoped that a similar change from the antithermic management of sunstroke to the 
stimulating method will take place. Fortunately, we have a therapeutic control experiment regarding sunstroke as convincing as that cited by Vogl in typhoid fever. In an epidemic of sunstroke in New York in 1S96, 648 cases died in one week. 'There were sent to the hospitals 510 hyperpyrexia cases. These were treated differently at the various hospitals, and it will be profitable for future guidance to study their management.

The statistics collected by Alexander Lambert were as follows:

Graduated baths, $110^{\circ}$ to $72^{\circ} \mathrm{F}$., in the Brooklyn Homeopathic Hospital, gave a mortality of 41 per cent.; later a number of cases were treated by spray at $75^{\circ} \mathrm{F}$, with a mortality of 11 per cent.

'Tub baths of $50^{\circ}$ to $75^{\circ} \mathrm{F}$. (with floating ice), a method practised in Bellevue Hospital, gave a mortality of 33 per cent.

Needle spray at $75^{\circ} \mathrm{F}$., stopped when rectal temperature registered $103^{\circ} \mathrm{F}$, at the Flower Hospital, gave a mortality of 11.5 per cent.

Affusions with ice-water over head and shoulders, followed by wrapping in warm blankets (O'Dwyer) till temperature was $103^{\circ} \mathrm{F}$., at the St. Vincent Hospital, gave a mortality of 6 per cent.

Ice-packs, recommended in Osler's text-book, show a mortality of 38 per cent., and, are, therefore, more fatal than the above methods.

We would urge the adoption of O'Dwyer's method of wrapping the patient in a cotton sheet and placing him on a stretcher, and dashing dipperfuls of cold water from a distance of several feet upon his body, until the rectal temperature falls to $103^{\circ} \mathrm{F}$. This is practically the sheet bath described on p. 231. A small stream of ice-water from a height upon the head increases the stimulating action. Good friction to warm the cooled part is required. Most of the text-books on practice advise the jce cold treatment, which this control experiment proves fatal. It should be abandoned.

Phthisis Pulmonalis.-Despite the fact that one of us (Baruch) called attention to the very positive favorable action of judicious hydrotherapy in the cases at the Montefiore Home in 1892, and the publication long ago by Brehmer and Dettweiler, of the improvement of nutrition from hydrotherapy, writers in this country appear to regard hydriatric procedures with indifference or contempt. 'The improvement of the nutrition and general vigor by the judicious application of water in tuberculosis has been confirmed on a large scale by Kuthy, of the BudaPest Sanatorium, and others. American sanatoria directors would do well to heed the warning of Kuthy, who controls an enormous material. "'The sanatoria for tuberculosis will never do justice to their difficult demands until they possess a well arranged water institute, conducted by properly instructed attendants." 'The daily neurovascular training, beginning with the ablutions, by affusions, drip sheet, and wet pack, gradually made of lower temperature, affords very gratifying results.

Neurasthenia.- In no disease is water applied more irrationally than in neurasthenia, if we may judge from the published reports of lectures and papers by otherwise well-informed nen. It is specially 
Imfortunate that physicians do not investigate the action of the various "tonie" procedures, which improve the nutrition of the cortical centres and contribute to more speedy recovery. It has long heen the rustom to regard nenrastlenics as beyond the pale of the genceral practitioner. There is no greater fallacy. While the neurologist may lo useful in establishing a diagnosis, specialists are no more competent to treat these cases than the well-informed general practitioncr, who has loarned to donbt the value of drug tonic's for the nervous system, and has no confidence in the nerve specifics vaunted in our journal advertisements. Dallying with these so-called nerve tonics causes loss of time and opportunity, and renders the sick and their friends impatient.

For the more preeise adaptation of water treatment to the individual ease we have divided neurasthenics into the erethetic type, the depressed type, and the intermediate type. In the first class lielong the excitable individuals, mostly women bordering on hysteria, school girls, society maidens, social climbers, love-sick women, and pets, male and female, who have been spoiled or thwarted, and who are the bane of family and friends by reason of their exactions, domineering trend, and impatience of restraint. 'The physician's tact plays an important rôle. Many of this type do not improve under the customary environment, and are benefited by removal from home. A rest cure is useful, but this treatment is not successful if given at the patient's own residence, where segregation is rarely strict. Many of this type are well nourished and even plethoric; these are the most difficult to treat. 'They are best managed by sedative hydrotherapy added to moral and physical hygiene. Hammock baths at $100^{\circ} \mathrm{F}$. , of half an hour's duration night and morning, present a method which is tonic. 'The morning bath should be followed by friction with a towel and the temperature of the water gradually lowered 2 degrees daily, until $70^{\circ} \mathrm{F}$. is reached. 'The patient must go ont for a walk immediately after the morning treatment. All dowehes or severely cold procedures must be avoided.

'The depressed type of neurasthenia also demands eareful attention as to the environment. While the other type requires diversion more than amusement, the latter is hetter adapted to the depressed type. Physical exercise, graded to the capacity, not the wishes, of the patient, is of great value. Gardening, walking, driving, or riding are more useful than dumb-bells or calisthenics or any indoor exercise. 'The chief point is to order exereise, yet in a systematic method, at stated hours, and in definite amounts. 'The patient's interest and obedience will be in proportion to the doctor's earnestness.

The hydrotherapeutic procedures should be a gradual but persistent "neurovascular training," beginning with a morning ablution at $\$ 5^{\circ} \mathrm{F}$., and reducing the temperature one or more degrees each day. More water should be left in the washeloth each day, until ablution with the coldest water of the entire trunk is followed by reaction, i. e., absence of chilliness. 'The next procedure is the affusion, described on p. $2: 30$, followed by the drip sheet, which is deseribed on p. 232, and applied with water at the lowest olstainable temperature. If these procedures 
given skilfully at home are insufficient, the patient would be benefited by daily douches in an institution, if possible under his physician's direction. If the patient has had neurovascular training at home he will bear at once a fan douche at $80^{\circ} \mathrm{F}$. for one minute over the entire body, followed by a jet douche over the back at $70^{\circ} \mathrm{F}$. for half a minute, then rubbed dry and sent into the open air. The water may be reduced daily one or more degrees until the lowest temperature is reached. The effect is enhanced by a pressure of 20 pounds, increased once each week, until 35 or 40 pounds are reached. The aim should be to arouse the nerve centres from their depression and to improve nutrition. In Philadelphia, New York, Chicago, Baltimore, Richmond, and Boston there are institutions in which a prescription ordered as here directed may be filled with the same precision as drugs are prepared by an apothecary. This method was introduced to enable the family physician to retain his patient under his own control. It will not be found difficult to follow the line of treatment here laid down, if a fair amount of attention be given to the subject. A resort to the specialist, or worse still, a resort to a quack, may often be avoided by early resort to hydrotherapy, exercise, diet, and positive yet tactful management.

The intermediate type of neurasthenia calls for both the above methods of hydrotherapy in accord with therapeutic indications.

Insomnia is one of the chief and most intractable phenomena of neurasthenia. Every well-informed physician avoids hypnotics. The usual prescription of water is "take a warm bath before retiring," without directions for the manner of taking it; the usual result is failure. A bath which produces cutaneous hyperemia should result in that anemic condition of the cerebral vessels which conduces to sleep. To produce this effect attention to the technique is demanded. The bed should be warmed by hot bottles, to be removed before it is occupied, the temperature of the bath-room should not be less than $75^{\circ} \mathrm{F}$., and a warm sheet should be at hand for drying the patient. 'The bath water, at not more than $102^{\circ} \mathrm{F}$., should cover the entire trunk for ten minutes; the patient then steps on a rug, wraps himself in the warm sheet, and goes to bed. Here he is rapidly dried, covered with blankets, and left alone. Perspiration must be avoided.

An excellent procedure for most cases of insomnia is the wet pack at $70^{\circ} \mathrm{F}$., described on p. 234. Patients who claimed to have suffered from insomnia for montlıs often fall asleep in the pack, despite the din of the clinic. If the patient feels chilly, hot bottles may be laid alongside the pack until he feels comfortable.

In many cases of neurasthenia there are evidences of intestinal autointoxication, indican being found abundantly in the urine. An intestinal irrigation twice or three times a week, administered secundem artem, has been found a useful procedure in such cases. (See p. 247.)

The douche is the only procedure that cannot be effectively administered at the home of the patient. It consists of a stream of water from the nozzle of a hose, having an opening of $\frac{1}{8}$ to $\frac{1}{2}$ inch, delivered at a distance of 12 feet under pressure, at a prescribed temperature. For 


\section{PLATE XVI}

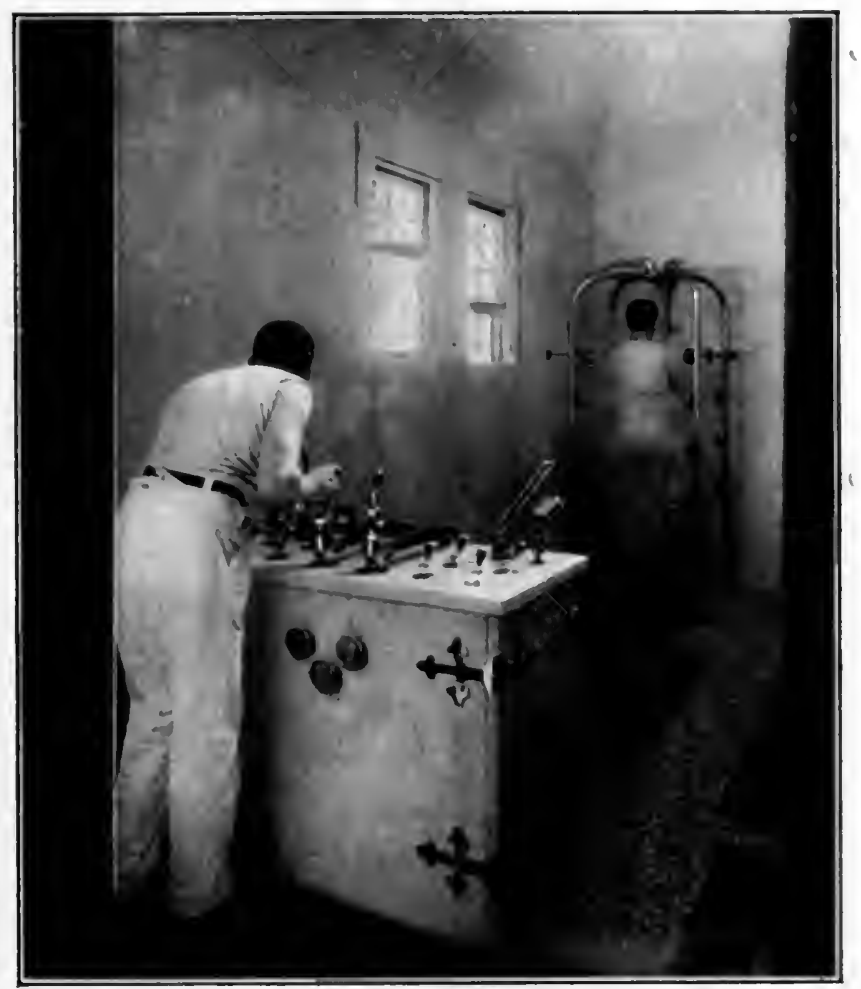

Baruch douche table at Hydriatric Institute. Paso Robles, California. 

the exact dosing of water a douche apparatus is indispensable. 'The usual shower or spray of the modern bath-room does not answer the purpose, because temperature duration and pressure cannot be arranged with precision. For this purpose a douche table and douche room, which have served as patterns for all institutions in this comntry, has been devised by one of us (Baruch). The douche room should have an asphalt sunken floor with a descent toward the sewer inlet. A corner (northeast if possible) of a room in a well-lighted basenent or, better, on the first floor, securing 15 feet square or thereabouts, suffices. 'The asphalt is covered by a woolen slatted floor, in sections of 4 by 6 feet, to permit the water to flow off readily. 'The walls are protected by marble or slate blocks 1 inch thick, 8 feet high, and 4 feet or less wide.

A circular or needle douche with an overhead shower is placed securely in one corner, and connected by pipes with the douche table at the opposite end of the room, about 12 feet from the back of the former to the back of the latter.

The douche table, devised about fifteen years ago, consists of a series of conduits so arranged that they are connected with the cold and hot water supply of the building. One precaution must be observed, viz., to connect the donche table directly with the main supply of the building, in order that the pressure and temperature may not be dininished at any time. Especially for the hot water there should be a separate heater (steam coils in a boiler) to make it independent of possible interference. Plumbers usually offer objections to this plan, but the supply need not be large. The diagram shows the arrangement of the hose for the jet douche described above, and the fan douche which is a stream scattered horizontally by placing the index-finger over the opening. 'There is a separate faucet for the rain (shower) douche, steam douche, and for the perineal douche. 'The water flowing over a thermometer bulb indicates its temperature, and its passage over a pressure gauge indicates the force with which it is delivered, while a small "second clock" indicates the duration, all of which may be instantly changed according to the physician's prescription. (See Plate XVI.)

'There is also a pipe connecting a hose and nozzle to the steam supply. 'This being of low pressure, it may be used safely at a distance of about 2 feet. Care must be taken that all water escapes from the nozzle before the steam is applied to the skin, in order to insure against scalding.

Therapeutics of the Douche.-Neurasthenia furnishes the majority of cases. Charcot's success in hysteria and melancholia were due to spinal cold douches. The neuralgias, rheumatism, and gout are diseases in which the judicious application of the douche has produced favorable results after failure of milder procedures. The douche apparatus is not adapted to hospitals in which non-ambulant cases predominate, while it is valuable in large out-door departments and dispensaries. There appears to be an impression among advanced hospital physicians and superintendents that a hydrotherapeutic plant is a sine qua non of a modern hospital. One excellent hospital is known, in which a costly plant is now used chicfly for the staff and 
employees as a substitute for the 'Turkish bath. In two other hospital out-door departments the masseur and masseuse in charge furnish the technique according to their more or less crude judgment. Until instruction in hydrotherapy becomes more general in our colleges the latter unfortunate condition must continue. The physician must give a precise preseription. For the average general hospital, instruction in bedside hydrotherapy is most urgently needed. 'The house staff and nurses should be instructed in the action and technique of water applications as they are in surgical asepsis and bandaging, and every student should be required to practice each procedure.

Rheumatism and Gout.-These diseases are grouped together because the hydrotherapeutic measures indicated are similar in many respects, and baths are applied in all the resorts for these diseases. While much benefit accrues from the change of environment and strict supervision of the patients' mode of life at the various hot springs, it is probably true that the mineral ingredients of many of these springs are overestimated. It has been established beyond cavil that the various constituents described in the analyses of these springs are rarely absorbed through the skin, except, perhaps, $\mathrm{CO}_{2}$, and possibly sulphur by inhalation. It would seem, therefore, that the effect of these baths is a purely hydrotherapeutic one, viz., by temperature, duration, and pressure. For this reason it is advised that physicians study the action of hot and cold baths, and apply them at home. Excellent results may be obtained in this way.

In subacute articular rheumatism, and in certain types of muscular pain, the hot-bath pack is a most useful procedure. It is described on p. 242 ; its technique must be followed with precision. Care should be taken that the eliminative action be not sought for at the expense of the patient's strength and nutrition. If the latter appears threatened, the hot treatment should be raried with alternating cold tonic procedures, affusions, ard ablutions. In cases resisting home treatment, methodically applied, the alternating douche at $60^{\circ}$ and $125^{\circ} \mathrm{F}$, the hot steam for ten seconds, and the cold for twenty, the procedure lasting one or more minutes, is very effective if terminated by a fan douche over the body of $85^{\circ} \mathrm{F}$. for half a minute. In cases of sciatica I have had striking results from the alternating steam and cold water douches-Scotch steam douche.

In the Vanderbilt Clinic obstinate sciaticas have afforded the most satisfactory results, as have cases of myalgia and neuritis. No result has been obtained in advanced cases of arthritis deformans, in the early stage of which only is there hope of improving general nutrition and elimination.

All cases of rheumatic and gouty character should be subjected to hydrotherapy early, without wasting time on the advertised specifics. Medication is practically useles except as palliative. 


\title{
ELECTRO-THERAPEUTICS, INCLUDING HIGH- FREQUENCY CURREN'TS.
}

\author{
BY GEORGE E. PRICE, M. I).
}

Elactricity as a therapeutic measure is not at the present time looked upon with great favor by the general practitioner of medicine. 'That this attitude is due for the most part to the adoption and abuse of electricity by quacks and advertising doctors I think there can be little doubt. On the other hand, many conscientious physicians become disappointed when they fail to achieve the brilliant results they expect, either from having formed a too optimistic opinion of its possibilities from a perusal of the literature published and disseminated by many of the electrical apparatus makers, or from an insufficient knowledge of its physiological and therapentic action, together with the closage and methods of application.

With the courses of lectures and practical demonstrations on the subject of electro-therapentics at present given in our la rger medical colleges there should result a more general use of electricity in the treatment of disease. As the student approaches the subject in the same manner as he studies the therapeutic action of drugs, he will learn that the effects of electricity are limited, as are the effects of drugs; like them, it must be given in definite amcunts and repeated at stated intervals. He will learn, noreover, that a single current, like a single drug, can produce only the physiological action peculiar to itself, and that a static machine cannot meet all electrical indications any more than calomel can take upon itself the office of opium or digitalis.

Although electricity was discovered twenty-five hundred years ago and mentioned by Pliny as a remedial agent, it was not until after the discoveries of Galvani and Volta in 1790 and 1800$)$, and the discovery of induction by Faraday in 1S:31, that electricity began to be established on a scientific basis as a therapentic measure of more or less general use.

While there is but one kind of electricity, it is manifested in three chief forms, known as the static, galvanic, and faradic currents. 'The high-frequency and simusoidal currents are but further modifieations of these forms.

Electricity may be employed as a therapentic measure in any of its forms or modifications, each having a definite plysiological action on the tissues of the body. 'Through its property of generating light, we have the Finsen light and the Roentgen rays, while its property of generating heat fumishes us with the useful electro-cautery and the thermophore. 
'The eleetric bath eabinet may be said to have resulted from the generation of both heat and light, and in vibratory massage, eleetricity acts by furnishing the motive power. As a generator of ozone, it also adds to its field of therapeutic usefulness.

Static, frictional or Franklinic electricity, is electricity at rest, but in a state of tension, tending to discharge suddenly; it is obtained by frietion between dissimilar substances, one of which becomes positively, and the other negatively, eharged or electrified.

Galvanic, voltaie, direct or continuous electricity, is electricity in a state of flow-uninterrupted, constant, and unaltering in direction; it may be obtained from the voltaie cell, or from the electrie-light circuit.

Faradic, interrupted or indireet electrieity, is vibratory or alternating, and obtained from the galvanie current by induction.

'The following article will be divided into two general parts, the first referring briefly to the more essential points of electro-physics and eonsidering the physiologieal action of electrieity also electro-pathology and electro-diagnosis. It will include a brief diseussion of therapeutie measures in which electricity aets as the motive power. The second part will have to do with the applieation of electricity in the treatment of disease.

\section{VARIETIES OF ELECTRICITY.}

Galvanic Electricity.- The galvanic eurrent, while not the first to be diseovered, is perhaps the most useful form of electricity from an eleetro-therapeutic standpoint.

Galvanic electricity may be obtained from a battery of wet or dry cells, the latter in recent years having practically superseded the equally effeetive but less conveniently handled wet cells. The current may also be obtained from the public main.

A wet eell eonsists of a vessel in which are placed two metals or a metal and another substance spoken of as elements, and a fluid electrolyte.

'The elements must be substances capable of reacting chemieally and in a dissimilar manner to the action of the electrolyte.

The electrolyte must be eapable of aeting upon or exciting the elements by chemical action, through which aetion electricity is generated. These conditions are fulfilled if we place zine and earbon in a solution of dilute sulphuric acid and connect them outside the vessel by means of a wire. The action of the acid upon the zinc generates an electromotive force which passes through the liquid from the zine to the carbon and from the carbon along the wire back to the zinc. 'The direction of the flow of an electric current is always from the positive toward the negative or from the higher to the lower potential.

When two dissimilar metals are brought in contact, one will become positively and the other negatively electrified. This difference varies with different metals, being greater, for instance, if zinc is brought in contact with copper than it would be if the contact were between zine and iron. 'The difference beiween the electropositive and the electronegative 
is what is known as "diflerence of potential," and as the electroposicive state is stronger or higher than the clectronegative state, we have the flow of the current in the direction of the least resistance, or, as al reasly stated, from the positive to the negative.

'The projecting ends of the elements above the surface of the liquid in the cells are spoken of as the "poles" or "electrodes," and have at tached to them the conducting cords.

Varieties of Cells. - There are many kinds of cells, in which a great variety of elements and electrolytes are used. Among th most practical forms are the Since, the bichromate, the I eClanche, the silver chloride, the Daniell, the Fleming, and the Grove.

'The Smee cell is composed of a plate of platinized silver placed between two plates of amalgamated zinc, with a dilute solution ( 1 to 10), of sulphuric acid. When this cell is not leing used, the rine should be withdrawn from the solution, otherwise it would be slowly eaten awa by the artion of the acid. 'The electromotive force of this cell has heen estimated at from 0.65 to 1 volt.

FIg. 41.

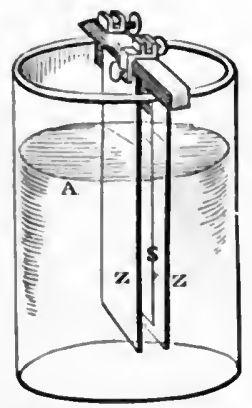

Smee cell: A, exciting fluid; S, silver plate, platinized; $\mathrm{Z} \mathrm{Z}$, zincs.
FIg. 42.

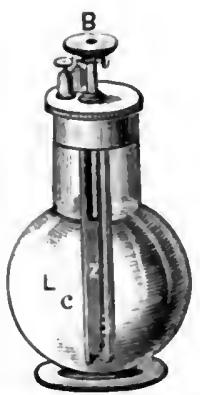

Grenet cell: I, liquid; C, carbon; $\mathrm{Z}$, zinc.

'The Grenet or bichromate cell consists of a plate of zinc (positive element) between two plates of carbon (negative element), acted upon by a solution of bichromate of potash and sulphuric acid (electrolyte). Chromic acid may be used in place of bichromate of potash in making the electrolyte. 'ithis is an exeellent cell, largely used, having an electromotive force of from 1.95 to 2 volts.

'The LeClanche cell has as its positive element a small rod of amalgamated zine and as its negative element a plate of carbon. 'The electrolyte is a strong solution of sal ammoniac. The carbon plate is surrounded by a mass of coarsely powdered carbon and oxide of manganese, enelosed in a porous cell. This cell is placed in a glass jar one-third full of sal ammoniac in which the rinc rod is immersed. 'The electromotive force of the LeClanche cell is from 1.40 to 1.45 volts.

'The chloride of silver cell has an ammonium chloride (sal ammoniac) solution as an electrolyte, with zinc as the positive element and silver 
as the negative element. 'The silver electrode is protected from coming in contact with the rine by being enclosed in a paraffin paper. 'The enclosing jar is comparatively small in size, hermetically sealed by paraffine wax or by an ebonite lid and rubber washer.

This cell does not deteriorate rapidly when not in use, owing to the fact that a coating of oxychloride of zinc forms about the zinc and thus increases the internal resistance of the cell. The electromotive force is from 0.9 to 1.7 volts.

'The Daniell cell is a double fluid cell. The negative element, copper, is enclosed in a porous cell filled with a saturated solution of copper, the whole being placed in a glass jar containing the positive zinc plate immersed in an electrolyte consisting of a dilute solution of sulphuric acid. The electromotive force is a little over 1 volt and the internal resistance is high.

FIG. 44.

Fig. 43.

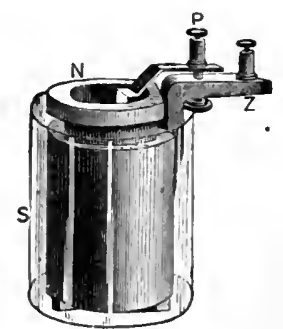

Grove's cell: S, glass cup; Z, zinc; $\mathrm{N}$, porous earthenware jar filled with nitric acid into which the platinum $(P)$ of next cell dips.

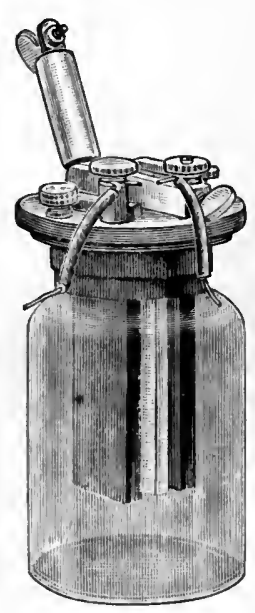

Fleming battery cell.

The Grove cell consists of a cylinder of zine as the positive plate, with platinum as the negative element enclosed in a porous pot partially filled with strong nitric acid and a strong solution of sulphurie acid as the electrolyte. Electromotive force about 2 volts.

'The Bunsen cell is like the Grove cell, except that carbon is used in place of the platinum. Both are double fluid cells.

The Fleming cell is still another form of the double fluid cell, consisting of a $U$-shaped tube with a rinc electrorle immersed in a zinc sulphate solution in one arm, and a copper electrode immersed in a sulphate of copper solution in the other arm. Electromotive force is a trifle over 1 volt.

Dry cells, so called, are cells in which the electrolyte is held in a jelly-like or paste-like mixture made by the addition of some absorbent material, the jar being sealed to prevent evaporation. 
Determination of Polarity, -A pole detector or prole tester, as it is termed, consists of a scaled glass tube containing a solution of phenolphathalcin in water. Platinum wires pass in through the cork and come in contact with the solution, which turns red when brought in contact with the negative pole.

A ready practical method is to ayply the poles, a short distance apart, to wet litmus paper, which will be discolored red at the acidl, positive pole, and blue at the alkaline, negative pole, In using a milliamperemeter, it will be observed that the needle is always deflected toward the positive pole.

Upon electrolysis of water, bubbles collect about either pole, but in larger numbers at the negative pole.

The Galvanic Battery. - In the formation of the galvanic battery, a number of cells are joined. This may be aceomplished in one of two

Fig. 45.

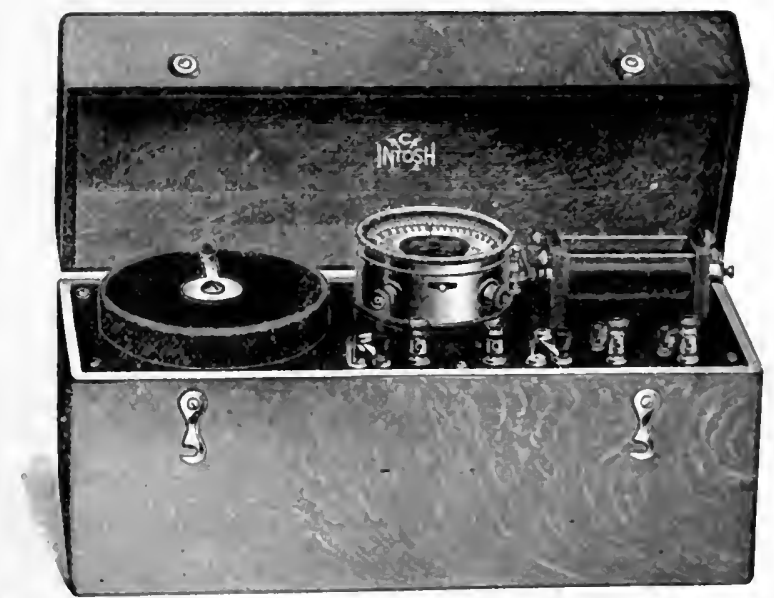

McIntosh "elaborate" 27 dry cell galvanic and faradic battery.

ways. 'The unlike elements may be combined, as, for instance, the zinc of one cell with the carbon of another, or the like clements may be joined, as zine with ginc and earbon with carbon.

When unlike elements are comnected, it is spoken of as joining in series, a battery so constructed giving a current of high voltage and low amperage, which is the character of current desired to overcome the body resistance in electro-therapeutic work.

When like elements are commected, it is spoken of as joining in parnllel. 'This method gives a current of high amperage, which is desirable for cautery work.

A battery composed of wet cells is satisfictory for office work, but in treating the patient at his home a portable battery is required, and for this purpose the dry cell batteries have proved to be lighter and more practical. 'There are very satisfactory portable hatteries upon the market 
containing from 20 to 40 cells, with which the operator can obtain excellent results. It has been my experience that the so-called "combination batteries" arranged to give both galvanic and faradic currents have proved far from satisfactory, separate batteries being more dependable.

A more reliable source of current supply when available is to obtain the current directly from the public main, utilizing for this purpose one of the numerous wall plates, tables, or cabinets upon the market, which also enables the operator to obtain the faradic and galvano-faradic currents as well. The direct or constant current is the one desired, so if the street current is alternating, it must be changed by a "transformer" to the direct current. From whatever source obtained, the current should

FIG. 46.

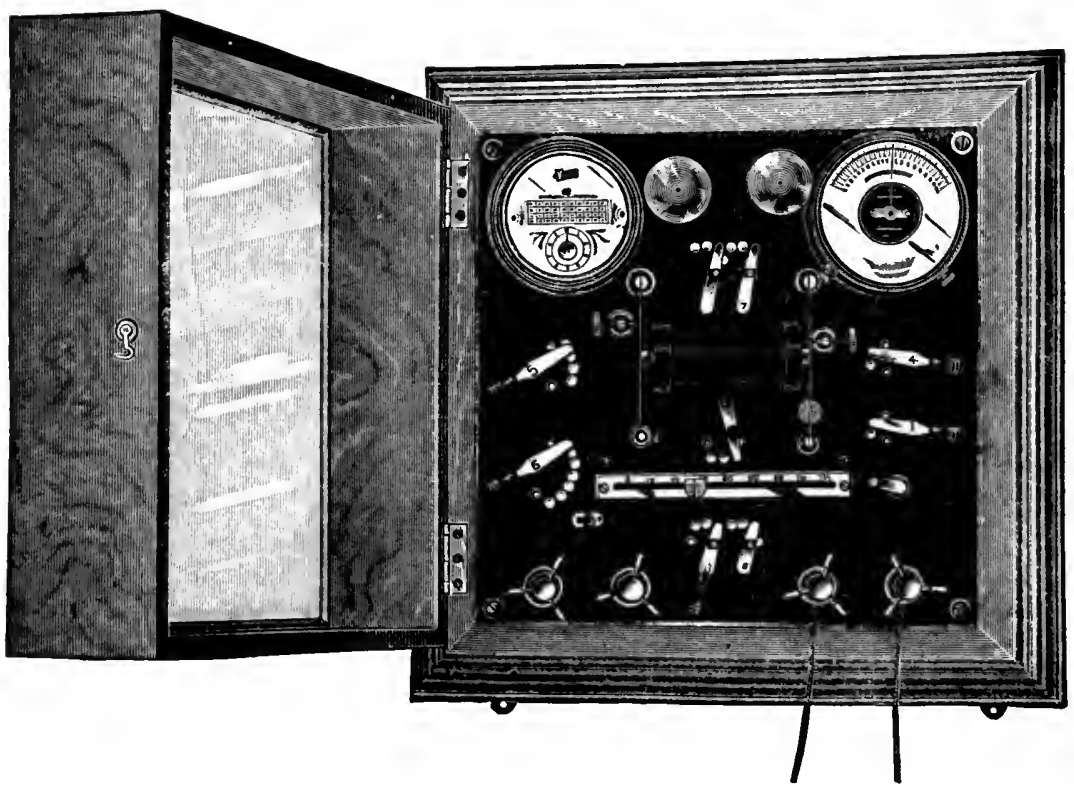

Victor ear phone massage instruments for attachment to wall plate.

be constant, with a maximum electromotor force of from 40 to 50 volts giving a current capable of varying from 0 to about 200 or 250 milliamperes. 'The battery or "plate" should be supplied with a rheostat and a milliamperemeter.

Rheostat.- The rheostat is an instrument so constructed as to enable the operator to control the current by increasing or decreasing the resistance to the flow of the current. Graphite strips or coils of wire are so arranged as to furnish variable resistance, the length of the resisting medium employed being regulated by means of a sliding contact. Of the two, the wire rheostat is the more reliable, as the current heats the graphite and deereases its resistance; it is also difficult to regulate the amount of graphite employed. 
Milliamperemeter.-A milliamperemeter is an instrument nsed to detcrmine how much current is passing through the patient. In other words, it tells us the dose or amount of current the patient is receriving.

Fig. 47.

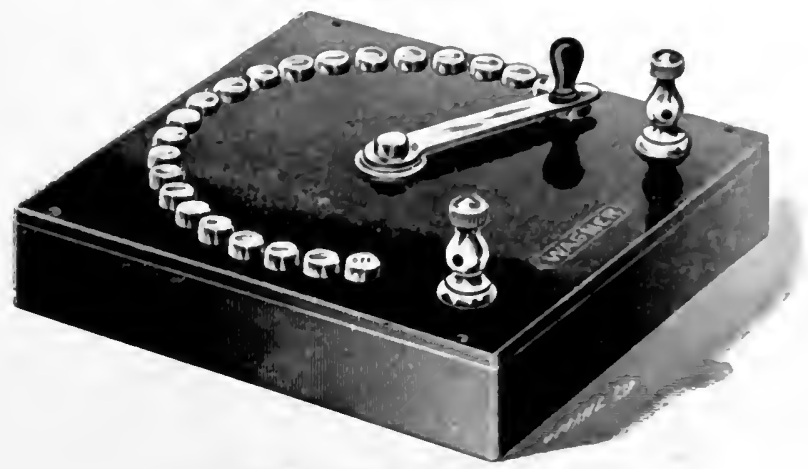

Storage cautery battery rheostat.

We cannot tell the dosage by the number of cells employed, but we control the current by using the rheostat, decreasing the resistance until the meter registers the amount of current we wish the patient to receive.

Fig. 48.

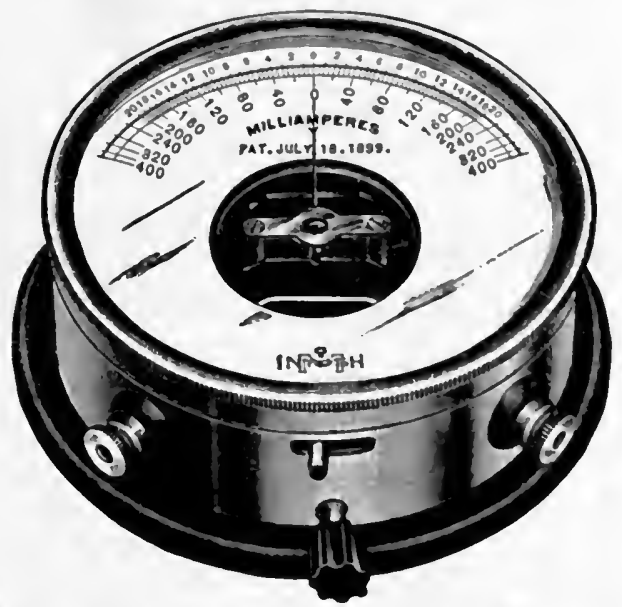

MeIntosh improved milliamperemeter with shunt.

Electrodes.-Electrodes vary in size, shape, and composition, de pending upon the purpose for which they are intended. 'Thus we have cutaneous, urethral, rectal, and vaginal electrodes, and electrodes for the eye, ear, and nose, etc. For general work, in addition to the usual small, round and flat metal electrode, I would advise the operator to have at least two of the large flexible electrodes to be found on the market, as the greater the area of cutaneous surface beneath the electrode, the more 
diffused the current and thus the greater the amount that can be given without blistering or other undesirable effects. An electrode with an in-

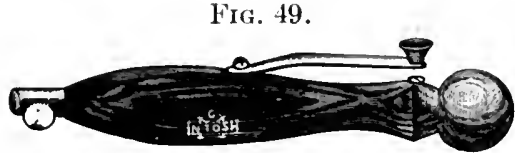

Universal handle, with interrupter.

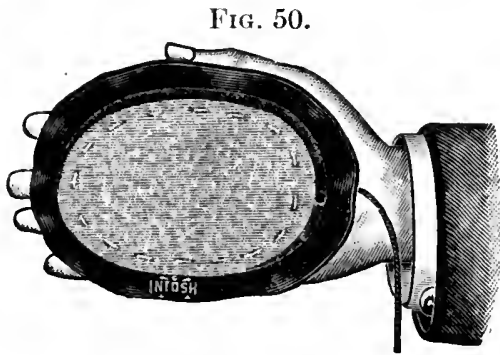

Sponge-covered electrode, insulated with soft rubber for general application with the hand.
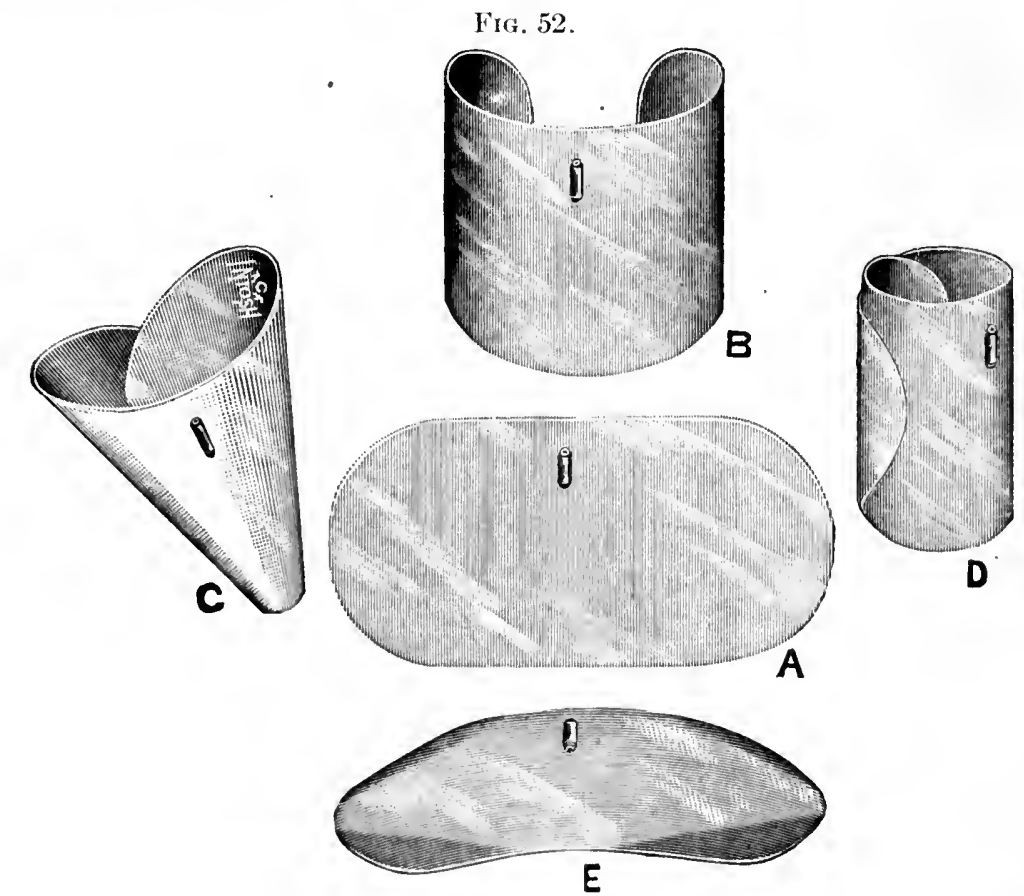

Parl electrodes.
Fig. 51.

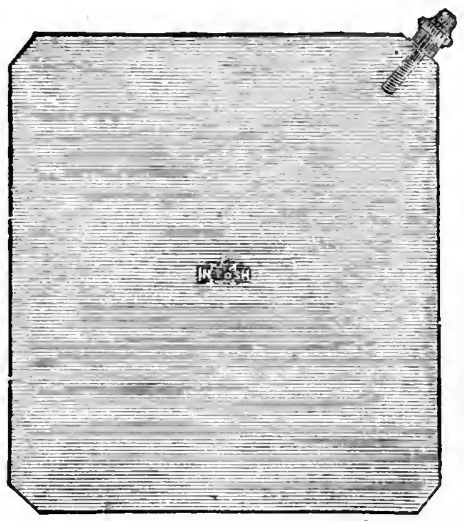

Metallic foot plate.

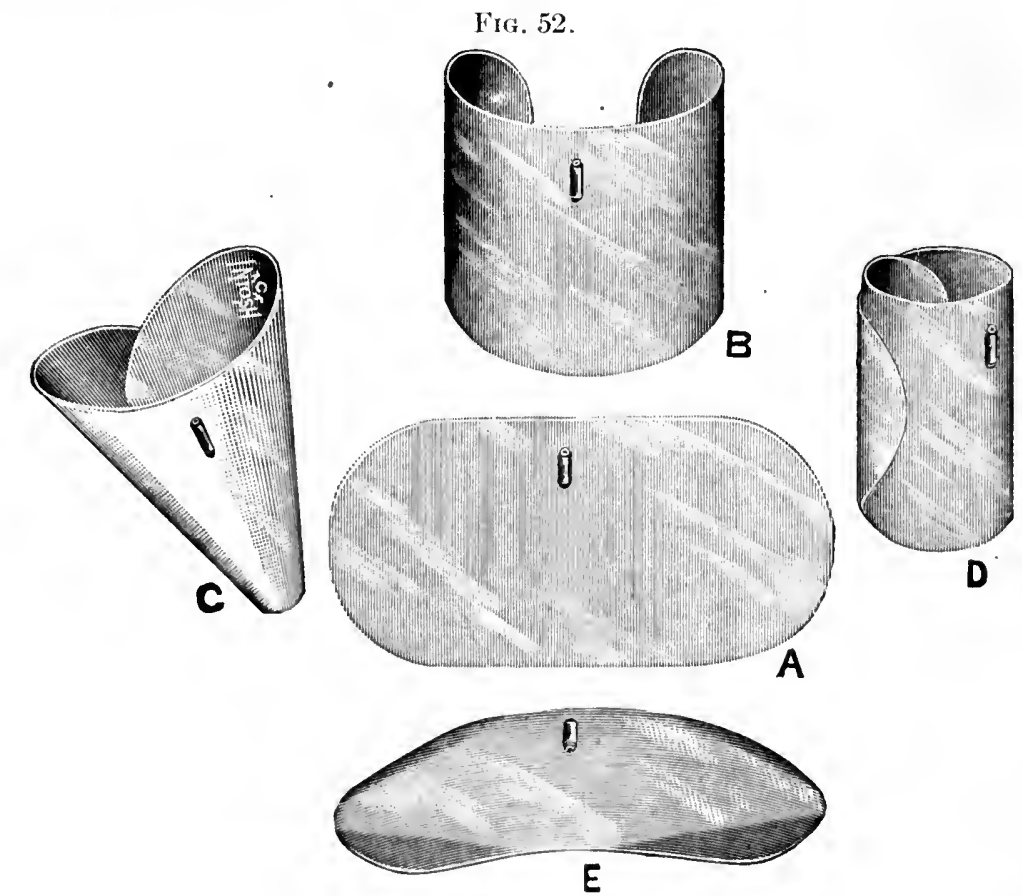

terrupter handle is indispensable, enabling the operator to make or break the current at will. 
Fig. 53.

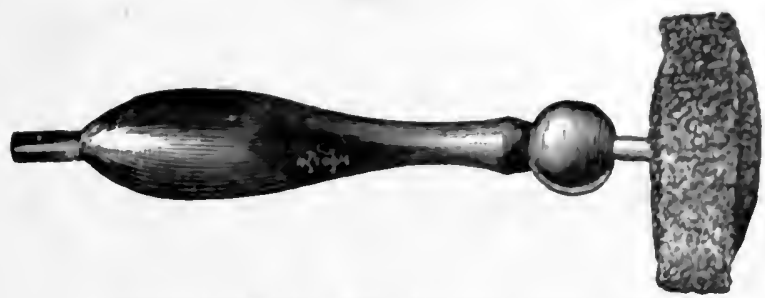

Universal handle, with sponge-covered discs.

Fig. 54.

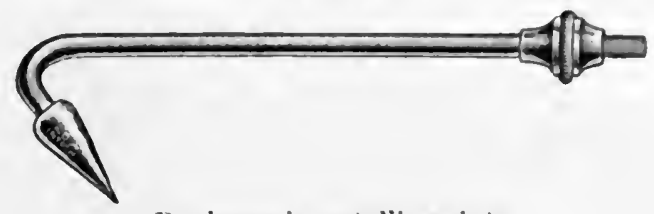

Duchenne's metallic points.

FIG. 55.

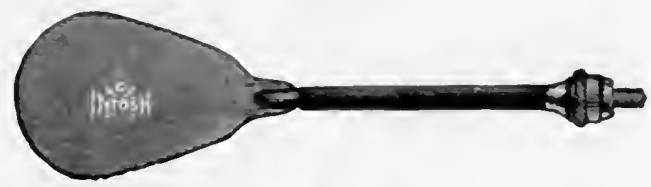

Copper tongue plate, insulated.

FIG. 56.

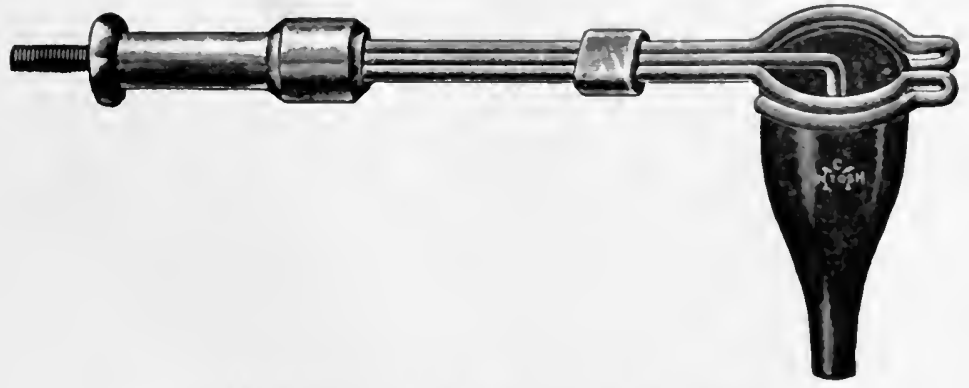

Ear eleetrode, with hard-rubber speculum.

FIG. 57.

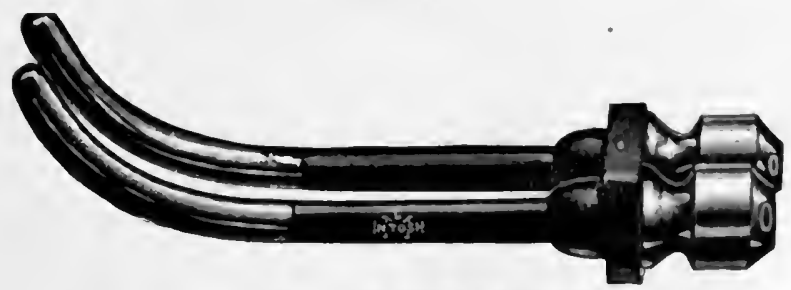

Double copper nasal electrode.

VoL. I-18 
FIG. 5S.

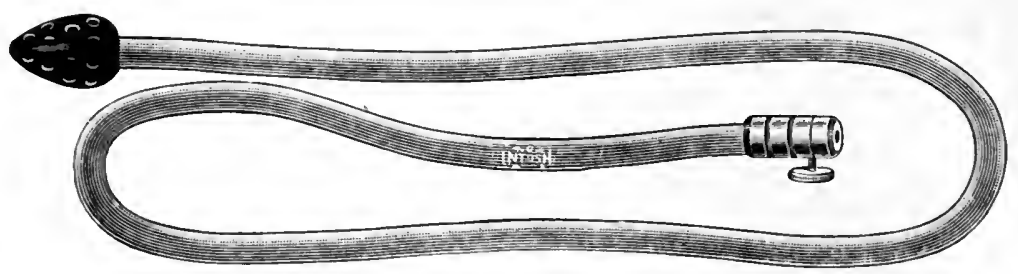

Stomach electrode.

Fig. 59.

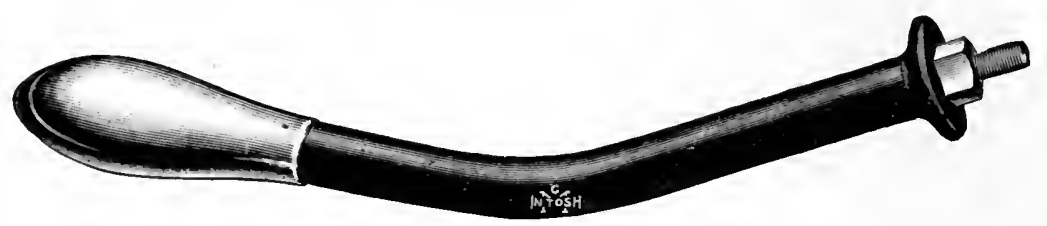

Curved vaginal electrode.

FIG. 60 .

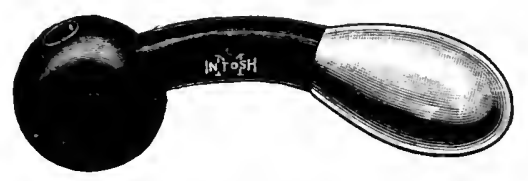

Copper rectal electrode for hemorrhoids.

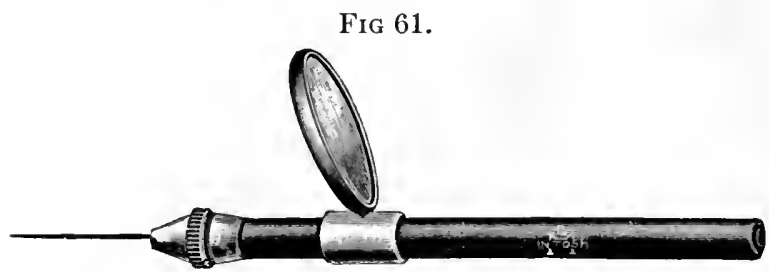

Needle holder with magnifying glass.

Rheophores.-Rheophores are the wires connecting the electrode with the battery. 'They are marle of insulated copper wire, the wire being usually composed of several fine strands twisted together which make the rheophore more pliable than if a plain wire of the same size were used. Insulation is obtained by a covering of silk, cotton, or India rubber.

Electromotive Force (E. M. F.).- This is the power which enables electricity to move from place to place and to overcome resistance. It results from the difference of potential, and the greater this difference, the greater is the electromotive force. It can be likened to heat which passes from the body of the greater to the body of the lesser temperature, to gases which move from the place of higher to one of lower pressure, or to the flow of water from the higher to the lower level. 
Ohm's Law.-Measurements of electromotive force are based upon the law of Ohm, which is as follows: "The strongth of the current passing through any part of a circuit varies directly as the difference of potential between its elements, and inversely as the resistance of the circuit itself."

Expressed in symbols, we would have-

$$
\mathrm{C}=\frac{\mathrm{E}}{\mathrm{R}} \text {; or } \mathrm{I}=\mathrm{C} \times \mathrm{R} \text {; or } \mathrm{R}=\frac{\mathrm{E}}{\mathrm{C}} \text {. }
$$

C (current), I (electromotive force), R (resistance).

$\mathrm{C}$ is expressed in amperes.

$\mathrm{F}$ is expressed in volts.

$\mathrm{R}$ is expressed in ohms.

The volt is the unit of electromotive force; the ampere, the unit of the rate of flow of the current; the ohm, the unit of resistance, and the coulomb, the unit of quantity of the current.

'The volt is that quantity of electromotive force or pressure that will force or drive a coulomb through a resistance of one ohm. 'The amount of electromotive force generated by a Daniell cell is approximately one volt (1.079 volts).

'The ampere is that amount of current strength furnished by an electromotive force of one volt driving electricity through a resistance of one ohm.

'The ohm represents the amount of internal resistance in a cell having an electromotive force of one volt and producing a current of one ampere.

'The coulomb is that quantity of electricity that will pass through a circuit when a current strength of one ampere is maintained for one second.

Physiological Action of the Galvanic Current.-The physicochemical effects of the passage of the galvanic current through the living body are electrolysis and eataphoresis.

Electrolysis. - This is a katabolic phenomenon-the breaking up of a substance into its original elements by the passage through it of a current of electricity. 'The substance acted upon is termed an "electrolyte," and must be a conductor of electricity and in the fluid or semifluid state. Another requirement is that there must enter into its construction a metal or the salt of a metal. Albuminoids, fats, or sugar are non-conductors, and, therefore, not electrolytes.

When "electrolytes" are disintegrated by the action of the galvanic current, decomposition products are formed which are supposed to represent dissoeiated portions of a molecule and are attracted, some toward the positive, and some toward the negative, pole of the battery. 'These substances or "dissociated portions of molecules" are termed "ions."

Ions which are attracted by the positive pole and repelled by the negative pole are termed "anions," while those attracted to the negative pole and repelled by the positive pole are termed "cathions."

The practical signifieance of these phenomena is, that the liquids or fluids of the body are electrolytic - that upon the passage through them 
of the electrical current the electrolytes in solution are disintegrated, the anions (acid molecules) liberated appearing at the positive pole, and the cathions (alkaline metals) at the negative pole, while in the interpolar region there is an exchange of ions, the cathions following the direction of the electrical current and the anions moving against the current.

Polarity Effects.-For electrolysis of living tissue, the cathode should be the active pole, as it is a vaso-dilator and tends to liquefy or disintegrate, while the anode is a vaso-constrictor and tends to harden tissue.

Other polarity characteristics of practical importance in using the galvanic current are as follows: The positive pole, having an affinity for oxygen, creates an acid reaction in the tissues and has a sedative effect, relieving pain, while the negative pole, having an affinity for hydrogen, brings about an alkaline reaction in the tissues and acts as an irritant.

'Therefore the positive pole should be used as the active electrode where the operator desires to induce vascular constriction and produce sedation, as in bleeding, inflammatory processes, or to relieve pain where there is irritation. Conversely, the negative pole should be used as the active electrode where the operator desires to produce vascular dilatation, congestion, or tissue destruction, as in counter-irritation, removing moles, tumors, etc.

Cataphoresis.-This is the phenomenon of the movement of a liquid or substance in solution without dissociation of the molecules, through the tissues in the direction of the current. That the galvanic current could be utilized for the purpose of introducing medicine into the tissues was first mentioned by Palprat in 1833. As Neiswanger points out, however, it must not be supposed that all medicaments must be placed upon the positive pole to be carried into the tissues by the direction of the current. Following the law of electrolysis, the medicine is broken up into ions, which, according to their character, have an affinity for one or the other of the poles. Thus the alkalies, being attracted by the negative pole, should be placed upon the anode, while the acids, being attracted by the positive pole, should be placed upon the cathode. In using a salt, the pole to be selected would depend upon what part of the compound is desired for the therapeutic effect. In using the salts of morphine or cocaine in solution, the drug should be applied from the positive pole, and in using potassium iodide; the medicine should be placed upon the negative electrode.

This method of administering medicine has a somewhat restricted field of usefulness. For general medication, the amount capable of being introduced into the tissues would be so slight in comparison with that which could be absorbed through the mucous membrane of the gastrointestinal tract as to render the method useful only when the stomach rebels or as an adjunct.

Where local introduction into the tissues is desired, it has a wider range, and is used by dentists for the introduction of cocaine: and is sometimes useful in introducing iodine into a gland or salicylates or allied drugs into the joirt tissues. 
Method of Application.-Pads of lint, cotton-wool, or other absortent material are attached to the electrode and saturated with the solution the operator desires to employ. Weak solutions (1 to 2 per cent.) should be employed, as ionic penetration is not increased by an incrouse in the strength of the solution. Where a strictly local effect is desirerl, I have found the following method of Guilleminot's very sitisfactory. Over the skin of the region to be treated apply a sheet of gutta-percha with a central opening, the edge of which opening is thickened so as to press upon the underlying tissue and thus limit the entrance to the tissue immediately under the aperture over which the electrode is applied.

Physiological Effects.- The sensory effect of the passage of a galvanic current consists of a burning or tingling which is directly proportionate to the strength of the current, and most marked at the cathode.

A steady, uninterrupted galvanie current passing through a motor nerve does not cause contraction of the muscle, but brings about a modification in the condition or excitability of the nerve, which is termed "electrotonus."

Elcctrotomus.-Near the anode, excitability is diminished (anelectrotomus) and near the cathode it becomes increased (catelectrotonus). Between the two electrodes is a neutral point, where the excitability of the nerve is unaltered; this, when a weak current is employed is nearer the anode, and with a strong current, nearer the cathode. With a weak zurrent, therefore, the greater part of the interpolar area is in a state of increased excitability, and with a strong current in a state of diminished excitability.

Effccts of Variations of Current.-Beside the sensory phenomena when the current is closed or opened, muscular contraction oceurs at both opening and closing because the nerve is stimulated. 'The stimulation, and hence museular contraction, is more pronounced at the cathode on closing than that obtained at the anode on opening. Nerves, therefore, are stimulated when made more excitable-i. $c$., when catelectrotonus is established or anelectrotonus is removed.

Muscular contractions appear in the following order as the intensity of the current is raised (Erb):

C C C contraction on closure when the active electrode is a cathode.

A C C contraction on closure when the active electrode is an anode.

A O C contraction on opening when the active electrode is an anode.

C O C contraction on opening when the active electrode is a cathode.

Non-striatcd Muscle.-Involuntary muscles are more sluggish in reacting than voluntary muscles, and react better to the galvanic than the faradic current. They also contract in response to a steady uninterrupted current.

The Brain.-When the positive pole is placed on the forehead and the negative pole at an indifferent point, the subject feels intellectually stimulated, whereas if the poles are reversed, he becomes drowsy:

When the galvanic current is passed through the temporal regions, the subject experiences giddiness and a feeling as if drawn toward one side 
-away from the cathode on closing and toward it on opening the circuit.

Therapeutics.-The galvanic current is of value for its effect upon metabolism through its electrolytic action, and in the administration of medicine through the integument or mucous membrane by means of cataphoresis. It is useful in relieving pain or local spasm when the current is flowing constantly, and is the best stimulus for nerves and muscles when it is properly interrupted.

It should be remembered that the smaller the electrode, the more concentrated the action of the current, therefore the active electrode should be small where a limited or superficial area is to be treated, and large when it is desired to reach deeply located organs. Moreover, the electrodes should be arranged so as to include the organ to be treated between them, that the organ may lie in the path of the current as it travels directly from pole to pole. As a general rule, the largest electrode is attached to the cathode, as the most pain is experienced at that pole when the current is strong enough to produce sensory effects.

Application.-The electrodes should be moistened with warm water (preferably salt water) to secure a better penetration of the current.

The current is said to be applied by the "stabile method" when the electrodes are not moved during the séance, and by the "labile method" when the active electrode is moved rapidly over the part treated. For a sedative effect, the current should be increased and diminished gradually by means of the rheostat, a voiding any shocks or muscular contractions. Vilhere stimulation is desired, the current should be interrupted.

Reversal of the current should be avoided as far as possible, especially about the head.

Dosage.-This varies, depending upon the part treated and the effect desired. 'The average is from five to ten milliamperes, rarely over twenty milliamperes. Séances usually last from five to fifteen minutes and may be repeated every second or third day.

For the use of the galvanic current in disease see latter part of this article.

Static Electricity.-Certain dissimilar substances when rubbed together become electrically charged, one positively and the other negatively. This electricity is termed "static." The amount of electricity obtained in this way readily diffuses itself along subtsances which are good conductors, as the metals, but may be collected and studied if imparted to a non-conductive substance like glass, which does not readily diffuse its static charge.

Conduction.-All substances do not conduct electricity equally. 'Those along which electricity diffuses itself readily-substances offering but little resistance to the electrical flow-are spoken of as "conductors." Those along which electricity passes with difficulty-substances offering great resistance to the electrical flow-are termed "non-conductors or insulators." 'Thus, silver, copper, iron, and other metals are good conductors, while the tissues of the body and ordinary tap water are poor conductors. Glass, shellac, vulcanite, and any dry wood are non-conduc- 
PLATE XVII

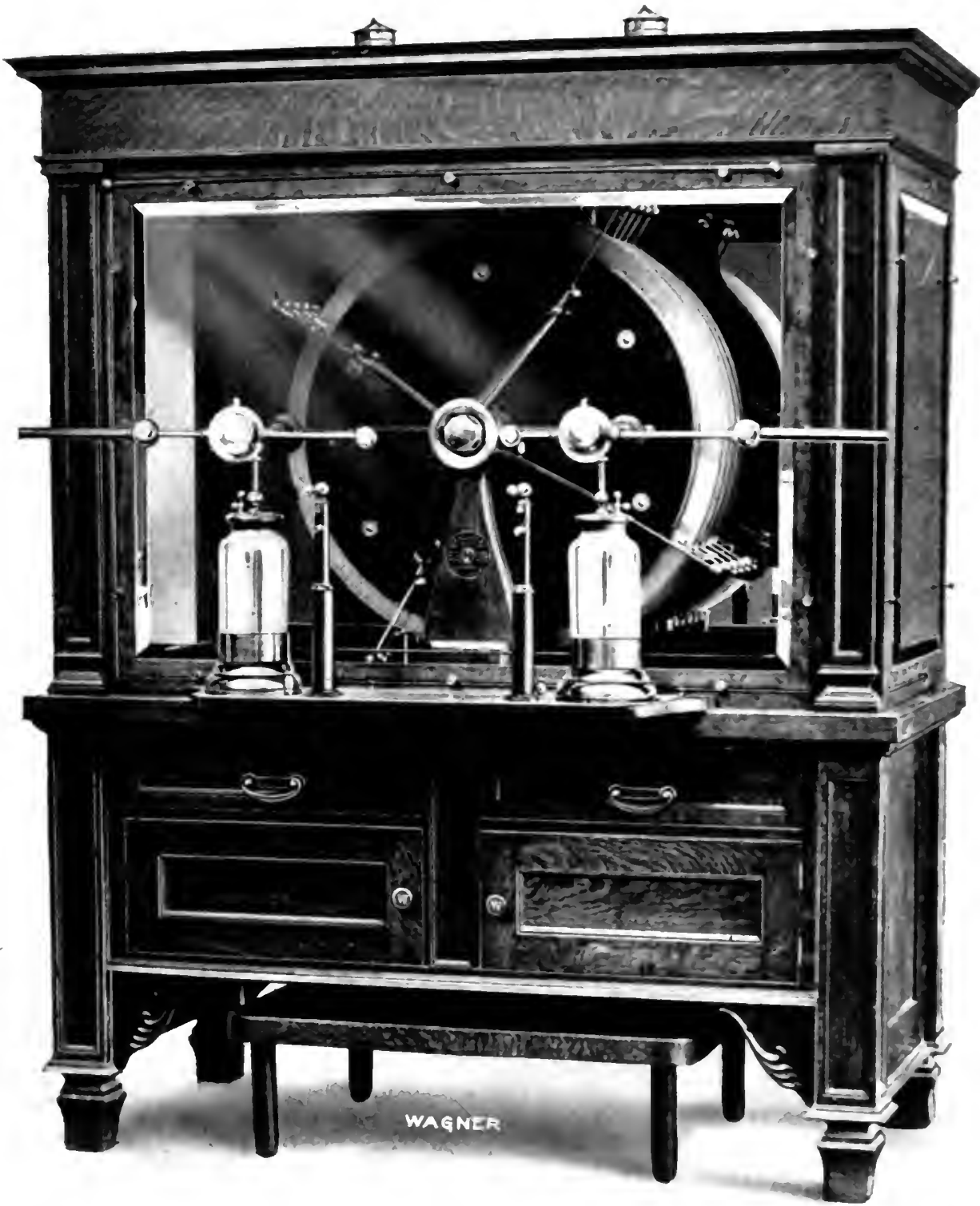

Wagner 20 mica plate high-tension electric generators. Special machine for rabiograph.: ar.k. 

tors or insulators. The skin when dry offers great resistance to the passage of electricity, but when moistened, this resistance is considerably decreased.

Induction. - A substance electrically charged can impart electricity to another substance without the bodies coming in actual contact; this is what is meant by "induction." A familiar example of this electrical phenomenon is that of the pith ball suspended by a silk thread, being attracted to a glass rod which has becn previously electrically charged by having been rubbed with a piece of silk.

Static Machines.-A continuous supply of statie electricity is obtained from "static machines," of which there are many varieties upon the market. In the older machines, the electricity was obtained by friction, but the modern types apply the principle of induction.

In the IVimshurst machine, two circular glass plates or dises of equal size are placed about one-eighth of an inch apart, and are rotated in opposite directions. 'The plates have attached at equal distances over their outer surface a series of tin-foil sectors.

The Toepler machine has a stationary and a revolving glass plate. Upon the back of the stationary plate are the "field plates," and upon the front surface of the revolving plate, a series of metal sectors. Metal brushes are so arranged that when the plate is revolved they brush against the sectors and the glass and generate enough electricity to charge the machine.

In the Holtz, as in the Toepler machine, one plate is stationary and the other revolving, or as in the modern machines used in producing static electricity for electro-therapeutic and $x$-ray work, there are several couples of stationary and revolving plates. The Holtz machine, while an efficient one, is

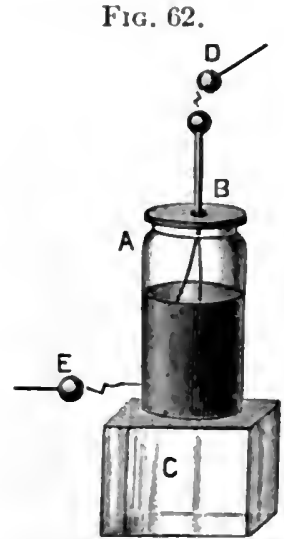

Leyden jar: $A$, Widemouthed bottle; $B$, wooden stopper for top; C, insulated stand; D, prime conductor of machine in action; E, knob in electric communication with the ground. not self charging, requiring the use of a small Wimshurst or Toepler machine within the case to supply the initial charge.

'The most commonly used machines are the Holtz or a modified Holtz known as the Toepler-Holtz machine.

Hard rubber and mica have been used instead of glass for making the plates, but are not considered as satisfactory.

A static machine with eight or ten plates, thirty to thirty-two inches in diameter, will give excellent results. It should be enclosed in an airtight case of glass and wood, and is usually driven by an electric motor.

The current obtained from such a machine would have a very high voltage and very low amperage.

As static machines are affected by dampness, it may become necessary at times to place some hydroscopic substance in the case, such as calcimm 
chloride, calcium oxide, or caustic soda. Whichever substance is used, it should be placed upon a plate or dish and changed as soon as it becomes saturated with moisture.

If dust collects on the plates, it interferes with the generation of electricity and should be wiped off with a dry, clean cloth wrapped about a lath. Should this fail, the plates may be washed with kerosene or gasolene.

Some other factors which interfere with the efficiency of a static machine are degeneration of the shellac coating of the plates, a faulty adjustment of the combs, or the plates may become loosened.

Condensers are used to store up or accumulate electricity. The most common form is the well-known Leyden jar, which consists of a glass bottle with an inner and an outer coating of tin-foil to within a quarter of its height from the top. The bottle is corked with an insulating substance, through which passes a brass conductor which is in contact with the tin-foil lining of the jar and terminates on the outside in a metallic ball or knob. In the I.eyden jar, the conducting surfaces are the tin-foil coatings, and the non-conducting surface, the intervening glass of the jar (Fig. 62). The glass is spoken of as the "dielectric."

A Leyden jar may be discharged by bringing one end of a bent wire in contact with the outer tin-foil covering, and approaching the other end to the metal knob until a spark passes between the knob and the wire. It may be discharged through the body by touching the outer covering with one hand and approaching the other hand to the knob.

Leyden jars may be attached to the poles of a static machine, where they act in the capacity of electrical reservoirs.

Accessories. - In the application of static electricity for therapeutic purposes certain accessories are necessary. An insulating platform is required upon which the patient may stand or sit. The platform may be of wood, and should have glass legs from six to nine inches long. When in use, this platform should not be in contact with the wall nor with any object about the room.

Brass chains are used as conductors, making connections about the machine wherever required.

Variously shaped electrodes are employed, with insulated handles, usually of vulcanite, and having a ring for the attachment of one end of a chain conductor, the other end of which would be connected with a pole of the static machine.

One of the electrodes in a set should terminate in a metal point, another in a number of small, parallel brass wires or rods. One electrode should have a two-inch brass ball at the extremity; another a twoinch brass roller. Sometimes a carbon-pointed electrode is of service, and sponge electrodes, while not important, may be of use.

'The crown is used for giving the head breeze, and consists of a brass ring nearly a foot in liameter, with a row of points projecting from the lower border of the band. It is connected to the top of the machine by a brass rod so arranged as to be capable of vertical and horizontal adjustment. 


\section{PLATE XVIII}

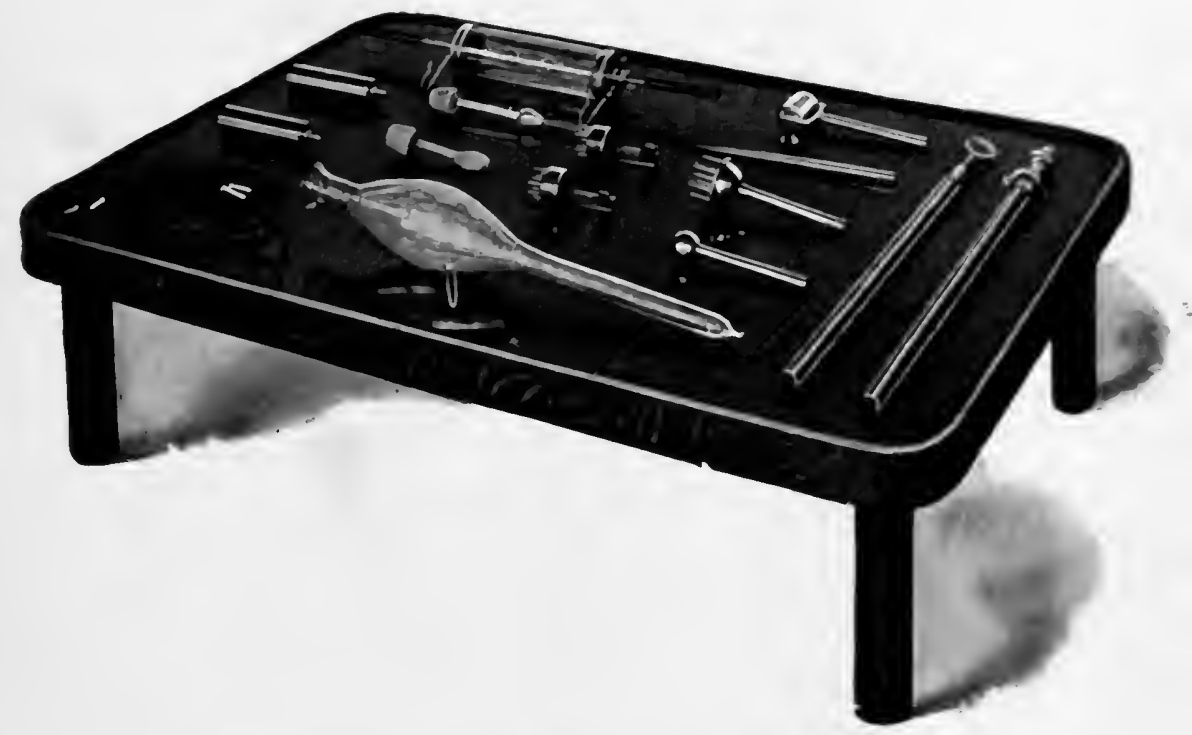

Insulated platform, ionizer, spark regulator, and static electrodes. 

A chain holder is used to keep the chain from conning in contanct with the patient, and a shepherd's crook of brass may be used where a rod is desired to make comnection.

Polarity. - The polarity of the terminals of a static machine maty be deternined by disconnecting the leyden jars and starting the machine with the prime conductors from one-half to one inch anpart. At the prositive pole the spark will be white, while at the negative pole it will be of a violet color.

If the prime conductors are placed some distance apart, and a lighterl candle held between them while the machine is in motion, the flane will be deffected toward the positive pole.

Physiological Effects of Static Electricity.-Static electricity may be applied in the form of the spark or friction, the aigrette, the effleuve, and the bath.

Elcctric Spark or Friction.-'The sensory reaction depends upon the character of the spark, and varies from the slight pricking sensation to a severe shock.

The motor reaction consists of muscular contraction, which is most marked at the negative pole and is in direct proportion to the square of the spark length. Voluntary muscles have been known to respond to static sparking when they had failed to contract under the influence of the other forms of electricity.

The vasomotor effects are first, vasomotor constriction- with accompanying pallor of the skin, which is followed by vasomotor paralysis with the accompanying phenomena of redness and heat. 'The effects are localized to the area over which the sparking is applied, and are most marked when the positive pole is used. In addition to these phenomena, it is claimed by some authorities that electric friction has a sedative effect upon the deeper tissues.

The Elcctrostatic Effleuve, Aigrette, Douche, etc.-'The electrostatic effleuve gives rise to a local sensation of coolness, which is accompanied by an actual lowering of the surface temperature. 'This effect is most marked at the cathode and is prolonged for some time after the application of the current.

The static breeze is sedative in its effect, tending to relieve pain. It is claimed by some writers that it also acts on the trophic nerves, increasing tissue vitality.

The Elcctrostatic Bath.-Tissue changres, glandular secretions and heat production are said to be increased by this modality. 'The most marked effect of the electrostatic bath upon the circulation is an increase in the arterial tension. 'Trichot claims that there is also an increase in the frequency of the pulse-rate which is maintained for a week after the treatment.

Methods of Application.-Electrical Friction or Sparking.-The patient is seated upon the insulating platform, which is connected with the positive pole. The electrode is attached to the negative pole and held a short distance from the skin or the clothing. 'The electrode should be moved rapidly, so that not more than one or two sparks are given at a time. 
Electric Effleuve (Douche, Breeze, Aigrette, Souffle).-The patient is placed as described for sparking, the platform being connected with whichever pole desired. An electrode with single or multiple points is held several inches from the patient, over the area to be treated, and not approachefl closely enough to cause sparking.

Electrostatic Bath.--'The patient is seated upon the platform, which is connected to one pole of the machine, the other pole being earthed. For a stimulating effect, the connection is made between the platform and the positive pole, the effect of the opposite polarity being depressant. While receiving the static bath, the patient is traversed by the current, which imparts a tickling or pricking sensation as it is given off from all parts of the skin. 'The duration of treatment by this method should be about fifteen minutes.

Faradic Electricity.-If a magnet or a current carrving wire is approached to or withdrawn from a closed circuit, there will be momentarily induced in that circuit a current of electricity. From the above discovery of Faraday are evolved the principles utilized in the construction of the ordinary faradic battery.

A faradic buttery consists of a primary coil of insulated wire surrounding a soft-iron core, and a secondary wire coil surrounding but being insulated from the primary coil. The ends of the primary coil are connected with a voltaic cell or cells, which have, however, no connection with the secondary coil. An automatic hammer is arranged to interrupt the current from the cells.

When in operation, the soft-iron core is magnetized by the current from the surrounding primary coil, and throws out lines of magnetic force which induce an electrical current in the wire coil, which flows one way when the magnetic lines are thrown out and in the opposite direction when they are withdrawn. The alteration in flow of the magnetic lines is brought about by the antomatic hammer or interrupter, which is so arranged that when its iron head is drawn by the magnetic force toward the central core, the spring of the hammer is pulled away from the point of a thumb screw which served as a part of the circuit. This breaks the circuit, the current reases to flow through the primary coil, the core becomes demagnetized, allowing the hammer to fall back until contact is made again with the screw point. This closes the circuit and the whole process is repeated.

The current in the primary coil as it is rapidly made and broken produces by induction an alternating current in the secondary coil. It is this circuit which is most generally employed medically under the name of the faradic current.

'The primary coil should consist of a few turns of thick wire, so as to keep the resistance as low as possible, while the secondary coil should consist of a great number of turns of a thin wire well insulated to prevent short circuiting.

Physiological Action of the Faradic Current.-The effects of the faradic current are mechanical, the phenomena of electrolysis and cataphoresis not being obtained through its use. 'The faradic current 
differs from the galvanic current in having high electromotive force, but low current strength.

Stimulation of a motor nerve produces museular contraction, which continues throughout the duration of the flow of the current.

'The opening current gives rise to a stronger musconlar contraction than the elosing current, and the reaction is greater at the cathode than at the anode.

FIg. 63.

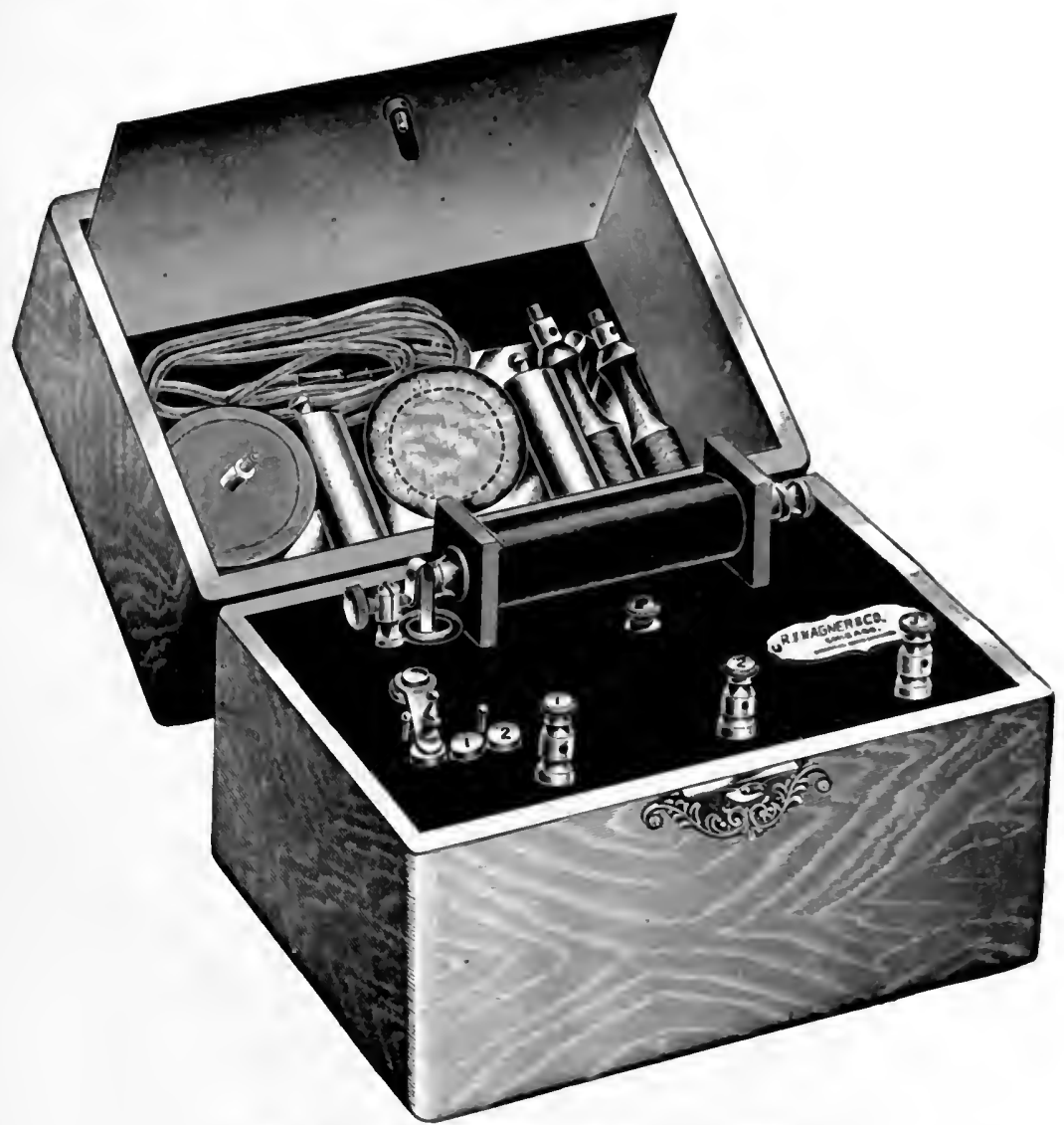

Portable faradic battery with rapid spring vibrator.

The experiments of Debedat upon rabbits have shown that numerous applications of a rhythmic faradic eurrent, each of short duration (four minutes), caused the muscles treated to increase in size and weight to a marked degree, the increase being due to development of the musclefibers, while tetanization, if prolonged, would cause atrophy of the muselefibers.

In using the faradic current to stimulate a wasted muscle, it is important, therefore, that the eurrent be interrupted and the séances not too long. 
For the purpose of interrupting the current, an "interrupter handle" may be used, or a "rheotome," the latter instrument securing rhythmic interruptions.

Upon sensory nerves, the effect of the faradic current is that of a burning or pricking sensation directly proportionate to the strength of the current up to a certain point, after which it is said to have a numbing sensation (faradic anesthesia). The current also gives rise to a sensation of warmth.

The faradic current is physiologically an excitant of functional activity, exercising the muscles and stimulating general metabolic activity.

Fig. 64.

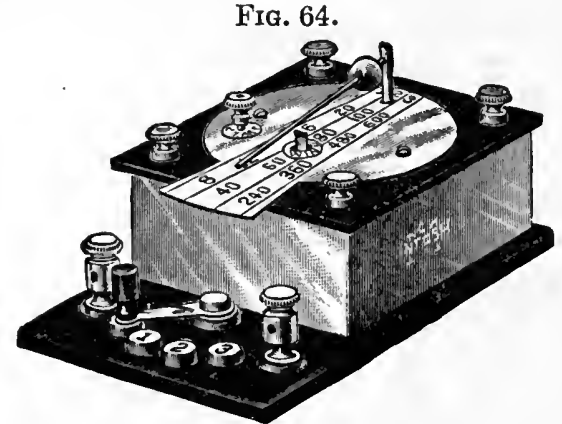

McIntosh graduated automatic rheotome.

Electrodes.-These are of various shapes, sizes, and construction, depending upon the use for which they are intended. The ordinary sponge-covered, flat metal electrodes are most commonly employed. I prefer, when using these electrodes, to cover the metal with a layer of absorbent cotton. This can be renewed for each patient, being moistened with water, or, what is better, salt solution.

Netal cylinder electrodes can be held in the hand, and metal plate electrodes are employed by placing them under the bare feet of the pa-

Fig. 65.

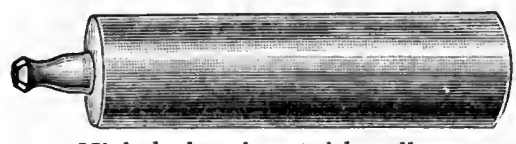

Nickel-plated metal handle.

tient. A bipolar roller electrode is used in giving faradic massage, and a wire brush electrode is useful where revulsive effects are desired. 'There are also different forms of bipolar rectal electrodes.

Methods of Administration.-The faradic current may be applied locally, generally, or in the form of the faradic bath. When employed locally, one electrode is usually placed at an indifferent point and the other applied over the part we desire to treat. The active electrode may be kept in one position (stabile) or moved about over the affected part (labile). 
The electrodes should be moistened and firmly applied if an effect upon the deeper structures is desired. If the superficial effect is wanted, the skin should be dried over the area to be treated, and dusted with talcum or other powder, the current being applied with a metallic brush as the active electrode. The indiflerent electrode should be moistented and placed over a remote part of the body.

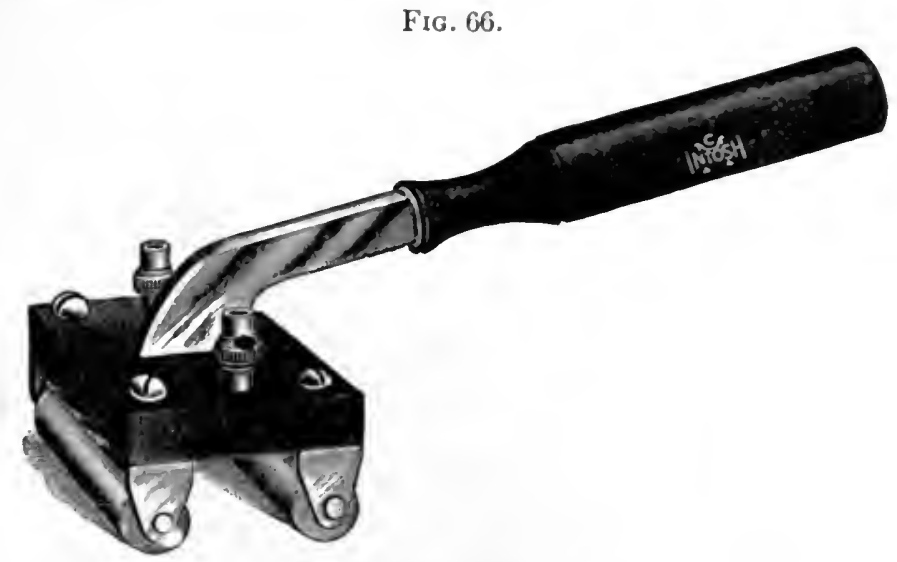

Bi-polar massage roller.

General faradization may be given by having the patient sit or place the feet on one of the plate electrodes, connected with one pole of the battery, while a hand electrode attached to the other pole is moved sy'stematically over the surface of the body and the extremities. Faradic massage is a form of general faradization.

The faradic bath is described in the section on Hydro-electric Baths.

Galvano-faradic Currents. - The combined current is sometimes spoken of as the "de Watteville current," and is ordinarily obtained by"

Fig, 67.

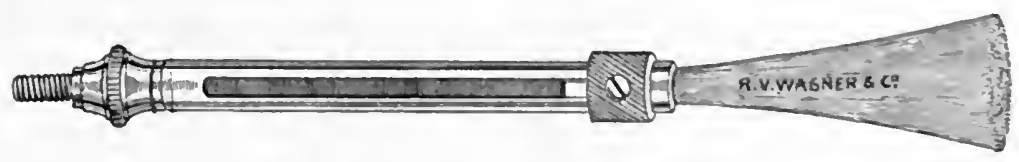

Metallic brush with protecting shield.

placing an induction coil in series with a galvanic battery connecting the positive pole of the galvanic battery with the negative pole of the secondary faradic coil. A combined current may also be obtained by connecting the positive pole of the battery to the positive pole of the coil. This would have the effect of opposing the currents.

Physiological Action.-The effect of the combined current (joined in series) at either pole, upon both striated and non-striated muscle, is greater than when the faradic current alone is used; with non-striated 
muscle, however, the anodal closing contraction is greater than the cathodal closing contraction. When the two circuits are joined in opposition, muscular reaction is diminished, owing to a decreased excitability, the galvanic current diminishing the effect of the faradic.

Upon sensation, the effect of the currents combined in series is to increase the reaction. When the two circuits are joined in opposition, sensation of pain is diminished in direct proportion to the amount of the galvanic current used.

A rapid increase in the growth of the muscle-fibers is the most marked effect of repeated applications (daily or every second day) of the combined current. This effect makes it one of the most valuable currents for treating such forms of muscular wasting as are responsive to electrical stimulation.

The Sinusoidal Current.-While many of the characteristics of the sinusoidal current have been recognized for some years, it remained for d'Arsonval to attract the general notice of the profession to the current's possibilities as a therapeutic measure.

The sinusoidal current is an alternating, induced current, in which the strength and direction vary, as represented by a sine curve. It differs from the ordinary faradic current in that the rise and fall of the intensity occur gradually instead of abruptly. Fig. 68 illustrates the change of the current with time.

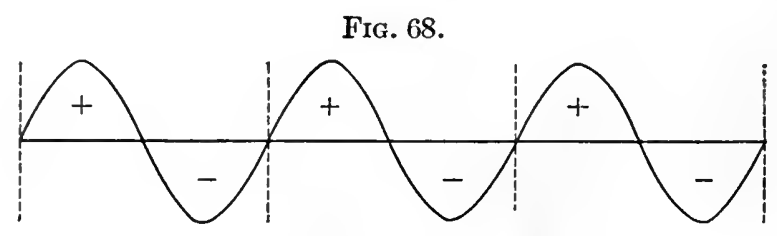

The current should vary gradually in intensity from zero to the maximum, from the maximum of intensity gradually to the minimum, then repeating with reversed polarity. As indicated by the diagram, the wave between the dotted lines would represent one complete cycle.

The sinusoidal current can be obtained by using a rotary transformer actuated by the current from a storage battery or the direct or alternating electric-light current.

Physiological Action. - Sinusoidal currents will cause contraction of both striated and non-striated muscle, the degree of excitation depending upon the amplitude and frequency of the cycles. Below a certain point (undulations too slow and too feeble) there is no muscular contraction; at another point (about 30 cricles per second) the muscle becomes tetanized, and with a frequency of 5000 per second the muscle ceases to respond. Owing to the gradual rise and fall of intensity of the sinusoidal current, the muscular contractions are unaccompanied by the sensation of pain.

Upon the sensory nerves, sinusoidal currents are said to have a sedative effect. 
The effect of the sinusoidal current upon general nutrition is to stimulate or accelerate. 'The pulse-rate is increased and the respriratory capacity of the blood increased.

Application.-Sinusoidal currents may be applied by means of the usual electroles that one would employ for the application of the galvanic or faradic currents, and also by means of the hydro-electric bath, as described in another chapter.

While useful in many conditions, the sinusoidal current is particularly valuable in treating wasted muscles, the painless character of the current permitting of a greater dosage than could be given of the faradic or combined currents.

High-frequency Currents.-We are indebted to Morton for the discovery of high-frequency currents and to d'Arsonval for the greater part of our knowledge of their physiological effect.

The spark which occurs when a Leyden jar is discharged is not a single spark, but a great number of sparks passing alternately with tremendous rapidity. It is possible for millions of these oscillations to occur a sccond, a rapidity of vibration unattainable by any mechanieal means. It is through the application of this principle that high-frequency currents are obtained. 'To illustrate by means of diagrams:

FIg. 69.

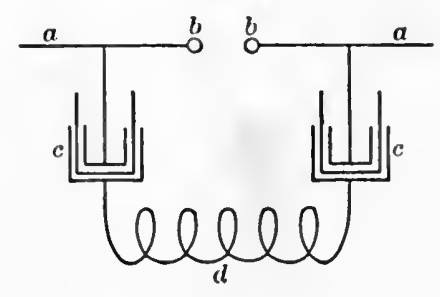

Fig. 70.

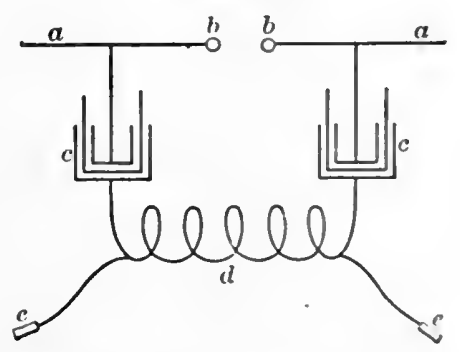

If, as in Fig. 69, a high-tension current from a static machine or an induction coil be conducted along the rods $(a$ a $)$ to the inner coating of the jars $(c c)$, there will occur, when the jars become sufficiently charged, an eruptive spark discharge across the knolss $(b \quad b)$ representing a great number of oscillations per second. With each oseillation there occurs a reversal of the charges of the inner and outer coatings of the jars, the wire $(d)$ connected with the outer coating of the jars being traversed by a current alternating with corresponding frequency.

If we connect electrodes $(e)$, as in Fig. 70, to either end of the coil $(d)$ by means of wires, and apply the electrodes to the boty, the latter will be traversed by this "high-frequency" current.

In the application of this principle several methods have heen $\mathrm{em}-$ ployed, those most frequently referred to being the d'Arsonval, the Oudin, and the Tesla, named after their originators. All the currents ohtained by these different methods are of high frequency, but vary as to their quality. 
The method employed by d'Arsonval is indicated in Fig. 70. 'This gives a current of comparatively low electromotive force.

'The Oudin apparatus (Fig. 71) may be described as the d'Arsonval apparatus, with the addition of a second larger coil, the arrangement greatly increasing the electromotive force.

'Tesla's apparatus employs a disruptive discharge coil or high-frequency transformer. It consists of a primary coil surrounded by a secondary coil composed of a great number of turns of fine wire. (See Fig. 72.)

For practical purposes there are high-frequency coils upon the market based upon the 'Tesla induction principle, which utilizes the current from the electric-light main. The current supply must be alternating, and if the operator is so unfortunate as to live where the current supply is direct, a rotary current converter must be employed. Some of the "high-frequency coils" are so compactly arranged as to be portable, and of sufficient power to enable their being used for $x$-ray work. I would advise the prospective buyer to carefully investigate the merits of

Fig. 71.

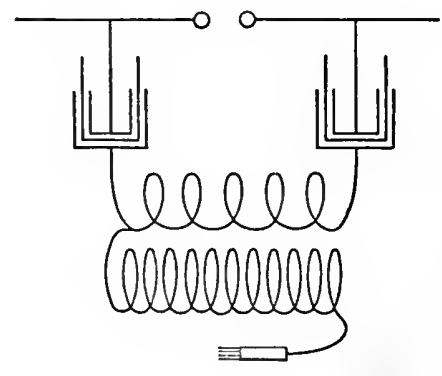

FIg. 72.

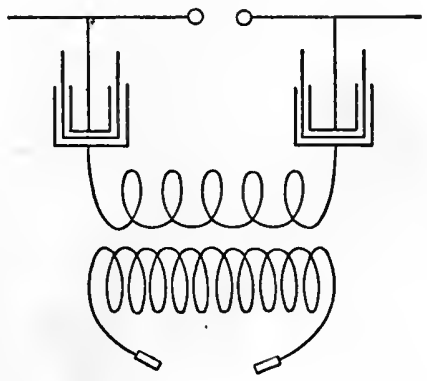

the apparatus before purchasing, as not all the so-called "high-frequency coils" give the true high-frequency current, and they also vary considerably with regard to their reliability.

Accessories.-Sponge-covered electrodes with insulating handles are of value for the direct application of high-frequency currents, but are not so much used as the glass vacuum electrodes. 'The latter are of various shapes and sizes, shaped so as to render them convenient for applying the current to the surface of the body or to the mucous membrane of its various cavities. The set usually supplied with the highfrequency coils contains a cutaneous bulb electrode, and vaginal, rectal, urethral, and nasal clectrodes. Metal electrodes, one pointed and the other terminating in a round knob or ball, are of servicc, as is also an electrode terminating in a series of metal points.

An autocondensation pad should be part of the armamentarium. This is in two sections, hinged together so that the pad may be placed upon a wooden chair. 'The "autopad," as it is called, is made of indurated fiber, with a sheet of metal placed upon the back and covered with an insulating cloth. One terminal of the d'Arsonval apparatus is connected 


\section{PLATE XIX}

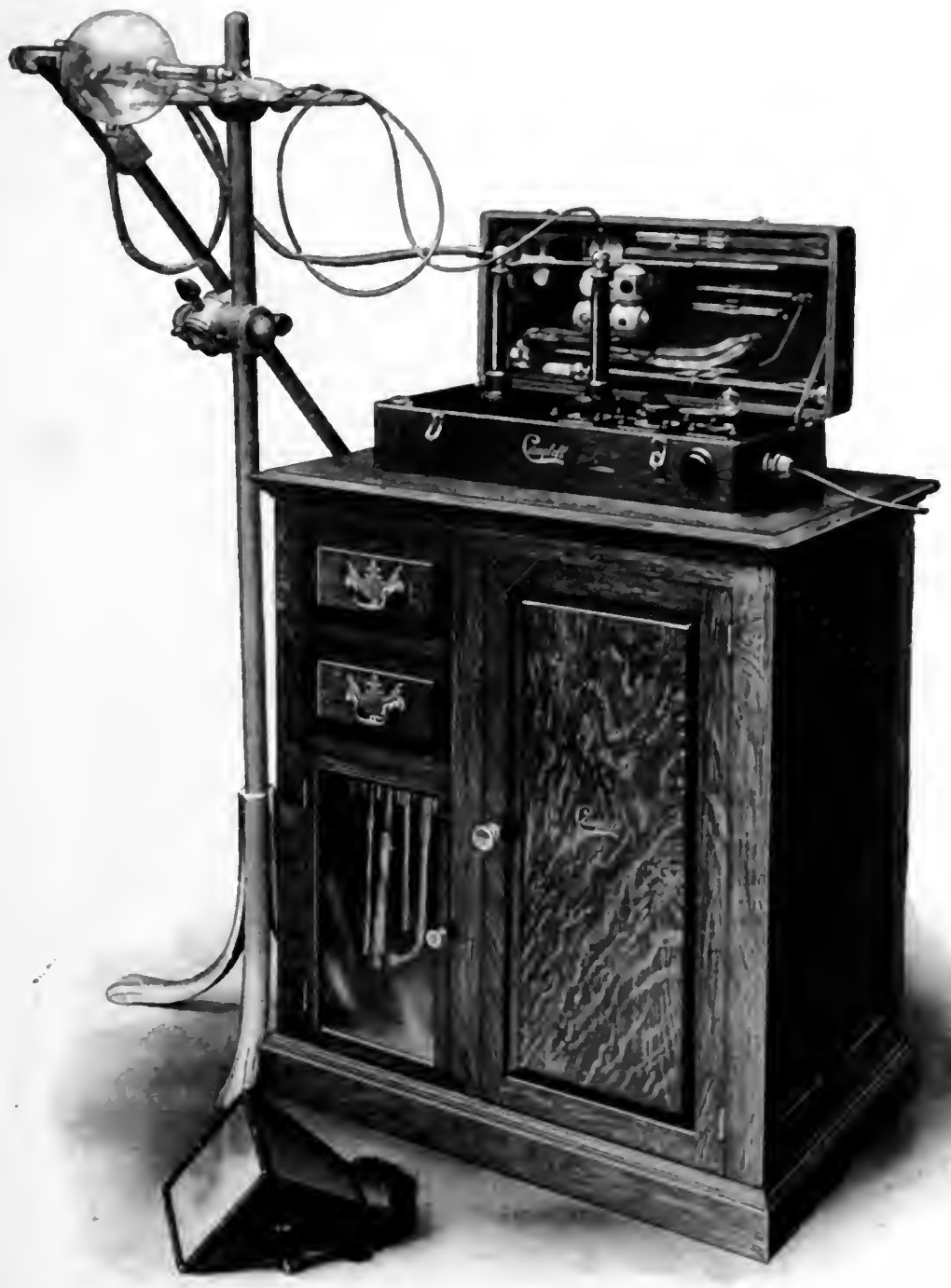

Modern office equipment. 

by an insulated wire conductor to both sections of the pad, while the patient holds in his hand a ronnd metal electrode connected to the other terminal.

The autocondensation couch is constructed along the sane principle, the patient lying on an insulating cushion which rests upon a metal plate connected with a terminal of the d'Arsonval transformer. An electrode which the patient holds in his hand is connected to the other terminal.

Physiological Action.-High-frequency currents penetrate the berly without registering any sensory impression, or producing any museular contraction. 'This non-excitability of the nerves is due to the fact that the oscillations of the current occur so rapidly that the nerves cannot respond. Motor and sensory nerves can only respond to stimuli occurring less than 10,000 per second, which is far below the rate of the undulations or oscillations of the high-frequency currents.

That high-frequency currents do not produce surface effects alone, but actually penetrate the body, has been shown by experiments of d'Arsonval and Maragliano, the latter placing an electric-light bulb in the thoracic cavity of a dog and connecting it with two metal plates, the latter being attached to the inner wall of the pleural cavity, one on either side. Upon the animal being subjected to the high-frequency currents, the lamp became incandescent.

Another effect of the high-frequency currents is that of causing a diminution of excitability in the tissues in contact with the electrode. This is shown by the fact that after the application of high-frequeney currents to a given part of the body the strength of the galvanic or faradic current required to cause contraction is greater than it was before the high-frequency application.

High-frequency currents applied in the form of autoconduction or autocondensation cause a fall in blood-pressure, owing to an inhibitory action upon the vasomotor system. 'This effect is more marked when the method of autoconduction is employed than it is with autocondensation.

High-frequency sparking causes a local anemia and rise of bloodpressure by its action on the involuntary muscle system (spasmodic contraction). Effleuvation causes local congestion primarily, followed by an oscillatory reaction above and below the normal arterial pressure.

Respiration.-Respiratory movements are increased in number and amplitude; there is an increase in the amount of $\mathrm{CO}_{2}$ expired and an amount of oxygen by weight absorbed in excess of the amount of $\mathrm{CO}_{3}$ given off. 'This has been proved experimentally by Bouchard, who found that after being subjected to high-frequency currents there was an increase of weight, although no food or drink had been ingested.

Blood.-It is claimed by 'I'ripet and Guilleminot that autoconduction increases the reduction of oxyhemoglobin where there is imperfect nutrition and diminishes it where it is already exaggerated.

Urine.-As shown first by d'Arsonval, high-frequency currents increase the elimination of urea, uric acid, phosphates, sulphates. and chlorides, also the total quantity of nitrogenous elimination; and deVoL. I-19 
crease the elimination of the non-oxidized sulphates. That the toxicity of the urine is increased has been shown from studies made by d'Arsonval, Denoyes, Martre, and Rouviere.

Thermogenesis.- 'That autoconduction increases the amount of heat given off by the body has been shown experimentally by d'Arsonval, Bordier, LeComte, and Bonniot.

It has been shown by experimentation that the virulence of toxins is diminished slightly under the influence of high-frequency currents. 'The activity of micro-organisms may be said to be slightly if at all inhibited by the action of this form of electricity.

Local Effects of the High-frequency Effleuve and Spark.-The first effect is a localized vasoconstriction, with resulting pallor, followed by hyperemia, and after long exposure blistering and ulceration may occur.

Therapeutic Action. - The chief field of usefulness of the high-frequency currents is through their general physiological action in increasing elimination and stimulating metabolism in such conditions as rheumatism and gout, diabetes, neurasthenia, etc.

Applied locally, high-frequency currents are of value in relieving pain in such conditions as neuritis and neuralgia, and in relieving congestion or venous stasis.

Application.- There are two methods for general bipolar application of high-frequency currents - the patient may be surrounded by a large solenoid coil (autoconduction), or he may be connected to an end of the solenoid and in contact with an insulated metal plate connected to the other terminal (autocondensation).

The latter method is the most practical, the apparatus required for autoconduction being expensive and unwieldy. The "autocondensation couch," in which an insulated couch acts as the "metal plate," is to be preferred, as the patient is more relaxed in the recumbent posture, but the less expensive "cushions" or "pads" are more convenient and very practical. The patient, reclining on the couch or seated upon the pad (the latter placed upon a wooden chair), connected with a terminal of the coil, holds firmly in his hands a metal electrode connected with the other terminal. It is not necessary in this method for the patient to remove the clothing. Séances should be of not over ten or at most fifteen minutes' duration, and repeated every other day.

For direct application, two sponge-covered electrodes, three or more inches in diameter, moistened with water or salt solution, should be employed. 'These should be applied directly to the skin, one on either side of the organ or area to be treated; they should be held firmly in position, and not moved during the séance. 'The duration of the treatment should be from ten to fifteen minutes.

For the local application of the high-frequency effleuve (monopolar), electrodes having numerous metal points or edges are required, such as the wire brush or multiple point electrodes. Unless superficial cutaneous lesions are to be treated, the effleuve may be applied through the clothing. 'The spark-gap shonld be kept as short as possible for surface treatment, and lengthened for deep-seated conditions where a coarse and more pene- 
- PLATE XX

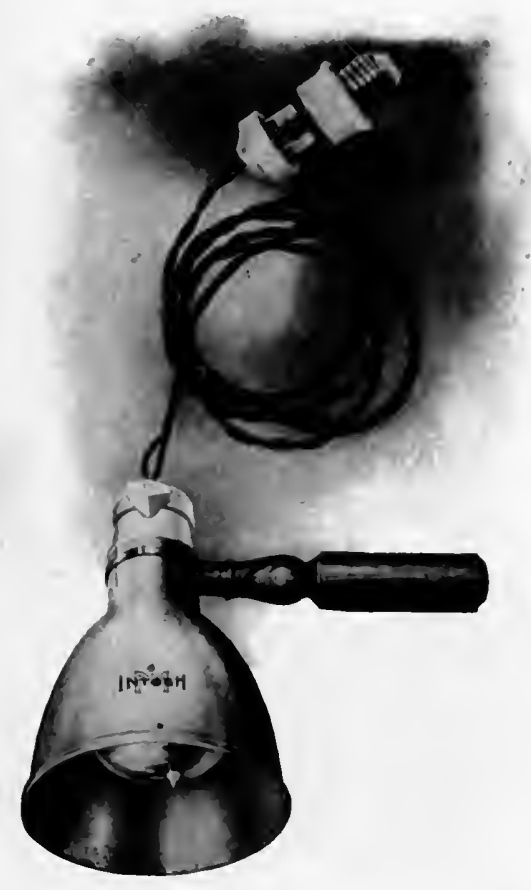

Mcintosh style " $B$ " therapeutic lamp. 50 candle power. 

PLATE XXI

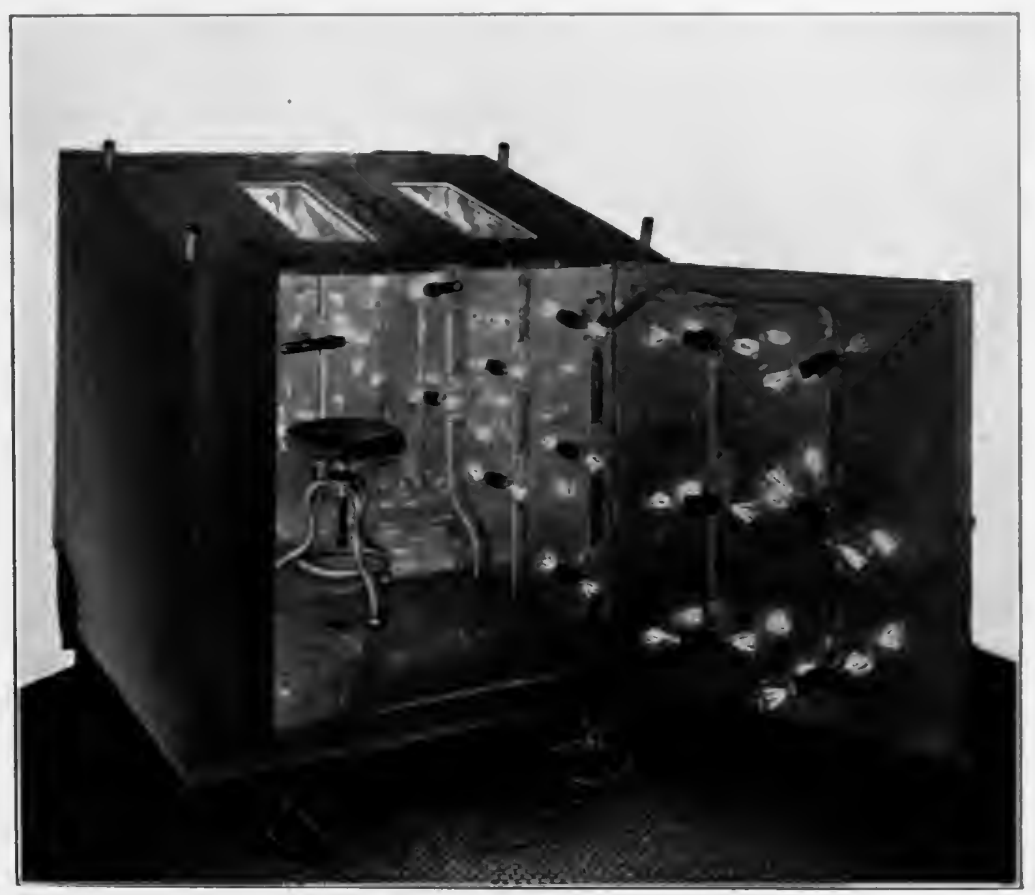

Electric light bath cabinet. (Jefferson Hospital, Philadelphia.) 

trating effleuve is desired. For the application of the high-frequency spark an electrode ending in a metal ball or a rounded proint should be used.

Glass vacum electroles being admirably adapted for the direct local application of high-frequency currents, have come into very general use. 'They should be used in an insulated glass or rublerer handle, and connected to the terminal of the coil.

'The vacum glass electrodes may be applied directly to the skin, through the clothing, or may be covered with a piece of chamois skin or cloth.

When the glass is applied directly to the skin, the patient experiences no sensation other than that of a feeling of warmth at the point of contact. Upon approaching the electrode to or withdrawing it from the skin, a stinging or prickling sensation is felt, which may be eliminated by the operator placing his hand on the tube until the latter is in contact with the patient, and removing the electrode in the same way. It may also be avoided if the vacuum tube is applied to the skin before turning on the current, and by turning off the current before removing the electrode. This method is the one to be employed unless the operator wishes to affect the deeper tissues or counter-irritation is desired, in which case I prefer covering the electrode with chamois skin or cloth of the desired thickness, instead of applying the current through the patient's clothing, as, owing to variations in thickness and differences in fabrics of the clothing, it is impossible to secure a uniform effect.

The effects resulting from the application of the monopolar vacuum electrode are local; treatment should not be continued for more than ten to fifteen minutes over any area.

A bipolar method seldom used is the application of two vacuum electrodes to the patient, each electrode being attached to a terminal of the coil. Still another method is to use the Friedlïnder vacuum condenser chair, connecting the cliair to the resonator terminal.

Hydro-electric Baths.-Hydro-electric baths may be either local or general. In the former, the baths form the electrodes, and possess the advantage of permitting an even application of the current over a larger area than conld be covered by the ordinary electrode.

'These local baths may be multiple, an excellent plan in certain conditions being to have four, one for each extremity. 'The vessels or tubs may be made of porcelain or glass, containing an electrode so arranged as to come in contact with the liquid used, preferably warm water. 'These cell-baths, as they are called, are very convenient in treating such conditions as Raynaud's disease and neuritis of the arm, hand, or leg.

It has been observed that when a limb is immersed in a hydro-eleetric bath, a ring of cutaneous redness and irritation appears at the junction of the licyuid with the air. 'To obviate this, Tousey has his patients wear a stocking or a gauze bandage, which extends for the distance of a couple of inches above and below the level of the liquid. A small amount of glycerine added to the liquid in the bath will also prevent the irritation.

In the general bath, the patient is immersed in a tub containing 
water, the electrodes being in contact with the fluid, but protected from direct contact with the patient. A metal-lined tub is unsuitable, but an enameled tub nay be used, provided there are no breaks in the enamel. Tubs of wood, porcelain, or glass may also be used. The electrodes may be attached one to the head and the other to the foot of the tub. $A$ shovel-shaped metal electrode with an insulated handle can be employed where it is desirable to bring the current in close proximity to a given part of the body.

Warm water is the best liquid for use in the bath. It las been estimated that from 20 to 30 per cent. of the total current employed is transmitted through the patient's body, the rest of the current passing through the water. The addition of any medicament to the bath increases the conductivity of the liquid, and thus decreases the amount of current transmitted through the patient.

The unipolar method, in which one electrode is in contact with the water and the other held by the patient, is not as satisfactory as when both electrodes are immersed.

Physiological Effects of Hydro-electric Baths.- The currents commonly employed are the galvanic, faradic, galvano-faradic, and the sinusoidal.

Gialvanic Current.-With the negative electrode cephalad and the positive electrode caudad, the effect is to increase motor and sensory irritability and to stimulate cerebral activity. There is also an increase of pulse-rate and arterial tension is raised. 'The galvanic current applied in this manner is said to be useful in depressed conditions, various neuritic pains and palsies, and in certain constitutional conditions.

With the polarity reversed, the anode being nearest the head, a general sedative effect is obtained, and this method may be employed for the treatment of insomnia.

The temperature of the bath should be between $85^{\circ}$ and $100^{\circ} \mathrm{F}$., with a current strength of 50 to 125 milliamperes. The duration of treatment is from twenty to thirty minutes.

Faradic Current.-With this current, polarity is not of any import. The effect produced is one of general stimulation, rendering the application of the current of value in neurasthenic states. The strength of the current should not be pushed beyond a point at which the sensation is agreeable to the patient.

The galvano-faradic current, giving, as it does, the combined effects of both currents, is indicated wherever the faradic or galvanic currents alone would be of any value.

Sinusoidal Current.-The effect of this current is to increase bloodpressure without increasing the pulse-rate. It is said also to increase metabolic activity and to liave a sedative effect upon the sensory nerves.

'The sinusoidal hydro-electric baths are indicated in insomnia, neuralgia, muscular atrophy of nerve origin, and the idiopathic type of muscular atrophy.

Electro-pathology.-Electricity, while capable of causing certain beneficial physiological effects, is also capable, under certain conditions, of producing deleterious or pathological effects and may even destroy life. 
Pathological effects may result from carelessness or faulty technique in the therapentic application of electricity, or from contact with a conductor of high-tension electricity. 'The effect of lightning upon the human body is, of course, but another illustration of the pathological effects of electricity.

In using the galvanic current for electro-therapentie purposes, the operator may very readily' produce a superficial burn if a strong current is used with small electrodes or if the metal is not well covered. 'T'(x) long an application of a moderate current will produce the same effect. 'The first phenomenon is a temporary pallor of the skiin, soon followed by hyperemia which is long persisting. These effects are not considered pathological, and occur especially at the cathode, whenever a current of any considerable intensity is employed. Following this, however, papules and vesicles appear, developing finally into a burn which in no way differs in appearance from a burn resulting from heat.

Burns caused by the galvanic current are not the result of heat generation, but are chemic in origin and may be considered as the result of ionization. 'The body is the electrolyte; salts collecting about the electrodes, the acids at the positive and the alkalies at the negative pole, and undergoing decomposition, may act as tissue irritants. 'The treatment is that of an ordinary burn.

Prevost and Battelli have shown by experiments upon animals, using a continuous current, that with dogs, death results from cardiac paralysis which may occur without the shock of breaking the current, or it may be caused by the "break" shock. Failure of respiration is secondary to cardiac failure. Guinea-pigs and rats, on the other hand, succumbed through respiratory failure.

With an alternating current of low tension, dogs and guinea-pigs died from cardiac paralysis, respiration continuing for some time longer, while with a high-tension alternating current death resulted from respiratory paralysis.

The same experimenters have shown that animals in which a low-tension current has caused cardiac paralysis may be sometimes resuscitated by a high-tension electrical current. 'The experiments of Cunningham would seem to prove that if a strong direct or alternating current traversed the entire body, death would result from cardiac paralysis, the current producing a fibrillation of the heart muscle. If the current was passed only through the head, death would result from asphyxia.

Prevost and Battelli's experiments are in harmony with those of Cunningham, inasmuch as when the voltage was relatively low (in the hundreds), they obtained primary cardiac paralysis, primary respiratory paralysis resulting only when the voltage was relatively high (in the thousands). 'The currents, as ordinarily used for industrial purposes, would correspond to their low voltage currents.

'The "static machines," as ordinarily used for electro-therapeutic purposes, do not generate a current of sufficient strength or amperage to eause any serious effects, except such functional nervous disturlances as might possibly result from the shock of unexpectedly receiving the full charge 
from the Leyden jars. A series of sparks if allowed to strike directly upon one point for a long time would ultimately produce a localized blister or burn, but this, of course, may be easily avoided.

High-frequency sparking, if directed long enough over a small area of the skin, will produce the same effect as the spark from a static machine.

Electrical currents used for industrial purposes are generally alternating currents of high voltage-from 110 to 220 volts in the electric lighting system, and 500 to 800 or more volts in the trolley system. A person coming in contact with such a current may be either shocked or killed, the effect produced by the current depending upon the conditions under which the contact occurred. The current may pass only through a part of the body, as an extremity, or if the individual is in contact with a good conductor of electricity the current may traverse the entire body. A dry wood floor is a poor conductor, while the earth is a good conductor; even the state of the atmosphere may be a factor. One effect of these currents is involuntary muscular spasn, which explains why a man who has hold of a "live wire" cannot let go, and why we sometimes read of a lineman hanging suspended by his hands.

Efforts at resuscitation should be promptly instituted, even though the victim of accidental contact with a current-carrying wire be apparently lead. While cardiac paralysis is the usual cause of death from industrial currents, treatment should be directed toward both respiratory and cardiac stimulation as the current may not have included the heart in its circuit. 'The patient should be wrapped in a warm blanket and given a full dose, hypodermically, of atropine and strychnine. Artificial respiration may be employed, also cardiac massage applied in the form of intermittent pressure below the lower border of the ribs. Faradization of the pneumogastric is another measure often of value.

Electricity as a Test of Death.-In electricity we have an agent not only capable of causing death, but also one which furnishes us with a reliable means of determining if death has transpired.

The test is based upon the fact that muscular response to electrical stimulation ceases shortly after death, and before the onset of cadaveric rigidity. The faradic irritability is lost before the disappearance of galvanic irritability, the difference in time, however, being slight.

Electro-diagnosis. - From a diagnostic standpoint, electricity is of value chiefly in the examination of the muscles and motor nerves. For this purpose we resort to the faradic and galvanic currents.

The examination should always be conducted where there is a good light, so that slight muscular response to the electrical excitation may be readily observed, and in this connection it is well to remember that touch may be used as an aid to sight in detecting faint muscular contractions. The patient should be in the recumbent posture, or seated with the back supported, and should relax the muscles in the part of the body which is under examination. The operator is advised when using the induced current to test it first upon his own person, so as to avoid any shock to the patient by commencing with a current of too great strength. He should also bear in mind the rule of comparing the reaction of the muscles of an 
affected extremity with the corresponding sound muscles of the opposite extremity, or if the pathological process is bilateral, to compare the reaction of the muscles under examination with those of healthy muscles. 'The electrodes should be moistened, preferably with a salt solution, to secure the best results.

Fig. 73.

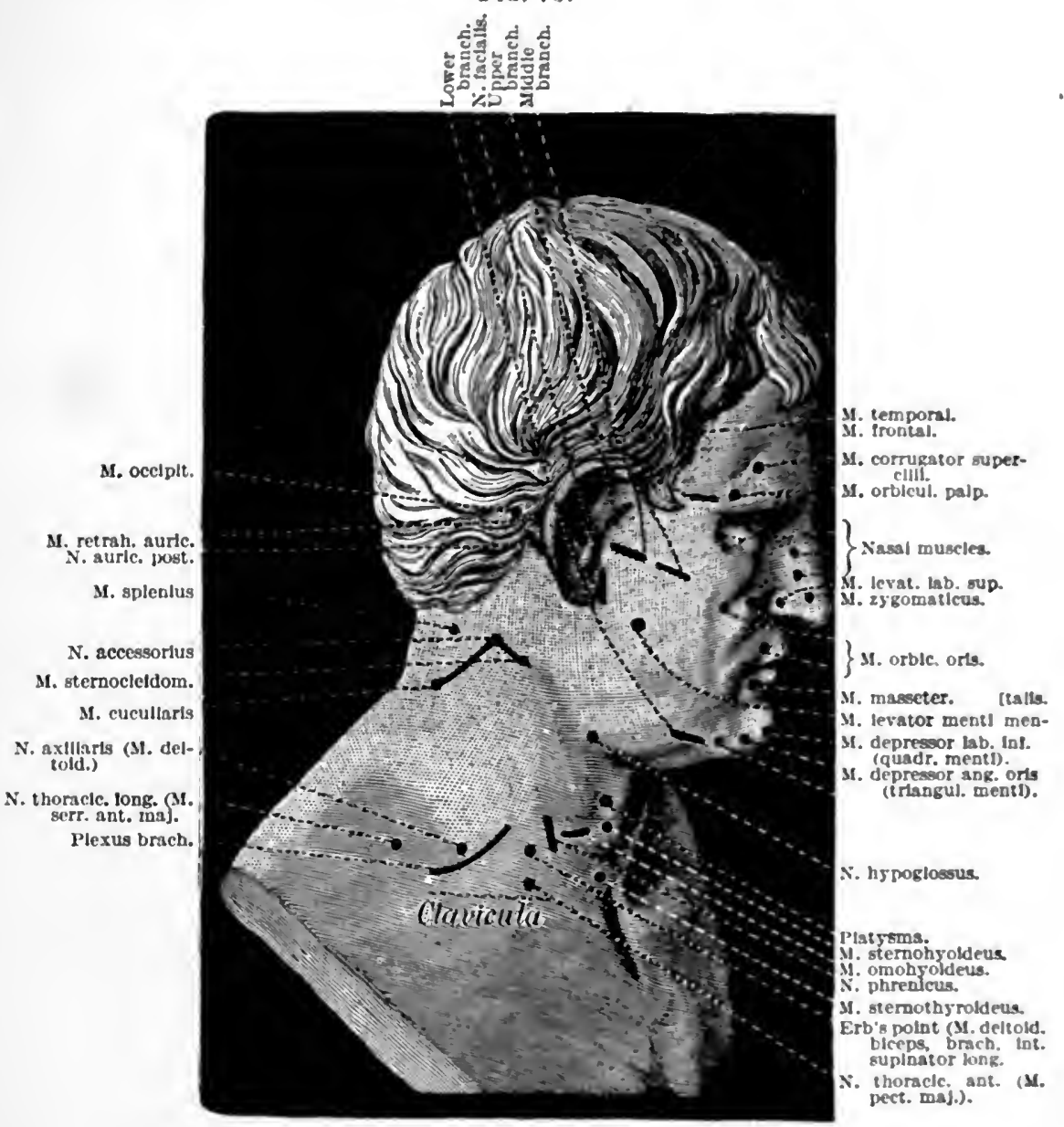

Motor points for the head and neck. (SAIILI.)

The examination should be systematic, beginning with the faradic current and ending with the galvanic current.

Faradic Reactions. - A furadic current of a given strength will cause a greater response in a muscle when applied over the motor nerve at its point of entrance than when applied over an indifferent part of the muscle. The point where the nerve enters the muscle is spoken of as the "motor point," and should be located and employed by the operator in making his examination. 
This method is not always satisfactory in testing single muscles, owing to the diffusion of the current causing contractions of adjacent muscles. When this occurs, the difficulty may be overcome by resorting to the method of Duchenne, and applying an electrode to either end of the muscle.

'The negative electrode should be selected as the active electrode, and the secondary current used. Commencing with feeble shocks, the rate of which may be regulated by a rheotome or by an interrupter handle, the operator increases the strength of the current gradually until sufficient to produce muscular contraction, which may then be compared with that of other muscles.

FIG. 74.

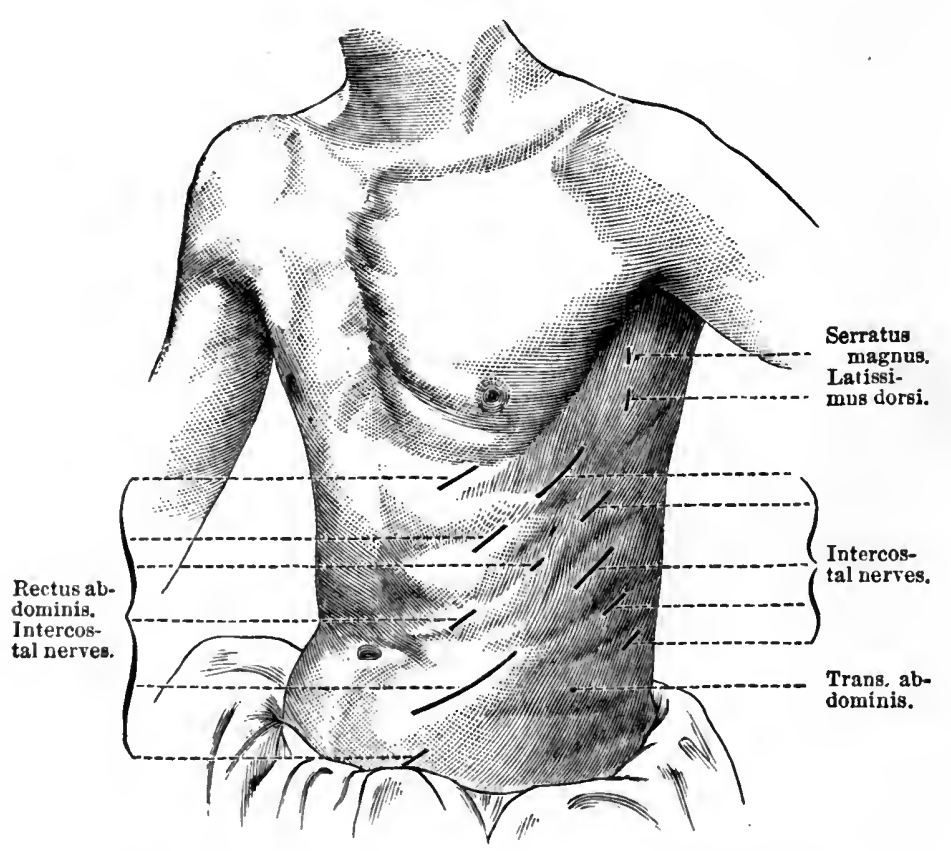

Motor points of the trunk. (From von Ziemssen.)

Galvanic Reaction.-For testing with the galvanic current, the battery should be equipped with a rheostat, a milliamperemeter, and with a reversal switch, as is is important that the poles be changed without its being necessary for the operator to alter the position of the electrodes. As the muscular response to the galvanic current occurs only upon closure and opening of the current, the first point to determine is whether or not these contractions occur in the normal sequence, as the current is gradually increased.

Starting with a current which is not strong enough to cause any reaction of the museles, and the strength gradually increased, reversing the switch each time the intensity is increased so as to change the polarity. 
In healthy muscles the first response will be at the cathore upon closing the current; then, as we further increase the strength of the current, the next response we note in addition to the $K$. C. C. is that at the atnode upon closing. Next in order would be contraction at the ande upon opening, and lastly, at the cathode upon opening. 'T'his normal reaction may be indicated therefore as follows: $\mathrm{C} \mathrm{C} \mathrm{C}-\mathrm{ACC}-\mathrm{AOC}-\mathrm{COC}$.

FIg. 75.

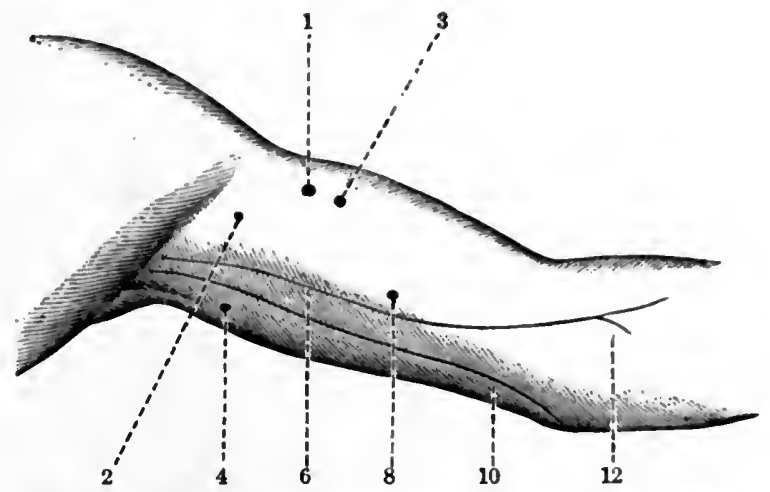

Motor points of the arm, under side. (From vos ZiEMssen.)

1. Musculocutaneous nerve. 2. Musculocutaneous nerve. 3. Biceps. 4. Internal nerve of the triceps. 6. Median nerve. 8. Brachialis anticus. 10. Ulnar nerve. 12. Branch of the median nerve to the pronator teres.

For practical purposes in testing the operator need only concern himself with the observation as to whether the cathodal closing contraction is greater than the anodal closing contraction.

FIG. 76.

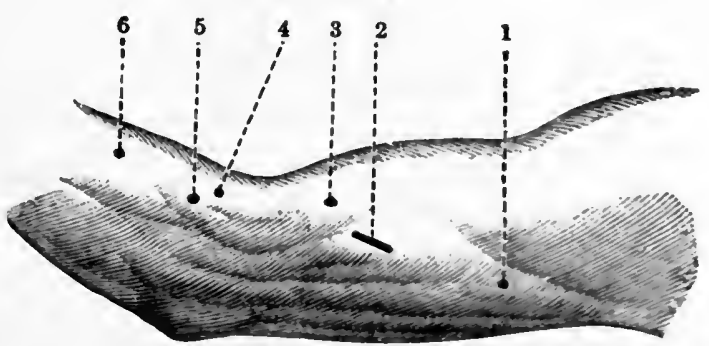

Motor points of the arm, outer side. (From ron Zremssex.)

1. External head of the triceps. 2. Musculospiral nerve. 3. Brachialis anticus. 4. Supinator longus. 5. Extensor earpi radialis longior. 6. Extensor carpi radialis brevior.

In testing with the galvanic current, the active electrode is placed as described under testing by the faradic current upon the so-called motor points. If the muscle does not respond, however, to a current the strength of which can be comfortably borne by the patient, the active electrode should be placed on the lower tendon of the muscle. 'This is the socalled "longitudinal reaction." 
To recapitulate, the normal reaction should be a prompt muscular contraction upon faradic stimulation, with prompt response upon closure

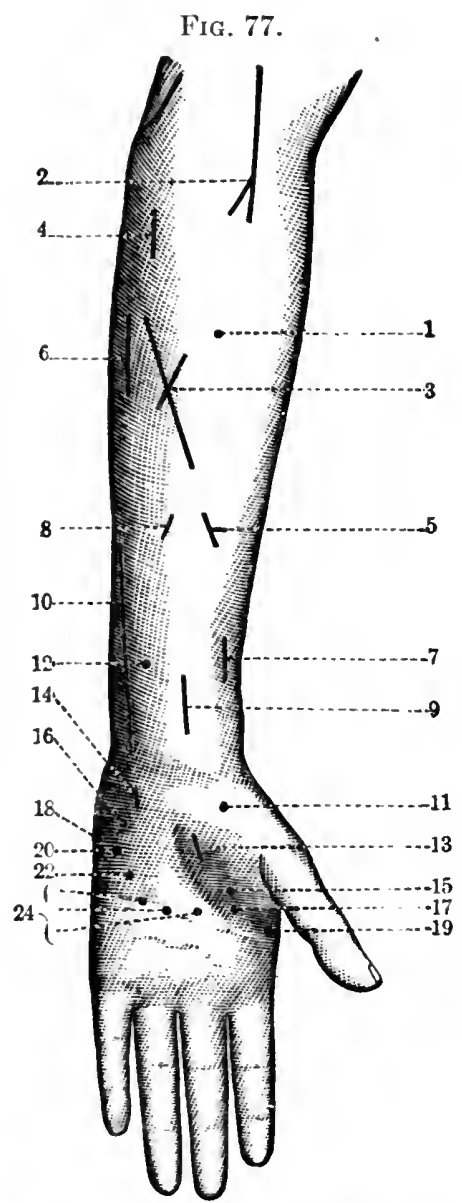

Motor points of the forearm, inner surface. (From von ZiEMSSEN.)
FIG. 78.

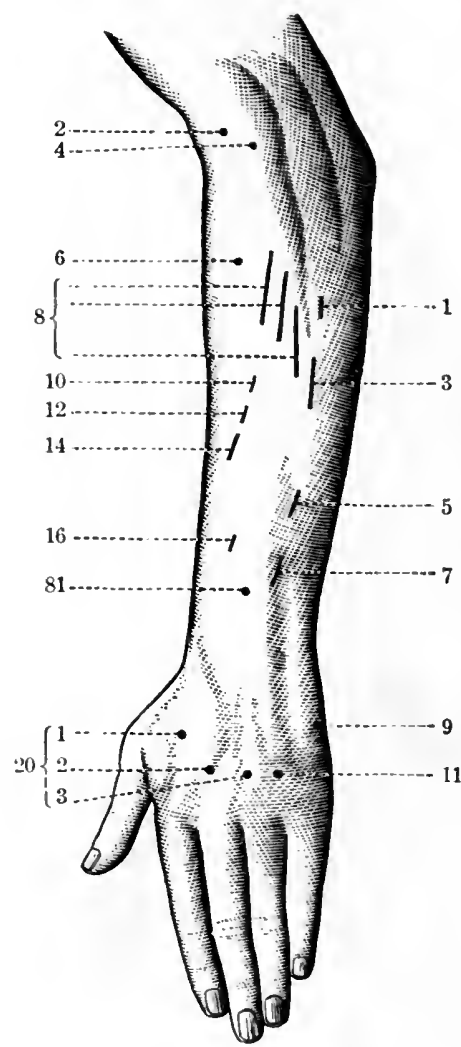

Motor points of the forearm, outer surface. (From von Ziemssen.)

Fig. 77.-1. Flexor carpi radialis. 2. Branch of the median nerve for the pronator teres. 3. Flexor profundus digitorum. 4. Palmaris longus. 5. Flexor sublimis digitorum. 6. Flexor carpi ulnaris. 7. Flexor longus pollicis. 8. Flexor sublimis digitorum (middle and ring fingers). 9. Median nerve. 10. Ulnar nerve. 11. Abductor pollicis. 12. Flexor sublimis digitorum (index and little fingers). 13. Opponens pollicis. 14. Decp branch of the ulnar nerve. 15. Flexor brevis pollicis. 16. Palmaris brevis. 17. Adductor pollicis, 18. Adductor minimi digiti. 19. Lumbricalis (first). 20. Flexor brevis minimi digiti. 22. Opponens minimi digiti. 24. Lumbricales (second, third, and fourth).

FIG. 78. - 1. Extcnsor carpi ulnaris. 2. Supinator longus. 3. Extensor minimi digiti. 4. Extensor carpi radialis longior. 5. Extensor indicis. 6. Extensor carpi radialis brevior. 7. Extensor secundi internodii pollicis. 8, extensor communis digitorum. 9. Abductor minimi digiti. 10. Extensor indicis, 11. Dorsal interosseus (fourth). 12. Extensor indicis and extensor ossis metararpi pollicis. 14. Extensor ossis metscarpi pollicis. 16. Extensor primi internodii pollicis. 18. Flexor longus pollicis. 20. Dorsal interossei.

when the galvanic current is used, the cathodal closing contraction being greater than the anodal closing contraction.

Abnormal Electrical Reactions.-Faradic Current.-With this 
current the question as to which pole is used as the active celectrode is of little practical importance, the main point to consider being whether or not the excitability is increased or diminished. Faradic liverexcitability occurs in tetanus and tetany, recent hemiplegia, infantile hemiplegia, and diplegia, and sometimes in chorea.

Faradic hypoexcitability ocrurs in long-standing cases of cerchral palsy, in tabes with marked muscular hypotonia, in various forms of muscular atroply, in neuritis, and sometimes in insular sclerosis.

FIG. 79.

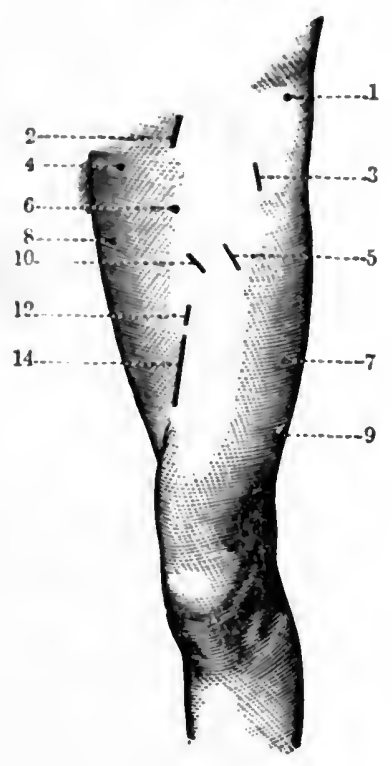

Motor points of the thigh, anterior surface. (From von Ziemssen.)

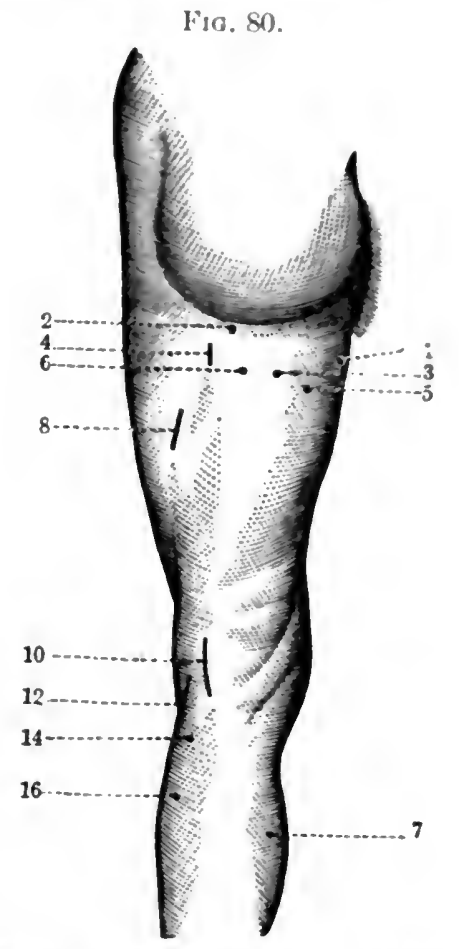

Motor points of the thigh, posterior surface. (From voN ZIEMSSEN.)

Fig. 79.-1. Tensor vagins femoris (branch of the superior gluteal nerve). 2. Anterior crural nerve. 3. Tensor vagina femoris (branch of the crural nerve). 4. Obturator nerve. 5. Rectus fernoris, 6. Sartorius. 7. Vastus externus. 8. Adductor longus. 9. Vastus externus. 10. Hranch of the crural nerve to the quadriceps extensor cruris. 12. Cruseus. 14. Branch of the crural nerve to the vastus externus.

Fig. 80.-1. Adcluctor magnus. 2. Inferior gluteal nerve for the gluteus maximus. 3. Semitendinosus. 4. Great sciatic nerve. 5. Semimemhranosus. 6. Long liead of the biceps. $\div$. Gastroctremius (internal head). 8. Sloort head of the biceps. 10. Posterior tibial nerve. 12. Peroneal nerve. 14. Gastroenemius (external head). 16. Soleus.

Galvanic Current.-In studying the abnormal response of nerve and muscle to the galvanic current, quantitative anomalies are not nearly so important as qualitative changes. Galvanic hyperexcitability is nearly always found where there is faradic hyperexcitability, but may also occur in the conditions which we shall consider under the heading of "Reactions of Degeneration," where there is diminution or loss of faradic excitability. 
Galvanic hypoexcitability is seen in the last stage of degeneration of nerve and muscle. There may also be changes or departures from the normal physiological order of the occurrence of contraction; these may be called "serial anomalies." These changes in the nature or mode of the muscular contractions, together with diminution or loss of faradic ex-

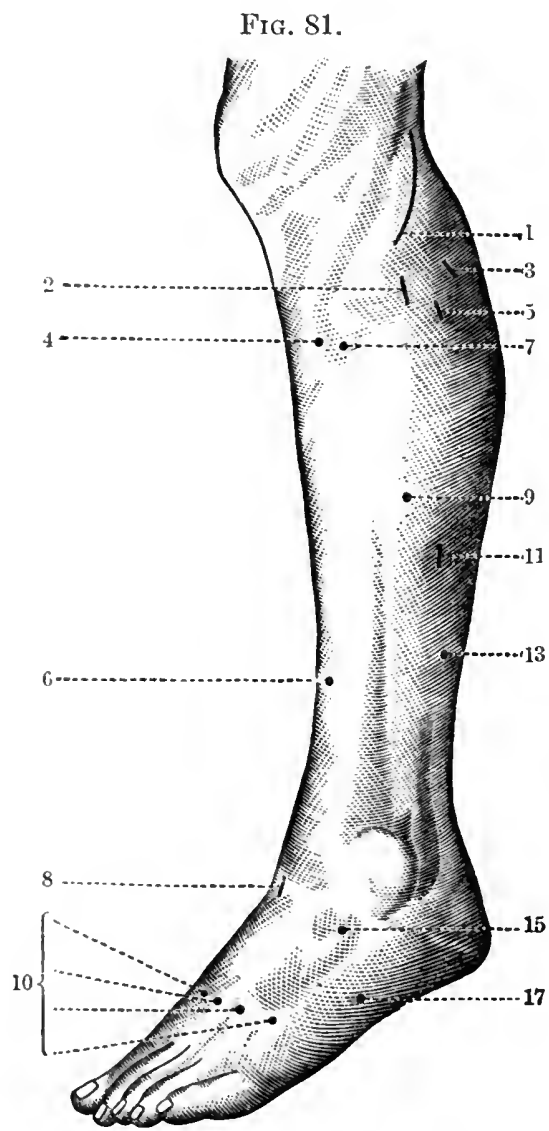

Motor points of the leg, outer side.

\section{(From von ZiemsSen.)}

FIG. 82 .

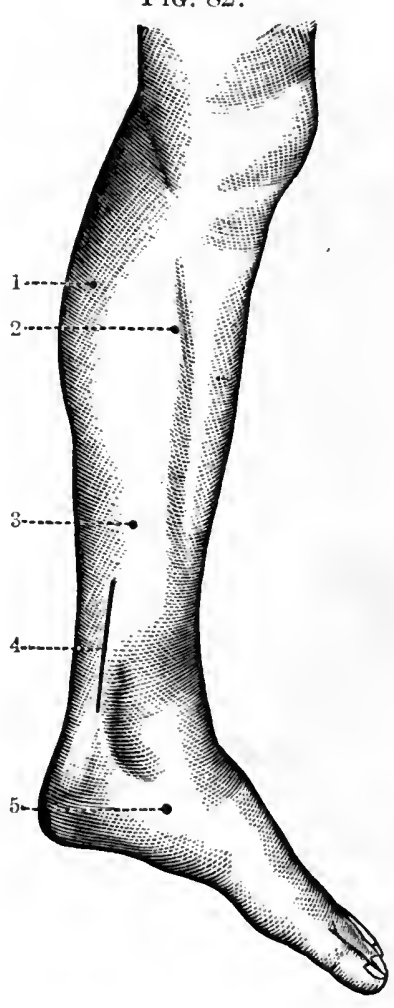

Motor points of the leg, inner side.

FIG. 81.-1. Peroneal nerve. 2. Peroneus longus. 3. Gastrocnemius (external head). 4. Tibialis anticus. 5. Soleus. 6. Extensor longus pollicis. 7. Extensor communis digitorum longus. 8. Branch of the peroneal nerve for the extensor brevis digitorum. 9. Peroneal brevis. 10. Dorsal interossci. 11. Soleus. 13. Flexor longus pollicis. 15. Extensor brevis digitorum. 17. Abductor minimi digiti.

FIG. 82.-1. Gastrocnemius (internal head). 2. Soleus. 3. Flexor communis dig!torum longus. 5. Posterior tibial nerve. 5. Abductor pollicis.

citability, constitute what is known as the reactions of degeneration, and will be considered more in detail under that heading.

Reactions of Degeneration.-This condition is present in disease in or destruction of any part of the lower motor segment. This includes that part of the motor pathway extending from and including the ganglion- 
cells in the anterior horns of the cord and the nerve-fibers leading from them to the muscles. 'Ihe cranial nerves, with their nuclear origin, also come under this head. Reactions of degeneration are to be found, therefore, in such conditions as:

Acute and chronic polio-encephalitis superior; acute and chronic poliomencephalitis inferior (bulbar palsy); aroute and chronic anterior poliomyelitis; amyotrophic lateral selerosis; single or multiple neuritis (whether from trauma, infection, or intoxication), and syringonyelia and insular sclerosis when these diseases involve the gray inatter of the anterior horns of the cord.

'The reaction of degeneration is absent in lesions involving the upper motor segment, such as in pure cerebral paralysis or in diseases of the cord involving only the white matter. It is also absent in the idiopathic muscular atrophies and, needless to say, in hysterical palsies.

The changes which follow a lesion causing degeneration of a motor nerve vary with the different stages of the affection. Immediately after the onset, faradic and galvanic excitability of the nerve is increased. and faradic and galvanic excitability of the corresponding museles decreases. 'There is then gradual diminution of the excitability of the nerve, with a corresponding diminution of faradic irritability of the muscles, but an increase of galvanic muscular irritability. In about ten days' time these changes reach their maximum, and the typical reactions of degeneration are seen. 'The nerve does not respond to faradic or galvanic irritability and the muscles do not respond to faradic irritability, but are hyperexcitable to galvanic irritability. 'There is inversion of the normal physiological formula of contraction with the galvanic current, anodal closing contraction now being stronger than cathodal closing contraction. 'The formula now becomes: $\mathrm{A} \mathrm{C} \mathrm{C-C} \mathrm{C} \mathrm{C-A} \mathrm{O} \mathrm{C} \mathrm{-}$ $\mathrm{C} \mathrm{O} \mathrm{C}$.

From this stage there is a gradual diminution of galvanic excitability, and the muscular contractions are sluggish and prolonged, the other features remaining the same. Should recovery ensue, voluntary motor power is regained first; next there is a return of faradic excitability, cathodal closing contraction gradually becomes stronger than the anodal closing contraction, and muscular contractions in response to the galvanic current become shortened and sharper. In some instances there may be only partial or incomplete reaction of degeneration, which condition is spoken of as "beginning reaction of degeneration." 'This consists of a diminution in reaction to the faradic current, but not a complete loss, while the galvanic changes remain the same as in complete reaction of degeneration. When this condition occurs, the degenerative process is less marked than when complete reaction of degeneration is present, and, therefore, gives a better outlook from a prognostic standpoint.

'To recapitulate, the test for the reactions of degeneration should not be made for at least ten days after the onset of an acute conditior. When present, they indicate a lesion of some portion of the lower motor neuron. They consist of the following phenomena: No response to the faradic current; increased excitability to the galvanic current, with a reversal of 
polar reactions, anodal closing contraction being stronger than cathodal closing contraction, and the response being slow and prolonged.

Myasthenic reaction, or the reaction of fatigue, is characterized by a gradual diminution in the muscular response to faradic shocks of a given strength. Furthermore, to produce museular contractions, repeated shocks of the faradic current must be employed, and the strength of the current must be progressively increased, until a period is finally reached at which the muscle will not respond at all to a powerful faradic shock. After an interval of a few minutes, faradic excitability reappears, but if the muscle response is again tested, it will be found that it becomes exhausted as before. Muscular reaction to the galvanic current in this condition is unchanged.

The myasthenic reaction is characteristic of myasthenia gravis, being seen but rarely in other conditions. As myasthenia gravis is considered of toxic origin, it is interesting to note that Gunn has demonstrated the same reaction in the muscles of frogs poisoned by yolumbine.

Myotonic reaction is characterized by the fact that upon faradic stimulation there is a hyperexcitability of nerve and muscle, the muscular contraction persisting for some time after the cessation of the electrical stimulation. There is also galvanic hyperexcitability of nerve and muscle and a modification in the form of the reaction-cathodal and anodal closing contractions becoming about equal. With either faradic or galvanic currents there may be peculiar undulatory or wave-like contractions of the muscles.

The myotonic reaction is pathognomonic of myotonia congenita or Thomsen's disease.

Rich's Reaction.-This is a condition in which anodal closing and anodal opening contractions tend to become equal and would be represented by the following formula: $\mathrm{C} \mathrm{C} \mathrm{C}-\mathrm{A} \mathrm{C} \mathrm{C}-\mathrm{A} \mathrm{O} \mathrm{C}-\mathrm{C} \mathrm{O} \mathrm{C}$. This reaction is said to indicate an insufficient blood-supply to the nerve.

Remak and Doumer's reaction, or the longitudinal reaction, occurs when the motor point is displaced, the muscle contraction being better when the active electrode is applied over the junction of the muscle with its distal tendon.

'This reaction appears early,--within two or three days after an injury to a nerve,- -and must not be confused with the typical reactions of degeneration which do not appear before ten days have elapsed. Remak and Doumer's reaction, however, like the reaction of degeneration, is most marked upon anodal closing contraction.

Electric Vibratory Massage.--Vibratory massage, like the bicycle, has had its day of popularity, and now the pendulum has swung, I believe, too far in the opprsite direction. Too much was claimed for this method, and when physicians failed to obtain the expected results, it fell into disfavor.

'I'he principle of vibration in the treatment of disease is an old one, and, indeed, much of the effect produced by the faradic and high-frequency currents depends upon vibratory stimulation. Compared with 
these methods, mechanical vibration, even from the best of "vibraturs," is coarse and slow, and cannot be expected to usurp the functions of these currents. 'The best vibratory massage machine can only produce thonsands of oscillations per minute, while high-frequency coils give millions of vibrations per second.

The best machine is one capable of giving true vibrations in all directions. 'The vibrations should be imparted to the patient, and not to the operator. 'The machine should be comparatively noiseless, readily' adjusted, and should give uniform vibrations. 'The source of power is generally the electic-light circuit, connection being made with the ordinary lamp socket.

Physiological Action.-Electric vibratory massage acts as a mechanical irritant upon muscles, causing contraction and increasing the growth of the muscle-fibers. 'The muscular contractions produce an increase in the local blood-supply and thus stimulate nutrition. 'The activity of the skin is also increased. It is doubtful if there is any considerable effect produced upon the nerves except such as would result from an increase in their blood-supply.

Some of the conditions capable of being benefited by vibratory massage are weakened or atrophied nuscles, constipation (especially atonic), subacute and chronic muscular and articular rheunatism, insomnia, and obesity. It is beneficial in the form of neuralgia which is relieved by pressure, but contraindicated in neuralgia which is unrelieved by pressure. It promotes absorption in contusions and allied conditions, but is contraindicated when pus has formed. It should not be given when the blood-vessels are the seat of such pathological changes as arterioselerosis or aneurysm. It should not be employed within the female pelvis, the rectum, or in the urethra, and should also be avoided in tuberculosis or where there exists a malignant growth.

Magnetism.-If a small compass is brought near a wire through which is passing a strong current of electricity, the needle will be deflected in the direction of the flow of the current. 'This is due to the fact that a field of magnetic force surrounds the wire, the lines of which are at right angles to the direction of the current's flow.

Another illustration of the magnetic force in a current-carrying wire is seen if the wire is brought in contact with iron filings. 'The filings will adhere to the wire as long as the current flows through the wire, but will fall off if the current is stopped.

The earth itself is a magnet, with the lines of magnetic force passing from the north to the south pole, and any magnetized substance, such as a bar of iron, is surrounded by magnetic lines of force flowing from one end or pole of the magnet to the other. 'These lines do not flow in a straight course, but outward in eurved lines, and after passing an imaginary line at right angles to and bisecting the magnet (termerl the mangetic equator) curve inward toward the opposite pole.

Magnetism, like electricity, is a foree, and Bachelet claims that hy means of an apparatus that he has devised he can utilize this force in the treatment of disease. 
His apparatus consists of two co-acting electromagnets which he places one on either side of the patient. 'The electricity required to energize them may be obtained from a battery of dry cells or from the street current. It is claimed by Bachelet that the magnetic lines of force thus generated will increase the electric potential of the living body 33 per cent., and that this energy is transformed into vital energy. It is further claimed that the magnetic wave will lower blood-pressure and act as a general sedative; also that it stimulates secretion and excretion.

The Bachelet "magnetic wave generators" have been recommended for the treatment of a host of conditions, varying from arteriosclerosis to melancholia, and including convulsions and neuritis.

It will be interesting to note whether or not Bachelet's observations and conclusions are confirmed. Certainly, unless Bachelet has solved the problem with his "generators" we can say, with Turner, that "It is a curious fact that, so far as it is at present known, no physiological or pathological effect is produced by even the most powerful magnets."

Photo-therapy.-'The physiological effects of light are due to the action of luminous, chemic, and heat rays.

'The various colors of the spectrum have various wave-lengths, running from ultra-violet rays at one extremity, with the shortest wave-length, to ultra-red rays at the other extremity, with the longest wave-length. Both the ultra-violet and ultra-red ravs are invisible, but between these are the visible colors of the spectrum-violet, indigo, blue, green, yellow, orange, and red rays.

Chemic or actinic rays are present throughout the spectrum, but most pronounced at the ultra-violet extremity, while the heat or caloric rays are found chiefly among the ultra-red rays.

It has long been known that the various rays affect the living organism in different ways. Thus, blue light acts as a sedative, while the effect of red light is to stimulate.

Without spending any more time upon elementary considerations, however, we will concern ourselves with the application of light as a therapentic measure. Ordinary incandescent electric light contains caloric rays, but practically no chemic or actinic rays, so it is utilized for the application of heat and light, both generally and locally. The most simple "therapeutic lamp" consists of a 50-candle-power incandescent globe mounted in a metal reflector. It can be attached to any lamp socket and is easily handled. More elaborate lamps consist of a series of incandescent lights so arranged as to permit of their being applied about a joint or extremity. Their value depends almost entirely upon the heat which they generate, and they are indicated therapeutically wherever the application of heat locally is desired. Perhaps their chief value is in cases of subacute or chronic articular or muscular rheumatism.

Electric-light bath cabinets are arranged for the general application of heat and light. 'The patient is seated within the cabinet, which fits about the neck so that the body but not the head will be suljected to the heat. Along the sides of the cabinet is a series of incandescent lights with selector switches on the outside, so that a few or all the lamps may be 
turned on as desired. A thermometer is, of course, connected with the cabinet, so that the operator may deternine and regulate the amount of heat employed. Colored lights may also be utilized in these cabincts, although, in my opinion, their efficacy depends entirely upon the heat generated.

Another form is the Beez portable electric-light hath, which may be upplied to the patient while in bed. 'These baths are excellent in producing diaphoresis, and obviously are a great improvement over the old "hot pack" method. 'The operator in using any of the bath calbinets should remember to keep the patient's head cool by the application of an ice-eap or cloths saturated with cold water. 'I'he physiological effect of the incandescent-light bath is to produce vasodilatition with profuse diaphoresis and a lowering of blood-pressure.

Uitra-violet or actinic rays applied locally produce the following physiological effect: 'The skin becomes swollen and reddened, the inflammatory process reaching its maximum in from twelve to twenty-four hours. It subsides in from four days to a week, and is followed by desquamation and pigmentation.

'This effect of the actinic rays has been utilized by Finsen in the treatment of disease by means of a lamp so constructed that a quartz condensor transmits the ultra-violet rays, while caloric and other rays are practically excluded. As blood diminishes the penetration of actinic rays, the tissues are blanched either by pressure from a quartz lens or by the introduction of adrenalin hypodermically or by cataphoresis.

Modern adaptations of the Finsen lamp are the Finsen-Reyn lamp, lamps devised by Bang and Gorl, in which iron electrodes are used, and Piffard's modifications, utilizing carbon electrodes containing iron filings. The reason for the introduction of iron into the composition of the electrodes is that iron is more rich in ultra-violet rays than carbon.

The Finsen lamp and its modifications are used chiefly in the treatment of lupus and allied conditions.

If arc lights are substituted for ineandescent lights in an electriclight bath, the patient is subjected to a certain anount of actinic radiation in addition to the luminous and caloric rays. The resulting effect is much the same as that produced by incandescent lamps, except that cutaneous hyperemia is more pronouncel. It is claimed that these baths possess some value in the treatment of chronic endocarditis.

Red Light.-It has been known since the observations of Picton, that cases of small-pox had less pustulation and resultant pitting if treated in a room in which there was no light except such as came through red glass. It is believed that this effect is the result of the exclusion of the irritant rays, rather than of any actual benefit derived from the red rays.

Blue Light.-It is of interest to note that blue light has been employed as a method of producing anesthesia during which tecth have been extracted, but from the description of the method and the reaction upon the patient I am strongly inclined to believe that the patients were simply hypnotized.

VoL. I-20 
At one time the experiment was tried of keeping patients in a room having only blue glass windows, for the supposed sedative effect of the blue light. 'This is mentioned merely as a matter of historical interest.

\section{THE SPECIAL THERAPEUTIC USES OF ELECTRICITY}

\section{Diseases of the Nerves.-Neuritis.-Multiple Neuritis.-No} electrical treatment should be instituted until the cause has ceased to act, and it should be discontinued upon the appearance of contractures.

The various currents may be used to meet the different stages and symptoms of the affection. For the pain, galvanism gives excellent results, with the anode as the active electrode on account of its effect in diminishing nerve excitability. A good rule to follow is to use large electrodes, the indifferent one over the nerve-roots, and the active electrode over the peripheral distribution. When acute symptoms subside, the cathode is to be preferred to the anode as the active electrode, as the former, according to Guil, has a resolvent action on chronic inflammatory processes and arrests the cicatricial process.

To combat the muscular wasting in multiple neuritis, the combined (galvano-faradic) current gives the best results. Next in value is the faradic current alone or the sinusoidal current. With reaction of degeneration present, the rhythmically interrupted galvanic current is best, applying the anode to the motor points.

The sinusoidal current has many advocates, but while more grateful to the patient than the more abrupt faradic shock, its results, in my experience, have not equaled those obtained by using the combined current. High-frequency currents, applied by the unipolar vacuum electrode method, may also be used to relieve the pain in multiple neuritis,

In old, long-standing cases of chronic multiple neuritis, counter-irritation may be applied over the nerve-trunks (not peripheral distribution) by the application of high-frequency sparking or static electricity. Multiple neuritis due to lead poisoning may require recourse to interrupted galvanism to secure muscular response, while for diphtheritic polyneuritis Erb and Duchenne advocate faradism.

Facial Neuritis.-The same general rules regarding selection of current and polarity that were discussed under "Multiple Neuritis" apply equally to inflammation of a single nerve. Several of the nerves are so frequently the site of inflammatory processes that they deserve special consideration.

In facial palsy electricity is an aid to diagnosis, and a very valuable factor in determining the prognosis should the palsy be of peripheral origin. When facial paralysis is of central origin, there is only a quantitative change in the response of the muscles to galvanic and faradic stimulation, the degree of excitability being diminished immediately after the palsy occurs, and is increased coincidentally with the increase of the reflexes.

If the paralysis is nuclear in origin or results from inflammation of the seventh nerve, reactions of degeneration may be present, appearing in from six to ten days after the onset of the palsy. Should no electrical 
changes appear by this time, the outlook is good for recovery in from three to four weeks. If within this time reactions of degentration are absent, but the response to the faradic current diminislied, and response to the galvanic current inereased, the prognosis is gool for recovery in from eight to twelve weeks. When partial or beginning reactions of degeneration are present, the paralysis will probably last for several months, while if complete degeneration is found, recovery should not be expected.

Electrical treatment for peripheral facial palsy may be commenced almost immediately with the galvanic current, using large, well-protected electrodes, the active anode covering the entire side of the face, the indifferent cathode being applied to the back of the neck or between the shoulder-blades. 'The duration of the treatment should be about ten minutes, using a current of from ten to fifteen milliamperes.

After ten days the affected muscles may be treated by the faradic current, or, better still, by the combined galvano-faradic or de Watteville current. In applying this current, the indifferent electrode may be held in the patient's hand or applied to the back, while the active electrode should be applied over the motor points of the paralyzed muscles. The current should be rhythmically interrupted by means of a rheotome. 'The application to each muscle should not be over a couple of minutes at each treatment, to avoid fatiguc of the muscles. With the appearance of contractures, the faradic current should be discontinued, although mild galvanism is still of some advantage.

Hunt has pointed out the association of herpetic inflanmation with lesions of the geniculate ganglion, and in treating this type, it is advisable to defer electrical treatment until all signs of herpetic inflammation have subsided.

While practically every variety of peripheral facial palsy is benefited by electrical treatment, cases caused by cold (rheumatic) respond most readily and, as a rule, give the most favorable prognosis.

Musculospiral Nerve. - Involvement of the musculospiral nerve alone is most frequently the result of pressure upon the arm, and constitutes the so-called "bridegroom's palsy" or the more frequent "Saturday-night palsy." As the nerve supplies the extensors of the forearm and wrist and the supinators, the patient has "wrist drop," with the hand held in the semiprone position. When the paralysis is the result of pressure, the prognosis is favorable and the application of the galvanic and faradic currents hastens the recovery.

Median Nerve.-It is comparatively rare for the median nerve to be affected alone: its treatment by electricity in no way differs from that of the nerves already mentioned.

Ulnar Nerve. - In contradistinction to the median nerve, the ulnar is frequently affected by reason of its exposed position, causing the characteristic "claw-hand" deformity. "The treatment is the same as that of other nerves.

Neries of the Lower Extremity.-These are less frequently injured than those of the upper extremity, and complete paralysis of the muscles supplied by a single nerve is also less common. 
Sciatic Nerve-Of the nerves of the lower extremity, the sciatic is the one of most importance to the electro-therapeutist, chiefly because of sensory disturbances, and, indeed, it is at times difficult to determine whether we are dealing with a neuritis or a neuralgia.

Electrical treatment for sciatic neuritis in the acute and subacute stages should, in the opinion of the writer, be secondary to rest, but with the patient in bed can be advantageously combined with this measure. Guilleminot advocates using the galvanic current with the indifferent electrode over the loins and a foot-bath or active electrode attached to the cathode. In my own experience the best results have been obtained by using the anode as the active electrode. This should be followed by rhythmic galvano-faradization of the muscles.

Neuralgia.-It is not within the scope of this chapter to discuss in detail the etiology of neuralgia or its differential diagnosis, but it may be of advantage to point out that neuralgia is characterized by paroxysmal attacks of severe pain, and while there may be present the "tender points" of Valleix, there is an absence of tenderness throughout the course of the affected nerve and an absence of muscular wasting. Treatment, therefore, is directed toward relief of the pain, and when the neuralgia is the result of a constitutional condition, toward relieving the underlying cause. Reactions of degeneration are not found in neuralgia unless this condition is accompanied by a neuritis.

When the neuralgia is the result of autointoxication from faulty metabolism, general electrical treatment is often of distinct value. General faradization, static electricity, the electric bath, and high-frequency currents in the form of autocondensation may be used, the selection of the current depending upon the general condition.

For local treatment, galvanism is the method of election, the positive pole being employed as the active electrode. Large, well-covered electrodes are best, and the usual dose is one-tenth to one milliampere per square centimeter of surface (Guilliminot). Bergonie, Bordier, Vernay, Guilleminot, and others advocate very large electrodes and strong currents, but this method requires great care and skill in technique, and in the opinion of the writer is less safe in the hands of the general practitioner and more severe for the patient than using a weaker current for a longer period of time. Neuralgia may also be treated by the galvanic current through cataphoresis-using such medicaments as cocaine, quinine, etc.

Rockwell recommends the furadic current for treating neuralgia in cases where pressure over the affected nerve relieves the pain. If the pain is increased by pressure, faradism is contraindicated. When desired, counter-irritation may be applied along the course of the nerve by the method of Duchenne, which consists of the application of the faradic current by means of a metallic brush electrode attached to the negative pole, with an indifferently placed positive electrode. To secure the best results, the skin should be dried by the application of a powder, such as starch, lycopodium, talcum, etc. Static electricity in the form of sparks may also be used to bring about revulsion.

High-frequency currents may be used in the form of the bipolar 
effleuve, followed by monopolar sparks along the course of the affected nerve, as recommended by Albert-Weill, or autocondensation followed by the application of a glass vacuum electronle along the course and distribution of the nerve.

In discussing the treatment of neuralgia, we should not overlook the relation existing between the viscera and certain cutaneons areas, as studied by Ilead and others. With an abnormal condition of a given viscus, there is a hyperesthesia in the corresponding cutancous "zone." In such conditions electricity is, of course, secondary in importance to medical treatment of the diseased organ, but may assist materiall! if applied so as to secure counter-irritation.

With reference to the so-called "visceral neuralgias," Neiswanger claims to be able to diagnose between ovarian neuralgia and pyosalpinx by means of electricity, stating that no pus is present in the pelvis if the pain resulting from a mild galvanic current within the pelvis can be relieved by rapidly interrupted faradism, but that pus is always present where the pain resulting from the galvanic current is unrelieved by faradism.

Standing out most prominently among neuralgias, because of their frequency and obstinacy are those of the trifacial and sciatic nerves and the brachial plexus.

Trifacial Neuralgia.-One, two, or all three branches may be involved, and the pain is sometimes associated with muscular spasm, constituting that most distressing condition - "tic douloureux." In treating trigeminal neuralgia electrically, it should be remembered that counterirritation is not indicated, and that galvanism promises the best results. Bergonie and Guilleminot advocate currents as strong as 60 to 75 milliamperes, applied by means of specially constructed metal electrodes, the active anode molded to cover the entire half of the face, and carefully covered with felt, clay, or kaolin moistened with a sodium bicarbonate solution. The indifferent cathode should also be of large size ( 300 to 500 square centimeters), and may be applied to the back of the neck or between the shoulder-blades. The duration of the treatment is from thirty to fortyfive minutes. Care must be taken to see that no metal touches the skin, or a severe burn may result. I have obtained excellent results, using a weaker current for a more prolonged period of time.

Tousey reports excellent and often prompt results in trifacial neuralgia following the use of high-frequency currents, and has observed that the pain is in some cases worse after the first one or two treatments, but subsequently disappears.

Brachial Neuralgia.-The nerves from the brachial plexus are not infrequently affected by neuralgia, the condition often being mistaken for neuritis or rheumatism. Herpes zoster is often a complication, and the neuralgic pains are most distressing.

Galvanism with a large electrode at the back of the neck and a smaller positive electrode passed over the course of the nerves may afford slight relief. The current should be mild, but long continued. "The high-frequency effleuve applied by means of a glass vacuum electrode may also be tried. 
Sciatic Neuralgia.- This is a rather common affection, found most frequently in adult males. The causes are numerous and varied, and are important from the standpoint of the electro-therapeutist, as it is obviously useless to treat electrically a neuralgia resulting from the pressure caused by a tumor, pelvic exudate, or disease of the pelvic bones as long as the cause is still active. Such neuralgias as result from exposure to cold or from constitutional disorders as gout or rheumatism are more amenable to electrical treatment, but the results are by no means as promising as in trifacial neuralgia. The same treatment may be used as advised for brachial neuralgia.

Meralgia Paræsthetica.-In this condition, the pain and paresthetic disturbances over the front and outer surfaces of the thigh have been treated by galvanism, faradism, static and high-frequency currents. One observer has used the metallic brush and faradic current for the anesthetic area often present. My experience with this affection has been limited, but in two cases treated with mild galvanism, there was prompt relief of the pain and a gradual disappearance of the paresthetic symptoms. The anode was used as the active electrode.

Diseases of the Spinal Cord.-Acute Anterior Poliomyelitis.Considered from the standpoint of electrotherapeutics, infantile spinal paralysis is the most important of all the spinal-cord diseases. First, because of the unfortunate prevalence of the condition, and secondly, because of the benefit derived from the application of electricity to the wasted muscles.

Before considering the electrical treatment of this affection, it may be well to briefly recall its pathology. We now know that it is not, strictly speaking, an anterior poliomyelitis, but a myelitis in which the brunt of the "insult," as well as the permanent changes, are found in the multipolar ganglion-cells of the anterior horns. We also know that not all the cells primarily affected undergo complete destruction, many regenerating after the acute symptoms have subsided. Moreover, in the muscle groups affected, there are usually some fibers or muscles that remain intact. 'Tousey aptly speaks of these as "the residual sound fibers."

Some electrotherapeutists advise galvanization as soon as paralysis appears, but in my opinion absolute quiet and rest are more important in the acute stage than any form of electrical treatment, and I believe it unwise to subject the child to unnecessary manipulation or any measure that might frighten it or cause struggling.

Treatment of the paralyzed muscles may be commenced from two to three weeks after the subsidence of the acute symptoms. If the muscles respond to the faradic current, they may be stimulated with the rhythmically interrupted faradic or galvano-faradic currents. If, on the other hand, they respond only to the continuous current, galvanism may be applied, using a theotome to secure interruptions; whichever current is used, the active electrode should be applied over the motor points of the muscles and the indifferent electrode over the spine or other point.

Many cases of acute poliomyelitis are mild in character, and tend to improve or even recover without treatment, while in others, the destruc- 
tion of the ganglion-cells and conseruent wasting of the muscies are so widespread as to leave the patient hopelessly erippled, despite the most persistent treatment. Between the two extremes may be found all grades of paralysis, yet even in the worst cases some goxd can be accomplished by thorongh electrical treatment, and in the milder cases recovery can be hastened. Results have been obtained even after many years have elapsed since the onset of the tronble.

In the above affection, electro-diagnosis is of value both from a prognostic standpoint and in informing the operator, by means of the modifications of the electrical reactions, as to the actual amount of degeneration that has occurred in individual muscles or groups of muscles.

Chronic Anterior Poliomyelitis.-In this condition, where there is progressive degeneration of the anterior horn cells and wasting of the corresponding museles, it is useless to expeet anything approaching recovery. Here the eleetrotherapeutist should devote his energies toward improving the general nutrition of the spinal cord, and in retarding, as far as possible, the progression of the muscular atrophy. For the first of these indications, Erb has advocated galvanization of the spinal cord; the current should be ascending, with large electrodes applied, one over the cervical region, and the other over the lumbar region. 'The intensity of the current should vary from ten to thirty milliamperes, with an average duration of ten minutes for the treatment. In this, as in other conditions, it is well to remember the general rule that the milder the current, the longer may be the duration of the treatment. Faradism should not be applied to the cord because of its irritating effects.

The second indication in this disease, stimulation of the muscles, is carried out by the applieation of the galvano-faradic, faradic, or sinusoidal currents, or if the reactions of degeneration are present, interrupted galvanism may be employed. 'The indifferent electrode should be placed over the back or at any point on the extremity above the affected muscles, and the active electrode placed over the motor points or over the distal tendon (point of longitudinal reaction).

Locomotor Ataxia.-Electrical treatment of tabes dorsalis is of value in relieving certain of the symptoms rather than toward retarding or curing the disease itself. Some writers advise galvanism of the spinal cord, but in my judgment any beneficial effects upon the cord itself can hardly be expected. Such symptoms, however, as are due to involvement of the posterior roots or ganglia may be amenable to electrical treatment. In using galvanism, the electrode should be applied in such a way as to include the nerve-roots of the trunk supplying the extremity or other part of the body affected. I arge electrodes should be used, and a current of from ten to thirty milliamperes should be applied for a period of ten minutes. Guilleminot, when there are lightning pains in the lower extremities, advises an indifferent anode of 300 or more square centimeters, applied to the neck with electrically connected cathodes of 150 square centimeters, placed under the thigh or calf of each leg. 'This method, in my opinion, is not the best, as we know from the phisiolog- 
ical effects of the galvanic current that the cathode acts as an irritant, while the positive pole has a sedative action, and should, therefore, always be used as the active electrode where the operator desires to relieve pain. Tousey applies the galvanic current to the spine by means of cell-baths. He seats the patient in a perineal bath connected with the positive electrode, with the forearms of the patient in baths connected with the negative pole. A current of from 25 to 30 milliamperes is given for a period of ten minutes, three times a week, for from four to six weeks.

In the treatment of the pains of locomotor ataxia, as in the treatment of any painful condition, the current should be increased gradually and diminished gradually, in order to avoid shock to the patient by suddenly making or breaking the current.

The extreme numbness or coldness of the feet and legs, which are very distressing symptoms in some cases of locomotor ataxia, I have relieved in two instances by the use of a strong faradic current, the effect lasting for five or six hours after the treatment.

High-frequency currents have also been recommended for the relief of pain, but the results seem hardly to be compared with the galvanic current. Static sparking along the spine, like faradic electricity, its contraindicated.

Primary Lateral Sclerosis.-In this disease, as there are increased muscular irritability and spasticity, without muscular wasting, electricity in any form is practically useless.

Syringomyelitis. - Should the characteristic lesion of this disorder extend to the anterior horns of the cord, the resulting muscular atrophy may be treated in the same manner as described under Chronic Anterior Poliomyelitis. Galvanism of the spine has also been recommended. Reymond and others have reported improvement following $x$-ray treatment.

Diffuse Myelitis.-The rules already outlined for the treatment of pain, atrophy, and paralysis occurring in the various systemic diseases of the cord may here be applied according to the symptoms present.

Diseases of the Brain.-In the vast majority of cerebral diseases the results of electrical treatment have been negative. Of the most frequently seen pathological conditions, hemorrhage, tumor, and abscess, the first named is the only one in which electrical treatment might be of some use, but even in this condition, electricity has a very limited sphere of usefulness. Whatever value this therapeutic agent may have is manifested in the resulting palsies occurring in consequence of the vascular lesion.

'The first rule of treatment in, for instance, hemiplegia accompanying a stroke, would be to withhold all electrical treatment for at least one month. The second rule is never to apply faradism or interrupted galvanism if there are any signs of spasticity. Mild galvanism may be employed to improve the general nutrition of the paralyzed extremities, and faradism is sometimes serviceable in stimulating individual muscles in such a way as to overcome the contraction of their antagonists.

Electro-diagnosis.-As the hemiplegia resulting from cerebral lesion 
is due to disturbance of the upper motor neuron, it, of course, follows that reactions of degeneration would be absent.

Cerebral Galvanization.- The experiments of Buckhardt, Erh, von Ziemssen, Ieduc, and others have proved conelusively that the resistance of the eranial bones and overlying tissues can be overcome ly electrical eurrents. Galvanization of the (erebrum may induce a sense of vertigo, and it is clained by I educ that with the anode placed over the forehead and the eathode over the neck or body, the galvanic current will cause intellectual stimulation, and that somnolence oceurs if the position of the poles be reversed. I believe, however, with I)igmet, that no absorbative effect can be obtained upon a cerebral blood-clot without using a current of so great an intensity that harm would result, especially as Vimmern, Bateli, and others have produced epilepsy by experimental cerebral electrization.

Functional Nervous Disorders.-Sydenham's Chorea.-'l'his is a self-limited disease, tending toward recovery in from two to four months, and I cannot but feel that many of the beneficial effects of electricity reported have been due to the tendency of the condition to improve, rather than as a result of the electrical treatment. In long-stinding cases, however, treatment tending to improve the general condition is certainly advisable. General galvanization or faradization may be used advantageously, also the static breeze, or the application of high-frequency currents over the spine by means of the glass vacumm electrodes. For the condition known as post-choreic hemiplegia, or where there is general weakness occurring as a sequel of this affection, rhythmically interrupted faradization, galvano-faradization, or the sinusoidal current may be applied to the extremities. In the early stages of chorea, electrical treatment in any form is entirely secondary to that most valuable therapeutic measure-rest.

Choreiform movements occurring as the result of other conditions are not considered in the above diseussion of the electrical treatment of chorea. Under this head would come hysterical choreiform movements and post-hemiplegic chorea.

Electro-diagmosis is of no value in differentiating true chorea from allied conditions, as the reactions of degeneration would be absent in all.

Hysteria.-This disorder is characterized by marked emotional instability and a proneness to react to suggestion. Tanet has even gone so far as to claim that all the symptoms are induced by suggestion. Without entering into a discussion of the etiology of hysteria, the important fact from the standpoint of the physician is that the varied symptoms are removable by suggestion.

For treating the protean manifestations of hysteria, the electrotherapeutist has a most valuable means of treatment at his command. The noise and sensory impression of the faradic current; the sparks and breeze of the static machine, with its huge size and revolving wheels; and the beautiful violet rays of high-frequency currents, when applied by means of the glass vacuum electrodes, are all pregnant with suggestive possibilities. 
Aside from the psyehic state, the symptoms with which one meets may be almost any form of sensory or motor disturbance. The sensory symptoms vary from pain to complete loss of sensation, and the motor symptoms from increased motion to complete paralysis. 'Thus tremors, spasms, convulsions, hemiplegia, monoplegia, and paraplegia may be presented, and it has been well said that hysteria ean simulate any known disease. The operator should, above all things, remember that in removing an hysterical symptom by electrical suggestion, he is only lopping off but one of the tentacles of the octopus, and that his whole manner and attitude toward the patient should be one of constant optimism. Discipline, a healthful environment and, in some instances, isolation are necessary, electricity being but one agent in effecting a cure.

The kind of current used should vary according to the symptoms presented; thus, anesthesia may be treated by the static souffle or breeze, by the static bath, or by high-frequency eurrents, using the glass vacuum electrode. If resistant to these methods, the faradic eurrent may be applied, using the faradic brush attached to the negative pole of the battery. 'The hyperesthetic areas or "devil spots" may be treated with galvanism, using the positive pole as the active electrode, by mild faradism, or by high frequency applied in the same manner as for anesthesia.

Hysterical paralysis may be treated in much the same manner, combining the electricity with passive movements, and, as motion is gradually regained, with voluntary reëducational movements of precision. Hysterical hiccough and vomiting frecpuently yield to galvanization of the pneumogastric. For the globus hystericus and hysterical aphonia the static breeze or the high-frequency effleuve constitute the best forms of electrical treatment.

The reader will bear in mind, I trust, that in any hysterical condition it is not so much the form of electrieity that is used, as the manner in which it is given, and that to secure the best results, one must judiciously combine psychotherapy with the electro-therapy.

Neurasthenia.-In this disease, characterized by exhaustion and imperfect nutrition of the nerve-centres, the symptoms are those of motor, sensory, and psychic fatigue, combined with evidences of imperfect metabolism, of which atonic gastro-intestinal disturbance is perhaps the most conspicuous. To meet these indications we have in electricity a means, not of securing rest, which is of first importance, but an aid in improving the general nutrition and of temporarily relieving some of the manifold symptoms.

In the treatment of neurasthenia, the static, faradic, galvanic, sinusoidal, and high-frequency currents are all of value, and each has its ardent advocates. The first general rule to remember is that the selection of the current to be used will depend largely upon the state of bloodpressure. With low arterial tension, static electricity or the high-frequeney spark or effleuve would be indicated on account of their physiological action in raising blood-pressure.

With high arterial tension or when arteriosclerosis is present, the autocondensation method of applying high frequency is indicated be- 
cause of its property of lowering blool-pressure. Both static and highfrequency currents are of benefit in stimulating or rogulating genceral metabolism, as are also the galvanic and, to a lesser degree, the faradic and sinusoidal currents.

'The static bath is perhaps the most generally preferred methor of applying static electricity in the treatment of neurasthenia. With the positive pole grounded, the patient is seated upon an insulated platform with his feet in contact with a metal plate attached to the negative pole of the machine. Commencing with five-minute treatments daily, or every other day, the duration may be gradually lengthened to thirty minutes or even longer. Should insomnia be present, the patient should be connected to the positive pole and the negative pole grounded.

Static sparking may be given along the spine, with the active electrode connected to the positive pole of the machine. 'This method is particularly' valuable in cases presenting low blood-pressure. 'The treatments should not be of long duration, and the sparks should not be allowed to impinge in succession on any one point, as it would cause the patient considerable pain and discomfort.

Neurasthenic headaches are sometimes relieved by the head breeze, and the constipation so commonly present in neurasthenia may be treated by application of the static spark to the left iliac fossa.

Gulvamism.- The continuous current is not used as much for the general effect in neurasthenia as are the static and high-frequeney currents. For relief of the headache and other cerebral symptoms, Castex and Leduc advocate the application of the galvanic current, using a large positive electrode at the back of the neck and a smaller cathode to the forehead. A current intensity of twenty milliamperes may be applied for ten or fifteen minutes. It is important that the current be turned on and off gradually. 'The backache so common in this condition is sometimes relieved by galvanism, the operator using a large positive electrode over the painful area, which is usually the lumbar region.

Galvanization is also recommended as being more or less effective in relieving cardiac palpitation, constipation, and sexual impotence.

Faradism.- While some writers entirely ignore the use of faradism in the treatment of neurasthenia, I believe it to be of considerable value, especially when applied to the spine or in the form of general faradization. In the latter method, the patient's feet are placed on a covered metal electrode, while the active electrode is passed rapidly over the general surface of the body. For this purpose the sponge hand electrode is useful.

An important point to remember in using faradism in this condition is that the interruptions should be as rapid as possible, as muscular contractions are not desired. Polarity is of but little importance.

High-freguency Currents. - Since the introduction of these currents, there have been many advocates of their use in neurasthenia. In cases with high blood-pressure or arteriosclerosis, the d'Arsonval autocondensation method is indicated. The patient reclines upon the couch or is seated upon the pad, which is connected with one pole of the d'Arsonval transormer, 
a block tin electrode connected with the other pole being held in the patient's hand. 'The duration of the treatment should be from ten to fifteen minutes. Gidon, Mounier, Challamel, Gay, and others have ealled attention to the marked reduction of arterial tension which follows the employment of high-frequency currents by this method, the reduction being permanent. Not only do the high-frequency currents lower the blood-pressure, but they aid in the climination of the waste-products, particularly carbon dioxide and urea. They increase respiratory movements and general eellular activity.

In treating cases with low arterial tension high-frequeney currents, either by means of the bipolar or unipolar method should be used. In the bipolar method a metal electrode is placed over the epigastrium, and sparks applied over the spine by means of a glass vacuum electrode. According to Mounier and Tousey, the monopolar method gives practically the same results, besides possessing the advantage of enabling the operator to apply the current through the patient's clothing.

Occupation neuroses are really localized neurasthenias, but the results of any form of electrical treatment in these conditions have been discouraging and unsatisfactory. The most rational form of electrical treatment would seem to be the galvanic current, with the active electrode connected with the positive pole.

The various ties probably also belong under this general heading, and may be treated in the same manner with about as unsatisfactory results.

Exophthalmic Goiter.-Perhaps no disease is so uncertain in regard to its eause, duration, and prognosis. Some cases will recover without any form of treatment, while others grow progressively worse, despite thorough medical and electrical treatment, going to the surgeon as a last resort.

Whatever may be the underlying cause of this interesting malady, we know that the symptoms result from an auto-intoxication caused by a disturbance in secretion of the thyroid gland. Just how electricity acts in this condition we do not know, but the fact remains that in a eertain proportion of cases galvanism brings about a deeided amelioration of the symptoms.

Various methods of application have been advised, such as placing the eleetrodes one on either side of the gland, or one on either side of the neck, behind the angles of the jaw. It has also been advised by some to place the cathode over the goiter and the anode over the back of the neck, but, in my experience, the best results have been obtained by using the positive electrode over the gland and the indifferent electrode at the back of the neck. A current of from five to ten milliamperes may be used for a period of fifteen minutes-treatments may be given every second or third day.

'Tousey elaims to have permanently cured a certain percentage of cases by the use of the $x$-ray and high-frequency currents.

Paralysis Agitans.-The reports of the results of electrical treatment in this condition are meager and not conelusive. Guilleminot has 
reported one case of improvement following galvanism, applied by using an arm bath for the anode, with the negative electrole at the barek of the neck, a current intensity of twenty milliamperes to be used for fifteon or twenty minutes every second day. I) muner and Maces report excellent results in a case following the daily application of high-frecpuency currents by the autoconduction method.

'This latter method, through its property of lowering the blowd-pressure, would seem at least to wamant a fair trial, as any mothod of treatment which offers the slightest hope of even temporary relief $\mathrm{in}$ this distressing condition should not be overlooked.

Diseases of Muscles.-Idiopathic Muscular Atrophy (Primury Myopathy). - This disease is essentially the same, whether pseudohypertrophy is present or absent, and the varions names attached to the different types of this affection indicate differences in location rather than any pathological divergence. 'Therefore, the rules for treatment that would apply to one form would be equally indicated in all.

Electro-diagnosis.-Unlike muscular atrophy of spinal or neuritic origin, the pure myopathies give no reactions of degeneration; there is, however, a diminished excitability to both the galvanic and faradie currents.

Elcctro-therapeutics. - As the condition is an abiogenetic one, the operator can only hope to retard the progression of the muscular wasting by careful exercise of the affected muscles. 'The best current for this, as already pointed out, is the rhythmic galvano-faradic, in the application of which a large indifferent electrode may be applied over the posterior cervical region for the upper extremities, or the lumbar region for the lower extremities. A small active electrode connected to the negative pole is applicd to the motor points of the museles. No one muscle should receive over two minutes' treatment at each séance, but the treatments may be given daily or every other day. 'The furadic current alone may be used in the same manner as the combined current, but the effects are not quite so good as when galvano-faradism is applied.

The galvanic bath or sinusoidal bath is recommended by some authorities.

Diseases of the Cardiovascular System.-- Endocarditis.-Owing to the doubtful value of electricity in the treatment of any variety of this affection, and the possibility of actual harm resulting from the use of the various currents, I am of the opinion that their use is not to be recommended. For the consideration of radiography or radioscopy as a means of diagnosis in endocarditis or cardiac hypertrophy, the reader is referred to the chapter on $x$-rays.

Aneurysm.-Although the treatment of ancurysm by electricity is really a surgical procedure, it may not be out of place to nention that advantage has been taken of the elot-forming property of the galvanic eurrent at the anode, first by the method of "galvanopuneture," in which a needle, insulated to within $\frac{1}{2} \mathrm{~cm}$. of its point, is thrust directly into the aneurysmal sac, and connected with the positive pole of it galvanic battery, while the other electrode, attached to the negative pole, is applied 
over some remote point of the body. A current of thirty milliamperes is allowed to pass for a period of one-half to one hour. 'Ihis has been followed by a better method in which clot formation is further aided by the introduction, into the aneurysmal sac, of a fine wire through a canula. The wire is connected to the positive pole of a galvanic battery and the cathode applied as in the first method. For details of the above methods the reader is referred to text-books dealing with electro-surgical procedures.

Arteriosclerosis.-As already indicated, high-frequency currents applied by autocondensation are of decided value whenever the bloodpressure is increased. Moutier and Challamel have reported a great change in blood-pressure following the autoconduction method, a reduction of 30,40 , or 50 millimeters in a series of cases treated, as against 15,20 , or 30 millimeters by the autocondensation method. As "autoconduction" requires an expensive and cumbersome wire "cage," the condensation method is more practical for the general practitioner, as an inexpensive folding pad may be employed.

Diseases of the Digestive Tract.-Riggs' Disease or Pyorrhoa Alveolaris.-A condition in which there is inflammation of the gums with suppuration and a tendency for the teeth to become loosened in their sockets. For this condition Snow and others have used high-frequency currents and claim to have obtained excellent results.

For the application of the current, specially constructed electrodes are employed, shaped to fit the gums and with insulated stems so that no current will come in contact with the lips. A current strength of 75 milliamperes may be used.

Tousey advises a combination of $x$-ray and high-frequency treatments for pyorrhea.

Regnier and Didsbury have shown that anesthesia may be produced in the teeth by means of high-frequency currents, and dentists have for many years utilized the cataphoric property of the galvanic current for the introduction of cocaine into a painful tooth.

CEsophageal Stricture.-This may be functional or organic, and the question of diagnosis should be settled before determining upon the treatment.

Functional or spasmodic stricture is often the result of hysteria, and will usually respond to some form of electrical treatment. Guilleminot suggests that galvanization of the pneumogastrics be tried first; failing in that method, he introduces an cesophageal electrode and attempts to exhaust the irritability of the unstriped muscle-fibers by prolonged application of the faradic current. As a last resort he tries intra-œsophageal galvanization.

The beneficial results obtained by galvanization for the relief of organic stricture of the cesophagus appear to have been thoroughly established. 'The following is a brief outline of the technique emploved. After ascertaining the caliber of the stricture, select an olive-pointed œsophageal electrode a couple of sizes larger than the caliber of the stricture, and introduce it into the osophagus, Attach the osophageal 
electrode to the negative pole of the battery. A large electrode attuched to the positive pole is applied over the body at an indifferent point, and a current strength of ten milliamperes is gradually turned on. About five minutes is usually suflicient for the electrode to pass the stricture, after which the current should be turned off and the electrode withdrawn. In from five to seven days an electrode a comple of sizes larger may be employed. A current of greater intensity than that indicated above would be apt to cause too great an inflammatory reaction.

Reflex Vomiting.-For this condition galvanism is the best form of electrical treatment. 'The anode should be applied above the right clavicle, between the heads of the sternomastoid muscle, and a large electrode connected to the negative pole is applied over the epigastrium. A current intensity of ten milliamperes may be given for a period of from fifteen to thirty minutes.

'The above treatment is also applicable for emesis gravidarum.

Dilatation of the Stomach.-When simple gastric dilatation exists, as from atony, we have in electricity a valuable adjunct to other forms of treatment. 'The unstriped muscle-fibers of the stomach-wall may be stimulated by intragastric electrization or by external application of the current over the viscus. 'This latter method is known as "percutaneous electrization."

Einhorn's Intragastric Electrification.-After fasting for a time sufficient to insure emptiness of the stomach, the patient is given a glass of water to drink, with which he swallows the special electrode. 'This is of metal, surrounded by an ebonite olive with numerous perforations, and attached to a well-insulated flexible electrophore. 'To faradize the stomach, the internal electrode is connected to one pole of the battery, and a roller electrode attached to the other pole is applied over the epigastrium. 'The stomach may be galvanized by passing a positive electrode over the stomach with the intragastric electrode attached to the negative pole of a galvanic battery.

Freund has studied the effect of intragastric electrization upon the function of the stomach, and concludes that secretion of the digestive ferments is not stimulated by this method.

The percutaneous method is easier of applieation and less disagreeable for the patient, besides producing equal or better results.

Static electricity may be applied as follows: The patient is not insulated, but reclines on a couch with the abdomen exposed. 'The external armature of the condensor at the positive pole is earthed, while the other is connected with a metallic electrode applied over the epigastrium. 'The spark rods are kept from two to six inches apart, the intensity being regulated by the length of the spark-gap. 'The shock with each discharge should be sufficient to cause a painful sensation and visible muscular contration. Duration of treatment should be from fifteen to twenty minutes, repeated daily or every other day.

The motor function of the stomach may also be stimulated externally by the faradic, galvanofaradic, or sinusoidal current. 'The writer prefers the combined current, applying the indifferent electrode between the 
shoulder-blades and the active electrode over the epigastrium. A rheotome is essential to interrupt the current. Tousey uses a rhythmic rheostat and pole changer, which he claims removes the shock to the patient and produces contractions which closely resemble physiological ones.

Secretion of the gastric juice is best stimulated by faradization. The indifferent electrode is applied to the back or other remote point, and the active electrocle passed over the epigastrium. Interruptions should be as rapid as possible, as intermittent muscular contractions are not desired. Duration of treatment, from ten to fifteen minutes, repeated daily or every second day.

Constipation.- The character of the electrical treatment to be employed in this condition depends largely upon the cause, as to treat all forms of constipation in the same manner electrically is about as senseless as to treat them all by the same means medically.

Atonic constipation, with sluggish peristalsis and relaxation of the abdominal walls, is best treated by static electrization. The method described for dilatation of the stomach should first be tried. If this fails to remove the condition, recourse may be had to intrarectal application.

To apply this method, a rectal electrode is connected with the positive condensor of the machine, the chain of the negative condensor being earthed. The electrode is inserted until grasped by the sphincter, but not allowed to pass beyond. 'The spark-gap should be gradually increased until it is from 5 to 8 inches in width. Duration of treatment, ten to fifteen minutes, at first daily, then every second day.

Next in efficiency to static electrization are galvano-faradization, galvanization, and faradization in the order named. Fither current may be applied in the manner advocated by Benedikt, which consists in placing a large positive electrode over the lumbar region and passing a smaller negative electrode over the course of the large intestine, using as strong a current as can be tolerated by the patient. I have obtained excellent results from the interrupted galvanic current, applying both electrodes a few inches apart over the abdomen.

Erb advises intrarectal faradization, inserting an olive-shaped electrode into the rectum, and applying the other electrode over the lumbar region or abdomen.

Spasmodic Constipation.-For this condition, Delherm, Tousey, Guilleminot, and others, recommend continuous galvano-faradization for a period of ten to fifteen minutes daily. Large electrodes are used, one being applied over the abdomen and the other over the lumbar region. The direction of the current is not of importance. The galvanic or faradic currents may also be applied separately in the same manner, but have not given as satisfactory results when used alone as when combined.

As relaxation of the unstriped muscle-fibers of the intestine is the effect desired, it is obvious that there should be no shock or intermittence of the current.

Intestinal Occlusion.-Electricity, applied in the form of the electrical 
douche or enema, as described by Boudet in 1854, is of value when the obstruction is due to chronic constipation resulting from spasm or atony of the bowels. It is not to be recommended when there is ulceration or perforation of the intestines, or suppuration or adhesions of the neighboring organs.

Technique. - The rectal electrode is inclosed in a perforated rubber tube, which prevents the metal from coming in contact with the intestinal wall, and eliminates the danger of sloughing of the tissues. 'I'he tube is so constructed as to allow the passage through it into the bowel of from one to two quarts of a normal saline solution. A large electrode is applied over the abdomen, and a galvanic current of twenty to forty milliamperes is turned on slowly and allowed to flow for five minutes, then suddenly reversed and allowed to flow in the opposite direction for a few minutes. 'This procedure may be repeated every few minutes and continued for a period of ten to twenty minutes. If effective, the movement may occur immediately after withdrawal of the electrode, or it may be postponed for two or three hours.

Hemorrhoids. - The galvanic current is indicated in the electrical treatment of hemorrhoids because of its properties of relieving pain, hardening tissue, and constricting the blood-vessels. It is best applied by means of a copper bulb electrode, carefully covered as advised by Neiswanger, with chamois skin, and moistened with a 10 per cent. solntion of ichthyol in glycerine. With the patient in the Sims position, the electrode is inserted carefully into the rectum until grasped by the sphincters, and then connected with the positive terminal of the batterv. A large electrode is connected with the negative terminal, is applied over the abdomen, and a current of ten to fifteen milliamperes slowly turned on. The duration of the treatment should be ten minutes and repeated every second day.

The use of the high-frequency currents in the treatment of hemorrhoids may be said to date from the publication of Doumer's report in 1597. Results of this form of electrical treatment have been excellent, especially in cases of recent development. Fither the glass vacuum rectal electrode may be used, or the metal electrode of Doumer. After being covered with lubricant, the electrode is introduced into the anus before the current is turned on. The operator should also turn off the current before withdrawing the electrode. Applications of five minutes' duration may be made, at first daily, later every second day.

Fissure of the Anus.- The treatment deseribed for hemorrhoids is also applicable for the treatment of anal fissure.

Pruritus Ani.--'lhis distressing condition is usually amenable to electrical treatment in the form of the static bath or efflewve, or by highfrequency effleuvation. Galvanism is also of value in pruritus, applied with the anode as the active electrode.

Diseases of the Urinary Tract.-Urinary Incontinence.-The nocturnal incontinence occurring in children is amenable to electrical treatment, and many cases of incontinence in adults resulting from weakness of the vesical sphincter can be decidedly benefited. When complete

Vou. I-21 
paralysis of the sphincter has occurred as the result of an organic disease of the spinal cord, improvement should not be expected any more than it would be looked for from medical treatment.

Galvanism may be employed, placing the anode over the perineum, and the cathode over the lumbar region. A current strength of ten to twelve milliamperes should be used for five minutes.

The faradic or galvano-faradic currents may be applied in the same manner.

Static or high-frequency sparking of the lumbar and suprapubic regions is recommended by some. 'Treatment by intra-urethral faradization is advocated by Guyon. The galvanic and static currents have also been applied by means of intra-urethral electrodes.

Nephritis.-The electric bath is an excellent means of inducing diaphoresis, and thus meets one of the indications in the treatment of nephritis. As used at the Jefferson Hospital, the "light bath" has proved to be both practical and satisfactory.

Regarding the application of the various currents in the treatment of diseases of the kidneys, it is not surprising to find that both the static and high-frequency currents have some enthusiastic advocates. I do not consider their use of much value, except in cases with high blood-pressure or arteriosclerosis, where high-frequency autocondensation would be indicated.

Constitutional Diseases.-In the treatment of the numerous disorders that arise as the result of imperfect metabolism, there is no doubt that in electricity, intelligently applied, we have a useful addition to our armamentarium. We can, through the various currents, raise or lower the blood-pressure, induce diaphoresis, exercise the muscles, stimulate the general functional activity of the various organs, and increase the elimination of the toxic waste-products.

Arthritis. - While opinions differ regarding the benefit to be derived from high-frequency currents in acute articular rheumatism, all are practically agreed as to their value in the passive forms of chronic arthritis.

In treating subacute or chronic arthritis I subject the patient first to high-frequency autocondensation for ten minutes, using the "auto-pad," then with the glass vacuum electrode make local applications over the affected joints for the same length of time. Treatments may be given at first daily, then every second day.

For the acute joint inflammation, ionization with salts of lithium has been used by Bordier, and with sodium salicylate by Bergonie, Guilleminot, and others. 'The amount of the medicament thus introduced into the tissues must, however, be insignificant in comparison to that which could be absorbed from the mucous membrane of the intestinal tract. For the relief of pain, galvanization of the joint is sometimes useful.

In myalgia, lumbago, etc., electricity is decidedly indicated. Galvanism is applied with a large positive electrode over the painful area and a large negative electrode over some indifferent point. A current of considerable intensity may be employed for a period of twenty to thirty minutes, repeated daily or every second day. 
Static electricity may be applied over the sore muscles in the form of the effleuve for from ten to fifteen minutes daily.

'Ihe high-frequency spark and effleuve are often effective, and the sinusoidal bath also has its advocates.

Gout.-Electrical treatment in gout is practically the same as that described for chronic rhematism. The electric bath, autocondensation, high-frequency effleuve locally, galvanism, and the sinusoidal bath have all been recommended.

Guilloz has reported beneficial results in a series of cases treated hy lithium ionization. 'The affected part is immerserl in a porcelain bath filled with a 2 per cent. solution of lithium chloride, to which sufficient lithium hydrate has been added to alkilinize it. 'The bath is connected to the positive pole of a galvanic battery and a large negative electrode is placed over the back or other remote point. A current of from one hundred to two hundred milliamperes is allowed to flow for a half-hour. Treatments may be given daily. In this method advantage is taken of the fact that uric acid is more soluble in a lithium solution than in normal body fluids.

Diabetes.-Opinions differ greatly regarding the effect of high-frequency currents upon glycosuria. d'Arsonval, Apostoli, Guilleminot, Boinet, 'Tousey, and others report decided reduction in the amount of sugar excreted, while Boedeker, Cohn, and others failed to obtain any modification of glycosuria with the use of these currents. No deleterious effects have been reported, however, and all observers agree that the general health has been improved by the application of highfrequency currents. While there are no contraindications to their use in diabetes, I believe it best to wait for further reports before making any definite statement as to their value or uselessness.

Obesity.-For the treatment of this condition I do not adrocate any form of electricity, unless it be the faradic exercise of the muscular tissue, and even this should be used with caution.

Cephalic galvanization, with a current of considerable intensity, will, it is claimed, cause a great reduction of weight, but I clo not consider its use justifiable, as a current sufficiently strong to accomplish the result desired would be apt also to cause injurious effects. Noreover, as myocarditis is a common complication in obesity, there is danger in using such methods as autocondensation and general galvanization.

Diseases of the Respiratory Tract._-Nasal Catarrh.-The local application of high-frequency currents by means of the nasal ylass vacuum electrode has a stimulating effect on the mucous membrane, and may be employed in the treatment of this condition. As the mucous membrane is more sensitive than the skin, only weak currents should be employed, and sparking should be avoided by inserting the electrode before turning on the current, or by the operator keeping his hand "pon the electrode while inserting the latter into the patient's nostril. 'The duration of its treatment should be from two to four minutes, repeated daily.

Ozena.-High-frequency currents may be employed in the same manner as indicated for nasal catarrh. Electrolysis is, however, the 
better method of treating this condition. The mucous membrane is cleansed and treated with a 5 per cent. cocaine solution. 'The positive electrode, consisting of a copper needle, is thrust into the middle turbinate and a negative electrode, consisting of a steel needle, is introduced into the mucous membrane of the inferior turbinate. A current strength of ten to fifteen milliamperes may be used; it should be turned on gradually, allowed to flow for ten minutes, then gradually reduced, and finally reversed to free the positive needle. This procedure should not be repeated for a week.

Anosmia.-Both extranasal and intranasal galvanization have been used in treating this condition, the latter method being the more effective and applied as follows: A negative electrode, consisting of an insulated probe, is wrapped with moistened cotton-wool and introduced into the nostril, the positive electrode being placed at the back of the neck. A weak current, not over five milliamperes, is used, the duration of treatment being five minutes.

Pulmonary Tuberculosis.-Electricity, like almost every other known remedy, has had its advocates for the treatment of this disease. I consider the inhibitory action of the electrical currents upon germ life too insignificant to be considered, and the beneficial results from electrically generated ozone too problematical to merit any consideration.

Asthma.-Results have been claimed in the treatment of asthma by galvanization of the pneumogastric nerve by the application of high-frequency currents over the chest-wall with a glass vacuum electrode, and general treatment by autocondensation; the latter method is at least worthy of trial should the blood-pressure be too high.

It may be interesting to note that Stembo has reported that galvanism will sometimes induce cough and expectoration in bronchitis and bronchopneumonia in cases unresponsive to medical treatment. With the negative electrode held at the back of the neck, he applies the positive electrode at the anterior borders of the scaleni muscles, using a current of two or three milliamperes.

Diseases of the Ear. - Tinnitus Aurium.-Imbert, Marques, and others claim to have had good results from the use of high-frequency currents in cases with "noises in the ears." The effleuve or fine sparks should be applied over the mastoid process, and just in front of the external auditory meatus. 'The treatment should not be continued over five or six minutes, and may be repeated every other day. Where high bloor-pressure or arteriosclerosis are associated with tinnitus, autocondensation may be advantageously combined with the above treatment. If recovery is to occur, improvement should be manifest after several treatments, and it is always advisable to continue the treatment for at least a month after the disappearance of the symptoms.

Otitis Media.-Mounier and Bergonie advise the use of faradism during the subsidence of an acute otitis media, claiming that it promotes absorption of the exudation. 'The current is applied by means of an aural electrode, the indifferent electrode being applied at the back of the neck. The current should be rhythmically interrupted and of a strength 
sufficient to cause faint contraction of the facial muscles. 'Treatment should be continued for fifteen to twenty minutes, repeated daily or every second day.

Galvanism may also be employed in the same manner, using a current of five milliamperes.

Diseases of the Skin.-Acne.-Electrolysis is a very useful method of treating acne and acne rosacea. 'The patient holds in the land a moistened electrode connected with the negative pole of the galvanic battery. A fine iridoplatinum needle, connected with the positive pole, is introduced into the skin parallel with the course of a dilated blood-vessel, and a current of from two or three milliamperes is turned on and allowed to flow until the needle becomes loosened. The procedure may be repeated for other vessels at subsecuent sittings.

High-frequency currents in the form of the spark or effleuve are also of some value in this condition. 'The latter is perhaps the better method, and should be applied from a small, flat, glass vacuum electrode. 'The duration of each treatment should be about ten minutes, repeated every second day.

Alopecia.--In this condition Bordet reports excellent results from high-frequency sparking. 'The Oudin metal electrode with a glass cuff may be used, or the ordinary glass vacuum electrode. The application should he continued until the scalp becomes decidedly reddened, but vesication should be avoided.

Faradization of the scalp is also a method of some value. Ordinary sponge electrodes may be used.

Eczema.-As eczema may be considered as the cutaneous manifestation of deranged metabolism (usually alimentary), electrical treatment should be both general and local. Central galvanization or high-frequency autocondensation is sometimes helpful, and the sinusoidal hydroelectric baths have been also adrocated by Guimbail.

In acute eczema high-frequency currents are effective in relieving the pain and itching, besides having a beneficial effect upon the course of the affection. 'They may be applied by the glass vacuum electrode, using a current of moderate intensity and avoiding sparking. The electrode may be applied directly to the skin or through a thin, evenly applied bandage. 'The duration of the treatment should be from ten to fifteen minutes, repeated every second day.

In the chronic forms stronger applications of the high-frequency effleuve may he made, and the static bath and effleuve may also be tried.

Urticaria.-Central galvanization, hrdro-electric sinusoidal baths, and high-frequency autocondensation may be used, with local application of the static or high-frequeney effleuve.

Pruritus. - The treatment is practically the sane as that for urticaria. An excellent plan is to combine high-frequency autocondensation with the local application of the effleuve, the former, for a period of fifteen minutes, followed by effleuvation over the affected area for a period not exceeding ten to fifteen minutes. Daily treatments may be given.

Lupus.-'The two most effieient methods in the hands of the electro- 
therapeutist in treating this condition are the $x$-rays and the Finsen lamp, or one of its modifications; for the $x$-rays treatment the reader is referred to the chapter devoted to that subject.

When actinic rays are employed, the duration of each treatment varies from twenty minutes to one hour, depending upon the form of lamp used, the more recent modifications requiring less time than the lamp originally designed by Finsen. The inflammatory reaction appears some hours after the exposure, and lasts seven or eight days. The treatment may be repeated in from seven to ten days.

High-frequency currents are less efficacious than the $x$-rays or actinic rays, but are of some value. They may be employed in the form of the spark or effleuve. In the aberrant form of lupus erythematosus, highfrequency applications have given fair results.

Hypertrichosis. - Superfluous hairs are readily removed by electrolysis, the method having been first employed by Michel in 1875 .

'The needles should be of gold or platino-iridium. I prefer the bulbouspointed needle introduced by Dr. Hayes as being less apt to leave a superficial scar, because the maximum of the current energy is expended at the point, and therefore within the hair-follicle, rather than at the point of entrance, as is the case with the ordinary shaped needle. It is best to have a magnifying glass, either mounted on the needle-holder or attached to a head-band; a small pair of forceps are also necessary. The indifferent electrode is connected with the positive pole of a galvanic battery and applied over the body at a point remote from the field of operation, or may be held in the patient's hand. The needle, attached to the negative pole, is introduced into the hair-follicle until it reaches the bottom of the follicle; a current of from two to five milliamperes is allowed to flow until a little foam gathers about the needle, or until the hair will come away without undue traction being exerted. From five to twenty seconds is usually sufficient. Care must be exercised in removing hairs by electrolysis, as too much current or too long an application is apt to cause scarring or pitting.

Electric Sleep.-Leduc, in 1902, produced in animals a condition of narcosis which he termed "electric sleep." He employed a low-tension intermittent current with from 90 to 110 interruptions per second, the cathode being placed over the head and the anode over the extremities, back, abdomen, or other indifferent point. Later, in experimenting upon himself, he produced a state of partial abolition of sensibility, motion being completely inhibited, although consciousness was not entirely lost. As it has been shown by experiments upon animals that an overdose may cause a marked fall of blood-pressure, respiratory and cardiac inhibition, epilepsy, and death, it is obvious that "electric sleep" is not at present of any practical value, and may be considered as being still in the experimental stage. 


\title{
THE TREATMENT' OF DISEASES BY T'HE $X$-RAYS AND RADI0-AC'IVE SUBST'ANCES
}

\author{
Br GEORGE E. PFAHLER, M.D.
}

'TuE Roentgen rays, and to a lesser extent other radio-active emanations, have been used extensively in the treatment of diseases during the past ten years. We should be in a position to-day to know fairly well their place in therapeutics. During this period most of the chronic diseases have been subjected to this form of treatment. For the most part, it has been by men who were totally ignorant of the underlying principles governing the treatment, or of the general biological effects to be expected. 'Technical knowledge was lacking, and practitioners who were generally well informed on medicine and surgery, but lacked this special knowledge, were carried away by the enthusiasm which led to the use of the rays, as a last resort, in cases in which there could be no reasonable hope of success. As a result, these same enthusiasts are now being overcome by their disappointments, and are being carried as far away from the truth in the opposite directions.

' $T$ 'he fact is, that these rays have a definite biological effect, and, when properly and skillfully used in suitable cases, they produce results that cannot be equalled by other methods. I believe that more uniformly good results are obtained to-day in the hands of skillful men than ever before.

Because of the brevity of this article one must presuppose a certain amount of technical knowledge and skill in this special work.

General Biological Effects.-In general, the effects produced depend upon the absorption and chemical effect of the rays upon the cells of the tissues exposed. 'The total effect will depend upon the degree of sensitiveness of the particular cells and upon the quantity of absorption. The glandular epithelial cells of the body are the most sensitive, then the protective epithelinm of the skin and mucous membrane, followed by the connective tissue, muscle, and nervous tissue.

Of the glands, the most sensitive are the testicles and ovaries, then the spleen, thymus and thyroid glands, the lymphatics, and liver. 'Therefore, these organs, when not under direct treatment, must be carefully protected.

'The effects produced by the rays must of necessity only involve the tissues through which they pass, since they move in straight lines from their point of origin. Any other symptoms or manifestations in remote 
parts must be secondary to changes in the tissues directly irradiated, or are due to secondary rays generated in the tissues, or to substances generated or liberated under the influences of the rays.

'The quantity of rays absorbed will depend upon the quantity striking the tissues, their duration, their quality, and the density of the tissue through which they pass. The quantity of $x$-rays striking the skin will depend upon the amount of milliamperage of current going through the tubes, the character of the target in the tube, the thickness of the glass, the distance of the tube from the patient, and the duration of the treatment. Therefore, when one speaks only of the duration of a treatment it means absolutely nothing as regards the dosage. This quantity of rays can be fairly and definitely measured by means of the barium platinocyanite disks of Sabouraud and Noire, which change color under the influence of the rays, or by means of the Holzknecht capsules, or the Kienböck quantimeter. The latter instrument is dependent upon a specially sensitized and standardized photographic paper, and divides the dose necessary to produce a mild dermatitis into 10 degrees, and when properly used is, I believe, the most accurate measure of the quantity of rays absorbed by the tissues. This also gives the quantity of absorption at 1,2 , and 3 centimeters in depth. Any one who has not become skillful by experience should to-day, depend upon one of these means of measuring the closage.

Soft rays are more readily absorbed than the more penetrating or hard rays, and, especially in dealing with soft rays, the greater amount are alssorbed in the skin and first centimeter of tissue. Therefore, the first effect will be produced upon the skin, and the skin must be our index of the maximum dose permissible in a patient.

The first noticeable effect upon the normal skin is the production of an erythema, which is often spoken of as a "reaction." This is also recognized as the first degree of a burn, and is the maximum or "full" dose, as measured by the above scales.

Even this stage is variable in degree, and at first appears as a slight blush, which increases until it becomes a deep red. 'This dose should seldom if ever be exceeded. If the full dose is given at one sitting, the redness will usually begin within a week and attain its maximum in the second week. 'This period of latency will vary somewhat with the softness of the rays and with the sensibility of the patient's skin. Blondes are more sensitive than brunettes. In the latter, the delay may be more than two weeks, and, if the dose has been divided, a tanning usually occurs, which seems to make the skin more resistant and permits of a greater dosage.

'The redness is accompanied by slight itching and a slight burning sensation. These symptoms will be relieved by the application of a simple ointment, such as cold cream, and afterward covered by talcum powder. The redness usually disappears within a week, and is followed by a slight desquamation within the following week. As a rule, it is best to interrupt the treatment for two weeks following such a reaction. Accompanying this reaction the hair usually falls out. 
The falling out of hair need give no special alarm, for when removed by a single maximum dose, it invariably returns, and the return growth is heavier than the primary one. 'This return growth occurs in two to three months.

'The second degree of burn, or Roentgen dermatitis, will occur at times as a result of one's anxiety to control a malignant growth which threatens the life of the patient, and the full dose is exceeded. 'This comes on in two to three weeks after the total dose has been given, or, if given in divided doses, may occur during the treatment. It consists of the appearances of the first degree, followed by the formation of vesicles, blebs, loss of skin, then crust formation, followed by healing, which requires several weeks. 'The result is the formation of a pink scar, and later this is likely to be infiltrated by telangiectases, especially if further treatment has been given on the same area.

'The treatment of this second degree of burn consists, in the early stage, in the applications of normal salt solution, or 1 per cent. picric acid solution. After the secretion ceases to be excessive, cold cream, covered by a soft cloth, will usually give relief and hasten healing. When crusts form, they must be removed from time to time by the application of a thick layer of cold cream on a cloth.

The third degree of $x$-rays burn should never occur. It occured early in the history of $x$-rays work as a result of prolonged exposures for diagnostic purposes, and in the hands of incompetent operators. It, of course, may occur as a result of excessive exposure for any purpose. It passes quickly through the two preceding stages, and then forms a deep yellowish gray ulcer. This either heals very slowly, or may never heal. It also becomes extremely painful.

The treatment consists primarily of the treatment of the second degree, until the ulcer limits itself and becomes stationary. 'The ulcerated area should then be excised without further delay, and followed by skingrafting or transplantation.

Chronic radiodermatitis occurs in operators who have been exposed to the Roentgen rays or radio-active substances extending over a prolonged period of time. It consists of a premature senility of the skin, and corresponds almost exactly to the atrophy, fissures, and multiple keratoses which are seen in certain old people, especially in those whose skin has been exposed to some chronic irritation, such as the sun's rays or chemical irritants.

The treatment of this distressing condition consists primarily in prevention, by avoiding the exposure to the rays. 'This will be discussed under technique. In the early stages of this affection the skin must be protected from any further exposure, and it must also be protected from exposure to irritations of any other kind, such as developers, fixing baths, disinfectants, the sun's rays, and exposure to heat and cold. 'The skin lacks moisture and lubrication, due to an atrophy of the glands. I have found that the wearing of ordinary kid gloves almost continuously, protects the skin from dirt, traumatism, cold, heat, and sun's rays, and serves to retain the moisture. 
If the keratoses and fissures show no tendency to disappear under this protection, or if they increase and become painful or inflamed, they should at once be excised and the areas covered by healthy skin. Otherwise the degeneration goes on to malignancy, followed by metastases, which have caused the death of at least a dozen Roentgenologists, and have resulted in the loss of the limbs of a number of others. A more recent, and apparently a more satisfactory, method of treating the keratoses and warts is by means of fulguration.

The biological effects of radium and other radio-active substances are almost identical with those described above, resulting from exposure to the Roentgen rays. There has been infinitely less investigation along this line with radio-active substances, and, therefore, our knowledge is less definite.

The Biological Effects upon Diseased Tissue are of the same general character. The effects are, for the most part, of an atrophic nature, and, therefore, Roentgentherapy or radiotherapy is indicated in the conditions which are characterized by hypertrophy or hyperplasia, hypersecretion, or new-growths. The diseased tissue or diseased cells respond more readily to the effects of the rays than normal tissue, and, therefore, one commonly sees diseased tissue react to the rays, and return to a normal condition without noticeable effect upon the surrounding or overlying tissue, which has been exposed equally as much or even more.

While the general and final effect is atrophic in nature, especially after prolonged treatment, if a single full dose is given, the reaction which is brought about may have a stimulating effect. This is illustrated by the increase in growth of hair following a temporary removal, resulting from a single full dose of rays. It is also illustrated by the growth of hair following Roentgen treatment of alopecia areata.

However, in contradiction to some authors, as a result of a wide experience in the treatment of malignant disease, I have never seen any stimulation of a new-growth by the rays. This may be due to the fact that in the treatment of such conditions it is my custom to give full doses.

The relief of pain by the rays is an effect which has been observed by most or all operators, but which has not been satisfactorily explained. It does not always occur, even in what would appear to be identical conditions. The pains of malignant disease are at times relieved, as well as those of chronic neuralgias, when the only alternative is stuporous doses of morphine.

General Technique.-The Tube and its Vacuum.-To do satisfactory work, one should have a number of tubes, for no single tube can be kept in proper condition for all kinds of work. They should each have a regulating derice, so that they can be made to emit the proper quality of rays, and this regulation should be automatic, so that the quality of rays may remain constant during a treatment.

Generally speaking, new tubes tend to produce softer rays, and should be used for superficial work, while old tubes tend to produce hard rays of a more penetrative quality, and should be used for deep-seated work.

The quality of rays produced by a tube should not be guessed at, 
but should be measured by means of the Benoist, Walters, or Wehnelt scales. 'These instruments measure the penetrative value of the rays by means of graded thicknesses of aluminum. I prefer the Benoist scale, which consists of twelve gradations, measured by the shadow cast by from 1 to 12 millimeters of aluminum, as compared with a layer of silver $0.11 \mathrm{~mm}$. in thickness. 'Therefore, if the shadow cast by the silver equals that cast by $5 \mathrm{~mm}$. of aluminum, one would say the quality of rays are Benoist 5 , etc.

In the treatment of superficial affcctions, especially skin diseases, one should use soft rays, varying from Benoist 3 to 5 , depending upon the effect to be produced. 'Ihe softer the rays, the more superficial will be the effect. In the treatment of deep-seated diseases-conditions beneath the skin-one should use more penetrative rays, varying from Benoist 6 to $S$ inclusive. 'This quality of rays will have proportionately less effect upon the skin, and will reach and be partially absorbed by the deeper tissue.

The Strength of Current passing through the tube will govern to a great extent the quantity of rays emitted. 'I'his is measured chiefly by a milliameter placed in the secondary circuit in series with the tube. Even with a given milliamperage, the quantity of rays emitted will vary with the voltage and the duration of each pulsation of current, being very much greater in value with the current from a static machine than from a coil. The coil current is more generally used, and with this I usually work with 1 milliampere. However, in the treatment of deep-seated affections, I commonly use from 10 to 20 milliamperes for short periods of time, changing the tube for a fresh one as soon as the vacuum begins to drop. I am sure that I get better results by this latter technique in the treatment of deep-seated disease. When a tube is used continuously for ten minutes or more, one cannot pass through it, as a rule, more than 1 milliampere without having a decided fall in vacuum and even valueless light.

Many of the poor results obtained from Roentgentherapy are due to absolute ignorance or carelessness regarding the quality and quantity of light used. One might as well expect uniformly good results from the use of a solution of strychnine of unknown strength and without measurement of dosage.

The Distance of the Anode, or Target, from the Skin deserves careful consideration in each case. The Roentgen rays obey the law of intensity, which governs the other ethereal vibrations, and, therefore, diminish with the square of the distance from its source. 'The intensity' of the rays, therefore, at a distance of 5 inches from the anode, is four times as great as at 10 inches' distance. So the duration of an exposure at 10 inches' distance would have to be four times as long to produce a given effeet, and at 12 inches about six times as long.

Here again it is clearly seen that the mere mention of the duration of a treatment which is so common in literature means nothing unless the other factors are given.

In the passage of the rays through the air many of the very softest 
rays are absorbed by the air; therefore, when the tube is very near the skin, there will be a greater proportion of soft rays. It is the soft rays that are absorbed by the skin; therefore, in the treatment of skin conditions one should bring the tube near to the skin, and the greater distance should be used for deeper work.

Another principle, based chiefly upon the law of intensity of radiation, which must be constantly borne in mind, is that the difference between the surface effect and the deep effect is proportionately less the greater the distance from the target or anode. 'Therefore, for deep-seated work the anode of the tube should be placed 10 or more inches distant. In this way one can produce a deep effect without so much irritation of the skin.

Filters.- Just as the air absorbs the very softest rays, so any interposed object will absorb a certain amount of the rays in proportion to its density and thickness, and also, to a certain extent, according to the peculiarity of the object itself. 'Therefore, clothing, aluminum, glass, etc., if placed between the skin and tube, will absorb a certain proportion of the rays that would otherwise affect the skin. Based upon the theory that each substance has a peculiarity in the character of the rays which it absorbs, I interpose sole leather near the tube, when treating deep-seated disease, in order to remove in great part the rays that are especially absorbed by the skin.

Five years ago I performed a series of experiments which proved that the use of this leather filter protected against what would otherwise produce a complete destruction of the skin, and yet not materially affect the deep dose. It is, however, possible to burn the skin, even when this filter is used, if sufficient exposure is given.

The Duration of a Treatment.-Based upon the above facts, it can now be understood why the duration of a treatment must depend upon the quantity of current going through the tube, its distance from the part treated, the quality of rays emitted (especially their penetrative power), and the sensibility of the tissue treated. Generally speaking, the duration of a treatment will vary from five to thirty minutes, being shortest for skin diseases (when the tube is close) and longest in deep diseases (when the tube is at a greater distance).

Frequency of Treatment.- This will vary with the dose given at each treatment, being more frequent in deep work and less so in skin diseases. In the former I commonly see my patients three times a week or daily; in the latter, perhaps once a week.

Protection of the Operator.-Nothing can be more important than the personal protection of the operator. At least a dozen $x$-ray workers have sacrificed their lives in this work, and many others have lost fingers and arms. The lesions produced have been previously mentioned. This can all be aroided by protection from the very beginning. The lead-glass shields and other opaque covers for the tube give a certain amount of protection, but certainly not sufficient when the more penetrating rays are used. Additional protection is given by a leaded wall between the patient, in one room, and the operator with his control 
switches in the other. For this purpose the wall may be covered with sheet-lead, $\frac{3}{3}$ to $\frac{1}{16}$ inch thickness. When this is not practical, a learl screen may be used to shield the operator. 'The small outfits that are sold to men who think they will "only do a little treatment" are sure to do much harm, for such nen will not be willing to spend more money for protection than they do for their instrument, and usually the same protection is needed. In addition, the men who "only do a little treatment" do not prepare themselves to do the work well.

Protection of the Patient.- The patient should only be treated over the diseased areas, or over those parts of the boly in which we are trying to produce a radiologieal effect. All other parts of the borly must be protected. 'This is best accomplished by surrounding the tube with a substance opaque to the rays, and allowing them to be emitted through a controllable diaphragm. In treating skin diseases, one should further limit the exposed area by cutting holes in thin sheets of lead, so as to expose only about $\frac{1}{8}$ inch of healthy skin beyond the diseased area under treatment.

Radio-active Substances.-The radio-active substances used in the treatment of disease are radium, uranium, thorium, etc. Of these, radium has been used most, and may be taken as the example. Its cost has prevented an extensive use. Its effects will vary with the units of radio-activity. 'The lower activities require a longer time to produce a given effect, and even then are probably less efficient.

Generally, the radium is used in the form of a capsule, which is introduced into a cavity affected with malignant disease, as the rectum, uterus, or the maxillary sinus, but at times is introduced (through an incision or otherwise) directly into a growth. It is allowed to remain in position from an hour to a day or more.

'These radio-active substances are also incorporated into pastes or ointments for external application, and at times water is rendered radio-active (Morton) and administered internally.

Diseases of the Pilo-sebaceous System.-As an Epilating Agent.The shedding of hair following exposure to the rays was observed soon after their discovery, and led directly to their use in the treatment of disease. It is probably due to a temporary atrophy of the hair follicle, from which it recovers, unless the exposure is repeated. The best rays to use for this purpose are those corresponding to Nos. 5 and 6 on Benoist's scale. The distance must vary somewhat with the area treated, but generally I use 5 inches, measured from the anode to the skin. One must give nearly a full dose, corresponding to 5 Holzknecht's units, 10 of Kienböck's, or 10 of Holzknecht's modification of Sabouraud and Noire's scale. It usually requires two to three weeks for the hair to fall out. This may or may not be accompanied by a reaction in the skin. Ordinarily, unless more treatment is given, the hair will return in about three months, and usually thicker than before.

Hypertrichosis. - The overgrowth of hair on women's faces is a tempting field for the use of the rays as an epilating agent, but usually the treatment must be carried to the extent of damaging the skin in order 
to make the effect permanent. This makes the skin look old and atrophied. Therefore, I do not recommend it in this affection.

Trichophytes. - In hypertrichosis we are not dealing with disease, and the treatment is only demanded for æsthetic reasons. In the treatment of ringworm, however, we are dealing with a troublesome disease of the hair follicles. 'The usual treatment is by the application of some form of antiseptic. Sabouraud wrote previously to the Roentgen treatment, "Not only is there no known cure for tinea tonsurans, but I think I am justified in saying there never will be a cure of this disease by the use of antiseptics. We may vary at will the chemical nature of the application we employ, but this will in no way alter their power of penetration. The root of the hair is inaccessible to external antiseptics." These same remarks regarding tinea will apply to favus. The parasites in these diseases affect and depend upon the vitality of the hair follicle. Therefore, if we destroy temporarily the vitality of the hair follicles, we eliminate the disease. 'The Roentgen rays are the ideal means for this purpose. The treatment is painless, and when carefully done, is both harmless and permanent. To Sabouraud is due the credit of a definite technique, by which the area treated is given a full dose at a single sitting. His formula is as follows: "In order to cure a patch of tinea, an exposure should be given with the focus-tube at a distance of 6 inches from the skin, with rays corresponding to No. 4 of Benoist's radiochromometer, and the total quantity absorbed should be 4 to 5 Holzknecht units, or to the scale B on the Sabouraud and Noire radiochromometer. These disks of Sabourand and Noire are more convenient and accurate. After this full dose the hairs fall out, the disease disappears, and then the hair returns in two to three months."

Favus.-Practically all that is said above applies to favus and need not be repeated. It is advisable in this disease to treat the entire scalp, and then keep it covered with a mild antiseptic solution, such as diluted tincture of iodine or carbolic acid in lanolin.

Sycosis and Folliculitis of the Beard.-In these conditions, one may usually expect a cure. The bare parts of the face and the nucous membrane should be covered with lead. The normal dose of from 3 to 5 Holzknecht units ( $B$ of the Sabouraud and Noire scale) should be given. After a latent period of about a week epilation commences, the inflammatory symptoms increase, the small abscesses around the hairs are evacuated, and after a time the skin heals completely. At times, in this disease, the skin is especially sensitive, and becomes much inflamed before epilation is complete.

Alopecia.-In quite a number of cases of alopecia there has been a return of a good growth of hair, especially in alopecia areata. That the return growth of hair was due to the rays was shown by the newgrowth being confined to the areas treated by the rays, while no change was seen in the parts not so treated. The treatment is usually followed by a complete alopecia and then by the new hair. Success cannot be expected in those cases in which there are no lanugo hairs, nor in those cases in which there is a universal alopecia, with loss of eyebrows and eyelashes. 
The treatment must not be carried beyond the hyperemic stage of inflammatory reaction, which will be equal to a dose of about 3 Holzknecht units on the bearded area, or 3 to 4 Holzknecht units on the scalp (equals 6 to 8 Kienböck units).

'The general technique for skin treatment may be followed. In alopecia areata the treatment should be confined to the affected arcas, while in the more general involvement this localization need not be made. 'This treatment has not heen used extensively.

Acne Vulgaris and Acne Rosacea.-In the treatment of these conditions the rays are one of the accepted agents, and in the more chronic cases, which have lasted over six months, and which have resisted the more simple methods, this form of treatment is probably second to none. In the early cases other methods should be given a trial.

'The treatment should be confined to the affected areas. It should not be carried beyond the hyperemic stage in its effects. 'I'his will correspond to from 3 to 5 Holzknecht units ( 6 to 10 Kienböck), and will be produced approximately by a current of 1 milliampere passing through a tube whose anode is 6 inches from the skin, which emits rays corresponding to 5 Benoist for ten minutes.

'This dose may be given at a single sitting or may be divided. When accurately measured, the single sitting is probably best. The whole face should not be exposed to the tube at one time, but in definite areas, so that the rays are evenly distributed. After a full dose has been given to any area, it should not receive more exposure inside of two weeks, or until all visible effects have disappeared. I usually estimate that it will require 15 to 20 treatments and three to four months to effect a cure. The final results are generally very gratifying, leaving the face smooth and soft. At first the scars from the old lesions (which were present before this treatment) become more noticeable, because of a hyperemia, but later they fade considerably as a result of the treatment.

Diseases Associated with Seborrhœa.-Psoriasis will usually yield to other external applications, together with internal medication, but in the obstinate cases radiotherapy may be used with success. However, even with this treatment the results are not always permanent. The general technique described for skin diseases may be used. 'The treatment should be carried only to the extent of producing a hyperemia. 'This is followed by desquamation and disappearance of the disease in the area treated. A full dose can be given at one sitting.

Seborrhœic eczema will yield in the same way, with the same dose, and with the general technique.

Pruriginous Dermatoses.-Vulvar and Anal Pruritus.-In these conditions one should, of course, search for a probable cause, and when possible, this must be removed. In the idiopathic cases, or when no cause can be determined, radiotherapy usually gives satisfactory results. The itching is relieved, and the thickened, indurated, ecrematous skin is restored to normal. In this disease the rays should be especially confined to the affected area. In men, the scrotum must he carefully: protected by sheets of lead, on account of the effects on the testicles. 
If the scrotum is involved, it becomes a doubtful method of treatment. In this disease the treatment is given in fractional doses ( 1 to 2 Kienböck units), and repeated two or three times a week.

Prurigo, Lichen Ruber Planus, and Lichen Corneus.-Like most of the skin diseases, radiotherapy may be recommended after the more common methods of treatment have failed to produce satisfactory results. 'The itching is one of the first symptoms to be relieved. This is followed by a reactive hyperemic desquamation and a return of the skin to normal. The technique recommended for pruritus will apply in these diseases.

Lupus Vulgaris.-This is a disease that requires much patience, no matter what method of treatment we may choose. However, I have succeeded in curing patients in whom the disease had resisted other forms of treatment for from ten to fifteen years. Success may be expected if the treatment is carefully and persistently given, in nearly all cases. Those cases in which large areas are involved, or in which most of the skin is diseased, and in which there is a coalescent, brawny, reddishbrown surface, success can hardly be expected. When the mucous membrane is involved, the outlook is less hopeful than otherwise, though I have had success even in these cases.

Most authors recommend this treatment very highly, and place it scarcely second to the Finsen treatment. It is especially preferable in those cases in which the diseased tissues cannot be satisfactorily compressed for the Finsen treatment.

The treatment is usually given in fractional doses, and must be continued over months. Hyperemia must be produced repeatedly, but not continuously. It is seldom necessary to give a dose that will produce more than a hyperemia, and this effect should then be allowed to disappear before renewing the treatment. If the entire area of the disease can be treated at a single seance, I usually only see the patient once a week, and give a dose of about 4 to 6 Kienböck units. When the disease is extensive, the patient must be seen oftener.

The first noticeabie effect is an increase in redness of the skin. This is followed by slight edema, which gradually fades and desquamation follows. The disease slowly limits itself to islands. These must then be especially treated, protecting the healthy scar.

When the disease is deep, or when the patients come from a distance, and can only remain for a short time, the treatment must be given more energetically. Under these circumstances the dose necessary for destruction of the thickened tissue by ulceration is given, and then the treatment is discontinued. In these cases a violent inflammation develops, crusts form, and under them serum accumulates. The crusts are gradually thrown off and reform. If the patient's natural resistance is good, the healthy epithelium gradually creeps over, leaving a soft pink scar which is most gratifying. This destructive dose will usually be about 10 Holzknecht, or 15 to 20 Kienböck units. One must consider conditions carefully before giving these large doses, for a slowly healing ulcer is most embarrassing to the physician and painful to the patient. 
Lupus Erythematosus. - A number of cures have been reported in this disease from Roentgentherapy, but it is considered generally less useful than in lupus vulgaris. Heavy closes are required. If a cure is obtained, it is only as the result of a violent reaction and consecutive cutaneous atrophy. Doses of 5 Holzknecht units are given in one or several seances, with rays of a moderate degree of penetration-4 to 5) Benoist. It should be remembered that in this disease the skin is very sensitive to the rays and is apt to react violently.

Scleroderma, being another obstinate disease, has been subjected, in a number of cases, to radiotherapy. 'The results are not brilliant, and require much time and patience, but in the end are perhaps as satisfactory as other methods. The patches are comparatively insensitive to the rays, and a full dose must be repeated every three weeks.

Keloid offers a much more favorable prognosis, and the treatment can be highly recommended. Successes are obtained in patients who have been operated upon repeatedly, or who have had other methods tried without success.

The treatment must be carried to the point of producing a distinct hyperemia; but violent reaction must be avoided; 5 to 6 Holzknecht or 10 to 12 Kienböck units may be given, either in one or several treatments. Then there should be an interval of three weeks before repeating the dose. The anode of the tube should be about 6 to 8 inches from the surface of the keloid. The remainder of the skin and body should be carefully protected. Gradually, after about a month, the keloid begins to soften and diminish in size, until it nearly or completely disappears. 'The treatment is not infallible, but the results are better than I have seen obtained by other methods. As a rule, one can obtain a soft pink scar.

Mycosis fungoides is a formidable disease, and, previous to the days of radiotherapy, it was looked upon as almost hopeless. Therefore, the good results obtained by means of the Roentgen rays become all the more important. Much patience and many treatments are necessary, but by persistence and care the disease can usually be made to disappear.

Each tumor or diseased area is treated separately, with the tube at 6 to 8 inches away, emitting rays corresponding to No. 6 Benoist; a dose of 7 to 9 Holrknecht units should be given at one sitting. Other areas may be treated on subsequent days, but an interval of fifteen to twenty days should elapse between any two treatments (of the above dose) on any single area.

The immediate result to be expected is a clisappearance of the pruritus within two or three days following the above dose. Within six or seven days the skin over the tumors becomes slightly erythematous, and gradually takes on a brownish tint. 'The tumors gradually become softer, and after a fortnight they are diminished in size. After a few repetitions of this dose (on each tumor or affected area), at intervals of two or three weeks, they should entirely disappear. At each repetition of the dose it should be somewhat diminished.

VoL $\mathrm{I}-22$ 
The size of the tumor will govern somewhat the amount of treatment needed. In the erysipelatoid areas a smaller dose will be required, and in the eczematous even less. Belot has observed unusual sensibility to the rays of the thenar and hypothenar eminences and of the sole of the foot in this disease. The treatment demands considerable expenditure of time and money, but it is painless and makes the patient more comfortable, by relieving the itching almost at once and without affecting the general health.

Verrucæ-Cutaneous Horns.-Excellent results have been reported in both of these conditions by many authors. I have treated a number of cases, and almost invariably with the very best results. I usually hope to get rid of them without producing a scar.

Warts have been treated by so many different methods that one is not limited to radiotherapy; but it is painless, almost absolutely certain in its results, and the cosmetic effect is perfect. I have succeeded in eliminating warts that have recurred after having been excised and cauterized, and this without even a scar or a trace of the former wart. These results are permanent after several years.

The surrounding tissue should be covered by lead sheets, in which an aperture has been cut large enough to expose the wart. The tube should be brought as close as possible; it should emit rays to No. 6 Benoist, and a dose of 5 to 10 Holzknecht or 10 to 20 Kienböck units should be given (a quantity equal to one or two times the full skin dose). This may be given in a single sitting or divided. About two weeks after this dose the wart becomes inflamed and then atrophies, or forms a crust and drops off. They disappear in a month to six weeks after the full dose has been given.

Cutaneous horns are treated in the same way, and with practically the same results.

Nævus.-Radiotherapy has been used in the treatment of nævus with only a moderate degree of success. These lesions are divided into the pigmentary and vascular. 'The first division includes nærus pilaris, nævus verrucosus, and nævus hypertrophicus. In each of these three some improvement has been obtained, but electrolysis or fulguration is so much more certain and rapid in its results that, while painful, it should receive preference over radiotherapy.

The "wine marks," which are sometimes very extensive and very troublesome, offer a little better field. The treatment must be carried to the extent of ulceration. This involves some risk, and cannot be recommended. Fulguration, or one of the other controllable destructive agents, is to be preferred. I have treated two cases with "wine marks" of an extensive nature, in which the dose was not carried to the destructive degree. I obtained a considerable fading, and this improvement in appearance has continued during the five subsequent years.

Epitheliomata.-A natural step from the consideration of warts, moles, and keloidal formation is the study of epitheliomata. In this disease, above all others, I believe the $x$-rays hold first place. This treatment offers the following advantages: 
(1) It is painless in its application, and, therefore, patients can be more easily persuaded to be treated early. 'This in itself would be sufficient advantage to recommend it most highly, for it is the delays that make the prognosis so bad from any form of treatment.

(2) The treatment does not open lymph-channels and make fresh wounds through which the disease may spread.

(3) If treated early, and before the deep tissues are invaded, the disease disappears, often without leaving any sear. When a scar does form, it approaches most nearly in appearance that of normal skin. It is soft, pliable, and of a pinkish color. If this disease can be made to disappear without scar formation, it accomplishes at once what no other method of treatment can hope for. If one avoids scar formations the tendency to recurrence is correspondingly lessened, because there is more tendency for epitheliomatous formations in scar tissue than in healthy tissue.

(4) If the cases are treated early and skillfully, one may hope for recovery in at least 98 per cent. I believe no other method of treatment can claim nearly so much.

(5) Epitheliomata commonly affects areas such as those about the inner canthus of the eye or the nose, where there is little tissue to be spared without producing marked deformity and much annoyance. The destructive methods of treatment in these regions, even if successful, produce scars which contract and cause ectropion, with the train of eye symptoms that naturally follow. By means of the rays I have treated patients upon whom caustic had been previously applied, and in which there had been scar formation and a recurrence. I have succeeded not only in eliminating the disease, but even the scar, which had formed and drawn the eyelid downward, was softened and relaxed, so that the ectropion practically disappeared.

(6) Even in advanced cases, in which further destructive treatment is out of the question, one can often destroy the diseases, and yet retain all the healthy tissue. It must be understood, however, that the deeper and the more advanced the disease is at the beginning, the less chance there is for complete recovery.

(7) In very advanced cases, in which one cannot reasonably hope for recovery, one may still retard the disease, prolong life, lessen discharge, diminish the odor, relieve pain, and make the remaining days of the patient more comfortable and more hopeful.

'The disadvantage that might be considered in connection with this form of treatment is the rather long time required to produce results I usually count on from two to four months for complete disappearance, and about six to twelve treatments in early cases. In the late stages the duration, the amount of treatment, and the outcome will depend upon the extent of the disease and the natural resistance of the patient. If the entire treatment is given within a few days, the results are obtained sooner, but I think less certainly.

Epithelioma of the mucous membrane is much less favorably influenced by this form of treatment. 'This statement will, however, apply to every form of treatment. 'The tendency for recurrence and metastasis 
is much greater. Success has been attained even in this location, but, I believe, the rays should not be depended upon alone to effect a cure.

Epithelioma of the mouth and lip may be taken as examples of involvement of the mucous,membrane, and the statements made can be applied to the same disease affecting the nose, vagina, and rectum.

Unless treated very early, the disease should first be removed by excision, fulguration, or by means of the thermocautery. 'The patient should then receive active $x$-rays treatment over the wound and the associated glandular area. By each of these methods of preliminary $x$-ray-treatment I have seen excellent results. One patient, who had an ulcerating epithelioma involving nearly the entire lower lip, with glandular cnlargement and cachexia, recovered after excision and $x$-rays treatment, and has remained well eight years since.

If the disease originates or is connined to the cutaneous half of the mucous membrane, the prognosis from $x$-rays treatment is distinctly more favorable. If it originates in the buccal mucous membrane, I believe $x$-ray-treatment should not be applied primarily. Early lesions of this kind ean be best destroyed, in my experience, by fulguration.

Rodent Ulcer.-This disease, which is so similar to epithelioma, may be considered in conjunction with it. It was the results obtained in the treatment of rodent ulcer by the $x$-rays that first attracted serious attention to radiotherapy. Generally speaking, I believe the results obtained are not quite so good as in epithelioma, but superior to any other forms of treatment. The reason for not obtaining as good results is probably explained by the fact that these cases tend to come late, after mucous membrane has become involved.

Technique.-In the treatment of epithelioma and rodent ulcer the most brilliant results are obtained, but much will depend upon the skill and judgment of the operator in adapting the technique to each particular case.

In the early cases, in which there is a small warty formation which has just begun to be annoying, and perhaps bleed a little, but which has not been treated by other means (attempts at destruction by caustics or pastes), as in the crust formations with sluggish ulcers at their bases, I use the following technique:

I first cut a hole in a piece of lead about $\frac{1}{64}$ inch thick, which exposes the epithelioma, and about $\frac{1}{8}$ inch of healthy skin on the outside, protecting all other parts of the patient. I then place the anode about 6 inches from the skin, and use rays corresponding to Benoist No. 5. I usually allow 1 milliampere of current to pass through the tube. The duration will vary with each case.

If the patient lives out of the city, I usually give in one dose, or in several treatments on successive days, what I believe to be a destructive dose, equaling 10 Holzknecht or about 20 Kienböck units. The patient is then allowed to go home, and return for inspection and possibly further treatment at the end of a month As a rule, in about two weeks the lesion becomes violently inflamed, followed by crust formation. 
A small amount of serum or pus collects beneath this crust. 'The crust should then be removed by applying a thick layer of vaseline or cold cream on lint over night. A new crust will form, and this will be repeated two or more times. Each time, however, it will be found that the skin has grown more nearly over the ulcer. At the end of a month, if all goes well, the ulcer will be closed over, and will he covered by scales or a superficial crust. One should then give about half the previous dose, which will usually suffice to bring about a cure. A physician should decide when the patient is well and not the patient. If, at the end of this first month, or ats a result of the first series of treatments, the ulcer is found to be healed and simply covered by a red sear, no more treatment should be given. The red sear will fate in about a month more, and will resemble very closely the skin itself. In fact, the lesions often disappear without leaving any scar.

If the patient lives in the city, or at the place where the treatment is given, I think a safer method is to give about 10 Holzknecht units during the first week, and then see the patient once a week afterward. In this way one can better quiet the fears of the patient and watch the progress more closely. Generally in early eases I count on from six to twelve applications of the rays and count on about three months for recovery.

If the disease has become more extensive, all of the above statements must be modified. In all cases the healthy tissues must be protected. If still superficial, very much the same technique must be used. If it has involved the deeper tissues, more penetrating rays must be used, at a greater distance, and more frequently. One must aim to produce a corresponding effect at the deepest point of the disease. When the deeper tissues are involved, it is advisable to treat the associated glandular area by the technique which will be described under diseases of the lymphatic glands.

Carcinoma.-It is a very short step from epithelioma to carcinoma. Yet the prognosis and the technique differ very much. Fewer patients must be expected to recover from carcinoma, since it involves deeper tissues, and under any form of treatment it is more likely to give rise to metastases. 'That the $x$-rays will cure carcinoma in certain cases cannot be disputed, except in so far as any cure can be disputed.

Primary Carcinoma of the Breast.-Comparatively few primary cases of careinoma of the breast have been treated. Most of these have either been too far advanced to permit an operation, or the feebleness of the patient precluded its consideration. Results obtained under such conditions cannot be fairly compared with those obtained by operative procedures. I believe that operable eases of earcinoma of the breast should be operated upon as soon as the disease is discovered and diagnosed, and this should be followed by a course of $x$-rays treatment, having in mind the destruction of any malignant cells that may have been left behind or been transplanted during the operation.

While this is the rule, there are a certain number of cases that cannot be operated upon because of advanced age, or beeause of the conditions of the heart, or some other chronic disease. 'These patients ean be made 
more comfortable, their lives can be prolonged, and at times a cure can be produced. I recall one old lady who has remained well over five years, who had a typical schirrus, but who could not be operated upon on account of disease of the heart. Another old lady of the same type lived comfortably for five years, and then developed carcinoma of the uterus and died of this disease. In a number of cases in which the disease was advanced $\mathrm{I}$ have seen marked reductions in the area of disease, odors and discharges ceased, pains were relieved, the patients were enabled to go about the usual affairs of life, and I am sure their lives were prolonged one or more years.

In one woman the disease involved the entire mammary gland, and presented a nodular mass, extending up to the axilla, with enlarged, hard, supraclavicular glands. 'The whole mass was firmly fixed, and some of the nodules seemed to be on the point of ulceration-clearly an inoperable case. I kept her under treatment and observation for a year. At the end of this time nothing was left of the disease but a mass of scar tissue, about an inch in diameter, which was freely movable, not in any way tender, and in every way seemed to be scar tissue. I advised the removal of this surgically, which was done. She has been well over a year since this operation. In this instance a clear inoperable case was transformed into an operable one. Therefore, another hope in advanced cases may be the transformation of inoperable into operable cases. This case is a strong argument in favor of anteoperative treatment.

Technique in the Treatment of Primary Inoperable Carcinoma of the Breast.-I must here refer to my general statements concerning the technique of the treatment of deep-seated disease in the early part of this chapter. A liberal admixture of good judgment and skill with those principles will go far toward producing good results.

'The entire area of the gland and the associated lymphatics should be treated. The other parts of the body should be protected. The disease should be attacked from every possible side and direction, always aiming toward the centre. In this way, each area of the skin will only receive a fraction of the total dose, and, as a result, a much larger dose can be conveyed to the interior without burning the skin. The treatment shonld not be carried beyond the production of a slight erythema. I usually try to reach this point, however, thus producing more certain and quicker results.

The distance of the rays should be from 12 to 15 inches from the skin. This diminishes the quantity of rays reaching the part considerably, as compared with closer treatment, and the treatment must be correspondingly longer in duration. I commonly give from 15 to 30 milliampere minutes in such cases, or its equivalent. One must give sufficient treatment to produce results or the energy is wasted, and yet burns must be avoided.

The quality of rays in this work must be most penetrative, so that the deep effect will approach more nearly that produced on the surface. I commonly use rays of the value of No. 6 to 8 Benoist. One must be 
most eareful to keep this value constant. This can be partially gauged by watching the milliamperemeter, which will register higher (with the same amount of primary current) as the rays become softer. It, therefore, should remain stationary. One can also form an idea by measuring the parallel spark gap frequently. Generally, but not invariably, the parallel spark will increase as the tube gets harder and will decrease as it gets softer. 'The actual scale should be used frequently' until the operator has become familiar with each tube and its variations. Careful attention to these details will make the difference between success and failure.

One should have a number of tubes, so that they can be allowed to cool off between treatments, and there should be a series for each class of treatments. No tube can be used continuously for all classes of work. When a tube becomes hot, it is not likely to produce a uniform or satisfactory quality of rays.

Frequency of Treatments.-Generally, I treat these patients three times a week, but if the tumor has developed rapidly, it may be advisable to treat the patient every day. When daily treatments are given, one must be very cautious in not producing a burn. On the other hand, if the tumor has developed slowly, or if the patient is feeble and the exertion of coming for treatments is exhausting, I often only see such patients once or twice a week. The number of treatments necessary will, of course, vary in each ease with the extent of the disease, the rapidity of growth, and the resistance of the patient.

Results to be Expected.-Generally no change will be noted for at least two weeks. Within a month, however, the area of disease becomes softer and gradually grows smaller. We must seldom expect the breast to be restored to a normal condition. There forms what appears to be scar tissue, which is freely movable, not tender, and not painful.

As stated at the beginning of this subject, this method of treatment is not generally recommended as the one of choice, but either because an operation is attended with too much risk, or because the patient absolutely refuses operation.

Ante-operative treatment of carcinoma of the breast deserves some consideration. If the disease is recognized at a stage when it can be removed completely, I believe that it is best to operate without delay, for any cause, yet even in such cases I have seen prompt recurrences, and a second operation seemed only to aggravate the condition. 'Therefore we need not condemn, at least, a suggestion of ante-operative treatment.

In the more advanced cases, where there is axillary and perhaps supraclavicular involvement, I believe that a course of treatment extending over a period of two weeks to a month preceding the operation can accomplish much. It should be applied over the glandular regions especially, with the object of destroying these glands, and especially the smaller ones, that may escape recognition at the operation.

'That ante-operative treatment will at times accomplish much is well illustrated by the case referred to at the beginning of this subject, where an inoperable case was made operable. 
Postoperative Treatment of Carcinoma of the Breast.-This line of action is generally endorsed. It is based upon the theory that, at the operation, a few outlying cells or nodules may have escaped the scrutiny of the surgeon, or a few cells may have escaped and been transplanted in the wound, thus leading to a recurrence. One aims to destroy such cells or nodules. The Roentgenologist, therefore, begins the postoperative treatment with the impression that there may still be disease present in the tissues. A line of treatment and sufficient dose must be given that would destroy the disease, if known to be present. Unless this line of action, based upon such theory, is accepted, I can see no reason for postoperative treatment.

A surgeon who condemns postoperative treatment because he has seen no results after having ordered four or five different applications of the Roentgen rays as postoperative treatment of his cases, is exactly in the same position as a person who condemns surgery because poor results are obtained after an incomplete operation by an indifferent surgeon.

The postoperative treatments should be given in the same general way as in the treatment of primary carcinoma. They should begin as soon as the patient can go for the treatment. They may be given through the dressings. As much or more treatment should be applied to the axillary and supraclavicular regions as over the line of the incision. I usually count on giving the patient 15 to 20 treatments. These are given three times a week or daily. I aim at producing a very slight erythema, but go no further. When daily treatments are given, the dose must be very carefully estimated, or one will give too little or too much.

It is difficult to estimate exactly the statistical value of postoperative treatment, because, as a rule, only the more malignant and more extensive cases are referred.

Recurrent and Metastatic Carcinoma Following Breast Operations. -In the treatment of recurrent and metastatic carcinoma following breast operations, I believe that Roentgen therapy stands first and almost alone. Early recurrences in the scar yield almost marvelously at times. Even those cases with an extensive subcutaneous infiltration, will often show a softening and a relief from pain and tenderness; but very rarely in these brawny recurrences can we expect the evidence of disease to disappear completely, and after a time it will develop rapidly. In those cases in which there are isolated nodules which have developed slowly, several months or longer after the operation, we may expect more favorable results, and usually a disappearance of the disease. In such cases one commonly finds one or more hard nodules in the supraclavicular region. In all of these cases the supraclavicular regions must be thoroughly treated.

These recurrent nodules usually disappear, but at times they are only reduced in size, become freely movable, and then remain stationary even with continued treatment. In such instances I usually advise excision, followed by a half-dozen postoperative treatments, in which the full skin dose is given, but not exceeded. 
Even when there have been axillary, supraclavicular, and puluonary metastases one need not despair. However, in such cases one can only hope for a retardation in the disease, with the production of at enicesent stange, which may continue for a mumber of years. I have had one case in which there was a local recurrence and metastases in the supraclavicular region sufficient to canse a palsy of the left arm, with medliastinal involvement, which was demonstrated both by physical signs and radiograph, and in which life has been prolonged, with a reasonahle degree of comfort, over a period of at least seven years.

'The recurrence and the supraclavicular swelling disappeared, but the palsy has remained. A radiograph shows in the upper portion of the left lung many isolated nodules, which are quite dense, and which I believe were, and perhaps are now, carcinomatous, but which have become encapsulated.

'The technique to be used in such cases is similar to that described for the primary disease. 'These patients must continue under observation. 'The longer they remain well or free from symptoms, the greater may be the interval between visits and treatments. One must also guard against producing a chronic radiodermatitis.

Carcinoma involving other parts of the surface of the body must be judged and treated very much along the same lines, as has been described under Carcinoma of the Breast. In most instances the disease will be found to have followed epitheliomas.

Carcinoma of the larynx has been reported cured by Roentgen therapy, but one cannot hope for this in many instances. 'The disease, however, will often diminish, the patient will have less pain, discharge will cease or be diminished, life will be prolonged, and the patient made more comfortable. The general principles governing deep treatment must be followed. 'The disease must be attacked from as many different points as is possible. It is in the treatment of cavities that radium finds its greatest field of usefulness.

Carcinoma of the accessory sinuses is usually secondary to the disease in the mouth or nasal cavities, and when this disease has infiltrated the maxillary or ethmoidal sinuses, one must expect little from Roentgen therapy or radio-active substances.

Carcinoma of the œsophagus, stomach, and bowels I believe may be retarded; pain is relieved, and the patient gets some general physical and mental relief, but I know of no genuine cure of carcinoma in any of these localities. In 2 cases of osophageal carcinoma I got so much improvement that I had hopes of a cure. The patients were practically free from symptoms for nearly a year. They swallowed their food almost normally, and there was improvement in the general health, but finally the disease grew rapidly and ended fatally.

The same remarks apply to carcinoma of the stomach. In only 1 case out of 15 did I see deeided improvement, and this seemed to be a symptomatic cure. 'The surgeon did an exploratory operation previous to Roentgen treatment, and found a large carcinoma involving the lesser curvature. After six weeks of treatment, three times a week, the par- 
tient was able to leave the hospital and take an hour's ride on the train. Later he came to the city three times a week for treatment. In six months he was able to eat most things, and was able to attend to some business.

At this time he developed some symptoms of appendicitis. The same surgeon was called upon to operate. He did not find appendicitis, and did not find the tumor which he had previously felt. He accounted for the pains by adhesions which were formed. In three weeks the patient died, but no autopsy was performed.

Sarcoma.- The treatment of sarcoma by means of the Roentgen rays has been generally more satisfactory than the treatment of carcinoma. Most of the patients that have come under my care have had inoperable sarcomas, or were suffering from rapid recurrences, yet $I$ have obtained recoveries in about 50 per cent., based upon an experience with 50 cases affecting different parts of the body.

Comparison with Other Methods.-Statistics so often depend for the final showing upon the kind of cases that have been excluded or included, that a review of the details of a large number of cases will give a truer valuation of a method than any simple numerical comparison. The above statements are based upon the results of 35 cases that had been treated previous to August, 1908. My observations upon the treatment of this disease have extended over a period of eight years. Of these 35 cases treated and reported two years ago, 15 have recovered and remained well from two to seven years: 4 others improved, and then remained stationary from two to six years. Practically all cases showed some improvement, but 16 out of the 35 cases have died. Some of these seemed to be recovering, when, without apparent cause, they progressively grew worse.

Most of these cases were either inoperable or rapid recurrences following operation. 'Therefore, operations in this class cannot be compared. 'The only treatment that can be compared in such cases is the injections of Coley's toxin, for it is in such cases that the toxins are also used.

Coley only claims 10 per cent. of recoveries from the injection of the toxins. The prostration, chills, fever, and constitutional symptoms that accompany or follow the injections of the toxins make this a less desirable treatment, even if the number of recoveries were the same.

Fortunately, the three methods of treating sarcoma may be used conjointly or in succession. An operable case is probably best operated upon. This should be followed immediately by a series of postoperative treatments. Indifferent postoperative treatment will accomplish little.

As stated under the subject of carcinoma, these postoperative treatments must be given with the assumption that discase may still be present. A striking example of the variation in results according to the radiotherapeutic technique used is shown in the illustrations of the sarcoma of the forearm (Plate XXII). 'This patient had a sarcoma excised from the right forearm, which recurred in three months, and was again excised by the same surgeon, who then gave 32 postoperative treatments 


\section{PLATE XXII}

FIG. I

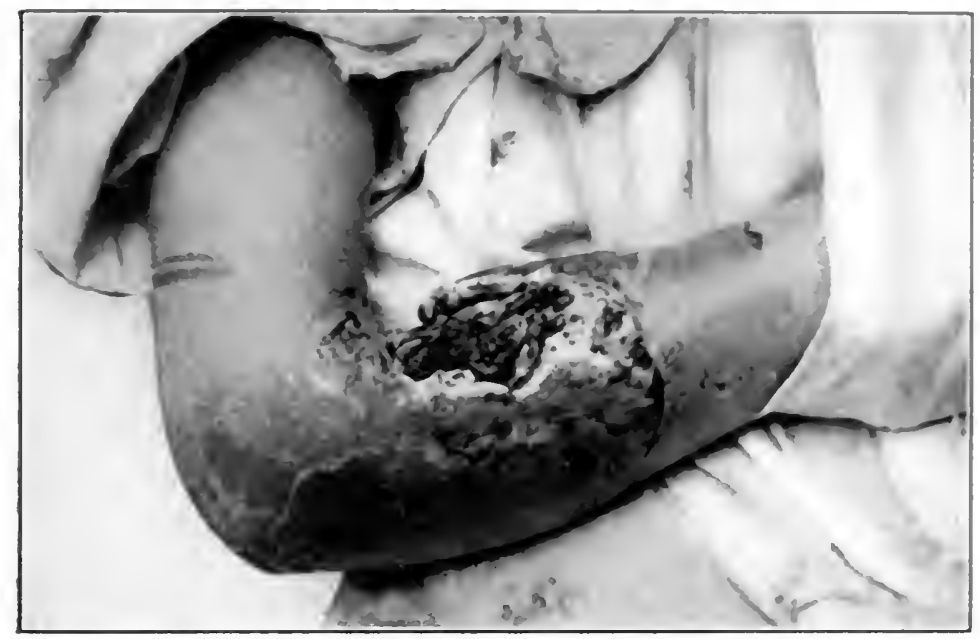

Sarcoma of the forearm, two weeks after the third operation, showing a very malignant recurrence. November 6, 1905.

FIC. 2

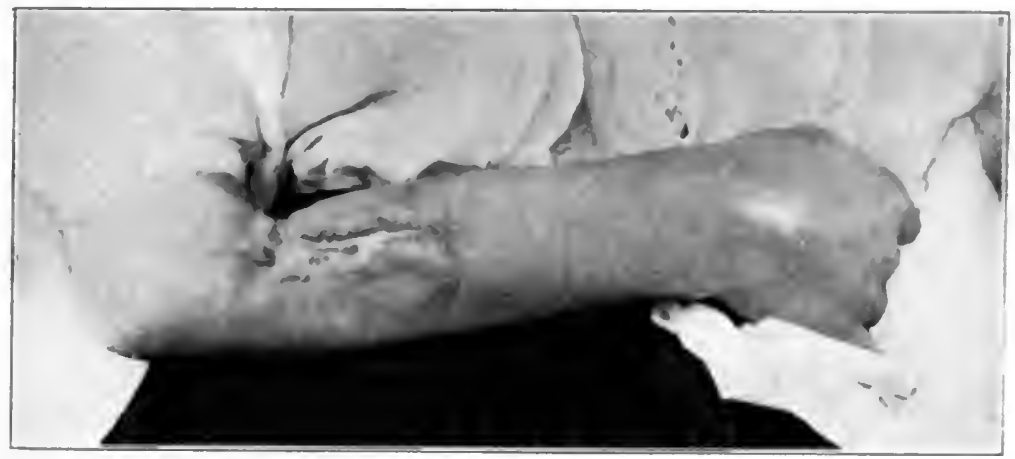

Same as Fig. 1, after 32 x-ray treatments. Photographed March 11. 1907. The patient has remained well over three years since. 



\section{PLATE XXIII}

FIC. I

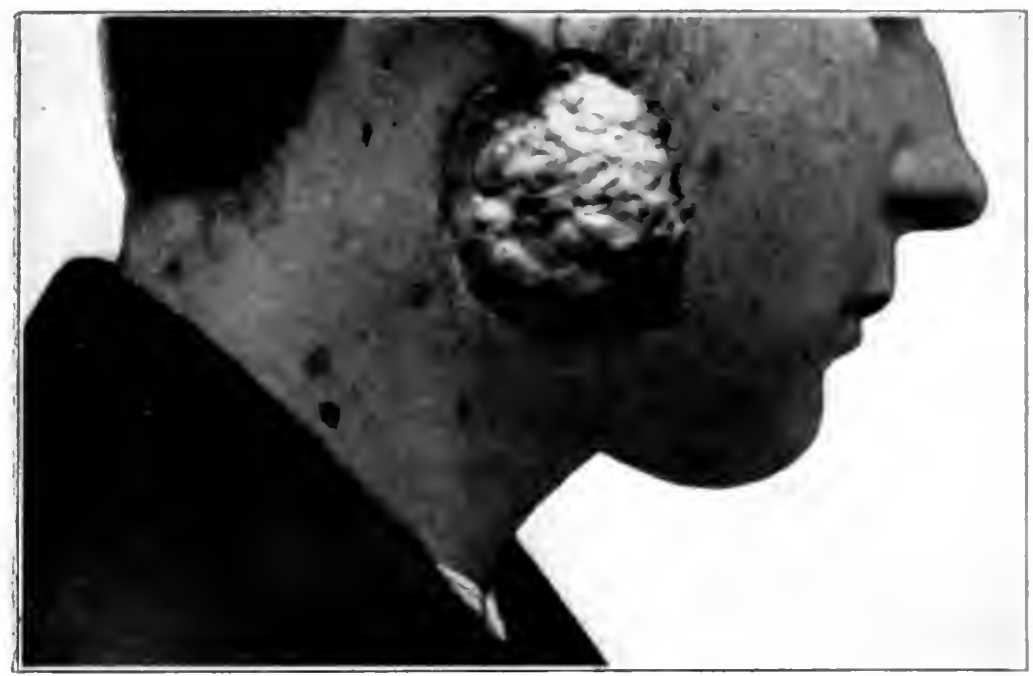

Sarcoma photographed on the fifth day after the second operation. The primary tumor had developed to the size of an apple in four weeks. It was excised, and then recurred to the original size in three weeks. In five days after the second operation it had recurred to the size of half a hen's egg.

FIG. 2

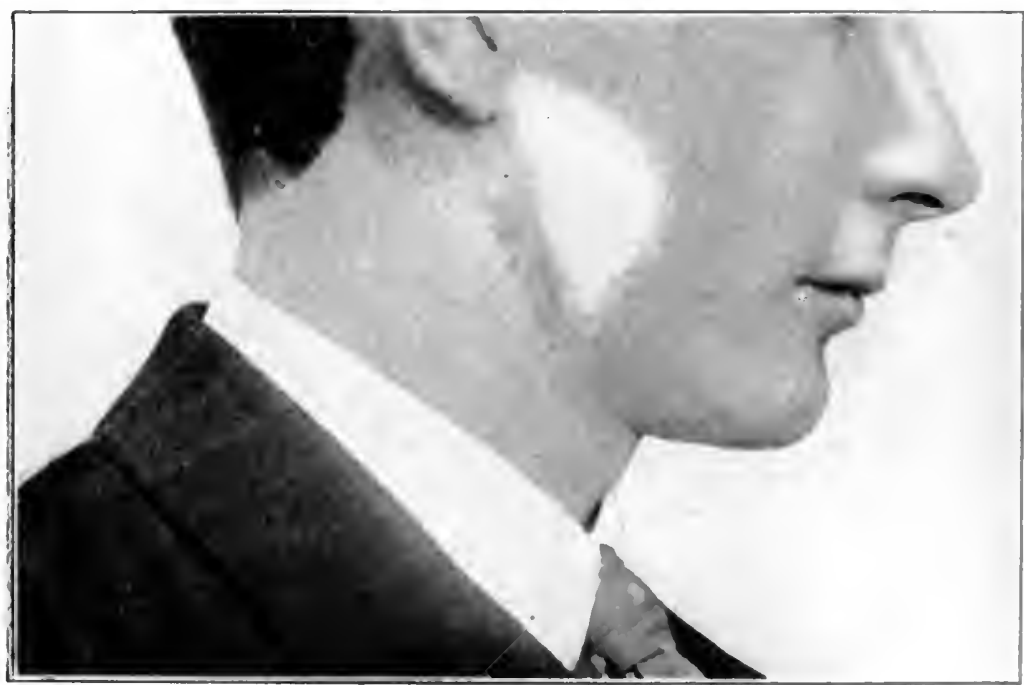

Shows complete disappearance of the tumor following nineteen applications of the $x$-rays. He has remained perfectly well over three years since. 

with the Roentgen rays, but recurrence took place under treatment. A scapulohumeral amputation was then advised, but the paticnt would not consent. Dr. W. W. Babcock was consulted, and carefully excised the tumor. 'Iwo weeks after this third operation there was a recurrence, with induration of the entire forearm. At this late stage, and with a pronounced malignancy, I began treatment, and gave 32 treatments with the Roentgen rays, which resulted in complete recovery, and which has lasted over three years. 'The patient has been able to carry on her general housework, and has been entirely comfortable.

In this group of cases, previously reported, there were 4 cases of osteosarcoma in which good results were obtained. 'To these I might atd 2 more. 'Three others involved the ethmoid cells, and good resilts were obtained. 'This would indicate that not simply superficial lesions are favorably influenced by the rays.

A careful analysis, therefore, of cases of sarcoma, treated by this and other methods, will convince any one of the value of this treatment. When one sees repeated recurrences follow operations, and then sees the growth gradually melt away, and the patient remain well for three or more years, one cannot help but feel that this treatment deserves serious consideration.

Recurrence.-In addition to the cases that have recovered, of those that have been reported, 4 others had been free from symptoms for a year or more, and then had a recurrence. One of these was a round-cell sarcoma, involving the orbit and ethmoid cells. After being apparently well for one year she had a slight operation upon the nose, followed by recurrent sarcoma which proved rapidly fatal. Another round-cell sarcoma of the lower jaw had a recurrence after the extraction of a tooth, ending fatally. A third case, one of sarcoma of the leg, had recurrences which have yielded to the rays. A fourth patient, with sarcoma of the groin, which appeared to be well for two years, had a recurrence and died.

It has been shown repeatedly that traumatism is an exciting factor in the etiology of sarcoma, so it is an exciting factor in the recurrence, and, therefore, operations in a sarcomatous area after apparent recovery should be undertaken only after due deliberation. 'The recurrences have been remarkably few considering the gravity of the cases treated.

Types.-More observations will be needed before one can draw definite conclusions from the classification of types treated, but, from my experience, I believe that the small round-cell sarcoma is most easily influenced by the rays, that the spindle-cell variety is most difficult, and that the mixed variety stands in an intermediate position. 'Three cases of melanotic sarcoma were treated with success. 'This latter type is unusually formidable to other methods.

Technique.-The mere possession of an $x$-ray apparatus is of the same general and comparative value as is the possession of a set of surgical instruments, and the sooner the profession realizes that no technique is more varied, more complicated, or more difficult to aequire, the sooner will we obtain uniformly better results, and more will be accomplished for the patients. 
In the description of Roentgen therapy much difficulty is encountered, because of the lack of accurate means of measuring the dosage. This is particularly true in the treatment of deep-seated lesions, to which sarcomata usually belong. Here we have not only the danger of a dermatitis, but the danger of a toxemia, when large growths are made to degenerate and toxins therefrom are absorbed. For this reason much of the result will depend upon the skill and experience of the Roentgenologist, not only in the application of the rays, but also in the close study of the constitutional condition of the patient.

In general, we have four fairly definite factors to consider, which will enable one to repeat a like dose and probably produce like results:

(1) Distanee of the Anode from the Skin.- This will vary with the depth of the tissue involved. In the treatment of dcep-seated lesions this should be about 10 to 12 inches.

(2) Time and Direction.- - This, too, will vary, but, in general, should be from ten to thirty minutes or its equivalent (10 to 30 milliampere minutes, which means 1 milliampere for ten to thirty minutes). Most of my patients receive 20 milliampere minutes at each exposure. 'The treatment should not be given continually from one direction, but each successive treatment should be given from a different direction, so far as is possible. The neglect of this point is the cause of many failures. If the treatment is given from all directions, all the malignant cells are more likely to be destroyed, and there will likely be produced a fibrous capsule, which will encase the malignant cells that remain. In addition to these advantages, and not to be considered lightly, there is less likelihood of a dermatitis.

(3) Vacuum or Quality of the Rays. - So far we are only able to estimate the penetrating quality of the rays. This is best done by a Benoist, Walter, or Wehnelt scale. The rays, measured by the Benoist scale, Nos. 6 to 8 , will give the best results in the treatment of sarcoma. An old, hard tube will be found most useful in the treatment of sarcoma. This is true because there is less likelihood of the old tube decreasing in vacuum. Frequent tests should be made to be sure the rays are uniformly penetrating.

(4) Amount of Current Passing through the Tube.-This is measured by means of a milliamperemeter placed in series with the tube. Measured by this means, I find it difficult to use a coil current of more than 1 milliampere, and keep the current uniform. One may use greater currents for shorter periods of time, changing the tube as soon as there is a change in vacuum. At present I commonly use 4 milliamperes from a static machine for a period of two minutes; using three or four different tubes. 'This is the equivalent of at least 30 milliampere minutes.

Kienböek Quantimeter.-Measured by this means, I usually give a skin dose (beneath the leather filter) of about 2 Kienböck units at each sitting. By varying my direction, and measuring everything carefully, I can usually give much deep treatment without affecting the skin.

Protection of the Skin.-'The skin can be protected by means of a filter, which I described five years ago, consisting of sole-leather inter- 
posed between the tube and the skin. In experiments upon rabbits I was able to protect the skin from any visible effect, under the lat ther, while a neighboring portion of the skin, exposed to the same rays, showed a characteristic Roentgen ulcer. It is used on the principle of sclective absorption of the rays. I place the leather close to the tube, in the protective tube case, so that at no time does it come in contact with the patient and thus become soiled. Aluminum is also used for the same purpose. It is possible to burn a patient even through this filter, but a much larger dose can be given to the deep tissues by this technique than otherwise without damage to the skin. The variation in the direction of the rays will also add to the protection. I ikewise, the greater the distance of the tube from the skin, and the higher the vacuum, the less the likelihood of a dermatitis.

Postoperative Treatment.-'The value of postoperative IRentgen treatment is being more and more recognized. I bclieve that it is especially valuable in the treatment of sarcoma, because of the tendency to rapid recurrence, and because any remaining cells are likely to be destroyed or encapsulated. It is especially desirable when combined with "subcapsular enucleation," as strongly recommended by Babcock. He recommends simple enucleation, and excision or resection of the discased area in long bones. His objections to major operations for Sarcona are: (1) The immediate mortality; (2) the mutilation, incapacity, and mental anxiety produced; (3) the fact that the most extensive operation yet devised gives no assurance against future disease, recurrence being the rule, and persistent cure the exception; (4) the operative trauma seems often to determine the point of recidivity.

The treatment should be given as soon after the operation as the patient is able to be taken to the laboratory. On the other hand, I have never seen any advantage in forcibly keeping the wound open for the purpose of treating through it. By this means one simply adds and repeats traumatism. The danger to be feared is an extension into the surrounding tissues, and this can be best prevented by general treatment of the involved area according to the technique given above. The wound should receive the same careful surgical attention that it would receive independently.

The treatment may be given daily, but in total should not exceed the normal full skin dose. 'This skin dose is not so easily reached when given through the dressings, because of the absorption of many of the softer rays by the dressings. 'The number of treatments necessary will vary with each case, but a fair average would be about twenty.

Finally, good technique and careful attention to details are as essential to good results in this work as in any other special field.

Leukemia.-Myelogenous Leukemia.-In about 90 per cent. of the cases of leukemia that have been treated by the Roentgen rays improvement was noted, and in many a symptomatic recovery was obtained. The improvement consisted of an increase in strength, improvement in all the subjective and objective symptoms, a decrease or almost complete disappearance of the swelling of the spleen, and a decided approach to the normal of the blood-content, both the erythrocytes and leukocytes. 
These results are obtained even in advanced cases, but, so far as I know, there has been no case in which the results have been permanent. With interruptions of the treatment there is often a recurrence of the sumptoms after several weeks. 'These symptoms usually disappear with renewal of the treatment. In some cases, however, the recurrent symptoms show no further effect from the exposure to the rays, and even with prolonged treatment the patients gradually lose weight and strength, the spleen and liver increase in size, the leukocytes increase, and the prostration becomes progressive.

However, some patients have lived for two or three years with the treatment.

As a whole, one can only expect life to be made more comfortable and prolonged by the treatment and not an actual cure.

Lymphatic Leukemia.-Here, too, there is often an improvement in the general health and comfort of the patient, but the blood-picture and the size of the glands are not greatly influenced. Life can be prolonged, and the patient rendered more comfortable, but a complete cure need not be expected. A marked anemia in this affection renders the prognosis less favorable, while in the myelogenous variety the blood-picture is of little prognostic value.

Technique.-In general, the technique that has been recommended for deep-seated therapy may be used here. Pancoast has recommended that the exposures be made over the long bones, but Kienböck sees no advantage in this, and recommends that the spleen be treated. This latter is in accord with my experience. Treatments over the spleen give more prompt and more positive results. In the lymphatic variety the spleen and all enlarged glands should be treated. The chest and abdomen must not be forgotten.

Diseases of the Salivary and Lymphatic Glands.-Tuberculous adenitis is a very common affection, and, while surgical interference is necessary at times, the majority of cases, if treated early, will get well without surgical intervention. 'The ugly scars which we see upon the neck, and the involvement of the deeper glands after operation, indicate that surgery falls far short of the results for which we should aim. If we have at our command an agent that will cause these glands to disappear, without the production of scars, it is our duty to use it.

In my experience, the earlier the treatment is used, the more prompt and satisfactory will be the results. If the glands have already undergone suppuration, of course, the rational method is to incise the gland and drain it, since we all know that an incision will leave a better scar than if allowed to rupture spontaneously. I believe, however, that only the softened gland should be incised and curetted, and that the other glands should be subjected to $x$-rays treatment. During the process of suppuration nature has already established a wall, which, if not disturbed, will prevent dissemination or secondary involvement. By Roentgentherapy, then, we lessen the danger of dissemination and obtain better cosmetic results.

Operations are dreaded by every one, and patients will postpone 
operation until the disease has advanced to a marked degrce. A large number of glands are likely to have become involved, and sorne of these have gone on to suppuration. If a less dreaded methol of treatment can be offered, these patients will seek the aid of the physicians earlier, and better results can be obtained.

In nearly every case of tuberculous adenitis that has come to my attention there was some evidence of pulmonary involvement. "Ihis is not usually to the extent that a positive diagnosis can be made, and of ten no cough is complained of, but the expiration is prolonged at one or both of the apices, and fine crackling rales may be heard at the end of inspiration.

At times the resonance is slightly impaired. For this reason the rays should not be confined to the glandular area, but should be allowed to play secondarily upon the apices of the lungs. Likewise, all the measures that are to-day recognized to be of value in combating tuberculosis should be adeled.

Results to be Looked For.-Usually there is no change to be seen for two or three weeks after the beginning of treatment. 'Then there is a gradual reduction in size, each gland becomes more movable, and gradually disappears. Occasionally in early cases, that have only lasted a few weeks, there is a distinct reduction within the first few days after the first treatment. Such cases recover more rapidly. If at the beginning of treatment a gland is already red and tender, the signs of suppuration will be hastened by exposure to the rays. If suppuration occurs, it should be treated just like an abscess, and a scar $\frac{1}{4}$ inch long will be the result instead of one of 4 inches.

Technique.-In this affection I believe that divided rather than massive doses are indicated. The results are due probably to an increased phagocytic action. It has been shown by Crane that under $x$-rays treatment the opsonic index is raised. 'Therefore, I usually treat these patients two or three times a week. A leather filter is interposed between the tube and the glands for the protection of the skin. The appearance of any redness of the skin will be an indication for cessation of treatment for a week or two. It usually requires from 12 to 20 treatments to get a patient well. 'This will vary, of course, with the extent of the lesions, their duration, and the natural resistance of the patient.

'The anode should be 10 to 12 inches from the skin, and about 15 milliampere minutes given at each treatment to the affected areas, with rays corresponding to No. 6 Benoist.

Hodgkin's Disease (General Lymphadenoma or Pseudoleukemia).'This disease is treated on the general principle that the rays will cause a reduction in glandular hyperplasia. The success in the treatment of this affection may at times seem brilliant. There is usually a reduction in the size of the glands, and at times a complete disappearance. 'The patient may remain well for several years, but sooner or later one must expect a recurrence. In this affection, too, the more recent the development, the more rapid will be the disappearance. In the older cases, in 
which the tumors have become very hard and fibrous, I have been able to obtain some reduction, but not a disappearance. While the results are not all that might be hoped for, they are probably better than is obtained by any other method of treatment.

The technique is similar to that which has been recommended in tuberculous adenitis, except that, on account of the usual extensive inrolvement, one must vary the area of treatment on successive days, and so far as possible vary the direction of the treatment successively on each area.

Chronic Parotitis (Mikulicz's Disease).-Only about 6 of these cases have been treated so far. I have had 2 under my care. There is a persistent enlargement of both parotid glands, which may give some pain and may be slightly tender. Other forms of treatment have accomplished little. The Roentgen rays were used on the theory that they reduce hyperplasia of glandular tissue in general. Haenish was the first to treat the disease with the rays, and obtained an apparent cure in 2 cases after a few treatments. In my 2 cases the swelling completely disappeared after about a dozen treatments in three months. One of these has remained well about two years. In both of these cases a diminution in the salivary secretion was noted, otherwise no unfavorable symptoms.

The treatments are given once a week, with the anode at a distance of 12 inches, the skin protected by a filter, the rays confined to the gland, and about 15 milliampere minutes given to each side at each treatment, with rays corresponding to No. 6 Benoist.

Exophthalmic Goitre.-There have been recorded in the literature nearly 100 cases of exophthalmic goitre treated by the Roentgen rays. Good results were obtained in about 75 per cent., with no risk to the patient and no great inconvenience. This number is still small, and one should not be hasty in drawing conclusions, but surely the results have been sufficiently good to justify further trial, since there seems to be nothing lost and a chance for much gain by the trial.

It has been observed that good results showed themselves in some instances within forty-eight hours, and, almost without exception, within a month. In most cases almost complete recovery was obtained in from three to six months. It would seem, therefore, that the trial of the Roentgen rays for a month, with from six to a dozen treatments, could. do no harm, and is likely to be followed by good results. If some improvement is not shown within a month, there should be an interruption for at least two weeks.

The treatment should be localized upon the goitre, and may be carried to the point of producing a mild dermatitis, but no more. 'The first rlose should not be excessive.

The permanency of the results is still a question, yet all the patients in which good results had been obtained had improved or remained well up to the time of the reports, which varied from a few months to three years.

'The earliest and most noticeable improvement is the increase in weight. This is followed by improvement in all the symptoms. The 
two symptoms that remain longest are usually the enlargement of the thyroid and the exophthalmus.

Technique.-I usually treat these patients three times a week, confining the rays to the enlarged gland, and attacking the enlargement from as many points as possible. 'The skin should be protected by the leather filter. 'The anode should be 12 inches from the skin, and the rays should have a penetration corresponding to No.6 Benoist. I give from 10 to 15 milliampere minutes.

Prostatic Hypertrophy.-It is but a natural step to pass from the treatment of the glandular enlargements, previously described, to that of prostatic hypertrophy. 'The frequency of this affection makes this treatment especially welcome, for there are many patients who either cannot or will not submit to a radical operation. Not a great number of cases have been treated by this method, but sufficient careful work has been done to justify its recognition as a form of treatment.

Good results have been noted in some cases within two weeks, but ordinarily the improvement is noted within a month. 'The obstructive symptoms, as well as the pain and tenderness, gradually disappear.

Technique.- The rays must be confined to the region of the prostate, and especially the scrotum and testicles must be protected. 'The patient should be placed in the knee-chest position. 'This can be done by having him rest his abdomen upon a stool, covered with a cushion. If the prostate is not too tender, a speculum may be passed into the rectum, and this should be opaque posteriorly and transparent to the rays anteriorly. 'This must be definitely centered with the tube and held firmly in position. By this means we attempt to treat directly upon the enlarged prostate. Usually this is impossible, and we must treat through the perineum. When this latter technique is used, one should employ a localizer which will confine the rays to the perineal region, and give protection to the rest of the body. Then all the principles governing deepseated Roentgen therapy should be followed. 'The skin should be protected by a filter. 'The anode of the tube should be 12 to 15 inches distant. The rays should correspond to No. 6 or 7 Benoist, and about 20 milliampere minutes treatment should be given at each seance. A dermatitis must be avoided. 'The treatments are usually given three times a week.

Chronic Bronchitis and Bronchial Asthma.-Bronchial asthma, which is so commonly associated with chronic bronchitis, is often so resistant to all forms of treatment that anything offering a reasonable hope of cure, instead of simply suppression of symptoms, must be welcomed by both the patient and physician. Shilling and Immelmann have done most work along this line, but at least a dozen authors report encouraging results.

Shilling has treated over 60 severe cases by means of the Reentgen rays. Many of them had suffered for a number of years and had resisted all other forms of treatment. Some had become habituated to the medicated cigarettes or to the "l'ucker" inhalation. His observations have extended over more than four years. He has obtained actual Vor. I-23 
cures in about 25 per cent. and relief in 50 per cent. Some cases show no relief whatever. Those cases that are associated with a chronic myocarditis, marked emphysema, or marked arteriosclerosis are least influenced by Roentgen therapy. The most favorable are the asthmas occurring in the roung.

A number of theories have been advanced to explain the relief obtained from Roentgen therapy. None are entirely satisfactory. It is claimed that the enlarged bronchial glands that are often associated with bronchial asthma are its exciting cause, and that these are reduced by the action of the rays, thereby removing the cause and bringing about relief. It is also claimed that the rays have an inhibitory action on the secretory functions of the bronchial mucous membrane.

The results are often noted early, some patients getting slight relief after the first treatment and within a few days. This has led to a claim of the results being due to mere suggestion. As a rule, the treatment must be given at intervals during several weeks or months, yet never giving a full skin dose. The patient first gets relief from the oppressed respiration and the persistent cough, with a gradual decrease in the quantity of sputum. The Curschman spirals disappear from the sputum, and the eosinophiles decrease to normal in the blood.

Technique.-The patient should be treated at first about three times a week, giving about one-fourth of a skin dose at each treatment. The rays should be allowed to enter the chest at a different point at each successive visit. For instance, the first treatment may be given through the right side of the chest anteriorly, the second through the left anterior, the third through the right lateral, the fourth through the left lateral, the fifth through the right posterior, then the left posterior. These visits may be made daily when occasion demands. As relief is obtained, the interval must be increased. Good judgment and skill will have its influence on the results here as in all others.

The focus point of the tube should be 10 to 15 inches distant. 'The rays should correspond to Nos. 6 or 7 Benoist, and 10 to 15 milliampere minutes should be given. The skin should be protected by interposing a leather filter. The rays should be confined to the chest, and it is advisable to protect the spleen and liver. 


\title{
THE REST-CURE
}

\author{
BY F. X. DERCUM, M.D.
}

Modern conception as to the nature of functional nervous diseases has done much to simplify their treatment and to put the latter upon a rational basis. It will serve our purpose best to consider briefly the indications afforded by neurasthenia, which may be taken in a sense as representative of functional nervous diseases as a group. What is true of it, is largely true of the others. In neurasthenia there is present a condition of chronic nervous exhaustion, of persistent overfatigue, and it is the nature of this condition which must for a moment claim our attention before we take up in detail the question of treatment.

The changes which take place in fatigue are closely related to those which take place as a result of the normal exercise of function. Every exercise of function, of course, presupposes a nutritive change. It is in the muscles, the nervous system, the glands, the blood, and the bloodmaking organs that this change is most evident; but changes likewise take place in such structures as the bones, though these changes are very gradual and are noted only after the lapse of years; the same thing is also true of cartilage, tendons, ligaments, and other connectivetissue structures. It is, however, the changes in the muscle and nerve substance which at present concerns us most closely, and it is especially in muscle that these changes can be most readily studied. While our knowledge of the subject leaves much to be desired, sufficient is known to indicate their general character. They may be readily grouped as chemical, morphological, and physical. It is the chemical changes that to us are most important. They may be briefly summarized as follows. During contraction a muscle absorbs more oxygen and gives off more carbonic acid than when at rest. Its juices, from being neutral or feebly alkaline, become acid, owing to the formation of sarcolactic and possibly also phosphoric acid. It loses glycogen and gains water. Its capillaries dilate, it contains more blood, and the blood in its veins differs chemically from that in its veins at rest. It evolves heat, mechanical and electrical energy. Finally, in general terms, the degree of these changes depends on the degree and duration of the contraction.

As regards nerve substance, our knowledge is much more meagre. However, when at rest, nerve substance, like muscle, is neutral or feebly alkaline, and when active it is acid. While we are still in the dark as to the detailed nature of the change, the facts justify the inference that, as in muscle, it is dependent upon oxidation. An interference with this oxidation by temporary compression of the arteries supplying the brain 
or cord means an immediate arrest of function. Again, a number of observers have noted physical changes in nerve-cells as a result of functional activity - e. g., Hodge, Vas, Nissl, Mann, Lugaro, and others. While their statements differ somewhat as to details, they agree as to the essential facts; namely, that during rest the chromatic substance in the cells increases in amount, and that during functional activity it is diminished. The nucleus and even the nucleolus participate in these changes which appear to be pronounced in proportion to the degree of the functional activity.

That waste substances are formed as a result of functional activity is, of course, well known. 'The exact facts as to the action of these substances are not in our possession. However, a valuable hint as to the role they play is afforded by the following experiment. If the gastrocnemius muscle of a frog which has been completely exhausted by electrical stimulation and refuses any longer to respond, is washed out by injecting its artery with normal salt solution, the muscle again responds to the electrical current, the responses being as before and for a time well maintained. It would seem that the presence of the waste substances prevents the electrical response; in other words, the waste substances impede or inhibit the contraction of the muscle. It is probable that the action of waste substances is everywhere the same, and that when they form in nerve substance, their action is likewise to inhibit, to interfere with, function.

Waste substances have no doubt a useful, a physiological, function under normal conditions. They induce rest and prevent the excessive exercise of function, i.e., they prevent the undue, the abnormal, consumption of tissue by inducing fatigue. Waste substances or fatigue substances, as they had best be called, thrown normally into the circulation, are probably sedative in their action and are among the direct causes of rest and sleep.

Fatigue, it would appear, is the result both of the consumption of tissue and of the formation of waste substances. The excessive exercise of function must, of course, mean an excessive consumption of tissue; this of necessity must mean the formation of waste substances in excess. Instead of exerting a normal or physiological action on the tissues, they now exert a toxic action-a fact proved by our clinical experience. Instead of acting as gentle restrainers of function, preventing an unphysiological consumption of tissue, they now appear to act as poisons and irritants. To them is probably to be ascribed the nervousness, the restlessness, the insomnia of neurasthenia. It would appear also that prolonged excess of function leads not only to an excess of fatigue substances, but also to a change in the chemical character of these substances, so that the problem becomes still further complicated.

The therapeutic indications are rendered, by the above considerations, very clear. If the symptoms are the result of overfatigue, rest is indicated; if there is loss of substance-as shown not only by physiological considerations, but also by loss of body-weight-food is indicated; if waste substances are present in excess, these must be eliminated. 
The question at once arises as to the mothod of employing rest, its degree and practical application in a given case. Rest may vary from that slight degree of rest-relative rest-which is secured by at modification or, better still, by a change of occupation, to that profound degree secured by absolute rest in bed for a prolonged period of time. Unfortunately, relative rest, although we are occasionally forced by the circumstances in which the patient finds himself to employ it, serves merely to tide over a given situation or to lessen for the time being the strain under which the patient is laboring. If we are able, on the other hand, to diminish the anount of work, we accomplish much more, for we may then be able to institute that degree of rest to which Weir Mitchell has applied the term "partial rest." Unfortunately, in a large number of cases, simple plans of treatment are ineffectual, so that, the circumstances of the patient permitting, we are forced to insist upon an absolute rest in bed. In many cases the nervous exhaustion is so grave that even a moderate exertion may be followed by a marked increase of symptoms. Frequently in such cases the bed has already been sought by the patient before the physician is called in.

If the patient and physician find themselves face to face with an absolute rest treatment, the rest should be made as nearly complete as possible and the closest possible attention given to details. As a rule, it suffices to place the patient comfortably in bed with the head resting upon a low pillow. He is instructed to lie quietly and is not permitted to sit up in bed save for the special purpose of eating his food, nor to leave his bed save to empty the bladder or bowels. Occasionally the condition of the patient is such that not even this degree of exertion can be permitted and the patient must be fed by the nurse while lying down.

Further, the rest must not only be physical, but mental as well, and in order to secure this, it does not suffice that the patient should not read or do other mental work, but it is necessary that all sources of mental and emotional excitement be excluded from the room. This means that the visits of relatives and friends shall be forbidden and that the patient shall neither write nor receive letters, $i$. $e$., the patient must be isolated. Not infrequently, especially in hysteria, the isolation is resisted by the patient, sometimes by some well-meaning but a misguided relative or friend. It is usually against just such a friend that the patient needs protection. In neurasthenia and hysteria alike the patient is apt to give herself over to dwelling upon her symptoms; and an overanxious or oversolicitous relative frequently re-enforces this nosophobia by unwitting but none the less harmful suggestions. By injudicious statements and questions, by a thoughtless display of sympathy, the patient's mind is constantly filled with ideas of illness, and to hope for progress under such circumstances is useless.

While it is imperative in many cases that the isolation be rigidly enforced and that none shall have access to the patient's room save the physician and the nurse, it occasionally happens that absolute isolation is neither feasible nor wise. Sometimes the patient has a young child concerning whose welfare the patient worries, and it may be wiser to 
permit brief visits by the child at stated periods. Sometimes, also, more especially in the case of male patients, an imperative business matter, such as the signing of a paper, demands attention, but with proper precautions, modifications and interruptions of the isolation are deprived of harm.

It oecasionally happens also that the patient's condition is such as to permit the visits at given times of some one friend or relative who has been previously instructed by the physician as to the things he or she may or may not do or talk about. It is also necessary in the case of some patients to break in upon the isolation at intervals for other reasons. The majority of patients bear isolation well, soon become attached to their nurses and used to their surroundings. On others again a too rigid isolation imposes a strain which may of itself retard progress. The physician must in individual eases use his best judgment. Other things equal, he should enforce isolation rigidly whenever practicable; he should cautiously modify it whenever this seems wise. He should also bear in mind that eases of hysteria necessitate a more rigid isolation than cases of simple and uncomplieated neurasthenia.

A properly instituted rest in bed reduces the expenditure of energy on the part of the patient to a minimum. However, we are confronted by the fact that absolute and long-continued rest in bed is not physiological. In its way it is as much open to objection as is the opposite condition of overwork, for it is not without its dangers. Just as the life of a joint depends upon the maintenance of its function, so does the integrity of other structures depend upon a persistence of their activity. Rest is an instrument powerful for both good and evil, and the problem presents itself as to how it shall be applied so that the patient may receive its benefits without suffering from any of its disadvantages. This may be accomplished by employing measures which, while they do not tax the strength of the patient, nevertheless stimulate tissue change and thus produce indirectly some of the effects of exercise. These measures consist of massage, electrical stimulation of the museles and bathing. Massage is of the utmost value in rest treatment and can never be dispensed with. It should, in the beginning, be very gentle and superficial in character and should be applied for a short time only. If deep and vigorous massage be given at first, it may greatly increase the general sense of fatigue from which the patient is suffering; this is probably due to the forcing into the eireulation of fatigue substances present in the tissues. Gentle massage soothes the patient and advantage may be taken of this fact by directing the massage to be given in the evening. At this time it greatly favors sleep and is one of the best agents for combatting the insomnia. Little by little the massage should be inereased both in depth and vigor until at about the expiration of a week or ten days full vigorous massage is given. Toward the latter part of the treatment there should be added passive movements of the limbs, these being flexed, extended, rotated and abducted from the body in various directions. Lastly, the movements should be converted into movements with resistance, the patient being instructed to gently resist the movements made by the nurse. 
Gradually the degree of the resistance should be increased. As a rule, one or two movements for each limb are sufficient in the beginning; later their number may be increased and elaborated so as to include movements of the trunk.

In many cases no other procedure is necessary than the intelligent application of massage, passive and finally resistive movements. However, electricity affords a useful adjuvant to the treatment. It should rarely, if ever, be used in the early period of the treatment; its application should be deferred until the treatment is well under way and in many cases not until the last weeks preparatory to getting the patient out of bed. As a rule, it should be applied in the form of the slowly interrupted faradic current, and each of the more important flexor and extensor groups, as well as the muscles of the trunk, should be made to contract a given number of times. 'The duration of the electrical treatment, for fear of inducing fatigue, should be limited to from twenty to forty minutes.

The third method of combatting the possible disadvantages of long-continued rest is that of bathing; the consideration of this measure is best studied in connection with the problem of elimination.

Having now considered the matter of rest and the necessary corrective procedures, let us next turn our attention to the question of food. From the facts that are presented as to the consumption of tissue in nervous exhaustion, it is quite evident that the amount of food administered must be large. Again, it must be adapted to the physiological needs of the neurasthenic, as well as to the fact that his digestion is, as a rule, enfeebled and delayed. Not infrequently also the presence of gastric catarrh still further influences the diet. 'The physical needs of the patient indicate first that he requires a mixed diet; secondly, inasmuch as the output of waste substances, more especially the alloxuric bodies, is increased, a diet including a large amount of the red meats is contraindicated; thirdly, inasmuch as the carbohydrates also, though indirectly, favor the formation of the alloxuric bodies, and inasmuch as their tissuc-building value is low, they should be given in limited quantity only.

At first the diet should consist largely of milk. In a few cases it is wise to limit the diet exclusively to milk for a few days or a week. More frequently other foods ean be permitted from the beginning, though the amount had best be small. Curiously enough, though milk is admirably adapted to the physiological needs of nervou patients, many of the latter strenuously object to it. However, in the majority of cases, the administration of milk or some of its modifications is imperative; it can rarely be dispensed with. It should at first be ordered in small quantities only", c. g., four ounces six times daily. If indigestion be very marked or if hysteria be present with its attendant vagaries of appetite, no other food for the time being should be given. In the presence of idiosyncrasies, of obstinate anorexia nervosa, we may comfort ourselves by calling to mind Pavlow's results as to the marvellous adjustment of the gastric secretions to the food that is given. Sooner or later the victory is gained. Little by little the amount of milk may be increased until eight ounces 
six times daily are given. Little by little other food can be added-bread and butter, a small quantity of rice, a cereal. Very soon a soft-boiled egg can be given at breakfast, a small piece of Salisbury steak at dinner, a little boiled rice or stewed fruit at supper. Chicken, fish, oysters, an occasional chop or steak may be permitted. Gradually the succulent regetables, spinach, squash, stewed celery, are added, and finally peas, tomatoes, onions, and the like until a full diet is reached. Potatoes should for a long time be excluded, as should also white bread in any quantity.

If it be found that the patient is unable to take solid food in quantity while she is taking milk, the amount of the solid food should be diminished in amount or restricted, and the main reliance placed upon the milk. The latter may be inereased until ten, twelve, fourteen, or even a pint six times daily is reached. However, such large amounts are only exceptionally indicated, and yet it is remarkable with what ease really enormous amounts of food can be digested and assimilated by the neurasthenic, provided the increase in the quantity has been very gradual and provided that the other details of the rest cure are being rigidly carried out.

Sometimes the milk palls upon the patient, or in spite of every effort that is made, we are obliged to suspend its administration or abandon it absolutely. Under such circumstances it is best to resort to egg feeding. Eggs are best given raw, and should be given in an increasing number daily. A raw egg is carefully opened and dropped into a cup in such a way that the yolk is not broken. The patient is then directed to swallow the egg with a single effort, and thus avoids experiencing any unpleasant taste. As a rule, it is best to administer the eggs without any attempt at flavoring. However, some patients insist upon adding a little salt, lemon-juice, or sherry. As a rule, it is wise to begin by ordering one egg between meals; then the number is increased to two, three, four or even more. Afterward raw eggs are added to each meal, the number being very cautiously increased. Sometimes it is wise for a time to abandon all solid food, and, as in the case of milk, rely on the eggs alone. Under such circumstances sixteen, eighteen, or even more eggs can readily be given daily. Now and then when egg-feeding is massive and long continued, the patient's skin assumes a distinct yellow tinge, and this exceptionally becomes so pronounced as to suggest jaundice; however, the tint is much lighter and does not invade the conjunctiva. It is a symptom of little consequence, the discoloration gradually fading when the eggs are discontinued.

Occasionally milk and egg-feeding may be advantageously combined; under such circumstances the milk and eggs should not be mixed, as in a milk-punch, but should be given separately; e.g., the milk with meals, the eggs between meals.

At times, to meet the peculiarities or idiosyncrasies of the patient, it is wise to modify the milk in various ways. 'Thus, the milk may be skimmed or a small quantity of bicarbonate of sodium, of lime-water or table-salt may be added. When there is a tendency to diarrhœea, which is rare, the milk may be boiled or "scalded." Hot milk is occasionally 
preferred by patients, and given at night, it favors sleep; at such timess also malted milk may be substitnted.

Sometimes the addition of some alkaline water, still or effervescing, such as Vichy or Seltzers, or of some simple carbonated water, such is Apollinaris or ordinary syphon soda-water makes the milk more aceeptable. Occasionally it is wise to predigest the milk, or to add the digestive powder to the cold milk just before the latter is taken. Buttermilk may also be of advantage, especially in overcoming constipation. In some cases whey proves useful, and though its nutritive value is not high, it may prove of service when milk is not tolerated. Milk and rennet prove of occasional service. Kumyss, $i$. $e$., home-made kumyss, is frequently of great value. It is readily prepared by the nurse, and is often gladly accepted by the patient. Cream I rarely make use of. Curiously, in moderate quantity, it can sometimes be given when milk is rejected. It is sometimes a useful addition to whey or may be added to a little hot water-_cambric tea."

Meat broths and soups are of little value in rest treatment. 'Their nutritive value is low and they occupy space that had best be given to more necessary food. However, there is no disadvantage in allowing the patient to have a small quantity at dinner; it makes the mid-day meal a little more acceptable to the average patient.

Sometimes, when the stomach is much disturbed, it may be necessary to resort for short periods exclusively to various meat, oyster or clam broths, or to some of the liquid beef preparations found in the market. The latter must be regarded as temporary makeshifts only; they contain, as a rule, a large amount of alcohol and a comparatively small amount of nutriment; they have value in proportion to the amount of peptones they contain.

Under rare circumstances, $e . g$. , in obstinate and protracted anorexia nervosa, we are compelled to resort to rectal feeding. For this purpose various articles may be employed. Warm peptonized milk is very serviceable. A very satisfactory material that I have employed consists in two parts by weight of finely scraped beef digested with one part by weight of finely chopped pancreas. 'The beef is thoroughly mixed with the gland, a little water is added, and the mixture warmed as in peptonizing milk. 'The quantity should be rather small, say two ounces. The rectum may be made tolerant by the previous injection of a few drops of laudanum or, better still, by giving, twenty minutes previously. a halfgrain opium suppository. Feeding with the nasal tube is often practised with success. If the stomach is very intolerant, so that even water is persistently rejected and the amount of urine becomes much diminished, it is wise to make free use of hypodermoclysis; enteroclysis may also occasionally be employed.

Full feeding having been once established, it can usually be continued for a considerable time. However, it is wise now and then to diminish the amount of food at intervals; indeed, I believe that this is quite important during the menstrual period, as during this time the patient should be neither rubbed nor bathed. Further, sameness in the diet should be 
avoided as much as possible. Now and then salted foods, e. g., minced ham, dried beef, grilled bacon, should be added to the diet. Salted wafers may be taken with the milk. Fresh fruit, $e . g$., oranges, may be given at breakfast; stewed fruits at supper. Many patients, especially those who have been ill for a long time, have adopted a very restricted diet by the time they come under the physician's care. Every effort should be made to expand this diet, and especially to prevent the patient from still further restricting it. As a rule, the object of the physician can be accomplished, provided he is not in too great a hurry to establish massive feeding; for example, the physician may anticipate the coming struggle-for it often is a struggle-by at once limiting the diet to milk and giving the latter in very small quantities a given number of times during the day. This plan usually succeeds, for the patient soon becomes quite hungry. Other food can then be sparingly added and gradually increased until the victory is complete. Even in anorexia nervosa this plan sometimes succeeds, and even if it fails, the situation is no worse than before.

'The treatment is best conducted from beginning to end without the use of stimulants. This rule applies especially to alcohol. Only under exceptional circumstances, as in the case of persons beyond middle life, who have been accustomed to its use, should it be allowed, and then only in very limited amount, $e . g$. , a glass of claret or Burgundy or a bottle of light beer at dinner, the milk being, of course, omitted from this meal.

Having considered the subject of rest, the corrective procedures, and the problem of feeding, we must next turn our attention to the question of elimination. The presence of an excess of waste substances in fatigue and their deleterious effect on function I have already discussed. These facts urgently demand our attention, the more so because of certain significant symptoms commonly noted in the neurasthenic. First, he usually presents a deficient thirst, in consequence of which he consumes too little liquid; secondly, he is usually constipated, the bowel movements being firm and rather dry; commonly his skin, and especially his mucous membranes, and even his joint surfaces, are dry; there is a deficient secretion of saliva; the amount of urine also is diminished. There can be no doubt that one of the advantages accruing from a milk diet is due to the fact that it is a liquid food; but in addition to giving liquids, and, among them, of course, water, and also to the obvious indication of keeping the bowels freely open, there is one avenue of elimination that especially demands attention; namely, the skin. This does not imply that we should make special efforts at diaphoresis or that we should subject our patient to elaborate hydrotherapeutic procedures. Indeed, such measures would inevitably add to the exhaustion of our patient and radically interfere with the rest we have established. A sponge-bath with warm water, given between blankets, answers every purpose. Gentle friction with the towel suffices, as a rule, to prevent any local depression of temperature. Sometimes, if the limbs tend to grow cold, it is a good plan for the nurse to rub them vigorously with her hands moistened with alcohol. The effect of the bath, if given successfully, is not only to stimu- 
late the action of the skin, but also the circulation, and in this way it distinctly increases tissue metabolism.

As the treatment approaches conclusion, a tub-bath may oceasionally" be given. The diminished power of resistance and feebleness of circulation in the neurasthenic forbids, especially at first, the use of the cold bath. The latter should never be employed until improvement has been firmly established; many if not the majority of cases are never tolerant of cold or very vigorous bathing.

'The progress made in a given case may be estimated by the subsidence of the fatigue symptoms and the increase in body weight. Progress, if made, is not, however, necessarily uniform; recrudescence or accentuation of various symptoms may occur from time to time; not infrequently: weeks elapse before they yield altogether. 'The length of time recpuired for a successful rest cure is upward of three months; if the case be unusually severe, it may be many more. 'The shortest time in which a really durable result can be achieved is six weeks, and this is infreguent; now and then great improvement follows even in a month, but this is very exceptional. As a rule, if the neurasthenia occurs in a young person and has not been of long duration, the period of time required is relatively short.

As the period of treatment draws to a close, it is noted that the weight is decidedly inereased, that the muscles are firm and the extremities warm, while the surface shows the normal flesh tints. At the same time a change is noted in the mental condition. After the first week or ten days of treatment, nervousness and restlessness begin gradually to subside. 'The increasing sense of physical well-being, the result of the full feeding, the quiet, and the various procedures, gives rise to a feeling of satisfaction and contentment. 'This condition is maintained for some time, but sooner or later a reaction sets in. 'The patient manifests a desire for activity. Placidity and indifference give way to spontaneity of thought and action.

Little by little the patient should be gotten out of bed. She should be permitted to sit up for a few minutes, say, five or ten-once daily, later twice daily; this time should be very gradually increased. Otherwise undue fatigue, with all its discouraging mental sequels, may be induced. Little by little the patient shonld be permitted to take limited exercise about the room, and from time to time light calisthenic movements should be instituted. By the time the patient is out of bed five or six hours out of the twenty-four, exercise in the open air by means of walking or an occasional carriage ride may be added. Later, the patient being up out of bed the greater part of the day, $i$. e., rising at 10.30 , lying down between 2 and 4, and going to bed shortly after supper. is ready for the final stage of her treatment, which had best be carried out in the country or at the seashore. As a rule, two weeks at the seashore or elsewhere are sufficient. Communication with relatives and friends need no longer be restrieted, and the patient, though still accompanied by her murse, should gradually be taught to be self-dependent. The nurse should tactfully and little by little withdraw her assistance, until the patient requires none but really necessary help in dressing, bat hing, or 
other personal matters. Before the patient returns home, she should be instructed to live conservatively and simply. One of the great benefits of a radical rest treatment is the acquisition by the patient of habits of normal physiological living. If the patient is a woman and married, her family and household afford the proper field for her interests and activities; if she be single, she should, if possible, be encouraged to take up some healthful occupation. Work is the best guarantee of mental and physical health-work within physiological limits and adapted to the physical and mental constitution and the social condition of the patient. Women have really a wide range of choice. For some there is the work of the stenographer, the librarian, the private secretary; for others there is art, literature, history, the charities, social work, the garden, business. For male patients the problem of occupation, ass a rule, solves itself; here the important thing is to prevent, if possible, the patient from drifting back into the ruts and habits which led to his illness.

Before dismissing this portion of our subject, it is necessary to say a few words as to the nurse. That each case demands a special nurse goes without saying; though now and then one nurse in control of a hospital ward occasionally does efficient work for a number of patients. In private work this is, of course, neither practicable nor desirable. The nurse must devote herself exclusively to her one patient. In the case of a male patient, the nurse must, of course, be a male nurse; it is inadmissible, for obvious reasons, both ethical and from the standpoint of efficiency, that a woman should be in charge of the rest treatment of a man.

The nurse should sleep on a cot, either in the same room with the patient or in a room immediately communicating. The introduction of the nurse into the room constitutes in itself a wholesome modification of the isolation. The nurse sleeping in the room or being within easy call at night is practically on duty all the time, with the exception of two hours in the afternoon; i.e., from 2 to 4 , during which she is to seek diversion and exercise in the open air. The period of from 2 to 4 is commonly spoken of as the "rest hour," for the patient is directed to rest quietly or, if possible, to sleep during this time. The success attending a given case depends not only upon the attention and care given by the physician to the details of treatment, but also upon the tact and good sense of the nurse. It goes without saying that the proper selection of the latter is a very important matter; other things equal, she should be chosen for her special fitness by training and education for the particular case in question. Finally, it is best that the massage, bath, and electricity be given by the nurse; the introduction of a masseur or other person into the room complicates the situation, and not infrequently upsets the patient.

The rest cure is essentially a treatment by physiological methods. It must ever be looked upon as a remarkable achievement. Weir Mitchell, whose name is inseparably connected with this procedure, devised it long before our knowledge of the nature of fatigue would have suggested it; and although other physicians have since elaborated and modified it in various ways, the general principles underlying the treat- 
ment remain unaltered. 'These principles, as just stated, are simple physiological principles, and stand in crass contrast to carlicr methouls of treatment which relied mainly, if not altogether, on medicines.

Medicines, while playing a subsidiary role, may le cmployed with advantage now and then to meet special indications; as, for example, in the atony or catarrh of the stomach, or the constipation frepuently inet with. At times these conditions are successfully combatted by the dict and the massage; at other times simple bitters, alkaline mixtures, bismuth subnitrate, nitrate of silver, or laxatives of various kinds may be indicated. Among the laxatives may be mentioned sodium phosphate, the mild laxative waters, such as Hathorn or Ficdrichschall; or it may be Abilena. Saline laxatives should not be too long continued. Cascara is an almost ideal laxative for neurasthenics, for it directly stimulates the muscular coat of the intestines; it should be given preferably in a liquid preparation and in small doses after meals, so that its action will be well distributed. At other times, laxative pills containing aloes, compound extract of colocynth, or other vegetable cathartic may be employed. Laxatives should, on the whole, be discontinued as soon as practicable.

Occasionally other special symptoms, such as headache, demand medical interference; here small doses of cannabis indica suggest themselves; at other times one of the coal-tar products, preferably a moderate dose of phenacetine, may be given or it may be one of the bromides. 'The role which the eyes play in the headaches of neurasthenia need only be mentioned, and that the eye should receive adequate attention goes without saying.

An annoying complication frequently present is insomnia. How this is to be combatted by the massage and feeding has already been pointed out. If general measures fail, drugs should be used; a few good nights of sleep under a simple hypnotic frequently suffices to restore the sleep habit, or it may be that a simple placebo, such as a capsule of starch, can be substituted for the capsule of trional or veronal. Space will not permit of the detailed consideration of sleep-producing remedies; suffice it to say that the milder remedies, trional, sulphonal, veronal, and the bromides should be given preference. Morphine and chloral are rarely required. Hyoscine and scopolamine are both exceedingly useful, especially as they can be given if necessary without the patient's knowledge -a matter occasionally of importance.

Sometimes the nervousness of the patient does not subside, but persists or even increases for a time after the patient has begun the treatment. In such case it is wise to give small doses of bromide three times daily after feeding.

'Tonies are not especially indicated, though sometimes they satisfy: a patient who feels that unless he is getting medicine, the treatment is not complete. It is a common custom to prescribe iron, and yet there is no anemia, no deficiency of hemoglobin; the bloot count is normal. 'The surface pallor, as noticeable in many neurasthenies, is merely due to feebleness of the surface circulation. A drug frequently prescriber is strychnine, yet it can only help temporarily; it is not a food and cannot 
build tissue, and it has the disadvantage that it frequently increases the nerrous tension of the patient.

The special schedule adopted in the rest cure must vary with every case; it should not be too rigid, but should permit of occasional changes. In general terms, the patient should have breakfast at 8 o'clock; occasionally in very weak patients a little milk may be given on awakening; others, though this is infrequent, are permitted a little milk during the night if awake. 'The bath should be given at 9 or 9.30 ; at 11 milk (or raw egg), at 1 o'clock dinner, 2 to 4 rest hour, at 4 o'clock milk (or raw egg), at 6 o'clock supper, at 7 or 7.30 massage, at 8.30 or 9 milk and sleep; if electricity is being used, it should be given at 4.30. If the time has arrived for resistive movements or other exercise, the electricity should be discontinued. The simpler the schedule, the better; a crowded schedule does harm by fatiguing the patient. I was forcibly impressed by the truth of this some years ago by one of my patients remarking that I was not giving her the rest cure, but the work cure.

'The rest treatment of hysteria requires but little modification from that employed in simple neurasthenia, save that suggestion by the physician and by the nurse plays a somewhat greater role. No better opportunity can possibly be afforded for the free use of a rational psychotherapy - suggestion without hypnotism - than the conditions afforded by a rest cure; psychotherapy, so much vaunted of late, has for many years been practised in Philadelphia under these most favorable conditions; it is perhaps not surprising that, when coupled with the restorative physical methods of the rest cure, suggestion has proved a useful adjuvant. That psycho-analysis can also be here applied, as nowhere else, goes without saying.

In conclusion it may be wise to point out that the application of the rest cure is not limited to neurasthenia or hysteria, but, indeed, is applicable to almost all the functional nervous diseases; many organic diseases also are benefitted by the rest, and appear at times to progress less rapidly by reason of the great uplift given to nutrition. It is interesting also to note the general application made of rest in bed to mental diseases, notably to melancholia, in which its benefits are most striking. The still wider application to mental cases made by Kraepelin and others in Germany is alike interesting and significant. 


\title{
NUTRITION AND FOOD
}

\author{
BY JULIUS FRIEDENWALD, M.D., aNd JOHN RLHRÂH, M.D.
}

Foon may be defined as anything that is taken to nourish the borly. Food is needed for various purposes. A given amount of food substance itself is used in the repair of the cells of the body, as there is always wear and tear going on, and a certain other amount is utilized by the growth of the body from birth until the body attains its full size. Some of it is also stored up as fat, which is utilized for the purposes of keeping the body warm and for furnishing kinetic energy, every movement, no matter how slight, requiring a corresponding amount of energy to be furnished from the food. Food cannot be utilized directly, but must be prepared for use by the processes of digestion, and afterward it is absorbed and then undergoes further changes which consist, roughly speaking, of the processes of oxidation, so that one might aptly compare the body with a furnace, in which the food represented the fuel; and these processes that have to do with the change of food in the body are spoken of as metabolism.

Foods are divided into a number of different classes for the purposes of study - proteins, carbohydrates, fats, mineral salts, and water. About 60 per cent. of the food consists of water, and this serves to keep the body of proper consistency. 'The water itself is not metabolized or burnt up, and so does not furnish any heat or energy. About 6 per cent. of the body-weight is made up of mineral salts, the greatest amount being in the lime salts in the skeleton and in the teeth. 'The amount of energy and heat furnished by the metabolism of salts is so slight as to be practically disregarded, but the salts play a most important part in the metabolism, and many diseased conditions are due to disturbances of this salt metabolism. Further study will undoubtedly reveal a great deal in this direction.

The proteins comprise all the food-stuffs containing nitrogen, and these may be divided into various classes. The albuminoids, which include such substances as the white of egg, the curd of milk, the lean of meat, and the gluten of wheat; gelatinoids (called albuminoids by some writers), of which gelatin is the most familiar example; and extractives which contain nitrogen, but which differ greatly from the above; they are the principal constituents of beef-tea and meat extracts, and are also found in certain vegetables. They add zest to the food, but have practically no fuel value. 
Fat, roughly speaking, makes up about 15 per cent. of the average individual, but there are great variations in the amount of fat found, and this depends more upon heredity and personal habit than on the character or amount of the food taken.

The carbohydrates are composed of carbon, hydrogen, and oxygen, the last two in the proportion to form water, as, for example, starch, $\mathrm{C}_{6} \mathrm{H}_{10} \mathrm{O}_{5}$. They include the starches, the sugars, and vegetable fibre or cellulose. 'The formation of these various food constituents differs slightly. The protein is the only form which may be used to build up tissue, and this, in the form of albuminoids, if one excepts fatty tissue, alone serves to build up the body in the process of growth. The protein may also be burnt up in the body to serve as fuel to yield heat and muscular power, and fats may be stored up in the body as fat, and also serve as a valuable source of heat and energy. The carbohydrates may be transformed into fat and stored in the body, and may also be used as fuel. The mineral salts are used to build up certain parts of the body and also assist in digestion and the various processes of metabolism.

Food has a definite fuel value, and it furnishes the same amount of energy whether it is utilized by the body or burnt in a properly constructed furnace, the law of conservation of energy applying to the metabolism in normal bodies as it does in mechanics. The fuel value of foods may be determined by the use of an instrument known as a bomb calorimeter and is expressed in calories. The calorie is the amount of heat that is necessary to raise the temperature of 1 kilogram of water $1^{\circ} \mathrm{C}$., or nearly the same as the amount required to raise 1 pound of water $4^{\circ} \mathrm{F}$. Expressed in mechanical force a calorie would raise a ton about 1.54 feet.

Fuel values of various foods have been determined by different observers, chiefly Rubner and Atwater. Atwater's figures are as follows: $1 \mathrm{gm}$. of protein furnishes 4 calories; 1 pound furnishes 18.20 calories; $1 \mathrm{gm}$. of fat furnishes 8.9 calories; 1 pound furnishes 40.40 calories; $1 \mathrm{gm}$. of carbohydrate furnishes 4 calories; 1 pound furnishes 18.20 calories.

Rubner's figures are slightly higher, and are universally used in calculating the values of food. They are: Carbohydrates and protein, 4.1 calories per gram; fat, 9.3 calories per gram.

The absorbability of a food is a very important point when considering its nutritive value, and has to be taken into consideration, as well as the actual fuel value; and the absorbability will depend very largely upon the digestibility of the food, and this in turn depends largely upon the skill with which it has been prepared and the appetite and digestive power of the individual who eats it. The amount of food-stuff that is retained in the body after it is used is commonly called the percentages or coefficients of digestibility. Protein is absorbed partly in the large intestine and partly in the small, and there are considerable variations in the absorbability, those from animal food being much more completely absorbed than those from vegetables. 'Thus, certain meats may have 97 per cent. absorbed, while some of the vegetable proteins, such 
as those from lentils, may only have about 60 per cent. absorbed. Fats are absorbed very completely, but only within cortain limits, individuals having a varying capacity for fat absorption, the atverage being placed at about $150 \mathrm{gm}$. a day, more than this being thrown off in the feces. The earbohydrates are quite completely absorbed, but there is usually much that is not absorbed, as carbohydrates are mainly taken in the form of vegetables, which contain large amounts of collulose. When foods are taken in combination in a properly mixed diet, they are absorbed very much more completely than when merely one kind of food is supplied.

The quantity of food that is required varies according to the individual and to the condition under which be lives, the grown person requiring more food than a child, and the person indulging in anctive physical exercise more than one who is at rest. One has to consider the total amount of food needed in twenty-four hours under any given condition, and this may be expressed in calories; and, further, the question of how much of this should be furnished by proteins, how much by fats, and how much by carbohydrates must be considered.

The proteins are the most important to consider, as they supply the material to repair the waste of the body, which goes on constantly. One might get figures for the amounts that were consumed by average individuals under ordinary circumstances, and then consider the minimum amount of protein that can be got along with and also the maximum that can be got along with without producing deleterious changes in the body. Voit's standards have ordinarily been accepted. 'They were based on averages of men weighing 70 kilograms (157.5 pounds); of course, heavier individuals requiring more and light individuals less. Voit arrived at his results from studying the food actually taken. Similar estimations have been made by Rubner and Atwater, and it will be seen from the following table that the average-sized man, doing a medium amount of work, requires about $120 \mathrm{gm}$. of protein.

\section{STANDARD DIETARIES FOR A MAN OF 70 KILOGRAMS}

Light work:

Voit.

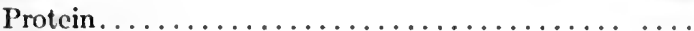

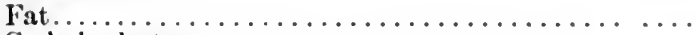

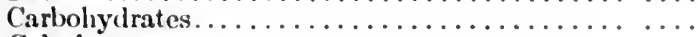

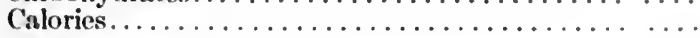

Medium work:

Protein ....................... 11s

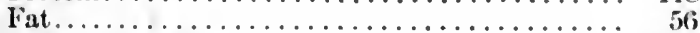

Carbohydrates................... 500

Calories........................... 3055

$\begin{array}{cc}\text { Rubner. } & \text { Atwater. } \\ 123 & 100 \\ 46 & * \\ 377 & * \\ 2445 & 2700\end{array}$

$\begin{array}{rc}127 & 125 \\ 52 & * \\ 509 & * \\ 2565 & 3400\end{array}$

Iard work:

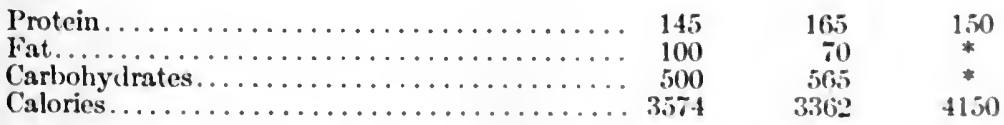

Vou. I-24

* Carbohydrates and fats to make up the fuel value. 
Under ordinary circumstances, an individual cannot take much over $150 \mathrm{gm}$. a day without producing symptoms of overfeeding, and also causing too great wear and tear on the excretory organs. As to the minimum anount of protein that can be gotten along with, the experiments of Chittenden are of particular interest. He found that he was able to reduce the amount of protein to 55 or $60 \mathrm{gm}$. a day, and that nitrogen equilibrium was thus established, in which there was a daily intake of from 8.5 to $9.5 \mathrm{gm}$. of nitrogen, and the same amount being excreted in the urine.

The average standard diet contains about $16 \mathrm{gm}$. of nitrogen, which is taken into the body daily, and about $16 \mathrm{gm}$. is excreted. If less is excreted than is taken in, it means that too much is being given, and that it is being stored up in the body; and if more is excreted than is taken in, it means that the excess must come from the cells of the body, and if this is kept up, emaciation will take place. This is commonly seen in fevers, but may also be noted in individuals with insufficient diets.

The important point to bear in mind, in considering the amount of nitrogenous food taken, is the amount on which the individual would get along best, and not the minimum amount nor the maximum amount that may be taken. The minimum amounts are of great interest in dieting individuals with certain diseases, as, for example, nephritis, where it is highly desirable to lessen the work of the kidneys as much as possible, and the maximum amounts have the same interest in the feeding of individuals with diseases, such as tuberculosis, in which it is desirable to increase the resistance of the body, and, at the same time, important not to overstep the limits of the body's ability to utilize protein. If too little nitrogenous food is constantly taken, the resistance of the body is apt to be somewhat lowered.

As regards the amount of carbohydrates and fats, Atwater leaves the figures open, allowing the total amount of food to be made up, using either carbohydrates or fats or a mixture of both. 'The other authorities usually place the average diet at $500 \mathrm{gm}$., to come from the carbohydrates, while the remainder is to be made up of fat, which will bring this between 50 and $100 \mathrm{gm}$.

The amount of fat and carbohydrate used in making up the dietary will depend largely upon the part of the world in which the individual lives, large amounts of fat being used in the cold northern climates, and comparatively small amounts being utilized in the tropics. The reverse is true of carbohydrates, large amounts being used in the tropics and small amounts in the cold countries.

Food varies very largely with the supply at hand, and the availability must always be taken into consideration in ordering diets. It happens only too of ten that expensive dietaries are given to individuals, who are unable to carry out the orders through their inability to purchase the food mentioned.

In young, growing animals the fats scem to play a very important part in the absorption of the mineral salts, and diets too low in fat are 
followed by grave disturbances of nutrition, especially the development of rickets.

As to the total number of calories needed to supply the requirements of the body, it will be found that this varies according to the amount of work being done by the individual in question. 'The following table, from Rubner, shows the daily needs of an adult weighing 65 kilograms.

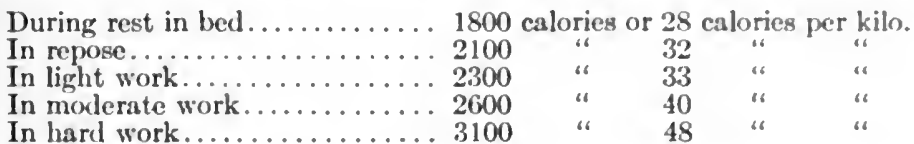

The requirements for children are proportionately larger than those for adults, and Atwater has given the following table, showing the quantity required in comparison with the amount needed by a man at moderately active muscular work:

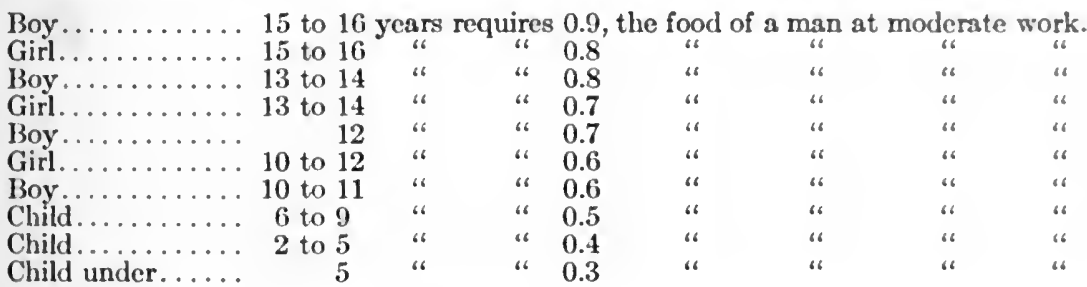

Infants require large amounts of food per kilo. During the first three months 100 calories per kilo (45.5 calories per pound), during the second three months between 100 and 90 (40.90 calories per pound), and during the latter half of the first year the requirement gradually sinks to $\mathrm{SO}$ or a trifle below ( 36.4 calories per pound).

Vegetarianism.- It has been determined by experiment that the animal protein can be replaced entirely by vegetable protein without any appreciable change in the nitrogen balance; but, be this as it may, from practical experience the average individual does much better on a mixed diet, the objections to an exclusive vegetable diet being that it makes the bulk of the food very large, and throws an unusual amount of work upon the gastro-intestinal tract, and in many individuals produces intestinal disease. It is also said that persons living on a pure vegetable diet are apt to lose strength and to have a lessened power of resisting disease. As carried out by most vegetarians, the diet includes milk and eggs, which makes it really a mixed diet.

Appetite and Other Factors. - There are a number of points in regard to feeding which should always be borne in mind, and anong these may be mentioned the fact that appetite is a very important factor. It has been shown experimentally that where the appetite is gool, the digestion is also liable to be good, and that food ingested where there is little or no appetite is liable to be digested slowly and often insufficiently: 'The proper variety in diet and care in the preparation of food is, therefore, 
especially desirable in feeding invalids. The interval between taking foods should be carefully regulated and sufficient time allowed between meals to have the stomach emptied entirely. This length of time will depend upon the character of food taken and the amount of it, four or five hours being necessary for the complete digestion of an ordinary mixed meal, and even longer times may be required by individuals with feeble digestions. Where small amounts of easily digested food are taken, the length of time between the meals may be shortened. The food should be neither too hot nor too cold, as extremes of temperature in the food are liable to give rise to gastric disturbances, especially gastric catarrh.

It is advisable to rest before and after meals, as violent exercise immediately after meals inhibits digestion, and it should also be remembered that sufficient time should be allowed to elapse between taking food and sleep, as the digestive processes are not as active during sleep as during the waking hours. Severe mental strain and emotion disturb digestion, while pleasant companionship and conversation improve it.

Certain conditions and discases, or the tendencies to them, are inherited; among these may be mentioned the tendency to leanness or to obesity, and the strong family tendency in diabetes, gout, and alcoholism. There are also curious food idiosyncracies, some of which are inherited. The urticaria or poisoning which follows the ingestion of strawberries, shell-fish, and the like are familiar examples.

Classes of Foods.-Milk.-The animal food which is the most important from the standpoint of an invalid is milk. Cows' milk contains on an average about 3.5 per cent. of protein, 4 per cent. of fat, and 4.5 per cent. of sugar, the mineral salts make up about 0.7 per cent., and the remainder is water. About five-sixths of the protein consists of casein and the remainder is lactalbumin. The large amount of casein present causes the milk to be precipitated in the stomach in large curds, which sometimes give rise to digestive disturbances. The formation of these curds may be prevented by the addition of lime-water or of barley or oatmeal water, or by the mixture of other foods.

The principal point about milk is that it should be produced under hygienic conditions, and kept clean and cool immediately after milking until used. All milk contains some bacteria. The best grades contain less than 5000 per cubic centimeter. . The arerage milk marketed in cities contains from 50,000 to 100,000 per cubic centimeter, and this latter number may be regarded as the limit in milk which is to be used for human consumption. The number of bacteria in some milk is almost unbelievable; over 20,000,000 per cubic centimeter have been noted in milk purchased as human food.

The best milk for almost all purposes is plain, fresh milk, to which nothing has been done. In cities this may be impossible to obtain, and to prevent the milk spoiling it may be pasteurized, that is, heated to a degree of heat sufficient to kill most of the bacteria without changing essentially the composition of the milk, which means heating to a point something under $170^{\circ} \mathrm{F}$. Sterilized milk is sometimes used for long 
journeys, and, where it is found impossible to keep the milk otherwise, as owing to lack of ice, it may be sterilized.

Milk is frequently adlulterated, usually by adding water, and milk sometimes carries infectious diseases, chiefly typhoid fever, but occasionally diphtheria and searlet fever and other diseases. Chemieal preservatives are frequently added, and this should be prohibited by law.

Buttermilk is frequently used as food, and whole milk, which has been inoculated with the lactic acid bacilli, is much in vogue at the present time, especially as an invalid food, and in certain forms of gastric and intestinal disorders. Condensed milk is much used as a substitute for fresh milk, and is of especial value in the tropies and in traveling. It is often used for food, both for invalids and infants, but it should be borne in mind that the continuous use of condensed milk as an infant food leads to the development of fat, pale, flabby babies, with a tendency to rickets, scurvy, and a lowered resistance to infections. 'This is due to the low protein and fat content and the excessive amount of carbohydrate. Fermented milks, koumiss, kefir, and matzoon are frequently used as invalid foods. 'The average cows' milk contains about 320 calories per pint and is free from purin nitrogen.

Eggs are also valuable food, especially in invalid feeding. 'They contain about 720 calories per pound, the whites alone yielding 250 calories per pound and the yolks 1705 per pound. 'The white is pure protein, while the yolk contains numerous substances, chief of which are 15 per cent. of protein, 20 per cent. of fats, besides lecithin, nuclein, salts of iron, and calcium, potassium, and magnesium. For human food eggs should be reasonably fresh. Ordinarily eggs are tested after having been in storage by candling; that is, holding the egg up against the light. A fresh egg appears unclouded and is almost translucent; if incubation has begun, a dark spot is visible, and eggs which are much spoiled are of a dark color.

The white of eggs, when used for food, should be slightly beaten and strained, and may be flavored with wine, orange, lemon, or grapejuice, or may be added to other foods, such as milk or cocoa. Whole eggs may be prepared in numerous ways, and for invalid feeding are frequently beaten up and added to milk.

Meats.-These vary greatly in composition and digestibility, the variations being chiefly in the amount of fat and water contined. Meat may be cooked in various ways, broiled or roasted meats, as a rule, being more digestible than meat prepared in other ways. It should be remembered that most bouillons and beef extracts consist chiefly of the extractives from the meat, and have little or no food value, but are chiefly useful in stimulating the appetite and flow of gastric juices. Nutritive beef juices may be obtained by pressing out the juice in a neat press or by other suitable methods. The following table shows the composition of some of the different varieties of meat, the proportions given being, of course, only approximate: 


\begin{tabular}{|c|c|c|c|c|c|c|c|}
\hline $\begin{array}{l}\text { Food-materials } \\
\text { (as purchased). }\end{array}$ & $\begin{array}{l}\text { Refuse. } \\
\text { (Perct.) }\end{array}$ & $\begin{array}{l}\text { Water. } \\
\text { (Per ct.) }\end{array}$ & $\begin{array}{l}\text { Protein. } \\
\text { (Per ct.) }\end{array}$ & $\begin{array}{l}\text { Fat. } \\
\text { (Per ct.) }\end{array}$ & $\begin{array}{c}\text { Carbo- } \\
\text { hydrates } \\
\text { (Per ct.) }\end{array}$ & Ash. & $\begin{array}{c}\text { Fuel } \\
\text { value per } \\
\text { pound. } \\
\text { (Calories) }\end{array}$ \\
\hline $\begin{array}{l}\text { Beef, fresh: } \\
\text { Sirloin steak..... }\end{array}$ & 12.8 & 54.0 & 16.5 & 16.1 & $\ldots$ & 0.9 & 975 \\
\hline $\begin{array}{l}\text { Beef, corned, canned, } \\
\text { pickled, and dried: }\end{array}$ & & & & & & & \\
\hline $\begin{array}{l}\text { Corned beef ........... } \\
\text { Veal: }\end{array}$ & 8.4 & 49.2 & 14.3 & 23.8 & $\cdots$ & 4.6 & 1245 \\
\hline Breast.... & 21.3 & 52.0 & 15.4 & 11.0 & $\ldots$ & 0.8 & 745 \\
\hline $\begin{array}{l}\text { Mutton: } \\
\text { Ieg, hind... } \\
\text { Lamb: }\end{array}$ & 18.4 & 51.2 & 15.1 & 14.7 & & 0.8 & 890 \\
\hline Breast.... & 19.1 & 45.5 & 15.4 & 19.1 & $\ldots$ & 0.8 & 1075 \\
\hline $\begin{array}{l}\text { Pork, fresh: } \\
\text { Ham...... }\end{array}$ & 10.7 & 48. & 13.5 & 25.9 & & 0.8 & 1320 \\
\hline Loin chops... & 19.7 & 41.8 & 13.4 & 24.2 & $\ldots$ & 0.8 & 1245 \\
\hline $\begin{array}{l}\text { Poultry: } \\
\text { Chicken, }\end{array}$ & & & & & & & \\
\hline Turkey........... & $\begin{array}{l}41.0 \\
22.7\end{array}$ & $\begin{array}{l}43.7 \\
42.4\end{array}$ & $\begin{array}{l}12.8 \\
16.1\end{array}$ & $\begin{array}{r}1.4 \\
18.4\end{array}$ & $\cdots$ & $\begin{array}{l}0.7 \\
0.8\end{array}$ & $\begin{array}{r}305 \\
1060\end{array}$ \\
\hline
\end{tabular}

Fish.-These differ widely in their nutritive and digestive qualities. Owing to the fact that they undergo decomposition rapidly, fish should always be eaten in as fresh condition as possible, although there are many modern methods resorted to to prevent these changes.

As compared to meat, fish contains somewhat less protein, and is frequently used in dietaries, where it is desirable to lessen the amount of nitrogenous food ingested.

Vegetables.-Vegetables differ from the animal foods chiefly in containing a large proportion of starch and sugar and comparatively small amounts of protein. Vegetables also contain cellulose, which, when old, cannot be digested, and hinders the digestion of the starches involved by it. Some vegetables contain pectin, which, when cooked, gelatinizes, and this is taken advantage of in preserving various fruits. 'The proteins in vegetables are glutens, as found in wheat; legumin, found in the legumes, and various globulins. Some vegetables contain considerable amounts of extractives and others contain considerable amounts of fat. Nothing further need be said concerning this class of foods, except to call attention to the fact that oatmeal contains considerable amounts of purin nitrogen, as do also all the legumes.

All the members of the cabbage family contain a considerable quantity of sulphur, and on this account are liable to produce flatulence. Green vegetables and the fruits are particularly desirable additions to the dietary, especially at the present day, when so many canned and preserved meats and foods are used.

Sugar is a valuable food, but there are limitations to the amount of sugar that can be utilized, excesses being liable to undergo fermentation and produce gastro-intestinal clisease. Individuals with little appetite, as a rule, do not care for sweets and, if taken, they produce a cloying sensation which prevents the taking of other foods.

Fats are obtained from both the animal and vegetable kingdom, 
and are taken chiefly in the form of cream and butter and the various oils. In too large quantities, and in people with feeble digestions, they are liable to cause gastric disturbance. 'They are particularly usefuil in certain forms of intestinal disease and in building up thin and rundown individuals.

Salts.- The amount of heat and energy supplied by salt metabolism is so small as to be practically disregarded, but they play a very important part in metabolism. If salts are withdrawn entirely from an individual, he will starve to death, much in the same way as if some apparently more important part of the food had been omitted. If excessive amonnts are taken, that which is not needed by the body will be exereted, and, if the amounts be very great, the salts may be retained in the body and produce pathological changes.

The metabolism of animal food tends to render the blood and tissues acid, while the metabolism of vegetables, with the exception of cereals, tends to render the tissues alkaline. Sodium chloride is taken apart from the food, and if the amount be increased, it causes great thirst, which is followed by the drinking of large quantities of water, which aids in the excretion of the excess of the salt. If salt is withdrawn from the diet of otherwise healthy individuals, certain symptoms may be noted, chief of which are lassitude, incapacity for work, and digestive disturbances. In certain nervous diseases, particularly epilepsy, salt-free diet is often used with considerable benefit, and it seems to increase the action of bromides.

Phosphorus is found in many foods in the form of phosphates, and also in the form of a more complex organic compound, as lecithin, nuclein, and the like. 'The most available foods containing phosphorus are the yolk of egg, fish-roe, and other glandular structures.

Iron plays an important part in animal economy, and is present in small amounts in many foods, and in anemia is frequently demonstrated, either in the form of foods containing large amounts of iron, as the yolk of egg, spinach, and meats, or in the form of either organic or inorganic preparations.

Calcium metabolism has been a matter of considerable study in recent years, and a number of pathological conditions are intimately associated either with excessive excretion of calcium or its retention in the boly. In osteomalacia, or pernicious anemia, there may be loss of caleium, while in arteriosclerosis and arthritis deformans there may be calcium retention. There is a close relation between calcium metabolism and tetany.

Water.-See article on " Mineral Springs."

Tea.- This much-used beverage contains eaffeine and tamic acid. The caffeine, being more soluble, is extracted rapidly, while the tannic acid, being less soluble, is not present in the infusion in any great amount until after the tea has stood for a number of minutes. The infusion of tea is best made, therefore, by pouring boiling water upon the tea-leaves and allowing it to stand for a few minutes. If tannic acid is present in large quantities, it precipitates the pepsin in the gastric juice, and even 
the weaker solutions retard digestion. Individuals suffering with gastric disturbances do much better without tea, and excessive tea-drinking is accompanied with ner:ous symptoms, sleeplessness, and excitability, disturbances of digestion and of the circulation.

Coffee.-Coffee acts much in the same way as tea, the stimulating effect being due to the caffeine present, and its excessive use being followed by the same train of symptoms. In many persons in which there is gastric disturbance the digestion may be affected.

Cocoa.-This contains an alkaloid, theobromin, and also is very rich in fat in the form of cocoa-butter, and contains starch, tannic acid, and various nitrogenous substances. It is a much milder stimulant, and it is not liable to produce the nervous symptoms produced by tea and coffee. It is a very useful and nutritious article of diet, but, when used in excess, is liable to produce digestive disturbances, owing to the large amounts of sugar and fat that it contains.

Alcohol.-While it is undoubtedly true that alcohol has been enormously abused in its use in medicine in the past, it is probably also true that its opponents at the present time have gone too far in their criticisms of its use in medical practice.

The effects are too well known to require more than a very short notice at this time. Atwater and others have determined that alcohol may be burned in the body similarly to other foods, and that each gram yields 7 calories. It cannot be utilized to build up the tissues, but furnishes a source of heat and energy, and acts as a protein sparer, much in the same way as fat or carbohydrate.

In addition to its food value, it has many other properties which render its use as a food dangerous, unless the quantity be limited well within the powers of the individual to metabolize the alcohol without affecting the tissues. Atwater places the limit of the average individual to about 2 ounces per day, but this certainly varies widely with different individuals.

'The effect of alcohol upon the heart is that of a depressant, unless there may be a certain amount of stimulation at the beginning due to the irritative effects of the alcohol locally. One of the most important changes that it causes is its effect upon the circulation, redistributing, as it were, the flow of blood to the various parts of the body, and so acting practically as a stimulant, although it may be depressing the heart at the same time. It acts upon the stomach by promoting an increase in the flow of gastric juice, and is frequently used for promoting appetite and aiding digestion. It should be borne in mind that where it amounts to more than 5 per cent. of the stomach content it interferes with digestion to a more or less marked degree. It interferes markedly with the amount of muscular work that can be done, with the accuracy of finer movements, and also lessens the amount of mental work that may be done and slows the association of ideas. It should be classed with the anesthetic and narcotic drugs.

Its use in medieine is partly as a food and partly for its effect upon the stomach, for its effect upon the circulation, and sometimes for its 
anesthetizing effeet upon the nervous system. As a foow, it is often valuable in tiding over periorls when the assimilation and digestion of ordinary foods has been so lowered as to render the use of ordinary nutrients impossible. In prolonged asthenic fevers it may be of very great service, and large amounts may be used with great benefit. In using alcohol it should be remembered that it should be given in small doses, repeated at more or less frequent intervals, and should be well diluted. 'The form in which it is used will depend largely on the taste of the patient, and eare should be taken to secure a pure and sufficiently aged article, as a large amount of the alcoholic beverages sold are adulterated or are not sufficiently old. 'The alcoliolic content of the various beverages is shown in the following table, compiled from the report of the Committee of lifty:

\begin{tabular}{|c|c|c|}
\hline & Percen & \\
\hline & 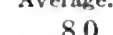 & \\
\hline Freneh white & $\begin{array}{r}8.0 \\
103\end{array}$ & $\begin{array}{l}6 \text { to } 12 \\
0\end{array}$ \\
\hline erman Rhine wines, & $\begin{array}{r}10.3 \\
8.7\end{array}$ & $\begin{array}{lll}9 & 612 \\
7 & 12\end{array}$ \\
\hline$\ldots$ & $\therefore 17.5$ & $16 \cdot 20$ \\
\hline ra......... & . . 15.4 & $15 \cdot 16$ \\
\hline ne. & . 10.0 & $8 * 1$ \\
\hline hampagne.... & 8.0 & 6 " \\
\hline & 9.0 & 6 “ \\
\hline & 12.0 & 10. \\
\hline & 3.8 & $1 "$ " \\
\hline . & 4.7 & 3 “ \\
\hline & 4.8 & 3 “ \\
\hline porter.. & 5.0 & 3 “ \\
\hline 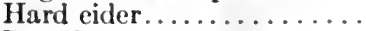 & 5.0 & $4 "$ \\
\hline Bral & 47.0 & $40 " 50$ \\
\hline & 43.0 & $41 "$ \\
\hline mon. & 35.0 & \\
\hline Irish...... & 40.0 & $36 "$ \\
\hline & 60.0 & 40 \\
\hline . & $\ldots 30.0$ & $20 \cdots 4$ \\
\hline
\end{tabular}

The amount of alcohol should never be sufficiently great to cause any mental symptoms in the patient, and the general condition and appearance of the patient is a very good guide as to whether the alcohol is being well borne and doing the patient good or not.

Rectal Feeding.- In certain instances, where the patient cannot take nourishment by mouth, or only in insufficient quantities, rectal feeding is sometimes employed. Such conditions are met with in diseases of the mouth, pharynx, and asophagus, which affect the patient's ability to swallow, caused most frequently by tumors. It is also seen in certain gastric diseases, where food does not enter the stomach, as in carcinoma of the cardiac end. In certain general conditions, where the patient will not or camnot take nourishment rectal feeding is also occasionally employed, as in eases of delirium, the insane, and the comatose, although in these cases feeding by means of a nasal or stomach-tube is, as a general rule, to be preferred. In certain diseases of the stomach, where it is desirable to give complete rest to that organ, rectal feeding may also be employed. It is sometimes of great service in cases of ulcer, nervous vomiting, dilatation, and in certain forms of nervous indigestion. 
It should be borne in mind that rectal feeding has certain definite limitations. 'The length of time the patient may be nourished by this method depends upon the amount of nourishment he has stored up in his body at the time the feeding is begun; and, secondly, upon his ability to have prolonged absorption from the rectum.

As ordinarily carried out, rectal feeding can only be used over a period of one or two weeks, although, with good technique, this period may be extended in many cases to as long as four or even six weeks, and there are on record instances where the feeding has been prolonged over many months. Von Leube has kept a patient alive for six months, and Riegel for ten months, exclusively on rectal feeding. At best the method is to be considered, however, as only a means to bridge over some critical period, and it should be borne in mind that the patient at the outset of the treatment has more strength than he will have later, as will be seen from the results of studies of these cases, so that rectal feeding is not to be used as a means for building up weakened patients preparatory to surgical operations or other procedures.

'The amount of nourishment that may be absorbed from the rectum varies considerably in different individuals, but probably, even under favorable circumstances, not more than one-fourth of the nourishment needed for the maintenance of the body can be absorbed, and Edsall places this as low as one-sixth, the patients absorbing from 240 to 645 calories per day. 'There are, doubtless, some exceptional individuals whose capacity for absorption is greater than this. The question of the absorption of the various foods from the rectum has not been definitely settled and is deserving of further study, but it would seem that the carbohydrates are absorbed better than any of the others, something like nine-tenths of the total quantity injected being absorbed when 10 to 20 per cent. sugar solutions are used; but not more than $40 \mathrm{gm}$. of sugar can be given by rectum, and, as a rule, a daily allowance of $30 \mathrm{gm}$. is about as much as the average patient can tolerate. Solutions of pure glucose, dextrose, or dextrine have given the best results, and boiled starch is also frequently used in conjunction with other things. The proteins are not absorbed very well, only about one-sixth of that given being utilized, although certain forms of dried casein seem to be absorbed to quite a remarkable degrce. Such products as sanatogen, proton, eucasin, and nutrose may be used in 10 per cent. solutions, or may be added to other foods intended for rectal absorption. Fats are absorbed with difficulty, but doubtless better than formerly supposed; they are particularly useful on account of their high food value. Various fats and oils have been suggested, both in emulsions and saponified, but the yolk of eggs seems to give the best results. Alcohol is usually easily absorbed in solutions containing from $\frac{1}{2}$ to 2 per cent., and in many iustances apparently aids in causing the absorption of other foods. An important addition to all nutrient enemas is ordinary salt, which should be added up to 1 per cent. This aids materially in the absorption, and also apparently favors reverse peristalsis, which causes the food to be carried upward in the bowel, and so brings it in contact with 
a greater surface of the intestine. 'Thymol, in proportion of 1 to 4000, may sometimes be added to advantage, especially where sugar solutions are being used, and if there is any irritation following the use of the solutions or if there is any sour fermentation taking place. 'The temperature of the solution injected is very important. If the solutions are either too hot or too cold, they will be quickly rejected. 'The solutions intended for retention should be between $90^{\circ}$ and $9.5^{\circ} \mathrm{F}$.

'The technique of rectal feeding is exceedingly important, and, to be carried on successfully over any period of time, must be done by some one having experience. The enema should be preceded by a cleansing injection, which ordinarily may be of normal salt solution, although bicarbonate of soda solutions of the strength of 1 dram to the pint may be used if there is much mucus in the rectum, and sometimes boric acid solutions may be employed to advantage. 'The first two or three cleansing injections should be given with an ordinary rectal tube; after that they may be given to great advantage through a return flow tube. The temperature of these cleansing injections should be between $95^{\circ}$ and $99^{\circ} \mathrm{F}$. The patient should be placed in as comfortable a position as possible, a somewhat modified knee-chest position being used, with a large pillow so placed as to elevate the hips, and a small rectal tube lubricated with oil introduced slowly as high into the rectum as possible. Glycerine should never be used as a lubricant. The tube should be passed well beyond the inner sphineter, and it is not necessary to push in great lengths of tubing, as it only curls up in the rectum, and only in exceptional cases passes into the sigmoid, and certainly not unless fluid is allowed to flow through the tube at the time it is introduced, and this cannot be done when the tube is passed for purposes of rectal feeding. The solution used should be allowed to flow in slowly, using a fountain syringe connected with the rectal tube, and laving it only slightly above the hips of the patient. 'The patient should remain as quiet as possible after the injection, and, if possible, should be diverted by conversation or other means. If necessary, a pad of gauze or a towel may be held over the anus for twenty or thirty minutes where the injections are poorly retained. Where there is irritability of the rectum, or when the injections cause pain, they may be preceded by a suppository containing opium, or a small enema of boiled starch, to which opium has been added, may be used. Where there are hemorrhoids which are painful, the mucous membrane may be painted with a 2 per cent. solution of cocaine. It should be borne in mind that gynecological dressings may interfere with rectal feeding.

'The number of nutrient enemata which may be used depends somewhat on the individual patient. Ordinarily, three or four may be given in the course of twenty-four hours, at about six-hour intervals. Where the patient is badly in need of food, the interval may he made five hours, and it is only in exceptional instances that they should he given closer together than this, as too frequent use of injections leads to irritability of the rectum. 'The amount to be given at any one time should be $S$ ounces or less. Various formulas have been suggested. It 
was formerly taught that it was an advantage to predigest the proteins by means of chopped pancreas or pancreas extract, but such solutions have no particular advantage over other forms of liquid protein. Mixtures containing milk as a basis, to which eggs, starch, and sugar have been added, are most frequently employed.

Egg-and-milk enema:

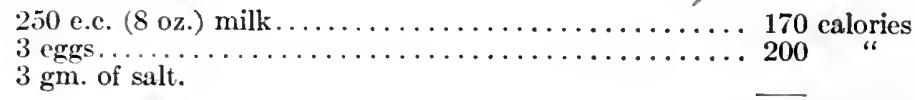

$\overline{370}$ calories.

Starch-and-milk enema:

60 to $70 \mathrm{gm}$. (about $2 \mathrm{oz}$. ) starch............... 250 calories

250 c.c. $(8 \mathrm{oz}$.$) milk............................. 170$

Sugar-and-milk enema:

420 calories.

$60 \mathrm{gm}$. (2 oz.) grape-sugar................ 246 calories

250 e.c. $(8$ oz.) milk........................... 170

Ewald's nutrient enema:

416 calories.

2 or 3 eggs,

1 tablespoonful of water.

A small amount of flour is boiled in half a cupful of 20 per cent. solution of dextrose, and a wineglassful of red wine added. The egg solution is stirred in, care being taken not to have the solution too hot lest the albumin be coagulated. Entire amount, 250 c.c. $(8$ oz.).

The Mouth.-Great care should be taken of the patient's mouth. The teeth should be regularly cleansed and mouth-washes used. If the patient complains of thirst, injections of water containing a small amount of salt may be given.

The feeding of normal infants and children cannot be considered in an article of this length, but feeding plays a very important role in certain diseases of infancy and early childhood about which a few worls may be said.

As a general rule, the weight of the child is the best index of its nutrition, but it should also be borne in mind that the general appearance, whether it is firm and healthy looking, is an important factor. It is easy to produce large, flabby, pale children by feeding them on excessive amounts of carbohydrate food, but even though these children are gaining in weight, they are not in as good physical condition as if the gain had been more slow.

In the cases that might be set down as difficult ones to feed, a very carcful study must be made of all the conditions surrounding the child in its daily life. In many instances the difficulty is not so much with the food as with some other factor. For example, the child may be fed on suitable mixtures when it is not getting a sufficient amount of fresh air or exercise, or is kept too warm or too cold, or is worried or disturbed in its rest by unseemly noises or a dozen and one other small matters may interfere. Where the conditions are reasonably normal, and the child is not gaining on any special diet, it may be assumed that this particular diet is not suitable for it and some other form of food should 
be substituted. Changes should not be made too frequently, however, as it may take a week or ten day's to determine whether the child will gain on any certain food, unless, of course, it disagrees actively and produces untoward symptoms.

In cases of acute inanition, that is, in cases of acute starvation, which are sometimes seen in young infants, due cither to insufficicont or improper food, the loss of weight may be very rapid and the (ondition accompanied with fever, so that the diagnosis may be a matter of some difficulty. Wherever possible, breast-feeding should be substituted at once, as it offers the greatest chance of recovery, and in many instances the only one. When this cannot be done, the use of proper dilutions of condensed milk, or of more or less completely peptonized cows' milk, may be tried.

In cases of marasmus, which may be put down as subacute cases lasting weeks or months, the life of the child should be carefully regulated, a wet-nurse obtained if possible, and, if not, the child may be given peptonized milk or condensed milk mixtures or suitable modifications of cows' milk. The child should be given a food suited to its age and condition as soon as possible.

In cases of malnutrition, which may be put down as chronic cases, lasting months or years, usually occurring in older children, it will frequently be found that the child has either inherited a bad constitution or the conditions followed some other disease, but a certain number of cases are due, however, to improper feeding, the child sometimes being on what is apparently a normal diet, but being unable to utilize what might be regarded for normal children as the proper amounts of carbohydrates, fats, or proteins. Many of these cases are suffering with overfeeding, usually in the line of fats or carbohydrates, and when put on a suitable diet do remarkably well.

Food Intoxications.-These are comparatively frequent, and may usually be suspected when the child is having recurring, more or less periodic attacks of any special symptom, vomiting being the most frequent, but sick headaches, asthma, and many other symptoms not ordinarily associated with dietetic conditions may be met with. In some instances the child is getting too much food of all kinds. This usually leads to recurrent attacks, which are characterized by ferer, coated tongue, foul breath, headache, malaise, and drowsiness; the liver is sometimes enlarged and tender, and there may be vomiting or diarrhoea or both. The condition is cured by a purge and a limited diet. Where protein is given in too large amounts, the symptoms are very similar-sometimes one symptom is especially permanent, as recurrent headaches or neuralgia. 'Too much fat is a frequent cause of trouble, some of these cases arising from the child's being given large quantities of butter, cream, col-liver oil, and other fats in an endeavor to increase the child's weight. 'The most striking features are the coated tongue, an exceedingly fetid breath, pale, muddy skin, frequent disturbance of the gastro-intestinal tract, and often the passage of large amounts of undigested fats in the stools. 'Too much carbohydrate 
is the commonest of all, owing to the fact that many children are given large quantities of starches and sugars, not only at meals, but between meals, and there are also many children who have a low capacity for utilizing sugar and starclies. Perhaps the most common form is recurrent or cyclic vomiting. This may be seen not only where too much carbohydrate food is given, but where too much protein is given as well. In other instances there are attacks of asthma, or of hyperacidity or of sick headaches. These cases do very well upon reducing the amount of carbohydrate food and using an occasional purge, such as phosphate of soda. The child should be on an otherwise suitable diet, and the meals should be taken at regular intervals.

Acute Gastritis.-In acute gastritis and other conditions accompanied with vomiting, the best method of treatment is to empty the stomach and give it a rest. Food may be withheld entirely in acute cases for twenty-four hours, allowing very small quantities of hot water and sometimes small quantities of albumin water or barley water. At the end of twenty-four hours small quantities of albumin water, barley water, or some other very easily digested food may be allowed. For several days after the attack food should be small in quantity, given at reasonably long intervals, and the child kept at rest. The return to the ordinary diet should be slow.

Diet in Diarrhoea of Infants.-For the practical purposes of diet the diarrhœas of infancy and childhood may be divided into two classesthe simple diarrhoeas, which are caused by eating articles of food that cannot be digested, to nervous causes, to drugs, and to extremes of temperature. In the second class there is a toxic condition present, and the disease is usually caused either by the ingestion of milk containing ptomaines, milk containing large quantities of bacteria, or milk containing pathogenic bacteria. All the above things may be ingested in some food other than milk. As it is impossible to tell at the outset which form one is clealing with, it is best to be exceedingly cautious until the diagnosis is made. The most serious of these diarrhœas occur in buttlefed babies, and nearly all the deaths due to diarrhoal diseases occur in bottle-fed babies. In the breast-fed baby the emptying of the gastrointestinal tract and lengthening the feeding to six hours is most important, giving small amounts of plain boiled water, or albumin water or barley water, if necessary, in the interval. In the bottle-fed baby the child should be given castor oil at once. If that is not retained, calomel may be substituted, and in some cases the stomach should be washed out and sometimes the lower bowel as well. For the first twenty-four hours the child should be given plain boiled water, and no attempt made to administer food, or, if it is thought desirable to give small quantities of food, dilute albumin water or barley water may be tried. It should be borne in mind that where there is much disturbance of the stomach and intestine there is usually very little digestion or absorption going on. After the first twenty-four hours the above-mentioned articles may be given at regular intervals, and, if improvement takes place, the amount may be increased and some other form of food given, such 
as malted milk or mixtures of condensed milk, or barley or rice water. As the child improves, the return may be made to its ordinary dict. This should be made with great caution, the milk being well diluted when first tried.

'I'wo mistakes are usually made in dieting diarrhora cases. One is that the cases are not promptly starved at the outset, and the second is that they are kept on a low diet too long. It must be borne in mind that, while the child may be starved for a day or two, a starvation diet lasting over long periods is not advisable; sufficient quantities of foord should be administered, care being taken to have the food in a form which can be digested and assimilated without increasing the gastro-intestinal disturbance.

Ileocolitis. - This term may be used to include the bowel conditions in which there are serious lesions in the intestine, and these are usually followed by summer diarrhoea. 'The diet is exceedingly difficult. 'The earlier stages are dieted like the acute diarrhoas; the more chronic cases should be fed on foods that leave as little residue in the intestine as possible-peptonized milk, whey, thoroughly cooked barley, or rice gruels, which sometimes may be malted to advantage. Malted milk is frequently useful. Mixtures of milk and barley water, and especially condensed milk and barley water, are of great service, and well-diluted alcohol may even be added to advantage. Broths from which the fat has been removed are valuable. Eggs may be given in various ways, and a return to the child's ordinary diet may he very gradual. It should be borne in mind that severe and often fatal relapses occur from dietary indiscretions. 'Too much food in the form of coarse and irritating vegetables may do very serious damage in these cases.

Chronic Intestinal Indigestion.- This may occur at all ages, and in bottle-fed babies is often due to the child's having been for a considerable period on a food containing too much of some one of the food elements, and frequently it is due to the use of articles of diet unsuited to the age or condition of the child. The diet in infants is usually more easily arranged than in older children. In older children an attempt should be made to determine what the child can digest and what it cannot. 'The use of peptonized or otherwise modified milk, kumiss, albumin water, or rice water, barley gruels, skimmed meat broths may all be used to advantage. Raw or rare meat seraped fine is often digested without producing any increase in the intestinal disturbance, and sometimes some of the concentrated protein foods that are on the market may be used to advantage. Later, junket and simple dishes, prepared with milk or milk and eggs, may be used. Small amounts of meat may be added, and very gradually small quantities of toast, zwieback, or crackers, and later the very soft and easily digested vegetables, the best of which are spinach, cauliflower tops, asparagus tips. As improvement progresses cream and butter may he added and other easily digested articles of food.

These cases require a great amount of patience, as they last for 
months or years, and it should be borne in mind that a change of climate always does these cases an enormous amount of good.

Constipation.-This causes a great deal of difficulty, and the most important feature in the care of constipation is the formation of correct habit. Infants as young as three months of age may be taught to have a stool regularly, and much of the difficulty experienced in both infants and older children is due to carelessness in this direction.

In infants certain additions may be made to the diet, comparing the amounts added according to the age of the child. Well-sweetened orange-juice, apple sauce, a baked apple, or prune juice, or sometimes grape-juice, may be used. Various fats and oils and the addition of more cream and the use of small quantities of olive oil, or the addition of some malted food, may be made to advantage. Sometimes the thick, sweet malt extracts are useful. Any article that upsets the stomach should not be used. Well-cooked oatmeal is often very valuable, and for children over a year of age a laxative; porridge, Graham crackers, brown bread, figs, and prunes may be used to advantage. In constipation the idea is to give a food which has a laxative effect, or a food which leaves a certain amount of residue in the large intestine, which will stimulate peristalsis. In some cases, where there is hyperacidity, the administration of alkalies is of great value. Of these, the citrate of soda and milk of magnesia are the most useful.

Rickets.-Rickets is not thoroughly understood. It most commonly occurs in the temperate zone in individuals from southern climates who have been transported north, but it is by no means limited to such individuals. Rickets may be produced experimentally by improper feeding, being produced by a diet wanting in fat, especially if the proteins at the same time are also deficient. The development of the disease is furthered by the use of too large quantities of carbohydrates along with the above, or by articles which upset the digestion. 'There seems to be an intimate connection between the absorption of lime salts and the proper amount of fat in the dietary. In the treatment of rickets the child should be put on what would be a normal diet for a child of its age and condition, with the addition of a suitable amount of fat. This may be administered in the form of cream, butter, or cod-liver oil, or some other form of oil. Crisp, fat bacon may sometimes be used. Care should be taken not to disturb the child's digestion by the ingestion of too much fat.

Diet in Pregnancy.-No especial diet is needed. It should be plentiful, nourishing in character, and about what the patient has been accustomed to under ordinary circumstances. Special diets are sometimes ordered when certain diseases are present, such as diabetes, gout. anemia, and others.

In certain conditions, where it is desirable that the child be as small as possible, as in cases of contracted pelvis and extreme obesity, and in women who have previously borne very large children, a special diet is sometimes advised, along the lines suggested by Prochownick. His results have been confirmed by Florschütz and Patton. The principle 
of the diet consists in reducing the carbohydrates and the amount of fluid given, and this diet may be begun from ten to twelve weeks before the time of clelivery. 'The diet usually contains from 140 to $160 \mathrm{gm}$. of protein, from 80 to $180 \mathrm{gm}$. of fat, and $100 \mathrm{gm}$. of carbohydrates, and 500 c.e. of fluid may be allowed daily.

Diet During the Puerperium.- There is no reason for restricting the diet after delivery, as is so frequently done. A cup of weak tea or a glass of milk may be allowed a short time after the birth of the child if there is no nausea, and after that light, easily digested food may be given at regular intervals. As a rule, the appetite is poor during the first two or three days, and the diet may be increased as the appetite returns, the ordinary diet being reached by the end of the first week in the average normal case.

If there is albuminuria or any signs of nephritis or a low urea output, the patient should be placed upon a milk or milk and cereal diet, to which bread and butter and similar articles of food may be added.

Diet in the Nursing Mother.-'The food should be that to which the mother is accustomed. It should be plain in character and well prepared, and should be well-balanced with sufficient protein and sufficient fluid. All indigestible, highly seasoned, and complicated dishes should be avoided, any abnormal craving that may be present should not be yielded to, and the mother should avoid any food which she knows, under ordinary eircumstances, disagrees with her. Very acid food should be avoided. Of great importance is a sufficient amount of exercise and fresh air.

In vigorous young women, if too much food is given, it sometimes results in unusually rich milk, or in the mothers gaining unduly in weight. If the milk is good in quality, and the quantity is below normal, it may be increased by massaging the breasts and by the adninistration of inereased amounts of fluid and the use of malt extracts. Beer, porter, or brown stout are often added to the diet to increase the quantity of milk, but should not be given unless they agree perfectly with the patient. If the milk is poor in protein, attempts may be made to increase it by increasing the protein in the diet. If the milk is poor in fat, an attempt may be made to increase the fat by feeding fatty foods to the mother, of which cream and yolk of egg are the most important.

Feeding in Fevers.--'The general conditions as regards nutrition are more or less alike in all febrile conditions; that is to say, the metabolism is increased and the digestive powers weakened. The necessity for food is really somewhat greater in any given individual during the fever, owing to the increased metabolism, while the patient's ability to take nourishment and to utilize food after he has taken it is lessened. 'There is more waste and the power of elimination is less. 'These conditions usually lead to a more or less rapid loss of weight in the patient, although, in many instances, by proper feeding, this can be partially or wholly prevented. The general indications are to support the strength of the patient, to have the feeding such that as little of the body weight is lost as possible, to see that the elimination is sufficiently free, and later,

Vou. I-25 
during convalescence, to remember that, to enable a patient to regain his normal weight, it is usually necessary to increase the feeding somewhat during this period.

It is impossible, in an article of this length, to speak of the various important factors in regard to the care of the patient and his food. Suffice it to say, that all the little matters which pertain to the sick-room play an important point in fostering the appetite of the patient. As far as possible, the patient's wishes should be consulted if he has any. The mouth should be kept clean, and the patient's position while taking food should be comfortable. Trays for the bed should be of the proper height, and, when the patient is unable to sit up, feeding-cups may be used. Everything pertaining to the food and dishes should be scrupulously clean, the food should be made as attractive in appearance as possible, and the nurse should be enjoined to remember the eternal fitness of things. Food should not be prepared in the sick-room where this can possibly be avoided, but preferably just outside of it, and, in prolonged illnesses, a small sick-room refrigerator, which can be kept near the patient's room, will be found of great service. The directions for feeding should be definite, and it is usually better to have them written so that there can be no possible mistake. Measured amounts of food should be given at definite intervals, especially where the patient is more or less unconscious, and the total amount of food given in the twenty-four hours should be sufficient to cover the needs of the patient. Foods can be calculated in calories without much difficulty, and this method affords an easy way of keeping check on the relation between the amount of food given and the patient's needs.

As a rule, patients with fever are unable to do much chewing, and the food should be such as can be taken easily and quickly. For this reason, and because of the great ease in digesting, liquid foods are usually chosen. Milk, eggs, the white of egg, meat broths, vegetable broths, milk soups, barley, and other cereal gruels, arrow-root gruel, corn-starch jellies, and meat juice, will all be found useful, and the protein content of these may be increased, if thought desirable, by the addition of some of the various preparations, as sanatogen, somatose, plasmon, eucasin, and the like. Gelatin, in the form of wine jelly, or the variously flavored fruit jellies, is useful in invalid feeding, and spares the protein to a certain degree.

In order that the elimination may be free it is important that the patient gets fair amounts of fluid, of which plain cold water is perhaps the most desirable of all. Aërated waters, especially the slightly alkaline ones, are useful in some cases, and care should be exercised in their use, as in too large quantities they may lower the patient's digestive power, and sometimes the gas accumulates in the stomach to an unpleasant degree. 'The various fruit-juices, lemon, orange, and grape, diluted with water, are found refreshing, and tea and coffee and cocon, given weak and well diluted with milk, may be used in some cases. A very useful drink, where elimination is deficient, is what is known as the imperial drink, which consists of a teaspoonful of cream of tartar, over which 
a quart of boiling water is poured; to this add the juice of a lemon, sweeten to taste, and serve cold. During convalescence a gradual return to solid food may be made. The first thing usually given is suall anounts of milk-toast or of sponge cake and milk; the broths and soups may be thickened with boiled rice or barley, or the various flours, and small] amounts of meat and vegetables may be added. Ovsters, soft-looiled eggs, well-made omelettes, well-cooked rice or other cercals may then be added, and the white meat of chicken will be fonnd the most satisfactory meat to begin on, owing to its digestibility. From this the patient may go to chops, steak, and other heavier food. Where the discase luas affected the gastro-intestinal tract, it may be necessary to exereise unusual caution in the return to solid food.

'The question of the use of alcohol in fevers is one about which there has been considerable discussion, and the swecping condemnation that it has received during recent years is doubtless undeserved. 'There can be no question but that alcohol as an invalid food was greatly abused in the past, but, on the other hand, it is the most easy of assimilation of all foods and yields 7 calories per gram. Furthermore, its effect upon the circulation in certain conditions is favorable. In acute diseases of short duration it would rarely be found necessary, but later in the more prolonged fevers, when the heart begins to lose its strength, when there is cyanosis and a tendency to congestion, it is found of great service. It may also be used in febrile conditions in the feeble and aged, and should be allowed habitures, as the withdrawal in these cases may lead to delirium. In hyperpyrexia, where little or no food is taken or assimilated, it may be found of use in tiding over a critical period. 'The dose should be varied from time to time according to the patient. It should be given in small doses and well diluted. If the pulse and general appearance improve under its use, it is a sign that it is being well borne by the patient. If the general appearance is not improved, or is made worse, it is a very good sign that it is doing harm. It should be remembered that the susceptibility of patients to alcohol differs tremendously, and that the amount given should never be large enough to produce any mental symptoms, nor should the patient smell of the drug any great length of time after it has been given. In children from $\frac{1}{2}$ to 2 ounces may usually be given in the course of twenty-four hours, and for adults, from 4 to $S$ ounces may be looked upon as a reasonable allowance in the same length of time. However, under certain conditions, this amount may be increased.

The feeding of refractory and unconscious patients may present some difficulties and may be accomplished in a number of different ways. Sometimes rectal feeding may be used, while in other cases the patient must be nourished by means of a nasal or stomach-tuhe. A sufficient number of attendants should be present in every case to iusure that the operation of feeding is done quickly and without unnecessary struggling on the part of the patient. 'The nasal tube is usually more easily' used than the stomach-tube, and should be well oiled and passed gently. back through the nares into the osophagus. Liquid food may then be 
poured through this tube. In the use of the stomach-tube a mouth-gag is necessary to prevent the patient from biting it, although a rolled-up towel or napkin in the hands of a person with experience may be substituted to great advantage. 'The tube is passed rapidly into the stomach and liquid food introduced as in the case of the nasal tube.

Typhoid Fever.-The feeding of typhoid fever is carried on along the lines outlined above, and if one has studied carefully the subject of feeding typhoid-fever patients, they are in a position to feed any of the other fevers. With certain exceptions, noted below, the food should be liquid during the entire period of fever. 'The food suggested above as being useful in fevers may all be used, and may be varied to suit the patient's requirement. Milk may be regarded as the standard diet, and, despite the fact that there has been considerable written to the contrary, it remains the most satisfactory and most available food for typhoid patients. It should be remembered that the milk should be pure, for, as Edsall has pointed out, milk teeming with bacteria is a food unsuited to typhoid patients, for it produces an increase in the symptoms. Pasteurized milk may be used to great advantage if there is any question about the character of milk used. From 1 to 3 quarts may be given daily, and, while children take milk over long periods of time with very little modification, it will be found that adults usually are unable to take milk unless it is modified in some way. This modification consists in making milk gruels by the addition of various cereals, barley water made out of barley flour being one of the best diluents, although practically any cereal gruel may be used. It may be further modified by making broths, which may be flavored with meats, oysters, or clams, the broth being strained before it is given to the patient. The addition of carbonated water agrees with some patients, and the addition of lime-water with others. Pancreatized milk may be found useful in patients of weak digestion. Gelatin may be used in typhoid patients often to advantage, as very palatable dishes may be made from it. Eggs may be added to the patient's dietary to a degree. As a general rule, they can only be used sparingly in typhoid patients. They may be used in the form of albumin water, or beaten up with milk in the form of milk-punch. Where there are digestive disturbances, it is usually necessary to place the patients upon an exceedingly simple diet, and peptonized milk will be found one of the most suitable; albumin water, barley water, and the various liquid beef preparations are also useful. In case of hemorrhage food should be withdrawn for a number of hours and the patient's gastro-intestinal tract allowed a complete rest, although small bits of ice may be given to suck to relieve thirst, or spoonful doses of cold water or cold weak tea may be used for this purpose. After some hours milk may be given in spoonful doses, and a gradual return made to the usual diet. If perforation occurs, all food should be stopped and the patient operated on at once.

'The management of the patient during convalescence from typhoid fever is of extreme importance. In the milder cases the diet should be liquid until the temperature has been normal for, at least, six or seven days, 
while for the severer cases ten days should be allowed to clapse. During this period the patient usually has a return of appetite, and sufficient soft food should be given to appease it. 'The return to solid food should be made along the lines indicated above.

Atypical and Complicated Cases.-One occasionally meets with an atypical case in which the fever is prolonged, apparently due to lack of assimilating sufficient food. In these cases feeding may be begun, even though there is some temperature. 'The diagnosis of this condition presents some difficulties. In cases where the fever is kept up by some complication, such as an abscess, the feeding need not be so careful as where it is dependent upon the typhoid process, as the restrictions of diet are made chiefly to avoid intestinal disturbance.

Scarlet Fever.-In scarlet fever the diet is of primary importance, as it is to proper feeding that one of the chief complications of scarlet fever may be almost entirely avoided. In cases that a re kept in bed, protected from cold and fed on a milk diet or a milk and cereal diet, nephritis is practically unknown. As long as the child takes milk well the diet may be restricted to milk; after that time, or where there is difficulty in getting the child to take milk, it may be given in the form of milk broths and soups flavored with various things, such as oyster broth, clam broth, and strained before they are given to the patient. Small amounts of well-cooked cereals may be allowed, with the exception of oatmeal, which should be avoided on account of its high purin nitrogen content. Milk-toast and bread and butter may also be allowed and similar articles of diet. Later on, if the diet becomes too monotonous, fruit may be allowed, using the same restrictions as would be used in the case of nephritis. Meat, beef extracts, peas, beans, and other legumens and oatmeal should be avoided. It is remarkable how free from nephritis patients properly dieted remain, and albuminuria is very much less frequent in these cases than in those in which the diet includes meats and other things.

Small-pox.- The diet in small-pox is the same as in any other fever, the only point to emphasize being that if the case is at all severe the patient may have a period of days or even of weeks in which the taking of food is apt to be very difficult, owing to the eruption in the mouth and throat, and during this period the digestion is very much lowered. 'There is also an enormous drain on the body, owing to the extensive suppuration of the pocks. In order to meet this, advantage should be taken of the period of remission which comes between the appearance of the eruption and the time when it begins to become pustular. During this period the patient usually feels comparatively well, and is able to take food, and as nutritious and generous diet should be allowed as the patient is able to take. If the patient is practically starved during this period, it makes a great difference in the way in which he goes through the hard period of the disease.

Whooping-cough.-Ordinarily, in whooping-cough the diet need not be restricted, but in some few cases it becomes a matter of primary importance. As long as the child is not vomiting and is taking its 
food reasonably well, the usual diet may be given. If the vomiting begins, the food should be liquid and given at short intervals-three hours or even less. If the child vomits one meal, it should be repeated shortly after one of the paroxysms, as immediately following the attack of coughing there is usually a period of quiet. Sometimes the application of a broad elastic belt over the abdomen will lessen the vomiting and facilitate the retention of foods.

Diphtheria.-The feeding of diphtheria may present some difficulties, owing to the patient's inability to swallow with ease, but, as a rule, he is able to take liquid food without much difficulty. Where the food causes pain, it should be of a very bland character, and is often given very cold. In cases in which an intubation has been done it may be difficult to feed the child for a day or two, until it learns that it is able to swallow as well with the tube in as with it out. If there is any difficulty experienced in swallowing, the Castleberry method may be used, which consists in having the patient suck the food through a tube, having the head lower than the rest of the body. In small children this is easily accomplished by having the patient lying in the lap in this position, while in older children the head may extend over the edge of the bed. Sometimes, where liquids are taken with difficulty, some solids, such as baked custards, omelettes, milk-toast, and the like, are easily taken. If paralysis of the muscles of deglutition occurs, the food may return through the nose, or sometimes swallowing may become impossible. In these cases feeding may be done at regular intervals, preferably by using a stomach or a nasal tube, or rectal feeding may be employed.

Asiatic Cholera.-During an epidemic of Asiatic cholera it should be remembered that many cases come from drinking contaminated water, or by taking infected milk or raw vegetables. For this reason all water should be boiled and all food thoroughly cooked, and all liquids that are taken should also be boiled. Raw food of all kinds should be avoided during the period of danger of infection. During the diarrhœal stage of the disease food should be given in small quantities, and of such character that it leaves a little residue in the intestine. Albumin-water, beef-juice, predigested beef solutions, barley water or rice water, or whey will be the best things to use. Milk is usually best avoided at this time. Weak tea, to which a little red wine is added, is often of value in checking the thirst. In the second stage, where there is more or less continuous vomiting and purging, no food should be given, as it will not be retained, and thirst should be allayed by the use of small quantities at frequent intervals of ice-cold water, which may be acidulated with dilute phosphoric or sulphuric acid, or small quantities of cold lemonade or lime-juice and water may be given, and sometimes carbonated water, and at other times small quantities of tea or coffee may be utilized to advantage. After the vomiting ceases very small quantities of food may be administered, albumin-water and cereal gruels being the best to begin on, and later peptonized milk, and then other easily digested articles of food may be added, remembering that the stomach remains irritable for days or even weeks after the attack, and 
that diarrhoea is easily caused by indiscretions in the diet. Convalescence is managed much in the same way as that of typhoid fever.

Tetanus. - Many cases of tetanus die from exhaustion, and the feeding of these cases is one of the most difficult problems in the treatment. In the milder cases fluid food is usually given without a great deal of diffieulty, as there is, as a rule, space enough between the teeth for it to pass, or, if the patient happens to have lost a tooth, the food passes through the jaws easily. If the teeth are very regular and fit closely together the liquids will not pass between them; nasal feeding or rectal feeding may be tried. Partially peptonized milk, with or without the addition of eggs, or cereals are usually found most scrviceable. 'The chicf difficulty in feeding the tetanus cases consists in the faet that as soon as the patient is approached or touched, especially in the severer cases, there is a convulsion which stops any attempt at feeding, and this condition of irritability sometimes persists over a considerable period of time. In these cases, if the patient cannot be quieted by the use of sedatives, chloroform may be administered in sufficient quantities to permit food being given.

Rabies.-As rabies is always fatal, and as attempts to feed the patient only result in increasing the suffering, it is usually best not to try to administer food in any form. Convulsions are frequently brought on by any attempt to do so, and the patients are usually unable to swallow, even if they do succeed in getting food in the mouth, which is rare.

Tuberculosis.-The feeding in tuberculosis is one of the most important parts of the treatment of the disease. One of Detweiler's favorite expressions was: "My kitchen is my pharmacy." The general aim is to increase the resistance of the patient, as far as possible, to improve the general condition, and, by doing this, to increase the recuperative powers. Tuberculosis is a disease of such numerous manifestations, and the conditions under which the patients live differ so much, that it is impossible to detail the diet for all the cases that one may meet. Special forms of tuberculosis may require special forms of dieting, as when the intestinal tract is affected, but, in a general way, what is said about the feeding of cases of pulmonary tuberculosis will apply to all the other forms. It should be borne in mind at the outset that the individual and the conditions under which he lives should be carefully considered, and the advice given each patient suited to his pocket-book and station in life. It is too often the fact that the patient is given advice which is useless owing to his inability to purchase the articles suggested. Great attention should be paid to the teaching of these patients, or their families, how to purchase food and how to prepare it.

Exact dietary standards for tuberculosis cannot be stated at this time, although those suggested by Bardswell and Campluell seem to meet the requirement better than any other. In order to determine the diet for the average case of pulmonary tuberculosis the normal diet of that individual in health should be considered, both in reference to the number of calories per day and also as to the proportion of fat, carbohydrate, and 
protein. It has been found that for the average-sized individual the protein should be increased from 25 to 30 per cent. over his ordinary physiologic needs, and this brings the average daily amount up to $150 \mathrm{gm}$. This is one of the most important points in the treatment, as patients who are getting an insufficient amount of protein have a lowered resistance in tuberculosis, and this cannot be increased by merely increasing the carbohydrates and fats. There may be an increase, too, in the carbohydrate and fat given to about 30 per cent., either in one or the other or in both combined, and this should be continued until the patient has gained a few pounds over his normal weight. when a decrease to about 15 per cent. may be made. Standards for average-sized individuals work out to about 3200 calories a day for men, containing $150 \mathrm{gm}$. of protein, and about 2700 calories per day for women, containing $128 \mathrm{gm}$. of protein. These standards are perhaps a little lower than those suggested by various writers in previous years.

Overfeeding patients does not produce as satisfactory results as proper feeding. During the past years, since diet has been recognized as one of the important means of treating tuberculosis, many errors were permitted in this line, both physicians and patients thinking that if a slight increase in food were good, a great increase must be better, and so many patients were stuffed far beyond their capabilities of utilizing the food given, with the result that gastro-intestinal disturbances were frequent, and what was popularly called bilious attacks also occurred. If the patient had a good alimentary tract in many instances the gain in weight was too great, and a fat, flabby, inert mass resulted. There is no advantage in going over $150 \mathrm{gm}$. of protein for the average sized individual.

The next point to be considered is how this increase is to be obtained, and if one realizes that 3 pints of milk contain 1070 calories, $50 \mathrm{gm}$. of protein, $60 \mathrm{gm}$. of fat, and $75 \mathrm{gm}$. of carbohydrates, and by adding this amount in addition to such ordinary diet as most well-to-do people take, the result is practically accomplisbed, or, if somewhat less milk is taken, the difference may be made up by increasing the amount of meat or by adding several eggs. Meat is a very important part of the diet, and about 10 ounces a day, as purchased, should be allowed for each patient, and, where it is possible to get the patient to take raw meat, it will be found of a distinet advantage, given very finely chopped or scraped. Many patients take it readily in the form of sandwiches. The fats may be increased by the use of cream, which may be taken over cereals and over desserts, by the free use of butter, and sometimes, especially if it does not upset the patient's digestion, by the use of cod-liver oil. Various emulsions of oils have also been suggested, but probably have no especial advantage over cream. For poorer people the protein increase may sometimes be made to advantage by increasing the amount of peas, beans, and lentils eaten. 'The greatest objection to using the vegetable proteins is that in so doing we increase the bulk of the diet considerably, and throw more strain on the intestinal tract, which many patients are 
unable to stand. It should be borne in mind that sweets should be reduced, as patients who have poor appetites almost invariably dislike sweet things, or, if they take them, they may produce a feeling of saticty which prevents their taking other food.

Hemorrhage from the Lungs. - In cases of hemoptysis, if slight, the fluid should be restricted, and there should be a slight restriction in the amount of solid food given. Where there has been severe hemorrhage an effort should be made to reduce the blood-pressure, and this is perhaps best accomplished by giving but little flud and by having whatever food is administered of as small bulk as possible. After severe hemorrhages thirst may be relieved, to a certain extent, by allowing the patient to suek small pieces of ice, and milk may be given in very small quantities. After two or three days of low diet, an inerease may be male, and this may be kept up from day to day until the patient gets back on his regular diet-about the tenth day.

Tuberculosis of the Intestine.-Where the intestines are involved, an effort should be made to lessen peristalsis by giving the patient food which leaves but little residue. The bulk should be as small as possible and fluid should be given in limited quantities. All these things tend to prevent irritation and to make the patient more comfortable. Milk should be so modified as to prevent the formation of large curds, and should be mixed with cereal gruels or be peptonized. Raw meat, eggs, the softer varieties of cheese, butter, and cream, zwieback, toast and crackers, custards, cornstarch puddings, blanc mange, milk and egg puddings will all be found suitable foods in these cases. Food may be given in small quantities at rather more frequent intervals than for the ordinary patients. Patients should avoid soups, should not take cereals in too great quantities at any one time, should avoid oatmeal, all the coarse vegetables, potatoes should be used sparingly, if at all, and green vegetables and fruit given in small quantities and usually not at all.

Gastric Irritability.--This may be avoided to a certain extent by seeing that the patient does not take nauseating drugs and that he does not swallow his sputum. If the stomach is very irritable, the patient is best placed at rest, given liquid diet, small quantities at a time. Milk that has been peptonized or diluted, or buttermilk or kumiss, is very serviceable. Albumin-water is useful, and sometimes fresh meat juices and broths may be given to advantage.

Alcohol in Tuberculosis.-As a rule, alcohol is best avoided in all forms, but oceasionally it may be found useful in habitues, whose? powers of assimilation will not work without it, and sometimes small quantities in anorexia and gastric irritability will be found useful. In late cases of tuberculosis, where recovery is impossible, it is frequently of considerable service in rendering the patient more comfortable.

Diet in Diseases of the Lungs.--There are no specific diets to be used in diseases of the lungs, and yet diet is of considerable importance, as much can be done toward lessening the intensity of certain symptoms, 
and also in relieving the discomfort of the patient. The chief point in view is to avoid flatulence, so common in the acute lung troubles, and so disagreeable and dangerous to the patient.

'The diet in tuberculosis has been considered elsewhere.

Acute Bronchitis.-The diet in this condition is like that of any acute fever; the same general plan of diet should be carried out as suggested for the ordinary febrile diseases. In the dry stage much relief may often be given by the free use of hot drinks. Hot lemonade, with or without the addition of barley water, is one of the best preparations to use. Very weak hot tea may be substituted if desired, and the oldfashioned remedy, which is passing into oblivion, linseed tea.

In chronic bronchitis, particularly forms occurring in fat people, much can be done toward relieving the frequency of the exacerbations by restricting the diet of the patient as suggested for obesity. Bronchitis occurs with great frequency in the obese, and a carefully undertaken reduction cure very often improves the bronchitis to a remarkable degree.

Lobar Pneumonia.-The diet in this condition is like that of any fever, and, as the disease is usually of short duration, the necessity for supplying the patient's every need is not an urgent one. As a matter of fact, they do better on rather a light feeding than otherwise. Easily digested liquid foods, that leave very little residue in the intestine, are to be chosen. Of these, milk, which should be peptonized or modified in some way, meat soups, soups with egg added, will all be found valuable. Weilcooked barley water, if it doesn't cause flatulence, may also be used. As a rule, farinaceous foods are to be avoided, as they tend to the production of gas, and the pressure of this on the lungs may impede the breathing very materially. Food should be given in small amounts and at short intervals of two or three hours. Well-nourished, vigorous patients, even if they do not take much, do not especially suffer for want of food, but weak subjects, and those who have no reserve to draw on, should be carefully looked after as regards the amount taken. Sufficient amount of fluid should be given to favor excretion.

The use of alcohol in pneumonia is still a moot point, and it is a question of individualizing, using it when the patient improves under its use and avoiding it in susceptible subjects. As a rule, it will be found of service where the pulse is small, irregular, and rapid, where the first sound is indistinct, where there is a dry mouth and tongue, and delirium. It is not needed in patients with a full, bounding pulse.

Pleurisy.-The lost diet for the pleuritic patient is to make it as nutritious as possible, the character of the food being varied according to the amount of fever present and the digestive powers. Dry diets have been suggested, but are apparently of very little practical value.

Asthma.- In a certain number of cases of this curious disease food plays a very important part. A certain number of cases seem to be due to food idiosyncracy, and, where the article or articles of diet which bring on the attacks can be discovered, the attacks may be lessened by 
elininating these things from the dietary. 'The other cases are due to obscure disturbances of metabolism, in which the patient is unable to utilize normal amounts of carbohydrates or of fats or of protein, sometimes one, sometimes one of the others. In these cases it is often difficult to find out what is causing the tromble. 'The best plan for any asthmatic patient is to be put on a diet of plain nutritious ford, aroiding all highly seasoned, indigestible, and complicated dishes. If the attanchs still continue, try the effect of cutting first the sugars and lessening the starchy foods over a sufficient period of time, to see whether the attacks are lessened. If they are not, the articles of diet so cut out may be allowed and the fats reduced; if they in turn are not at fault, the proteins.

Gouty individuals are prone to asthma, and these individuals should be placed upon the diet recommended for the gouty, and should be given plenty of exercise, if possible. In younger individuals, in which the asthma is more or less associated with their taking on weight rapidly, the effect of the reduction cure may be tried.

Diet in Cardio-vascular Disease.-In individuals with heart lesions, in whom there is perfect compensation, the diet should be that of the average normal individual, the only care being to avoid excesses of all kinds and at all times. When bordering on the line of broken compensation, extreme care must be used, as serious results may follow overindulgence in food or drink, or attacks of indigestion, especially those accompanied with flatulence. Feeding in cases where the compensation is broken is a difficult problem, the patients often having little or no appetite and suffering considerably with gastric disturbance.

In cases of myocarditis the welfare and the comfort of the patient depend very largely upon the care with which he is fecl. 'The food in heart disease should be simple in character and carefully prepared. 'The meals should be small, not more taken at one time than can be perfectly digested, and sufficient time should be allowed between meals. 'The meals should be about the same weight, the one in the middle of the day being slightly larger than the others. Care should be taken to avoid flatulence and gastric disturbance, which are liable to be brought on by the meals being too large, by indigestible artieles of food, or by too large quantities of carbohydrates, especially sweets. 'The amount of fluid taken with the meals should be restricted, and thirst relieved by the use of hot water apart from the meals. As a rule, alcohol is not allowerl, but in certain cases in habitués small quantities may be used with bencit. When food is taken in very small quantities, the meals may be brought closer together. When there is gastric disturbance or nausea, complete rest and the use of a milk diet will often afford relief.

In arteriosclerosis the diet should be somewhat the same as in heart disease, and in the severer cases the diet, consisting largely of milk, is usually borne better than anything else.

In aneurysm special diets have been recommended, consisting chiefly of great restrictions in the amount of food, and especially in the amount of fluid while the patient is at absolute rest, the idea being to lower the 
blood-pressure, and so favor the regulation of the blood in the aneurysm itself. 'The results of these diets are not very encouraging.

In angina pectoris the diet is of extreme importance. The patient should be enjoined to lead a simple life, to eat sparingly the plainest varieties of foods, and to avoid excesses of all kinds, as many fatal attacks follow overindulgence in food or drink.

Diet in Diseases of the Blood.-Pernicious Anemia.-The feeding of these patients is a matter of considerable difficulty, owing to the presence of gastric disorders and also intestinal disturbances. As a rule, it will be found that the milk and carbohydrate diet is borne rather better than any other, but the results are unsatisfactory, no matter what plan is followed. It has been suggested that bone-marrow from the long bones be taken raw, in daily doses of from 1 to 3 tablespoonfuls.

Chlorosis and Secondary Anemia.-The diet of these two conditions is essentially the same, and, in addition to the diet, iron should be given in some palatable form. The meals should be small, not more than the patient can digest at one time being given. They should be given at regular intervals and nothing allowed between meals. Care should be taken that the patient does not ruin her appetite by the eating of various indigestible articles, for which there is often an unusual craving. Meat and eggs should be given two or three times a day as well, as the usual constituents of an ordinary diet, in addition to fruit and green vegetables. Milk mav be given, but is usually used in these cases in too large quantities. It should be given at the end of the meal, as a glass of milk taken the first thing usually destroys the appetite in these cases. A glass of milk may also be given at bedtime and a cupful of hot milk early in the morning. Small amounts of beer, porter, or red wine are often of service.

Diet in Gout.-Gout is caused by excessive quantities of food and too little exercise, by the overindulgence in alcohol, and there is also a marked hereditary influence, the tendency to acute gout being directly transmitted by individuals who have transgressed the rules of right living. The disease is also furthered by indigestion and constipation, and may be induced by certain poisons, chief of which is lead. Gout is in some way closely associated with the formation of uric acid, but the theories about this replace each other so rapidly, one upsetting the other, that it is scarcely worth the time of the practising physician to follow them. Further studies are needed along this line to clear up the question, especially concerning the purin nitrogen and its relations to the formation and excretion of uric acid. The purin bodies are found in meats, and especially the glands, sweet-breads, and liver, and fish, in the legumes, such as peas, beans, and lentils, asparagus, onions, mushrooms, oatmeal, and also in coffee, tea, and chocolate. In the present state of our knowledge, it would seem a good idea to limit the ingestion of these articles which are forerumners of uric acid.

In acute gout the diet should be limited to milk or milk and cereals. The amounts should be small, and, as a rule, there is not much difficulty experienced during this time. 
In dieting patients with chronic gout the problem is exceedingly difficult. In order to succeed, it is necessary to study the needs of the individual patient, as gouty patients vary enormonsly in the way in which they will be able to utilize the various foods. One cannot lay down hard-and-fast rules. An effort should be made to preserve a normal digestion, to insist upon the patient chewing his food well, and to choose articles of diet such as the patient finds easy of digestion. Food should be simple in charaeter, the meals small enough to be perfeetly digested, or as perfectly as possible. Iarge meals are liable to upset the digestion and lead to imperfect metabolism, and the same is true of indigestible articles in the diet. Meat may be allowed once daily, and fish may be used in addition if desired. Milk is a very valuable food. Vegetables may be used, especially those that the patient finds he is able to digest well, although it is usually necessary to restrict somewhat the use of starchy vegetables. Fats may be used in moderation, and fruits, green vegetables, aceording to the patient's desires and idiosyncracies. Sweets should be given very sparingly, if at all. All rich, complicated, highly seasoned dishes should be avoided, and alcohol is generally forbidden. Most gouty patients are alcoholic habitues, and follow their own desires in this regard, more or less, despite the commands of the physician. Care should be taken to avoid hyperacidity, which is usually produced by indigestible articles of diet and by overindulgence in sweets.

Nephritis.- There are various diseases of the kidney in which diet plays a very important role in the treatment. 'The most common of these are the various forms of nephritis. It should be remembered that too much stress is usually placed upon albuminuria in these cases, and that, where it is used as a guide, the amount in a twenty-four-hour specimen should always be estimated.

In cases of acute nephritis the albuminuria is a more or less reliable guide as to the condition of the kidney, and in the subacute conditions it is also a fair guide, while in the chronic conditions the albumin furnishes but little information. There is at present no easy method of estimating the excretory power of the kidney. It should also be borne in mind that changes in the food may cause temporary albuminuria in individuals with diseased kidneys. 'To the skilled elinician the general appearance of the patient is the most reliable guide, and the amount of latent edema, which may be determined by weighing the patient, may also furnish valuable information.

In general, it may be stated that food given to individuals with diseased kidneys should be easy of digestion, and, at the same time, of such nature that the end-products of metabolism are excreted without difficulty, and those artic'es whose end-products.are excreted with difficulty should be avoided as far as possible. Among the articles whose end-products are excreted with difliculty may be mentioned the browned surfaces of grills and roasts, strong sauces, spices, tea and coffee, and foods containing hippuric acid, which is found in some of the green vegetables, fruits with kernels, and cranberries. While the uric acid is rela- 
tively well excreted, it may produce toxic effects at times, so that it is a good plan to include in the forbidden list all the foods containing purin bodies in large amounts, such as glands, kidney liver, sweet-breads, very strong meat broths, as well as oatmeal and the legumes. This whole subject of the excretion of the end-products of foods is a matter for further study.

As a rule, alcohol is best avoided, but in certain cases, with cardiac weakness and nausea, it may be of value. As regards the use of water, it should be borne in mind that in the early stages of acute nephritis, where the excretion of urine is greatly reduced or is excreted with great diffieulty, but small amounts of fluid should be given during this period, as it only increases the edema and throws an extra burden upon the heart. As soon as the amount of urine passed is increased, and the water is excreted easily, the amount may be increased, and at this time the water acts as a very good diuretic, carrying with it the products of metabolism.

In acute nephritis, where there is nearly complete anuria, but very small amounts of food should be attempted, small quantities of milk, small amounts of ice to quench the thirst, or very small quantities of water. In cases where the heart is failing, brandy or whisky in small, infrequent doses may be tried. Such a diet cannot be continued long, but at the end of a few days the patient is either dead or in a very much improved condition. In cases where the amount of urine is reduced, if the patient's condition is not immediately dangerous, the milk diet is the most satisfactory. A litre and a half of milk a day, with the addition of 350 c.c. of sweet cream, may be given, and it should be remembered that excessive amounts of fat should not be administered to patients with nephritis, and if more food is desirable, either on account of the patient's appetite or general condition, cereals, except oatmeal, crackers, and zwieback, or bread and butter, and similar articles may be given. Grape-juice may also be allowed, usually to advantage. The amounts of these articles may be increased, and later on digestible meats and the more easily digested vegetables may be given, as well as various gelatin preparations. 'The Imperial drink, which consists of cream of tartar, lemon-juice and water, is much used as a beverage where a mild diuretic is required, and small amounts of carbonated waters or mineral waters may be used. Milk may be modified, as suggested above, if necessary. A return to the normal diet should be gradual, the amounts of protein food being limited to the needs of the patient, bearing in mind the low limits which have been determined by Chittenden.

Diet in Diseases of the Stomach.-In the treatment of gastric disorders the greatest care must be taken as to the selection of a proper diet, this being even more important than the administration of drugs. In the selection of the diet the food prescribed must be regulated, not only in regard to the particular disease with which the patient is affected, but the taste and peculiarities of the individual must be considered. Changes in the diet may often be necessary, and must be gradually and cautiously made in conformity with the patient's power to digest 
food. While it is a simple matter to determine the digestibility of food under normal conditions, as the motor and secretory functions of the stomach are normal, and the effect of various fool-stuffs upon either of these functions is easily ascertained, it becomes more difficult in the various gastric disturbances, as either or both of these functions may be disturbed.

In arranging a diet-list for patients affected with gastric disturbance, it must be remembered to have it contain food in a very digestible form, in order to lessen the work of the stomach, and of such a character as to increase the nutrition of the patient. 'The well-known Leube's diet scale, arranged so as to present the various foods in the order of their digestibility, forms the basis of the well-known Leube ulcer cure, and is of great service in other conditions as well.

Leube's Diet Scale.-Dict I.-If the digestion is very much reduced, the following articles of food are most easily digestible: bouillon, meat solution, milk, raw or soft-boiled eggs.

Dict II.-Somewhat less digestible than Diet I are the following articles of food: boiled calves' brain, boiled thymus, boiled chicken and pigeon. The different forms of meat are enumerated in the order of their digestibility. Gruels, and in the evening milk mushes made with tapioca and white of egg, may also be placed in this list. The majority of patients can digest boiled calves' feet in addition to the various meat foods already enumerated.

Diet III.-If Diet II is well borne, Diet III may be given. 'This consists in adding cooked or raw beef to Diet I. Leube gives the following method of preparing beefsteak and believes that beef cooked in this way is very easily digested: 'The meat should be kept for some time, and is then scraped witl a dull spoon; in this way a pulp is obtained, consisting only of the delicate parts of the muscle, and not containing any of the tough, hard, and sinewy portion. 'This pulp is roasted in fresh butter. Raw ham is also to be recommended. In addition to meat a small quantity of mashed potatoes may be given, some stale wheat bread, and small amounts of coffee or tea with milk (cantiously).

Diet $I V$.- This list is so arranged that if the patient can digest the articles of food mentioned under this head for some time, he can then begin with his usually accustomed diet: Roast chicken, roast pigeon, venison, partridge, roast-beef-medium to raw (particularly cold), veal (from the leg), pickerel, boiled shad (trout, even when young, is very difficult to digest), macaroni, bouillon with rice. Snall quantities of wine may be taken one to two hours before eating; gravies are contraindicated. Young and finely chopped spinach is allowable; other vegetables, such as asparagus, may be tried cautiously, although I fube considers this a rather risky procedure. After this fourth diet the patients are allowed to take a more liberal diet, but the increase shculd be gradual. They should refrain from eating vegetables, salads, preserves, and fruits for some time; and when they are resumed, a baked apple is the first of these articles to be eaten.

In this connection, too, the tables given by Penzoldt are most valu- 
able. His results were obtained by determining the exact time which the stomach required to empty itself after partaking of a given quantity of a particular food:

\section{One to two hours:}

100-200 gm. pure water.

$220 \mathrm{gm}$. carbonated water.

$200 \mathrm{gm}$. tea, alone.

$200 \mathrm{gm}$. coffee, alone.

$200 \mathrm{gm}$. cocoa, alone.

$200 \mathrm{gm}$. beer.

$200 \mathrm{gm}$. light wines.

$100-200 \mathrm{gm}$. boiled milk.

$200 \mathrm{gm}$. meat broth, alone.

$100 \mathrm{gm}$. eggs, soft.

Two to three hours:

$200 \mathrm{gm}$. eoffee with eream.

$200 \mathrm{gm}$. cocoa with milk.

$200 \mathrm{gm}$. Malaga wine.

$200 \mathrm{gm}$. "Ofner" wine.

$300-500 \mathrm{gm}$. water.

300-500 gm. beer.

$300-500 \mathrm{gm}$. boiled milk.

$100 \mathrm{gm}$. eggs, raw and serambled, hard-boiled or as omelet.

$100 \mathrm{gm}$. beef-sausage, raw.

$250 \mathrm{gm}$. calves' brains, boiled.

$250 \mathrm{gm}$. calves' thymus, boiled.

$72 \mathrm{gm}$. oysters, raw.

$200 \mathrm{gm}$. carp, boiled.

$200 \mathrm{gm}$. pike, boiled.

$200 \mathrm{gm}$. shellfish, boiled.

$200 \mathrm{gm}$. cod, boiled.

$150 \mathrm{gm}$. cauliflower, boiled.

$150 \mathrm{gm}$. cauliflower, as salad.

$150 \mathrm{gm}$. asparagus, boiled.

$150 \mathrm{gm}$. potatoes, boiled in salt water.

$150 \mathrm{gm}$. mashed potatoes.

$150 \mathrm{gm}$. stewed eherries.

$150 \mathrm{gm}$. raw cherries.

$70 \mathrm{gm}$. white bread, old or fresh, dry or with tea.

$70 \mathrm{gm}$. pretzels.

$70 \mathrm{gm}$. zwieback, fresh or stale, dry or with tea.

$50 \mathrm{gm}$. Albert biscuits.
Three to four hours:

$230 \mathrm{gm}$. young chicken, boiled. $230 \mathrm{gm}$. partridge, boiled.

220-260 gm. pigeon, boiled.

$195 \mathrm{gm}$. pigeon, fried.

$250 \mathrm{gm}$. beef, raw, boiled, lean.

$250 \mathrm{gm}$. calves' feet, boiled.

$160 \mathrm{gm}$. ham, boiled.

$160 \mathrm{gm}$. ham, raw.

$100 \mathrm{gm}$. veal, warm and cold, lean.

$100 \mathrm{gm}$. beefsteak, broiled, cold or warm.

$100 \mathrm{gm}$. beefsteak, raw, seraped.

$100 \mathrm{gm}$. tenderloin.

$200 \mathrm{gm}$. Rhine salmon, boiled.

$75 \mathrm{gm}$. caviare, salted.

$200 \mathrm{gm}$. sardines in vinegar, kippered lerring.

$150 \mathrm{gm}$. blackbread.

$150 \mathrm{gm}$. barley bread.

$150 \mathrm{gm}$. Wheat bread.

100-150 gm. Albert biscuits.

$150 \mathrm{gm}$. potato, as vegetable.

$150 \mathrm{gm}$. rice, boiled.

$150 \mathrm{gm}$. kohlrabi, boiled.

$150 \mathrm{gm}$. carrots, boiled.

$150 \mathrm{gm}$. spinach, boiled.

$150 \mathrm{gm}$. eucumber salad.

$150 \mathrm{gm}$. radislies, raw.

$150 \mathrm{gm}$. apples.

\section{Four to five hours:}

$210 \mathrm{gm}$. pigeon, broiled.

$250 \mathrm{gm}$. fillet of beef, broiled.

$250 \mathrm{gm}$. beefsteak, broiled.

$250 \mathrm{gm}$. beef tongue, smoked.

$100 \mathrm{gm}$. smoked beef in slices.

$250 \mathrm{gm}$. hare, broiled.

$240 \mathrm{gm}$. partridge, broiled.

$250 \mathrm{gm}$. goose, broiled.

$280 \mathrm{gm}$. duek, broiled.

$200 \mathrm{gm}$. herring, salted.

$150 \mathrm{gm}$. lentils, mashed.

$200 \mathrm{gm}$. peas as purée.

$150 \mathrm{gm}$. string-beans.

By a careful study of these details much information can be gained as to the foods which are suitable in a given case of disease in which careful dietetic treatment is essential. 
Penzoldt formulated this series of diet-lists, based upon the principle just described, which, for the most part, agree with Leube's diet-list:

\section{Penzoldit's Difi-listy}

Diet I. (about Ten Days)

\begin{tabular}{|c|c|c|c|c|}
\hline Food or drink. & $\begin{array}{c}\text { Largest } \\
\text { quantity to } \\
\text { be taken at } \\
\text { one time. }\end{array}$ & $\begin{array}{l}\text { Method of } \\
\text { preparation. }\end{array}$ & $\begin{array}{l}\text { Special require- } \\
\text { ments. }\end{array}$ & Ifow to be caten. \\
\hline Meat broth..... & $250 \mathrm{gm}$. & & $\begin{array}{l}\text { Without fat, or not } \\
\text { salted. }\end{array}$ & Slowly. \\
\hline Cows' milk..... & $250 \mathrm{gm}$. & $\begin{array}{l}\text { Well boiled or ster- } \\
\text { ilized. }\end{array}$ & $\begin{array}{l}\text { Entire milk (or } \\
\text { lime-water 1; milk } \\
3 \text { ). }\end{array}$ & $\begin{array}{l}\text { If desired, with a little } \\
\text { tea. }\end{array}$ \\
\hline Eggs.......... & 1 or 2. & $\begin{array}{l}\text { Very solt, just } \\
\text { heated, or raw. }\end{array}$ & Fresh. & $\begin{array}{l}\text { If taken raw, should be } \\
\text { atirred into the warm, } \\
\text { not boiling, meat broth. }\end{array}$ \\
\hline $\begin{array}{l}\text { Meat solution } \\
\text { (Leube-Rosen- } \\
\text { thal). }\end{array}$ & $30-40 \mathrm{gm}$. & $\ldots \ldots \ldots$ & $\begin{array}{l}\text { Should have only a } \\
\text { slight meat broth } \\
\text { odor. }\end{array}$ & $\begin{array}{l}\text { In teaspoonful doves. } \\
\text { stirred in meat broth. }\end{array}$ \\
\hline $\begin{array}{l}\text { biscuits) } \\
\text { Water............ }\end{array}$ & $\begin{array}{l}6 \\
1 \text { liter. }\end{array}$ & $\ldots \ldots \cdots \cdots$ & $\begin{array}{l}\text { Without sugar. } \\
\text { Ordinary water or } \\
\text { natural carbon- } \\
\text { ated water with a } \\
\text { small percentage } \\
\text { of } \mathrm{CO}_{2} \text { (seltzer). }\end{array}$ & Not too cold. \\
\hline
\end{tabular}

Diet II. (about Ten Days)

\begin{tabular}{|c|c|c|c|c|}
\hline Calves' brain.... & $100 \mathrm{gm}$. & Boiled. & Freed from & Best taken in meat broth. \\
\hline Thymus (calt).. & $100 \mathrm{gm}$. & Boiled. & membranes. & \\
\hline Pigeon & 1 & Boiled. & $\begin{array}{l}\text { Only if young, with- } \\
\text { out skin tendons, }\end{array}$ & Best taken in meat broth. \\
\hline $\begin{array}{l}\text { Chicken ..... } \\
\text { Raw beef.... }\end{array}$ & $\begin{array}{l}\text { As large as } \\
\text { a pigeon. } \\
100 \mathrm{gm} .\end{array}$ & $\begin{array}{l}\text { Boiled. } \\
\text { Chopped fine or } \\
\text { scraped, with a }\end{array}$ & $\begin{array}{l}\text { As above (no fat- } \\
\text { tened chicken). } \\
\text { From the tender- } \\
\text { loin. }\end{array}$ & $\begin{array}{l}\text { Best taken in meat broth. } \\
\text { To be eaten with cakes. }\end{array}$ \\
\hline $\begin{array}{l}\text { Raw beef-saus- } \\
\text { age......... } \\
\text { Tapioea....... }\end{array}$ & $\begin{array}{l}100 \mathrm{gm} . \\
30 \mathrm{gm} .\end{array}$ & $\begin{array}{l}\text { Without any addi- } \\
\text { tions. } \\
\text { Boiled with milk to } \\
\text { make gruel. }\end{array}$ & Smoked a little. & To be eaten with cakes. \\
\hline
\end{tabular}

\section{Diet III. (about Eight Days)}

\begin{tabular}{|c|c|c|c|c|}
\hline Pigeon ......... & 1 & $\begin{array}{l}\text { Broiled with fresh } \\
\text { butter. }\end{array}$ & $\begin{array}{l}\text { Only, young bird, } \\
\text { skin, etc. }\end{array}$ & Without gravs: \\
\hline Chicken. & i & $\begin{array}{l}\text { Broiled with fresh } \\
\text { butter. }\end{array}$ & $\begin{array}{l}\text { Ouly young birl, } \\
\text { shin, etc. }\end{array}$ & Without gravs: \\
\hline Beefstenk. & $100 \mathrm{gm}$. & $\begin{array}{l}\text { With fresh butter } \\
\text { half rare (Fing- } \\
\text { lish). }\end{array}$ & $\begin{array}{l}\text { Frm the tender- } \\
\text { loin, well beaten. }\end{array}$ & Without gravy: \\
\hline Ham... & $100 \mathrm{gm}$. & & $\begin{array}{l}\text { Smaked a little, } \\
\text { without the bone. }\end{array}$ & With white bread. \\
\hline $\begin{array}{l}\text { Milk bread, } \\
\text { Zwieback, or } \\
\text { Frieberger } \\
\text { pretzels. }\end{array}$ & $50 \mathrm{gm}$. & Crisped, bakedl. & $\begin{array}{l}\text { Stale (so-called } \\
\text { rolls, etc.). }\end{array}$ & $\begin{array}{l}\text { To be carefully masti- } \\
\text { rated and well sali- } \\
\text { vated. }\end{array}$ \\
\hline I'otatoes........ & $50 \mathrm{gm}$. & $\begin{array}{l}\text { (a) Mashed: (b) } \\
\text { boiled in ealt } \\
\text { water and } \\
\text { mashed. }\end{array}$ & $\begin{array}{l}\text { The potatces should } \\
\text { be mealy and } \\
\text { crumble on crush- } \\
\text { ing. }\end{array}$ & \\
\hline Cauliflowe & $50 \mathrm{gm}$. & $\begin{array}{l}\text { As a vegetable } \\
\text { boiled in salt } \\
\text { pater. }\end{array}$ & $\begin{array}{l}\text { Use only the flow- } \\
\text { ers. }\end{array}$ & \\
\hline
\end{tabular}


Penzoldt's Diet-Lists

Diet IV. (about Eight to Fourteen Days)

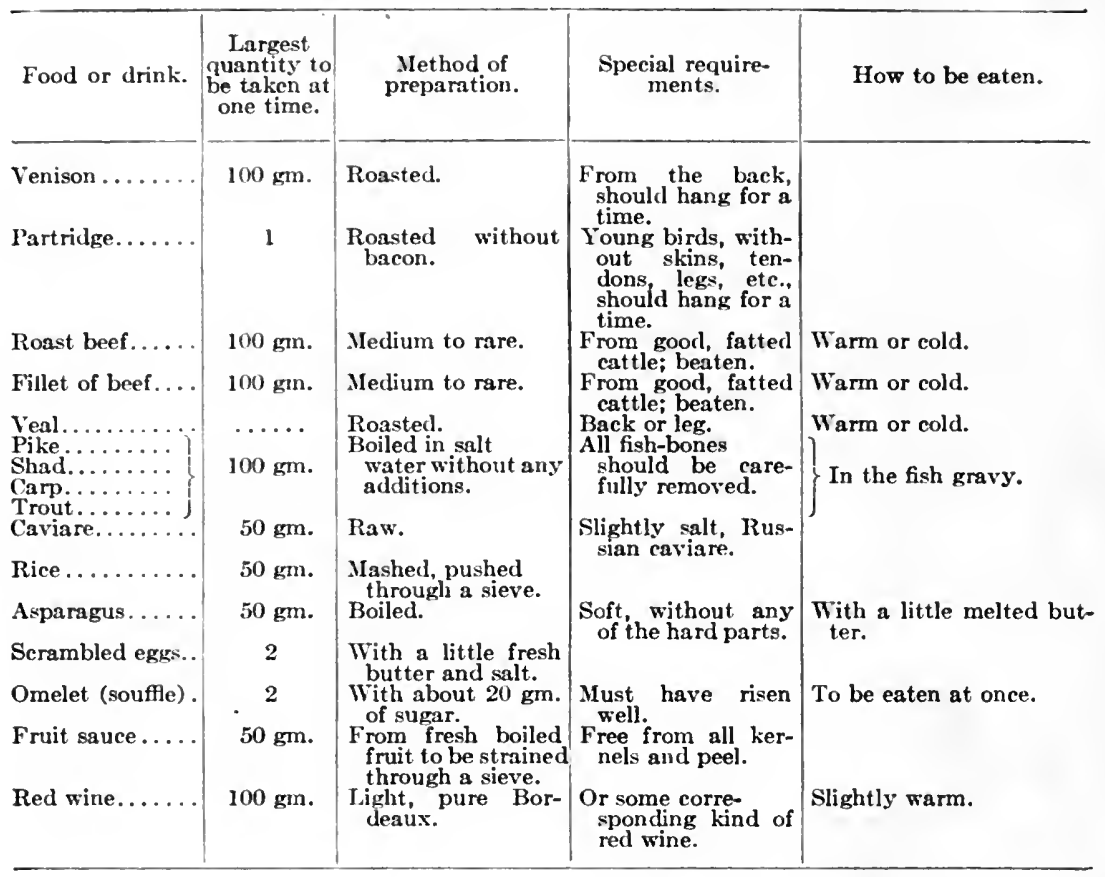

In the selection of a diet suitable for patients suffering from gastric disturbances it is important that the food be rapidly dissolved in the gastric juice, that it be passed into the intestine and quickly absorbed, and that it does not ferment abnormally. There should be no discomfort during digestion. The digestibility of any particular food depends, too, largely upon the individual taste, for, no matter how digestible a food may be, it cannot be properly utilized if it is distasteful to the individual. It is, therefore, important to conform the diet according to the special manifestations, whether of the motor or secretory functions. The effect of the food upon the motor function depends largely upon the physical structure and chemical composition; the more finely divided the food, the less it stimulates the secretion of hydrochloric acid, and the less it taxes the motor function. Those foods which have little effect in stimulating the gastric juices, are, as Bickel has recently shown, water, cocoa, eggalbumen, fats, starch, and vegetables in purée form, also boiled meat, and fish when finely minced. 'The gastric juice is stimulated by coffee, alcoholic drinks, beef-extracts, condiments of all kinds, all raw, roasted, or lightly boiled cooked red meats, and vegetables not thoroughly cooked and finely divided. In general, it may be said that in acute conditions the food should be of such a nature that the stomach be spared as much work as possible, and that, while it is important in all chronic disturbances to spare the stomach, it is most important to supply, in an easily digestible 
form, a sufficient quantity of nourishment to maintain the body weight. Inasmuch as at rest an individual requires 35 calories per kilo of weight, while in performing light work he requires 40 calories; in determining the exact amount of nourishment required it is only necessary to know the weight of the individual. In so far as the proteins can be replaced to a certain amount by the carbohydrates and fats, an interchange of any of these three food-stuffis may be made according to the patient's condition.

König's tables giving the composition of various food-stuffs and the number of calories they produce are important in this connection. Keeping a record of the weight of the patient gives a clear idea, too, as to whether the amount of nourishment consumed is sufficient. It has been a method, practised many years by the authors, to weigh every patient suffering with stomach disorder as soon as treatment is instituted, and to repeat this from time to time, to determine whether the patient is gaining or losing flesh.

\section{Composition of the Most Common food Surstances}

1. Dairy Products

\begin{tabular}{|c|c|c|c|c|}
\hline & Protein. & Fat. & Carbohydrates. & Calories. \\
\hline Cows' milk. & $\begin{array}{c}\text { Per cent. } \\
4.00-4.30\end{array}$ & $\begin{array}{l}\text { Per cent. } \\
3.00-3.80\end{array}$ & $\begin{array}{c}\text { Per cent. } \\
3.70\end{array}$ & $\begin{array}{r}\text { Per } 100 . \\
64.00\end{array}$ \\
\hline Cream.............. & 3.61 & 26.75 & 3.52 & 276.01 \\
\hline Butter................... & 0.50 & 90.00 & 0.50 & 837.00 \\
\hline Whey $\ldots \ldots \ldots \ldots \ldots \ldots$ & 0.50 & 0.30 & 3.60 & \\
\hline 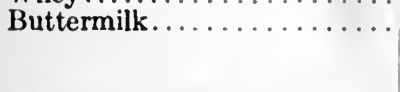 & 3.00 & 1.30 & $\begin{array}{c}3.00 \\
0.70 \\
\text { lactic acid }\end{array}$ & 3.67 \\
\hline Kumiss (of cows' milk).... \} & 3.35 & 2.07 & $\begin{array}{c}1.90 \\
\text { alcohol } \\
0.80 \\
\text { carbonic acid }\end{array}$ & 32.99 \\
\hline Cheese (eream) ............ & 25.00 & 30.00 & 3.00 & 394.00 \\
\hline Cheese................ & 33.00 & 9.00 & 5.00 & 240.00 \\
\hline Egg $\ldots \ldots \ldots \ldots \ldots \ldots \ldots$ & 12.50 & 12.00 & 0.50 & 165.00 \\
\hline
\end{tabular}

II. Meat and Game

\begin{tabular}{|c|c|c|c|c|}
\hline Beef (fat) $\ldots \ldots \ldots \ldots \ldots \ldots$ & 17.19 & 26.38 & & 315.81 \\
\hline Beef (lean) . . . . . . . . . . . . & 20.78 & 1.50 & & 99.15 \\
\hline Veal (fat).... & 18.88 & 7.41 & 0.07 & 146.61 \\
\hline Veal (lean)................ & 19.84 & 0.82 & .. & 86.97 \\
\hline Mutton (very fat) .......... & 14.80 & 36.39 & 0.05 & 399.31 \\
\hline Mutton (leaner) ............ & 17.11 & 5.77 & $\ldots$ & 120.81 \\
\hline Pork (fat) .......... & 14.54 & 37.34 & $\cdots$ & 406.SS \\
\hline 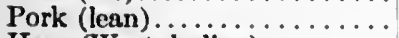 & 20.25 & 6.81 & & 146.36 \\
\hline Ham (Westphalian)......... & 23.97 & 36.48 & 1.50 & 453.69 \\
\hline Sweet-bread.............. & 22.00 & 0.40 & & 93.92 \\
\hline Pulverized meat ............. & 64.50 & 5.24 & $2.2 S$ & 322.53 \\
\hline Poultry $\ldots \ldots \ldots \ldots \ldots$ & 22.00 & 1.00 & & 100.00 \\
\hline Spring chicken . . . . . . . . . . & 18.19 & 9.34 & 1.20 & 167.59 \\
\hline Duck (wild) . . . . . . . . . . & 22.65 & 3.11 & 2.33 & 131.36 \\
\hline 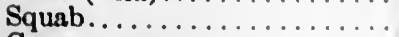 & 22.14 & 1.00 & 0.76 & 100.07 \\
\hline 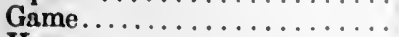 & 23.00 & 1.00 & & 103.60 \\
\hline Hare................. & 23.34 & 1.13 & 0.19 & 107.05 \\
\hline Venison................ & 19.77 & 1.92 & 1.42 & 105.44 \\
\hline
\end{tabular}


Composition of the Most Common Food Substances

III. Fish

\begin{tabular}{|c|c|c|c|c|}
\hline & Protein. & Fat. & Carbohydrates. & Calories. \\
\hline Pike & $\begin{array}{c}\text { Per cent- } \\
18.50\end{array}$ & $\begin{array}{c}\text { Per cent. } \\
0.50\end{array}$ & Per cent. & $\begin{array}{r}\text { Per cent. } \\
83.57\end{array}$ \\
\hline Carp & 20.61 & 1.09 & & 94.64 \\
\hline Shellfish. & 17.09 & 9.34 & & 156.93 \\
\hline Salmon.. & 15.01 & 6.42 & 2.85 & 132.93 \\
\hline Sardellen. & 22.30 & 2.21 & 0.45 & 113.83 \\
\hline Oysters. & 4.95 & 0.37 & & 24.00 \\
\hline Salt herring & 19.50 & 17.00 & 0.50 & \\
\hline Caviare..... & 28.04 & 16.26 & 7.82 & \\
\hline
\end{tabular}

II. Cereals and Vegetables

\begin{tabular}{|c|c|c|c|c|}
\hline Sago.................. & 0.50 & Trace & 86.50 & 356.70 \\
\hline Wheat flour........... & 8.50 & 1.25 & 73.00 & 345.78 \\
\hline$\ldots \ldots \ldots \ldots$ & 10.00 & 2.00 & 69.00 & 342.50 \\
\hline Wheaten bread........... & 6.00 & 0.75 & 52.00 & 245.00 \\
\hline Rye bread............ & 4.50 & 1.00 & 46.00 & 216.00 \\
\hline Roll........... & 6.82 & 0.77 & 43.72 & 213.87 \\
\hline Zwiebaek. & 9.50 & 1.00 & 75.00 & 356.00 \\
\hline Cauliflower. & $2.00-5.00$ & 0.40 & 4.00 & 35.00 \\
\hline Carrots..... & 1.04 & 0.21 & 6.74 & 33.85 \\
\hline Asparagus $\ldots \ldots \ldots \ldots \ldots$ & 2.00 & 0.30 & 2.50 & 21.00 \\
\hline 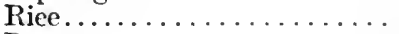 & 5.50 & 1.50 & 76.00 & 348.10 \\
\hline Beans........... & 19.50 & 2.00 & 52.00 & 311.75 \\
\hline Peas. & 19.50 & 2.00 & 54.00 & 319.95 \\
\hline Potatoes.. & 1.50 & & 20.00 & 88.00 \\
\hline Oatmeal. . & 12.50 & 5.26 & 66.77 & 338.80 \\
\hline Barley meal. & 8.31 & 0.81 & 75.19 & 323.00 \\
\hline Spinach...... & 3.49 & 0.58 & 4.44 & 38.00 \\
\hline Piekles. & 1.02 & 0.09 & 0.95 & \\
\hline
\end{tabular}

I. Soups and Beverages

Milk soup, with wheat flour. .

Meat broth (ordinary) .......

Meat-juice (pressed) ..........

Beef-tea.

5.00
0.40
$6.00-7.00$
0.50
$9.00-11.00$
protein
$+1.79-6.50$
peptone
$8.00-10.00$
1.50
8.80
3.12
12.38
0.50
0.70

3.25

0.60

0.50

0.50

Leube's meat solution

n....

Malt extraet

VI. Fruits

Rice pap, with milk........

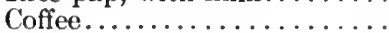

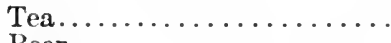

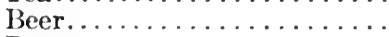

Porter.

\section{Fruits}

\begin{tabular}{|c|c|c|c|c|}
\hline & Free acid. & Protein. & Fat. & $\begin{array}{c}\text { Carbohy- } \\
\text { drates. }\end{array}$ \\
\hline Apples. & $\begin{array}{c}\text { Per cent. } \\
0.82\end{array}$ & $\begin{array}{c}\text { Per cent. } \\
0.36\end{array}$ & Per cent. & $\begin{array}{r}\text { Per cent. } \\
7.22\end{array}$ \\
\hline Pears. & 0.20 & 0.36 & & 3.54 \\
\hline Plums. . & 1.50 & 0.40 & $\ldots$ & 4.68 \\
\hline Peaehes. . & 0.92 & 0.65 & - & 7.17 \\
\hline Grapes.. & 0.79 & 0.59 & & 1.96 \\
\hline Strawberries............... & 0.93 & 0.54 & 0.45 & 1.01 \\
\hline Chestnuts. . & $\ldots$ & 5.48 & 1.37 & 38.34 \\
\hline Sugar-cane. & $\ldots$ & & $\ldots$ & 3.40 \\
\hline Honey ................ & $\ldots$ & 1.20 & $\ldots$ & 5.28 \\
\hline
\end{tabular}


We are in the habit of considering the chatracter of the diet from the standpoint of gastric secretion, whether we have, on the one hand, the condition of hyperchlorhydria and hypersecretion or, on the other hand, hypochlorhydria or anacidity. In hyperchlorhydria an abundant protein diet is indicated, the excess of hydrochloric acid being neutralized by this elass of food. 'This is not always the case, however, for, while proteins are usually best adapted, we have observed that patients suffering with hyperchlorhydria at times do better on bread and amylaceons food. 'The usual proteins of greatest value to patients suffering with hyperchlorhydria are the red meats and eggs, while the carbohydrates must be administered in the most easily digestible form. In cases of hypochlorhydria the proteins are, as a rule, more difficult to digest, while the carbohydrates are more easily digested. In these cases we are accustomed to give the meat only in a very tender form, as scraped beef, boiled sweet-breads, brains, etc., while the vegetables may be eaten in quite large quantities. In hoth hyper- and hypochlorhydria a certain amount of fat must be eaten in the form of good butter. 'This form of food is especially useful in the hyperacidity eases, as it has a marked tendency to diminish the amount of hydrochloric acid secreted. The diet, wherever there are motor disturbances, depends largely whether there is an excess or diminution of the gastric secretion. If there is an excess in acid an increase in protein food is advisable. If there is a diminution of the secretion, the protein must be given in the most digestible form, while the carbohydrates and lighter vegetables may be given in larger proportions. We agree with most writers that the ingestion of fluids in all motor disturbances of the stomach should be reduced as far as possible. 'They should be taken in frequent but small quantities. and principally between meals.

While under normal conditions, the appetite is usually a fair indication of the number of heat calories required, this cannot be said to be true whenever gastric disorder exists. Insufficient nourishment is often taken, inasmuch as the appetite is usually impaired. 'The fact that a patient at rest requires but one-sixth of the number of calories of that which is necessary when at hard work should always be borne in mind as a matter of advantage to the patient.

It has been our custom for many years in patients suffering with gastric disorders, which have been protracted and aecompanjed by great loss of flesh, and in which the patient is taking insufficient nourishment, to place such patient in bed for a certain period of time. 'The splendid results of this form of treatment is often remarkable.

Liquid Foods. - Whenever it is necessary to give liquid foods in large quantities, as is often the case when one wishes to spare the stomach as much as possible, milk is the food which is to be most highly recommended. When taken alone, it must be given in rather large quantities, in order to supply sufficient nourishment. It ean be diluted with Vichy. barley water, or lime-water, so as to render it more digestible; or it may be flavored with cocoa, tea, coffee, or vunilla, to make it more palatable. When milk is not well borne, kumiss, kefir, or buttermilk may be used 
as a substitute. Other liquid foods recommended are chicken, mutton, or beef broths, beef-tea or beef juice, and of these, beef juice is by far the most nutritious.

Gelatinous Forms of Foods.-Of these, gelatin, calves'-foot jelly, and chicken jelly are quite easily digested.

Meats.-Beef or other meats can be made more digestible by scraping, grinding, and chopping.

Eggs.-Raw and soft-boiled eggs are most digestible, though poached and scrambled eggs are sometimes very acceptable.

Fish.--Those with but little fat are to be most recommended. Halibut, carp, trout, and shell-fish belong to this class.

Vegetables.-Inasmuch as the salivary and intestinal secretions play an important part in the digestion of these substances, they must be thoroughly masticated. In patients suffering with gastric disturbances, on account of the danger of fermentation, they must be given with great caution. It is best in most instances to have them mashed and strained. Inasmuch as leguminous foods are apt to cause considerable fermentation, they, too, must be prescribed with great caution. Potatoes are best eaten mashed or baked. Cabbage should be entirely omitted from the dietary of patients suffering from gastric disturbances. Amylaceous food can be best given as toast, tapioca, oatmeal, etc. Fruits, while they give relish for other food and increase the intestinal movement, have but little nutritive value.

Fats.- When this form of food is taken in the form of fat meat, greasy gravies, etc., it causes indigestion, and should be omitted from the dietary of patients suffering with stomach disorders. In the proper form, however, fat is to be recommended, as it increases the weight of the patient, and also has a tendency to decrease the acidity of the stomach. Fat can be utilized in the form of good butter spread on bread. Chocolate contains a good percentage of fat, and on this account is to be recommended. Mehring's vigor chocolate is a useful preparation. Olive oil is an excellent form of fatty food in a certain form of gastric disorders, especially those associated with a hyperchlorhydria.

Special Factors Relating to the Diet of Patients Suffering with Gastric Disorders. - It is a well-known fact that the intestine will vicariously perform the work of the stomach when the secretory function of the latter is lost, so that a patient with this form of disturbance may maintain his weight. When it is necessary to spare the stomach, as in those instances where food is romited or is not digested, nutriment may be administered through channels other than the stomach, as by means of rectal feeding, or by giving concentrated food preparations, as somatose, nutrose, Mosquera beef meal, or plasmon flour. It is important that the food be thoroughly masticated, not only to thoroughly divide it, but also on account of the important effect of salivary secretion and digeston upon the gastric functions. The temperature of the food is also of primary importance, and it should be taken at a temperature of $98^{\circ}$ to $100^{\circ} \mathrm{F}$. As a rule, the meals should be moderate in size and taken at regular intervals. The question of rest or exercise is a matter 
of considerable importance to those suffering with gastric disturbances. From our observations the following conelusions are drawn: (1) In persons with normal digestive powers it makes little difference whether the individual rests, exercises, or sleeps after meals, though after violent exercise or sleep the gastric digestion is very slightly impaired. (2) In patients suffering with superacidity and subacidity it is best to order rest after meals; after violent exercise or sleep the digestion is impaired in these cases. (3) In patients suffering with motor disturbances of the stomach it is best to prescribe molerate exercise after meals, for rest, violent exercise, or sleep disturbs the digestion under these conditions.

Diet Cures in the Treatment of Gastric Disturbances.-Of these, we have the rest cure, milk cure, grape cure, and forced feeding.

A rest cure, as first devised by Weir Mitchell, is a most important form of treatment in gastric disturbances. We are constantly employing it in the various forms of gastric neurasthenia accompanied by severe anorexia and cmaciation, with very excellent results. It is also a most useful form of treatment in cases of ulcer, gastritis, atony, and gastroptosis. The method of carrying out the rest cure in the treatment of gastric conditions has already been described under the head of the section dealing with this subject.

Milk Cure.-'This cure consists in the administration of large quantities of milk, taken either alone or together with other foods. In certain disorders of the stomach milk may be given alone, which allows the stomach to regain its normal functions. It is especially useful in the treatment of ulcer of the stomach and in some forms of chronic gastritis. We use it largely in the treatment of the secondary forms of gastritis, especially those dependent upon tuberculosis and anemia. In certain forms of gastric neurasthenia the milk cure produces excellent results, while in others milk quickly disturbs the digestion and cannot be employed. In those instances in which there is a decrease or absence of acid in the stomach, milk is usually not well borne. The milk cure should not be employed in severe forms of atony and dilatation, or in intestinal conditions associated with extreme flatulence, or in chronic diarrhoea. When milk is taken in large quantities, in addition to other food, it is much less apt to disagree. One of the disagreeable features of milk is the obstinate constipation which it is liable to produce. Milk is often rendered more digestible by the addition of lime-water, barley water, milk of magnesia, or small quantities of coffee, tea, or cocoa may be added to it. When milk disagrees, kefir, kumiss, or buttermilk may be substituted for it.

Forced Feeding or Gavage.-'This method consists in introducing milk, eggs, and beef juice into the stomach through the stomach-tube. We have frequently employed it in aggravated cases of nervous anorexia, where there seems to be danger of starvation.

Grape Cure.-In this form of treatment grapes are exclusively given as food. They are useful in plethoric patients in whom it is necessary to diminish the weight, in certain forms of nervous dyspepsia, and in chlorotic girls suffering with dyspepsia. 
Acute Gastritis.- In the treatment of this condition diet plays a most important part. It is important in all cases to secure an absolute rest for the stomach, as well as a total abstinence from food, for at least twenty-four hours. 'This is not always an easy procedure, for most patients, believing that food is necessary for them, are unwilling to abstain from nourishment for a sufficient length of time. Nausea and vomiting, which are always present in this disorder, are so aggravated by the taking of food that the patient himself will soon be convinced of the need of abstaining from food. For the thirst, which is intense in this condition, the patient may be allowed to rinse his mouth with water frequently, or even partake of very small quantities of Vichy or Apollinaris. It is our experience that, according to this plan of treatment, the patient usually recovers in three or four days. After the first twenty-four or forty-eight hours we begin to give small quantities of milk, diluted with lime-water or Vichy, plain egg-albumen, and clear broths. 'These are gradually increased in quantity, and during the following days scraped beef, sweetbreads, chicken, and toast may be added. We have been accustomed to prescribe the following $\operatorname{diet}^{1}$ about the third or fourth day after an attack of acute gastritis:

Calories.

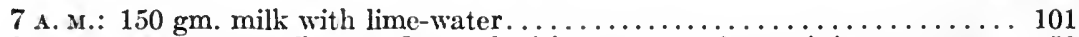

9 A. M.: $100 \mathrm{gm}$. egg-albumen flavored with orange- or lemon-juice.......... 53

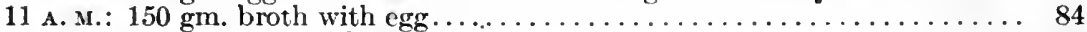

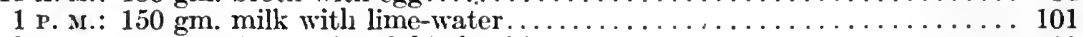

3 P. м.: $5 \mathrm{gm}$. Armour's soluble beef in water................... 10

5 P. M.: $100 \mathrm{gm}$. egg-albumen flarored with orange- or lemon-juice....... 53

7 P. ... $150 \mathrm{gm}$. milk with lime-water............................. 101

503

After the fourth day the diet is increased as follows:

Calories.

7 A. M.: $150 \mathrm{gm}$. milk (101) with $70 \mathrm{gm}$. toast $(182) \ldots \ldots \ldots \ldots \ldots \ldots . \ldots 283$

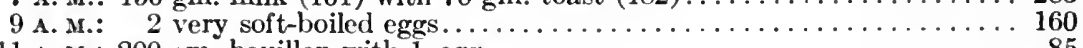

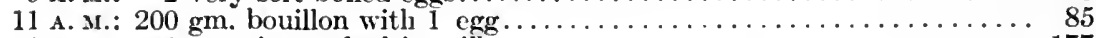

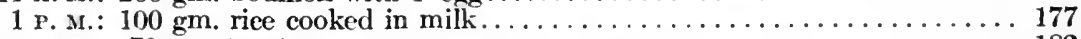

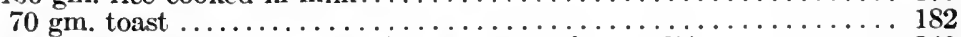

3 P. s.: $100 \mathrm{gm}$. egg-albumen (53) with $50 \mathrm{gm}$. erackers $(187) \ldots \ldots \ldots \ldots .240$

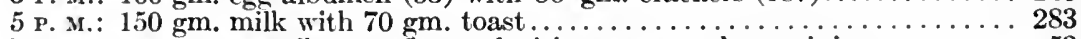

7 P. м.: $100 \mathrm{gm}$. egg-albumen flavored with orange- or lemon-juice........ 53

1463

Chronic Gastritis.-The dietetic treatment is of greater importance than the use of drugs. 'The most easily digestible foods are liquids, such as broths, and their food value may be increased by the addition of beef extract, rice, barley, eggs, somatose, or plasmon. The diet is to be varied, as a rule, according to the form of gastritis. Whenever the entire gastric secretion has disappeared, the proteins are digested with great difficulty, and these foods must, therefore, be given in a most digestible form. Sंuch foods as scraped beef, steamed chicken, boiled sweetbreads, broiled squabs, and steak are especially useful. Vegetables are best taken when mashed and strained. In most cases milk is well

1 This and the other diet-lists are from Diet in Health and Disease by Friedenwald and Ruhräh. 
borne and is a very useful food, and it may be modified as suggested above. When the gastric juice still contains acid, meats form a very important part of the dietary, and any of the more easily digested meats may be utilized. Fish and eggs may be added to the dietary, and such vegetables as spinach, baked or mashed potatoes, and carrots, and peas, when mashed and strained, are to be recommended. Cereals, when well cooked, are also useful fools in this condition. In order to supply the necessary amomut of fat, butter, cocoa-butter, and Vigor chocolate are useful. We find it hest, as a rule, to order small meals at regular short intervals. Mineral waters play a certain role in the treatment of chronic gastritis, and of these the saline waters and alkaline saline waters are especially useful. Kissingen, Homburg, Carlsbad, Marienbad, and Saratoga (Congress and Hathorn) are among the important waters which we have found of considerable benefit in the treatment of this disease. In chronic gastritis water should be taken in small quantities between meals, alcoholic stimulants or any strong stimulants should be forbidden, and strong coffee or tea, as well as condiments, as a rule, should be omitted from the dietary. We have found the following diet-list of great value in the treatment of cases of chronic gastritis:

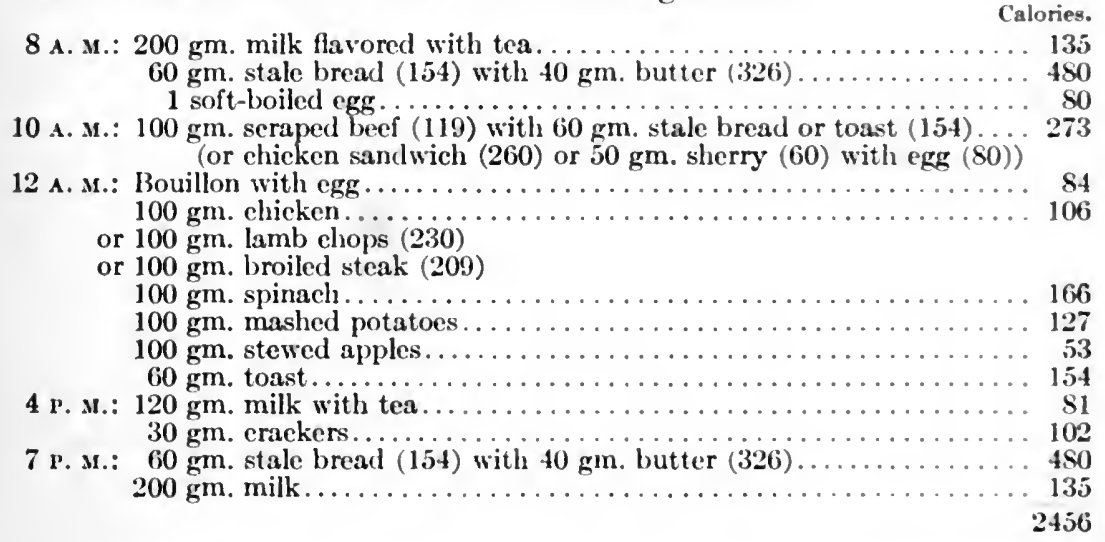

Dilatation of the Stomach.-Fluids are not well borne in this condition, and are, therefore, to be given only in small quantities-not over 1 to 2 quarts a day. Of these we utilize milk, cream, buttermilk, tea, coffee, and broth in small quantities. 'The intense thirst which is often present in this disorder may be overcome by allowing the patient to suck bits of ice, or by giving rectal injections of water or normal salt solution. In order to increase the general nutrition, mutrient enemata must at times be administered. In order to increase the nutritive value of the milk, rice, tapioca, or eggs may be added. Concentrated beef juice or meat extracts may also he used, as well as broths thickened with rice. barley flour, tapiocal, or eggs. 'The only meats to be allowed are those which are the most digestible, such as sweetbreads, stewed or baked chicken, calves' brains, and scraped heef. Vegetables must be given only: in the purce form, and carrots, potatoes, peas, and spinach are the best. 
Bread must be taken stale or toasted, or in the form of zwieback. Stewed fruits, such as baked or stewed apples, stewed prunes, may be allowed. Butter is given, but only in small quantities. Alcohol is to be taken cautiously and only in the form of light wines. Spices and condiments should be avoided. It is important to prescribe small concentrated meals, and large quantities of fluids should never be taken. We have used the following diet in many cases of dilatation of the stomach:

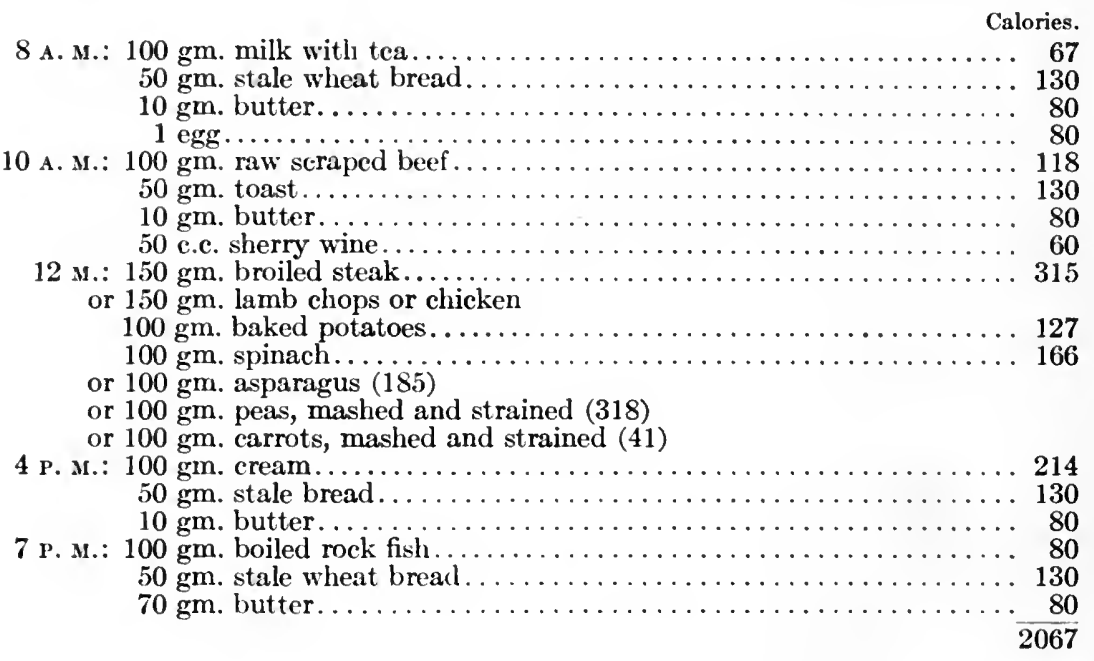

Atony of the Stomach.-In patients suffering with atony of the stomach, great care must be exercised to prescribe food in small quantities at frequent intervals. The quantity of fluids should be limited to a quart and a half per day, and should be given always in small quantities. If the thirst continues to be excessive, rectal injections of salt solution may be administered. We do not advise the use of large quantities of milk, as is often prescribed in this disorder, as when the patient is up and about the weight of excessive quantities of fluids overdistends the stomach. When, however, the patient is in bed, as during a rest cure, milk is borne better even in large quantities. Whenever there is a hyperchlorhydria associated with atony, the meats, vegetables, and cereals advised in dilatation may he given. Butter will be found useful. As a rule, alcoholic stimulants should be prohibited, though in a small proportion of cases light wine may be permitted to be taken as a stomachic. In cases of atony of the stomach with hypochlorhydria, the light meats, such as chicken, sweetbreads, scraped beef, or the white meat of fish, are to be recommended. Vegetables are to be prescribed, on the other hand, in large quantities. For the chronic constipation, which is usually associated with this disease, diet plays an important role, and such foods are to be recommended which increase the intestinal peristalsis. Among these foods are certain vegetables, as carrots, tomatoes, beans, peas, turnips, as well as stewed and raw fruits, cider, honev, and Graham and 
rye bread. We have used the following diet-list in the treatment of atony of the stomach with a great deal of success:

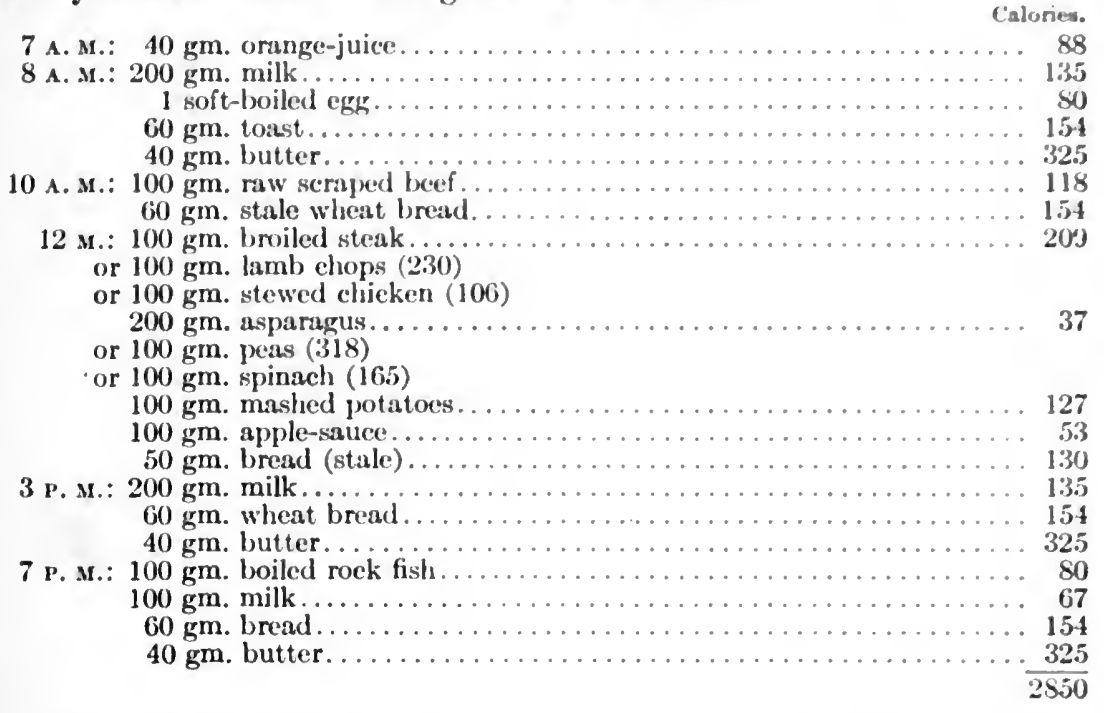

Ulcer of the Stomach.-The onset of ulcer of the stomach may he prevented if careful attention be directed to the very first symptoms. As soon as these are manifest, the patient must be placed upon a milk diet, which should not be given either too hot or too cold. 'The anemia, as well as the hyperchlorhydria, which bear so important an etiologic relation to this disease, must be combated by the usual remedies. It is generally admitted by most authorities that the rest treatment should always be instituted in all cases of ulcer of the stomach, in order to place the patient in conditions most favorable for rapid healing. 'The patient should be put to bed, and kept at complete rest for a variable period of time, depending upon the severity of the symptoms-ordinarily from two to five weeks. During this period the diet is limited to liquids. 'The rest in bed, as well as the rigid diet, not only assists in the healing of the ulcer, but also in a measure guards against the onset of such accidents as perforation and hemorrhage. 'The additional advantage of the rest lies in the fact that a patient when in bed requires but a sixth the number of calories that he requires when about, and, therefore, much less nourishment is needed under these conditions, thus entailing less labor upon the diseased stomach. 'The stomach should be spared all possible irritation, and the food should be taken ne ther too hot nor too cold $\left(9 S^{\circ}\right.$ to $100^{\circ} \mathrm{F}$.). The excess of acid in the stomach should, as far as possible, be lessened, and all irritation from drugs should be avoided. Milk can ordinarily be given without being peptonized, but it should usually be diluted with lime-water. In the beginning, small quantities may be given at twohour intervals, 1 to 2 quarts being consumed in twent $y$-four hours.

After the first week, when the nausea and pain have subsided, some well-cooked wheat preparation, strined barley, oatmeal, somatose, or 
plasmon may be added to the milk to increase its nutritive value, or clear broth, with an egg thoroughly beaten up, may be added to the diet. If the patient has improved, and is free from nausea or vomiting, a soft diet, such as rice cooked in milk, milk-toast, or soft-boiled eggs, may be allowed at the beginning of the third week. At the beginning of the fourth week solid food in easily digestible form, such as raw scraped beef, stewed chicken, broiled sweetbreads, calves' brains, baked potatoes, may be allowed, and gradually the vegetables, such as spinach, carrots, peas, and turnips in the purée form. Later, broiled steak, chops, or roast beef, if well cooked, can be given more liberally, but raw fruits, salads, pastry, and the heary vegetables should be avoided for a long period of time. The patient should remain in bed from two to four weeks, according to the severity of the symptoms. Ordinarily, at the beginning of the third week he may be allowed to recline on a couch, and toward the end of this week to sit up for a short time, and, finally, for a longer time each day. In the fourth week the patient is usually permitted to walk about. Hot poultices are applied to the abdomen for several weeks during the early part of the treatment, and 200 to 300 c.c. of hot Carlsbad water are taken morning and evening.

The Leube and Penzoldt diets, already given, may be followed in the treatment of ulcer of the stomach, the first dietary for ten days, the second for the succeeding ten days, and the third for eight days. The severity of the case must, of course, determine the length of time during which each dietary must be continued. In all cases of ulcer of the stomach of a severe type, accompanied by excessive nausea, vomiting, and pain, or accompanied by vomiting of blood, all nourishment by mouth should be withheld for a period varying from five to ten days, the patient being fed by means of nutrient enemata given from three to five times daily. During this period the thirst may be partly allayed by allowing bits of crushed ice, or permitting the patient to rinse his mouth with water. MeCall Anderson and Donkin first called attention to this plan in the treatment of ulcer of the stomach. Whenever it is possible it is best to begin treatment in all cases of ulcer, according to this method, for four or five days, then gradually allowing small quantities of milk, and, finally, continuing the rest cure diet, as has already been described. It is a much more simple matter now to determine the length of time a patient should remain on a strict milk diet and at rest in bed than formerly. By means of the examination for "occult hemorrhages" we are enabled to establish the individual term of treatment, dismissing the patient only when repeated tests fail to disclose further occult hemorrhages. Rarely milk does' not agree, and other foods must be substituted for it. Of these, buttermilk, kefir, matzoon, and kumiss are especially useful.

While this plan of treatment is usually recommended, it must not be forgotten that there are some objections to an exclusive milk diet extending over a number of weeks. The anemia frequently found accompanying ulcer is often very extreme, and the small quantities of milk which are consumed eannot prevent the onset of rapid emacia- 
tion. For this reason, Fuetterer recommends the juice expressed from 5 pounds of beef, to be taken daily, to combat this condition. Lenhartz, too, has cautioned against the strict abstinence diet in the treatment of ulcer, even in those instances in which there is hemorrhage He bases his conclusions on the fact that, since ulcer of the stomach is most frequently accompanied hy superacidity, and ulso by a generally enfeebled condition, it is best to give protein food early to overcome the acidity as well as to build up the system. 'The accompanying table illustrates his method of feeding.

The egg and milk are given ice cold and in teaspoonful doses. By means of this method we have treated many cases with gratifying results. In those cases where there has been hemorrhage the patient is kept in bed for fourteen days, and an ice-bag placed on the abdomen for ten days.

There are a number of mild cases of ulcer of the stomach in which it is impossible to carry out the rest treatment. Under these conditions the ambulatory form of treatment may be undertaken according to one of three methods. In all cases the patient is permitted only to take liquid or semisolid food: (1) Nitrate of silver is prescribed in solution in $\frac{1}{6}$ to $\frac{1}{3}$ grain doses for a period of three weeks, or (2) bismuth subnitrate may be administered in large doses (one teaspoonful) three times a day. (3) The oil cure has been recommended by Cohnheim in the treatment of ulcer of the stomach. Olive oil is taken three times daily, from half to one hour before meals, in wineglassful doses in the morning and in dessertspoonful doses at noon and in the evening. In very mild cases an emulsion of sweet almonds may be substituted for the oil. 'The oil fulfils several indications. It forms a coating over the stomach, and thus assists in overcoming pylorospasm, and by relieving friction it overcomes pain, it checks the excessive secretion of acid, and improves the general nutrition. Mineral waters are often employed in the treatment of ulcer of the stomach. Of especial service in this regard are the waters of Carlsbad and Saratoga (Hathorn), which have been utilized by us with considerable benefit in the treatment of this disease. The mineral-water treatment is only of service, however, after the ulcer has

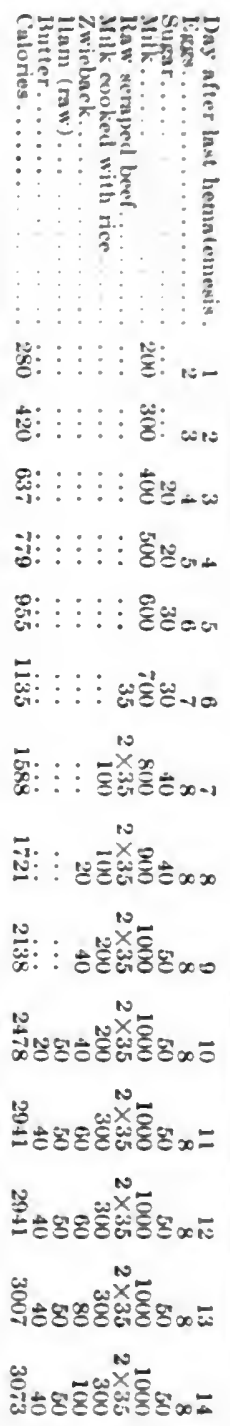
entirely healed, as a prophylactic measure, and can only be undertaken at the spring itself. After the ulcer is entirely well, it is of importance to overcome the anemia present. In most instances this can be best accomplished by means of diet (see Diet in Anemia) as well as by sending the 
patient to some invigorating watering-place, the seashore, or the mountains.

Carcinoma of the Stomach.-In those instances in which the cardiac portion of the stomach is involved the diet should be of such a form as to prevent any irritation of the stomach or osophagus which is stenosed. The food is, therefore, best given in liquid form. Milk is the form of food usually best borne and most nutritious. It can be made even more nutritious by adding somatose, plasmon, eggs, etc., and in addition all forms of broths, cocoa, coffee, and buttermilk, kefir, and kumiss may be taken. When great difficulty in deglutition arises, gastrostomy must be performed, and feeding continued through the new opening. Nutrient enemata may also be employed. Whenever cancer attacks the body of the stomach, milk also remains the most useful form of food. In addition, the more digestible foods, such as sweetbreads, scraped beef, boiled chicken, are to be recommended, but there is usually so great a distaste to meat in this disease that fish or other forms of food must be substituted for it. Of these fish, boiled rock, trout, and mackerel are the most useful. In addition, the light vegetables, as baked or mashed potatoes, carrots, peas, beans if mashed or strained, as well as spinach and asparagus, are to be recommended. Farinaceous food and eggs are also useful. For the thirst, iced milk alone, or flavored with tea or coffee, cocoa, whisky, may be ordered. It is frequently necessary to assist the general nutrition by the use of nutritive enemas in this disease. Inasmuch as but little is to be expected from food in cancer of the stomach, it is hardly necessary to urge upon the patient the necessity of taking a very large quantity. We have found the following diet table useful in some cases of cancer of the stomach:

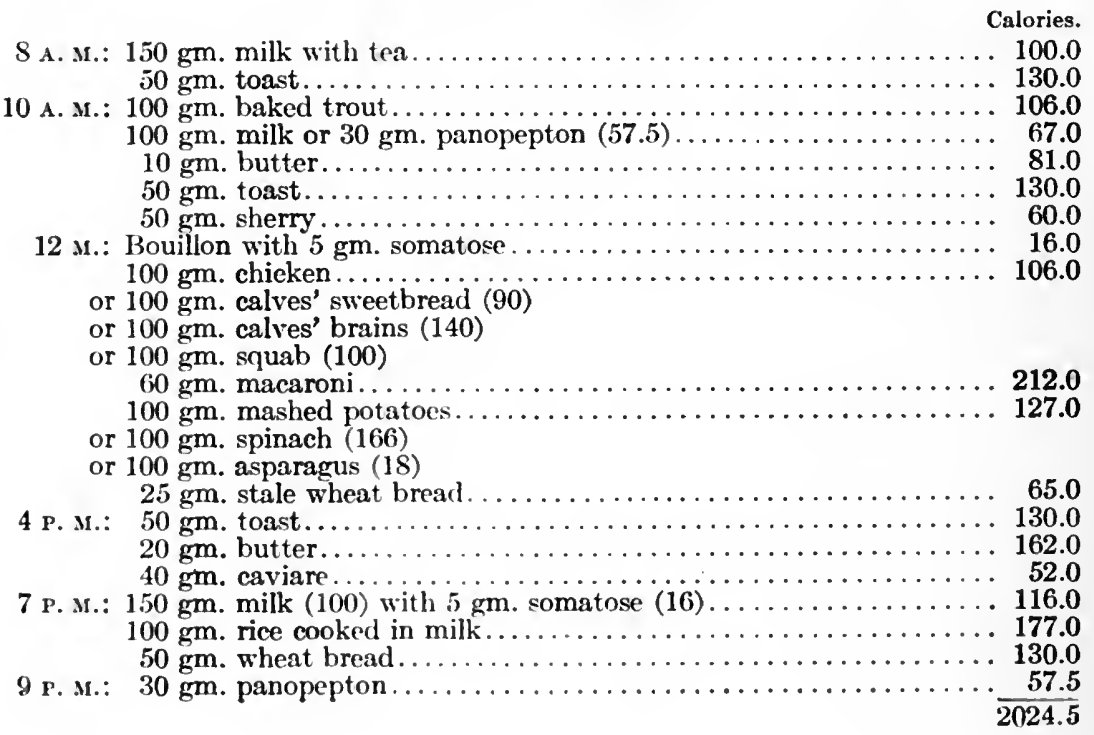


Gastroptosis and Enteroptosis.-In addition to the usual treatment to be undertaken in the treatment of this disease, much ean be accomplished by regulating the diet. In a large number of cases the rest cure is indicated, with the patient in the recumbent position, and consuming large quantities of food. As a rule, patients suffering with this disorder can accomplish much by lying down after meals. 'The diet must be of the most nourishing form, and should contain a considerable proportion of fatty food. When milk is well borne, it should be given in rather large quantities. Of the solid food, all forms of digestible meats, as chicke+n, roast beef, lamb chops, and broiled steak, are permissible. Eggs, either raw or cooked, are useful. 'The vegetables to be reconmended are spinach, asparagus, potatoes, cauliflower, and carrots. 'The fats which are especially useful are butter. crean, Vigor chocolate, and olive oil. In order to overcome the constipation accompanying this disorder, such foods as stimulate the intestinal movements are to be employed. Anong these may be mentioned grape-juice, buttermilk, honey, fruit, and cider.

Nervous Dyspepsia.- In this condition it is important not to diet the patient too rigidly. Strengthening food should be given, but no attempt should be made to maintain a rigorous diet. Nilk is by far the most nourishing food in this disorder, and, if well digested, may be ordered in large quantities; if not well borne, buttermilk, kefir, or kumiss may be substituted for it. It is important to properly nourish the patient, and for this reason we have found it most advisable to allow any food that the patient can digest. Coffee, tea, as well as alcoholic stimulants, should be avoided. In severe forms of nervous dyspepsia a rest cure is indicated. In those patients suffering with nervous anorexia the patient should be isolated and placed in bed, and milk should be administered or nutrient enemas may be given. All food must be given in as nutritious and concentrated form as possible, and somatose, plasmon, or sanatogen may be added to the milk.

Hyperchlorhydria and Hypersecretion.-In the treatment of these conditions great care must be exercised to prevent an increase of the hydrochloric acid of the gastric juice. It is important to avoid all irritating food, as condiments and spices, the use of all strong alcoholic drinks, and of substances apt to irritate the stomach, as muts. Food must not be given too hot or too cold, and must be thoroughly masticated. 'The diet should be largely composed of proteins, since they combine' with and neutralize the excess of hydrochloric acid. Such foods are milk, meat, eggs, and fish. On the other hand, carbohydrates must be given only in small quantities, and only in the most digestible form. Fleisher, in investigating the subject of the combining effect of hydrochloric acid with the various food-stuflis, has shown that beef, veal, ham. and mutton bind twice as much hydrochloric acid as calves' brains and sweetbreads. 'The first-mentioned forms of meat are, therefore, best given in patients suffering with hyperchlorhydria and hypersecretion. In addition, pork, cheese, sausage, Graham bread, milk, and coroa are capable of binding large quantities of hydrochloric acid. Farinaceous 
foods are not so easily digested in this condition, and must be given in the most digestible forms. Of the vegetables to be recommended in this condition only the most digestible are to be allowed, as mashed and baked potatoes, asparagus, spinach, peas, and carrots, strained and taken in the purée form. 'The fats which have a tendency to decrease the acidity of the gastric secretion, and which are to be recommended, are butter, olive oil, and cream. The alkaline mineral waters are useful in lessening the acidity, and are to be given as Vichy, seltzer, and Appolinaris. The following table, taken from Fleischer, shows the ability of various foods to combine with hydrochloric acid:

Fleischer's Table, Showing the Power of Foods to Combine with HCl

\begin{tabular}{|c|c|c|c|}
\hline Meats (100 grams). & Pure HCl. & $\begin{array}{l}25 \text { per cent. } \\
\mathrm{HCl} \text {. }\end{array}$ & $\begin{array}{c}\text { Dilute } \\
\text { muriatic acid. }\end{array}$ \\
\hline 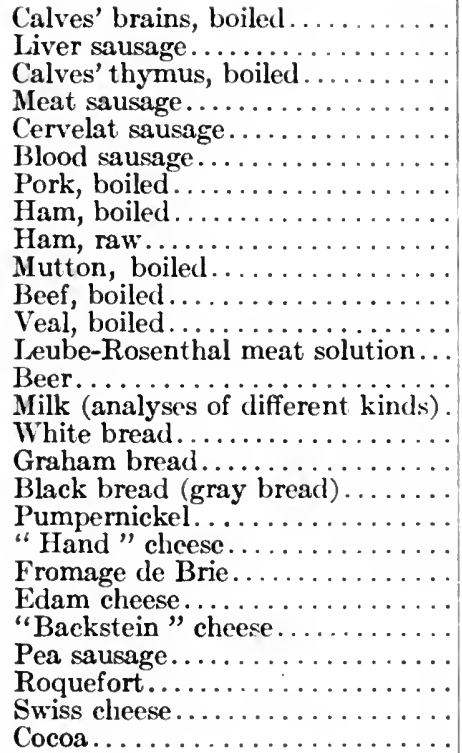 & $\begin{array}{l}0.65 \\
0.80 \\
0.90 \\
1.00 \\
1.10 \\
1.30 \\
1.60 \\
1.80 \\
1.90 \\
1.90 \\
2.00 \\
2.20 \\
2.20 \\
0.10 \\
0.36 \\
0.30 \\
0.30 \\
0.50 \\
0.70 \\
1.00 \\
1.30 \\
1.40 \\
1.70 \\
1.70 \\
2.10 \\
2.60 \\
4.10\end{array}$ & $\begin{array}{r}2.60 \\
3.20 \\
3.60 \\
4.00 \\
4.40 \\
5.20 \\
6.40 \\
7.20 \\
7.60 \\
7.60 \\
8.00 \\
8.80 \\
8.80 \\
0.40 \\
1.44 \\
1.20 \\
1.20 \\
2.00 \\
2.80 \\
4.00 \\
5.20 \\
5.60 \\
6.80 \\
6.80 \\
6.40 \\
10.40 \\
16.40\end{array}$ & $\begin{array}{r}5.20 \\
6.40 \\
7.20 \\
8.00 \\
8.80 \\
10.40 \\
12.80 \\
14.40 \\
15.20 \\
15.20 \\
16.00 \\
17.60 \\
17.60 \\
0.80 \\
2.80 \\
2.40 \\
2.48 \\
4.00 \\
5.60 \\
8.00 \\
10.40 \\
11.20 \\
13.60 \\
16.80 \\
16.80 \\
20.80 \\
32.80\end{array}$ \\
\hline
\end{tabular}

It is manifest that the combining power of some foods renders them particularly useful in such conditions.

It has been our experience in dealing with patients taking but little nourishment to allow them to eat at frequent intervals. If, however. large meals are taken, it is advisable to permit only three meals a day, We have utilized the following diet table in cases of hyperchlorhydria and hypersecretion: 


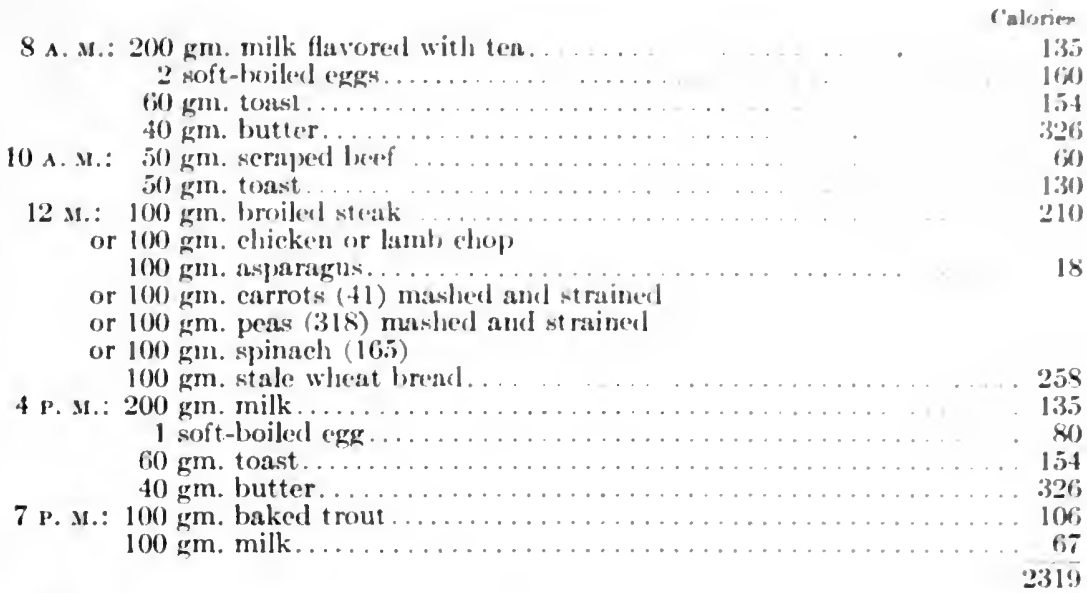

Diet in Intestinal Diseases.- The diet is quite as important in the treatment of intestinal disease as in the treatment of diseases of the stomach. In many instances by diet alone a cure can be effected, which often makes the use of drugs superfluous.

The question as to the digestibility of food in the intestine is one which is often difficult to determine, as the process of digestion in the intestine is very complicated. The diet should always be of such form as will not produce annoying symptoms. The food must be easily absorbed, and one can learn much from the experiments of Rubner, who determined the degree of absorption of various foods in the intestines. His results are embodied in the following table:

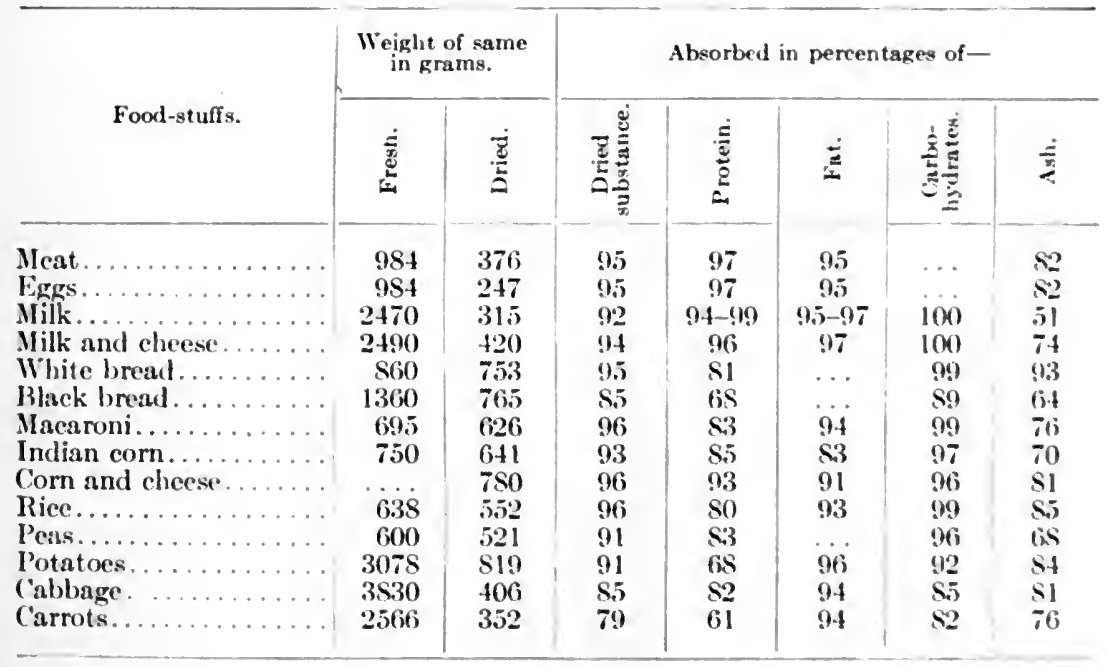

It is evident that, notwithstanding the large proportion of protein matter in certain foods, its absorbability is so slight that the nutritive 
value is far less than that of foods containing far less protein. The absorbability depends, to a large degree, upon the method of preparing the food, as, after mashing and straining, vegetables become much more easily absorbable, inasmuch as some of the cellulose is removed. The digestibility of various food in the intestine differs in different individuals, which makes it impossible to formulate exact rules for all cases. The diet must be regulated personally for each individual.

Foods may be divided into three classes, according to their effect on the peristaltic movements of the intestine: foods which produce constipation, those producing a laxative effect, and those having no effect either way. Among the constipating foods are red wines, cocoa, tea, rice, tapioca, barley, macaroni. In the class of laxative foods are fruits, tomatoes, cabbage, cider, beer, buttermilk, and alkaline carbonated water; while those foods having no especial effect on the movements of the bowels comprise meat, fish, eggs, and wheat breads. However, while in some patients certain foods may have a laxative effect, they may have a constipating effect in others, so that no exact general rules can be formulated, but the tendencies of each person must be especially taken into account, a point that cannot be too strongly insisted upon.

Acute Intestinal Catarrh.-The regulation of the diet in this condition is of the greatest importance-by far more so than the use of drugs. The patient must be kept at rest in bed, and for several days exclusively upon liquid food, consisting of clear broth, egg-albumen, gruels, weak tea, and cocoa cooked in water. We have found it best, as a rule, to forbid the use of milk in this disorder. The carbonated waters may be used as a means of allaying the thirst, but should be allowed only in small quantities. It is best to relieve the thirst by allowing small pieces of crushed ice to melt in the patient's mouth. As soon as the patient is relieved of his pain and discomfort it is our habit to allow soft and light solid food, consisting of cereals, toast, stewed chicken, soft-boiled eggs, and baked potatoes. Such food as raw fruit, fatty and acid foods, and the heavy vegetables should be avoided for a considerable length of time after the patient is well.

Chronic Intestinal Catarrh.-The dietetic treatment of this condition depends largely upon the question as to what portion of the intestine is involved; that is, whether the condition is associated with chronic constipation or chronic diarrhœa, or whether diarrhœa alternates with constipation. In the cases in which there is chronic constipation it is important to order such foods as will stimulate the intestinal movements. Astringents should be carefully avoided. Those foods which are of most value are rye and Graham bread, buttermilk, cider, beer, honey, fruit (stewed or raw), such vegetables as cabbages and sour-kraut, as well as salads. Fats are especially useful, and olive oil is of great value in this disorder. Salty foods have a tendency to increase the intestinal movements, and such foods as caviare and herring are to be recommended. Sugar, too, is of a considerable value in this disease, and milk-sugar is to be especially recommended. Cold water is also useful when taken in the morning on an empty stomach. 
Whenever diarrhoea exists, the patient should be kept quiet in bed, and food administered in small quantities at frequent intervals. 'Ilhe foods of most value in this condition are broths containing barley and rice, stewed ehicken, broiled steak, soft-boiled eggs, boiled fish, toast, baked and mashed potatoes, light tea, cocoa, boiled milk, and port wine. At times milk disagrees, even when boiled, and must be avoided.

Whenever diarrhoa alternates with constipation, it is most important to treat the most prominent symptom, whether it be diarrho'a or constipation, and vary the diet frequently, according to the condition at hand. Mineral waters play an important role, frequently, in the treatment of chronic intestinal catarrh. When constipation exists, the Marienbad and Saratoga (Hathorn and Congress) waters are most useful, and when diarrhoea exists, Carlsbal and Vichy waters are most beneficial.

Dysentery.--The diet in acute dysentery is largely that preseribed in acute intestinal catarrh. 'The patient is kept quietly in bed upon liquid foods, consisting mainly of broth, egg-albumen, and tea. As the patient improves he is given semisolids, as milk-toast, rice cooked in milk, or broth and gruels, and solid food should only be given after this disorder has entirely disappeared. In ehronic dysentery the nourishment is to be given in small quantities at frequent intervals, and all heavy food is to be avoided. The diet is quite similar to that given under the head of chronic intestinal catarrh.

Ulcers of the Intestine.-(1) Duodenal Ulcers.-The same dietetic treatment is to be instituted in the treatment of duodenal ulcers as in gastric ulcers, already described. Whenever there is hemorrhage, the patient must at once be placed at rest in bed and fed by means of nutrient enemas. After a week the usual Leube rest-cure treatment is inaugurated, milk being allowed first. In severe forms rectal feeding may be continued for two weeks.

(2) Other Forms of Intestinal Ulcers.-In any form, whether it be tuberculous, dysenteric, or syphilitic, the diet must be very digestible and not irritating. Such foods as milk, broths, eggs, cereals, toast, sweetbreads, and stewed chicken, baked and mashed potatoes, cocoa. and tea may be allowed.

Acute Intestinal Obstruction.-Operation is usually indicated in this disorder. Before operation is undertaken the patient inust be kept quiet in bed and must abstain from all nourishment. The thirst may be allayed by allowing small bits of ice to melt in the mouth.

Chronic Intestinal Obstruction.- The food in this condition should be administered only in the liquid or semisolid forms. The patient must avoid all heavy foods, especially those leaving a large residue in the bowels, such as salads, fruits, and heary vegetables. 'Those foods which are to be recommended in this condition are broths, milk, eggs, broiled meats and fowl, boiled fish, cereals, stale bread, and toast. In severe forms rectal feeding may be resorted to.

Appendicitis.-As surgical treatment is usually indicated in this disease, the dietetic treatment can only be earried ont until this procedure 
is undertaken, or when operation cannot be undertaken. The patient is placed at rest in bed, and usually upon liquid food, such as eggalbumen, weak tea, thin broths, rice and barley water, and small quantities of milk diluted with lime-water. After the acute symptoms have disappeared, the diet can be increased; eggs, gruels, scraped beef, stewed chicken, and toast may gradually be added to the dietary, and, finally, the light vegetables, mashed and strained, may be added. We have treated, according to the plan of Ochsner, a number of cases of appendicitis in which operation could not be undertaken with very satisfactory results. According to this plan, in all cases of acute appendicitis all food is prohibited by the mouth and all cathartics must be avoided. Whenever there is nausea or vomiting, gastric lavage is practised. In very mild cases the patient is allowed to rinse his mouth with cold water, and to drink a very small sip of hot water, but in the severer forms he is not permitted to drink either cold or hot water for a few days until the acute attack has subsided. If the nausea persists, gastric lavage may be practised every four hours, in order to remove all substances which have regurgitated into the stomach from the bowel. The patient is supported by nutrient enemas, consisting of 1 ounce of concentrated, predigested, liquid food, as is found in the market, dissolved in 3 ounces of warm normal salt solution, introduced through a catheter. In those instances in which no water is given by the mouth, enemas of 8 ounces of normal salt solution are given four to six times daily in addition to the nutrient enemas. When the pain has subsided for four days, the patient is permitted to take 1 to 4 ounces of beef-tea every two hours. In a few days one of the commercial predigested foods, dissolved in water, is substituted, and, still later, equal parts of milk and lime-water, then general liquids, then light diet, and, finally, after the patient has fully recovered, full diet is given.

Mucomembranous Catarrh of the Intestine.-The various authors differ as to the character of diet to be recommended in this disorder. Fleiner recommended a simple diet, von Noorden a very coarse diet. We agree with Einhorn, that a moderate diet is best, and, therefore, prescribe a nutritious mixed diet that is not too coarse. With such a diet the movements of the bowels usually become normal and the amount of mucus secreted becomes lessened. Von Noorden's diet consists "largely of Graham bread, $250 \mathrm{gm}$. a day, in addition to a great variety of leguminous vegetables, including the husks, and vegetables containing much cellulose, fruit with small seeds and thick-skinned, like currents, gooseberries, and grapes, besides large quantities of fat, particularly butter and bacon. The permanent effect of the diet is chiefly due to the amount of cellulose it contains. This cellulose undergoes decomposition in the intestines, and thus softens the movements." In connection with the diet mineral waters are utilized; those containing sodium chloride are especially useful, and in particular those of Weisbaden and Kissingen.

Nervous Affections of the Intestine.-At times the most indigestible food is well borne, while digestible food causes discomfort, and it is 
best, therefore, to regulate the diet according to the patient's digestive power. As a rule, the patients are much run down, and a liberal diet is, therefore, indicated; often a well-regulated rest-cure is indicated in order to restore the patient to liealth.

Hemorrhoids.- Inasmuch as chronic constipation is often associated with this disorder, it is important that this condition be correcterl. Pattients having hemorrhoids should eat moderately, and should avoid all excess of food and drink. Moderate exercises should be indulged in, and the bowels should be evacuated daily. Patients suffering with hemorrhoids should avoid spiced food, strong coffee and tea, alcolrolic drinks, cheese, beans, cabbage, and fruits containing seeds. 'Ihey may indulge in carrots, spinach, potatoes, and asparagus. Stewed and raw fruits, meats, eggs, and fish are to be recommended. 'The waters of Carlsbad, Kissingen, and Saratoga are often beneficial in this condition.

Chronic Diarrhœa.- The diet in chronic diarrhoea varies with the type of the disorder. In the nervous form the patient must be taught to restrain his bowel movements except early in the morning. All food having a tendency to increase the bowel movements must be excluded, and for this reason food containing much cellulose must be forbidden. Especially to be mentioned are pickles, salads, turnips, cabbage, carrots. Carbonated drinks should also be avoided. 'To be recommended are tea, red wine, broth, cereals, and boiled milk. At times milk disagrees and causes diarrhoea, and must be excluded even when boiled. In a certain number of cases we have had excellent results in the treatment of chronic diarrhoca by an exchusive milk diet. In other cases a systematic rest-cure was found necessary to bring about good results.

Our Diet-list fol Monerate Cases of Chronic Diakhoea

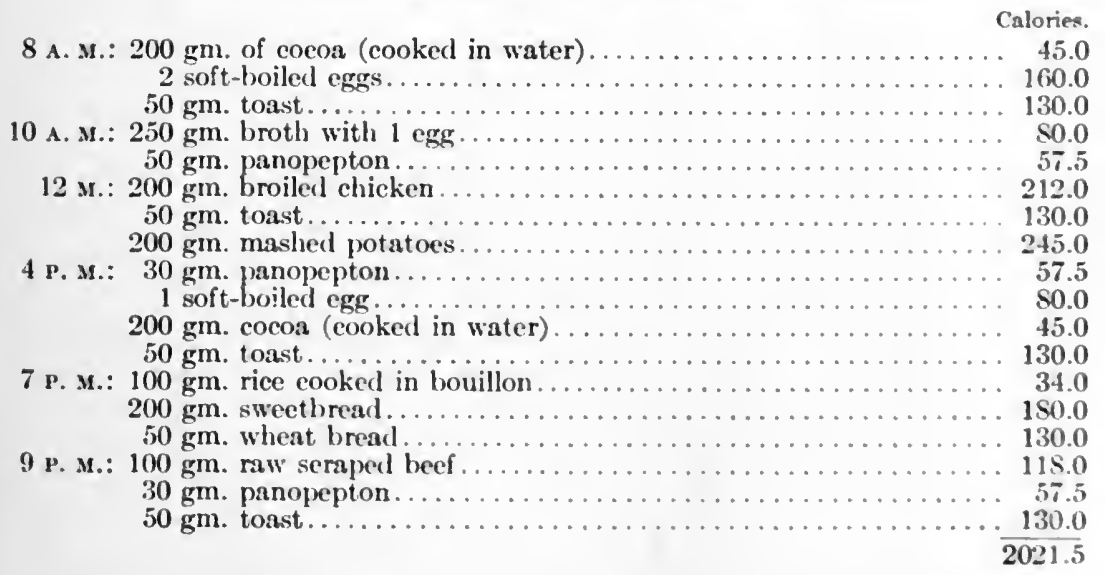

Chronic Constipation.-It is necessary in prescribing a diet in this condition that the food consumed should be of such a nature as to cause intestinal movements. 'Those foods containing a large percentage of 
cellulose, and, therefore, producing a large residue in the intestines, are especially useful in this condition. A glass of cold water to which a pinch of salt has been added taken before breakfast is useful in assisting the movements of the bowels. Raw and stewed fruits aid materially when taken on an empty stomach morning and evening. It is most important that the patient realizes the necessity of having a movement of the bowels at the same time each day. The smoking of a cigar in the morning will often stimulate the peristaltic movements. Chronic constipation is due, in many instances, to a certain dyspeptic condition, and is of ten relieved by treatment of the gastric disorder. The use of drugs should, as far as possible, be avoided, as this condition is often induced by the too frequent use of cathartics. Proper exercises should always be taken in addition to the dietetic treatment of this condition. Of the foods that are useful in the dietetic treatment of chronic constipation are cauliflower, peas, cabbage, asparagus, spinach, tomatoes, celery, onions, salads, and oatmeal. Whole wheat, corn, Graham and rye bread, and bran bread, as well as honey, molasses, cider, and acid fruits, as peaches, apples, pears, oranges, and cherries. Berries are also included in this list, on account of the acid and seeds they contain. Dates, figs, and prunes are especially useful. It is always important to prescribe an abundance of water in chronic constipation, as it is often due to taking insufficient quantities of water. Foods which are constipating should be avoided, as tea, cocoa, chocolate, port wine, rice, barley, and farina gruels, and huckleberries. Milk acts in some instances as a laxative, in others it produces diarrhœa, and it must, therefore, be tested in every case. Buttermilk has usually a laxative effect. By a careful regulation of the diet many cases of chronic constipation can be entirely relieved. We frequently prescribe the following diet in chronic constipation:

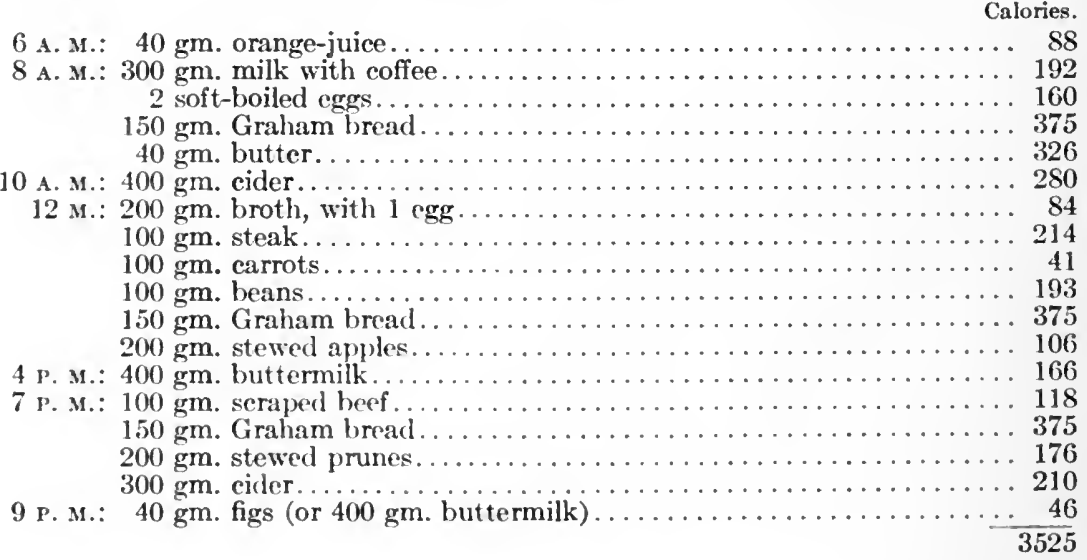

Peritonitis.-In acute peritonitis the diet is only of importance until operation is to be undertaken. The patient should abstain from all food by mouth, and nutrient enemas should be resorted to. If the 
patient no longer vomits, and the operation is not performed, fluids, as milk with lime-water, bouillon, and egg-albumen may be given. Solid food should not be allowed for some weeks. In order to stimulate the patient small quantities of sherry, whisky, brandy, or champagne may be utilized. In chronic peritonitis the diet should consist of foor given in the most easily digestible form, such as boiled meats, eggs, milk, cereals, and vegetables, mainly in puree form. Food should be taken in small quantities, but at frequent intervals.

Liver Diseases.-Biliousness is a term usually applied to a class of symptoms associated with disturbances of the liver and is often due to immolerate and reckless eating. It is very frequently associaterl with constipation. In the treatment of this condition a restricted diet must be insisted on, and calomel administered, followed by a saline purgative; after this a simple but a carefully regulated diet of protein, fats, and carbohydrates may be prescribed. In those affections of the liver in which the liver function is markedly impaired the fats and carbohydrates must be restricted for some days. In all liver affections overeating is apt to cause annoying symptoms, and the abuse of alcohol is also apt to cause serious changes in the liver. It is, therefore, important to avoid the use of alcohol in all such cases. The foods producing an irritating effect on the liver are spices, pickles, mustard, peppers, concentrated meat extracts, and meat broths, radishes, onions, and horse-radish. These, as well as strong coffee and tea, are forbidden.

In serious liver disturbances the diet is usually limited to broth, diluted or peptonized milk, egg-albumen, buttermilk, kumiss, or kefir. In milder forms the diet can be enlarged, including broiled lean meat, eggs, toast, crackers, the green vegetables, baked potatoes, stewed fruit containing but little sugar, and fresh fruit, as oranges, grape-fruit, peaches, pears, and green salads without oil. Mineral waters are useful in the treatment of liver affections if dropsy is not present, and are to be taken early in the morning before breakfast; at times hot water is an excellent remedy in this affection. 'The food must be eaten slowly, must be well masticated, and must be taken in small quantities, and best at frequent intervals.

Catarrhal Jaundice.-In the early period of this disease, when the patient is nauseated and is suffering with indigestion, he should be kept in bed and upon a liquid diet. Milk is the best form of food in this condition, and should be diluted with lime-water or Vichy. Kumiss, kefir, and buttermilk are often also well borne, and may be taken, as well as clear broth, beef-juice, and egg-albumen. Food is best given in small quantities every two or three hours. As soon as the stomach is less irritable, stale bread, zwieback, broiled lean meat, as chicken, sweetbreads, and steak, may be allowed, and, later, thickened flour soup. mashed and baked potatoes, digestible fish, green vegetables, and stewed fruit may be added to the dietary. All coarse forms of regetables and heary raw fruit must be aroided. The meats should be given in small quantities at regular intervals. All heavy food must he avoided for a considerable lengtlı of time after the jaundice has disappeared. Coffee 
and tea, which may be allowed after the acute stage of the disease, should be quite weak; alcoholic stimulants should be avoided, and fats, as butter and cream, should be withheld for a considerable period of time, as also should an excess of starch or sugar. Mineral waters are of considerable service during the entire course of the disease, and of these Carlsbad, Vichy, Saratoga, and Bedford Spring waters are most useful.

Cholelithiasis. - In the dietetic treatment of this disease the meals should be arranged so as not to be too widely separated. The patient should be advised against prolonged fasting, as the ingestion of food increases the flow of bile, while fasting causes bile to be held in the gallbladder. Kehr considers it advisable to eat late at night, while others believe it best to awaken the patient in the middle of the night to administer food. 'This latter, however, seems an entirely unnecessary procedure. Naunyn advises a large breakfast, and a large meal should follow a period of fasting. It is our experience that patients are apt to err in the opposite direction, and take more food than can be thoroughly digested, and this produces indigestion, which is often the immediate cause of an attack of colic. Certain food must be avoided in this condition. Fats must be reduced greatly in quantity, and should be given only in the most digestible form. Herter has shown that animals fed for months largely on a fat diet and a low protein diet are apt to have concretions in the gall-bladder. Excess of starches and sugar must also be avoided, as well as all rich foods. Of meats, only the lean varieties should be utilized. Milk and eggs are permissible, and green vegetables and fresh fruits may be taken. Cereals may be taken in moderate quantities, and potatoes, either baked or mashed, may be given in small quantities. 'The heavier vegetables, as turnips, beets, and cabbage, must be forbidden. Bread should be eaten stale or in the form of toast or zwieback. Alcohol must be forbidden, but light tea or coffee may be allowed. The use of hot water on arising in the morning is useful in this disease. Certain mineral waters are especially useful, as Carlsbad, Vichy, Bedford, and Saratoga. The best effects are obtained when the water is taken at the spring itself.

Cirrhosis of the Liver-As soon as the disease is recognized, if ascites is not present, the patient should be placed on a milk diet or a diet of milk foods. Buttermilk and kefir are especially to be recommended, and eggs, albumin, and cereals added to milk are also valuable. The saline mineral waters are of great service, and should be taken on arising. As the patient improves, the easily digested meats, as chicken, broiled steak, lamb chops, the green vegetables, baked potatoes, fruit, eggs, and stale bread, as toast, are to be recommended. When ascites exists, the salines and a restricted dry diet are of great value, and should be utilized, provided the kidneys are in good condition. Hot water may be allowed in small quantities at intervals during the day, but the remainder of the diet should be, as far as possible, dry. Should kidney disturbance intervene, it is no longer advisable to attempt to relieve the ascites by restricting the intake of fluids. Alcoholic beverages are forbidden. 
Scurvy.-Scurvy is not thoroughly understool. It oxcurs wherever there is a deficient amount of fresh food. In adults it is not common in America, but is occasionally met with in individuals who have been forced to live on preserved foods. Scurvy has been the seourge of armies that have been forced away from their base of supplies, and formerly was very common in sailors, as well as in prisons and other institutions where the diet was improper. 'The prophylactic treatment is very important, as the disease can be easily prevented by the use of lime-juice or lemonjuice, and the addition to the diet of small quantities of fresh vegetables, among which onions are of practical value. When seurry is once developed, the treatment is simply dietetic. 'The diet should consist of good nutritious food, plenty of fresh milk, fruits, and green regetables; it often being necessary to use soft foods, owing to the condition of the patient's mouth. Fresh fruit juices, lime-juice, lemon-juice, or orangejuice should be given freely.

Infantile Scurvy.-It is curious to note that, while scurvy in adults has grown less common, scurvy in infants has become of rather frequent occurrence. Most of the cases occur between the sixth and fifteenth month, and the usual time of onset is between the seventh and tenth month. 'The disease is due to the use of dried, preserved foods and sterilized foods. 'The proprietary foods which are mixed merely with water, sterilized milk and condensed milk produce the greatest number of remaining cases. 'The prognosis is favorable if seen reasonably: early. 'The child should be put on a suitable mixture of fresh milk, and should have some form of fresh fruit-juice added. Sweetened orange-juice usually agrees best of all, but scraped ripe apple or grapejuice, or even lemon-juice, may be substituted if necessary. For older infants small quantities of baked potato, mixed with milk or cream, may be added to the diet, and other forms of fresh food given.

Diabetes.-Diabetes is a disease in which the power for utilizing sugar is lowered; the sugar not being utilized in the body is passed in the urine, but it should be borne in mind that not every person who has sugar in the urine is a diabetic.

'There is a limit to the power of utilizing sugar in all individuals, which varies slightly. with the individual and also with the kind of sugar taken. From 150 to $200 \mathrm{gm}$. of cane-sugar will produce in the average individual a certain amount in the urine. If taken with other food, the limit is somewhat higher. Some diabetics are able to utilize a certain amount of sugar, while others cannot take any without an increase in the symptoms. Some can take a fair amount of starch, while others can take very little, without increasing the glycosuria, and in severe cases. even though the individual is on a sugar-free diet, a certain amount of glycosuria may be present, the sugar being manufactured in the body from the tissues.

One should bear in mind that every diabetic should be dieted separately. One can lay down general rules and general principles, but to obtain success, one must apply these in each case according to circumstances Efforts should be made to maintain the strength of the patient 
if possible, to increase the sugar-utilizing power of the body, and to avoid complications.

Where large quantities of carbohydrates are taken, degenerations of the various tissues of the body are liable to take place, and tendencies to complications are greatly increased. It should also be borne in mind that the sugar passed in the urine has not been utilized as a food, and this amount must be made up in the food in other things. In order to obtain a proper diet of what food the patient may be safely allowed it is necessary to do a certain amount of experimenting. The percentage of sugar in the urinc should be estimated, and the total quantity of sugar passed in each twenty-four hours determined. The patient should then be placed for about five days upon a carbohydrate free diet. Carbohydrates should not be withdrawn from the diet suddenly, but the amount diminished from day to day. Von Noorden's carbohydrate-free diet is as follows:

Brealfast: $5 \mathrm{gm}$. of tea steeped in 200 c.c. of water; $150 \mathrm{gm}$. of ham; $1 \mathrm{egg}$.

Luncheon: $200 \mathrm{gm}$. cold roast beef; $60 \mathrm{gm}$. fresh cucumbers, with $5 \mathrm{gm}$. vinegar, $10 \mathrm{gm}$. olive oil, and salt and pepper to taste; 20 c.c. brandy with 400 c.c. Apollinaris water; 60 c.c. coffee without milk or sugar.

Dinner: 200 c.c. clear bouilion; $250 \mathrm{gm}$. beef (weighed raw), basted with $10 \mathrm{gm}$. butter; $80 \mathrm{gm}$. green salad with $10 \mathrm{gm}$. vinegar and $20 \mathrm{gm}$. olive oil, or 3 tablespoonfuls of some well-cooked green vegetable; 3 sardines in oil; 20 c.c. cognac, with 400 c.c. Apollinaris water.

Supper: 2 eggs (raw or cooked); 400 c.c. seltzer water.

As soon as the urine has remained free from sugar for several days definite amounts of starch are added, usually in the form of white bread, and the point noted at which the sugar reappears in the urine. If these amounts of starch are taken, definite amounts of sugar may be added in the same way. These may be noted in the record as:

Tolerance equals standard diet plus gm. of starch.

Tolerance equals standard diet plus gm. of sugar, etc.

The diet of the patient could then be arranged, allowing proteins, fats, and, in chosen cases, a certain amount of alcohol; green vegetables, and just under the amounts of starch for starch and sugar that the individual has found to be capable of metabolizing.

The urine should be examined from time to time, and the diet varied to suit the changing conditions, owing to the value in the prognosis, as what Mandel and Lusk have described as the dextrose, and nitrogen ratio, which consists of placing the patient on a meat and fat diet, then estimating the amount of dextrose and the amount of nitrogen. If the ratio is 3.65 to 1 , it means a complete intolerance for carbohydrates, and there will probably be a rapidly fatal outcome. The weight of the patient is a fairly good guide to the general nutrition.

In families in which diabetes is present, it is a good plan to limit the amount of carbohydrate food taken, especially where there is a tendency to obesity as the individual grows older. 
While the diet in each case must be made a matter of individual study, the cases may be grouped under the following classification:

Mild forms of glycosuria, in which the urine should be free from sugar, notwithstanding the administration of 50 to $150 \mathrm{gm}$. of carbohychate:

In Eilderly P'ersons. - In these cases the amount of sugar excreted is small, varying from about 0.5 to 2 per cent., and the patients are usually over fifty, with a tendency either to obesity or to gout. 'The outlook in these cases is usually good as long as the patients pay a reasonable amount of attention to their diet, and they get along very comfortalbly.

Care should be taken not to frighten these patients by giving too many directions. 'The patients should have it explained to them that sugar and sweetmeats and all such things are to be given up, and all starchy foods, with the exception of bread and potatoes. 'This includes, of course, all cereals and various puddings and things of a like nature. 'The patient is allowed to take all sorts of meats, all sorts of fats, eggs, and cheese as freely as desired, and to eat all the various green vegetables, concerning the starch content, of which he need not bother. 'The starch taken may be in the form of either bread or potatoes, and, in addition, small amounts of light wine or some other alcoholic beverage may be allowed if thought desirable. 'The corpulent patients usually lose weight on this treatment, which should not be allowed to continue until the patient is too thin, and thin patients should be encouraged to take sufficient amounts of food to protect the body from loss of weight. Unless contraindicated, the patients should take sufficient exercise.

In Young Persons. - The mild cases of diabetes in young people usually occur in nervous individuals, and, knowing the deleterious effects of too much carbohydrates in such patients, the starch tolerance should be estimated from time to time, the amount of bread and potatoes allowed being only the amount that can be given without producing glycosuria, or only that of a most trifling character.

Milk is of great service in these cases, and, notwithstanding the fact that it contains some sugar, it is a good plan to allow a liter of milk a day to the standard carbohydrate-free diet, and then ascertain the amount of bread and potatoes that may be added in addition to this. As in the older individuals, the patient is told to omit all sugars and sweets and all starchy foods, except bread and potatoes, fresh vegetables and fruit being allowed in moderation. The amounts of bread and potatoes allowed should be taught the patient by weighing the quantities a few times; afterward the amounts can be judged by the eye without much difficulty. Where the diet becomes very irksome, and the craving for sweets very great, small amounts of other various farinaceous preparations may be allowed in place of part of the bread or potatoes. Foods that might be mentioned in this connection are chocolate, various fruits, and the various jams and preserves. Foods containing fats, as butter and oil, are very valuable, and the varieties of cheese containing fat may all be used.

'These patients are particularly difficult to manage, as they are young, usually not very ill, and often inclined to overindulgence in 
sweets, alcohol, or in other directions, the dangers of which should be carefully explained to them.

Moderately Severe Forms of Glycosuria.-These are the cases in which sugar occurs in the urine unless all, or nearly all, the carbohydrates are withdrawn. 'The tolerance for carbohydrates varies greatly from time to time, and, as a rule, usually decreases as time goes on. 'These patients should undergo periods of more or less complete abstinence from carbohydrates two or three times a year if possible, this being carried out in a hospital or a private institution, until the patient learns something about the diet. During this period of abstinence the patient should be at as complete mental rest as possible, and only a very limited amount of physical exercise taken. As much of the time should be spent in the fresh air as possible. 'These periods of abstinence from carbohydrate food increase the patient's resistance, and usually cause an increase in the tolerance for starch and sugar. At the end of the period of abstinence the exact toleration of carbohydrates may be determined.

During the intervals the patient should be on a reasonably strict diet, consisting of all sorts of fresh and preserved meats, fresh and preserved fish and shell-fish, eggs, all sorts of fats, either animal or vegetable, green vegetables, such as lettuce, endives, cress, spinach, onions, asparagus, cauliflower, various varieties of cabbage and green beans, olives, and canned asparagus, pickles, and sour-krout. The fatty cheeses and cream cheeses are also very useful. In addition to these things, limited amounts of food containing starch may be allowed in certain moderate quantities. 'The exact amounts should be carefully measured, and this is best done by some one who looks after the patient. The amount of bread or potatoes allowed daily may be replaced by other foods containing a like amount of carbohydrate, as, if the the patient wishes, a liter of milk may be given in place of $50 \mathrm{gm}$. of bread, or this changed for certain amount of beans or other things.

Severe Forms of Glycosuria.--These usually occur in individuals under forty, generally run a rapid course, and end fatally. 'The patient should undergo frequent periods of rigid dieting, as suggested above, if possible, three or four months out of the year being spent in a sanitarium or hospital under strict supervision.

Diabetic Coma.- If the patient is actually stricken, the diet is not of any special importance, but in the threatened cases much may be done to prevent the onset of the coma. 'The patient should be put to bed, milk may be given to drink, usually fairly large quantities of alcohol, preferably in the form of dry champagne, should be given frequently, and large doses of sodium bicarbonate, administered either by mouth or by rectum. If the urine is not passed freely, salt solution should be administered subcutaneously, and von Noorden has suggested adding from 8 to 10 per cent. grape-sugar to this, with the idea of stimulating excretion. In case the patient recovers, he should be allowed a mixed diet containing a certain amount of carbohydrate, care being taken that too much is not given.

Diet Cures.-Various diet cures have been suggested, all of them 
depending upon the fact that if a patient is kept from one to two weeks on some one form of food containing starch there is often established an increased tolerance for carbohydrates. 'This may be done by the use of oatmeal, as suggested by von Noorden, and potatoes, as suggesterl by Mosse; milk, rye bread, and many other things have been suggested.

We have found that the oatmeal diet is usually well borne, and produces good results, especially in the severe cases in which it is impossible to get the urine free from sugar even on a strict diet, and in cases in which there is diacetic acid and acetone in the urine. 'The gatmeal is cooked very thoroughly, and the white of egg or some form of vegetable albumin added to increase its food value, roborat being a palatable preparation. Butter is allowed, but no meat. 'The meals are given at short intervals. Cognac, light wines, and black coffee are allowed. Once a week a vegetable day is given, on which other things are allowed to break the monotony of the diet, and small amounts of meat and fish may be allowed if the patient finds the diet unbearable. 'The oatmeal treatment is usually kept up from one to two weeks, after which, in the severe cases, there is usually found very beneficial results. It should not be used in mild cases.

We have also found that the tolerance for utilizing carbohydrates may be increased in the severe cases by placing them on a strict diet, and adding large quantities of soya beans. 'These beans may be served in many ways, and are palatable when prepared as the small navy beans; that is, in soups, boiled beans, or baked beans. 'These beans contain only $\&$ per cent. of sugar and no starch and a very high percentage of protein, and make a particularly valuable addition to the diet of diabetics.

Substitutes for Sugar.-Various things have been suggested as substitutes for sugar, most of the preparations being furnished in small tablets, each having the sweetening power of an ordinary lump of sugar, saccharin, garantose, dulcin, and saxin being most frequently used.

Substitutes for Bread.-Many have been suggested, but none of them are very satisfactory. Gluten bread is the most popular, but most gluten breads contain nearly as much starch as the ordinary varieties. Certain manufacturers prepare sugar-free foods, some of which are reliable. Almond cakes and cocoanut cakes and dishes made with aleuronat or peanut flour may be tried. Soya-bean flour may be also utilized for making muffins and other articles of diet. Care should be taken in using the various diabetic foods and flour to see what the composition of the food is. A. L. Winton has published a report to the Connecticut Agricultural Station which contains quite a complete list of those on the market.

Obesity.-The causes of obesity are numerous, but perhaps about 50 per cent. of the cases are due to hereditary influences. Overfeeding, especially when accompanied by insufficient exercise, is sometimes a factor, and some people have the tendency to take on weight as they grow older. In some instances the gain in weight is associated with the too free use of alcohol. 
Reduction cures of various kinds have been in vogue for many years. This method of treating obesity is frequently spoken of as banting, taking the name from a reduction-cure suggested by a Dr. Harvey for a Mr. Banting, who subsequently published an account of the treatment.

Various modern authorities have studied the subject of obesity, among which may be mentioned Epstein and Oertel.

In considering the question of reduction-cures, it is necessary to take into account whether the patient is plethoric or anemic, and it is also of extreme importance to consider the age, the condition of the heart and arteries, the condition of the liver and digestive organs, as well as the general appearance of the patient. As a general rule, reduction-cures are not necessary in patients under twenty years of age, but the diet should be so arranged as to prevent any further increase in the deposition of fat.

Diet cures may be undertaken with great benefit in many classes of cases, and they are particularly useful in patients between twenty and fifty years of age who are excessively over weight.

If the patient is not too fat, is in good health, and is in no way incommoded, it is only necessary to limit the diet so that the patient does not take on any additional flesh; but if there are any symptoms accompanying the overfat condition, or if there is disease of the circulation, such as myocarditis, valvular heart lesions, or deposits of fat about the heart, or hypertrophy of the heart, reduction-cures will be found of great value.

In certain diseases of the respiratory system associated with obesity, such as bronchitis, emphysema, or where there are deformities of the chest, reduction cures may also be of great service. In fat people with chronic interstitial nephritis the proper reduction is most useful in maintaining the general health of the patient. Reduction-cures may also be undertaken when the patient is suffering from any disease affecting locomotion, as arthritis deformans or hemiplegia or other nervous diseases, and it is occasionally necessary in the after-cure of tuberculosis cases that have been unduly fattened in the course of the treatment. 'These patients should be reduced slowly to get over the fat, flabby condition in which many of them find themselves.

Another very large class of cases in which reduction-cures are undertaken is chiefly in women, on account of the personal appearance. In families in which there is a distinct predisposition to obesity the diet should be restricted so as to prevent any undue increase in weight.

Under some conditions reduction-cures should not be undertaken. The slight increases in obesity are best managed by restricting the diet so as to prevent any further increase. Patients who have been fat for twenty or thirty years, and who are growing older with but a slight iricrease in the obesity, need not be reduced, as the tendency after certain ages are reached is toward greater leanness. In cases where there is atheroma the patient should not undergo a cure.

The question which is frequently asked is that regarding the reduction of especial parts of the body, and this will depend largely upon 
whether the reduction is undertaken slowly or rapidly. If the reduction is undertaken slowly, the fat is absorbed from all over the loody, while in rapid reductions the loss of weight comes chicfly from the neck, the breasts, the arms, and the calves of the legs.

Massage is frequently used in connection with reduction-cures, but it should be remembered that the effect is to rather increase the part massaged than to reduce it, although general massage is of ten of great use in patients who are unable to take sufficient exercise.

Certain symptoms may follow reduction-cures. 'They may also be the cause of hernias, displacements of the kidney and uterus, and occasionally of gastroptosis or enteroptosis. Gall-stone colic is said to be more frequent in women who have undergone reduction-cures than in others.

Reduction-cures are usually best given over a period of four or five weeks, having the patient lose from five to fifteen pounds during this interval, and then to restrict the diet so there will be no further gain, and, after keeping the patient on this for a while, to reduce him still further by another period of restricted diet. In all cases the amount of protein food should be increased. In plethoric patients the protein is increased while the fat-forming foods, the carbohydrates, and fats are greatly reduced. 'The amount of fluid taken is limited to about the normal amount, that is, about 1500 c.c. a day or a little below this. In cases where there is anemia the protein is also increased and the fat-forming foods decreased, and there should be a decrease in the amount of fluid given. In the third class of cases, sometimes called hydremic cases, where the protein and fat of the body are gradually decreasing, the protein should be increased and sufficient fat-forming foods given to prevent any further waste in the body, while the amount of fluid ingested should be diminished.

The amount of food given in any instance should be well within the limit of that which can be completely used. Oertel has suggestcd the following minimum and maximum standards during the periods of reduction:

$\begin{array}{lcccc}\text { Proteins. } & \text { Fats. } & \text { Carbolyjdrates. } & \text { Minimum calories. Maximum calories. } \\ 156-170 & 25-45 & 75-120 & 1180 & 160 \mathrm{~S}\end{array}$

If the patient is taking sufficient exercise, from 2500 to 3500 calories daily will be utilized by the body, and the difference between that taken and that utilized will come from the body-fat. Exercise is a very important factor in reduction cures. It should be regulated to the patient's strength and gradually increased. Often patients with certain forms of heart disease who are being reduced may take exercise with great advantage, walking being the form ordinarily chosen, the distance in any given case being carefully regulated. The patient is instructed to take as much time in making the distance as he wishes, always heing careful not to go too fast, and not to produce palpitation or other heart symptoms. After the patient is able to go these distances on the level, slight grades may be given him to climb, varying the distance and the grade with the condition of the patient. Individual attention to each 
ease and constant supervision are necessary to prevent doing damage to the heart, especially in patients who already have heart lesions and who are too ambitious. Exereise is contraindicated in myocarditis where there is pronounced albuminuria or general oedema. In such cases rest and proper medication are advisable.

There are many other methods of reduction, the Schweninger method, which is much like the above, except that the use of fluids at meals is absolutely prohibited, the fluid taken being consumed at least two hours after the meal.

Scé usesan increased amount of fluid in certain cases, especially where there is a gouty or so-called uric-acid diathesis, claiming that the increased amount of fluid stimulates metabolism and aids elimination.

The Salisbury method consists of putting the patient upon a diet which is chiefly composed of meat and giving him hot water. One other method may be mentioned, which in certain cases may be of especial value, and that is reduction by means of a rest-cure, as suggested by Weir Mitchell. The patient is placed at rest and put upon a milk diet, and later upon a skimmed milk diet. The amount is gradually reduced until the patient is losing a half a pound a day more or less, as may be thought best in the ease under observation. Nassage, Swedish movements, baths and packs, and electricity are useful adjuvants to the treatment. Care should be taken not to reduce the patient too rapidly or to produce weakness by too severe dieting. In certain cases the restriction of the diet need not be to milk alone.

Diet Cures.-There are a large number of special methods, or special diets, which are used extensively in the treatment of various conditions, usually spoken of as cures, although in many instances all that is intended to be produced is an amelioration of certain symptoms.

Milk Cure.-Milk has always been extensively used in the treatment of disease, and exclusive milk diets have been especially urged by Karell, Weir Mitchell, and others, particularly in the treatment of various nervous conditions, but it may also be used with excellent effect in dropsies, either of renal, hepatic, or cardiac origin, in disease of the liver, and various stomach and intestinal diseases, such as chronic indigestion and colitis, disturbances of metabolism, and the various troubles resulting from it are often relieved by this method of treatment. It is often a good plan to start with skimmed milk, especially where there is difficulty in digesting whole milk, and sometimes modifications of milk, buttermilk, kumiss, or similar preparations, are used. If diarrhœa results, the milk may be boiled, especially with the addition of small amounts of some cereal gruel. The milk is taken slowly, in definite quantities at definite times, starting usually with 3 to 6 ounces three or four times a day, and then the amount is gradually increased until something like 2 quarts a day are given. Later on in the treatment other articles of food are gradually added, and the patient finally returns to normal diet. During the first week or two of the treatment there are usually drowsiness, slight loss of weight, and a coated tongue. Later on there is considerable gain in weight and an increase in the amount 
of urine passed, while the stools are of light yellow, and have a peculiar odor of the milk stools of infancy.

In place of the milk cure, a whey cure is sometimes used, and a kumiss cure has also been advised in many different conditions. More recently, owing to Metchnikoff's publication, buttermilk, and particularly milk which has been inoculated with the Bulgarian lactic acid bacillus, are frequently used, either ulone or in connection with other food. These preparations seem to have especial value in certain forms of malnutrition, in fermentative diarrhoeas, and certain of the chronie dyspepsias and in cases where plain milk is not well borne.

Other cures are the egg-yolk cure, which has been recommended in diabetes, in malnutrition, and in tuberculosis; the grape cure, which consists of eating from 4 to 6 pounds of grapes daily, and other fruit cures. 'There are numerous other methods, such as the dry cure, the meat and hot-water cure, and the Kneipp cure which need not be considered at this time.

The Salt Free Diet.-It has been suggested in a number of different conditions, particularly where the nervous system seems to be hypersensitive, in epilepsy, in diseases of the kidney where there is a tendency to oedema or even latent oedema, and some other conditions.

Two methods may be used, the first, or milder form, consists of removing the salt cellar from the table, and having the patient avoid articles known to contain mueh salt; the second or strieter diet may be arranged with the coöperation of the kitehen. Fresh meat should be used and no salt should be used in the cooking. Fresh-water fish may be allowed, but the salt-water variety should be avoided. Fresh eggs may be taken in any form desired; vegetables should be prepared as far as possible without the use of salt, potatoes, and rice being the most palatable without the addition of it. Bread should be made without salt. Green vegetables and various sweets are all useful, and chocolate will be found of especial value. Fresh unsalted butter, fresh cream, and tinsalted cheese may all be used.

VoL. I-28 



\title{
HYGIENIC MEASURES: THE MANAGEMENT OF EPIDEMICS AND DISINFECTION
}

\author{
BY JOHN WALTER KERR, M. D.
}

TrE ultimate object of all hygienic measures is the prevention of morbidity and mortality. On their observance depends not only individual health and happiness, but ultimately the material prosperity and continued existence of the state itself. In scope, such measures are personal, communal, or national. They react favorably not only on the individual and, through him, the state, but modify prenatal influences on which to some extent at least the phenomenon of natural immunity depends.

Knowledge regarding the practical application of the principles of hygiene is accordingly an essential part of the equipment of every physician, and his profession should be able to exercise its legitimate funetion as conservator of the public health. The medical profession is rightly' expected not only to advise the individual regarding his sanitary welfare, but, as far as possible, to protect the community from the deleterious influences of heredity, and from among its members should come the public officers on whom devolve administrative duties pertaining to preventive medicine.

It is proposed, therefore, to outline those hygienic measures of a fundamental character which the physician is daily called upon to prescribe. They may be logically considered, first, in relation to the individual; second, the community; and third, the state. Since, however, disinfection is perhaps the most important single measure applicable in the interest of all three, it may properly be given separate consideration, as may also the management of epidemics.

\section{HYGIENIC MEASURES-INDIVIDUAL}

From an etiological standpoint, diseases may be classified as constitutional or environmental, the former being due to congenital defects or errors of nutrition, and the latter to outward deleterious influences, such as the attacks of pathogenic micro-organisms. In order to aroid congenital defects it is necessary that hygienic measures in the interest of the individual shall long antedate his birth. It naturally follows, therefore, that such measures must first react favorably on the lives of those responsible for his existence. 
From the moment of birth, the effect of prenatal influence upon development receives no further accretions, and, aside from those states of debility that are manifest from the outset, the health of the individual is almost wholly dependent on his habits and environment. The latter in many instances may be influenced by him only in so far as relates to his own habitation, but the former are capable of great modification, and it is the exercise of these measures that comprises personal hygiene. They include the maintenance of bodily cleanliness; protection from extremes of heat and cold; temperance in the use of foods and drinks; regulation of the excretions; enjoyment of pure air and sunshine; resort to a reasonable amount of exercise; the securing of needful rest and sleep; and the location and care of his home in order that it shall promote health.

Maintenance of Bodily Cleanliness.-People of intelligence recognize generally the value of personal cleanliness both from the rsthetic and hygienic standpoints, but among squalid and ignorant classes there is difficulty in fairly understanding the necessity of this measure. Their education, therefore, becomes necessary, and this is best accomplished through the public schools. Such a method is primarily beneficial to the children, and cannot but be expected to react favorably also on other members in the home.

At the present time too large a proportion of the people do not practise regular bathing, and some even regard it as injurious to health. It, therefore, becomes important to combat this belief.

Bathing is important to health, not only by reason of its removing dirt and infectious matter, but because it has an influence in keeping the skin free from waste-products of metabolism and in condition for the proper exercise of its natural functions.

Baths are classified both as to the temperature of the water and its mode of application. A cold bath implies the use of water at a temperature of $40^{\circ}$ to $70^{\circ} \mathrm{F}$; ; a cool bath from $70^{\circ}$ to $S 0^{\circ} \mathrm{F}$; a tepid bath from $80^{\circ}$ to $90^{\circ} \mathrm{F}$.; a warm bath from $90^{\circ}$ to $100^{\circ} \mathrm{F}$.; and a hot bath from $100^{\circ}$ to $110^{\circ} \mathrm{F}$. Baths are also referred to as graduated, the temperature of the water to begin with being $90^{\circ} \mathrm{F}$., and gradually reduced.

Childhood is the proper time to be taught the importance of bathing, and if parents will see to it that their children have their regular morning baths for the first few years, afterward the child will think no more of it than most children do of daily bathing their hands and face. While very young, a child can readily be taught also to brush its teeth, and later the care of its hair and nails.

The initial bath after birth is preceded by anointing the body with olive oil. 'The bath water should be at a temperature of $100^{\circ} \mathrm{F}$., and the body entirely submerged except the head. As age advances, the temperature of the bath can be reduced from time to time, and in later childhood cool baths are to be preferred.

A cool bath acts as a general stimulus to the body. At the beginning, the cutaneous vessels contract, the respirations are deepened, the heart's action is somewhat slowed and increaser in force, and the nervous system is favorably stimulated. If the bath is continued not over long, there 
follows what is known as the "normal reaction," which manifests itself by dilatation of the cutaneous vessels, redness and warmth of the surface of the body, and return to normal of the heart and respiration rate.

Cool baths are best taken in the early morning, and if the normal reaction does not follow, but instead blueness of the skin, shivering and continued eold, they should be discontinued. 'This is likely to happen only in the case of delieate children and adults and old people.

Whole baths may be taken by means of the sponge-bath, the tub-bath, the shower-bath, or the surf-bath. The latter is especially beneficial because of the exercise and pleasure attendant upon it, and not due to any inherent property of sea water.

The shower-bath is also attended by a certain amount of exercise, and more stimulating than tub-bathing, because of the impact of the falling water upon the skin.

'The cool tub-bath is most convenient and generally used. 'The addition to it of commercial sea salt is valueless.

Warm baths are more cleansing and sedative, and best taken before retiring.

Should facilities for complete bathing not be at hand, the spongebath and foot-bath should be employed daily, particular attention being paid to cleansing of the face, hands, feet, groins, genitals, and axilla.

Protection from Heat and Cold.-By reason of the nervous mechanism regulating the production and loss of heat, and the conserving of heat with clothing, man is able to withstand any climate. 'The use of wearing apparel, therefore, becomes a hygienic measure. Choice of clothing depends on the season, and the availability of the different materials, which include cotton, linen, wool, silk, furs, leather, and rubber. While cotton garments do not shrink in washing, they are not very hygroscopic, and rapidly conduct away heat. In summer, on account of their lightness, they are preferable to woolen ones as a means of protection against the sun's rays, but on account of its property of conductivity, cotton is not the most suitable material for underwear. Its property of dissipating heat may be overcome, however, by using loosely woven fabrics, or two or three garments over one another, as air is a poor conductor and the body is thus wrapped in superimposed blankets of air. Iinen likewise favors the dissipation of heat, and does not absorb moisture as readily as wool. Its use in summer is advisable, but not for underwear.

Wool is a poor conductor of heat and very hygroscopic. On account of these properties and because of its lightness it is especially suited for garments used in any climate. It protects from cold and prevents too rapid evaporation from the skin. Its use as a material for underwear is accordingly advised, and in the tropies this is especially applicable for the prevention of chilling, which may give rise to dysentery and congestion of the internal organs. Woolen underwear requires frequent washing because of its property of absorbing odors and effete matter from the body, but because of its liability to shrink and harden, such washing must be carefully done. 
Silk is also an excellent material for clothing, especially underwear, on account of its being very hygroscopic and a poor conductor of heat, and because of its "lightness." It has the additional advantage that it is more cleanly, does not shrink, and is less irritating to the skin than wool.

Leather and water-proof clothing are sometimes of service on account of their property of protecting from winds, rain, and exposure to injuries, and the indications for their use are to promote comfort and prevent disease.

Care in the selection of clothing is especially necessary during childhood. The clothing at this age should be warm and loose; it should cover the body, and the undergarments should preferably be made of silk or soft wool. Undue exposure of the body, such as the knees and legs, with the view to hardening the child, really diminishes resistance through loss of heat, and results in congestion of internal organs, with accompanying catarrhal affections.

The clothing of adults should not interfere with free movements of the body, nor with respiration, digestion, or circulation of the blood. Tight fitting garments should be avoided, especially those interfering with normal expansion of the thorax.

Since white and light colors absorb less heat from the sun's rays, they are cooler in summer and particularly suited for tropical wear. In the tropics care is necessary to avoid undue exposure to the direct rays of the sun. For this purpose a helmet should be worn, made of white, gray, or khaki material, and the outer garments should be of the same colors. In order to avoid chilling, wool next to the skin is advised, and Simpson advises the use of a cholera belt or knit woolen belt over the abdomen both day and night in the tropics.

The shoes are perhaps the most important article of dress to be considered. Their shape should conform to that of the foot. High heels should be avoided, and the material of which the upper is made should be soft and pliable.

Regulation of Excretion.-The exercise of hygienic measures has a potent influence on the excretions. By means of proper diet and exercise much may be done to insure the free action of the skin and kidneys and prevent constipation. Young children should be taught early the habits of regularity. The desire to evacuate the bowels or bladder should always be granted, but with care the performance of this function may be accomplished at a regular hour each day, preferably shortly after the morning meal. The drinking of an abundance of water is to be encouraged, as, in addition to combating constipation, this element of food flushes the urinary tract, and promotes the excretions through the skin.

Pure Air and Sunshine.-In order that the function of respiration shall be properly performed, pure air is necessary. The oxygen must be in normal proportion, and the maximum of carbon dioxid should not be allowed to exceed 6 parts in 10,000. A moderate amount of watery vapor is also essential to man's well-being; unusually low humidity 
of the air giving rise to dryness of the skin and mucous membranes, and great moisture preventing evaporation and consequent cooling of the body.

'The greatest danger to health, however, comes from vitiated air, as is shown by the development of "colds" in stuffy rooms and the undue prevalence of respiratory affections among those whose lives are mostly spent indoors and in overcrowded quarters.

'The breathing of foul air gives rise to headache, drowsiness, and bodily depression, and if long continued, results in the lowering of resistance to disease, especially pulmonary tuberculosis. It appears that the effects of breathing impure air are due in part to the organic matter given off during respiration, and in part to the bacterial growth in the compartment brought about by increased heat and moisture. Carbon dioxide may also play a minor part, as may also carbon monoxide, the latter originating in the heating apparatus, or as a result of incomplete combustion of illuminants.

Regardless of the factors bringing about vitiation of air, the necessity of a pure supply is clear. 'The individual must see to it that he obtains an abundance of pure air both day and night. 'The windows of his sleeping apartment should always remain open, his place of occupation should be well ventilated, and when, by reason of vicious inheritance, there is lowered natural immunity, selection of outdoor occupation should be advised. The increase of urban population tends to limit the availability of pure air in city residences, and can be overcome only through prevention by the state of overcrowding. But practically every individual is free to spend some of his time in the open air, and those predisposed to affections, such as tuberculosis, will do well to make this a regular practice.

Outdoor life also permits of exposure to sunlight, which acts as a mental stimulus and exerts a favorable influence on metabolism. It is known also that direct sunlight is a powerful disinfectant and beneficial to health. While excessive exposure to light on the part of the individual may be injurious, it is practically certain that this does not oceur under ordinary conditions, and that persons who live in the open air are healthier than those who live indoors and follow sedentary occupations.

Rest and Sleep.-Following exercise and periods of mental activity, there is necessity of rest and recreation. This applies to all the tissues of the body with the possible exception of certain gland-cells and musclefibers, and even these slacken their activity from time to time.

Fatigue is the indication for rest, and experience has shown that the hours of the day are best apportioned regularly for alternate activity and repose. 'The amount of rest necessary must depend on the character of work and the capabilities of the individual. In the case of children, more rest is required in which to permit of building up of the tissues, and the hours of labor of adults have been progressively diminished in recent years. Persons engaged in monotonous occupations should be provided with more recreation on account of the mental effect.

While the mental activities of individuals vary greatly, rest and 
recreation are essential to all to prevent nervous exhaustion and mental degeneration. The cultivation of mental control will do much to prevent worry, which is more productive of nervous breakdown than work, and the annual vacation, which is becoming more common among all classes of workers, is of the greatest benefit in maintaining the health of body and mind.

As to the amount of sleep required, no hard-and-fast rule can be made. It depends on age, occupation, and climate. Eight hours may be said to be sufficient for the average man, but some persons require more sleep. On the other hand, it is said that Napoleon, in the heyday of his life, never slept more than four or five hours in the twentyfour, and five or six hours was the usual allowance of Frederick of Prussia during the greater part of his long and active life.

Sanitation of the Home.-Hygienic requirements in the interest of the individual must take into account also the sanitation of the house in which he lives. If choice is practicable, the house should be located on rising ground, high enough to avoid fogs and dampness, and protected from strong winds by trees and shrubbery. The soil should be porous and capable of easy drainage, and the location should be removed as far as possible from swamps and breeding-places of flies and mosquitoes.

An ample supply of potable water should be provided, and there should be adequate facilities for the removal of refuse. In the absence of sewage connections it is important that facilities be provided for the proper disposal of excrement. For this purpose, a well-screened dry earth closet is well suited.

A southern exposure is desirable, especially if the house is in a tenement or apartment, and in any event it should be well lighted, well ventilated, and thoroughly screened and rendered rat-proof. An abundance of pure air and sunshine is requisite to the maintenance of health, and since air is vitiated by respiration, combustion, and putrefaction, care must be taken in order that their end-products shall not exert a deleterious influence on those occupying the dwelling. The heating and illuminating apparatus should receive special attention in this respect, and thorough cleanliness should be maintained and garbage promptly removed, so that it shall not become a nuisance.

The cleanliness and ventilation of the dwelling is as important as that of the hospital or operating-room, and windows should be so arranged as to admit of the entrance of an abundance of sunshine. It would be well if some of the sleeping rooms in every house were located without the walls. The floors should be of hard wood, polished, and only partially covered by rugs. The walls should be painted rather than papered. 'The furniture should be plain, and all hangings should be washable. Brooms covered with moistened bags should be used for cleaning. Feather-dusters should be abolished, and whenever practicable, vacuum cleaning should be adopted.

Electricity is to be preferred for artificial illumination, and heating is best accomplished by hot water or steam under low pressure. Open 
fires, however, aid ventilation and render more checerful the living rooms. Every house should have a bathroom, and the kitchen and servants' quarters should be capable of thorough sanitation. Lnder no eircumstances should soil pollution be allowed to oceur, and the soiling of the surroundings with human excretions, such as sputum, should not be allowed to occur.

In the selection of a habitation, overcrowded tenements and districts are to be avoided, since it has becn shown by statistical studies that the morbidity rate increases with density of populations. In general, it may be estimated that the dwelling should contain 1000 cubic feet for each occupant, though conditions of poverty may require a very considerable reduction from this standard. The by-laws under the London Public Health Act of 1891 require a minimum of 300 culbic feet to each adult occupant in sleeping-rooms of tenements, and 400 cubic feet in rooms not used exclusively as sleeping-rooms.

The individual living in rented quarters is under the same obligation to the community to preserve thorough cleanliness and otherwise observe the laws of personal hygiene as is the owner of his own dwelling. In addition he has rights under the laws of the community, and such laws also place restrictions upon him in the interest of the public health. These sanitary laws and ordinances represent the hygienic measures necessary for the protection of the community, and will be considered under that head.

\section{HYGIENIC MEASURES-COMMUNAL}

Under modern conditions of life the individual himself is unable to exercise the full measure of protection against disease, and it is only through official agency that this can be accomplished. Community of action in this respect has brought about a marked reduction of the death-rate, which during the last century has declined one-half and the expectation of life increased by ten or twelve years. These results, however, are due almost entirely to diminution of the infectious diseases, little or no reduction having occurred in the case of organic and other diseases of advancing life.

It has been estimated that by reasonable and practicable measures of prevention, based on existing knowledge, the amount of illness now known to be preventable could be further reduced from 30 to 50 per cent. But in order to do so, combined effort on the part of national, state, and local health authorities will be required, and on the latter will devolve the greatest duty in this respect. ${ }^{3}$

The town or township is generally the unit of sanitary administration, but it derives its powers in this respect from the state, and may be said to

1 On account of our dual form of government, and because sanitary police powers within the states, by whatever authority exereised, are in the interest of the community, "Hygienic measures-communal" will be taken in this chapter to mean those measures properly enforeel under state law. On the other hanil, "Iygienic Measures-National" will be taken to menn those measures devolving mion the national government by resson of its sovereignty. 
represent the latter in the community. In some instances it has assumed great importance in health matters. These results on the part of the municipality predicate the adoption and enforcement of efficient measures for the prevention of communicable diseases and the inauguration of measures having for their object the improvement of hygienic conditions by means of which life will be rendered more productive.

Among the more important hygienic measures necessary in the interest of the community may be considered the following: Registration of cases of sickness and deaths; care of cases of communicable diseases and prevention of their spread; maintenance of asylums; supervision of water and food supplies; removal of sewage and wastes; supervision of dangerous and offensive trades; regulation of housing; prevention of nuisances, and disposal of the dead.

Registration of Cases of Sickness and Deaths.-Statistics of morbidity and mortality are the fundamental basis of effective sanitation, and their registration now devolves primarily on state and local authorities. The laws recently enacted for the collection of death statistics are in general uniform, and require the prompt reporting of deaths. The burial or removal of any body without a permit issued by a local registrar is forbidden, and no permit can be issued in those states until a complete and satisfactory certificate of death has been filed. On the undertaker is placed the responsibility of filing the certificate, and the medical certificate must be made and signed by the physician last in attendance on the deceased. Through such provisions and their enforcement, returns of all deaths will be made possible, and the responsibility for reporting them to the local authority is fixed. The local registrar is in turn required to report regularly to the state authority, the latter being given supervisory powers under the law for the faithful execution of its various provisions. Falsification or failure to record either permit or death certificate should be, and generally is, a punishable offense. 'The data thus collected are enduring records of the effectiveness of preventive measures, and serve as a guide for further sanitary laws and ordinances.

Morbidity statistics are of even greater importance to the sanitarian than mortality statistics. They not only indicate the severity of disease and its damage to society, but serve as a guide to prompt sanitary action. A complete system of morbidity registration would be invaluable both from epidemiological and therapeutic standpoints, since it would be possible to determine the "expectation of sickness" and efficacy of treatment under different conditions.

Three methods have been proposed for the collection of morbidity statistics, viz.: enumeration, estimation, and registration. The latter method is the only feasible one, but its success thus far has been limited on account of a lack of recognition of the value of such data to the community. Nevertheless, the systematic registration of cases of preventable diseases should be required by law, and, as public opinion is moulded, other remotely communicable diseases should be added to the list. In Pennsylvania notification is required to be made to local health 
authorities of 31 diseases, as follows: Actinomycosis, anthrax, bubonic plagne, cerebrospinal meningitis, chicken-pox, cholera, diphtheria, epidemic dysentery, erysipelas, German measles, glanders, hydrophobia, leprosy, malarial fever, measles, mumps, pneumonia, puerperal fever, relapsing fever, scarlet fever, small-pox, tetanus, trachoma, trichiniasis, tuberculosis, typhoid-fever, typhus fever, whooping-congh, prellagra, hookworm disease, and yellow fever. 'The reports are made on postal cards by physicians in attendance and serve as a basis for the institution of preventive measures by local health authorities. Provision is made, however, for the making of such reports by telegraph when the necessities of the case demand.

Measures with Respect to Communicable Diseases.--The measures to be taken on notification of an infectious disease, endemic in the community, are essentially the same as during epidemics, the management of which is considered on p. 458. 'The local health officer or inspector should visit the house to determine whether the patient can be properly cared for therein or should go to hospital, and whether necessary precautions are being taken to prevent the spread of infection to others.

In the interest of the community, the health authority should also ascertain whether there is overcrowding in the house, and whether its occupants in consequence are unable to observe necessary preventive measures if the case remains. In addition, it should be determined whether there is accumulation of house refuse or fouling of the premises that may affect the incidence of the disease.

An inspection should be made of the sewerage system or other means of disposal of excreta and wastes to determine whether there are proper ventilation of sewers, trapping of house-drains, removal of wastes, and cleansing of basement and yards.

Every effort should be made to ascertain the origin of the infection in order that prompt measures may be taken to deal also with the original foens of infection. 'This will necessitate inquiry regarding the date of onset of the illness and the patient's whereabouts immediately previous thereto, the association he has had with others, the condition of the premises, the number of occupants in the house, the possibility of previous cases in the same house, and the character and source of food and water supplies.

Such information is necessary in the specific instance and of value also for the improvement of sanitary conditions in the community as a who'e.

A most important measure is the giving of instructions regarding prevention to the members of the household, which may be done in person or by means of hand-bills, and the public should be warned by means of placards bearing the name of the infection. 'The question of isolation of the case, surveillance of contacts, administration of prophylactic serum, cleansing and disinfection from day to day, will then be decided, depending on conditions. It is not sufficient, however, to leave the enforcement of necessary measures entirely to the attending physi- 
cian and family, but the health authority should give the matter supervision, and on the termination of the illness be prepared to carry out necessary disinfecting procedures. In the mean time, if there is reason to suspect the water or food supplies, they should be investigated. In the case of typhoid fever and diarrhœal diseases, if the only available. water supply is not above suspicion, the water should be boiled, and it would be better if all milk not "certified" were pasteurized before use by heating for twenty minutes at a temperature of $145^{\circ} \mathrm{F}$.

Other detailed measures will suggest themselves, depending on the character of the infection, but they should be reasonable, and wisely enforced. The difficulties surrounding the handling of long-continued infections, such as tuberculosis, diphtheria, and typhoid fever, must be met, but unreasoning fear of persons so affected should not be encouraged. They should be held under supervision of the health officer, and, especially in the case of "bacillus carriers," authority should be had to regulate their appearance in public as well as their occupation, so that it shall not involve the handling of foods and beverages intended for public consumption.

Supervision of Water Supplies.-In communities of any size there is likely to be one or more common water supplies, and these should be subject to supervision by the local health authority or other official agency. In small communities such supplies are usually derived from stored rain or ground water, the latter including springs and wells.

Shallow wells are often polluted, and it is the duty of the community to adopt measures to prevent the pollution of any well or spring. When there is reason to believe that ground water is injurious to health, the well or spring from which it comes should be closed by authority, but if this is impracticable, an order should be issued that the water be boiled.

The water supplies of large communities are usually surface in character, and, therefore, liable to contamination. While natural purification of water during flow occurs to some extent, it has not been definitely determined the length of time typhoid bacilli will live in streams under different conditions. It thus becomes necessary for commun ties to provide for artificial purification by means of either chemicals, bciling, distillation, or filtration.

Alum has long been used in the proportion of $\frac{1}{2}$ to 1 grain per gallon, which, by uniting with the $\mathrm{CaCO}_{3}$ in the water forms a flocculent precipitate, thus carrying down the bacteria. Other chemicals, such as permanganate of potash, lime, iron, sodium hypochlorite, and ozone are also sometimes used on a large scale. The use of hypochlorite of calcium has recently attracted much attention, and appears to have yielded excellent results. On account of the free chlorin available, the process has a high germicidal action, and the cost is exceedingly low. It has accordingly been adopted in a number of cities, as Jersey City, Minneapolis, and Montreal.

While efficient as a means of sterilizing water, boiling is not applicable on a large scale. Distillation can often be used, however, in institutions and on board ships, and is thoroughly efficient. 
For general application in communities using surface waters filtration is lest adapted, either slow sand or mechanical filters being used. Hazen has estimated that in 1904 there were in the Linited States 560,000 people supplied with water from sand filters, and 2,600,000 with water from mechanical filters. In view of the increasing pollution of streans, the official supervision of artificial purification becomes important, and whatever method is adopted, expert supervision is necessary to attain hygienic efficiency. 'The mechanical filter consists of an iron or wooden cylinder filled with moderately coarse sand, through which water is forced with pressure, the process being supplemented oftentimes by the use of a coagnulant.

Sand filtration may also require previous treatment of the water with a coagulant or sedimentation, especially when there is ligh turbidity. 'The filter-beds contain layers of coarse and fine gravel and coarse and fine sand, superimposed on each other in the order mentioned, to a depth of from 3 to 5 feet. After the water has been applied, its rate of flow is regulated so that generally not more then $2,000,000$ gallons will be allowed to pass daily through each acre of filter-bed.

Whether the filter-beds are operated by the community or not, they should be carefully supervised, and daily chemical and bacteriological examinations should be made to test their efficiency. The filters should be required to be cleaned frequently, and, according to Koch, when the water contains more than 100 bacteria per cubic centimeter, it should not be distributed for the use of individuals.

Regulation of Foods. - Since certain kinds of food may become contaminated with pathogenic micro-organisms and convey the same to man, and since food may become unwholesome by reason of adulteration and changes due to age, its supervision becomes of great importance to the community. Those foods and beverages that enter into interstate traffic come under the provisions of the national pure food and drugs act of 1906. Articles of food originating within a state are subject only to the laws of that state, which laws may be enforced by central or local authority. Not only the food itself should be subject to control, but the conditions under which it is offered for sale, and this devolves upon local health authorities.

The proper authority should have power to inspect at all times any foods or beverages offered for sale, and the market, stall, or manufactory in which they are kept. Such authority should prevent the sale of meat derived from animals dead of disease, or that are found to be diseased.

Meats that enter into interstate traffic come within the purview of the national meat inspection act, under which regulations have been issued specifically defining the necessary sanitary conditions surrounding the preparation of such meats, and those parts of carcasses that should be condemned. Such regulations may well serve as a guide for local health authorities. ${ }^{1}$

The meat of calves under four weeks, pigs under five weeks, and lambs under eight weeks of age, should not be sold, nor should any meat be ${ }^{1}$ U. S. Dept. of Agriculture, 13. A. I., Order 150. 
allowed to be exposed to the dust of streets. The latter rule should apply as well to the products of bakeries and confectionery establishments. Not only the shops and markets where food is sold should be required to be kept clean, but boarding-houses, restaurants, and all public places where the food is used should be subject to sanitary inspection at the discretion of the health officer.

Due care should be exercised to prevent the sale of adulterated foods that may be dangerous to health, and they should not be kept in such a manner as to render them unwholesome or poisonous. Especially should supervision be had over the handling of milk from the farm to the consumer, and where there is reason to believe that it is infected it should be destroyed. Milk should not be allowed to contain preservatives, and it should not contain less than 3 per cent. of butter-fat nor less than 12 per cent. of milk solids. 'The minimum bacterial content required will depend on local conditions, but milk should be free from pathogenic organisms, such as tubercle bacilli, and where this cannot be guaranteed, it should be pasteurized. There would appear to be reason for the issue of permits to sell "skim" milk, provided it is sold as such, but adulteration or underweight of any article of food should be forbidden.

Authority should be granted to the proper official to condemn any article of food, such as meats, vegetables, milk, or breads, found in the market and unfit for use, and the owner should be required to destroy it.

Removal of Sewage and Refuse.- Whether removed by the householder or by contract entered into by the community, there is necessity of such official supervision as will prevent sewage and wastes from becoming a nuisance or detrimental to health. In the absence of a sewerage system, every householder must, of necessity, provide for the disposal of effete matter, but the method should be subject to sanitary regulation, and ordinarily one of two methods may be prescribed, viz.: biologic purification by means of the septic tank, or the pail system for slops and dry earth closet for human excrement. Garhage should be required to be burned, buried, or carted away to a designated place. When the use of the dry earth closet is approved, waste water from kitchen and bathroom may be distributed over land where it will do no harm, but soil pollution with human discharges should not be allowed to occur.

The sewerage systems of communities should be under official control, in order that they shall remain efficient, and no part be diverted from its proper purpose. Sufficient water should be provided for their operation, and necessary inspections should be made to determine adequacy of ventilation of sewers and trapping of drains. Ordinances regulating plumbing and building should be enforced, and no person or firm should be allowed to discharge any sewage, drainage, or factory refuse into any body of fresh water excepting under such conditions that no nuisance will result.

The discharge of crude sewage into streams should be prohibited, as otherwise natural bodies of water will be converted into sewers and endanger health. 'The purification of sewage of towns thus becomes 
imperative, and it may be accomplished by methods to be determined by expert engineers after full consideration of local conditions. Among the methods may be mentioned: Chemical precipitation in tanks; liquefaction by means of anaerobic organisms in septic or cultivation tanks; and liquefaction by means of aerobic biologital filters. Following any of the above methods, the liquefied sewage requires treatment by land irrigation or biological filtration to render the efluent safe. In any event, the system of sewage disposal to be adopted is a highly technical problem, and in this matter communities should be guided only by expert advice.

After the disposal of liquid sewage there remain street sweepings and house refuse that must be removed. In communities reasonable ordinances should be enforced requiring such removal in order that there shall be no nuisance. In small communities each individual may be expected to do his part of the work, but in municipalities of any size, the local authority should become responsible for the daily or weekly removal of refuse.

The question of final disposition will arise, and it may be said that burning is the safest and most efficient method. For this purpose communities should be provided with incinerators, but householders can render great assistance by burning house refuse, as far as possible, in the heating apparatus of their homes, and the sanitary authority should encourage them to do so. In addition all householders should be required to provide proper receptacles for collecting and retaining, without leakage, all remaining ashes, garlage, and refuse, which should be promptly carted away to a proper place, and no person should be allowed to fill in any land within municipal limits with garbage, dead animals, decaying matter, or unwholesome materials.

Regulation of Dangerous and Offensive Trades.-In the interest of individuals, the community should supervise by factory inspection and otherwise, dangerous occupations so as to minimize as much as possible their ill effects. Occupations may be dangerous to the community, but particularly to the persons engaged therein by reason of the following: Accidents in the operation of machinery; breathing excessive quantities of dust; working under conditions of excessive temperature and moisture; poisoning from certain metallic preparations, such as lead and phosphorus; breathing fumes of non-metallic chemical substances or noxious odors of decomposition, and bacterial infections through handling infected materials. All these conditions, including exclusion of women and children from exhausting occupations, may be met in great measure by regulations properly enforced by either local or state authority.

In general, licenses or permits should be required for the carrying on of dangerous trades, and similar restrictions should also apply to offensive trades. Offensive trades are usually objectionable to the community on account of attending noise, smoke, dust, or odors, but in some of them the emanations given off may possibly become dangerous to life. 
If practicable, offensive trades, such as bone boiling, bone grinding, rendering, tanning, manufacture of fertilizer, rag sorting, or chemical processes in which an organic basis is used, should not be carried on in urban districts. If such trades are carried on in municipalities, they should be under sanitary supervision, and the local sanitary authority should have power to inspect at ali hours such establishments, and require that they be kept clean, properly ventilated, and not overcrowded. 'Trades that are not necessarily objectionable may become so by reason of neglect or mismanagement, and, as in the case of stables, much may be done by inspections to prevent unhygienic conditions which foster disease.

On the whole, there is necessity for systematic investigations of the various occupations in order to determine their influence on health and the further safeguards necessary for the protection of those engaged therein.

Regulation of Housing.- In the interest of the community, special attention should be paid to proper housing, especially of the working classes. Habitations should be free from dampness and organic pollution. They should be required by ordinances to be so situated and constructed as to insure adequate light, heat, and ventilation, and an abundance of potable water should be supplied, as well as a proper system of plumbing for the removal of sewage. Such provisions should apply also to schools, factories, and workshops, and they should be required to be kept clean and free from overcrowding.

The plans and specifications of all buildings to be erected in urban districts should be subject to official approval. The minimum amount of air space required varies in different ordinances in force. In the District of Columbia it is required that "no room in any tenement or lodging house shall be occupied as a sleeping room unless there are at least 400 cubic feet contents for each person therein not less than ten years of age." Tenements and lodging houses especially should be subject to official supervision and inspection, and the right should be granted to the sanitary authority to regulate the number of occupants therein. Owners of such structures should be required to secure cleanliness of basement and courts, keep the drains in order, and prevent obstruction of ventilation. Animals that cause filth should not be harbored in tenements, and such buildings should be required to be cleaned periodically in the interest of the public health.

In general, the height of dwellings should not exceed the width of the street, but in deciding this question, consideration should be given to the depth of the open space in the rear.

Power should be granted the sanitary authority to prevent the use of tenements and lodging-houses for other than living purposes, and the storage therein of combustibles or other articles prejudicial to health should be forbidden.

Buildings that are found on inspection to be unfit for human habitation and incapable of being placed in sanitary condition should be condemned by proper authority, and when so condemned should be required 
to be demolished by the owner or at his expense. Authority should also exist for dealing with alleys and alley houses, in order that they may be made to conform to a reasonable sanitary standard. It is only through the enforcement of such hygienic measures that the incidence of tuberculosis and other so-enlled "house diseases" can be favorably affected.

Prevention of Nuisances.-One of the most useful hygienic measures in the interest of the community is the albatement of nuisances. They have been defined as "whatever is dangerous to human life or health, and whatever renders soil, air, water, or food impure or unwholesome." From this it is evident that they include most insanitary conditions that require official supervision, but detailed mention of each one would be impracticable. Nuisances may be generally classified, however, to include: premises that are in an insanitary condition and dangerous to the public health; stagnant waters, drainage courses, sewerage systems, streets, out-houses, or refuse bins in such condition as to be prejudicial to health; animals kept in such a manner as to be offensive or detrimental to health; manure piles or other accumulations or deposits that may be offensive; overcrowding of human habitations and work-places to the injury of the occupants; manufactories so managed as not to prevent insanitary conditions, and poisonous emanations from industrial and other establishments that affect unfavorably the inhabitants of a community.

Local health officials should endeavor, by periodical inspections, to discover the muisances in their districts that require suppression, and upon receiving complaints of nuisances they should investigate the same with the view to their abatement. In addition, the inhabitants of a community should coöperate with the sanitary authority by giving information regarding conditions injurious to health.

The method of abatement of nuisances will depend on their nature, and the provisions of the law or regulations under which action is to be taken, but generally the sanitary authority will notify the persons, committing or maintaining the nuisance, to abate the same within a stipulated time, and if the necessary action is not taken, the health authority will do so at the expense of the responsible persons. In any case the circumstances will be considered in order that any action taken will not work a hardship on the individual without commensurate benefit to the community as a whole.

Hygienic Disposal of the Dead.-In the interest of the community, provision must be made for the prompt disposal of the dead, especially those due to the infectious diseases. In urban districts this includes the maintenance of mortuaries, regulation of burial places, and conduct of funerals.

An analysis of the public health laws reveals the facts that practically all of the states and territories have laws upon this subject. In generai, these laws require that no disposition of a body can be made without a permit, and in the case of contagious diseases, special precautions must be taken to prevent the spread of infection.

$$
\text { liot. I-29 }
$$


Only two methods of disposal need be considered, viz.: cremation and earth burial. From a hygienic point of view, the former is far preferable, and to be encouraged in a community, yet earth burial is practically universal, and should be subject to sanitary supervision. Burials should not be permitted except in such grounds as have been specifically set aside for the purpose, the depth of interment should be prescribed, and should not be less than 4 feet from the surface of the ground. Public funerals should be forbidden in the cases of contagious diseases, and no unnecessary delay should occur that will jeopardize the health of the community.

The Relation between State and Local Authorities.-The foregoing hygienic measures are necessarily enforced within the communities of a state under authority of the state itself, by whatever agency employed. But wherever practicable, executive power in local sanitary matters has been delegated to the local community, thereby making it responsible, in so far as possible, for its internal sanitary condition. It thus becomes evident that the powers of state and local health authorities vary in degree rather than kind.

On account of rapid transportation and consequent intimate relation between communities, however, it is impracticable for each in itself to exercise the full measure of protection. 'There is accordingly necessity of coördination in sanitary matters, and this should be brought about by state authorities acting in a supervisory capacity. Whenever necessary, state authorities should also exercise their broader powers to enforce hygienic measures when local authorities fail or refuse to do so, and in addition regulate sanitary affairs between communities, and conduct investigations for the benefit of the state as a whole.

\section{HYGIENIC MEASURES-NATIONAL}

While police powers with respect to sanitation within the states have been reserved to the states themselves, they are without extra territorial jurisdiction. The national government, therefore, has responsibilities with respect to interstate as well as international sanitary matters, and must enforce those hygienic measures devolving upon it by reason of its sovereignty. In considering such measures, account must be taken of the jurisdiction of the national government in sanitary matters, which may be said to cover: foreign intercourse; interstate intercourse; federal territory, and federal administrative affairs, including protection of the Indian tribes.

The hygienic measures taken in federal territory, and in the interest of the Indians, however, are generally the same as for communities, except in so far as outward influences are concerned. Only those measures will be considered, therefore, that are peculiar to the federal government, which thus far have been taken in the interest of commerce under authority of the "interstate clause" of the constitution. Such measures as are now being enforced will be considered, as well as those that should be taken in the interest of the public health. 
Foreign and Insular Quarantine.-By the act of February 15, 1893, all international as well as interstate quarantine relations were placed under the national government. The measures taken under this law include the issue of regulations for the prevention of the introduction of plague, cholera, typhus fever, small-pox, leprosy, and yellow fever.

Under existing regulations consular officers abroad are required to report the occurrence of transmissible discases, and issue bills of hoalth to all vessels destined to United States ports. 'These regulations require that the officer issuing the bills of health shall satisfy himself that the conditions certified therein are true, and that all necessary measures have been taken to insure freedom of the vessel, cargo, passengers, and crew from infection. In infected ports the inspection of vessels is recpuired, and the president is authorized to detail officers of the L.S. P'ublic Health and Marine Hospital Service for duty in consular offices abroad for the purpose of making such inspections.

Vessels, prior to storing cargo, are required to be clean, and any portions liable to have been infeeted must be disinfected. 'The air space, ventilation, food, and water supply and hospital accommodations aboard must be adequate. Cargo that is presumably infected must be disinfected prior to loading, and no person suffering from a quarantinable disease is allowed to ship.

Special precautions are observed in cholera-infected ports, with respect to water and food supplies, and steerage passengers are held under observation five days prior to sailing. In yellow-fever ports, special measures are taken to prevent access of mosquitoes aboard, and "nonimmune" passengers, if exposed, are held six days. In plague ports special precautions are taken to exclude rodents from vessels, and passengers presumably exposed are required to be held seven days. Crews coming from districts where small-pox prevails, and all steerage passengers, are required to be vaccinated or show evidence of recent successful vaccination, and typhus fever suspects are held twelve days prior to embarkation.

Masters of vessels destined to United States ports are required on the high seas also to observe certain regulations to preserve health, and, when sickness occurs, to take necessary precautions to prevent its spread. On the observance of such regulations depends largely the stringency of quarantine measures on arrival.

Vessels arriving at domestic ports are subject to quarantine inspection; if from a foreign port (except Canadian); if sickness has occurred on board; if from domestic ports where cholera, plague, or vellow fever prevails, or small-pox or typhus fever prevails in epidemic form, and if from a yellow-fever infected port, via a port north of the southern boundary of Maryland and destined to a port south of that latitude.

Vessels arriving under the following conditions are placed in qumrantine: With quarantinable disease aboard, or if considered to be infected; if from a tropical American port, and arriving at a port sonth of the southern boundary of Maryland between April 1 and November $1:$ and if from a tropical American port, and destined via a northern port to one south 
of the southern boundary of Maryland during the close quarantine season.

The regulations prescribe in detail the hygienic measures to be taken with vessels in quarantine to prevent communication with shore; to isolate the sick; to observe contacts and eradicate the infection so that it will not be carried ashore.

The measures of disinfection, which are necessarily thorough, are outlined on p. 469.

By means of special precautions in Central American and West Indian ports, and on ressels en route, such vessels are admitted without detention, thus aiding an enormous fruit trade that would otherwise be impossible.

International Sanitary Agreements.-As a means of simplifying quarantine procedures and rendering them more effective, an international sanitary agreement was signed in Paris, December 3, 1903, and ratified by the President, August 2, 1905. This agreement codified the regulations necessary to guard against the invasion of plague and cholera, and its execution is one of the fundamental hygienic measures taken by the national government.

Recognizing the necessity of similar provision in the Western Hemisphere, there was signed in Washington October 15, 1905, a second international sanitary agreement codifying the regulations with respect to cholera, plague, and yellow fever. 'This treaty, to which the United States is a party, is now in force. In accordance with the former treaty, the United States contributes to the support and participates in the benefits of an international sanitary office in Paris, which is charged with the collection and dissemination of facts respecting the progress of epidemics. An International Sanitary Bureau of Washington has for its object the performance of a like duty in the Americas.

Medical Inspection of Immigrants.-As a further means of excluding exotic diseases, medical inspections are made by the U. S. Public Health and Marine Hospital Service of all arriving aliens. For the purpose of enforcing the immigration law with respect to physical and mental conditions, aliens are divided into three classes: In the first class are included those whose exclusion is mandatory under the law by reason of specified diseases or defects, such as idiots, imbeciles, feeblemincled persons, epileptics, insane persons, persons afflicted with tuberculosis, and persons afflicted with loathsome contagious or dangerous contagious diseases, such as favus, syphilis, or trachoma. In the second class are included those cases who present diseased conditions that materially affect their capacity for self maintenance or tend to call for institutional care or treatment, such as organic heart lesions, permanently defective nutrition, chronic arthritis, nervous affections, malignant growths, defective vision, and deformities. In the third class are included those who present diseased conditions that may affect their ability to earn a living; such conditions being certifiable as to the extent of the disability.

'The ship's papers are required to contain correct statements with 
reference to the physical and mental condition of each alien at the lime of embarkation, and the ship's surgeon is reyuired, on arrival, to make reports of all diseases, injuries, hirths, or deaths occurring during the voyage.

'The medical examination consists of primary and secondary inspecetions. 'The former is a line inspertion and intended to segregate those presenting physical or mental defects for further cximination. 'The secondary inspection consists of as careful an examination as will enalole the officer to arrive at a definite and correct diagnosis, and whenever necessary, aliens are detained in hospital for the purpose for such time as may be required. In the first class of cases the certificate of the medical officer brings about deportation. 'The necessity of careful diagnosis is, therefore, apparent, and while it is impracticable to attempt to refer to all of the procedures prescribed, mention mav be made of the necessity of everting the eyelids of every alien to detect trachoma, and careful studies to detect signs of mental or nervous diseases.

Not only may diseased aliens be deported on arrival, but at any time within three years if it is shown that they have become public charges or are suffering from excluded diseases, both of which conditions existed prior to landing.

'That such measures have an important bearing from a hygienic standpoint is shown by the number of deportations annually, and the rarity in our country of certain diseases, such as favus, which are very common in localities from which arriving aliens come.

Interstate Quarantine.-Having jurisdiction over interstate commerce, the national government becomes responsible for the hygienic conditions under which such traffic is conducted, and its influence on the sanitary welfare of the country as a whole.

Should cholera, yellow fever, small-pox, plague, or typhus fever occur in any state, territory, or the District of Columbia, and there be danger of the spread of such diseases to other states, the President has power to cause sanitary regulations to be made and enforced to prevent their spread, and appropriations are made annually for the suppression of epidemics of these diseases.

Laws have also been enacted for the prevention of the spread of contagious and infectious diseases from one state or territory to another through coöperation of the federal health agency with state and nunicipal health authorities in the enforcement of rules and regulations made by such authorities. In case additional measures become necessary, the Secretary of the Treasury may prescribe them, and when such regulations are issued, they may be enforced by the states and municipalities, but in case this is not done, the President has power to take the necessary steps and adopt such measures as shall be necessary to prevent the spread of such diseases.

Under these provisions, certain hygienic measures have been in force, which relate to cholera, vellow fever, small-pox, plague, and typhus fever. During outbreaks of yellow fever at New Orleans and plague at San Francisco, such measures have been especially valuable. 'The regulations 
in force provide in effect for the notification of the quarantinable diseases to the Federal Bureau of Public Health, the isolation of persons afflicted, and those exposed to the infection; the disinfection of their effects, and the disinfection of infected apartments. Common carriers are forbidden to accept for interstate transportation persons suffering with any of the quarantinable diseases, or any infected article of clothing, bedding, or personal effects. The bodies of persons dead of quarantinable diseases are not allowed under the regulations to be accepted for transportation except in hermetically sealed coffins, and not then except by order of the state or local health authority. In case of outbreaks of small-pox, vaccination is prescribed, or isolation for fourteen days. Special regulations have also been issued for the prevention of the spread of cholera, yellow fever, piague, and typhus fever, and are based on those hygienic measures subsequently outlined in the discussion of the management of epidemics.

In addition to the above-mentioned diseases, uniform provisions should be made for the prevention of the spread of other communicable diseases, such as tuberculosis, typhoid fever, scarlet fever, and diphtheria, and there should be sanitary surveillance over trains and vessels engaged in interstate traffic so that passengers and crews would not be liable to become infected.

Since interstate and intrastate traffic are so intimately associated, there is necessity of close coöperation on the part of federal, state, and municipal health agencies, and all hygienic measures should be uniform in character and operate to bring about improved sanitation.

Not only should the above quarantine measures be enforced during outbreaks of communicable diseases for the prevention of their spread from one state to another through the agency of persons and merchandise, but there should be instituted hygienic measures and devices for the sanitary conduct of interstate traffic. These measures should include proper ventilation of trains and vessels and their thorough cleansing; the providing of pure water and food supplies en route; the proper disposal of excreta, so as not to pollute water courses and railway tracks; the regulation of conditions under which the employees of common carriers work, and the exclusion of dangerous or infected articles from transportation.

In order to insure sanitary protection of passengers and those conducting interstate commerce, frequent sanitary inspections should be made of terminals, stations, and equipment. Common carriers should be required to display standard sanitary rules, which employees would be required to observe. By these means, wharfs, terminals, and especially small way-stations would be kept in better sanitary condition. In addition, data should be collected of all conditions fostering disease.

Investigations of Communicable Diseases.-While epidemiological studies and field investigations of matters pertaining to the public health are indirect hygienic measures, they are among the most important public health functions of the national government. Under the present interpretation of the constitution, they are carried on in the 
interest of interstate commerce, but the results attained should affect favorably the greater portion of the population.

Such studies shonld relate primarily to those diseases of national moment, such as tuberculosis, malaria, and typhoid fever, and the conditions favoring their spread in order that they may be prevented.

In the case of tuberculosis, federal measures have thus far been confined to the care of certain of its beneficiaries, the inspection of government buildings, the disinfection upon repuest of infected quarters aboard ships in the merehant marine, and the issue of regulations for the prevention of the disease among government employees.

In the case of typhoid fever, leprosy, and other matters pertaining to the public health, laboratory studies have been and are heing made by the public health service, but there is need of field investigations to determine the extent of the migrations of tuberculous and other patients from one locality to another, to ascertain the geographical distribution of communicable diseases, and to determine the means for the prevention of their spread, as well as the division of responsibility in relation thereto of federal, state, and municipal authorities. 'Through such measures also there would be brought about improved sanitation of ports and consequent reduction of expensive quarantines.

On account of its insidious onset and fatal nature, special investigations are being made of leprosy to determine its modes of transmission, and with the hope that an improved method of treatment will be evolved. Important epidemiological facts have already been deduced, among them being the probable relation between school age and the spread of the disease in Hawaii, which indicates the desirability of systematic inspections in infected localities.

Prevention of Pollution of Interstate Waters.-Prevention of the pollution of interstate waters is closely allied to the prevention of typhoid fever and intestinal diseases, and should receive legislative consideration. 'The common law grants to riparian owners the rights to water in its natural state, and no community or state has the moral right to defile interstate waters with sewage or manufacturing wastes to the injury of its neighbors.

No national statutory control exists, however, for the prevention of this muisance, and data should be collected as to the extent of such pollution and the means necessary for its prevention. This data, when collected, should be used as the basis for comprehensive legal enactments, the enforcement of which would obviate the necessity of prolonged litigation, such as that between the states of Missouri and Illinois.

'The extent of national intervention when it becomes effective will depend partly on the activities of the states, and in the mean time no municipality should be permitted under state law to discharge raw sewage or wastes into bodies of fresh water without purification.

Collection of Sanitary Reports and Statistics.-The compilation of statistics of sickness and deaths must be mentioned as a valuable hygienic measure, and while such duty now devolves primarily upon the states and municipalities, the central government is engaged 
in compiling the data received from them through voluntary coöperation. In addition diplomatic and consular officers are required to give notice, by telegraph or otherwise, of outbreaks of communicable diseases abroad.

Mortality statistics are compiled by the Census Bureau from duplicate records of deaths as they are received, and not as is believed by some in connection with the taking of the decennial census. Sanitary reports and morbidity statistics are compiled by the Bureau of Public Health by means of blanks and weekly reports. While of distinct value, neither the statistics of sickness nor deaths represent the complete facts, and there is need of additional provisions whereby the central government will acquire full data, as is the case in taking the decennial census.

By such means the geographical distribution of diseases would become known. In no field of preventive medicine is there greater lack of definite information, notwithstanding its acquisition is essential to the complete understanding of the problems of prevention of diseases, such as cancer, dysentery, and malaria. Such data would also be of service in determining correctly the influence of climate in relation to disease. The physical features of the subject have long been provided for by generous appropriations, but in order that these data may be utilized, the hygienic aspects of the subject should be made presentable through accurate morbidity statistics.

Educational Measures.-The collection of sanitary reports and statistics and discovery of scientific facts are valuable hygienic measures only as they are made public. A considerable amount of scientific information on sanitary matters is constantly being disseminated by the bureau of public health, but popular bulletins should be issued in larger editions as are now farmers' bulletins. In addition, fundamental research should be extended and the results made immediately available. By this means the central government would contribute largely in establishing the scientific basis upon which future hygienic measures must rest.

As a further means of improving sanitary administration, it has been suggested that the facilities of the national public health laboratories should be extended to state and municipal authorities for their benefit, and thus bring about more efficient coöperation between the various health agencies. In order also to improve the public health, it has been suggested that sanitary exhibits should be prepared, and a national museum of hygiene might with advantage be maintained.

Finally, as a means to secure further uniformity of sanitary administration, there should be regularly collected by the central government copies of all health laws and ordinances as they are enacted, which would be published and distributed, and this hygienic measure has been recently undertaken.

Supervision of Biologic Products in Interstate Commerce.Under the law of July 1, 1902, viruses, serums, toxins, and analogous products intended for the cure of diseases of man, and entering into interstate traffic, are required to be propagated in licensed establish- 
ments. Prior to the issue of lierenses, inspections are made of each establishment by officers of the Public Health and Marine Hospital Service, and licenses are not renewed until after reinspections, which are required to be made annually. In addition, samples of the products are secured in the open market from time to time and examined in the hygienic laboratory as to purity and potency, the laboratory reports being also considered in connection with the granting of licenses.

Regulations have been issued under the law governing the issue, suspension, and revocation of licenses, the inspections of establishments, and the examination of viruses, serums, and toxins. Standards have been established for testing the strength of diphtheria antitoxin and tetanus antitoxin, and the standard units for these products made in the hygienic laboratory are distributed bimonthly to the several establishments, and the serums offered for sale must conform to these standards. All other licensed products must be satisfactory as to purity. In addition, close watch is kept of vaccine virus, and every lot is required to be tested in order to determine its freedom from tetanus bacilli and other pathogenic micro-organisms. 'The names of the establishments, and the products for which they are licensed are published from time to time, and in case contamination of any product is discovered, its immediate withdrawal from the market is demanded.

The importance of such supervision from a hygienic standpoint is evident, and practitioners can rest assured that these products are satisfactory as to purity, and that packages of antidiphtheric serum and antitetanic serum contain the number of units labeled.

Control of Foods and Drugs in Interstate Traffic.-While passed in the interest of commerce, the law to prevent the manufacture, sale, or transportation of impure foods or drugs is of importance from a hygienic standpoint. Under its provisions, it is illegal to manufacture within any territory or the District of Columbia any article of food or drug which is adulterated or misbranded, and the importation or transportation from state to state of such foods and drugs is prohibited. Regulations are authorized under the law to cover the collection and examination of specimens to determine whether they are adulterated or misbranded. Any drug bearing a name recognized in the U. S. Pharmacopocia and National Formulary withont any further statement respecting its character, is required to conform in strength, quality, and purity to the standards laid down in those publications. Regulations are in force containing those measures in detail for the prevention of the adulteration and misbranding of foods. A general guaranty may be filed with the Secretary of Agriculture by manufacturers or dealers, and in return, serial numbers are supplied, which are required to appear on each package of goods sold, together with the words "Guaranteed under the Food and Drugs Act of June 30, 1906," and the examinations of samples are made in the bureau of chemistry.

Provision was also made in a law, passed June 30,1906 , to prevent the use in interstate or foreign commerce of meat and meat products which are unsound, unwholesome, or unfit for human food. Inspections 
are required of all slaughtering, packing, meat canning, and similar establishments, the products of which enter into interstate or foreign commerce, except such small establishments as may be exempted. All establishments liable to inspection are given a serial number, and this number is required to be used to mark meat and meat products. All animals used for human food are subject to inspection, as well as the meat produced therefrom. As previously stated, detailed regulations have been prescribed to secure the sanitation of establishments engaged in the handling of meats.

On inspection, carcasses, and parts thereof that are found to be sound, are passed and so labeled, but diseased meats are required to be disposed of in an appropriate manner, as outlined in the regulations. Carcasses showing the lesions of anthrax, black-leg, hemorrhagic septicemia, vaccine, rabies, tetanus, generalized actinomycosis, or malignant catarrh are condemned. Special provisions are made in the regulations for guidance in passing on carcasses affected with tuberculosis and other diseased conditions. Provision is also made for the safe transportation of meats, and common carriers are forbidden to accept for export or transportation in interstate traffic any meat-food product until a certificate shall have been furnished showing that the same has been inspected and passed, or exempted from inspection under the law.

Coöperation with State and Local Sanitary Authorities.Certain other functions of the federal government, and the manner in which they are performed, have an indirect bearing on the pubic health, but their enumeration as hygienic measures will be omitted. The enforcement of those already mentioned presents a broad field of activity, which, however, may be extended in the future as a result of judicial interpretation of the constitution.

As is shown, the fundamental hygienic measures devolving upon the central government are: the investigations of preventable diseases, the conditions favoring their spread, and the methods necessary for their prevention; the collection of sanitary reports and statistics; the dissemination of information regarding sanitary matters; the prevention of the introduction of contagious and infectious diseases, as well as their spread from one state or territory to another, and the management of epidemics of communicable diseases that threaten the country as a whole.

All these functions involve coöperation on the part of national, state, and local authorities, and this fact must be borne in mind in perfecting the organization which has to do with sanitary administration.

\section{THE MANAGEMENT OF EPIDEMICS}

The communicability of certain diseases and their faculty of becoming epidemic have long been known, but not until their causes and methods of transmission became better understood was there foundation for scientific measures of prevention. In consequence of this lack of knowledge there is history of repeated pandemics of plague, cholera, small-pox, 
and typhus fever. 'There is also history of epidemies of typhoid fever, diphtheria, yellow fever, and similar diseases, some of which have become endemic partly through insufficient knowledge as to their modes of transmission and partly on account of public apathy brought about by long years of prevalence.

Although the specific virus has not been isolated in every case, the communicable diseases are all believed to be due to micro-organisms of animal or vegetable origin. In character they are, therefore, "infectious," and owe their propagation to the passage of the specific virus directly or indirectly from one person to another.

Communicability is an essential factor in the origin and spread of epidemics, but it is only one of many component factors that enter into the problems of epidemiology and render them exceedingly complex.

Assuming that every case of communicable disease has its origin in a previous case, there is indication for the institution of rational measures of prevention. For their successful elaboration, however, it is desirable to know the character, virulence, and viability of the particular organism, and, above all, its avenues of transmission from person to person. Other important considerations are atmospheric and telluric conditions, the influence of seasons, the relationship of the population to the lower forms of animal life, and especially the susceptibility of individuals.

Bodily susceptibility is perhaps the most potent single factor determining the occurrence of epidemics, and one that is worthy of careful study. Not every person that is exposed contracts typhoid fever, and it is reasonably certain that some time in the life of every individual there is exposure to tubercle bacilli, yet many escape infection. On the other hand, very few persons have a natural immunity to the infectious exanthemata, such as measles and scarlet fever. Consequently such diseases recur periodically, the extent of the epidemic depending upon the degree of susceptibility of the population afiected.

It is the consideration of these multiple facts that comprises the science of epidemiology, and in proportion as they are known and practised will success attend preventive measures.

'The successful management of an epidemic from whatever cause predicates, first, thorough understanding on the part of the people and government as to the sanitary, social, and economic significance of the particular disease that threatens; second, the existence of constituted authority possessed of power and wisdom necessary to enforce rational ordinances; and, third, intelligent coöperation of the people and medical profession witi local authorities, and the latter with the central authority when operative.

Educational Measures.-In order to guard against the occurrence of epidemics, there is necessity of educating the public regarding their propagation and the principles underlying their prevention. In fact, the watchword of preventive medicine is education. It should be under the clirection of the sanitary authorities, but they should utilize every private organization and means available, and in turn endeavor, through scientific research, to extend the knowledge of the disease previniling. 
In times of epidemics educational measures are especially demanded. Unreasoning fear of yellow fever in the southern states, on the one hand, and lack of appreciation on the Pacific coast of the dangers of plague, on the other, have in the past prevented intelligent coöperation on the part of the people, and hence, defeated prompt suppressive measures.

Lectures, pamphlets, and exhibits are fertile means of disseminating information that can be utilized in the homes and among small assemblies, both by officials and private individuals. But the fountain-head of sanitary education should be the public school, and if definite school hours were set aside for public health instruction, the rising generation would acquire in advance of epidemics the essentials regarding their control, and in turn impart them to their elders for application during outbreaks.

The Sanitary Organization.-In view of the possibility of epidemics there is necessity for the enactment of comprehensive laws to meet emergencies as they arise. These in general may not be specific, but they should confer authority on the executive to issue regulations which will have the force of law.

In the several states and territories such laws now exist, and their enforcement generally devolves on a central sanitary board or head. On account, however, of greater necessity and more frequent recourse to such provisions in congested centres of population, these powers have in most instances been delegated to local authorities, and it is only when they fail or refuse to enforce them that the state intervenes.

By reason of its control over interstate and foreign commerce the national government has a vital interest in the occurrence of contagious and infectious diseases, and has laws providing for coöperation with state authorities in their measures of suppression. As previously stated, should the latter fail or refuse, the national laws authorize the President to execute and enforce measures necessary to prevent the spread of communicable diseases from one state or territory to another.

By virtue of such laws the U. S. Public Health and Marine Hospital Service becomes directly responsible for the exclusion of exotic diseases, and prevention of the spread of plague, cholera, yellow fever, and smallpox in interstate traffic, its operations in the latter instance being conducted in coöperation with state and local authorities.

Democratic ideals demand that the exercise of sanitary police shall in so far as possible rest with the local authorities or sanitary units. Their powers should accordingly be clearly defined, and there is necessity of adequate organization to meet emergencies.

If an epidemic is localized, the command of all sanitary operations may be vested in a single officer, who becomes responsible for the entire campaign. This is usually the health officer, who has the assistance of statisticians, inspectors, clinicians, and disinfectors.

With a sufficient force, and properly trained, it will be practicable to keep informed of the progress of the epidemic, and by charting cases as they occur, the necessities of the situation can be met from day to day. If the epidemic becomes widespread, coördination of effort is indi- 
cated. 'The infected area should then be divided into districts, each in cliarge of a sanitarian under authority of a central office.

Coopperation of the Public.-Intelligent coöperation on the part of the people during epidemics is a fundamental necessity, without which prophylactic measures cannot be expected to yield their best results. It depends on the people's understanding of the requirements of the situation and the moderation and tact of those in authority.

Above all, there is necessity for hearty coöperation on the part of the medical profession, and, while this can be required by law in as far as notification of cases is concerned, it is desirable that it be rendered in a spirit of helpfulness to the patients and community.

Detection of Infected Foci.- The prompt discovery of infected foci is an absolute essential to effective prophylaxis, and in most diseases these are the homes of the sick. 'The people of the infected locality must, therefore, be kept under constant surveillance. In some epidemics this implies frequent sanitary surveys, but they should be made with as great consideration as the situation will allow, or there will be concealment of cases and resistance to preventive measures.

Since it is persons harboring infection that must receive primary attention if epidemics are to be suppressed, notification of cases to the authorities becomes essential, and should be required to be made within a specified time after the development of suspicious symptoms of the disease.

During minor epidemics, information regarding the occurrence of cases comes almost entirely from physicians, but in this duty the householder should also be made to share, and penalties should be provided for its neglect.

Notification on the part of the physician and householder, however, will not locate every case of infectious diseases, such as cholera and typhoid fever. It is only by means of thorough sanitary surveys and laboratory examinations, conducted by competent authorities, that this will be accomplished, and subsequent prophylactic measures will also devolve upon those authorities.

In the case of some of the communicable diseases, confirmation of diagnosis is indication for placarding the residence. While there may be objection to this procedure, it is sound in principle and a valuable measure during times of epidemics. It not only serves as a means of avoiding exposure and minimizing the difficulties of maintaining isolation, but the educational effect is good.

Having briefly referred to the legal provisions required to meet epidemics and the organization necessary for their enforcement, as well as the attitude of the public, and means of determining foci as they occur, it remains to consider the several measures available in the suppression of epidemics.

There may be said to be two broad principles, upon which prophylaxis is based: first, immunization against infection, which contemplates wholesale inoculations to confer temporary or lasting immunity upon those hitherto susceptible; second, protection against exposure to infec- 
tions, under which may be grouped all measures designed to prevent exposure and to destroy infection.

Protective Inoculations.-Recovery from attacks of certain of the communicable diseases is known to be followed by a more or less complete immunity to that particular infection, and on this vital phenomenon is based the use of viruses, serums, and toxins in the prevention and cure of diseases. The immunity established by these products is "active" or "passive," depending on their modes of production, and while their field of usefulness is limited as yet, it is believed to be capable of great extension in the prevention of epidemics. The discovery of cowpox lymph by Jenner in 1798 marked the beginning of scientific preventive inoculations and provided a reliable method of combating small-pox. Through vaccination almost complete immunity has been conferred on entire populations, and by its use alone small-pox can be eradicated from a community. In the Philippine Islands there were performed from 1906-08, 4,905,040 vaccinations, and Heiser in 1908 stated that, after the completion of vaceination in six provinces a year previously, not a single death from small-pox had been reported in those provinces. ${ }^{1}$

On the appearance of small-pox, wholesale vaccinations should be instituted for the prevention of its spread, and if thoroughly enforced, the necessity of auxiliary measures will be practically removed.

The experience with cholera in India has led to the employment of an anticholera vaccine, which consists of living or dead cultures of comma bacilli of uniform virulence. When injected subcutaneously into an individual, this vaccine is said to establish an immunity in about four days, that may last as long as two years. The excellent results secured by these inoculations paved the way for the development and use of Haffkine's antiplague vaccine, which is injected in doses of from 2 to 5 c.c., and diminishes the incidence of attacks during outbreaks of the disease. Antiplague serum has also been employed as a prophylactic with varying degrees of success.

In the event of outbreaks of diphtheria, tetanus, or cerebrospinal meningitis, the use of the respective antitoxic serums will be found to be of great value, and in persons actually exposed to tetanus infection, antitetanic serum affords practically the only hope of prevention of the disease.

'The work of Wright and others with antityphoid vaccine appears likewise to have established its value in diminishing the incidence of attacks of typhoid fever among inoculated troops. Its voluntary use has been recommended by the medical authorities of our army, and its use would probably be advantageous among civilians who are obliged to visit heavily infected localities. In the present state of our knowledge, however, its use can hardly be expected to become general, and none but healthy persons should be inoculated. Its use during epidemics is not advised, on account of untoward results that might follow in those already infected. Other communicable diseases of man in which preventive

' Annual Reports, Bureau of Health, Philippine Islands, 1906-8. 
inoculations have been employed are pneumonia, streptococcus infections, and hydrophohia. In those exposed to the latter disease antirabic virus has proved of great value. 'I'wo methods of adininistration are employed in the hygienic laboratory, the mild and intensive, differing in the strength and rapidity of injections of the cords used. 'This measure can only be regarded as a prophylactic in the interest of persons actually bitten, however, and for the protection of the community at large preventive measures must aim at the control of rabid animals.

The Sanitary Survey.-During epidemics there can be no relaxation of those general measures intended for the sanitary defense of the community. In addition, aggressive measures are necessary to determine insanitary conditions affecting the incidence of the prevailing disease.

Careful sanitary surveys should accordingly be made as occasion requires to investigate infected foci, to trace the origin of infection, and ascertain what additional sanitary precautions are demanded to prevent further spread. House to house inspections may sometimes be made to inquire as to prevailing illness, the quality of food and water supply, the presence or absence of vermin, fleas, and mosquitoes, and the precautions taken to exclude them. Such inspections should not be perfunctory in character, but be made with the greatest care, and discovered cases should be regarded as suspicious until all doubts regarding diagnosis are cleared away.

'The systematic investigations of typhoid fever inaugurated in Germany in 1903 emphasized the necessity of seeking out infected persons, and brought to notice the influence of children in the propagation of this disease. In spite of heavy infection in a district it was possible to eradicate typhoid infection in the course of a few months by this plan, as had been previously done in the case of cholera. 'The results attained clearly indicated the necessity of systematic sanitary surveys applicable not only in typhoid fever, but other infectious diseases.

Isolation of the Sick.-Upon the discovery of a case of any of the graver communicable diseases, prevention of the spread of infection from that individual is a comparatively simple matter, provided there is exercise of proper control from the time of onset of illness to the time of recovery, as determined by laboratory tests or otherwise. Failure in any particular case rests with those responsible for his care, or with the public, because it is not alive to the necessity of isolation of infectious cases. Such control may be exercised certainly without detriment to the patient himself, and without much inconvenience to him until convalescence is well established. It is only in those cases of diphtheria, cholera, and typhoid fever, which become bacillus carriers that the problem becomes difficult and its solution almost impracticable. In measles, also, in which the pre-eruptive stage is infectious and the infection highly diffusible, isolation offers less hope of success, but in view of its predilection for the young, and the danger of sequele. such isolation as is possible is nevertheless advisable.

Isolation is such control as will prevent the dissemination of the infec- 
tion from the sick and permit of its destruction as it leaves the body. It accordingly varies with the character and mode of transmission of the infection, and is not the same for all diseases. In cases of small-pox, scarlet fever, and other air-borne diseases, rigid isolation is essential. But in plague, cholera, typhoid fever, and similar diseases of known bacterial origin the restrictions need be only such as will permit of certain destruction of the infection and prevent its being carried from the room. In yellow fever the problem is even simpler, since a case is only infective during the first three days, and the infection conveyed only by the Stegomyia calopus. Here the prevention of the spread of infection depends entirely on exclusion of this species of mosquito from the sick. In the case of typhus fever, also, recent researches associate the body louse as the probable agent of transmission, the confirmation of which will greatly simplify preventive measures. The necessary supervision in any case cannot be left to untrained attendants, but must be exercised by competent authority to assure that the proper precautions are carried ont.

In some diseases isolation may be safely practised in the home, both in the interest of the patient and the public. During the Hong Kong epidemic of plague in $1902 \mathrm{I}$ had the opportunity of witnessing the successful adoption of district isolation, which was made necessary on account of Asiatic opposition to removal to the contagious disease hospital.

Well-to-do patients and those of tender years will probably desire to remain in their homes with ordinary infections, such as measles, scarlet fever, and diphtheria, and without there are special reasons to the contrary, the health authority will do well to accede to this desire. There should be authority, however, for the removal to hospital of all cases that cannot be properly cared for elsewhere, and official supervision should be had over the methods exercised in homes and at hospitals to prevent the spread of infection from the sick, No one should be allowed to come in contact with patients in isolation except those who will exercise the proper precautions. Association with such patients should be limited almost entirely to persons concerned in their care and treatment.

Upon the discovery of a case of infection during an epidemic an official should risit the patient for the purpose of determining whether there are proper facilities for the management of the case. During this visit it should be determined whether the sick room selected is clean and well ventilated, free from vermin, and thoroughly screened to exclude insects, and one that can be used exclusively for the care of the patient. It should be as far removed from the living room as possible, and preferably on an upper floor. Such arrangement will be found to be reasonably safe, and will offer opportunity of continued surveillance of the surrounding as well as other members of the family. Persons suffering from the communicable diseases should not be domiciled in houses any part of which are used for the manufacture, storage, or sale of foods or drugs. This is especially applicable to typhoid fever, diphtheria, and scarlet fever, more than 500 epidemics of these diseases having been cited 
by 'Trask as having been traced to milk alone. In case housing under such conditions is unavoidable, it becomes the duty of the proper authority to close the building until after the recovery or removal of the patient to hospital. Isolation hospitals should generally be provided in advance of epidemies, at least among urban populations. Parkes estimates that such accommodations should represent one bed to every 1000 of the population when this is largely composed of the industrial classes.

The hospital buildings should be isolated, eapable of thorough disinfection, thoroughly screened from insects, and plague hospitals should also be rendered absolutely rat-proof by concrete or galvanized iron inclosures extending 18 inches under ground.

Separate pavillions are required for each disease, and should be as comfortable as are other hospitals. 'The word "pest-house," as well as all it stands for, should be abolished.

Cases of small-pox, leprosy, cholera, and typhus fever should invariably be required to be placed in hospitals. 'The same action should be taken in cases of scarlet fever, diphtheria, typhoid fever, plague, and yellow fever in which the necessary precantions cannot be carried out at private residences, and in other infections when, by reason of poverty, no adequate care can be had at lome.

The period of isolation in any case will depend on the nature of the disease, and examinations to determine that the danger of transmission of infection is over. In general the period of detention will be as follows: plague until complete recovery; small-pox from four to eight weeks; scarlet fever six weeks, or until complete desquamation has occurred and throat symptoms disappeared; diphtheria until two examinations three days apart show absence of diphtheria bacilli from the throat and nose; measles three weeks; typhus fever four weeks; yellow fever seven days; and typhoid fever until two examinations three days apart show absence of typhoid bacilli from the urine and feces.

Surveillance of Contacts.-While the campaign against any disease centres around its victims, after their proper isolation and care, consideration must be given also to other persons who have been exposed, to the infection. 'Two methods of surveillance suggest themselves depending on the character of the disease. Quarantine, on the one hand, implies segregation and restraint, and observation, on the other, contemplates a reasonable degree of liberty to carry on ordinary avocations, but under such sanitary supervision as will permit of prompt isolation on the appearance of suspicious symptoms.

Better knowledge of the communicable diseases and recognition of the rights of individuals has resulted in the gradual abandonment of irksome quarautine for observation.

In diseases such as measles, except during sharp epidemics, it is generally impracticable even to exercise any very close observation of those exposed, and it is the infected foci, such as schools, that must receive the attention of the authorities. These and other places of public assemblage being at times required to be closed, or those exposed being refused attendance.

$$
\text { VOL. I }-30
$$


In small-pox, after thorough disinfection and vaccination of contacts, there is no material advantage in shutting them up at their residences, provided they submit to inspection daily to determine that they continue free from suspicious symptoms.

In other diseases, such as plague and cholera, observation of contacts may with advantage be substituted for quarantine in order to accomplish with less resistance specific measures of prevention which are absolutely necessary to success. But the decision of this point must depend on local conditions, and to some extent upon the attitude of the public during the epidemic. In yellow fever this question will also depend on like considerations, and here quarantine of contacts should give way to house to house inspections of infected districts, such being the wiser procedure, the disease being capable of rapidly becoming epidemic, and the prevention of its spread depending largely on the detection of actual cases within the first three days of illness.

It must be remembered, however, that the measures taken during epidemics are in the interest of the community as a whole, and should quarantine become advisable, it must be wisely enforced in order to be effective. House quarantines are, on the whole, unsatisfactory, and in congested centres, where there are tenement and floating populations, they are practically impossible of adequate enforcement. For such classes, therefore, isolation barracks are required.

Fear of grave epidemics has occasionally given rise to the establishment of sanitary cordons which aim to prevent the escape of infection from infected districts into clean territory. They can rarely be successful and are only to be tolerated until such time as effective measures can be taken within the infected district or its orderly depopulation brought about.

Depopulation of Infected Areas.-In areas heavily infected with plague or yellow fever, this measure recommends itself both on account of the severity of the infections and because they are transmitted by agents which are difficult of speedy control. Depopulation is a valuable measure for the suppression of plague, and in many instances has been essential to the control of localized epidemics. In Hong Kong, and other centres of plague infection, evacuation of habitations and districts is regularly practised, with excellent results. Inasmuch as the disease is primarily one of rodents, which are responsible for its continuance in a locality, this measure is in reality a means of escaping contact with rats and their parasites.

Simpson cites an instance where, in one afternoon in Cape Town, a thousand natives were removed from an infected district to the outskirts of the city and inoculated against plague, with the result that the disease was eradicated from among them. Instances have also come under my observation in which plague cases continued to occur in infected houses until evacuation was brought about. It is the first measure to be enforced when infection among rodents within a house is demonstrated. and should be carried out without delay, otherwise fatalities among humans will occur. 
Depopulation of yellow-fever infected areas, on the other hand, has the double object of removing susceptible persons from attacks of the Stegomyia calopus, and allowing them to proceed in an orderly manner to non-infectible territory or localities where this species of mosquito is known to be absent.

Infected localities and localities contiguous thereto should be depopulated as rapidly and completely as possible under official supervision, persons not having been exposed being allowed to depart without detention, and persons having been exposed being required to undergo observation for six full days in a camp of probation properly located. Persons having been exposed may be allowed to proceed, however, without detention to non-infectible territory, provided they can be held under observation at destination for a period of six days from the date of last possible exposure to infection. This measure is prescribed in the United States quarantine regulations, and has been employed time and again by the public health service, both in the interest of the individual, and with entire safety to the country.

In order to safely depopulate an area, however, detention camps are necessary, and it must be done under strict medical supervision. 'There must be also adequate train inspection, by which measure the dissemination of infection may be reduced to a minimum and the avenues of com. merce kept open, the latter being an important consideration in these days of interurban and interstate communication.

Supervision of Traffic.-Supervision over traffic includes measures that will insure the cleanliness and freedom from infection of cars and vessels, as well as the merchandise and passengers to be transported in them.

The sanitation of cars and vessels is deserving of greater consideration, and during epidemics this is doubly important. Consequently, at such times, in addition to thorough cleansing, they should be required to be disinfected before every trip, and authority should be had to cut a car out of a train en route for purposes of disinfection.

During epidemies of small-pox and scarlet fever routine inspections and disinfection of those cars and parts of ships in which cases have occurred may reasonably be demanded. During plague outbreaks great care will be required also to guard against the transportation of rodents, some of which may be infected. On trains conditions are not so favorable for the transportation of rodents, but on ships the danger is imminent, and no ressel should be allowed to leave an infected dock without first having been thoroughly fumigated, or measures taken to absolutely prevent the access of rats aboard.

During yellow-fever epidemics the greatest care is required to free all vessels and trains leaving the infected district from mosquitoes. Frequent fumigations are, therefore, required, and in some instances it may even then be inadvisable to permit such carriers to proceed to uninfected territory. In the latter case, resort may be had to "shuttle trains," which run only from within the infected area to a quarantine station, on the outer zone of infection, where passengers and freight may 
be transhipped to cars or vessels known to be free from infection. This measure has frequently been enforced by the public health service in times of epidemics with excellent results.

The possibility of merchandise and fomites acting as carriers of infection must be borne in mind, but epidemiological experience goes to show that this danger is not so acute as was formerly believed. 'This especially applies in the case of plague, which is spread principally by rodents, and to yellow fever, which is known to be propagated only by the Stegomyia calopus. New merchandise, and properly packed cargo that has originated in uninfected localities, may generally be permitted to be shipped without disinfection, but where there is reason to believe that any article has been exposed to infection or is liable to convey the same, its disinfection should be required.

Articles of clothing, soiled linen, and household effects that have been subjected to exposure to infection should be disinfected. In the case of plague this means the destruction of plague bacilli and vermin, and in the case of yellow fever rendering such articles free from mosquitoes. Any infected article that cannot be thoroughly disinfected should be destroyed or stored in a safe place for a period sufficiently long to cover the viability of the infecting organism.

Within infected areas proper supervision over infected persons will reduce the danger of infection of merchandise and bring about prompt disinfection of articles as contamination occurs. In other words, necessity of general disinfection will be minimized in proportion to the thoroughness of isolation of patients and continuous cleanliness of their surroundings. With the present state of sanitary practice, however, disinfection of infected houses and their contents cannot be safely neglected during epidemics.

Special Measures against Rodents and Insects.-The role of insects and vermin in the transmission of yellow fever and plague is now established, and the eradication of these diseases depends largely on the control of such agents of transmission.

During yellow-fever epidemics systematic measures against mosquitoes are demanded. Since the patient is the primary source of infection, the all-important precaution is to prevent their biting him and transmitting the infection to healthy persons. Rooms in which patients are to be confined must be given preliminary fumigation to kill mosquitoes, and screening of such rooms must be accomplished by the use of 18 mesh wire cloth. 'The rest of the house then requires thorough fumigation and screening. Following this measure the contiguous buildings should be fumigated, and this may mean the systematic fumigation of entire city blocks.

All breeding of mosquitoes should be prevented. Water containers, such as cisterns and barrels, should be carefully screened, and those that cannot be so treated should be oiled, to destroy larvæ. When neither screening nor oiling is practicable, as in gutters, salting may be resorted to, as was done by White in New Orleans, using rock salt for the purpose; or some other preparation, such as lime or copper 
sulphate. In order to be successful, efforts at mosquito destruction must begin immediately upon the discovery of an infected focus and be directed to that locality.

The removal of waste, draining of swamps, and clearing away of underbrush will suggest themselves as additional measures depending on local conditions, and must be utilized to their fullest extent.

During plague outbreaks, the control of rodents is essential, and for this purpose several measures are available, namely, poisoning, trapping, exposing them to natural enemies, cutting off their food supply, destroying existing nests, and preventing the making of new ones. "The poisons that may be used are phosphorus paste, arsenic paste, strychnine, and carbon bisulphide. All food-stuffs must be proteeted from rats, and the utmost care taken in the disposition of garbage in order that it shall not be accessible to them.

Experience has shown that rat-proofing is the most valuable antiplague measure, and that it should accompany auxiliary measures, such as trapping and placing of poisons. Rat-proofing is accomplished by the elevation of buildings from the ground or the adoption of concrete construction, and screening. Rat-proofing has the advantage of being a permanent improvement, and by such means rodents may be builded out of human habitations.

Upon the discovery of plague-infected rats in a city or town, that particular locality must be regarded as a centre of infection. As a means of dealing with such localities in Manila, Heiser has practised the trapping of rats along radiating lines from the centre of infection. 'The examination of these rats determines the extent of infection. The entire rat-catching force is then concentrated along the outer border of the infected area, from whence they commence to move toward the centre, catching the rats as they go. Behind them thorough rat-proofing is carried out. One section after another is treated in this manner until all are freed of plague-infected rats. This plan is intensive in character and practicable of application in any city.

\section{DISINFECTION}

Disinfection implies the destruction of micro-organisms outside the body, in order that they may not infect healthy individuals. It was resorted to for the prevention of communicable diseases long before their etiology was known, but with a better understanding of the various infections and their modes of transmission, its practice became more exact, and its field of usefulness enlarged.

From a theoretical standpoint, disinfection should be applicable to the destruction of disease germs wherever found, but practically this is not the case, except within the narrowest limits, as those agents that have the power of destroying vegetable micro-organisms cannot be employed for this purpose within the body on account of their injurious action on the tissues themselves. Disinfection is, therefore, a preventive measure and not a curative one, and is brought about by both 
natural and artificial means, designated as disinfectants. Those substances, on the other hand, that inhibit the growth of micro-organisms without necessarily destroying them, are known as antiseptics.

By preventing putrefaction, disinfectants and antiseptics may act as deodorants, but the latter term is applied to preparations because of their property of counteracting offensive odors, and not necessarily because of any deleterious effects exerted over bacterial life. Notwithstanding this fact, there remains in the lay mind the belief that one requisite of a disinfectant is that it must have a strong, disagreeable odor to be effective. Consequently, many so-called disinfectants offered for sale in the market owe their popularity to this property rather than to any germicidal power, thus engendering feelings of false security.

In order that disinfection shall be effectively performed, it is necessary to have a knowledge of the germicidal powers of the disinfectants used and their limitations under natural conditions. This has been determined to some extent by practical experience, and laboratory studies have demonstrated the germicidal action of many preparations under artificial conditions, but until such work is greatly extended, there will be a decided limit to the choice of disinfectants in actual practice.

Many natural processes favor the destruction of pathogenic microorganisms, among which may be mentioned the influence of air, desiccation, filtration, light, and heat. With the exception of heat, however, they must all be regarded as adjuncts to more powerful means in the presence of infectious diseases.

The artificial means employed in disinfection include a large number of chemical agents used in either a gaseous or liquid form. Some of them are much more powerful than others, but all have limitations in actual practice. On this account, and because of the variable results secured by different sanitarians, there is need of a standard by which disinfectants can be tested as to their relative efficiency under natural conditions.

Rideal and Walker have devised a method by which the disinfectant to be tested is compared with a standard solution of carbolic acid. By this method 0.1 c.c. of a twenty-four-hour broth culture of bacillus typhosus is added to 5 c.c. of a particular dilution of the disinfectant to be tested. From this mixture subcultures are then made every two and one-half minutes until six are obtained. These are incubated for from forty-eight to seventy-two hours at $37^{\circ} \mathrm{C}$. The dilution of the disinfectant which is found to have the same germicidal action under like conditions as the standard carbolic solution is divided by the latter, which gives a quotient known as the carbolic coefficient. While relatively simple to perform, this test has decided limitations, as variable results are obtained, depending on the character of the disinfectant and the amount of organic matter present.

In nature, infectious organisms are always associated with more or less organic matter, which limits the power of disinfectants to destroy them. The selection of a disinfectant accordingly, depends on the 
natural conditions under which the organisms are found and the properties of the disinfectant itself.

The limitations of this chapter forbid detailed discoussion of the large number of disinfectants that have been exploited. It is intended rather to consider the principles of application of the more important of those that have been found eflicient under natural conditions.

'The infections of the communicable diseases originate from some previous case, and on the care exercised over such case's depends the prevention of the spread of infection. 'The area of infection can be greatly restricted by thorough cleanliness and an abundant supply of sunlight, pure air, food, and water, and reduced to a minimum by the judicions use of disinfectants for the destruction of infection as it leaves the body.

Light is a powerful germicide and practical of application to some extent in every sick-room. It owes its action largely in this respect to the ultra-violet rays, which probably produce chemical disassociation of the atmosphere, with the production of ions of oxygen. 'This probably also accounts in part for the lesser number of bacteria in high altitudes, and some of the beneficent influences of such altitudes on tuberculous and other cases, for it is known that sunlight is richer in actinic rays as ascent is made to higher levels.

It has been shown by Koch that tubercle bacilli soon die from exposure to direct sunlight, and similar observations have been made with respect to typhoid bacilli, cholera vibrios, and many other organisms. The same is also true to lesser extent of those forms of artificial light rich in ultra-violet rays, such as the mercury arc, which suggests their possible use under certain conditions.

Pure air exerts a beneficent influence by dilution of the infection and its partial oxidation. But the germicidal action of light and air is largely dependent on the number of bacteria and their protection by organic matter. 'These agents can, therefore, be regarded only as adjuncts in the disinfection of the sick-room and its contents.

The destruction of infection by means of chemicals as it leaves the body is a comparatively easy matter. An ample supply of appropriate disinfectants should, therefore, be provided upon the appearance of infectious diseases for judicious use under the direction of the physician or proper health authority.

Infection leaves the body through the feces and urine; the discharges from the mouth, nose, and conjunctiva; the skin, and by means of coitus. With a knowledge of the particular disease and the avenues through which its infection leaves the body, therefore, the necessary procedure to be adopted is made plain.

Disinfection of Feces.-Since the bowel discharges from cholera. dysentery, intestinal tuberculosis, typhoid fever, hookworm disease, and probably other diseases contain the respective infections, they should be burned or received in a ressel containing $S$ to 10 ounces of disinfecting solution, with which they should be thoroughly mixed and allowed to stand for one hour. 
Liquid disinfectants find their greatest field of usefulness is the disinfection of excretions, particularly feces, but to be all that is desirable they should meet the following requirements: They should not be too bulky and should be easy to use under all conditions of weather and temperature; they should be stable and readily soluble or miscible with water; they should not have an unpleasant odor, and should be nonpoisonous to man; they should be capable of penetrating fabrics and organic matter, and should not stain or corrode. They should kill all bacteria promptly, and be cheap enough to render their free use practicable. It is safe to say that none in use will meet all of these conditions.

For the disinfection of feces a 10 per cent. solution of formalin is very effective. Anderson has shown that feces exposed to 10 per cent. formaldehyde solution were rendered practically sterile immediately, and that the spore-bearing organisms were destroyed after forty minutes. It has the additional advantage of overcoming odors by chemical union, and its own odor is not particularly offensive.

Carbolic acid in 5 per cent. solution, when properly mixed with feces, is effective; crude carbolic acid appears, however, to be more reliable than the pure product, probably on account of the presence of cresols. Its power is enhanced by the addition of common salt. Solutions of the different cresols, such as creolin, lysol, and saprol, may be substituted for carbolic acid, 8 to 10 ounces of a 5 per cent. solution being used.

Milk of lime, which consists of slaked lime in 4 volumes of water, is efficient but inconvenient to use on account of its bulk and the danger of clogging water-pipes and closets. A 4 per cent. solution of chlorinated lime is likewise efficient for disinfection of excreta in the bed-pan.

When used in sufficient amount, lime in its various forms is useful also for disinfection of the contents of privy vaults or latrines. The lime must be fresh, it must be thoroughly mixed with the mass, and there must be sufficient moisture present to slake it. Excreta known to be infected should not be thrown into privy vaults until after thorough disinfection.

Disinfection of Urine.-In typhoid fever the urine often contains typhoid bacilli and may be disinfected by the addition of one-twentieth of its volume of formaldehyde solution. The same measure should be adopted to disinfect the urine of typhoid bacillus carriers, and in addition they may be given medicinal doses of urotropin, which will sometimes cause the organisms to disappear from the urine.

In order to disinfect urine, it may also be diluted 50 per cent. with a 5 per cent. solution of carbolic acid, creolin, or lysol. In the absence of the more suitable preparations a 5 per cent. solution of chlorinated lime may also be used in equal volume of urine.

Disinfection of Buccal and Nasal Discharges.-The mouth discharge of patients suffering from tuberculosis, pneumonia, plague, typhoid fever, and other diseases in which the infection may be contained in such discharges should be received into sputum-cups or cloths or paper, which, with their contents, can be burned or thoroughly sterilized 
by boiling. Burning is the most efficient disinfectant known and can be resorted to in practically every houschold. Boiling for twenty minutes is likewise very efficient and readily applied.

Sputum and other mouth discharges may be collected also in metal or glass spit-cups in which has been placed 4 or 5 ounces of a 5 per cent. solution of formalin, carbolic acid, creolin, or lysol. Mercuric chloride, on the other hand, is not reliable for the disinfection of sputum, for reasons to be given later, and should not be used.

In leprosy, diphtheria, cerebrospinal meningitis, and like diseases the nasal discharges should be collected on cloths and immediately burned. In order to lessen the danger of infection through this channel, nasal sprays consisting of acetozone or other solution may be used, but this practice should under no circumstances prevent collection and burning of the discharges.

Disinfection of Conjunctival Discharges.-In infectious diseases of the conjunctiva, such as gonorrhoeal ophthalmia, trachoma, and purulent conjunctivitis, the discharges, as they are removed from the eyes by means of drops or douches, should be collected on pads of absorbent cotton or gauze and burned or treated with double their volume of 5 per cent. solution of formaldehyde. By this means, and the confining of patients to bed, with appropriate instillations of argyrol and douches of boric acid, I have treated many patients in hospital without spread of infection to other occupants of the wards.

Disinfection of the Skin.-In the infectious exanthemata the infections are thrown off during desquamation. 'Thorough cleanliness of body and surroundings from day to day limits their spread and, therefore, becomes a part of the disinfecting process. Disinfection of the hands of those in attendance upon all cases of communicable diseases is also essential after every contact with the patient or his discharges, and may be practised by thorough washing with green soap and water, to be followed by immersion in 3 per cent. solution of formaldehyde, 2 per cent. solution of lysol, 1 to 1000 mercuric chloride solution, or 40 per cent. alcohol.

Mercuric chloride is a powerful germicide under favorable conditions, acting as a direct poison to micro-organisms as well as coagulating their protoplasm. It is this latter faculty that precludes its use for the disinfection of masses containing much organic matter, such as feces; for the salt is preeipitated as an albuminate, thus limiting its action. Acidulation by the addition of 5 parts of hydrochloric acid to every part of sublimate in 1000 , however, will prevent precipitation, and in preparing the solution for disinfection this practice should be invariably followed.

Mercuric chloride has the additional disadvantage that it is highly poisonous and will corrode metals and fix stains. It is probably not as eflicient for disinfecting the hands as formaldehyde or lysol, but formaldehyde has the disadvantage that its continued use roughens the skin.

Disinfection of Clothing and Bedding.-Even with the greatest care the clothing and bedding coming in contact with infections cases, 
and to a less extent the curtains and room-hangings will become infected. 'Their disinfection before being removed from the sick-room, therefore, becomes essential, and may be accomplished by either soaking for one hour in a disinfectant solution, boiling, or exposure to steam in a chamber for thirty minutes. The solutions to be recommended for this purpose are 5 per cent. solution of formaldehyde, carbolic acid, or lysol, and the clothing and linens should be immersed therein immediately after use.

Since boiling is usually done after removal of the articles from the room, it must be preceded by soaking in one of the solutions mentioned above. Stains from any cause, such as blood, urine, or feces are more or less fixed by boiling, and they should be removed as far as practicable before this method is applied; previous soaking in a cold disinfectant solution in great measure accomplishes this purpose.

In hospitals and under special conditions infected articles may be removed under cover to a disinfecting room and immediately sterilized by steam. Whenever the necessary apparatus is available for its application, steam under pressure is the most efficient means of disinfecting clothing, bedding, and other textiles and articles not injured by it.

While such apparatus is not ordinarily available in private practice or in small conmunities, every hospital, detention station, and large town should have one conveniently located in case of outbreaks of the infectious diseases. The apparatus consists of a boiler for generating the steam, and this is connected by pipes with a jacketed chamber which should be large enough to admit bundles and mattresses, and the doors at either end should open into separate compartments or rooms.

After the articles to be disinfected have been placed in the chamber, it is closed by a close-fitting door provided with a gasket. Steam is then introduced into the jacket until the temperature within the chamber reaches $70^{\circ} \mathrm{C}$., the object being to bring the articles to a temperature at which the steam, when admitted, will not be condensed on the fabrics; such condensation not only damaging them, but also preventing penetration of the steam.

With a vacuum apparatus which is attached to the chamber a vacuum of 10 to 15 inches is then obtained, after which steam from the boiler in introduced into the chamber until a pressure of 10 pounds is present, as indicated on the pressure gauge. When this pressure and temperature are reached, drip cocks communicating with the interior of the chamber are opened and the circulation of steam is allowed to continue for at least twenty minutes.

Upon completion of the disinfection, the steam is shut off from the interior of the chamber, a vacuum is again produced, and the door is kept closed for ten or fifteen minutes longer to allow of drying of the articles, and thus prevent any condensation of steam that might injure them.

In the absence of special apparatus flowing steam not under pressure may sometimes be available, and is an efficient disinfectant. Here, the time of exposure must be at least thirty minutes after the temperature has reached $100^{\circ} \mathrm{C}$. 
Articles injured by steam, such as leather, gutta-percha, rubber, furs, hats, silks, and fine woolens, should not be disinfected in this manner, but by some gaseous disinfectant or by immersion in a germicidal solution. 'Those articles which will be injured by wetting must be exposed to formaldehyde gas. For this purpose the steam chamber may be used, provided it has a formaldehyde generator attached.

Such an attachment is provided on all steam chambers at the federal quarantine stations. After the chamber is filled with leather goods or other articles that may be damaged by steam, the jacket is heated until the temperature in the chamber is raised to $60^{\circ} \mathrm{C}$. A vacuum of 10 inches is then obtained inside the chanber, after which formaldehyde gas is introduced. The gas is evolved under a pressure of 45 pounds in the generator from a solution of formaldehyde to which has been added a neutral salt, such as calcium chloride 20 per cent. 'Thirty ounces of formaldehyde solution should be used for every 1000 cubsic feet of space within the chamber, and a small amount of steam should be also introduced to supply moisture, which renders the gas much more effective. 'This method is to be recommended because of its efficiency and rapidity of action, the time of exposure required being one hour under the conditions ontlined.

In a majority of cases, however, the employment of special apparatus is impracticable, but formaldehyde gas is effective when generated by other means, such as the formalin-permanganate method, formalinaluminum-sulphate-lime method, sheet-spraying, and the autoclave. Successful disinfection with formaldehyde is dependent more on the condition under which the work is done than the method of evolving the gas, and these conditions will be deseribed when discussing room disinfection, as will also the various means of generating the gas.

Disinfection of Instruments and Utensils.-Instruments and other utensils used in the sick-room may be ordinarily disinfected by boiling or exposure to live steam for not less than thirty minutes. 'Those articles, such as rubber and leather goods, that will be injured by either of these methods, must be disinfeeted by one of the germicidal solutions. 'The addition of 1 per cent. of carbonate of soda renders boiling applicable to polished steel, cutting instruments, or tools.

All china-ware and kitchen utensils should invariably be sterilized by boiling before being removed from the sick-room, and articles of food that have not been used should be destroyed. An Arnold sterilizer is a very efficient means of disinfecting with steam, and may be conveniently used in the ease of many small articles in daily use in the sick-room.

Disinfection of Rooms.-Previons selection and preparation of the room in which the patient is to be isolated and the constant application of hygienic measures throughout the illness will reduce greatly the amount of infection, but not forego the necessity of disinfection of the room itself and the furniture it contains. In order to be effective, it must be thoroughly done.

Room disinfection is usually accomplished by the use of gaseous dis- 
infectants, but a number of precautions are necessary in preparing for their application. All windows and doors should be closed, and all holes and cracks sealed by pasting over them pieces of paper. If the paper on the walls is damaged, it should be removed as thoroughly as possible and burned. Any rugs or carpets that may be in the room should be removed for steam disinfection or placed on chairs. 'The drawers of bureaus, lockers, and other furniture should be opened, and all textile fabrics that have not been disinfected by immersion in a disinfecting solution should be hung over lines or hooks, to allow the greatest possible exposure to the gas. All articles of little value, such as paper, rags, and cheap books, should be burned, other books being placed on end in such a manner that the leaves will not remain in juxtaposition. The temperature of the room should be raised to over $60^{\circ} \mathrm{F}$., and maintained there throughout the process. Provision should also be made to provide moisture, which is necessary in all gaseous disinfection, a relative humidity of over 60 per cent. being highly desirable.

The selection of the method of disinfection will depend somewhat on the infection to be destroyed, and largely on the character of the room and its contents. A choice may be made of formaldehyde gas, sulphur dioxide, or hydrocyanic acid gas.

Formaldehyde is produced by the oxidation of wood-alcohol. It is soluble in water to the extent of 40 per cent.; such a solution being known in the pharmacopoia as liquor formaldehydi. While the gas is very irritating to mucous membranes, it is not poisonous to the ligher forms of animal life, and fails to kill vermin, such as rats, mice, roaches, bedbugs, and lice. On the other hand, it is very destructive to vegetable micro-organisms, and is the most efficient gaseous disinfectant known against the ordinary infections. In the case of yellow fever, plague, and typhus fever it should not be depended on, however, since the infection of yellow fever is transmitted by the mosquito, the infection of plague by the flea, and the infection of typhus fever probably by the bodylouse.

Formaldehyde gas has the additional advantage that it does not attack metals or fabrics, nor will it injure colors except very delicate pinks. It is, therefore, of great practical value in disinfection, and may be effectively applied in inclosed spaces of moderate size, such as rooins, by means of a generator lamp that brings about partial oxidation of wood-alcohol by passing its fumes over heated platinum. With this method a special generator is necessary; the room must be rendered as tight as possible, and 24 ounces of wood-alcohol must be used to every 1000 cubic feet of space in the room. The wood-alcohol should be of 95 per cent. strength, and should not contain more than 5 per cent. acetone. The generators are of necessity placed within the room. The danger of fire, must, therefore, be guarded against, and the room must remain closed at least six hours.

In order to avoid the use of lamps, and for the purpose of generating the gas outside the room, the autoclave was invented by Trillat, and variously modified by others. The gas is evolved under a pressure of 
not less than 45 pounds, and led into the room through a pipe inserted into a keyhole or other opening. In the apparatus 10 ounces of formaldehyde solution, to which has been added calcium chloride ( 20 per cent.), is used for every 1000 cubic feet of space, and the room must be kept closed for at least six hours. For large rooms the gas must be introduced at several points as far apart as possible.

Formerly, spraying of formaldehyde solution was used in the absence of apparatus for generating the gas, and is effective if properly carried out. 'The formaldehyde solution must be spraved on shects suspended in the room in such a manner that the solution will remain in small drops.

Not less than 10 ounces of formaldehyde solution should be used for every 1000 cubic feet. Used in this manner, a sheet will hold about 5 ounces without dripping or the drops running together. 'The room must be tightly sealed for twelve hours. 'The methorl should not be attempted in rooms of over 2000 cubic feet, and may be supplemented by thoroughly spraying the solution upon the walls, floors, and objects in the rooms. 'The advantage of the sheet spraying method is that it is rather simple and economical, and is applicable for disinfection purposes where long exposure is required.

'The formalin permanganate method, which was advanced by Evans and Russell, and sometimes known as the "Maine method" has obviated the necessity of any but simple apparatus that can be improvised at practically every house. It depends for its action upon the fact that when formalin is poured over crystals of permanganate of potash a vigorous reaction takes place, accompanied by a strong ebullition of the liquid, and heat sufficient to produce a large quantity of formaldehyde gas and watery vapor. For the generation of the gas there should be a galvanized iron pail of a depth of 12 inches, and with a diameter at its base of 10 inches, the top flaring in such a manner that the outlet will be 18 inches. As considerable heat is generated during the process, this pail should be set in a tub or other vessel containing water or sand.

When the room is made ready for disinfection, the above-mentioned apparatus should be placed in the centre of the floor. The permanganate of potash is then placed in the pail in the proportion of $S$ ounces for every 1000 cubic feet of room space to be disinfected. The solution of formaldehyde is then poured over the crystals of permanganate, using 16 ounces of the solution for every 1000 cubic feet of space. Since the time for the reaction to begin varies from only a few seconds to a minute or two, the operator must at once leave the room and close the door.

The reaction is over in a comparatively short time, thus providing the maximum volume of gas at the very beginning of the process. For these reasons, and because a considerable amount of moisture is produced, the formaldehyde permanganate method is highly efficient and generally to be preferred to all other methods.

The formalin-aluminum-sulphate-lime method, also known as the Walker method, may also be used, and is preferred by some hygienists. One part of aluminum sulphate is added to 2 parts of hot water. One 
part of this solution is added to 2 parts of formaldehyde solution (both by volume); 1 part of this second solution is then poured on 2 parts of unslaked lime broken into fine particles. The liberation of the formaldehyde gas is completed in about twenty minutes. Because of the longer time required to evolve the gas, and because less than half the amount of gas is given off compared with the formaldehyde-permanganate method, the latter is regarded as very much the more desirable. With the formalin-aluminum-sulphate-lime method 2 pints of formaldehyde solution per 1000 cubic feet of space should be used, and the temperature should be not less than $60^{\circ} \mathrm{F}$. Care should also be taken to guard against fire, but this danger is decidedly less than in the permanganate process, on account of the large amount of watery vapor coming off with the gas.

Formaldehyde gas may also be evolved by heating paraform tablets or pastiles in a special apparatus. Each tablet usually contains $1 \mathrm{gm}$. of paraform, which is polymerized formaldehyde, and from 60 to 75 tablets are required for each 1000 cubic feet of space. Since the development of the formaldehyde-permanganate method it is to be preferred.

Regardless of whatever method is selected, great care must be taken to have the required amount of gas generated, and the temperature and moisture must be controlled so as to insure the full efficiency of the gas. The room must also be kept closed at least six hours to permit of complete disinfection.

Upon completion of disinfection, the windows and doors should be thrown open, and, if desired, the remaining formaldehyde gas may be neutralized by sprinkling ammonia water through the room. 'The articles of furniture may be then aired, or washed with a disinfectant solution as an additional precaution.

Sulphur dioxide may necessarily be resorted to at times for room disinfection, although for this purpose it is inferior to formaldehyde, because it is not as highly germicidal, and because it attacks colors, metals, and fabrics, thus injuring them.

In the presence of moisture, however, sulphur dioxide is highly destructive to vegetable organisms, but will not kill spores. In order to provide the necessary amount of moisture, means must be taken to volatilize water to the extent of $\frac{1}{2}$ pint for every 1000 cubic feet of space, and this may be done by the heat generated during the combustion of the sulphur itself.

Sulphur dioxide is also very destructive to all forms of animal life, which renders it especially applicable for the destruction of infectious organisms transmitted by rodents, lice, mosquitoes, flies, and other insects. During outbreaks of plague, yellow fever, and malaria, therefore, its use may become necessary to rid houses of vermin and insects, and for the fumigation of ships, granaries, warehouses, and similar large spaces to kill vermin, it has no equal.

'Three methods may be resorted to for the generation of sulphur dioxide, namely, burning in pots, volatilization of liquid sulphur dioxide, and the use of the sulphur furnace or other apparatus. 
By burning the sulphur in pots disinfection can be done efficiently, and it has the advantage of being cheap and easy of application. 'The number of pots required will depend on the size of the room and amount of sulphur to be burned, it being necessary to use 5 pounds to each 1000 cubic feet of space. 'This amount of sulphur will supply to the atmosphere in which it is burned 4.5 per cent. per volume of sulphur dioxide, which has been shown to be effective for the destruction of non-sporebearing organisms after an exposure of less than eighteen hours.

'The pots should be shallow and each one should contain not more than 10 pounds of sulphur. 'They should be distributed throughout the compartment, and elevated from the floor by placing on boxes or tables, as sulphur dioxide is heavier than air and gradually sinks to the bottom of the room. 'The sulphur should be in a fine state of subdivision, and ignition is best accomplished by alcohol, but great care must be taken to guard against fire. By placing the pots in tubs containing water fire is guarded against and the necessary moisture furnished by evaporation that takes place. After the sulphur in the several pots has been ignited, the doors should be closed and remain so for twenty-four hours.

By means of the sulphur furnace which can be transported from house to house on trucks sulphur dioxide can be generated in large amounts, and a larger percentage of the gas can be supplied in a given space than is possible with the pot method. The sulphur furnace resembles the fire-box of an ordinary stationary horizontal boiler, and contains a large shallow pan under which can be placed a fire. 'The furnace is started by placing a small fire under the sulphur pan on which have been placed rolls of sulphur. When the pan becomes suiniciently hot to ignite the sulphur, its combustion will be continued by the heat produced. By means of an ordinary draft air is made to pass over the burning sulphur, and the fumes are compelled to take a devious course through the furnace to an attached cooling oven. From this oven they are conducted by means of an exhaust fan operated by a small engine through ordinary fire hose to the compartment. Only enough air should be admitted through the draft to keep the sulphur burning with a low blue flame, and the fan should not be run too rapidly, otherwise a portion of the sulphur will be sublimed. The conducting hose should be led into the compartment as near the floor as possible, as the gas is heavier than air, and thus made to displace the latter, which should he allowed to escape through a small opening near the ceiling. Since sulphur dioxide generated in this manner contains no moisture, it is necessary to provide the same, which may be done by adnitting a small jet of steam from the boiler.

'The sulphur furnace is necessarily cumbersome, and, therefore, impracticable of use in ordinary disinfection, but for the disinfection of ships, docks, and warehouses, it is of great value, and has been used with advantage in San Francisco to fumigate sewers.

Various types of sulphur furnaces are in use, the different points in their construction having to do mostly with the cooling of the sulphur 
fumes and their delivery to the compartment. As previously stated, a drum is attached to the ordinary furnace for the purpose of cooling the fumes, while in the Clayton apparatus, this is accomplished by means of a gill pipe and condenser. 'This latter apparatus is also provided with a return pipe, by means of which air can be exhausted, and the gas delivered to the apartment may be subsequently agitated to favor penetration and prevent the settling of the gas to the bottom. Practically the same amount of gas can be evolved by either apparatus, and if sufficient moisture is present, it will destroy non-spore-bearing organisms, but not spores. In any event, but little penetration of the gas can be expected, and in the presence of moisture, it is very destructive to colors, fabrics, paper, and metals.

Liquefied sulphur dioxide may be used for disinfection, but 10 pounds per 1000 cubic feet will be required. If the liquid is contained in tins, the leaden pipes in the tops should be cut and the tin inverted in a washbowl or iron pan, when volatilization occurs. By this means, a large amount of gas can be liberated rapidly, but at much greater expense than with other methods. A special apparatus has been designed for using liquid sulphur dioxide, which aims at the exhaustion of the air in the compartment and its replacement by the gas. The Marot apparatus accomplishes this, and aims to evaporate the liquid at a constant rate by means of heat. By means of this apparatus electric sparks are passed through the sulphur dioxide, which, it is claimed, increases the bactericidal power of the gas on account of the formation of small amounts of sulphur trioxide. On account of the greater expense involved, such apparatus is impracticable in ordinary room disinfection.

Hydrocyanic acid is included among the gaseous disinfectants on account of its poisonous effects on all forms of animal life. Aside from the fact that it is not injurious to fabrics and furniture, it has no advantage over sulphur dioxide for the destruction of vermin and insects, and it is very much inferior to formaldehyde for germicidal purposes. Hydrocyanic acid is extremely poisonous to man, and it should not be used for disinfection purposes except in rare instances and by a thoroughly experienced operator. It should never be used in occupied houses.

Upon opening a room after the completion of gaseous disinfection, it is always advisable to follow with thorough mechanical cleansing. The furniture should be sunned and aired, and woodwork of the room repainted. The floors and walls should be painted, or the walls and ceilings may be calcimined, whitewashed, or papered.

Room disinfection may also be carried out by thorough washing of every article in it with a disinfecting solution, spraying the floors, walls, and ceiling with the same solution, and afterward washing them down, but great care must be exercised in order that the disinfecting solution shall reach every surface that may possibly harbor infection.

Disinfection of Cars.-The disinfection of street-cars and passenger coaches can be accomplished by the same procedures as outlined for rooms. When the disinfection is done on account of the occurrence of one of the communicable diseases, formaldehyde gas is the most 
suitable agent to apply. In the case of sleeping cars, the mattresses, linens, and hangings should have been previously removed and disinfected by steam, and the car itself should receive thorough mechanical cleansing after the gaseous disinfeetion is completed, During outbreaks of plague, the routine disinfection of cars with sulphur dioxide may be considered advisable to kill rodents, and for this purpose at least 2 pounds of sulphur per 1000 cubic feet should be burned.

During yellow-fever epidemics, routine disinfection of all cars leaving the infected centre is required to kill mosquitoes. 'T'wo pounds of sulphur per 1000 cubic feet is efficient for this purpose, but its destructive action must be borne in mind. In those ears where there are articles liable to be injured by the use of sulphur, the fumes of burning pyrethrum may be used to destroy mosquitoes. 'This agent may also be used for a like purpose in rooms and cabins on ships.

Four pounds per 1000 cubic feet of space will kill practically all mosquitoes after two hours' exposure, but precautions should be taken to sweep up and destroy any that may have escaped being killed.

As previously stated, hydrocyanic acid gas is a powerful insecticide, and not injurious to materials. It is best generated by mixing cyanide of potash 4 parts, sulphuric acid 6 parts, and water 9 parts. 'The acid should first be diluted, which must be done in some vessel capable of withstanding the heat; the whole amount of the cyanide of potash must be put in the acid at once, and as the evolution of the gas is very rapid, the operator must be prepared to leave immediately. 'Ten ounces of cyanide of potash should be used for every 1000 culic feet of space. Its poisonous effect on man must be constantly borne in mind.

Disinfection of Ships.-'The principles underlying the eradication of infection aboard ship are the same as in the case of houses, but it must be remembered that the passengers and crew constitute a community which is more or less congested and housed under unusual conditions. In case of infection, therefore, the measures to be enforced will depend on its character and extent.

Having determined the maximum limits of infection, the ship should be remanded to quarantine, where there are proper facilities for disinfection. 'The passengers and all members of the crew, except the captain, an engineer, and two or three petty officers, are then removed to the disinfecting station. 'Their treatment, as well as that of the ship, will depend on the infection.

In case of yellow fever only the disinfection of the baggage to kill mosquitoes will be required. In the case of plague, typhus fever, cholera, small-pox, or other exanthematous infection, the passengers and crew should be required to take a bath and have their personal effects disinfected by appropriate methods.

With the assistance of those left to care for the ship, the quarantine personnel then remove to the disinfecting chambers all presumably infected bedding, linens, rugs, curtains, and other hangings. Attention is next given to the ship, disinfection being begun in the forecastle?

In the case of ressels, when treated for yellow-ferer infection, the Vou. I-31 
process should be a simultaneous fumigation with sulphur dioxide, a 2 per cent. volume gas, and two hours' exposure, in order to insure the destruction of all mosquitoes; the fumes of pyrethrum being used in compartments, such as the cabins, where there are articles that would be injured by sulphur. This should be followed by disinfection of all casks and tanks containing water to kill the larve of mosquitoes.

In the case of plague infection, there should be a simultaneous fumigation with sulphur dioxide, not less than 2 per cent. being used with six hours' exposure. where there is no cargo. Where cargo is present, the gas should be present in 4 per cent. volume with not less than twelve hours' exposure. Such fumigation is to be followed by thorough washing down of all parts of the ship with mercuric chloride solution or other disinfectant.

The forecastle is usually disinfected first, which may be done with formaldehyde gas or a solution of mercuric chloride; the latter, combined with mechanical cleansing, being preferred; because the solution can be thoroughly applied with a force-pump driven by an engine. The decks, petty officers' quarters, and similar apartments are next washed down with mercuric chloride solution. The floors and walls of bathrooms and water-closets are also hosed, and the hoppers flushed and treated with 5 per cent. solution of formaldehyde or carbolic acid.

The cabins, saloons, and other apartments require to be disinfected with formaldehyde gas. The sick-bay should be given special attention, and may be flooded with mercuric chloride solution after gaseous disinfection is completed.

The holds should be invariably fumigated with sulphur, when there is reason to believe them infected, as in plague. Infected vessels may require partial or complete discharge of cargo, and fractional fumigation with sulphur for efficient deratization. The oxides of carbon, as used at Hamburg, are efficient to destroy rats, but do not kill fleas or other insects. They are produced by burning carbon, coke, or charcoal in special apparatus, the gas produced consisting of about 5 per cent. carbon monoxide, 18 per cent. carbon dioxide, and 77 per cent. nitrogen. While not injurious to cargo, this method is inferior to sulphur because of the failure to kill fleas or micro-organisms: After each disinfection all dead rats must be collected and burned in the furnace.

In cholera-infected ships all water ballast should have been previously pumped out at sea, and sand ballast may be required to be disinfected or discharged in deep water. The bilgewater should be disinfected with 5 per cent. carbolic acid solution, and then pumped out. The water-tanks should be emptied and disinfected by potassium permanganate, formaldehyde solution, or by steam.

The cargo is rarely ever infected, and may generally be disregarded by the quarantine officer, except in ships harboring infected rats, and then, if properly packed, surface disinfection is all that is required. Vegetables and other food-products coming from cholera-infected districts may require special treatment, however, but such articles should not be shipped during outbreaks of the disease. 


\title{
SERUM THERAPY
}

\author{
BY WILLIAM H. PARK, M.D.
}

\section{HISTORICAL}

Serum therapy is largely confined to the use of serum containing specific immune bodies produced in animals through active immunization.

Practical immuno-therapy may be said to date from the introduction of vaccination by Jenner, in 1796 , although the production of immunity from small-pox by inoculation was practised long before that in the Orient. In the middle of the eighteenth century two English physicians called attention to the fact that persons who had had cow-pox were immune against small-pox, but the significance of this observation appears to have escaped them. 'The same observation had passed into tradition in parts of England, and was carefully investigated by Jenner, a physician of Gloucestershire. He found that dairy-maids and others having to do with cows suffering from cow-pox developed a peculiar localized pustule (man-pox), and that the material from such a pustule inoculated into other persons produced similar localized lesions at the point of inoculation. Persons so inoculated, he found, were immune to smallpox. The results were so striking that the practise of "vaccination," as it was called, soon spread to all parts of the world.

While the practise of immunization was thus empirically established, it was not until about seventy-five years later that any insight was gained into the underlying principles. Pasteur, with keen intuition, gainerl the idea that cow-pox was small-pox, the virus of which had been attenuated by its passage through the cow, and that consequently when man was vaccinated he was inoculated with a benign form of the disease. But might not this be a general law? He determined to investigate the question in connection with anthrax, the bacillus of which had just previously been isolated, both by Koch and himself. From some work by'Toussaint it was evident that a weakening or attenuation of the bacteria or virus must first be obtained before it could safely be injected into animals for the purpose of producing immunity, for, if the unaltered virus were injected, a virulent infection would result. After much experimentation Pasteur hit on the plan of growing the bacillus at a temperature of $42^{\circ} \mathrm{C}$., obtaining in this way a culture of the fully developed organism which had a low virulence. His first experiment was most dramatic. He injected half of a flock of sheep with this attenuated anthrax culture, and then, two weeks later, injected all of them with a virulent culture. All of the sheep not previously injected died, while not one of the others was even dangerously sick. 
It was perhaps only natural, in view of his discoveries in fermentation, that Pasteur should have believed that the production of immunity required the action of the living virus. He, therefore, vigorously combated the idea that immunity could be brought about by means of dead virus or of lifeless products of growth of the virus. Touissant had previously held out for the latter possibility, but the imperfections of his technique were such that his views were not accepted. To Salmon and Smith, of this country, belong the credit of first clearly demonstrating the possibility of immunization with dead cultures. All of these methods required active immunization, and, therefore, time for the production of immunizing substances in those vaccinated.

'The discovery of diphtheria antitoxin by v. Behring, in 1890, inaugurated an entirely new method of immunization; namely, by means of injections of serum from immunized animals. Although practical serum therapy may be said to begin with $\mathrm{v}$. Behring's discovery, the work of Gscheidlen and Traube, Fodor, Nuttall, and Buchner had shown that blood-serum possessed marked germicidal properties. Moreover, Richet and Hericourt had immunized rabbits against staphylococcus infection by injecting them with serum from those previously inoculated with these organisms. The results obtained when these sera were used to cure an infection already in progress were most disappointing, and it is probable that serum therapy would have received but scant consideration at the hands of scientific investigators had it not been for the remarkable success achieved by antitoxin in the cure of diphtheria.

\section{UNDERLYING FRINCIPLES}

Immuno-therapy concerns itself mainly with the treatment of infectious diseases. An infectious disease may be defined as one caused by living micro-organisms which have gained access to the tissues of the body. It is obvious that the pathogenic effect of the micro-organisms must be due to deleterious substances, in some way given off to the invaded tissues. In fact, following the discovery of diphtheria toxin by Roux and Yersin, in 1888, and of the toxin of tetanus shortly after, it was thought that all bacteria secreted powerful poisons whereby they produced their injurious effect. Further investigation, however, showed that such "extracellular" poisons are the exception; most bacteria appear to have their poisons so intimately bound up with their protoplasm that it is impossible to isolate the poison in cultures. Such intracellular poisons are spoken of as "endotoxins." The infecting agent may remain localized, giving rise to a local infection, or it may be widespread through the body, causing a general infection. The absorption of poisons from a local site of infection may produce general symptoms; this is called an intoxication.

Infection is usually followed by a remarkable alteration in the susceptibility to disease of the tissues of the host. Representing as it does all the tissues of the body, it is natural that these changes are strikingly exhibited by the blood. As far back as 1889 Buchner showed that the 
serum of animals artificially immunized against a certain hacterium possessed increased bactericidal properties for that particular organism. In studying immunity on aninals successfully immunized against diphtheria infection, Behring noted that, although the blood-sermm of the animals possessed no bactericidal power, it was highly efficacious in proterting other animals against diphtheria infection. Further investigations showed that this serum contained a substance capable of neutralizing the diphtheria poison very much as a base neutralizes an acid. "lhis substance is called an antitoxin. In addition to the bactericidal substances and antitoxins, the body produces still other substances in response to the introduction of micro-organisms; namely, agglutinins, precipitins, and opsonins. Moreover, it has been found that antibodies are produced in response to the injection of the most varied kind of cells, such as bloorcells, spermatozoa, epithelial cells, ete. Antibodies can also be produced against unformed elements, such as dissolved albumins of various kinds, ferments, etc.

Since the mechanism of the action of these various antibodies differs, and since the differences are of considerable practical importance, it will be well to briefly describe each class of antibody.

Antitoxins.-If an animal is injected with gradually increasing doses of a toxin-e.g., with diphtheria toxin-its serum will gradually acquire powerful antitoxic property. Small doses of the serum injected into another animal will protect the second animal against large doses of the particular toxin. 'The action is strictly' specific, the serum neutralizes only the toxin used for immunization; it is absolutely inert against any other. 'The antitoxins are comparatively stable substances, the serum maintaining its neutralizing strength for a considerable time. On long standing (months or years) there is a gradual loss of antitoxic power, and this loss is permanent. 'The sera can be heated to $55^{\circ} \mathrm{C}$. without destroying the antitoxin. The action of the antitoxins appears to be a simple neutralization, comparable to that of an acid by a basc. 'The action follows the law of multiple proportions, so that if a given quantity of toxin is neutralized by a certain amount of antitoxic serum, double the quantity of toxin will require double the amount of scrum. Antitoxins appear to play the deciding role in the immunity against diphtheria and tetanus; their role in other diseases is less pronounced, and there are many bacterial infections in which they appear to play almost no part whatever.

Agglutinins.-Following the injection of certain bacteria- $\varepsilon . g$. , typhoid, dysentery, cholera-the serum of the injected individual acquires the property of causing a peculiar clumping of the organisms. 'This may be observed with the naked cye in the test-tube mixtures or, under the microscope, in hanging-drop preparations. 'The action is specific, though it usually extends to nearly related organisms. It is not ret clear what the purpose, if any, of the agglutinating function is. Agglutination does not appear to injure the bacteria in any way. The anglutinins are of considerable importance in the serum diagnosis of infections and in the identification of bacteria. 
Precipitins.-When an animal is injected with dissolved albuminous substances-e. g., with serum from another animal, egg white, milk, etc. - the serum of the injected animal acquires the property of forming a precipitate when mixed with the solution used for injection. The action is highly specific. 'Thus, when a dog is injected with human blood, the dog serum will give a precipitate when mixed with human blood, but not with the blood of any other animal. This fact is made use of in identifying blood-stains, in determining the animal species of meat in sausage, etc. Precipitins are also developed by the injection of certain bacteria, e. g., typhoid bacilli; the serum of the injeeted animal causing precipitation with bacteria-free filtrates of old cultures. This is readily understood, for such filtrates contain the autolytic products of the bacteria originally present. Whether or not the precipitins have any function in immunity is unknown.

Bacteriolysins.-This is an extremely interesting group of antibodies. In 1894 Pfeiffer called attention to the fact that enormous numbers of living cholera spirilla could be injected into the peritoneal cavity of guinea-pigs previously immunized against these organisms. $\mathrm{He}$ showed that the spirilla injected were rapidly disintegrated by the peritoneal exudate and the serum of the animals, and that by injecting normal, untreated animals with this serum these could be protected against infeetion with living cholera spirilla. This action was strictly specific: -i. e., the serum acted only against the organisms used for immunization. Its protective action was likewise limited to infection with that particular organism. Bordet showed that the destructive action of such a serum could be demonstrated even in a test-tube, but that it was necessary for the serum to be fresh. On standing, or on exposure to $55^{\circ} \mathrm{C}$, the serum rapidly loses its bactericidal power. Metchnikoff showed that the addition of fresh serum, even serum from a normal animal, restored the activity of the serum. It should be remarked that Bordet injected animals with red blood-cells, and found that the serum of the animals so immunized exerted marked solvent action of these cells. The serum acted only when fresh, but the activity of an inactive serum could be restored by the addition of fresh serum from a normal animal. It was evident that the injection of the bacteria or of the red blood-cells had caused the production of an antibody in the serum of the immunized animals. The action of the antibodies was strictly specific; a serum obtained by immunization with cholera spirilla acted only against those organisms, one made by means of typhoid bacilli acted only against typhoid bacilli, one made by means of bovine blood corpuscles acted only against bovine blood corpuscles, etc. Since the activity of the inactive sera could be restored by the addition of fresh normal serum, it was evident that the destructive effect depended on the combined action of two substances-a stable, specific substance, present only in the immune serum, and a labile non-specific substance, present in all fresh sera, both immune and normal. 'The stable substance is called the immune body (amboceptor, sensitizer, substance sensibilatrice); the labile substance is called complement (alexin, addiment). 'The combination of 
the two is spoken of as a bacteriolysin or hemolysin, according as its action is directed against bacteria or against red blood-ells.

Opsonins.-In 1895, Denys and I ecelef called attention to the fact that serum may act on bacteria so that they are more readily taken up by phagocytes. Iittle attention seems to have becn paid to this observation, and it is to Wright's extended researches that we owe our appreciation of the significance of this property of the serum. 'The substance in scrum which prepares bacteria for phagocytosis Wright calls opsomin: it is present in all normal serum, and can he specifically increased for a particular bacterium by immunization with that organism. I3y means of absorption tests Wright showed that the opsonin has a specific affinity for the bacteria and none for the lencocyte. The opsonin for staphylococcus prepares only staphylococei for phagocytosis; that for tubercle bacilli, only these bacteria, etc. Wright has devised a comparatively simple means of measuring the changes in the opsonic power of a serum. By means of smear preparations he determines the average number of bacteria ingested per leucocyte when bacterial suspension, emulsion of leucoeytes, and scrum are mixed together, and compares this with the average number ingested when the same bacterial suspension and leucocyte emulsion are mixed with normal serum. 'The result thus obtained is the "opsonic index" Others have modified Wright's methods, and estimate the degree of dilution which a serum will stand without losing its opsonic power. Wright and his followers belicve that the degree of immunity can be readily followed by studying these changes. Our own experiences have led us to believe that while the opsonic index affords some indication of the changes going on within the body of the immunized animal, it reflects but one phase of the immunity reaction, and, therefore, does not always indicate the serum's immunizing power. In this respect it is like the agglutinin determinations; these also represent only a part of the immunity reaction withont reflecting the condition of immunity as a whole.

Phagocytosis and Leucocytic Extract.-The discovery of the various antibodies in the serum of immunized animals went far to explain the nature of immunity against infectious diseases. In fact, in the first enthusiasm following the discovery of the antitoxins and of the bacteriolysins many were rash enough to believe that the entire wonderful process had been cleared up. However, it soon became apparent that immunity was due to more than merely one or more serum antibodies, and attention was once more turned to the doctrine of phagocytosis. 'The significance of this phenomenon was pointed out by Metchnikoff in 1S83. He showed that the wandering cells of the animal organism possessed the property of taking up, rendering inert, and digesting microorganisms which they may encounter in the tissues. According to this view, susceptibility to or immunity from infection is essentially a matter between the invading bacteria on the one hand and the leucoerte of the tissues on the other. According to Metchnikoff, many of the antiborties present in the serum, and especially the complement (alexin), are derived from the leucocytes. A number of investigators have experinented 
with the injections of leucocytic extracts into the bodies of diseased men and animals. Our present conception of immunity tends to combine the original phagocytic theory of Metchnikoff with the original humoral theory of Ehrlich, and to regard the phenomenon as a complex coördination of the activities of many different tissues of the body.

Immunization.-In order to better understand the therapeutic application of specific scra and of bacterial vaccines it will be well to glance for a moment at the phenomena accompanying experimental immunization. Immunization may be accomplished passively or actively. The former is usually produced by the injection of a quantity of serum from a highly immunized horse. Here we merely transfer, by means of the blood-serum, the immunity actively produced in the horse in response to bacterial or toxin injections. The effect of the serum injection is the transfer as soon as it is absorbed of whatever available antibodies the serum contains. In the case of diphtheria antitoxin the transfer of the

FIG. 83.

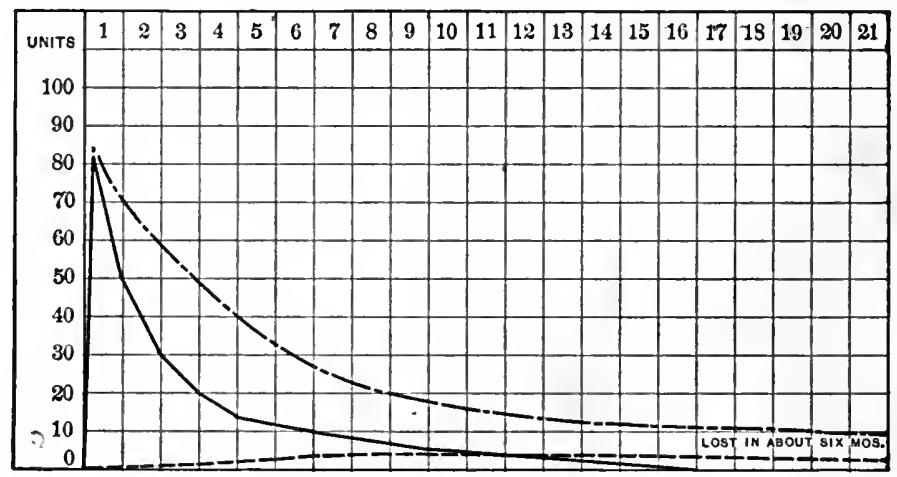

Dotted line shows passive immunity produced by homologous serum. Continuous line shows passive immunity from injection of heterologous serum. Interrupted line shows development of active antitoxic immunity after toxin injection.

antibodies is practically the transfer of complete immunity against the disease, but this is not always the case. For example, by immunizing a horse with typhoid bacilli we can produce an immune serum showing marked agglutinating, opsonizing, and bactericidal powers. 'The injection of such a serum into another individual, however, will not always prevent the development of typhoid infection. Whether this is because it lacks sufficient accumulation of antibodies or because of other more complex reasons we do not know.

In contrast to the immediate effect produced by the injection of serum is that produced by the injection of bacterial vaccines and toxins. In typical cases the first effect of an injection of vaccine is a decrease in the degree of immunity. 'This is spoken of as the negative phase and is followed by a rise in the immunity. This, in turn, is succeeded by a very gradual return to the normal. 'The following diagrams, one showing the development of the opsonins in a case following the injection of 
a dose of bacterial vaccine and the other contrasting the development of antitoxic immunity, bring out the contrast between the secfucnce of events in the two kinds of immunization.

According as injections of antitoxin or toxin are made, it will be noted that the injections of antitoxin produce within twelve hours, when given subcutaneously, their maximum effect, while the toxin injections produce no antitoxin for several days, and that the greatest amount which is reached on about the ninth day is very small as compared with that produced by the antitoxin injection. The persistence of the antitoxin in the blood is seen to be much longer in animals actively im-

Fig. 84 .

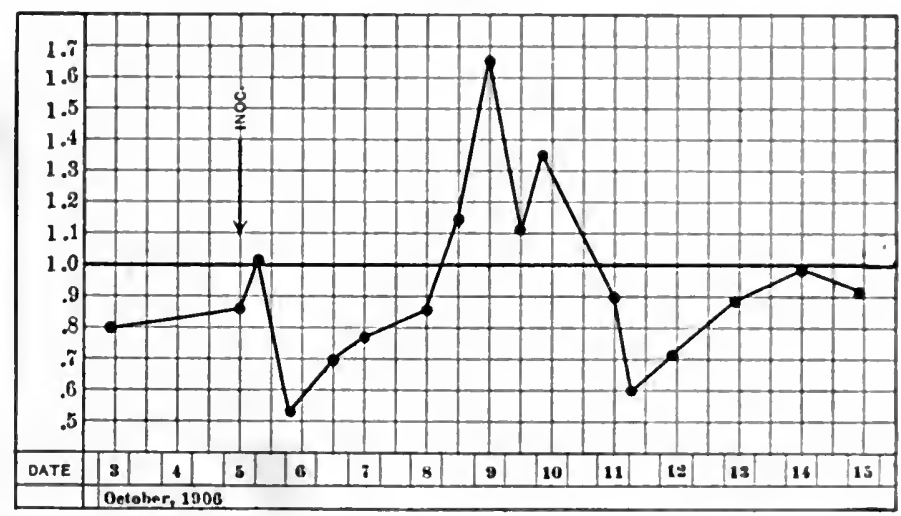

The first curves give the amount of antitoxin in the blood at different periods of time after subcutaneous injections of antitoxin or toxin.

munized or immunized by antitoxin produced by their own species than by other species.

We may summarize the advantages and disadvantages of active and passive immunization as follows:

Active immunization is not effective at the start, but when developed is more lasting, and is perhaps a more complete immunity. It is sometimes difficult to bring about, and may involve some risk to the patient.

Passive immunization is accomplished as soon as the serum is absorbed, that is, within a few hours when given subcutaneously, and instantaneous when produced by intravenous injections. It is convenient, and involves practically no risk to the patient. As it is usually due to antibodies produced in a different species of animal, it does not last over three weeks unless the injections are repeated.

\section{SERUM THERAPY OF DIPHTHERIA}

The term "diphtheria," as at present employed, is limited to infections caused wholly or chiefly by the Klebs-Loeffler bacillus. 'This bacillus is constantly present in the false membrane and the tissues just 
beneath it. Only in rare eases does it invade distant regions, such as the blood and viseera, and then it does not increase in numbers. The constitutional effeets observed in diphtheria are due to a powerful toxin, produced at the seat of the lesions and carried to all parts of the body. 'This'appears to be a true secretion of the bacilli. While the ability to produce toxin is common to all true diphtheria bacilli, there is some variation in the amount produced by different cultures. Toxin production is dependent not only on the individual culture employed, but also on the eharacter and composition of the medium and the conditions under which the culture is grown.

As the result of animal experiments, we know that immunity against diphtheria can be produced by the action of less than fatal doses of toxin on the cells. These are thus stimulated to produce substances antidotal to the diphtheria toxin. Behring, in conjunction with others, showed that the blood of immune animals contains a substance which neutralizes the diphtheria toxin. The blood-serum of persons who have recovered irom diphtheria has been found also to possess this protective property, which it acquires about a week after the beginning of the disease, and loses again in a few months. Moreover, the blood-serum of many individuals, usually adults, who have never had diphtheria often has a slight general antitoxic property.

Diphtheria antitoxin has the power of neutralizing diphtheria toxin, so that when a certain amount is injected into an animal before or together with the toxin it overcomes its poisonous action. There is a direct action of antitoxins upon their corresponding toxins.

The various attempts to separate the toxins and antitoxins from neutral mixtures have been failures, and it is found that neutralization takes place according to the law of multiple proportions-i. $e$., to save an animal from 1000 fatal doses of diphtheria toxins requires little more than a hundred times as much antitoxin as is required for 10 fatal doses, the resistance of the animal itself accounting for the difference.

Nature of Diphtheria Antitoxin.-This has until recently been known almost wholly from its physiological properties. Experiments have seemed to show that it was either closely bound to the serum globulins or was itself a substance of proteid nature closely allied to serum globulin.

Atkinson found that in the case of antitoxic serum the globulin precipitate carries with it all of the antitoxic power of the serum, leaving the filtrate without any neutralizing power against the diphtheria toxin. Independently of Atkinson, Pick obtained similar results. 'These experiments were continued later by Gibson and Banzhaf, and they proved that the globulins which were insoluble in saturated sodium chloride solution carried with them no antitoxin. The soluble globulins which on heating become insoluble also contain no antitoxin. With this knowledge a practical method of eliminating much of the non-antitoxic portion of the serum was perfeeted. Antitoxin is but little injured by prolonged moderate heat $\left(56^{\circ} \mathrm{C}\right.$.), but is destroyed by short exposure to 
higher temperatures $\left(95^{\circ}\right.$ to $100^{\circ} \mathrm{C}$.). It is less sensitive than diphtheria toxin.

Antitoxin Unit.- The power possessed by a definite quantity of antitoxin to neutralize a certain amount of toxin is utilized in testing the amount of antitoxin in any serum or solution. We measure this anount in units. A unit may be defined as the amount of antitoxin which wili just neutrali\%e 100 minimal fatal doses of toxin for a 25)-gram gnineapig.

There are certain peculiarities in the composition of toxins which require us to use certain precautions in selecting the one to be used for testing. 'This we call a standard toxin.

In order to facilitate testing, we consider that a guinea-pig which lives after injection more than four days is protected. 'The test is carried out as follows: Guinea-pigs of about 250 grams' weight are subcutaneously injected with the equivalent of 100 fatal doses of a carefully preserved standardized toxin, which toxin has been previously mixed with an amount of antitoxin believed to be sufficient to protect the animal. If the guinea-pig lives four days, the amonnt of antitoxin added to the 100 fatal doses of toxin is considered to have neutralized it and to measure at least 1 unit. If the guinea-pig dies earlicr than four days, less than 1 unit of antitoxin was in the mixture.

Production of Diphtheria Antitoxin for Therapeutic Purposes.-As a result of the work of years in the laboratories of the Health Department of New York City, the following may be laid down as a practical method:

A strong diphtheria toxin should be obtained by taking a very virulent, actively growing culture and growing it in slightly alkaline broth in wide-neeked flasks for a period of eight days. 'The toxin is then filtered from the bacilli, stored, and tested.

'The horses used should be young, vigorous, of fair size, and absolutely healthy. 'The horses are severally injected with 10,000 units of antitoxin and then the following day with toxin sufficient to kill 5000 guinea-pigs of 250 grams' weight. After from three to five days, so soon as the fever reaction has subsided, a second subcutaneous injection of a slightly larger dose is given. 'The following figures give the actual injections in a horse which produced an unusually high grade of serum:

First day, 12 c.c. toxin ( $\frac{1}{400}$ c.c. fatal dose). This followed an injec. tion of 10,000 units of diphtheria antitoxin.

Second and later injections of toxin without antitoxin at three-day intervals as follows: 15 c.c., 25 c.c., 35 c.c., 45 c.c., 55 c.c., 65 c.c., 80 c.c., 95 e.c., 115 c.c. (twenty-eighth day), 140 c.c., 170 c.c., 205 c.c., 250 c.c., 300 c.c. (fortieth day). 'The injections were gradually increased until, on the sixtietl day, 675 c.c. were given.

The antitoxic strength of the serum was on the twentv-eighth dav, 225 units; on the forticth day, 850 units; and on the sixtieth dav, 1000 units. Regular bleedings were made weekly for the next four months when the serum had fallen to 600 units in spite of weekly, gradually increasing doses of toxin. 
In order to obtain the serum the blood is withdrawn from the jugular vein by means of a sharp-pointed cannula, which is plunged through the vein wall, a slit having been made in the skin. 'The blood is carried by a sterile rubber tube into large Erlenmeyer flasks, held slanted, or into cylindrical jars, and allowed to clot. 'The serum is drawn off after four days by means of sterile glass and rubber tubing, and is stored in large flasks. Instead of this process, when the globulins are to be separated, the blood may be added directly to one-tenth of its volume of a 10 per cent. solution of sodium eitrate. This prevents clotting of the blood. With the serum or globulin solution small phials are filled. 'The phials and their stoppers, as, indeed, all the utensils used for holding the serum, must be absolutely sterile, and every possible precaution must be taken to avoid contamination. An antiseptic may be added to the serum as a preservative, but it is not necessary except when the serum is to be sent to great distances, where it cannot be kept under supervision.

Kept from access of air and light and in a cold place it is fairly stable, deteriorating not more than 30 per cent., and often much less, within a year. Diphtheria antitoxin, when stored in phials and kept under the above conditions, contains within 10 per cent. of its original strength for at least two months; after that it can be used by allowing for a maximum deterioration of 2 per cent. for each month. The antitoxic power in old serum is just as effective as in that freshly bottled, only there is less of it. 'The serum itself is less apt to produce rashes. All producers put more units in the phials than the label calls for, so as to allow for gradual loss of strength.

The Use of Diphtheria Antitoxin in the Treatment of Diphtheria. -It is very difficult to correctly estimate the amount of antitoxin which should be given in a case, as there is no way of knowing how much toxin has been absorled by the blood and taken up by the tissues. We know that the larger the amount of antitoxin injected into the tissues the quicker will a considerable amount be absorbed into the blood and pass into the body fluids. Only a small percentage of what is in the blood passes out of the vessels into the tissue fluids. It is for this reason that, if we would neutralize toxin which has passed from the blood stream, Fut has not yet united with the tissue cells, very much more than the amount of antitoxin must be given than would be required in the testtube to neutralize the toxin. It is also debated as to whether all the antitoxin required slould be given in one dose or should be given in clivided doses. Theoretically, it would be proper to give sufficient in the first dose to suffice, but, practically, it is diffieult to judge the necessary amount, and, unless we give larger doses than are required, we fail to give sufficient for the needs of the exceptional cases. It has, therefore, become the practise to give sufficient in the first dose to probably eover the needs of the ease, and then to repeat the dose in six to twelve hours if it becomes necessary. 'The antitoxin is usually injected subcutaneously, but in severe or toxic cases it should be given intravenously.

There are only two real objections to giving larger amounts than 
necessary - one is the cost of the antitoxin, and the other the somewhat greater liability of rash production and the other after-effects of the serum. 'The new refined globulin solution proluces much less of these after-effects, so that the avoidance of rashes is largely a yuestion of cost, and this is becoming of less importance to the individual, since liealth authorities are providing more and more free antitoxin for those under their charge. 'The following doses are those nsed in the diphtheria hospitals in the city of New York. We have adopted these after a great deal of investigation.

The ordinary laryngeal cases, and all cases that are quite severe or show signs of poisoning, are given 10,000 units. 'Those cases which appear malignant, or show a very large amount of exudate with swelling, or appear decidedly toxic, are given 15,000 or 20,000 mits. 'These malignant cases should be injected intravenously. If the cases are not decidedly better in about twelve hours, this dose is repeated, and if the symptoms still indicate it, another dose is given in twelve hours. Moderate cases, especially those in older children and adults, are given 3000 to 5000 units, the dose to be repeated as in the more severe cases. McCollom, of Boston, advises that the antitoxin injections be repeated every six hours until the symptoms improve. Clinically, the action of the serum is very prompt, especially in cases treated early. Often within twelve hours there is a fall in temperature, accompanied by an improvement in the pulse and in the nervous condition of the patient. 'The diphtheritic membrane stops spreading, and soon begins to soften and loosen. The swelling of the mucous membranes subsides, the nasal secretion and fetid odor and all the signs of the local disease abate. Upon complicating infections, such as bronchopneumonia, otitis media, etc., the antitoxin has, as a rule, no effect, as these diseases are due to other organisms. During the first few hours following the injection there is apt to be a slight rise in temperature and acceleration of the pulse. As all diphtheria antitoxin is produced by horses, it is rapidly eliminated when injected into man, but this is of great importance only when prevention and not cure is sought. 'The question of sensitization is only important when more than two weeks between doses elapses. Even then the risk of any serious after-effects is almost negligible. 'The earlier in the disease the antitoxin is giventh e more the effect it will produce. Given late in the disease it often does little or no good. We cannot tell, however, whether it is too late or not, so that one should always give it. Diphtheria occurring at unusual situations, such as on the conjunctiva or vagina, is treated successfully by one or two full-sized injections of antitoxin. In these cases cultures should be used in order to make the diagnosis.

The Use of Diphtheria Antitoxin in the Prevention of Diphtheria.1000 units of antitoxin are usually given when the danger of infection is great and 500 when it is slight. By the end of five days fully nine-tenths of the antitoxin will have been eliminated, and by the end of two weeks, 99 per cent. (See chart, p. 4SS.) When danger of infection continues, it is well to give a second injection at the end of a week, or certainly at 
the end of two weeks; 500 units, given every ten days, would suffice to indefinitely prevent infection. I have supervised the use of immunizing injections to prevent infection in many institutions. It has given great satisfaction. Recently, in one institution, where some 60 cases had developed within four days, over 2000 persons were immunized, with the result of immediately stopping the development of new cascs. Similar results have always taken place when all persons were immunized. I believe that there is no need of closing schools when immunization can be carried out.

The local effects of the injection are a slight redness and pain, which rapidly subside.

Results as Shown by Statistics.-At the present time the evidence of the value of diphtheria antitoxin is overwhelming. Studying this question some years ago, the writer collected statistics from nineteen large cities in the world, and found that the introduction of diphtheria antitoxin everywhere caused a marked drop in the mortality from that disease. The mortality varied somewhat from year to year in each city, but in every one it tended to grow less each year as antitoxin was more generally used. New York City had a mortality of 153 per 100,000 in 1894 and one of 36 in 1909 . During six weeks I watched the result of treating only every other case admitted to the hospital with antitoxin. The results were so much better in those treated with antitoxin that we felt compelled to use it in all cases. (See article on Diphtheria.)

Rash Production.-It was early noted that injections of antitoxin were sometimes followed, after a few days, by a peculiar train of symptoms, principally rashes, pains in the joints, and fever. Investigation has shown that these are due to the injection of serum as such-i.e., to the injection of an alien proteid. The symptoms come on after a period of incubation, which varies from two or three days to a week or more. The average interval is about a week. The rashes are extremely varied in character, the most common being urticarial in type. Other types resemble the rashes of measles and scarlet fever. The first appearance is usually at the site of inoculation, and frequently this local rash is the only symptom, so far as the rash is concerned. The rashes on the rest of the body usually develop symmetrically. The regional lymph-nodes are rather constantly swollen and are tender to touch. Some of the cases complain of joint pains, the joints of the hand and the knee being the most frequently affected. 'The mucous membranes hardly ever take part in the serum reaction, and this constitutes a valuable aid in their differential diagnosis from scarlet ferer and measles. Treatment for the rashes is cntirely symptomatic, and consists of the application of prolonged warm compresses to the affected joints, local applications to the skin to relieve the itching, sponging for the fever, etc.

Realizing that the rash production is due to the alien proteid as such, and not to the antitoxin, attempts have been made to produce as powerful a serum as possible. Compared to the sera first produced by v. Behring, much has been accomplished in this direction. Whereas, the first sera contained 50 and 100 units per cubic centimeter, few sera are at present 
marketed containing less than 500 units per conbie centincter. A decided advance was made when Gibson succeeded in perfecting a practicable method of purifying antitoxic serum by ridding it of some of the non-antitoxic proteid constituents. By this metherd serat containing 1200 units or more per eubic centimeter can casily be producad. A still further purification is effected by the procedure introbluced by Banzhaf; by means of this, sera containing as high as $25(x)$ units per eubic centimeter can be produced. 'The use of such purifierl and concentrated sera has been attended with excellent results, and severe rashes are much less frequent than with native sera.

Serious Accidents. - A very small number of persons feel faintness and nausea after the injection. 'The pulse becomes rapid and foethle and there is a feeling of impending suftocation. 'These cases usually improve after a few minutes, but at times stimulating injections and artificial respiration may have to be employed. Still more rare are the cases of sudden death occurring within a few minutes of the injection of the serum. 'These rare accidents have occurred in persons suffering from the status lymphaticus. No such case has been reported during the past three years in New York City. 'There are also a few cases that develop some hours after the injection, with or without an accompanying rash, a severe attack of asthma. 'These attacks are very disagrecable, but not dangerous.

The Administration of Diphtheria Antitoxin by the Mouth.-There are a number of reports that diphtheria antitoxin has done good when given in large and frequent doses by the mouth. Whatever good is accomplished must be due to the local contact of the serum with the diseased tissues. During the first few days of life a small percentage of antitoxin which is swallowed is absorbed. After ten days absorption practically ceases, unless by means of opiates and alkalis digestion and peristalis is interfered with. Even then only about 1 or 2 per cent. is absorbed.

\section{SERUM THERAPY OF TETANUS}

Tetanus is produced by infection with the tetanus bacillus or its toxin. 'The bacillus occurs in nature as a common inhabitant of the soil, at least in places where manure has been thrown.

Natural infection is probably in most instances an infection with the spores of the organisms, the conditions favoring infection being badly bruised or laccrated tissues and inoculation with ordinary saprophytic germs. In tetanus neonatorum, infection is introduced through the navel; in puerperal tetanus, throngh the inner surface of the uterus. 'The toxins of the tetanus bacillus are extracellular-i. $e_{\text {. }}$, ther are given off in culture fluids. The quantity of poison produced varies according to the age of the culture, the composition of the medium, reaction, completeness of the exclusion of oxygen, ete. Under the best conditions the amount produced in cultures by the fifth day is such that 0.00005 c.c. is the fatal dose for a 15-gram mouse. 
Methods by which Infection Takes Place.-A wound of some kind has occurred, penetrating at least through the skin, though perhaps of a most trivial character, such as might be caused by a dirty splinter of wood, and the tetanus bacilli or their spores are thus introduced from the soil in which they are so widely distributed. If, in any given case, the tissues being healthy, the ordinary saprophytic germs are killed by proper disinfection at once, a mixed infection does not take place, and tetanus will not develop. If, however, the tissues infected be badly bruised or lacerated, the spores may develop and produce the disease.

It is a comparatively rare disease except after the Fourth of July celebration, when throughout the United States a considerable number of cases develop. In some years more than 100 persons have dedeveloped tetanus after blank-cartridge wounds. On examination of an infected individual very little local evidence of the disease can be discovered. Generally at the point of infection, if there is an external wound, some pus is to be seen, in which, along with numerous other bacteria, tetanus bacilli or their spores may be found. Although rather deep wounds are usually the seat of infection, at times such superficial wounds as an acne pustule or a vaccination, may give the occasion for infection. Not only undoubted traumatic tetanus, but also all the other forms of tetanus, are now conceded to be produced by the tetanus bacillus. It should be borne in mind that when there is no external and visible wound there may be an internal one.

Action of Tetanus Toxin in the Body.-After the absorption of the poison there is a lapse of time before any effects are noticed. Following the injection of an enormous amount into animals, such as 30,000 fatal doses, this incubation period is about twelve hours; with ten fatal doses, thirty-six to forty-eight hours; with two fatal doses, two to three days.

Presence of Tetanus Toxin in the Blood of Infected Animals.The blood usually contains the poison, as has been proved experimentally. on animals. Neisser showed that the blood of a tetanic patient was capable of inducing tetanus in animals when injected subcutaneously. In St. Louis the serum of a horse dying of tetanus was given by accident in doses of 5 to 10 c.c. to a number of chilaren, with the development of fatal tetanus. In this connection Bolton and Fisch showed, by a series of experiments, that much toxin might accumulate in the system before symptoms became marked. The demonstration that the toxin is abundant in the blood is extremely important from the standpoint of treatment, and teaches the necessity of neutralizing this poison at the first practical moment. It is true that the nerve-endings in the region of the infection are the first to absorb toxin, but this is only a small part of the whole amount. This passes into the blood-current and then, passing through the capillary walls, comes in contact with the nerve-cells throughout the body.

Tetanus Antitoxin.-Behring and Kitasato were the first to show the possibility of immunizing animals against tetanus infection. The entire procedure is analogous to immunization against diphtheria. 
'The treatment of tetanus is directed against the action of the toxin, and this is accomplished by the neutralization of the toxin by antitoxin in the body.

'The immunioing experiments in tetanus have borne practical fruit, for it was through them that the principle of serum therapeutics first became known - the protective and curative effects of the bloof-serum of immunized animals. It was found that animals could be protected from tetanus infection by the previous or simultaneous injection of tetanus antitoxin, provided that such antitoxic serum was obtained from a thoroughly immunized animal. From this it was assuned that the same result could be produced in natural tetanus in man. Unfortunately, however, the conditions in the natural disease are very much less favorable, inasmuch as treatment is usually commeneed not shortly after the infection has taken place, but only on the appearance of tetanic symptoms, when the poison has already attacked the cells of the central nervous system.

The tetanus antitoxin is developed in the same manner as the diphtheria antitoxin-by inoculating the tetanus toxin in increasing doses into horses. 'The horses receive, subcutaneously, 10,000 units of antitoxin and then the following day an injection of 5 e.c. of toxin which kills 250,000 grams of guinea-pig. In five days this dose is doubled, and then every five to seven days larger amounts are given. 'The dose is increased as rapidly as the horses can stand it, until they support 700 to 800 c.c. or more at a time. After some months of this treatment the blood of the horse contains the antitoxin in sufficient concentration for therapeutic use.

Antitoxin Unit.-'Tetanus antitoxin has not as yet been obtained in pure form, and is known only by its power to neutralize tetanus poison. Its amount is estimated in units exactly in the same manner as diphtheria antitoxin, except that the unit is different. A unit is defined as the amount of antitoxin required to be mixed with 1000 fatal doses of tetanus toxin for a 350 -gram guinea-pig in order to neutralize it. The United States government has adopted this unit and supplies the different producers with standardized toxin. 'The amount of antitoxic serum which neutralizes an amount of test toxin which would destroy 40,000,000 grams of mouse contains 1 unit of antitoxin by the German standard. In the French method the amount of antitoxin which is required to protect a mouse from a dose of toxin sufficient to kill in four days is determined, and the strength of the antitoxin is stated by determining the amount of serum required to protect 1 gram of animal.

Persistence of Antitoxin in the Blood.- Tetanus antitoxin, whether directly injected or whether produced in the body, is eliminated wit! equal rapidity from the blood of an animal provided that the serum was from an animal of the same species. If from a different species, it is much more quickly eliminated. From this we see an explanation of the fact that immunity in man, due to an injection of the antitoxic serum of the horse, is less persistent than immunity conferred in the horse by a similar injection. (Sce chart, page 4S8.)

VoL. I-32 
The Union of Toxin and Antitoxin in the Living Organism.-The studies of H. Meyer and Ransom as well as those of Marie and Morax in Roux's laboratory have thrown considerable light on the much disputed phenomena observed in poisoning by tetanus toxin. The investigations of Gumprecht in $1895^{\circ}$ had made it highly probable that all the pathological symptoms were due to a poisoning of the central nervous system. 'The paths, however, by which the poison reached its central points of attack were still doubtful. The experiments of Meyer and Ransom and of Marie and Morax have proved to them that the poison is transported to the central nervous system by way of the motor nervesand by no other channel. 'These authors thought the essential element. for the absorption and transportation of the toxin is not the nerve-sheath or the lymph-channels, but the axis-cylinder, the intramuscular endings of which the toxin penetrates. Marie and Morax were able to demonstrate the poison in the corresponding nerve-trunk (sciatic) one and a half hours after the injection. Absorption, however, and conduction are dependent to a large extent on the nerves and their lymph supply being intact. In other words, we see that section of the nerve prevents the absorption of the poison by way of the nerve-channels.

Ascending centripetally along the motor paths the poison reaches the motor spinal ganglia on the side of inoculation; then it affects the ganglia of the opposite side, making them hypersensitive. The visible results of this is the highly increased muscle tonus-i. $e$., rigidity. If the supply continues, the toxin next affects the nearest sensory apparatus; there is an increase in the reflexes, but only when the affected portion is irritated. In the further course of the poisoning the toxin as it ascends continues to affect more and more motor centres, and also the neighboring sensory apparatus. This leads to spasm of all the striated muscles and general reflex tetanus.

A different explanation of the passage of the toxin up the nervetrunks has recently been discovered. It is well known that the lymph flow in nerves is from the periphery to the centre, and Field, in our laboratory, has shown that not only tetanus toxin, but diphtheria toxin, and inert colloids can be demonstrated in the sciatic nerves after they have been injected subcutaneously or intramuscularly, and after varying periods may be found in the spinal cord. He believes that the toxins are absorbed by way of the lymphatics of the nerves, and not by way of the axis-cylinder.

A recent experiment of Cernovodeanu and Henni almost proves this contention. They ligated all the muscles and blood-ressels in a guinea-pig's leg, leaving intact only the sciatic nerve, skin, and bone, and then injected a large amount of tetanus toxin below the point of ligation. 'The animals in which this was done never developed tetanus.

In this case there was only a very slight flow of lymph into the ligated area, and so there could be only a slight flow up the nerve.

The major part of the toxin elaborated in the infected area gets into the blood, finally reaching the central nervous system by way of the various motor nerve tracts. There seems to be no other direct path, as, for 
example, by means of the blood-vessels supplying the central nervous system.

Rapidity of Absorption of Tetanus Antitoxin from Tissues.-The complete absorption of a given quantity of antitoxin administered subcutaneously takes place slowly. 'The maximum quantity passes into the blood only after twelve to twenty-four hours. Fom that time on the amount again steadily decreases, so that by the sixth day less than one-third the maximum quantity is present. When antitoxic serum from the horse is injected into some other species of animal, appreciable quautities cannot be found after four weeks. 'These facts emphasize the necessity of giving the first dose in a case of tetanus intravenously.

Naturally, the time during which these changes take place varies with the application, the conditions of absorption, and the concentration and amount of the preparation injected. When injected intravenously, a portion of the antitoxin very quickly passes into the lymph. Ransom was able to demonstrate it in the thoracic duct of a dog a few minutes after intravenous injection. Only after very massive intravenous doses are small traces found in the cerebrospinal fluid. 'This fluid never contains more than 2 per cent. of the amount contained in the blood.

A rapid and plentiful appearance of antitoxin in the blood is dependent on the injection of a large number of antitoxin units. The more units the blood contains, the more thoroughly will the tissues be saturated with the antitoxin.

From the foregoing it is not difficult to formulate the conditions under which an antitoxin introduced into the organism can exert its neutralizing power on the toxin. We see that the poison deposited at any given place takes either of two paths to the central nervous sistem-the smaller amount, a direct path by way of the local peripheral nerves, and the larger amount, an indirect path through the lymphchannels and blood to the end-plates of all other motor nerves. Hence only that portion can be neutralized which $(a)$ still lies unabsorbed at the site of inoculation, or $(b)$ which, though it has passed into the blood and lymph, has not yet been taken up by the motor nerve-endings. A curative effect can therefore result from antitoxin introduced subcutaneously or intravenously only so long as a fatal dose of poison has not been taken up by the nerves.

So long as the toxin circulates in the blood it is neutralized by antitoxin in about the same proportion as in test-tube experiments. 13y means of intravenous injections of antitoxin Ransom was able to render the blood free from toxin in a very few minutes. According to Marie and Morax, toxin injected into the muscles is readily demonstrable in the nerve tissue at the end of one and a half hours-i. e., a part of it has passed where it is probably no longer reached by the antitoxin. There must, however, be a condition or locality in which the toxin can still be neutralized by means of large doses, though with difficulty. This is indicated, among other experiments, by some older researches of Dönitz. 'This observer injected various rabbits 
intravenously each with 1 c.c. of a toxin solution containing twelve fatal doses. Thereupon he determined the dose of antitoxin which, when intravenously given, would neutralize this poison after various intervals of time. The antitoxin was of such a strength that in test-tube experiments 1 c.c. of a $1: 2000$ solution just neutralized the amount of toxin employed. He found that at the end of two minutes double the dose required in vitro would still neutralize the poison; at the end of four minutes about four times the dose was required, and at the end of eight minutes ten times. When one hour had been allowed to elapse, forty times the original dose just sufficed to protect the animal from death, but not from sickness: In order to explain these results, the correctness of which has been confirmed by many analogous observations, the conception "loose union of toxin" has been introduced. By this is meant a state of union between toxin and susceptible cell constituent which can still be disrupted by means of large doses of antitoxin. In this particular instance we do not need to make use of this conception, for the reason that the tetanus toxin is not at all combined during the first hour. Personally, I should regard it as more probable that the interval during which the toxin can still be neutralized, though with difficulty, corresponds to that time during the passage of the toxin in which, after leaving the capillaries, the poison is held up in the fine interstices of the connective-tissue, which it must penetrate before it can be taken up by the nerves.

Results of the Antitoxin Treatment in Tetanus.-The course of tetanus varies so much with the individual that it is difficult to judge by statistics or a slight personal experience as to the value of the antitoxic treatment of the developed form. It is interesting to note that two late authoritative reviews by American writers differ greatly in their conclusions. Anders and Morgan state: "The present status of the serum question leaves no room for doubting that, when given in a well-developed case of tetanus, antitoxin does not have any appreciable beneficial effect, neither the mortality being reduced nor recovery hastened thereby." McFarland states as his opinion: "It would seem, therefore, that we have in tetanus antitoxin not a specific, because it fails too often to merit that name, but a valuable remedy in the treatment of the disease, and one that ought not to be neglected until a better one is supplied." My own opinon, founded on reading and a considerable personal experience, coincides with that of McFarland. In fact, since I have seen the results in cases given large intravenous injections I have become even more optimistic. I have seen cases of generalized tetanus that after several intravenous injection have markedly improved and finally recovered, and these cases have certainly done far better than apparently similar ones receiving small injections or palliative treatment alone. Lambert, who a few years ago made an exhaustive study of tetanus, states that in a total of 114 cases of this disease treated with antitoxin, mostly subcutaneously, according to published and unpublished reports, there was a mortality of 40.35 per cent. Of these, 47 were acute cases-that is, cases with an incu- 
bation period of eight days or less and with rapid onset, or cases with a longer period of incubation, lut intensely rapid onset of symptoms; of these, the mortality was 74.46 per cent. Of the dhronic typethose with an incubation period of nine days or more, or those with shorter incubation with slow onset - there were 61 cases, with at mortality of 16.39 per cent. L. Vaillard quotes statistics covering 592 cases treated by antitoxin as follows: Cases treated, 592); died, 265; mortality, 44.7 per 100 . He believes that mortality without treatment would have been 70 per cent. The cases having an incubation period of over eight days gave a recovery percentage of 27 .

Method of Administering Tetanus Antitoxin.-For immunization, a single subeutaneous injection of 1500 units of a serum of medium strength will suffice unless, on account of possibly infected materials remaining in the tissues, the danger persists, when the injection is repeated at the end of a week. For treatment, according to the severity of the case, 10,000 to 20,000 units should be given intravenously, and the smaller amount should be repeated every six hours until the symptoms abate. 'The same amount should then be given every twelve hours until the danger seems past. It is well to continue subcutaneous daily injections of 4000 units until recovery is complete. In the gravest cases no curative effect will be noticed from the serum. 'The first injections should unquestionably be made intravenously or otherwise many valuable hours may be lost before the full effect is produced, as the antitoxin is absorbed from the subeutaneous tissues slowly. Some advise that injections should also be made into the nerve-trunks leading from the infected region. 'These injections should be made as near the trunk as possible and distend the nerve so as partly to neutralize and partly mechanically interrupt the passage of toxin to the cord or brain. "The method of injeeting from 3 to 15 c.c. of antitoxic serum into the lateral ventricles has not, in the writer's opinion, shown itself to be advisable. No bad results have followed the injections when the serum was sterile and the operation was performed aseptically; but several brain abscesses have already followed the intracerebral injections. 'The use of antitoxin in no way prevents the giving of other forms of treatment.

Immunization of Persons Possibly Infected.-The striking results which have been obtained, particularly in veterinary practice, with the prophylactic injection of tetanus antitoxin, would seem to warrant the treating of patients with immunizing doses of serum-at least, in the neighborhoods where tetanus is not uncommon-when the lacerated and dirty condition of their wounds may indicate the possibility of a tetanus infection.

Splendid results have followed this practice in many places. It is the custom at many dispensaries in New York city and elsewhere to immunize all Fourth of July wounds by injecting 1000 units. None of these have ever developed tetanus. Even the small number of eases of human tetanus reported as occurring in Europe after single injections of antitoxin prove the value of immunizing injections, for the mortality was very low. They teach also that where tetanus infection 
is suspected, the antitoxic serum should be given a second and even a third time at intervals of seven days. It must be remembered that the antitoxin developed in the horse is a foreign substance in man and does not remain as long as if injeeted in the horse. The antitoxin is mostly eliminated within fourteen days.

Experimental Evidence of the Value of Enormous Doses of Antitoxin. -In coöperation with Dr. Cyrus W. Field, I have recently tried a number of experiments upon guinea-pigs to test the importance of intravenous and of intraneural injections of antitoxin in animals in which tetanus had already developed. Forty guinea-pigs have been experimented upon. These were injected in the lower part of the hind leg with ten to twenty times the fatal dose of a mixture of tetanus toxin and bacilli. Within from one to two hours after the development of the first definite symptoms of tetanus the animals were operated upon and given antitoxin. The experiments show clearly that moderate doses of antitoxin given after the development of tetanus do not save the animals from death or even prolong life, while very large doses usually did both. Seventy-five per cent. of those receiving 500 units recovered. The surprising result developed that amputation of the infected leg at the hip-joint hastened the death of the animals in every case. Control animals which had not been infected stood the amputation perfectly well, and made good recoveries. Without antitoxin, excision of a piece of the nerve did not materially prolong life, nor did ligation of the nerve. In the guinea-pigs receiving antitoxin the ligation of the nerve seemed to be of benefit. The results of the experiments showed that large doses of antitoxin given shortly after the development of tetanus usually saved the animals, and that most of the toxin was absorbed by the blood and not by the nerves of the infeeted part. Every minute of delay after the appearance of tetanus was of importance. I feel convinced that in human tetanus the most important thing is to give at the earliest possible moment after diagnosis a very large intravenous injection of antitoxin. From 50 to 75 c.c. of the most potent serum obtainable should be given. During succeeding days injections can be given either intravenously or subeutaneously, until marked improvement or death has taken place. If a surgeon is at hand, intraneural injections into the nerves supplying the infeeted portion of the body may also be given, but these, I believe, are not usually necessary if the large intravenous injections have been given. All hospitals should have a supply of tetanus antitoxin on hand, so that the usual delay in obtaining the serum, which frequently amounts to several hours, can be avoided. The serum should be injected as soon as possible after the disease has been suspected.

\section{SERUM THERAPY OF CEREBROSPINAL MENINGITIS}

Cerebrospinal meningitis, as is well known, may be eaused by a number of different organisms. In children many of the cases are due to the tubercle bacillus; secondary infection of the meninges is most often as- 
sociated with streptococeus and pnenmococeus. 'The eppidenic form of the disease is almost invariably due to the diplocerous intracellularis, first described by Weichselham. 'The recognition of the particular variety with which one is dealing is, of course, of paramount importance in applying specific sera, ete. ${ }^{1}$

'The only certain method of doing this is by an examination of the cerebrospinal fluid drawn off by lumbar punceture. 'The naked-eye appearance is often sufficient to recognize whether the process is tuberculous or not, the fluid from tuberculous meningitis being usually very clear, while that from the other forms of meningitis is cloudy. Sinned specimens, however, should always be examined. 'Tubercle bacilli are easily recognized by their appearance and staining characteristics. If cocci are present, it is necessary to determine whether they are Gram positive or Gram negative. Only in the latter case can any benefit be expected from antimeningococeus serum.

Despite the extensive investigations carried on in the past few years, both in this country and in liurope, the exact mode of infection in epidemic cerebrospinal meningitis is not yet known. Our own experiences in the large epidemic in New York in 1904 and 1905 showed that meningococci were present in the nasal secretion of over half of the patients suffering from the disease, especially in the early stages. We also found that about 10 per cent. of persons in direct contact with cases of the disease harbored meningococe in the nose. But we do not know whether infection of the meninges results by the direct spread of these organisms up through the cribriform plate of the ethmoid, or whether infection occurs otherwise. 'Thus, Elser established the fact that meningococei occurred in the blood in the early stages of the disease.

Following the work carried out in connection with the epidemics of cerebrospinal meningitis in recent years, a number of specific antisera were produced. Of these, the best known are those of Flexner in this country, and of Wassermann and Jochmann in Europe. The sera are obtained from horses, and it has been found advantageous to immunize with autolyzed cultures alternated with either dead or living cultures.

The meningococci are grown on serum agar, to which a little glucose has been added. The autolysates are prepared by growing the cultures for twenty-four hours in large, wide-mouthed flasks at $37^{\circ} \mathrm{C}$., pouring 10 to 20 c.c. sterile physiological salt solution over the surface of the growth, and adding a few drops of toluol. By gently shaking the flask, the growth is loosened and mixed with the salt solution. "The flask is returned to the incubator and allowed to stand twenty-four hours, at the end of which time the toluol has evaporated and the fluid is found to contain merely a few disintegrated bodies of meningococei. The main bulk of the bacteria has been dissolved br an autolitie ferment. Wassermann prepares extracts by adding sterile distilled water to the cultures, and agitating the mixtures for twenty-four hours in a shaking apparatus. In either case the solution obtained possesses toxic properties for animals.

1 See also article by Dr. Dunn. 
The horses are injected subcutaneously, beginning with small doses of extract, alternating with small doses of dead culture. The injections are repeated weekly, and it is well to make them in different parts of the body, in order to secure as much antibody formation as possible. After a month or two the dead culture can be replaced with living culture, and the dose, both of this and of autolysate, inereased. 'The entire course of the immunization occupies from four to six months.

Considerable difficulty has been experienced in measuring the strength of the serum. It is generally beliered that the anti-endotoxic effect is one of the chief properties of the serum. However, there is no way in which this can be even approximately determined. It is true that the serum does neutralize the toxic effects of the autolysates, but the fatal dose of the latter varies so enormously as to render this unsuited for testing. The use of living cultures is exeluded, because of the marked variation in their virulence. Kolle and Wassermann thought to measure the strength of the serum by means of the complement fixation test, but it was soon found that there was no parallelism between the complement binding power and the therapeutic action of the serum. Recent investigations appear to show that the opsonic power of the serum is a fair measure of its therapeutic value. This does not mean that the activity of the serum is principally opsonic; the opsonin content seems, however, to parallel the content of the principal effective antibody or antibodies. The opsonic content is measured by the dilution method.

It is difficult to apportion the credit for the production of the first protective serum. Bonhoff and Lepriere produced in animals a serum which showed definite protection. 'The world-wide epidemic, beginning in 1905, stimulated a number of laboratories to produce sera in horses with the idea of treating human cases.

Thus, in 1905, ${ }^{1}$ Kolle and Wassermann, Jochmann, and ourselves immunized horses.

The Therapeutic Use of Serum.-In 1905 there was inaugurated in Hartford, Connecticut, the use of subcutaneous injections of diphtheria antitoxie serum. This influenced me to prepare and try the subeutaneous injection of an antimeningococeus serum. The results, reported by the physicians in some 20 cases, did not seem to establish any beneficial effeet, so that no further serum was issued. Later, Kolle ${ }^{2}$ and Wassermann reported somewhat more favorable results in a number of cases from the subeutaneous injection of a serum prepared by them. Previous to their use of the serum, however, Jochmann had used his serum by the intraspinal method in a series of cases.

The first successful use of an immune serum in cases of human cerebrospinal meningitis, by the intraspinal method, should, therefore, so far as we know, be credited to Jochmann ${ }^{3}$ and the physicians who used his serum in the winter of 1905 and 1906 . He reported a series of eases, treated by the intraspinal method, before the Congress for Internal

${ }^{1}$ Deutsche med. Woch., 1906, xxxii, No. $16 . \quad{ }^{2}$ Ibid., 1907, xxxiii, p. 1585.

${ }^{3}$ Deutsche med. Woch., vol. xxii, No. 20, p. 788. 
Medicine held in Munich in April, 1906, and published his paper on May 17, 1906.

'The serum was prepared by injecting horses with increasing doses of meningococcus killed at about $58^{\circ} \mathrm{C}$. 'The doses were given every eight days, beginning with a loopful, and increasing until the growth on the surface of ascitie agar covering two Petri dishes was used. After this dose was reached living eultures were given.

'The serum was shown to possess both bactericidal and opsonic power. He reported 40 cases had been treated, but gave details concerning 17 patients, which all occurred in one hospital and were treated by Kromer. Five of these patients died, and 12 recovered. He dirceterl that, after lumbar puncture, 20 to $50 \mathrm{cec}$. of fluid should be removed and then 20 c.c. of immune serum injected. 'These injections were to be repeater once or twice if the fever did not alsate or if it returned. He noticerl, in general, a bettering of the headache, stiffness of neck, and mental condition. Jochmann showed that, in animals, colored fluids injected into the spinal canal in the lumbar region passed the full length of the canal.

Although serum, prepared in several laboratories in Europe, was used in a few localities after Jochmann's report, it did not receive much attention in this country until Flexner, through his important experiments on infected monkeys, which demonstrated the value of the intraspinal injections of the serum, aroused public interest and paved the way for him to try out the serum on a large scale. Eighteen months later, Flexner and Jobling published their report, which fully corroborated the earlier results of Jochmanu. 'The serum prepared at the Rockefeller Institute for Medical Research has been generously sent to many places, both in this country and in Europe, and results obtained have been of the utmost value in arriving at the value of the intraspinal treatment. 'The results of its use, and the manner of its employment, will be found discussed in the article by Dr. Dunn, on cerebrospinal meningitis, in this volume. My personal experience has been limited to watching the results in a small number of cases. On the whole, it has been very favorable. The poorest results have been in severe late cases, in which the exudate was so extensive as to prevent the proper application of the serum.

\section{SERUM THERAPY OF DYSENTERY}

Following the researches of Shiga, Kruse, Flexner, and others, whereby the specific character of baeillary dysentery was established, attempts were made to produce a specifie serum for the disease. 'To Shiga belongs the credit of being the first to be successful in this direction. By the subcutaneous inoculation of dead cultures into horses, followed after a time by living cultures, he produced a serum which was able to save guinea-pigs when injected, even as late as ten or fifteen hours after infeetion. Encouraged by these results, he used the serum in dysentery in humans. Out of 298 cases treated exclusively with serum, in doses of 
20 to 50 c.c., 31 died, a mortality of 10.8 per cent. Of 2599 cases treated according to the usual methods, 957 died, a mortality of 35.4 per cent.

While many favorable reports were published concerning the use of the serum, the experiences of other workers, especially those in America, was not so favorable. One reason for this lies probably in the fact that, clinically, typical dysentery is produced by a number of related organisms, and a specific serum produced against one variety has only a moderate effect on infections due to another. According to their fermentation and antibody characteristics, at least three different varieties of dysentery bacilli in this country must be distinguished, namely, the original variety, identified by Shiga as the chief factor in the Japanese cases and the most prevalent form in the largest epidemics in all countries, the variety isolated by Flexner, and the one isolated by the writer and also by Hiss. Another reason is that the serum was given in cases of ordinary enteritis which the dysentery bacilli had little or no part in producing.

By autolyzing cultures of dysentery bacilli a number of investigators have obtained solutions of what they consider the specific endotoxins of the organisms. By immunizing animals, both with the toxins and with living bacilli from the three strains, a serum is obtained which possesses both antibacterial and antitoxic properties. The results obtained with this polyvalent serum have been excellent. 'Thus, in one series of cases, out of 157 treated with the serum only 7 died-a mortality of 4.5 per cent. At the same time the mortality was over 10 per cent. in those treated according to the usual methods, without serum. Good results were also reported from the use of such a serum in Manchuria during the Russo-Japanese War.

For purposes of regulating the dosage, Vaillard and Dopter divide the cases of dysentery into four classes: namely, mild, moderately severe, severe, and extremely grave, taking as their principal guide the number of stools in the twenty-four hours. Thus:

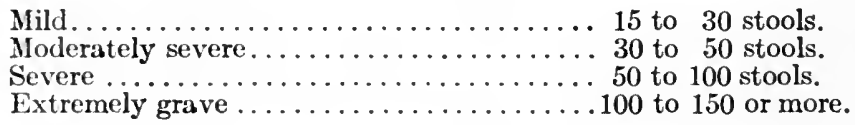

Such a classifieation, to be sure, is rather arbitrary, and the degree of prostration, frequency and character of the pulse, and signs of collapse must also be taken into account. On the whole, however, the number of stools is a valuable indication of the gravity of the case.

For mild cases a single injection of 20 c.c. subcutaneously often suffices to quickly ameliorate the symptoms and bring about prompt recovery. Howerer, if, after twenty-four hours, the symptoms persist, or if, aiter an initial improvement, they return, a second injection of the same dose should be made. In the more severc cases it may be necessary to give daily injections for three or four days to bring about recovery. In the severe and extremely grave cases the dose must be large. In the latter, as much as 100 c.c. in the course of the day may be necessary. As with the 
other sera, the injections are kept up, in decreasing doses, according to the degree of improvement noticed. 'The doses for infants are somewhat smaller, though not nearly in the proportion guiding the doses of ordinary drugs. My personal experience has been limited to something over 20 eases of characteristie dysentery. 'The patients certininy secmed to be benefited by the serum, and within twenty-four to forty-right hours the constitutional symptoms, as well as the abdominal pain and the tenesmus, usually were much lessened. 'The ordinary severe summer diarrhceas, which do not present the symptoms of dyentery, are noi due to any of the dysentery bacilli, and are not benefited by serim injections. As we have no units of measure, we have to depend upon the reliability of the producer to give us an effective serum.

\section{SERUM THERAPY OF TYPHOID FEVER}

The history of the serum treatment of typhoid fever begins with attempts to utilize the serum of typhoid convalescents. On the supposition that such a serum represented the curative properties of the organism against typhoid infection, a number of authors-Hammersehlag, von Jaksch, Jez, and others-made attempts in this direction, but obtained only disappointing results. Klemperer and Lery used the milk of animals artifieially immunized against typhoid, but this likewise yielded poor results. Next, treatment was undertaken with sera obtained by immunizing horses with either living or dead cultures of typhoid bacilli, and again the results were disappointing. Believing that the reason for this was probably a lack of sufficient complement, it was suggested that the sera be injected fresh, but this was impracticable. Pfeiffer and Kolle held that the injection of an antibacterial sermm in typhoid fever might even constitute a distinct menace to the individual, by a sudden large liberation of endotoxins. A number of investigators, therefore, sought to produce a serum possessing increased antitoxic properties. 'Thus, Shaw kept cultures of typhoid bacilli in the incubator for three weeks, and then filtered the liquid. By inoeulating this filtrate into animals, he produced a serum which possessed some antitoxic power. MacFadyen immunized horses with typhoid bacilli, expressed juice of which had been broken up by freezing with liquid air. The resulting serum possessed strong neutralizing powers when tested against the toxin, and gave good results when tested in animals, both in preventive and in curative tests. Chantemesse cultivated a virulent strain of typhoid bacilli in a special broth made with defibrinated blood and ox spleen. At the end of a week the culture is heated to $55^{\circ} \mathrm{C}$., centrifugalized, and decanted. The clear fluid is his "typhoid toxin." By long immunization of horses with this "toxin" he produces a serum for which he claims marked curative properties. 'Thus, out of 5621 cases of typhoid fever treated by the expectant method, the mortality was 17 per cent., while among 1000 cases treated with this sermm the mortality was 4.3 per cent. The patients receive very small doses of the serum, five or six drops, and the close is repeated only two or three times. Wright 
believes that the serum may contain toxins and not antibodies, and that its action may possibly be that of a vaccine rather than that of an antiserum. The serum has been used to such a limited extent outside of the cases treated by Chantemesse that it is impossible to form any accurate judgment as to its value.

'There has been almost no use of serurn in the treatment of typhoid fever in this country. The statements made concerning the use of a serum in infections due to the typhoid bacilli apply equally to those due to the members of the colon group.

\section{SERUM THERAPY OF CHOLERA}

If in any disease more than another a curative serum is needed, it is cholera. Clinically, the disease is a pure intoxication, the organisms remaining, at least until the later stages of the disease, in the superficial layers of the mucous membrane and in the bowel, where they multiply and produce their peculiar poison. The course of the disease is so rapid that there is little hope in effectively combating it except by passive immunization. In spite of this, and in spite of the large amount of careful experimental work that has been done in order to produce an effective antiserum, the goal is still far off.

It is the simplest thing imaginable to produce a serum possessing marked bacteriolytic powers; in rabbits, for example, a single injection of dead cholera culture will often suffice to bring this about. But such a serum is without the least curative value against the disease. On the contrary, there is evidence that the administration of a bacteriolytic serum increases the intoxication by suddenly liberating a large amount of endotoxin. The more the question has been studied, the more has grown the conviction that a serum, to be effective in cholera, must possess strong antitoxic powers, and efforts have, therefore, been concentrated in devising means to produce a strong toxin with which to immunize. A large number of different methods have been employed, but the "toxin" has never been of more than very moderate strength. Moreover, the poison shows evidence of not being a "toxin," in the strict sense, for its strength, when tested in animals, varies enormously, and when tested against the antiserum, the neutralization does not follow the law of multiples.

Some success has been claimed with a serum preparation recently made by Krause. He employs the El Tor strain, an organism isolated in $1905^{\circ}$ from the body of a Mecca pilgrim who had died of dysentery, not cholera. Culturally and biologically, this is identical with true cholera spirilla, except that in bouillon cultures it produces a hemolysin and a toxin which acts acutely. The toxin thus produced neutralizes not only El Tor toxin, but also that of true cholera. This antitoxic serum has been used in a limited number of cases in Russia, but the reports are still too meagre to permit a proper estimate of its value. The dose recommended by Krause is from 60 to 100 c.c. intravenously in salt solution, to be repeated if necessary in twelve hours. 
Salimbeni ${ }^{1}$ reports somewhat favorable results with a serum prepared from horses strongly immunized "against the soluble cholera toxin." 'The mortality in the cases treated was 24 per cent.

\section{SERUM THERAPY OF STREPTOCOCCOUS INFECTIONS}

From what has already been said, it is apparent that the specificity of the action of immune sera sets very definite limitations to their employment. In the ease of the streptococcous infections this has given rise to considerable discussion as to the unity or plurality of streptoroeci in different infeetions. It is well established that there are marked differences in the behavior of various strains of streptococci-differences in virulence, in agglutinating characteristics, in the production of hemolysin, etc. According to the partisans of the pluralistic view, these differences are so great as to constitute really different species. 'This causes us to immunize with streptococei of different kinds. Sera thus obtained are said to be "polyvalent."

Another important question was whether a more effective serum could be produced by immunization with streptococei whose virulence had been enormously increased by frequent passage through animals. Following investigations in this direction, 'Tavel came to the conclusion that animal passage was a disadvantage, in that it caused the development of substances on the part of the streptococeus which differed from those concerned in producing the elinical effects in man. Tavel, Moser, and others, therefore, immunize with freshly isolated streptococci from various sources which have passed through animals as little as possible. 'This view, however, is not shared by all the investigators in this ficld, and so we find a number of sera produced by immunization with strains whose virulence has been increased by frequent passage through animals. In the production of Aronson's antistreptococcus serum, the animals are immunized with mixtures of strains which have not passed through animals, and strains whose virulence has thus been enormously increased.

The mechanism of the immunity to streptococcous infection is still very obscure. In fact, but little is known of the manner in which the germ effects its pathogenic action. Many of the strains produce a strong hemolysin, but, curiously, this hemolytic power does not measure the virulence of the organism. 'Thus far it has been impossible to isolate any specific toxin from streptococei; we speak of their poison as being probably an "endotoxin." By the various methods of immunization just mentioned it has been found possible to produce sera which exert definite protection in experimental streptococeous infection in animals. When these sera were studied, it became elear that they represented something more than specific baeteriolysins. 'They did, to be sure, possess some bacteriolytic power, and there was also some evidence that they contained something akin to an antitoxin. 'The discovery of the role of serum in aiding phagocytosis by means of opsonins did inuch to aid our understanding of the immunity against these infections, and we

1 Ann. de l'Inst. Pasteur, vol. xxiv, 1910, p. 24. 
now know that phagocytosis plays perhaps the most important part in the mechanism of this immunity.

Antistreptococcus serum may be used either locally, subcutaneously, or intravenously. So far as the local application is concerned, this method is indicated in infected wounds, and in conditions like septic endometritis or septic peritonitis. The serum can be applied in fowder form, but more conveniently in the form of a dressing soaked in serum, or, in the case of septic endometritis, as an injection directly into the uterine cavity. Similarly, in streptococcous peritonitis the serum can be poured or injected directly into the peritoneal cavity. In all these various local applications liberal quantities should be employed-from 25 to 250 c.c.

Between subcutaneous and intravenous injections, the latter are much to be preferred in all severe cases, such as septicæmia, broncho-pneumonia, extensive cellulitis, and erysipelas, where large injections of 50 to 100 c.c. are given. 'They are usually easily carried out by plunging the needle directly through the disinfected skin into one of the large veins at the elbow. While it is not necessary to cut down on the vein, it is generally more satisfactory to do so. Studdiford injects into the vein 50 to 75 c.c. of serum, together with 100 c.c. of warm isotonic salt solution. We have found the apparatus used to inject toxins in horses very suitable to inject these large doses. We use a wide burette, plugged at its upper opening by a perforated rubber stopper, to which is attached a hand air-pressure apparatus. The lower end is attached to a flexible rubber tube. It is well to have the tube attached to the needle when plunging the latter into the rein; if one inserts the needle alone, and waits for a drop of blood to flow, thus showing that the vein has been entered, one will often find that the blood has clotted in the needle when one comes to inject the serum. With a little practice one is usually able to tell when the needle is in the vein. Moreover, the question is quickly decided when the injection is commenced; if the needle is in the vein, the injection is followed by a sudden blanching of the vein some distance beyond the syringe, while if it is only in the subcutaneous tissue, a characteristic swelling at once ensues. 'The serum-container should be capable of holding 200 c.c. Subcutaneous injections are made where there is difficulty in carrying out the intravenous injection. The intravenous injections should be from 50 to 100 c.c. of serum. Ordinarily about 25 c.c. can be given subcutaneously at one time in one place; if more is to be injected, the injections should be distributed over several areas. 'The resulting swelling should be left to absorb spontaneously, and not massaged. There has been considerable diversity of opinion concerning the therapeutic value of antistreptococcus serum. It is difficult to arrive at a definite conclusion from a study of the cases in the literature because of the variety of sera employed and their divergent methods of production. From our own experiences we believe that there is a distinct field of uscfulness for antistreptococcus serum, but the dose should be large and the scrum used early. Our first experience was with a serum from a horse immunized with one strain only. The doses 
given were small and the results unsatisfactory. We therefore stopperl the production of serum for several years. A year ago we immunizerl three horses with al large number of strains of freshly isolated streptoxecri. Since then we have had favorable results. 'The cases in which the results have been best are those of puerperal sepsis due to streptecoceri. We have tested the serum in some thirty cases of scarlet fever. In some cases no apparent effects followed the use of the serum, but in some a striking fall in temperature followed the injection, with general improvement in the symptoms. We have used the serum in a number of cases of erysipelas. 'T'he majority received small doses of 20 c.e. subcutaneously. These cases showed no appreciable results. In several grave cases 75 to 100 c.c. were given intravenously, with apparently good effect. The temperature dropped to nearly normal, and the erysipelas ceased to extend.

\section{SERUM THERAPY OF STAPHYLOCOCCIC INFECTIONS}

Antistaphylococcic sera have been prepared which contain agglutinins and opsonins. 'The results of the use of the serum have not been encouraging.

\section{SERUM THERAPY IN PNEUMOCOCCIC INFECTIONS}

The pneumococci are really varieties of streptococci, and what has been said concerning the latter can be in a general way applied to the former. 'The Klemperers many years ago made use of the injection of filtrates to prepare antitoxic sera. The results of the use of serum in the treatment of pneumonia were practically negative. In ulcus serpens of the cornea good results have been claimed from the local application of the serum. Although since then good results in pneumonia and septicæmia have been claimed by some, they have been doubted by most. It is possible that with suitable polyvalent sera and large doses better success may be obtained.

\section{SERUM THERAPY IN GONOCOCCUS INFECTIONS}

The pathogenic effects exerted by the gonococeus are ehiefly due to endotoxins. Several observers elaim that they have obtained slightly toxic exotoxins. 'Torrey has prepared a serum which has powerful bactericidal and agglutinating properties, and also opsonins. This serum has been used to advantage in the complieations of gonorrhara, especially in gonorrhceal arthritis. 'The doses are small, 3 to 5 c.c., and repeated every day for several days. In acute gonorrhoa the serum has been without results.

\section{SERUM THERAPY IN TUBERCULOSIS}

Many attempts have been made to produce a serum which would have curative value, but without avail. A number of investigators have believed that they have produced such a serum, but when others 
have put it to the test the results have been negative. Maragliano's serum has been tried as much as any, and is still used to some extent in Italy. Marmorek and others have also produced sera, but none so far prepared appear to be of any real value.

\section{SERUM THERAPY IN BUBONIC PLAGUE}

The plague bacillus produces a powerful endotoxin and feeble extracellular toxins. Sera are prepared by immunizing horses at first with dead cultures and later with living ones. Yersin's serum is prepared at the Pasteur Institute, and this is tested for potency by estimating the amount of serum which will protect a mouse from a fatal dose of living bacilli. We have had no opportunity in New York of testing this or other sera. Those who have used it in other countries differ in their opinion as to its use, but the majority think that it does good. The amount given at first was evidently too small. At present the doses given are from 100 to 200 c.c. in adults and 20 to 100 c.c. in infants and young children, these amounts to be repeated in twelve to twentyfour hours. When given early in the disease, and in sufficient amounts, the serum probably reduces mortality by from 10 to 20 per cent.

\section{SERUM THERAPY IN ANTHRAX}

Sclavo obtained a serum from horses by immunizing them at first with vaccines attenuated by growing them at higher temperatures than their optinium. Later, they were injected with fully virulent cultures. Sobernheim and others have produced efficient sera by somewhat different methods. The serum treatment has been used but little here, and I have no personal experience with it. In England and on the continent good reports are made of the results of serum treatment in malignant pustule. The dose is 20 to 40 c.c., repeated in twenty-four hours if necessary. In the grave forms of the disease attacking the lungs and intestines no results of serum treatment have been published.

\section{SEROM THERAPY IN RABIES}

A serum has been prepared by injecting sheep and other animals with the brain tissue of rabid animals. This has considerable protective effect. This serum is sometimes used in combination with the use of vaccines in very dangerous bites, where it is feared there will not be sufficient time to immunize by the vaccines. In the great majority of cases the vaccines prepared from the spinal cord of a rabbit are alone used.

\section{THE SERUM TREATMENT FOR SNAKE POISONING}

Sewall found that pigeons could gradually be accustomed to increasing doses of venom, until as nuch as ten fatal doses could be given without producing death. 
Calmette carried the investigation further, and produced immunity in guinea-pigs and rabbits against the venom of different varietics of poison. He also proved that the serum of these animals had protective power. He later, after much difficulty, immunized horses to obtain a protective serum for man. He required as much ass six months toimmunize a horse suffieiently to obtain a potent antitoxic serum. 'The scrum is quite stable, and retains most of its efficacy for a period of two years.

Dried antivenin in sealed packages will last almost indefinitely. 'The serum is tested by giving the rabbit an intravenous iujection of 1 c.c. of serum, and then, five mimutes later, a fatal dose of venin. 'The average amount of poison injected by the severe bite of snakes is believed to be about 0.25 ; this would require from 100 c.c. to 300 c.c. of the usual strength of antivenin placed on the market to neutralize it. If the antidote is injected directly into the vein, its action will be much quicker than if given simultaneously. 'The longer the delay, the greater will be the amount required to neutralize the poison.

Calmette considers that a serum, of which $1 \mathrm{cc}$. neutralizes $1 \mathrm{mg}$. of cobra venom in vitro, is sufficiently strong for practical purposes.

Noguchi found that the action of the antivenin was almost wholly specific for the neurotoxic and hemolytic poisons of the species of snake from which the inmunizing poisons were obtained, and had little effect on the poison of other speeies. 'This difficulty is overcome by injecting the same animal with several different poisons, so as to make a polyvalent serum. The only trouble is to get the venins of the snakes of different countries. At present it is difficult to obtain a serum which is concentrated enough to be very efficacious in the more dangerous bites. This, and the diffieulty of having it at hand, restrict its usefulness.

\section{THE USE OF BLOOD-SERUM IN THE PREVENTION OF HEMORRHAGE IN CASES OF HIEMOPHILIA AND OTHER CONDITIONS}

In a number of instances it has been found that little infants, showing a tendency to bleeding, were greatly benefited by the transfer of blood from the parent or other individual. Later, it was found that subcutaneous injections of rabbit serum and horse serum produce much the same effect. I have come in touch with a number of cases in which horse serum proved to be very beneficial, while in some it had little or no effect. 'Thus, in a case in which a tooth was extracted, and it was impossible to stop the oozing of blood, the injection of 10 c.e. of horse serum caused the blood to clot and all oozing to cease. Trembier had a patient, a girl of thirteen, who was known to have been a "bleeder" since the age of five, although otherwise in good health. 'The child had been in the hospital several times on aceount of serious hemorrhages, which had yielded after tamponing and injection of gelatin. In February, 1909, there was menacing hemorrhage from the nose, gums, left ear, and throat. Epistaxis a few days later required tamponing anew. About 5 c.c. of sheep's blood-serum was then injected subcutancously and four hours afterward the bleeding was arrested. 'I'wo

VoL. I-33 
days later it recurred, but was stopped by a tampon of gauze soaked in the serum. 'The bleeding from the gums was also arrested by local serum applications. A severe hemorrhage from both ears occurred in the night and 10 c.c. were injected into each thigh. 'These injections were repeated two weeks later and again three weeks later. The child was released in good health after gaining 20 pounds.

'The amount of serum to be injected has not yet been definitely established. In little infants 10 to 20 c.c. have usually been given, while in older children and adults 20 to 30 c.c. These injections are usually repeated daily for three to four days, and then stopped unless another hemorrhage occurs. The serum does not need to be fresh, as it probably depends on stable constituents, and not upon any of the ferments. I have used serum kept for six months with good results. Some prefer rabbit or sheep to horse serum. I have not noticed any special difference between them. Beef serum should not be used. The serum may be employed not only to check existing hemorrhage, but to prevent hemorrhage where operations are to be performed. 'The effect of the injection lasts for several weeks. Where normal serum cannot be obtained, diphtheria antitoxin serum can be substituted. This should be serum and not the globulin solution. 


\title{
OPSONINS AND VACCINE THERAPY
}

\author{
BY NATHANIEL BOWDITCH POTTER, M.D.
} AND OSWALD T. AVERY, M.D.

\section{OPSONINS AND THE OPSONIC INDEX}

Tre phenomena accompanying the bacterial invasion and infection of the animal body have occupied and perplexed the mind of man since the dawn of medical history. Even in antiquity it was observed that certain species of animals were known to be invulnerable to certain diseases; that, although a number of individuals might be equally exposed to a common epidemic disease, the majority escaped infection, and, of those contracting the illness, many came to spontaneous recovery; and, further, that a recovery from diphtheria, small-pox, and typhoid fever, for example, usually protected an individual against a second attack of the same disease.

The mechanism by which the body resisted, overcame, or survived infection, although submitted to diligent investigation, received no reasonable explanation until the close of the nineteenth century. 'The first suggestive light came with the discovery of bacteria by Pasteur. From that time on there continued a rapid expansion of our knowledge concerning the nature of bacteria, their distribution, their disease-producing ability, and pathological sequels. It was not until the first years of the new century, however, that medical science was enable to define with any degree of certainty the biological forces at work in somatic antagonism to bacterial invasion.

Developing step by step, and owing their growth to the evidence furnished piecemeal by a multitude of detailed researches in varied realms of biological science, there were formulated two distinct theories of bacterial immunity. Antithetical as they are in their basic conceptions, they, however, elucidate, in a way hitherto unapproached, the nature of the vital processes that underlie the body's system of defense against microbic infection. The humoral theory, elaborated to its present form by Ehrlich, seeks to resolve the resisting powers of the body into a series of chemical reactions, in which an array of substances existing in the body tissues-particularly the blood serum-participate. Metchnikoff, on the other hand, sees in the phagocytic power of the leucocytes the body's chief defense against invading bacteria. 'This conception has been termed the cellular theory of immunity. The positive terms of these two widely divergent hypotheses for a long time seemed to preclude the possibility of a conciliation. It remained for Wright to show how the 
terms of the one complement the terms of the other. The theory of opsonins, therefore, serves to fulfil the office of mediator, and, in the scheme of immunity, occupies a place midway between the humoral theory of Ehrlich and the cellular theory of Metchnikoff.

History of Opsonins.-Metchnikoff, by discovering the power of the white blood-corpuscles to derour pathogenic bacteria, first established the importance of phagocytosis in immunity. The phenomena, both of susceptibility to infection and resistance against disease, were all interpreted in terms of phagocytic activity of the leucocytes. 'This doctrine of phagocytic immunity is still ardently supported by the French school, which, although recognizing the significance of certain protective substances in the blood, believes that they serve to "stimulate" the leucocyte in its function of bacterial destruction.

In 1895 Denys and Leclef first called attention to the role played by blood-serum in phagocytosis. By demonstrating the fact that leucocytes exhibited phagocytic activity in vitro as well as in vivo, they opened a way to the solution of many important problems in the field of immunity. They definitely proved that, in the process of immunizing an animal against a specific micro-organism, there is no marked change in the behavior of the leucocytes per se. Contrary to the view of Metchnikoff, these show themselves to be less active as phagocytes than do the leucocytes of normal serum. One of the most far-reaching of their observations was the discovery that, in the blood of animals thus immunized there exist substances which are capable of altering microbes in such a way as to permit of their being ingested by the leucocytes. In other words, the immunity acquired is referable to a developed function of the serum, and not to any change in the leucocytes, and, in subsequent investigations, is was further developed that the action of the serum consisted, not in a "stimulation" of the leucocytes, but in an acutal combination with the bacteria, which thus prepared them for subsequent phagocytosis.

Somewhat later Leishman, in an endeavor to determine the degree of immunity acquired in the course of staphylococcus infectionsMalta fever, cholera, anthrax, and plague-found that the measure of the agglutinating or of the bactericidal power of the serum, so marked in typhoid fever, was here of no value. He, thercfore, sought to determine whether the estimation of the "stimulin" content of the serum would prove a reliable criterion for measuring the amount of resistance developed. 'To this end he devised a method, simple in detail, yet one which in a large measure served to lay the foundations of the opsonic theory. This method consisted in mixing equal amounts of a suspension of bacteria and the blood-serum of the individual whose resistance was to be measured. The use of capillary pipettes permitted the employment of minimal amounts of blood. This mixture was placed on a glass slide, covered with a cover-glass, and incubated for a quarter of an hour. Stained preparations were then made, the intracellular bacteria were counted, and the average number contained in each leucocyte determined. With the aid of this method, Leishman was able to demonstrate a paral- 
lelism between the intensity of the phagocytosis and the resisting power of the body; but the true theoretical significance of this demonstration escaped him, inasmuch as he considered his results as affording confiumation to the "stimulin theory" of Metchnikoff.

Besides repeating and confirming I.eishman's work, Wright and Douglass so modified the former's method as to permit the separation of the cells from the fluid part of the blood, and thus, through suitable combinations of bacteria, cells, and plasma, to enable one to inquire into the significance of each separate component. 'Their modification consisted in the introduction of a means for preventing the coagulation of the blood, which thus facilitated the isolation of the corpuscles from the serum. 'This separation was further effected by repeated washings and centrifugalization. To these washed corpuscles were added the serum of the patient and the bacterial suspension. These three components were thoroughly mixed in a capillary tube and incubated a quarter of an hour at $37^{\circ} \mathrm{C}$. Smears of this mixture were then prepared and stained, and the number of phagocyted bacteria enumerated. This count-that is, the average number of phagocyted bacteria per leucocyte-was termed the "phagocytic count," while the ratio of the phagocytic count, obtained with the patient's serum to a similar count with the serum of a normal individual, was designated as the "opsonic index." This method, altered and refined in its details from time to time to meet the developed theoretical requirements, made possible the employment of human blood. Since it was observed that the phagocytic activity of the blood of different individuals varied in intensity, the following theoretical considerations presented themselves: Does the phagocytosis take place independently of the fluid part of the blood, or with the aid of it, and does this action concern particularly the bacteria or the leucocytes? With the view of bringing a solution to these questions, and with the added purpose of developing a method for accurately measuring the degree of resistance exhibited by an individual during or following infection or vaccination, Wright carried out a series of studies, the results of which, among other things, enabled him to make the following dogmatic statements, which constitute the more important fundamental contributions to the theory of opsonins:

(1) In the fluid part of the blood-and this holds true for the serum as well as for the plasma-there is present a substance upon which the beginning of phagocytosis is to a large degree dependent.

(2) This substance acts upon the bacteria; the bacteria combine with it and thereby become phagocytable.

(3) This substance is not identical with any other known immune bodlies. It was shown that opsonins are present in sera which contain no bactericidal substances; further, when serum is heated to $60^{\circ} \mathrm{F}$. for ten to fifteen minutes, its opsonic power is destroyed or greatly diminished.

(4) After the opsonins have become bound to the bacteria, they (the opsonins) are no longer destroyed by heat.

Among all the data developed in regard to the nature and function of opsonins, there are no facts of greater significance than the early ob- 
servations of Leishman and of Wright in cases of staphylococcus infections in the human subject. These two observations-and they are of first importance-reveal that:

I. In men suffering from staphylomycosis the phagocytosis of staphylococci is abnormally small, and-

II. 'That in the course of artificial immunization with killed bacterial cultures, according to the vaccine method of Wright, this phagocytic activity increases.

These constitute the very foundations of opsonic theory and vaccine therapy.

From the nature of these substances, contained in serum, that possess the property of rendering bacteria ingestible by leucocytes, Wright designated them by the name "opsonins," from opsono or obsono, "I prepare for the meal " or "I prepare food for."

The Nature of Opsonins.- The foregoing account briefly reviews the principal considerations which have aided in establishing the theory of opsonins. In order to gain a clearer conception of the practical significance of opsonins, as well as to understand their relation to the other immunity-producing substances of the blood, it is necessary to inquire into the nature of their specific identities. Upon finding that the opsonic strength of any serum was independent of either the bactericidal or the agglutinating strength, Wright and his co-workers inclined to the view that opsonins were to be looked upon as hitherto unknown substances. Stimulated by the early investigations of the Wright school, there has arisen a lively and wide-spread interest, not only in the scope of the practical applications of this method for determining the degree of natural or acquired immunity, but also regarding the nature and constitution of this new class of immune bodies. Sauerbeck, in discussing the identity of opsonins, classifies the principal considerations in the form of the following questions:

(1) Are opsonins to be demonstrated as the cause of phagocytosis? The plasma as well as the serum of normal and, to a high degree, of immune blood, has the ability to render bacteria phagocytable. This ability depends upon a change in the bacteria: bacteria which have once come in contact with the plasma or serum, even after the removal of the plasma or serum, are quite as phagocytable as in their presence. It is impossible to demonstrate any direct action of the plasma or serum upon the leucocytes. Without the coöperation of the immune serum the leucocytes of an immune individual are no more actively phagocytic than those of a normal individual. The relation of bacterial virulence to opsonic activity and the accompanying phagocytosis is a factor which would seem to detract from the importance of opsonins as the sole causative agent in inducing phagocytosis. Hektoen, Rosenow, Löhlein, and others found that virulent streptococci and pneumococci, after the addition of normal serum, were eagerly devoured, while virulent strains, under similar conditions, resisted the engulfing action of the leucocytes. Rosenow found, further, that, by artificially depressing or exalting the virulence of an organism, he was able respectively to increase or decrease its 
phagocytability. If as these experiments would seem to show, opsonins are important to render virulent bacteria vulnerable to phagocytes, then their role as defenders of the human organism against disease would seem to be an insignificant one. However, the pancity of data and the contradictory nature of the reported experiments leave this a question demanding further investigation. A further specificity of opsonic action in phatgocytosis has been read into the phenomenon of spontaneous phagocytoisis exhilited in the case of anthrax bacilli, cholera vibrios, and certain other organisms. 'This leucocytic ingestion of bacteria without preliminary opsonic intervention has also been interpreted as arguing for the leucocytic origin of opsonins. In diseussing the processes in the phagocytic destruction of micro-organisms, Löhlein points out that the ultimate ingestion of any given species of bacteria is dependent, not only upon the presence of opsonizing substances, but also to a greater or less extent upon the nature of the organism ingested. With respect to this peculiarity all bacteria might be differentiated into:

Bacteria which are phagoeytable independent of any action of the serum.

Bacteria which, under the influence of serum, become more easily phagocytable.

Bacteria which, even though acted upon by serum, do not become phagocytable.

Such a classification would provide for variations provoked by virulence and for the appearance of spontaneous phagocytosis.

A further proof of the speeificity of the part played by opsonins in phagocytosis is to be found in the absorption experiments of Wright, Bullock, and Atkin, and others. It was their experience that the addition of bacteria to serum diminished the opsonic power of the serum for the particular bacterial species employed, and that a more or less complete exhaustion of the opsonin can thus be effected. This observation offers additional evidence that opsonins, in effecting the destruction of bacteria through the action of leucocytes, exert their action upon the bacteria, and do not, as the Metchnikoff school holds, stimulate the leucocyte to phagocytic activity.

(2) Is there one single opsonin or are there many and specific opsonins? 'The opsonins of normal serum, as well as those of immune sera, would seem to follow the rule of specificity already established for other immune bodies, such as agglutinins, lysins, precipitins, etc. 'Thus, in immunizing an individual against a given organism by means of injections of a bacterial vaceine, the bodily resistance is increased only against the organism employed, while the opsonic content of the serum for other species remains unchanged.

(3) Do opsonins occur in increased amounts in immune sera? Relying upon the opsonic index as an indicator, Wright finds an inerense in the serum content of opsonins in the human subject after artifieial active immunization. 'The curve of increase does not follow the curve for agglutinins, preeipitins, or bacteriolysins throughout the course of a like period of immunization. The final opsonin titre may represent an 
increase of 100 , or perhaps as high as 400 , per cent., while in the case of agglutinins, etc., a multiplication in potency of a 1000 or even 50,000 fold or more is possible. This anomalous course in opsonin production might be construed as supporting a negative answer to the question-

(4) Are the opsonins of normal and immune sera identical? At the present time no definite answer is possible. Normal opsonins are thermolabile (destroyed by ten minutes' heating at $60^{\circ} \mathrm{C}$.); the immune opsonins appear to be thermostable. Whether this is due to their greater concentration in the serum is disputed. If a difference can be demonstrated, then support would be lent to the theoretical unity of immune opsonins and "bacteriotropins," as claimed by Neufeld and others.

(5) Are opsonins hitherto unknown substances, or is opsonic action to be considered as a hitherto unknown action of already known substances? Wright early proved that the opsonic activity was entirely independent of the bactericidal power of a serum. It is now generally accepted that a difference in kind exists between opsonins and the better known agglutinins and bacteriolysins. 'The exact identity of opsonins, according to the system of Ehrlich, is still a mooted question. While the opsonin of normal serum resembles complement in many of its manifestations, the opsonins of immune sera, on the other hand, bear marked resemblances to amboceptors, or immune receptors of the second order. Dissimilarities of equal importance are also exhibited. The many manifest contradictions leave one in doubt in denoting the exact immunological identities of this class of substances.

(6) Do opsonins possess significance as protective substances? Are virulent bacteria strongly subject to the opsonic action of serum, while virulent bacteria, on the other hand, are only weakly or not at all affected. A brief recapitulation of considerations, already referred to under Question 1, is that the resistance of any micro-organism to the action of opsonins varies in proportion to the degree of virulence possessed by that organism.

From the standpoint of the dependence of bodily welfare upon the protective action of opsonins, the question of greatest importance may, perhaps, be expressed thus:

(7) Does the phagocytosis, which is the result of opsonic activity, lead to a destruction of the bacteria? Unfortunately, this question has not received the attention devoted to other phases of opsonic phenomena. At present the most that can be said is that opsonized bacteria are destroyed in the ensuing phagocytosis, the extent or degree of their destruction depending somewhat upon the nature of the bacteria ingested, and, among other conditions, upon the proportion of organisms to leucocytes.

Method for Determining the Opsonic Index.-The method for determining the phagocytic power and eventually the opsonic index of any serum is as follows: 
The following essentials are required: 1 (1) Pipettes: (2) washed blowd-corpuscles (3) bacterial emulsion; (4) serum to be estimatcd; (5) normal serum or posled sera for control.

(1) Pipettes.-These are glass capillary tubes, one end of which is of sufticiently large diameter to fit into a rubler teat. All tubes should be of approxinutely the same calibre, slightly tapering toward the point, and stiff cnough to prevent vil, ration. For use the ends are cut square, and marked with a paraftin pencil about ? inch from the extremity.

(2) Washed Corpuscles.-Two small balanced centrifuge tubes are used. 'The'y are cleansed with 25 per cent. sulphuric acid, washed with water, and nixed with 1.5 per cent. sodium citrate solution. Fill one of these tubes two-thirds to threequarters full with 1.5 per cent. citrate solution, and then run in blood to the full from a puncture in the finger. Mix hlood and citrate by inverting the tube several times-not by sliaking; then centrifugate for tle minimum period of time compatible with the settling of the corpuscles. Pipette off the plasma with a special fine-pointed bulbed pipette of fine calibre, add physiological salt solution, mix, and centrifugate, then repeat this washing process a second time. The tube is rolled between the hands to effect a mixture, and the corpuscles are then ready for use.

(3) Bacterial Emulsions.-With the exception of tubercle bacilli, the emulsions are mude from fresh live cultures. The age for coliform organisms may be from four to ten hours, the younger the better, as it is difficult to disintegrate the masses or clumps in older cultures. Cultures of Gram-positive organisms may be as old as twenty-four hours. All growths are made on agar slants. The diluent used is 1.5 per cent. sodium chloride solution for tubercle bacilli, M. neoformans, and the nongramming coeci. For all other organisms, 0.85 per cent. sodium chloride is used. A small loopful of the agar culture is placed in a small volume of the diluent in a watch-glass, or small staining dish, and the mixture is emulsified by being alternately sucked into and forced out of a stout bulbed pipette (fitted with a rubler bulb or teat) witi a square end, which is firmly held at a right angle to the bottom of the mixing dish. Emulsions of cocci should be slightly opalescent to the eyc, bacillary enulsions slightly thicker. They should be used when fresh, and a "trial trip" made in order to determine the thickness of the emulsion.

Tubercle Emulsion.-A portion of dried or moist bacilli from the manufacture of Koch's old tuberculin, or washed sterilized bacilli from a fresh culture, is rubbed up in an agate mortar, first alone to a dry powder, and then 1.5 per cent. sodium chloride solution is added drop by drop. In this way a paste, and subsequently a comparatively thick emulsion, is made. The excellence of the final emulsion largely depends upon the smoothness of the paste and thick cmulsion at this stage. The latter is sealed in a tube and well shaken. On inverting the tube, the clumps settle into the drawn-off end, and may be removed en masse by cutting off that portion of the tube. For use a small portion of the resultant cmulsion is centrifugated unt il the upper layers are slightly opalescent only. These layers are pipetted off and thoroughly mixed. The enulsion should then be free from clumps. A "trial trip" should show whether further dilution is necessary.

Streptococci may be occasionally rubbed up in a mortar with 0.85 per cent. sodium chloride and subsequently centrifugated. As a rule, however, vigorous pipetting into a watcli-glass, rith subsequent centrifugation for a few minutes, is enough to remove all chains and leave a satisfactory emulsion.

The "phagocytic count" for tubercle bacilli should be between 1.5 and 2 per cell-for other organisms not less than 3 per cell.

(4) Serum for Examination.-This is usually collected and contained in a glass capsule. The blood after clotting is centrifugated.

(5) Control Sera.-The index for tubercle bacilli is worked out against the average phagocytic counts or two or more normal sera, most other indices being calculated from the count of a pooled serum of four or more normal persons.

We now have the three necessary factors for estimating the opsonic index, viz., washed corpuscles, bacterial emulsion, and serum. A volume each of these, in the order given above, is taken into a pipette and thoroughly mixed on a clean slide, eare being taken that no bubbles get in. The mixture is taken into a pipette, followed by an air space, the end sealed off, and the pipette placed in an opsonizer. Ccliform organisms and non-gramming cocci (particularly gonocecei) should not be incubated longer than seven to ten minutes. Tubercle bacilli and other organisms require fifteen minutes, more or less, according to the strength of the enulsion. "The

1 This detailed description of the method is practically identical with the directions given by Sir Almroth Wright to his pupils at St. Mary's Hospital, london. 
time should be noted exactly. At the end of the incubation the contents of the pipette are blown on to a slide, previously roughened with fine emery paper on its convex side. A film is made by means of a broken slide having a concave edge. The curve, the sharpness of fracture, and cleanliness of this "spreader" are important. The spreader should be held lightly, and a thin smear with a sharply defined final edge made. In this straight edge are found most of the leucocytes.

The films are fixed for one to three minutes in a saturated solution of mercuric chloride. Tubercle films are then stained with carbol fuchsin $(1: 5)$, heated to steam, washed, decolorized in 2.5 per cent. sulphuric acid, covered for a short time (thirty to sixty seconds) with 4 per cent. acetic acid, then washed and counterstained with Loeffler's methylene-blue. Care should be taken with the carbol fuchsin, as the carbolic acid tends to rupture or make vacuoles in the leucocytes. Most other films are stained best with carbol thionin $(0.25$ per cent. thionin in 1 per cent. carbolic acid) in the cold.

A minimum of 50 polymorphonuclear lcucocytes are examined, and their microtic contents enumerated. If 50 cells be counted, the total number of bacteria multiplied by 2 and divided by 100 , the result will represent the average phagocytic content or "phagocytic count." Normal sera or pools require the enumeration of at least 100 leucocytes, and the phagocytic index of the pool, or the averages of the phagocytic indices of a series of normals, divided into the phagocytic index of any serum, gives the "opsonic index" of that serum. Normal sera should not differ from one another in a tubercle determination by more than 10 per cent.

When testing bloods for opsonic index which contain specific agglutinins or lysins, heat the bacteria in the emulsions to $72^{\circ} \mathrm{F}$. or over. This destroys their agglutinability and lysability, while power to combine with opsonins is only destroyed at $115^{\circ} \mathrm{C}$.

Suggested Improvements in the Method.-Among the more important changes suggested may be mentioned those proposed by Simon. Instead of counting the average number of bacteria phagocyted, this author would determine the number of leucocytes participating in the phagocytosis, and he further would determine the influence exerted by diluted as well as by concentrated serum. This method-termed the method of the "procental index"-has received the approval of various subsequent investigators. The estimation of the index with varying serum dilutions has also been advocated by Dean, who recommends that the point in such dilutions at which a phagocytic action equal to the action of the normal serum takes place be taken as the true opsonic titre. This suggested method resembles the procedure adopted by Neufeld and Huhne in their "bacteriotropin" investigations. Others suggest a simplification of the Wright technique, by omitting entirely the separation of leucocytes from their respective sera. They advise adding the bacterial emulsion directly to the blood to be examined, using a similar mixture of emulsion and normal blood as control. These advocated changes, besides facilitating or rendering more accurate the determination of the opsonic index, may also serve to point the way to a more feasible method for gauging the degree of bodily resistance to microtic infection.

Significance and Practical Value of the Opsonic Index.-Ever since the discovery of opsonins, it has been Wright's assertion that the estimation of the opsonic index was to be looked upon as the chief guide in the diagnosis, prognosis, and specific therapy of infectious diseases. Briefly stated, the claimed practical application of the opsonic index is as follows: If the index to any given micro-organism be and continues normal, then an infection with that particular micro-organism can be excluded; if, on the other hand, it is persistently low, than a local 
infection by this micro-organism is indicated; if it fluctuates, then there is the likelihood of an infection in which the whole bodily organism is involved; if it continues high, then the infection is being suecessfully resisted, or an active immunization is being accomplished; if thronghonit the process of artificial immunization an enduring low index is exhilited an unfavorable prognosis is manifest.

'Thus it will be seen that this method, if wholly reliable, would afford invaluable aid in combating bacterial infections. 'There are several factors, however, which would seem to militate against its value, and, therefore, against its unqualified acceptance at the worth ascribed to it by Wright. 'The limit of error involved in the technical exceution of index estimations now appears to be sufficiently great to make specific fluctuations due to the progress of the infective processes, and to cause confusion in the interpretation of results. A second factor consists in causes which may markedly affect the index, and which are attributable to conditions other than those associated with the infection. It may lee further argued, that the phagocytic phenomena displayed in test-tube experiments are by no means to be taken as indicating the possible phenomena occurring in the human body. In addition to the above factors, the observation that virulence in any given bacterial species, while being an essential to infection and resulting disease, at the same time inhibits phagocytosis - at least in in vitro experiments - offers another stumblingblock to the ready subscription to the claims of the Wright school.

The carrying out of opsonin estimations, according to the Wright method, requires long and constant practice, and demands a high degree of technical skill. 'This at once limits the scope of its availability, since the services of men trained in its proper execution are not always at hand. Under optimum conditions, and in the hands of those skilled in its technique, the method yields results which are not always to be considered as entirely trustworthy. Simon, Potter, Park and Biggs, Bolduan, Thomas, Saathoff, Weinstein, Armit, Ball, and Browne, Reyn and Kjer-Petersen, Kinghorn, 'Twitchell and Carter, and Glynn-and these names include those who learned the technique at first hand in Wright's laboratory-either failed to attain the accuracy alleged by Wright, or were unable to corroborate the claimed parallelism between index variations and clinical manifestations. A more or less general abandonment has thus come about.

It is not to be imagined, however, that the last word has been said on the subject of the true value of the opsonic index as a measure of bodily resistance to pathogenic bacteria or of bodily response to artificial immunization. What has gone before suggests rather than details the arguments for and against the utility of this method, without any implication or inference as to finality. Whatever may be the views held to-day concerning the nature or identity of opsonins or their role in immunological processes, or whatever may be the future application or development of the present method for measuring opsonic indices, to Wright, as the discoverer of opsonins and inventor of this mique method, must be granted a full meed of credit and distinction for the service rendercy 
humanity in establishing the use of bacterial vaccines in the treatment of bacterial infections.

\section{VACCINES AND VACCINE THERAPY}

Immunity against bacterial disease may be naturally acquired or artificially induced. Recovery from certain infectious diseases insures a more or less effective protection against reinfection - as a result of an immunity naturally conferred by an original attack. The infecting agent in disease, by virtue of the very properties which cause it to be infectious, $i$. $e$., by the poison it secretes, or through its other metabolic products, stimulates the tissues of the infected host to a neutralizing or protecting reaction. In this reaction not only are the bacterial cells and their toxins rendered innocuous, but at the same time there is called forth an excessive production of neutralizing substances. These substances, the so-called immune bodies or antibodies, by combining with the toxin, by dissolving or killing the invading bacteria, or by rendering them more susceptible to phagocytosis, are respectively known as antitoxins, bacteriolysins, bacteriocidins, opsonins, etc. It is the persistence of these substances in the blood-serum or other tissue fluids, long after recovery from the initial attack, that renders the animal body resistant to or immune from recurring infection. This principle is exemplified in the well-known immunity resulting from an attack of typhoid or scarlet fever, small-pox, and certain other infections. Such a state of increased resistance to reinfection constitutes one form of active immunity.

In combating bacterial infections, with a view toward aborting or limiting their course or preventing exacerbations, the attempt is made to utilize or reinforce this same protective mechanism. To artificially induce this state of increased resistance or active immunity is the purpose and basis of vaccine therapy. Active immunity may be thus established by the inoculation of a specific virus which has been modified by biological, physical, or chemical methods. Any virus, regardless of its derivation, which is thus used to induce immunity is properly spoken of as a vaccine. Originally employed in connection with the immunizing material from cow-pox, the word vaccine-being derived from vacca, meaning cowwould, therefore, strictly speaking, be etymologically incorrect when applied to a suspension of dead bacteria. However, on account of the use of the French verb "vaccine" by Pasteur to denote the inoculation of any attenuated virus, and its establishment by custom, the derived noun "vaccine" may be considered as the most satisfactory term for designating all immunizing viruses, including bacterial emulsions. Such newly coined names as "bacterins," etc., have nothing beyond their novelty or trade value to recommend their adoption.

Acquired immunity of the active type may be brought about by the use of various agents. Living vaccines, $i$. e., live bacterial cultures of altered virulence, or vaccines composed of dead bacteria, or bouillon filtrates of cultures, in which the toxins have been freed from the bacterial bodies, may be used. 
The virulence of living eultures employed as vaceines may be attenuated or altered in various ways. Jenner, by passing the virus of smallpox through a less susceptible animal-the calf-successfully induced active immunity against that disease in the human subject, by incrolating the latter with lymph from the infected animal. Pasteur, by desircation of the specific virus, established protection against rabies by injecting the virus thus diminished in virulence. By growing the organism of anthrax at a temperature above the optimum, and thus greatly modifying its pathogenicity, Pasteur produced his anthrax vaceines. Castellani, in preparing an antityphoid vaccine, uses attenuated living bacilli, the virulence of which is still further artificially depressed by heat. Attempts have also been made to induce active immunity by the use of minute doses of living bacteria of normal virulenee. Such a method has been employed successfully in anthrax and symptomatic anthrax. Recently Webb, Williams, and Barber have used this principle clinically, and report favorably on the production of a certain degree of immunity in tuberculosis by the inoculation of increasing numbers of bacteria, heginning with one living tubercle bacillus. Although interesting, this method is obviously not without danger.

The vaccines of so-called opsonic therapy consist of suspensions of dead bacteria in known dilutions. This method of immunity production, elaborated by Wright and his pupils, renders available certain prophylactic and curative agents in the treatment of many diseases not amenable to other forms of immune therapy. This use of dead cultures of bacteria permits of greater accuracy in the standardization and administration of dosage than is possible in the case of a living virus, and by its very nature precludes the possibility of inciting, per se, a severe general infection as the result of inoculation. The mode of action, methods of preparations, and principles of administration of these vaccines will be discussed later.

It is evident, therefore, that the elaboration of immunes or antibodies may be stimulated in response to an attack of tisease by the inoculation of an attenuated or otherwise modified living virus, or by the injection of dead bacteria, their toxins, or other soluble products. Any of these methods may produce an active immunity, which implies that the individual's own mechanism of immunization has been actively engaged in establishing this state of more or less complete protection. In certain diseases it is also possible to create a passive inmunity in an individual by the injection of the serum of an actively immunized animal. This type of transferred immunity is familiar in the use of antitoxin and other specific sera. 'The distinction between vaccine therapy, on the one hand, and the treatment of disease by immune sera on the other, is based upon the difference, not only in the chemical nature of the substance used, but also in the kind and nature of the immunity produced. Yaccines represent antigenic substances in which the organisms, devitalized by heat, have suffered changes in their toxic and infective properties, without impairing their power to stimulate the production of protective or immune bodies. They eroke active immunity, hence their suceess 
presupposes that the patient is not already overwhelmed with the poison of his own infection, but possesses the ability to respond to the added stimulus. On the other. hand, immune sera, of which diphtheria and tetanus antitoxin are two well-known examples, represent protective substances already available in the serum of an animal previously subjected to active immunization. They confer passive immunity, which, although immediate, is brief in duration, never so potent as active immunity, and in many diseases still impossible. Active immunity is slower in production but more permanent. The former occurs as the immediate result of the injection of a curative serum elaborated in the body of another animal, the latter as the mediate, accumulating result of the patient's own bodily response to graduated doses of a vaccine or other antigenic substance, given at definitely spaced intervals.

In order to intelligently interpret the phenomena of immunity, and to define their relation to vaccine therapy, it is necessary not only to understand the physiology of the immunizing mechanism, but also to appreciate the conditions under which bacteria invade, multiply, and produce disease in the human body. The physiological response of the body's protective power receives its initial stimulus from the very bacteria against which it is directed. Upon the nature and extent of this bacterial invasion, as well as upon the degree of bodily resistance, depends the success or failure of nature's attempt to cope with infection. The interrelation of these two factors also determines the advisability of artificially supplementing or stimulating this defensive endeavor by the use of bacterial vaccines.

A basic principle in the economy of natural defense is the primary attempt, on the part of the organism, to localize bacterial infection. An analysis of the biological forces involved in the mechanism of vital resistance and defense may, perhaps, be best approached by stating the problem in the form of the following questions: What is the significance of the localization of bacterial infection? Under what circumstances do bacteria or their toxins escape from these localized foci into the general circulation, and how does the body respond to the liberation of this toxic material into the blood and lymph stream? In the answer to these questions lies the rationale of vaccine therapy:

(1) What is the significance of the localization of bacterial infection? Adami, emphasizing the importance of tissue immunity as a fundamental principle in vaccine therapy, says, "when bacteria gain entrance to the system they find but a limited opportunity for growth; nay, indeed, they are destroyed in, if not by, the majority of the tissues." He predicates this fact with the corollary that "there is a natural or indifferent or nonspecific immunity toward most bacteria exhibited in most of the tissues of the organism." Limited by the nature of the tissue invaded, and overcome by the excessive number or exalted virulence of the bacteria gaining entrance, this natural immunity may give way, and the seat of inoculation become a focus of bacterial infection. These localized foci, by their existence, evidence the lack of bacterial resistance in that particular tissue. Wright has called such foci "areas of lowered bacteriotropic 
pressure." This local diminution, or even absence, of antibacterial substances has been repeatedly demonstrated in inflammatory cxudates and serous eflusions. 'The deficiency of defense against microbie activity in any localized area of infection is, in general, dependent upon conditions which hinder the free access of fresh lymph and serum into the focus of disease, or else render inert such bacteriotropie substances as may al ready be present. 'The walling off of an infected focus by a zone of inflammatory infiltration, or by the process of encapsulation, as in abscess formation; the dense growth of exuberant granulations, clotted lymph, and fibrin which ensheath the chronic sinus, these are conditions which offer a more or less effectual barrier to the free coursing of lymph and serum through the infected focus. Such antibacterial elements as may reach the seat of infection are either inadequate in amount or are absorbed by contact with the bacteria. In suppurative foci, with accumulation of pus, the disintegration of the leucocytes liberates a tryptic ferment, which inhibits the phagocytic power of white blood-corpuscles, chemically antagonizes the protective substances, and exerts a digestive action upon adjacent tissues.

It is evident, therefore, that the processes of natural defense, which seek to limit the spread of infecting bacteria, by their very nature create conditions which favor the chronicity of the lesion. 'The graver dangers of a generalized septicemia are averted at the expense of the initial focus. If is obvious that the mere inoculation of bacterial vaccines in such cases, without regard to local conditions, must be futile. The processes of immunization must be supplemented by measures which will activate the local lesion with the full bacteriotropic power of the blood. 'The deficiency of local tissue immunity must be supplied by aid from without. Effort should be made to establish free circulation of blood and lymph through the focus of infection, in order that these fluids, rich in the newly formed antibodies, may be brought into direct contact with the infecting organisms. Among the measures which may be utilized to accomplish this end are active hyperemia, by the application of heat, poultices, etc., passive congestion, by the use of Bier's method, and the release of tension and liberation of pus from the focus. These measures serve to remove a stagnant inactivated fluid, rich in tryptic ferment and poor in protective substances, and, at the same time, favor the free ingress of fresh lymph of higher potency. In order to facilitate the transudation of lymph through the fibrin-coated, infiltrated and granulating walls of a sinus or abscess cavity, or in brawny swelling of the subcutaneous tissues, Wright advocates a dressing, wet with a solution of 0.5 per cent. sodium citrate and 4 per cent. sodium chloride. 'The citrate of soda, by its decalcifying action, prevents the coagulation of lymph and subsequent bloeking of the lymph-channels; the salt, by osmosis, promotes the transudation of serum from the blood-vessels. This solution insures the constant and copious bathing of these tissues with fluid fresh from the hemolymph system-the carrier of the body's immune forces.

(2) Under what circumstances do bacteria or their toxins escape from 
these localized foci into the general circulation, and how does the body respond to the liberation of this toxic material into the blood and lymph stream? The passing of bacteria or bacterial products from any localized area of infection into the systemic circulation constitutes autoinoculation. Such diffusion of toxins and other bacterial products may occur as the result of natural processes or may be artificially brought about. Spontaneous autoinoculation results from the direct and unobstructed escape of these bacterial substances from a localized area of infection into the adjacent blood and lymph-vessels, and is favored by conditions which increase the permeability of the encapsulating membrane and effect changes in the vascularity of the surrounding tissue. Spontaneous autoinoculation is the natural and dominant feature of all septicemic diseases, as ulcerative endocarditis, typhoid fever, pneumonia, and some types of pulmonary tuberculosis. Artificial autoinoculation may be induced in cases where the focus of infection is accessible to direct or indirect local irritation, by massage, exercise, and increased hyperemia of the affected part. Massage of a tuberculous gland, active or passive motion of a gonorrhœal or tuberculous joint, excessive use of the voice in tuberculous laryngitis, or even deep breathing and percussion of the chest incident to the examination of pulmonary cases, may result in the diffusion of toxic and infective substances, with consequent constitutional reaction. The liberation of this toxic material calls forth both symptoms of intoxication and evidence of immunizing response. The toxic condition is manifest in the febrile reaction and the aggravation of symptoms which follow; the immune response is exhibited in the measured increase in opsonic and other antibacterial substances, which are evoked by stimulation of the processes of immunization. The correlation between the amount of intoxication produced and the resulting degree of immunity attained by this method, however, is not proportionate. The patient may suffer a maximum of constitutional reaction for a minimum of immune response. This sacrifice is made at the expense of the patient's own resisting power, and is dependent, not only upon the excessive amount of free circulating toxin, but also upon the overtaxing of those body tissues concerned in and responsible for the elaboration of antibacterial agents.

Every focus of infection is potentially a source of autoinoculation. A comparatively trivial local infection may give rise to profound systemic disturbance. 'The constant effusion of bacterial elements from the seat of infection into the general circulation, as in septicemia, is marked by symptoms of intoxication, continuous temperature, and the more acute and septic course of the disease. Autoinoculation is conspicuously absent in those cases where the natural defense of the body has effectively localized the infected area by process of encapsulation, or where the microbic focus is confined in a dense, indurated, and poorly vascular tissue, which, by virtue of these conditions, prevents the diffusion of bacteria. In this type of infection the absence of autoinoculation is characterized by the lack of febrile reaction and by the chronicity of the lesion. Between the extremes of these two types of cases, in which auto- 
inoculation is continuous, on the one hand, and absent, on the other, there is an intermediary class, in which this phenomenon oceurs intermittently. Here an occasional irregular temperature reaction serves to mark the periodic release of bacteria or their products from the local focus.

Autoinoculation is nature's method of bringing about spontaneous recovery from bacterial disease. 'This intermittent introduction of varying amounts of bacterial substance (antigen) into the hemolymph system stimulates the physiological mechanism of the body to the production of immunizing substances. The effects produced by antoinoculation are comparable to the similar phenomena evoked by bacterial vaccines. While the ultimate results achieved by either method are analogous, the vaccinating substances differ in each case. Autoinoculation consists in the release of living baeteria or their products in unmeasured dose, directly into the blood-stream, while vaccination consists in the injection of dead bacteria, in measured dose, into the subcutaneous tissues. Autoinoculation is uncontrollable, and is not void of the danger of exciting an active and disseminated infection. Vaccination, on the other hand, by the use of dead bacteria in known quantity, provides a controllable method for artificially producing those conditions which nature provides for the combating of microbic disease.

Preparation of Vaccines.-A necessary preliminary to the use of bacterial vaccines is the establishment of an accurate and complete diagnosis of the micro-organism, or micro-organisms, inciting the infection. There may be instances where the urgency of the case requires that a hypothetical diagnosis be ventured, since the delay incident to the isolation and identification of the infecting bacteria may be detrimental to the welfare of the patient. Such a procedure, no matter if it yicld an apparently true diagnosis, should be permitted in no case to supplant the actual determination of the exact nature of the infection. When the condition is such that a proper bacteriological diagnosis by isolation is impossible, it is obvious that the solc means of attaining guidance is by inference. It is only in such instances, however, that one may be justified in substituting a guess in place of bacteriological methods. 'The careless practice of surmising the nature of the infection by analogy has no justification, and cannot be too heartily condemned. Given the culture, the technique devised by Wright for the preparation of the corresponding vaccine-with the exception of the tubercle bacillus-is as follows:

Bacterial Emulsion.-Several transplants on agar slants, or similar media, suitably enriched as the nature of the organism may require, are incubated the minimum period of time necessary to produce sufficient growth. The bacterial mass from the surface of thrce or four of these cultures, depending upon the luxuriance of growth, is carefully washed off with sterile physiological salt solution and transferred to another sterile test-tube. This tube, containing about five cubic centimeters of the collected emulsion, is sealed off in the blast-flame to form a closed capsule, the fused end of which is drawn out to a much narrower calibre. This operation requres care. Heating the emulsion should be avoided,

VoL. I-34 
by applying the flame well above the line of fluid. A thick-walled testtube of soft glass is best suited for this purpose. Clumps of bacteria are broken up by agitation in some mechanical device, or emulsification completed by thoroughly shaking the capsule by hand. Sterile glass beads may be added to the emulsion to facilitate this process.

Standardization. - This homogeneous suspension of bacteria must next be standardized. This may be accomplished by the red bloodcell method of Wright, by actual enumeration of the bacteria with a hemocytometer, by McFiarland's nebelometer, or by Gabritschewsky's method. By the first of these methods one mixes known volumes of bacterial emulsion and normal human blood. Knowing the number of red bloodcells per cubic centimeter, and counting the ratio of bacteria to red corpuscles, a simple arithmetical calculation enables one to estimate the number of micro-organisms in each cubic centimeter of the emulsion. The capsule is opened by severing the constricted neck of the tube at its distal end. The application of gentle heat-the warmth of the handto the body of the inverted tube suffices to expel a drop or two of the contents of the tube, or, preferably, a minute quantity of the emulsion may be withdrawn by means of a sterile fine-calibered pipette and the capsule immediately resealed. In the next step a Wright capillary pipette, controlled by a rubber teat, similar to those employed in the technique of determining the opsonic index is used. A point arbitrarily chosen, one or two centimeters from the end of the capillary tube, is marked with a wax pencil. Blood from the punctured finger-tip of a normal individual is drawn up in the pipette to the mark, the point of the tube withdrawn, and the column of blood allowed to ascend a short distance. An equal volume of salt solution is similarly taken up, and, finally, a like quantity of the bacterial suspension. These three components are then thoroughly mixed on a glass slide by alternately expelling and then redrawing them up into the tube. A small drop of the mixture is uniformly spread upon a glass slide, after the manner of an ordinary blood film, and stained by any of the usual blood stains. The number of red blood-cells and bacteria in successive fields are counted under the microscope until at least 500 red blood-corpuscles are enumerated. The process of counting is greatly facilitated by reducing the microscopic field with an Ehrlich diaphragm or similar device. Having determined the ratio of red bloodcells in a given number of fields, and reckoning the normal red cell count as $5,000,000,000$ per cubic centimeter, it follows that the bacterial content of the same unit of emulsion may be expressed by an equation, thus: let $R B C$ represent the number of red blood-cells counted, $B$ the number of bacteria counted, and $X$ the number of organisms to be determined in one cubic centimeter of the emulsion; then $R B C: B:: 5000$ million $: X$. Having thus determined the number of organisms in each cubic centimeter of bacterial suspension, the concentration required for appropriate dosage may be obtained by dilution. The numerical method of vaccine standardization yields only approximate accuracy in the enumeration of the bacterial content, and affords no measure of the variable virulence exhibited by different strains of any organism. 
Sterilization.-After standardization the emulsion is sterilizerl by heat. 'This sequence is followed in order to avoid any inaceuracy in the count that might result from autolysis as a result of heating. 'Ihe capsule containing the emulsion is completely immersed in a water-bath at $60^{\circ} \mathrm{C}$. for one hour. Where large volumes of vaceine are to be sterilized, an additional fifteen or twenty minutes may be allowed to hring the total volume of emulsion up to the required temperature. 'This temperature is sufficient to kill the non-spore-bearing pathogenic bacteria, and is within the limit of heat beyond which the chemical properties of the vaccines are altered and their immunizing power impaired or destrovect. 'Therefore, the lower the effective temperature to which the emulsion inay' be subjected in sterilization, the greater the potency of the vaceine. Sterilization may also be accomplished by various chemically indifferent agents. Weaver and Tunnicliff found that in rabbits a streptococcus vaccine, in which the bacteria were killed by suspension in 25 per cent. galactose solution, produced a certain protection against subsequent injection of homologous, living, virulent organisms, while a corresponding vaccine, sterilized by heat, afforded no such immunity.

Vaccine Containers.-Vaccines may be conveniently dispensed in small bottles of suitable capacity. In preliminary preparation these bottles are plugged with cotton and autoclaved. The requisite amount of sterilized bacterial emulsion is then transferred to one of these sterile containers, and the required dilution obtained by adding 0.85 per cent. solution of sodium chloride. The addition of lysol to the final product in concentration of 0.25 per cent. acts as a preservative. Phenol or tricresol may be used for this purpose. 'The cotton plug is replaced by a strong rubber cap, which has been sterilized by boiling in 0.5 per cent. lysol. These rubber caps, especially made to fit the style of bottle used, have the advantage of permiting easy access to the fluid without the risk of contamination possible in the removal of an ordinary cork or stopper. Before the vaccine is used, control cultures must be made from the bacterial emulsion immediately after sterilization, and from the final bottled prodıct before the addition of an antiseptic. These cultures are grown aërobically and anaërobically, to preelude the possibility of incomplete sterilization or the chance of contamination in technique. When large quantities of vaccine are prepared, animal inoculation may be resorted to as a further safeguard. Lamentable accidents may follow neglect of sufficient precaution. After disinfection of the exposed surface of the rubber cap with a drop of lysol or other suitable germicide, the desired dose of vaccine may be readily withdrawn from the bottle by plunging the sterile hypodermic needle through the cap while holding the bottle in an inverted position.

Kinds of Vaccines.-Various terms, descriptive of the source and nature of their bacterial content, have been applied to vaccines. A vaccine composed of a single strain of an organism is spoken of as univalent, one composed of two or more strains as polyvalent. A mixed vaccine is one containing two or more bacterial species. The term autogenous vaccine denotes a vaccine made from the specific or- 
ganism isolated from the patient's own infection. The name stock vaccine is applied to such as may be made from stock or heterologous cultures and kept ready at hand to supply the immediate need pending the preparation of an autogenous vaccine, or for use in cases where it is impossible to secure a culture of the patient's own organism. Stock vaccines also include those bacterial emulsions prepared in quantity, and sold under the same conditions as other biological products, such as antitoxin and other sera, etc.

Administration of Vaccines.--Site of Inoculation.--Vaccines exhibit their maximum therapeutic potency when administered subcutaneously. Any area of loose cellular tissue with an active blood-supply may be chosen as the site of inoculation, such as the region about the scapula, the outer surface of the arm, about the insertion of the deltoid, or, in fact, any site which may be recommended by convenience, or which, should any local reactions arise, would cause the patient the least discomfort. Care should be taken to avoid any veins, since the introduction of a bacterial emulsion directly into the blood-stream is not unattended with danger. The immediate liberation into the systemic circulation of even minute quantities of bacterial substances in concentrated form may excite a profound toxemia. A further advantage of the subcutaneous injection lies in the fact that their comparatively slow absorption permits of a more gradual introduction of the vaccinated substances into the circulation. Thus, it is possible to obviate the sudden overwhelming of the body with an excess of antigen.

Dosage.-There are no fixed rules concerning the dosage of bacterial vaccines. The variability of the immunizing response in different individuals may be due to personal idiosyncrasies or contributing physical disabilities, to the nature or severity of the infection, or to the kind and potency of the vaccine used. Such variations preclude the possibility of formulating definite laws governing the appropriate dose in any given case. However, as a result of accumulated experience, based upon repeated estimations of the opsonic content of the serum of those vaccinated, and upon clinical observations, certain general principles have been established which govern the practical application of vaccine therapy. The degree of immunity produced by the inoculation of bacterial vaccines bears no proportionate relation to the size of the dose administered. Small doses may serve as active stimuli to the mechanism of immunization. On the other hand, doses, excessive in amount or too frequently repeated, may overtax these protective processes. The danger of a large dose lies in the possibility of inciting a toxemia. The bodily resistance may be overwhelmed by the immoderate use of these modified bacterial substances, and thus furnish an opportunity for the infecting organisms to multiply and increase their activity at the site of infection, or becoming disseminated, to produce metastatic foci or even septicemia. The injudicious repetition of injections at inappropriate intervals may likewise depress the patient's power of protective response. The optimum dose of vaccine, as emphasized by Wright, is the minimal amount that will evoke a farorable immunizing response without inducing systemic re- 
aetion. It should be repeated only when its effects are diminishing, and increased only when it no longer suffices to elicit a satisfactory response. It is desirable to employ a small initial dose and gradually to inerease the amount with subsequent inoculations, as the nature of the case may warrant. In a mixed vaccine, where more than one organism is used, the combined doses should not exceed the maximum for any one of its constituents, but should be sufficiently large to include the initial dose of each. 'The interval between inoculations in chronic infections is generally from five to ten days. 'This period corresponds with the appearance in the blood of the maximum quantity of opsonins and other demonstrable inmune bodies. In the acute general infections smaller doses of vaccines at more frequent intervals have been found more satisfactory.

In systemic infection the rule of smaller doses and shorter intervals finds its endorsement in certain theoretical considerations which justify the use of bacterial vaccines in septicemias in general. It may be argued that in such conditions the blood-stream is already surcharged with free cireulating toxins, and that the injection of an additional amount of similar substances by vaccination can serve no purpose, and may even result in positive harm. It may, with equal reason, be asked how benefit can accrue from the local injection of an increment of bacterial substance which is infinitesimal in comparison to the total amount of toxic elements already present in the general circulation. It is now generally maintained, and there is considerable experimental evidence to corroborate the view, that the circulating blood serves merely as a vehicle for the lody's protecting forces, that this medium is not directly concerned in the elaboration of antibacterial substances, and that their production is the unique function of certain highly specialized tissue cells. Wright believes that these immune bodies are produced locally at the site of inoculation, and that the subcutancous injection of vaccine, therefore, forms an immediate and concentrated stimulus to these tissues. A similar unit of vaccinating substance, inoculated intravenously, must needs be diluted by the total blood volume before being brought into direct contact with the tissues, and hence furnishes a less potent stimulus less effectively applied. He further reasons that the whole immunity reaction implies a holding back of the bacterial substance in the tissues, and that this local, temporary retention permits of a slow absorption of toxins into the general circulation, and hence minimizes the danger of aggravating the existing toxemia. Therefore, the smaller the dose the less the possible absorption, and the period of lowered resistance is correspondingly diminished. Since the immunizing response to a minimal inoculation is necessarily transitory, these doses must be more frequently repeated.

This tentative theory assigns provisionally a cause for the recognized clinical fact, that in many cases marked improvement follows the use of bacterial vaccines in septicemic diseases, and that the course of many of the more acute generalized infections are favorably modified by the judicious administration of these therapeutic agents. Summarizing the clinical indications for the use of raccines in such cases, Adami expresses the belief "that, on the one hand, a sinking temperature, 
accompanied by an increased weakness of pulse and respiration and advancing 'toxic' state, is a clear sign that the reactive powers of the patient are being overcome and exhausted, so that vaccination can only be harmful, and, on the other hand, if the fever be somewhat high, provided that the pulse is steady with no signs of caridac weakness, then vaccination may be safely undertaken." Hyperpyrexia contraindicates their use.

The following table gives the doses of vaccine recommended by Wright:

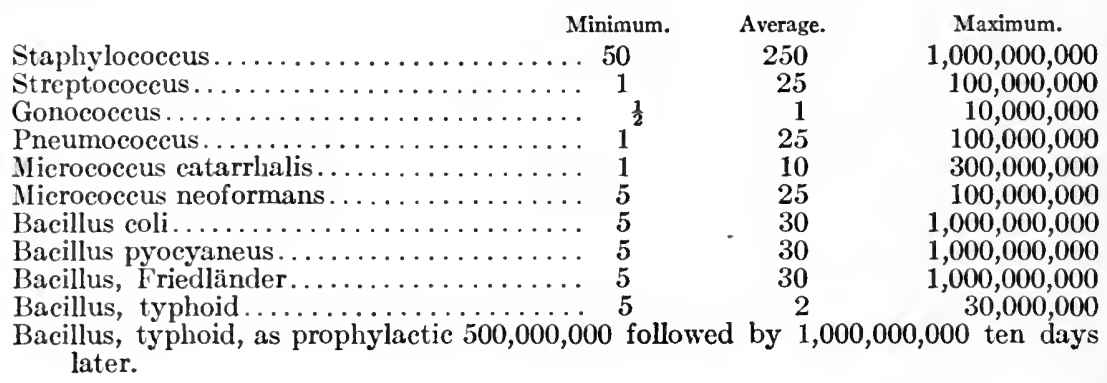

Tileston has recently compiled from the writings of various authors the following table of dosage:

In local infections.

Staphylococcus... . . . . 100,000,000-1,000,000,000

Streptococcus ......... . 5, 5,000,000- 200,000,000

Pneumococcus . . . . . . 10,000,000- 200,000,000

Gonococcus.......... . 5, 5, . $\quad 500,000-500,000,000$

Bacillus coli........ 10,000,000-200,000,000
In general infections.

$5,000,000-25,000,000$ $20,000,000-50,000,000$ $5,000,000-100,000,000$ $10,000,000-50,000,000$

A cursory examination of the above tables suffices to indicate the wide variations in dosage. This lack of uniformity emphasizes further the futility of attempting to reduce the quantitative administration of bacterial vaccines to exact formulæ.

General Considerations. - The question of the strain of any given micro-organism to be used in the form of a vaccine is of no little importance. At the present stage of our knowledge any answer must be considered as tentative. Generally speaking, whenever possible the autogenous vaccine is to be used. Pending the preparation of such a vaccine from the patient's infecting bacteria, or in those cases where laboratory facilities are lacking, recourse must be had to such vaccines as may be at hand. A purely theoretical consideration of the conditions involved gives the preference to a vaccine prepared from the particular strain of the infecting organism against which the immunizing attempts are directed. Such a view has recently been expressed by Adami: "Such vaccines, liberating a particular order of toxins and other bacterial products, will more surely stimulate the production of just the particular order of anti-bodies necessary to counteract and destroy the infective microorganisms." 
The opsonic index, as a criterion of dosage, has been largely discarded in this country. Opsonins constitute but one of the immune bodies evoked by vaccination, hence the opsonic index does not represent an accurate measure of the total immunity produced, while the tedious technique, and the possible sources of error inherent in the method, render it impracticable in most eases. 'There is a general parallelism, however, between the opsonic indices and the clinical course of most cases, so that these determinations should be made when the results of inoculation cannot be controlled by the ordinary clinieal methods of observation, when there are several organisms present, or when the patient does not improve under the usual methods of inoculation.

Following the inoculation of an appropriate dose of bacterial vaccine certain vital phenomena occur which influence quantitatively the amount of protective substances in the blood. Subsequent to the injection of any vaccine there occurs a diminution in these antibacterial elements. This period, lasting one to three days, is called the "negative phase." The degree and duration of this period are proportionate to the dose and potency of the vaceine administered. An accentuated negative phase, as the result of a large dose, may manifest itself clinically by symptoms of intoxication, fever, and general constitutional disturbance. 'Theoretically, the negative phase is associated with a lessened resistance to infection, although experimental evidence and clinical experience tend to show that the importance of this danger has been exaggerated. The exact nature of the negative phase is not well defined. It may be attributed to the neutralization by the vaccine of antibodies already present, or to some obscure action upon the cells concerned in the production of immune bodies. 'The negative phase is followed by an increase in the antibacterial power of the blood, associated with heightened resistance to infection. This period of reinforcement Wright calls the "positive phase." The curve of increased immunity representing the positive phase usually ascends during a period of eight to twelve days, when the maximum is reached, and then gradually descends. If the process of immunization has been successful, and if no untoward conditions arise, the descent terminates at a point higher than the initial immunity index. The curve then becomes a horizontal constant, indicating that the body resistance to infection has been effectively raised and the increase maintained. This curve of immunization, with its sequence of negative and positive phases, constitutes what Wright has ealled "the law of the ebb, flow and reflow, and subsequent maintained high tide of immunity." Fig. 85 , selected from an article by Wright, illustrates this law.

This train of events succeeds the inoculation of sufficient vaccine to cause a constitutional disturbance. When only a small dose of vaccine is given, the negative phase may be so insignificant and of such short duration as to be incapable of measurement, and the positive phase will be correspondingly diminished. On the other hand, if the dose of vaceine is too large, the negative phase will be unduly prolonged and the positive phase may not appear. The question of dose, then, is of the 
greatest importance, for, if too little is employed, no appreciable therapeutic effect will result. On the other hand, too large a dose is dangerous, and so lowers the resistance of the host as to favor the multiplication of the bacteria. Such dangers in a single inoculation are, of course, greatly magnified by repeated inoculations. If a series of such inoculations is attempted during the negative phase, there will result a further diminution in the protective substances or a "cumulation in the direction of the negative phase." Sooner or later, a point will be reached where the organism will cease to respond to inoculation, and further doses of vaccine will not only be of no avail, but may bring the amount of the protective substances in the blood to a lower level than that from which it originally started. Wright considers that the result of the vaccination cannot be

FIG. 85.

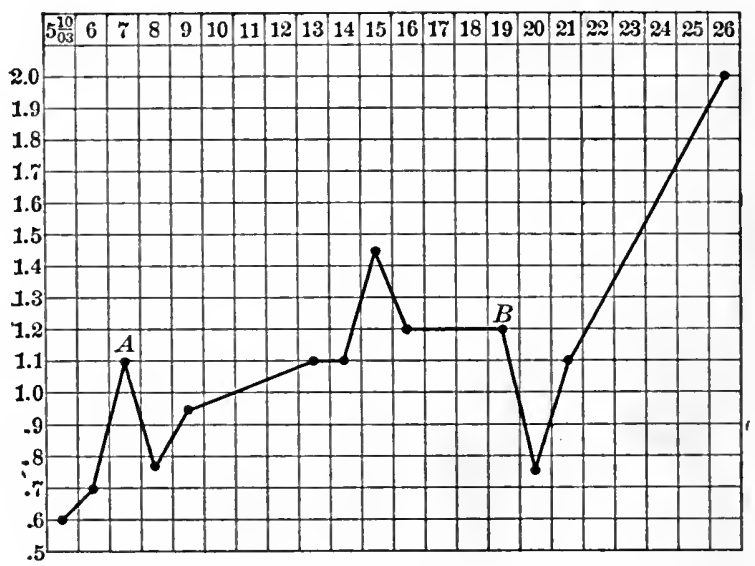

Case of a physician who had suffered from boils for four years (Proc. Roy. Soc., $1904-5$, vol. xxiv, p. 150). A, Inoculated with 2,000 millions sterilized staphylococci derived from patient. B, The same repeated.

estimated from the character of the constitutional disturbance. $\mathrm{He}$ advises the smallest possible initial dose sufficient to produce any negative phase, and reinoculation only after the subsequent positive phase has begun to diminish.

To recapitulate, therefore, the ideal train of events to be striven for in a course of active immunization should be somewhat as follows: The existence of an infection presupposes a low degree of resistance on the part of the infected individual. The goal to be attained is the exaltation of this resistance to a degree which permanently inhibits the multiplication of the invading bacteria, and, therefore, annihilates the infection. The initial dose should be gauged with a view to eliciting the greatest immunizing response with the least constitutional disturbance. Unfortunately, it is at present impossible to dogmatize concerning the means best adapted to the accomplishment of this end. Empiricism must still be relied upon to establish the course to be pursued. The 
endeavor is now directed to the progressive elevation and maintenance of the antibacterial resistance, care being taken to avoid any temporary depression of the body's defenses. 'The measure of the sulsequent doses will depend upon the degree of the reaction called forth by the initial quantity. 'The progression should be of such a nature that the immunizing mechanism is never overwhelmed or even stimulated to the peint of strain, as evinced by severe local or constitutional reaction. 'The interval between injections is to be determined by the nature of the infection, and by the readiness with which the body responds to previous doses. Acute infections demand haste. It is of the greatest moment to effect an increase in resistance before the patient reaches the fastigium of the disease. Since an ordinary dose, under these circumstances, becomes jotent to depress the already ozertaxed defenses, the quantity infected must be diminished, and small doses, to become effective, must be repeated at frequent intervals. 'The greater the chronicity of the infection, the less the importance, within certain limits, of the time factor. Injections should be so spaced as to occur during the ebb of the immunity flood toward normal. The quiescence and disappearance of the infective process are not to be considered as positive indication for the cessation of treatment, as it is highly advisable to continue inoculations during a space of time sufficient to insure against exacerbation.

The amount of vaccine to be administered in any particular case is a problem requiring careful discrimination. What constitutes a moderate immunizing dose in one individual becomes an intoxicating dose in another. The introduction of comparatively large amounts of bacterial substance into an uninfected or normal body may be tolerated without the manifestation of toxic symptoms. Nor does such an injection elicit a toxic response if administered to an individual infected with a bacterial species different from that vaccinated. In an infected individual, on the other hand, the exhibition of what might be considered a moderate dose of an homologous vaccine may produce a more or less severe degree of intoxication. In such a case the response elicited will depend upon the nature and character of the infection, the amount of toxin already circulating in the blood-stream, and the degree and frequency of autoinoculation. In other words, a cardinal rule in dosage shouid be-the more acute the symptoms, the smaller the dose. Whenever doubt arises in the treatment of any particular case, the tendency should be to diminution rather than increase of dosage.

In every case the determination of the quantity and frequency of vaceine dosage demands a full realization of the true nature of the vaceinating substances employed. Chemically, these bacterial substances are essentially toxins; physiologically, they are more than poisons. From a primi considerations one might suppose that in an infected person the injection of a given amount of toxic substance-in the form of a bacterial vaceine-would preduce an additional intoxication proportionate merely to the amount of toxic material vaccinated. In a normal individual the total intoxication produced would correspond to the sum of the effects of the separate toxin units injected. In the in. 
fected individual, however, a totally different situation prevails. Here the body is not only already intoxicated, but the physiological mechanism by which the body neutralizes the intoxicant is capable only of a more or less limited response. If the amount of already circulating toxin is sufficient to tax this mechanism to its full capacity, then the introduction of any added toxin, in the form of a dose of bacterial emulsion, no longer acts as a mere superimposition, but, instead, lays a strain upon the bodily defenses out of all proportion to its mere toxin content. The responsive mechanism is overwhelmed and the body cells stand exposed, with no intervening protection, to the full action of the combined toxins. And this is not all. In addition to the toxemia thus established, the body so attacked, by the giving way of its defenses, offers ideal conditions for the multiplication and dissemination of the invading bacteria and the infection extends. Hence, an inordinate dose of bacterial vaccine by the excess toxin it contains does not act as it would if injected into a normal body. Its action is in no way comparable to the action of any other class of poisons. 'The danger attendant upon the use of an excess of bacterial substance is, therefore, not so much attributable to the primary intoxicating effect as to its irresistible and overwhelming stimulus to the protecting mechanism. The dosage, therefore, is not to be light-heartedly prejudged. What has been said concerning the amount of vaccine to be employed applies in a measure to the frequency of administration.

The success of the immunization will depend upon the ability of the immunizator to read and correctly interpret physical signs, upon the quality of his judgment in determining the quantity and interval of doses, and upon the application of such subsidiary measures as may increase the bodily welfare of the host, as well as militate against the activity of the invading horde.

Local, Focal, and General Reactions.-The injection of bacterial vaccines into infected individuals may be followed by a more or less marked reaction at the point of inoculation. The appearance and severity of such a local reaction is dependent upon the bacterial species in the vaccine, preemminently by the size of the dose, and upon the condition of the patient. Normal individuals rarely manifest any local reaction to vaccine injections. This is also true in the case of infected individuals when vaccinated with suspensions of a heterologous bacterial species. Exceptions may be noticed in the case of prophylactic typhoid vaccination, and, occasionally, with certain strains of streptococci. The exhibition of tuberculin in apparently normal individuals is frequently attended by a local inflammatory reaction of more or less pronounced severity. In such instances the response is probably due to the presence of a healed or latent tuberculous lesion.

An analysis of the circumstances of the local reaction, provoked by the injection of bacterial emulsions, would make it seem that it is due to an anaphylactic sensitization brought about by the previous autoinoculation or autointoxication emanating from the infected focus. In addition to the possible effect at the point of inoculation, bacterial vaccination may produce rcactionary changes at the seat of infection. This focal reac- 
tion is evidenced by the pain and increased inflammation of the affecterl joints in gonorrhoeal arthritis evoked by the administration of gonocoecus vaccine, and by he temporary increased infective activity in the casce of staphylococeus, streptococeus, tuberculous, and other infections. 'The focal reaction may be of far greater moment to the economy. By means of the hyperemia and increased permeability of the encapsulating wall brought about by such injection, an autoinoculation may be induced, with possible consequent dissemination and extension of the infection. Such a possibility constitutes the chief hazard in tuberculin administration, and is a factor to be duly considered in all injections of hacteria or bacterial products, It is to be remembered, however, that both local and focal reactions may be wanting when the dose given is sufficiently small. When the quantity given is excessive, the effect produced may be so great as to constitute a general reaction. 'This maty vary from a mere temporary rise in temperature and general malaise to a more severe intoxication.

The specificity of these local, focal, and general reactions would seem to point to the possibility of the use of bacterial vaccines in diagnosis of obseure bacterial infections. In equine glanders the injection of mallein, and in human glanders the inoculation of mallei vaccine, produces local swelling and tenderness which have become of valued diagnostic aid. Since, as a rule,-antityphoid vaccination being the most conspicuous exception,-reaction follows only when the bacterial vaccine injected corresponds with the bacterial species infecting, it may be possible, by selective vaccination with proper doses, to determine the nature of any given infection. 'The physiological mechanism underlying this manifestation offers an interesting field for the prosecution of anaphylactic studies.

Prophylaxis.-The prophylactic use of bacterial vaccines in infectious diseases occupies an important place in preventive medicine. The earliest attempts to establish protection against disease by preventive inoculation were made by Pasteur in 1880 . By the use of vaccines of attenuated living micro-organisms he successfully protected animals against chicken cholera and anthrax. Some years later Haffkine, using living cultures, extensively employed anticholera vaccination in an epidemic in India. In over 100,000 persons inoculated, the incidence was reduced one-tenth, although among the inoculated, who subsequently contracted cholera, the course of the disease was apparently uninfluenced by inoculation. This same investigator, using an antiplague vaecine composed of bouillon cultures sterilized by heating, achieved marked success in prophylactic inoculation against bubonic plague. The incidence of disease among those inoculated was 1.S per cent; among the inoculated, 7.7 per eent. The mortality was ten times greater among those uninoculated.

Antityphoid Inoculation.-Prophylactic inoculation against typhoid fever by the injection of dead typhoid bacilli has been extensively employed among the British and German Colonial troops, and more recently has been recommended in the United States army. Both the morbidity 
and the mortality of this disease have been decidedly decreased by this preventive measure, and an immunity established which is active for about two years. Wright, following the suggestion of Pfeiffer, demonstrated the practical efficacy of antityphoid vaccination by actual test in India and South Africa. Although statistical records are unfortunately incomplete, Wright concludes that the incidence of typhoid fever was diminished one-half and the mortality from the disease reduced two-thirds. Leishman, succeeding Wright as head of the Royal Army Medical Corps, has further confirmed the value of vaccine prophylaxis by convineing statistical evidence. British statistics, in general, show that, under similar conditions of exposure, for every case of typhoid fever among the inoculated there were 10 cases among the uninoculated. In the Herero campaign, in 1904, 8000 men of the German Colonial army were vaccinated by the method of Pfeiffer and Kolle. About one-half as many cases of typhoid, and only one-quarter as many deaths, occurred among the troops subjected to preventive inoculation. Under Major Russell, antityphoid vaccination is now being carried out among the enlisted men of the United States army. This work was begun in February, 1909, and recent reports show that 1400 persons have been immunized. With one exception, no case of typhoid has occurred among those vaccinated. On the other hand, 135 cases of this disease have been reported among the 75,000 enlisted men since the introduction of vaccination.

Antityphoid Vaccines.-Antityphoid vaccine, as prepared by the Royal Army Medical Corps, consists of a broth culture grown for fortyeight hours at $37^{\circ} \mathrm{C}$., and killed by exposure to $53^{\circ} \mathrm{C}$. for one hour and ten minutes. The original method subjected the vaccine to $62^{\circ} \mathrm{C}$. for one hour, but this temperature was found to impair its immunizing properties. The increased potency of this "new vaccine," prepared at the lower temperature, has been proved by laboratory experiment and clinical observation. This vaccine is standardized by the red bloodcell method of Wright. An initial dose of 500,000,000 is followed in ten days by a second inoculation of $1,000,000,000$. The outer surface of the arm is chosen as the site of inoculation. The antityphoid vaccine used in the United States army is prepared from eighteen to twenty-hour agar cultures, the surface growth of which is suspended in physiological salt solution, standardized by bacterial count and sterilized at $60^{\circ} \mathrm{C}$. for seventy-five minutes.

Castellani has used an antityphoid vaccine, consisting of a twenty-four hour bouillon culture of a virulent bacilli heated at $50^{\circ} \mathrm{C}$. for one hour. Although this living vaccine induces a high degree of immunity, its use is not unattended with danger, and it may possibly convert the individual inoculated into a typhoid carricr. Castellani, from a comparative study of 416 cases, concludes that a greater and more permanent immunity is obtained by using an initial dose of dead vaccine (Wright), followed in a week by one cubic centimeter of living vaccinc prepared as above.

Reaction.-A reaction of variable severity follows antityphoid inoculation. Locally, reduess and tenderness may appear at the site of inocu- 
lation in from six to ten hours following injection. In the more severce forms this area of redness may extend five to ten contimeters in dianeter, with lines of injected lymphatics radiating from the inflaned areola. Adjacent lymphatic glands may be involved. These local manifestations disappear after forty-eight or seventy-two hours, although a hard, shot-like nodule may persist for one or two weeks, Constitutionally the reaction is still more variable. It may be absent entirely, or a sense of languor or general malaise may come on one to six hours after inoculation. Occasionally a chill may supervene, and the temperature rise to $101^{\circ} \mathrm{F}$, or, more rarely, to $103^{\circ} \mathrm{F}$. 'These symptoms disappear in from eighteen to twenty-four hours. Russell has observed that the tendency to a more marked or severe reaction is greater in those who have suffered from a previous attack of typhoid, and that both local and constitutional symptoms are exceedingly mild in children. Spooner vaccinated 103 individuals against typhoid at the Massachusetts General Hospital and the United States Naval Hospital at Chelsea. By using small doses, he was able to elicit a high agglutinating response with a minimum of constitutional reaction. The vaccine userl was prepared from agar cultures, sterilized at $53^{\circ} \mathrm{C}$. for one hour and the bacterial content enumerated by the Zeiss blood-platelet counter. An initial dose of $50,000,000$ was followed at five-day intervals of $100,000,000$ and 200,000,000. Slight local reaction, with soreness and induration, occurred in a few hours, but constitutional symptoms were not common and rarely severe.

Wright associated the negative phase immediately succeeding vaccination with a period of increased susceptibility to the disease. Because of this theory, a prejudice naturally arose against using vaccines during an epidemic, since this procedure would render the patient exposed less resistant to typhoid infection. Experimental and clinical observation tends to show, however, that no definite relationship has been established between the negative phase and a state of hypersusceptibility, and that these two are not necessarily coincident. Pfeiffer and Friedberger have shown that the inoculation of guinea-pigs with bacterial vaccines does not produce an immediate increase in susceptibility, but that, on the contrary, an increased resistance is manifest even in the first twelve to thirty-six hours following injections.

Because of the degree and duration of the immunity produced, hecause of its harnlessness, and the facility with which it nay be carried out, antityphoid vaccination is a valuable measure against the spread of typhoid fever and an additional aid in its prevention. Its prime application consists in preventing those epidemics which are the scourge of army camps, and which usally attend the mobilization of any considerable number of men. In the prophylactic vaccination of physicians, nurses, hospital attendants, and others exposed to active disease, or of individuals inhabiting regions where typhoid is endemic or epidemic, the sanitarian possesses a formidable weapon against the inception and spread of this unnecessary disease.

Scarlet Fever.-The prophylactic use of streptococcus vaccine in scar- 
let fever by Gabritschewsky and other Russian investigators has been brought to more general notice in this country by a recent resume of their results by Smith. The vaccine employed consists of a concentrated bouillon eulture of streptococcus isolated from a case of scarlet fever. The culture is killed by heat at $60^{\circ} \mathrm{C}$., and preserved by the addition of 0.5 per cent. carbolic acid. Since these vaccines are prepared and standardized according to the method of Gabritschewsky, it is impossible to compare their potency with that of vaccines standardized by the Wright method. Six to eight hours following the injection of this vaccine a local reaction commonly occurs at the site of inoculation. This reaction is temporary, and is usually accompanied by a slight rise of temperature, more or less headache, and general malaise. Less frequently, in 10 to 15 per cent. of cases, there appears a punctate, erythematous eruption over the body, which disappears in one to three days without desquamation. Swelling of the glands, sore throat, and the so-called "strawberry tongue" may occur. This reaction simulates a mild scarlatiniform condition, and, together with the subsequent immunity response, serves to emphasize the possible role of the streptococcus as the specific organism in the etiology of scarlet fever. Over 50,000 raccinations have been made. From the outcome of these cases it appear that the careful use of the vaccines is unattended with harmful results. Usually three immunizing doses are given at seven- to ten-day intervals, the amount being increased at each injection one-half to two times the previous dose. It is claimed that after three doses of the vaccine, and usually after two, there is established a complete immunity against scarlet fever. 'The duration of this protective period is as yet undetermined. Statistical evidence warrants the wider application of these prophylactic vaccines, in order that their value may be definitely established in controlling the spread of epidemics of scarlet ferer.

With an advanced knowledge of the physiology of the mechanism of immunity, with a better understanding of the etiology of infection, or with more delicate technique in the preparation of vaccines, it is not inconceivable that prophylactic vaccination may be successfully directed against many other epidemic diseases.

The preliminary administration of vaccines, either before or after surgical operations, with the intention of protecting the patient against any post-operative infection, is a procedure which may find future application.

To immunize the patient against a hidden but suspected lesion, to produce an effective resistance against bacteria already present which surgical measures might tend to disseminate, or to guard against the invasion of pathogenic micro-organisms from within or without, would be to eliminate one of the chief dangers of operative surgery.

Clinical Application of Vaccines.-A systematic consideration of the therapeutic application of bacterial vaccines may be best effected, perhaps, by pointing out the correlation existing between the various lesions produced by any given genus of micro-organism. The infections produced 
by the staphylococeus, for instance, bear certain corresfonding resemblances to one another, but certain dissemblances to infections probluced by other kinds of bacteria. 'These resemblances may lic due to a selective action on the part of the specific genus in respect to the particular tissue of implantation, and to the specific behavior of the bodily tissues toward each genus.

'The kind of vaccine to be employed in the treatment of any given infection, therefore, is to be selected with reference, not to the kind of lesion produced, but to the nature of the infecting bacteria. 'This specifice treatment is clirected more against a bacterial agent than a pathological condition. The kind of vaccine appropriate to any particular infection is to be determined by the species of micro-organisms isolated; the guantity and frequency of dosage by the nature and extent of the lesion produced.

Staphylococcus Infections.-There are two species of staphylococci principally concerned in the production of infective processes in the human subject-the Staphylocoecus pyogenes aureus and the Staphylococcus pyogenes albus. In conditions incited by these micrococci, a vaccine to be effective must contain staphylococci of the same species as those present in the infected focus. Iocal infections caused by the staphylococei include furunculosis, carbuncle, acne, sycosis, and other pustular skin affections, osteomyelitis, abscesses, cellulitis, and surgical wound infections. General infections, such as multiple abscesses and septicemia, may also be due to these organisms. As a rule, the Staphylococcus aureus is provocative of the more severe infections, while the Staphylococeus albus is usually the sole or dominating species in the more superficial lesions.

Vaccine therapy found its initial application in staphylococcic infections, and to-day it is in these cases that bacterial vaccines yield the most satisfactory results. Where the infection is localized, the exhibition of a corresponding vaccine is usually followed by a prompt immunizing response attended by the arrest of the infective process. The lower grade of toxicity manifested by the staphylococeus permits of the employment of larger doses of vaccine than is the case in the majority of the other infections. In furunculosis and carbuncles, vaccines may be considered as most effective therapeutic agents. 'The evacuation of pus by' a small incision, the stimulation of the lymph flow by means of the sodium citrate and salt solution of Wright, and the use of other measures supplementing the proper administration of an appropriate vaccine will generally insure the prompt relief of pain, hasten resolution, and promote healing. A few inoculations in the after-treatment of these conditions is advised as a preventive measure against exacerbations. By this method recurrence is less frequent, and subsequent lesions, if they occur at all, are less severe and yield more readily to treatment.

Acne is not always so amenable to vaccines. Many types of this disease exhibit great obstinacy to treatment, and may require patience and persistence if the treatment is to be successful. 'ilhis is particularly true of the indurated and seborrhoic types. One reason why some of the forms of acne have failed to respond readily to vaccines may be 
that usually it has been taken for granted that staphylococci alone were responsible for these infections, and vaccination has been directed solely against these organisms. In certain types of this disease, particularly those characterized by comedos, with a few indurated papules and pustules, the Bacillus acne has been found to be the sole cause of infection. In other cases, especially those exhibiting pustulation, the Bacillus acnes has been found together with the Staphylococcus albus. Gilchrist demonstrated the presence of this bacillus in a large series of cases of acne, and succeeded in cultivating it in a number of these, either alone or in symbiosis with the coccus. Such evidences emphasize the necessity of a bacteriological diagnosis. Whenever the Bacillus acnes is found to be present in an acne lesion, the corresponding vaccine is indicated. Fleming reports success in the treatment of such cases with vaccines of the bacillus alone or in conjunction with the Staphylococcus albus. $\mathrm{He}$ advises injections of $5,000,000$ to $10,000,000$ of acne vaccine at intervals of one to two weeks.

Streptococcus Infections.-Although all disease-producing streptococci are included in three or four species, yet the various strains of streptococci encountered in infections display so many and such wide variations in their biological characteristics, that any campaign directed against infections produced by them must be carried on with a full knowledge of the importance of these variations. The greatest hope of success in the vaccine treatment of these infections lies in the employment of a vaccine containing the particular strain causing the lesions. Recognizing the importance of type variations, the attempt has been made to treat streptococcus infections with polyvalent stock vaccines, prepared with a number of different strains obtained from as diversified sources as possible. Such vaccines, however, should be used only in those cases where bacteriological isolation and corresponding vaccine preparation are pending or impossible. The principal infections which may be due to streptococcus invasion are septic wounds, cellulitis, erysipelas, and septicemia, including a large proportion of all cases of puerperal sepsis and malignant endocarditis. The streptococcus also appears to play an important, though not wholly understood, role in scarlet fever, and in the suppurative complications of certain other contagious diseases.

Streptococcic infections are characterized by their fulminating nature, and by the severity of the intoxication they produce. These two factors may be largely responsible for the clinical fact that the efficacy of bacterial vaccines is not so great inn ifections of this nature as in those referable to other organisms. These unsatisfactory results may in part be attributable also to the present method of preparing vaccines. It is now quite generally believed that the immunizing power of streptococcus vaccine decreases as the exposure to heat is increased. Weaver and Tunnicliff sought to avoid this effect by exposing the cocci to the devitalizing action of galactose, and found that vaccines thus sterilized yielded much better results than corresponding vaccines prepared by the usual method of heat sterilization. Leary concludes from his results that the efficacy of streptococcus vaccines is increased by a shorter exposure to heat. 
Streptococcus vaccines yield their most favorable results in limited infections, such as abscesses, cellulitis, ete. In erysipelas vaccines appear of limited value, although Ross and Johnson report that their administration tends to shorten the course of the disease. In all these infections vaccines should be used in conjunction with other routine treatment. In streptococcus septicemia all that has been said of this class of infections has here its most forceful application. In order that an immunizing response may be obtained without adding to the already severe constitutional disturbance, the greatest care must be exercised in determining dosage and interval. 'The more severe the symptoms, the less the tolerance for larger doses. 'The septicemias in general offer the least promising field for the application of vaccine therapy. Positive results have been obtained in septic endocarditis, but the cases recorded are far too few to establish the curative value of the vaccines. In puerperal sepsis more encouraging success has been obtained. Here the success of vaccination may be due to the fact that in many cases this condition is not a pure septicemia, but rather a toxemia with a local focus of infection which is accessible to surgical treatment. 'The results of Leary and Hartwell, Streeter, and Green in streptococcic puerperal infections warrant the further use of vaccines in postpartum sepsis, particularly in those cases resisting the usual treatment.

Dose of Streptococcus Vaccine.-In general infections 1,000,000 to $25,000,000$; in local infections, $5,000,000$ to $200,000,000$.

Pneumococcus Infections.-Besides the affections referable to the Diplococcus pneumoniæ, one might properly include here those infections due to the Diplococcus or Streptococeus mucosus. Although all the organisms of this type may be comprised in two species, the various individual strains differ so widely in their pathogenicity that what has been said regarding the advantages of autogenous vaccines is valid here. Although its prime manifestation is in the pneumonias, the pneumococcus may be concerned in the etiology of empyema, abscesses, arthritis, and other metastatic conditions. Pneumococci, like the streptococci, are characterized by the severe degree of intoxication they produce, and, where the infections prove fatal, death is almost invariably due to this toxemia. The indication, therefore, is for the early administration of vaccines in acute cases before the intoxication has lecome established, and for their exhibition in small doses at shorter intervals.

Recent observations seem to show that pneumococcus vaccines possess marked curative properties when properly administered in acute pulmonary infections of this organism. Leary, Harris, Wilcox and Morgan, Allen, Wolfe, and others used have vaccines in the treatment of aeute pneumonia, and their results are sufficiently encouraging to warrant a wider investigation of the value of vaccine therapy in these cases. According to these observers, the course of the discase is favorably influenced, the crisis occurs earlier, and there is marked relief of toxic symptoms and a lowered mortality.

Two facts are to be borne in mind in administering pneumococcus vaccine; first, there are large and constantly increasing amounts of toxin

VoL. I-35 
circulating in the body, and, second, that, owing to the great tendency of the pneumococcus to undergo disintegration or autolysis in the tissues, there is a more rapid absorption of endobacterial substances from the point of inoculation.

In acute cases, such as lobar, bronchial, and double pneumonia, an initial dose from $1,000,000$ to $20,000,000$ is recommended. This amount may be repeated within twenty-four or forty-eight hours if no unfavorable reaction follows the initial dose. Some authors recommend that smaller doses be repeated at three- or four-hour intervals. While benefit may accrue from such frequent injections it must be remembered that it is advisable to await the effect produced by the initial dose before deciding upon such a course. The temperature may serve as an index of the reaction produced by an injection. When in acute infections a temporary rise of two or more degrees repeatedly follows the administration of vaccine, it may be considered that the limit of safety is being reached, and that no immediate increase should be made. Larger doses of $50,000,000$ or more are to be employed only in the more chronic infections.

Gonococcus Infections.- The marked variation in the cultural and biochemical features of different strains of Micrococcus gonorrhœa emphasizes the importance of using autogenous vaccines in these infections. The biological specificity of homologous strains is corroborated clinically by the increased efficacy of such vaccines. Several authors, however, have obtained satisfactory results with stock vaccines. Gonococcus vaccines have been used in urethritis, orchitis, prostatitis, epididymitis, vulvovaginitis, iritis, and septicemia of gonorrhœal origin. Vaccine treatment of acute and chronic urethritis has yielded less satisfactory results than those obtained in metastatic infections. Although the administration of appropriate vaccines may be of little service in acute gonorrhœe, Allen and Eyre and Stewart propose their use after the subsidence of the more acute symptoms to prevent secondary complications.

The results of Cole and Meakins, Eyre and Stewart, Irons, and Hartwell not only justify the use of vaccine in gonorrhœal arthritis, but attest their value as therapeutic agents in many of these obstinate joint infections. In the majority of these cases injections of vaccine are followed by the amelioration of symptoms and the restoration of the joint to its former function. The amount of vaccine given in these infections varies, from moderate doses of $5,000,000$ to $50,000,000$, to relatively larger doses of $300,000,000$ to $500,000,000$. Cole and Meakins employed injections of $300,000,000$ to $1,200,000,000$ of stock vaccine, at intervals of seven to ten days. Eyre and Stewart gave 1,000,000 to 25,000,000 at intervals of three to twenty days. Irons administered $50,000,000$ to $500,000,000$, interspacing these injections three to seven days. Hartwell used $10,000,000$ to $100,000,000$, at two- to four-day intervals, and in the more chronic cases the dosage was larger-up to $600,000,000$ at intervals of five to six days. Where autogenous vaccines are employed, or when the symptoms are acute, the dose must be proportionately smaller.

In such obstinate infections as vulvovaginitis in young children gonococcus vaccines have been proved to possess decided worth. The 
tedious term of treatment is markedly shortened and medical interference greatly simplified. Hamilton, Hamilton and Cooke, Butler and Iong, and Clurchill and Soper report most encouraging results. 'They variously recommend amounts from 1,000,000 to 50,000,000. Initial doses of $1,000,000$ to $10,000,000$, or at most $50,000,000$, should be chosen. Hamilton gave $50,000,000$ every five days, increasing $10,000,000$ until five injections were given, then repeated this maximum dose at seven- to tenday intervals. Hamilton in no case observed any constitutional reaction following these vaccine injections, and in very few cases only an extremcly slight local reaction appeared. In the adult, on the other hand, Evre and Stewart, vaccinating against acute urethritis, noted increased discharge, pain, and slight general malaise. In gonorrhoal arthritis a temporary focal reaction may be expected, which consists in increased pain, tenderness, and swelling of the joint. Since such a focal reaction is specific-that is, it results only when the gonococcus is present both in lesion and vaccine, Irons has suggested the use of vaccine in diagnosing obscure cases of arthritis of suspected gonorrhoeal origin.

The immunity produced by gonococcic vaccines is transient. Although cure of an affected joint may occur, the possibility of subsequent exacerbation is not precluded. Recovery from acute gonorrhoeal infections confers no immunity against reeurrence, and a fresh infection from without may serve to reëstablish old metastatic foci.

Meningococcus.- So far the attempts to create an active immunity by vaccines in patients suffering from meningococcic infections have met with little success. The use of antimeningococcus serum in the treatment of cerebrospinal meningitis yields the most prompt and satisfactory results.

Micrococcus Catarrhalis.-The infections due to this organism comprise some of the more acute catarrhal inflammations of the respiratory tract. Many of the conditions commonly spoken of as "colds" or "grip" may often be due to infections with this organism. Webb has suggested the prophylactic use of catarhalis vaccines in immunizing individuals against these affections, and thus affording protection against epidemics due to this organism.

Micrococcus Neoformans.-According to Doyen, this micro-organism is an important contributory factor in causing or aggravating the ulceration accompanying some malignant growths. Wright, using stock raccines of this coccus, found that in such cases vaccination was followed by a decided lessening of the ulcerative process. Such treatment, although purely palliative, may give great relief, and add to the general welfare of the patient. Wright uses doses ranging from 5,000,000 to $100,000,000$ at approximately weakly intervals.

Bacillus Coli. - The group of colon organisms includes several welldefined types. These types exhibit certain intermediary varieties with slight cultural or biological peculiarities, which acquire importance, however, when the production of a specific immunity is concerned. The immune bodies produced by any given strain of Bacillus coli appear to be spccific for that strain. Thus, from the standpoint of laboratory 
experience, it would seem that autogenous vaccines would yield the most farorable results, a supposition which is supported by clinical evidence.

Although a normal inhabitant of the intestinal tract, the colon bacillus, when implanted in tissues foreign to its normal habitation, may acquire increased virulence and corresponding pathogenicity. Such manifestations occur commonly in the secondary complications following suppurating appendicitis, in infected sinuses, particularly those which are fistulous in nature, in ischiorectal and pelvic abscesses, and most frequently in pyelitis and cystitis. Colon infections of the urogenital tract are characterized by their chronicity and obstinate resistance to the usual therapeutic procedures, and in these conditions bacterial vaccines may effect a symptomatic cure even though the bacilluria persists. Hartwell and Streeter, from their observations of such treatment, conclude that colon vaccines are efficient in relieving symptoms in mild forms of cystitis, but have less effect on pyuria; they are without value in severe forms of cystitis; they probably hasten the recovery of pyelitis; they have no influence on bacteriuria. Favorable results have been reported by Billings and McDonald in colon infection of the urinary tract.

The dosage of colon vaccine varies from $5,000,000$ to $200,000,000$. Some authors recommend doses sufficiently large to produce a general reaction. This nay be permissible in those cases where the chronicity of the lesion is marked or where no toxic symptoms are present. In general, such a course would seem to be of doubtful expediency.

Bacillus Typhosus and Bacillus Paratyphosus. - While the usual medical therapy is similar for all infections due to either the typhoid or paratyphoid bacillus, the treatment of such cases by means of vaccines demands cognizance of the particular type or species of organism causing the infection. Since these diseases exhibit a constant toxemia, accompanied by a more or less continuous autoinoculation, the administration of bacterial vaccines must be so regulated as to cause a minimum of constitutional disturbance. The theoretical considerations which have seemed to contraindicate vaccines in acute systemic infections may be responsible for the general disinclination to apply this mode of treatment in enteric fevers. However, this method has its advocates. Richardson regards the use of typhoid raccines as efficacious in preventing relapses, but he was unable to note any marked change in the regular course of the disease. Sappington, on the other hand, in a study of 22 cases, observed distinct and progressive improvement in 75 per cent. following vaccination. The dosage of typhoid vaccine is variously recommended as between 5,000,000 and 50,000,000. The bacteriemia and toxic symptoms accompanying these infections demand the administration of small doses. It is recommended that a small, safe initial dose be given and its effects observed. Subsequent injections should be determined by the reaction and condition of the patient.

The vaccine treatment of all intestinal infections caused by typhoid, paratyphoid, colon, paracolon, or the dysentery bacilli at present rests upon experimental data too meagre to permit of trustworthy conclusions. 
Bacillus Pneumoniæ, Friedlander.-'The most frequent forms of infection caused by this bacillus are croupous pncumonia and empyema. Like the pneumococeus, under favorable conditions, the Friediander bacillus may escape from the primary focus, and give rise to a general infection or localized metastases, as peritonitis, pericarditis, otitis media, meningitis, etc. Although vaccines have been employed in the treatment of infections of this bacillus, the cases are too few to warrant any definite conclusions. This clinical data, meagre as it is, when supported by theoretical considerations suggests the desirability of this treatment. Initial doses of $5,000,000$ to $10,000,000$ and not exceeding $30,000,000$ are recommended. 'The subsequent maximum dose is 1,000,000,000, according to Wright.

Bacillus Pyocyaneus.-Characterized by the green coloration which it imparts to pus, the Bacillus pyocyaneus is perhaps best known as associated with other bacteria in septic wounds and sinuses. In persons of low resisting power it may exhibit increased pathogenicity and set up local and even general infection of marked severity. When present with other organisms, it may disappear spontaneously during the administration of vaccines against the accompanying infecting organisms, However, it may be advisable at times to employ vaccines against the pyocyaneus itself. Clinical experience has shown that frequently such a course has been followed by a distinct improvement in cases which had previously resisted treatment. The initial dose recommended by Wright is $5,000,000$ to $30,000,000$, with a maximum of $1,000,000,000$ for subsequent doses.

Bacillus Mallei.-Cases of human glanders are usually considered of rare occurrence. Owing to the difficulties in diagnosis is is probable that many infections treated as pyemia, recurring abscesses, etc., may, in reality, be due to the Bacillus mallei. The infection may assume an acute fulminating course or display an obstinate chronicity with acute exacerbations. The mortality is high, and the usual curative measures appear to be futile. Although only two cases of human glanders treated by homologous vaccines are recorded-Wright, and Bristow and White - the successful outcome in both cases is highly suggestive of the value which bacterial emulsions may possess in controlling infections of this nature. In the latter case the initial dose was $10,000,000$ and after fourteen injections, at intervals of four to nine days, a maximum of $300,000,000$ was reached. While the injections were followed by local and febrile reactions, no untoward effects were noted, and the course toward recovery was consistently favorable.

To appreciate the value of vaccine therapy one must be acquainted with its limitations as well as its ficld of application. It is not to be supposed that bacterial vaccines constitute a catholicon for all bacterial infections, nor must it be imagined that vaccines possess unlimited virtue even in those cases where their use is indicated. 'They are intended to supplement and not to supplant other therapeutic measures. Although an appropriate vaccine be employed, and although the dosage and interval of administration be properly judged, there still remains a factor, 
independent of specific therapy, which may render such treatment futile. This involves the defensive resources of the patient. When the body's defenses have been overwhelmed or exhausted by the acuteness or longcontinuance of an infection, and the immunizing mechanism rendered incapable of response, then the stimulus of a vaccine becomes impotent to call forth any effective immunity reaction.

The disappointment which frequently follows the application of this treatment is in many cases due to a disregard of the theoretical considerations involved. To vaccinate with scant knowledge of the nature of the agent employed, or of the process to be evoked, to vaccinate in ignorance of the nature of the infecting organism, in the hope that by some lucky chance the infecting and the injected species will correspond, is to invite discouragement and bring disfavor upon this mode of therapy. It must be frankly acknowledged that there exist unexplained facts, many inexactitudes, and seeming inconsistencies in the theory and practice of vaccine therapy. Whatever the limitations inherent in the method, or imposed upon it by the restrictions of our present knowledge, immunization, by the injection of dead bacteria, must be recognized as having gained an established position. When one realizes their already great service in benefiting and curing many of the localized infections, their possible application in systemic infections, their supporting aid in surgery, and their future promise in anticipating epidemic, endemic, and casual infection, it must be granted that the use of bacterial vaccines, in combating bacterial disease, constitutes one of the most splendid and valuable contributions to the science of medical therapeutics. 


\section{GLANDULAR THERAPY}

By S. P. BEEBE, Pн.D., M.D.

General Considerations. - The work of the last twenty years has placed organotherapy upon so firm a foundation that it can no longer be ignored as a valuable method of practical therapeutics. At various times in the history of medicine there have been sporadic attempts to treat disease by means of extracts of animal glands, but these have been, until recently, without scientific foundation and based upon the most unintelligent empiricism.

Beginning with the work of Sehiff upon the thyroid gland, there have been, during the last sixty years, a series of brilliant discoveries, which have placed at our disposal facts of fundamental importance, and have made it possible to use some of the glandular preparations with an approach to scientific accuracy. It has been found that certain comparatively insignificant anatomical structures furnish to the body substances whieh are vital. If these small bits of tissue, such as the adrenal, parathyroid; or pituitary, are removed, an acute death or chronic nutritional disturbances of various grades and character may ensue. 'These various tissues have been grouped together under the name of ductless glands, because their products are not emptied into the blood or lymph stream through a definite tube, and the products of their activity are spoken of as internal secretions, in distinction to the activity of such a gland as the salivary glands or the kidneys. Our knowledge of the number and eharacter of these internal secretions has increased rapidly during the last few years, and to such an extent that we are now accustomed to think of most active tissues as forming substances which, on entering the blood and lymph stream, may exert marked physiological activity upon distant tissues. Many of the most important reactions of the body are evidently under the chemical control exercised by the circulating medium. Each month new substances, or reactions, are described in various scientific journals, which are in the nature of chemical mechanisms for the control of some important function, and we are passing from the belief that internal secretions may be formed only in certain specialized bits of tissue to the belief that, through the medium of the blood and lymph, many functions work harmoniously. It is probable that during the next decade many advances in practical therapeutics will be based upon these facts. Certain well-marked pathological conditions are recognized now to be due to the faulty activity of certain of these special ductless glands, and it will be the purpose of this paper to describe briefly some of the best methods of treating these conditions and the therapeutic precautions that are to be observed. 


\section{THE THYROID GLAND}

Schiff made the first important contributions to the physiology of the thyroid gland in the middle of the last century, when he discovered that if the thyroid was completely removed from dogs the animals in many cases died with acute symptoms, which we now recognize as those of tetany. Not all animals so operated died. Some of them lived for varying lengths of time, but the operation nearly always produced severe nutritional disturbances, resulting in cachexia, susceptibility to infection, and occasionally one would be found to recover. These observations were repeated by other observers on differing species of animals, and it was found that herbivorous animals, such as the sheep, goat, rat, and rabbit, might have the thyroid totally removed without suffering serious consequences, but in foxes, cats, and dogs this operation was nearly always followed by serious consequences. It was, therefore, for many years believed that the thyroid gland in carnivorous animals had an entirely differert function fro that in herbivorous animals, but we now know that such is not the case. Since the discovery of the parathyroid gland by Sandstrom in 1880, we know that the thyroid apparatus consists of two glands, the thyroid and the parathyroid. In dogs, cats, and foxes these glands are so near together anatomically that both were removed at the same operation, while in the other animals, such as rabbits, rats, sheep, and goats, the two are distinct structures, and only the thyroid was removed, with a consequent difference in symptoms. When the thyroid gland is removed, and only the thyroid, a series of nutritional disturbances ensue which are not necessarily fatal for some months. In fact, they may not be fatal directly at any time, because of the development of fragments of thyroid tissue having atypical distribution and known as accessory thyroids. The further discussion of the effects of parathyroid removal will occur in the section devoted to that subject. It has been found further that if fragments of fresh thyroid are implanted beneath the skin, they do not grow, but the symptoms characteristic to thyroid removal may not develop for some time. The next step was to discover that subcutaneous inoculation of extracts of the glands had the same effect, and that, to a considerable extent, the beneficial results of thyroid grafting or thyroid injection could be duplicated by feeding the fresh or dried gland by mouth. No other tissue is capable of replacing the thyroid for this purpose. In order to prevent the disastrous consequences of thyroid removal, we have not yet been able to substitute any synthetic or artificial product for the secretion formed in the thyroid gland.

The first important chemical studies of the thyroid were made by Baumann and Roos, and their observations have remained to a considerable extent undisputed at the present time. They found that the gland contained an abnormally large quantity of iodine. No other tissue in the body has been found to contain so much. They found that the quantity of iodine in the gland varied in different species of animals and in different types of thyroid glands, the goitres in general containing 
relatively less iodine than the normal gland, and the gland of young animals relatively less than that of older animals. 'These results have been abundantly confirmed by the later studies of Oswald, Jolin, Marine, and in my own laboratory. 'They found that, by digestion with artificial gastric juice or by hydrolyzing the thyroid glands with mineral acids, it was possible to digest away most of the tissue and leave as a residue a brownish powder, soluble in alkaline solutions and containing from 8 to 10 per cent. of iodine. Moreover, this organic iodine componnd, which they called iodothyrine, they believed to be the particularly active substance in the thyroid, and stated that it answered all the requirements for the function of the gland. From more recent work, we know that this iodine-containing fragment occurs in the gland as a part of a proted, thyreoglobulin, and that the physiological activity of the latter substance is dependent upon the amount of iodine thus combined. In the space of this article it is not possible to enter into details regarding this matter, but it may be stated, as a result of recent work, particularly that of Hunt, that the functional activity of thyroid extract is in proportion to its iodine content.

For purposes of therapeutic administration at the present time a large variety of thyroid products are offered. The whole gland dried and powdered, in tablet form, in solution, and even the jodothyrine of Baumann has been to some extent used. Up to the present the glands from sheep have been most commonly employed for these preparations, but there are certain objections to the use of these glands for this purpose, since it is difficult to obtain any quantity of them free from goitres, and they generally have a low iodine content, with consequent small physiological action. Thyroids from pigs and beef are more uniform in their histology and iodine content. I have used large quantities of the latter glands for therapeutic purposes, and can see no objection to them. There has been thus far no agreement as to the best method for preparing thyroid for therapeutic purposes, the dosage to be employed, or the method of administration. As obtained from the glands, the iodine is in combination with a proteid forming thyreoglobulin, and we know that proteids have specific biological characters. This iodized proteid does not enter the blood through the stomach, but directly, so that, if we would wish to imitate exactly the condition in nature, it would be desirable to administer to the human subject by hypodermic injection the secretion from a normal human thyroid gland. Such a procedure is a little more than a physiological or a therapeutic curiosity, but during the last two or three years the writer has had an opportunity of administering thyroid in this manner to several patients, and comparing its effects to that of thyroid administered in the usual way, and, without question, a better therapeutic effect with less material is obtained when human thyroid gland is employed. To get uniform results in thyroid therapy it is necessary that a product having uniform activity be employed. If animal glands are used for this purpose, we at once encounter the difficulty occasioned by the wide variation in iodine content found in such glands, and, since the physiological and therapeutic activity of the 
substance are dependent upon the iodine content, we cannot be certain of the efficiency of a product unless it is made in some uniform manner and standardized by the iodine content. The writer has recently published what he believes to be an improved method for the production and standardizing of thyroid extract for therapeutic purposes, and during the last three years has had this substance tested in a large number of different conditions, and believes that the method should be widely adopted for this purpose. The method briefly is as follows: Thyroid glands as nearly normal as can be found are ground in a hashing machine, and extracted with four times their volume of normal saline solution made slightly alkaline. The extract is filtered, and the dissolved proteid precipitated by heating to $44^{\circ} \mathrm{C}$. and acidifying with acetic acid. This proteid is washed free from all adherent impurities, redissolved and reprecipitated several times, and finally dried at a low temperature and its iodine content estimated. As a standard we employ the proteid obtained from normal human thyroid glands which contain 3.5 of a milligram of iodine per gram of fresh gland. The purified proteid may be used for hypodermic injection or made into tablet form for administration by mouth. Other iodine-containing proteids than the one described may be found in the thyroid gland, and even in extracts from normal glands certain iodine-containing fragments are found which have tonic effect. It may be objected that the method of preparation gives only one kind of iodized proteid. This is true, but from results determined experimentally and therapeutically, I believe this iodized proteid has all the normal physiological effect of the gland and is all that is necessary for therapeutic purposes. If the total dried gland is employed for therapeutic purposes, many products of decomposition and autolysis are certain to be included. It does not seem probable that the thyroid forms a large number of different kinds of substances which act upon different tissues.

The various uses to which thyroid extract is now employed therapeutically have been derived by two kinds of observation-by comparing pathological conditions with the symptoms obtained by thyroid removal, and by knowing the effects produced in healthy animals by administration of thyroid extract. Thyroid removal causes marked metabolic disturbances of a character which we recognize as being similar to the conditions found in myxœdema and cretinism. Its administration to healthy animals produces effects which it may be desirable to obtain for therapeutic reasons, and its effects in what are known as normal animals vary somewhat with the size of the dose and the type of the animal. In small doses it may slow the heart; in large doses it generally accelerates it. It is said to increase the irritability of the vagus nerve and to decrease the irritability of the accelerator and vasoconstrictors. Such effect is not always to be expected. In large doses muscular contraction is weakened; in very small doses it may be strengthened. The effects produced by single doses are quite different from those that may be expected with continued administration. In most animals there is marked increase in the nervous excitability, tachycardia, palpitation, certain vasomotor disturbances follow, increased appetite, diarrhœa, sleepless- 
ness, marked increase in the output of carbon dioxide, nitrogen, and, with large doses, there may be a diminished ability to burn carbohydrates. In addition to these mentioned, there are many others that are occasionally to be observed in therapeutic use, which are not to be regarded as general effects, but as an idiosyncrasy to the reaction of that particular individual.

It is a general rule, in beginning thyroid administration in any patient for any purpose, that the patient should be for three to four weeks under the immediate observation of a physician, and the beginning doses should be small. For the first few days not more than 5 grains of the commercial extract should be given each day, and this quantity should be gradually increased until a desired closage has been obtained. 'This rule should apply in all but what may be regarded as emergency cases, for the reason that many persons are very susceptible to thyroid extract and distressing disturbances will occasionally be caused. A physician who gives a preseription ealling for 15 to 20 grains of thyroid extract in a day to a new patient, with directions that this programme should be followed for three or four weeks, is following a progranme which is distinctly elangerous and quite unjustifiable. 'The dosage which may be required to effeet any desired result is a matter which cannot be determined except by trial. I have seen a patient suffering from myxodema made completely comfortable by giving each clay three 2-grain tablets containing 5 per cent. of the pure proteid heretofore described; whereas in a cretinous child, weighing not more than 30 pounds, it was necessary to give double this number to get favorable effects, and in another case of myxœedema it has been necessary to go as high as 12 tablets three times a day to get favorable effects. In one case of general obesity 30 of these tablets were given each day, for a period of some weeks, under daily observation, without causing any distressing symptoms. 'The necessity for proceeding in the manner described cannot be too strongly emphasized. No established dosage for producing desired effects in any distinct disease can be given, but it should be remembered that a person taking thyroid extract is taking a powerful medicine, and cannot safely be allowed to continue in it for any length of time without being examined frequently by a physician.

Myxœdema and Cretinism. - It hardly seems necessary to state that thyroid is a specific for myxoedema and cretinism. Its use should be begun as soon as a diagnosis of these conditions can be made, and in most instances it will be necessary to continue their administration during the remainder of the life of that particular individual, though in some instances there are well-defined periods when the patient has a myxoedematous condition alternating with one of health or even of too much thyroid activity. Although the effeets of thyroid in these conditions is one of the most striking things in medicine, I have failed to see any case of well-defined myxoedema or cretinism restored to a completely normal condition by thyroid therapy. In these cases I believe the pure proteid which I have described is a more suitable medicine than the dried gland. I have had many instances in which the commercial thyroid, given in 
sufficient dosage to alleviate distressing features of myxœedema, has proluced fairly definite thyroid poisoning in some particulars. If the pure proteid is substituted, desirable effects without toxic manifestation have been obtained, and it is not at all unusual to find that a commercial product may answer very well most of the time, while, for some unknown reason, a new sample may be totally ineffective. It seems to me probable that this variability is due to a lack of standardization. It must be borne in mind that even with these conditions of distinct diminished function of the thyroid gland it is possible to get marked evidences of thrroid overdosing.

Other Metabolism Disturbances in which Thyroid may be Beneficial.-D During the last few years there has been a very noticeable increase in the number of pathological conditions in which thyroid therapy has been found to be efficacious. It is possible in some of these instances to find some analogy between the pathological conditions and the experimental conditions produced by thyroid removal. In others no such analogy exists. For instance, an individual somewhat obese, with a dry skin, suffering markedly in cold weather from cold hands and feet, fairly comfortable in warm weather, somewhat slow and indolent, with marked chronic constipation, is not a typical myxœdema, but such a patient is often rery markedly benefited by thyroid treatment.

In many cases a somewhat imperfectly developed cretinism may be found. Such a child will be dull mentally, physically inclined to inactivity, and, in a large percentage of cases, below the normal in physical development. They rarely do well in public schools, and it is occasionally found that they have enlarged thyroid glands. They are often backward in attaining development, and an infantile type of physique may persist for some years beyond the usual age of puberty. We cannot always get definite proof that such cases are related to a deficient thyroid function. It is simply a clinical fact that such children frequently are very much benefited by thyroid treatment; to such a degree, in fact, that there can hardly be any doubt as to the origin of the condition.

The thyroid function is undoubtedly influenced by menstruation and pregnancy, and it is not always changed in the same direction. There are many indications that, with some women, the thyroid function is decidedly increased during menstruation. The gland itself increases in size, and this change is accompanied by many of the phenomena of hyperthyroidism exhibited in mild form. With other individuals, there are just as definite reasons for believing that the menstrual function requires more thyroid secretion than the normal condition, and that, without the corresponding increase in the total quantity of thyroid available, the result will actually be a diminution in the external evidences of thyroid activity. Many cases of Graves' disease have decided exacerbations during menstruation, while others have just as decided an improvement, though the latter instances are comparatively rare. It is likewise true in pregnancy that the thyroid gland may increase in size and be accompanied by increased function. At least 50 per cent. of Graves' patients are to some extent relieved during pregnancy, but the distressing con- 
ditions are very likely to recur immediately following parturition. On the other hand, what have apparently been mild cases of Graves' diseuse are lighted up into an active, distressing condition by pregnancy, so that it is not at all unusual for a mother to date the development of the disease with the birth of one of her children. I have recently observed a young woman, who had a mild case of Graves' disease complieated by epilepsy, who during pregnancy was entirely relieved of all the symptoms of hyperthyroidism as well as of evidences of epilepsy. She gained in weight 65 pounds during the term of pregnancy, and was perfeetly well throughout. At its ternination, both conditions recurred, and both were again entirely relieved, at least during a period of some months, by the use of antithyroid serum. It is, therefore, of very considerable importance for a practitioner to study carefully the type of the metabolic disorder which accompanies menstruation and pregnancy. During the past two years I have followed a number of eases of toxic menstruation in women generally over thirty-five years of age. During a period of two weeks a month they would be entirely well. Prior to the onset of menstruation very marked nervousness developed,--sleeplessness, vomiting, almost a maniacal condition, accompanied by a marked alteration in the nitrogen partition,- -which, perhaps in some of these cases, was to be explained simply by the starvation which they underwent, but certainly in others was not to be so explained. In those which were carefully followed it was found that the administration of thyroid in tablet form ox hypodermically, beginning about four to six days before the expected onset of menstruation, would afford entire and absolute relief of all distressing features of the period, and the patient would pass through this phase with practically a normal metabolic condition. It is, therefore, I believe, decidedly worth while, and this has been the experience of many others, to study carefully the type of menstrual disturbances, and, if it is apparently related to a diminished thyroid function, to try to relieve the distress by the thyroid administration. In my own experience this has been most certainly accomplished by giving thyroid hypodermically, but tablets may in many cases serve as well. Likewise, I believe that many pathological conditions which accompany pregnancy may be entirely. or very largely relieved by the administration of the suitable amount of thyroid.

As an excellent example of this form of treatment, I may refer to one case which was recently published by Ward, in which a patient who had previously had Graves' disease became pregnant, and suffered from all of the distressing phenomena of toxic romiting, but whose condition was completely relieved, and the pregnancy allowed to go to full term, by the use of thyroid extract. In this instance it was given hypodermically. I have been interested in trying thyroid extract in a few cases of puerperal eclampsia, which were not seen until the eclamptic sumptoms were well developed and the patient in a desperate condition-in some cases before, and others after, delivery. In four of these cases there was a high bloodpressure, the patient was unconseious with stertorous breathing, temperature by rectum from $103^{\circ}$ to $105^{\circ} \mathrm{F}$., and convulsions frequent and severe. 
In these cases the hypodermic administration of from 6 to 12 c.c. of a 1 per cent. solution of thyroid extract was followed by relief of all symptoms. I do not wish to maintain, by any means, that this is the only treatment which these cases should get, or that it is by any means to be looked upon as an infallible and universal remedy for such a condition, but I think it quite worth while to use it in an emergency, inasmuch as it will not interfere with other means of treatment and will do no harm if it does no good. For many metabolic disorders accompanying menstruation and pregnancy, thyroid therapy is, therefore, to be regarded as a method having considerable value.

Its use has been advocated by many therapeutists in the treatment of epilepsy. Epilepsy, however, includes such a diversity of conditions that one can scarcely take the space in an article of this sort to state when it may be of value and when it may not. In fact, that question can only be answered in a large percentage of cases by actual trial. I have seen many patients who have convulsive seizures, perhaps as an accompaniment of some metabolic disorder, who have experienced great relief by the use of thyroid extract. This relief was improvement in the general metabolic condition of the individual, a diminution in the number of convulsive seizures, and improvement in the mental condition. There are no definite rules which can be laid down in regard to the dosage, nor can we yet give an accurate clinical description of the type of cases that is to be benefited. There is no question that, in many instances, a patient is made decidedly worse, and an increase in the number and severity of seizures may follow thyroid administration. Such a result is more likely to occur in the high-strung, nervous epileptic than in the rather dull and stolid individual.

Blood-pressure in some cases of arteriosclerosis may be reduced, and the patient made more comfortable by thyroid administration. 'The metabolic disorders which are associated with lack of thyroid function may express themselves in various ways. In some individuals it appears that the toxic products cause a chronic arthritis. In others, the skin is the sensitive tissue and prurigo or psoriasis may result. There is no question in my mind that many cases of so-called rheumatism, which are in fact conditions of chronic arthritis of metabolic origin, may be completely relieved by thyroid treatment; and, in some instances, when the condition resembles the so-called arthritis deformans, or has progressed so far that ankylosis or complete disorganization of the joint has occurred, accompanied by evidences of inflammatory reaction and severe pain, thyroid therapy may be extremely valuable. In this type of case it is that we need a sharper differentiation and a more accurate diagnosis before attempting or condemning a given therapeutic measure. During the last two years I have administered thyroid to a large number of cases of chronic arthritis with good results, and I am inclined to believe that this is due to an improvement in the metabolic disturbance which was precedent to the difficulty.

Thyroid therapy may be a useful measure, or perhaps an absolutely essential measure, in the treatment of certain skin diseases of metabolic 
origin. Johnson has recently treated, in a Cornell clinic, a number of cases of prurigo, by thyroid extract which I furnished him, with most gratifying results. One case, which had lasted thirteen years, was after some months' treatment completely cured and has staid so. Other cases of psoriasis which had resisted all the usual means was likewise cured, and I think there can be no doubt that it should be used in this type of disturbance.

'Thyroid has likewise been used with some benefit in the treatment of malignant growths. In my opinion a considerable percentage of cases may be relieved of pain, the rate of growth markedly diminished, and a great deal of relief to the patient obtained. It cannot, by any means, be looked upon as a specific for this purpose, and many patients cannot take it at all, because the heart or nervous system is too easily disturbed by it; but I have administered thyroid to a large number of cancer patients in the last two years, and, from my experience, all that can be promised is that, if the patient can take considerable doses of it, relief from pain, diminution in the rate of growth, and, in some cases, a very marked diminution in the size of the growth already attained, may be expected, but it is in no sense curative.

Thyroid has been a favorite remedy for obesity, and some of the most distressing physical wrecks have been caused by excessive dosing for this purpose. 'There is no question that thyroid increases the rate of oxidation, and that it may cause marked diminution in weight. Most of the patent anti-fat remedies depend upon this substance for their effectiveness, and there can be no more dangerous use of a valuable medicine than its sale in this form. If a physician uses it for this purpose, he should not attempt a rapid reduction. A majority of the patients who will come to him for this purpose will be women, and they, in most instances, wish to reduce rapidly and without the annoyance of exercise or restricted diet. In a great many cases thyroid administration in fairly moderate amounts will result in a diminution of 20 to 30 pounds fairly promptly, but to go beyond this point it is necessary to use an excessive dosage-therefore, a dangerous dosage. I believe it is unwise and unwarranted to rely upon thyroid alone for the purpose of reducing weight, and I believe it is dangerous to attempt a rapid loss of weight. I never wish to see a patient lose more than two pounds a week, and much prefer that they shall lose a pound a week, and that this loss shall be accomplished by diet, exercise, bathing, and such measures quite as much as by the use of thyroid. It requires patience, both on the part of the physician and patient, to accomplish such a result. I have recently treated a young woman of twenty-two for this purpose. In the beginning she weighed 254 pounds, and was extremely weak physically. She had had up to this time an enormous appetite, which she liad gratified. During the next nine months she redliced to 176 pounds and was in good health. The largest dose of thyroid which she took at any time was ten 2-grain tablets, containing 5 per eent. of the pure thyroid heretofore described.

Most of the conditions for which thyroid is efficacious develop because of a metabolic disorder, and the reason thyroid is beneficial is prob- 
ably because this metabolic disorder is to some extent corrected by the use of thyroid. In addition to these specific conditions which have been mentioned, thyroid has been used for a great variety of purposes, but they are all to be looked upon as metabolic in origin, and as having some relation to a disordered or diminished thyroid function.

\section{THE PARATHYROID GLAND}

It is only since Sandstrom's discovery in 1880 that we have known of the existence of the parathyroid gland; and that the differing histological structure of this tissue corresponds to an independent function is a fact which has been established within the last three years. Even yet there are a few investigators who believe, with Forsythe, that "the parathyroids are glands essentially thyroid in nature, possessing no peculiar function, but engaged in the active secretion of the same substance as the thyroid gland." In view, however, of the very positive results which have been attained by careful operative work within the last three years, Forsythe's conclusion is entirely wrong, and the consensus of opinion at the present time agrees with the generalization of Jeandelize, that removal of the parathyroids causes death from the acute development of tetany, while simple thyroidectomy is followed by a chronic cachexia. It is only when all the parathyroid tissue is removed that these acute symptoms develop; and the varying opinions have been caused in part by the different anatomical relationships which these glands have in various species of animals. Certain classes of animals show the symptoms somewhat more promptly than others. Thus, the symptoms are more easily demonstrated in cats and dogs than in rabbits and rats, but, even in herbivorous animals, the complete removal of parathyroid tissue will result in the development of these symptoms in more or less typical fashion. In collaboration with Berkeley I have recently completed a series of experiments, using dogs, which entirely confirm the conclusions of Jeandelize. Parathyroidectomy, when complete, is followed so directly and invariably by tetany that those investigators who deny this relationship could not have been successful in their operative methods.

The precise origin of symptoms following complete parathyroidectomy is yet somewhat obscure, and the relationship which these symptoms may have to certain clinical conditions, such as tetany in children, eclampsia, epilepsy, gastric tetany and tetany occurring in pregnancy, and lactation, is as yet undefined. These clinical conditions have certain resemblances to the disturbance following parathyroidectomy, but there is no satisfactory demonstration yet, physiologically or pathologically, that they are of the same origin. We may summarize the present ideas in regard to this matter by stating that one set of investigators maintain that a deranged calcium metabolism is the origin of the disturbance, while others believe that it is an essential damage to the nitrogenous metabolism. McCallum and Voegtlin find that parathyroidectomy is followed by an actively increased excretion of calcium, amounting to a calcium diabetes. The cridence in favor of what may be called the calcium deficiency theory may be summarizcd as follows: 
1. There is a low retention of calcium during the eclamptic period of gastric tetany, a condition probably similar to that of tetany parathyreoprivus (Cybulski, Pineles).

2. Surgical removal of the parathyroid gland is followed by an increased excretion of calcium, and the brain of an animal dead of tetany contains less calcium than is found in the brain of a normal animal (McCallum and Voegtlin).

3. Intravenous administration of a soluble calcium salt promptly relieves the symptoms of tetany parathyreoprivus.

4. The inactivation of the circulating calcium by means of intravenous injections of trisodic citrate is accompanied by muscular twitching and convulsions similar in character to those of tetany, and these may be relieved by the injection of soluble calcium salts in sufficient quantity to restore the equilibrium (Sabbatini).

The evidence in favor of the symptoms having their origin in a metabolic toxin may be summarized as follows:

I. The symptoms have a central origin.

II. Symptoms are shown best in young animals, and are more severe if the animal has been kept on a meat diet.

III. The symptoms have close relation to certain clinical conditions which are accompanied by severe nutritional disturbances.

IV. Gastric tetany is accompanied by severe metabolic disturbance, it has similar symptoms, and is promptly relieved by intravenous calcium injections and by parathyroid nucleoproteid.

V. Bleeding, followed by intravenous infusion, relieves the animal, a procedure well suited to free the body from a circulating poison.

VI. Symptoms are promptly relieved by the injection of fresh parathyroid nucleoproteid.

VII. Injection of known simple poisons, such as ammonia and xanthin, produce symptoms which can be promptly relieved by injection of calcium or strontium salts, similar to the relief obtained by the same means in tetany.

VIII. Parathyroid tetany has a deranged metabolism, accompanied by a large increase in the excretion of ammonia.

It is certain that the symptoms are caused by removal of the parathyroids, and that they may be relieved by administration of parathyroid proteid. For therapeutic purposes, the most effective method of adninistering the gland extract is by hypodermic injection, and I give below the method which I have found effective for preparing this proteid, and the properties and the physiological action which it has.

Method for Preparing Extract.-'The carefully cleaned glands should be cut into small pieces with scissors, and the comminuted tissue then ground to a fine pulp in a large mortar, with the help of enough sand to give the whole mass a moist, pasty consistence. The grinding with sand is a slow, laborious process, but the success of the extraction depends in no small degree upon the care with which this portion of the work is done. 'The crushed glands are next shaken for two hours at room temperature with six to eight volumes of physiological salt solution, to which have

VoL I-36 
been added two drops of 10 per cent. sodium hydroxide solution. 'The jar should now be transferred to the refrigerator, and the extraction allowed to continue for eighteen to thirty-six hours. Filtration first through gauze, to remove the fat and coarser particles of tissue, and then through moderately thick paper, will give a clear extract which should be preserved by the addition of chloroform.

The Properties of the Proteid.-1. 'The nucleoproteid of the parathyroid, when freshly prepared, is equal to the whole gland in relieving the symptoms of acute tetany in dogs.

2 . The globulin is of no value in relieving tetany.

3. Boiling the nucleoproteid solution, or heating it to $80^{\circ} \mathrm{C}$. for onehalf hour, completely destroys the activity of the nucleoproteid.

4. The nucleoproteid is most active when freshly prepared, and rapidly deteriorates when kept in solution or in suspension at refrigerator temperature. Freezing also destroys its activity, although not so rapidly as room temperatures.

5. Tryptic digestion or the action of pepsin and hydrochloric acid for forty-eight hours severely injures but does not completely destroy the activity of the nucleoproteid.

6 . The nucleoproteid will relieve tetany if given by mouth, but is much more quickly and certainly effective when given subcutaneously or intraperitoneally.

Therapeutic Use of Parathyroid Products.-The particular condition in which parathyroid extract is of value is undoubtedly in the treatment of tetany following operations upon the thyroid gland, in which, presumably, the parathyroids have been removed, with the resultant characteristic symptoms. The acute condition is a dangerous one and demands immediate treatment. There is no question that instant relief may be obtained by the injection of the calcium lactate. This is only temporary, however, and should not be used to the exclusion of other therapeutic measures.

The subcutaneous injection of the parathyroid proteid mentioned above is likewise indicated. The dose of calcium lactate that may be given to an average-sized individual intravenously for this purpose varies from 2 to 6 grams. It is probable that the smaller injections will for a few hours control the active symptoms. In two cases of gastric tetany I have given an intravenous injection of 3 grams of calcium lactate in 500 c.c. of normal salt solution every twenty-four hours, for a period of four days, with the result of a complete control of the symptoms. In case of acute tetany developing after the thyroid operation, an injection of 1 c.c. of a 1 per cent. solution of the parathyroid proteid was given every three hours for two days, with a result of complete control of the symptoms and recovery of the patient. It is presumable that in this case there was only a temporary hypofunction of the parathyroid.

The symptoms of tetany are very closely related to those of eclampsia. We have found that after complete parathyroidectomy a diet of bread and milk tends to delay the symptoms, while a heavy meal of meat is likely to cause their earlicr development. In fact, I have occasionally 
fed an operated animal a heavy meal of meat in order to bring on the symptoms of tetany at a convenient time.

Because of these relations, it is worth while to compare the clinical with the experimental conditions. I quote from a publication made elsewhere on this subjeet: "The nitrogen partitions in the urine in such patients are in most cases abnormal, with relatively high ammonia, high rest nitrogen, notable quantities of kreatin, but with diminished urea and kreatinin in excretion. Such findings indicate severe nutritional disturbance, and the possibility of metabolic toxins being responsible for the symptoms in such cases is commonly accepted. The means of relief in acute conditions by the very active use of the hot pack, diaphoreties, purgation, the high saline irrigation, stimulation of excretion through skin, bowel, and kidney through the vigorous diuresis, and occasionally by bleeding and saline infusion, are all based on the belief that the symptoms are caused by an active toxic substance in the circulating blood. A similar method of treatment is of elinical value in the treatment of parathyroid tetany. 'The fact that, under such disturbed conditions of nitrogenous metabolism, a meat diet gives rise to metabolic products which may provoke convulsions suggests that the meat diet has more than a passive role in producing the symptoms of parathyroid tetany." The administration of calcium salts or parathyroid extract by stomach is not a suitable and effective means of controlling symptoms in these cases. The chronic tetany following thyroid operations may need parathyroid treatment extended over some months. Halstead and Poole have reported satisfactory treatment of such cases by the hypodermic administration of parathyroid proteid which I furnished them.

The relation between parathyroid tetany and the other forms mentioned above is so close that it may be worth while to attempt treatment of some of those conditions by similar methods, but there is no satisfactory experience as yet available upon which to base any predictions regarding the possible success of such a method.

Berkeley has used parathyroid extract in the treatment of a large number of cases of paralysis agitans. Such a method is based upon the belief that the parathyroids have a deficient function in this condition. The evidence, from autopsy material reported by 'Thompson and others, does not support this contention, but we cannot always draw accurate conclusions in respect to the functional capacity of a tissue by examination of its histological condition. Berkeley states that, to be successful, a fresh, active preparation must be given in small doses over long periods of time. Of 26 cases treated, 18 grew progressively better during the whole period of the treatment. Other elinicians have not had so favorable results, but this has, in part, been due to the fact that they did not have satisfactory preparations, and, furthermore, that they did not follow the method for a sufficiently long time. Recently many successful attempts at parathyroid grafting have been reported, and it is not improbable that such a method of replacing an inactive gland may become a valuable therapeutic method. 


\section{THE PITUITARY}

The pituitary body is one of the ductless glands whose function is very imperfectly known. There are two portions, an anterior and a posterior lobe, the anterior lobe having the histological structure of a gland, and the posterior lobe resembling nerve-tissue to some extent in its structure. 'These two lobes have a different embryological origin, and, as far as our information at present goes, it seems certain that they have quite different functions.

The space available, and the purpose of this article, do not permit detailed description of our present knowledge of the normal function of this tissue. Its anatomical location is such that experimental removal has been accompanied by extraordinary difficulties, and only within the last two or three years has the work of Cushing in this country, and Marinesco and Paulesco abroad, given a satisfactory degree of harmony in experimental results.

The total removal of the pituitary body leads, in a vast ntajority of cases, to a development of symptoms of characteristic cachexia, invariably followed by death in from thirty-six to ninety-six hours. Cushing has been successful in removing the two portions separately or successively, and his conclusion is in agreement with the other investigators in the belief that the anterior lobe is responsible for the nutritional disturbances consequent on removal, and that extracts of the posterior lobe contain substances acting on the heart, blood-vessels, and temperature control. Oliver and Shaffer and Howell made the first studies on the blood-pressure-raising constituents of the gland, which has an action somewhat similar to that of the adrenal. Our present knowledge in regard to the function of the pituitary has been well summarized by De Lille. We have not isolated the active principle of the gland.

Although the histological structure and the results of operative removal agree in emphasizing the importance of the anterior lobe, the physiological results obtained by administering extract show, on the contrary, very marked effect with posterior lobe preparations, and very little effect from that of the anterior lobe.

Extract of posterior lobe causes elevation of arterial pressure, strengthens and slows the heart-beat. This effect is slower in its development, but it is much more lasting than that produced by the adrenal. Its effect upon the kidney is especially marked. After a short period of vasoconstriction there is a period of vasodilatation, accompanied by a very marked diuresis. There is apparently slight elevation of temperature accompanying these phenomena. Pituitary extract likewise stimulates metabolism of nitrogen, phosphorus, and possibly that of calcium, so that there may be some ground for Schiff's belief that the gland exercises special control over the development of the osseous system. Bell has studied the excretion of calcium, as influenced by pituitary extract, and comes to the conclusion that calcium excretion is markedly stimulated. He, therefore, believes that the overdevelopment of the bones in acromegaly is due to a loss of this function. From histological examina- 
tion of other organs, De Lille concludes that continued treatment with pituitary extract results in hypersecretion from the hypophysis, transformation of the thyroid activity amounting to a hypofunction in some of its activities at least; hypertrophy and hyperplasia of the adrenals; congestion of the kidney, with increase in volume of the glomerulus. We know very well that the glands of internal secretion have quite remarkable interrelationships, which account for the complexity of the disturbances which arise in the course of their lisorders.

Many diverse clinical conditions have been associated with abnormalities in function of the pituitary. Marie was the first to call attention to the apparent relationship between acromegaly and tumor of the pituitary, and in nearly all instances there have been tumors of the anterior lobe corresponding somewhat to a goitre histologically, so that it would appear that hyperactivity of the anterior lobe results in a fairly definite disease, acromegaly. As accompaniments of pituitary tumors, other phenomena, such as polyuria, glycosuria, infantilism, impotence, and a very marked pathological obesity and marked muscular weakness, have been described.

Therapeutic extracts on the market at the present time afford the following preparations: (1) The whole gland dried in the form of powder or tablets. (2) The anterior and posterior lobe separately dried and powdered, or in the form of tablets. (3) Fluidextracts of the whole gland. (4) Extracts of either the anterior or posterior lobe.

Such clinical observations as are available indicate that extracts of the anterior lobe have very little, if any, therapeutic effect, while the extracts of the posterior lobe have marked effects, equal in character and extent to that of the whole gland. The pituitary extract in suitable doses causes a marked elevation of arterial tension, decrease in weight, a marked diuretic effeet, diminishes profuse perspiration, diminishes excessive pathological appetite, induces sleep, relieves marked asthenia, and relieves certain psychic difficulties, and exercises a marked control over the nutrition of the bones and muscles (De Lille). Below is given a brief table, showing the symptoms which are usually associated with increased and with decreased pituitary activity.

Decreased ACtivity.

Low blood-pressure.

Tachycardia.

Sudden heat sensations.

Prof use sweating.

Diminished secretion of urine.

Anorexia.

Profound asthenia.

Inereased obesity with some skin difficulties.

Insomnia.

Occasionally, signs of intercranial pressure.
InCreased ACtivity.

Increased blood-pressure.

Polyuria.

Glycosuria.

Nutritional difficulty, occasionally amounting to obesity.

Hypertrophy of the bones.

Psychic difficulties.

Pronounced somnolence.

Impotence.

Evidence of diminished thyroid activity.

Occasionally, indications of a pituitary tumor.

The clinical conditions deseribed rarely occur in a pure form. 'The conditions are generally extremely complex, and it is not possible to give 
definite rules in regard to the dosage which must be employed in any given instance. 'This is particularly so because the various preparations on the market vary in their activity. Each case must be carefully watched, and the administration of the extract guided accordingly. Much more prompt effects will be obtained when the extract is administered by hypodermic injection. The effects come on quickly. Doses vary from 10 to 30 minims, and the control of the effects is much more accurate when given in this way.

In other conditions than those associated directly with the pituitary the extract has been used with varying success. De Lille recommends it highly in the treatment of exoph thalmic goitre, and other clinicians have used it with reported success as a cardiac stimulant in infectious conditions. Until its physiology is somewhat better known than at present, it should be used with the greatest care, because it is a powerful substance which has great capabilities for doing harm.

\section{THE ADRENAL}

The adrenal gland has an especial interest in organotherapy, because from it there has been obtained a definite chemical substance which has been prepared synthetically, and which expresses in its physiological action a large portion of the function of the gland. Anatomically, the adrenal has two distinct portions, the cortex and the medulla. Concerning the function of the cortex we know very little. The characteristic blood-pressure-raising constituent is found in the medulla. If the vagus nerve is in a functional condition, adrenal extracts are said to cause a slow heart-beat with rise of blood-pressure. A very small amount of the epinephrin hydrate, first isolated by Abel, is sufficient to do this. The action is a temporary one, and in a few minutes the blood-pressure and heart-beat return to normal, probably indicating that the substance causing these effects is destroyed. The substance producing these effects is found in the blood taken from the adrenal vein, thus giving strong probability that there is a continuous production of this substance, and Dreyer has shown that the amount produced can be considerably increased by stimulation of the splanchnic nerves. The adrenal extract causes a marked constriction in the blood-vessels, probably due to a direct effect upon the muscular wall in the vessel, since it may be obtained after complete disconnection of the vasoconstrictor centre. This action, however, is not exhibited on all varieties of plain muscle, but only upon such as are innervated by the sympathetic nerves.

Removal of the adrenals invariably causes death in from twelve to sixty hours, with symptoms of profound prostration, great muscular weakness, and loss of vascular tone. 'The function that the gland serves is not definitely known. There are two theories in this respect-first, that the gland furnishes to the circulation an internal secretion absolutely necessary to the functional activity of many tissues. The second, which has much less evidence in its favor, is that the gland destroys certain metabolic toxins, and that, when a gland is removed, the accumulation of these toxins causes death. 
Various adrenal preparations are now on the market, such as: (1) The whole gland dried and powdered. (2) Varied preparations of the active principle of the gland which pass under various trade names, such as adrenalin, suprarenalin, suprarenin, adrenin, etc. 'T'hese active principles may be obtained in solutions of various strengths; the most common form is a dilution of 1 in 1000 . In addition, there are now various ointments, tablets, suppositories, etc., containing these active substances.

'These preparations are administered in a variety of ways-by stomach, by absorption from the mucous membrane of the mouth, by suppositories in the rectum, by local application to mucous membranes, and by subcutaneous and intravenous inoculation. 'There has been a dispute among physiologists and clinicians as to the proper method of administering the extract and the effects it may be expected to produce. 'The physiologist has been primarily concerned in its reaction upon the heart and blood-vessels and not so much its effect in disease. Its effects are both local and general. When used upon the mucous membrane, the most pronounced local effect is a blanching of the same, due to the local constriction of the blood-vessels, and some of its most valuable uses are found in this particular property. Advantage is taken of this fact to treat hemorrhages of mucous membranes, reduce a congestion in an inflamed mucous membrane, to render bloodless a small field in operation, to delay absorption of local anesthetics, thereby prolonging their effects.

When given intravenously, the purpose is to act as a tonic to the vascular system in cases of shock or sudden heart failure. In addition, there can scarcely be any doubt that something further than the local effects on the mucous membrane may be expected when adrenalin is given by the stomach. There is little physiological evidence upon which to base this conclusion, but the clinical evidence is abundant, and it is at present scarcely to be questioned. Certain degrees of sensitiveness to adrenalin are found in different patients, corresponding somewhat to the sensitiveness which many persons show to thyroid, and for this reason, when adrenal extract is to be given a patient in other conditions than those of emergency, its administration should be begun in very small doses, gradually increased until the desired effect is obtained. For lypodermic injection, a dose of 10 to 15 minims of a 1 in 1000 solution is sufficient to produce the desired results.

In the special lines of therapeutics adrenalin is put to a variety of uses. I shall not describe these in detail. In inflammatory conditions of the eye, ear, nose, and throat it has been used, either alone or in combination with other substances, such as atropine, eserinc, cocaine, etc., to diminish the congestion and reduce inflammation. In the nose and throat it is commonly used in the form of a spray by mixing a quantity of it with normal saline solution, and is very effective in diminishing the turgescence of a mucous membrane in the simple inflammatory conditions. For hemorrhages of the mucous membrane, such as bleeding from the bladder, from nose, from intestinal ulcers, and from the uterus, it has been used with very beneficial results, but it must be remembered that 
the constriction of the vessels which it causes is only temporary, and is often followed by a marked dilatation, so that, unless a coagulum forms during the constricted phase, harm may result. Furthermore, for the control of hemorrhage, the use should be local. If given intravenously, the tremendous rise in blood-pressure which follows is likely to increase the hemorrhage and may prove exceedingly dangerous to the patient.

As a cardiovascular stimulant, its most important use is in the treatment of shock, and Crile has shown that an injection backward toward the heart through an artery of a saline solution containing adrenalin will often revive the heart some minutes after it has ceased beating. In certain diseases it has come to have almost the value of a specific in relieving the attack. In the treatment of hay-fever, adrenalin will prove effective in relieving for a time the severe paroxysms of sneezing. For this purpose, it should be used either as a spray or in an irrigator in a mixture with normal salt solution. If the nasal cavity is irrigated with a mixture of 2 or 3 ounces of normal salt solution, in combination with 30 minims of adrenalin, at as high a temperature as can be comfortably borne, there will be immediate relief. The mucous membrane will diminish in size, the breathing is easier, and marked relief obtained. The relief is not permanent, however. The irrigation will need to be continued at intervals in order to prolong the effect. If used in combination with cocaine, the effect of the irrigation is generally more lasting. Adrenalin therapy in hay-fever, although it produces a very marked relief, and is a valuable therapeutic method, should not be looked upon as being in the same category as the serum treatment devised by Dunbar. There have been some untoward results from the continuous use of adrenalin in hay-fever, and adrenalin treatment of hay-fever should, therefore, be considered only an emergency treatment to tide over an attack.

Asthma.-In severe paroxysms of asthma almost complete and immediate relief can be obtained by the subcutaneous injection of 10 to 15 minims of 1 in 1000 adrenalin. 'This, however, is only a temporary relief. It is in no sense permanently curative. In some instances relief will last only one-half to three-quarters of an hour, and again it may last three or four hours to a day. At any rate, the relief is only temporary. No definite rule can be given as to how frequently adrenalin may be administered to an asthmatic for this purpose. Such a question can only be answered with respect to a definite case when all the conditions are known.

Addison's Disease.-Addison's disease resembles in many respects the phenomena observed on removing the adrenal glands in animals. It is, of course, not an acute process In most instances it pathologically is accompanied by some lesion of the adrenal-in a large majority of cases a tubercular destruction of the adrenal. From this standpoint, adrenal therapy is indicated. No definite promises can be made as to what may be expected from such treatment. Statistics show that a very small percentage of patients with Addison's disease are reported as cured by this treatment. Most of them are either not cured or somewhat improved. It is a difficuli matter to replace, by therapeutic administration of adrenalin, the continuous function of the gland. In most of the cases 
treated by adrenalin or adrenal extracts, these have been given by mouth, whereas in nature the secretion enters the circulation directly through the adrenalin vein, so that the logical method is to use hypodermic administration of small doses of adrenalin administered frequently. As far as I can learn, no cases have been treated by this fashion except one which I treated a year ago, giving 5 minims of 1 in 1000 adrenalin ever: hour, except during six hours of sleep. 'This required, of course, a constant attendance from the nurse and was somewhat troublesome, but I am convinced that the effects were very favorable. 'The patient inproved to such an extent that he left the hospital and passed out of my observation, and what his future was I am unable to learn. 'The dried whole gland given by mouth has been used in many of these cases, and I have myself treated a number by administering the proteids from the adrenal gland by mouth with some apparent benefit, the blood-pressure gradually increasing, general muscular tone improving, the gastro-intestinal disturbance elearing up, and the patient being mueh better. 'This proteid certainly did not contain adrenalin, and I should at present be unwilling to make any statements as to the therapeutic value of the method. I believe, however, that the most favorable method of treating these cases is to give adrenal extract in small doses frequently by hypodermic administration. It hardly seems probable that the isolated adrenalin represents the whole function of the gland.

Adrenalin is used in a great variety of conditions other than those which have been deseribed here-as a cardiae tonic in infection, as a specific for certain atypical types of Graves' disease, and to control paroxysms in whooping-cough.

\section{THE THYMUS GLAND}

We, as yet, have very little knowledge as to the exact function of the thymus gland. During embryonic growth, and during the growing period of the young animal before puberty, it has a size and structure which indicate that it has some important function to fill in the organism. With the development of the sexual glands the thymus retrogrades, and may be almost completely absorbed or replaced by fatty tissue. This is the usual course of events. In some individuals the thymus does not atrophy, but remains persistent. There appears to be some definite relationship between these two sets of glands. If the sexual glands be removed in the young animal, the thymus does not atrophy, and there are certain peculiarities of growth which such an animal shows. 'The bones are larger, the animal is heavier in weight, and has less of the peculiar characters incident to the sex. On the other hand, if in the young animal the thymus gland be removed, the sexual glands are certain to develop at an earlier period. However, concerning this point there is yet some dispute. The thymus does not fill that vital function in the body which the parathyroid, the adrenal, and the pituitary do. In case it is removed, there is not an immediate and dramatic death. In fact, the observations differ as to whether any serious results at all may follow such 
a removal. The most recent experiments indicate that, if the thymus be completely removed, a chronic nutritional disturbance follows, which may not in itself be fatal; but which renders the animal an early victim to his usual surroundings. We cannot as yet associate a distinct disease with the overdevelopment or lack of development of the thymus gland. It apparently has close interrelationship with other glands and with the diseased conditions which affect other glands. We know that in a normal animal lack of food, starvation, or infection causes a very rapid decrease in the size of the glands. We know that indviduals in whom the thymus persists in an enlarged form sometimes suffer sudden death for no discoverable cause. They, likew ise, easily succumb under unfavorable conditions, and are not resistant to infections. In certain diseases it is nearlv always enlarged, as status lymphaticus, acromegaly, exophthalmic goitre, and often in Addison's disease.

Because of our lack of knowledge in respect to its pathology and function, there has been very little therapeutic use at the present time. Some years ago it was regarded as a specific in the treatment of exophthalmic goitre, and at the present time there are reports in literature occasionally of cases which have been markedly benefited by the use of thymus extracts. Because of its apparent functional condition during a growing period in the young animal, it has been used to influence the growth in children who are undersized and have been underfed. Animal experiments made in my laboratory during the last year seem to indicate that it may, under such circumstances, have very beneficial effects. Certain animals were treated with intraperitoneal injections of fairly large doses of thymus extract, and were observed to increase in weight and health more rapidly than the control animals kept under the same conditions. Some years ago its use was advocated in the treatment of cancer (Fotheringham). Recently, Gwyer has advocated its use for this purpose. I have personally used it in combination with thyroid and pancreas. in the treatment of malignant growths. The preparation which I have used has been the pure proteid from the glands in the following proportions -5 per cent. thyroid; 20 per cent. pancreas; 75 per cent. thymus-given in doses as high as 50 to 60 grains a day, with a result of marked diminution of pain in a very large percentage of cases. How such a result is accomplished we know absolutely nothing. Thyroid alone, or thyroid with pancreas, will accomplish the same results, but in many instances the heart will be somewhat disturbed by a dose of sufficient size to accomplish good results. It is not in any sense to be regarded as curative in this condition.

\section{PANCREAS}

For a number of years the islands of Langerhans, in the pancreas, have been believed to furnish an internal secretion having marked physiological value in carbohydrate metabolism. We cannot as yet draw any definite conclusions from our knowledge of the physiology and pathology of this glaud as to what this secretion is or how it acts. Cohnheim main- 
tains that, if pancreatic extract is added to muscle extract, the mixture causes much more rapid destruction of sugar than would otherwise occur, and his experiments have been widely quoted in support of the existence of a definite internal secretion. Attempts to repeat these experiments by other investigators have not been successful.

'Total removal of the pancreas will in most cases produce an acute death, accompanied by many of the symptoms of diabetes. Such a death and such symptoms cannot he averted by the use of any kind of a thymus extract with which we are now familiar.

With the above facts as a basis, pancreatic preparations of various sorts have been used extensively in the treatment of diabetes, but as yet we have no convincing evidence that they are distinctly beneficial. When facing so desperate a condition as diabetes, it may be excusable to use any remedy which promises benefit, but under no circumstances should the use of pancreatic extracts take the place of other measures, such as diet and hygienic eare, which we know to have a distinct effect in modifying the course of disease.

\section{SEXUAL GLANDS}

There is no question that the sexual glands produce an internal secretion. 'This secretion is not one vital to the organism. Attention has already been called to the interrelationship between the activity of sexual glands and the thymus. If the sexual glands are removed before the age of puberty, the thymus does not atrophy as it normally does at this time, and there is, in addition, a lack of development of distinct sexual characteristics. Muscular strength does not develop to the extent it normally does, and such animals are generally fat. Brown-Séquard studied the effects of the administration of extracts of the sexual glands many years ago, and his results attracted wide attention at that time. He stated that when extracts of the fresh testes were injected subcutaneously there resulted a remarkable physical and mental vigor. Under the name of the Brown-Séquard Elixir of Life this extract for a time had wide popular use, and was one of the most potent factors in bringing study. of the internal secretions into scientific disrepute. Pochl has in many respects confirmed Brown-Sequard's opinions, and aseribes them to a definite chemical substance-spermin-and claims for this substance a remarkable action as a physiological tonic. \%oth and Pragel have made ergographic studies, which seem to indicente an inereased ability to do muscular work and a diminished feeling of fatigue after the use of these extracts.

We know that in the sexual glands there are two types of cells, the reproductive cells and the interstitial cells. 'The reproductive cells may be killed or injured by exposure to the $x$-rays, while the interstitial cells remain, as far as histological examination shows, in a functional condition. After destruction of the reproductive cell with the $x$-rals there does not develop any of those changes consequent upon the removal of the organ as a whole. It seems, therefore, probable that interstitial cells furnish the internal secretions. 
The ovaries certainly furnish a secretion which is of vast functional importance. 'The function of menstruation and the development of the decidual tissue certainly have its origin in substances furnished to the circulation by the ovaries. Removal of the ovaries before the menopause is followed by distressing physical and mental symptoms, and there are many reports in the literature which indicate that administration of ovarian extracts relieve these distressing symptoms and also relieve painful or scanty menstruation. Ovarian transplantation has been practised in animals, and in a few instances in the human subject, with the result that the sexual functions have again been renewed. Oxidation may be stimulated by both extracts of the testes and extracts of the ovary.

These substances are furnished directly to the circulation, while therapeutically they have been given in most cases by stomach. Again, there is discrepancy between the method of the physician and that of nature. For therapeutic purposes, sexual glands are obtained in the form of powder or tablets. In most cases the whole gland is ground and dried for this purpose. It seems probable that the corpus luteum has a somewhat different function than the interstitial cells, and some few preparations, said to be those of the corpus luteum alone, are now upon the market. At the present time ovarian extract cannot be recommended for other purposes than the treatment of disorders due either to the premature removal of the ovaries or to a probable functional inability of the ovaries. During the natural menopause the organism, as a whole, is readjusting itself to the new conditions, and ovarian extract may be a valuable aid in temporarily relieving the disturbance while these readjustments are going on. No authentic dosage can be recommended. Preparations of different manufacturers vary so widely, and patients are so differently susceptible, that each case must be treated by itself. 


\title{
TUBERCULIN AS A THERAPEUTIC AND DIAGNOSTIC AGEN'T
}

\author{
By FRANCIS M. POTTENGER, A.M., M.D., LL.D.
}

\section{TUBERCULIN AS A THERAPEUTIC AGENT}

IN the discussion of tuberculin, I make neither excuse nor apology for its employment, and what I write shall not be in the nature of a defense, but a plain statement of the facts which I have been able to gather from careful study and observation during fifteen years of practical experience in its employment as both a diagnostic and a therapeutic agent.

'To be sure, there are those who will disagree with me, claiming that tuberculin is still on trial. For such there is convincing proof in the literature of the last ten years if they will accept it. Scientists might still be expending energy in proving that the world is round if they would attempt to convince every opponent. The time for defense is past. It now remains for the friends of tuberculin to continue studying their remedy and the method of its administration, so that better results may be obtained, and so that it may be made a safe measure for general use.

At the beginning of this discussion, I wish to emphasize the fact that tuberculin is only one of many measures to be used in the treatment of tuberculosis, and that it should always be reinforced by correct living, open air, suitable diet, proper rest, and exereise, and all such measures as tend to keep the patient in a high state of nutrition.

It should be understood that tuberculin is a cure for tuberculosis, and not for consumption. Much of the misunderstanding of this remedy depends upon the fact that many clinicians are expecting tuberculin to replace dead and decaying tissue with new cells, to fill in cavities with functionating lung, to make scar tissue equal normal lung tissue in its functionating powers, to renew degenerated heart muscle so that it can overcome an increased pulmonary resistance, to restore crippled and weakened digestive and assimilative powers to such an extent that they are as good as new; in fact, many have expected tubereulin not only to cure tuberculosis, but to remove all those conditions which have resulted from advanced consumption. This cannot and should not be expected of any remedy.

It is not the province of tuberculin to cure consumption; it is the province and the duty of the medical profession to see that tulerculosis does not become consumption. 
In spite of the good results which have been produced by the proper use of tuberculin, faulty use and failure to realize its limitations are likely to bring disappointment to many medical men who, without sufficient understanding of the remedy, are taking up its employment and expecting it to do the impossible. By such a course there is danger of again bringing reproach upon the remedy and once more casting gloom over the cause of tuberculosis.

What is Tuberculin?-The term tuberculin is used to designate a number of remedies, differing from each other in their method of production, but resembling each other in that they are all prepared from the tubercle bacillus and the culture medium upon which it has grown. 'These preparations differ in the strain of bacilli from which they are made, and in their content in toxin. The same preparation of tuberculin differs when made from bacilli of different virulence; also, when made from the same strain of bacilli grown on different media.

Discovery of Tuberculin.-The discovery of tuberculin was made possible by two observations of Koch: first, that guinea-pigs reacted differently to inoculations of pure culture of tubercle bacilli when they had previously been infected with tuberculosis than when they were healthy. A healthy pig, when inoculated with a pure culture of tubercle bacilli in quantities sufficient to cause an infection, shows no signs at the seat of inoculation at first; but, in the course of ten days or two weeks a nodule appears which breaks down and remains an open ulcer until the death of the pig. If a pig, which is already tuberculous, on the other hand, receives a secondary inoculation of tubercle bacilli, a nodule forms which sloughs out and heals without even showing infection of the neighboring lymph-glands.

The second observation, which bears more particularly upon tuberculin therapy, is the one relating to the behavior of guinea-pigs toward sterilized cultures of tubercle bacilli. When considerable quantities of sterilized cultures are injected into healthy pigs, suppuration occurs, but no constitutional symptoms appear, while even small quantities of the sterilized cultures will kill pigs which are already tuberculous within a short time. However, if the infected pigs are injected repeatedly with very small doses of the sterilized cultures, instead of death resulting, the pigs improve and their lives are prolonged and in some instances a cure is attained.

These two observations show:

(1) That a primary infection with virulent tubercle bacilli fortifies guinea-pigs with an immunity which is sufficient to protect them from a second infection with virulent bacilli.

(2) That there is also an active principle in sterilized dead cultures of tubercle bacilli, which, when administered in repeated small doses to pigs which are infected with tuberculosis, will increase their immunity and aid them in withstanding and overcoming their infection.

The search for the active principle contained in the sterilized cultures which produce this curative effect has given us the many preparations of tuberculin which are at our disposal to-day. 
Basis for Curative Action of Tuberculin.-'The curative action of tuberculin depends, on the one hand, on its powers to increase the patient's immunity to the tubercle bacillus and its toxins; and, on the other, to its action in stimulating the tissue about the foci of infection.

Animal experiments have shown that the greatest degree of immunity is attained by the use of living virulent tubercle bacilli in doses which the animal is able to overcome. In the act of overcoming the virulent bacilli the organism forms sufficient antibodies not only to cure the infection, but to fortify the animal against further infection. Experiments of Römer, Calmette, Vallé, and others show that, when an animal has once recovered from tuberculosis, it possesses a high degree of immunity, and even an animal which harbors an unhealed disease, though it may not be able to heal out its own infection, yet has immunity against an infection from without. Cultures of weakened virulence will also produce an immunity, but of lesser degree, while dead cultures produce one of still lesser degree.

It seems impractical to attempt to treat human beings with virulent cultures of tubercle bacilli or even with cultures of weakened virulence; so, up to the present time, except for a few isolated instances, the attempts to raise the specific immunity of human beings to tubercle bacilli has been confined to the employment of dead cultures and extracts made from the tubercle bacilli.

Many attempts have been made to confer immunity on animals against virulent tubercle bacilli by the use of dead cultures and the various tuberculins. Most of these have been unsuccessful, although a few men have reported successes. 'This failure has been used as an argument against tuberculin, but it does not hold, for the conditions are different. As noted by Koch, in one of the observations above, the animal which is already tuberculous, and the one which is healthy, reacts very differently toward dead cultures of bacilli. 'Tuberculins are inert when injected into healthy animals unless the dose be very large. It would seem that a previous infection with virulent bacilli is probably necessary in order to produce the necessary antibodies to call out the specific action of dead eultures or tuberculins; and it may be that those animals which have been successfully immunized against virulent cultures by tuberculins had a previously healed tuberculous focus which furnished this principle, whatever it may be, which is necessary to call out the specific immunizing action of dead cultures.

Action of Tuberculin.-Koch originally was of the opinion that tuberculin acted by causing necrosis of the tuberculous tissue and a separation of it from the healthy tissue.

Wassermann and Bruck have described an antituberculin which they find in tuberculous tissue and in the blood of patients who have previously been treated with tuberculin. According to them, this antituberculin is found especially in the tissues at the seat of the infection, and its combination with the tuberculin causes the reaction. 'This theory has not been accepted, although the presence of antituberculin has been confirmed by other observers. 
The theory of Wolff-Eisner has much in its favor and is heartily championed by Sahli. He considers that all tuberculins contain fragments of bacilli, and that the tuberculin reaction depends upon the tuberculin being acted upon by the tuberculolysins, which are found in the body of individuals affected with tuberculosis. The injection of tuberculin also stimulates this formation. When tuberculin enters the tissues, it is at once split up by the action of the lysins into other substances, which are far more toxic than the tuberculin itself. 'These very toxic products probably have a part in the causation of the general and focal reactions which accompany the injection of tuberculin. We also assume that they stimulate the body cells to the production of antitoxic bodies, which combine with not only themselves, but with the toxins engendered by the bacilli in the focus of the disease.

Whether this theory is correct or not, it furnishes a working hypothesis which seems to come more nearly satisfying the conditions surrounding the tuberculin reaction than any previously offered.

The specific action of tuberculin depends, first, on its power to stimulate the cells of the organism to the production of immunizing agents; second, on the local hyperemia produced about the tuberculous focus.

Early in the life of the tubercle the blood-vessels are cut off. A patient may have a high degree of immunity and still not be cured of his tuberculosis, because of the inability of the immune bodies to reach the focus of the disease. We often observe patients whose focus of disease, although not healing, yet remains stationary without spreading for long periods of time, the bacilli which pass out into the tissues beyond the limits of the old infection being destroyed by the protective bodies found in the body fluids; but, sooner or later, the disease is apt to spread, because more bacilli will escape from the original focus than the protective bodies can destroy. 'Tuberculin not only increases the immune bodies in the blood, but it also materially aids healing by the hyperemia which it produces. This undoubtedly brings the antibodies into as direct application at the seat of the disease as is possible. It also stimulates absorption of the products of inflammation, and probably in this way aids in the formation of healthy scar tissue.

Clinical Advantages of Tuberculin.-From theoretical considerations, tuberculin should be a valuable aid in the cure of tuberculosis. Practically, if one does not expect the impossible, he will find it so. Many patients who are treated with tuberculin do not get well, but this cannot be counted against the remedy, because a large number of the patients so treated are in the advanced stage of the disease, which offers great difficulties in cure. It is also a fact that tuberculin is rarely given to the best advantage. There are a few men here and there who have been able to get better results than others. 'The probable reason is because they know better how to use the remedy. This is true in all branches of medicine. One surgeon surpasses another, because he knows better how to operate, and also because he handles his cases better; so it is with the physician who administers tuberculin. There is a time to 
give a dose of tuberculin and a time not to give it. 'There is a time to increase the dose and a time when not to increase it. 'The reconnition of this comes only with much experience and close study. We must judge the actual value of tuberculin clinically by the results which are ohtained by men who use it most successfully, the same as we judge the actual value of a surgical operation by the results of the best operators. 'The practical value is another thing, depending upon whether or not it can be employed generally. 'Tuberculin is being employed generally with a measure of success; but the results must not be expected to be the best that tuberculin can produce.

'The practical therapeutic application of tuberculin in several hundred cases of tuberculosis, during the past fifteen years, has convinced me that it is a remedy of great value. Its value is shown by:

(1) A greater percentage of favorable results are obtained where tuberculin is used than where it is not used.

(2) Patients whose condition is stationary and often when progressive, who will not improve under ordinary hygienic measures, often improve at once on the addition of tuberculin to the treatment.

(3) Patients who are treated with tuberculin do not seem to develop as many tuberculous complications and suffer as many extensions of the disease as those who are not treated by it.

(4) 'Tuberculous complications are more often cured when the patient is treated by tuberculin than when he is not so treated.

(5) Patients improve faster when tuberculin is added to the hygienic régime, thus shortening the period of necessary treatment.

(6) Patients who have been treated by tuberculin, and who have obtained an apparent cure, do not suffer relapses nearly as often as those who have not been so treated.

With reference to the effect of tuberculin upon the various tuberculous complications, I consider the following observations as worthy of mention:

Tuberculous laryngitis yields very readily to tuberculin treatment, even many of the severe infiltrations and ulcerations healing. In treating several hundred cases of tuberculosis, most of whom were in the advanced stage of the disease, I have found it necessary to aspirate for pleural effusion only about half a dozen times; and, in patients who have come under treatment when suffering from effusion, with the exception of the few instances mentioned, it disappeared quite readily. Careful measuring of the urine, before and after treatment, showed a rapid increase in the quantity after tuberculin was begun. Fistula in ano, enlarged glands, tuberculous otitis media, and tuberculous conditions of the genito-urinary tract have almost universally improved, and a fair proportion of them have been cured under tuberculin treatment. Even tuberculous enteritis has been improved, and in a few exceptional cases it seemed entirely cured.

Choice of Preparation of Tuberculin.-There are many preparations of tuberculin which have been used in the diagnosis and treatment of tuberculosis. I shall mention only the ones with which I have had per- 
sonal experience, or which I have had the opportunity to study in the practice of other physicians.

I am convinced that tuberculosis can be successfully treated by most preparations of tuberculin. Although some seem to be more valuable than others, yet I believe there is far more in the method of using the remedy than in the individual preparation. When asked what preparation of tuberculin I would advise in a given case of tuberculosis, I almost invariably answer "the preparation which the physician in charge of the case knows how to give," believing that it is more advantageous to be treated by a preparation which may not be so active, but the administration of which the physician understands, than by a better preparation whose administration he does not understand.

It seems, as previously mentioned, that living bacilli produce some substance which is essential to the establishment of immunity. Whether the treatment of tuberculosis could be carried on successfully or not by the use of living bacilli is a question to be settled in the future. While, as mentioned above, it has only been in rare instances that experimenters have been able to immunize apparently healthy animals against infection by the use of tuberculin, yet, when tuberculosis is already present, the organism reacts differently and tuberculins are able to stimulate the cells to the production of an increased immunity.

Recognizing the fact that living virulent bacilli produce the greatest immunity, and realizing the impracticability of using them therapeutically, it seems that in our choice of tuberculin we should prefer those preparations which come nearest containing all the toxins of the bacillus. This would consist in using a remedy containing both the toxins which are given off during the growth formed in the tissues or in the culture fluids, and those found in the bodies of the bacilli. Preparations which meet these conditions are tuberculin (Béraneck); watery extract (von Ruck); TBV. and PV. (Spengler); and a mixture which is often used of B. F. and T. R. or B. F. and B. E., or of Koch's old tuberculin with T. R. or B. E.

Heat does not destroy the power of tuberculin to produce its specific reaction, but it causes a precipitate which probably deprives it of some of its value. I have long been of the opinion that a preparation which has not been acted upon by heat should have greater immunizing value than one which has been subjected to it.

I have had a very satisfactory experience in the employment of tuberculins made from bovine bacilli. I have found them attended with fewer and less marked reactions, and have especially noted their superior influence in lowering temperatures and reducing the rapidity of the pulse. I have accounted for this by the fact that in the administration of tuberculin from the bovine type of bacilli, we are not introducing the same toxin, but one so closely allied to it that it will set the machinery of immunization in motion without increasing the amount of human tubercle toxin in the organism.

As already stated, I believe there is far more in the manner in which tuberculin is administered than in the individual preparation that is 
used; yet I have found it extremely advantageous to have at my command several different preparations. On finding that a patient is not doing well on one preparation, I ehange to another, usually with a favorable result. Especially have I been impressed with the advantage of having the same preparation made from both the bovine and human strains of bacilli. It is rare, in my experience, to find a patient who is sensitive to tubereulins made from both the bovine and human bacilli. He will usually tolerate both, but I have found that the average pulmonary case tolerates the preparations made from bovine bacilli best.

In a few cases, where the patient suffered from active tuberculosis of both the lungs and glands and the lungs and peritoneum, I found that the pulmonary condition improved under the bovine tubereulin, but was irritated by the human, while the glands and peritoneum improved on human, but were irritated by the bovine. Skill is required in adapting the tuberculin to the patient. Long experience demonstrates that some patients will do better on one preparation than on another; and yet, if one were called upon to tell why, he could not do so. If a patient does not do well on one preparation, the inference is often drawn that he is not suited to tubereulin treatment. 'This is erroneous. If I find that a patient does not do well on one, I try another, and by this method I have never found a patient who was suited to treatment to whom I could not suit a tuberculin. 'The reason for the change is, as mentioned above, that preparations vary when grown on different media and when made from different strains of bacilli.

Preparations of Tuberculin.-Old tuberculin (O. T.), Koch's original preparation, is the beef broth, containing 5 per cent. of glycerine, slightly alkalinized, upon which human bacilli have grown for a period of from six to eight weeks, filtered and treated by boiling for an hour, and then concentrated over a water-bath to one-tenth its volume. It is a variable product, depending on the virulence of the strain of bacilli, the development of their growth, and the medium upon which they grow. It contaius 50 per cent. of glycerine. 'The initial dose is 0.001 to $0.1 \mathrm{mg}$.

Bacillus filtrate (B. F.), Denys, is the bouillon culture of human tubercle bacilli filtered through porcelain. It is neither concentrated nor has it been subjected to heat. Denys claims that it is more active than $\mathrm{O}$. 'T., because the latter has been deprived of some of its active principles by heating. The initial dose is from 0.0001 to $0.01 \mathrm{mg}$.

Perlsucht tuberculin (P. 'T. O.), Spengler, is a filtered bouillon culture of bovine bacilli, concentrated in an incubator, at $37^{\circ} \mathrm{C}$. to onehalf of its original volume. It is then restored to its original volume by the addition of glycerine. One cc. equals $100 \mathrm{mg}$. of P.'T. O. 'The initial dose is from 0.001 to $0.1 \mathrm{mg}$.

Tuberculin Béraneck is a 20 per cent. solution of equal parts of an unheated precipitate, caused by the action of 60 per cent. alcohol upon a culture of tubercle bacilli grown upon a special unneutralized medium containing glveerine, but no peptone, and an extract made by treating tubercle bacilli by a 1 per cent. solution of orthophosphoric acid. The 
preparation contains both the intracellular and extracellular toxins. Those who have had experience in the employment of this preparation are convinced that it is less toxic than $\mathrm{O}$. 'T. or 'T. R. 'The initial dose is 0.05 cc. $\frac{A}{128}$ to 0.05 cc. $\frac{A}{320^{\circ}}$

T. B. V. and $P . V$., Spengler, are preparations containing both the extracellular and intracellular toxins, the former being made from cultures of human bacilli and the latter from cultures of bovine bacilli. The bacilli from which these preparations are made are grown upon a special medium. The initial dose is 0.00000001 to $0.0000001 \mathrm{mg}$.

Tuberculin R., Koch, is an emulsion made from living virulent human tubercle bacilli, which have been ground in a mortar and first extracted by water. It is standardized so that each cubic centimeter contains $10 \mathrm{mg}$. of solid substance. 'The initial dose is 0.001 to $0.002 \mathrm{mg}$. of the solid substance.

Watery extract, von Ruck, is made by extracting with water the pulverized bodies of bacilli, which have been previously washed free from culture fluid with water, and then extracted with alcohol and ether for the purpose of remoring fat. The initial dose is 0.001 to $0.01 \mathrm{mg}$. solid extract.

Bacillen emulsion (B. E.), Koch, is a glycerine emulsion of living unheated virulent human tubercle bacilli pulverized, containing $5 \mathrm{mg}$. of solid extract to each cubic centimeter. The initial dose is 0.00001 to 0.0001 solid substance.

Perlsucht emulsion (P. E.), Spengler, is a glycerine emulsion of crushed bodies of bovine bacilli, made in the same manner as B. E. The initial dose is 0.00001 to 0.0001 solid substance.

$N a s t i n-T b-A$ and $T b-L$, Deycke and Much.-While I do not wish to discuss these two preparations from a standpoint of therapy, I feel that it is my duty to call attention to them as probably throwing some light upon artificial immunization by products made from the tubercle bacillus. It would seem that these preparations have given more satisfactory results in the artificial immunization of animals than any other preparations made from the tubercle bacillus.

Method of Administration.-A cardinal principle in administering tuberculin is to give a definitely measured dose. While it may be used in all the various ways employed for the introduction of remedies into the system, such as by the mouth, inhalation, inoculation, intravenous injection, and subcutaneous injection, yet the necessity of exact dosage excludes all but the last two.

Latham and others have reported favorably on the administration of tuberculin by the mouth, showing opsonic curves which indicate that the action is the same as when it is administered subcutaneously.

'The fact that the gastric and intestinal juices alter the remedy, however, together with the unavoidable inexactness of dosage, speaks against the method. The latter oljection also holds in the case of inhalation and inunction methods. Reactions may be produced by all of these methods, which shows that the remedy is absorbed. 
Intravenous injections have been used by a few clinicians. I have never been able to see any advantage in this mode of administration; on the contrary, I see decided disadvantages.

'The pathology of tuberculosis shows that there is no lack of formation of toxins in the body. Products having the same general character as tuberculins marle in the laboratory are present in the tuberculous foci; but it is probable that the colls of the tubercle and those adjacent to them are but faintly able to respond to their stimulus and produce the specific antibodies necessary to the cure of tuberculosis. It is probable that specific immunizing bodies may be formed by any of the tissuecells. It is accepted as a fact that when we inject tuberculin sulxcutaneously into the arm or back, or any other part of the body, that the local cells whieh come in contact with the remedy at the site of injection react and produce the antibodies. It would seem that these, being removed from the focus of the disease, would be better able to respond. When the remedy is used intravenously, on the other hand, it is carried directly to the lungs, and the first cells stimulated are those, at least in part, which are already overstimulated and unable to respond fully in the production of antibodies.

Another disadvantage, although this can be overcome by care in dosage, is the rapid action of the remedy when administered in this manner. It would seem to me that the slower absorption from the subcutaneous injection would be more desirable. While the danger of infection through faulty technique in the intravenous injections can scarcely hold in this day of aseptic methods, yet the great ease of the subcutaneous injections must recommend them. I can see no valid reason for the therapeutic employment of tuberculin in any other manner than subcutaneously, unless it be for some temporary cause.

Method of Diluting Tuberculin.-For convenience in administering tuberculin a series of dilutions should be made, each one of which is one-tenth the strength of the next higher. 'This solution can be made either by the use of a hypodermic syringe or a pipette graduated to the metric scale; but it is important that the dilutions always be made with the same measure, beeause a slight variation in the amount of tuberculin contained in one of the more concentrated solutions wonld make a marked difference in the amount in one of the higher dilutions.

For diluting fluid sterilized normal salt solution, plus .4 per cent. phenol, can be used for most all of the preparations, although Denys prefers that his preparation, B. F., be diluted with normal salt solution. Where no phenol is used, however, the solution is liable to become infected by contaminating bacteria, and extreme care must be used. 'T. R. is best diluted by 20 per cent. glveerine solution.

I rarely make more than one cubic centimeter of the various dilntions at a time. This makes the solutions fresh, prevents waste, and reduces the danger of contamination. 'The following is the method used by me for diluting the preparations of tuberculin: 


\section{Directions for Diluting O. T. Koch and B. F. (Denys).}

(Diluent: Sterilized normal salt solution +.4 per cent. phenol for $0 . T$., and stcrilized normal

1. cc. original solution contains $1 \mathrm{gm}$. or $1000 \mathrm{mgs}$. of tuberculin . . . . . . Sol. No. 0

.1 cc. Sol. No. $0+.9$ diluent: .1 ce. $\left(1 \frac{1}{2} \mathrm{~m}.\right)=.01 \mathrm{gm}$. or $10 \mathrm{mgs}$. tuberculin $\ldots \ldots$ Sol. No. I

.1 cc. Sol. No. I + .9 diluent: .1 cc. $\left(1 \frac{1}{2} \mathrm{~m}.\right)=.001 \mathrm{gm}$. or $1 \mathrm{mg}$ tuberculin.... Sol. No. II

, 1 cc. Sol. No. II +.9 diluent: .1 cc. $(13 \mathrm{~m})=.0001 \mathrm{gm}$. or $.1 \mathrm{mg}$. tuberculin $\therefore$. Sol. No. III

.1 cc. Sol. No. $1 \mathrm{II}+.9$ diluent: .1 cc. $\left(1 \frac{1}{2} \mathrm{~m}\right.$. $)=.00001$ gm. or $.01 \mathrm{mg}$. tuberculin $: \ldots$ Sol. No. IV

.1 cc. Sol. No. IV + .9 diluent: .1 ce. $\left(1 \frac{1}{2} \mathrm{~m}.\right)=.000001 \mathrm{gm}$. or $.001 \mathrm{mg}$. tuberculin $\cdots$. Sol. No. V

\section{Directions for Diluting P. T. O. (Spengler).}

\section{(Diluent: Normal salt solution +.4 per cent. phenol.)}

1. cc. original solution $=.1 \mathrm{gm}$. or $100 \mathrm{mgs}$. of tuberculin . . . . . . . . Sol. No. 0

.1 cc. original solution +.9 ce. diluent: .1 cc. $\left(1 \frac{1}{2} \mathrm{~m}.\right)=.001 \mathrm{gm}$. or $1 \mathrm{mg}$. tuberculin : Sol. No. I

$.1 \mathrm{cc}$. Sol. No. I +.9 cc. diluent: .1 cc. $\left(1 \frac{1}{2} \mathrm{~m}.\right)=.0001 \mathrm{gm}$. or $.1 \mathrm{mg}$ tuberculin . . Sol. No. II

.1 cc. Sol. No. II +.9 cc. diluent: $.1 \mathrm{cc} .\left(1 \frac{1}{2} \mathrm{~m}.\right)=.00001 \mathrm{gm}$. or $.01 \mathrm{mg}$. tuberculin : . Sol. No. III

.1 cc. Sol. No. III + .9 cc. diluent: $.1 \mathrm{cc}$. $\left(1 \frac{1}{2} \mathrm{~m}.\right)=.00000 \mathrm{gm}$. or $.001 \mathrm{mg}$. tuberculin " Sol. No. IV

\section{Directions for Diluting T. R. (Koch) and Watery Extract (von Ruck).} (Diluent: Normal salt solution +20 per cent. glycerine for $T$. $R$. and normal salt +.4 per cent. phenol for Watery Extract.)

1. cc. original solution $=.01 \mathrm{gm}$. or $10 \mathrm{mgs}$. of solid substance . . . . . . . Sol. No. 0 .1 ec. orig. sol. +.9 cc. diluent: .1 ce. $\left(1 \frac{1}{2} \mathrm{~m}.\right)=.0001 \mathrm{gm}$. or $.1 \mathrm{mg}$. baciliary substance : Sol. No. I .1 cc. Sol. No. $1+.9$ cc. diluent: 1 cc. $\left(1 \frac{1}{\mathrm{~m}}\right.$. $)=.00001 \mathrm{gm}$. or $.01 \mathrm{mg}$. " " "Sol. No. II .1 cc. Sol. No. II. +.9 cc. diluent: 1 cc. $\left(1 \frac{1}{2} \mathrm{~m}\right.$. $)=.000001 \mathrm{gm}$. or $.001 \mathrm{mg}$. “ " " Sol. No. III

\section{Directions for Diluting B. E. (Коch) and P. E. (Spengler).}

(Diluent: Normal salt solution +.4 per cent. phenol.)

1. cc. original solution $=.005$ gms. or $5 \mathrm{mgs}$. of bacillary substance . . . . . . Sol. No. 0 .2 cc. orig. sol. +.8 cc. diluent: .1 cc. $\left(1 \frac{1}{2} \mathrm{~m}.\right)=.0001 \mathrm{gm}$. or $.1 \mathrm{mg}$. bacillary substance. Sol. No. I 1 cc. Sol. No. I +.9 ce diluent: 1 cc. $\left(1 \frac{1}{2} \mathrm{~m}\right.$. $)=.00001 \mathrm{gm}$. or $.01 \mathrm{mg}$ " " " " Sol. No. II .1 ce. Sol. No. II +.9 ce. diluent: 1 cc. $\left(1 \frac{1}{2} \mathrm{~m}\right.$. $)=.000001 \mathrm{gm}$. or $.001 \mathrm{mg}$. " "

Injection of Tuberculin.-Time of Day.-The time of day when the therapeutic injection is given is probably of less importance than is sometimes believed, and practically resolves itself into the time of greatest convenience for both the physician and the patient. As an argument in favor of the evening it may be cited that the quiet of the patient during sleep lessens the tendency to reaction, while, if given in the morning or afternoon, the activity of the patient favors the reaction. This apparent objection to the administration of the remedy in the morning or afternoon may be overcome by cautioning the patient to remain quiet after injection. I prefer the morning as a matter of convenience and because I am more apt to have a record of the reactions that occur than when the remedy is given at night. The time of reaction after the dose has been administered varies with the nature of the disease, probably with the individual and the preparation of tuberculin used. In some it comes on in four hours, while in others it may be delayed for two days. In the great majority of cases, however, when the injection is given in the morning, the reaction shows before night and may be recorded on the day's chart. If the reaction is not sufficient to cause a rise of temperature, but only shows in the form of some of the subjective symptoms, such as malaise, nervousness, and aching, the patient will usually note them before retiring at night. On the other hand, if the dose is given at night, either slight temperature rise or slight subjective symptoms may be unrecognized. 
Site of Injection.-The site of injection is likewise largely a matter of convenience. I formerly gave a great many injections in the loose tissues between the scapulat, also in the tissues covering the upper portion of the abdomen and deep into the muscles of the upper arm. At present I am using the flexor surface of the forearm as recommended by C. Spengler more than any other site, and injecting the tuberculin subcutaneously. 'This site is convenient and the subcutaneous injection offers excellent opportunities of olserving the degree of reaction of the tissues to the dose, and affords an exedlent control for dosage. Since it is probable that all of the fixed cells have the power to form antibodies, it makes little difference beyond that of convenience where the injection is made as long as it is in tissue which is vascular. It seems to me, however, that it is best to change the site with each injection, thus insuring that the tuberculin cloes not constantly stimulate the same cells.

Deep Muscular and Subcutaneous Injections.-Deep museular and subcutaneous injections are of equal value therapentically as far as I have been able to judge, although the latter give more useful information regarding the time and size of dosage than the former. Whether or not it be accepted as true, as C. Spengler has suggested, that the reaction in the tuberculous focus and that in the subcutaneous tissue at the point of injection be coextensive in point of time, nevertheless, I have found that, when tuberculin has been given with the proper aseptic precautions, and the method of ascending dosage has been used, this local tissue reaction does not appear, as a rule, until a comparatively large dose has been employed. 'This seems to suggest that the limit of safe dosage is approaching. If such a local reaction clears away with a few days, the same dose may usually be repeated with safety, and not infrequently, by the use of caution in increasing and spacing the dosage, the treatment mav be continued. I am in the habit, however, after one or two distinct local reactions, of changing the tuberculin. If the reaction is due to tuberculin made from human cultures, I change to one made from bovine cultures and vice versa. I have often noted where a distinct local reaction has been produced, that a small dose of the same tuberculin is apt to cause another local reaction, and by changing to a tuberculin made from another variety of bacilli, from human to hovine, and vice versa, it is possible to gradually increase the dosage angin without producing loeal reactions until a higher dosage has again been attained.

More important than the manner of administering the injection is the accuracy of dosage. If a number of patients, who are taking doses of different strength of tuberculin are being treated with the same syringe, it at once becomes clear that it is very important to be sure that the syringe is thoroughly washed of all remains of the higher concentration before a dose of lower concentration is administered. Neglect of this is sometimes followed by a severe reaction.

Tuberculin Reaction.- The action of tuberculin is closely bound with what is termed "tuberculin reaction." While there is some question as to the cause of the tuberculin reaction, yet its manifestations are very definite. We assume that the tuberculin reaction is just the same 
as the reaction which occurs every now and then in tuberculous patients when the bacilli themselves or their toxic products are acted upon by the defensive elements which have been produced by the cells of the body, producing general malaise, rise of temperature, and the other symptoms which are so well recognized in the course of tuberculosis. Whatever the exact explanation is, we are safe in assuming that it is due to a reaction between the tuberculin and the protective substances of the body.

'Tuberculin reactions are of three kinds, the local reaction at the site of injection, the focal reaction in the focus of the disease, and the general reaction.

The local reaction is of special importance in diagnosis, as noted in the conjunctival test as described by Wolff-Eisner and Calmette, the skin test by von Pirquet, Morro, and Lignieres, and the intracutaneous by Moussu and Mantoux. It is also often of importance, as I mentioned above, in determining the point of safety of dosage when tuberculin is being used therapeutically.

The focal reaction is the reaction which occurs in and about the focus of infection. This can be readily studied in visible tuberculous infiltrations and ulcerations. The degree of reaction depends upon the dosage; if the dose of tuberculin be very small it may only appear as a very slight hyperemia. If the dosage be a little larger, it appears as a distinct congestion with exudation; and, if it be still larger and the action be prolonged, it may even cause destruction of the tuberculous tissue. The clinical symptoms which follow a focal reaction vary according to its severity and the site of the disease.

A slight focal reaction may produce no symptoms at all. However, if the dosage be a little larger, symptoms varying in intensity may be noticed. A focal reaction in the lung may produce a slight feeling of constriction or an increased tendency to cough, or an increase of expectoration. In the larynx it may produce a feeling of uneasiness, discomfort, or actual pain. In the intestines it may produce pain or diarrhea. In the joints it may produce pain.

The term "general reaction" is used to designate the symptoms which are produced by a dose of tuberculin entering the tissue, which is sufficiently large to call forth a manifest reaction on the part of the organism, in contradistinction to the lack of symptoms produced by small doses which set into action the machinery of immunization without producing any recognizable disturbance on the part of the organism. The symptoms which make up the general tuberculin reaction depend upon the size of the dosage and the peculiar manner in which the patient reacts toward the remedy. The symptoms which I have noted most frequently in slight general reactions are as follows: malaise, slight nerrousness, feeling of heaviness in the limbs, slight tendency to ache, either with or without a fraction of a degree of elevation of temperature. If the reaction be a little more pronounced, the symptoms are more severe and more nearly resemble those of la grippe; the patient may feel slightly chilly, may have a severe aching, and may 
even feel nauseated. 'These symptoms are usually accompanied by a marked rise of temperature and increased pulse-rate. If the reaction be still stronger, the patient may have a distinct chill and a very high temperature and pulse-rate. 'The symptoms of a general reaction usually pass off, according to their severity, in from two to five days.

Schemata for Dosage.--Many writers have attempted to give accurate instructions for the use of tuberculin. 'There has been a tendency ever since tuberculin was first discovered to try to make the method of administration conform to simple rules. It has heen suggested that doses be given so many days apart, and that the size of the dose should be increased each time by a certain amount according to a set rule. Such a plan can never give the best results in the use of tuberculin. I never treat two patients exactly alike. One paticnt may bear increases much more rapidly than another, and also bear the dosage more often. If one wishes to derive the best results from the administration of tuberculin, he must first have a definite idea of what tuberculin is and how it acts, and then study tuberculosis carefully, acquaint himself thoroughly with his patient, and then be guided entirely by the cireumstances at the time, as to the interval and increase of dosage. I realize full well that physicians want some definite way to give tuberculin, but I can no more see how there can be a definite way of giving tuberculin than there can be a definite way of operating and treating patients in severe surgical affections. 'The best results ean only be obtained by strict individualization.

Prior to beginning the administration of tuberculin, it is necessary to observe the patient for a few days, to make a careful diagnosis of his tuberculosis condition, and to form as accurate an opinion as possible of his reactive powers. During this time, as well as during the entire course of treatment, the temperature and pulse should be taken every two hours and carefully recorded.

It is best to have such a record, covering a period of from three to ten days, before the first dose is given. The carefully recorded symptoms during these first few days of observation give us a record with which to compare the condition after beginning the administration of the remedy.

It is also necessary during this time, as well as during the entire period of treatment, to keep a careful record of the prominent symptoms, in order that we may note any change upon them produced by the tuberculin. The amount of sputum, the feeling of malaise, aching, nervousness, condition of appetite, sleep, and pain at the seat of infection should be carefully noted, to see whether or not they are influenced by each dose administered.

I would suggest the following as a safe plan for the administration of tubereulin. First, no matter what preparation is given, begin with a dose so small that it is practically certain no reaction will be produced. This dose, of course, will vary materially according to the preparation that is used, and according to the condition of the patient. Second, always keep the dose short of producing a general reaction. 'The patient who has very slight symptoms of tuberculosis is more apt to 
react than one who has more marked symptoms. I usually begin to treat patients who are extremely nervous and those who have had recent advances in their disease; also those who are inclined to run a temperature or whose reactive powers are low, with smaller doses than I do others. I would also give smaller doses to a young individual than I would to an older one. If the first dose is borne well, the dosage can usually be increased as follows: $1,2,3,4,5,6,7,8,9,10,15,20,25$, $30,40,50$, etc.

The same preparation in careful and experienced hands can be advanced much faster than in inexperienced hands. If one is to err in dosage, let it be on the side of advancing too slowly rather than too rapidly. The intervals between doses should be gradually increased as the dose becomes larger. My custom has been to begin with the small dose, administering it usually every second day, and then increasing the interval so that by the time the large doses are given the interval has been increased to from seven to ten days, according to the preparation used. I consider it very important in beginning the administration of tuberculin to increase the dosage as rapidly as the patient will bear it. By increasing the amount of tuberculin rapidly, the tolerance of the patient is materially increased and the danger of hypersensibility is decreased. I have noticed many patients who have been treated by small doses, given at infrequent intervals, who finally developed a state of hypersensibility which manifested itself by either a local or temperature reaction every time a dose was given. Very often this is interpreted to mean that the patient bears tuberculin poorly. The proper interpretation to my mind is that the patient has been treated in a faulty manner and has become hypersensitive instead of tolerant to the remedy. Such patients can usually be treated very successfully by changing to another preparation of tuberculin, and increasing the dose rapidly.

Large and Small Doses.-The question of whether it is better to treat tuberculosis by repeated injections of small doses of tuberculin or by rapidly increasing doses is one which will bear considerable thought and discussion. Ever since tuberculin was first introduced, the men who have used it most successfully, such as Koch, Petruschky, C. Spengler, von Ruck, and 'Trudeau have given it by the method of gradually increasing the dosage; and I believe we can safely say that men who are most successful in the treatment of the disease to-day are using this same method. I am sure that this will hold true as far as pulmonary tuberculosis is concerned.

Wright and his followers, on the other hand, have adopted the method of giving small doses, injecting them infrequently and increasing the dose very slightly, if at all. 'This has proved quite successful in the treatment of surgical tuberculosis, such as that of the joints, bones, glands, and genito-urinary system; but, if the results are carefully compared with those obtained by gradually increasing the dosage, I am sure that the results by the latter method will compare favorably. In the treatment of pulmonary tuberculosis I have not been able to produce as good results with small doses as with larger ones. I have always felt 
that I was doing my patient the most good when I was giving a dose just short of reaction and increasing his tolerance to the highest degree possible. Large doses, however, should never be given according to a definite scheme, but according to the patient's condition and his tolerance to the remedy.

It has been my good fortune to treat many cases of tuberculosis of the larynx, and some of the pharynx, tonsil, and tongue. 'The healing of these visible lesions has probably tanght me more of the action of tuberculin than any other experience which $I$ have had. It has always seemed to me that the healing is hastened in these cases by administering a dose sufficiently large to produce a local reaction, allowing the reaction to pass off, and then increasing the dose so that a distinct focal reaction is again obtained. In this way the part is kept in an intermittent state of reaction. In order to produce this result $I$ have found it necessary to increase the dosage. A dose that will produce a local reaction to-day will probably not do so a few days later. From the theoretical stand point there is a decided advantage in the increased dosage. Wolff-Eisner and others have shown that antibodies (lysins) are found in much greater numbers after large doses of tuberculin than after small doses. In the establishment of immunity the degree of resistance which is produced seems to depend, at least to a certain extent, upon the amount of the specific stimulating substances which are employed. Of course, this may be true only up to a certain point, beyond which the reactive powers of the patient may be overtaxed; nevertheless, it does seem to hold good, if we may judge from the effect upon animals in the production of antitoxins.

We know that small repeated doses fail to produce hyperemia in the focus of infection, while constantly increasing doses are nearly always attended by it. We must look upon this local hyperemia as a very favorable condition for cure, both in its effect in stimulating the absorbing power of the cells, and in its increased opportunity of applying the immune bodies at the focus of disease.

The small doses are especially favorable, as mentioned above, in circumscribed foci; but, it seems that where the disease is more extensive, the larger doses are much more active. 'This may be due to the fact that small doses are capable of stimulating the machinery of immunization sufficiently where the disease is not very extensive, but unable to meet the needs where the disease is more extensive.

'There has been considerable discussion regarding the maximum therapentic dose of the various preparations of tuberculin. In my practice $I$ have no set maximum dose. I have found this to vary with the patient and the condition to be treated. It seems to me that we must use common sense above all else in the employment of tuberculin, and recognizing the various degrees of susceptibility of different patients, we cannot help seeing that the maximum dose differs for different individuals. It is well, however, to know what is considered the maximum dose of the various preparations, for it is wise, as a rule, not to exceed them. These are O. T., 1 cc.; P. 'I. O., 1 cc.; B. F., 1 to 2 cc.; 'T. R., 
10 to $20 \mathrm{mg} . ;$ B. E., $5 \mathrm{mg}$.; Watery extract, 1 cc. of the 1 per cent. extract; T. B. V., $\frac{1}{100}$ mg.; and P. V., $\frac{1}{100} \mathrm{mg}$.

Guide to Dosage.-A. question of some importance is whether or not it is safe to administer tuberculin without the laboratory determination of the opsonic power of the blood. While clinicians had developed a safe and successful method of administering tuberculin, depending entirely upon clinical observations, and were producing excellent results, their work was threatened with discredit after the publication of Wright's monumental work on the effect of tuberculin upon the immunizing content of the blood. 'Those of us who had employed the gradually increasing dosage, believing that the increase of the patient's tolerance to the remedy was of value either in itself or in certain conditions which the larger doses produced, were suddenly confronted with a mass of criticism, and told that our apparent cures and arrestments had not been produced in a scientific manner; there seemed to be some question for a time whether or not the ultra advocates of the new method were going to permit the patients who had been successfully treated to remain cured. After the effect of the first announcement had passed over, however, and the scientific world had again gained its equilibrium, it was found that tuberculosis could be successfully treated with both large and small doses, and while the estimation of the opsonic content of the blood, when done by men whose technique is accurate, is of value as a guide to dosage, yet it is not necessary. Wright's epoch-making work must not be underrated, however, for it has accomplished much for the establishment of the principle of vaccine therapy upon a rational basis. At the same time the opsonic index, as generally estimated, probably gives far less accurate information upon which to base the tuberculin dosage than clinical data obtained by careful observation. It seems to be generally accepted by those who are committed to the gradual increase in dosage that the best results are obtained by pushing the remedy to the point of tolerance and keeping just short of a reaction. Since tolerance is established quite readily, as a rule, this method warrants a fairly rapid increase.

Hypersensibility.-This is a condition which sometimes arises during the course of tuberculin treatment. Sometimes it is met at the beginning of treatment, under which circumstances those who use only one variety of tuberculin are prone to declare that the patient is not suited to tuberculin treatment; while those who have at their command several different tuberculins, and especiallly those made from both bovine and human cultures, will consider it as an indication to change to another preparation. While it may occur at the beginning of treatment, on the other hand, it may not occur until after the patient has been under treatment for some time. I recall one patient who had taken ${ }_{4}^{3}$ cc. of von Ruck's watery extract, which is a preparation containing 1 per cent. of solid extract of the tubercle bacillus, when, without warning, he became so sensitive that he could not tolerate the smallest dose without violent temperature reaction. Another patient; after several months of treatment, became hypersensitive to tuberculin made from bovine 
cultures, but bore those made from human cultures well. I have seen in a number of instances hypersensibility, manifested by local reactions at the site of injection and by slight temperature reaction. I an of the opinion that the very best way to avoid this condition is to begin treatment with small doses, and, with short intervals elapsing between the doses, to increase the quantity of tuberculin at each injection quite rapidly. If the patient seems to be sensitive, I change the preparation. It seems especially eflective to change from a preparation from bovine culture to one of human and vice versa. Since adopting this method of treatment I have had no difficulty with this troublesome complication.

Hypersensibility is not inconsistent with immunity. In fact, it must be looked upon as a specific reaction dependent upon the presence of immune bodies; nevertheless, it is a troublesome complication when it develops in the course of tuberculin treatment, and one to be avoided if possible.

Choice of Patients for Tuberculin Treatment.-Koch insisted that patients should be treated in the early stage. Every man who has had elinical experience in the treatment of tuberculosis, whether with or without tuberculin, insists on the same thing; yet, in order to obtain early cases in public institutions three-fourths of the applicants must be rejected, and private institutions would be unable to run without accepting advanced cases. Nevertheless, in spite of this fact, great headway is being made in the treatment of this disease.

Formerly, it was believed that only non-febrile cases of tuberculosis were suited to tuberculin treatment. In fact, this rule is adhered to by many to-day. It is true that non-febrile cases respond best to all forms of treatment, but tuberculin in experienced hands does not need to be confined to such narrow limits. I believe that it is safest and best for beginners, if they are going to use tuberculin at all, to confine their administration of it to non-febrile cases. 'The expert, however, who knows his remedy well and has his patients under full control, as is the case in a sanatorium, will find a very valuable aid in the careful administration of tuberculin in many febrile cases. He must not expect tuberculin, however, to do impossible things. He must be satisfied if it lowers the temperature or pulse-rate, or relieves some of the other more prominent toxic symptoms, or, if it stimulates the absorption of some of the inflammatory products in the area of infection or aids in preventing the spread of the disease to other foci.

Febrile cases are the most difficult to treat, no matter by what method. Advanced febrile cases of pulmonary tuberculosis, whether treated or untreated, whether treated properly or improperly, run an uneven course. Their temperature rises and lowers, not only because of the tuberculous process and the associated infections that may be in the lung, but because of the absorption of poisons from dead tissue and because of the various tuberculous complications. It also raries with external influences. These conditions make it very difficult for one to give an opinion as to the effect of a given therapy and demand that the most serere cases be 
treated only under the most favorable circumstances, and if they are to be treated by tuberculin at all, that it be given only by men who are expert in the use of the remedy.

It is often pointed out that advanced progressive cases of tuberculosis do not do well under tuberculin treatment. A casual observation of these cases reveals the fact that this same type does not do especially well under treatment when tuberculin is not used. My personal belief is that this class of cases can often be helped immeasurably by the careful administration of tuberculin. I believe, however, that their treatment should be left to men who are expert in the use of the remedy, because greater skill in dosage and greater judgment are required than in the treatment of ordinary cases. Whoever wishes to treat advanced progressive cases must make as accurate a diagnosis as possible, so as to know what to expect from the disease and what from the remedy. $\mathrm{He}$ must expect some of the densely infiltrated areas of the lung to go on to destruction, and the cheesy degenerated areas to form cavities. He must expect the absorption of the material from the diseased areas to produce fever and its accompanying symptoms, and he must not be discouraged if the disease shows some tendency to spread. From the proper use of tuberculin, however, one can expect many of the products of inflammation to be absorbed, relieving the severe congestion and saving some of the lung tissue from going on to destruction. He can expect many of the areas in the lung which are less advanced to heal even while the temperature continues and the most severely diseased areas are breaking down. He can also hope, with a reasonable degree of certainty of fulfilment, that the tuberculin treatment will aid in preventing the extension of the disease.

To determine these points in tuberculin treatment requires far more careful diagnostic methods and more accurate records than are usually employed.

'The reason usually given for not employing tuberculin in such cases is that such patients are already saturated with tuberculin. This reason ignores other sources of fever, such as that caused by associated bacteria, the absorption of necrotic material, advancement of the disease process, and other complications. It also disregards the common theory, which seems quite plausible, that the tuberculin which escapes from the foci of infection probably expends most of its force upon the adjacent injured cells, which, because of their injured condition and because of the more or less continuous action of the tuberculin upon them, fail to respond in the production of antibodies.

Against the above reason I would offer the following observation: Patients who have visible tuberculous infiltrations and ulcerations, such as those in the larynx, even although running continuous temperatures which are thought to be due to saturation with tuberculin, do not show continuous focal reactions; but a reaction shows promptly when tuberculin is injected subcutaneously.

The probabilities are that when tuberculin is injected subcutaneously into cells that are remote from the seat of infection, they are better 
able to react with the production of specific immune bodies which are necessary for cure.

'The reckless, ignorant use of tuberculin is to be condemned under all circumstances. I would suggest that beginners, after carefully studying the remedy and the disease, confine their efforts to early and non-febrile cases. I would also suggest that experts cautiously extend their employment of tuberculin to active cases.

It is usually considered that a high pulse is a contraindication to the employment of tuberculin. With this opinion I cannot agree. On the other hand, I consider tuberculin our best remedy for the reduction of temperature and pulse when the cause is a tuberculous toxemia. I submit chart I in illustration of this point. I was pleased in a recent visit to find that Prof. Neusser, of Vienna, is using tuberculin in fever cases. His assistant praised it as one of the best antipyretic remedies at our command.

Illustrating the antifebrile action of tuberculin and especially of the bovine tuberculin. This patient had run a temperature varying from $101^{\circ}$ to $103^{\circ} \mathrm{F}$. and a pulse from 110 to 130 for three months before entering the institution, and had been on small doses of human tubereulin given at infrequent intervals. Besides this she had been treated by rest and hygienie measure; in her home. In less than a month's time after the beginning of bovine tubereulin, alministered in gradually increasing doses, the temperature was reduced to the point shown in the chart.

Key to Dosage.-ión M, mg.
Fic. 86.

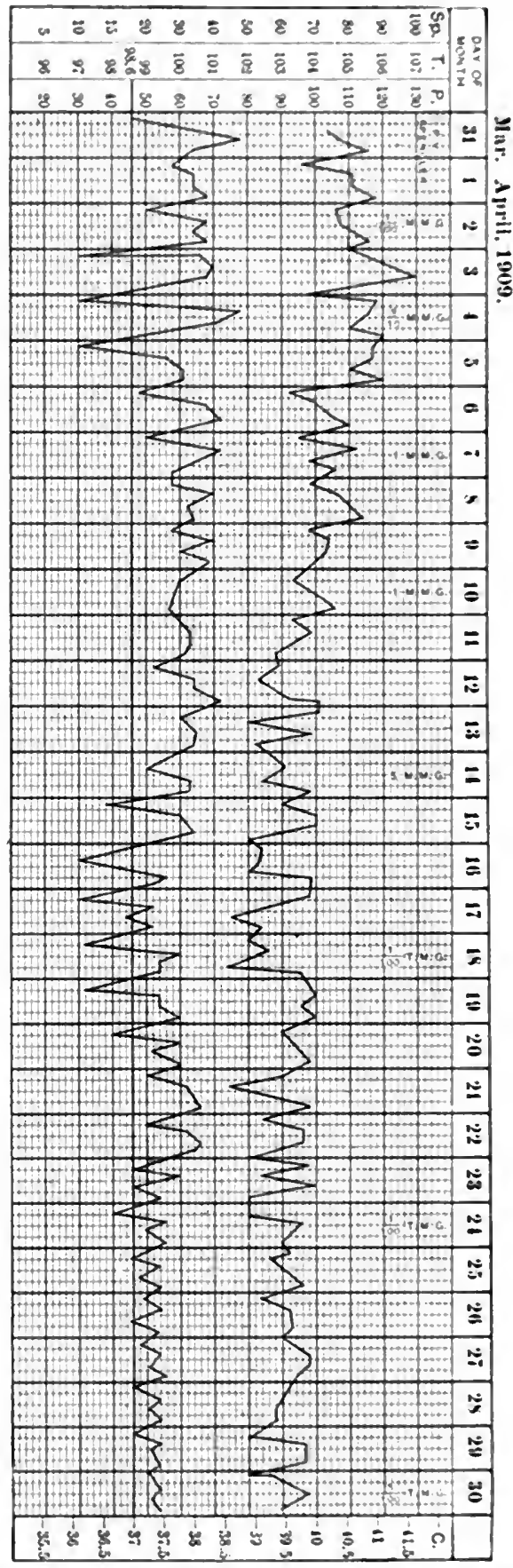

$=.00000001 \mathrm{mg}$; if M. mg. $=.0000001 \mathrm{Mg} . ; 1 \mathrm{M} . \mathrm{mg} .=000001 \mathrm{mg} . ; 5 \mathrm{M}$. mg. $=$

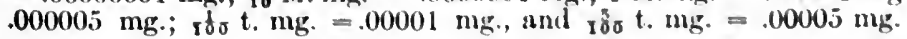


Favorable Effects upon Symptoms Shown when Tuberculin is Administered.-When a patient is put upon tuberculin treatment, if the preparation is properly adapted to him, there usually takes place, within a very short time, an amelioration of the symptoms which are of a toxic nature. Nerrousness and malaise lessen and disappear, the appetite improves, the patient sleeps better, and the temperature gradually approaches normal. 'The extent to which this relief of symptoms takes place depends upon the nature and severity of the infection and the individual peculiarities of the patient. The improvement of symptoms is often noted in patients who have carried out the hygienic, open-air treatment for a time without attaining a satisfactory result, and then are treated with tuberculin. The improvement in some cases is almost magical, the injections seeming to furnish the stimulus which has been lacking.

Duration of Treatment.-One often hears tuberculin treatment spoken of as a "course" of so many weeks, or so many doses, or of a sufficient length of time to attain a certain dosage. This is at variance with my ideas of tuberculin and its action. Individualization is the only plan to follow. Each patient has his own dose and his own time of dosage, and the length of treatment is an individual matter. My plan has been to treat my patients until I have obtained the best result possible. This varies from four to six or eight months in incipient cases to one year or more in advanced cases. I am of the opinion that the method of Petruschky, somewhat modified, would be the best, but it is practically impossible of attainment. His plan is to treat the patient for a time, arriving at a certain dosage, then waiting for a few months and repeating this, then waiting for a few months more and repeating again. I would suggest that it would be wiser to treat the patient until a cessation of symptoms and removable signs has been reached, and then wait for a time and resume injections. The difficulty with this plan is that it impossible to have patients do as one wishes them to do. As a rule, patients will not take a course of treatment, resume their natural way of living for a time, and then return to treatment again. Necessity demands that we keep them under treatment until the best result possible has been attained, whether it requires few months or many. The generally understood time of treatment for tuberculosis is far too short. Tuberculosis, from the nature of its pathology, is a slow disease to heal. Healing is produced through the blood; and the nature of the tubercle is such that the blood cannot enter it at all or only with difficulty. The entire area affected and surrounding tissues are the seat of a congestion and a retardation of the flow of lymph and blood. Then when scar tissue begins to form, it is also poor in its blood supply. Therefore, cure is obtained at a great disadvantage, and the time of cure is necessarily prolonged.

Is Tuberculin a Safe Remedy for General Use ?-It is very desirable that as many patients as possible be given the benefit which the intelligent use of tuberculin affords, yet friends of this remedy cannot help viewing with regret the present reckless employment of it by men who are not trained in its use. The administration of tuberculin requires careful 
discernment, and the best results can only come through experience. It is a very dangerous remedy when wrongly used. I believe it is just as essential that men who use tuberculin should be trained in its use as those that are contemplating performing surgical operations should first see the operations performed. How many times when we ask a physician what preparation of tuberculin he uses, and in what dosage, we receive some such reply as this: "It is a preparation by - and I give 5 drops from such and such a bottle." One feels like asking, "Have we forgotten the harm that can be done by the ignorant use of tuberculin and have all the warnings of the first era of disaster been given for nought."

The man who treats tuberculosis successfully will not know dosage by so many drops of such and such a solution. He must remember that he is dealing with a remedy which is very powerful, and a drop of one solution may contain one million or one hundred million times more of the bacillary extract than that of another, so whoever administers tuherculin should know definitely how much of the product he is giving at each dose, and should not remember it as drops, but as milligrams or a certain part of a milligram.

During the last few years a number of instances have come under my observation where laymen were treating themselves with tuberculin. I feel that I camnot protest too strongly against such a practice. Few laymen realize fully the dangers which they are subjecting themselves to in this practice. No physician has any right to place tuberculin in the hands of a layman for self-dosage.

I believe the following principles should govern those who use the remedy:

(1) Any man who has intelligence and training necessary to practise medicine should be able to administer tuberculin, provided that he will first study the preparations and know their action; second, study tuberculosis in the light of recent investigations, so as to know the condition with which he is dealing; and provided he familiarizes himself with the administration of the preparations perfectly by following their use in the hands of men who are expert in their administration.

(2) No physician should attempt to employ tuberculin if he has only an occasional case to treat, for it requires experience in the constant use of it to produce results and avoid dangers.

\section{TUBERCULIN AS A DIAGNOSTIC AGENT}

The cure of tuberculosis depends upon the earliness of the diagnosis and the promptness and intelligence of the treatment. In our zealous searching for a perfect remedy for this disease we are failing to take advantage of the best that we already have. 'The treatment of incipient cases of tuberculosis under the best circumstances restores from 65 to 90 per cent. to health; but the sad fact is constantly emphasized by all writers that patients are not seeking treatment when they are in this curable stage. The reason for this is both lack of apprecia-

Voz. I. -38 
tion of the early symptoms of tuberculosis and inability to establish a diagnosis when the disease is suspected.

The diagnosis of early tuberculosis is based upon the data obtained from-(1) A carefully taken clinical history; (2) a thorough painstaking physical examination; (3) a tuberculin test; and (4) a microscopic examination of any secretion that may be present from the suspected lesion.

Importance of the Tuberculin Test in Diagnosis.-The diagnostic value of the tuberculin test depends upon the fact that an individual who has a tuberculous infection within his body reacts differently to the products made from the tubercle bacillus, from an individual who has no infection. The tuberculin test is specific for tuberculosis. This point needs no discussion. Although it has been reported that syphilis and some other conditions react, yet our knowledge to-day is such that we feel sure that in all such cases tuberculosis was also present.

The tuberculin test, if positive, proves that tuberculosis is present within the organism. Unfortunately, it does not locate the lesion. Many unwarranted diagnoses have been made by failing to appreciate this fact. A short time ago a patient came to my notice who was suffering from an abdominal lesion which was thought to be tuberculous. A skin test had been given, the patient had reacted, and on this the lesion was pronounced to be tuberculous. In another instance a patient was operated on for tuberculosis of the kidney because of suspicious symptoms and a positive conjunctival test, to find that the kidney was not tuberculous. Another patient was assured that he had pulmonary tuberculosis because he reacted to tuberculin, the lung not even having been examined.

The tuberculin test, when positive, unless it be accompanied by a focal reaction, is nothing more than proof that tuberculosis is present somewhere in the body. It still requires all the judgment and skill derived from experience to locate the lesion. No test that has yet been devised can dispense with careful expert clinical examination.

There are several different ways in which tuberculin may be used to determine the presence of tuberculosis: the cutaneous, percutaneous, intracutaneous, conjunctival, and subcutaneous.

Interpretation of the Tuberculin Test.-It requires considerable skill and experience to properly interpret the results of a tuberculin test.

Recent pathological investigations (Naegeli, Burckhardt) show that tuberculosis is present in nearly all adult bodies. Clinical investigations in childrens' wards where those who are not clinically tuberculous have been subjected to the tuberculin test, reveal the fact that the great majority of children are infected with tuberculosis and react to the tuberculin test by the time they are fourteen years of age. Hamburger found a positive reaction in 94 per cent. of children between the ages of eleven and fourteen years in the St. Anne Children's Hospital in Vienna. Ganghofner, of Prague, found a positive reaction in 80 per cent. of children of thirteen and fourteen years of age.

Many of the infections in early childhood heal out and show only a scar in the adult body when examined postmortem. A very large 
per cent. of them remain quiescent, yet do not heal out entirely; while probably less than 20 per cent. cause clinically recognizable symptoms.

'The clinically active cases, with exceptions to be noted later, and the quiescent cases will react to tuberculin; and we are not sure just how long after a thorongh healing the protective antibories which canse the specific reaction will remain in the tissues and produce a reaction.

It is well understood that patients suffering from andvanced tuberculosis, especially those who are eachectic, lose their reactive powers. It has been reported that some intercurrent discases, especially measles, interfere with the patient's reactive powers. 'The reaction depending upon the specific protective bodies in the blood, the fact that children do not react during measles probably indicates that the specific immune bodies for tuberculosis are temporarily in abeyance, and may account for the frequency of clinical tuberculosis following this disease.

Realizing the fact that nearly all children are infected with tuberculosis during their early years; and interpreting the animal experiments which have been performed that go to show that these early lesions, if they heal, afford a certain amount of immunity against further infection, as indicating that these infections in children should be treated seriously, it would not be surprising if the treatment of tuberculosis in the future would consist of determining the presence of an infection, even though it be latent, during the early period of childhood, and resorting to proper treatment, and curing the discase before it becomes a clinical entity.

Even in adults to-day I am positive that a more general use of the tuberculin test in many obscure conditions, especially in neurasthenic patients, would give information of immeasurable value in the handling of such cases.

We have long recognized syphilis as being one, if not the particular, cause of nervous affections. My observations would lead me to place by the side of syphilis, tuberculosis. 'The chronic toxemia produced by tuberculosis seems to spend much of its force upon the nervous system, and it is surprising to see what wonderful results can often be prodiced by recognizing this fact, diagnosing the condition, and treating the patient with tuberculin along with other appropriate measures. It requires little thought to appreciate the fact that tuberculous lesions existing during a long life-time and throwing out toxins intermittingly, must produce conditions which can best be combated by measures directed toward the cause. 'The tuberculin test is of great value in clearing up the diagnosis in such cases.

From what has been said before it can be seen that patients who are pathologically tuberculous, though showing no elinical signs, may react to tuberculin, and also that patients who have advanced tuberculosis, or have some intercurrent disease which temporarily deprives them of their reactive powers, may not react; consequently considerable judgment must be used in interpreting the result of the test. 'The test, as such, if properly made, does give valuable information, but it must always be considered in accordance with the limitations which I have mentioned. 
If a patient has suspicious symptoms of tuberculosis of a given organ, we should use every method that we have to clear up the diagnosis; and if to other positive evidence we can add a positive tuberculin reaction, this knowledge is not without value, and if we can add a focal reaction, the diagnosis is beyond question.

Wolff-Eisner has attempted to differentiate between active tuberculosis and latent tuberculosis by the character of the reaction. If the reaction comes on quickly, he considers that it is more apt to be an active lesion. If, however, it be late in its appearance, he considers it to be a latent lesion, and accounts for the late appearance of the reaction as being due to the fact that few antibodies are being formed, consequently it takes longer for a sufficient number of them to gather at the point of inoculation and combine with the tuberculin used in making the test, to produce a reaction. He also considers that the tuberculin reaction has a prognostic significance. If the reaction is strong, he considers that the patient's reactive powers are good, but does not conclude from this alone that the patient will recover. If the reaction is weak, he looks upon it as of bad prognostic significance.

The Subcutaneous Test.-'Tuberculin has been used for diagnostic purposes ever since Koch announced its discovery in 1890 . Until recently it was used exclusively by the subcutaneous method. Many different schemes of dosage have been suggested and used by different workers, all, however, aiming at the same general result. The subcutaneous use of tuberculin, if it produces a positive reaction, causes two distinct phenomena, the one the reaction in the diseased area, which is known as the focal reaction, the other the constitutional symptoms, which is known as the general reaction. In order to produce a general tuberculin reaction the dosage must be quite large or advanced quite rapidly, unless the reaction of hypersensibility as used by Loewenstein is to be employed. Koch determined that the normal individual is uninfluenced by moderate doses of tuberculin and arrived at the conclusion, which has been generally adopted, that $10 \mathrm{mg}$. of old tuberculin will not produce a reaction in the normal individual, consequently if an individual reacts after a dosage of $10 \mathrm{mg}$., he must be considered as suffering from tuberculosis. While it is not always necessary to use doses as large as this, at the same time it is questionable whether it is safe to exclude tuberculosis unless at least $10 \mathrm{mg}$. have been given.

The subcutaneous test is not applicable excepting in patients who are running a low temperature. While it is usually considered available only when the temperature is practically normal or $99^{\circ} \mathrm{F}$., personally I believe it can be used with entire satisfaction even with a temperature of $100^{\circ} \mathrm{F}$., although since we have the newer methods this is unnecessary, because the cutaneous or conjunctival methods can be used in preference.

Preliminary to giving a diagnostic dose of tuberculin it is necessary to have a carefully taken two-hourly chart of the patient's temperature for a period of at least three days. During these days the patient should be under the same conditions which he will be under when the test is given; for example, it might give a faulty reading to have a patient work- 
ing while he is taking the control temperature, and then have him resting in bed when he is given the injection; so it is better to have the patient quiet two or three days before the injection is given if he is to remain quiet afterward or let him go about his usual duties after the injection, the same as before. Of course, wherever it is practical, rest both before and during the period of test is preferable and should be insisted upon.

The original dosage for the tuberculin test was first to give the patient $1 \mathrm{mg}$, , then after two days 5 mg., followed about three days later with $10 \mathrm{mg}$. In case no reaction occurred, the latter dose was repeated again after three or four days. Another method was to give $1 \mathrm{mg}$., two days later $2 \mathrm{mg}$., two or three days later $5 \mathrm{mg}$., and then in three or four days more $10 \mathrm{mg}$. Loewenstein has advocated a definite plan, beginning with a very small dose of $0.2 \mathrm{mg}$., to be repeated in threeday intervals until it has been given four times. He claims that this, by producing hypersensibility, will quite often produce a reaction. If it does not, however, he would increase the dosage to 5 or $10 \mathrm{mg}$.

There is some likelilıood of the gradual ascending doses producing a tolerance, so that a patient might have tuberculosis in a stage where he should react, and yet even no reaction occur with $10 \mathrm{mg}$., but the likelihood of such is not very great. Bearing this in mind some clinicians have thought best to give one dose of $10 \mathrm{mg}$., or one dose of $5 \mathrm{mg}$., followed a few days later by $10 \mathrm{mg}$. 'This, however, is rarely used at the present time.

I have no set rule for the tuberculin test. While I have used the subcutaneous method for fifteen years, I am always guided entirely by the condition of the patient. Sometimes I begin with $0.1 \mathrm{mg}$., sometimes with $1 \mathrm{mg}$., sometimes with $2 \mathrm{mg}$., but rarely above $2 \mathrm{mg}$., usually allowing one day, but not more than three days, between doses. My advance depends considerably on the initial dose. If $\mathrm{my}$ first dose was $0.1 \mathrm{mg}$., my second dose would most probably be $1 \mathrm{mg}$. If my first dose was $1 \mathrm{mg}$., my second dose would most probably be $3 \mathrm{mg}$. If my first dose was $2 \mathrm{mg}$., my second dose would probably be $5 \mathrm{mg}$. I nearly always give $10 \mathrm{mg}$. as $\mathrm{my}$ final dose before excluding tuberculosis.

After having given this test many times, I consider it absolutely safe in careful hands. I have never scen harm result from it, and when we consider the fact that it produces not only a general reaction, but a foeal reaction as well, which we are often able to detect, we must accept it as being the most reliable of all the tubereulin reactions. My impression after long experience in its use, together with eonsiderable experience in the newer tests, convinces me that the subcutaneous method is the most reliable test that we have for the detection of active tubercuiosis. I believe that a much smaller percentage of patients will react to $10 \mathrm{mg}$. subcutaneously than will react to either the skin or conjunctival tests; and where the question of latency must be determined, I believe that the subcutaneous test will give us our most reliable data.

The phenomenon which makes up the general reaction should be well understood; formerly it was thought to be necessary to have a rise in temperature of 2 degrees in order to have a positive tuberculin reac- 
tion. I was early convinced that a temperature rise of only a fraction of a degree is reliable in the hands of an expert in the use of tuberculin. The temperature reaction is only one phase of the reaction. Short of a 2-degree temperature reaction, the patient will often show nervousness, malaise, feelings of heaviness of the limbs, aching of the head or back, or actual pain. If such a train of symptoms should occur and should not be interpreted as a positive reaction, it would be better to repeat the dose which had produced it or make the increase in dosage very small, for a pronounced reaction would be almost sure to follow the next increase in dosage. The focal reaction should also be carefully looked for. If one suspects tuberculosis of the lungs, the focal reaction may show in an increase of the physical signs. If there previously was a roughened or harsh respiratory murmur, it might be increased; if there were no rales, rales might be produced; if there were rales, the number would probably be increased; or, there might develop on the part of the patient a feeling of constriction, actual pain, or a tendency to cough. If the infection be visible, an area of hyperemia or even congestion may appear. If it be in the joints, kidney, testicle, or ovary, pain may be felt. All these focal signs are very suggestive, and while they require care in their interpretation, yet they add very valuable diagnostic evidence.

While it is possible to produce general and focal reactions by the introduction of tuberculin through the skin or mucous membrane, yet the other methods of administering the tuberculin test, which I shall describe, depend for their value upon the reaction at the point of contact between the tuberculin and the body-cells. The general and focal reactions are left out of account. This fact has made the more recent tests very popular, and has robbed the tuberculin test of the fear in which it was formerly held. The old idea of the mobilization of bacilli through a tuberculin reaction has been slowly driven from the minds of the medical profession. The fear to-day, however, should not deter any one from securing the benefit from the subcutaneous test provided due care is used in its administration.

Cutaneous Test.-Von Pirquet announced in 1907 that tuberculous patients react to tuberculin when tuberculin is administered by scarification of the skin, in a manner similar to that employed in vaccination against small-pox.

'The test is given by cleansing the skin with alcohol or ether and drying it thoroughly before applying the tuberculin. For the test, old tuberculin of various strengths is used. Von Pirquet himself experimented with many different strengths, and found that a 25 per cent. solution of old tuberculin is probably the best. A drop of this solution should be placed on the skin, and then with a small lancet a superficial abrasion is made. Care should be taken not to cause bleeding. It is only desired to bring the tuberculin in contact with the superficial lymph-spaces. A control scarification should also be made at a convenient distance from the point where the test is to be made. Extreme care should be used that no tuberculin comes in contact with the control 
scarification. The best lancet to use is one like that suggested by von Pirquet himself, in which the entting surface is on the end of the blade. A simple twist on the skin produces the searification. 'The scarification can be made with the cutting surface of a bistoury', but it is not so good, as it is more diflicult to make the same tramma in the control and in the vaceination; but, with a little turn of the instrument suggested by von Pirquet, the skin is abrated, and the same pressure can be used at both points. If the reaction is negative, the control and the point of inoculation will appear the same, simply showing the result of trauma. If the reaction is positive, it shows, as a rule, as a slight raised area of reduess, in the middle of which a small seab may form. 'This area of reaction may be very slight or it may be $2 \mathrm{~cm}$. in diameter. 'The advantage of this method is that there is no pain comnected with it, neither before nor after the test. In only one or two instances have I seen vesicles form following the scarifieation. It is to be especially recommended in children.

This method is so simple that it was adopted at once and tested generally. 'Those who have used it have interpreted it very differently from von Pirquet himself. Realizing the fact that the incidence of tubereulosis in childhood inereases as age advances, and that probably all individuals who have an infection, unless it be thoroughly healed, react to the cutaneous test, von Pirquet laid little stress upon its diagnostie value, except during the first two years of life; in fact, he showed very early that nearly all children will react by the time they are twelve or fifteen years of age. In adults he did not consider it of special value. I believe, however, that it is of value even in adults, but that it must be interpreted in the right way.

The Conjunctival Test.-In June, 1907, Wolff-Eisner published the fact that tubereulin when instilled into the conjunctival sac would produce a local reaction. During the same month Calmette published the same fact. While Wolff-Hisner was prior in his work, yet there has been some tendency to call this reaction the Calmette reaction. If it should be ealled by the name of an individual, it would certainly be fair to call it the Wolff-Eisner reaction or the Wolff-Eisner-Calmette reaction, but it seems more appropriate to call it by the name "conjunctival reaction."

The conjunctival test is produced by the instillation of 1 or 2 drops of a dilution of old tubereulin in sterilized physiological salt solution into the eye. The strength of the solution varies. Wolff-Eisner used a 1 per cent. solution, later a 2 per cent. solution. Baldwin recommended the initial dose to be of a 0.35 per cent. solution, while other writers have used from 1 to 4 per cent. 'This solution should be made fresh every day.

In dropping the solution into the eye, the patient should hold his head backward, the lower lid should be drawn down, and the drop instilled. 'The lid should not be released at once, but held for a moment so that the liquid becomes well applied to the surface and is not pressed out, as it is more apt to be if the lid is allowed to resume its normal position. 'The patient should then hold his head back for a few minutes 
and not rub the eye. This test may be used in all cases where the eye is normal, but if there is a disease of the eye, it seems best not to use it. It is probable, however, with careful technique in the preparation of tuberculin, and by making the dilution fresh each day, that no danger will result to the normal eye. Wolff-Eisner himself has made the test in some 5000 cases without harm.

A positive reaction consists in the production of conjunctivitis. If it be a slight reaction, it may amount to only a slight hyperemia; if it be marked, it may produce a severe conjunctivitis which may last for a number of days. Such severe reactions are exceptionally rare.

An analysis made by Wolff-Eisner of many cases where both the conjunctival and cutaneous tests were employed, indicated that the cutaneous test is much more sensitive than the conjunctival, and that it is much more apt to show a reaction in the presence of a latent focus than is the conjunctival. Wolff-Eisner claims, after careful study of many cases, that the conjunctival reaction is not apt to occur unless the disease be active. His explanation of the difference in frequency of reaction between the cutaneous and conjunctival test is that when the tuberculin is dropped into the conjunctival sac, the blood supply is so rich that unless there be many antibodies present, the tuberculin is absorbed and carried off before the reaction can occur, while in the cutaneous test the blood-supply is not so great, consequently the tuberculin remains at the point of scarification until sufficient antibodies collect to cause the reaction. If further experiments bear this out, it will be very much in favor of the conjunctival reaction as compared with the cutaneous.

The Morro Reaction.-In 1908 Morro published the fact that a tuberculin test could also be made by an ointment consisting of 50 per cent. tuberculin in lanolin. This ointment, when made, may be preserved in a refrigerator for months without deterioration. For making the test, a portion of the ointment about as big as a pea should be used. Any portion of the skin may be used, although some portion where the skin is thin is best, for example, the skin covering the chest, abdomen, or flexor surface of the forearm. The ointment should be rubbed into the skin for one-half to one minute. It is best not to use too large a surface for the inunction. A reaction consists of a number of small red spots usually coming on from twenty-four to forty-eight hours after the inunction, and disappearing after a few days. In some cases I have seen quite a severe reaction follow the Morro test. It would seem to me that this test is not as satisfactory as the percutaneous, which, if made after the method described by von Pirquet himself, leaves no doubt of the tuberculin coming in contact with the lymphspaces. When the Morro test is made, however, it may be that the ointment is not rubbed in sufficiently, and consequently the test may fail where it should be positive. The test is harmless unless too large a quantity of the ointment is used, and like the cutaneous, is especially useful on that account. To my mind, however, it is a question whether it is sufficiently trustworthy to be relied upon in case of a negative result, owing to the failure of absorption. The experience of most men who 
have used this is that the percentage of reactions is much less than with the other methods.

Lignieres has also described a percutaneons test. Insteat of using an ointment, 5 or 6 drops of undiluted old tubereulin is rubbed on a well-shaved skin. 'The disadvantage of this test to my mind is the likelihood of producing a reaction. I have seen very severe tuberculin reactions follow the rubbing in of tuberculin through the skin of the forearm. Carl Spengler his used this method in treatment, especially in children, for several years.

The Intracutaneous Test.-The intracutaneous test, as defined by Mendel and Moussu and Mantoux, is also of value in diagnosis. It consists of injecting the tuberculin into the skin itself, a positive reaction showing as a distinct reddening of the skin, sometimes with induration. Koch's old tuberculin is used in a dilution of 1 to 5000 . Of this dilution, 1 drop, which equals $0.01 \mathrm{mg}$., is used for the test.

Wolff-Eisner suggests that febrile cases be treated with intracutaneous injections, believing that the tuberculin injected into the skin itself, being absorbed very slowly, is less apt to eause reaction.

Detre's Differential Test.-Following the work of Koch and C. Spengler, Detre suggested that a differential test might be made by the use of tuberculins made from both the bovine and human eultures. In case the patient reacted to the bovine tuberculin and not the human, he inferred that it was a case of infection from bovine tubercle bacilli. If a patient reacted to the human and not the bovine, he inferred that it was an infection from human tubercle bacilli; while if he reacted to both, it was probably an infection from both the human and bovine tubercle bacilli. It is probably impossible to draw conclusions from the result of this differential test; at the same time it is surprising to see how often patients will react to one of these (the human), without reacting to both.

'The administration of this test, at least, offers evidence favoring the fact that tuberculins made from bovine and human tubercle bacilli are different; although it is questionable if, as a differential test, it is beyond question.

\section{SERUMS}

Maragliano's Serum.-In the efforts to find a passive immunizing serum against tuberculosis, the name of Maragliano stands out prominently. He published his first results in 1895 , and he has been working continuously in this line since. His serum is that of an animal (usually horse or cow) which has been subjected to repeated increasing doses of a watery extract of tubercle bacilli and a pulp of virulent tuberele bacilli until such a time as its blood contains a certain amount of specific antibodies. 'The time of treating the animal is usually about six months.

This serum is injeeted subeutaneously, as a rule, the dose being 1 cc. every second day for ten days, then 5 cc. every second day for ten days, then $10 \mathrm{cc}$. every second day for twenty days more.

The opinions of clinicians on this remedy differ as do the opinions of medical men on all remedies. Some have reported excellent results 
with it, while others have nothing but condemnation for it. It seems to merit a further trial.

Marmorek's Antitubercular Serum.-Marmorek announced in 1903 that tuberculin is not the real toxin of tuberculosis, and that the young bacilli do not excrete tuberculin, but another substance which he believes to be the true toxin. He thereupon devised a special medium containing leucotoxic serum and extract of liver tissue, which he claims has the power of preventing the bacilli from developing rapidly; thus keeping them in the "primitive forms" for a longer time and allowing them to produce larger quantities of the "true tuberculous toxin." Horses were immunized with these cultures, and their serum used therapeutically. Owing to the fact that the subcutaneous use of serum produces urticaria and other annoying symptoms, this has more recently been used per rectum, by which method these annoying symptoms, for the most part, are avoided. The dosage is 5 to $10 \mathrm{cc}$. every day for three weeks, then omitted for two weeks and then repeated again.

The reported results are conflicting, but seem to warrant a more extended use.

Immune bodies (J. K.), Spengler, is a preparation which is described by its author as containing both lysins and antitoxins, 1,000,000 of each to the cubic centimeter. Spengler has not published his method of making this yet, because he does not consider it perfected.

The action of "J. K," as described by Spengler, is twofold, first to produce a lysis of the tubercle bacilli, and second to combine with the toxins and prevent them from causing symptoms. An important feature of "J. K." is that it also contains antibodies against streptococci and other ordinary bacteria associated with tubercle bacilli. I have used "J. K." constantly from the time of its discovery. My early impressions of it were unfavorable, but I found that I was not using it correctly. My recent experiences with it are very satisfactory, and I believe we have in it a remedy of unusual merit.

The administration of " $\mathrm{J} . \mathrm{K}$. ", is difficult. It requires even more judgment than tuberculin in order to produce the best results. Many of our old principles of tuberculin therapy spoil us somewhat for this; for example, the intervals between doses in "J. K." are greater at first than they are later. As the treatment progresses, the doses become larger and the intervals between them decrease. We do not give tuberculin when the temperature is rising, but many times I have seen the proper dose of "J. K." prove effective in reducing fever when the temperature was on the rise.

"J. K." is diluted for administration by the following solution, in which the carbonate of soda is used to alkalinize the mixture, experience having shown that alkalinization decreases the lytic action and increases the antitoxic action of the remedy.

R. Sod. chlorid.,

Acid. carbolic cryst.;

Sol. sod. carb. sat.,

Aq. dest.,
0.5

0.5

gtt. i-iv;

q.s. ad. 100.0.-M. 
The reports in literature so far have, for the most part, been adverse; but the method of dosage where given has been almost universally faulty. Spengler's first publication as to dosuge gave a wrong impression; and while he has corrected this in his own practice and in his later publications, yet many physicians who began its use early, used it wrongly and were disappointed.

“J. K." is prepared for use by making a series of dilutions, using the formula given on p. 602. Each succeeding dilution is one-tenth as strong as the preceding, thus:

$$
\begin{aligned}
& \mathrm{O}=\text { original solution } \\
& I=\}_{0} \text { cc. original solution }+{ }_{1 \%}^{9} \text { cc. diluting fluid }= \\
& \text { II }=\frac{1}{10} \text { cc. I } \quad+9 \text { cc. diluting fluid }= \\
& I I I=\frac{1}{10} \text { cc. II } \quad+90 \text { ce. diluting fluid }= \\
& \text { IV }=\text { If cc. III } \quad+\frac{9}{10} \text { cc. diluting fluid }= \\
& V=\frac{3}{30} \text { cc. IV } \\
& \mathrm{VI}=\mathrm{x}^{3} \mathrm{ccc} . \mathrm{V} \\
& V I I=\frac{1}{10} \text { ce. } V I \\
& =1,000,000 \text { lytic and anti- } \\
& \text { toxic units. }
\end{aligned}
$$

Treatment is usually begun with solutions No. VII, VI, or V; $\frac{1}{10}$ cc. or $\frac{2}{10}$ cc. being given. After the first dose a pause of eight to fourteen days is usually observed to watch the effect. 'Then the dosage is increased every four to eight days according to circumstances. If the first dose were $\frac{2}{\sigma_{0}}$ of $\mathrm{VI}$, the sequence in an carly uncomplicated case should be about as follows: $\frac{2}{10} \mathrm{~V}, 1_{10}^{2} \mathrm{IV},{ }_{10}^{2} \mathrm{III},{ }_{10}^{2} \mathrm{II}, \mathrm{1}^{2} \mathrm{I}, \frac{2}{10}-0,5-0$, 10-0.

It requires care and judgment to administer " J. K." No scheme can be given. It must be learned either from some one who knows, or at the expense of the best results.

It seems to have a very marked antitoxic effect, as is shown in reduction of temperature and pulse-rate, and the relief of those subjective symptoms which are usually considered as toxic, such as malaise, nervousness, and lack of appetite.

The following chart illustrates the effect of the remedy upon the temperature and pulse. Chart II is one of the most instructive and convincing charts that I have seen. It will be noted that coincident with the administration of every dose there was a maried reduction in the pulse-rate, so that in the space of one month it had dropped from 100 to 72 per minute. Such a marked result must not be expected regularly, yet a diminution in the pulse-rate is the rule in a large percentage of cases. Chart III illustrates the effect of "J. K." on both temperature and pulse-rate.

Regardless of the adverse opinions which have been given, I believe it is one of our most valuable remedies and would urge that it be given a careful trial according to Spengler's later method.

Specific Treatment of Mixed Infections.-In approaching the subject of mixed infection in tuberculosis, I realize that I am entering upon a subject which is still unsettled. There was a time when it was thought that tuberculosis itself did not destroy life, but that death was practically always due to some concomitant infection. Since then the pen- 
Fig. 87.

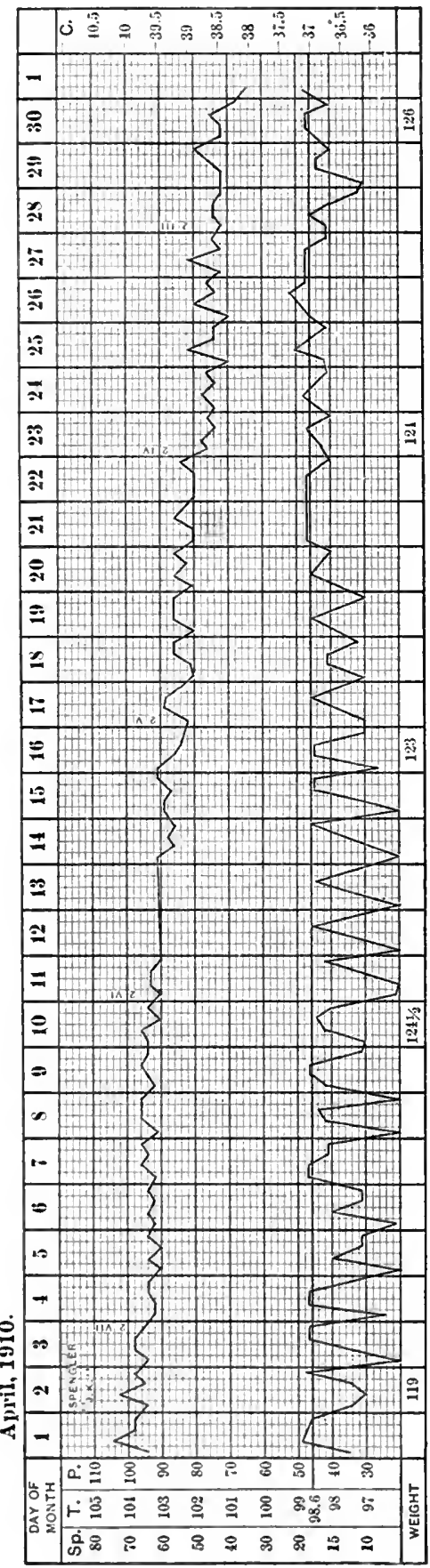

dulum has been swinging back, and we recognize to-day that while mixed infection is sometimes a very important complication in tuberculosis, yet the tubercle bacillus and its toxins are able to produce the same symptoms that have been assigned to the various associated bacteria.

A simple microscopic examination of the sputum after staining and the cultures taken from sputum as it comes from the mouth nearly always show the presence of other bacteria; but this does not mean that they are necessarily pathogenic. Many erroneous diagnoses of mixed infection have been made, based on the examination of microscopic slides and disregarding this fact. It is even doubtful whether the cultures taken from a washed specimen of sputum which has been removed from the center of a so-called "cavity mass" are to be regarded as unquestionably pathogenic.

One's ideas of a mixed infection will depend very much upon the strictness of the rules which he adopts to determine whether or not the bacteria are pathogenic. Much of the confusion on mixed infections comes from the fact that different workers use different methods, without publishing them along with their results. The determining of pathogenicity is somewhat difficult; for example, in dealing with the streptococcus; a streptococcus isolated by most careful washing methods will grow upon ordinary agar, while pathogenic streptococci should not do this.

Illustrating the effeet of "J. K." in the lowering of pulse. It will be noted that at each dose there was a distinct influence upon the pulse, so that in the space of one month its rate had lowered from 100 to 72 per minute.

KEY TO DOSAGE: 2 -VII $=\frac{2}{10}$ cc. of VII dilution, J. K., etc. (see text). 
$\therefore$

䒠

0

产苛

然

당

$\because=0$

ن?

-

ज坷

II

吅

รᄋํㅇ

응

需

四

喝。

Es

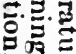

응

के

क्ष

대 象象

雪

है흘

E

글.

ह

홍.

을

5

용

"⿹

음

哭

$\rightarrow$

实

릉

ह

롤

क.

की

혹

o

ב.․․

它
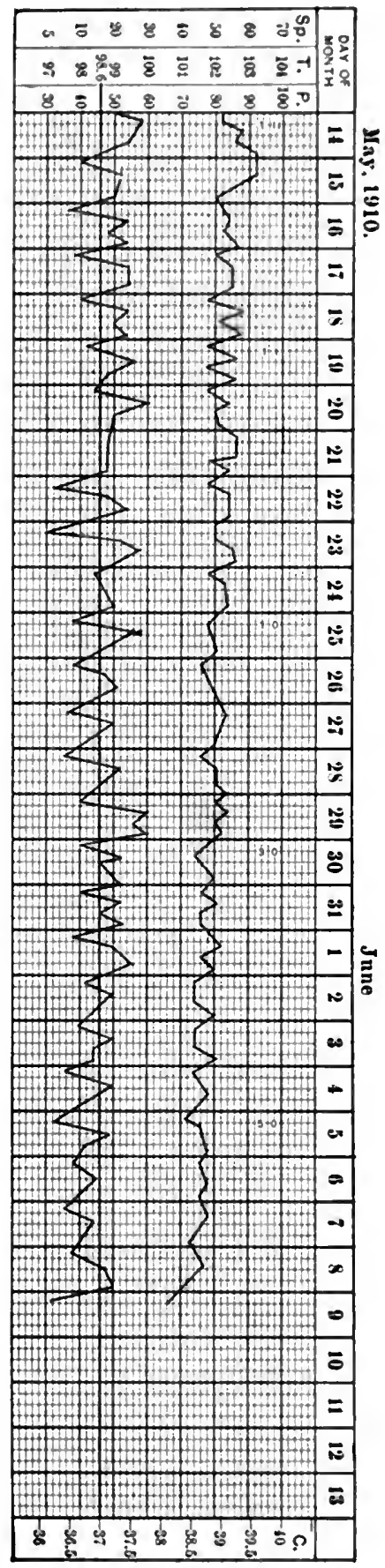
It would be well for those who are working along this line to always publish carefully their method of determining the pathogenicity. The most common bacteria found in the sputum are the staphylococcus, streptococcus, pneumococcus, Micrococcus catarrhalis, Friedländer's bacillus, Micrococcus tetragenus, and influenza bacillus. The relative frequency with which these micro-organisms are found depends upon the condition of the patients who are examined, the locality in which they live, the presence or absence of various epidemics, and the season of the year.

T'le same bacteria which are found in the sputum have been cultivated from cavities postmortem; but the presence of these bacteria in the cavities does not necessarily indicate that they are pathogenic. 'Tuberculous sinuses in various parts of the body, however, show the presence of secondary bacteria, which would indicate that tuberculosis of the lungs might also show secondary infections.

Whether the various non-pathogenic bacteria found in the sputum are totally harmless is a question for future determination.

In the treatment of the various secondary infections, our advancement has been somewhat disappointing. When vaccine therapy received its impetus from the masterful work of Wright, we hoped that we had found a means of relieving tuberculous patients of many of their most troublesome complications; but, up to the present time, efforts along this line have not been crowned with great sticcess. The working out of the specific treatment of mixed infections should be for the present left to the specialist who has associated with him careful laboratory workers. 


\section{PART III}

\section{TREA'IMENT OF THE INFECTIOUS DISEASES}

\section{TYPHOID FEVER}

BY DAVID RIESMAN, M.D.

'TYPHoIn fever stands as the type of acute infectious disease; a mastery of its epidemiology and treatment gives in a large measure control over other infectious fevers. Its history, especially during the last three decades, epitomizes the development of medical science. In the discovery of its cause, in the elucidation of its modes of transmission, and in the study of its prevention, we have an inspiring picture of what is happening in the whole realm of medicine. Especially in the field of prevention has signal progress been made; and if the work along this line is as intensively continued as it is being pursued today, the result will be the disappearance of typhoid fever from the civilized countries of the world. Preventive medicine, it should, however, be realized, is not the sphere of the trained sanitarian alone, but must be the daily thought of every practising physician, for it is the latter's duty not only to treat the ease in hand, but also to see that no others arise from it. As Whipple ${ }^{1}$ tersely says, he should act in a dual capacity, as medical adviser and as sanitary guardian $e x$ officio.

In order to devise and to apply preventive measures against typhoid fever, a thorough understanding of its modes of infection and transmission is indispensable. Infection in this disease takes place through the alimentary eanal, and arises from the ingestion of typhoid bacilli, which are invariably derived, directly or indirectly, from a previous case of the disease. A typhoid fever patient discharges typhoid organisms with the feces, and, in a large proportion of cases, with the urine; and it is through these excretions that all the agencies through which the disease is contracted become contaninated. 'This being the case, it follows that if typhoid fever is ever to be entirely stamped out it can only be done by killing the bacilli as soon as they leave the human body; in other words, by sterilizing the dejecta containing them.

1 Typhoid Fever, 1908, p. 24. 
As will be shown further, the bacilli may gain access to drinking water, to milk, and to other food, and through them may find entrance into the body. They may also cling to the hands of those coming in contact with typhoid fever patients, especially when in attendance upon them, and may be directly carried to the moutl or taken in with the food. It has, furthermore, been demonstrated that flies, by feeding upon the fecal discharges of typhoid fever patients, and then alighting upon food, may act as transmitters of the disease. In dry climates the discharges may, if thrown upon the surface of the ground, become desiccated and pulverized withont destruction of the bacilli. A wind storm may then carry the germ-laden dust to considerable distances and deposit the bacilli on food or in milk. 'This seems to have been the cause of several outbreaks of typhoid fever among the British troops during the South-African War, and was considered a factor in the spread of typhoid fever in our own army camps during the Spanish-American War.

The infective agent in all these modes of transmission is, in the final analysis, the excreta of the typhoid fever patient. For many years it was the current belief that the bacilli were contained in the feces only during the course of the disease, but brilliant and painstaking studies made within the last few years have shown that many persons discharge virulent bacilli long into convalescence-and some even after they have apparently recovered completely. These latter, which, according to Lentz, constitute about 4 per cent. of all cases, are the so-called "typhoid carriers"-persons who, while in apparent health, eliminate daily large numbers of living typhoid bacilli in the feces, more rarely in the urine, and act as foci of origin for local outbreaks of the disease. Inasmuch as a large proportion ${ }^{1}$ are women, the danger is greatly augmented through the domestic occupations of such typhoid carriers.

The reasons for persistence of typhoid bacilli in the feces after recovery are not altogether clear. It may be that slight ulceration remains here and there in the intestines, or in the appendix. More probably, however, the germs come from the gall-bladder, in which they may live for many years. ${ }^{2}$

Virulent bacilli may also be found in the urine long after convalescence is established. Young ${ }^{3}$ records a case in which they were present five years after an attack of typhoid fever. The number of bacilli in cases of bacteriuria is at times enormous. Petruschky found as many as $72,000,000$, and Gwyn over $500,000,000$ to the cubic centimeter.

I have spoken of typhoid fever carriers as persons who have had typhoid fever. Carriers have, however, been discovered who had never had the disease. In a locality in which typhoid fever is epidemic many persons ingest bacilli withont becoming infected. The organisms occasionally may find a lodging place, and may continue to live and to multiply in a symbiotic way in the host. In this dangerous class belongs

1 Four-fifths, aceording to Forster, Münch. med. Woch., 1908, No. 1.

${ }^{2}$ Neufeld (Kolle-Wassermann, Handbueh d. pathog. Mikroorg.), five, fifteen, and seventeen years.

${ }^{3}$ Johns Hopkins Hosp. Rep., 1909, viii, 401. 
the now famous "'lyphoid Mary," reported by Park, a cook who, in her numerous migrations, was the unknowing cause of twenty-six cases of typhoid fever. Her sinister career has been traced by Soper ${ }^{2}$ in a series of masterly investigations.

Another factor in the epidemiology of typhoid fever is of great practical importance, namely, the occurrence of mild, abortive, and anbulatory cases (short duration typhoid ${ }^{3}$ ) - cases that usually escape detection. Such types are especially common in children, ${ }^{4}$ although they occur in adults, and explain some of the limited outbreaks for which the water or milk supply cannot be blamed. In these cases contact infection plays the principal part. It goes without saying that unrecognized cases may also become the source of endemic or epidemic outbreaks, through the medium of water or milk contamination.

Sources of Infection and their Control.-Drinking Water.-Outbreaks affecting entire communities or large parts of a community are nearly always water-borne. 'The evidence of this is of the strongest character. I need cite only the historic epidemics of Maidstone, England, and of Plymouth and Butler, Pennsylvania. Almost equally strong circumstantial evidence is afforded by the experience of many cities throughout the world, in which improvement in the water supply has been followed by a tremendous fall in the incidence of the disease.

The drinking water of many cities is taken from the same streams into which sewage is emptied. In rural communities the water of wells, as Dutton has graphically shown, may become contaminated by a leakage from cesspools placed near by and at a higher level.

A large city can obtain an absolutely pure water supply only by protecting the source of intake and the water in transit against contamination. 'This is difficult, but not impossible, and is the ideal for which some cities are striving; as, for example, New York, in its magnificent undertaking to bring a pure water supply from a great distance. Where the water is, of necessity, taken from open streams and unprotected lakes, filtration by the municipality is the best method of rendering the water pure. 'The results upon the incidence of the disease of improvement in the water supply is well shown in the accompanying table of Kober. ${ }^{6}$ 'To the statistics in the table I might append those of Philadelphia. In 1908 the number of cases of typhoid fever was 3564 , which was less than half the average number for all the years tabulated, a result for which the filtration system is largely responsible. 'There were in that year a few sections that received unfiltered water. In these sections 337 cases of typhoid fever occurred per 100,000 of population. In the sections receiving filtered water the rate varied from 115 to 172 per 100,000 of population.

'Jour. Amer. Med. Assoc., 1908, li, 981.

${ }^{3}$ Coleman, Amer, Jour. Med. Sci., Jume, 1909, p. 781.

'Ibid., xviii, 2019.

- Koch (Die Bekampfung der Typhus, Berlin, 1903) found that 49 out of 64 unrecognized eases of typhoul fever in Treves (Trier) oecurred among children.

bour. Aner. Med. Assoc., 1910, Iv, \$54.

- 'Transactions Assoc. Amer. Phy's., 1909. voL. 1. -39 
DEATH RATES PER 100,000 OF POPULATION

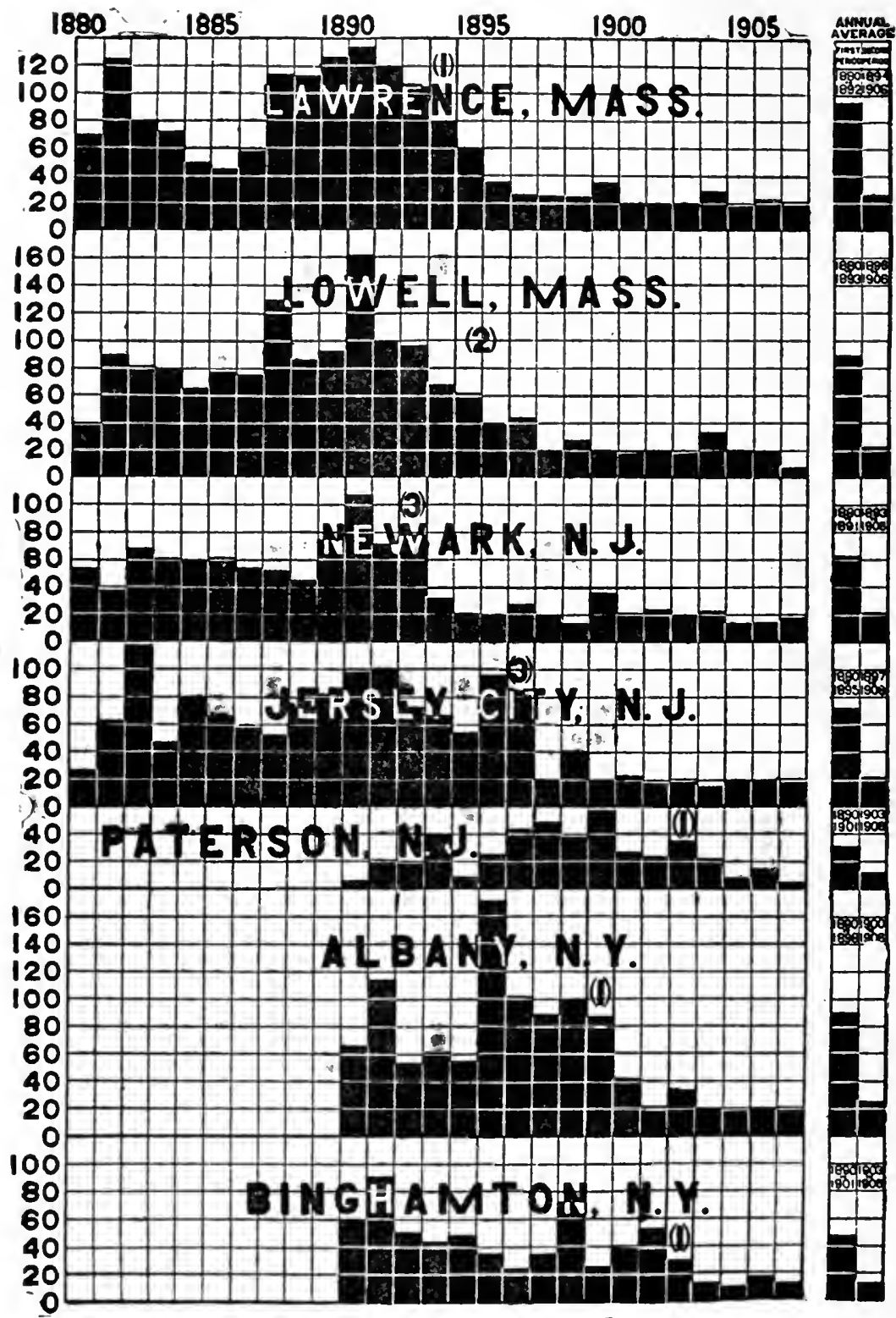

CHANGE IN WATER SUPPLY

(1) From unfiltered river supply to filtered river supply

(2) From unfiltered river supply to wells

(3) From polluted river supply to conserved river supply 
The experience of Cincinnati, Richmond, and many other cities is similar.

Some of the large cities of the world are not only filtering their drinking water, but are also purifying, or preparing to purify, their sewage. Sot much good is to be expected from this so long as other localities empty raw sewage into the stream higher up; that is, proximal to the intake. Iet us hope that eventually no contaminated sewage will be allowed to flow into streams supplying drinking water to riparian communities. Of course, where the body of water receiving the sewage is relatively large, the dilution the latter undergoes may be sufficient to prevent any decided pollution of the water supply. 'This fact is demonstrated by the experience of Milwaukee, which empties its sewage into, and takes its drinking water from, Lake Michigan. When the intake in 1995 was carried far out into the lake $(6000$ feet) and placed at a point three and a half miles from the sewage ontflow there was an immediate drop in the typhoid rate to about half. ${ }^{1}$ Slowly, however, the growth of the city asserted itself by an increasing volume of sewage poured into the lake and the consequent increased contamination of the water. For the present year the rate promises to be as high as before the extension of the water intake from its original point of 2100 feet from shore. From this we may conclude that it is wise at all times to purify all sewage before it is emptied into a body of potable water, no matter how large.

A number of cities, among them Washington and Philadelphia, have been chagrined to find that even after filtration had been established, occasional outbreaks of typhoid fever occurred. Several explanations are possible: the filtering plant may temporarily break down; cases may be imported, as usually happens in Philadelphia at the end of the summer vacation; or the outbreak may not be water-borne at all, but due to typhoid carriers, to milk contamination, or to contact infection.

The individual can best protect himself against water-borne typhoid fever by drinking only boiled water, or spring water, even in cities where filtration obtains. Cheap filters attached to spigots are dangerous. Boiled water should be used not only for drinking, but also for other purposes, such as cleansing the teeth, washing vegetables, fruits, etc. Persons who are travelling should avoid drinking local waters. It is better to depend upon some well-known safe bottled water.

The typhoid bacillus is probably not capable of multiplying in water, but it can live in it for from two to three weeks. ${ }^{2}$ 'Tavel ${ }^{3}$ records an instance in which bacilli survived in the blind end of a water main for six months.

Inasmuch as typhoid bacilli are not killed by freezing, ice may become a carrier of the disease. 'Thus Park reports an epidemic caused by ice from a certain pond. It was proved that the dejecta of a typhoid fever patient had been thrown on the surface when the pond was frozen.

1 Jour. Amer. Med. Assoc., July 16, 1910.

2 LoefHer, Weyl's Handbuch der Hygiene, 1S96, i, 636, 676.

3 Cent. f. Bakter., 1903. 
Milk.-Outbreaks of typhoid fever due to contaminated milk are not rare in literature, and seem to be increasing in frequency with improved methods of tracing the sources of epidemics. Kober, ${ }^{1}$ in 1901 , collected records of no less than 195 epidemics of enteric fever traceable to milk supply, in 148 of which the disease had prevailed at the farm or dairy. Trask, ${ }^{2}$ in 1909, found 179 typhoid fever epidemics reported as spread by milk. In the Washington epidemic of $1907,3.17$ per cent. of the cases were attributable to milk infection; in that of $1906,11.3$ per cent. In the Stamford epidemic, ${ }^{4}$ one dairy supplied 225 households, in which 352 cases of typhoid fever occurred; a café, among the frequenters of which 12 cases developed; a bakery, whose patrons furnished 2 cases; and there were 2 other typhoid fever patients who had obtained the milk in other ways. In Munich where, through the rapid and thorough removal of discharges for the protection of the drinking water, typhoid fever has been almost stamped out, the disease broke out in a certain quarter of the city and continued to prevail for seven years. It was then traced to a particular milk. When the sale of this milk was prohibited the outbreak ceased. ${ }^{5}$

During the recent epidemic in Budapest not only was the milk held to be an important factor, but also certain milk products, such as butter and soft cheese. In butter, according to Heim, ${ }^{\circ}$ typhoid bacilli may survive twenty-one days; in buttermilk ${ }^{7}$ they live forty-eight hours. They also live in ice-cream if the milk used is not boiled, since, as already mentioned, freezing does not kill them.

Every localized outbreak of typhoid fever should be traced to its source. Jensen ${ }^{8}$ states that where the water supply is safe and hygienic, local outbreaks come principally through milk. If the milk supply is suspected, the place whence it is derived and the people handling it should come within the scope of the investigation. The sale of milk from an infected dairy or farmhouse should be prohibited.

Other Foods.- Several small epidemics have been traced to oysters and other shellfish. In certain sections of the country it is customary to place oysters for a short time in fresh or brackish water to fatten them. If the water is contaminated by sewage, which probably happens rarely, the oysters may take up typhoid bacilli, and, when eaten raw, cause infection of the consumer. Experiments have shown ${ }^{\theta}$ that in oysters placed in artificially infected water, the typhoid bacilli may live twenty days - long after they have disappeared from the surrounding water.

Among other foods, vegetables, like celery, lettuce, and watercress, and all such fruits as are eaten raw, may act as typhoid carriers. They

1 Amer. Jour. Med. Sci., May, 1901.

2 Milk and its Relation to the Public Health, Hygienic Laboratory Bulletin, No. 56.

${ }^{3}$ Report on the Origin and Prevalence of Typhoid Fever in the District of Columbia, Hygienic Laboratory Bulletin No. 35, Rosenau, Lumsden, and Kastle.

4 Herbert E. Smith, Connecticut State Board of Health Report, 1895.

5 Mandelbaum, Münch. med. Woch., 1908, No. 1.

- Arb. a. d. Kaiserl. Gesundheitsamt, Band v.

'Fraenkel and Kister, Münch. med. Woch., 1878, No. 7.

8 Essentials of Milk Hygiene, 1907, English ed., p. 106.

- Horcicka, Wien. med. Woch., 1900, Nos. and 3. 
may be contaminated by the hands of the huckster, grocer, or cook, or through being washed in infected water. Both raw and cooked food may, furthermore, become infected through the angency of insects, as will be shown later.

Investigations made in the Imperial Health Office at Berlin seem to show that eggs may at times be the source of paratyphoid infection in man. Paratyphoid bacilli remain viable on the eggshells for sixteen days or longer, and may even penetrate into the egg itself. 'The eggs, of course, do not contain the bacilli when laid, but, like milk, lecome contaminated in handling.

Insects.-Flies, it is now definitely proved, play an important role as typhoid carriers. 'They feed, when proper sanitary measures are not observed, on human excrement, and earry, purely mechanically, on their bodies, virulent bacilli to food. 'There is reason to believe that they constitute a special danger in army camps. 'They were, in large measure, responsible for the epidemics of typhoid fever that ravaged the American army camps during the Cuban War, and that prevailed among the British troops in South Africa during the war with the Boers. The striking distance of a fly factory, to use the words of an unknown writer, ${ }^{1}$ is 200 yards. 'There should, however, be a space of not less than 300 yards between refuse depots and houses.

Insects other than flies have little to do with typhoid fever, and there is no evidence that animals of a higher order communicate typhoid bacilli to man, although experimentally it has been shown by Barbaschi² that snails and slugs, which live on vegetables usually eaten raw, may act as typhoid carriers.

Guiart $^{3}$ and others have attributed to intestinal worms, especially to the Trichiuris trichiura ('Trichocephalus dispar), a role in the production of typhoid fever. 'The worms are supposed to cause a mechanical lesion of the mucous membrane, through which the oft-present typhoid bacilli find a ready entrance. The theory, to my mind, is fanciful. Lacomme and Vaulaude, Chantemesse and Rodriguen, ${ }^{5}$ and Stiles ${ }^{6}$ find no evidence for it, nor does my own experience bear it out.

From what has been said regarding the menace of flies, it follows that these insects must not be allowed to have access to human feces. In rural districts where there is no sewage system, cesspools and privies must be covered and screened. From dwelling houses flies should be excluded by means of screens; and those that enter should be killed with the same real as that with which mosquitoes and bedbugs are exterminated. The breeding places of flies must be hunted out and destroyed. Whether the suggestion of Dr. Perey I. Jones, of the United States Army, that a Philippine ant, destructive to fly larva, and itself

'British Med. Jour., May 9, 1908.

2 Gazz. degli Osperl. e delle Cliniche, 1910, No. 37, 355.

3 Areh. de parasitol., 190-4, p. 122.

Jour. de Phys., 1908, No. 1.

3 Bull. de l'Aead., April $7,1908$.

- Bull. Hygienic Laboratory, No. 35, p. 195. 
innocuous, be introduced into the United States, should be adopted or not, is a question requiring careful and scientific research.

Contact Infection.-I have already alluded to this. It plays a much more important role than hitherto has been supposed, and places typhoid fever among the truly contagious diseases. Contact infection is especially common among children. It also accounts for a very large proportion of the cases occurring among physicians, nurses, and others of a hospital's personnel. 'There are also on record cases in which trphoid fever was conveyed by a clinical thermometer that had been used to take the rectal temperature of a patient. ${ }^{1}$

Nurses and orderlies should wear gloves when handling discharges, and should, in addition, disinfect the hands after every kind of service to a typhoid fever patient. In the world at large contact infection may be lessened by educating the laity concerning the dangers of typhoid fever, especially as to the ease with which it may be transmitted from the sick to the well. Furthermore, it is important that mild cases should be recognized, and that all typhoid carriers should be searched out. How to do this is not the province of this article to discuss. I need refer only to blood culture, examination of the feces for bacilli, and the Widal reaction and other blood tests for typhoid antibodies. During the summer months, especially in cities and communities in which typhoid fever is endemic, every case of acute enteritis persisting for more than a few days should be considered suspicious.

Specific Methods of Prevention.-Immunization; Vaccination; Bacterination.--The question is often asked by intelligent laymen and by students and physicians not familiar with immunology, "Why is there no antitoxin for typhoid fever?" 'The answer is to be found in the peculiar nature of the toxins of the disease.

Bacterial toxins are of two kinds: (1) Those that are easily diffusible; hence, are found in the culture media and in the fiuids and tissues of the infected animal; these are called toxins, or exotoxins. (2) Those that are not easily diffusible, called intracellular toxins, or endotoxins. The former are true secretory products, elaborated by the living bacteria. 'The latter are so firmly united with the bacterial bodies that they are set free only when the microörganisms die and disintegrate. It is not impossible that they, too, are secretory products, but their difficult diffusibility differentiate them from toxins proper. When the latter are injected into animals or spontaneously elaborated in disease they induce the formation of antitoxins which have the power of chemically neutralizing the toxins without exerting any direct action upon the bacteria themselves. On the other hand, when endotoxins are injected into susceptible animals, or are formed spontaneously through the disintegration of pathogenic bacteria, they produce not antitoxins, but so-called antibodies which have a specific influence upon the corresponding bacteria.

${ }^{1}$ Leube, Speeiel. Diag., ii, 404; Bormanns, quoted by Neufeld (Kolle and Wassermann, Handbuch d. patholog. Mikroorganismen, ii, 296). 
In a general way the action of antibodies is fourfold:

(a) Agglutinative (agglutinins).

(b) Opsonic (opsonins).

(c) Bactericidal.

(d) Bacteriolytic (bacteriolysins).

Some endotoxins may produce anti-endotoxins.

It is conceivable that a sermm rich in antibodies might be harmful when injected into an animal infected with the corresponding bacteria, in that it might cause a great destruction of the latter, and in that way set free the endotoxins. A given bacteriolytic serum may thus become at double-edged sword-while it kills the bacteria it also by this very act liberates their poisons.

'The typhoid bacillus belongs to the class that does not elaborate toxins. It exerts its pathogenic effects through the action of powerful endotoxins. 'The blood of the typhoid fever patient, as well as that of the experimental animal, contains the antibodies referred to above, in large quantities, but no antitoxins. According to Wassermann the antibodies, or immune substances of typhoid fever, are derived from the spleen, the bone marrow, and the lymph glands. Experiments seem to show that they can pass through the placenta and that they may be conveyed to the offspring by the mother's milk.

From what has been said above it would seem to follow that the toxemia of typhoid fever is an endotoxinemia. 'There is, however, it seems to me -and I have pointed this out elsewhere ${ }^{1}$-another source of poisons to be considered, namely, poisons of metabolic origin. 'To understand this phase of the subject it is necessary to recall that the principal pathological change in typhoid fever consists of an extraordinary proliferation of lymphoid cells-in the intestines, the spleen, the lymph glands, and the bone marrow-the new cells being numerically as much beyond computation as the typhoid bacilli themselves. The cell proliferation is necessarily accompanied by the formation of metabolic products that are thrown into the blood stream. They may be the same substances as are formed in health, or they may be different. After a few days the countless new cells die and disintegrate, their constituent elements or compounds finding their way into the blool. Now, it is my belief that the metabolic products formed during this proliferation of cells, which is characteristic of typhoid fever, and the disintegration products accompanying the death of these same cells, act as poisons and add themselves to the intracellular poisons of the bacteria. 'There are as yet no experiments on record actually demonstrating the existence of these hypothetical metabolic poisons, and for the present our methods of treatment and prophylaxis leave them out of account.

'To return to the subject of immunity against typhoid fever. Unlike diphtheria, it camnot, for the reasons above given, be induced by transferring to a human being serum from an animal rendered artificially immune. It is brought about, in practice at least, by injecting dead

'Jour. Amer. Med. Assoc., January 29, 1910. 
typhoid bacilli or extracts obtained from them directly into the individual to be immunized. The method employed originated with Fraenkel; it was elaborated by Pfeiffer and Kolle, but was first tried on a large scale by Sir A. E. Wright. Strictly speaking, it is nothing more than an adaptation of Pasteur's fundamental discovery of anthrax vaccination and of Koch's tuberculin immunization.

The immunizing substance, the vaccine or bacterin, is a definite quantity of dead typhoid bacilli. The bacilli are usually killed by heat, although indifferent chemical substances may be used to kill them. In general, the method of preparing and using the vaccine is similar to that employed in the vaccine therapy of infectious diseases established by Wright. 'The basis for the vaccine is a strain of typhoid bacilli that will produce large quantities of antibodies when injected into human beings or animals.

Preparation of the Vaccine.-The following method is largely that described by Major F. F. Russell. ${ }^{1}$ Agar slant cultures eighteen or twenty hours old are washed off into a small quantity (about 2 c.c.) of normal salt solution. The emulsion is well shaken to break up clumps, and is then transferred to large tubes $(50$ c.c.), which are sealed in the flame of a blowpipe. The sealed tubes are then sunk in a water bath, which is furnished with an electrically driven stirring apparatus to secure an equal temperature throughout. 'The bath is heated to $60^{\circ} \mathrm{C}$., and the tubes are kept submerged for seventy-five minutes, since experiment has shown that it takes fifteen minutes for a large quantity of material to reach the temperature of the bath. The bacteria are therefore killed by heating to $60^{\circ} \mathrm{C}$. for one hour. After the cultures are thus killed, the concentrated emulsion is diluted to from 15 to 20 c.c. for each agar slant. The quantity varies somewhat as the bacterial count serves as a check, and may indicate that a little more or a little less salt solution to the tube is necessary to give a product containing $1,000,000,000$ bacteria to the cubic centimeter. Before the vaccine is put into ampullas, 0.25 per cent. of tricresol is added as a matter of safety. The vaccine is administered to at least two animals - a mouse and a guinea-pig-before any is used on human beings.

Dosage of the Vaccine.-Russell's vaccine contains $1,000,000,000$ dead bacilli to the cubic centimeter; 0.5 c.c. is used for the first injection, 1 c.c. for the second, and the same for the third. 'The injections are given ten days apart. Spooner ${ }^{2}$ prepares a vaccine containing 400,000,000 bacilli to the cubic centimeter, and uses for the first injection 100,000,000; for the second, 200,000,000, and for the third 300,000,000. Wright ${ }^{3}$ advises large doses $-750,000,000$ to $1,000,000,000$ for the first injection, and from $1,500,000,000$ to $2,000,000,000$ for the second. For therapeutic purposes, as will be shown later, much smaller doses must be employed. The injections are given subcutaneously at the insertion of the deltoid muscle, the area having first been washed with alcohol.

'The injection of the vaccine is followed by a local reaction taking the

1 Johns Hopkins Hospital Bulletin. March, 1910.

2 Monthly Bulletin, State Board of Health, Mass., February, 1910.

s A Short Treatise on Antityphoid Inoculation, 1904, p. 27. 
form of a painful nodule at the point of inoculation. This reaches its height in about twelve hours, and disappears gradually by the second or third day. In rare instances it spreads a considerable distance; the glands in the axilla become tender, and motion in the arm is restricted. But even these symptoms seldom last more than twenty-four hours. In a certain proportion of cases there is also a general reaction, characterized, in the milder cases, by headache and lassitude, and in the severer ones by nausea, chill, and fever of from $100^{\circ}$ to $104^{\circ} 1 \%$ ( $37.5^{\circ}$ to $40^{\circ} \mathrm{C}$.); at times there is herpes labialis. After a second injection both the local and the general reactions are milder. 'Those who have already had typhoid fever usually show a tendency to a stronger reaction. "This phenomenon, in Russell's opinion, ${ }^{1}$ is coupled with an immunity to typhoid fever, though it may indicate residual typhoid affections of the biliary, urinary, or intestinal tract. An attempt has recently been made to introduce the typhoid vaccine into the system by way of the mouth. ${ }^{2}$ The dead bacteria were guided past the dangers of the stomach by being administered in pills suspended in a matrix of fat and stearin. 'The results were, however, unsatisfactory.

Effect of Vaccination.-'The blood after vaccination shows very significant changes, to wit: (a) a marked increase in the agglutinative power; (b) a rise of the opsonic index; (c) an increase in the leukocytes. 'The agglutinative index begins to rise rapidly on the fourth or fifth day, and remains high for about six weeks, then gradually declines, reaching normal at the end of about fifteen months. Variations are, however, frequent. The phagocytic index follows an analogous course. It is thus definitely established that after typhoid vaccination immunity reactions occur that are identical with those found in the natural course of the disease.

The practical prophylactic results of vaccination are striking and full of promise. Nevertheless, the method has made but little headwaya rather surprising fact-for which there are several reasons. First, the slight fear of typhoid infection as compared with that entertained for smallpox, for example; second, the novelty of the method and its apparent complexity; third, the belief in the existence of a "negative phase." According to the teaching of Sir A. E. Wright, the injection of a bacterial vaccine depresses for a short time the phagocytic power of the blood and increases susceptibility. 'This depression of the phagocytic or opsonic index is known as the negative phase. If such a negative phase does occur, and if it represents a temporarily heightened susceptibility, then the use of typhoid vaccine at the time of an epidemic is dangerous. Pfeiffer and Russell. however, have shown that the importance of the so-called negative phase has been greatly exaggerated; and even Wright admits that when small doses are used for the primary injection the negative phase is a negligible quantity.

The degree of immunity following vaceination bears no relation to the violence of the reaction. It is, however, greater the larger the number

1 Loc. cit.

2 Leishman, The Practitioner, September, 1910. 
of injections. In practice it is unnecessary to exceed three doses, which, as already mentioned, are given at intervals of ten days.

In the German experiments in Southwest Africa the immunity seemed to last about a year; observers elsewhere found it to endure for as much as four years; statistics relating to the British garrison in India make it probable that it persists during the third year.

Statistics of Typhoid Inoculation. - Wright, ${ }^{1}$ up to 1902, had succeeded in collecting, chiefly from army reports from Africa, India, and Egypt, 22,825 inoculations, including 84 from an asylum for the insane. Among the inoculated the incidence of the disease was diminished by at least one-half. In some instances the reduction achieved varied from sixfold to twenty-eight fold. A striking effect was also noticeable upon the case mortality. Among the inoculated it was rather less than half that among the non-inoculated, 1758 inoculated persons furnishing 142 deaths ( 8 per cent.); 10,980 non-inoculated, 1800 deaths ( 16.6 per cent.). The combined effect of the diminished incidence and diminished case mortality manifested itself in the diminution of the death rate from typhoid fever among the inoculated. The statistics show a fourfold reduction in favor of the latter. Russell ${ }^{2}$ reports one case of typhoid fever among 1400 inoculated, while among 75,000 non-inoculated there were 135 cases.

The results of antityphoid vaccination in the German Colonial Army in the Herero campaign of 1904, in Southwest Africa, were also quite favorable. Eight thousand men were vaccinated, and the difference in the incidence and death rate is shown by the following table:

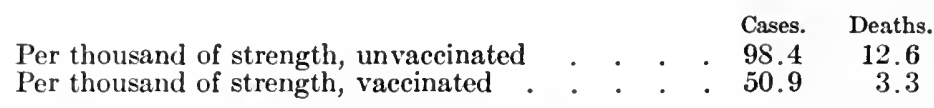

There were thus about half as many cases and only a quarter as many deaths among the vaccinated.

Treatment.-General Considerations.-The essence of the treatment of typhoid fever may be summed up in the word "watchfulness." Watchfulness on the part of the physician is, however, not sufficient. It must be supplemented by that of a thoroughly trained nurse, who should have experience in fever cases.

A typhoid fever patient is best cared for in a hospital. Not only is that to his own advantage, for he is under the constant supervision of nurses and internes, but it is to the best interest of his family and the public at large, since the methods of disinfection upon which the control of typhoid fever depends can be better carried out in an institution. Moreover, there are certain measures of treatment, such as the administration of oxygen, hypodermoclysis, etc., as well as diagnostic procedures, that may be demanded which present great difficulties in the ordinary private home. When the number of cases is sufficiently large, they should be isolated in a special ward. Where the cases are few in

${ }^{1}$ Antityphoid Inoculation, p. 52 et seq.

${ }^{2}$ Loc. cit. 
number, they should be placed together in a section of the ward, and the nurse having them in charge should have nothing to do with the other patients - nothing, at any rate, with the preparation of their meals.

If the patient is treated at home, he should, whenever circumstances permit it, have two nurses-a night and a day nurse. Among the very wealthy three nurses are sometimes employed, whose hours of service are then so arranged that two are always on duty.

'The patient should immediately be put to bed in a large, airy, wellventilated room, preferably one with a bathroom adjoining. I have elsewhere urged that private homes and apartment houses should have what might be called a "hospital room," a room especially designed by the architect to serve as a sickroom. 'The temperature of the room should not be over $65^{\circ} \mathrm{F}$. In summer it may be cooled artificially; if there is a porch or veranda adjoining the room, on which the bed might be wheeled, the patient may spend part of the day outdoors. 'The windows and doors should be sereened, and fly-paper and other traps should be spread to catch any flies that may get in. If the patient's mental condition is sufficiently bright to permit him to enjoy flowers, any sent to him may be left in the room during the day, but should be removed at night. If there is the slightest sign of delirium or other mental disturbance the flowers must be banished. It is also well to have hangings, screens, fans, and other things used in the sickroom, of a neutral color. I once saw a patient who had post-typhoidal mania. She cried out constantly that she was surrounded by fire and was being burnt. After convalescence she told me she knew exactly whence the illusion came. The wall paper was red, the screen of the bed was red, she was fanned with a red fan, and red flowers were brought to her, and it was this concentrated redness that caused her indescribable mental agony.

'The sick bed should be single, and preferably white enamel. Such a bed, aside from being more sanitary, makes the work of the nurse and the examinations by the doctor easier. 'The bedcovering should be light, the best being a thin blanket between two sheets. A draw-sheet should be placed over the lower sheet, under the patient, and a mackintosh on top of the mattress. There should be a comfortable cot in the room for the uurse. A basin containing an antiseptic solution, for the disinfection of the doctor's and nurse's hands, should always be in readiness. 'The family should be most minutely instructed in the necessity for disinfection and personal care, and should be informed that typhoid fever is now known to be a contagious disease. As soon as the diagnosis is definitely established the case should be reported to the Board of Health.

From the very outset the patient must be kept as quiet as possible. He must be urged to use the bedpan and urinal, and any objections he may raise should be overcome by tactful persuasion. Visitors should be kept away during the febrile period and for the first week of convalescence. No one should read to the patient nor discuss with him business or household aflairs; but, of course, there can be no ironclad rule in such matters, as each temperament requires individual handling. 
Unless absolutely necessary, I oppose the moving of patients any great distance; yet contingencies may arise that make it advisable to transport a patient to another place rather than keep him where he cannot have proper care. I may say that I have not seen any serious consequences from transportation during the early stages of the disease. The most rigid disinfection should be carried out on the journey.

In an ordinary uncomplicated case the patient should be seen by his physician once a day; an intelligent nurse and the telephone render additional visits unnecessary. In serious cases, two or even more visits may be required. The physician should arrange for himself a definite routine to be followed on his visits. He should note the general appearance of the patient-the tongue, the degree of abdominal distention, spots, condition of the bladder, size of the spleen and liver, the position and strength of the apex beat, the character of the heart sounds. He should auscult the lungs, especially posteriorly, and examine the back for possible signs of bedsores. He should carefully note any tender areas, without, however, too much manipulation of the abdomen, particularly of the right iliac fossa, and from time to time he should inspect the bowel movements. In a case in which the symptoms suggest a possible perforation he should call in a surgical consultant. He should make frequent urine examinations and such diagnostic tests-Widal reaction, blood examination, blood culture, etc.-as modern scientific medicine demands. After every visit he should wash his hands with soap and water and disinfect them in carbolic acid or bichloride solution.

Whether the patient should be informed as to the nature of his ailment is a matter to be freshly decided in each individual case. It is, however, my custom not to tell him if I can avoid it. The physician should be guarded in his prognosis in even the mildest case, for typhoid fever is the most treacherous of all diseases. Relapses are so common that it is unwise to pronounce a patient entirely well and out of danger too soon.

Duties of the Nurse.-The general duties of the nurse need not be discussed. All specific instructions bearing on the management of the case in hand, including disinfection, should be given to the nurse in writing. Although she may be informed of the contagiousness of typhoid fever, the physician should not fail to impress upon her the importance of reasonable precautions for self-protection and the protection of others. In hospitals, nurses who have perhaps a number of typhoid fever cases to take care of should wear rubber gloves when handling excreta, and should religiously wash their hands after any service to the patients. Neither the nurse nor anyone else but the patient should eat or drink in the sickroom.

Careful records must be kept by the nurse. They should show (1) temperature, pulse, and respiration every three hours, as well as the highest and lowest for the day; (2) daily quantity of urine; (3) hours of sleep; (4) quantity of food and water taken; (5) number and character of bowel movements; (6) any noteworthy features, such as spots, cough, hiccough, hemorrhage, delirium, incontinence, etc. In the case of hem- 
orrhage or vomiting, the discharged material should be saved in a covered vessel, unmixed with disinfectant, for the inspection of the physician. If there is any unexpected change in the temperature or pulse, or if there is sudden abdominal pain, or signs of intestinal hemorrhage, the plysician should be summoned at once.

The routine attention to the patient comprises a morning and evening sponge bath with tepid water and alcohol, gentle combing of the hair, and cleansing of the mouth with an antiseptic solution. 'The care of the mouth is of great importance. 'To cleanse it properly the nurse wraps a piece of sterile gauze over the index finger, dips it into a solution of boric acid or of lifuor antisepticus alkalinus diluted with four parts of sterile water, and wipes the tongue, the gums, and the inside of the cheeks. If able, the patient should himself gargle freely with either of the solutions. 'To prevent bedsores, the patient should not be allowed to lie too long in one position. 'The part subject to pressure should be bathed with alcohol or spirits of camphor, and afterward thoroughly dried. If redness appears, an air cushion should be used, and the parts dusted with boric acil, talcum powder, or a powder of salicylic acid and chalk. After every movement the buttocks should be thoroughly: washed with soap and water. For this purpose, it is best to use clean rags or pieces of gauze, that are afterward burnt.

The patient's finger-nails must be kept clean and cut short. If he tries to pick his nose or lips, he should be restrained. He should not be left alone. If he is delirious, he should be controlled with gentle firmness. Mechanical devices for restraining should be avoided. In case of extreme restlessness the sheet may for a short time be drawn fairly tightly across the chest and fastened down at the sides. It is unnecessary to cut the hair of female patients while the disease lasts. If the patient is young, the hair may be cut ofl at the beginning of convalescence; in older patients, it may be left to itself. Massage of the scalp is very useful.

Care of the Bowels. - The profession is not agreed on the use of purgatives in the beginning of typhoid fever; my own practice is never to give a purge after I suspect a case to be one of typhoid fever. Frequently, however, one sees fever cases in which the diagnosis is uncertain and in which calomel is indicated. I have not seen that such patients, if they afterward proved to have typhoid fever, were hurt by the initial purge.

If constipation is present, which in my experience is the rule, a soap and water or saline enema should be given every other day. In the later weeks of the disease, and especially during convalescence, the bowels are often extremely sluggish, even to the point of impaction. An enema of from 4 to 6 ounces of warm cotton-seed oil should then be tried. If unsuccessful, a digital examination of the rectum should be made, and if there is impaction the mass should be removed with the finger. Thereafter, if there is no fever, a gentle cathartic may be used-10 to 20 minims of aromatic fluid extract of cascara, or $\frac{1}{2}$ to $1 \mathrm{dram}$ of compound liquorice powder. 
Disinfection.-This is of such great hygienic importance, that it becomes the sacred duty of every physician to see that it is properly done. For the disinfection of stools, the cheapest and, withal, a perfectly satisfactory agent is milk of lime, made by adding water to freshly slaked lime, everywhere easily obtainable. 'The floor of the bedpan is covered with milk of lime, and after the stool has been received, an equal amount of milk of lime is added. 'The mixture is well shaken and allowed to stand two hours. For the urine, milk of lime may be used; likewise, carbolic acid ( 5 per cent. solution) or corrosive sublimate. 'The internal use of hexamethylenamin (urotropin) in 5 or 10 grain doses three times a day, in capsule, is an excellent means of sterilizing the urine during convalescence.

Instead of milk of lime, chloride of lime or chlorinated lime is frequently employed. Though more expensive, a much smaller amount is required-only about 1 per cent. of the contents of the bedpan. Its odor is somewhat objectionable.

Water closets or privies into which the discharges are emptied should be kept clean and should receive a plentiful supply of milk of lime. The wash water and the bath, if the patient is tubbed, should also be disinfected. It may be done with milk of lime (6 liters to a bath of 300 liters), or with chlorinated lime ( 250 grams to 300 liters of water). ${ }^{1}$

Disinfection of Bedclotbing.-Bedclothing, towels, handkerchiefs, underwear, night-gowns, etc., should be disinfected before they are laundered. 'To this end they are at once put into a tub or basin containing a 3 per cent. carbolic acid solution, or are wrapped in a sheet soaked in 1 to 2000 bichloride solution, and afterward boiled in soap and water. Members of the family and servants handling the bedclothing should disinfect their hands with soap and water and corrosive sublimate.

All food and drink left by the patient should be destroyed. After the disease has run its course, the sickroom should be thoroughly disinfected.

Diet.- The profession has divided itself into two schools in the matter of diet - those advocating an absolute milk diet, on the one hand, and those giving semisolid and solid food, on the other, representing the extremes of the two camps. My own practice has been to give virtually a liquid diet, not restricted to milk, but including broth, albumen water, cream, and ice-cream. Junket and calf's-foot jelly I also allow. The lowest amount of milk is three pints per day; whenever possible, two quarts are given. 'The patient should be fed every two hours during the day, but should not be disturbed more than twice at night, unless the quantity of food taken during the day is insufficient. At each feeding from 4 to 8 ounces of milk are given, but occasionally ice-cream, junket, or broth replaces a milk feeding. I personally feel that, when it is tolerated, milk or one of its modifications, kefir, kumiss, etc., has more virtue than broth or any other liquid food. It can be made more palatable by flavoring it with coffee or by mixing it with carbonated water, in the

'Babucke, Cent. f. Bakteriol., 1900, xxvii. 
proportion of 2 parts of milk to 1 of the water. 'I'his, however, is not advisable if there is any distention. Milk is best taken through at sorda straw (a glass tube is apt to become sour). I have followed the suggestion of Edsall' to have the milk, both in private and hospital practice, pasteurized, so as to eliminate any possible typhoid bacilli, as well as other bacteria causing gastro-intestinal disturbances.

If there is much distention, or if curds appear in the stools, I substitute albumen water for every other feeding. If the distention does not promptly yield, the milk is stopped entirely for twenty-four or thirty-six hours and replaced by albumen water. If necessary, an occasional broth feeding may be given. While on albumen water the patient receives the whites of ten eggs a day, made more agreeable by being mixed with orange or lemon juice. As soon as the fever begins to decline, the patient may have a little calf's-foot jelly. When the temperature has reached normal or subnormal-a subnormal temperature always gives one a sense of greater security - the patient may have the yolk of a poached egg, strained rice, or oatmeal. One week later, mashed potatoes, custard, or well-soaked toast may be given, and gradually. other easily digestible foods are added. On the tenth day of convalescence the dietary usually includes chops, spinach, and boiled rice.

I find that the tongue is cleaner and the desire for food keener since I advise patients to chew chewing gum.

The dietetic regimen detailed above has given me very good results. The few deaths that I have had have been due to complications, such as perforation and hemorrhage, rarely to toxemia or exhaustion. White the patients on this diet are much reduced in weight at the end of the febrile period, the appetite during convalescence is so enormous and the digestion so excellent that they quickly pick up all they have lost. and more. One almost feels that the pleasure the convalescent patient finds in his food and in the thought of it is some slight compensation to him for his weeks of starving.

It is contended that the caloric value of a pure milk diet is too small, and that in consequence the body consumes a large amount of its own fat and protein. This is undoubtedly true, for two quarts of milk represent only 1300 calories, while a typhoid fever patient requires at least 3000 , if we wish to compensate for the energy loss. This destruction of body protein, as is pointed out by Shaeffer and Coleman, ${ }^{2}$ may be retarded or prevented by a dietary made up of a moderate amount of protein (10 to 15 grams) and a large amount of carbohvdrates. Of the various carbohydrates the most useful and convenient is lactose. $\mathrm{By}$ adding 175 grams, or about 6 ounces, of this (equal to 700 calories) to two quarts of milk the total caloric value of the diet is raised to 2000; and by the addition of a little cream the heat value of the food may be still further augmented.

These considerations are logical and may be safely put in practice.

'Trans. Assoc. Amer. Phys., 1907.

' Archives of Internal Medicine, December 15, 1909. 
The following is a specimen dietary based on these principles. I have not had enough cases on this diet to have formed a final opinion, though I am much impressed with the recent reports of Coleman: ${ }^{1}$

Milk, 7 ounces; cream, 1 ounce; lactose, 1 dram. Gradually increase cream and lactose until in about four days the formula is: Milk, 6 ounces; cream 2 ounces; lactose, $\frac{1}{2}$ ounce, which is given the patient at 6 and $S_{\text {A.M. }}, 2,6$, and $S$ P.M., and twice during the night.

At 10 A.M. and 4 P.M., and once during the night the patient is given: Albumen water, 10 ounces; lactose, 2 ounces, and lemon juice. Add cane sugar or more milk sugar if not sweet enough.

At 12 noon the same as at 6 A.M., except add one egg, with or without whisky to flavor.

'Total in twenty-four hours: Milk, $4 S$ ounces ( $1 \frac{1}{2}$ quarts, or 1000 calories); cream, 16 ounces (1 pint, or 1200 calories); lactose, 10 ounces (1200 calories); egg, one.

Shattuck ${ }^{2}$ allows crackers, finely minced meat, the soft part of raw oysters, puddings without raisins, and macaroni. Robertson ${ }^{3}$ gives three regular meals a day, with milk feedings between. On his diet list are, among other things, toast, wheat bread, fish, baked apple, cooked tomatoes, salad, oysters, and baked potatoes. Both men have had good results with their liberal diet, but I cannot help feeling that an intestinal canal in a state of inflammation, such as that present in typhoid fever, should not be overtaxed. Moreover, there is need for shielding the kidneys, and a milk diet accomplishes this better than any other food.

Water.-Water is an important adjunct to feeding in typhoid fever, and should be given freely-4 to 6 ounces every two hours-and no effort should be spared to get the patient to take the largest amount possible. It may be flavored with grape juice or lemon. I do not greatly favor carbonated waters, as they may increase distention. While on a licyuid diet and on large amounts of water, patients often pass extraordinary quantities of urine -100 ounces or more a day. While we have no definite knowledge of the presence of specific toxins in the urine in typhoid fever-some studies undertaken on this point at my suggestion by Dr. H. C. Wood, Jr., have given negative results-it seems to be proved that cases with polyuria do better than those with oliguria. This point has been emphasized by Cushing and Clarke. ${ }^{4}$

Hydrotherapy.-Ever since the days of Currie and Todd some form of hydrotherapy has been used in the treatment of typhoid fever. 'The simplest and oldest method is that of sponging. In the early stages of the disease sponging makes little difference in the temperature, but it lessens nervousness and lowers the pulse rate. I Later, the effects upon the temperature are more pronounced and persist longer.

The water may be of any temperature, but the majority of patients feel best with a temperature of $85^{\circ} \mathrm{F}$. Lower temperatures, down to $70^{\circ} \mathrm{F}$.,

1 Jour. Amer. Med. Assoc., October 9, 1909.

2 Quoted by Fussell, Amer. Jour. Med. Sci., 1909, cxxxviii, 532.

3 Penna. Med. Jour., 1909, p. 204.

4 Amer. Jour. Med. Sci., 1905, exxix, 187. 
may be used, the patient's own attitude to some extent controlling the choice.

In sponging, only one part of the body should be exposed at a time. Careful attention should be bestowed upon the back. From fiftcen to twenty minutes should be given to each sponge; a large amount of water should be used and a thin film left on the surface for evaporation.

The sponges are not given oftener than every three hours, and only when the temperature is $102.5^{\circ}$ and over.

Packs.-For persistently high temperature a wet pack may be used. 'The patient is wrapped in a sheet wrung out of cold water, the bed being protected by a mackintosh. If there is hyperpyrexia the patient may be rubbed with pieces of ice over the sheet, or the sheet may be sprinkled with ice water. 'This is conveniently done with a whisk broom dipped in the water. In some cases I have used a limited instead of a general wet pack. In this, towels soaked in ice water are wrapped around the limbs one at a time.

Tub Bath.- The tub bath was introduced by Brand, of Stettin, and has probably had a larger degree of popularity than any other method of treatment ever advanced for the disease. 'There is no question that it constituted a tremendous advance, and through it the mortality of the disease has been appreciably reduced. With the rest of mankind, I used tub baths for several years in my hospital practice, but finding that during the same period my private cases, which were not tubbed, did equally well, I came gradually to abandon the baths, and found that, as regards the proportion of recoveries, the rarity of complieations, and the general well-being of the patients, the results were no less satisfactory.

'The world gradually outgrows each of its advances, and I personally: feel that today the management of typhoid fever has made large enough strides along other lines to warrant us in dispensing with the troublesome tub bath. ${ }^{1}$

However, the bath is still in use by many authorities, and one hesitates to discourage a practice that has such ardent advocates. McCrae, for instance, in his masterly article on typhoid fever in Osler's System, Yol. II, declares it to be almost a specific in typhoid fever. For this reason I will include a description of the method.

'The tub is moved to the side of the bed, the patient is encouraged to

${ }^{1}$ The following table, adopted from Hare and Beardsley (The Medical Complications, Aecidents, and Sequels of Typhoid Fever and the Other Exanthemata, 1909), shows the results of general and of bath treatment:

\begin{tabular}{|c|c|c|c|}
\hline & Cases. & $\begin{array}{l}\text { Mortality. } \\
\text { per cent. }\end{array}$ & Treatment. \\
\hline Maidstone, England. & 1,855 & 7.5 & General. \\
\hline Boston (Mason).... & 676 & 10.4 & General. \\
\hline Homerton (Collie). & 677 & 9.5 & General. \\
\hline Glasgow (Collie)......... & $61 \mathrm{~s}$ & $S .2$ & General. \\
\hline Jaccound ........... & 665 & 10.5 & General. \\
\hline Riess .... & 900 & 7.5 & Tepid baths. \\
\hline Boston (Shattuek). & 237 & 9.5 & $\begin{array}{l}\text { Expectant and cold } \\
\text { sponging. }\end{array}$ \\
\hline Collected by Brand...... & 19,017 & 7.5 & All kinds of cold baths. \\
\hline
\end{tabular}
vol. 1. -10 
pass water, and after being undressed and dried is covered with a sheet and lifted by two nurses gently into the tub. If the patient is weak, his head is supported by the nurse. While in the tub the patient is continually rubbed through the sheet and is encouraged to rub himself. 'The extremities, chest, and back (not the abdomen) are rubbed. The first bath lasts only about eight or ten minutes, and has a temperature of $85^{\circ} \mathrm{F}$. Thereafter, if the patients stands the tubbing well, the temperature is gradually lowered by means of ice from $85^{\circ}$ to $70^{\circ} \mathrm{F}$. while the patient is in the tub, and the duration is increased to fifteen minutes. Shivering and a slight degree of cyanosis are no contraindications to the bath, but if the patient is always greatly prostrated afterward, it is best not to continue the tubbing. At the end of the bath the patient is lifted back into the bed, wrapped in a warm sheet, covered with a blanket, and allowed to rest for a short time before being dried and dressed. Half an hour after the bath the temperature is again taken. If the patient should be slow to react, he may have a half teaspoonful of aromatic spirits of ammonia or a dram of whisky. Hemorrhage, perforation, phlebitis, and pneumonia are contraindications to the bath.

Various devices have been used to facilitate the lifting of the patients into and from the bath. In some hospitals the patients are. supported while in the bath by canvas strips attached to the sides of the tub.

The Murphy Treatment.-Impressed by the splendid success of the Murphy treatment in surgical conditions affecting the abdomen, I began a little over two years ago to use the same procedure in the treatment of typhoid fever, the principal lesions of which are also in the abdomen. The method as I now employ it differs a little from that described by Murphy. 'The patient is in the recumbent and not in the Fowler position. The solution contains only sodium chloride, 1 dram to a pint, and the ordinary rectal nozzle is used, which I find no difficulty in retaining in place. The container, kept warm by hot-water bags, is hung a foot above the bed, and the solution is allowed to flow steadily during the day; at night the flow is usually intermitted. If the patient bears the treatment well, it may be continued to the end of the fastigium. Hemorrhage, perforation, and great irritability when the tube is in place are contraindications. The amount of salt solution that can be easily absorbed varies; generally, from two to six quarts can be given in twelve hours.

I have found that this method of treatment, as might be expected, promotes diuresis and lessens toxemia. The number of cases is too small for final judgment. In order to test any treatment in a disease like typhoid fever, large numbers of cases are necessary. However, the results so far are excellent; delirium is rarely seen and the cases are less toxic. The duration of the disease is not shortened.

Drugs.-Antipgretics.-When the coal-tar antipyretics first came into vogue they were hailed as the long-sought remedies for the combating of the fever of infectious diseases. 'Time has shown, however, that they are distinctly harmful. It is true they reduce temperature, but they do so by causing a profound systemic depression bordering on shock. They 
are, therefore, no longer used routinely to control fever. If employed at all, it is for the relief of headache and in cases of persistent hyperpyrexia not yielding to hydrotherapy. In either case a few small doses of antipyrine (2 to 5 grains) or of acetphenetidin (3 to 6 grains) may be given. Some clinicians praise pyramidon; others, aspirin-their use can only be a restricted one.

Intestinal Antiseptics.-Much has been written for and against their employment. Some deny any antiseptic value, others are enthusiastic in their praise. My own faith in them is sufficiently strong to make me use them more or less regularly in severe cases-my preference is for phenyl salicylate (salol) in doses of from $2 \frac{1}{2}$ to 5 grains three times a day.

Many other drugs have been acclaimed as specifies in typhoid fever, but not one of them has stood the test of time. It is therefore unnecessary to mention them. As the few remedies with which the physician of the present day contents himself are inexpensive, the druggist's bill in a protracted case of typhoid fever is insignificant-a welcome change from two decades ago.

The only drug that I have used routinely is dilute hydrochloric acid, in doses of from 10 to 15 minims every four hours. It is a known fact that in typhoid fever there is a diminution of hydrochloric acid in the gastric juice, which the drug is intended to remedy. It also seems to keep the tongue moist.

Serum Treatment.-For reasons given in the section on vaccination, not much is to be expected, theoretically, at least, from serum treatment. Nevertheless, the experience of Chantemesse ${ }^{1}$ commands attention. Chantemesse obtained his serum from horses inoculated with filtered cultures of typhoid bacilli grown on a medium of splenic pulp and defibrinated blood. In 545 cases treated with the serum at the Hopital du Bastion 29, the mortality was 4 per cent., while in other Paris hospitals the contemporary mortality was from 12 to 18 per cent. Other serums, as well as the antityphoid extract of Jez (which is prepared from the spleen, bone marrow, thymus, and brain of animals highly immunized to the thyroid bacillus), have yet to prove their value.

Vaccines.-Wright's theories and experiments stimulated the vaccine treatment of nearly all bacterial diseases. In a number the results were good. In typhoid fever, however, they have so far been disappointing. A consideration of the nature and source of typhoid poisons, which I have discussed on page 614 , will give a partial explanation for this failure.

Autogenous vaccines made from bacilli obtained from the case to be treated, as well as stock vaccines, have been used.

Richardson, ${ }^{2}$ instead of a vaccine prepared from typhoid cultures killed by heat, has used the filtrates of bouillon cultures in one series and in another the non-toxic residues of Vaughan." 'This is made by

1 La Presse med., 1904, 6S1.

${ }^{2}$ Treatment of Typhoid Fever with Specific Sera, Filtrates, and Residues (Vaughan) (Reprint).

Amer. Jour. Med. Sci., 190S, cxxxvi, 330. 
treating the bacterial cells with an alcoholic solution of sodium hydroxide. No noteworthy results were achieved.

In a later communication, Richardson ${ }^{1}$ reports treating 122 cases with bacterins and bacterial toxins without obtaining much effect upon the disease. He found, however, that relapses were less frequent in the inoculated cases. The dose employed by him varied from 10,000,000 to $100,000,000$ bacilli.

Smallman ${ }^{2}$ at first used inoculations of $100,000,000$ bacteria every ten days, but as this had no effect, he increased the dose to $300,000,000$, and reduced the interval to three days. His mortality was 8.3 , which is no less than that of many large American hospitals where the vaccine treatment is not employed.

Sappington ${ }^{3}$ prepared autogenous vaccines, controlling the administration by a study of the opsonic index. The results in 22 cases were not conclisive.

In Leishman's ${ }^{4}$ opinion typhoid vaccine offers great hope in the treatment of typhoid fever. With doses of from 300,000,000 to $400,000,000$ repeated every fourth or fifth day good results are obtained. The injections are followed by a rise of temperature of $1^{\circ}$ or $1.5^{\circ} \mathrm{F}$., which is, however, succeeded on the next day by a considerable fall. The temperature remains comparatively low for two or three days after the inoculation; then it begins to rise again, and if at this moment the injection is repeated the same sequence of events occurs, and after three or four inoculations the temperature falls to normal and usually does not rise again.

'To sum up, the vaccine treatment in its present state is not a decided improvement over the treatment now in vogue. It is, however, more than probable that a specific remedy will be found, and that its discovery lies in the hands of the laboratory investigator.

Treatment of Special Symptoms and Complications.-Toxemia.All the general measures that have been detailed, hydrotherapy as well as drugs, have as their final aim the lessening of the toxemia. But there are cases in which, either because of low resistance on the part of the patient, or because of an extraordinarily large quantity of toxins, or because of complications, the patient seems so profoundly poisoned that all functions are depressed to the danger level. These toxemic cases are far less common than they were in former times, probably because of better nursing and less interference with the natural processes. When treating such a case, every effort should be made to introduce as large an amount of water into the system as possible; by the mouth, by the rectum - either by the Murphy method or by intermittent enteroclysis-by hypodermoclysis, or by venous infusions. Hypodermoclysis often gives excellent results. A pint of salt solution should be slowly introduced in the region of the breasts, in the flank,

1 Boston Med, and Surg. Jour., 1910, i, 255.

2 Jour. Army Med. Corps, 1909 , xi, 136.

Jour. Med. Research, $1910, \mathrm{v}, 435$.

- The Practitiouer, september, 1910. 
or in the thigh; it may be repeated. In hospitals, where proper assistance ean be had, the salt solution may, in grave casces, be injectred into a vein. Oxygen inhalations are also beneficial.

Alcohol still has many warm advocates. I formerly gave it in large amounts, more or less routinely, but now I give small doses and only when the toxemia is very profound and in the case of confirmed alcoholic subjects.

Occasionally when alcohol is given in large amounts it may lead (o) a condition resembling delirium tremens, and I also know of a case in which the patient, a physician, was made so profoundly drunk by the injudicious use of champagne that he was thought to be dead.

Strychnine becomes, in toxic cases, a routine remedy, and should be given hypodermically, in doses of $\frac{7}{60}$ to $\frac{1}{40}$ grain, every three or four hours, day and night. In several desperate cases I have seen abscesses. formed spontaneously or at the site of the hypodermoclysis injection, eoincide with an improvement of the symptoms that led on to recovery. 'This confirms the ideas of Fochier with regard to the abscès de fixation, an artificial abscess, which I have several times induced, and which is readily obtained by the injection, usually into the calf, of 5 minims of sterile turpentine. A tender, red, hemispherical swelling forms, which should not be opened. 'The abscess is supposed to stimulate the phagocytes or in some other way to rouse the defensive powers of the system.

Cardiovascular System.-Death in typhoid fever in over ninety per cent. of cases is due to failure of the circulation. Until recently this failure was ascribed to the heart, but the work of the I reipzin school has made it clear that the vasomotor system is an important factor. 'The vascular tension is always lowered in typhoid fever, and constant attention is necessary to anticipate a fall to a dangerous level. 'The physician should be familiar with the early signs of falling circulation, and, if possible, should make blool-pressure estimations.

The treatment in the milder cases consists in giving strychnine, prefer-

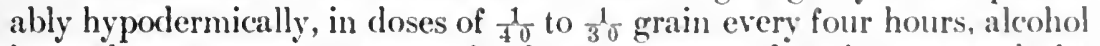
in moderate amounts, except in those accustomed to its use, and the injection of salt solution under the skin.

Sudden heart failure is much more rare in typhoid fever than in diphtheria. It must be met promptly by injections of from 10 to 20 minims of sterile camphorated oil, by rectal injections of strong coffece. and by the administration of stimulants, such as aromatic spirits of ammonia and brandy by the mouth, and strychnine and digitalin hypodermically. Oxygen may prove useful, if it ean be quickly obtained. I have found it a wise precaution in grave cases to keep a tank of oxygen in readiness.

Headache.-Headache is one of the most constant of the early symptoms; as a rule, it is not present in the later stages. It is frequently. severe, and may reach such degrees of intensity as to suggest meningitis. 'The ordinary measures employed against it are the ice-cap, a mustanl plaster to the back of the neck, a mustard foot bath in bed, small doses of antipy rine or phenacetin-3 or 4 grains of each. In very severe cases 
the best measure is leeching. Three leeches to each mastoid process usually stop the headache at once, or, at the most, in a few hours.

Insomnia.-This is so common that I am accustomed to attach a distinct diagnostic value to it. It is present early, and may persist throughout the disease. Sodium bromide ( $7 \frac{1}{2}$ or 10 grs.), at night, in peppermint water, usually suffices. In severe cases, one of the newer hypnotics, such as veronal (5 or $10 \mathrm{grs}$.) in milk; or medinal, in similar doses, as a rule, brings sleep without doing any harm.

Delirium and general nervous excitement seem to me less common than in former years. 'They are combated by the various hydrotherapeutic measures, especially by sponging, tubbing, or the Murphy treatment. An ice-cap may be applied to the head, though its value is probably small. In a few instances I have seen patients soothed by inunctions of olive oil. If drugs have to be used, sodium bromide or chloral, or both together, may be given in the milder cases.

If the excitement is extreme, if the patient wants to get out of bed, or is very talkative, nothing takes the place of a hypodermic injection of morphine; a small dose usually suffices $\left(\frac{1}{8}\right.$ gr.). The effect is often magical, especially if the excitement is due to some extraneous cause rather than to toxemia, as, for example, the menstrual period. ${ }^{1}$ The periods are usually suppressed, but at the time at which they might be expected the erythism is greatly increased.

Nausea and vomiting are rare in typhoid fever, but not unknown. When present they add to the patient's peril. A change of diet may suffice to allay the condition. Albumen water may be used instead of milk. The milk may be peptonized or may be replaced by kefir, kumiss, or buttermilk. Carbonated water may be given in small amounts, either by itself or mixed with milk. Cracked ice is also useful. The best agent in several cases $I$ have found to be champagne on cracked ice. A mustard plaster over the epigastrium, or gentle massage of this region, may also prove useful. In obstinate cases, bismuth or cocaine may have to be employed.

Diarrhea.- It is rather strange that typhoid fever, from having always been described as a disease with diarrhea as one of its chief symptoms, is now a disease usually characterized by constipation. This may be due to the almost universal use of milk in the dietary or to a change in the type of the disease. Diarrhea does, however, occur. If the number of movements is not over three or four, nothing need be done. If that number is exceeded, an attempt should be made to change the character of the movements by altering the diet. The milk should be pasteurized. If that fails, scalder milk should be tried, or the milk entirely excluded and replaced by albumen water. If the diarrhea persists, bismuth subnitrate or bismuth salicylate, in 5 grain doses, in capsule or powder, or milk of bismuth, in 1 dram doses, may be used.

Tympanites.-One of the most distressing symptoms, and in a sense a real complication, is tympanites, or meteorism. It is a sign of marked

${ }^{1}$ Stengel, Univ. of Penna. Med. Bull., December, 1909. 
toxemia, and may be present in cases of diarrhea or of constipation. By pressing the diaphragm upward against the heart and longs it interferes with the function of these organs. Naturally, it also leads to arrest of absorption from the bowel.

'The measures I have found most useful in combating it are change in diet, turpentine stupes, suppositories of asafetida, or injections of milk of asafetida (2 ounces), and turpentine enemas. Internally, turpentine, in 5 or 10 minim doses, in emulsion, is given by many physicians. I have not seen much benefit from it, nor from the use of the long rectal tube. I believe that eserin, given hypodemically, has some virtuc. I have employed the salicylate in doses of $\frac{1}{40}$ grain tlaree times a day.

Puncture of the bowel has been recommended. I have never had occasion to use it.

Hemorrhage.- This is a frequent symptom, occurring in about 7 per cent. of the cases (McCrae $\left.{ }^{1}\right),\left(5.8 S\right.$ per cent., Phillips ${ }^{2}$ ); the amount of blood lost varies greatly. It may be small, and the patient be no worse for it; yet, if bleeding has once begun, it is impossible to foretell when it will stop, or whether or not it will put the patient's life in jeopardy. It may be, without any other complication, the cause of leath. 'The routine treatment of hemorrhage is as follows: Stop all food by the mouth; give a little cracked ice if there is much thirst; elevate the foot of the bed on blocks or bricks; place an ice-cap to the abdomen, and administer morphine ( $\frac{1}{6}$ or $\frac{1}{4}$ gr.) hypodermically. If the bleeding persists, it is well to give 1 grain opium pills, three times a day, watching the pupils and the urine. The abdomen should not be palpated, but gentle percussion may be practised.

In cases in which the hemorrhage is large one way use high rectal injections of normal salt solution, gelatin by the mouth, or the sterile tetamus-free gelatin hypodermically, or calcium lactate (10 grs.) every four hours, in cinnamon water or an infusion of gentian, turpentine, ete. Occasionally, after the use of one of these, or some other remedy, the hemorrhage comes to a sudden stop, and there may be a tendency to give the credit to the last remedy employed. Post hoc ergo propter hoc is, however, not always good logic; only repeated tests in similar cases can determine whether a certain remedy had virtue.

Recently, Harte ${ }^{3}$ has advocated operation, as in the case of perforation, for serious hemorrlage not yielding to medical treatment. 'The thought of operation must have come to more than one clinician who has stood helplessly at the bedside and seen a patient slowly bleed to death. Harte's suggestion raises the hope that a method of localizing the hemorrhage and of dealing with it surgically may be found.

In one case of apparently uncontrollable hemorrhage I found transfusion of blood to be followed by immediate cessation of bleeding. 'The patient was a young man, who in the course of forty-eight hours had lost over three quarts of blood by actual measurement. He seemed

Osler's System, ii.

'Trans. Coll. Phy's of Phila., 1909, xxxi, 294. 
exsanguined; the hemoglobin had dropped to 29 per cent; he was restless and delirious. At my suggestion a brother volunteered to furnish blood for transfusion. The operation was performed by Dr. Dorrance and Dr. Ginsburg. Though the patient had been bleeding up to that moment, the bleeding stopped, the mind became clear, and recovery followed in due course. It seems probable that in such a case the alien blood supplied some deficiency in the factors of coagulation. The experiment suggests the possibility of using horse serum, if human blood is not obtainable. I have not space, nor is it necessary, to discuss here the dangers of transfusion and of the use of heterologous serum.

If the amount of blood lost is very large, hypodermoclysis should be given; in the case of moderate hemorrhages it is unnecessary, perhaps even unwise.

Hemorrhage from the stomach occurs, but is rare; ${ }^{1}$ the treatment is in general like that for bleeding from the intestines.

Perforation.-Perforation is the most dreaded complication of typhoid fever. Fortunately, it occurs in less than 3 per cent. of the cases. In Chantemesse's ${ }^{2}$ collection of 8160 cases of typhoid fever there were 2.6 per cent. with perforation. The only treatment that promises anything is immediate operation. Delay enormously increases the death rate. Without operation, recovery may occur. If it ensues, there is always the lingering doubt that there may not have been a perforation. Nature, however, at times achieves a spontaneous cure by plastering a bit of omentum, more rarely a coil of bowel, at the crucial moment against the hole.

Early diagnosis is of paramount importance. Sudden abdominal pain is the most significant symptom, but it is not pathognomonic. Its occurrence should at once put us on guard to look for confirmatory signs-rigidity, distention, a drop in the temperature, a rise in the pulse rate, and an increase in the number of leukocytes. Such a case should be seen at short intervals, and, if it is possible, the leukocytes should be counted every hour. If vomiting supervenes, and if the patient grows restless and thirsty, and sucks his lips, it is probable that peritonitis is present.

With the first appearance of suspicious symptoms a surgical colleague who has had operative experience in perforation cases should be called in consultation, so that he can be prepared to perform the operation should the diagnosis be confirmed. While the case is under suspicion all food should be withheld. Stimulation should be continued actively if the pulse is failing, strychnine being given hypodermically.

Typhoid fever patients bear operation well. 'The great mortality is due to the delay in operating rather than to the operation itself. When in doubt, it is probably better to operate, the pros and cons having been made clear to the patient and his family.

The results of operation are constantly improving. Of 816 cases collected from the literature, 249 recovered, a percentage of 30.5 .

I Schlesinger, quoted in Münch. med. Woch., 1908, 1191.

2 la Presse méd., 1904, 681. 
Appendicitis.-Appendicitis may occur in typhoid fever, and may present all the pathologieal conditions seen when it occurs independentls: Its treatment no way differs from that of ordinary appendicitis, except that the indications for operation must be imperative. Of 3.5 operated cases, gathered from recent literature, 27 recovered; of these 35,7 showed perforation of the appendix. Out of 4.3 cases not operated on, 41 died; 33 of this series were perforation cises.

Cholecystitis.-Mild cases may be treated by the use of ice bags or turpentine stupes locally, and such other measures as the patient's general condition may indicate. Recovery usually ensues. If, however, the symptoms are stormy and progressive, operation should not be delayed. In 21 cases collected by Ashhurst, including two personal instances of perforation of the gall-bladder, operation was followed by recovery in eight. Great difliculty is at times experienced in differentiating between cholecystitis and appendicitis.

Parotitis. - This is an interesting complication, usually bilateral, which in my experience very rarely suppurates. Ichthyol ointment is the most useful application. If suppuration ensues, incision is necessary.

Respiratory System.-Epistaxis is seldom sufficiently severe to require treatment, but it may become alarming. Applications of ice to the nose and instillations of adrenalin chloride, 1 to 1000 , and in severe cases packing of the nose, constitute the treatment. Occasionally the bleeding ceases when ice is applied to the nape of the neck.

Bronchitis in rare instances dominates the clinical picture to such a degree as to throw the physician into diagnostic error. Usually treatment is not required. If the case is severe it may be controlled by mustard to the chest, by Dover's powder, gr. ij, or heroin, gr. $\frac{1}{2}$, every four-hours. 'The sputum should be disinfected.

Pleurisy is rare. Strappings of the side arrests the pain. If there is fluid, tapping may be necessary.

Pneumonia is also rare. Preventive treatment consists in turning the patient from side to side, so as to lessen hypostatic congestion. Established pneumonia is treated like pnemmonia in other circumstances. 'The bath treatment, if used, is generally suspended, though some authorities contimue bathing even when there is inflammation of the lung.

Boils.-Boils occur in severe and protracted cases, especially in those that are tubbed. In hospitals the infection may be carried from one patient to another by the use of the same sponge, bedpan, or other utensils. Boggs' has called attention to the value of alum as a preventive. The larger boils, which are often the cause of a continuance of the fever, are best treated by incision and antiseptic dressing.

Tender Toes.- This condition is rare, but extremely annoying. It is best treated by supporting the bedclothes on a cradle away from the toes, and by the application of a solution of sodium bicarbonate, or painting 
the toes with a mixture of tincture of iodine and tincture of opium ( 3 drams each), and tincture of aconite ( 2 drams).

Retention of Urine.-Retention of urine is common, but is often overlooked because accompanied by overflow. I have found, at times, the sheet soaked with urine and the bladder, nevertheless, distended halfway up to the umbilicus. The treatment of retention is catheterization, which should be done with the greatest aseptic precautions.

Scanty urine requires no other measure than the free introduction of water by the mouth and regular saline enteroclysis-a pint once or twice daily. Certain mineral waters I have found at times to act most satisfactorily. Polyuria is common in typhoid fever, and may be looked upon as a favorable symptom. It requires no treatment. It may reach extreme degrees, especially when the Murphy treatment or systematic daily enteroclysis is used.

Bacilluria is present in many cases. It may be combated by the use of hexamethylenamine (urotropin), in doses of 5 or $10 \mathrm{grs}$., three times a dar, given in the later weeks and for a short time (about two weeks), after the temperature is normal. Too long continued and in too massive doses it may produce tenesmus, hematuria, and even hemorrhagic nephritis.

Phlebitis. - This is a fairly common complication, though one may go for a long time without seeing a case. It begins suddenly, with agonizing pain in the calf; swelling promptly appears and rapidly extends up the leg to the groin. The limb is helpless; the slightest movement or touch is painful. Usually unilateral, phlebitis may be bilateral. The limb should be lifted up on pillows, cloths soaked in lead water or laudanum should be applied, and over these an abundance of cotton, the whole being held in place by a loose bandage. 'The bedclothes should be kept from the limb by a cradle. Massage is not to be used. The swelling subsides quickly, as a rule, but if the patient gets up too soon it may return; in that case a firm bandage or an elastic stocking is required.

Typhoid Meningitis. - This is a rare complication, of which, according to Cole, ${ }^{1}$ three types are distinguishable: one in which there are symptoms without lesions; one in which the meningitis is serous in character but in which the spinal fluid contains typhoid bacilli; and lastly, a purulent meningitis. Lumbar puncture is of value both in diagnosis and in treatment.

Relapses.-Relapses are, unfortunately, quite common, and we have no definite means of preventing them. So long as the spleen is enlarged, even if the temperature has been normal a number of days, there is a danger of relapse.

The treatment of relapse differs no way from that of the original disease.

Bone Lesions.-These are rarely complications of the disease, but are more frequently sequels. ${ }^{2}$ The treatment consists in operation, if possible before fluctuation is present.

'Johns Hopkins Hospital Reports, 1904, xii, 379.

${ }^{2}$ Keen, Surgical Complications and Sequels of Typhoid Fever, 1898. 
Post-typhoid Insanity.-I'sychoses as sequels to typhoid fever are rare, but exceedingly interesting to the clinician. 'They may' take a depressing or an exalted type. Usually they are exaggerated expressions of the patient's normal personality.

The prognosis is good. All the cases I have seen have recovered. 'The patient should he isolated, and if he cannot have quict and the care of at least two good nurses at home, he should be sent to an institution. If the insomnia is intractable, which is a rule in maniacal cases, the newer hypnotics, as well as morphine, ma be given in large doses, as these patients bear them well. It is advisable, however, in serions cases, to call an expert alienist in consultation.

Typhoid Psychoses in Children.-While as an infection typhoid fever usually runs a mild course in children, it leaves not infrequently some mental disturbance in its train. 'This disturbance mav take the form of insanity, such as mania, dementia, or melancholia, or it may be delirium or hysteria. Recovery takes place, according to the statistics of Edsall," in 62.3 per cent. of cases. I have seen one pronounced case of posttyphoid hysteria which ended in recovery. 'The treatment of typhoid insanity in ehildren, barring cases of a violent type, is probably best carried out at home.

Typhoid Spine.-'This extremely painful condition is very rare. I have seen only one pronounced case. 'The patient, a trained nurse, was totally helpless; the slightest passive movement caused her agonizing pain. 'There is always a large neurotic element in such cases. I'sychotherapy and the Paquelin cantery usually achieve a cure.

Neuritis.-Neuritis is rare. It occurs both during the course of the disease and as a sequel. Hot compresses, the heat of which is maintained with hot-water bags or a thermophor, often give relief. In the later stages, after general convalescence is established, electricity and, if necessary, the analgesics, like antipyrine, may be employed.

Typhoid Fever and Pregnancy.-Pregnancy has little or no influence upon the course of typhoid fever; but the reverse is not true, for in from 68 to 85 per cent. of cases, according to the statistics of rarions reporters, ${ }^{2}$ abortion or premature labor occurs as an effect of the disease. The treatment of abortion or premature labor need not be discussed in this article.

General Management of Convalescence.-Vigilance must not be relaxed simply because the temperature is normal. Aside from relapse, there are all sorts of possible accidents that imprudence may invite. No visitors should be allowed until the eighth day, and then only for a few minutes. On that day the patient may have an extra pillow; on the tenth day he may recline on a bed-rest for twenty minutes in the morning and in the aftemoon. 'The next day the time is doubled, and on the thind day increased to an hour. With every change of this kind the pulse should be watched. If it rises suddenly the recumbent posture is to be resumed 
at once. At the end of two weeks the patient may sit in a Morris or wheel chair. By the eighteenth or twentieth day he may walk a few steps. He should, however, not do anything to fatigue himself. When walking is more freely attempted, he should wear firmly laced shoes to protect the pedal arches, the ligaments of which are weakened by the disease and the long rest in bed. After the patient is able to care for himself the nurse is dismissed. Sometimes a very strong attachment is formed between patient and nurse. In one case, the patient being a young woman, the departure of the nurse was immediately followed by a relapse and profound emotional disturbance.

In some cases there is during convalescence a persistent slowness of the pulse - the rate may be as low as 40 - a condition alarming to the members of the family, who consult the nurse's records. I have seen no harm from this bradycardia; it always disappears as the patient's strength increases. Drugs have but little effect upon it-neither the stimulants nor atropine raises the pulse rate permanently. While the condition lasts it is best to keep the patient in bed.

As the patient gains strength the desire to return to his daily work grows upon him. It is then the physician's duty to restrain him until restoration of bodily and mental vigor is complete. In a severe case a long time may be required for this-rarely less than three months, often six or twelve. Among the laboring classes the long period of invalidism is fraught with great hardship, and no charity is more urgently needed than one which looks after the welfare of the poor during convalescence. What I have said of work also applies to social life. The convalescent should devote himself exclusively to the task of regaining his strength, and should spend his time as far as possible outdoors.

Children, who often grow one or more inches while in bed, should be allowed to "fill out" in proportion to their stature before being sent back to school. Inasmuch as in the predisposed typhoid fever may prepare the way for tuberculous infection, the lungs of such patients should be carefully watched. At times a weakening of the memory manifests itself during convalescence, but fortunately the physician can give full assurance that this is only a passing symptom.

The increasing frequency with which typhoid carriers are being discovered makes it incumbent upon us not to discharge a patient as cured, either in private or in hospital practice, until we know that stools and urine are free from bacteria. In Prussian hospitals two bacterial examinations of the feces must be negative before the patient can be dismissed. Just as in diphtheria, so in typhoid ferer, municipal laboratories should be prepared to make the cultural tests; and until the bacterial examinations are negative, unremitting care must be exercised to disinfect the discharges. If the nurse has been dismissed the physician must instruct some member of the family in this responsible duty.

The Control of Typhoid Carriers.-The control of typhoid carriers is a serious problem, since they are a great menace to the public health. When they are inmates of institutions, such as insane asylums, their isolation presents no difficulties; but in the world at large their 
confinement, barring here and there an exceptional case, is impractical. Medical treatment has been almost devoid of results, and intestinal antiseptics are useless. Purgation and careful dieting were found by Park and others to diminish but not to banish the typhoid bacilli from the stools. Yoghurt, for which so much is claimed at present, has not proved efficacious. ${ }^{1}$

In two cases Delher opened and drained the gall-bladder, with good results. How many typhoid carriers, however, will subnit to such an operation, especially as we cannot promise them that it will put an end to their infectivity, since the bacilli might come from the intestine and not from the gall-bladder.

Irwin and Houston ${ }^{2}$ treated a carrier successfully with typhoid bacterin (vaccine). 'The bacilli were in the urine, not in the feces. The blood gave the Widal reaction. A vaccine was prepared from the bacilli in the urine. Its injection in doses up to $200,000,000$ was ineffectual. Sodium lactate was then given, to render the urine alkaline, and the injections resumed in increased dosage, with complete cure. For the last injection, 1,000,000,000 bacilli were used. Other cases treated successfully by this method are recorded by Houston. ${ }^{3}$

In determining whether a typhoid carrier is finally cured of his infectivity, it is well to bear in mind the observations of Davies and Hall" that there is a seasonal variation in the "effectivity" of carriers. In the early part of the year "ineffectivity" usually obtains, but with the beginning of May the bacilli reappear in large numbers.

'Typhoid carriers should be informed of their condition, and should have pointed out to them in the strongest possible way the importance of thorough disinfection of their excreta.

Paratyphoid Infections.- The treatment of these infections, the diagnosis of which can be made only by laboratory methods, is identical with that of typhoid fever.

${ }^{1}$ Forster, Münch. med. Woch., 1908, No. 1.

2 Lancet, 1909, i, 311.

3 Brit. Med. Jour., 1909, ii, 1056.

- Lancet, November 18, 1908. 



\section{SCARLATINA, MEASLES, RUBELLA, VARI- CELLA, AND VARIOLA}

BY S. DANA HUBBARD, M.D.

\section{SCARLATINA}

Prophylaxis.- Under this heading will be embraced all the methods of the skilled sanitarian. Sanitarians and municipal health authorities have well-recognized regulations for the control of patients ill with this disease, and the possible prevention of its occurrence in others of the community.

All cases of scarlatina should be reported promptly; this should be compulsory, with severe penalties against the parent, guardian, or medical attendant who violates this rule, and thereby unnecessarily exposes others to the disease.

A case once located should be effectually isolated, and all concerned should coöperate with but one end in view, and that is, the prevention of others contracting the disease. Physicians should be the first to accept this as a responsibility, and with their best influence should endeavor to secure the family's assistance in controlling and preventing the spreading of searlatina.

Placarding.-Placarding should be done by means of a large gummed poster put up in a conspicuous place, but unexposed to the elements. Any one desiring to enter the premises may then know that scarlatina exists, and knowing, may avoid infection. If the placard be exposed to the elements, then a red flag, with large letters across the middle, should be tacked across the entrance gate. In the corner, notice of the penalty for removal, should be printed in smaller type. Oftentimes the fear of a penalty will insure isolation, whieh otherwise would be neglected. Placarding homes is sometimes objected to by physicians, as they feel it makes their visits too conspicuous. "Diserimination" in placarding by municipal authorities is only a term for laxness, and should not be tolerated. A law that is just and equable should be exercised toward all exactly alike.

Isolation.-The presence of scarlatina in a home which in any way has connection with the sale of food-stuffs should be guarded with extraordinary vigilance, and every known precaution observed most mimutely. Should the apartment be in the rear of, or in any way connected with, a store, either the store should be closed or the patient removed at once to an isolation hospital. 'The premises should be fumigated and the goods 
disinfected before opening of the store is permitted. Practical isolation in such a place is next to impossible, and simply encourages an epidemic.

Public Schools.-Public schools have a bad name for many reasons, chief among which is that when the schools open, up jumps the number of cases of scarlatina, and when the schools close, down goes the number of cases. At times certain schools seem to be the center of many epidemics. When it is known what is possible, and oftentimes does happen, this is not to be wondered at, but the blame should be placed where it belongs, not on the school, but on the people who make such a condition possible. Lists of contagious diseases in each school district should be publicly posted in the schools, so that each school could note their particular number of contagious cases.

Families disregarding the usual precautions should be posted conspicuously so that others may be warned. This is the great source of danger. Personally, I do not consider schools as infecting places aside from above-mentioned infractions. The infectious diseases soon decrease in number in mild weather, when the homes are well aired and ordinary hygienic precautions are more easy to observe.

In congested districts, in large cities, and especially among those who live very unhygienically, a large number of cases of scarlatina are the rule rather than the exception. I ask, in all fairness, how is it possible to properly isolate any patient in our modern apartments, much less in our tenements? Then, in addition, consider the many times that all attempts at isolation are utterly disregarded, especially during convalescence. To my mind, it is wonderful that there is not much more of the disease. Every possible effort should be made by the authorities to combat this tendency, and I am sure if it was taken up and put squarely before the community when scarlatina prevails, then better results toward preventing the spread of the disease would be possible.

Also, if school authorities would give particular attention to all scholars who are absent for a period of three days or more many cases of scarlatina would be found. These should be risited and personally observed by a medical officer.

From my experience I deem it far safer for all concerned that children be sent to school and kept from an infected home rather than to be forcibly excluded. An idle child is in great danger at all times, and add to this the possibility of playing with its sick companion and then going at large only adds to what we are trying to prevent. At home an idle child is oftentimes of more trouble than is the invalid and only places additional burdens upon the parents. If, however, the child is at school in well-ventilated rooms, and his mind occupied, there is less possibility of his having time to see the sick, with just that much less opportunity for carrying infection. At present we are working in this direction, and it will not be long distant, I hope, ere this old-time superstition is broken through.

At present the procedure regarding schools in most cities is for the public authorities, after receipt of a notification of a case of scarlatina, to at once notify the school of the presence of the disease, giving 
the name, age, and address. 'This is sent to each class-room, and all] scholars from that family (not that house) are at once excluded, and kept excluded, until armed with a school certificate from the public authorities. In addition to this, there is a special permit, which so far has worked successfully, and this, if properly executed, will shorten the period of enforced idleness and will work an advantage to the school attendance. 'This is what is known as a special school permit, and is as follows:

This certificate in force only during the absenee of persons named thereon from premises .................. where contagious disease exists. Any return to premises mentioned before fumigation is performed renders this certificate void.

'This rule is applied as follows: 'The child makes personal application to a specially appointed public officer-one who is able to discriminate and whose ability for carefulness is well known. The name and address of the invalid are taken, together with the name of the applicant and the name of the party with whom the child is to stay. Verification of this change of residence is then made by one of the various inspectors. 'The child returns to the special officer at the end of five days and if the verification is found proper and the child is healthy, the permit is issued. If for any reason at all the child is found to be visiting its home, the permit is revoked at once, the child excluded from school and as a penalty is not given further consideration. This rule has been in use now for two years and has been of great assistance and has very materially aided in forming the opinion given elsewhere about exclusion of school children.

Regarding the other members of the household, it is hardly necessary to isolate and quarantine them. The bread-earner should be permitted to continue his duties. Formerly, in the municipal service, when scarlet fever occurred in the home of a police officer, the patrolman was not permitted to continue his cluties for the reason that, at times, he has to live in barracks for a certain number of hours, thereby exposing his fellow officers to infection. 'This has been proven unnecessary.

Regarding the sending away of children, who have apparently. escaped infection, to distant relatives a word of caution is necessary: 'The possibility of the child contracting the disease is in the majority of cases slight, but it should never be sent from home until after the five-day period of incubation, for the reason that should it come down with the sickness it will be very awkward and inconvenient to have two of the family ill in separate places.

A child who has scarlatina should be excluded from school for a minimum period of thirty-five days. This should be always enforced. Patients who present nasal discharges, or rumning ears or scabs on the skin surface, and who occasionally have recurrent exfoliation, should not, under any circumstances, be readmitted to sehool, and if readmitted, should be at once excluded and the school-room at once disinfected. In many cities there are medical officers attached to the schools whose duties consist in frequent examinations of the pupils. Extra precautions should be taken by these examiners at all times to Vol. I-41 
discover the existence of a sore throat, or nasal discharge, pain in or discharge from the ears, or exfoliation of skin, particularly of the palms. The presence of any of these should at once cause the exclusion of the pupil. 'Too much care cannot be exercised in this respect.

In those schools not so fortunately situated as to have medical supervision, the teachers should be taught ordinary hygiene, and, with but slight attention to this detail, many epidemics of scarlatina could be avoided.

The earlier the recognition of scarlatina, the less danger of infecting others, for, unlike measles, scarlatina is very slightly contagious in its onset.

Closing of Schools-Of all unnecessary hardships, this is the limit of endurance. It is about as unnecessary as anything can possibly be. It is also an acknowledgment of inefficiency, to my mind. With practical and modern hygienic methods, the room can be quickly cleansed and rendered safe.

Prompt exclusion of suspects, efficient cleansing of room or building, efficient disinfecting of.books and papers in the school, and proper ventilation will, as a rule, secure and maintain effectively healthy conditions. Careful and conscientious regard for these very simple rules will effectually guard against any infection.

The liability of children contracting scarlatina from an early or brief exposure, and then carrying the infection, is a very remote possibility.

Isolation Hospitals.-The more cases removed to an isolating hospital and treated there, the less will be the number of cases in the community; also the greater number treated under such conditions, the better will be the statistics regarding mortality.

The average home is ill fitted, as a rule, to isolate a case for any length of time, and the less isolation, the greater danger there necessarily is toward the spreading of infection to others. Every municipality should have an isolation hospital for this disease. This should be semiprivate; that is, a general service for ward cases, with rooms for private cases. The more patients under such supervision, the better will be the results.

Patients treated at home, especially among the poor and ignorant, to say nothing of the wilfully careless, who do not recognize their responsibility to others under these conditions, do not, as a rule, do well, and are further a positive menace to the community. These should receive extra care, and the severest enforcement of isolation should be exacted of them. A very large number of people contract scarlatina from individuals who are up and going about.

Mild unrecognized cases which continue to go about, or cases released prematurely,-and it is at present impossible to determine accurately when the danger of infection ceases in a scarlatinal case,-add to the danger.

Relapses.-Relapses are rare, and when they do occur, cause considerable confusion and bring down unfavorable criticism, which is often unmerited, upon the heads of the authorities. 
The change from the hospital to the home, the change of diet, and the nervous reaction oftentimes are the main reasons why such relapses occur. The same thing is observed in typhoid fever and other diseases. The principal reason, however, is the premature discharge of the patient. I have observed infection take place in a home after the patient had been eighteen weeks in the hospital ill with aural disease.

Irrigation of Throat.-In my experience the infection in most late cases comes from the discharges from the throat. 'Too much care cannot be given to this one organ, which ordinarily escapes attention.

Frequent irrigations with a solution of peroxide of hydrogen or permanganate of potash solution will ordinarily make the throat as nearly sterile as is possible. 'This is a precaution that is not ordinarily observed, and I cannot lay too much stress upon it, as it is very necessary. Such convalescents should not be kissed on the lips for weeks after returning home from an infectious hospital.

Desquamation.-'The contagiousness of the scales of the desquamated epithelium is open to question. Personally, I do not believe the scales themselves are contagious except as carriers of infection. 'They have been in close contact with an infected subject, and necessarily cannot be entirely free from infecting material. It is on this point that the freedom from infectivity must rest and on it alone.

I have tried, on several occasions, to reproduce scarlatina in the human subject, and have so far failed, except in two instances, and these were accidents. 'They occurred some years ago, and showed the principle, only too well known, that exposure in a room with a scarlatinal subject is commonly followed by infection. In one of the large municipal hospitals, while patients were being removed to new quarters, a stupid orderly took a patient into the wrong ward. The patient, convalescing from measles, was left on a clean bed only for a few minutes, but exactly five days later he came down with scarlatina, and fourteen days later measles broke out in the scarlatinal ward. This accident has happened in the experience of others, but it proves nothing special.

Scarlatina is in all probability due to a micro-organism not as yet recognized. The danger of infection ordinarily disappears about the time that desquamation ceases. At other times, and this is frequent, in my opinion, it persists for an indefinite term, usually much longer than is ordinarily imagined. Therefore, if we are to err in future cases of scarlatina, let it be on the safe side, and continue isolation for a long rather than for a short period.

Certain patients also appear to retain the infectious principle for some time. These cases are hard to determine, and unless recognized, are apt to cause very disastrous results. It is this type of case that we should be constantly on the lookout for.

Under ordinary conditions, in the mild scarlatinal cases which desquamate early, thirty-five days' isolation will be sufficient, but in the severe cases at least sixty days should be required. No case should be discharged or exposed to others until all discharges from the ears, nose. or elsewhere have ceased, and the skin has resumed its normal condition. 
Before discharge each patient should undergo a complete and thorough cleansing in every respect, and be given a complete change of clothing which has not in any manner been exposed. After passing out of the infected quarters, the patient should not for some time be exposed to other susceptible persons.

Many cases of scarlatina will desquamate twice or even three times or more; these should be held as long as the desquamation continues. Recurring desquamation may be benign and impossible to transmit the disease, but, in the view of the present-day knowledge, I prefer to take no chances.

The Apartment of the Scarlatinal Patient.-This is at times a very vexatious question. When a patient is taken ill, and it is suspected to be scarlet fever, he should be immediately isolated in a room where he can remain throughout the illness. The room selected should be one as remote as possible from other dwelling quarters. In a flat, the room should be at the front or rear, and preferably one that opens into the street rather than one that opens into a courtyard. In a house a room on the front or rear of the top floor is preferable. Ventilation without a draught should be at once considered and arranged for. The bed should be protected by a curtain or screen, so that at no time does the patient get chilled by sudden changes.

A wide board placed under the lower sash and overlapping the two sashes sufficiently to direct the draughts away from the patient will effectively ventilate the room.

All dispensable articles of furniture, ornaments, draperies, rugs, and carpets should be removed before the room is occupied. The floor may be covered with carpet lining, and over this an improvised covering may be made of unbleached muslin, tacked down in strips the full length of the room. The carpet lining deadens the noise of foot-steps and the muslin gives a hard finish which will bear considerable wear. It is inexpensive, saves the carpet, and can be destroyed later by burning. All doors leading to other rooms or closets not used should be sealed with strips of gummed paper. The entrance door to the sick chamber should hare a sheet tacked over the inner and outer portal. This can be improvised from the unbleached muslin. The sheet should be tacked across the top and down one side to the floor. If the outer sheet is left loose on the left side, the inner sheet should be left free on the right side, so as to prevent a direct opening between the outer and inner shields. The sheet will thus prevent draughts into other rooms as well as air-currents, which may carry infected material into the interior of the home.

The inner shield in the sick chamber should be spraved with a solution of carbolic acid, 1 to 20 , to which is added a small amount of glycerine to make it slightly adhesive and also hygroscopic.

Near the entrance of the sick chamber, should be kept gum shoes, a covering gown, and towels for making a head-cap, also a stand with basin, water pitcher, soap, towels, and disinfecting solution. To make this outfit complete rubber gloves should be added. Any one 
that enters should be made to put on the gown, cover the head, put on rubber shoes and the gloves, so as to be properly protected against infecting themselves or their clothing and transmitting the disease to others.

Men before entering should, in addition, turn up the bottom of their trousers.

'The woodwork in the room and the furniture should be oceasionally washed down with a mop soaked in a bichloricle of mercury solution, 1 to 1000 , and if the floor is not covered, it should be mopped daily with this solution. 'The toilet used should be frequently flushed with this solution. No one except the nurse and the medical attendant should be permitted to enter the siek quarters. Should the mother act as nurse, she should devote her entire time exclusively to the care of the patient and delegate her household duties to another. 'The nurse should not, under any circumstances, leave the sick chamber, and, should such become necessary, then only after a complete change of clothing and a shampoo, followed by soaking in an antiseptic solution. Garments worn by attendants should be of such material as can be easily cleansed by boiling.

'The hair should be at all times while in siek chamber covered with a protecting cap. 'The ordinary nurse's cap does not do this, and it should be exchanged for one that does.

Medical Attendant.-Precautions.-If the following directions are carried out there will be but slight possibility of transmitting infection to others.

Keep as far as possible from the patient as the necessities of the case will permit. Handle the patient as little as is possible. Always wear a protecting gown that entirely covers all parts of the person and extends well down to the floor on all sides. The gown should have a pursestring of rubber elastic about the lower ends of the sleeves so as to prevent opening and exposing the parts to infection. Such a gown or one that either has no openings, slipping over head, or which opens in back is to be preferred. Wear overshoes over the boots and have the lower ends of the trousers turned up. Have the head and face except the eyes covered by a towel. Wear rubber gloves.

All instruments needed, thermometer, tongue depressor, and stethoscope, should be left in the sick chamber to be sterilized on recovery, or have the patient furnish the same of an inexpensive type.

The throat should be sprayed with an antiseptic solution before leaving the patient.

All articles coming in contact with the patient should be subjecterl to thorough disinfection.

In the sick-room, or if there is a bath attached, so much the better, there should be a pan or large vessel containing a solution of phenol, 1 to 20 , in which all utensils should be immersed and soaked for a period of at least twenty minutes or longer.

The bed-linen when changed should be left to soak over night. wrung out, placed in a boiler, taken to the kitchen, and boiled for at least one-quarter of an hour, when it can then be washed safely. 
The dishes and eating utensils, and such articles as are necessary for the attendants, should be retained within the sick chamber. The nurse should not come to the dining-room for her meals, nor should she be permitted to mingle with other members of the family.

Such articles as are necessary to send out of sick-room should be soaked in the phenol solution for at least one-half hour. Before others are permitted to use them, they should be boiled for at least one-quarter of an hour.

Discharges from the patient's ears, nose, or throat should be received on bits of old cloths or cheesecloth, cut about size of a lady's handkerchief, put in paper bags, or a cornucopia made of newspaper, and then burned. Sputa cups, urinals, and bed-vessels should have a solution of phenol, 1 to 20 , in them at all times. If the odor is objectionable, chloride of lime may be substituted.

Have the nurse add warm solution to the urinal or bed-chamber before using, as it materially adds to the comfort of the invalid.

Visit scarlet fever cases early in the morning or late in evening, and let as much time as is possible elapse after leaving these cases before visiting other patients. 'Take a walk of some distance in open air after leaving the fever-infected apartment. Always thoroughly wash the hands on leaving the apartment.

It is best for physicians who happen to have obstetrical and scarlatinal patients at the same time, if they can afford to do so, to delegate to an associate the care of the one or the other, as there is more than ordinary susceptibility to infection in parturient patients.

Some physicians do not believe in the possibility of physicians transmitting infection, but it is possible, and no less an authority than Murchison, as well as other eminent observers, have noted it. Certain lax habits in the profession are difficult to eradicate, but the slightest possibility of a physician carrying infection should be eliminated.

It is an interesting fact that in the private hospital for contagious cases in New York not a single case of reinfection has taken place in any one of the homes to which patients have returned. Not a single person has ever been permitted to enter or visit a patient in this hospital without being clad as directed above, and there has not been a case of mixed infection or an instance of a ward being infected with another disease-a truly wonderful record when it is known what takes place in most municipal hospitals.

Adult members of the home where scarlatina exists should safeguard the interests of others by avoiding contact with the outside world as much as is possible, and should particularly avoid social gatherings and attending places of amusement where there is congestion and the ventilation is not of the best. Here is a splendid opportunity for a living exemplification of the Golden Rule.

The Diet.-This will vary with the severity of the disease. If the case is an extremely mild one, then no variation from the normal diet is necessary. During the early stages of scarlatina, when there is ordinarily considerable elevation of temperature, a milk diet is the best and most 
acceptable to the patient, as a rule. Cool milk soothes the throat and quenches the thirst which is present. As long as there is any elevation of temperature, and until it has returned to the normal and showed evidences of remaining there, I insist upon a inilk diet.

Should the patient be unable to take milk for any particular reason, then broths or strained soups may be used. At times one of the liquid proprietary foods may be tried with benefit, but in my treatment patients have first to prove to me that milk will disagree before I accept their unsupported statement made from caprice. Nothing will ordinarily agree with a patient so well as milk.

Many objections to milk can, with persuasion, be overcome, and, in the ease of young children, altering the flavor or slightly sweetening with saccharine, or, if necessary, coloring, will often overcome the dislike.

Milk should be given liberally. I give it as often as patient will take it, ordering one glass ( 3 viij) every two hours.

When the fever is lower, plain bread and butter is added, with at times plain puddings and stewed fruit once a day. While I do not overfeed, I never starve scarlatinal cases. If the milk has a tendency to constipate, milk of magnesia ( $\bar{s} s$ ) may be added to each glass, or it may be peptonized just short of bitterness, which will at times overcome this tendency. 'To the milk may be added raw fresh eggs, and occasionaliy the diet may be varied by substituting for the milk an oceasional cup of broth or a dish of plain or flavored gelatine.

'The plain juices of such fruits as oranges, limes, lemons, or grapefruit may be given, as they are appetizing and very acceptable. emonade is very refreshing, and during convalescence is of assistance in irrigating the kidneys. A pitcher of water may be placed near the bed, and the patient encouraged to drink freely. In infants and young children an exclusive milk diet is preferable to any other, and should be insisted upon. In older children, when they show a craving for food, the above solids should be added.

As the patient's condition warrants, a change from liquid to a few solids is made, and later, as he convalesces and shows a craving for additional changes, a more liberal diet is given, until a return to normal or regular diet is made.

Always see to it that if dainties are given they are digestible and nourishing. Pastries and fried foods, especially the excessively fatty and oily ones, have a tendency to derange the stomach and intestines. If the diet should disagree, administer salines, in small doses, repeated at intervals, so as not to weaken the patient by overpurgation.

General Care of Patient.-Confinement to bed in all scarlatinal cases should be insisted upon, and this should be continued until well on into convalescence. 'This is especially desirable in avoiding many. unpleasant complications, and it is my invariable rule, even in the mildest cases. The rest is very necessary in scarlatina, and, in addition, the variations of temperature are avoided by keeping the little one quiet. Nephritis also occurs less frequently. In hospitals all cases should be kept in bed until exfoliation is completed. I always inform the parents of 
this rule at the onset, and explain that unpleasant complications can only be safely avoided by so doing.

In the municipal hospital of New York the percentage of nephritis in scarlatina is less than one-half of 1 per cent., a result that is certainly worth the trouble of obtaining. Before this regimen was insisted upon nephritis was invariably present in about 5 to 10 per cent. of the cases.

Catching cold in the absence of the ward attendant has, in my opinion, been the cause of changing a normal safe convalescence into a complicated one with disastrous results.

'The skin, which has important functions to perform, should in scarlet fever receive very careful consideration. Sponge-baths with tepid water, bathing only a part of the body at a time, the remaining parts being protected by wool covering, should be given at least twice a day. Later the child should be massaged with cocoa-butter or olive oil and phenol, 1 or 2 per cent.

'The careful rubbing of the limbs is very restful, and assists in quieting the irritability of the nervous system. In the cases where there is an intense eruption, we practically have a dermatitis to deal with, and the burning or itching may be very annoying; here local cooling solutions may be safely used, such as extract of witchhazel or calamine solution (calamine, $5 \mathrm{ij}$ to $\mathrm{Oj}$ aqua calcis), applied on cloths or mopped to a part. When watery solutions are objectionable, an ointment of ichthyol, menthol, or eucalyptol may be substituted.

Later, as exfoliation commences, olive oil with phenol, 1 or 2 per cent. strength, should be used; if this dries too quickly, then petrolatum with phenol of the same strength may be substituted. The frequent application of ointments assists exfoliation, is mildly antiseptic, relieves itching, and prevents cracking and fissuring of the skin.

Do not let patient pick off the dead skin too soon, it will only cause hang-nails about the distal parts of the fingers and open up cracks in the new skin. The loose exfoliated part should be lifted with forceps and trimmed away with cuticle scissors. The susceptible derma should be guarded most carefully, and all local infections prevented if possible.

Applying a coating of salve and then bandaging will produce softening and maceration, so that, with soaking in warm water, the loose skin is, as a rule, easily removed.

All methods that are used outside of these to hasten desquamation should be interdicted as harmful, especially sand-papering, scraping with knives, or the rubbing with pumice, once in use for scarlatinal cases.

This forcible peeling is often followed by secondary desquamation, as the too early removal of the superficial layer does not give nature a chance to restore the outer surface, and the too tender cells exposed thereby only die and are later cast off. This no doubt accounts for some of the repeated infections on returning home as well as the recurrent desquamations.

Internal Medication.-The internal or medical treatment of scarlatina is entirely symptomatic. We possess no medicament which is a specific in either preventing the appearance of this disease or of curing 
it when it has appeared. In fact, aside from symptomatic medication, we are unable to abort or influence the course of scarlet fever in any way, either directly or indirectly. Still our lay friends often ask us to give them something to prevent their catching the disease when scarlatina has appeared in their vicinity. Being in the open air, exercising properly, eating judiciously, and sleeping regularly will prevent any disease. Anxiety, worry, and fear will depress and reduce vitality and render an individual susceptible, so that if exposed to the disease he is likely to contract it.

In mild cases of scarlatina internal medication is often unnecessary, the disease running its course to a pleasant and favorable termination unassisted. Good nursing, constant care, and a simple diet may be all that is ordinarily required. During the febrile stage a febrifuge may be administered, such as sweet spirit of nitre or liquor ammonii acetatis. For vomiting, if prolonged or accompanied by nausea, the abstaining from food and a few doses of calomel in fractional amounts ( $i^{\frac{1}{0}} \mathrm{gr}$. half hourly for four doses), followed by milk of magnesia, diluted and in small amounts, frequently administered, will usually suffice. Bicarbonate of soda, with peppermint water, at times is of service. If constipation is present, it should, of course, be corrected by laxatives, either cascara or rhubarb or phenolphthalein in powder form.

In the onset of any disease, Barker's old adage is very applicable, "Clean out and clean up." A saline, to my mind, is almost always necessary. Rochelle salts in aromatic spirits of ammonia and water is a favorite remedy of mine. It is cooling, sweetens the alimentary canal, lessens the formation of gases, quiets the nervous system, and relaxes tension. It is best given in small doses half hourly, and is not followed by copious exhausting movements.

For the throat, a mild antiseptic should be used, such as Dobell's or Thiersch's solution or diluted peroxide of hydrogen. 'This cools the parts, eleanses the mouth, and sweetens the breath, and, to a certain extent, relieves congestion and soreness and limits the liability to secondary infection. One should be extremely gentle in the use of gargles or sprays; do not use any irritants, as they will not be well borne, and are liable to be followed by an extension of the infection. Also in cleansing be careful not to spread the infection to the middle ear.

In scarlatinal cases there is a specialized tendency toward the formation of an exudate, either diphtheritic or pseudodiphtheritic (streptococcic), and here antitoxin is indicated. I use it empirically, because I have found that it limits the formation of this membrane. Concentrated antitoxin, 2000 units, are injected, under antiseptic precautions, into the back, and if there is no improvement in six to eight hours the dose is repeated. I have never seen it do any harm, and have frequently seen it followed by excellent results, even in those cases which, after repeated culturing, failed to show the Klebs-Loeffler bacillus. I have not obtained similiar results with antistreptococcic serum (20 to 40 c.c., and six hours later a like amount), even though I have given it just as early, and even when the culture showed numerous streptococci. 
Hyperpyrexia necessitates special attention, on account of the debilitating influence and the causing of nervous phenomena. Scarlatina is characterized by a very high initial fever, which naturally tends in a few days to decline. If, however, the temperature lingers around $103^{\circ} \mathrm{F}$., or just above that, I endeavor to combat it, using sponge-baths, followed by alcohol rubbing. Cool wet cloths to the chest or forehead are very acceptable. Extra vigilance must be taken where coal-tar products are used to safeguard the heart from depression. Cold baths are not well borne by scarlatinal patients, being followed too frequently by cardiac depression, and require considerable safeguarding.

In infants and very young children, tincture of aconite, $\frac{1}{5}$ to $\frac{1}{2}$ of a drop every hour for three or four doses, of ten is quieting, and assists greatly at times in reducing the fever and relieving distressing nervous phenomena.

If the fever continues high, and cannot be controlled by these methods, a wet-pack is given with an ice-cap to the head. In children the ice-cap is soothing, and quiets excessive nervousness when other means fail. Do not use an unprotected bag, as it chills and is not well borne by the irritable child. The ice-bag should be protected by a layer or two of thin toweling. In infants a warm bath, $95^{\circ} \mathrm{F}$., will at times be followed by quieting sleep, from which the patient awakens refreshed. In older children the graduated bath will give better results. Commence with fairly warm water and cool by degrees; at the first evidence of depression remove the child and administer stimulants. (See article on Hydrotherapy.)

The warm bath in children may with advantage be followed by an alcohol rub. Any treatment that irritates or unduly excites the child does more harm to the nervous system than good to the febrile condition. There is no danger of the patient's "catching cold" or the fever "striking in," and the old superstition regarding the use of water in scarlatinal cases is not borne out by modern experience. Bathing at all times is comforting and exhilarating, and is necessary, not only for hygienic reasons, but for the prevention of certain conditions likely to arise in scarlatina. More often nephritis has arisen from the lack of the use of water rather than from its abuse.

Antipyretic drugs may be used from time to time, and, if observed carefully, act without depressing unduly the cardiac system. Small doses of the coal-tar synthetics, accompanying a bath, have at times given considerable comfort and have helped allay nervous irritability.

The Throat.-From the very commencement I always use mild antiseptic lotions, either in form of spray or gargle or as an irrigation.

It should be remembered that the throat is always attacked in scarlatina; it is here, normally, bacteria reside and the lymphatics in this locality offer a wide portal of entry for any infection. 'Too much care cannot be given to the throat, and it cannot be commenced too early. But too much care cannot be exercised to perform the same as gently as is possible, every care being exercised at all times to prevent the spreading of infection to the Eustachian openings of the nasopharynx and thus exciting middle-ear disease. 
Preferably I use Dobell's solution, 'Thiersch's solution, or diluted peroxide of hydrogen in the form of a good strong stream of spray with the patient reclining, or with a moistened swab. The throat may also be irrigated with 2 per cent. salt solution.

Under ordinary conditions the spraying or swabbing may be done morning and afternoon, but if there is severe involvement, as often as every hour, or as occasion may demand and the condition of patient will warrant. It must be remeinbered that the parts of the throat are very sore and are extremely sensitive, so that the utmost gentleness must at all times be used, not only for the comfort of the patient, but to prevent making openings for infection to enter. Children often resist on account of unnecessary force used, and I have frequently irrigated throats when nurses have failed, simply because of remembering this condition. In the case of children, try to gain their confidence, as when they find that the treatment is followed by pleasing and comforting results, you will experience no difficulty. All physical resistance, no matter what the disease, is accompanied by more or less depression and weakness, and should on this account alone be avoided if possible. Should there be false membrane treat as directed above, by the injection of antitoxin or use of antistreptococcic serum.

For the local cleansing of a throat with considerable exudate, peroxide of hydrogen should be used and followed by irrigations of a 2 per cent. salt solution, warm. In septic cases with ulceration an acid solution of chlorate of potash may be used. I prefer cleansing with dilute muriatic acid, half a dram to a pint of warm water, and occasionally painting with nitrate of silver solution, $20 \mathrm{gr}$. to the ounce.

The Nose.-When the nose is involved, as shown by the nasal râles and sanious discharge from the anterior nares, it should be cleansed frequently with salt solution ( 2 per cent.) in the form of a spray, or, if the involvement is severe, by irrigation. When irrigating the nasal cavities, be sure to have patient keep the mouth open. The salt solution should be allowed to flow by gravity from a funnel with rubber tubing and a glass nasal nozzle. The funnel may at times have to be elevated to four or five feet above the head of patient, but ordinarily twelve inches is sufficient height. The greatest care should be used in irrigating the nose, as many unpleasant complications, such as ear involvement, are likely to result. Severe involvement, with odor and excoriation of skin surface, demands this kind of treatment. Ordinary involvement demands but little attention. Gargling and spraying are of doubtful efficiency, but if the irrigations are not well borne, these should be given a trial. Whenever it is possible to get along without nasal irrigations do so, on account of the frequency with which such cases are complicated with mastoid trouble (as high as 25 per cent. in one season).

The swelling of the nasal structures is at times considerable, thus interfering with respiration and enforcing mouth breathing. If the discharge becomes purulent after cleansing, occasional painting of the interior with stimulating applications is often beneficial, especially in the declining stages, when the inflammation tends to become chronic. I 
use nitrate of silver, 5 to $15 \mathrm{gr}$. to the ounce. The application is best made by means of a nasal applicator. A head light is essential. The application lessens the discharge and leads to a healthier condition. Should the mucous membrane of the nose become gangrenous, nothing will avail, and a fatal termination is likely. Such cases should receive irrigations with a very weak solution of permanganate of potassium (color of solution, a very dilute claret). If they can be reached, localized patches of gangrene may be painted with tincture of iodine.

Noma.-Noma is very rare in scarlatina, but it occasionally occurs. 'The condition should be anticipated, and, when recognized early, treated drastically by surgical interference. Curet away the jelly-like deposit and cauterize the base with nitric acid. Owing to the pain of the operation the patient had better be slightly anesthetized with ether. If the skin is attacked, then excision is none too radical.

Enlarged Glands.-The glands at the angle of lower jaw are the ones usually affected and attain the greatest size. They often break down and suppurate. Abscesses are very frequent, and are to be expected in this type of scarlatina.

At the first intimation of enlargement of the glands a small, lightweight ice-bag or bladder-skin should be applied. The ice-bag should be covered with a thin woolen cloth, as it makes it less chilling to the superficial nerves.

Later, when the glands show evidences of suppurating, heat in some form should be used-moist heat is the more preferable. Flaxseed poultices, or a hot cataplasm of kaolin, may be used to hasten the process of suppuration.

As soon as pus is detected, the gland should be incised and drained.

It is much more advantageous to incise too early than to delay too long, for by so doing the involvement of the periglandular tissues may oftentimes be prevented.

Cellular infiltration, when detected, should be treated by free incision, even before pus is detected. By so doing, burrowing, which is a'most always fatal, may be prevented.

The Ears.-Aural discharges are very frequent in scarlatina, and every possible precaution should be observed in the treatment of the disease to anticipate them, and, if possible, prevent their occurrence. I have given this one complication much careful study, and think that it can often be avoided by the exercise of care and patience. There are cases of fever and scarlatina, however, in which middle-ear trouble must be considered as part of the disease and not due to faulty treatment. I believe irrigations are responsible for a large percentage of mastoid cases, and when they can be avoided, aural complications are not so frequently observed.

At times ear pains appear early, are persistent, and are oftentimes followed by spontaneous rupture of the tympanum. With the appearance of the discharge the pain ceases or is less intense.

Scarlatina has been credited with 27 per cent. of the deafness present in the world. In an institution for the care of the deaf in New York 
City I once investigated the cause of the deafness, and found that in 45 per cent. of those present an attack of scarlatina in childhood was the exciting cause. 'This is indeed appalling, and should call forth every possible effort for the prevention of this condition.

Prophylactic measures, which are designed to keep the nasopharynx clean and free from infection, should be early instituterl, and particular eare should be given in each case not to spread infection through the Enstachian openings. Unfortunately, in severe involvement of the nasopharynx this is next to impossible, bit with care and the proper performing of the irrigation with the patient's mouth always kept open, the danger of infection may be avoided. It is mainly on this account that I prefer to let patients have some discomfort before I start irrigating too early, for in many cases nature will resist the infection unided better than with assistance improperly given. 'The liability to ear complications is in direct proportion to the severity of the involvement of the throat and nose, so that any treatment which moderates or relieves the infection in these localities lessens the danger of ear involvement.

Pain at the onset is best relieved by the application of heat. 'The external auditory canal should be syringed with alcohol, or water heated as hot as can be borne. 'The anditory canal is then dried out and heat, in the form of a hot salt bag, hot plate, or hot-water bottle (covered with a woolen cloth) is applied. A few drops of a 4 per cent. cocaine solution, placed in the ear with a dropper, will at times act like magic.

The canal should never be obstructed by plugging with cotton-wool; this, while frequently done, only increases the tension which we are trying to relieve. Pain, increasing in spite of these efforts, indicates that suppuration is to be expected, and we should at once try to locite it. There are now on the market convenient and portable electric appliances which render exposure of the tympanum easy. If the tympanum is dull and bulging, it should be incised with a bistoury, the blade being protecter with adhesive plaster, or by a tenotone. A quick short stroke will easily puncture the drum. Following the puncture there is consiclerable relief from the pain. In very young children this procedure may have to be performed under slight anesthesia, or it may be delayed, when spontaneous rupture will later appear. At times spontaneous rupture, with a copious diseharge of pus, will be the first evidence given of the involvement of the ears. 'Then careful cleansing and the avoidance of plugging with gauze or cotton-wool will be followed by sulssidence of the symptoms. Often the tympanum is ruptured, and heals without affecting the hearing; at other times, the destruction of the tympanum, followed br adhesions, will completely destroy the function of this organ. After paraeentesis it is very necessary to keep drainage established and the external canal clean and free of pus and alwars open.

'The best solution for general use is diluted grain alcohol, but if this is too harsh or drying, sterile water or 'Thiersch's solution may he substituted. A splendid wash is one of sterile water suds from Castile soap. to which are added a few drops of phenol. 'The solution should be warm and injected very gently, preferably with a soft-bulh ear siringe 
with a soft-rubber nozzle. It requires time and patience, but it is worth the effort to try, if possible, to save the hearing.

A sudden rise of temperature indicates oftentimes the stoppage of drainage or the extension of the inflammation to adjacent structures. A careful examination should be made for mastoid involvement, as indicated by pain on pressure or by swelling. When detected, there should be no delay in operating and the antrum of the mastoid should be either trephined or chiseled and drainage established.

Do not overlook examining both ears, as oftentimes both are equally involved.

The Joints.- We hear frequently of scarlatinal arthritis, and in certain epidemics many cases of tendinous synovitis, usually of the wrist and ankles, are observed. At times, in the convalescent stage of scarlatina, acute oedema with pain in many of the large muscles appears. Not much is known about these conditions. They have been called scarlatinal rhemmatism, though they are not true rheumatism, and are not much affected by antirheumatic treatment. The condition runs a course similar to ordinary rheumatism. The joint is hot and painful on movement. The trouble is, as a rule, self-limited, and rarely ever becomes subacute or chronic; it usually runs its course in about seven to fourteen days.

Treatment has so far failed to abort or limit this trouble. The pain can be eased by rest and protecting the joint from pressure, by wrapping it in cotton-wool, and applying well-fitting splints so as to completely immobilize the parts. 'This will, as a rule, be all that will be necessary. The coal-tar syntheties, if not too depressing to the heart, will to a certain extent relieve the pain.

In my experience the salicylates or antigout remedies have had absolutely no effect, and only upset the stomach without giving any relief. I have given up trying to use them, contenting myself with rest, immobilization, and sedatives, hoping that the trouble will run a short, mild course.

Rest should be absolute, the patient using a urinal and commode in bed in a reclining posture, and never permitted to get up. The part should be supported on a soft pillow and protected from all pressure. If the heat and pain become more severe, I use cooling lotions or salves and internally a saline flush is administered. Some have painted the parts with tincture of iodine, others have applied finely-powdered epsom salts, but $I$ have experienced no results from them. In the joints pus may form, and this is indicated by increased pain, chilly sensations, and rise of temperature, with general aggravation of former symptoms. Surgical measures should be at once instituted. If streptococci are detected in the pus, antistreptococcic serum may be administered. During convalescence passive motion, gentle exercise with massage, may preserve the function of the joint, otherwise mobility may be materially impaired. In all cases of scarlatina with synovitis or arthritic conditions carefully examine for cardiac complications, as they are very common in these conditions. Every precaution should be exercised to pre- 
vent them. It is wise, when these arthritic complications appear, to advise the parents of the likelihood of serious after-effects.

The Kidneys. - The kidneys are frequently attacked, even in the mildest cases, and oftentimes at a period when the patient is considered well. As advised before, it is a wise plan to confine to bed all patients ill of scarlatina until exfoliation is completed and all discharges have ceased, or this complication will continue to be more frequent than it should. Even in the mildest cases of scarlatina I invariably insist, from the very commencement, that the patient be confined to bed, and kept in bed until convalescence is well established.

The urine should be frequently examined-every day is none too often. At the bedside I always have an outfit, consisting of a test-tube, dropper, and nitric acid, and perform the Heller cold test as part of my routine work at each visit. 'The presence of albumin is thus quickly.detected, and measures may be early adopted to prevent further unpleasant conditions arising. If albumin is detected, I at once have a microscopic examination made, and have it repeated from time to time. At times-and happily such instances are rare-sudden and alarming symptoms develop, indicative of the occurrence of kidney involvement without having any prodromata at all.

It is a wise plan, when nephritis is suspected, or albumin is present in small amounts, to have entire amount of urine carefully measured for the twenty-four hours, starting with an empty bladder at a certain hour and stopping at a similar time twenty-four hours later.

When albumin appears in the urine, the patient should be kept absolutely quiet and positively in bed at all times. 'The room should be kept warm and the temperature equable. Variations, especially on damp and foggy days, are to be particularly avoided. The room should be well ventilated, but always with an idea toward preventing draughts. If it is warm and the weather changes suddenly, the room should be kept at its normal temperature by artificial heat, either gas or oil stoves being called into use. The diet should be limited to sweet milk, and water should be imbibed freely. Salines assist in eliminating irritating poisons and help the kidneys by taking their burdens away. 'The functions of the skin should be increased by bathing and friction (warm baths daily are absolutely necessary). Should the urine continue to be scanty and the constitutional symptoms not improve, more active treatment is demanded. Diuretics may be employed, such as theobromine with salicylic acid (diuretin), $5 \mathrm{gr}$. for an adult, every two or three hours, or the fresh infusion of digitalis, in tablespoonful doses, at the same interval, alone or in combination with acetate of potash. Calomel, $\frac{1}{10}$ gr. up to $1 \mathrm{gr}$., three times daily, in order to increase the activity of bowels, should be occasionally given. Hot flaxseed poultices may be applied over the kidney region, and these should be repeated every two hours at least.

A high-tension pulse, flushed face, with nausea and vomiting. calls for nitroglycerine to lower the tension. Hot-air baths also relieve the function of the kidneys by increasing the activity of the skin.

Should these measures fail, it indicates a very grave condition, and 
other measures should be at once tried. Pilocarpine, $\frac{1}{60}$ to $\frac{1}{15}$ of a grain, may be given hypodermatically. To a child five years old $\frac{1}{40}$ grain may safely be given. This drug is very depressing, and should always be preceded by stimulants. It increases the salivary flow, and this should be anticipated, for at times it is enormous and to some is very objectionable. Should there be coughing present, pilocarpine is contraindicated, as likely to cause pulmonary oedema. Should odema occur, it may be combatted with atropine, grain $\frac{1}{100}$, in combination with morphine, grain $\frac{1}{s}$ in adults, or smaller doses in proportion for younger patients. Convulsions are likely to develop, and should they be expected, chloral hydrate may be administered by mouth or by bowel. If persistent, a hypodermic of morphine may then be given, or they may be controlled by chloroform inhalations.

In desperate cases bleeding should be performed ( 4 to 6 ounces or more), to be followed by the subcutaneous injection of a pint or more of a 2 per cent. salt solution. High enemas of hot sterile salt solution should also be given. These may be repeated frequently. Alarming symptoms are likely to appear should effusions take place into any of the serous cavities. When this happens, these should be treated promptly by paracentesis.

In all cases of nephritis anemia to a greater or less degree will invariably follow, and for this condition iron in some form is indicated. Convalescence will be quickened by a change to a warmer climate. Every possible precaution should be taken to protect the patient against any exposure or sudden changes of temperature. The patient should be under observation for months after all symptoms have disappeared.

The Heart.- Scarlatina appears to have a special predisposition to attack the heart and its membranes. In mild cases this is oftentimes indicated by a weakened first sound and a rapid, small, and irregular pulse. Scarlatinal subjects require the use of stimulants, and we should not hesitate, even in children, to use them promptly. Brandy or whisky should be given in full doses when such depressing symptoms manifest themselves.

If the stomach will tolerate digitalis or strophanthus, either in combination or singly, we should give them, for oftentimes they will be of considerable assistance. Strychnine in small doses, $\frac{1}{200}$ of a grain, repeated hourly, may at times be used. Should the skin show that the cutaneous circulation is enfeebled, owing to abnormal tension, then nitroglycerine, $\frac{1}{300}$ of a grain by hypodermic, may be administered and repeated as indicated. Should collapse threaten, the subcutaneous injection of camphor in oil may be used to advantage. Endocarditis and pericarditis are likely to appear, especially in those cases in which scarlatinal rheumatism has appeared. Involvement of the joints in scarlatina should cause increased concern and make the physician extra cautious concerning his prognosis as to the heart.

At the first suspicion of cardiac involvement light-weight ice-bags should be applied to the precordium. The patient should at all times be kept in recumbent posture, as sudden dilatation may occur, with 
disastrous results, on the slightest exertion. All physical effort on the part of the patient should be interdieted, and the family should be warned in order to secure the proper precautions and cooperation. Salines should be used to flush the alimentary canal and help eliminate some of the irritating toxins. Should pericardial effusion oceur, the withdrawal of the exudate should be undertaken surgically before it crowds the heart to any extent.

The Gastro-intestinal Tract. - This tract is rarely affected to any extent in scarlatina. 'The early vomiting is rarely persistent, and disappears without inconveniencing the patient. 'The vomiting generally follows the ingestion of food or drink, and appears so suddenly that oftentimes observers are taken off their guard and think it due to what was recently taken. If the vomiting should be persistent, then withdrawal of food, and the partaking of cracked ice in small amounts, or at teaspoonful of milk of magnesia or a pineh of bicarbonate of soda in peppermint water will under ordinary conditions control it. The vomiting stops ordinarily when the stomach is emptied.

Diarrhoea is occasionally present, and unless it is severe and exhausting, it is best to let it run its course, as it is only eliminating some gastro-intestinal irritant. Small fractional doses of calomel, $\frac{1}{10}$ of a grain every fifteen minutes for five or six doses, will, as a rule, answer. Should it, however, be more severe and persistent and depress the patient's physical condition, then it may be controlled by vegetable astringents combined with some form of opium.

A favorite prescription of mine is as follows;

I). Tr. opii, Tr. kino, Tr. catechu, Acidi sulphurici aromatici,

Tr. zingiberis, Aqua dest.,

Sig.-One drachm every half-hour, diluted.

gtt. xlviij;

fวิiv;

fร̃iv;

f $5 \mathrm{vj}$;

f

q. s. ad f

In place of this, if more drastic action is needed, morphine may be administered.

Later in the course of scarlatina, should diarrhoa occur, then a saline should be administered, and if not checked when the irritant is flushed out, it may be controlled with the combination just given or by morphine administered either by mouth or by hypodermic injection. At times irrigation of the colon with a 2 per cent. sterile salt solution will be beneficial, and should be followed by some mild astringent and antiseptic.

The Hemorrhagic Type of Scarlatina.-'True purpura hemorrhagica, seen in the malignant types of scarlatina, is invariably fatal, and all treatment will in the end prove unavailing. We should meet symptomatically the indications as they arise, but the family should be warned of the inevitable result to be expected.

In some forms of scarlatina petechix appear on the dermal surface, occasionally on the mucous membranes, about the end of the second or third week of the disease. They indicate disintegration of the blood, Vol. I -42 
with changes in the tissues, and are of grave omen. The patient is usually very anemic, and the debility may be so severe as to result seriously at any time. These cases should be stimulated freely, and all nourishment possible, in concentrated form, should be administered. Iron in some form is usually indicated, and will be well borne. In the bleeding cases, turpentine, 5 to 7 drops, in an emulsion of acacia has been suggested, and at times is successful. It should be administered every two or three hours. It may be disguised with any aromatic elixir. Morphine is necessary if there is severe bleeding. It should be given hypodermatically in small doses, $\frac{1}{50}$ to $\frac{1}{8}$ of a grain, and repeated as indications warrant. Hot mustard baths or hot stupes will aid in relieving internal congestion and improve the terminal circulation.

Should pulmonary œdema threaten an adult, hypodermic injections of morphine, grain $\frac{1}{4}$, and atropine, grain $\frac{1}{1} \frac{1}{0}$, should be administered.

For acute anemia, the intravenous injection of a half pint or a pint of $\frac{1}{2}$ or 1 per cent. sterile salt solution should be given.

\section{MEASLES}

Prophylaxis. - When a case of measles has once developed in a family, and has not been recognized until the eruption is well out, all in that family have been more or less exposed. It is useless then to upset the routine of the house by strict measures to control the spread of the disease. All measures for control will, in all probability, be of but little avail: the damage has been done, and if any of the children escape, it may be said to be due more to an act of Providence than to any preventive measure adopted. To send children to other quarters is simply to invite the spread of the disease. The sick should be isolated on the top floor of the house. Should there be members of the family whom it seems desirable to protect from contagion at any time, these should be segregated on the lower floors of the house. Comparative safety from attack cannot be assured, as the length of exposure makes more certain the possibility of others contracting the disease. Complete isolation in the home is always more or less uncertain, unless all communication with the afflicted is absolutely prohibited. As a rule, if a child shows drooping, or has a slight "cold" in the head, much can be done, so far as school life is concerned, if it is at once segregated; but at home the possibility of preventing the spread of the disease to others susceptible to the contagion is very difficult.

In the absence of an epidemic of measles an early diagnosis is at times impossible, for the family or the physician are so taken off their guard that the mischief is done before precautions can be thought of, and we must then accept the situation as best we may.

The subject of nursing cases of measles is a study of itself, and calls for the exercise of the utmost care and patience. Careful nursing oftentimes is the deciding factor between success and failure in this disease.

Treatment.-In the treatment of measles, let me early and prominently call to the attention of my readers that the advice given concerning 
this disease by our forefathers, and more particularly by our greatgrandmothers, was that a patient ill with measles must be placed in a room almost black dark, enveloped in thick coverings, and steeped with hot drinks. 'This was done for the purpose of protecting the eyesight and "drawing out the rash." 'There is not a particle of doubt but that many a precious life has been sacrificed in this way.

The room should be pleasantly warm and well ventilated, and the child protected by suitable bed-clothing. Sudden changes are no doubt very detrimental, and the susceptibility of measles patients to respiratory complications has evidently led to the extreme caution.

A room should be selected, preferably on top floor, and one affording plenty of sunlight. If in a flat, which in New York is like a chimney laid flat and full of draughts at all times, an end room, preferably one opening on to the street, rather than one opening upon a court or shaft, should be chosen.

The room should be kept comfortable and well ventilated at all times. The air which the patient is to breathe should always be pure and uncontaminated. Owing to the irritation of the mucous membranes of the respiratory tract, lung complications are most frequent in patients supplied with deficient and improper air. A patient ill of measles, owing to the mucous membranes being more or less congested, needs fresh air even more than one suffering from any other acute disease accompanied by fever. The most suitable room temperature is one not under $63^{\circ} \mathrm{F}$. and not over $70^{\circ} \mathrm{F}$.

In changing the air, one should be particularly careful to avoid strong draughts, and, if the air is dry, to supply moisture by means of steam. Much care not to overheat the room is necessary when adding moisture to the air. Should the patient feel chilly, do not cut off the ventilation, but add more bed-clothing or warmer personal clothing.

In winter it is not necessary to keep the windows open day and night, but simply to provide a constant change of air. At night more air is required, owing to the lights consuming a considerable quantity of oxygen, and also because they give off irritating products. 'The nurse should bear this in mind. Should it be windy and stormy out-of-doors, a sudden change in the sick chamber should be anticipated by altering the openings.

Regarding the use of steam, one should not supersaturate the air with moisture, as this is as undesirable and uncomfortable for the patient as when the air is too dry. A good way to gauge the amount of moisture is to place a large mirror in the room, or, if there is an interior window, to note how quickly moisture gathers on its surface. When this occurs, the steam supply should be reduced. If the window frosts, there is not sufficient ventilation. 'The room should be as free as possible of furniture.

As before mentioned, while it is wrong to darken the room, the light should not glare into the patient's face. 'The bed can be so placed that the light is indirect, and if the eves are sensitive, colored shades or screens can be used, or the patient can wear dark-colored glasses. At 
night the room may be kept dimly lighted, the light being screened so that it does not shine into the eyes of the patient. Sunlight is a necessity of life and is imperative at all times, particularly in the infectious diseases.

'The arrangement of the patient in bed will depend on his condition. If there is severe throat involvement, or the uvula is elongated or catarrhal pharyngitis irritates and annoys the patient, it is more comfortable for the head to be more or less elevated. Many a distressing attack of coughing may be avoided by keeping the head raised. If there is a severe bronchitis present, the patient should be raised on pillows, at times almost to a sitting posture, this being the only posture which will relieve the respiratory system.

I deem it wise, even in ordinary cases, to insist always upon patients with measles being put to bed, and kept there as long as the temperature is above normal, and for several days after it has shown evidences of remaining so. Many unpleasant phenomena are avoided if this plan is studiously followed, and the patient of tentimes makes a much more sure and quick recovery. In the mild cases, or those commencing with mild phenomena, ordinarily too much latitude is given, and this indulgence is oftentimes paid for by very distressing complications. The length of time in bed will, of course, ordinarily depend upon the patient and his surroundings.

In the homes of the well-to-do and intelligent more liberality can be safely shown than in the homes of the poor. The poorer classes are particularly liable, owing to impaired and impoverished constitutions, to complications, and these can best be met by being anticipated by proper prophylaxis.

During the warm days of summer our rules may, to some extent, be appreciably relaxed. The condition of the lungs is by far the most important point to be considered in forming a conclusion in this regard. As long as any catarrhal symptoms persist, it is best to keep the patient quiet and at rest in bed.

The Diet.-Ordinarily, the diet in measles is the same as would be applied to any acute disease accompanied by fever and lung complications. Usually measles is of short duration, and all that is necessary is to sustain the patient with suitable nourishment until the disease has run its course. I believe it unnecessary to force nourishment on a patient whose appetite is impaired only temporarily. The appetite will return usually soon after the fever subsides to normal, and if the stomach and intestines are in good order, the patient's desires as to food, unless capricious, may safely be complied with.

Milk is the staple article of diet in this disease, being both food and drink, and capable of sustaining the strength indefinitely, provided it is given in sufficient amounts. It may be administered plain or diluted with cold or carbonated water. At times it is best to slightly salt the milk, as it makes it slightly more palatable and a little easier to digest, and adds a chemical needed at this time.

Stimulants are not ordinarily indicated. In children alcohol, in the 
form of a strong sweet wine, may be administered, and will oftentimes be cheerfully taken. In older patients sherry may be given, but whisky diluted with either milk or water, either cold, in form of "high ball," or hot, in form of a "toddy," is better.

If the disease has been complicated by bronchopneumonia, complete recovery cannot be expected for weeks, even in favorable cases. 'The general nutrition will necessarily suffer considerably, and the patient under these circumstances should be given as much suitable nourishment as is possible from the beginning. Fluids (soups, broths, milk, etc.) alone should be given, and these in small amounts, frequently repeated. 'The extreme dyspnoa precludes mastication, and at times it is extremely difficult for the patient to even swallow, owing to the frequent attacks of violent coughing. Rectal feeding may be tried.

The Fever.-At times this may be so slight as to require little or no consideration, and is usually of short duration. If the fever is excessive and accompanied by nervous phenomena, we may safely reduce it by hydropathic measures. (See article on Hydrotherapy.)

The indications for reduction of the temperature depend more on the patient's general condition than on the fever itself, and for this reason I do not give a general order for a bath if the fever reaches such a point. Special indications for reduction of the temperature, in my opinion, are a temperature that shoots up suddenly, and is accompanied by cerebral symptoms, such as delirium, convulsions, ete. A short cold douche, the water not warmer than $60^{\circ} \mathrm{F}$., and lasting about one-half and not over two minutes, applying the water to the face, neck, and head, is often efficient.

Should the symptoms not improve, and the fever remain about the same or even go higher, then a more active bath may be given.

If a very young child is to be given a bath, it is best to use a graduated bath, owing to the irritable nervous system. Start with water a little over the temperature of the patient, and cool it down quickly, not occupying over five minutes. 'The bath should be followerl by vigorous friction of the skin and the patient covered with hot cloths until warm and dry. 'The bath, thus cautiously given, may be repeated as often as the nervous symptoms indicate. For older children, the bath may be started at a lower temperature, and may be somewhat more prolonged, but the other conditions should be followed closely.

It is well to bear in mind that in the use of a bath for heat abstraction it is specially depressing to the cardiac system, and this should always be anticipated by the slightest suggestion of weakness, as evidenced by the increased frequency of the pulse.

'The temperature should be taken by rectum every quarter of an hour, several times after the bath.

In malignant cases of measles hydrotherapy should be our mainstay for the control of cerebral symptoms and the temperature. Those who hesitate or falter about the use of the bath are more than likely to suffer the loss of their patient.

During convalescence, should eatarrhal symptoms give evidences of 
" hanging on," a warm bath, once daily, followed by a short cold douche, splashing with a glass of cold water on chest, or poured down the spines of vertebræ, or quick friction of the chest with a small piece of ice, will very materially assist in relieving this condition. After the cold shock the skin should be vigorously rubbed until the glow returns.

While upon the subject of hydrotherapy in measles it might be well at this time to call attention to the fact that frequent bathing is of service, not only for the reduction of high fever and control of unpleasant nervous symptoms, but also for other conditions. The skin of the convalescent patient should be cared for with warm baths, of short duration, followed by vigorous friction of the skin. While rubbing, use some oil or grease, like mentholated oil, carbolated oil, or plain cocoa-butter. If the bath is followed by a short cold douche, the liability of the patient taking cold is reduced to a minimum, and is oftentimes avoided by such treatment. Exfoliation can be very materially hastened by frequent bathing, and if followed by carbolized oil, lessens the danger of the disease being transmitted to others.

Convalescents usually enjoy warm baths as well as the mild short douching, and the advantages of a careful toilet are at all times most desirable.

It is my almost invariable plan to order at least one bath daily, part by part being bathed, followed by a short cold douche, and to use the same up to the time of complete convalescence, when the patient is able to be up and about the house. The short cold douche is also indicated in bronchopneumonia (old-fashioned capillary bronchitis), even if attended with atelectasis. The method to pursue is that which will cause the lungs to expand to the greatest degree possible. In very mild cases of measles the baths, aside from reasons of cleanliness, are not necessary, but if used, it will be found that they give comfort, and when given in the evening, aid in securing refreshing slumber. At these times the temperature of the bath should be only a few degrees below the temperature of the patient, and should be of short duration-not over ten minutes.

If the heart is good, and there is no tendency toward cyanosis, a small dose of phenacetine, 2 or 3 gr., may be given in combination with caffeine.

If there is cardiac depression, then stimulation is imperative; for a small child, strychnine, $\frac{1}{200}$ of a grain, repeated every hour. For older patients larger doses may be given. If the syncope becomes more acute, then a hypodermic injection of camphor in oil may be administered, and later sustained with caffeine or strophanthus.

Stimulants, in form of a light wine, sweet Tokay, or strychnine, should be administered just before and just after a bath. In children it has been recommended that 5 to 10 drams of wine should be given just before the bath and again immediately after the same. In older patients hot strong coffee is sometimes an admirable remedy and very satisfying.

Affections of the Pharynx and Larynx.-These two organs are attacked more or less severely in nearly every case of measles, and should receive special consideration at all times. 
The catarrhal inflammation of the nasal mucous membranes oftentimes seriously interferes with respirations and necessitates mouth breathing, whereby the mucous membrane of the mouth and pharynx quickly becomes the seat of inflammatory processes incident to the drying and cracking, offering thereby a most favorable opportunity for the implantation and entrance of various infectious bacteria. 'The nostrils must be frequently cleansed, and kept open as much as is possible in order to assist free breathing.

On the appearance of hoarseness of the voice the patient should have the head elevated somewhat. In very young patients only warm milk should be given, inasmuch as it is most difficult to care properly for their throats.

In older persons, who are able to permit of gargling or irrigation, it is wise to cleanse the nasal and throat passages from time to time by this means. 'The patient should avoid, if possible, breathing through the mouth, and this will at times severely tax the ingenuity of both nurse and physician. Should the nares become dry and obstructed, they may be frequently cleansed by swabbing with oil-saturated applicators or sprays of oil (albolene or a direct application of petroleum jelly).

The percentage of moisture in the atmosphere of the sick chamber at this time must be increased, and it will be found very comforting if a tent made of sheeting is erected over the bed, and the air saturated with a spray of medicated steam. This should be continued both day and night. At times a wet cloth, or a single thickness of cheese-cloth, placed over the lips, will give some comfort and assist in keeping the parts moist.

If the throat is very irritable, a wet cloth applied about the neck and covered with a dry towel will materially relieve at times. This wet cloth should be wrung out in cold water, $60^{\circ} \mathrm{F}$., and changed about every third or fourth hour. At times the cold water is not well received by the patient; then it is best to try water as hot as can be borne and renew it frequently.

Pharyngeal irritability, as indicated by an almost constant cough, can sometimes be allayed by the application of a very weak solution of cocaine, applying it to the pharynx by gentle swabbing or in the form of a short copious spray. A very mild solution, just sufficient to control the irritation, should be used; if not effective, it should be discontinued, as the anesthetized throat is at times very uncomfortable to the patient. Opiates will be found objectionable, though at times, especially in older patients, they will have to be resorted to as the choice of two evils.

Laryngeal spasm may at times be relieved with Dover's powder in doses of 1 or 2 grains, frequently administered, until an appreciable effect is noticeable. If after at least three doses no effect is produced, then it is wise not to delay, but to at once administer diphtheria antitoxin5000 units - and repeat in six to eight hours, depending on amount of obstruction.

A culture from the laryngeal surface should be immediately taken to confirm the diagnosis of complicating diphtheria.

Frequent experience with these cases, particularly in municipal 
work, has led me to believe that the croup of measles is in a large majority of cases a diphtheritic complication, and should be promptly treated with large doses of antitoxin, repeated until effective.

While waiting for the Dover's powder to take effect, it is wise to hasten relieving measures by trying a hot bath, during which vigorous friction of the skin is made; the bath should last about a quarter of an hour to be at all effective, and should be as hot as can be well borne.

Should symptoms of cerebral congestion appear, as would be indicated by twitching or convulsions, then, in addition to the prolonged hot bath, apply ice or cold to the head.

If the stenosis is due simply to nervous spasm, it will disappear as the result of the hot bath, and, unless the symptoms point to danger of suffocation, the hot bath should be tried before other measures are instituted.

In very young children an emetic may be tried, either apomorphine by hypodermic, or tartar emetic by mouth. Do not give too small a dose, for one that does not act within a few minutes is apt to upset the stomach.

Bronchitis.-In measles there is a marked tendency toward congestion of the respiratory tract, and more or less cough is constantly present. As long as the inflammation is limited to the large bronchi, no special treatment will be necessary. When there is present considerable hard cough, and the sputa is scanty and tenacious, I use apomorphine, fresh, in suitable-sized doses, varying with the age of the case, the smallest doses being frequently repeated until the effect is evident. A mixture I employ is made of apomorphine, dilute hydrochloric acid, and licorice syrup in water. A constant irritating cough in an adult may be controlled by the use of morphine, but in children heroin in suitable dosage is preferable.

'The essential point in the treatment of the bronchitis is to stimulate the patient and increase the expulsive power of the cough, and at the same time allay irritability. Douches, as a rule, can accomplish more if commenced early, and are then much more effectual than drugs.

If coarse mucous râles are heard over a large area of the lungs, these can oftentimes be removed by an emetic. 'This can also be very materially assisted by douching, either immediately before or just after the administration of the emetic. In very neurotic cases, if the douche cannot be given for any particular reason, a limited cold wet-pack may be used. This is applied by means of a wet 'Turkish towel, folded and placed on chest and abdomen, the other parts of the body being kept warm. A hot-water bottle at the feet will prevent the distal parts from becoming chilled.

A very wise precaution is to see that the wet compress does not to any extent constrict the chest and interfere with respiration. If the pack is not well borne on the front part of the body, then it may be well to apply the wet-pack over spines of the vertebræ, with a dry warm blanket over the front part of chest. In using water about the bedside in measles be more than careful not to get the bed wet at any time, and if st:ch should happen, see that it is dried at once by being ironed with a 
hot flat-iron. Frequent changing of sheets, unless previously warmed, is likely to chill the patient too much. Should it be desired to make a considerable reduction of the body heat by this method, it can be made eflective by frequent changing of the wet-pack and soaking in graduated cold water.

General bronchitis will oftentimes be present, and is a most irritating complication, and causes increased frequency of respirations, with more or less superficial inspirations, oftentimes in proportion to the height of the temperature. In such cases the bath is of the highest value, and should be continued as long as the fever is high. If the respirations become rapid, out of proportion to the height of the fever, lung complications are evidently starting, and should be carefully sought for, and if discovered, can be better remedied by cold douching than by any other plan of treatment.

The marked tendency to recurrences of bronchitis which will be manifested by the patient sometimes for years after a severe attack of measles should be borne in mind. All suitable precautions should be taken, and oftentimes the necessity of sending these patients to different localities on a visit of more or less extended duration can be avoided by the careful use of bathing and particularly douching with cold water. Of course, understand by the above that while I consider a change desirable in that it always benefits a convalescent from any acute disease, I am speaking here of the necessity for such an action. It has always been recognized that an attack of measles, especially the severe forms, is apt to be followed by tuberculosis. It is apparent, without comment, that this should be prominent in our minds, and these patients induced to take such precautions as will reduce such a possibility to a minimum. 'The relationship is indeed intimate, and, on investigation, it is striking how many cases of severe measles are followed by tuberculosis.

Bronchopneumonia.-The old-fashioned capillary bronchitis of measles has in later-day investigations been made to give place to the more proper condition mentioned above. It is a frequent complication of measles, especially in the very young, and is more to be feared than any of the other complications. 'The large mortalities of the past have, in my opinion, been due to the methods of treatment more than to the severity of the complication itself.

With proper treatment, bronchopneumonia can many times be avoided, and the early care of the breathing and the proper methods used to secure sufficient and uncontaminated air will accomplish this, as a rule.

The sweating process, in darkened chambers, of our ancestors was, to my mind, the cause of this disease more often than can possibly be reckoned. I appreciate the fact that this complication is no doubt due to the lung being affected by special organisms, but this is rendered possible, to a great extent, by reducing the patient's vitality through insuflicient fresh air. Prophylactic measures, such as have been frequently mentioned in this article, will in the vast majority of cases, if instituted early, almost certainly prevent this complication. 
Hutinel has written an admirable account of this condition. "Children having bronchopneumonia should be isolated by themselves. They should not be near other children having simple measles. Not only developed cases of bronchopneumonia, but cases that show bronchitis, or those that have been exposed to the contagion, may be considered as threatened with bronchopneunionia."

Children in hospitals should be kept in small isolated groups; those in full eruption being together; those with bronchitis separated, and lastly, those with bronchopneumonia should be isolated together, separate and apart from the others.

"Measles patients should never be densely crowded, but always kept in well-aired ample quarters. The patient should be kept in a condition of extreme cleanliness. The patient's skin should always be in a most satisfactory condition; if excoriated, eczematous, and otherwise soiled or unclean, he should be given antiseptic baths, and kept thoroughly clean at all times. Sores, crusts, ulcers, impetiginous patches, should be antiseptically dressed with surgical minuteness.

"There are normally micro-organisms in the mouth, the pharynx, and nasal fossæ which are capable of becoming virulent and provoking bronchopneumonia. One should take care of the mucous membranes covering these regions not less carefully than the skin of the patient. Nasal lavage is more often harmful than useful. It is liable to irritate the delicate epithelium and very often leads to otitis media. Applications of boric acid in petroleum jelly or weak solutions in tepid water may be gently applied by swabbing."

To which my experience leads me to add the following: Children in hospitals, especially in the large cities, do not do well when they contract measles. These children are considerably below par, and, contracting measles, they quickly develop complications which are a menace to others.

Children contracting measles do not do well in hospitals, and when it is possible, such cases should, by preference, be treated in their own homes.

Children convalescent from measles are more than ordinarily susceptible to other infections.

The Heart.- This organ is rarely affected by the toxin of measles. It is sometimes influenced by the hydrotherapy, or there may be cardiac depression, owing to incidental complications, such as bronchitis or pneumonia. The strength of the heart is severely taxed in the latter conditions, particularly when they are severe, and in such cases special stimulation is necessary. Wine, coffee, tea, or whisky may be used for general stimulation. Camphor in oil, hypodermatically, should be used when a quick, speedy effect is desired. Later, strophanthus, either alone or in combination with digitalis, is useful.

The management of the heart in measles, therefore, resolves itself into careful observation of the patient, anticipating weakness, and taking every precaution that will prevent a waste of the patient's strength. 
The Gastro-intestinal Tract.- This tract appears to suffer particularly in measles, especially the upper part. 'The condition of the mucous membranes should receive early and constant attention.

Aphthous inflammation of the buccal mucous membrane is of frequent occurrence, and is most of ten due to neglect of the care of the mouth. 'The mouth should be cleansed, particularly after the taking of food.

The lower the vitality, the less the power of resistance displayed by the patient, and complications are thus most likely to arise. 'These conditions should be anticipated, and every endeavor made to improve the patient's general condition and sustain strength with proper nutrition. 'The mucous membranes of the mouth, pharynx, and nose should be kept in as good condition as possible in order to prevent further microbic development.

Ulcerative stomatitis, should it develop in spite of careful attention, or if it has developed before the case has been seen, will have to be met with mild antisepties, irrigations of a saturated solution of chlorate of potash, or a weak solution of biborate of soda or permanganate of potash; later peroxide of hydrogen may be used, dilute or pure, followed by warm salt solution. If the condition does not improve, painting the affected area with a solution of nitrate of silver, 10 grains to the ounce, once daily will be of material assistance. Should noma appear, it will have to be dealt with surgically, and, in addition, every attention given to improving the patient's general vitality. 'T'onics may be given, such as compound tincture of cinchona with tincture of nux vomica, followed by iron in some form, preferably the syrup of the iodide of iron, together with milk, cream, raw eggs, whisky, nutritious soups, broths, etc.

Severe gastric disturbances in measles do not differ from the same conditions met under other circumstances, and should be treated similarly.

The diarrhœa so often met with in measles is, as a rule, an effort to remove injurious material from the bowel, and, in my opinion, should not be interfered with unless it becomes too exhausting. An improvement is oftentimes observed in the patient's entire general condition, coincident with the frequent diarrhcal passages, and this improvement is especially noticeable in the severe cases of measies.

Purgative drugs fail to exert such a beneficial effect. 'This has been tested time and again, so as to make it superfluous for the physician to experiment further along this line at the expense of the patient's strength.

If the diarrhœa appears when the measles is at its height, and continues to persist, then treatment should be instituted for its relief, and this should not be delayed too long, as intestinal derangement may appear which is not directly related to the measles process. Under these circumstances the complication may be treated just as if it had appeared independently.

The treatment of the diarrhora of measles should first consist of a gentle irrigation of the lower bowel with a 2 per cent. salt solution, at 
about body temperature. The irrigation should be done immediately after each evacuation, and may be repeated about three or four times in the twenty-four hours without any injury to the patient.

A solution of gum acacia, 1 to 200 , may also be used, which serves as a protector of the mucous surface. The acacia is more efficient if it is preceded by a saline irrigation of from 1 to 2 pints. The injection should be made very slowly and at very low pressure. About $\frac{1}{2}$ or 1 pint of the acacia solution is usually injected about one-half hour after the irrigation.

Should the diarrhœa not yield to these measures, vegetable astringents may be used.

I prefer the following:

R. Tincture of kino, Tincture catechu, Aromatic sulphuric acid, Tincture opium, Elix. auranti, Aq. cinnamomi vel, aq menth. pip., q. s. ad f $3 j$.-M. Sig.-Give every half-hour or of tener.

The dosage should be adapted to the age of the patient.

If there is much gas or colic with the diarrhœa, then a mixture of Hoffman's anodyne, chloroform, and camphorated tincture of opium, equal parts, may be used in doses suitable to the age of the patient. To the acacia irrigation turpentine may be added when gas is present.

The main indication is to prevent the loss of nutritious fluids by too frequent intestinal evacuations, and to decrease peristalsis; this, as a rule, can only be done by the use of sedatives. In very young children, tincture of opium in acacia may be injected very slowly into the bowel under low pressure, or if this is impractical, opium suppositories may be used.

Warm wet compresses to the abdomen are at times very useful, and occasionally hot turpentine stupes may be necessary. A suitable diet must be prescribed, and in addition small doses of brandy well diluted. The old-fashioned home-made blackberry brandy, which is known to have a very strong astringent as well as stimulating action, should be used if obtainable.

Severe secondary intestinal disorders demand appropriate treatment, such as is ordinarily used without reference to the measles infection, as they usually develop after the measles has run its course.

The Skin.-The eruption in measles is in the nature of an inflammatory process-a dermatitis, as it were. The congestion and œedema followed by exfoliation of the superficial cells in flakes, are occasionally accompanied by a burning sensation, at which times cooling lotions may be applied.

'The face in the early stages, particularly in adults, is severely affected, and is occasionally much distorted. At times there is considerable pruritus, for which phenol lotions are indicated or carbolized oil-about 2 per cent. If the surface is much involved, a modified calamine lotion is very acceptable, such as the following: 
P. Phenolis,

Pulv. zinei oxidi,

Pulv، amyli,

Pulv, ealamina

Glycerini,

Aq. calcis,

gtt. $x x-x l$;

ăa $\mathbf{3}$ ij;

f כ̄ij;

Sig.- Mop on with a moistened cloth or apply wet cloths held in place by bandaging - never rub with the lotion.

'The face usually gives the most annoyance; a mask may be made and kept wet with the above solution.

During the exfoliating period a bath should be given daily and followed by friction of the entire body with warm 2 per cent. carbolized oil. 'To hasten the desquamation rubbing with mittens made of 'lurkish toweling is oftentimes very effective.

'The rubbing should take place immediately after a prolonged warm bath, and this should be followed by the oiling.

The Kidneys.-In measles the urine should be regularly examined for albumin, especially if the rash is intense and there is considerable hyperpyrexia. At the first appearance of kidney involvement the usual treatment for nephritis should be instituted.

Eye Complications. - These are at times frequent in measles, and aside from controlling blennorrhagia, it is better for the general practitioner to consult a specialist. For the photophobia, shaded glasses, either of dark or amber tint, should be constantly worn in the day-time, and at night special regard must be given to protect the eyes from the direct rays of the artificial light.

Also the eyes should be bathed from time to time with a dilute boric acid solution, preferably cold.

For the mild catarrhal symptoms of the eyes, in addition to the above cleansing, a mild astringent may be used from time to time. A drop of alcohol in an ounce of water, or 2 or 3 drops of extract of witchhazel, or 1 drop of a solution of zinc sulphate of the strength of 1 grain to 1 ounce may be instilled once daily.

\section{GERMAN MEASLES (RUBELLA)}

German measles, or, as I prefer to call this discase, rubella, provokes discussion, more on account of the diagnosis than any necessity for treatment, for in truth very little treatment is needed in this disease.

I have observed many hundreds of cases of rubella in the past nineteen years, and I have yet to see a severe type of this malady. Symptomatic treatment must, however, at times be considered. If there are indications of nervousness, constipation, intestinal indigestion, or other condition, one should act accordingly.

'The disease is self-limited, and, as a rule, will quickly disappear without any interference. A mild saline laxative never does harm. I personally use $\frac{1}{2}$ ounce of a solution of citrate of magnesia, diluted, every two hours until effective.

If there is restlessness or irritability, tincture of aconite, $\frac{1}{5}$ of a drop every fifteen minutes, for five or six doses, may be given; the dose may be repeated from time to time as appears necessary to quiet the patient. 
Because of this disease affecting the mucosa I do not approve of the use of irritating drugs, as, for instance, oleum ricini, so frequently used as a household remedy.

The child should be secluded and fed on a light diet (milk, gruels, etc.). Within a very few days the disease will have spent itself, and in a week it will be safe to permit the child to go out.

Municipal departments do not consider it necessary to disinfect for this disease, but at times overcautious families insist upon this being done, and here it is wise not to oppose their desires.

\section{VARICELLA (CHICKEN-POX)}

Varicella or, as it is popularly called, chicken-pox is, as a rule, so mild as to run its course without needing any particular attention from the therapeutist. In most cases it may be disregarded.

Children frequently have varicella and recover from it without the slightest inconvenience; oftentimes, were not more severe cases present, one would doubt the accuracy of the diagnosis. I have seen, in families where several children had the disease in a well-recognized form, other members of the same fanily become attacked, and have not more than five to ten small spots, with not the slightest constitutional evidence of anything being the matter with them. On the other hand, there are at times cases of a severe type, which, while giving much concern from a diagnostic point of view (because resembling variola), almost invariably tend toward recovery. In many thousands of cases, covering an experience of nineteen years, I have never seen a case of death from varicella.

Isolation.--Exclusion from school should be required, but in other respects the isolation of a patient with varicella need not be as complete as is required in other infectious diseases. The child should not be taken into street-cars or stores or to places of amusement where people, especially children, congregate. If the child feels comfortable and is well enough to be up and about, it should be permitted to get all the fresh air possible and to play at large, but away from other children who have not had this disease.

Adults are as susceptible to infection as children, and the contracting of this disease is at times most awkward and inconvenient.

Varicella, as a rule, is such a mild disease that the question may be asked, in all seriousness, if it is not best to permit other children to contract it by being exposed, so as to have it while young. I always tell parents that in any disease there is some uncertainty and some risk, be it ever so slight, but if it is their wish to permit exposure of the well children, they may do so without my consent.

When children are weakly or are convalescent from some other malady, they should be most positively protected against contracting any other disease, however mild.

When isolation is necessary, for any well-recognized reason, it should be continued until all scabs or crusts are detached and the skin has returned to its normal condition, except for the localized discoloration. 
I purposely use the term "all," for I consider any crust-primary, secondary, or even tertiary-able to carry the infection. Never consider a child well and fully recovered until it is free from a shadow of a possibility of conveying infection. In some cases of varicella it is wise to frequently examine the patient's urine for any evidences of nephritis; if present, the nephritis should be treated according to the usual principles. In the severe forms of varicella, and those where pus-formation is general, this complication is a rather frequent occurrence and of tentimes is overlooked.

Local and Constitutional Measures.-Inasmuch as the latter are of but little concern in the vast majority of cases, we will consider them first.

In the invasion stage the symptoms are, as a rule, very slight and insignificant and require no internal medication.

The febrile disturbance may at times demand attention, and here a mild saline laxative, like citrate of magnesia, followed by a cool sponge-bath, is all-sufficient.

The patient should be put to bed and kept there as long as any fever persists.

The diet should consist of milk or soups, and the patient should be encouraged to drink water in small amounts at frequent intervals.

In the later course of the disease, if there are severe constitutional symptoms, they will have to be met symptomatically.

'The same may be said regarding any of the complications which are likely to occur.

Conjunctivitis is the most frequently observed complication, and here darkened quarters, the use of an antiseptic wash, such as Thiersch's solution, or 25 per cent. boric-acid solution in sterile water, with cold cloths to eyelids, generally answer the purpose.

Local treatment is, in all surface inflammations, very necessary, and particularly so in this disease, as it opens the way for outside infections. Absolute cleanliness is the key to the situation. The itching, which is usually present, offers opportunities for infection through rupture of the tiny vesicles with soiled hands and nails.

Itehing of the skin is at times very intense. In this condition cooling lotions are indicated; they should be mopped on, as any friction of the surface is likely to rupture the vesicles. Witchhazel extract, eau de cologne, or spirits of camphor have rendered me good service. Menthol, 10 per cent., in albolene or olive oil, will be found very comforting, by reason of its cooling the surface of the skin.

The calamine lotion of the dermatologists also has been very serviceable and frequently relieves this distressing condition:

R. Pulv. calamin,

Pulv, zinci oxidi, Glycerini,

Aq. calcis,

Aq. rosa, gr. $\mathbf{x x}-5 \mathrm{j}$;

วิss-j;

f.5s:

fวัiv;

q. s. ad f 3 vj. - M.

Sig.-Apply localiy. Soak a picce of cotton-wool or an old piece of linen. and apply. If the surface is too large, apply locally by mopping with a cloth wet with the solution. 
'I'o the calamine lotion may be added, with very good results, $\frac{1}{2}$ or 1 dram of pure phenol.

At times the irritation may be so persistent as to necessitate sedatives, such as one of the bromides. In children, the U. S. P. elixir bromide of potassium may be used with very satisfactory results.

I would suggest the avoidance of opium in any form for dermal irritations, as it usually produces a worse condition than the one it was meant to relieve.

Should the above suggestions not meet the indications, then the following may be tried:

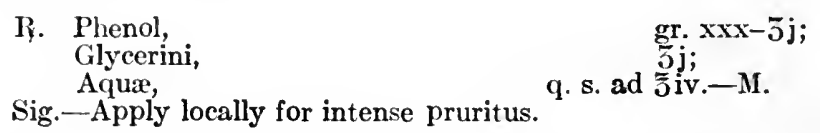

Let me again insist that perfect cleanliness is of the first importance. Neglect of this turns a simple, insignificant malady into one of grave import, and scars which last a life-time may result, to say nothing of the occasional loss of eyesight, or disfigured beauty.

Should the vesicles change to pustules, as sometimes occurs, they should be opened with a clean flat needle and the contents evacuated. Lesions of this character on the face demand particular consideration. Ordinarily, in these cases, after evacuation I order peroxide of hydrogen, dropped into the opening, and after it has effervesced, wash with alcohol, and then apply white precipitate ointment by means of a clean blade or spatula, never with the hand, for at best it is next to impossible to sterilize some attendants' hands.

In place of the white precipitate the following may be used:

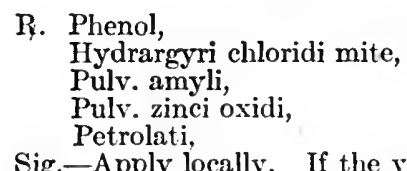

Sig.-Apply locally. If the vesicle dries up quickly after evacuation, it should be sealed up by means of flexible collodion.

In some cases of varicella the skin becomes hyperesthetic and very sensitive, and most lotions or salves used are likely to cause additional irritation. In such cases it is preferable to use Lassar's paste:
R. Pulv, zinci oxidi, Pulv. amyli, Petrolati,
Sig.-Apply locally.

$$
\text { q. s. adā } \underset{\text { วิ }}{\mathrm{ij}} \mathrm{iv} ; \text {-M. }
$$

To this there may be added salicylic acid as an additional antiseptic. Scratching, especially with long, dirty nails, is very likely to break the vesicle and cause infection. In such cases the nails should be cut closely, and, in addition to the application of soothing lotions, salves mav be used. If these measures do not relieve the condition, then it will be necessary to tie soft mittens on the hands, or to apply splints 
to the elbows to prevent the child from reaching its face. 'There are on the market celluloid sleeves, which I lave used frequently in eczemas of the face in small children. I would recommend them, as but few parents will tolerate the tying of a cliild's lands to the sides of a crib, however necessary it may be.

It is frequently observed that after hearty meals, or the ingestion of rather hot fluids, the itching is very much aggravated. Food likely to cause intensified itching, especially fruits and berries, should be avoided.

Gangrene of the skin has been observed in varicella, but, in my experience, it has generally been due to secondary infection in very unfavorable subjects.

Some lesions of varicella will, in spite of our best endeavors, leave permanent indelible scars. 'This may be due to an early necrosis involving the papillary layer of the derma, and under these circumstances pitting cannot be prevented. In the large majority of cases, however, the scars observed have been due to ulceration, the direct result of secondary pyogenic infection of the varicella lesion, and the exercise of proper precautions would have prevented the pitting.

I would urge that in the more severe forms of varicella, and in those cases with lesions that are deeply situated in the derma, to be as nearly aseptic as is possible; every attention should be given to the proper care of the skin in order to prevent infection. 'The hands of the patient should be kept clean. Frequent bathing has a very decided influence for good in hastening desiccation and in preventing pus-formation. 'The bedding should be kept clean, and the child should not be overheated with an excess of heavy bedding. A freely ventilated room is essential.

\section{VARIOLA}

General Treatment of Variola.-Ordinarily in variola the treatment is purely dietetic and symptomatic, varying with the case, whether mild or severe.

'There is no therapeutic method or agent which is a specific in any stage of the disease. Numerous attempts have at times been made, and some have apparently for a time appeared successful, but, in the light of experience, all measures for checking or aborting variola have invariably failed; that is, excepting recent successful vaccination. I have tried numerous methods, with the coöperation and assistance of many expert sanitarians, and I am sorry to state that we are still trying.

'The nearest to success so far has been with autogenous vaccines and serum therapy. Blue light, red light, prolonged warm-water baths, saline, carbonated, and other medicated baths, have all been tried, and considerable money has been spent experimentally for this purpose, but to-day we are just where we were when we commencel close personal supervision, and meeting symptomatic conditions with the therapeutics of our fathers still seems to be the proper and only method.

Antisepsis has, no doubt, made a great change in the treatment, for no disease has to be guarded more against sepsis than does vari-

Vol. I -43 
ola. Sepsis is the great Evil Spirit. Prevent this, and variola is a simple condition to care for. Small-pox, like scarlatina and some other diseases, renders the human subject especially susceptible to any infection.

Aside from the hopes we have in serum treatment, which is yet in its embryonic state in variola, I do not believe that there is a specific method of preventing, checking, or controlling variola that will stand the test of an open, fair trial.

Prompt and efficient vaccination, if exposure is known, may cut short or possibly abort variola, and, under these conditions, it is not only the proper, but about the only, thing we can do. Vaccination will take in about three to five days, and renders a certain amount of immunity, whereas variola takes ordinarily about fourteen days to incubate. Persons exposed and not vaccinated successfully within two years should be immediately vaccinated. If the vaccination does not take, it should be immediately reperformed. Vaccination after known exposure should be obligatory, and should be enforced or the person removed to a proper isolating place, and held there until all possibility of contracting the disease is past-usually a period of at least twenty-one days.

The longer the exposure, and the closer the contact, the graver the danger, for variola is not only contagious extremely early, but the longer it has existed, the graver the danger. If the patient is shedding, he is likely to leave scales, scabs, or crusts about, which are very dangerous, and the utmost care should be exercised to destroy them.

Ventilation.-One of the first essentials to successful treatment in variola is proper and sufficient ventilation at all times. Owing to the musty, disagreeable odor constantly present, due to the decomposing pus, a change of air is very necessary, or the ward soon becomes so stifling that it is not only disagreeable for those coming into it, but for all the occupants as well.

Variolous patients should never be crowded in a ward, and more than the usual allowance per capita of cubic feet of air space should be furnished. I deem it absolutely necessary not to have less than 2000 cubic feet for each case-man, woman, or child.

Ventilation should be obtained by artificial means, as a rule. It is not safe to use windows, for obvious reasons-wilful patients may escape, besides making irregular and dangerous drafts.

If the air in the ward is not repeatedly changed, it soon becomes foul, and is a positive menace to others, in that it carries infection from one to the other, and mild cases may change quickly for the worse, owing to complications. In dense, congested wards suppuration is more frequent, and this is the great danger in these cases.

The Temperature of the Room.-A very necessary consideration should be given to ventilation, as most wards are overheated, and thus are a hindrance toward success. The temperature best borne by bed patients is about $63^{\circ} \mathrm{F}$. 'Those convalescent and up and about the ward will require about $70^{\circ} \mathrm{F}$. In children's wards it should never at any time be over $65^{\circ} \mathrm{F}$. 
Variations are dangerous, and the temperature should be carefully observed and kept equable. A good attendant will look out for this, but ordinarily such are rareties.

Variations of temperature are very likely to cause bronchitis and pneumonia.

The Diet. - The diet in variola varies with the character and intensity of the disease and with the stage of development. In the mild cases no change from the normal is necessary, and this can be given with safety. Owing to the patient being more or less forced into confinement, he will, as a rule, be hard to plase, and his appetite will be capricious. On this account a frequent change, with a pleasing, well-cooked diet is absolutely necessary.

It is a peculiarity of this class of cases that, no matter how hard one will try to please, owing to the personal caprices it is indeed difficult to satisfy them. Patients that tolerate the roughest and most irregular feeding in their daily lives will complain about food in isolation hospitals, apparently simply because there is nothing else to do. And once the discontent arises, it is a very difficult matter to break up the grumbling. Personal attention and the frequent appearance of the superintending officer at the time of serving the meals will help a lot toward controlling this condition.

If, in the onset, there is considerable fever and general malaise, the patient should be put to bed on a restricted diet. Milk freely given is the best article of food. Cool water should be given liberally at all times, and a pitcher of ice-water with a glass should be on each patient's serving table. 'This mark of respect to his comfort often catches the eye on admission, and it is also important in relieving the murse of considerable work. Give water freely and at all times. It is of very material assistance. If plain water is unpalatable, substitute lemonate or carbonated waters.

Cracked ice or, better, shaved ice, is very cooling to the inflamed mucous membranes of the throat. Patients often will take this when they will refuse milk and water.

With hyperpyrexia there is always more or less intense thirst. 'The free use of liquids will assuage the thirst, and oftentimes the relief of this will be reflected in the nervous system. Children are no exceptions, and should be given as much consideration, if not more, in this regard than adults, who can and do make their desires known.

Should the child drink too much or too fast and the stomach rejects it, use shaved ice, and when the gastric organ is less sensitive, return to the milk and water.

To convalescents I give milk freely-at least a glass every two hours, in addition to the regular diet. At times it is constipating, but when it is. milk of magnesia, a half to one ounce, should be added to eight ounces of milk. Peptonizing the milk just short of bitterness will oftentimes overcome this unpleasant condition.

At times small doses of Rochelle salts, repeated at short intervals, will be necessary to relieve the constipation. If a generous diet is given, 
and considerable good sweet milk is taken, the patients recover, looking considerably improved in general condition, rather than thin, emaciated, and played out. The physical condition of discharged patients has its influence reflected in the community. A man may complain about his treatment, but if his physique belies his statements, the public will accept his complaints with a grain of salt. It is very expensive to treat such cases and to keep them well fed, but it is more than counterbalanced by the good that is accomplished.

At the onset, as before stated, the condition of the temperature controls and regulates the diet.

If the fever is slight, it will not cause much variation, but if there is considerable fever, such cases should be placed on a restricted diet of milk and water. During the progress of the disease milk will be the mainstay. 'To this may be added at times the whites of eggs beaten, or if the condition of patient warrant it, a whole egg raw may be added. A milk-punch with an egg in it or an egg-nog or egg-shake may also be given.

In the pustular stage stimulants, particularly alcohol, are absolutely necessary. In this condition the system will tolerate considerable quantities of whisky. A good blended rye whisky is, as a rule, better taken than a raw article. Scotch whisky is not well borne. Irish whisky is not appetizing to some. Whisky is best given in water, freely diluted. Very occasionally it may be given in milk, but not over once a day, or the patient may rebel against the very nourishment that we place our greatest reliance on. Three times a day (at eight-hour intervals) it is wise to add a few drops, three to five, of diluted muriatic acid to the whisky. It does not add to the palatability of the whisky, but it assists digestion, which is materially disturbed by the disease.

At times patients rebel against the whisky; under these circumstances I change to a good, old sherry wine. It may be given plain, or diluted with shaved ice or carbonated water. At times the stomach is so irritated that only champagne can be given; it is best to chill the wine thoroughly and give it in very small amounts, frequently repeated.

It is impossible to accurately estimate the amount of alcohol necessary, but my plan is to use as much as the system will tolerate. It is remarkable how much these patients will tolerate without the least effect of intoxication or unpleasant phenomena. I have given a woman with a severe septic condition, from which she recovered, the astonishingly large amount of 24 ounces in twenty-four hours for a period of fourteen days. I firmly believe this saved her life.

Frequently, a pint is consumed every twenty-four hours by this class of cases. Push stimulants for their effect, and as long as they are tolerated and show results the amount is not to be considered. As a rule, dilute the whisky with cold sterile water. At times a hot "toddy" may he given. The white of an egg may occasionally be added to the whisky and water.

Under ordinary circumstances, from the beginning of the eruption in the more severe cases of variola, it is best to establish a routine milk diet, 
and at times the changes in the mucous membranes of the mouth and throat will render such necessary. 'This dict should be continued until the digestive apparatus functionates properly.

Variolous patients with severe forms of the discase are very difficult to nourish, but with fluid foods, such as soups, milk and water, and whisky much can be accomplished. Owing to the severity of the lesions and the weakness of the patient very little at a time can be taken, so that it will require the entire services, very nearly, of two attendants to properly care for such a case.

Feeding had best be done by means of a piece of rubber tubing with a glass mouthpiece; never molest the patient by having him lifted or turnerd at each feeding; in fact, as little disturbance as is possible should be the rule. Swallowing at times is very difficult and painful. Oftentimes rectal feeding will save a patient who would otherwise succumb. Nutritive enemata of milk, or milk, whisky, and eggs, should be resorted to in order to sustain the patient. Unfortunately, as a rule, the bowel is almost as intolerant as is the throat.

When the fever subsides and the appetite shows signs of returning, then, in addition to the milk, such food as will be digested without discomfort may be given, such as calf's-foot jelly, whey, kumiss, buttermilk, remet, and later custards and other soft puddings, like bread, rice, and others.

Later in convalescence, tender, juicy steak, roast-beef, boi ed regetables, and thick, strong, and nourishing soups, may be given. During this stage I always give wines and malt liquors with the lunch and dinner. In fact, I crowd fluids and foods so fast that a regular stuffing process is undertaken. I do not let a man go out of the service until I feel assured that he is going into better conditions than I have to offer, and what I offer is the best the community can afford. 'The diet is advanced, day by day, on regular schedule, as the health returns. Foods that are difficult of digestion or ordinarily disturb the digestive apparatus, like cabbage, cauliflower, and beans, are avoided, likewise spices, aromatics, berries, and certain fruits.

Medicinal Treatment of Variola.-Headache.-For the pain in the head, which at times in the stage of invasion is acutely painful, cold wet cloths, tied about brow, rubbing with camphor water or eau de cologne or witchliazel, are very comforting. An ice-bag to the temples, with absolute rest in a darkened room, at times gives relief.

Phenacetine, 5 grains, or a capsule of phenacetine 3 grains, in combination with caffeine citrate 2 grains, may be substituted if there is fear of cardiac depression.

If camphor water is used, rub the surface dry, and do not cover. as it is likely to blister or irritate the surface, and later the eruption will be most intense at this site. Any irritation of the skin is apt to be fol. lowed by an intensification of the eruption in the irritated area.

Backache.-For the backache the same remedies may be tried as for the headache; irritating plasters or liniments should be avoided. Rest in bed is best, with dry heat, in the form of a hot-water bag or bag of hot 
salt. Every movement of the body will excite and aggravate the pain. The burning sensation in the skin may be relieved by repeated sponging of the entire body with plain water or acidulated water, acetic acid, 1 dram to 8 ounces, or household vinegar may be used as a substitute for the acid.

Fever--The initial fever may be slight and not necessitate any consideration. Should it, however, be intense and discomforting, it may be controlled by coal-tar synthetics or cool baths.

The stage of suppuration, with its general toxemia, will give the most concern in the treatment of variola, and here the general indications for treating septicemia and pyemia apply, in addition to what has been mentioned heretofore. The high fever should be treated with wet packs, or cold wet cloths placed on chest and abdomen, or cold wet cloths applied to the extremities and changed from time to time. Bathing with warm water gradually cooled down may be used also.

The coal-tar synthetics, like phenacetine, in combination either with caffeine, camphor, or strychnine, will at times successfully control the fever. Jaquet recommends lactophenin in 5- to 10-grain doses, but I have never used it. Other observers speak well of its merits, especially if cerebral symptoms are present.

Nervous Manifestations.- These are, as a rule, in proportion to the severity and intensity of the fever, the reduction of which will ordinarily control the nervous phenomena. At times it may, however, be wise, in the presence of excitement and irritability of temper, to give frequently repeated small doses of bromide of potassium. If there is in addition insomnia, chloral may be added to the bromide. Codeine in small doses, repeated at two- or three-hour intervals, will also frequently control excitement and allay irritability.

Unless very positive indications are present, morphine should not be used in variola, particularly in the early stages, as it is not ordinarily well - borne, and, owing to its irritating effect on the skin, intensifies the eruption. Frequently mental excitement can be controlled by local dermal applications, such as a prolonged warm bath, or the application of oiled bandages or soothing ointments.

The Heart.-If there are evidences of cardiac involvement, as shown by a rapid and irregular pulse, camphor or caffeine may be administered, followed by strophanthus, either alone or in combination with digitalis. Ordinarily, as variola progresses, the initial symptoms retrogress-almost invariably as the eruption appears this retrogression is marked; it may be only for a short period or it may be permanent. 'The temperature usually, on the appearance of the eruption, will drop at least several degrees. This is rather characteristic of variola, and is one of the cardinal diagnostic points. In many cases of variola the disturbances of the initial stage gradually recede, and when the rash appears, the patient is fairly convalescent, and continues his course with apparently but slight constitutional disturbance; here very little consideration is necessary aside from the diet and personal hygiene. In the severe cases of variola important 
therapeutic indications will constantly present themselves as the discase progresses, and will have to be met and solved as they appear. The astute clinician will in many instances be able to anticipate them by proper measures.

The Skin.- The prevention of abrasions, affording sources of infection, either of the skin or of the mucous nembranes, must at all times be kept in the mind, and measures adopted to prevent them, and, if such should unfortunately occur, antiseptic lotions or ointments should be applied. The skin of the variolous patient must be kept clean, and the bedding must be given constant attention. Frequent warm baths, the application of lotions to the skin, either alcoholic or watery vegetable extracts, together with free use of dusting-powders, will meet the usual conditions in this regard.

Artificial evacuation of the pustules is tedious, painful, and is not followed by the success which one desires, and on this account it is best not to undertake it. The risks encountered also more than counterbalance its advantages. In an individual case, under favorable circumstances, one might be justified in treating a patient in this manner, but in a general ward it is positively out of the question. In the more severe cases, with numerous lesions, what was once thought to be in the nature of a specific was the application of quicksilver, either in form of an ointment or a plaster, but in the light of experience I can state that it cannot be regarded as such.

If a salve is to be applied to the face, the ordinary white precipitate ointment, applied in form of a mask, with openings for the nose, mouth, and eyes, is about the best that can be recommended. It is a soothing antiseptic, relieves tension and the burning sensation, and has all the advantages of the so-called specifics, with none of their dangers.

In young women and girls it is particularly desirable that everything be done to avoid sepsis and deep ulceration of the individualized pocks, and for this purpose white precipitate meets the indication-if there is evidence of too much absorption, I change to ordinary ointment of zinc oxide for a few days. The desirable results are more often due to the macerating influence of the fats than to any specific virtue of any particular salve.

A very comforting application, which at times affords considerable relief, is a face-mask of cotton-wool soaked in a cold, watery solution of glycerine ( $5 \mathrm{j}$ to $3 \mathrm{j})$. 'This may be bandaged on, with openings made for the nose, eyes, and mouth. 'To prolong the effect, it may be covered with oiled silk or rubber tissue.

Scabs may be removed from the face, if they have been allowed to form, by making a mask of several layers of lint soaked with vaseline. After the crusts have macerated, remove the softened masses and reapply the mask until all the crusts have desquamated, and then apply the zinccalamine salve. The thick crusts should not be allowed to exfoliate, as infection is likely to occur, with ulceration and considerable disfigurement.

I sesions about the eyes have to be treated very carefully, and at times are very annoying. I coss of eyesight may result from corneal opacities. 
Lesions in the mouth are to be treated by cleansing washes, such as Dobell's or 'Thiersch's solution, or a plain gargle of dilute witchhazel extract. If there is a bad odor, then it is best to use a solution of permanganate of potash. Should sordes form and accumulate rapidly, which is likely to occur in nearly every case of any severity, cleanse the mouth with peroxide of hydrogen, followed by warm-water irrigation. The teeth and gums should be frequently wiped off with lemon-juice, diluted about equally with water. A saturated solution of chlorate of potash also is of service here.

In children the mouth is at times very much affected, and it is very difficult to treat properly. Irrigations with a dilute solution of permanganate of potassium will give the most satisfaction. Swabbing with a piece of cheese-cloth, wrapped about the handle of a fork and wetted with the solution, may occasionally be possible. Extra care should be taken not to injure the already swollen and sensitive tissues. Tincture of myrrh, a few drops in a tumbler of water, is a pleasant mild astringent for irrigation. Should ulcers form, they should be touched occasionally with a solution of nitrate of silver, 20 to 40 grains to the ounce.

Compresses of ordinary cheese-cloth soaked in olive or sweet oil, with the addition of carbolic acid, 1 or 2 per cent. strength, will be found very comforting. It is a good plan to envelop the extremities as much as possible with this oily dressing. Keep the dressings soaked with oil as fast as they dry out, and when soiled, a change should be made. Ordinarily, they can be worn for two or three days at a time. They relieve tension, allay irritability, prevent rupturing of the vesicles or pustules, and in this way prevent infection. Poultices, unless applied to a localized condition, do harm and macerate too extensively. For furuncles, poultices are necessary, and it is wise to add to the poultice bichloride of mercury to render it as aseptic as is possible.

Any application that will irritate or cause localized congestion should be avoided, for later, when the eruption comes out, it is most intense in that site, and if we have unwittingly caused a plaster to be applied at a point where there is apt to be pressure it is not only uncomfortable to the patient, but may endanger his life by opening a portal for sepsis. I have frequently observed that where hot-water bags or mustard plasters have been applied to the lumbar region to relieve the usual backache of variola in its onset, a mass of pustules frequently develop which quickly become confluent. Besides, being in a most uncomfortable locality when crusting takes place, they are extremely difficult to care for. 'Too much consideration cannot be given to this one item alone, for it is oftentimes overlooked, and frequently causes much harm and unnecessary suffering.

Many writers have felt that there was much virtue in a solution of tincture of iodine used as a wash. Except for its mild antiseptic qualities, I have never found it of any particular use. Unless used very much diluted, it is likely to do more harm than good.

Phenol and bichloride of mercury have their places in preventing 
sepsis, and the former in allaying itching; further than this, they have no special virtue in the treatment of variola.

Phenol is also a splendid deodorant, and is useful as such, as the drying, musty odor of small-pos is a thing to be controlled. 'The odor can be controlled much easier and with much less danger by the use of permanganate of potassium. I much prefer it to phenol, creolin, or lysol. The disagreable tanning caused by the permanganate can be easily removed by washing with a mild solution of oxalic acid. Permanganate is innocuous, is a splendid deodorant, and is a goor antiseptic, and for these purposes can be used liberally and withont fear. As a mouth-wash it is unexcelled in variola; a light claret-colored solntion should be used. Cloths kept moist with a warm solution of permanganate can be applied to the body. 'This keeps the body clean and free from odor, and, unlike phenol, does not substitute one unpleasant odor for another.

Internally, disinfectants have not exerted any influence on the course of variola, and, in my opinion, should not be used, owing to the possibility of adding further irritation to the already inflamed mucous membranes.

Applications of moist cold compresses, especially if the solution used is antiseptic without any irritating properties, will very materially assist in comforting the patient by relieveng tension and burning and prevent the rupturing of the pustules. When this is done, the lesions are apt to be more superficial and scarring is not so prominent. Any means that will diminish the suppurative process will diminish its penetration and inhibit the loss of the papillary structure. 'This will not prevent pock-marking by any means, but it will prevent deep pitting, by preventing deep ulceration, which nearly always follows infection. Hebra suggests ice-compresses applied to the head, face, hands, and feet during the advancing period of suppuration. 'They should be changed frequently and made very light; at times they are pleasing and well borne, but, as a rule, patients will not tolerate them. When the fever is at its height, and the patient is made uncomfortable thereby, he will, as a rule, tolerate the chilling, but I have been unable to use them for any length of time. Intermittent applications I have often used with splendid success, and at times have applied them to the body, particularly when fever seemed uncontrollable and the heart showed evidences of embarrassment. This plan in children is much more acceptable than using drugs for the control of the suppurative fever.

The suppurative fever cannot be controlled by cold applications nor by internal antipyretics for any length of time; either the patient gets chilled or the cardiac system will show evidence of depression. It is a well-recognized fact that in variola the cardiac system is markedly affected, and danger of collapse must be constantly anticipated, and if possible prevented.

In all stages of variola, especially in the earlier ones, antiseptic and astringent dusting-powders are very soothing, and will relieve considerable distress. Fuller's earth, lycopodium, finely powdered rice. cornstarch, or subgallate of bismuth, may be used alone or in combination. 
A good plan is to make a small sac of cheese-cloth, fill this sac about one-half full, and tie the top with a string, and as it is wanted shake the bag over the part on which the dusting is desired.

Never dust the skin by touching it with anything, or you are apt to rupture the tender pustules.

A powder formula which I have used is made of equal parts of boric acid, lycopodium, corn-starch, and subgallate of bismuth. This is inexpensive, can be used liberally, and is very soothing.

An excellent dusting-powder where there is a discharge of moisture, as in crusting stage, is as follows:
R. Phenol, c. p.,
Pulv. zinci oxidi, Lycopodii,
Sig.-Apply liberally.

$$
5 \mathrm{ss-j}
$$$$
\bar{a} \bar{a} \overline{5} \mathrm{j} .-M \text {. }
$$

At times in variola the eruption is so discrete and occasions so little inconvenience that no local application is necessary. For such cases a daily antiseptic warm bath should be given. Bichloride of mercury, 1 to 10,000 , is what I use. After being dried, the patient is dusted from head to foot with a drying powder.

If thick crusts form on the lesions, particularly where confluency has occurred, then these should be softened by being macerated with a bichloride flaxseed-meal-poultice, or a starch poultice with phenol or flaxseed meal with iodoform. When softened, remove the poultice and cover the cleared area with a mild astringent ointment, such as zinc oxide, to which has been added calamin, 1 or 2 drams or if foul, use 5 or 10 per cent. salicylic acid in the salve.

In the very severe cases of variola, an air- or water-bed is absolutely necessary for the comfort of the patient.

If insufficient urine is voided, the spirit of nitrous ether well diluted may be given. Warm wet compresses should be placed over the bladder region. The urine should be measured for the twenty-four-hour quantity and tested for albumin, the persistent presence of which in any quantity is of grave omen.

In purpuric cases of variola, death, as a rule, ensues in a few hours, or at best a few days. These cases are not affected to any extent by treatment. Turpentine has been administered, but so far without a favorable result. Every possible symptomatic indication should be met and combated, but the family and relatives should be warned in advance what to expect. Morphine hypodermically should be used if the patient's delirious condition demands sedatives.

Complications.-The complications of variola are numerous. These should be met symptomatically as they appear.

Furunculosis is frequent. The furuncles should be poulticed until softened, and then incised and treated antiseptically. Painting the infective area with pure phenol, leaving it on until the tissues are porcelain white, and then washing the acid off with pure alcohol, will sometimes prevent recurrences in the immediate vicinity.

Alscesses when accessible should be treated surgically. When fur- 
uncles or abscesses do occur, compound tineture of cinchona should be given internally, with occasional aperient doses of a saline cathartic.

Pleural and pericardial effusions shonld be withdrawn before they cause embarrassment to the heart or lungs.

Arthritis, especially if followed by pus, should be drained surgically.

Corneal ulcers should receive close attention, and everything done that is possible to preserve the eye-sight.

General alopecia may be expected, for it is the usual rule, but the hair will return in the great inajority of cases.

Abscesses of the tonsil and peritonsillar tissues are not uncommon, and should be opened early and cleansed with peroxide of hydrogen irrigations.

Edema of the glottis should be met with prompt intubation, and a set of instruments for both adults and children of varying ages should be in every isolation hospital armamentarium.

Should intubation not be possible, tracheotomy should be performed early.

During convalescence the skin should be bathed twice a day and massaged with cocoa-butter or any pleasant oil.

The baths may be of either plain water and Castile soap or they may be medicated. If itching is present, the bran or starch bath, with or without bicarbonate of soda, may be used. If pruritus is intense, the bath may be acidulated with acetic acid, or carbolized oil cloths may be applied to the parts until exfoliation is complete. 'These, applied to the maturating parts, will also assist in relieving the nauseating odor.

Frequent bathing assists very materially in drying of the lesions and exfoliation of the scabs. I permit the patients to soak in the bath for twenty minutes or more several times a day. 'The seabs, unless thoroughly' dry, adhere tenaciously, but not as much as in chicken-pox, nor are they as sunken in the skin. When thoroughly dry, they drop off or may be scraped off with a cuticle knife. Should the crusts be removed too early, the ulcerated surface should be dressed antiseptically or it is sure to become infected. Granulations should either be cureted or burned down with some caustic, preferably nitrate of silver, either the pure stick or in strong solution.

Pock-marking of the Face.-In the moderately severe forms of variola, as well as the very severe, pitting of the skin of the face, due to a destruction of the papillae, is in nearly every instance more or less unavoidable. Any treatment that will prevent irritation and infection, however, will very materially reduee the intensity of the lesion, and in that proportion reduce the tendency to pock-markings. Scar tissue once formed is irremediable.

Buri's method of removing small-pox pittings consists in the repeated application of a strong resorcinol paste to the affected parts. I have had occasion to use the method, but cannot recommend it, for it is painful and very uncertnin. Unprincipled persons advertise this cure, but of the many cases that I have had reason to investigate in my clinic, I have never seen a single successful result. 
Of several cases investigated, not only were they not improved, but their facial appearance was made much worse than it was before its use.

The safest way to avoid pitting, particularly deep pocking, is to destroy the human susceptibility by vaccination and revaccination, and if any one is so unfortunate as to contract variola after this, then it may reasonably be assured that the disease will be so attenuated as to attack the individual in a very mild form, which, as a rule, never marks. 


\title{
CEREBROSPINAI، MENINGITIS
}

\author{
BY CHARLES HUNTER DUNN, M.D.
}

THE term cerebrospinal meningitis is descriptive of a certain pathological anatomy found in the central nervous system, and belongs to that classification of diseases which is based upon the lesions found at postmortem examination. It refers to an inflammatory condition found in the covering of the brain and spinal cord, and bears no reference to the various etiological factors which may produce such morbid changes. The most notable of the advances in the modern treatment of disease is the increasing tendency to direct therapeutic measures at the underlying causes of diseased conditions, instead of, or in addition to, directing them only at the anatomical changes and their symptomatic manifestations. In this respect treatment is becoming more and more specific in its character, its plan being based on a classification of diseased conditions which is etiological rather than anatomical.

'The treatment of disease is, therefore, to be divided into two classes: first, specific treatment, directed at the specific cause of the disease; and, second, general symptomatic treatment, directed at the morbid changes and their symptomatic manifestations. 'The general symptomatic treatment of cerebrospinal meningitis has little or no dependence upon varying etiological factors, and will, therefore, be here described as applying to all forms of cerebrospinal meningitis, whatever may be the cause. The modern progress made in the specific treatment of cerebrospinal meningitis has been so great, and is so directly dependent upon recent advances in the knowledge of causes that it must be described separately with regard to the various etiological forms of the disease.

The Various Forms of Cerebrospinal Meningitis.-Cerebrospinal meningitis represents no etiological entity. It may be caused by any of the pathogenic organisms; it may be caused by the tubercle bacillus; and it may appear as a manifestation of syphilis. "There is wide difference of opinion as to the relative frequency of oceurrence of these various causes. 'There are probably marked variations according to locality. In my own experience the order of frequency is as follows:

(1) Tuberculous meningitis.

(2) Epidemic meningitis (Diplococcus intracellularis).

3) Pneumococcus meningitis.

(4) Streptococcus meningitis.

(5) Influenza meningitis.

(6) Other forms.

In some text-books the term cerebrospinal meningitis is applied 685 
only to the epidemic form, caused by the meningococcus (Diplococcus intracellularis), the forms due to other pyogenic organisms being called "purulent," or simple meningitis, while the tuberculous makes a third form, and the syphilitic, a fourth. This is a bad classification, because the epidemic form is purulent, and in all the other forms (except perhaps the syphilitic) the process is usually cerebrospinal. Also, this classification is not convenient for the classification of specific treatment.

For the purpose of a convenient description of the therapeutic measures applicable to these various forms, I shall adopt the following classification:

(1) Epidemic meningitis (Diplococcus intracellularis).

(2) 'Tuberculous meningitis.

(3) Pneumococcus and streptococcus meningitis.

(4) Staphylococcus meningitis.

(5) Syphilitic meningitis.

(6) Meningitis due to other micro-organisms.

Comparison of Various Methods of Treatment.-The term epidemic cerebrospinal meningitis refers to that form caused by the meningococcus or Diplococcus intracellularis of Weichselbaum. The name of the disease is derived from the tendency to occur in epidemics, although the disease is not exclusively epidemic in its occurrence, but may be found practically at any time, and is constantly present in many localities. It is frequently referred to as "spotted fever." The pathology of this disease has been thoroughly elucidated by the work of Councilman and others, and the recognition of its specific character has led to the successive trials of various methods of specific treatment, some of which have been discarded as of no value, others of which have been retained as of possible contributing usefulness, and one of which has been almost universally adopted as of the highest efficacy. These methods are: (1) Lumbar puncture; (2) diphtheria antitoxin; (3) vaccine therapy; (4) leucocyte extract; (5) serum therapy.

Before the year 1906 the only method of treatment in any way approaching a specific character was the employment of lumbar puncture as a therapeutic measure. There has been no convincing evidence based on mortality statistics that this procedure is of value, but many cases of improvement following its use have been reported by various observers, and a number of authorities advocate repeated lumbar puncture as an effective method of treating this disease. I have seen cases in which improvement seemed to follow immediately upon this treatment, and I believe that it has some value. In view of the definite proof now available of the value of serum therapy, lumbar puncture alone as a therapeutic measure should be discarded, except in certain rare cases presenting peculiar conditions. The indications for therapeutic lumbar puncture will be discussed below, under the lieading "The Treatment of Persistent and Chronic Cases."

In 1906, for a short period, the subcutaneous administration of diphtheria antitoxin in large and repeated doses was advocated as being an effective measure in epidemic cerebrospinal meningitis, The belief 
in the efficacy of this treatment was based on comparatively fow observations, but it was for a time quite widely tried, and entirely failed to establish the claims of its first advocates. It has been finally proved to be of no value.

At one period the great progress made in vaccine therapy led to a widespread trial of a vaccine made from the meningococcus. No evidence based on mortality statisties has been fortheoming in support of this method of treatment, although cases have been reported which appeared to show a favorable influence from this procedure. My own experience with vaccine therapy has given no evidence of favorable action. It is, however, possible that vaccine therapy may, at times, have some value, and we may find indications in certain cases in which serum camnot be used; these indications will be discussed helow under the heading devoted to chronic and resistant cases.

'There have recently been reports of cases of epidemic meningitis treated with leucocyte extract, which have shown some evidence of a favorable result from this method of treatment. The treatment is a new one, and the evidence in favor of its value is as yet too slight to warrant the drawing of any final conclusions, for in comparison with the proved value of serum therapy, the only possible indications for leucocyte extract will be found in the discussion of resistant and chronic cases.

Serum therapy is now recognized by the majority of authorities as an effective specific method of treating epidemic meningitis. Thanks to the work of Flexner, the proof of the value of this treatment has been placed beyond the possibility of reasonable doubt.

It is possible to throw some light on the relative value of these various methods of treatment by comparison of the mortality figures of various years at the Children's Hospital in Boston. While the average number of cases treated each year varies, the average yearly number treated for the last ten years is 20 . Up to 1903 the treatment was symptomatic, no specitic measure being employed. In 1903, 1904, and 1905 lumbar puncture was employed as a routine therapeutic measure, and was often frequently repeated. In 1906 the diphtheria antitoxin measure was used as routine treatment, and in 1907 , vaccine therapy. Each year is regarded as beginning on November 1st of the preceding calendar year, as serum therapy was begun as the routine treatment in November, 1907. There was no notable variation in the type of the disease from year to year. Fig. 90.

FIG. 90.

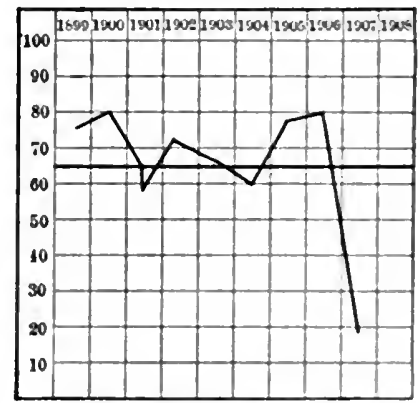

Chart showing death-rate from cerebrospinal meningitis at the Children's Hospital from 1899 to $190 \mathrm{~S}$.

The figures are shown in It appears that the lowest mortality of any year before 1905 was $5 S$ per cent., and that it varied between $5 S$ and $s 0$ per cent., but that in 
190S, under serum therapy, it showed the remarkable drop to 19 per cent., and in 1909 was only 25 per cent. I believe this is strong evidence of the slight value of any of the other measures of specific treatment in comparison with serum therapy.

The Serum Treatment of Epidemic Cerebrospinal Meningitis. -Evidence of its Value.-Beginning in 1906, epidemic meningitis in various parts of the world began to be treated with the serum of animals inoculated with the Diplococcus intracellularis. The results obtained by European observers are of value chiefly as confirmatory evidence, when considered in connection with the results obtained in this country and in England, with the antiserum prepared by Flexner at the Rockefeller Institute for Medical Research.

For proof of the value of the Flexner antiserum the reader is referred to the various publications of Dr. Flexner and his associates, and the reports of the various investigators who have becn using the serum. Through a careful method of distribution Flexner has been able to collect an overwhelming set of statistics, based on carefully studied cases properly proved to be true epidemic meningitis, which show a reduction in the average mortality of the disease under the use of the serum from 75 to 25 per cent.

Flexner's conclusions are now based on more than 600 cases. The conclusions drawn by Flexner and Jobling from their former analysis of 400 cases are as follows:

"It is our belief that the analysis of histories of cases of epidemic meningitis which have been presented in this article furnish convincing proof that the antimeningitis serum when used by the subdural method of injection, in suitable doses and at proper intervals, is capable of reducing the period of illness; of preventing in large measure the chronic lesions and types of the infection; of bringing about complete restoration of health in all but a very small number of the recovered, thus lessening the serious, deforming, and permanent consequences of meningitis; and of greatly diminishing the fatalities due to the disease."

That this reduction in the mortality of the disease has largely failed to be evident in various local health board reports is due to the following facts: (1) 'Treatment with the serum has not yet become general; (2) cerebrospinal meningitis is not everywhere a notifiable disease, and few cases are reported to health boards except fatal cases; (3) the diagnosis of cerebrospinal meningitis cannot be made on a purely clinical basis without bacteriological examination. In most cases reported to health boards no such examination is made, and very many cases reported are other forms of meningitis or other diseases which clinically simulate meningitis.

What Antimeningitis Serum Is.-The antimeningitis serum prepared at the Rockefeller Institute for Medical Research, like diphtheria antitoxin and other immune sera, is horse serum. 'The horses have been treated with successive inoculations of preparations of the Diplococcus intracellularis until, as shown by experiment, their blood-serum contains the maximum amount of immune body. The antimeningitis 
serum is not primarily an antitoxin, although it contains a small amount of specific antitoxin with neutralizing power. It differs from diphtheria and tetanus antitoxins in that it is bactericidal rather than antitoxic in its nature. Although the theory of its action is not entirely clear, it is evident that its chicf effect is exerted upon the diplococci themselves. It appears to injure them in such a way as to cause restraint of multiplication, with rapid death and disintegration, while at the same time phagocytosis is greatly increased, and within the phagocytes the toxin is broken up and rendered harmless. Concentration is a necessary factor in obtaining gool effects.

How Antimeningitis Serum is Obtained.-The making of antimeningitis serum has not yet been taken up by the manufacturing chemists, and it is not yet on the market. At present the serum is made only at the Rockefeller Institute for Medical Research, Dr. Flexner having wisely elected to keep the use and distribution of the serum under his personal supervision. 'The quantity of serum which can be made at the Rockefeller Institute is hardly sufficient to supply the demand for its use in actual proved cases of epidemic meningitis. For this reason the Institute cannot distribute the serum to hospitals or physicians to be kept on hand for cases as they may develop; such a practice would take up so large a proportion of the output as not to leave enough to supply the actual cases reported.

'The serum is distributed to physicians whose hospital connections are of such a character as to give them the largest amount of material for the use of the serum under the proper conditions of investigation. It is the duty of these physicians to keep a fresh supply of the serum on hand, and to supervise its further distribution and the conditions under which it is used. When any physician or any hospital encounters a case in which it is deemed advisable to use the antimeningitis serum, the physician in charge of the case should communicate with whomsoever in his section is charged with the distribution of the serum. In case the name and address of the distributing agent is unknown, they can be ascertained by communicating with the Rockefeller Institute for Medical Research in New York. It is the duty of the distributor of the serum to assure himself that the physician who is to use it thoroughly understands the indications, method, and technique for its administration, that he is competent to make or have made proper bacteriological examinations of all the cerebrospinal fluds obtained, and that he will forward to the distributor a full report of the case, to be transmitted to Dr. Flexner. If these conditions are fulfilled, an amount of serum sufficient to meet the requirements of the case will be supplied without cost, other than that of sending it. In case the attending phrsician is not sufficiently familiar with the technique of lumbar puncture and the bacteriological examination of the cerebrospinal fluid, he may call on the distributing physician to assist him.

My own experience, as the physician having charge of the use and distribution of the serum over a large part of New England, has led me to believe that it would be of great advantage if every physician, having 
under his care a suspected case of epidemic meningitis, would perform a lumbar puncture and a bacteriological examination of the cerebrospinal fluid before communicating with the physician who supplies the serum. This procedure takes very little time-not enough to endanger the chances of the patient, and it might be the cause of avoiding delay in receiving the serum. It assures the distributing agent that the attending physician can fulfil a large part of the requirements, and that the case is actually one of epidemic meningitis, as shown by the bacteriological finding, and not a case of some other form of meningitis upon which the serum would be wasted. I believe the use of this procedure would avoid much more delay than it would cause.

It is by following this method of distribution that Flexner has been able to present such convincing evidence of the value of the antimeningitis serum, for by these means he has prevented the serum from falling into incompetent hands and from being improperly used, and has insured the scientific value of the cases forming the basis of his reports. This method is still being followed, in order to obtain evidence of the value of the serum based upon a series of cases so large that the proof shall be incontestable. It is probable, however, that in the course of time the production of the serum will be taken up by the manufacturing chemists, and possibly by the State Boards of Health. ${ }^{1}$

Technique for the Administration of Antimeningitis Serum.The antimeningitis serum is injected directly into the spinal canal. Experimental evidence showed that concentration was one of the most important factors in its efficacy, and led Flexner and his associates to waste no time with subcutaneous injections. All the clinical evidence goes to show that the subcutaneous injection of the serum is practically of no value in comparison with the subdural method. It is possible that there might be some favorable influence from subcutaneous injections, but such influence is so slight that serum should never be wasted with subcutaneous injections except in those very rare cases in which it cannot be given by the subdural method. These cases will be described below.

The technique is that of ordinary lumbar puncture. Some authorities prefer to perform lumbar puncture with the patient in a sitting position. I prefer to have the patient lying on his side, on a firm surface, with the region of the lumbar spine brought as near the edge of the bed or table as possible. Several needles, two test-tubes with corks to fit, and the syringe are boiled. The hands of the operator and the lumbar and sacral regions of the patient extending around as far as the

1 Since writing this paragraph, I have learned that an antimeningitis serum, made by the H. K. Mulford Company, has been placed upon the market. This product is advertised as being the Flexner serum. It is not the serum prepared by Dr. Flexner at the Rockefeller Institute, but it is prepared by the same metlod as that used by Dr. Flexner. I have had no experience with this serum, but see no reason why it slould not give just as good results as the serum prepared at the Rockefeller Institute. As it is possible that the Roekefeller Institute will not go on indefinitely making antimeningitis serum, and as the supply is already insufficient to meet the demand, it is fortunate that the making of serum has already been taken up by the manufacturing ehemists. 
highest point of the crest of the ilium are disinfected. An attendant then places one hand on the back of the patient's neck and the other in the bend of the knees, and, drawing up the knees while pressing the neck forward, flexes the spine as much as possible without the use of unreasonable force. 'The operator takes as a landmark the level of the highest point of the crest of the ilium as the upper limit of safety. 'The spinal eanal can be punctured anywhere below this level without danger of piereing the cord. Some authorities count the lumbar spines, giving as the proper points for puncture the spaces between the second and fourth lumbar spines. I do not take the time to count and locate the spines by number, but take as first choice the second space below the upper limit of safety described above. 'The spaces are located with the forefinger of the left hand. Some operators insert the needle directly in the median line, to pass between the spines, others, a little to one side of the median line, to pass between the lamina; for the latter case the needle must be inclined slightly toward the median line, so that after insertion the point of the needle will be in the median line. 'The advantage of the former method is that the proper direction is much easier to follow, and of the latter method, that there is more room for the needle to pass between the lamine than between the spines. I am accustomed to use the latter method, but believe that both methods are equally good, the choice depending upon the individual operator.

In either case the general direction is horizontally forward, and the needle is pressed in until the resistance opposing its progress is suddenly diminished, when the fluid will immediately begin to drop or spurt from the outer end of the needle. 'The fluid is caught in one of the sterile test-tubes. 'The advantage of boiling two test-tubes lies in the fact that part of the fluid will sometimes be blood-stained and part clear. As blood-stained fluid interferes somewhat with the diagnosis, if at any time the character of the dripping fluid changes from clear to blood-stained, or from blood-stained to clear, the second testtube should be substituted, in order to separate the clear fluid from the blood-stained.

If the lumbar puncture is undertaken for the purpose of administering antimeningitis serum, or to relieve excessive intradural pressure in chronic cases, as much fluid should be allowed to escape as will run freely. It is of little use to continue the withdrawal after the fluid is running no faster than four drops to the minute. If the lumbar puncture is undertaken for diagnosis only, not more than 5 cc. should be withdrawn.

If the lumbar puncture is performed properly, there will be no difficulty in obtaining fluid in the great majority of cases. Care should be taken that the needle is not pushed in far enough to touch the anterior wall of the spinal canal, as in this case the fluid will probably be blood-stained, and if the needle enters the tissues of the anterior wall, no fluid will flow. On the other hand, care must be taken that the needle is pushed far enough to enter the canal. If the needle strikes bone before entering the canal, it means either that the direction of the needle is not 
right, or that the spine is not sufficiently flexed to permit the needle to pass between the bones. In young babies, or in patients with very rigid spines, it is frequently necessary to use smaller needles, as the opening between the bones is very slight. Occasionally, patients have to be anesthetized in order to allow sufficient flexion of the spine.

A "dry tap," or failure to obtain fluid, may be due to several causes. In such a case the first thing to be thought of is that the needle has become plugged in passing through the tissues, and its lumen should be cleared by passing in the stylet of the needle. It may mean that through some fault of technique, or some peculiar anatomical condition, the needle has not entered the spinal canal. In this case the puncture should be repeated, with the technique corrected as far as possible. If fluid still fails to appear, the intervertebral spaces above and below the one first chosen should be successively tried. Even when the operator is sure that the needle has entered the canal, a dry tap occasionally occurs, being due to some peculiar anatomical cause, or to the fact that the fluid is too thick to flow through the needle.

When serum is to be administered, a sterile syringe should be filled with the proper dose of serum, which has previously been warmed to about blood temperature. The needle remains in place. After the air is expelled from the syringe, the latter is connected with the needle, and the serum is injected through the same needle by which the spinal fluid has escaped. The injection should be made slowly, so as to consume about the same amount of time as that taken by the fluid in escaping. During the injection the patient should be closely observed, particularly as to respiration, pulse, and color. Signs of dangerous raising of the intradural pressure are very rare, but are occasionally seen, and should be watched for. As I have seen them, these signs occur in the following order: disturbed or stertorous respiration, cyanosis, cold sweat, and failing pulse. When they appear, the serum should be allowed to run out again through the needle until these symptoms have disappeared. I am in the habit, after finishing the injection of serum, of holding the needle and syringe in place for three or four minutes before withdrawing the needle. This does away with the necessity of making a second puncture in case pressure symptoms appear. 'There has as yet, so far as I know, been no fatal case reported from the raising of intradural pressure in giving antimeningitis serum.

The ncedles used are the hollow lumbar puncture needles, which may be obtained from instrument-makers in all sizes varying between the small antitoxin needles, suitable for lumbar puncture in very young babies, to the larger needles used for thoracentesis. Trocars are sometimes employed, but I much prefer the needle. I use a metal syringe with a metal plunger, large enough to hold a full dose of serum without refilling. A sinall rubber connecting tube which screws into the end of the syringe and the outer end of the needle, by means of a thread, may be used. The needles are also threaded to fit the syringe. Some physicians use needles which are not threaded and a glass syringe with a ground-glass tip which fits directly into the lumen of the needle. 
The rubber connecting tube is difficult to sterilize, but convenient when the patient struggles. 'The only glass syringes which I have seen to fit lumbar puncture needles are too small to hold a full dose of serum without refilling.

I prefer to give the serum without administering an anesthetic, as the patient's condition during the injections can be much better observed. 'The operation is not a very painful one. An intelligent patient who, while in the hospital, had subcutaneous injections of diphtheria antitoxin, thoracentesis, and lumbar puncture, told me that the diphtheria antitoxin was the most painful, and the lumbar puncture the least painful, of the three. Meningitis patients also are frequently unconscious, or too mentally dull to notice pain. In the case of nervous patients, or those likely to struggle excessively, an anesthetic may be used, its indications varying with the particular case.

A few patients complain of pain, either in the head or along the course of the sciatic nerves, during the injection of the serum. Sciatic pain may be due to failure to have the serum warmed before it is injected. Neither form of pain is a contraindication to injection, as neither lasts long.

After the withdrawal of the needle, the puncture is covered with a little piece of sterile absorbent cotton held in place by collodion.

Indications for the Administration of Antimeningitis Serum.- In considering this division of the subject, a distinction must be drawn between those cases in which the diagnosis of epidemic cerebrospinal meningitis has been established by the finding of the Diplococcus intracellularis in the fluid obtained by diagnostic lumbar puncture, and those cases in which the diagnosis is suspected upon clinical grounds alone. In the former class of cases I believe that the antimeningitis serum is positively indicated in every case, except those cases which have advanced so far into the chronic stage that the meningococci can no longer be found in the cerebrospinal fluid.

In the latter class of cases the early administration of the antiserum in epidemic meningitis is so important that no time should be lost in confirming the diagnosis by lumbar puncture. I believe that in every case in which any form of meningitis is suspected, when meningitis cannot be excluded by fully explaining the symptoms suggesting meningitis by some other diagnosis, lumbar puncture should be performed at once. If the serum is at hand, the physician should be prepared to give it at the time of this first lumbar puncture, without waiting for the result of the bacteriological examination of the cerebrospinal fluid, unless the fluid obtained is clear, like distilled water. Clear fluid means either tuberculous meningitis or no meningitis, except in those rare cases in which meningococci are found in clear fluid. If the fluid is bloodstained, the serum should be given at the first lumbar puncture only if the case is a rapid or severe one, and strongly suggestive of meningitis. Blood-stained fluid throws no light on the diagnosis until examined bacteriologically. If the fluid is cloudy, with a cloudiness varving from a slight opalescence to a distinctly purulent fluid, serum should be given 
at the first lumbar puncture without waiting for the bacteriological examination. Cloudy fluid means that some form of cerebrospinal meningitis is actually present. It may be any etiologic form, but the chances are somewhat in favor of its being the epidemic type, and the advantages to bc derived from the early use of the serum are great enough to warrant its being given at once. There is little or no harm in wasting a dose of serum on one of the other forms of meningitis. Unless the spinal fluid shows that the meningitis is caused by the Diplococcus intracellularis the dose should not be repeated, as the serum is of no value against any of the other forms.

If the serum is not at hand, the lumbar puncture should be diagnostic only, and should be made as early as possible, in order that the diagnosis may be established before the serum is sent for, yet without undue loss of time.

Size of the Dose.-In the earlier days of the use of the serum the rule was to inject a quantity of serum no greater than that of the cerebrospinal fluid withdrawn, up to a maximum of $30 \mathrm{cc}$. It was soon recognized, however, that those cases in which only small amounts of cerebrospinal fluid could be obtained by lumbar puncture, and in which only small doses of 10,15 , or $20 \mathrm{cc}$. of serum were given, did not do so well as the cases in which full doses of $30 \mathrm{cc}$. could be given. So marked did the difference appear that I became convinced that the benefits to be derived from larger doses were great enough to counterbalance the possible risk of increasing the intradural pressure, and I began to use larger doses, even in cases where only very small amounts of cerebrospinal fluid could be obtained. Up to the present time this practice has resulted in no disturbance, except in one case. I then raised the maximum dose, in cases in which plenty of cerebrospinal fluid could be obtained, to 45 cc. without bad effects. Doses of $30 \mathrm{cc}$., in most cases, appeared to give sufficiently good results, but doses of less than $30 \mathrm{cc}$. appeared almost constantly to give less good results.

My present belief is that the larger the dose the better is the result, and that as large a dose as possible should be given in all cases. The limit is still the risk of disturbance from increased intradural pressure, and just how great this risk is, time alone can determine. I believe that $30 \mathrm{cc}$. should be a minimum rather than a maximum dose, and that in most cases this quantity can and should be given. The exceptions are found in very young babies, and in a few other cases in which only a small amount of cerebrospinal fluid can be obtained, and in which, on attempting the injection of a full dose, after a certain point further injection appears to encounter an abnormal resistance. This last point of resistarce can only be judged fully by practice. After giving a sufficient number of subdural injections, one becomes accustomed to a normal standard of the feeling of resistance and sensitive to any increase above the normal.

In all cases it is absolutely essential to withdraw as much cerebrospinal fluid as can possibly be obtained before injecting the serum. 'There are two reasons for this: first, so that as large a dose as possible 
may be given with the greatest safety; and, second, because it is probable that the creation of negative pressure in the upper parts of the cerebrospinal system favors the diffusion of the serum to the remoter parts affected by the disease. After the withdrawal of the cerebrospinal fluid, if the quantity withdrawn is $30 \mathrm{cc}$. or under, the routine dose

Fig. 91.

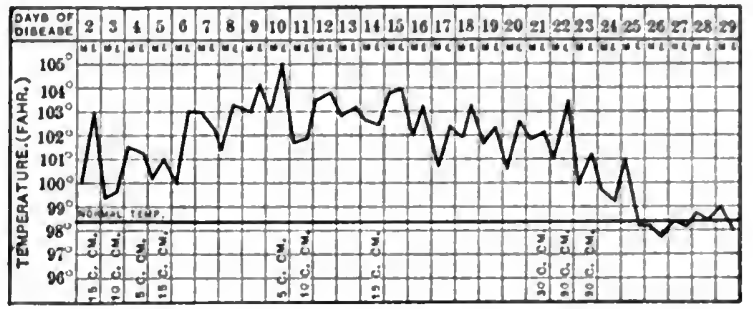

A case illustrating the result of too small doses. It was a case of average severity, in which injections were begun on the second day. As only small quantities of cerebrospinal fluid were obtained, small doses of serum were given daily for four doses. Symptoms improved, but were not completely relieved, and the temperature rose. On the tenth day the patient's condition was worse, and three small doses were given, after which the condition remained stationary. On the twentyfirst, twenty-second, and twenty-third days suffieient cerebrospinal fluid was obtained to permit the giving of full doses of $30 \mathrm{cc}$. This was followed by rapid permanent relief of the symptoms and fever.

should be 30 cc. except in case of the exceptions mentioned above. The aim should always be to give $30 \mathrm{cc}$. if possible, and a smaller quantity should not be given unless a distinct feeling of resistance is encountered. If the quantity withdrawn is more than $30 \mathrm{cc}$, the quantity of serum injected should be equal to the quantity of cerebro-

Fig. 92.

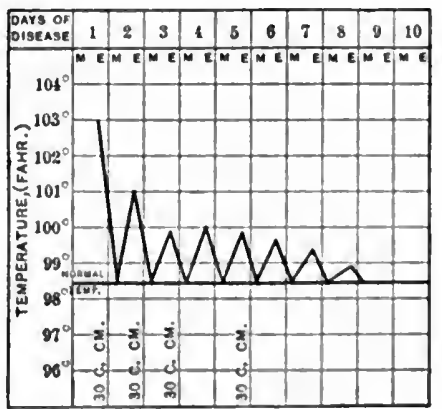

A severe case, in which the use of four full doses was followed by an tnusually rapid convalescence.

spinal fluid withdrawn. In very severe or fulminant cases, especially in adults, in which the quantity of fluid withdrawn is between $30 \mathrm{cc}$. 
and $45 \mathrm{cc}$., I believe that $45 \mathrm{cc}$. should be given, provided that no selsation of abnormal resistance is encountered.

Frequency of Administration.-A second point in the method of using antimeningitis serum is, when and how often should the injections be repeated. Here the earlier work with the serum was more or less experimental. The majority of cases in which the serum was used early in the course of the disease showed a distinct and often very marked reaction after one dose, this reaction consisting in a rapid fall of temperature and a marked improvement in all the symptoms. This was partieularly true of the mental condition, which at times changed within twelve hours from one of unconsciousness to one of complete consciousness. In some of the cases this improvement after one dose was permanent and progressive, and recovery was uninterrupted without further doses being given. In other cases the improvement after one dose was not complete, and the condition remained stationary or tended after a time to get worse again. It seemed then that the best method of using the serum would be to repeat the dose at those times when the limit of improvement from any one dose should have appeared to have been reached, that is, when the condition was stationary or beginning to get worse. I treated a number of cases in this way, and it soon appeared that those cases in which there was little immediate improvement, or which early showed a tendency to remain stationary or relapse, in which, in consequence, frequently repeated doses were given at the outset, did better than those cases in which the initial improvement was more marked, and in which repetitions of the dose were postponed and later strung along at intervals. This method of repeating the dose when the effect is exhausted, of striking at the disease, as it were, each time it attempted to raise its head, is a plausible and attractive method, but I am now of the belief it is not a good one.

At present I am convinced that it is better to repeat the injections a number of times at the outset in all cases, and that by so doing the improvement is more likely to be permanent, the course of the disease is more likely to be short, and that the liability to relapse is greatly lessened. As to just how often the dose should be repeated at the outset, the last word cannot yet be said, and for the present the number of repetitions must, to a certain extent, be arbitrary. The best guide is found in the disappearance of the diplococci from the cerebrospinal fluid, although this disappearance is no proof that diplococci have disappeared from all parts of the cerebrospinal tract.

As a routine, I believe that in all cases the injections should be repeated daily so long as diplococei are found in the cerebrospinal fluid, no matter how many doses must be given. The finding of diplococci in cover-slips is a more reliable test than the results of culture, as the ability of the Diplococcus intracellularis to grow in cultures is variable and very easily influenced by the serum, one dose often being sufficient to produce a sterile culture, even when diplococei can be found in the fluid withdrawn at the next lumbar puncture. In cases in which diplocoeci disappear early from the cerebrospinal fluid, the injections should be 
repeated daily for four doses. 'This number is an arbitrary one, but is based on the experience of a number of observers. While it is true that

Fig. 93.

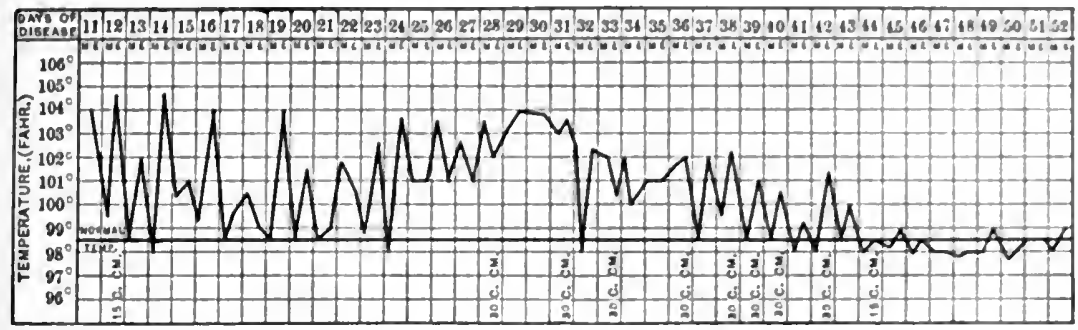

Illustrating improper use of the serum, both at the outset and after the case became prolonged. At the outset, on the twelftl day, one small dose was given, and was followed by no effect. The injections were not repeated until the twentyeightl day, at which time the disease was still in an active stage. Injections were then begun in full dose, and were not repeated on successive days, but strung along at intervals. On the thirty-eighth, thirty-ninth, and fortieth days full doses were given, with marked improvement. An additional dose was given on the fortysecond day, probably because of an evening rise of temperature, after which recovery was uninterrupted. This chart shows no improvement until the serum was used in successive doses.

in some cases fewer doses might suffice, yet, as one can never know beforehand, I believe four to be the safest number to be used in this class of cases to insure obtaining the most permanent improvement and the greatest freedom from relapse.

FIG. 94.

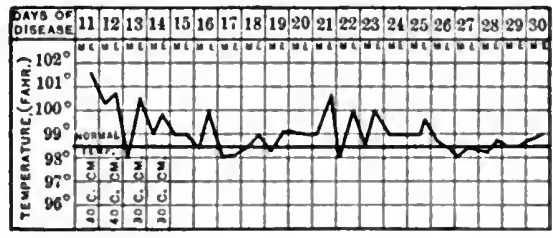

In this case the serum treatment was begun on the eleventh day, about the same stage in the disease as in the cases shown in Figs. 92 and 93 . The case was one of greater initial severity than the other two, but the injection of four full successive doses was followed by rapid improvement and recovery.

The Treatment of Fulminating Cases.-In certain fulminant or very severe cases the injections should be repeated oftener than once in twenty-four hours. My belief is based on two fulminant cases in my series which ended fatally. In both the first injection was followed by a distinct period of improvement occurring within twelve hours, and in one this improvement amounted to a return to consciousuess. In both cases, when I returned in twenty-four hours to give a second injection, the patients were already moribund, having become rapidly worse during the few hours preceding my arrival. On the other hand, 
in another fulminant case, the second injection was given during the period of greatest improvement, ten hours after the first, and in this case improvement was maintained and complete recovery occurred. I think it very possible that the two fatal cases might have been saved if the injection of the serum had been repeated sooner. The very fact that the effects of the serum appear so soon after its injection is an argument for its more frequent repetition, as a more rapid reaction is more apt to become sooner exhausted in so severe a disease. Therefore, I believe that very severe or fulminant cases should be very closely watched, and that the physician should stand by ready to give a second injection the moment the period of initial improvement shows any tendency to cease, and if they cannot be so watched, or if there is no period of improvement, the injection should be repeated in twelve hours instead of twenty-four.

The Treatment of Prolonged Cases.-In some cases, even when the initial treatment has been carried out as outlined above, recovery does not immediately occur. In a few, organisms may continue to be found in cover-slips. In such cases the only treatment is to continue to give daily doses indefinitely until either the diplococci disappear or the disease ends fatally. I have only known one such case. So long as diplococci can be found in the cerebrospinal fluid there is hope for a good result from repeated injections of the serum. The majority of prolonged cases are those in which treatment has been begun comparatively late in the course of the disease, and in which diplococci disappear, but in which symptoms are not entirely relieved. When such cases are progressing steadily, even though slowly, toward recovery, nothing further need be done. When, however, the condition of such patients shows a tendency to remain stationary, with certain symptoms still persisting, or becoming worse, then further treatment is indicated. In this connection the persistence of retraction of the head, rigidity of the neck, and Kernig's sign are not of great importance, as these symptoms tend to persist longer than others without influencing the final outcome. In some of the most favorable cases they persist long after all other symptoms have disappeared, and their persistence is not an indication for further treatment. When, however, any subjective symptoms, such as pain, or any fever, or any abnormality of the mental condition persist, then further treatment is indicated. After the initial course of treatment has been given, one may wait about four days, unless the persisting symptoms are serious, when one should wait only two or three days. Then the injections should be repeated. If diplococci have reappeared in the cerebrospinal fluid, then full doses should be repeated daily until the diplococci have again disappeared. If diplococci have not reappeared, four full doses should be injected on successive days, when one may again wait for a few days and then again repeat four full doses, and this routine should be continued as long as the patient's condition is stationary or becoming worse, with fever, subjective symptoms, or a mental condition other than full normal consciousness, or until the chronic stage of the diseasc is fully established. 
The Treatment of Relapses.-In one form of relapse the patients have not become entirely free from symptoms and fever, although they have shown marked improvement before they began to get worse again. The treatment of this form of relapse has been described under prolonged

lik: 95.

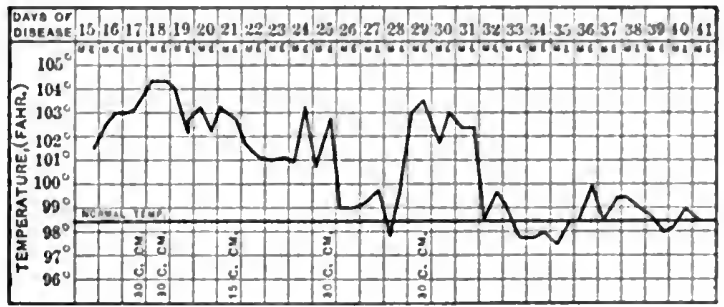

This ease illustrates the improper management of relapses. The first two doses of the serum were followed by a marked improvement in the patient's mental condition and subjeetive symptoms. On the twenty-first day these symptoms became notably worse, and one injeetion of $15 \mathrm{ec}$. was given, without effeet on the symptoms. The patient's condition remained stationary until the twenty-fifth day, when one full dose was followed by a fall of temperature and rapid relief of symptoms. A relapse oeeurred on the twenty-ninth day, when one injection of $10 \mathrm{ce}$. was followed by steady though slow improvement. I believe that in this case relapses might have been prevented if the serum had been properly used.

cases, but if the relapse begins while one is waiting four days, treatment should be begun at once. In another form of relapse, a true relapse, the patient, after being without fever or important symptoms, becomes worse, as shown by a rise of temperature, a recurrence of symptoms,

FIG. 96.

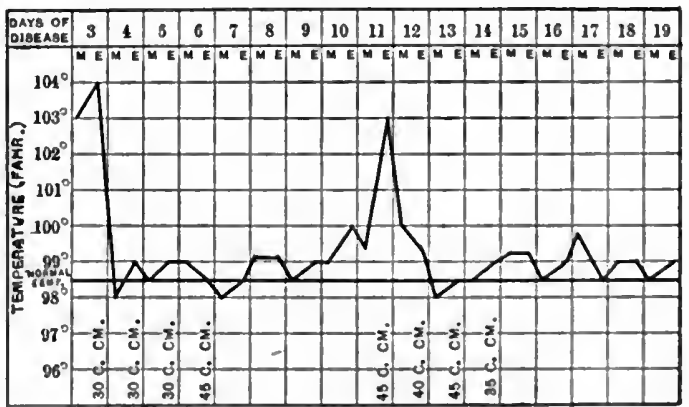

A ease in eontrast to that shown in Fig. 95, showing the proper management of a relapse.

or both. Cases in which a rise of temperature is the only manifestation are not nearly so serious as those in which there is a recurrence of a change in the mental condition. The latter is quite serious, especially if there is no fever or reappearance of diplococci, and may be the precursor of the unfavorable chronic stage. 
The treatment of a true relapse is the same as that of the form described under prolonged cases, immediate lumbar puncture and injection of full doses of serum, the injections to be repeated as long as diplococci-or symptoms persist; or in the absence of such persistence, until four full doses have been given; or, in the presence of continued symptoms, until the chronic stage is established.

Too much stress cannot be laid upon the necessity of giving several repeated full doses rather than a single one, or smaller ones at all times when the injection of serum is indicated. My experience has led me to believe that this is especially important in relapses. The cases in my series in which single doses were given in relapses did not do as well as those in which the relapses were combated by repeated full doses.

The Treatment of Chronic and Resistant Cases.-In the chronic stage the consciousness remains affected, but the temperature is normal and the patient's condition remains indefinitely stationary; no diplococci are found in the cerebrospinal fluid. In this stage the serum appears to be powerless. I think it is as well, however, even in this stage, to perform occasional lumbar punctures, to see, if by any chance, diplococci have reappeared in the cerebrospinal fluid, when there would be some hope of good effect from several successive doses. At these times an occasional dose of serum should be given, though little good is to be expected from its use if diplococci are absent.

It is probable that in some chronic cases there is a condition of chronic hydrocephalus, and it is a question whether this may not be largely responsible for the patient's condition. This would be shown by the fact that at lumbar puncture an increased amount of fluid is obtained under considerable pressure. When this is the case, I would suggest that the older treatment by repeated lumbar puncture, without the injection of serum, should be tried. The condition is a very serious one, and almost the only hope is to keep the pressure reduced as far as possible. I believe that a daily lumbar puncture should be done, withdrawing as much fluid as possible. I cannot base my advocacy of this method on experience, as it has not been employed in a sufficient number of cases. In the few cases in which it has been tried, no good result appeared. But severe chronic cases are rather hopeless, and I think daily lumbar puncture is worth trying when pressure is high.

Resistant cases are distinguished from the prolonged cases described above by the fact that the prolonged cases show some good effect from the serum, though later they may remain stationary, while the resistant cases appear to be entirely uninfluenced by the serum. In one class of resistant cases there is some obstacle to the proper administration, such as inability to obtain sufficient cerebrospinal fluid to permit the injecting of serum without pressure symptoms. Under such circumstances, if the first lumbar puncture has given a dry tap, and the diagnosis has failed of being established by bacteriological examination, it is better to inject serum into the canal with great caution, watching for the manifestations of pressure symptoms. If the diagnosis has been established by a previous lumbar puncture, but later gives either a dry tap or a very small 
amount of fluid, I believe the physician should be bolder in the effort to give serum, although ready to desist at any moment if pressure symptoms supervene. Although I have been able to give 30 (c. to children after a dry tap without pressure symptoms, it is safer, in children under two years of age, not to try giving more than 15 cc. under these circumstances, unless the patient is rapidly getting worse. Resistant cases of this form are, however, fortunately very rare.

When it is impossible, on account of pressure symptoms, to give intradural injections of the serum, subcutaneous injections should be tried. Vaccine therapy, with a homologous vaccine, if possible, may be employed as an additional measure.

In another form of resistant case, no obstacle to the successful administration of the serum is encountered, but no improvement follows in the clinical symptoms. Here there are two classes which call for different treatment. In one class, while there is no improvement in the clinieal symptoms, the organisms found in the spinal fluid show the usual effects of the serum treatment, viz., a progressive diminution and an eventual disappearance. In this class of cases there is evidence suggesting that the failure of the treatment is due to some anatomical change, such as the closure of the foramen of Magendie, which prevents the serum reaching the diplococci developing in the ventricles. In these cases subcutaneous injections of the serum should be tried, giving from 30 to $60 \mathrm{cc}$. at a dose. Vaccine therapy should also be tried.

In these cases the injection of the serum directly into the cerebral ventricles has been suggested. I believe that in a resistant case, growing progressively worse or failing to improve, in spite of the routine serum treatment, this procedure is worthy of trial.

In the other type of resistant case, the diplococci in the spinal fluid show no effect from the serum. These cases can only be explained on the ground that there may be subspecies or strains of the diplococcus intracellularis, which resist the serum. In these rare cases nothing is to be gained by giving the serum subcutaneously. Vaccine therapy should be tried with an homologous vaccine, and the routine serum treatment should be continued, in spite of its apparent failure, as these cases sometimes yield after a time.

Summary of the Serum Treatment of Epidemic Cerebrospinal Meningitis.-1. Perform lumbar puncture and be prepared to give the serum as soon as meningitis is suspected. If the fluid is cloudy, give the first full dose of serum at once, without waiting for the bacterial examination, although further doses are only to be given in case the Diplococens intracellularis is found in the cerebrospinal fluid. 'The serum is of no value in other forms of meningitis.

2. Always withdraw as much cerebrospinal fluid as possible. At every dose give as much serum as possible. Give $30 \mathrm{cc}$. in all cases in which the amount of fluid withdrawn is $30 \mathrm{cc}$. or less, unless a distinctly abnormal sense of resistance is encountered after as much fluid has been injected as has been withdrawn. In all cases in which the amount of fluid withdrawn is more than $30 \mathrm{cc}$, give as much serum as 
the quantity withdrawn. In very severe or fulminating cases, in which the amount withdrawn is between $30 \mathrm{cc}$. and $45 \mathrm{cc}$, give $45 \mathrm{cc}$. unless abnormal resistance is encountered.

3 . In very severe or fulminating cases, repeat the dose within twentyfour hours as soon as the patient begins to get worse again, or at twelvehour intervals.

4. In average cases, repeat the dose daily, until four full doses have been given in all cases.

5. If diplococci persist after four full doses have been given, continue the injections until they have disappeared.

6. If subjective symptoms, any impairment of the mental condition, or fever persist, after diplococci have disappeared, or after four full doses have been given without progressive improvement, wait four days if the condition of the patient is stationary. At the end of four days, or before if the patient becomes worse, repeat the treatment with four daily full doses, and continue as if this were the original attack.

7. When a relapse occurs, as shown either by a reappearance of diplococci in the cerebrospinal fluid or by a reappearance of symptoms, give four daily full doses and continue treatment as if this were the original attack.

8. Treatment along these lines should be continued until the patient is symptom-free, without diplococci in the fluid, or until the chronic stage is established.

9 . In the chronic stage, watch for the possible reappearance of diplococci by performing an occasional lumbar puncture. If diplococci reappear, resume treatment with the serum, as outlined above. In chronic cases with excessive cerebrospinal fluid under marked pressure, try daily lumbar puncture without the injection of the serum.

\section{TUBERCULOUS MENINGITIS}

Tuberculous meningitis is regarded generally as an absolutely fatal disease, although a few cases of recovery have been reported in which tubercle bacilli were found in the cerebrospinal fluid. I believe the absolute fatality of this disease is not proved, although all the evidence points toward there being a very slight possibility of recovery.

In two cases of apparent recovery, reported from Copenhagen, the treatment consisted of repeated lumbar punctures. In one of my cases in which tubercle bacilli were found in the cerebrospinal fluid, but in which the fluid was not injected into a guinea-pig, early lumbar puncture was followed by recovery. I believe lumbar puncture should be performed early, when tuberculous meningitis is suspected, without waiting for the stage of unconsciousness to supervene. If the spinal fluid shows the characteristics of tuberculous meningitis, that is, an excess of lymphocytes, with or without tubercle bacilli; I believe the lumbar puncture should be repeated, with the withdrawal of as much fluid as can readily be obtained. If the first lumbar puncture is followed by demonstrable improvement, it should be repeated whenever the con- 
dition of the patient shows a tendency to remain stationary or to grow worse. If the first lumbar puncture is not followed by improvement, or if the cerebrospinal fluid is under abnormal pressure, I believe the puncture should be repeated daily. I do not believe that the use of tuberculin offers any hope in a process so acute as tuberculous meningitis.

\section{PNEUMOCOCCUS AND STREPTOCOCCUS MENINGITIS}

Pneumococeus and streptococeus meningitis are considered absolutely fatal. If there is a possibility of recovery, it has not yet become manifest. When the diagnosis of one of these fatal forms is established, I believe we are justified in using any method of treatment which offers, even on theoretical grounds only, any prospect of a cure, provided that the measures used do no harm. Serum therapy and vaecine therapy are the only measures offering such theoretical grounds for hope. Serum therapy appears particularly attractive, since the work with the Flexner serum has demonstrated that concentration is so essential a factor in the efficacy of this serum against epidemic meningitis. It occurred to me that the antipneumococeus and antistreptococcus sera might prove more efficacious against meningitis if given in a more concentrated form by intradural injection. I have tried intradural injections in a few cases of pneumococcus and streptococeus meningitis, but so far without result. I believe, however, that in these fatal conditions, this treatment is worthy of trial, and should be carried out as is the serum treatment of epidemic meningitis, using antipneumococeic serum for the pneumococeus cases, and antistreptococcic serum for the streptococeus cases.

Vaccine therapy offers a less attractive theoretical prospect, and the most recent evidence tends to show that it has comparatively little value in streptococcus and pneumoeoceus infections. Nevertheless, in view of the fatal character of these forms of meningitis, I believe vaceine therapy should also be employed.

\section{STAPHYLOCOCCUS MENINGITIS}

It is now pretty generally admitted that the greatest successes of vaccine therapy have been scored against infections caused by the various varieties of the staphylocoecus. Therefore, on theoretical grounds, vaccine therapy would seem to be the most promising method of attacking cerebrospinal meningitis caused by this organism. Although the disease is a rare one, cases of staphylococeus meningitis have been reported, which have been suceessfully treated with homologous staphylococcus vaccines, and in which the administration of the vaccine was followed by a distinctly favorable reaction. I believe that vaccine therapy should be employed in all cases of staphylococcus meningitis. 'The size of the dose and the frequency of its administration are still in the experimental stage, to be determined finally by further experience. I believe that, aceording to present evidence, $100,000,000$ bacteria should be a minimum dose, and about $600,000,000$ a maximum. Doses at least as large as $900,000,000$ have been success- 
fully giver: in cases of staphylococcus septicemia. As to the frequency of repeating the vaccine, I believe, judging from the effects of the vaccine in other severe staphylococcus infections, that the best method is to be guided by the behavior of the temperature-curve and other symptoms. When effective in severe general infections, the administration of the vaccine is followed by a favorable reaction, which is an evidence of the production of some degree of immunity. This immunity tends to wear off, with an accompanying rise of temperature and a recrudescence of symptoms. The administration of the vaccine should be repeated at these periods. At present the evidence on the subject seems to show that the periods when repetition of the dose is indicated occur every two or three days in cases of severe staphylococcus infections.

If at the diagnostic lumbar puncture, the cerebrospinal fluid is found to be under pressure, I believe that the puncture should be repeated at intervals in the course of the disease, the length of these intervals being determined by the amount of cerebrospinal fluid obtained at each puncture.

\section{SYPHILITIC MENINGITIS}

The treatment of syphilitic meningitis, being identical with that of syphilitic processes in other organs, will not be discussed here. (See article on Syphilis.)

\section{OTHER RARE FORMS OF CEREBROSPINAL MENINGITIS}

There is no evidence in favor of the value of any specific treatment in the rarer forms of cerebrospinal meningitis, such as those caused by the typhoid bacillus or the bacillus of influenza. I am inclined to look with favor on the procedure of repeated lumbar puncture in these forms, especially if the intradural pressure is high.

\section{THE GENERAL AND SYMPTOMATIC TREATMENT OF CEREBRO- SPINAL MENINGITIS}

In the discussion of the measures employed in the general treatment of cerebrospinal meningitis, and those employed to combat particular symptoms, it must be remembered that none of these measures can properly be looked upon as in any way curative. There is no evidence that any of them exercise any notable effect upon the course and termination of the disease.

A disease of this character, leading to such severe emaciation through difficult nutrition, vomiting, persistent fever, and probably specific trophic disturbances, requires the most careful nursing. The nourishment of these patients is also of the first importance. Fluid in sufficient quantities is necessary, but it should be remembered that, except in infants, the diet should not consist exclusively of milk. Broths and soft solids which are easily digested should be given in addition. In certain cases the coma is so marked that the patients are unable to swallow, and under these conditions liquids must be given by means of gavage. The tube may be passed either through the nares or through the mouth. I have found that the frequent passage of the tube is apt to set up a 
catarrhal inflammation of the mucous membrane. On this account it is well to change the method of passing the tube every few days, from the mouth to the nose, and vice versâ.

'The bowels should be kept freely open by calomel or saline cathartics. If these drugs are vomited, enemata should be employed.

Although ergot and iodide of potassium have been recommended, there has never been the slightest evidence that either of these drugs exercises any influence upon the course of the disease, except the wellknown effect of jodides in syphilitic meningitis. I believe there is no reason for using them in any other form of meningitis.

Other general measures which have been recommended are the application of cold to the head, neck, or spine, the employment of baths, and counter-irritation. Concerning the application of cold by means of ice-caps to the head, or ice-bags to the neck and spine, I believe that the proper procedure is to be guided by the sensation of the patient, relinquishing this treatment if it affords no relief or is unpleasant. I believe it to be quite valueless as a means of influencing the pathological processes of the disease. On account of the extreme sensitiveness of the patients, I believe cooling baths should not be employed. Warm or hot baths have been highly recommended by some authorities, but I do not think the evidence presented in their favor is sufficiently convincing to warrant their use as a routine measure. As to counter-irritation by means of tincture of iodine, blisters, or the Paquelin cautery applied to the nape of the neck and along the spine, there is no evidence pointing toward any value in this procedure.

Of the symptoms which call for special treatment, the most prominent one is pain, which when severe requires morphine, sometimes in fairly large doses. Other nervous symptoms, such as delirium or sleeplessness, are treated with bromides, trional, sulphonal, or chloral. Among these drugs, I should place the bromides in the first rank in meningitis.

Spasm or rigidity of the limbs is the second symptom of importance requiring treatment. The bromides are decidedly the best means of combatting this symptom, and should be given in doses sufficiently large to produce a visible result. If the bromicle is vomited or is not well taken by mouth, or disturbs digestion, it may be given by rectum. Convulsions also are best met by the administration of bromide, preferably, by the rectum. If the convulsions are prolonged, severe, and repeated, as is occasionally seen in tuberculous meningitis, ehloral should be given by rectum in addition to bromide. At times prolonged severe convulsions require inhalations of ether.

In many cases, stimulants are required at some time in the course of the disease. 'They are indicated when there is weak, rapid, or irregular pulse, or stertorous respiration. I believe that caffeine is the best drug under these circumstances. At times digitalis or strophanthus produces good results. Stryehnine should not be used, except in case of collapse following the administration of serum. As to alcohol, the weight of modern opinion appears inclined to deny it any stimulant value. Nevertheless, I believe alcohol is frequently a clesirable addition to the treatVoL. I -45 
ment of cerebrospinal meningitis, particularly in prolonged cases, with marked emaciation. Possibly its value lies in its aiding the nutrition.

For the residual paralysis, which was formerly a common sequel of epidemic meningitis, but which is only rarely seen under serum therapy, massage, warm baths, and friction should be employed; electricity should be used only when all symptoms of central irritation have subsided.

One new drug in the treatment of cerebrospinal meningitis remains to be discussed. 'This is urotropin, the use of which in meningitis has been widely advocated in the most recent literature. Its theoretical value lies in its disinfecting properties, and in the fact that it is excreted into the cerebrospinal fluid. In addition, there is some clinical evidence of its value. As its use is harmless, I believe this drug should be tried in all resistant forms of cerebrospinal meningitis. The resistant forms in which it may be tried are the forms due to the tubercle bacillus, the pneumococcus, the streptococcus, the influenza bacillus, the typhoid bacillus, and any other rare form. It would not be indicated in the epidemic form, except in a thoroughly resistant case, nor in a case of staphylococcus meningitis which was progressing favorably under vaccine therapy. 


\title{
PNEUMONIA AND PLEURISY
}

\author{
By FRANK SHERMAN MEARA, M.D., Ph.D.
}

\section{PNEUMONIA}

THE greatest achievement of modern therapy has been the recognition of the fact that the body has its own methods for correcting disturbances in its operations, whether they arise from without or within; disturbances to which we apply the generic term "disease"; and that these methods cannot be improved upon in kind, though their efficiency may be reinforced; and, moreover, that so-called "symptoms" of disease are not merely to be looked upon as expressions of deviations from the norm, as diagnostic of a morbid state, but rather, in many instances, betoken of a parposeful effort to overcome an invasion or compensate for a defect.

With this knowledge, then, the following quotation of von $\mathrm{Wyss}^{1}$ is a logical sequence.

"The fundamental principle of all treatment consists in this: to study the organism closely ${ }^{2}$ to determine how it seeks, itself, to gain mastery over the disease; what protective measures are operating inefficiently, as a result of an ascendency of the disease, and what are failing which need energetic support; how the waning of forces may be anticipated by procedures, which, while they find no analogues in the body, can do no harm. The sine qua non of all intelligent therapy is to do what the defender of a threatened stronghold would do, gain an exact knowledge of the same, its strength, its weakness, and attend to the distribution of its protective forces in accordance."

Applying these principles to pneumonia, we find certain broad generalizations which must be observed in our procedure.

(1) To facilitate the body's positive efforts in its own behalf, by affording rest and proper rules of hygiene and dietetics; and, equally important, by maintaining an attitude of non-interference, avoiding malicious meddling and pernicious drugging when the organism is demonstrating its ability to cope successfully with the disease.

(2) To reinforce nature's own procedures along her own lines, by applying what the study of immunization has brought to hand, which entails the provinces of serum and vaccine therapy, as yet, in pneumonia, but touched upon.

1 Ein Beitrag zur Y'athologie und Therapie der fibrinosen Pneumonie, Zeits. f. klin. Med., Bd. Ixx, Heft 1 and 2, p. 172.

"The meaning is expressed exquisitely in the text by the word "ablauschen," to "eavesdrop," to "spy upon," the orgauism. 
(3) To support such organs, or functions of organs, the impairment of whose integrity threatens a fatal issue. Herein, in addition to the measures mentioned above, are to be considered drug therapy and physical therapy.

Expressed a little differently, our procedures depend on the severity of the disease. We recognize groups of pneumonias, which, from a prognostic standpoint, are fairly well defined:

(1) Those in whom, and often in the presence of massive local involvements, the evidences of intoxication or of implications of other vital organs is at no time obvious. These cases get well. The organism bears its own burden without difficulty. The only treatment indicated here is insistence on rest, the establishment of an intelligent dietary, and good nursing; beyond this emphatically non-interference.

(2) 'Those who are overwhelmed by the poison, perchance with a local lesion scarcely recognizable. To combat with such intoxication is like contending with a cataclysm of nature. We fight at the dictates of moral obligation, rather than from anticipation of result.

(3) 'Those who fall between the other two, representing varying degrees of severity, and in whom the issue depends on the skilful application of every legitimate measure.

While from the pathological standpoint we recognize different forms of pneumonia, such as lobar pneumonia and bronchopneumonia, and from the etiological, appreciate the varieties of invading organisms, such as the pneumococcus of Fraenkel and the pneumobacillus of Friedländer, the streptococcus, and so on, I shall make no attempt to observe these types in discussing the therapeutics of the disease, except so far as the severity of one or the other kind may suggest this or that procedure. cance.

The prognostic value of such knowledge is, however, of vast signifi-

I have already hinted that the symptoms of disease should be scanned as much for their therapeutic value as for the diagnostic import. The first symptom of pneumonia, as, indeed, of any acute infectious process, is malaise, carrying with it disinclination for work, that is, establishing a measure of vast conservative significance, namely, rest.

Rest is too often a vague term in the mind of the practitioner, connoting in a case of pneumonia simply "going to bed."

Rest means a comfortable bed, well made and well cared for; it means a competent nurse, to exercise every nicety of her profession; it means mental quiet, the exclusion from the sick-rooms of unnecessary visitors, of friends, and even of members of the family, except for such brief moments as will afford comfort to the patient.

One must remember, too, that an uncomfortable or strained position means muscular action to maintain it, and is work in the true sense of the term.

When the restlessness and uneasiness of delirium intervenes, drugs or other measures must be used to diminish them.

Last of all, but often first in importance, there must be exercised infinite tact and patience by physician and nurse, to instruct and persuade 
many patients, and especially the old, who, in obedience to the life-long habits of self-help, will of ten insist on rising to use the toilet or to do for themselves in one way or another.

With sicker patients, even the efforts to pull themselves up in bed, lift themselves on the bed-pan, ete., should be prevented, while those whose circulation is prejudiced, should not be allowed to turn over in bed without assistance.

Much of the success in securing rest depends on the bed.

The bed should afford the maximum of comfort for the patient and the maximum of convenience for the attendant. 'The ideal is the iron bedstead of one-half or three-quarters width, which, if not at hand, may be secured at no great expense. 'This is best exemplified by the standard hospital bed, which is usually 6 feet 6 inches long, 36 inches wide, and 24 to 26 inches high. 'The springs should be of woven wire, so stiff as to prevent sagging; the mattress of hair, firm and resilient. Over the mattress may be laid a folded blanket, which eliminates any irregularities in the mattress, rendering the surface perfectly even. 'The sheet is spread upon the blanket, drawn taut and smooth, and secured with pins. Over this sheet is placed a rubber sheet across the bed, wide enough to reach from the pillow to the bend of the patient's knees, and long enough to tuck under the mattress nicely. 'The object is, of course, to prevent the soiling of the under sheet and mattress. Over this rubber is laid a draw-sheet - a long sheet folded lengthwise, but wide enough to overlap the rubber above and below.

One end of this draw-sheet is tucked far under the mattress, so that it can be drawn little by little to the other side, affording a cool and refreshing surface to the body and the means of removing a soiled sheet without disarranging the bed, as a whole, or the patient to any degree. One thing in the making of such a bed must be observed-the scrupulous avoidance of wrinkles.

The room should be stripped of all unnecessary paraphernalia, in terms of ornament and superfluous furniture, which only serve to catch and distribute the dust; the walls, and the floors, too, if of hard wood, being made bare.

The temperature should be from $65^{\circ}$ to $68^{\circ} \mathrm{F}$., and the bed so placed as to avoid drafts, but not air.

Such a room may be secured by the use of roofs, verandas, porches, or balconies in fair weather, and wide-open rooms in storms.

The degree of cold in open-air treatment is immaterial.

Open-air Treatment. - The patient may not be exposed to the open air on a cold winter's day without the observance of certain precautions. In the first place, the bed must he so prepared that, no matter how cold the day or blustering the wind, the surface of the body will not be chilled nor inadyertently exposed.

The technique is as follows: Over the wovell-wire sping is spread at blanket large enough to spread well beyond the sides and below the foot of the bed.

Over the blanket is spread a rubber shect, paper, or other light 
material impermeable to air. Upon these two materials is placed the mattress, upon which the bed is made in the usual manner, as just described, except that the quantity of coverings is adapted to the weather. Now over this bed (the mattress and its appropriate coverings) in which the patient lies, the rubber sheet and blanket are drawn up and folded, as one would make an envelope, and secured with pins, thus enclosing the whole bed. The result of this manouver is that the blanket is on the outside, the rubber next, and then the bed-coverings.

The object to be attained is to so enwrap the patient that the cold air cannot work under or into the bed-clothes, as with the shifting currents of out-of-doors it easily does when the bed is made without these precautions.

The patient wears, instead of the cotton night-shirt opened all the way down the front or back for convenience of examination and local applications, as when treated indoors, a suit of light flannel underclothing and socks or stockings.

The hot-water bottle should always be put at the feet, and on the head a cap or hood suited to the degree of cold.

One must take care that the bed-coverings are of the lightest material compatible with warmth, as flannel blankets or eider-down quilts, for excessive weight may embarrass respiration.

The amount of covering, too, must be carefully regulated to the weather and the patient's comfort, as it is not desirable that he should be kept in a constant perspiration. In the warmer weather the rubber sheet may be dispensed with and later the enclosing blanket.

When there are high winds, shelter from protecting walls or screens should be sought.

If one does not have the conveniences for getting the patient out of the house, such a room as I have described may be used, every window being thrown wide open, or even the sashes removed, day and night, regardless of the weather. If it snows or is dusty, cheese-cloth screens may be used in the windows, and only a driving rain determines the closure in that direction.

As a rule, at night the patient is withdrawn from the open air to such a room.

The nurses must be dressed for out-of-doors-in winter in coats or furs, gloves or mittens, leggings, and hoods.

The fact that the patient is out-of-doors demands double vigilance; first, to prevent his exposing parts of his body to the cold, as may occur from disarrangement of the clothes by a restless patient, and second, especially when delirium is present, to guard against the carrying out of self-destructive impulses, accidents which form so lamentable a chapter in the disease.

When, for purposes of examination, local applications, the bath, the use of the bed-pan, etc., the body must be exposed, the windows are closed long enough for the temperature of the room to permit the procedure.

I am conversant with no measure in the treatment of acute infectious 
diseases that impresses one more promptly or more decidedly with its worth than this.

The most enthusiastic advocate of open-air treatment is the patient himself. 'The change in him begins almost immediately; respiration is easier, the pulse of better quality, nervous manifestations, restlessness, and delirim diminished, and sleep is improved.

On my service at Bellevie Hospital we have adopted this line of treatment as a routine, and I have never yet seen a pneumonia patient who wished to go back from the open air into the ward.

'To what this procedure owes its efficacy is not yet entirely clear. One might assume that it was to the greater content of oxygen in the air respired out-of-doors, but I have seen patients treated in rooms, with all the windows wide open in winter, show embarrassment of breathing within ten minutes after the windows were closed for one of the purposes specified above, and ask that they be opened again at once, entire relief ensuing. Certainly there could be no impoverishment of the air in the room in such a brief period.

Observations at Bellevue Hospital have demonstrated two facts of importance: first, that exposure to the open air will increase the bloodpressure 10 to 20 millimeters of mercury promptly, and that after a half hour in the ward, though it be well ventilated, a corresponding fall occurs, with again a rise on further exposure; second, that this rise is more marked when the temperature is low. ${ }^{1}$

Personally, I feel that the cold air, or the moving air, which has the same effect, stimulating the nerves of the face and nasal mucous membrane, causes a reflex effeet on the vasomotor nerves of the deeper vessels, as the splanchnics, to increase blood-pressure. It is highly probable that this is by no means the only factor in the beneficial results.

Care of the Body.- This important field of therapy, in which the trained nurse plays the dominant role, is too frequently and without excuse a "terra incognita" to the physician, who, when a trained nurse is not at hand, is compelled to leave the patient's comforts and needs to the chance of the environment, often with unhappy results.

A tepid sponge-bath, using Castile soap, should be given daily, both for purposes of cleanliness and confort. In a room at $65^{\circ}$ to $70^{\circ} \mathrm{F}$., little risk is run in exposing the body for the bath, but, if the patient or the family entertain fears, it may be done between blankets, exposing one part at a time.

Only at eritical periods, when the most rigid rest is enjoined, should the bath be omitted.

'The care of the mouth is of prime importance, as the coated tongue, the sordes upon teeth and lips, bits of food lying in dead spaces, contribute putrefactive processes and invite pathogenic invasion of the mucous membrane and adjacent structures.

Cotton swabs on wooden tooth-picks or other applicators, wet with a solution of borie acid (2 per cent.), or saturated (4 per cent.), should

${ }^{1}$ See Circulatory Failure in Acute Infections of Children, Causes, and Treatment, by John Howland, M.D., New York. 
be used on the tongue, cheeks, lips, and teeth, especially searching the dead spaces. Half-strength Dobell's solution may be used for the same purpose.

If there is a thick coat upon the tongue, or sordes on teeth or lips, half-strength peroxide of hydrogen will be found very useful, while the edge of a whalebone proves a valuable adjuvant in clearing the tongue.

When the mouth is very dry, equal parts of albolene and 2 per cent. boric-acid solution, with lemon-juice added, is a grateful application, while in case fissures are present, the breath fetid, and evidences of stomatitis are apparent, a good mixture for local use is the following: Carbolic acid (watery solution, 1 to 20 ),
Glycerine, (saturated watery solution),
Boric acid (saturation $\bar{a} \mathbf{a} f \bar{s} j ;$

f

Equally scrupulous attention should be given to the nose, lest by its obstruction the patient be converted into a mouth-breather, with an aggravation of conditions just mentioned. Dried secretions may be softened by sweet oil or vaseline, and the nose cleansed with boric-acid solutions of 2 to 4 per cent. strength, using the cotton swab on a tooth-pick as an applicator. The external nares, especially if there be fissures and excoriations, should then be anointed with the oil or vaseline.

The eyes must be kept free from collecting secretions by the use of boric-acid solutions of the same strength as for the mouth and nose. The genitals, parts sometimes neglected, should be attended to, especially in the very sick.

Diet.-In acute infectious diseases we know that the febrile condition, the pyrexia per se, increases the need of caloric intake some 20 to 30 per cent.- average 25 per cent. This, added to the requirement in health in the man of average weight, would make the demands of our patient some 2800 to 2900 calories.

Moreover, in acute infectious disease, proteid loss is increased by the effect of the fever itself and by the destructive effects of the toxins, a loss which is still further added to by starvation.

The theoretical quantity of food, then, is 33 calories plus 25 per cent. for fever, or about 40 calories per kilo, or, for the man of average weight of 70 kilos or 154 pounds, 2800 calories, which shall contain 70 to 100 grams of proteid.

Milk should furnish the basis of our dietary, and is far and away the best single food which we can offer our patient, but no single food quite meets our theoretical needs.

The analysis of good milk will show about 4 per cent. of fat, 4.5 per cent. of sugar, and 3.5 per cent. of proteid.

Using Rubner's figures, we should find that a litre of milk contained 700 calories. Average milk will run about 640 calories to the quart, or 20 calories to the ounce.

Now, it will be seen that in order to obtain the theoretical needs, 2800 calories, from milk, we should have to use over a gallon a day, which at once stamps the effort as impracticable. Moreover, that amount 
of milk would contain some 150 grams of proteid, an amount unnecessarily large, and perhaps even burdensome. If we furnish him with 70 or 80 grams a day, a sufficicney in his condition, we shall require but little more than 2 quarts of milk to obtain that amount.

It is now apparent that, to make up the necessary ealories, something must be added to the milk, or given in addition to the milk. 'This could be done by adding eream, one ounce in each glass or two ounces in each glass. Reckoning gravity cream (16 per cent.) as equal to about 100 calories per two ounces, in the first instance our milk would be enriched to 960 calories to the quart; in the latter, to 1280 calories to the quart. I have seen even richer milk and cream mixtures given to patients without harm, but, recogniring fat as the most difficult of the food-stuffs to digest, and its tendency to induce flatulency, and knowing that it does not rank with carbohydrate as a sparer of proteid, I prefer to add sugar to the milk, or cereals or other carbohydrate to the diet.

If 6 per cent. of milk-sngar were added, an amount which does not make the milk disagreeably sweet to most patients, one will get a milk of about 900 calories to the quart. Even larger quantities of sugar are well borne by many patients. Using this as a basis of our dietary, we may add cereals, such as oatmeal, one large serving of which, 5.5 ounces, affords 100 calories; or hominy, one large serving, 4.2 ounces, equaling 100 calories; or rice, an ordinary cereal dish of three ounces furnishing 100 calories. 'Two ounces of cream (100 calories) and three teaspoonfuls of gramulated sugar (100 calories) over such a cereal makes the total amount to 300 ealories.

Other foods which may be added during the febrile period are eggs, raw or soft cooked (each 70 ealories), cup-custard (ordinary eup, 100 calories), broths of cereals and milk, gelatine jellies containing sugar, drinks containing sugar. In prolonged cases bread and butter (one slice of white bread, one and a half ounces, 100 calories; one pat of butter, half an ounce, 100 calories), and in defervescence scraped meat and vegetables.

When milk itself is not well borne, some of the milk products may be substituted with success, but it must be remembered that they have a lower caloric value than the milk. Among these I may mention kumyss (one glass of 7 ounces, 100 calories), buttermilk (one and one-half glasses, 91 ounces, 100 calories), whey (two glasses, 13 ounces, 100 calories). Matzoon, zoolak, and other fermented milks may be given in place of kumyss. ${ }^{1}$

If the patient craves meat soups, or the appetite is improved by them, they may be allowed in moderation.

In considering the dietary, it must he remembered that at the onset, and when the intoxication is most intense, there is some impaiment of digestion, diminished saliva, and hydrochloric acid. 'The ferments and motility of the stomach and intestine, except in the distal end, are not much affected, and the alimentary canal soon regains its functions. to 1325 .

1 See Fisher's Tables, Journal Ameriean Medical Association, vol. xlviii, pp. 1316 
For the first day or two, when anorexia prevails, I should consider this symptom as a hint to desist from urging food, simply offering every two or three hours that portion of a glass of milk that can be taken without disgust or distress. If the food is taken when offered, and is well borne, inerease the milk up to two quarts a day, adding sugar to the milk later, and then cereals and eggs, the necessity for approximation to the theoretical caloric need increasing with the duration of the disease, thus obtaining in the prolonged bronchopneumonias and certain influenza pneumonias.

If vomiting, diarrhœa, or inereased flatulency oecur, cut out the cream or the milk-sugar, if either is being used, and, if this is not effectual, skim the milk, boil the milk, use buttermilk, or stop all food for a time.

If diarrhœa or flatulency occur when cereals are administered, stop them until their influence on the eondition is determined.

Drinks.- Water should be given in amounts suffieient to satisfy the patient's thirst, or if the patient is stuporous, should be offered him at regular intervals.

A study of the intake of water in a large number of cases of pneumonia by Wyss showed astonishing amounts-3000, 4000, 6000 cubic centimeters, and even more, which greatly exceeded the measurable output. Evidently, then, there is a very great water utilization in pneumoniawhether because of increased output through the lungs, as a result of increased respiration, or because retained in the tissues for other purposes, is not quite clear; if correlated with the retained chlorides, it would look as if water was retained for useful purposes. An increased viseosity of the blood in pneumonia, adding resistance to a weakened heart, and the knowledge of the disorders of metabolism incident upon a deprivation of water undergone by the tissues, make the administration of sufficient fluids imperative. In addition to water one may give lemonade, orangeade, or the juice of other fruits, as grape or grape-fruit. A good drink, a mild diuretic, and gentle laxative is the Imperial drink, made of $\frac{1}{2}$ ounce of cream of tartar to 3 pints of boiling water (to get it in solution), with the addition of sugar and lemon-juice or peel to the taste, given hot or cold ad libitum. These drinks carry with them in the sugar a considerable amount of nourishment.

Bowels.-The patient should be given a eathartic at the beginning of the treatment, if for no other purpose, to assure the physician that his patient shall not have poisonous materials absorbed from a constipated gut to add their deleterious effects to those of the toxins. Calomel and salts, or salts alone, may be administered. Calomel, on aceount of its anti-cmetic property, is especially indicated if there is nausea or vomiting. Give $\frac{1}{4}$ grain every fifteen minutes for four to six doses, or $\frac{1}{10}$ grain at the same intervals for ten doses. Three or four hours later follow the calomel with salts-Epsom, Rochelle, or Glauber's-in doses of $\frac{1}{2}$ to 1 ounce, in one-half or three-quarters of a glass of water.

The bowel should be emptied every other day by enemata or salines, but no drastic purging should be permitted, as it effects no good, and may induce exhaustion. 
I have never been convinced that a large dose of calomel at the beginning of pneumonia had an effect to abort the process, and so do not advise it.

But the state of the bowel demands our attention, not only for the avoidance of constipation and its results, but also beeause the intestine is specifically affected by the toxins of the disease, which, impinging on musculature or nerve supply, induce in the distal portion a condition of paresis, with consequent collection of gases - that is, meteorism. Meteorism occurs in over half of the cases and often very early in the attack. It is most marked in the severely ill, in alcoholics, in the aged, and is particularly common in infants.

The danger lies not only within the bowel, the paretic state of which encourages fermentative and putrefactive processes, but also in the fact that the collecting gases push up on the diaphragm, encroach upon the respiratory space, encourage atelectasis, and embarrass the heart action. For these reasons the relief of this condition often becomes the most urgent demand upon our therapentic endeavor.

The treatment aims at preventing the formation of gas by regulating the food, avoiding especially excesses of fats and sugar by attending to the proper evacuations. Relief is afforded by catharsis, by enemata, especially those containing the oil of turpentine, $e . g$., $\frac{1}{2}$ to 1 ounce of the turpentine to 1 pint of soap-suds enema; by hot stupes, or by adding a dram of turpentine to the hot water in which the flannel of the stupes is to be dipped, or by brushing the abdomen lightly with absorbent cotton saturated with turpentine before the stupes are laid on; by inserting a soft, pliable rubber catheter 12 to 15 inches into the bowel; or by oil of turpentine, given by the mouth in doses of $10 \mathrm{minims}$ in capsules or in emulsion, flavored with cinnamon or other aromatic, every four hours.

The tone of the intestinal wall may be improved by strychnine in doses of $\frac{1}{30}$ grain three or four times a day.

I will mention one other measure, the success of which, in the few cases that have come under my observation, has been exceedingly gratifying. It is the electric injection.

The bowel is filled with physiological salt solution (one teaspoonful to the pint), and a metal conductor is inserted into the rectum. The conductor must be covered with an insulator. A metal probe or applicator inside a catheter may answer, or a Kemp rectal tube, with a hard-rubber outside and a metal core. A faradic current, furnished by an induction coil or a galvanic current, completes the outfit. 'To the metal-conductor in the rectum is applied one pole (positive), and to the abdomen the other (negative), using a broad sponge electrode. With the faradic current one may use the whole strength of the battery of one cell if needed; of the galvanic, 5 to 20 milliamperes, or possibly a little more, avoiding the causation of any pain.

The response is usually prompt and striking. One may appreciate it by leading the end of the rectal tube under water to observe the discharge of gas. ${ }^{1}$

I I am indebted to Dr. Leander Shearer for the details of this procedure. 
Hydrotherapy.-Hydrotherapy is but a branch of the great field of physical therapy. This branch of our treatment, which includes air, water, light, heat, cold, counterirritation, electricity, massage, exercise, etc., I consider to have been grossly neglected in the plan of our medical education. That these measures afford comfort is a matter of daily experience; that they exert curative influences I am convinced. Of air I have spoken; of water I will add a word, because, though I would not sacrifice the benefits of the open-air treatment to the demands of hydrotherapeutic measures, there are times, as during the heated season, and places where the former cannot be carried out or reap the full measure of success, that invite the application of water.

Good results can only follow nicety of technique, and many measures of physical therapy have been condemned because of slothfulness of acquisition or slovenliness of application.

To no one do we owe more than to Baruch for the persistent and consistent advocacy of hydrotherapy in this country. I shall content myself with following his instructions closely. (See also article on Hydrotherapy.)

Water is used for purposes of cleanliness and comfort, also for curative effects, and it is of its use in this latter sense that I now write.

Baruch's preference is for the chest compress. For its preparation and application see article on Hydrotherapy.

It should fit snugly, but not embarrass breathing. A fresh compress should be ready for immediate application, as the chest should not be left exposed.

The compress is to be renewed each hour, if the rectal temperature is above $99.5^{\circ} \mathrm{F}$. and if the finger inserted under the compress finds it warm; if cool, it is not to be changed.

Dr. Baruch gives the following important advice: "Should the patient evince stupor or muttering delirium, a lower temperature should be adopted and the chest should receive one or more dashes of colder water before the renewal of each compress. The same procedure is indicated in bronchopneumonia when the bronchi are blocked by secretions or cyanosis exists. .. A higher temperature than $60^{\circ} \mathrm{F}$. may be used if there is much jactitation, insomnia, or excitability. In the latter event, great benefit will accrue from allowing the compress to remain two hours, and moistening it more thoroughly before application, thus converting the compress into a soothing fomentation that is not relaxing like a poultice."

If the patient remains cold and blue, and the compress does not get warm, then the flannel alone is applied until the skin is warm and a hot mustard foot-bath is given.

It is asserted that, after the momentary contraction of the superficial vessels on the application of the compress, a tonic dilatation ensues, "an active hyperemia by which the blood is propelled more vigorously through . . the vessels."

Moreover, it has been asserted that the pulmonary vessels are supplied with nerves, coming off from the second to the seventh dorsals, 
whose somatic supply is distributed to the skin of the chest and abdomen as far as the unbilicus.

Through this medium it is fair to assume that influences are exerted by the compress upon the vascular supply to the lungs.

The improvenent in the patients is seen in the deeper respiration, better quality of pulse, diminution in nervous manifestations, better sleep and appetite, moister skin, and freer diuresis.

I have given much space, and gone quite into detail in the consideration of the eare of the patient, because, with this and the assistance of fresh air or cold water, the great majority of our patients get well, and because without it some of our patients do not get well who otherwise might, and, finally, because the man who busies himself much with the care of the patient indulges himself little in the medication of the patient.

Symptomatic Treatment.-All symptoms do not need treatment, and not all of those that do require drugs. I believe that other measures of relief should be exhausted, as a rule, before we have recourse to drugs. In that way we come to use but fow drugs and learn to use the few well.

Fever.-The views on the significance of fever afford an interesting study, not always in agreement, but I think the trend of opinion to-day is voiced by Dr. W. G. MacCallum, who, in his Harvey Lecture, ${ }^{1}$ says of fever, that it "is in its essentials a protective reaction." Many physicians assume the correctness of the lay expression, "the patient is burning up with fever,"- and feel impelled to quench the flame.

'The truth of the matter is that the amount of heat produced during fever is trivial compared with that freed by many ordinary muscular activities of daily life. 'The significance of fever, so far as heat production and elimination are concerned, seems to be that the balance is not disturbed to any appreciable degree, but that this balance is established on a higher level by the heat-regulating center, which center, under these unusual demands, shows an instability, the degree and character of which are expressed by the charaeter of the temperature curve.

If this be so, there is no call or interference if the temperature is not excessive, and temperatures of $104^{\circ}$ and $105^{\circ} \mathrm{F}$., if the patient is otherwise doing well, are not to be considered as such.

'This does not mean that hydrotherapeutic measures are not indicated, for their effects on heart and lungs and other organs are of primary importance, and that upon the temperature is incidental.

However, it is possible for the heat-regulating mechanism to fail, and a degree of temperature to prevail that constitutes a real menace to life; we speak of this as hyperpyrexia.

I believe that the only safe and rational way of combating hyperpyrexia is by the use of cold-that antipyretics entail a danger to vital centers that renders their use warrantable only as a dernier resort.

One may have recourse to one of several procedures, such as a cold bath in the tub, after the manner of using a Brand bath in typhoid fever; a bath in bed, a trough being derised with the aid of a rubber sheet, whose

\footnotetext{
1 Arehives of Internal Medicine, vol. ii.
} 
edges are raised by rolled blankets or are fastened to a rope or clothesline run around the bedposts; a cold pack, the patient being enwrapped in a wet sheet over which cold water is poured or ice rubbed; or sponging with ice water, the patient lying on a dry blanket.

It is to be remembered that it is not desired or desirable to lower the temperature to normal, but to bring it within safe limits, and when the temperature, which should be taken from time to time, is at or about $102^{\circ} \mathrm{F}$. by the rectum, one should desist. The baths are to be repeated when the temperature approaches the danger-point.

Rectal injections of cold water are efficacious to some degree.

Cough.- The cough of pneumonia may be due either to the accompanying pleurisy or to bronchitis.

When due to pleurisy, it occurs early, is nagging, exhausting, and accomplishes nothing. It should be alleviated. This is accomplished by measures aimed at the pleurisy, such as counterirritation, application of heat and cold, or strapping the chest. If these are not efficacious, codeine in doses of $\frac{1}{8}$ to $\frac{1}{4}$ grain, should be given at two-hour intervals. In the most severe cases it is necessary to have recourse to morphine. One uses small doses to begin with, $\frac{1}{16}$ grain every four hours, increasing the quantity until results are obtained.

When the cough is due to bronchitis, it is purposeful, empties the bronchi of secretions, and should not be interfered with, unless it is exhausting, keeping the patient awake, and endangering a faltering circulation. The inhalation of steam from a croup-kettle or similar device, or steam medicated with the compound tincture of benzoin, pouring 1 or 2 drams on the hot water in the kettle, may be tried, or such a mixture as the following, first made known to me by Dr. Francis Delafield, which I have found very efficacious in some instances:

Alcohol,

Chloroform,

Creosote,

āā f ว̃ss.-M.

II. et Sig.-For inhalation. Pour ten drops on the moistened sponge of a Robinson's inhaler, ${ }^{1}$ a perforated zinc device fitting over the mouth and nose, and to be worn as long as the patient desires.

If the cough is persistent and exhausting, codeine or morphine may be used, as just described.

In very weak patients, where the signs of accumulating secretions are marked, the effort should be made to render the cough more efficacious, by turning the patient on his side to facilitate the discharge of mucus, and by the use of strychnine in doses of $\frac{1}{60}$ to $\frac{1}{30}$ grain, four times a day.

Personally, I object to the so-called expectorants, first, because I fear the disturbance to the stomach that may ensue, with impairment of appetite and nutrition, and second, because I am skeptical as to the accomplishment of what they are supposed to do. There are so many able clinicians opposed to my views, however, that I will mention ammonium chloride as the drug most commonly recommended, which may be used in 3-grain doses every two or three hours.

1 Practically the same inhaler is sold under other names. 
Pain.-If pain occurs in pneumonia, it is an early manifestation and due to pleurisy. Its relief is effected by such local measures as were referred to above, the use of the ice-bag and the splinting of the chest on the affected side by adhesive strips being particularly efficacious. Sometimes the pain is so severe as to demand an anodyne, of which the best are codeine and morphine, the choice and the dosage depending on the intensity of the symptom.

Toxemia. - While the whole picture of the disease, of course, expresses the action of the toxins, it is their attack upon certain centres, or organs of vital signifieance, that concerns us, such as the brain, the cardiovascular, and the respiratory apparatus. How rest, diet, and the care of the body limit the force of their attack I have touched upon, but I have also to consider the means of eliminating the toxins already elaborated. 'This means attention to the emunctories and their functions, as the skin, the kidneys, and the bowel. For the proper performance of their tasks nothing is comparable to a sufficiency of water. We have hinted at what is probably the role of water in pneumonia in maintaining the proper consistency of the blood, thus sparing burden to the heart; in maintaining accuraey of metabolic detail, thus preventing autogenous toxemia; at the increased demand for water from the increased respiration; and how jealously the tissues store up water, retaining sodium chloride to accomplish it, for useful purposes, doubtless, though they are as yet unknown.

For all these reasons water must be given the patient frequently and abundantly, satisfying the least demands of his thirst and anticipating its needs when the patient is too stuporous or weak to demand it.

Water is the best diuretic we have. If enough cannot be given by the mouth to encourage the kidneys, it may be given as an enteroclysis of physiological salt solution, or by the very excellent way suggested by Murphy, of a slow continuous irrigation, the inflow and outflow through a two-way rectal tube so regulated that it simply drips. This may be continued as long as it is not irksome to the patient. In the rare instances where the bowel fails as well, a hypodermoclysis of physiological salt solution may be used.

Of the drugs, my own choice is diuretin (the double salt of sodium salicylate and theobromin) in doses of 10 grains three times a day, or one of the alkaline salts, such as potassium acetate or citrate, or sodium bicarbonate, singly or in combination, in doses of 15 to 30 grains every two hours, until the urine reaction is alkaline, then the dose is gradually decreased.

It has been suggested by Wyss that, in consideration of the uses to which the body puts sodium chloride during a pneumonia, and appreciating how poor in the salt the diet is, that salt be added to the diet, and he advises, as the result of his observations, that 10 grams (5iiss) a day be given. He is convinced that the patients have benefited by this item.

Delirium.-We are prone to let the psychic aberrations of our patients crowd out of our minds the vast physical significance of delirium. Delirium is incompatible with bodily rest; it nullifies our most valuable 
therapeutic measure; it adds tremendously to the work of the heart and lungs; it entails delusions which lead to self-destruction. It cannot be too emphatically stated, then, that delirium is a serious condition that needs treatment. The amelioration of the cerebral manifestations when the patient is put in the open air is extremely gratifying. Hydrotherapy, too, is efficacious in this direction. Enough food, enough water, a clean skin, and effectual movements of the bowels are potent factors in diminishing delirium.

An ice-cap to the head is often of benefit.

When in spite of these measures, the restlessness of delirium and the exhaustion of sleeplessness are sacrificing our patient, morphine, and morphine alone, answers our needs. The depression of the respiration, the thickening of bronchial secretions, the danger of a habit in so short a disease, are objections that do not obtain if the drug is properly used.

The drug should be used hypodermically, and in the least doses that will accomplish the desired result, beginning with $\frac{1}{16}$ to $\frac{1}{12}$ grain.

In cases where sleeplessness rather than restlessness has been the dominant manifestation of the cerebral toxemia, one may use some of the milder hypnotics.

My own preference is for trional, 10 grains in a warm drink, or a little wine or whisky, to be repeated in two or three hours if needed; or chloralamid, 20 grains, to be given in a cold solution, as heat decomposes it, which, too, may be repeated, if needed, after the same interval.

In the delirium of alcoholics, paraldehyde, in doses of 2 drams to $\frac{1}{2}$ ounce, is a good hypnotic and sedative.

In these cases our chief reliance is morphine, and it must be used a little more liberally.

Of the use of alcohol in these cases a word will be said later.

Chloral, on account of its depressant effect upon the heart, vessels, and respiration, I advise against, holding its use legitimate only in the sthenic period of the early days.

Cardiovascular Symptoms.-Circulatory failure is a dread that never ceases to haunt the course of a pneumonia.

It may occur early when the toxemia is great; its advent is more frequent as the crisis approaches, and it does not always pass, even with the crisis. I use the expression "circulatory failure" advisedly, because, desirous as it is from the therapeutic standpoint to know what part of the cardiovascular apparatus is primarily at fault, we are still shuttlecocks between the battledores of conflicting opinions. There are some who believe that the seat of attack is the heart, undergoing anatomical changes as a result of toxic action, or, if not anatomical, at least functional, under the same influences. On the other hand, there are those who are profoundly convinced that the vasomotor system is at fault. It will probably be found that both are partly, neither wholly, right, but it has seemed to me that the evidence of animal experimentation and clinical observation points to the vasomotor centre in the medulla oblongata as the site of election for the toxins of the disease, while in the vast majority of circulatory deaths the heart has maintained its functional integrity up to the 
last. I am so far convinced of this that I urge therapeutic measures to be carried out on that hypothesis especially.

During the early or sthenic period of the disease, when the pulse is bounding and the heart strong and overacting, many practitioners use circulatory sedatives, such as aconite and veratrum viride. That they are not of value I am not ready to assert, but they are two-edged swords, these circulatory and respiratory depressants. I do not believe the benefit that may accrue in a few skilfully selected cases offsets the damage that may be done where the judgment has been lame.

In the occasional case, with bloated, deeply suffused face, laboring heart, and rapidly advancing lesion, seen in the early hours, these drugs: might seem indicated, but I prefer in such cases the withdrawal of blood by venesection. Eight to twelve ounces may be taken from the veins at the bend of the elbow, or wherever a prominent superficial vein makes the procedure an easy one.

With each day the possibility of circulatory failure increases; usually it begins insidiously, but sometimes with startling rapidity. 'The pulse, whose increase of eight to ten beats per minute for each degrce of temperature is looked upon as normal under the circumstances, now advances in rate and loses in quality, and that, too, though the temperature does not advance, or, more ominous still, when it is falling. It is more empty and gaseous, later thready; perhaps it intermits. 'The blood-pressure falls, and this study of blood-pressure during the disease I consider of the greatest importance, not only to determine a prognosis, but also as a guide in usage of drugs. 'The heart, under these evidences of circulatory failure, may remain strong in its action and regular almost up to death, bespeaking the fact that the vasomotor system is at fault. On the other hand, the first sound may be muffled, the second less accentuated, the cardiac outline increased, and that, too, without necessarily connoting anatomical change; but yet, of course, leaving us in doubt as to how far the impairment of the circulation is due to the heart or to the vasomotor system.

In considering the treatment, one must again reiterate the importance of rest, secured by attention to the bed, by niceties of nursing, and by treatment of delirium; the importance of a sufficiency of food, and especially water; of sleep and of relief of constipation; then comes the choice of drugs.

These latter may be divided into those whose chicf action is on the vasomotor system - caffeine, camphor, and adrenalin-and those which operate more particularly on the heart, as digitalis and strophanthus.

Of certain other drugs which have received a rather extensive usage as circulatory stimulants-alcohol, stryehnine, and nitroglycerine-I will say a word later.

Our procedures vary somewhat with the degree of rapidity with which the deterioration in the circulation manifests itself.

We will consider, first, the more insidious form. As soon as one assures himself of the waning of the cardiovascular forces, he should remember the virulency of the toxin he has to deal with, and how rapidly

VoL. I -46 
a structure attacked goes from bad to worse. Nothing is to be gained by temporizing. Of the drugs, my own preference is for caffeine, using the double salt of sodium salicylate or sodium benzoate and caffeine, on account of their ready solubility, in doses of 3 to 5 grains, hypodermically, at four-hour intervals. Its value rests especially in the fact that it stimulates the vasomotor centre, presumably just that point in the vascular mechanism most harrassed and most weakened. It raises bloodpressure in this way, and its effect may be appreciated by the sphygmomanometric readings. It is credited with action upon the heart, but we have not yet sufficient pharmacological data to make us place great reliance on that property. We do know, however, that it stimulates diuresis, acting on the kidney apart from or in addition to the effects wrought by the bettered circulation.

For all these reasons, and because so little toxic in therapeutic doses, it is a most satisfactory drug.

If caffeine fails to meet the demand made upon it, my next choice is camphor. This drug is less certain in its action than caffeine, and does not act as long, but often gives great satisfaction. The mode of administration is by the hypodermic needle, using a 10 to 20 per cent. solution in sterile olive oil. I believe it is ordinarily given in too small a dose. I use 3 to 5 grains every four hours, and have no hesitancy in increasing this dosage if necessary. 'The literature contains references of cases of pneumonia in which 4 and 5 grams (60 to 75 grains) a day have been used without bad effect. I have had no experience with such dosage.

The pharmacology of camphor has not been worked out in detail, but it seems to act upon the central nervous system especially, stimulating it from the beginning, so it may be concluded that its beneficial effect on the circulation is largely due to its action on the vasomotor centre.

The extract of suprarenal glands is the third member of this group of vasomotor stimulants. Trade names have been attached to the active extracts, such as adrenalin, suprarenalin, etc.

Adrenalin is indicated when a very rapid action is desired, as in threatened or actual collapse; it is, in a sense, an emergency drug, though it has been used at regular intervals, over a considerable period of time, when other drugs have failed. The dose is 10 to 15 minims of the 1: 1000 solution, intramuscularly.

It should be given straight down into a muscle, because the network of vessels in the muscle are such as to make the procedure practically equivalent to an intravenous one, as Meltzer has shown. The drug increases the blood-pressure strikingly and promptly, but the action is evanescent. Its main effect is on the vasoconstrictor nerveendings in the splanchnic area, while operating less on the vessels of the heart, lung, and brain; these organs thus receive a better supply of blood, and probably undergo a more lasting improvement than the short rise in the curve of blood-pressure would indicate. The drug also acts upon the heart like digitalis, slowing and strengthening it, but the effects are momentary.

I have expressed my opinion that, in the vast majority of cases of 
circulatory failure, the vasomotor centre is at fault, but also called attention to the difficulty of excluding the heart as sharing in the disturbance.

When, therefore, the sounds of the heart lose in distinctness, the cardiac outline increases, and arhythmia or intermittencies occur, although we know that in some instances the autopsy has shown such a heart to be anatomically intact, I feel that we should not neglect the possible need. and should have little fear of the effects of unnecessary stimulation on a sound organ.

In such a case digitalis is indicated. Any Galenical preparation may be used - the powdered leaf, the tincture, the fluidextract, or the infusion. The sine qua non is a freshly prepared drug from an active leaf. Nore important than any particular preparation is the knowledge of how to use some particular preparation. Personally, I am fond of the infusion, using 2 drams three or four times a day.

In the prolonged attacks one must keep in mind the possibility of accumulation, and intermit on the first expression of it, or after the third day of its use, for a day or two, as a precautionary measure.

I prefer to use the larger doses, intermitting occasionally, than to use small ones continuously, as one is sooner or later in doubt whether not enough or too much has been given.

Of the active principles, one can say that none represents the whole drug perfectly, and any one is a poor substitute for a Galenical preparation.

Various preparations, representing more or less satisfactorily the virtue of the leaf, have been put upon the market. I will mention two, because in fairly extensive use at present-digalen and digitpuratum.

It is very questionable whether these preparations contain what is claimed for them, but that they contain some of the active principles of the leaf there is no doubt. I have used them both and with results, but am not persuaded that they have any advantage over the official preparations.

Much work is being done on strophanthus at the present time, the result of which will be to place the drug in a new light, and make of it one of the most valuable members of our medical armamentarium. So far, one may say that the active principle, strophanthin, represents the total virtue of the drug; that, given hypodermically, it acts quickly, in thirty minutes to two hours, and in some cases apparently in ever less time, and that its effect is that of digitalis. By the mouth its absorption is so uncertain as to make its administration in this way difficult and even hazardous. I advise that it be given in doses of $\frac{1}{2}$ milligram ( $r \frac{1}{3} \gamma$ grain) to 1 milligram ( $\frac{1}{60}$ grain) hypodermically, to be repeated in twelve hours, but rarely again, thus giving digitalis a chance to operate. It is to be used where cardiac weakness and dilatation rapidly advance, and is an emergency drug for digitalis. 'This cautious advice is given in the knowiedge of its high toxicity in accumulation, and in the absence of clinical observation sufficient to define its limitations.

Alcohol has been much used in pneumonia, I believe without justification. I believe that it is never a true stimulant. I believe that its 
chemical structure, its pharmacological action, and its clinical effects stamp it a depressant. There are periods of excitation in pneumonia, constituting the so-called typhoid state, when alcohol apparently does good, but it does it as any other depressant or sedative, as morphine, would do it. In the delirium of alcoholic pneumonia it seems to do good, quieting as the restoration of a drug does for the time being to a drug habitué, or acting as a sedative, as paraldehyde will do. If, then, it does good under such circumstances, it should not be looked upon as a stimulant to be administered in conditions of circulatory failure. Indeed, I believe there are few cases in which the patient would not be better off without than with alcohol. The argument for its use as a food should be met by arguments for more rational dietaries in pneumonia.

Strychnine is another drug much used as a circulatory stimulant. It undoubtedly has a stimulant effect on the nervous system, and Cushny calls it more truly a general tonic than any other drug, but I believe that its stimulating action upon any part of the circulatory apparatus is feeble, and that it is too often relied upon to the exclusion of better drugs.

'The use of nitroglycerine is absolutely contraindicated, for it is a depressant to the vasomotor centre, and yet it is a common thing to see this drug given in pneumonia as if it were a stimulant of the highest value.

Upon caffeine and digitalis we will place our chief reliance in combating circulatory failure.

Collapse.-At any time during the attack, but more especially as the crisis approaches, collapse may occur. This is evidenced by the rapid prostration, chilly sensations, ashen face, clammy skin, cyanosis, soft, compressible pulse, and low blood-pressure.

The condition is urgent and relief must be prompt. Heat is applied to the extremities by hot cloths, hot bottles, or hot-water bags. Diffusible cardiac stimulants must be given. One of the best is the stronger water of ammonia on a towel flicked before the nose. Follow this with an injection of 10 to 20 minims of adrenalin deep into a muscle or into a vein. Give hypodermically caffeine, 5 grains, or strychnine, ${ }^{\frac{1}{0}}$ grain, and strophanthin, $\frac{1}{120}$ or $\frac{1}{60}$ grain, into a muscle. This should later be followed by digitalis, and the caffeine continued to maintain the circulation.

One of the most powerful stimulants to the vasomotor system is a high hot saline irrigation at $110^{\circ} \mathrm{F}$.

Hot coffee added to this increases its value. The effect of the heat locally is to constrict the splanchnic vessels and so increase blood-pressure.

Dyspnœa.-Dyspnœa is due in the early stages of pneumonia in some cases to pleurisy, the pain inducing a voluntary diminution in the breathing and insufficiency of oxygenation. This may be relieved by the measures taken to relieve the pain. Only when a very considerable amount of the lung is involved is the lesion responsible for dyspnœa, but the commonest cause is the intoxication of the respiratory centre. 
To relieve this condition, nothing is comparable to the open-air treatment, and the oxygen-tank should never be made a substitute. Indyspnoea the accessory muscles are ealled into play, and, as Magnus-lary has pointed out, this excess of museular action increases the demand for oxygen; thus, a vicious circle is established. It is possible that the slight increase of oxygen in the blood that can obtain when pure oxygen is inspired instead of pure air (18.3 per cent. hy volume instead of 15.3 , Magnus-Levy) may break this circle, as morphine does, by inducing muscular rest. When these measures fail, we may have recourse to such respiratory stimulants as we have at hand, in the shape of caffeine, strychnine, or atropine, but without much anticipation of result.

Pulmonary Edema.- Edema of the lungs is an occasional accident. Place the patient in the position in which he can breathe most easily, which is usually a semi-recumbent one. One local measure of value is cupping the chest, which should be done thoroughly. As the cedema is a circulatory phenomenon, relief is to be sought by measures aimed at it-injections of adrenalin, caffeine, strophanthin, and atropine. Venesection has, in some instances, been followed by satisfactory results. A measure lately advised, as the result of animal experimentation, and successfully applied in the clinic, is artificial respiration.

With the patient in a semi-recumbent position, the Sylvester method, elevating the arms slowly above the head and lowering them to press upon the sides of the chest and abdomen, is carried out twelve to fourteen times to the minute. It facilitates the movement of blood in the thorax and the discharge of secretions from the bronchi.

Specific Treatment.-Numerous efforts have been made to elaborate an antitoxic serum for pneumonia, but, in spite of an oceasional enthusiastic report, I do not think any encouraging results have yet been obtained.

The use of the extract of leukocytes, as described by Hiss, is now going on experimentally. Its value is sub judice.

Vaccination, using the dead bodies of the pneumococcus, seems to me the most promising field of specific therapy at present, but the results are too uncertain so far to allow of detailed mention here.

Convalescence.-Great judgment has to be exereised here, and tact, too, when the impatience of the convalescent has to be combated. The more severe the illness, the greater the eaution to prevent any untoward accident, the most dreaded of which are pulmonary embolism and cardiac dilatation. If the ease has been of average severity, the patient may be allowed to leave the bed a week after resolution has been completed. If there has been evidence of circulatory weakness, the getting up must be carefully regulated, the effect on the pulse and pressure being noted after being bolstered up in bed, and, when this is well borne, the patient allowed to sit up and then to walk. If the pulse is made unduly rapid by any effort, rest should be enjoined until strength has improved.

Feeding is an important item in convalescence. The food should be simple in selection, abundant in quantity, and well cooked. 
When the patient is up and about, his improvement may be bettered by a change of climate or even locality, but it must never be at the sacrifice of comforts and conveniences.

Prophylaxis.-As I have said elsewhere, prevention begins with the State and extends to the individual.

It concerns the national, State, and municipial boards of health. It involves clean streets, regulations to prevent spitting in public places, building laws to enforce sufficient light and air and prevent overcrowding, dissemination of knowledge through many channels, reports of cases, and statistics of disease.

Prevention in the individual means knowledge that the disease is due to the entrance of bacteria into a body rendered susceptible to their action. It means care to destroy the sputum of patients ill with the disease, to avoid careless contact with the sick, as kissing, exposure to the spraying of a cough, using contaminated dishes and spoons, etc., giving entrance to air and light, both deadly to the organism, appreciating that patients convalescing from disease may still carry virulent bacteria in their sputum for a long time, and that many of the common colds may convey the organism of pneumonia. It means the knowledge that the resistance is lowered by fatigue, mental worry, dissipation, alcoholism, and any other illness, all of which conditions make it doubly incumbent to be careful about exposing oneself to patients sick with the disease; that chilling by exposure to draughts, especially when overheated, may in all these states render one susceptible to the bacteria that we often harbor in health. We should not, in the hospital, expose the very sick, the aged, and the young to the direct environment of the pneumonia patient.

Maintaining the body in good health by proper hygiene, exercise, and food, keeping the mouth rigorously clean, and attending to disturbances of the upper air-passages, as tonsils, adenoids, teeth, hypertrophied turbinates, all of which facilitate the admission of the organism, constitutes true prophylaxis.

\section{PLEURISY}

Pleurisy, or pleuritis, as defined by the mere presence of adhesions or other pathological change in the pleural membranes, constitutes the rule rather than the exception as an autopsy finding; but clinically it constitutes only 2 to 3 per cent. of the admissions to a hospital service. These figures, however, make it a common disease, and, of course, the majority of lighter but clinically recognizable cases do not get into the hospital at all.

The significance of pleurisy has increased with the knowledge of the part the tubercle bacillus plays in its etiology. The vast majority of so-called primary cases, especially those with effusion, are due to this organism, and, indeed, in no case of the latter can we with confidence exclude it. Even in dry pleurisy a very large number of the primary cases are tuberculous.

Of the other organisms, the pneumococcus and the streptococcus are the most common, the former in the pleurisies accompanying lobar 
pneumonia and in the primary empyemata of children, the latter in empyemata accompanying both lobar and bronchopmeumonia, abscesses, and other suppurative processes in more remote parts of the body.

'The pleurisies are divided into three classes - the fibrinous, or "dry," the sero-fibrinous, or "pleurisies with effusions," and purulent or "empyemata."

From the standpoint of treatment they may be considered all together, since so many identical measures are directed toward each, though it may be said that the key-note of the treatment of "dry pleurisy" is expectant; of effusion, thoracentesis; of empyema, operative procedure. Empyema will not be considered in this section, as its treatment is solely surgical.

If the attack is a severe one, the patient will seek the bed without urging, as the pain and discomfort are great. Here he will instinetively seek the position that will give him the greatest relief, which is usually on the affeeted side, thus limiting its movements.

If there is fever, the bed should be insisted upon, even though the patient objects.

In the milder cases without fever the patient may be allowed to lie about or sit up.

The care of the bed, the choice of the room with reference to light and air, the measures directed toward the care and comfort of the patient, should all receive that attention to detail which has been set forth in the article on Pneumonia.

As for the open-air treatment, I earnestly advocate it in pleurisy, for it must not be forgotten that these cases are often cases of tuberculosis, and argument is no longer necessary to urge the open-air treatment of tuberculosis.

The bowels should be freely moved when the patient is first seen. A saline should be used, or calomel, followed by saline, $e$. g., calomel, $\frac{1}{4}$ grain, every one-quarter hour for six doses, followed in four to six hours, or the interval of the night, by $\frac{1}{2}$ to $\frac{3}{4}$ ounce of Rochelle or Fpsom or Glauber's salts in one-half to three-quarters of a glass of water. A milder saline, such as the liquor magnesii citratis, $S$ to 12 ounces, or a Seidlitz powder, or one of the many laxative waters on the market, or a simple enema, may be given every second day to assure freedom from stasis in the colon and rectum and the discomforts incident to constipation.

For the use of drastic purges to influence absorption in effusions I have no sympathy, for I believe the discomfort, exhaustion, and impairment of appetite that follow such procedures are in no way compensated for by the results that reward the theoretical consideration that dictated them.

The least satisfactory advice we can obtain with reference to the treatment of pleurisy is in the matter of the dietary. It ranges all the way from the dry diet of 'Tufnell, advocated for the treatment of aneurisms, to a pure milk diet, which is ss per cent. of water.

As for the dry diet, I can only state my conviction, that, if such pro- 
found changes are made by a dry diet in the fluids of the body as to influence the absorption of an effusion from an inflamed serous sac, it must producc other disturbances in organs and tissues, the extent of which we are not yet in a condition to appreciate, and may do much more harm than good.

If the fever is high, the pain acute, the patient suffering from anorexia and the malaise of the toxemia, the diet may be milk or milk preparations, koumyss, matzoon, acidified milk or buttermilk, in such quantities as the patient takes willingly and bears without distress; this obtains for the first day or two. Later, his food should be sufficient to meet the theoretical demands, and include milk, eggs, cereals, breadstuffs, soups, meat or vegetable, and, with the subsidence of the fever, fish, meat, and vegetables. If the fever is prolonged, these last may be granted, even before the temperature is normal, if the patient desires them, and they be given in moderation.

I have not the slightest hesitation in allowing the patients all the fluids that their thirst demands, and have no fear of accumulating the fluid in the pleura on that account. Besides water, the patients may be given lemonade, orangeade, or other diluted fruit-juices. The sugar added to these drinks affords no mean addition to the caloric intake.

There is no drug that may be considered as specific in the treatment of pleurisy.

Pleurisy has been considered in a certain number of cases as rheumatic in origin, and certainly does occur as an occasional complication of acute inflammatory rheumatism. In these cases, or when the patient has suffered from rheumatic fever before, it is entirely justifiable to try the salicylate treatment, and then in such doses as one uses in rheumatism, giving 15 grains of sodium salicylate, aspirin, oil of gaultheria, or other preparation every two hours, until one is convinced of the utility or futility of the treatment. The salicylates are well combined with alkalies, using 2 grains of the alkali to 1 of the salicylates, or 20 grains of the bicarbonate of soda or the citrate or acetate of potash, every two hours until the urine is alkaline, then reducing to just keep the urine alkaline.

The salicylates are recommended in this condition, not merely to combat any rheumatic element that may enter into the case, but because it gives relief to the pain, as it does in other painful conditions, and, whether rightly or wrongly, it has been credited with expediting the absorption of an effusion. The salicylates have the advantage of being the least harmful of the drugs administercd to relieve pain, and so fulfil a purpose in the treatment of pleurisy.

It is possible that certain local measures may have some influence on the inflammatory process, but, as they are all much more commonly used to modify the most frequent and characteristic symptom-painI will consider them under this heading.

Perhaps there is no effort to relieve the pain of pleurisy more simple or more effectual than strapping the chest. The object is fixation of the affected side. Fixation affords rest, by preventing the respiratory excursion more or less completely on that side, thus preventing the rub- 
bing of the pleural surfaces, which so aggravates the pain; moreover, it gives the processes of repair the best opportunity for accomplishment. This procedure is especially indicated in dry pleurisy and in the early painful stages of pleurisy with effusion.

When fluid has collected in the pleural cavity to a degree that embarrasses respiration, such fixation may interfere with the movement of the lung on the sound side to some degree, and should be dispensed with. Moreover, the rubbing of the pleural surfaces and the pain is removed, as a rule, at this stage and fixation is no longer indicated.

The method of application is as follows: A strip of adhesive plaster -the zine oxide plaster is the best, because the least irritating-about 5 inches wide, that is, wide enough to reach from the axilla to the edge of the ribs, and long enough to pass around one side of the chest, overlapping about 3 inches in front and behind, is held smooth and taut between the hands of the operator. 'The patient is in a standing position, if his condition warrants, or sitting up in bed. 'The operator, facing the patient's affected side, applies one end of the plaster to the back, about 2 or 3 inches to the sound side of the spinal processes, then, asking the patient to breathe out as completely as possible and ceasing to breathe, to turn slowly toward him, while the plaster is applied as tightly and snugly as possible during the rotation, overlapping about 3 inches in front.

Sometimes the shape of the cliest is such, either from adipose tissue, large breasts in women, or from other cause, that the plaster is better applied in three or four strips, overlapping like clapboards on a house. If there is any reason why a plaster may not be applied, a tight binder may be substituted.

If the chest is hairy, it should be shaved previously, to facilitate the removal. The removal should be by a sudden, sharp pull, unless the hair is abundant and adherent, when benzine or ether will soften the plaster and permit removal.

If strapping does not give relief, or for any reason it be deemed advisable not to try it, we may have recourse to cold, as, perhaps, the next most satisfactory means of effecting our purpose. 'The ice-bag is the best mode of application. 'There are ice-bags of many shapes, but the one I prefer, and which adapts itself nicely to the chest, is a circular one with a metal cap. 'The bottom of the bag should be covered with ice cracked to the size of the end of one's thumb. Over this is poured enough water to just cover the ice. The bag is then compressed until the water just comes to the level of the opening, thus expressing all the air. The cap is then screwed down. By this maneuver the bag is rendered flaccid and pliable, while, if it contains air, it is difficult of application. 'The bag is covered with a layer of linen or gauze, to intervene between it and the skin, and held in place by a towel or binder. It should be taken off at intervals of two or three hours, for continuous cold can damage the skin and underlying tissues. When taken off, a layer of oil or vaseline may be applied.

There are, however, a number of patients to whom cold is intensely 
disagreeable, or in whom the cold does not accomplish the desired result, and who do find relief in the application of heat.

'The simplest way to apply heat, the easiest to prepare, and the least disturbing to the patient is the hot-water bag. It has the disadvantage of being impossible to apply over an extensive area, and is difficult to retain in position, and so is often less efficacious than other measures. It may be tried first, however, and the others later.

Next we may try hot fomentations or compresses.

'Two or three thicknesses of flannel are wrung out of hot water as dry as possible. This is often best accomplished by using a dry crash towel for a wringer, twisting the ends to squeeze the water out of the contained flannels. The skin is smeared with vaseline or oil, and the flannels applied with dry flannels outside and kept in place by a binder. These are renewed when they become cool. They may be applied at intervals, a dry flannel being kept on in the meantime, or may be renewed as the indication, returning pain and discomfort, arises.

This method has the advantage over that next mentioned in the matter of neatness and ease of preparation, but it is difficult to attain prolonged heat, which may be accomplished by the flaxseed poultice.

The results obtained from a poultice depend much on the way it is made and the way in which it is applied. If we have a competent nurse, we naturally leave that to her, but, if we do not, we too often take it for granted that any woman in the household may do as well, or at least hope so, in the appreciation of our own ignorance on the subject.

For a poultice large enough for an adult chest we need flaxseed meal and water, in the proportions of two and one-half cups of the meal to three of the water. To the boiling water the meal is slowly added, stirring all the time with a spoon. When the mixture is just thick enough to drop, not pour, from the spoon, it is taken from the fire, beaten quickly to make light, and spread evenly, about one-quarter inch thick, on muslin or gauze, leaving a margin of some two inches all around to turn back over the edge of the poultice. The poultice is then covered with gauze, with a margin of three inches, to fold in and turn under the muslin between it and the protector, which is a piece of oiled silk or old flannel, which serves to keep in the heat. ${ }^{1}$

A binder is placed under the patient, the poultice brought to the patient in a towel or on a hot plate, to keep it warm, the heat tested against the face to prevent burning the patient, applied slowly, so that he can better bear the heat, the towel withdrawn, and the binder brought up around the poultice to keep it in place.

The poultice should not be kept on longer than one hour, as it will not remain warm longer than that. When it is removed, if the skin is very red, the site is smeared with oil or vaseline and covered with dry flannel.

Next to the applications of cold and heat, we may have recourse to the time-honored usage of counterirritants.

${ }^{1}$ I am indebted for descriptions of many procedures commonly applied by the nurse, to the excellent little work "Praetical Nursing," Naxwell and Pope, and to the courtesy of the Training School at St. Luke's Hospital, New York. 
Of the counterirritants, those in most universal use are sinapisms, applications which have mustard as their basis. 'I'hey are best applied either as the leaf or the paste.

'The mustard-leaf or plaster (charta sinapis) is dipped into lukewarm water and covered with gauze in such a way that one layer covers the mustard surface and three or four the back. 'The mustard surface is applied to the skin. 'The warm water permits the ferment, myrosin, to operate on the glucoside, sinigrin, setting free the volatile oil, which is the active irritating substance. 'The leaf is left on until the part is well reddened, which usually takes about fifteen to twenty minutes. It is then removed, the part washed with soap and water to remove the particles of mustard, and covered with a thin film of vaseline or oil.

The paste, a favorite method of applying the mustard, is made by mixing one part (one tablespoonful) of mustard with four of flour, and adding enough tepid water to make a paste of such consistency as will spread nicely on gauze or cheese-cloth. 'This is covered with gauze and applied to the chest. It is left on the same length of time as the leaf. Tepid or cold water is used in making the paste, because hot water destroys the ferment, and so prevents the evolution of the volatile oil.

If the skin is tender or the patient is a child, five, six, or more parts of flour may be used; if thick and tough, three or two, or even equal parts of flour and mustard, may be mixed.

'The white of an egg, or a little oil or vaseline, added to the paste, makes it less irritating to a sensitive skin.

These applications may be made twice or three times a day or every four hours.

Tincture of iodine has been used as a counterirritant, but it is not so easily regulated as the mustard, and far more likely to set up an annoying dermatitis.

At times a far more decided impression on the sensory nerves is needed than mustard affords, and this is found in the application of the cautery: The cautery is lightly flicked over the surface of the chest to which the pain is referred, care being taken not to more than redden the surface or, at the most, to raise a slight blister. After the application the part is covered with a thin layer of oil or vaseline and gauze.

Still another method of counterirritation has to be mentioned, namely, blisters. Personally, I prefer not to use them in this region and condition, first, because I believe that little may be anticipated from their use that may not be attained by the other two methods just described; second, because they leave a sore and often raw spot, the discomforts of which replace, if they are not added to, those they sought to relieve.

Another local measure that time and experience have demonstrated to be of value is cupping. The analysis of its modus operandi is not yet satisfactory, but it may be efficacious in part by modif ying the distribution of blood, and in part by operating through the nerve reflexes. Perhaps the best results are obtained when the attack is acute and sudden and the measure applied early.

Cups are made for this specific purpose, but any small glass-egg- 
glasses, wine-glasses, or medicine glasses-will answer. They should be of thin glass, that their own weight may not drag them off, and have as wide a bottom relative to rim as possible, so that a large volume of air may be displaced in forming the partial vacuum. There are several ways of exhausting the air, any one of which is good. One of the simplest is to insert in the bottom of the cup or glass a very small shred of absorbent cotton, light it with a match or alcohol flame, and, when it is nearly consumed, clap the glass on the part. The flame is quickly quenched in the rarefied air, and if it falls on the skin it is too light and evanescent to do harm, or the inside of the glass may be moistened with alcohol by means of a swab and set alight. Here precautions must be taken to use but little alcohol, lest a drop fall on the skin, when a severe burn may ensue. Moreover, the edge should be wiped dry before lighting. Still another way is to set afire a swab soaked in alcohol and held up in the inverted glass, or even hold the inverted glass over the flame of an alcohol lamp.

The skin to which the cup is to be applied should be clean, and when the cup is to be reapplied it must be wiped dry, as the moisture from combustion gathers on the inside of the glass and makes its application more difficult.

The cups should be left on until the skin is well congested; one can easily rupture the superficial capillaries and draw serum in large beads through the surface of the skin; but this is no advantage. To take off the cup, depress the skin at the edge of the cup and let the air in.

Wet cups and leeches have both been used for this condition, but I think they are of questionable utility.

If, for somes eason or other, these local measures may not be used, or if they prove of " 10 avail, one must have recourse to drugs.

In the mild $r$ cases we may use some one of the coal-tar seriesphenacetin, an syrine, or acetanilid. These may be given in single large doses of $15,8, \mathrm{r} 5$ grains respectively, as the occasion demands, or in smaller doses of 3,2 , and $1 \frac{1}{2}$ grains respectively, every hour or until relief is obtained, or the combined doses amount to about double the single large dose. I prefer the smaller doses, a favorite prescription being:

R. Acetanilid,

Citrated caffeine,

Bicarbonate of soda,

Sig.- To make one capsule. given cvery hour or two.

gr. iss;
gr. ss;
gr. j.-M.

If these drugs are not effectual, codeine, in doses of $\frac{1}{4}$ to $\frac{1}{8}$ grain, at intervals of one to two hours, may be used.

If, however, relief is not obtained after a few doses of codeine, or if the pain is intense to begin with, morphine should be used hypodermically; the dose may run from $\frac{1}{12}$ to $\frac{1}{4}$ grain, according to the degree of suffering.

Cough and dyspnoea are in the early stages due to the same cause as the pain, and the indications for local applications and for drug administrations are the same. 
Such is the treatment of a dry pleurisy during its onset and its course, and such is the treatment of a pleurisy with effusion during its early stages, before the effusion has become well established; but when that occurs, we have to turn our attention to measures that will facilitate the removal of the fluid from the pleural sacs. 'The measure par excellence is thoracentesis, and I think that the teaching of Dr. Francis Delafield, that it should be early, that there is nothing to be gained by delay, and much to be lost by waiting, is a correct one.

However, the patient may object to the procedure or flatly refuse it, or other reasons may obtain for its omission or delay, and under these circumstances one may have recourse to less certain efforts by drug administrations, or the applications of counterirritants, as already described.

Besides the salicylates, which I have already mentioned, one may try the diuretics, with no great anticipation of results, however. Of these, the best are diuretin (the double salt of sodium salicylate and theobromin) in doses of 10 grains three times a day, and the alkaline diuretics, potassium acetate or citrate, or bicarbonate of soda, 15 to 30 grains, three or four times a day. 'These latter are often combined in equal parts to make the same dose collectively.

Thoracentesis. - Whatever be the individual opinion as to the advisability of early tapping, there is a consensus of opinion among practitioners the world over that thoracentesis is indicated whenever there is such an accumulation as to embarrass either the heart or lungs, as shown by eardiac weakness, cyanosis, and dyspnea; when there is enough fluid in the ehest to dislocate the heart considerably; when a moderate amount of fluid is persistent, defying other measures for its removal.

But there is a growing acceptance among elinicians of the teaching of Dr. Delafield, whose words I quote: "I believe that aspiration is to be used not simply to remore fluid from the ehest, but to eure pleurisy as a morbid process," and further, "this means that, as aspiration is the treatment of pleurisy, it is to be performed as soon as the presence of fluid in the chest is to be made out." 1

With this opinion was published a report of 200 eases so treated, and the figures will convince any one who will take the trouble to look up the referenee here given that the opinion was well based.

Not only does the treatment advocated shorten very materially the course of the disease, but the extensive pleuritic adhesions that characterized expectant treatment with the subsequent bronchiectatic cavities are avoided, as well as the chronie interstitial pneumonia and secondary tubereulous involvement of the lung that finds so easy progress when that strueture has been long compressed.

When the signs of fluid nppear in the chest, an exploratory puncture should always be made before aspiration is done, for two reasons: first, because physical signs are no guarantee of the certainty of the diagnosis, while the fluid withdrawn is; and, second, because the nature of the fluid withdrawn must be taken into aceount in the procedure that follows.

1 American Journal of Medical Sciences, 1902, vol. cxxiv. 
If the fluid is clear, a simple thoracentesis is indicated; if pus, the chest is to be opened by a surgical operation; but if it is turbid, the decision as to the procedure is not so easy.

The fluid should be submitted to microscopic and bacteriological examination. If the culture shows streptococci, the case should be treated as an empyema; if there are pneumococci, which grow easily on culture, and there are a predominance of polynuclear cells in the exudate, it too, demands operation, but if they do not grow well on culture, and the polynuclears are but moderately increased, thoracentesis should be tried.

It has been repeatedly observed that the withdrawal of such minute quantities as one takes in an exploratory puncture, the matter of a few drops determines the beginning of an absorption of the effusion that may go on uninterruptedly until complete. A satisfactory explanation is not forthcoming, so that any obvious change in the level of the fluid from the day of the exploration to the next might seem to warrant waiting, but its doubtful occurrence should not be set against the advantages of the earlier removal of the fluid.

There are two ways of emptying the chest-by siphonage, the method in vogue in Germany, and by aspiration, in almost universal usage here.

For aspiration, some modification of the Potain apparatus is commonly utilized. The essentials of a successful aspirator are a needle or a trocar and cannula, to which a rubber tube is attached, passing to a bottle or other container, which in turn can be exhausted by a pump or some other device.

It is well to have a piece of glass tubing introduced into the rubber tube to enable one to see how the fluid is running.

'The needle is the easier of introduction, but has the disadvantage of wounding the lung if it comes in contact with it, and of easily getting plugged with fibrin. The trocar and cannula are more difficult of introduction and of more intricate mechanism, but free from the above objections.

'The needle should be about three inches long, and longer for a thick chest, and one-sixteenth to one-twelfth inch in diameter.

The tube should be of thick, firm rubber, and about a yard long. There should be a clamp attached with which to regulate the flow-an artery clamp will do.

The bottle should be of 1500 to 2000 cubic centimeters' capacity, and can be readily graduated by applying strips of adhesive plaster at the levels of given quantities previously determined.

The bottle may be exhausted by a pump, or, better yet, by a clever device of Dr. Karl Connell, ${ }^{1}$ namely, by burning alcohol which has been run in small quantity about the inside of the bottle and stoppering as the flame dies out. The stopper is of rubber or of cork, and is perforated by a glass tube, to which the rubber tube is attached; the rubber tube is clamped, of course, to prevent the advent of air into the bottle.

$$
{ }^{1} \text { Medical Record, July 4, } 1903 .
$$


All this apparatus must be earefully sterilized. 'The operator's hands and the field of operation should receive the same precautions with reference to the cleanliness as in surgical procedures of greater magnitude. Boiling for the instruments, and soap and water and alcohol for the skin, are the best, and any antiseptics that may interfere with the cultures desired are, of course, to be avoided.

A hypodermic needle, with such stimulants as may be needed, should be at hand.

'The best position for the patient is in a sitting posture, with the hands on the opposite shoulders, to widen the intercostal spaces. 'The site of election is in the fifth or sixth intereostal spaces, in the midaxillary line, or in the seventh space, between the posterior axillary line and the scapular line. 'This site avoids the dome of the diaphragm, which rises as high as the fourth space in the nipple line, the sixth in the midaxillary, and the eighth in the scapular.

Another site advised is one inch in front of the posterior axillary line, in the space that lies just below the angle of the scapula. It is easy to reach, the space is wide, and it is remote from important organs.

The average chest-wall will vary from four-fifths inch to twice that thickness. Musser has given the distance of vessels at the root of the lung at a level of the fourth costochondral junction and the seventh dorsal vertebra as 4 inches on the right side in the midaxillary line and 23 inches on the left. In the posterior axillary line the distance on the right is $5 \frac{1}{4}$ inches, on the left, 5 inches. As our sites are below this level the danger of puncture is less.

A local anesthetic is rarely neeessary, but, if the patient is insistent or nervous, an ethyl chloride spray may be used to freeze the part, or a dilute cocaine solution used to infiltrate the underlying tissue.

'The space to be entered should be clearly palpated, the thumb of one hand determining the edge of the rib below, while the nail serves as a guide for the point of the needle, so that it shall be kept away from the intercostal artery that hugs the rib. The needle or trocar, with the blunt end held against the palm of the hand, and the forefinger marking off the probable thickness of the ehest-wall, is thrust quickly and firmly in until the non-resistance to its advance tells of the entrance into the cavity.

In some cases the skin may be so thick that a slight incision may be needed to introduce the point of the needle.

The coupling should now be made, or, if already made, the clamp should be loosened gradually, that the flow may be slow.

The amount of fluid to be withdrawn depends on the individual case. It depends on how early it is done, how much fluid is in the chest, and how the patient reacts to the operation.

Most of the accidents may be attributed to the sudden return of bloxd to the vessels of the compressed lung. Hence, if the fluid is withdrawn very soon after its appearance, the probability of much compression is lessened, and, if no untoward symptoms set in during the procedures, all may be allowed to drain out that will. If the amount of fluid is 
large, the compression will be greater, and still more if it has been left for a long time.

Much, too, depends on the rapidity of the aspiration, for, if the fluid is drained off slowly, the expanding lung and its filling vessels have time to readjust themselves to the new condition in some measure.

If there is much fluid and everything goes on well, it is rarely necessary to take away more than 1500 cubic centimeters, and the better part of a half hour should be consumed in doing so. The balance of the fluid will often disappear promptly; if not, another tapping can be done.

If, however, either violent coughing or severe pain, or dyspnœa or hemoptysis occurs, or heart weakness ensues, the procedure should be stopped at once and the patient put in a recumbent position.

A slight feeling of faintness may arise from fear of the operation. This is to be relieved by a little whisky or a glass of cold water.

Pneumothorax may arise from puncture of the lung, entrance being made into a bronchus or cavity, or air enters by the needle or trocar.

While fatal cases are known to the literature, they are very rare, and the accident need not cause undue alarm. Sometimes subcutaneous emphysema may be caused by air following the needle from a punctured lung on its withdrawal. I have seen these results on more than one occasion. Sometimes the absorption of air was rapid; again it lingered for some days, but in no case have I seen alarming symptoms.

More rarely and more formidable is the appearance of pulmonary œdema with abundant albuminous expectoration. This may occur during the tapping or just after, and, in rare instances, later. The most plausible explanation is transudation from the congested capillaries, whose walls have undergone impairment of their integrity during compression.

It is more likely to occur after the withdrawal of large amounts of fluid, and especially when rapidly withdrawn. There may be cyanosis, dyspnœa, rapid, weak heart, and fall of blood-pressure.

Another accident, fortunately rare, but frequent enough to make its mention of importance, is the sudden onset, without warning, of cyanosis and dyspnœa with weak heart, fall of pressure, unconsciousness, and death. Numerous theories have been advanced to account for it, but none have been so suggestive as those of Joseph Lewis and Dean Capps, as the result of experiments carried on in the Laboratory of Experimental Therapeutics of Chicago University. ${ }^{1}$

They attributed these accidents to the result of irritation of the inflamed pleura by the instruments used.

The lessons they draw are that great care should be taken not to wound the pleura by scratching with the needle, and for that reason the cannula is preferable to the needle.

This condition is to be met like that of shock or collapse from other causes, the most valuable drug being adrenalin.

After the thoracentesis has been performed, one may anticipate, not only an amelioration of the urgent symptoms that may have been present,

${ }^{1}$ American Journal of Medical Sciences, 1907, vol. cxxxiv. 
but also a more rapid disappearance of temperature and the absorption of such part of the effusion as may have been left behind. However, we are not always so fortunate, and may have to repeat the tapping and have recourse to the effects of a change of climate or of drugs.

Our measures may have been so delayed that, even after the removal of the fluid, we still have to deal with a compressed and adherent lung. Under these circumstances our efforts must be directed toward developing it.

Even if everything goes well, the patient ought not to be up and about for a week after the thoracentesis, and if there is a pain, accumulating fluid, fever, or pulmonary complications, none of the measures for expanding the lungs should be begun.

'The most valuable effort to expand the lung is in establishing breathing exercises. 'They must be done very gradually, and on the appearance of pain cease. For the technique I quote from the excellent article on Pleuritis for Osler's System by Dr. Fred Lord: "Forced inspiration from six to twelve times every two hours may serve as a beginning. Later, with each inspiration, the outstretched arms on the diseased side may be elevated to a horizontal, then to a vertical, position, with coincident compression of the sound side by the other arm. Lateral deviation of the sound side during inspiration more forcibly puts the diseased side on the stretch. It may be combined with a similar movement, holding both arms outstretched at right angles with the body. This, as well as torsion of the trunk with the arms similarly placed, should, however, be preceded by simpler exercises and practised only late in the course."

Another method advised to make forced inspiration effectual is to sit in an arm-chair with the sound side against the arm of the chair, holding oneself by the grasp of the hand upon the rung of the chair on that side, while deep inspirations are taken, which, as the sound side is fixed, will be expended on the compressed lung.

A favorite method of expanding the lung is by blowing into a bottle, transferring water in that bottle into another, connected by a rubber tube, by the pressure of the expired air. These bottles should contain about a gallon each.

After-treatment.-It must not be forgotten that a very large majority of the cases of pleurisy are of tuberculous origin, and, for that reason, constant vigilance must be exerted to detect the earliest evidence of involvement of the lung in such a process, and, as this possibility exists, an abundance of fresh air, a sufficiency of diet, and suitable hygienic measures are of the same importance as in a known case. A change of climate and the effect of new scenes and new interests are no mean factors in hastening complete recovery.

VoL. I -47 



\title{
TUBERCULOSIS
}

\author{
BY H. R. M. IAANDIS, M.D.
}

The first step in the treatment of tuberculosis is to explain to the patient the nature of his trouble. In many instances the verdict is anticipated, and causes no surprise. But no matter whether such an opinion is looked for, or whether it comes as a disagreeable shock, the truth should be told. One is not infrequently cautioned, either by a member of the family or by the attending physician, not to tell the patient that he or she has tuberculosis. I have never consented to do this, and I have yet to see the patient who was harmed by being told the truth.

While there is only one kind of truth, there are a number of ways in which it may be imparted. 'To those who demand a true statement as to their condition it should be given frankly and without hesitation. If there is reason to believe that the patient is unaware of the possibility of having tuberculosis the truth can usually be conveyed in an indirect way, so that the initial shock is lessened; and in some instances it may be advisable to see the individual a second or even a third time before the true nature of the illness is divulged. Cheerfulness and encouragement as to the outcome will do much toward relieving the apprehensions of the patient.

In any event the physician, both for his own sake and for the sake of the patient, should avoid concealment. "To fool a patient is tyranny, not guidance. He must understand what is being done for him if he is to do his part properly. 'This means team-work of doctor and patient." (Cabot.) Sooner or later he is going to find out what the trouble really is, and when he does he is sure to resent being fooled. Furthermore, a patient who is deceived by being told that he is nervous or run down, or that he has stomach trouble or bronchitis cannot be made to observe the precautions necessary for recovery and for the safety of those about him.

Individual Prophylaxis.-The prevention of tuberculosis should always be uppermost in the minds of those dealing with the disease. The needs of each case should, of course, be first considered, but in every instance the simple rules of individual prophylaxis should be explained. 'This should never he omitted. While the necessary precautions are simple and easily carried out they are frequently neglected, and it is only by constant reiteration that some patients are educated in this respect.

Tuberculosis can be and is acquired from sources other than the sputum, but it is pretty generally acknowledged that if we can insure the destruction of the suptum the danger of spreading the disease is reduced to a minimum. Without in the least minimizing the danger, one should 
avoid exaggeration. Phthisiophobia has become so prevalent, largely through exaggeration, that there is urgent need of explaining to the family and friends under just what circumstances infection may occur. The careless consumptive is a menace but the careful one is not.

In no phase of the treatment is it so necessary to explain the reasons for the precautions ordered. 'The mode of infection by means of the sputum should be explained, so that the patient will have a clear conception of what it means, not only to hinself but to others.

'The mouth should always be covered during the acts of coughing and sneezing. For this purpose paper napkins should be used. Washable handkerchiefs are as objectionable for this purpose as they are for receiving the sputum. Control of the cough, especially in public, should be practised (see page $7 \$ 2$ ).

The most effectual method of destroying the sputum is by burning, and whenever possible this should be the method of choice. A most efficient method of disposing of the sputum is by the use of paper nakpins (toilet paper will answer the same purpose). Several thicknesses are folded once, so as to receive the sputum; the paper is again folded and the ends folded over so as to enclose the expectorated material, and then placed in a grocer's bag (about 6 by 12 inches). The bag can be pinned to the side of the bed or clamped to the small bed table. Several times a day, depending on the amount of sputum, the bag and its contents should be burned.

If a cup is used it should preferably be one that can be destroyed by burning. There is now on the market an impermeable pasteboard cup which fits into a tin holder. This pasteboard cup comes already creased, so that it can readily be fitted into the holder and renewed as required. The tin holder should be placed in boiling water at least once a day.

In disposing of the sputum by burning it should be seen that there is sufficient fire to quickly and completely destroy it. The addition of sawdust, newspapers, or kindling wood facilitates this. Unfortunately, with the general adoption of gas ranges, destroying the sputum by burning is often not feasible.

If non-destructible earthenware cups are employed they should contain four or five ounces of a 5 per cent. solution of formalin, creolin, lysol, or phenol; a 2 per cent. solution of chlorinated lime is equally efficient, and, besides, is much cheaper. The cup should be thoroughly scalded with boiling water each time it is emptied.

For ambulant patients a pocket cup should be carried. The flat sanitary pocket cuspidor manufactured by Seabury \& Johnson is one of the best of this type. This cup is destroyed by burning. The paper bag and paper napkins can also be used by ambulant patients. Rubber pocket linings are not to be recommended.

After coughing the mouth should be wiped with a fresh paper napkin, which should then be placed in the paper bag. Bed patients should not be allowed to crumple the napkin in the hand and retain it for future use.

Floor cuspidors should not be employed by a consumptive in the 
home. Indeed, he should always have about him the necessary means for disposing of his sputum, and never expectorate on the pavement or in public cuspidors.

Patients must especially be warned against using washable handkerchiefs for receiving the sputum, and also against swallowing their sputum. 'The use of handkerchiefs for this purpose not only endangers the individual who has to wash them, but also exposes the patient himself to the danger of reinfection through contaminating his hands or inhaling the particles of dried sputum if he again uses the handkerchief. A washable handkerchief that has unavoidably been used to receive the sputum should be soaked in boiling soap-suds, or a 2 per cent. chlorinated lime solution, or a 2 per cent. lysol solution, before going to the laundry.

Swallowing the sputum, besides being a filthy practice, is apt to cause gastric disturbances and the still greater danger of intestinal tuberculosis.

If sputum is accidentally spilled on the floor it should be wiped up with a paper napkin and a solution of formalin (10 per cent.) or phenol ( 5 per cent.) poured on the infected spot.

Male patients who wear a beard should be urged to remove it. 'The moustache should either be removed entirely or at least cut close; if it hangs down over the lips and the sputum is profuse, removal is imperative.

'The mouth should be thoroughly rinsed with plain water or some simple mouth wash (equal parts of listerine or euthymol and water or hydrogen peroxide and water) after the morning attack of coughing. 'The same thing is also advisable during the day if large amounts of sputum are brought up at intervals. Swallowing should be avoided until the particles of sputum which may adhere in the mouth are removed by the rinsing.

After rinsing the mouth the teeth should be cleaned. Toilet articles used in the care of the teeth should be kept apart from those of other members of the family.

Kissing by the tuberculous patient should be forbidden, and the danger from this source should be impressed upon parents, especially if it is the mother who is tuberculous. 'The father rarely comes into as close contact with the child as the mother, who is the one who usually administers to its wants. 'The mother should be especially warned against the practice of testing the temperature of the food and then feeding the child with the same spoon she has had in her own mouth. A tuberculous woman should not nurse her baby; even if her disease is quiescent, the practice is questionable, and should be avoided if possible.

'The patient should never share a bed with another person. 'The practice of having small children sleep with an adult is a bad one, under any circumstances; but if tuberculosis is present it is doubly important to prevent it.

When treated at home the consumptive should always use the same eating utensils. 'These should be washed separately, and kept apart from those used by the rest of the family. After each meal the dishes, 
knife, fork, spoons, etc., should be thoroughly scalded with boiling water; then washed with soap and warm water, and finally rinsed with clean water.

A patient who observes the above precautions is practically of no danger to those about him.

The feces, as a rule, are disregarded. They may be sterilized by putting in the bed-pan eight to ten ounces of a 10 per cent. solution of formalin. A cheaper and equally efficient agent is chlorinated lime (4 per cent.). Milk of lime is efficient, but bulky, and is apt to clog the water-closet.

Those attending tuberculous patients should exercise the same care regarding their hands as they would use if caring for a case of typhoid fever.

In communities where compulsory notification is required, physicians should see to it that the requirements of the law are fulfilled.

Financial Resources.-In order to intelligently advise the best course to pursue it is necessary to ascertain what the resources of the patient are. This precaution is commonly neglected, and constitutes what Cabot has termed "blindness to backgrounds." "We must go into the matter of his income and outgo and see if the two ends cannot be made to meet." The physician who does not do this is frequently guilty of giving advice that is useless, because of the inability of the patient to carry it out.

"I have heard physicians giving advice to patients not to worry, advice that would be laughable if it were not so pathetic: 'Just stop worrying' (you might just as well say stop breathing) 'and take a long rest. Avoid all mental and physical strain.' What his wife and children are to do meanwhile never occurs to this type of physician. The wife and children are in the background, out of range of his vision, and so for him they play no part in the case." (Cabot.)

The best advice is that which can be followed, and nothing can be worse than to tell a man without resources that he must go away if he hopes to recover. Either this advice is neglected entirely, or, as sometimes happens, the patient goes where he has been directed, but without the means of support after he arrives at his destination. 'The result is that he commonly becomes a charge on the community. It is now an accepted fact that a patient should not be sent away for climatic treatment unless he can obtain the same degree of comfort as that to which he is accustomed at home. Pure air does not compensate for discomfort and poor food. Furthermore, no patient should be sent away with the hope of obtaining employment in some health resort. Such places, as a rule, are overcrowded with work-seekers from all walks of life. Even an arrested or quiescent case should not seek a climatic change, with a view of permanent residence, without having sufficient means to support him until he can secure suitable employment.

Communities in the West and Southwest to which many tuberculous patients go have entered a strong protest against the practice of sending to them individuals far advanced in the disease, particularly when they 
are without the means of support. While many of threse patients seck these places on their own initiative, not at few of them do so on a doctor's advice. It is estimated that thousands who have no chance of recovery go to these places, and that many of them, owing to lack of funds, become public charges.

For those whose circunstances are known to be comfortable these preeautions are, of course, unnecessary; but for those only moderately well-to-do, and for the poor, the character of the advice griven will depend entirely on what they can aflord. 'To this extent tuberculosis is a discase controlled by the poeket-book.

Inquiry should be made as to sick benefits, savings, and the possibility of securing aid from other members of the family or from employers. In addition, it should be known whether the money, if olstained from any of these sources, is avalable for the patient alone or whether during the period of his inactivity a wife and children are to be cared for.

In advising a change, the physician should be able to inform the patient of the cost of the journey to and the cost of living in the particular place recommended. Information of this character may be found in the Tuberculosis Directory, issued by the National Association for the Study and Prevention of 'Tuberculosis.

'The length of time necessury to remain away should also be stated, even if it can only be approximately estimated. It is team-work of this kind that enables the doctor and patient to secure results that would otherwise be unattainable.

The Physical Status of the Patient.-In coming to a decision as to whether the patient should be advised to go away, one must also take into consideration the extent of the disease. Judging from some of the cases I have seen sent to sanatoria, there is too little consideration given to the possibilities of recovery. Patients so extensively diseased as to preclude any chance of bringing about an arrest of the trouble are sent away with the idea that they will recover. Often they come considerable distances, and owing to their weakened condition cannot return, and so it happens that not a few die away from their home and friends. Such occurrences, besides being very distressing, also add greatly to the expense of the family.

It not infrequently happens that a patient who refused to go away during the early and curable stages of the discase is only too eagrer to go anywhere when there is no chance of recovery. If money is no consideration, and other members of the family can accompany the patient, so much the better. The family, however, should be warned of the probable result.

To foretell the ending in any given case is not easy, and most of us can recall patients who recovered in spite of what seemed an almost hopeless outlook. Still, it must be borne in mind that there are limits beyond which there can be no hope. In coming to a decision as to whether recovery is impossible, careful attention should be given to what Brown has termed the prognostic triad, $i . e$., temperature, pulse, and weight.

A continuous high temperature, with marked remissions, is almost 
always indicative of septic absorption, cavity formation, and far-advanced disease. Recovery rarely takes place under these circumstances.

Single observations in the office do not mean much as far as the pulse is concerned. If, however, the pulse is constantly over 100 , with the patient at rest, it is of serious import; if constantly over 110, and associated with the type of temperature just mentioned, a fatal ending is almost certain. Cyanosis of the lips and extremities and edema of the lower extremities also indicate that the disease has progressed beyond our power of averting a fatal termination.

Marked emaciation is an almost constant feature of advanced phthisis. When the loss exceeds one-third of the body weight there is little hope of recovery.

In addition, serious complications should be given consideration, and if the disease in the lungs is at all advanced, such cases had best be kept at home.

Medical Supervision.- There is a very prevalent belief among the laity, and, it must be confessed, among some physicians, that the treatment of tuberculosis consists of fresh air and the administration of milk and eggs. Simple as the requirements are, they must be applied in an intelligent way; and while the underlying principles are much the same in all cases, success in the individual case depends on how skilfully we use the means at our command. "The best law to follow is that patients with tuberculosis should be treated by the physician, as nearly as possible, individually, both psychically and physically, as if there were in each case a separate malady." (White.)

Under no circumstances should a patient with tuberculosis be sent or allowed to go to the country, or to a summer or winter resort, without proper medical supervision. Skilful medical attention in the home, even with inferior climatic advantages, is preferable to the best climate possible and no medical supervision. As Cornet has stated, "Merely transferring the patient to a better climate does not mean much for his recovery."

The important thing in sending a patient away should be the assurance that he will have proper medical care, and this requirement should be, to my mind, the essential feature in the selection of a place. I am convinced that, consciously or unconsciously, it is this factor that influences most physicians in their choice, and that praise that is meant for some particular climate can usually be construed into a tribute to the ability of the doctor who has had charge of the case. Quite recently I listened to two physicians discussing the best place to send a patient. It was interesting to hear that their choice of a certain well-known resort was made, not because of the climatic advantages of the place, but because of their faith in the ability of the physician residing there.

While it cannot be denied that some individuals do recover when left to their own devices, the practice is a bad one, and should never be permitted. Those who have been properly instructed during the active period of the disease may prolong the treatment without being under medical supervision; but even this is questionable.

The common objection offered to going to a sanatorium or health 
resort devoted to the treatment of tuberculosis is that it will be depressing to see so many sick people abont. It never seems to oecur to many who raise this objection that they themselves may be in a worse condition than those among whom they are about to be thrown. While it is a very common occurrence for patients in a sanatorium in the beginning to have an aversion for their surroundings, in the great majority of instances this feeling quickly disappears.

Air and Environment.- It is not the function of this chapter to deal with the therapeutic qualities of the various climates. Inasmuch, however, as a residence at one of the climatic resorts is a method of treating tuberculosis, some mention must be made of its advantages and its disadvantages.

'The one feature in the treatment of tuberculosis which has outlasted all others is fresh air, or, as it is generally known, the climatic treatment. Indeed, until quite recently it was universally accepted that tuberculosis could not be successfully treated unless the patient availed himself of the advantages of some particular climate. One of the most interesting chapters in the history of tuberculosis is the development of the climatic idea and the gradual transition of climatic therapy into the modern fresh-air treatment.

During the last century the climatic idea rapidly expanded so as to embrace nearly every known variation-ocean voyages, sea and coast climates; inland regions, with a high altitude and those with a moderate or low altitude; hot, dry climates, with a mean equable temperature; to say nothing of the healing properties of pine forests. Every conceivable variation was advocated, and extravagant claims set forth as to the advantages of this or that locality. It is difficult to trace to their original sources many of the theories concerning climate in its relation to tuberculosis; nor are we able always to assign a reason for the selection of this or that locality.

'The one feature regarding climate that seems to have existed from the beginning and the one which has apparently determined the selection of many localities is warmth. As Jaccoud states, "From the existence of some impressions believed, rather than proved, to be correct the idea of this disease is intimately associated as regards treatment with that of a warm elimate." It would seem that the original selection of warm climates was determined by the fact that it was a matter of common observation that respiratory disorders were less frequent during the warm, dry seasons than during the cold and wet months. 'Then, too, it was noted that a residence in a warm, dry region of ten had a beneficial effect on pulmonary disorders. It is apparent from the claims that gradually arose concerning the influence of climate that it was not appreciated in the beginning that climate per se had little to do with it. 'The fact that the mildness of the climate was an incentive for people to live in the open air and also to keep the windows and doors of their house open, thus insuring free ventilation, was lost sight of. It also seems highly probable that the present views regarding the favorable influence of sunlight had their origin in the same way. Sunlight has always been regarded as 
one of the most essential features of a favorable climate, and much emphasis has been laid on this point. Later, with the discovery of the tubercle bacillus and the knowledge that its tenure of life was very short when exposed to the direct rays of the sun, the importance of sunlight was increased. It should be borne in mind, however, that in some of the cold winter resorts the sun is of ten concealed by heavy clouds, during the winter months, for days at a time, and yet during these winter months patients show the greatest improvement.

The disinfecting properties of sunlight are, of course, important, but proper personal prophylaxis and fresh circulating air can be made almost equally efficient. I mention the influence of sunlight at this point because in carrying out the home treatment it is utterly impossible in many instances to obtain a southern exposure with a maximum of sunshine.

While warmth has undoubtedly been the determining factor in many instances, it does not, of course, explain the selection of localities in which equableness of the temperature is not the dominant feature. I am inclined to believe that in not a few instances the personality and skill of the physician residing in a given locality have had much to do with its selection. This is certainly true of at least one very popular resort in the United States.

At the present time there are three views regarding climate: (1) That which still considers that climate is the sine qua non in the treatment of tuberculosis. The number holding this extreme view has dwindled to a very small minority during the past few years. (2) That which denies that climate per se has any influence whatever. This view, once scoffed at, is constantly gaining new adherents. (3) That which recognizes the advantages of a residence at some climatic resort, but appreciates the fact that such advantages are for the favored few, and that for the great majority climatic advantages must, of necessity, be dispensed with.

In regard to the relative merits of these views, it may be stated that there are not many, at present, who claim for the air of any health resort the marvellous healing properties it was once supposed to have. Modern experience has shown that climate, so far from being the all-essential factor, is at best of no more importance than diet, rest, or, indeed, of any other of the features necessary in the treatment.

'The second view-namely, that removal to a more favorable climate can be disregarded absolutely - does not sound nearly so radical as it once did. It is always a difficult matter to change or even modify an established belief, and those who have the temerity to make the attempt must expect to be regarded, in the beginning at least, as cranks and dangerous meddlers. There is no doubt that the argument of the adherents of home treatment is a difficult one to combat; if the poor man can recover under conditions anything but ideal there is no reason why his more fortunately situated neighbor cannot do the same. It is reasonably certain, also, that the modern crusade against tuberculosis in the masses would not have been possible had it not been for those who fought for the treatment of the disease in the home environment. While there are 
PLATE XXIV

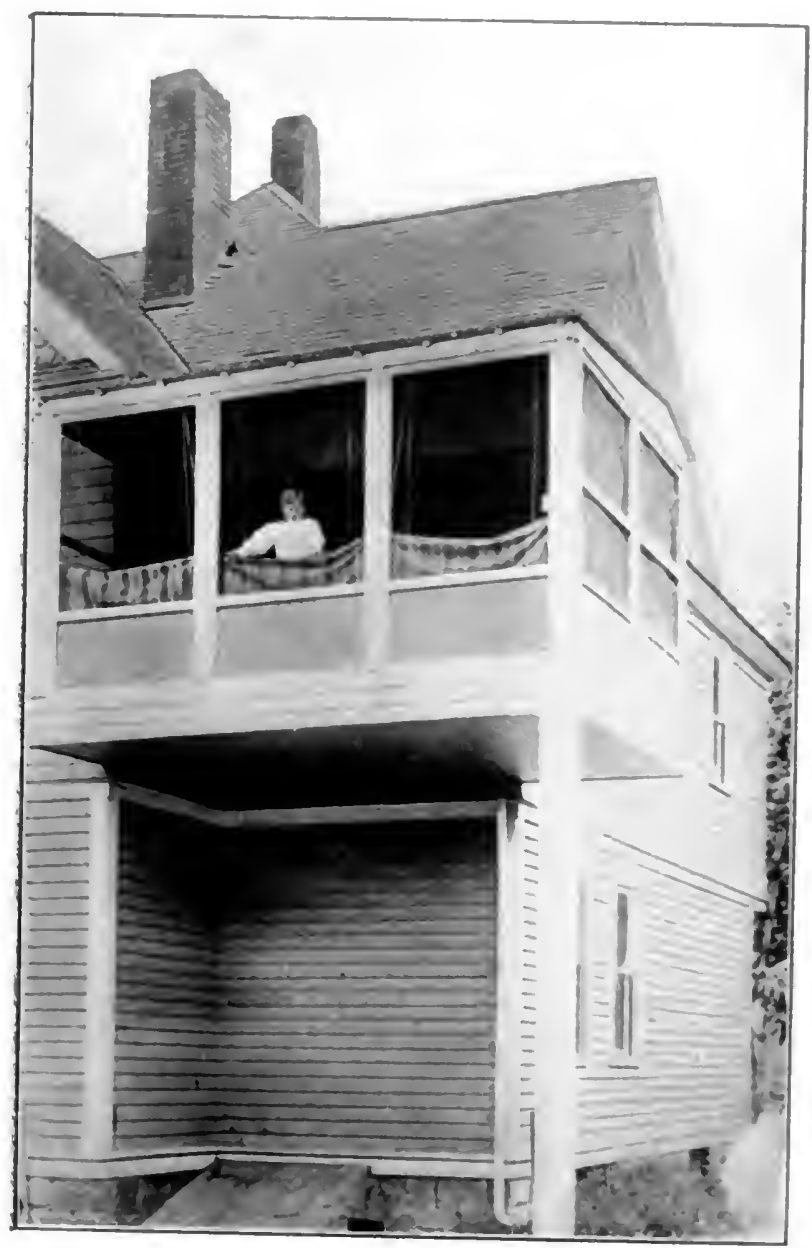

Outside sleeping room. (T. S. Carrington.) 

some who assert that eventually everyone will be treated in or near their homes, I believe that the class which has always availed itself of the aid of some salubrious climate will continue to do so. Dot for the reason that the locality selected possesses any curative properties, hut because, in the first place, it oflers a change, and in the second place, the patient is removed from the distractions of business or social duties.

Although it is now generally admitted that climate no longer occupies the place it once did, and that tuberculosis may be treated with or without its advantages, under what circumstances should we recommend a removal to some climatic resort?

Assuming that the patient is financially able to bear the expense, and that the disease is not too far advanced, there are several factors to be considered before deciding for or against such a step. If, for instance, a man allows his business or profession to interfere with the treatment he should be urged to go away and free himself, for the time being, from business worries. So, too, a woman who allows her household or social duties to interfere with the treatment should be advised to go away.

'The changeable weather which prevails in many localities during the winter and early spring months and extremely hot spells during the summer offer a good reason for a change.

'These indications are, of course, dependent to a great extent, on the inclinations of the patient. He should be urged to make the change, but if he strongly objects to the separation from family or friends, the depression and homesickness which may ensue render the advisability of such a measure doubtful.

A great deal has been written regarding the selection of a suitable climate for the particular individual. In a few instances this may be done; but for the majority it may be disregarded entirely. Certainly, we have all seen patients recover, show slight improvement, or steadily progress to a fatal issue in every variety of climate. I doubt very much whether the common-sense opinion expressed long ago by Austin Flint can be improved on: "My studies seem to lead to conclusions at variance with the prevailing popular and professional belief in a special elimatic influence. In the first place, it does not appear from the analysis of $\mathrm{my}$ cases that changes of climate have in a marked degree a beneficial influence as compared with the hygienic measures available at home. In the second place the improvement following a change appears to pertain alike to different climates and places. Hence it seems a fair inference that the benefit derived from the change is due not so much to a climatic influence per se as to the circumstances incidental to the change. 'I'hese circumstances are of ten a change of habits from those which are sedentary and confining within doors to those involving out-of-door life and activity, freedom from cares, anxieties, and annovances of business at hone, and, it may be added, the moral effect of the hope or expectation of being benefited by climatic influences."

It has long been held that the tuberculous patient stands a better chance of recovery if treated in a locality differing from that in which 
the disease was acquired. In recent years, however, the tendency has been to treat the disease under the same conditions (climatically, at least) as those in which it dereloped, the contention being that there is less likelihood of a relapse. 'Thus it is believed by some that a patient who has recovered at a high altitude runs a risk if he return to a lower level; and while this is the exception rather than the rule the possibility of such an occurrence should be borne in mind, as business reasons or family ties may prevent a permanent residence in the place selected.

In the great majority of incipient and moderately advanced cases the character of the climatic resort can be disregarded, as they will do well no matter where they go. It is, of course, recognized that there are a few who fail to improve in one place and make a good recovery in another.

Patients in whom the disease is advanced often bear the cold badly, and are, as a rule, best adapted to the warm resorts. A high altitude should not be selected if there is disease of the cardiovascular system, nephritis, anemia, marked nervousness, or cavity formation. Hemorrhage cases are generally regarded as unsuitable for a high altitude, although there are some who believe that the danger has been exaggerated.

Personally, I believe that if a patient can go away to a sanatorium or health resort, if only for a month or two, he should be encouraged to do so; partly because of the educational value and partly because a change of scene is often beneficial.

Home Treatment.-Home treatment in the great majority of instances is a necessity, not a fad. It has been estimated that not more than 2 or 3 per cent. of all tuberculous individuals are financially able to avail themselves of the advantages of a residence in some climatic resort. Even with the great increase in the number of free and part-pay sanatoria and hospitals for the care of early and advanced cases there are accommodations for but a small number of existing cases. Furthermore, it must be borne in mind that not all of those who can avail themselves of climatic or sanatorium treatment are willing to do so, but prefer, for one reason or another, to remain at home. And finally, after eliminating the small number of those who can go where they please and stay as long as is necessary, the great majority of patients treated in sanatoria or health resorts must return to their homes with the disease only partially arrested. 'The absence from home is generally from three to six months, and the rule at most sanatoria is that the patient must remain free from symptoms for at least two years after discharge before the disease can be considered as arrested. This is, of course, an arbitrary rule, and applies only to the very early cases. 'The more extensive the pulmonary damage and the more persistent the symptoms the longer will be the time necessary before the case can be considered as free from the danger of a relapse.

It is commonly believed that a stay of from six months to a year is all that is necessary to cure tuberculosis, and many a patient has relapsed because of this mistaken idea. The truth of the matter is that the limited time spent in the sanatorium by most patients only starts them 


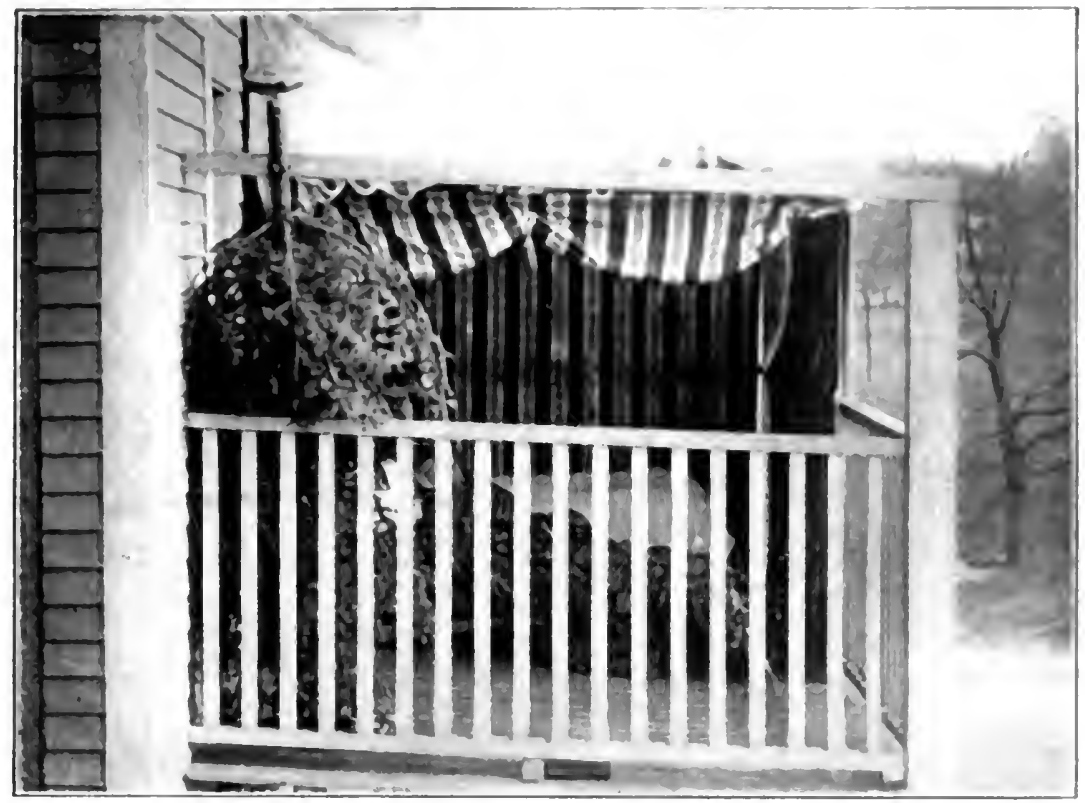

Fig. 1. - A cheap porch protected by awning, built on roof of first-siory veranda. (T. S. Carringion.)

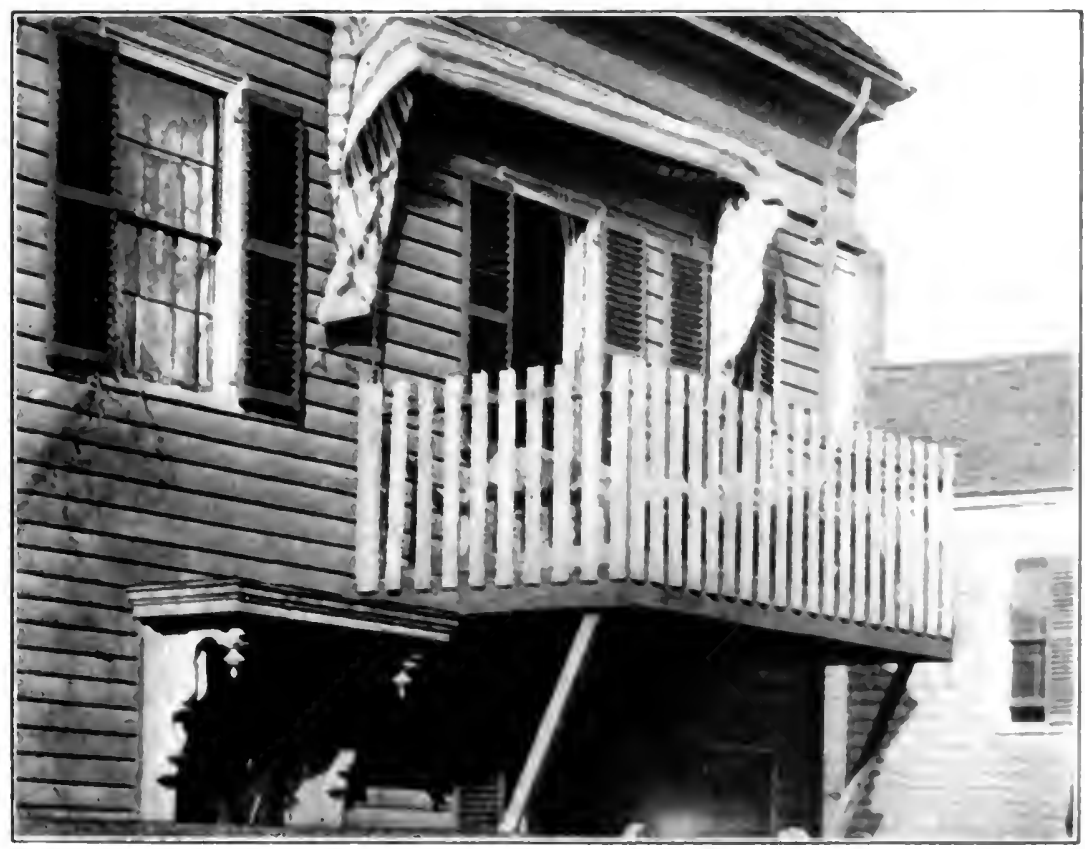

Fig. 2.-A cheap temporary porch protected by an awning and supported by braces at an angle (T. S. Carrington.) 

on the road to health. Recovery and the maintenance of health in the majority of instances depend on a continuance in the home of the life led at the sumatorium or health resort.

'This brief review of well-known facts simply emphasizes not only the importance, but the necessity of home treatment at some time diring the course of the disease.

For the individual who has just developed tuberculosis, my own practice is to exhaust every possible resource before adopting home treatment. We all recognize the benefit derived from a vacation, with its change of scene and food and freedom from business cares. A similar change, even if it is only for two or three months, and providing there is proper medical supervision, is equally beneficial to the tuberculous individual. If however, this is not possible, we must and should make the best of existing conditions.

Otis states that there are four classes in which home treatment is especially appropriate: (1) Patients in far-adranced stages; (2) those whose income ceases when they stop work, but who can, at least for a time, have good food and rest at home: (3) those who are averse to going away and prefer being treated at home; and (4) the very poor.

Success in carrying out home treatment is dependent to a great extent on three factors: (1) The willingness of the patient to faithfully obey instructions; (2) the possession of sufficient resources to enable the patient to secure proper food and rest; (3) the character of the home.

1. 'The first requirement is variable. Some patients readily fall into the required routine with little or no effort; others can be controlled only with difficulty, as it takes more strength of character to make the fight alone than when associated with people who are engaged in the same effort. 'Then, too, there is a group which is represented by the grossly ignorant and the wilfully neghigent who cannot or will not realize the necessity of following directions. The control of the last-named class is difficult. The only solution I can see at present is forcible isolation. In New York City compulsory isolation is permitted by law. It is obvious, however, that in applying such a law, tact and diseretion are most necessary, or the measure will quickly become inoperative. In New York compulsory isolation has been resorted to in but a few instances, and then only when it was absolutely necessary. 'The occasional enforcement of such a law also has an excellent effect on the community at large, as it is thereby warned that the lives of the majority must not be endangered by the negligence of the few.

2 . The second requirement-namely, the resources of the patientshould be carefully investigated. If assistance is not forthcoming from other members of the family, the employer, charitable organizations, or sick benefits, the patient should be sent, if possible, to a State or municipal institution.

3. The third requirement relates to the house itself. In small towns and in the suburbs of the large cities the house can almost alway's be adapted to home treatment. In the large cities, however, it of ten takes considerable ingenuity to furnish the patient with a maximum of fresh air. 
The first requisite is that the home shall be hygienic and that the immediate surroundings are sanitary. 'The house may be unhygienic because of slovenly housekeeping; this may be corrected by proper instructions. In other instances the unhygienic condition is due to no fault of the tenant, and, if such is the case, the landlord should be forced to remedy conditions, or the patient should be advised to move. 'The Board of Health should also be notified. In the larger cities the greatest drawbacks to home treatment are the unhygienic conditions of the houses and overcrowding.

An advantage of home treatment lies in the fact that an individual who has recovered in that locality in which he has lived and will continue to live, is apt to remain well, as there is no abrupt change from a favorable to a less healthful environment. Furthermore, in the sanatorium or climatic resort the prescribed rules are easily lived up to, partly from necessity and partly from the example set by others. Many patients after their return home are apt to lapse into faulty methods of living. In some instances this is due to the erroneous belief that they are cured, and so can ignore their health; others fail to grasp the underlying principles of the treatment. If these principles are gradually acquired in the course of home treatment there is less danger of the patient reverting to his former method of living, as he has learned that it is how he lives rather than where he lives that counts.

The underlying principles in the treatment of tuberculosis are the same no matter whether applied in the sanatorium, the health resort, or the home. If there is any difference, it is in the greater necessity for strict attention to detail in home treatment. Here nothing can be left to chance. What the patient learns he learns from the doctor or visiting nurse, not from the routine practice of the sanatorium. Furthermore, in addition to medical treatment the problem is not only hygienic and social, but an economical one, and all these factors must receive attention.

Of home treatment, more than any other method, it is true that "No single element in the cure-neither air, nor food, nor hydrotherapy, nor medicine-affords any guarantee of success; it is only the satisfaction of all the physiological and hygienic demands of the organism, from the greatest to the least significant, that can bring about this result, and of this fact the physician should not for a moment lose sight." (Cornet.)

Supervision of the Patient.-Home treatment cannot be successful if the doctor sees the patient in his office only. He must at least once visit the home and make himself familiar with its possibilities; in the case of dispensary patients this may be done by a visiting nurse who has been trained in this sort of work and its requirements.

The Home.-If there is a porch, it can be utilized for sleeping purposes; or on a flat roof a tent or light wooden shack can be erectedeven a sloping roof will admit the erection of a platform for a tent or shack. In the rear or at the side of the house it may be possible to erect a sleeping balcony; or the yard may be sufficiently large for the placing of a tent or shack. 


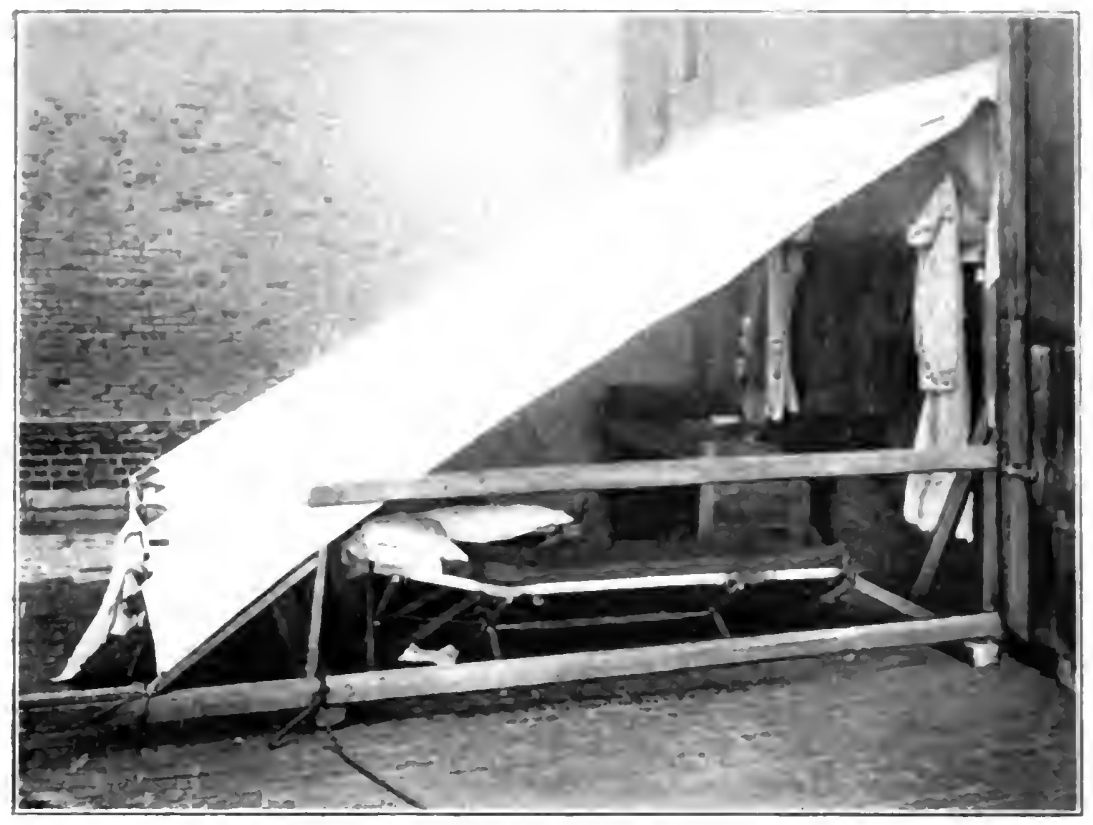

Fig. 1-A lean-to tent on roof of an apartment house in New York City. (T. S. Carrington.)

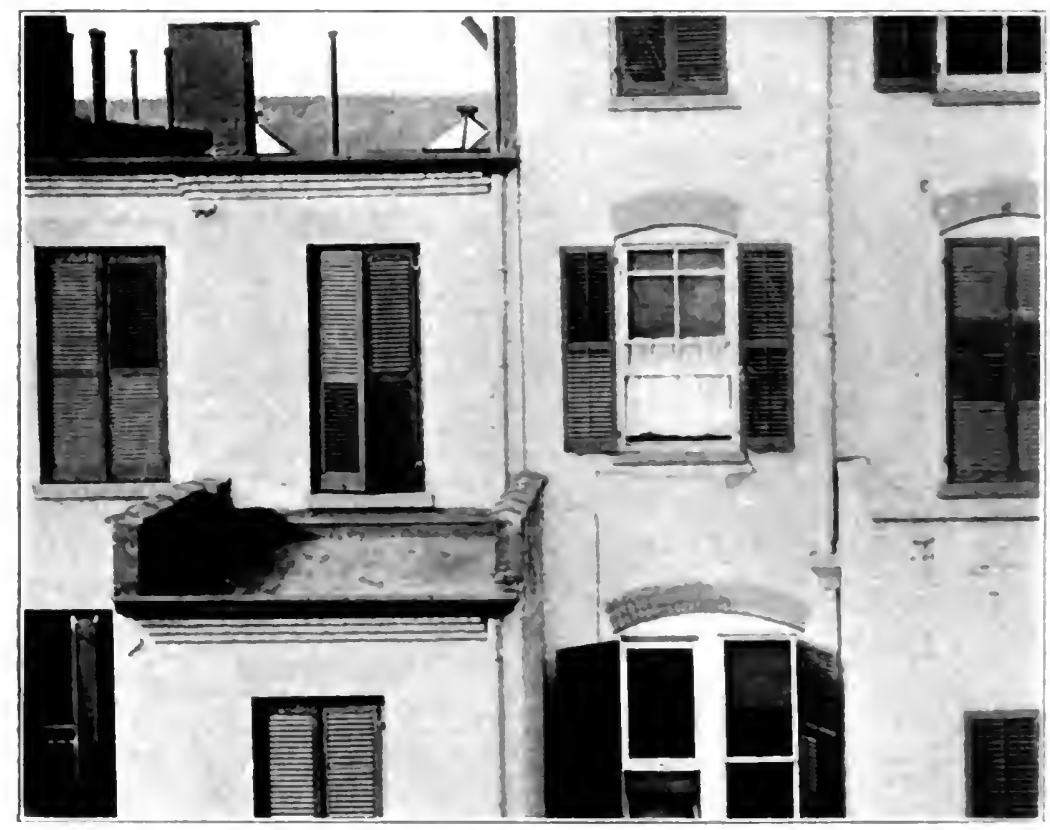

Fig. 2.-A small extension roof on which a tent or shack could be placed. (T. S. Carrington.) 

In the absence of any of these facilities it may be possible for the patient to move to a house where such advantages can be obtained.

When none of these measures are feasible, a room, capable of free ventilation, should be selected; one, if possible, in which there are windows on two sides, so that a free circulation of air can be obtained. Even this last requirement is not alway's available, especially in the homes of the poor, so that we may have to content ourselves with a room having two or even but one window in the same wall. 'The size of the room is not the principal consideration. 'The essential thing is to secure constantly changing fresh air rather than cubic air space. A small room which fulfils this requirement is preferable to a large one that does not.

Window tents which are so constructed that the head and upper part of the body are enclosed in a tent-like arrangement, so that the air breathed by the patient is constantly fresh, may be used to advantage. A cheap and serviceable window tent may be constructed by using the framework of the ordinary window awning and draping over it canvas or blankets. 'The framework is fastened on the inside of the window, so as to hang down over the patient's head.

Curtains should be removed, and if the floor is covered with carpet, this should be taken up. 'T'o completely dismantle a room, however, is not necessary. 'lo exclude everything, even plainly framed pictures, savors, as Brown says, of hygiene run mad.

No matter what arrangement is finally decided upon as the most practical for the particular individual, it must be insisted upon that there can be no half-way measures in the matter of fresh air. Practically the entire twenty-four hours, winter or summer, rain or shine, must be spent in the fresh air. If the patient is confined to a room, the windows must be kept open to their fullest extent. During a storm the flaps of the tent, the curtains of the balcony, or the windows may be closed if the rain or snow beats in. When the storm is over, however, the same conditions which existed before must be returned to.

There is a popular notion that night air is harmful particularly to those suffering from pulmonary trouble. This is not so. Indeed, so far as cities are concerned, night air is purer than that of the day, since there is little or no traffic, and hence less dust is stirred up.

Equally prevalent is the popular fallacy regarding "draughts," and it of ten requires considerable time and patience to convince people that their ideas on the subject are wrong. It has been repeatedly pointed out by Bridge and others that the fear of a draught compels numberless people to breathe bad air constantly, thereby lowering their vitality and making them an easy prey for microörganisms. As a result they get all sorts of diseases that constant fresh air might enable them to escape. As a matter of fact, such individuals are more susceptible to catching cold than the people who either ignore draughts altogether or clothe themselves so that they can bear them. It is now almost universally believed that "colds" arise in one of two ways: (1) That the individual from living in overheated rooms has so lowered the vitality of his mucous membranes that they cannot resist the efforts of bacteria normally 
present in the upper air passages, and which under ordinary conditions are perfectly harmless; (2) colds are extremely contagious, and spread rapidly from one person to another. It is a matter of common observation that an individual with a cold is apt to quickly infect his immediate associates. For this reason tuberculous patients should not be exposed to those with acute colds, as such infections are quite capable of producing very serious damage in these individuals.

Accessory Details of Treatment.--It is, of course, understood that a tuberculous individual should always sleep alone, and that under no circumstances should small children occupy the same bed with a tuberculous father or mother. 'The bed should be single, preferably made of iron, and so placed that the light does not shine directly in the patient's face. A small bedside table for the sputum cup, bell, urinal, etc., should also be provided. A bed-rest is important, so that the patient may change his posture from time to time.

Arrangement of the Bed.-During the winter months the patient confined to bed must be made comfortable. It is not generally appreciated that unless the bed is properly made the patient may become uncomfortable from the cold air coming up from beneath the bed. Northrup's directions as to the proper way to prepare the bed for a pneumonia patient taking the fresh-air treatment are applicable for the tuberculous patient also. He directs that over the mattress there be spread a layer of newspapers, and over this a blanket; the sides of the blanket should be brought up over the patient and the bedclothes covering him and the free edges pinned along the middle. This will keep the patient perfectly warm, and is quite as efficient as a sleeping bag. The latter, however, may be recommended if the patient can afford it.

The patient should sleep between blankets or canton flannel sheets instead of cotton or linen, and the night-dress or pajamas should also be made of canton flannel. The patient may be protected from cold currents of air getting under the bedclothes by arranging the two pillows in the shape of a $\mathrm{V}$, the head resting to the apex; or three pillows may be used, one extending down either side of the body and a third for the head. In order to prevent chilling, in case the shoulders become exposed during the night, a cardigan jacket, a sweater, or flannel jacket should be worn.

If the feet and lower extremities become cold, long woollen stockings may be worn. Many women do not require any covering for the head even in zero weather; but men, especially those who are bald or who have thin hair, should wear a cap which covers the head and back of the neck. If the patient does not occupy the bed during the day it may be warmed before retiring by means of hot-water bags, hot sandbags, or hot bricks.

Arrangements for Sitting Out in Cold Weather.-In order to take the cure in cold weather the patient must be made comfortable, otherwise he will shirk the treatment. Furthermore, more harm than good is done unless the patient is so protected that he is oblivious to the cold.

The personal clothing should be that which is ordinarily worn in winter. Any reinforcement that is needed in case the weather becomes 


\section{PLATE XXVII}

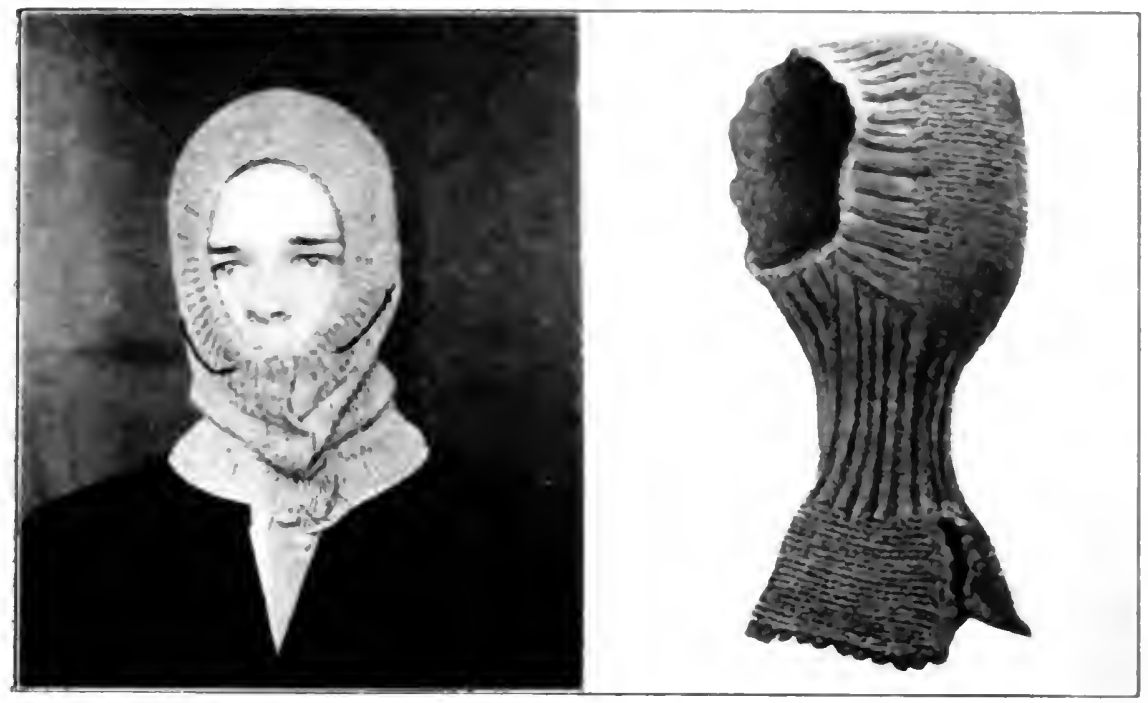

Fig. 1.-A knitted helmel for protecting the head. neck, and shoulders.

(T. S. Carrington.)

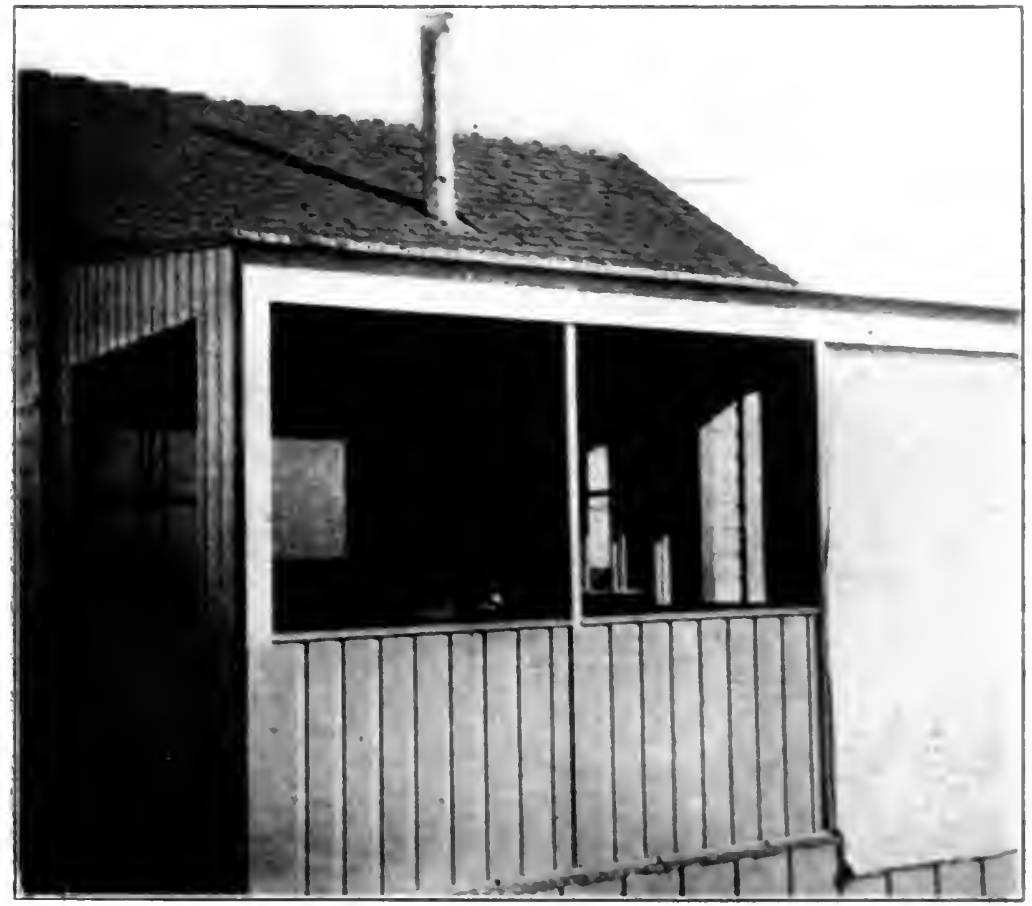

Fig. 2.-A good method of building a porch on the back of a cottage for country use. (T.S. Carringion.) 

colder should be in the form of outside wraps, which ean be added or removed as the temperature ehanges demand. A fur coat (one with the fur outside, and now known as an automobile coat) offers the best protection possible against the most extreme degrees of cold. Although comparatively inexpensive, an automobile coat is out of the question for the poor patient. 'Tlue patient can be made comfortable with an ordinary winter overcoat reinforced, if necessary, by a blanket or comfortable thrown over the slioulders.

The lower part of the body and the legs should be protected by blankets; in very cold weather two are needed. The blankets are spread out over the seat and arms of the chair and extended their full length over the extension of the steamer chair or on the ground. After the patient is seated the free edge of the blankets on the right side is given a quick jerk to the left, covering the lower part of the boxly, the legs, and the feet. 'The same thing is then done with the opposite side. 'The free end should extend beyond the feet from a foot to a foot and a half. The procedure is completed by raising the feet and eatching the free end beneath them.

Probably more people suffer from cold feet in taking the cure in winter than anything else. In the cold resorts all sorts of footwear are advised-felt shoes, moceasins, lumbermen's arctics, heary waterproof shoes. Some advise one thing and some another. 'The secret of keeping the feet warm, however, lies in having them loosely clad. A shoe which under ordinary circumstances is perfectly comfortable usually fits too snugly, as the slightest constriction will impede the circulation. What is wanted is a loose-fitting stocking and an equally loose-fitting shoe. In addition the feet should be kept off the floor or ground, so as to escape the currents of cold air. 'The feet either may be elevated on the extension of a steamer chair, or they may be placed in an ordinary soap-box filled with hay or loose newspapers. Foot warmers or hot bricks may also be placed in the box. Long woollen mittens are the best means of protecting the hands.

In addition to protecting the patient from the cold it is important to see that the chair is comfortable. 'The most comfortable chair obtainable is the "Adirondack recliner," and when the patient can afford it, it is the one of choice. The ordinary steamer chair is the one generally employed; besides being comfortable, it is cheap. No matter how poor the patient may be a rocking chair is always available, and in lieu of anything better, this can be used.

Arrangements in Hot Weather.-The hot summer months also present difficulties in the way of carrying out the treatment. Unfortunately, we can do less for the comfort of the patient during the hot than during the cold months. Cold can be overcome by means of proper clothing. Heat, on the other hand, is difficult to combat in hot, crowded cities. Electric fans will make an almost unbearably hot room comfortable. They are a luxury, however, that depends on the size of the purse and the presence of the proper facilities for their installation. For those who can afford it there is now on the market a fan driven by dry cells, which can be renewed from time to time as needed.

roL. $1 .-48$ 
The best we can do for the patient who cannot get away during the hot spell is to place him in the coolest place in or about the house, see that he is lightly clad, and that he is not exposed to the direct rays of the sun. Under no circunstances should the head be exposed to the sun.

During the hot waves, and especially in localities where the humidity is great, the patient should be kept at rest. If he is taking exercise it should be stopped, or at least greatly reduced.

Fig. 1, Plate XXVIII, illustrates a cheap and efficient method in which two half-barrel hoops are tied to the head and foot of the bed and mosquito netting draped over them to protect from flies and mosquitoes.

During the summer months patients sleeping out of doors or in a room which is not darkened may be wakened too early. Bandaging the eyes with some soft, black material will of ten prevent this.

Dispensary Treatment.-The part played by the tuberculosis dispensary has been one of the most important features in the modern crusade against tuberculosis, even though it has fallen far short of accomplishing what was originally hoped for in the way of results.

It is becoming more and more apparent that the tuberculosis dispensary best serves its purpose by acting as the educational centre for the community in which it is situated, and also as a clearing house in which, after the diagnosis is made the applicants are disposed of according to their individual needs. 'Thus the advanced case is sent to a hospital or is provided with suitable medical and nursing attention in the home; those offering a prospect of cure are placed in sanatoria, or, where this is impossible, are given closer supervision in the "tuberculosis class," the night camp or the day camp, and in the case of children, in open-air schools.

To render such service possible, the dispensary must be equipped with a social service department. 'This new department in dispensary treatment owes its inception to Richard C. Cabot, of Boston, and has as its object the highest type of philanthropy; philanthropy that is dispensed intelligently after a careful investigation of the individual's needs, both physical and psychical.

Heretofore the dispensary has been concerned, for the most part, with diagnosis only. Each patient has been looked upon entirely from the medical point of view, and while he has been treated, if the use of stock formulas can be called treatment, the physician has no knowledge of the "background" of the case. 'To supply this deficiency is the function of the social worker, who puts the physician in possession of all the details of the patient's life; his family relationships, the character of his environment and employment, his assets and his liabilities, his amusements, and, over and above all, the character of the individual is investigated.

Through social service we can look forward to the dispensing of charity in a rational way. Under our present system there is too of ten a failure to realize the exact needs of the individual; in some instances too much 


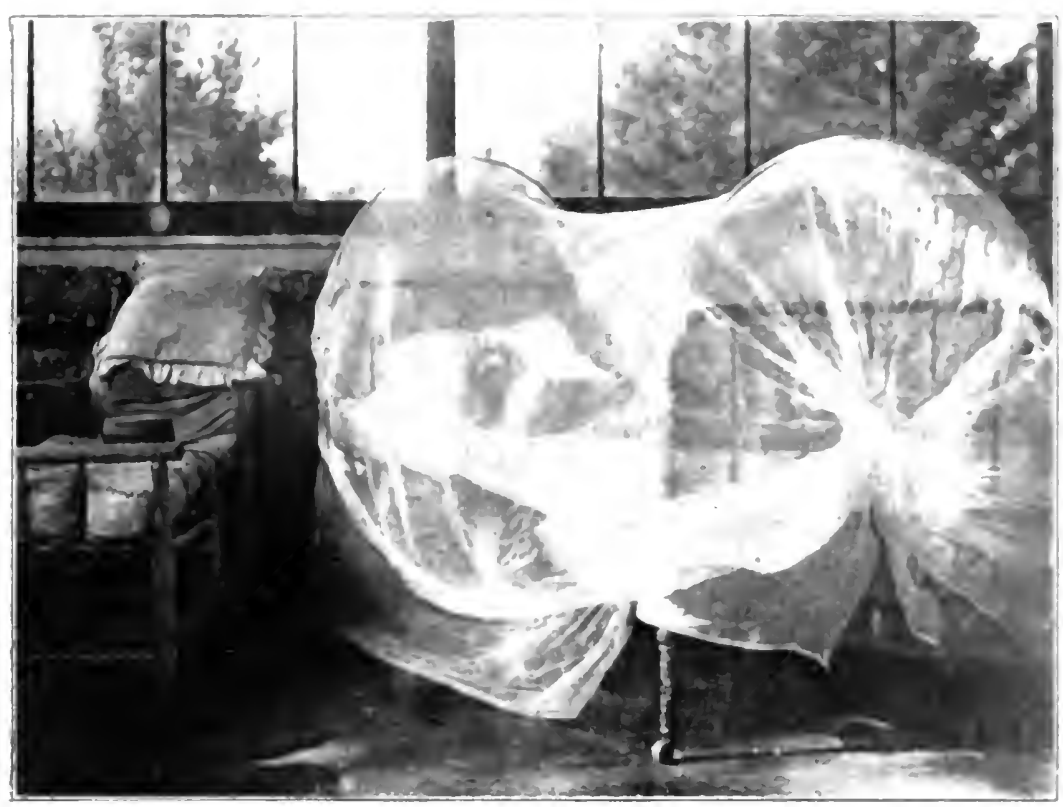

FIG 2

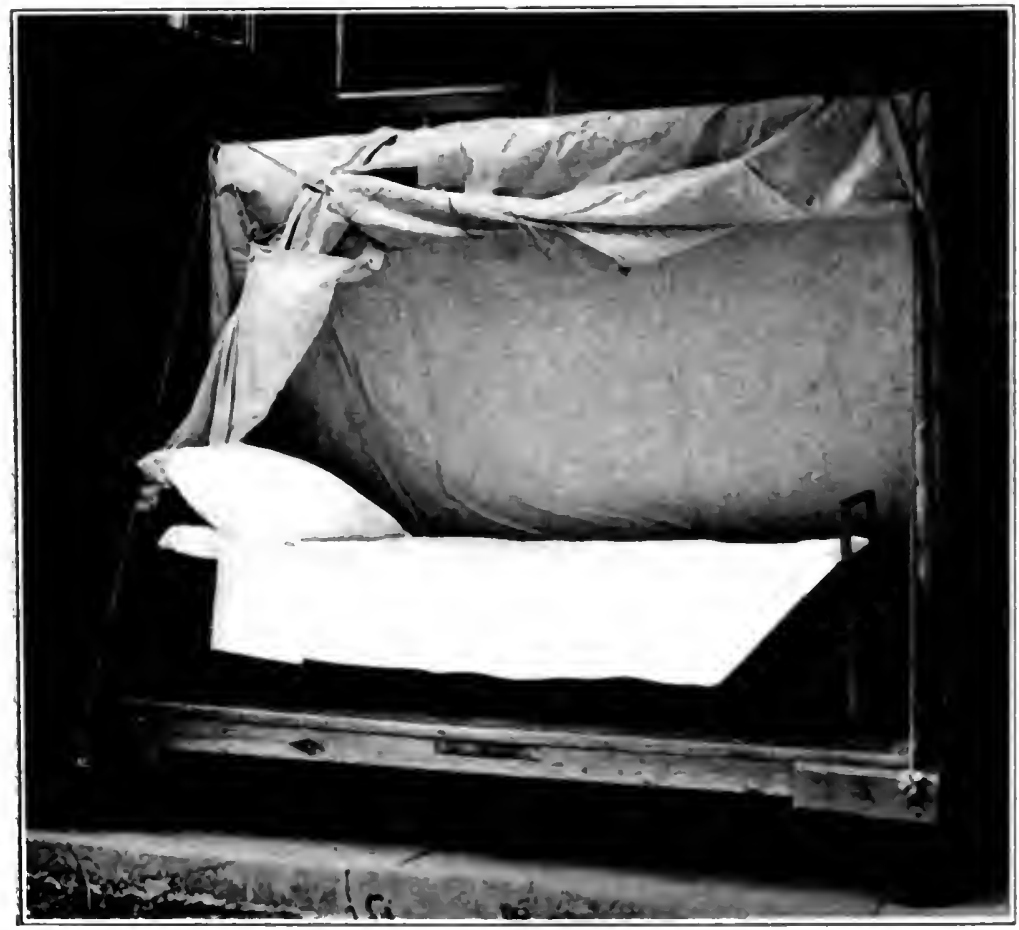

Tent in small back yard. Tuberculosis Class. Phipps Instifute. 

assistance is given, and in others not enough; while the charity of individuals is for the most part determined by sympatly which is frecuently misplaced.

In no disease should the dispensing of charity be more careful than in tuberculosis. Many of the working class sooner or later leecome in need of assistance when tuberculosis strikes the home, but in furnishing them aid we shomld always keep in mind the preservation of the individual's self-respect. Make him help himself in every way possible, and furnish only what is absolutely needed. I know of nothing more deplorable than to see a self-respecting man or woman cured of his or her tubereulosis and yet ruined by indiscriminate aid. Many resent aid at first, but once taken, and especially if easily obtained, their future usefulness is too often destroyed.

The Tuberculosis Class. - The elass method which was introduced by Pratt, of Boston, is the ideal way of treating the dispensary patient, although the plan is usually associated with a church and not a dispensary. 'This is because P'ratt's original class was financed by the Emmanuel Church, of Boston, and because most of his followers have adopted the same method.

The advantages of the tuberculosis class are that it furnishes careful supervision, and by having a small group of patients (fifteen to twentyfive) gather together once a week, a spirit of coöperation and interest is developed; those who gain in weight and otherwise improve inciting the others to make greater efforts.

In the selection of patients for class treatment, physical fitness is the first requirement. Patients who ofler at least some hope of improvement should be chosen. The second recuirement is the ability of the patient to secure proper food, to take the rest treatment, and also that the home permits plenty of fresh air.

'The second requirement is in my experience the most important, as many cases which are desirable from the physical standpoint either cannot afford to stop work or secure the proper food. In addition the home surroundings in the poor yuarters of large cities are of ten such that the requirements cannot be fulfilled. In these instances the class "fund" may be drawn upon or the aid of charitable organizations invoked. At any rate, no patient should be taken into the class from whom we cannot exact full obedience to the rules; otherwise diseipline, which is the keynote of the whole system, fails.

Each patient is furnished with a record book and a thermometer. In addition to keeping a record of the pulse and temperature the patient i: required to record the charaeter and quantity of food taken cach day, the hours spent at rest in the fresh air, the time of retiring, and also any unusual occurrence, such as blood-spitting, pleurisy, ete.

This feature of making the patient a partner, as it were, in the undertaking was first employed by Minor, of Asheville, who has for some years employed it in the treatment of private patients. It also exemplifies what Cabot calls team-work between the doctor and patient. Miller, of New York, has still further developed the plan by giving at each meet- 
ing of the class a short talk on the various phases of the disease, and the reasons for the measures employed in treating it.

One of the most important requirements for the successful conduction of a class is a competent nurse, or, as Pratt has called her, a "friendly visitor." As a matter of fact, the "visitor" need not have received the training of a nurse, but she must have a knowledge of social service, and she must, in addition, be possessed of enthusiasm and tact. On her shoulders rests most of the responsibility of instructing the patients and of seeing that they live up to the rules.

A patient having been selected as suitable for class treatment, the "visitor" calls the following day and explains to the candidate and the family the object of the class, the nature of the disease, the manner of its spread, methods of disinfection and prevention, and how to get well. The financial problem is also taken up. If relief is needed the employer is visited, also the priest or minister, and their aid solicited. The aid of charitable organizations may also be invoked. Failing to obtain aid in this way the class fund must be drawn upon; but it should be a rule never to touch this unless absolutely necessary.

The nurse's visits should not be made routinely; some patients need not be seen oftener than once in a week or ten days, while others must be seen more frequently, and especially in the beginning, when a daily visit for a week or more is of ten required before the patient can be made to understand the requirements.

The details in regard to rest, diet, and facilities for sleeping out are considered under the sections relating to these subjects.

The Night Camp.- Another useful accessory to the dispensary, provided the dispensary is a part of a hospital, with a roof garden or sufficient space in its grounds, is the night camp, a method that was introduced by White, of Pittsburg. The night camp is especially desirable for those who for some reason or another have to work.

"The patients report at the end of the day's work; their temperature and pulse are taken and recorded, when they retire to their reclining chairs or bed to rest until supper. After supper they are at rest until bedtime, 9 P.M., when they sleep out of doors. The time of their return to the hospital varies in compliance with the requirements of their work. The work is arranged with the employer to suit the case as nearly as possible. In the morning they rise, dress, have their temperature and pulse recorded, have their breakfast, and go to work. 'They arrange for lunch out of the hospital. Chest examinations are made and recorded, as with all cases in the hospital. The advantages to the patient are evident-constant medical supervision and advice, two nourishing meals daily, compulsory rest, and open-air sleeping facilities."

The Day Camp.-Still another accessory of the dispensary is the day camp, first employed in Boston. 'The camp may be situated in the hospital grounds or it may be placed in some easily accessible place in the city or suburbs. 'The principal objection to the day camp is that the major portion of the twenty-four hours is spent in the home, where control of the patient is chiefly desirable. 


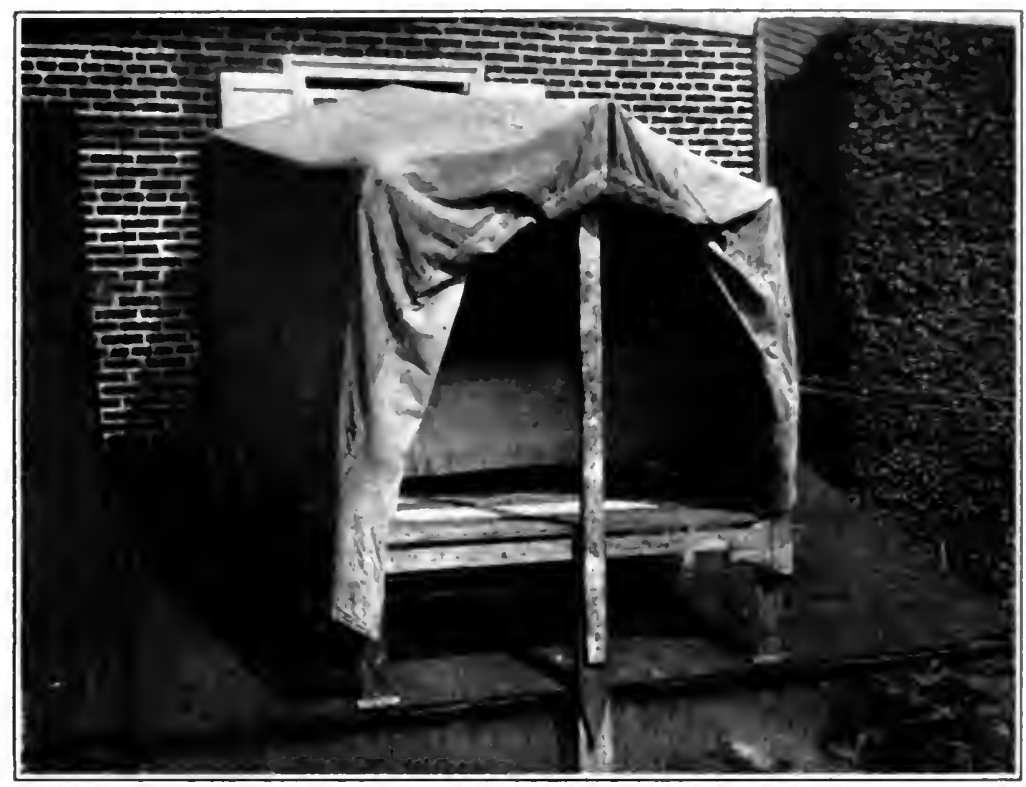

Tent made of old sail cloth, erected on sloping roof: entrance from second-siory window. Tuberculosis Class. Phipps Institute.

FIC. 2

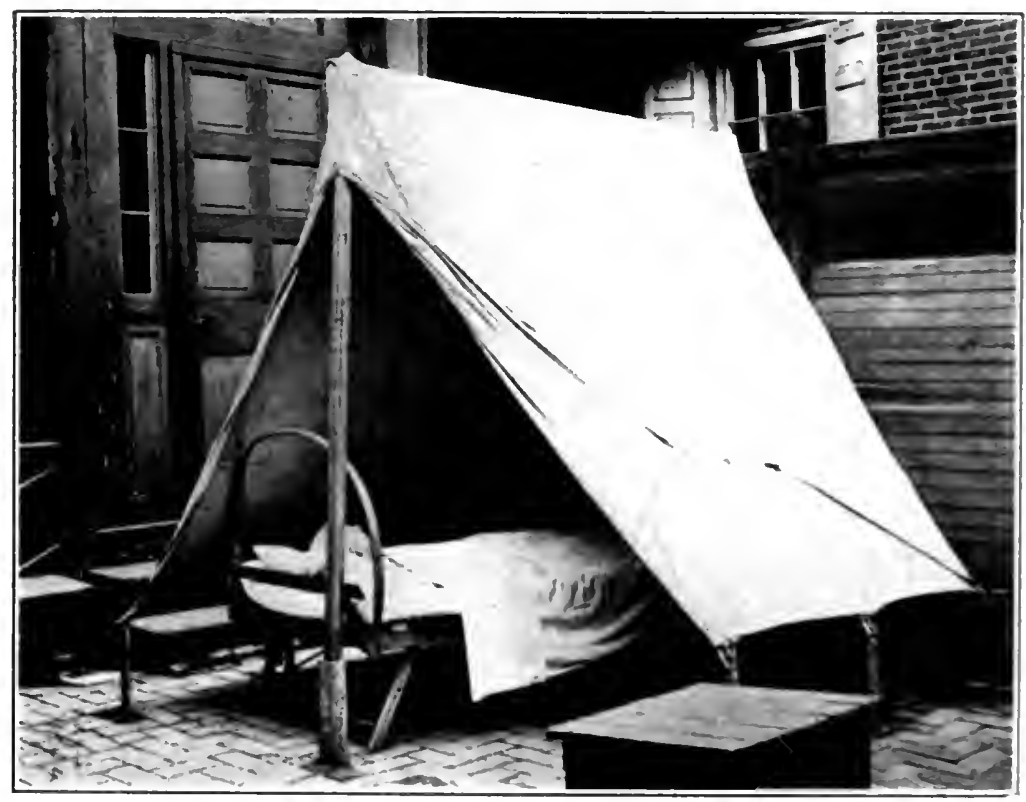

Tent in back yard. Tuberculosis Class. Phipps institute. 



\section{PLATE XXX}

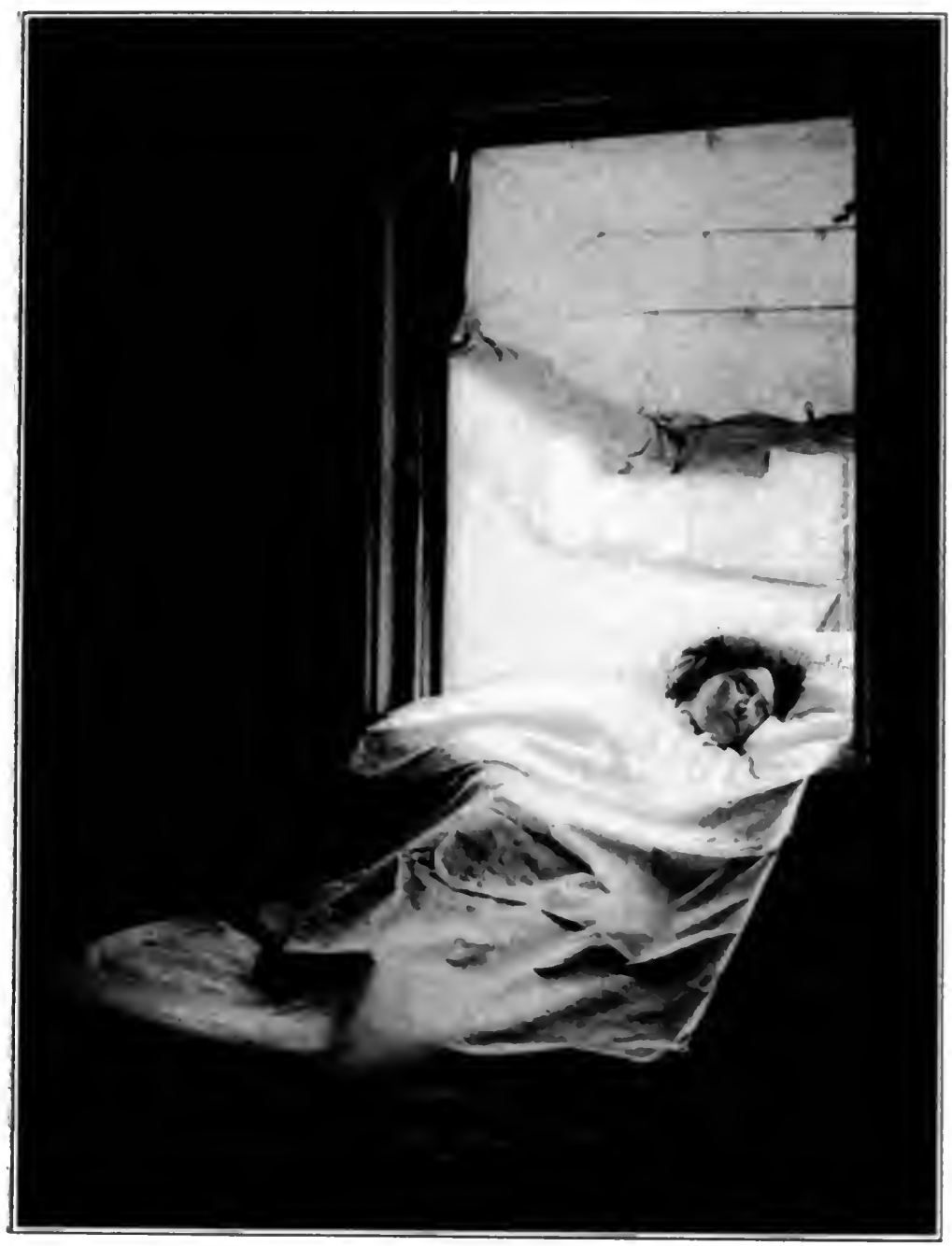

Window tent (looking out). Tuberculosis Class, Phipps Institute. 

Rest and Exercise.- "I 'Te healthy man sits down because he is tired; the tuberculons man should sit down so as not to become tired." (Brehmer.)

'The value of fresh air and a generous diet in the treatment of tuberculosis is now universally recognized. 'The importince of rest, however, is not fully appreeiated. As Hillary has very aptly said: "In the vast majority of eases the general practitioner is the man who is called on to diagnosticate and treat these patients, and so has been lectured, criticised, and cajoled into making an early diagnosis only to meet with failure in the treatment. I do not think it amiss to point out the error that is responsible for failure in nine cases out of ten, in otherwise curable cases-viz., exereise."

In order to obtain fresh air, patients are advised to stay out of doors, which is usually construed to mean, walk in the open air. 'The idea that exercise in the open air is beneficial seems to rest on no better basis than that if exercise is an excellent measure for those in health it should do twice as much good for those who are ill. It was this theory that in the past led many patients to try "roughing it" in the West. Fortunately this idea is less prevalent now than it was a few years ago. 'That the idea still exists in the minds of some was brought to my attention quite recently by a young man who refused to be treated here in the East and insisted on going West to live on a ranch. 'This he was doing on the advice of a friend, who had survived the method. Several years ago, Pogue, of Colorado, reported the ultimate results in 62 patients, with tuberculosis in various stages of the disease, who took the so-called "roughing it" cure, or active exercise, for the most part on the advice of friends. Of these 62 cases, 23 had died and only 2 showed signs of having their disease arrested, with a prospect of ultimate cure.

Violent exercise is not only to be avoided during the period of activity of the disease, but if health is to be preserved it must be eschewed for years. Sports which entail such severe exertion as tennis, baseball, football, rowing, rackets, wrestling, fencing, boxing, etc., are almost certain to lead to a fatal result if the disease is active. While it cannot be denied that in some instances these violent sports can be and are indulged in after the disease is arrested, everyone who does so runs a risk of eausing a relapse, a risk which is in no way commensurate with the pleasure obtained. It cannot be too firmly impressed on patients that their future health is more dependent on the avoidance of fatigue and overexertion than anything else. Rest, no matter how prolonged, rarely does any harm, while indiscretions in regard to exercise have brought more patients to grief than anything else.

It should be carefully explained to patients at the very beginning of the treatment that they are suffering from a disease the essential feature of which is tissue waste, hence the old Greek term, "phthisis," or wasting. Success in combating the disease depends to a great extent in overcoming this tendency. A generous diet and fresh air, even in the most favored places, cannot accomplish this if to the waste incident to the disease itself tissue combustion is further increased by exercise. Patients are quite 
apt to rebel against the idea of rest, especially in bed, on the score that it is weakening. When, however, it is pointed out that even a splendidly trained athlete of ten loses five or six pounds in an athletic contest, the possibilities of exercise in consuming tissue becomes apparent. In the tuberculous individual the balance in his favor may be so slight that the exertion of standing when he should be sitting, or sitting when he should be lying down, is often sufficient to prevent improvement.

One should be guided by the activity of the disease as to the character of the rest and the length of time necessary to continue it. Incipient cases or chronic latent cases with few or slight constitutional symptoms do not need absolute rest for a very long time. When, however, there are active symptoms present (fever, rapid pulse, severe cough, etc.), rest should be persisted in until these symptoms have disappeared. Naturally there can be no definite time set; for one it may be a month, and for another six months.

'The treatment is best started in all eases with one or two weeks' absolute rest in bed, during which time the temperature, pulse rate, respiratory rate, degree of emaciation, the existence of complications in heart, kidneys, intestines, pleura, bladder, etc., and the tendency to hemorrhage are considered.

A temperature of the continuous type, $100^{\circ} \mathrm{F}$. or above, indicates acuteness and progression of the lesion, and calls for absolute rest in bed. The occurrence of an intereurrent cold or other complication, which gives rise to a temperature of $100^{\circ} \mathrm{F}$. or above, also calls for rest in bed. With a normal or subnormal temperature in the morning and a temperature of $100^{\circ}$ in the afternoon the patient should be confined to bed; in the same type with an afternoon temperature under $100^{\circ} \mathrm{F}$, but above $99^{\circ} \mathrm{F}$., the patient should be kept quiet in a reclining chair.

A pulse rate of 120 or higher in the morning ealls for rest in bed; if it is 110 the patient may sit in a reclining chair for a part of the day. A pulse rate of 110 or lower, in the absence of complications, admits of exercise.

Exercise should not be attempted if the respiratory rate is 30 or higher.

Until the weight is within four or five pounds of the normal, exercise should be moderate and its effects carefully noted.

The presence of complications always demands attention. If they dominate the situation, exercise should be omitted, and in any event such eases should receire less exercise than an uncomplicated case.

If exereise causes an increase in the cough, or if vomiting oceurs afterward, the exereise should be reduced or omitted entirely.

Hemorrhage demands absolute rest in bed. For oceasional streaks of blood in the sputum, rest in a reelining ehair is sufficient, but it must be borne in mind that it occasionally happens that blood-streaked sputum is the forerunner of a large liemorrhage.

It cannot be too plainly stated that lifting heavy weights and running or walking rapidly, if the latter causes shortness of breath, must be avoided. A single indiscretion in this regard has more than once changed a favorable into an unfavorable case. 
Always err on the side of prolonging the rest period. It rarely does harm.

'The results of exercisc except in the very early cases are always somewhat problematical. One must not be too sanguine over the rapid disappearance of symptoms under rest. It frequently happens that patients will rapidly lose their symptoms under rest in bed, only to have them return if exercise is attempted too early. I camnot cmphasize too strongly the fact that exercise is an uncertain agent in the treatment, and requires the utmost care. Above all, the patient's desire for exercise must be disregarded. Patients, almost without exception, object to keeping quiet, and are constantly demanding that they be allowed to exereise. In those instances in which I have allowed myself to be persuaded to permit exercise I have almost always had reason to regret it.

When shall the patient be started on exereise? 'There is no absolute rule. As a general thing, the patient with any of the svmptoms which contraindicate exercise should have been free from such symptoms for at least two weeks, and if after exercise is started there is the slightest indication of recurrence, rest must again be instituted.

'The form of exercise almost universally employed is walking. 'This is largely due to the fact that walking requires no trained supervision and is easily regulated.

In beginning exercise the patient is permitted to walk five, ten, or fifteen minutes for the first day, and to increase this three or five minutes daily. 'The walk in the beginning should be on the level. When the time has been extended to half an hour or an hour, the walk should be arranged to include hills. 'The ascent of the hills should always be taken on the outgoing trip in order to make the return easy and free from fatigue. Climbing mountains or steep hills is to be avoided at all times. While walking the patient should not, on the one hand, saunter along, or, on the other, go at a racing gait, and he should be urged to sit down if he becomes tired or short of breath.

Either a nurse or the patient himself should take the temperature and pulse rate at the completion of the walk, and compare the results with those taken when at rest. If there is a rise in the temperature of a degree, or the pulse rate is increased fifteen or twenty beats, and these persist for an hour or more, the exercise should be reduced. If, however, these changes disappear within an hour the exereise may be continued the following day.

As to the best time of day for taking the exercise, my own preference, in the beginning, is the morning, not less than an hour after breakfast. When the exercise has reached an hour's time I usually divide it, half in the morning and half in the afternoon, making the increase alternately every morning and afternoon.

At the end of six months the patient should be able to walk at least four hours daily, and be up and about on his feet from six to eight hours daily.

Carriage riding is an excellent method of providing a mild form of exertion. An hour and a half in the afternoon, besides giving a certain 
amount of exercise, also provides a pleasant break in the daily routine. If the patient drives himself he should use a horse that does not pull on the reins.

Massage I have never employed. It has been recommended as a means of improving the general nutrition in patients who are confined to bed. Massage is contraindicated if there is great bodily weakness, hemoptysis, an intercurrent infection, severe complications, or an acute exacerbation of the disease.

Can the rest treatment be carried too far? In some instances I believe it can. 'There are undoubtedly some individuals who arrive at a point when improvement ceases in spite of the most strict enforcement of rest. They become irritable, a slight degree of fever persists, the pulse rate remains unduly high, the weight remains stationary, and the appetite becomes capricious. The condition is analogous to that seen in convalescents from typhoid ferer as the result of a too prolonged stay in bed; bed fever it has been termed. Getting the patient out of bed of ten causes the disappearance of these symptoms. The same thing is seen in tuberculosis, and a change from the bed to a reclining chair, or from the chair to carefully regulated exercise, will sometimes result in immediate improvement.

Unfortunately, there is no way of recognizing this type of case in the beginning, so that one has to be guided by what transpires as time goes on. After weeks or even months of persisting in the rest treatment it is of ten a great temptation to take a chance with exercise; and while it sometimes works like magic, it is quite as capable of making the patient worse than he was before. 'This is the type of case in which tuberculin has been especially recommended.

Admitting the existence of such cases does not alter the fact, however, that rest is the safest procedure in the great majority of cases, and adherence to it will, in the long run produce the best results.

Graduated Labor.-In dealing with tuberculous patients the social status of the individual must be kept in mind constantly. In the case of the well-to-do, rest and a residence in a favorable environment can be prolonged indefinitely and the course of the patient's life adapted to accord with his physical requirements. For those who live on a daily wage, however, the problem becomes more difficult, especially with those who have no trade and. must earn a livelihood by manual labor. Coddling tactics must, to a great extent, be avoided in treating these patients, and in many instances a certain amount of risk taken.

'To free patients from their svmptoms is not difficult under favorable conditions. 'The real test is whether they can earn a living and at the same time maintain their health. Unless this requirement is fulfilled the treatment, from an economical point of view, has been a failure and the individual becomes a charge on the community. The most favorable time to determine the fitness of the patient for work is while he is under observation. 'This is best accomplished by having him work as a part of the treatment. If he remains free from symptoms under these conditions the chances of his remaining so are much increased. The results on 
immediate discharge are undoubtedly better under less vigorous methods, but the ultimate results are not nearly so good, as the abrupt change from rest with a certain amount of walking, to a full day's work, will often lead to a breakdown.

So far as the working class is concerned, graduated labor as a means of providing exercise is not only feasible but also preferable to walking. 'This has been demonstrated by the admirable results obtained by Flick at the White Haven Sunatorium, Pennsylvania, and later by Paterson at the Frimley Sanatorium, England. 'The principle employed in both these institutions is the same, although the method of application is different.

When the White Haven Sanatorium was opened, in 1901, there was not available sufficient money to provide for salaried employees, and in order that the daily tasks about the institution should be performed it was necessary to employ the patients.

The method then instituted and still in use is as follows: On admission the patient is sent to the infirmary for observation. If at the end of from two weeks to a month the temperature, pulse rate, and general condition are satisfactory the patient is given an hour's work, and this is increased five, ten, or fifteen minutes daily. 'The increase depends on the amount of pulmonary damage and the severity of the constitutional symptoms on admission. Patients are kept at the sanatorium until they are able to do eight hours work a day for one month and remain free from symptoms. For the average case this involves a stay of about six months. Incipient cases frequently are able to leave the institution in three or four months.

'The work performed by the patients is as follows: For women: Waiting on the table, cleaning table and kitchen ware, preparing vegetables, mopping floors, sewing, and making beds.

For men: Carrying meal trays, moving beds, making sputum boxes, sweeping, mopping floors, delivering ice, cutting firewood, and work about the poultry farm, garden, stables, and roadways.

A few years ago Paterson introduced in the Frimley Sanatorium an admirable system in which not only the time spent at labor but also the severity of the work was carefully graded. Paterson's method is as follows:

After it has been determined that the patient is free from any of the symptoms denoting activity of the disease, he is started with a two-mile walk, and this is gradually increased to ten miles daily. If the preliminary stages are passed through without untoward symptoms arising, the patient is assigned a definite task. 'The work consists of carrving firewood, carrying baskets of stones or gravel, hauling a roller, pushing a lawn-mower, digging unbroken ground, and shovelling.

The labor is gradually increased in two ways: (1) By gradually lengthening the time spent at work, and (2) by increasing the severity of the work. The latter is increased by the use of baskets of different capacities and increasing the distance they are carried, and also by the use of picks, shovels, and spades of different sizes. 
The most important feature of Paterson's system is that the walk of life from which the patient comes is kept in mind. For patients who earn their living by manual labor the tests are severer and of longer duration. Clerks, salesmen, and inside workers in general, while hardened by the same preliminary tasks, are stopped several grades below those demanded for the laborers.

Paterson requires as a final test a maximum of six hours' work daily, on an ordinary diet, and without rest hours, for a period of three weeks.

In addition to the value of hardening patients and fitting them for a return to work, graduated labor is an economical method of administering a sanatorium. Money that is ordinarily spent on salaries is saved by having the patients work, thus reducing the cost of maintenance. This has been demonstrated at the White Haven Sanatorium.

"The work which is done by fourteen patients in the dining room, eight patients in the pantry, and six patients in the kitchen is approximately equal to what would be done by thirteen paid employees; the work which is done by eight patients serving and helping in infirmaries and six patients carrying dinners to various buildings is equal to the work of four employees; the work which is done by sixteen patients housecleaning, mopping, and so on is equal to the work of four employees; the work which is done by four patients making sputum boxes is equal to the work of one employee; the work which is done by eight patients in the laundry is equal to the work of one employee; the work which is done by one patient on the chicken farm is equal to the work of one employee; the work which is done by one assistant in the stable and one patient collecting sputum boxes is equal to the work of one employee; the work which is done by two patients removing bedding to and from the rooms is equal to the work of one employee; the work which is done by two patients repairing roads is equal to the work of one employee; and the work which is done in the clerical department, in the drug department, and in the office is given no value, as it could be done by persons employed otherwise." (Flick.)

Paterson, who has emphasized the value of graduated labor as a therapeutic measure rather than an economical one, has, nevertheless, utilized his patient labor in performing repair work and in making extensive improvements about the sanatorium. This has, of course, greatly reduced the cost of maintenance and improvements.

The rules which govern the application of graduated labor are the same as for walking.

My own experience has led me to believe that while some cases are harmed by graduated labor who would, perhaps, otherwise have recovered, the ultimate results are better. I am also convinced that in some instances well-to-do patients would be better off at some form of work as a part of the treatment. Certainly, after the disease has been arrested the patient, no matter what his position in life may be, should be urged to take some employment. As Cornet has said, "Nothing is so deleterious to the subsequent career as years of invalidism spent in institutions and health resorts." 
Employment aiter Arrest of the Disease.-With the tuberculous working man or woman one of the most vital problems is the question of employment after discharge from the sanatorium or after a course of treatment carried out in the home. Our efforts have been so centred on the early recognition of the disease, and on methods of arresting its course, that we have lost sight of the fact that the crucial test really comes when the patient resumes his place as a wage-earner. 'The ability to work and at the same time remain well is a real test of our methods of treatment. A high percentage of "disease arrested" at the time of discharge from treatment means very little if these arrested cases cannot resume their place in life and maintain it.

'The prevailing practice at the present time is to instruct the patient to get an outside position, one which will keep him in the open air. During the first two or three years of my connection with the White Haven Sanatorium I gave advice of this sort, as a matter of course, and without the least suspicion that it was not sound. After I hagd the experience, however, of having discharged patients report to me their inability to obtain outside employment, or when they did obtain it, find it was totally unsuited to them, I was finally convinced that, however desirable outside employment might be, it was, in the great majority of instances, impracticable. Furthermore, experience has shown me that outside employment is not only not necessary for the maintenance of health, but that in some instances it may be of such a character as to cause a breakdown.

The most superficial examination of the facts will, I think, convince anyone of the truth of this assertion. In the first place, outside positions are limited in number; and in the second place, and this is really the important point, they too commonly require severe muscular exertion, which is the one thing the tubereulous individual must avoid. 'Thus, employment as a driver may at the first glance seem suitable, but if this position also requires loading and unloading heavy boxes or barrels it is not desirable, although it is an outside position. Work causing physical fatigue is also objectionable, and for this reason bill collecting, soliciting orders, etc., which require constant walking, are not always desirable, although they too are outside positions. Only recently a patient, who was by training a bookkeeper, abandoned his position, against my advice, for an outside position which required walking about all day soliciting orders. At the end of a few weeks a loss of weight, slight fever, and a constant tired feeling convinced him that he was safer at his old employment.

The great essential for the arrested case is avoidance of fatigue. His occupation should be one that is free from incessant hurry and great effort. It is obvious that a man will do with the least effort that which he has always been accustomed to do. If he be a tailor, a clerk, or a salesman he can spare himself and be less hurried at his old work than if he undertake unfamiliar employment.

Another point of equal importance is that most people relapse not because of the character of their work, but because of their indiscretions 
during the non-working hours. It is not so much the eight or ten hours' work that kills, but the method of living during the remainder of the twenty-four.

To a great extent, common-sense must be our guide, and while the sine qua non is not outside employment, one must also remember that certain forms of inside work are not desirable, either because of hygienic faults or because of long hours. 'Thus, cigar-making is a notoriously unhealthy occupation so far as tuberculosis is concerned, and employment as a barber, at least among the poorer classes, one in which the hours are unduly long. Individuals who have followed such occupations or ones with similar objections had better seek a different employment.

For the last five years I have invariably advised patients returning to work to take up their old employment for the reasons just stated. In addition, I have emphasized the importance of rest and fresh air when not working. 'This means for at least a year that the patient must go to bed in a well-rentilated room as soon as he returns from work and remain there until the next morning. Such a life is undoubtedly irksome, but for the man with the responsibility of a family it is the price he must pay if he would maintain his health and wage-earning capacity. No time limit can be put on this period of his existence; for many it means attention to their health for years. Such patients should be warned that at least for a time the ambition to make large wages must be put aside. Better far to accept a remuneration that can be earned without impairing the health than to relapse from the effort to excel. This applies especially to those capable of but a few hours' work daily, as their temptation is to undertake more than they are capable of.

For five years I have been pursuing the course of having patients return to their original employment, and $I$ have seen no reason to regret it. And while I personally camnot give exact figures as to results, very convincing information dealing with this phase of the subject was presented by Lyman, of Wallingford, Connecticut, at the meeting of the National Association for the Study and Prevention of Tuberculosis in 1910. Lyman was able to determine the ultimate results of all but a very small percentage of his discharged patients for a period extending from one to five years. These he divided into advanced, moderately advanced, and incipient cases, and in each instance he ascertained those who had returned to their old employment and those who had taken new positions in the open air. In each class the percentage of those who had remained well and were at work was in favor of the ones who had returned to their original employment. 'The work involved included almost every variety of employment; factory-work, foundry-work, office-work, store positions, school-teaching, house-work, etc.

There are many who believe that the problem of after care is to be solved by convalescent farms, and that the permanency of the arrest of the disease will in this way be insured. Those who advocate this scheme claim that it will provide outside employment, and that in this way patients may be kept under favorable conditions for a longer time, and that later they can be induced to follow farming as a livelihood. So far 
as the sanatoria are concerned it is also clained that the farm can be made not only' self-supporting, but a source of income.

'There are a number of objections to this method of (aring for arrested cases, not the least of which is that farm-work is not casy. Furthermore, there is a large elass who have neither the inclination nor the capability to earn a living in this way, and farming, to be successful, recpuires quite as much ability as any other form of employment.

I see no objection to advising this mode of life for a man who is physically able and who really desires to follow it, but to attempt to make a farmer out of a Jewish or Italian tailor will almost certainly end in failure.

So far as employment is concerned, what is most needed torlay is an improvement in the character of working places, and, above all, the education of the people in the simpler rules of hygiene. Solve these two problems, and the character of the work need not greatly concern us.

Pulmonary Gymnastics.-Pulmonary gymnastics once occupied a prominent place in the treatment of pulmomary tuberculosis, ats it was believed that deep breathing improved the pulmonary circulation and increased the supply of oxygen to the lungs. They were also prescribed for the purpose of improving the shape of the ehest, which in individuals suffering from pulmonary tuberculosis is not uncommonly badly formed, either through faulty development or as a result of the disease. Now that we have a better conception of tuberculosis and the means necessary to secure its arrest this form of exercise is being less used.

As Cornet points out it is questionable whether the improvement in the pulmonary circulation and the increased supply of oxygen to the lungs is desirable, as it is under just such conditions that the tubercle bacillus flourishes. On the other hand, there is the old clinical observation of the bencficial effect of pleural exudates which lower the nutrition of the compressed lung and yet bring about an improvement in the tuberculous lesion. It is this last-mentioned fact that has led to various methods of splinting the affected lung, and, so far as is possible, of putting it at rest. Bridge, for instance, advises strapping the affected side with adhesive plaster in order to limit the respiratory movement. Murphy has adrocated and practised the radical measure of injecting into the pleural cavity nitrogen gas, thus aiming at the effect produced by an effusion. Then, too, we have the experience of surgeons in the treatment of tuberculous joints, where rest is the main consideration and exercise, especially forced exercise, is never employed. While it is obvious that we camnot put the affected lung at absolute rest unless we resort to Murphy's method, we can, however, limit the movements to those necessary for quite ordinary breathing.

One of Nature's methods of limiting and overcoming the inroads of the tubercle bacillus is in the overproduction of fibrous tissue, whereby. the infected focus is walled off and the activities of the germs are, so to speak, strangled. Anything which tends to strengthen these natural barriers increases the chances of arresting the disease.

Whatever of benefit there may be in pulmonary gymnastics is more 
than offset by the following disadvantages: In the first place there is the direct physical injury that forced breathing may do to the delicate, newly formed fibrous tissue-lung-stretching as it has, not inaptly, been termed by Bridge. In the second place, forced breathing is a form of severe exercise, and hence is open to the same objection as any other form of exercise that produces deep breathing, because of the danger of hemorrhage. We know that the ascent of a moderately steep hill increases the depth of the respirations. Indeed, this procedure is advised by some in order to expand a lung which has been compressed by a pleural effusion. 'The steeper the hill the deeper the respirations, and this is one of the reasons that mountain-climbing and the ascent of very steep hills is prohibited. The principle being the same, it is difficult to understand why one should be advised and the other forbidden.

Minor, of Asheville, believes that respiratory exercises are useful for the purpose of improving the conformation of the chest. In a disease in which lowered nutrition and lack of nervous tone play such an important part it is hardly likely that the lungs also are not subject to the same lack of tone. 'Therefore, with improvement in the general condition of the patient one would also expect better expansion of the lungs, even without respiratory exercises. This I believe to be true. I have made chest tracings after the method recommended by Minor, when the patient was first seen, and repeated the tracing at intervals of a month or six weeks. In those that were improving I found that the chest not only increased in size, but that it also tended to become more symmetrical, the affected side gradually approaching the confirmation of the unaffected side. This does not happen when in the course of the arrest of the disease there is a great overgrowth of fibrous tissue in the lung. In such cases more or less deformity of the chest commonly results.

Personally, I am opposed to the use of respiratory exercises in tuberculous individuals. 'That the position is a sound one is evidenced by the fact that Brehmer, Cornet, Trudeau and others of wide experience have warned against their dangers.

As a prophylactic measure the value of respiratory exercises is great. 'This is especially true in the case of children, in whom deformities of the chest, as the result of rickets, whooping cough, and other causes, are common. 'The correction of these faults while the chest is soft and pliable will of ten spare the individual much future trouble. Individuals with the flat chest of the habitus phthisicus, but no tuberculous trouble, should also be subjected to a course of pulmonary gymnastics.

While it is, of course, not true that only individuals with badly formed chests develop tuberculosis, it cannot be denied that a faulty chest development is commonly associated with delicate health, and experience shows that many of these individuals do become tuberculous.

Mental Occupation.--Rest of mind and later the exercise of its functions must be considered as truly a part of the treatment of tuberculosis as are physical rest and exercise.

Before a patient begins treatment everything possible should be done to free the mind from worries. I have repeatedly seen patients who, after 
improving satisfactorily, ceased to progress, or lost ground, or apparently had unexplainable rises in temperature, in which the underlying cause was mental worry. 'The source of the worry varies from that of the most trivial and foolish character to that of a serious nature. In many instances, unless the mind can be put at rest, it is uscless to expect any improvement. I recall one instance in which the patient became so homesick that he rapidly becane worse, and although removed from a splendid environment in the mountains to an inferior one in the city, he at once began to improve, and eventually made a complete recovery.

During the acute stages of the disease, when physical rest is imperative, the mind must also be kept at rest. Excitement of all kinds must be avoided, and much the sume regime followed as in carrying out the rest cure for nervous patients. Visitors must be excluded, and disquieting news kept from the patient.

We must also guard against allowing the patient to become nervous or self-centred and morose, beeause of physical inactivity. Mental occupation may be provided by reading if the patient is at rest, or, in the case of women, sewing or embroidery may also be allowed. Whenever possible the reading should be directed along definite lines. Books of a sensational or exciting nature should be forbidden.

Later, card-playing, checkers, pool, billiards, and shuffle-boards are permissible. One must be guided in permitting the various forms of amusement by the eflect they produce. Persons of a nervous temperament are sometimes unduly excited, especially by card-playing.

In institutions, entertainments of various sorts should be provided for the diversion of the patients.

Diet.-'The most characteristic feature of tuberculosis is loss of weight, and it is rare, indeed, that this symptom is absent. Sometimes it is alarmingly rapid. Usually, however, it is slow and takes place so gradually that the patient fails to notice that he has grown thinner. 'The habit of being weighed at regular intervals should be a common practice. 'The majority of people neglect this precaution entirely, and it is no uncommon experience to see patients who have suffered a loss of from ten to twenty pounds without the least suspicion that it had happened. It is only exceptionally that one is consulted because the weight is gradually decreasing.

'The loss of weight is due to increased metabolism, and it is to check this excessive waste that our efforts are chiefly directed.

Rest conserves energy and aids in preventing future waste.

Fresh air, acting as a general tonic, not only improves the appetite, but is our main reliance in controlling the symptoms of the disease (fever, rapid pulse, cough, ete.).

Diet, however, is the principal factor in preventing the continuance of loss of weight and in replacing what has already been lost.

Without in the least underestimating the importance of rest, exercise, and fresh air it may be said that proper food plus a good digestion are the patient's best assets. "I am convinced that we have been placing proportionately too great emphasis upon the open-air treatment and too little upon the side of the nourishment. What avails the reclining chair 
and the sleeping out of doors if at the same time the food supply is inadequate to the needs of the patient and the disease, and is of inferior quality and badly prepared?" (Otis.)

Eating the wrong kind of food (not necessarily a bad quality of food) and irregularity of the meals are often the exciting factors in arousing a quiescent lesion, and a change in the character of the food and regularity in taking it are not infrequently all that is needed to promote restoration of health.

Much has been written on what constitutes the proper diet for tuberculous patients, and many attempts have been made, and still are being made, to place the subject on a scientific basis. It can be stated without much fear of contradiction, however, that a dietary acceptable to everyone has not yet been discovered, and in no phase of the treatment is there such diversity of opinions. One of the curions features of the dietaries in use is that in spite of great discrepancies in caloric values they all seem to lead to practically the same results.

The objection to most of the dietaries recommended is not that they contain too much or too little of some particular food, but that they almost, as a rule, fail to take into account the social position of the patient. 'The first and most important consideration regarding diet is the purchasing power of the individual. One should aroid ordering articles of food that the patient cannot afford to buy. Most diets are designed for the well-to-do, or are in use in institutions maintained by private or public funds, the difference between what the patient can pay and the actual cost being made up by the institution. In most instances there is failure to take into account that the patients, even under the most favorable conditions, leave the sanatorium with the really serious part of their treatment before them. It is certainly not good policy to give patients in sanatoria, devoted to the working classes, food that they cannot afford to buy when they return to their homes.

With the well-to-do the price of food can be dismissed at once, but, as we descend the social scale, one must always have this fact in mind. For, paradoxical as it may sound, the less an individual has to spend the more he has to pay for whatever he purchases. One would naturally think that the very poor would spend less on the necessities of life than the well-to-do, while, as a matter of fact, they spend from two to four times as much proportionately to their income. Such necessary articles of diet as potatoes, flour, sugar, etc., are practically never purchased except in small quantities. 'The result is that where the rich man pays 50 cents per bushel for potatoes the poor man pays at the rate of $\$ 2$, more or less. This ignorance of economics must be kept in mind, and instructions as to how to purchase food should be given.

Another great fault in the dietetic treatment of tuberculosis is the lack of individualization. 'This is particularly true in institutions where, for the most part, an unvarying rule is applied to all cases. 'To a great extent this same principle is followed by physicians in private practice, each and every case being given food of the same character and in the same quantity. 
Just as one has to consider the social position of the patient, so must the physical condition be taken into account. We should first know what has been the highest weight, the average weight, and, if possible, the amount of weight lost. We should also note what the weight for the particular case should be. If the patient already weighs what he normally should, or has lost but a few pounds, it is not only not necessary, but bad practice to force him to take large quantities of food. On the other hand, if weight is being rapidly lost and the patient already has dropped twenty-five or thirty pounds, the feeding must be forced to the point of tolerance.

Another physical factor to be considered is the sex of the patient. A man will, as a rule, under any circumstances, be able to take greater quantities of food than a woman; and the fact that they may be in the same stage of the disease and have lost, proportionately, the same amount of weight does not necessarily mean that both should be given equal quantities of food.

Laxness in giving directions as to the kind of food, the quantity and the time of taking it, is another fault, and one which, I believe, is responsible for many failures in treatment. 'The patient is instructed as to the necessity of fresh air and rest, but is generally dismissed with rather vague ideas as to his diet. It too frequently happens that the patient is simply told by word of mouth to take plenty of milk and eggs. No one would think of giving such loose directions even for the most harmless of medicines. Everyone is careful to stipulate the exact quantity of medicine to be taken, and to give precise and written directions as to the time for taking it. 'The same attention to detail should be given in the ordering of food. The directions should be written and the articles of food, the quantity, and the time of taking specifically stated. 'Tuberculous patients should never be given vague or indefinite orders in any phase of the treatment.

Forced feeding is as necessary in some cases of tuberculosis as is fresh air or rest. Unfortunately, at the present time forced feeding is under a cloud. 'This is largely due to the fact that the method has been applied in a careless way and without regard to the needs of the individual case. "Eat all you can and take as much milk and as many eggs as possible" is not forced feeding. Rarely an individual is encountered who is blessed with a digestive apparatus which will survive such advice. But, as a rule, such directions, if followed, lead to a disordered stomach.

The amount of food at first ordered should be the minimum from which we can hope for a gain in weight. If the patient gains on what seems a small quantity it is $m y$ rule not to increase the amount. If, however, no gain occurs, and the appetitie is good and there are no digestive disturbances, the quantity may be gradually increased. Another point to bear in mind is the fact that the capacity for taking food varies in tuberculous individuals just as it does in normal people, and what would be forced feeding for one might be only a normal quantity for another.

The usual custom is to provide three meals daily, supplemented, as a rule, with lunches. 'The method advised by Flick consists of one meal voL. 1. -49 
(dimner in the middle of the day), the remainder of the dietary being made up of milk and, if the patient can afford them, eggs. Having had considerable experience with this method I can recommend it as one capable of giving excellent results. From an economical standpoint it is one of the best methods in use, and is especially adapted to the poorer classes. For details as to dietary formulas, see page 775 .

The extent to which we must cater to the pleasures of the table depends largely on the social position of the patient. The way in which a meal is served means much to those accustomed to the refinements and luxuries of life. In such individuals an appetite can be ruined utterly by slovenly service, and one of the requirements in sending patients away is to be sure that they can obtain well-cooked food, which is served in an attractive manner.

With the poor treated in their homes, such requirements are, of necessity, not of ten attainable. 'The principal considerations with these patients are that the food ordered is within their means; that it is properly cooked; and that it is taken at regular hours.

As Detweiller long ago pointed out, the most efficient pharmacy in the treatment of tuberculosis is the kitchen. Here, again, we can largely disregard the well-to-do, but advice regarding the preparation of food is urgently needed by the dispensary patient. W. G. Turnbull, in an investigation of the dietetic errors of dispensary patients, found that the most evident error was improper preparation of food, usually because of laziness, but of ten through ignorance. Food requiring long cooking is rarely used, or if used, imperfectly cooked. Frying is the common method of preparation. Turnbull found that it is useless to order food that requires baking, for the simple reason that the ovens very often will not bake. Another factor to be borne in mind is the amount of coal required to keep the food cooking a sufficient length of time. It must be remembered that most of the poor buy their coal in small quantities and pay a very high price for it. Hence, they are not apt to use more than is absolutely necessary.

In order to orercome the difficulty of prolonged cooking, and at the same time furnish these poor patients a suitable dietary, the "fireless cooker" is especially to be recommended. With the use of a properly constructed "fireless cooker" less coal is needed for the stewing of meats and the prolonged cooking of vegetables and cereals than is required for the frying and boiling now being done.

"No home is too poor to afford these cooking devices. The simplest and cheapest one is merely a box filled with hay, straw, or even excelsior. 'The food to be cooked is brought to a boil in a covered vessel, which is quickly removed from the stove and buried in the hay or straw. It is left there for several hours, and the food will then be found hot and thoroughly cooked. This cooker is perfectly satisfactory for cooking quantities of two quarts or more. A more convenient cooker and one more efficient for smaller quantities can be made by securing two tin pails, one fitting fairly closely into the other. 'The larger pail is buried permanently in the box, the smaller one is used for cooking the food, and is then slipped 
inside the larger pail and covered with a pad of newspaper or straw. The cooker in use for demonstration at the Pennsylvania Sute Dispensary at l'rankford, Philadelphia, was constructed at a total cost of twenty cents, the packing used in it being newspapers. I)ry ashes or sawdust are also perfectly satisfactory as packing." ('T'urnbull.)

'The calloric value of cereals, stewed neats, and many of the vegetables is high, but are of little use unless properly cooked. A cooker such as that just described, besides being economical, enables one to extend the dietary of poor patients very considerably.

Milk.- Taking into consideration the many advantages of milk, there is no other article of diet which can approach it in value. Milk contains two very important constituents-protein and fat-both of which are highly desirable for the tuberculous patient. Each pint of milk represents about three hundred and fifty calories, so that from a caloric standpoint, milk alone can be made to furnish almost sufficient heat units.

'The constituents of milk vary in diflerent samples, and while for practical purposes no fixed standard can be set, we should, so far as possible, demand the following proportions: Protein, 3.5 per cent.; fat, 4 per cent.; sugar, 4.5 per cent.

Another and perhaps even a more important requirement is purity, or rather relative purity, for no milk is absolutely so. Few samples contain less than 5000 bacteria per cubic centimeter, and the best that can usually be hoped for is a count of about 300,000 to 400,000 bacteria per cubic centimeter. 'The milk supplied to the poor is sometimes contaminated to an incredible degree, 5,000,000 to $6,000,000$ per cubic centimeter. In order to kill the dangerous organisms in milk, pasteurization is being extensively practised. This is an excellent plan, but one must bear in mind that pasteurized milk, unless properly kept, admits of the rapid multiplication of germs.

As an article of diet among the poor, nothing can replace milk, because of its cheapness, its high food value, and also the fact that there is no waste. 'The last factor is an important one when we take into consideration that meats represent a waste of from 8 to 21 per cent. of the total purchased.

'The value of milk is further increased by the fact that it is acceptable to practically all patients. It has frequently been stated that many patients cannot take milk. While it is true that at the outset many will state that they cannot take it, the objection is usually more fancied than real. I cannot recall more than half a dozen patients in the last five years who could not be induced to take milk in fairly large quantities.

In some instances when the distaste for milk is real it ean be overcome by giving small quantities at first. I have started with as little as a tablespoonful every two hours, and gradually increased the anount until the patient could take one to two quarts daily. Fortunately, it is almost the rule that milk can be taken even when the sight of other forms of food is repuguant.

Constipation sometimes results when the diet is largely made up of milk. Curiously enough, constipation is more npt to occur if the daily 
amount is small (two to three glasses) than when the patient takes large quantities (two to three quarts). It is not a serious objection, and one that can easily be overcome.

The opposite effect-namely, diarrhea-is one I have encountered only a few times. The addition of lime water to the milk will sometimes correct this tendency. The lime water should not be taken beyond ten days or two weeks. I have never had the experience of having to discontinue the milk because of diarrhea. If there is a tendency for the milk to form curds in the stomach, this may often be overcome by the addition of small quantities of lime water, oatmeal water, or barley water. Peptonizing the milk may also be tried.

Nilk, as a rule, should be sipped slowly. Many people, however, can drink a glassful at once without producing any discomfort.

Gastric distress after drinking the milk may be due to the milk being too cold.

For some years fermented mill has been used (kefir, koumiss, matzoon). It is claimed that these fermented milks are more easily assimilated, and thus especially adapted to patients with an impaired digestion. I have at various times given these preparations a trial, not, however, with any great success. 'Taken constantly, patients soon tire of them. Occasionally they are useful as a complete change, or they may be used alternately with the sweet milk for a time. Their special field of usefulness seems to be in intestinal disorders associated with putrefactive changes.

'The cheapest and most available form of fermented milk is buttermilk. If this cannot be obtained fresh, good results may be obtained by inoculating sweet milk with lactic-acid bacilli. This can be done by means of lactic-acid bacilli tablets, several forms of which are now on the market.

Eggs.-Milk and eggs have come to be almost synonymous with the dietetic treatment of tuberculosis.

Raw eggs as an article of diet for tuberculous patients have a very high food value, the white consisting of pure protein, while the yolk contains a high percentage of both protein and fat.

Eggs when taken raw should be broken into a glass and taken whole. Varrious means are taken to disguise the taste. For this purpose a small amount of plain water, milk, or sherry may be poured on top of the egg. Eggs should not be beaten up with milk, as the taste soon disgusts the patient. A few people prefer them this way, but, as a rule, this method should be avoided.

The popularity of eggs is, I believe, on the wane. It has been my experience that they are more frequently the cause of bilious attacks than anything else. 'This is due apparently to the fat in the yolk, for if only the white is given the bilious attacks are less apt to occur. I rarely use more than four raw eggs daily, and with poor patients have abandoned them entirely.

A great objection to eggs is their cost and the difficulty of obtaining them fresh. For at least six months in the year absolutely fresh eggs are very difficult to obtain in the larger cities, and at the same time the price is prohibitive. For dispensary patients who cannot get eggs, suffi- 
cient additional protein can be obtained by using vegetables, while the fat may be supplied by some form of oil.

Meat.-It has long been held that rare red meat was especially beneficial to tuberculous patients, and most of the dietaries for tuberculosis are constructed with this object in view. Some advise meat three times daily, and there are few who do not recommend it at least twice daily.

At present there seems to be a reaction against a heavy protein diet, and much less meat is being advised than was formerly the case. King, who has given much study to the dietary problems of tuberculosis, has found that equally good results can be obtained by giving less protein and fat and correspondingly larger quantities of carbohydrates. His patients not only did well, but the saving in the cost of food was from 10 to 14 cents a day for each patient. This last factor is of extreme importance in dealing with the poor, for with them dietary requirements must be adjusted to the pocket-book.

Practically, it has been shown that a satisfactory dietary can be supplied at a cost within the means of all but the very destitute. This cannot be accomplished, however, unless meat is restricted to once daily, or, in case milk and eggs are being taken, omitted altogether.

Whenever possible, however, meat should be taken at least once daily. Beef and mutton are usually to be preferred, while fresh veal and pork, because they are difficult of digestion, should not be eaten too frequently, and should never be given a patient with a poor digestion. In addition, chicken, fish, and shellfish may be given several times a week.

Poor patients should be advised to purchase the inexpensive cuts and cook the meat in the form of a stew, to which vegetables (potatoes, onions, and carrots) are added. The "fireless cooker" answers the purpose admirably in preparing these stews.

The diet may be varied from time to time by substituting for the milk at the lunches, sandwiches made of raw, scraped beef. For the same purpose pure beef-juice is often desirable. The beef-juice is prepared by taking small blocks of rump steak, one inch square, and cooking them quickly in a skillet. 'The pieces should be turned constantly while cooking. 'They are afterward cut into smaller pieces, and the juice extracted by means of a meat presser, a lemon squeezer, or by compression in a piece of cheese-cloth. 'The juice is seasoned with pepper and salt, and served either hot or cold as the patient prefers.

The various commercial beef extracts cannot be recommended; they are worthless from the standpoint of nutrition.

Fats.-Fats form an essential part of the dietary in tuberculosis. 'They must be used with more discrimination, however, than any other form of food, as they can very easily be given in excess. Individuals vary greatly in their ability to take and absorb fat, and gastric disturbances not uncommonly arise from giving it too freely. Fat intolerance, as it has been termed, may occur in any condition, but is quite commonly seen in tuberculosis by reason of the large quantity of fat in most dietaries (see page 800 ). Eggs, which are the most frequent offenders in my experience, should be omitted, or, at least, the yolks and the milk should be 
skimmed. If oil is being taken it should be omitted for a time or reduced in amount.

Formerly fat was supplied, to a great extent, by the use of cod-liver oil. While cod-liver oil has gone out of fashion, it is well to remember that it was an agent productive of much good in the treatment of tuberculosis, and I do not think there can be any doubt but that many individuals recovered from its use. That it is no longer employed may be attributed to the fact that fat can be furnished in a much more palatable form, and also because less importance is attached to the small iodine content of the oil.

Fat is now supplied by means of butter, pure cream or unskimmed milk, eggs, and meat, especially bacon. Certain varieties of fish, such as salmon, Spanish mackerel, sardines, etc., are also rich in fat. Pecan nuts are highly recommended because of their richness in oil.

Just as the protein in eggs can be supplied in a cheaper form, so can their fat value be replaced by less expensive food. Pratt, in his Tuberculosis Class work, has abandoned eggs and substituted in their place olive oil or cottonseed oil; the latter is very satisfactory, and is, besides, very cheap.

Olive oil or cottonseed oil should be given at first in small doses, a teaspoonful three times daily. If the patient tolerates it well the amount may be gradually increased to a tablespoonful three times daily.

Carbohydrates. - As I have already stated, carbohydrates are more of ten included in the dietary for tuberculous patients than was formerly the case. In this class of foods are the cereals, oatmeal particularly, which furnishes a cheap and desirable addition to the dietary. The use of a "fireless cooker" removes the principal objection to their use.

Rice is a very valuable food, and one that is too little used. It can be eaten as a vegetable, or may be served with sugar and milk or cream.

Carbohydrate food is also represented by corn-, graham-, and wheatbread, corn-meal mush, puddings, starches, and pastries of all kinds. It can also be furnished in a palatable form by the use of honey and maple syrup. Candy for dessert is allowable several times a week.

Vegetables.-Vegetables, especially during the season when they are fresh, should be used freely. Peas and beans, because of the protein they contain, are desirable, especially in the preparation of thick soups.

I know of no restriction in the use of vegetables except when there are intestinal disturbances. If diarrhea is present, and there is reason to believe it is tuberculous in character, vegetables containing much coarse fiber (cabbage, beets, parsnips, etc.) should be avoided. On the other hand, if constipation exists, the coarse fibrous vegetables are desirable.

Drinks.-Tea and coffee are allowable once a day. Whenever possible, chocolate or cocoa should be substituted, at least part of the time. Among the poor tea is drunk to an amazing extent. Its use should be limited in this class of patients to a small cupful once or twice daily.

The following memus will be found of service in prescribing a definite dietary schedule. The first contains a larger amount of carbohydrate food than is usually in use, and is the result of a careful study made by Herbert Maxon King, of Liberty, New York: 
SUNDAY.-Breakfast.

"Pettijohn Food"

Grams.

Sausage

Corn-bread

Oranges, bread, butter, coffee, cocoa, milk, cream.

Fricasseed chicken

Dinner.

Sliced tomatoes

Mashed potatoes

Boiled rice

Vanilla ice-cream

Bread, butter, milk.

\section{Supper.}

Boston baked heans, with catsup

Marmalade

Chocolate cake

Prunes

Bread, butter, tea, milk, cocoa.

Bread for day

Summary.

Butter for day

Milk for day

(ream for day

Cocoa for day

Sugar for day

Simmary.

Protein.

145
Fat.

123
Carbohydrates.

419

\section{Monday.-Breakfast.}

"Cream of Wheat," with cream

Poached eggs

Bread, butter, coffee, cocoa, milk.

Dinner.

Roast ribs of beef, with grary

Mashed potatoes

Turnips
Apple tapioca, with milk

Bread, butter, milk.

Supper.

Corned beef hash

Swoet and sour pickles

Plain cake

Bananas, with cream

Bread, butter, tea, cocoa, milk.

Bread for day

Summary.

Butter for day

Milk for day

Cream for day

Cocoa for day

Sugar for day

201
45 
Tuesday.-Breakfast.

"Quaker Oats," with cream

Grams.

Minced meat

Graham muffins

Bread, butter, coffee, cocoa, milk.

\section{Dinner.}

Soup

Roast shoulder of veal

Mashed potatoes

Sieva beans

Tapioca, with cream

Bread, butter, milk.

Cold sliced beef

Supper

Creamed potatoes

Sweet and sour pickles, bread, butter, tea, cocoa, milk.

Bread for day

\section{Butter for day}

Milk for day

Cream for day

Cocoa for day

Sugar for day

Average per patient per day

\section{Summary.}

29

927

158

364

74

Protein.

126
Fat. 117 369

\section{Carbohydrates. Calories.}

3117

\section{Wedxesday.-Breakfast.}

Cereal, with cream

Codfish cakes

Bread, butter, coffee, cocoa, milk.

Soup

Dinner.

Wafers

Roast leg of lamb

Peas

Mashed potatoes

Steamed pudding

Brandy sauce Bread, butter, milk.

Chicken salad

Supper.

Macaroni and cheese

Prunes

Bread, butter, tea, cocoa, milk.

. . . . . . 74

Bread for day

Summary.

Butter for day

Milk for day

Cream for day

Cocoa for day

Sugar for day

S. 
Thunsds Y.-Breakfast.

Figs

Grasne.

70

43

40

Catmeal, with cream

Corn-cake

Maple syrup

Bread, butter, tea, cocoa, milk.

Dinner.

Soup

Sirloin steak

Mashed potatoes

String beans

Bread pudding, with raisins Bread, butter, milk.

Supper.

Cold lamb

Fried potatoes

Baked apples, with cream

Bread, butter, tea, cocoa, milk.

Summary.

Bread for day

Butter for day

Milk for day

Cream for day

170

Coeoa for day

Sugar for day

347

63

Average per patient per day

Protein.

130
Fat. Carbohydrates.

400

FrIDAY.-Breakfast.

Prunes

Grams.

"Quaker Oats," with cream

Bacon

Corn-muffins

Bread, butter, coffee, cocoa, milk.

Bouillon, with wafers

Dinner.

Baked halibut.

Mashed potatoes

\section{Stewed tomatoes}

Iemon jelly, with cream.

Bread, butter, milk.

Creamed beef

Baked potato.

Supper.

Dutch cheese

Biscuit

Baked apples, with cream Bread, butter, tea, cocoa.

Bread for day

Summary.

Butter for day

Milk for day

Cream for day

Cocoa for day

Sugar for day

Average per patient per day

$:$
$:$
$:$

127
SO

32

1430

160

250

is

Fat. Carbohydrates. Calories. 
Prunes

"Shredded Wheat"

Hominy, with cream

Liver

Bacon

Muffins

Bread, butter, coffee, cocoa, milk.

Grams.

60

10

16

14

10

28

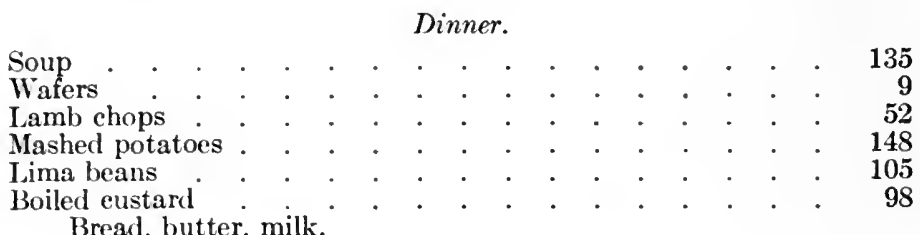

Supper.

Cold tongue

Fried potatoes

Rhubarb sauce

Bread, butter, tea, cocoa, milk.

135

52

148

05

98

Summary.

Bread for day

Butter for day

Milk for day

Cream for day

Cocoa for day

Sugar for day

Average per patient per day

Protein.

132

$$
\begin{aligned}
& \text {. . . . } 104 \\
& 36 \\
& 1600 \\
& 210 \\
& 235 \\
& 69
\end{aligned}
$$

Another method of feeding tuberculous patients is that devised by Flick, a sample day being as follows:

Breakfast, 7.30 A.M.-One raw egg and three glasses of milk.

Lunch, 10 A.M. - Two raw eggs and two glasses of milk.

Dinner, 12.30 Noon.-Soup, meat (steak, roast beef or lamb), potatoes, steamed rice, stewed onions, pudding or ice-cream, fruit.

Lunch, 4 P.M. - Two raw eggs and two glasses of milk.

Supper, 6.30 P.M. - One raw egg and two glasses of milk.

Lunch, 8.30 P.M. - Two glasses of milk.

Summary. Six raw eggs, three quarts of milk, and one full meal.

This diet admits of much variation. Milk is its basis, but this may be reduced to ten or eight glasses daily by substituting a light breakfast and supper. Personally, I have almost given up the use of eggs, the objections to which I have already pointed out. If additional fat is needed it can be furnished by using some form of oil. Inasmuch as a good appetite is a most desirable thing, nothing should be allowed to interfere with it; therefore, it is sometimes desirable to omit the morning or evening lunch.

The following dietary schedule is given as an illustration of what one encounters when dealing with the poor. It is for a family of four-father, 
mother and two children, aged three and five years. The father was making \$10 per week, about \$5.50 of which was available for food. 'The mother was tuberculous, and a member of my tuberculosis class at the Phipps Institute. Instructions in buying and preparing the food were given by the visiting uurse.

The outlay on Monday and Saturday is larger than the other days, because many of the supplies for the week (in italics) are purchased on Monday, and Sunday's buying is included with that of Saturday.

\section{Monday.}

Breakfast.-Oatmeal, skimmed milk, bread, butter, coffee, sugar.

Lunch (Midday).-Potato soup, bread, butter, milk or tea, syrup.

Dinner.-Hamburg steak, boiled rice, bread, fruit.

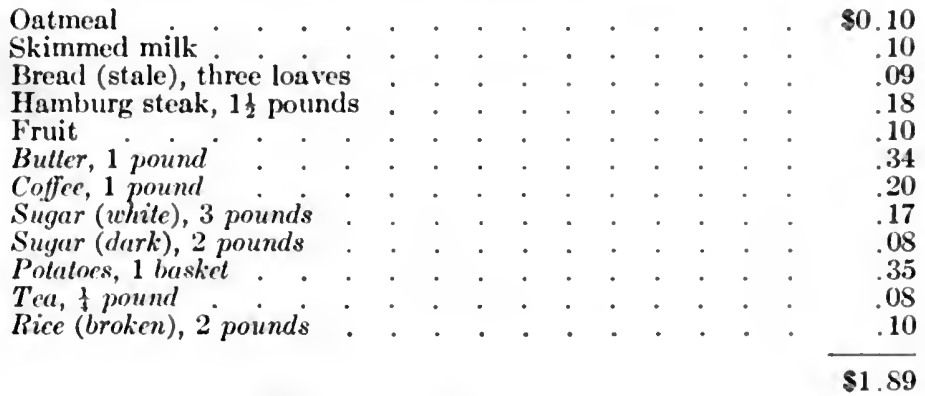

Tuespar.

Breakfast.—"Cream of Wheat," corn-meal mush, skimmed milk, toast, butter, coffee, sugar.

Lunch (Midday).-Boiled rice, sugar, milk, bread, butter.

Dinner.-Beef-stew, with potatoes and earrots, bread, butter.

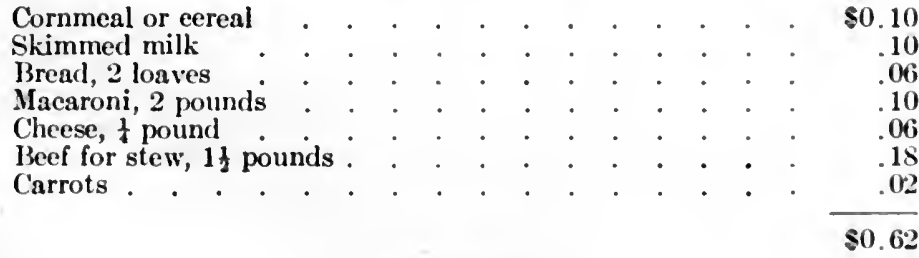

WEDNESDAY.

Breakfast.-Poaehed eggs (four), breal, butter, skimmed milk, coffee, sugar Lunch (Midday). - Bean soup, bread, butter.

Dinner.-Boiled mutton, mashed potatoes, vegetables, bread, butter.

Eggs, $\frac{1}{2}$ dozen

\$0. 15

Sikimmed milk

Bread, 2 loaves.

.06

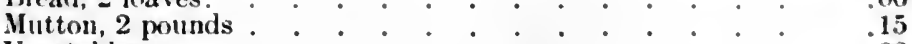

Vegetables

. as

Beans 


\section{THursday.}

Breakfast.-Oatmeal, skimmed milk, bread, butter, coffee, sugar.

Lunch (Midday).-Pea soup or maearoni and eheese, bread, butter.

Dinner.-Round steak, potatoes or vegetables, baked apples or fruit.

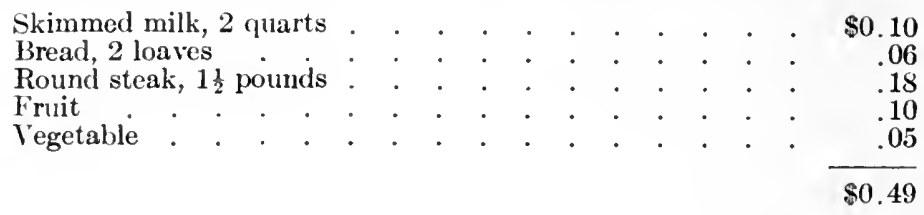

FRIDAY.

Breakfast.-Soft-boiled eggs (four), toast, butter, skimmed milk, eoffee, sugar. Lunch (Midday).-Baked potatoes, bread, butter, milk.

Dinner.-Fish, boiled rice, vegetables, bread.

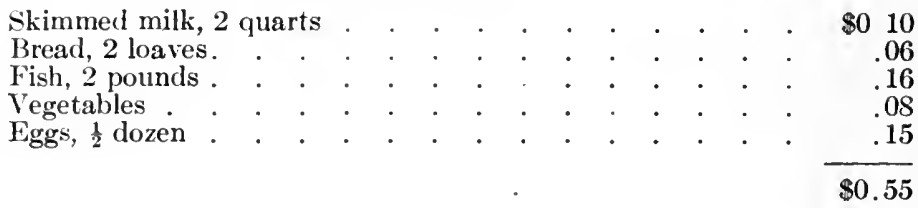

SATURDAY.

Breakfast.-Cereal, bread, butter, skimmed milk, eoffee, sugar.

Lunch (Midday).-Vegetable soup, bread, butter.

Dinner.-Stewed mutton, boiled beans, bread, fruit.

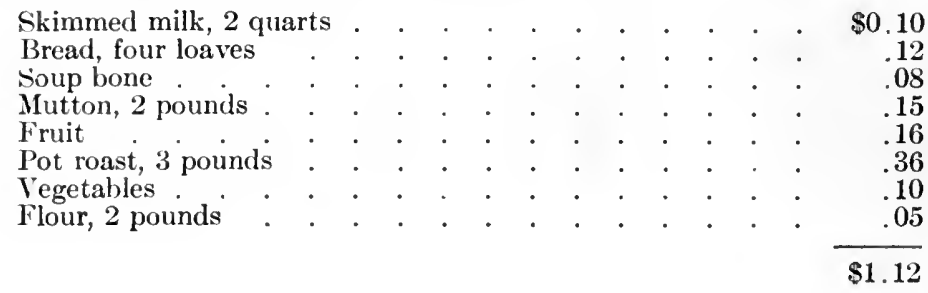

SUNDAY.

Breakfast.-Oatmeal, bread, butter, skimmed milk, coffee, sugar.

Dinner.-Pot roast, with browned potatoes, vegetables, bread, cake, coffee. Supper.-Bread, cheese, fruit.

Total expenditure for week, \$5.26.

As skimmed milk is used, fat should be added to the diet of the tuberculous individual in the form of olive oil. This can be done at an additional expenditure of 25 cents per week.

Hydrotherapy.-A sponge bath every morning, followed by brisk rubbing with a coarse towel, is desirable for all incipient and most moderately advanced cases. I usually direct that the water be used as it comes from the spigot, except during the winter, when it should be tempered by the addition of hot water. The bath should be taken in a room with the temperature not lower than $60^{\circ} \mathrm{F}$. 'The bath should be omitted if it causes chilliness or cyanosis, and if there is blood-spitting, or if the morning 
temperature is unduly low (below $97^{\circ} \mathrm{F}$ ). One occasionally encounters a patient who cannot stand the cold water; in some instances the distaste for the cold water may be overcome by starting with luke-warm water and gradually reducing the temperature, or by the use of water to which alcohol, salt, or vinegar has been added. A warm bath may be taken once a week, at bedtime.

For advanced cases with poor circulation and feeble reaction, cold baths are not advisable.

Sponge baths are sometimes of service for the control of fever (see page 757 ).

Clothing. - The tendeney of tubereulous patients is to wear too many clothes, fearing that they otherwise will " catch cold." During the warm months no special precaution is needed, except to see that the clothing is not so heavy that it will cause undue perspiring.

In the winter months the patient should wear moderately heary flannel underwear, woollen stockings, and the usual heavy winter suit. Some advocate, instead of flannel, linen-mesh underwear, believing that the latter is a protection against "colds." 'The secret of dressing properly during the cold months lies in the addition of outside garments, which can be removed if the weather moderates, or when the patient goes indoors for his meals.

Chest protectors are abominations, which the patient should never be permitted to wear.

For details as to nightclothes and the arrangement of blankets, etc., when taking the "cure," see page 752 .

Symptoms.-Cough.-Most patients demand, above all other things, a prescription for their cough; if it were not for the cough and expectorition they feel quite sure that they would be entirely well. It is by catering to this desire that the great majority of "consumption cures" become popular.

It must be carefully explained to the patient that the cough is simply one of the manifestations of the disease and not the disease itself. Furthermore, the cough is not necessarily an evil, but within certain limitations it is beneficial, as it is by coughing that the products of the disease are thrown off. Anything that prevents the cough without at the same time improving the pulmonary lesion is harmful.

'The two most potent factors for controlling the cough are fresh air and rest, particularly the latter. An increase in the cough, it will be recalled, is one of the contraindications for exercise. The patient who suffers from a cough serious enough to require interference is one who should be at rest, either in a reclining chair or in bed, depending on the severity of the cough and the presence of other symptoms.

Early cases of tubereulosis rarely suffer from a cough which requires the use of drugs. It is wonderful how many of these cases can be carried through a course of six months' treatment, especially in sanatoria, without medication of any kind, so far as the cough is concerned. In a few of these early cases, when first seen, the cough is irritating and annoying, especially at night, thus interfering with sleep. In such cases it is permis- 
sible to administer small doses of heroin $\left(\mathrm{gr} \cdot \frac{1}{12}\right)$ or codeine sulphate (gr. $\frac{1}{3}$ ), either alone or in combination with some expectorant, three or four times daily. A single dose at bedtime may be sufficient. 'The administration of these remedies should not be persisted in, however. Usually, a week or ten days is sufficient, as by that time the fresh air and rest will have exerted a favorable influence, and, besides, the patient should have learned to aid in controlling the cough.

Control of the cough on the part of the patient is a feature that is too often neglected. Some patients acquire this faculty very quickly, while with others it takes considerable patience and insistence on the part of the physician to gain their coöperation. Neurotic, excitable patients are especially prone to react to cough stimuli, and unless carefully trained will do a great deal of harmful and needless coughing. 'The number of unteachables in this regard depends a good deal on the amount of time spent on them.

Inasmuch as the object of the cough is to remove the secretion from the bronchi, any cough which fails to accomplish this is, to a certain extent, unnecessary. Violent, paroxysmal, unproductive coughing is not only unnecessary, but harmful, entailing, as it does, attacks of reflex vomiting and the danger of hemorrhage.

In teaching a patient to control his cough, he must be urged to resist the inclination to cough as long as possible. This may be aided by fixing the mind on some pleasant thought; by taking a deep breath slowly and holding it five or ten seconds; by taking a moderately deep breath and immediately starting to exhale slowly; by a moderately forced expiration or inspiration alone. A few sips of hot water or hot milk of ten are of service, and the morning cough is of ten diminished in severity by taking a cupful of hot water before rising. Unnecessary talking and loud and sustained laughing should be avoided. That the cough is amenable to control on the part of patients is evidenced by the fact that many consumptives who in private will give way to violent paroxysms of coughing are in public able to restrain their cough, because of the wish to avoid attracting attention to themselves.

Persistence in these simple measures for controlling the cough will often give the most gratifying results. I have seen patients, who had been educated in this way, have little or no cough in spite of the fact that they expectorated large quantities of sputum, and had most advanced pulmonary lesions.

Posture will also increase or diminish the cough, as the case may be. 'This is especially true in advanced cases, with cavity formation. Patients themselves will usually discover that by lying on one side or the other the tendency to cough is lessened. This is due to the fact that they lie on the side on which the cavity is situated, thus preventing the secretions from constantly irritating the bronchial mucous membrane. The cleaning out of these cavities is often facilitated in the morning by having the patient stoop over and lower the head.

Another way in which the congh may be controlled without resorting to cough remedies is by attention to the gastro-intestinal tract. Any case 
that suffers with gastric disturbances is quite apt also to have an exaggeration of the pulmonary symptoms. It is useless to expect any improvement in the cough so long as the gastric derangement remains unchecked. The management of these gastro-intestinal disturbances will be considered under another heading.

Local Measures.-Iocal measures in the way of counterirritation may be tried. A mild comnterirritant effect can be obtained by means of mustard plasters applied daily for four or five days, or by painting the skin with tincture of jodine every second or third day. Counterirritation is best obtained, however, by the use of a fly blister (emplastrum cantharidis) applied at diflerent points every third or fourth day.

In applying the "fly blister," a hot compress should first be placed over the site to be blistered, and allowed to remain until the skin has become hyperemic. 'The blister (preferably 2 by 2 inches) is then placed over the hyperemic area and allowed to remain from two to four hours, the length of time depending on the toughness of the skin and the blistering power of the plaster; in some cases it may take even longer.

On the removal of the blister a large bleb filled with serum will form. Some observers state that this bleb should be protected and not allowed to rupture, as the absorption of the serum is believed to exert a favorable influence on the tuberculous process. It is certainly interesting to note that many afebrile cases which have been blistered have a rise in temperature for a day or so afterward, possibly indicating a reaction from the absorption of the serum. 'The blister should be protected by a sterile gauze pad held in place by adhesive strips. If the blister breaks, zinc oxide ointment is a soothing and efficient application.

In dealing with the cough of advanced cases the same general principles used in the early and curable cases should be adhered to. A good deal of needless therapy can be avoided if it is kept clearly in mind that the pathological conditions in these advanced cases prohibits the hope of entirely abolishing the cough and expectoration. 'The best that can be hoped for is that the cougl can be sufficiently controlled to secure comfort and reduce to a minimum the dangers incident to this stage of the disease.

In the selection of a drug which will ameliorate the cough and render the expulsion of the sputum easier, one is confronted with a long list of remedies. None of them, however, exert any lasting beneficial effect. 'That which aids one case often fails in another.

Personally, I have never had the experience claimed by some, of finding any drug or combination of drugs that can be routinely employed with good results.

Of the various drugs used in treating the cough of tuberculosis, creosote holds the first place, and is undoubtedly of service in some cases. This drug has suffered and still suffers from a misapprehension as to its limitations. It is in no sense a specific, and has no place in the treatment of early uncomplicated cases of tuberculosis. Furthermore, it has no antiseptic value whatever, and no matter how administered is incapable of exerting any influence on the tubercle bacilli within the lung. 
Its great field of usefulness is in cases with profuse yellowish or greenish-yellow expectoration, extending over weeks or months, and containing large numbers of staphylococci and streptococci. In some cases of this type, but not in all, creosote will frequently diminish the quantity of sputum and render its expulsion easier.

Some contend that the dose of this drug should never exceed three to five drops; others assert that it only produces its best results when given in large doses; while still another group condemns the drug absolutely, because of the gastric disturbances which sometimes follow its use.

In the type of case I have indicated, creosote, in my opinion, is frequently of service, and, if properly administered, will not of ten disorder the stomach. I usually administer the drug after the method recommended by Flick. Pure beechwood creosote should be used, the initial dose being one drop, three times daily, fifteen to twenty minutes before taking food. For each drop of the creosote there should be a tablespoonful of hot water, and the mixture stirred until thoroughly emulsified. 'The dose should be increased one drop daily, the increase of the creosote being accompanied by a corresponding increase of one tablespoonful of water. When the larger doses are reached the water need not exceed a tumblerful. Given in this way the dose may be carried as high as twenty or thirty drops three times daily. I rarely exceed ten drops three times a day.

Of the derivatives of creosote, guaiacol and guaiacol carbonate seem to be the best. Jacobi claims that under their use the expectoration becomes more mucous, the cough looser, and the patient gains in weight. All of this, he says, happens in the arerage case among the poor, who have none of the advantages of the rich. He uses the following formulas:

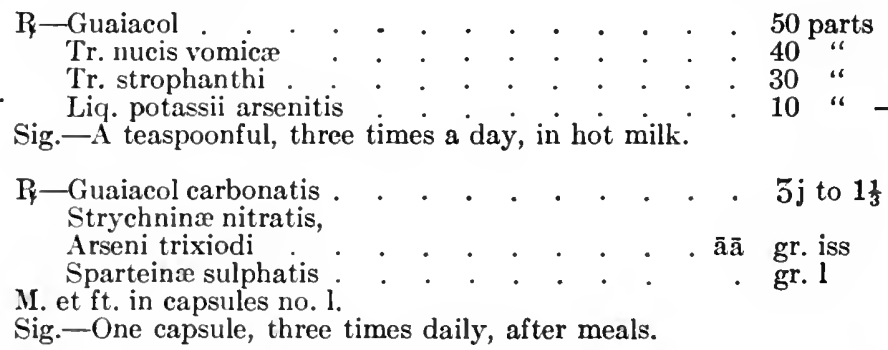

I have employed guaiacol and guaiacol carbonate alone, and in the combinations recommended by Jacobi. Just as with creosote, I have never found that they were of service except in certain selected cases.

A mixture containing ammonium chloride is sometimes of service if an intercurrent "cold" develops (see page 796).

Finally, in the terminal stages opium in some form must be resorted to. Its use, however, should be delayed as long as possible, and the dose employed be just sufficient to allay excessive coughing. If too much is used the effort to raise the morning sputum is so violent and exhausting that the patient suffers quite as much as from being awakened during the night at frequent intervals. 
I cannot too strongly emphasize the importance of combating the cough, as far as possible, without the use of drugs, and, above all things, the avoidance of using cough remedies as a matter of routine.

Fever-Fever is a most importint symptom in tuberculosis, and a study of its manifestations yields much information, as from this one symptom we can trace with reasonable certainty the destructive process of the disease as it progresses from its incipiency to the terminal stages.

It is now generally acknowledged that fever may be due to the tubercle bacillus alone, although it is never as violent as when the pus organisms (streptococci, staphylococci, etc.) are added to the process.

In the early stages of the disease, when the infection is presumably purely of a tuberculous nature, the temperature is but moderately elevated in the afternoon or evening, although in acute miliary tuberculosis, where the dissemination of the tuberele bacilli is widespread, the temperature may be high, is usually continuous, and may be inverted in type, that is, the rise occurs in the morning and the fall in the evening. 'The same phenomenon may occasionally be seen in the pulmonary cases when considerable areas of the lung are freshly invaded. As the disease advances and the pus organisms become associated with the tubercle bacillus the temperature assumes the familiar hectic type, the difference in the morning and evening temperatures sometimes amounting to $6^{\circ}$ or $7^{\circ} \mathrm{F}$.

From the standpoint of treatment, fever is very important. Indeed, the management of the fever constitutes, for the most part, the treatment of tuberculosis, for no matter what is done the results are largely judged by the effect produced on this one symptom. So long as the temperature continues to show an evening rise, no matter how slight, the process must be considered as still active, and if after a period of quiescence the temperature is affected by exertion, the visits of friends, or excitement of any kind, it must be assumed that the disease is not yet arrested.

As I have already pointed out, the importance of rest in controlling the manifestations of tuberculosis are not fully appreciated, and indiscretions regarding exercise are entirely too frequent. It is, of course, understood that during the period of enforced rest the patient is in the fresh air the entire time, either in a tent, a shack, a sleeping balcony, or in a room capable of being freely ventilated.

While we may, on occasion, temporize with some of the symptoms of tuberculosis, fever admits of no compromises. With the possible exception of tuberculin, rest and rest alone is the one measure on which we can place any reliance for permanently reducing the fever. Other agents will at times temporarily control it and add to the comfort of the patient, but they do not remove or affect the underlying cause.

The rules regarding rest, which are to be followed when fever is present, have been given on page 75S. 'The reasons for rest must be explained fully, not only to the patient, but to his friends as well. Probably more difficulties are encountered in enforcing rest than any other feature of the treatment. Neither the patient nor his friends can, voL. 1. -50 
as a rule, understand the necessity of absolute rest when he feels so well. Most people associate rest, especially in bed, with a serious illness, and so long as they can be up and about see no reason for such a radical step. In many instances success is obtained only after the greatest perseverance. One must expect and be ready to combat every conceivable excuse to escape the rest treatment. It often happens that after a few days in bed the patient gets impatient at the restraint, or he feels so much better that he is quite sure a short walk around the block or an afternoon spent in a neighboring park will do him good.

In addition to the rest the patient must be prevented from becoming excited or worried. So unstable is the temperature equilibrium of these patients, even after the fever has disappeared, that the slightest thing will often cause a recurrence. Visits of friends, letters, exciting books, or card-playing should be prohibited if the temperature is affected adversely.

Gastro-intestinal disturbances associated with faulty elimination through the bowel are not only capable of causing the persistence of fever, but also of producing sharp rises in a temperature previously normal. Constipation must be avoided, and care taken not to produce bilious attacks by overtaxing the digestive apparatus. Any intercurrent infection or complication associated with a rise in temperature calls for rest.

No definite time can be set as to how long the rest must be persisted in. The following table, published by Burton-Fanning, shows very well the effect of rest on temperature:

\begin{tabular}{|c|c|c|c|}
\hline $\begin{array}{l}\text { Average maximum temperature } \\
\text { for first week of treatment. }\end{array}$ & $\begin{array}{l}\text { Number } \\
\text { of cases. }\end{array}$ & $\begin{array}{c}\text { Percentage } \\
\text { in which } \\
\text { temperature } \\
\text { became normal }\end{array}$ & $\begin{array}{l}\text { Average } \\
\text { number of weeks } \\
\text { required to } \\
\text { reduce fever. }\end{array}$ \\
\hline Between $99.0^{\circ}$ and $99.5^{\circ} \mathrm{F}$. & 37 & 87.5 & 3.6 \\
\hline Between $99.5^{\circ}$ and $100.0^{\circ} \mathrm{F}$. & 18 & 54.4 & 5.7 \\
\hline Between $100.0^{\circ}$ and $100.5^{\circ} \mathrm{F}$. & 11 & 46.6 & 4.8 \\
\hline Between $100.5^{\circ}$ and $101.0^{\circ} \mathrm{F}$. & 9 & 34.2 & 6.5 \\
\hline Between $101.0^{\circ}$ and $101.5^{\circ} \mathrm{F}$. & & 37.5 & 17.5 \\
\hline
\end{tabular}

From a study of 716 patients under sanatorium treatment, BurtonFanning found that 50 per cent. lost their fever in an average of one month.

'The length of time necessary to enforce the rest after the temperature has become normal varies. In general terms it may be said that the rest should continue for two or three weeks longer. At the end of this time, providing there are no other contraindications, the patient may be allowed to sit up for half an hour or an hour, and increase this daily by the same length of time. At the end of a week or ten days exercise may then be started. If the fever has originally been slight (under $100^{\circ}$ F.), and the rest has been taken in a reclining chair, exercise may be started after the temperature has been normal for two weeks. In exceptional cases a slight evening rise of temperature persists even after prolonged rest. In such cases one may try the effect of exercise. It sometimes happens that the exercise will cause a disappearance of the fever. 'This step should never be taken, however, except as a last 
resort, and if the exercise causes an increase of the fever, the rest treatment must again be enforced and persisted in.

When it becomes apparent from the symptoms and physical signs that the outlook is hopeless, absolute rest need not be enforced. Our chief concern must be to make the patient comfortable, and it is in this elass of cases that hydrotherapy and antipyretic drugs may be found of service.

Hydrotherapy. - Some form of hydrotherapy is at times of service when the fever is high $\left(103.5^{\circ}+\mathrm{F}\right.$.), and while the effect on the temperature is only temporary, it often makes the patient more comfortable. 'The water should not be too cold in the beginning, as many of these patients do not react well, and often complain of chilliness for some time afterward. A sponge bath with the water at a temperature of $98^{\circ} \mathrm{F}$. should first be tried, and if no discomfort follows its use the water at the next bath may be colder and gradually reduced if necessary to $50^{\circ}$ or $60 \mathrm{~F} .^{\circ}$ Instead of plain water, some patients find salt water, vinegar and water (vinegar, 1 ounce; water, 1 pint), alcohol and water (equal parts), or alcohol alone, more agreeable.

Drugs.-Alcohol, once popular in the treatment of tuberculosis, has been largely abandoned. Brehmer advised its use in cases of persistent fever. While I have occasionally prescribed one to two ounces of brandy three times daily when the fever persisted, my experience has not given me much confidence in the drug.

Antipyretic drugs are not as much used as formerly. 'They should never be prescribed by routine, and it should be clearly understood that they do not produce anything more than a temporary reduction of the fever. Their continued use is dangerous. The following drugs have been recommended: Antipyrine, gr. iij; aspirin, gr. v; phenacetin, gr. v; acetanilide, gr. v; the latter being especially dangerous. 'The drug selected should be given an hour or so before the expected rise in the temperature. Pyramidon has been recommended as the safest and most efficient antipyretic drug. It should be given in $2 \mathrm{gr}$. capsules, at 9,10 , and 11 A.M.

Personally, I rarely resort to any of these drugs for their antipyretic effect.

Hemoptysis.- Of the many drugs that have been recommended for the control of pulmonary bleeding there is not one that can invariably be relied upon. 'This is in all probability to be explained by the fact that the nature of the hemorrhage varies greatly in different cases.

'The simplest form of pulmonary bleeding is that due to oozing, the result of congestion, or from granulations which line the wall of a cavity. Under these circumstances the sputum may be blood-streaked, or if the blood and sputum become thoroughly mixed the latter may be red, brownish, or salmon colored. By some the term pulmonary hemorrhage is restricted to those cases in which pure blood is expelled; this may vary from a teaspoonful to a pint or more. Such hemorrhages are usually the result of the rupture of one of the pulmonary bloodvessels, either venous or arterial. According to Minor, early hemor- 
rhages are always venous, and since the pulmonary veins carry arterial blood, are always bright red in color. In the later stages of the disease the bleeding is usually arterial and the blood dark in color.

'The occasion of hemorrhage in the early stages of tuberculosis cannot always be determined, although often it may be traced to factors which raise the blood pressure, such as overexertion, worry, and excitement.

In the advanced stages of tuberculosis the bleeding can usually be explained by the pathological changes in the lung. In the formation of a cavity the arteries, being more resistant than the pulmonary tissue, withstand better the eroding effects of the tuberculous process, so that it frequently happens that they are laid bare in the walls of the cavity, or may even cross it. It has furthermore been shown that these vessels are commonly weakened by small aneurysms, which are apt to rupture if the blood pressure is suddenly raised.

It is a matter of common observation that hemorrhages often occur in a number of individuals within a very short time. This epidemic occurrence of hemorrhage has been explained in two ways: (1) That it is due to meteorological conditions, and (2) that it is the result of an acute infection.

Minor, of Asherille, for instance, has gained the impression that hemorrhages are more apt to occur during a close, damp, hot spell, with a low barometer, and that they are less common in winter.

Several years ago Flick, Ravenel, and Irwin made a study of hemorrhage cases, and their results seemed to indicate that the pneumococcus was the offending agent. My own observation is that many of these hemorrhages are strongly suggestive of an infection, as it has been repeatedly noted that when a case suffering from blood-spitting occurs in the White Haven Sanatorium other instances are apt to follow. Only recently eight successive cases occurred within ten days.

Having in mind the various factors which may produce pulmonary bleeding, the treatment of this condition should really begin before it occurs. By this I mean that the patient should be warned against the things which we definitely know render the accident possible; such, for instance, as sudden or severe exertion, fatigue, violent paroxysms of coughing, contact with anyone having a cold (so far as this is possible), or the neglect of a cold aiready acquired. If there is reason to believe from the history of the case or from the physical signs that a hemorrhage may occur, long railway journeys should not be permitted. The fatigue incident to such trips is not infrequently a cause of hemoptysis.

In patients who give a history of having had a number of hemorrhages, often without any obvious exciting cause, our efforts toward prevention should be redoubled. Such cases require the strictest supervision, for the slightest indiscretion may cause a recurrence.

Finally, in view of the fact that there is some reason to believe that hemorrhages are, at times, infectious, hemorrhage cases should be isolated, and the same procedure should be followed with those having acute colds. 
There is no feature of the disease which the average patient dreads more than the occurrence of a hemorrhage, and when it actually does occur he is usually an object of terror and demoralization. Although the laity is of the opinion that hemorrhage is a frequent cause of death in tubereulosis, it is, as a matter of fact, one of the rarer terminations of the disease. 'There can be no doubt, however, that the occurrence of a pulmonary hemorrhage, even if slight, is a very disquieting thing to most people, and our first duty should be to allay the apprehensions of the patient. Cheerfulness and the assurance that the bleeding is not dangerous and will soon stop will do much toward calming the patient. For those of a nervous temperament a hypodermic injection of a quarter or an eighth of a grain of morphine is often advisable, as the chief danger in many patients is the aspiration of infected blood into healthy parts of the lung by reason of their excitement and exertions.

The principal object in the treatment of pulmonary hemorrhage is to hasten the formation of a clot, and in order to bring this about absolute rest in bed is necessary. In regard to the posture of the patient in bed, that which is most comfortable is the best, provided he does not sit up straight.

If the patient has to expectorate, paper napkins or small squares of linen which can be burned should be used, as they do not require any change of posture. Cough, if present, should be restrained as much as possible, and, if necessary, small doses of Dover's powder (gr. iij), heroin (gr. $\frac{1}{12}$ ), or codeine sulphate (gr. $\frac{1}{8}$ ) may be given to aid in its suppression.

Small pieces of ice held in the mouth may be tried, but too much should not be used, as it may upset the stomach. Placing an ice bag over the precordium is a common procedure, but one of doubtful value so far as the pulmonary bleeding is concerned. In some cases it is of service in quieting an overacting heart.

Talking must be forbidden, and care taken to see that the apprehensions of the patient are not increased by the presence in the room of anxious and frightened relatives.

As the slightest disturbance of the patient may eause a recurrence of the bleeding, there should be no examination of the lungs other than superficial auscultation of the front of the chest. In order that the patient or his relatives may not feel that he is being neglected, the reasons for the omission of the examination should be explained.

'The character of rest depends to some extent on the amount of bleeding. If the sputum is simply blood-streaked, rest in a reclining chair is usually sufficient. It must be borne in mind, however, that large hemorrhages are sometimes preceded by blood-spitting, a feeling of tightness across the chest, and a tickling sensation in the throat.

No matter how small the amount, if pure blood is expelled the patient should be put to bed and kept at rest. Rest should also be insisted upon until the sputum has been free from any trace of blood for at least three or four days.

The diet need not be restricted because of blood-streaked sputum, 
but for moderate-sized hemorrhages the food should be reduced in amount. For some hours after the occurrence of the hemorrhage food should be omitted, and for the first day limited to six glasses of milk and four to six raw eggs. Solid food may then be added to the dietary, and at the end of three or four days, if there is no recurrence of the bleeding, the patient can be allowed the full diet.

In cases of severe hemorrhage our principal consideration is to arrest the bleeding and prevent its recurrence. Except for three or four ounces of milk at intervals of three hours there should be no attempt to feed the patient for the first twenty-four or thirty-six hours. If the hemorrhage has not recurred at the end of this time the quantity of milk may be increased, and in addition raw eggs and solids may be added to the diet gradually.

The hemorrhages that occur in the early stages of tuberculosis do not, as a rule, require very active interference. 'They are usually the result of congestion or the rupture of a small vessel, and all that is necessary is to keep the patient at rest.

In the advanced stages of the disease hemorrhage is a much more serious matter. While many individuals pass through the entire course of the disease with only the most trifling evidences of hemoptysis, there is a possibility of a large and serious hemorrhage in any case in which cavitation has occurred. A patient who has passed into the moderately advanced or adranced stage of tuberculosis should be cautioned against overexertion and shouting or loud singing. Violent paroxysms of coughing should, if possible, be controlled, and anything that tends to produce attacks of coughing, such as smoking, the inhalation of irritating fumes, or sitting in dusty, smoky rooms, should be avoided.

Of the many drugs which have been recommended for the control of hemorrhage, those which have withstood the test most satisfactorily are the nitrites. 'The nitrites are administered on the theory that by lowering the blood pressure in the general arterial system the tension is reduced and the formation of a clot facilitated. Whether there is a corresponding lowering of the pressure in the pulmonary vessels is not yet determined. Although the administration of the nitrites may not be physiologically sound, there seems to be sufficient clinical evidence that they are often of service in checking pulmonary bleeding.

Flick, who first introduced the nitrites, has recommended spirit of glonoin, one minim every hour for four or five doses; the drug is then given in one-minim doses three or four times daily for a few days. He believes that the accentuation of the second pulmonic sound can be utilized to determine the frequency of the dose, and that the sharpness of the accentuation is also of service in anticipating a hemorrhage.

Francis Hare introduced amyl nitrite in the treatment of pulmonary hemorrhage. 'This form of the drug is probably the most useful agent we have for the immediate checking of the bleeding. If, however, one administration fails it is best not to repeat it. Pearls of amyl nitrite containing from three to five minims may be given to the patient, with 
instructions to crush one of the pearls in a handkerchief and inhale the fumes if a sharp hemorrhage should occur. A patient who has never inhaled amyl nitrite should be warned of the disagreeable seusation it causes, otherwise his fear and nervousness may be increased. Nitroglycerin, hypodermically, may also be used to advantage when the hemorrhage is taking place. For the prevention of a recorrenee, nitroglycerin is usually given by the mouth; and sodium nitrite in doses of $\frac{1}{6}$ to $\frac{1}{2}$ grain, three or four times daily, may be used for the same purpose.

While the nitrites seem at present to be the most popular means of controlling hemorrhage, there are other drugs which some use hy preference, and which even the adherents of the nitrites are at times forced to employ. Among these may be mentioned tincture of veratrum viride, in doses of five minims two or three times daily; atropine sulphate, hypodermically, in doses of from $\frac{1}{120}$ to $\frac{1}{50}$ grain; ergot, the use of which is more than questionable; the old-fashioned remedy turpentine, two or three drops of which may be given on a lump of sugar; and digitalis, which, although it raises the blood pressure, is sometimes of service in cases of prolonged or slight hemoptysis presumably due to congestion.

The use of a blood-pressure instrument has been advised in order to determine whether a vasoconstrictor or a vasodilator is indicated.

In addition to the drugs used for the immediate hemorrhage others have been recommended for the purpose of increasing the coagulability of the blood and thus preventing recurrences. For this purpose the calcium salts, especially the lactate, have been adrocated. Calcium lactate may be given in dose of from five to twenty grains in water, three times daily. Boggs advises that if the coagulation time cannot be taken regularly the calcium should be taken for three and omitted for two days. Gelatin by mouth and lime water added to the milk have also been recommended for increasing the coagulability of the blood. Bandaging the lower extremities has also been recommended when the hemorrhage is large. I have never seen that these measures accomplished any good.

Night Sweats.-Night sweats may occur in any stage of tuberculosis. In incipient cases, however, they are comparatively infreguent. As the tubereulous process advances, and especially when pus organisms become associated with the tubercle bacillus, night sweats become increasingly frequent.

It must be borne in mind that sweating is due to the action of a toxin (tubercle bacillus or other bacteria) on the sweat or heat-regulation centres. (Cornet.) Formerly it was the custom to treat sweating symptomatically, but in the majority of cases symptomatic treatment is irrational, and can at best give only temporary relief. So long as the underlying cause of the sweating remains unchecked the symptoms will persist.

In the curable stages of the disease sweating is rarely so profuse or obstinate as to demand medication. Rest in bed, in the fresh air, proper feeding, and the avoidance of too many bedclothes will cause a disappearance of the sweating, with but few exceptions, in a very short 
time. Night sweats occur less frequently in patients whose emunctories are active. Constipation should be avoided, and gastric derangements corrected as far as possible in all cases. If the sweats persist or are unduly severe, symptomatic treatment may be employed temporarily.

Even for the advanced cases the treatment just outlined may suffice; but for the drenching "colliquative sweats," which soak the bedclothes and occur several times during the night, medication is necessary. Every effort should be made to lessen the discomfort of the patient as much as possible. The bedclothes should be light and just sufficient to prevent chilling. If they become wet they should be changed at once and the patient rubbed dry.

Sponge baths at bedtime often prevent or lessen the severity of the sweating. The patient may be sponged off with equal parts of water and alcohol or an ounce of eau de cologne to a pint of water. Vinegar, an old-fashioned household remedy for night sweats, may be used pure or in the proportion of an ounce to a pint of water.

If these measures fail, internal medication must be resorted to. The following drugs have been recommended: Camphoric acid, grains $\mathrm{xx}$ at bedtime; aromatic sulphuric acid, minims $\mathrm{xxx}$ at bedtime, or minims $\mathrm{v}$ three times daily; agaricin, grain $\frac{1}{30}$ at bedtime; and atropine sulphate, grain $\frac{1}{200}$ two or three tmes daily. The last-named drug is the most efficient.

Brown recommends the following combination:

R-Atropinæ sulph.
Morphinæ sulph.
Acid. sulphur. aromat.
Syrup tolu
Sig. -To be taken at bedtime.

$$
\begin{aligned}
& \text { gr. } \frac{1}{100} \\
& \text { gr. } \frac{1}{6} \\
& \text { mxv } \\
& \text { f } 3 \mathrm{j}-M .
\end{aligned}
$$

Dyspnea.-Dyspnea is not often a symptom that demands attention in the early stages of disease, and even in the later stages, when the destruction of pulmonary tissue is great, it does not usually require medicinal treatment.

The dyspnea that occurs in the early stages of tuberculosis disappears as the pulmonary lesion improves, rest and fresh air being the most important factors. The presence of dyspnea is a contraindication for exercise, and a patient who when placed on exercise becomes short of breath should at once be returned to rest, or the amount of exercise should be reduced to a point short of that which produces shortness of breath.

As the disease advances, dyspnea becomes increasingly frequent, although surprisingly few cases develop the symptom to such an extent that it requires treatment. When the dyspnea seems to depend on curtailment of the breathing space, exertion, with its demands for an increased supply of oxygen, must be reduced to a minimum. So, too, if there are indications of a weakened heart muscle, rest rather than a cardiac tonic is needed in these cases. Although digitalis, strophanthus, or strychnine may be tried, I cannot say that I have ever found them 
particularly serviceable. Nitroglycerin has at times seemed to exert a favorable influence on the dyspnea, whether because it relieves pulmonary congestion or produces alterations in the intrathoracic pressure I cannot say.

Dyspnea may be a prominent symptom in chronic fibroid phthisis, and in such cases the only course to pursue is the restriction of physical exertion to a point that will not produce undue shortness of breath. Dyspnea dependent on a thiekened pleura following an effusion is subject to the same rules as that occurring in fibroid phthisis. Some advocate breathing exercises in order to expand the restricted lung.

It is useless to expect that any drug will reduce the number of respirations in an individual whose breathing space has been seriously encroached upon. Morphine, it is true, can be administered in doses sufficiently large to produce such a result, but I do not think anyone would advocate such a course. The only thing possible is to reduce the demand for oxygen by keeping the patient in the fresh air, at rest, either in bed or in a reclining chair. 'The extreme dyspnea that sometimes occurs in the terminal stages of the disease may be relieved by a hypodermic injection of morphine or by inhalations of oxygen.

Dyspnea due to acute plastic pleurisy requires no special treatment, as it disappears under the appropriate treatment for the pleurisy (page 794). The same is true of dyspnea associated with pleural effusions, serous or purulent in character.

Asthmatic attacks sometimes develop in tuberculous cases, especially those with the chronic fibroid type of the disease. 'The treatment does not differ essentially from the non-tuberculous asthmatic cases.

Tachycardia.-The pulse furnishes valuable information not only from the standpoint of diagnosis, but, in addition, its behavior after the patient has been placed on treatment aids in forecasting the probable outcome of the disease; thus, a rapid pulse rate (110 or higher), which is unaffected by rest, is of serious import, while a pulse rate that is comparatively low or one that gradually falls renders the outlook more favorable, other things being equal.

It must be borne in mind that acceleration of the pulse rate is a very common feature of tuberculosis while the disease is active, and that it is not, as a rule, due to disease of the heart itself, but is caused by the absorption of toxins. Therefore, our efforts should be directed not primarily to the heart, but to the underlying condition which is producing the trouble.

The treatment of tachycardia in its relationship to exercise has already been discussed.

I can only repeat that rest in bed or in a reclining chair is inperative if the pulse rate is high, and that exercise or the mental excitement incident to card-playing, receiving visitors, or even reading must be prohibited if they increase the pulse rate. Coffee, alcohol, and tobacco should also be forbidden. If the pulse rate is to be controlled in cases which offer any hope of arresting the disease it will be accomplished largely by rest and the avoidance of excitement. In rare instances 
when the pulse rate remains high in spite of prolonged rest, exercise may be cautiously tried; its effect on the pulse should be noted carefully.

Not much is to be expected of drugs, and this is especially true of digitalis and strophanthus, although both are frequently employed. The bromides, valerian, and menthol have been recommended as useful drugs. Tuberculin should be tried. Abrams recommends the Schott treatment. George W. Norris demonstrated, several years ago, that the pulse rate in tuberculosis could be reduced by the Schott method. In the rare instances, in which in an otherwise favorable case the pulse rate remains abnormally high, these baths might be tried. Their field of usefulness is, however, limited.

In cases steadily progressing to a fatal termination the pulse rate is almost invariably high, and is not affected much by rest. At times these advanced cases suffer from distressing palpitation; this is often helped by placing an ice bag orer the precordium.

Cardiac Weakness.--Symptoms referable to the heart itself are not common in tuberculosis in the early or moderately advanced stages of the disease. In the advanced stages weakness of the heart muscle is common, although, curiously enough, sudden death as a result of cardiac failure is rare; it has not been observed in nearly five hundred deaths at the Phipps Institute.

Patients subject to fainting attacks or sudden dyspnea and cyanosis, the result of cardiac dilatation, should be kept quiet in bed; for the attacks themselves one of the rapidly diffusible stimulants, such as aromatic spirit of ammonia, Hoffman's anodyne, or brandy, should be given by mouth, or strychnine or camphor in oil hypodermically.

Pleuritic Pain.--Pleuritic pain of varying degrees of severity is one of the most frequent symptoms in tuberculosis. The character of the pain varies all the way from a dull aching sensation to pain of a stabbing character, which is aggravated by a deep breath or by coughing.

In the great majority of cases the treatment consists in either immobilizing the affected side of the chest or the application of a counterirritant. When the pain is confined to the lower part of the chest, where the respiratory excursions are widest, immobilization by means of adhesive straps will usually afford relief. In some individuals the adhesive plaster cannot be used, as it causes marked irritation of the skin.

In the upper part of the chest our chief reliance is in some form of counterirritation. The simplest form of counterirritation is obtained by means of mustard plasters, which may be applied once or twice daily for several days. The prepared mustard plasters now on the market, if fresh, are efficient. They should always be applied to the chest with a piece of tissue paper intervening between the skin and the mustard, otherwise a brown stain, which is exceedingly difficult to remove, may result. The mustard plaster should be kept on sufficiently long to cause a burning sensation and a distinct hyperemia.

For the severer grades of pain the affected area may be painted with 
tincture of iodine. 'The iodine may be applied night and morning for several days. A good plan is to paint several small areas at a time, leaving a clear space for the next application. Because it not only relieves the pain, but in addition exerts other favorable effects, the "fly blister" (emplastrum cantharidis) is a most valuable aid, and the one on which I place the most reliance. For the method of applying a "fly blister," see page 783. 'The "fly blister" should be applied every third or fourth day as long as the pain persists. 'The actual cautery lightly touched to the painful area has also been recommended.

Of the drugs available, sodium salicylate, in 5 to 10 grain doses, has been long employed. Aspirin, in doses of 5 grains, three or four times daily, I have found serviceable, providing the pain is not too severe. It is preferable to the other salicylates, as it does not disorder the stomach. For the sharp stabbing pain which is aggravated by coughing, Dover's powder in 3 grain doses, or heroin in $\frac{1}{12}$ grain doses, every three or four hours, may be used.

Occasionally the pain is so severe as to require the hypodermic injection of morphine, but this does not of ten happen.

Anemia.-Even in the early stages of tuberculosis anemia may be a marked feature, but it is remarkable that in spite of the ravages of the disease, anemia is, in the majority of cases, a negligible factor.

As a rule, the blood picture in tuberculosis resembles that seen in chlorosis; that is, there is little or no decrease in the number of erythrocytes and a moderate loss of hemoglobin, giving a low color index. 'This fact has led some to question whether true chlorosis ever exists, and to assert that a low color index is always indicative of tuberculous infection. Brown, of Saranac Lake, recommends that a case of chlorosis which fails to respond to a course of iron should be injected with a diagnostic dose of tuberculin.

In most instances the anemia of tuberculosis needs no special attention, as it soon disappears under the hygienic-dietetic method of treatment. In incipient cases this nearly always suffices, and the same is largely true of the advanced cases. If the general condition of the advanced case improves the anemia will also share in the improvement, irrespective of the administration of iron or arsenic. I think most phthisio-therapeutists place their chief reliance in the treatment of anemia on fresh air, in combination with rest and a generous diet.

In view of the type of the anemia, iron in some form is the drug of choice, if medication is to be employed. Blaud's pill is the most popular and the most efficacious preparation of iron for this purpose.

Arsenic has long been employed in the treatment of tuberculosis, partly because it exerts a favorable influence on the general nutrition and partly for its effect on the anemia. 'There are a number of preparations available - the well-known Fowler's solution or arsenic trioxide; cacodylate of sodium, introduced a few years ago as a specific, and the recently invented atoxyl. I frequently employ arsenic, in the form of Fowler's solution, for its general tonic effect, but never specifically for anemia. 
Complications.-Colds.-In - cities especially the tuberculous individual is often exposed to acute colds, and even when the greatest precautions are used these colds, because of their contagious nature, cannot alwars be escaped. Still every care should be exercised, and the patient should be warned to avoid anyone suffering from a cold.

I always advise patients that at the first intimation of coryza or irritation of the pharynx, with which many of these attacks are ushered in, they are to go to bed at once, take a dose of salts or castor oil, and use mustard plasters over the chest, especially that part of the chest overlying the site of the tuberculous trouble. The mustard plaster may be repeated for several days. For the congested mucous membrane of the upper air passages, inhalations of steam impregnated with compound tincture of benzoin are useful. If the pharynx is much inflamed and irritated, the following astringent gargle can be recommended:

R-Potassii chloratis,

Acid. tannic. . . . . . . . . . . . . $\bar{a} \bar{a} ~ 3 j$

Glycerini,

Aquæ rosæ . . . . . . . . . . . . $\bar{a} \bar{a}$ f hours.

Sig.-Dilute with equal parts of water and use as a gargle every three or four

During the early stages of the cold, when the cough is unproductive in character, and especially if it interferes with sleep, small doses of Dover's powder (grains iij), codeine sulphate (graine $\frac{1}{8}$ ), or heroin (grain $\frac{1}{12}$ ) may be employed. Later a stimulating expectorant, such as ammonium chloride, may be used. The following mixture in use at the Phipps Institute is very serviceable in this condition:

$\mathrm{P}$-Ammon. chloridi .

$3 \mathrm{ij}$

Spts. glonoini

Spts. ammon. aromat.

Tr. nuc. vomic.

Elix, calisay:e

Sig.-Teaspoonful, three times daily, after meals.

mXxiv

fó $j$

f 5 iv

The importance of instituting treatment at the earliest possible moment cannot be too strongly emphasized, for while an acute cold is a simple matter in the perfectly healthy, it is capable of causing a great deal of damage in the tuberculous, individual. Every effort should be made to prevent the infection descending the respiratory tract, for once it does, it is apt to become localized in the damaged portion of the lung and stir into activity a latent tuberculous infection. For this reason acute colds are a not infrequent means of bringing about a relapse.

'The really important thing in dealing with these infections is the rest in bed in a well-ventilated room, or, for those who can afford it, a few days' stay at some place free from dust. Residents in Philadelphia, I have found, are frequently able to abort a cold in its early stages, or cut it short when fully developed, by a week's stay at Lakewood or Atlantic City. 
The old adage, "a stitch in time saves nine," can often be amply demonstrated in these infections. Arrested cases who have returned to work are liable to object to going to bed, involving, is it does, a sacrifice of time and wages. 'Those who neglect the andvice, however, expose themselves to the clanger of a relapse, and in the end often lose more time than they would had they heeded the original advice.

Hoarseness.-Hoarseness occurring in the course of pulmonary tuberculosis must always be viewed with suspicion. While not invariably of a tuberculous nature, it is so frequently so that an examination of the larynx is imperative. 'The treatment of laryngeal tuberculosis will be dealt with elsewhere. What I wish particularly to emphasize is the great frequency of laryngeal lesions, even in cases in which there is no suspicion of their presence. Every case of pulmonary tuberculosis should have the larynx examined, and even in the absence of symptoms the proper precautions taken to prevent extension of the trouble.

Were it not for the fact that I have not infrequently seen hoarseness in tuberculous individuals either entirely ignored or, what is even worse, treated by means of gargles, any mention of this symptom could be omitted from this section. A gargle cannot from the very nature of things exert the slightest beneficial effect on the larynx, and if the attending physician is not himself accustomed to examining and treating the larynx, the patient should be seen by a competent laryngologist. 'To neglect a case with a laryngeal lesion on the ground that tuberculosis of this organ renders the outlook hopeless is no longer warranted. It has been amply demonstrated that tuberculosis of the larynx, if not neglected and allowed to progress too far, is as amenable to treatment as tuberculosis in almost any other portion of the body.

Pleural Effusion.-Small effusions and even many moderately sized ones do not need to be aspirated. Sometimes they are absorbed without interference, while in other instances the absorption may be hastened by the use of a diuretic, such as acetate of potassium; counterirritation in the form of a "fly blister" (emplastrum cantharidis) also seems to stimulate absorption.

Large effusions (extending above the angle of the scapula) should, in my opinion, be removed whether they are causing inconvenience or not; if they cause shortness of breath or other evidences of pressure, there should be no hesitation in performing paracentesis.

'There are some who advocate allowing an effusion to remain, unless it is very large and is producing pressure symptoms, because it is supposed to exert a favorable influence by compressing the lung and thus limiting its movement. 'Then, too, it is believed that the absorption of the pleural exudate has a beneficial effect on the tuberculosis.

Empyema. - The treatment of purulent effusions is surgical, and as soon as the aspirating needle reveals the nature of the fluid the case should at once be seen by a surgeon.

Pneumothorax.-'This accident may occur at any time during the course of tuberculosis, but is most frequently seen in the advanced cases. It may be expected in from 5 to 10 per cent. of all cases. 
As the accident may occur as the result of severe exertion or severe paroxysms of coughing, the patient should be protected as far as possible in this regard.

In some cases the pneumothorax takes place without any acute symptoms, and may be discovered accidentally. In the great majority of cases, however, the rupture is attended with considerable shock, and this may be so severe that death ensues at once or within a few minutes. Accompanying the symptoms of shock there are usually pain and dyspnea. These symptoms demand immediate attention. They are best treated by the hypodermic injection of morphine and such stimulants as strychnine, digitalis, or digitalone. Strapping the affected side will sometimes give relief. If the air within the chest is under high tension, it may be advisable to tap the chest.

If the patient does not succumb within the first few days, the initial symptoms are apt to disappear, and the subsequent treatment then depends on the rapidity with which the effusion forms.

Stanton advised that the effusion be left alone unless it increases to such an extent as to produce pressire symptoms. In case operative interference is required, Carnett practises the following procedure: The skin is infiltrated with Schleich's solution, as the weakness of these patients makes the use of a general anesthetic inadvisable. Following the skin incision in the fifth interspace, in the anterior axillary line, the intercostal tissues are infiltrated and a large trocar and cannula are thrust into the pleural cavity. After withdrawing the trocar a rubber tube is inserted through the cannula, and after the withdrawal of the cannula the tube is stitched to the skin and clamped with a hemostatic forceps. 'This operation does not require more than five minutes. In one instance a portion of a rib was resected. This operation allows of the gradual withdrawal of the fluid.

The subsequent treatment is that ordinarily employed in tuberculosis. Stanton advised that these patients be removed from a hospital as soon as possible and placed in a sanatorium.

Gastric Disturbances.-I have been impressed repeatedly with the fact that physicians in treating tuberculosis are too much inclined to centre their efforts on the pulmonary phenomena. It is not sufficiently appreciated that success in overcoming the disease in the lung is for the most part achieved by indirect rather than direct methods; for, except to a limited extent, we can do nothing directly to influence the pulmonary process or the constitutional manifestations it gives rise to. Indirectly, however, by means of fresh air, rest, and diet the general nutrition of the patient is improved, and the extent to which this is accomplished largely determines the arrest of the disease in the lung.

I know of no phase of this indirect method of treatment, if I may so term it, that is so commonly neglected as the stomach. A good digestion is one of the best assets a tuberculous individual can have, and it should be our constant care to see that it remains so. Every effort should be made to correct digestive disturbances, for unless a sufficient quantity of 
food can be taken and assimilated, a favorable result is doubtful, no matter how efficiently the other details of the treatment are carried ont.

'The stomachs of many patients are disordered through faulty methords of feeding. 'The neerls of each individual should be carefully studied and care taken to avoid overtaxing the stomach with too inuch food or with food difficult of digestion. When foreed feeding is employed, special care must be taken to avoid stuffing the patient with unnecessarily large quantities of food.

'The gastric disturbances occurring in tuberculosis are not peculiar to the disease itself. 'They are such as may be met with in any condition, but demand more than ordinary care because little can be accomplished without a good digestion, and, in addition, the slightest intercurrent affection is apt to aggravate the pulmonary symptoms.

Gastric analyses have shown that in the early stages of tuberculosis the gastric juice is normal, or hyperacility is present; but as the disease advances there is a tendency for the gastric juice to become deficient, either in quantity or quality. In the far-advanced cases the gastric juice is commonly ineflicient. In addition to alterations in the character of the gastric juice, anatomical changes in the stomach occur, with increased frequency, as the disease progresses. In an analysis of the changes found in the stomach of advanced cases dying in the Phipps Institute, I found that many of them showed evidences of gastritis. Many of the stomachs had also undergone ptosis. 'These facts explain to a great extent the gastric symptoms of tuberculosis. In the early stages of the disease gastric symptoms are usually to be ascribed to errors in diet, while in the advanced stages the alterations in the character of the gastric juice and the anatomical lesions are additional factors. Fever is also believed to have an adverse influence on the gastric juice.

An important phase in the trentment of tuberculosis is the prevention of digestive disturbances. 'This is accomplished by carefully regulating the diet and seeing that the bowels are freely opened every day. The teeth should also be examined, and if found in bad condition and incapable of properly masticating the food, they should be attended to or false teeth should be obtained.

No matter what form of gastric disturbance the patient suffers from, rest is essential. 'This is one of the fundamental principles in the treatment of tuberculosis, and applies to gastric symptoms quite as much as to the symptoms referable to the lungs. Rest not only tends to improve the appetite, but also aids digestion, and even after patients have been placed on exercise it is always advisable, for a time, at least, to have them lie down for an hour or two after the heary meal in the middle of the day.

Anorexia is by far the commonest gastric symptom in tuberculosis. It may occur alone or in combination with one or more additional symptoms, and may vary from simple loss of appetite to a positive loathing of food.

Ordinarily, in the incipient stage, loss of appetite is restored by rest 
in the fresh air. Rest, if too prolonged, however, may itself be a cause of anorexia, and under these circumstances regulated exercise is indicated. If anorexia is pronounced, the dietary should consist of liquid foods, which do not require the stimulus of an appetite to be taken. Patients sometimes complain that the morning lunch takes away their appetite for dinner; when this occurs the lunch had better be omitted. Bitter tonics are useful. I usually employ the tincture of mux vomica, in doses of from 5 to 10 minims, three times daily, before meals. 'Alcohol in the form of malt is serviceable at times, but should never be employed unless other methods have failed.

One of the best means of restoring the appetite is, when possible, a change of scene and food. When the milk-and-egg diet is being used it also is sometimes advisable to change for a time to three meals a day.

Other symptoms commonly met with are pain, or a heavy feeling, in the epigastrium after taking food, distention, and nausea, with or without vomiting. Symptoms due to hyperacidity are rarely encountered.

To insure accuracy a gastric analysis should, of course, be made in all cases presenting digestive disturbances. Practically, however, this is not always feasible, nor for clinical purposes is it necessary. From the work already done in this respect it is reasonably. certain that in most instances when the advanced stage of the disease is reached the trouble is due to insufficient gastric juice, and that diluted hydrochloric acid or pepsin, alone or together, are indicated. I usually give the hydrochloric acid in ascending doses, starting with five drops, three times a day, after meals, and increasing one drop daily up to twenty drops, three times a day. The acid is taken well diluted with water. Peptonized milk is often very serviceable. Homemade kefir and koumiss may also be tried for the same purpose. The fermented milks are especially useful if there is much flatulence. Distention coming on some time after eating is often helped by pancreatin. When this symptom is complained of I often employ the following:

$$
\begin{aligned}
& \text { R-Pancreatin .. . . . . . . . . . . . . 3ss }
\end{aligned}
$$

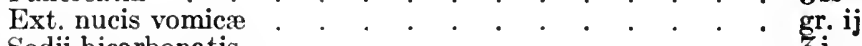

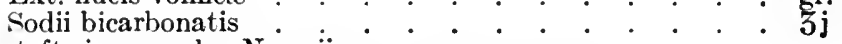

M. et ft. in capsules No. xii.

Sig.-One capsule, three times daily, before meals.

In the early stages of tuberculosis stomach symptoms are not so frequently encountered as they are later, and they are not, as a rule, due to changes in the stomach itself, but to errors in diet. If patients are given too much to eat, especially in the form of fat, bilious attacks are apt to develop. Individuals vary greatly in their ability to assimilate fat, and it has been my experience that bilious attacks are more often due to fat intolerance than anything else. Raw eggs, the yolks of which are very rich in fat, are especially apt to cause these attacks. The symptoms are as follows: Anorexia; nausea, with or without vomiting; constipation; a heavily coated tongue, and a foul breath. The skin frequently presents a sallow appearance, and there may be a sharp rise 
in the temperature. 'The treatment of this condition consists of absolnte rest in bed, the administration of a saline, and a reduction in the guantity of food taken. Eggs should be omitted entirely, and the fat may be reduced still farther by using skimmed milk. The length of time the patient should be on a reduced diet will vary from three or four davs to a week, depending on how quickly the tongue clears up and the appetite returns. Small doses of magnesium sulphate are especially useful at this time, and should be continued until the tongue has become elear.

R-Magnesii sulphat.

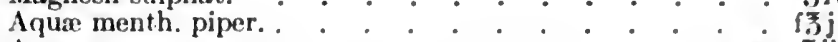

Sig. Aqua teaspoonful every three hours.

q. 8. 3ij-M.

After the bilious attack has been relieved the quantity of food should be gradually increased, but the amount taken should be less than that originally given. If an excess of 'fat in the diet is believed to have been the cause of the attack, this variety of food must be given in small quantities. Raw eggs especially should be omitted.

Diarrhea.-Attacks of diarrhea may oceur in tuberculous individuals under the same circumstances as they do in the healthy-namely, as the result of some indiscretion in diet. The condition is relieved by removing the offending material from the intestinal tract by means of a dose of castor oil or calomel followed by a saline. This will usually end the attack. If after the oil or calomel has acted loose movements continue, bismuth subnitrate, in doses of thirty grains, may be administered three or four times daily. The diet should also be limited to milk for several days.

Diarrhea due to the ingestion of too much fat, while in no way peculiar to tuberculosis, is more frequently met with in this disease than in any other condition, because forced feeding is so generally in use. 'The symptoms and treatment of fat intolerance have been considered. (See above.)

Diarrhea due to intestinal tuberculosis is a much more difficult condition to combat, and is at times beyond our control. In the present state of our knowledge there is no means by which we may be certain of the presence of tuberculous ulceration of the intestines. Inasmuch, however, as there is always associated with the ulceration some catarrhal inflammation of the mucous membrane of the bowel, the treatment does not differ essentially from that of chronic intestinal catarrh.

Rest in bed and an appropriate diet are of the most importance. Boas asserts that by these two measures alone he has been able to control some cases of tuberculous diarrhea. Rest, according to Boas, is essential, as it puts a stop to the external influences so often conspicuous in chronic diarrhea-namely, cold, wet, mental or physical exertion, and, above all, dietetic excesses or transgressions.

His requirements as to diet are: (1) To avoid injurious substances which might increase the intestinal catarrh; (2) to select such nutrients voL: $1 .-51$ 
as will be assimilated in the particular case; (3) to combine the greatest variety of those nutrients whose physiological action is astringent or which reduce secretion-in other words, those which have a tendency to constipate.

Foods to be avoided are those which are coarse and indigestible, particularly those containing much cellulose, such as cabbage, salad, pickles, beets, and other root vegetables. Also organic acids and sugar. For sweetening the food or drink in these cases, Boas recommends saccharin, as it has the additional value of being to some extent an intestinal antiseptic.

Cold drinks are to be avoided, especially cold milk. The milk should be heated, and may with advantage have added to it lime water, barley, rice, Indian meal, or oatmeal. Eggs should be omitted.

Foods which tend to diminish peristaltic activity should be used. Of these, Boas especially recommends farina, rice, barley, and oatmeal made into soup or a gruel.

The following dietary (somewhat modified) is one which Boas recommends as a guide in treating these cases of chronic diarrhea:

8 A.M. - Cocoa, one saccharin tablet, toast, and butter ( $\frac{1}{2}$ ounce).

10 A.м. - One cup (six ounces) of rice gruel. In addition one and onehalf ounces scraped beef or fried fish or cold meat (avoid salt or strongly pickled ham).

1 P.M. - Pea or bean soup or purée of oatmeal, farina, or cornstarch. Rice or farina bouillon, well thickened by cooking (six ounces). Green vegetables or potatoes in purée (six ounces). Meat and fish, fat excepted, (one-half to three ounces). Butter sauce allowed; highly seasoned sauces forbidden. Stewed fruits, with the exception of huckleberries and cranberries, are forbidden. Custards and cornstarch, with a little yolk of egg and saccharin, are allowed.

4 P.M. - Tea, with saccharin, or cocoa. Toast. Zwieback, with butter.

7 p.M. - Oatmeal gruel. Cold or warm meat (two ounces). Toast and butter (one-half ounce).

Recently, milk which has been subjected to the fermentative action of lactic-acid bacilli has been highly recommended in intestinal disorders. For this purpose buttermilk, kefir, or koumiss may be used. In addition there are now on the market a number of lactic-acid bacilli tablets which may be taken alone or may be added to the milk. These various forms of fermented milk are most useful in cases in which the diarrheal movements are foul smelling, the result of putrefactive changes. They may be used also in cases of intestinal tuberculosis. In addition, if the diet has been exclusively a milk one, the buttermilk or kefir or koumiss offers a welcome change in some cases.

Failing to control the movements by means of rest and diet, resource must be had to internal medication. For this purpose bismuth subnitrate (grains $\mathrm{xxx}$ ), alone or in combination with salol (grains $\mathbf{v}$ ), or beta-naphthol (grains v), three or four times daily, may be used for the milder cases. Creosote in three to five minim doses, three times daily, has also been recommended. 
The following formula used at the Phipps Institute is very efficient:

R-Tr. opii deodorati

Emul. ol. rieini

f 5 ij

Sig.-A dessertspoonful, two, three, or four times daily, as the case requires.

For the severer forms one grain of powdered opium in the form of a suppository may be used once or twice daily. A very good astringent mixture which may be used in extreme cases is the following:

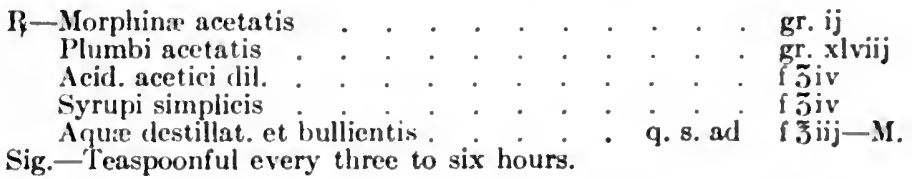

(The water must be distilled, or the salts in it will preeipitate the mixture; the water should be boiling, or the carbonie acid gas in it may also precipitate the mixture.)

Abdominal pain is not frequent when tuberculous diarrhea is present; but if it is, hot fomentations, a mustard plaster, or a turpentine stupe should be applied to the abdomen.

Constipation.-Under any circumstances there should be at least one free bowel movement daily, and this becomes even more essential if forced feeding is being employed. If the patient is inclined to be constipated the dietary should contain plenty of fruit and coarse fibered vegetables. Water drunk between meals is also of service. Massage over the course of the colon is of help in some instances.

'The best drug to employ is cascara sagrada, in the form of the fluidextract. I usually direct that thirty drops of the fluidextract of cascara sagrada be taken at bedtime; if this is not sufficient the same amount is also taken before breakfast. This dose may be increased or diminished according to the effect produced. At the end of a week the patient can usually omit the morning dose. A few days later the evening dose is taken every other night only, and finally stopped altogether.

In a few instances, when the constipation is marked, the more powerful laxatives will have to be employed.

In patients who are on forced feeding it is a good plan to thoroughly empty the intestinal tract once a week by means of a dose of salts or castor oil.

Fistula-in-ano and Peri-anal Abscesses.- The treatment of these conditions is surgical. It may not be amiss, however, to point out the fact that the operation should be thorough; never be satisfied with anything but complete obliteration of the fistulous tract, otherwise there may be a recurrence.

It is well to bear in mind that fistula-in-ano and peri-anal abscesses, in addition to complicating tuberculosis, are frequently the forerunners of pulmonary trouble. Collier F. Martin has investigated this latter phase of the subject. In his experience a very high percentage of these conditions are tuberculous, and unless proper pre- 
cautions are taken pulmonary tuberculosis is apt at some future time to develop. Following the operation the patient should lead the same life as the one frankly tuberculous, $i$. $e$., early hours, fresh air, avoidance of fatigue, and a'generous diet. This need not interfere with the individual's occupation.

Insomnia.-Insomnia should be relieved, if possible, without resorting to drugs. A sponge bath at bedtime, with tepid water, equal parts of alcohol and water, or alcohol alone, should first be tried. If this fails, one of the hypnotic drugs, preferably veronal, trional, or sulphonal, may be given. Veronal is best given in a capsule in doses of from 5 to 7 grains at bedtime. The trional and sulphonal are given in doses of from 10 to 15 grains, in powder form or in a cachet, and followed by a glass of warm water or milk to hasten absorption. An ounce of whisky or brandy in a glass of warm milk may also be used.

If the sleeplessness is due to coughing, Dover's powder (grains iij), heroin (grain $\frac{1}{2}$ ), codeine sulphate (grain $\frac{1}{8}$ ), or sodium bromide (grains $\mathrm{x}$ to $\mathrm{xv}$ ) may be sufficient. It goes without saying that the use of any of these drugs should be abandoned as soon as possible.

Tinea, or Pityriasis Versicolor.-Tinea, or pityriasis versicolor, is not infrequently seen in tuberculous patients. It has no special significance, occurring, as a rule, in those who bathe infrequently. It is usually removed by the use of castile soap and warm water. Several applications of vinegar or diluted acetic acid, followed by sodium hyposulphite, a dram to an ounce of water, may also be used.

Diabetes Mellitus and Tuberculosis.-Diabetes mellitus and tuberculosis is one of the most fatal combinations that can occur. The tuberculous process usually pursues a very acute course, and resists all efforts to control it. 'The treatment should be symptomatic, and the patient, so far as possible, made comfortable.

Syphilis and Tuberculosis.-This is a vicious combination, and is generally recognized as such by both syphilographers and phthisiotherapeutists. Experience has shown that a tuberculous patient who acquires syphilis has his chances of recovering from the tuberculous infection decidedly lessened, because in order to control the syphilitic manifestations, mercury and, later, the iodides are necessary.

Neither of these drugs are well borne when tuberculosis is present, and this is especially true of the iodides, which, it will be recalled, have been advocated for the purpose of procuring a specimen of sputum in suspicious cases which were free from cough and expectoration. A number of instances are on record in which a latent and slightly tuberculous infection of the lungs has become active as a result of this procedure. I myself have reported such an occurrence which came under my observation several years ago.

Mercury, while not acting as quickly as the iodides, produces much the same effect after it has been administered for several months. In the beginning both the syphilitic and the tuberculous infection seem to respond favorably to the mercury, but after a time the tuberculosis commonly becomes worse. 
Syphilis, of course, demands the use of mercury, but in treating syphilotuberculous patients every effort should be made, through dietetic and hygienie measures, to increase the resistance of the individual and in this way reduce, as far as possible, the unfavorable effect of the mereury on the tuberculous process.

Menstrual Disturbances. - Suppression of the menses is common in tuberculous women, and may be one of the first manifestations of pulmonary trouble. No special treatment is needed. As the patient's general condition improves the menstrual flow will reappear, at first scantily and irregularly; later, with the return of health, the function becomes normal.

The beneficial effect of the fresh air and regular life incident to the treatment of tubereulosis is sometimes strikingly exemplified in menstrual disturbances. I have frequently seen women who were incapacitated for days, at the time of their periods, become entirely free from pain and nervousness as a result of this mode of life.

Leucorrhea.-Leucorrhea is not infrequently present in tuberculous women, and is sometimes one of the first evidences of the deterioration of health. As a rule, no special treatment is required, the discharge disappearing or greatly diminishing with the improvement in the general health. In advanced cases leucorrhea is at times sufficiently annoying to require attention. A mild antiseptic vaginal douche given daily or several times a week usually answers the purpose.

Pregnancy.-If pregnancy occurs in a woman who has had tuberculosis, she should be watched most carefully and made to conform to much the same rules of living as when the tuberculosis was active. If she manifests no symptoms or signs in the lungs the pregnancy may be allowed to proceed to term. Women who have had tuberculosis and recovered may go through a number of pregnancies. Repeated pregnancies, however, are dangerous even in those who have apparently entirely recovered. It cannot be denied that pregnancy, and particularly labor, may awaken into activity an old, quiescent lesion or incite a fresh one into renewed activity; and with each succeeding pregnancy this danger increases.

It has been a matter of common observation for years that a woman with tuberculosis may improve during pregnancy only to have the disease break out violently after delivery. Lawrason Brown has pointed out that "the prolonged muscular exertion, the loss of blood, the attendant exhaustion, the inhalation of infectious material during the pains are always serious. It is difficult to estimate, even approximately, the mental and physical strain that many patients undergo during labor. 'This is unquestionably the cause of the rapid spread of the disease following childbirth."

The violent exertion in these cases should be aroided just as any other forms of violent exertion. 'That the woman can be spared the exertion incident to labor does not seem to be generally known. One of two procedures may be followed: Either allow the pregnancy to go to full term and at the end of the first stage of labor anesthetize the patient 
with chloroform (see p. 807) and deliver with forceps, or induce labor two or three weeks before full term. Edward P. Davis, of Philadelphia, has, for several years past, been following the latter course in tuberculous women, with the most gratifying results. He informs me that in none of his cases has there been an acute exacerbation of the tuberculosis after delivery.

The question of ending the pregnancy must at times be considered. So long as the disease remains quiescent the pregnancy may be allowed to continue. The woman should, however, not be allowed to deliver herself. If the tuberculosis is progressing, or there is a complicating tuberculous laryngitis, or there is marked nausea and vomiting, thus interfering with nutrition, a therapeutic abortion should be performed, particularly if the pregnancy has not advanced beyond the third or fourth month.

Marriage.-Shall a tuberculous individual be permitted to marry? This is an important question and one the physician is often called on to decide. Some extremists hold the view that the existence of tuberculosis should constitute an absolute barrier to marriage, and that the marriage of tuberculous persons should be forbidden by law. On the other hand, those who have had the widest experience in treating tuberculosis take a much more conservative view of the subject, and with certain restrictions sanction marriage. Certainly, so far as children are concerned and the fear that they may be delicate, the absolute prohibition of marriage is needlessly severe. I am quite sure it has been the experience of many to have seen children, resulting from such marriages whose health and development differed in no particular from children born of healthy parents. The chances of the child being born tuberculous are almost negative.

The children of tuberculous parents develop the disease because they are infected after birth by the diseased father or mother, or, indeed, through any other source of infection to which they may be exposed. So long as the child is protected in this respect the danger is very slight.

In passing judgment on the fitness of an individual for marriage who has or has had tuberculosis, there are several factors to be considered.

The activity and extent of the disease are the most important considerations. No one, either man or woman, should be given permission to marry if there are active symptoms of the disease; neither should any one in an advanced stage of the disease receive permission to marry. If the disease has been arrested, the sex of the individual has an important bearing, as there are fewer objections to a man undertaking marriage than there are to a woman. 'Thus, a man who is well-to-do and who has been free from symptoms for a year or two can safely marry. Indeed, in many respects such an individual is perhaps better off married than single, as he will have more comfort and better care than he otherwise would have. On the other hand, a man without means and who is dependent on his earning capacity runs a risk, with the increased responsibilities marriage brings. He should have either some inde- 
pendent means or be earning enough to permit of his laving aside a fund which can be drawn on in case of a breakdown.

I am well aware that such considerations do not always have much weight in those about to enter the married state, still it is our duty to explain the situation and then trust to the patient's own good sense.

With a woman an additional consideration is whether she can safely bear children. For this reason the period of absence from symptoms should be much longer for a woman than in the case of a man. ()ur judgment in these cases must be based on the absence of symptoms, the amount of damage to the lung, and the general condition of the woman. 'Thus, if the disease had progressed sufficiently to have pror duced a cavity, one should be very guarded, even though there are no symptoms. I should say that, as a general thing, a woman who had had an attack of tuberculosis, no matter how slight, should not undertake the duties of motherhood until she has been free from symptoms for from two to four years.

In sexual matters absolute continence should be the rule if there are any active symptoms of the disease. Iater, when the disease has become quiescent, moderate sexual indulgence is allowable. 'This, I confess, is rather vague, but it is not a matter that admits of any definite directions. Fever, a rapid pulse rate, hemoptysis, etc., slould not, however, be aggravated by the exeitement incident to the sexual act.

Choice of an Anesthetic for Tuberculous Patients.- In the northern section of the United States ether has by common consent become the anesthetic of choice, and, so far as immediate accidents are concerned, it is undoubtedly one of the safest agents for producing general anesthesia. It is not, however, a safe anesthetic to employ in a case suffering from tubereulosis 'This applies not only to those who have demonstrable pulmonary disease, but also those with tuberculosis in other parts of the body. Experience has shown that many of the cases of surgical tuberculosis have in addition a pulmonary lesion which may be incited into activity by the inhalation of ether.

It is now a matter of common observation that a trifling and inactive lesion is often turned into an active one, with a rapid extension of the tuberculous process, in those who have inhaled ether. Surgeons, as a rule, have failed to appreciate the danger of ether in this class of cases, and continue to administer it routinely. 'To some extent this is due to the fact that with the operative recovery the patient passes out of sight and the evil eflect of the ether, which appears later, is not seen.

In a recent paper Joseph Walsh has called attention to the dangers of ether in tuberculous patients. He urges that chloroform or nitrous oxide gas be employed.

II. Warren Buckler, who has had a wide experience as an anesthetist, always uses nitrous oxide gas and oxyen in these cases. In his opinion this is the safest of all methods, both for non-tuberculous and tuberculous patients; in the latter he has never seen the slightest ill-effect produced on the tuberculous process by nitrous oxide gas.

Spinal anesthesia has also been employed in operations about the 
rectum, such as fistula-in-ano. Those who employ this method assert that it is most satisfactory and safe. Most surgeons, however, are opposed to it.

It cannot be too strongly insisted upon that any case with a frank pulmonary tuberculosis or one in which a lesion is suspected should not be given ether. If the operation cannot be performed under local anesthesia, chloroform or nitrous oxide gas, preferably the latter, should be employed.

The Use of Tobacco.-At the outset of the treatment smoking had best be forbidden, especially if there is an irritating cough, fever, or a rapid pulse. In very early cases, in which the constitutional symptoms are slight, and in those in which the symptoms have diminished in severity, moderate smoking may be permitted. By moderate smoking I mean one mild cigar, three times a day, after each meal. This does no harm, providing the smoke is not inhaled. A long cigar holder should be used, so that the smoke may become cooled before entering the mouth. Cigarettes should be forbidden, not only because the smoke is almost invariably inhaled, but because it is hot and irritating.

The chewing of tobacco should not be permitted if tubercle bacilli are present in the sputum, as the frequent expectorating increases the danger of infecting others.

Management of the Hopeless Case.-Incipient tuberculosis is not difficult to manage providing one observes the essential principles of the treatment. The symptoms are not severe, and, as a rule, disappear under hygienic and dietetic measures. As the disease progresses, however, the symptoms usually increase in severity and require more attention; while in the terminal stage the treatment is almost entirely symptomatic. The principal object in this stage of the disease is to make the patient comfortable, and to do so often taxes one's therapeutic resources to the utmost. Even though the outlook is hopeless, one should preserve, at least in part, the same régime that is employed in the curable stages of the disease; not because the result can be altered, but because the measures employed make the patient more comfortable.

The diet should preferably consist of simple, digestible food, but caprices of the appetite should be humored, providing, of course, the food craved is not likely to produce disturbances which will increase the discomfort.

Relaxation in the matter of rest may also be allowed, even though fever is present and the patient is weak. If the patient desires to sit up, or even walk about, it may be permitted to a limited extent.

Fresh air, however, should be insisted on, because it influences favorably symptoms which must otherwise be controlled with drugs. The measures which have been indicated for the relief of the different svmptoms and complications relate, for the most part, to the advanced stages of the disease.

A word as to opium. I believe that, as a general rule, the best results will be obtained by avoiding opium in any form in the curable stages of the disease. In the terminal stages, however, opium is frequently 
a necessity. While one should defer using opium as long as possible, there should be no hesitation in employing it if the patient is harassed by pain or the sleep is interfered with by reason of constant coughing.

The most important service that the physician can render to the hopeless case, however, is that of sympathy, or, as Dr. Trudeau has so well expressed it, optimism. "The practising physician and surgeon must have optimism if he is to develop a full degree of efficiency in meeting the terrible emergencies of acute illnesses and accidents, or the longdrawn-out struggle with lingering and hopeless disease, and at the same time inspire his patients with a degree of optimism which means everything to them in the ordeals they have to pass through. In his hour of need the patient has no means of judging of the physician's intellectual attainments; it is the faith that radiates from the doctor's personality that he seizes upon and that is helpful to him. Any encouragement that emanates from the physician will help keep up the patient's courage and carry him through long days of illness and suffering to recovery; and, where recovery is impossible, if the doctor's optimism-that is, his faithis of the kind that extends to the future, not only here but hereafter, it may dispel for the patient much of the darkness and despair which brood over the end of life, and perhaps even illume for him that vast forever, otherwise so shrouded in impenetrable gloom."

Treatment by Drugs.- The art of using remedial measures lies in knowing when to use them, and not in the employment of a drug or combination of drugs as a matter of routine. Some physicians attribute much of their success in the treatment of tuberculosis to some favorite remedy, while others achieve equally good results by restricting their use of drugs to mect certain definite indications. 'The proper course to pursue is to use drugs when they are indicated and when no occasion arises for their employment to maintain a position of "masterly inactivity."

A patient who adheres to the preseribed rules of the treatment, who has a good appetite, who sleeps well, who is free from pain, whose gain in weight is satisfactory, and whose cough is only moderately severe and for the most part limited to the morning, does not, in my opinion, require medicine. Drugs will certainly not improve what is a perfeetly satisfactory state of aflairs. If the patient feels that he is being negleeted because medicine is not given, he should be told why it is being withheld. In my experience even the very ignorant can be satisfied in this regard.

Concerning the employment of remedies which have been from time to time exploited as exerting a specific influence in tuberculosis, it may be said that not one of them, with the possible exception of tuberculin, has withstood the test of time.

Creosote and its Derivatives.-Standing foremost among the "false specifics" are those drugs of which creosote itself, guaiacol, and guaiacol carbonate are probably the most popular. In recent years there have been added to the list creosotal, thiocol, duotal, ete. I can only repeat what I said in the treatment of eough: Creosote is in no case a specific; it has no antiseptic value, and no matter how administered is ineapable 
of exerting any influence on the tubercle bacilli within the lung. The indications for creosote have been given on page 783. In addition it may be employed as a stomachic, in doses of two or three minims three times daily, and in intestinal tuberculosis as an intestinal antiseptic.

Arsenic.-Arsenic has long been held to exert a specific influence in tuberculosis. 'This, however, is doubtful. 'The good results obtained by arsenic can be explained by its well-known effect in improving the general nutrition. Administered for its tonic effect, arsenic is best employed in the form of Fowler's solution (liquor potassii arsenitis), in doses of two minims or more, three times daily after meals, or as arsenic trioxide, gr. $\frac{1}{30}$, alone or in combination with iron.

Cacodylate of sodium, administered hypodermically, was recommended a few years ago as a means of treating tuberculosis. It is now rarely employed.

Iron.-Iron is of ten of great benefit, particularly in young girls, when the anemia is marked and of the chlorotic type. Blaud's pill given in ascending doses is as efficient under these circumstances as it is in pure chlorosis. It is generally held that iron should be avoided if there is a tendency to hemorrhage.

Alcohol.-Alcohol exerts no specific influence in tuberculosis, and the indications for its use are extremely limited. Indeed, one may go farther and say that even in the few instances in which alcohol is recommended other remedies, equally efficient, are available. Furthermore, it is well to remember that far more people with tuberculosis have been injured by the use of alcohol than have been benefited by it.

In addition to the administration of alcohol for its supposed curative effect, it has been recommended for certain symptoms, notably fever, insomnia, and anorexia. I have never seen that it exerts any favorable influence on reducing the temperature, and in insomnia other remedies are equally efficient and less dangerous. Occasionally I have prescribed alcohol in the form of beer, stout, or bitter ale, for its effect on a flagging appetite.

The Hypophosphites.-It is extremely doubtful whether anyone was ever benefited from the use of the hypophosphites. On the other hand, it is reasonably certain that many people have been injured from their use; not that the hypophosphites themselves are injurious, but because reliance on their supposedly good effects has led to the neglect of other more important measures.

Nux Vomica.-Aside from their tonic effect, nux vomica and its alkaloid strychnine exert no influence on the tuberculous process. In the form of the tincture, nux vomica, in doses of five to fifteen minims three times daily before meals, is an excellent stimulant for the appetite. Strychnine (gr. $\frac{1}{30}$ ) may be employed for the same purpose; it is also useful when the nervous tone is poor.

Iodine.-Iodine is still believed by some to exert a favorable influence on the tuberculous process. With this idea in view it has been given internally in the form of hydriodic acid, iodopin, iodoform, and potassium iodide. 'The dangers of the latter I have already called attention 
to (see p. 804). In addition, various combinations have been devised for administering the drug by inunction. I have never seen that iodine or any of its compounds, administered either by mouth or by inunction, had any beneficial eflect in tuberculosis.

Iodine in the form of the tincture is a valuable counterirritant in cases of pleuritic pain. For the same purpose iodine petrogen, 10 per cent., may be rubbed into the skin.

Mercury.-Mercury is the most recent "specific" in the treatment of tuberculosis. 'This use of mercury was introduced a few years ago by Wright, who employed the drug hypodermically in the form of the succinimide. Since the appearance of Wright's original paper many observers have given his plan a trial. While some have reported favorably on its use, the majority have condemned it. My own experience is distinctly unfavorable to its use.

Inhalations.-Inhalations of sprays have long been employed in the treatment of tuberculosis, with the hope of bringing substances antagonistic to the tubercle bacilli into direct contact with the diseased portion of the lung. So far the method has failed. For tuberculosis of the larynx and acute affections of the upper air passages, inhalations of medicated sprays often give great relief to the patient, although it is doubtful whether they exert any curative effect. 



\title{
DIPHTHERIA
}

\author{
BY B. FRANKLIN ROYER, M.D.
}

As soon as the diagnosis of diphtheria has been made antitoxin should be administered to the patient and the public health officials should be notified of the existence of the disease. 'The specific action of diphtheriat antitoxin has been so clearly and conclusively demonstrated by animal experiments and its effect on mortality statisties has been so often shown that it is unnecessiry to print elaborate tables for the purpose of proving its efficacy. 'Two illustrations and a statistical table will suffice. Critics of this method of treatment have been silenced and the doubting ones have long since taken up its administration. 'The question that arises with each individual case of diphtheria is what dose of antitoxin shall be administered. In America all commercial houses and most departments of health furnish antitoxic serum in sealed syringe packages of varying numbers of units, with needles and containers ready for the administration of the remedy.

'Two kinds of antitoxin are widely used by the profession. Whole horse serum of great antitoxic strength and antitoxic serum refined and concentrated by Gibson's method. 'They are equally potent as curative agents. With the first product 30 to 45 per cent. of the patients treated will develop in from seven to thirteen days some sort of skin rash. These eruptions are most frequently urticarial, are often morbilliform, and sometimes appear as simple erythemas or as scarlatiniform erythemas. They are never dangerous, but are sometimes extremely annoying. 'The refined and concentrated serum gives reactions of a much milder type in about 6 per cent. of the cases treated.

It is but fair to state that the refined product is not absorbed from the site of injection with as great rapidity, and the first improvement noted after its injection is apt to appear a few hours later than if the older type of serum is used. The advantages of the former, however, are so numerous that it is always to be preferred.

Antitoxin Dosage. - It is my custom to determine the size of the dose after a careful examination of the affected part discloses the extent of involvement. Where but one tonsil is in good part covered with pseudomembrane an initial dose of from 2500 to 3000 units, to be repeated in from twelve to twenty-four hours and later at intervals of twenty-four hours until the membrane has largely disintegrated, is all that is required. With both tonsils well covered with pseudo-membrane, from 5000 to 6000 units is given at once, and this dose is repeated in like manner. If the pseudo-membrane extends across the half-arches or to the superior pharynx, a dose of 7500 or 8000 units is given, and if much nasal involve- 
ment is seen or if the larynx has been invaded, 10,000 units are administered, and in each instance the dose is repeated in from twelve to twentyfour hours, and later is repeated in twenty-four-hour intervals until the pseudo-membrane largety disappears. Occasionally I find it necessary to give a third dose twelve hours after the second dose has been injected, and sometimes the dose may be decreased in size in the latter days of treatment.

A word of caution may be offered in discussing intervals between doses. One should avoid, if possible, giving serum four or five days or a week after having discontinued its use. It is always better to continue giving anti-

Frg. 97

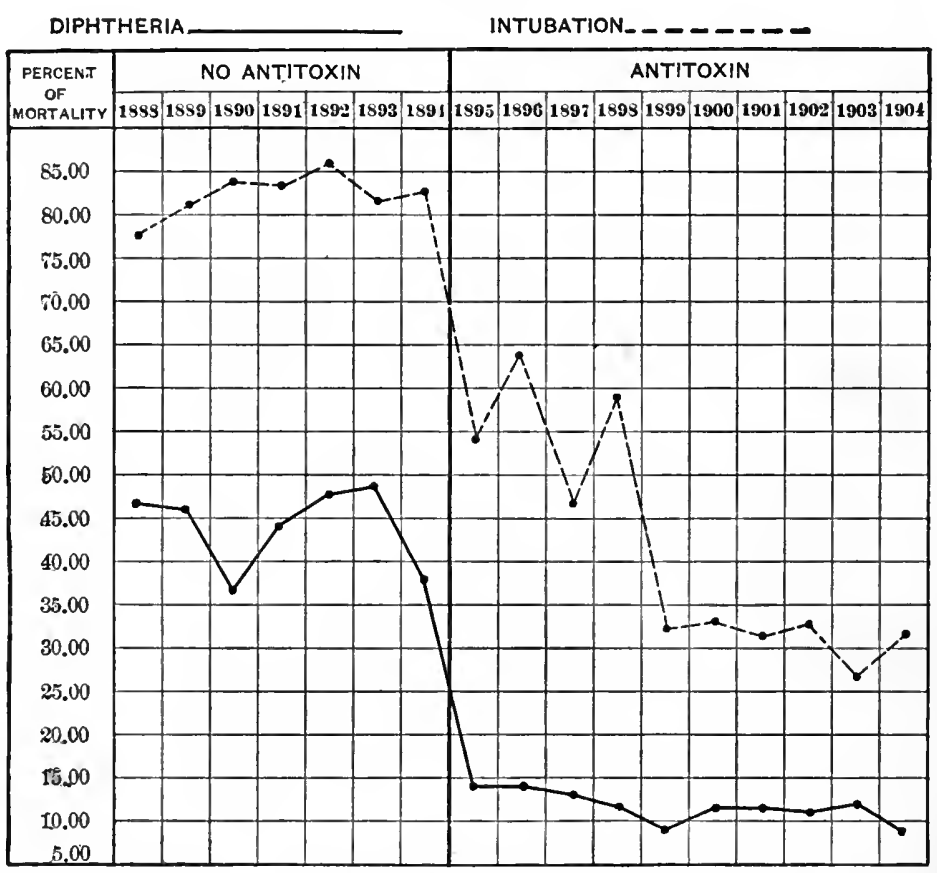

Per cent. of mortality of diphtheria at the Boston City Hospital, proper, and at the South Department from 1888 to 1904 , inclusive. Solid line, per cent, of mortality of intubations for the same time. 1888 to 1894 , no antitoxin. 1895 to 1904 , antitoxin.

toxin at intervals of from twelve to twenty-four hours until the patient has received enough to effect a cure rather than to await developments and begin treatment a second time during the illness.

I arrived at this system of dosage after months of variation both in the number of units and in intervals between doses, and after testing the treatment in hundreds of patients. 'The basis for this system of administration seems to me to be rational, and was in good part founded upon reports of Park's experimental work. It has been definitely proved by him that but a small amount of the toxin absorbed before serum was injected could be antidoted, and his further experiments proved con- 
clusively that but little antitoxin can be recovered from the blowel stream twenty-four hours after serum has been injected.

A large part of the first dose of serum may be used in taking care of toxins already in the circulation. Enongh should be given, however, to anticipate what may be absorbed during the next twelve or twenty-four hours, and subsequent doses should be given of suffieient size to neutralize toxins thrown into the circulation from the affected area during the interval. As soon as the pseudo-membrane has largely disappeared it seems reasonable to suppose that the organism is taking care of the invading bacilli, and serum may no longer be required.

I have used this system of dosage in treating the last three or four thousand patients coming under my care, and see no good reason for changing it. In fact, I find myself constantly leaning toward a larger dosage rather than toward lessening the number of units given. 'The age and size of the child is disregarded in determining the number of antitoxin units to be given.

Importance of Giving Antitoxin Early in the Illness.-During four years of my service in the Philadelphia Municipal Hospital for Contagious Diseases our statistics were tabulated in a way that shows the influence of treatment begun early in the illness. 'The following table proves that delays in beginning treatment are dangerous and often fatal:

\begin{tabular}{|c|c|c|c|c|c|}
\hline & 1904 & 1905 & 1906 & 1907 & Aver \\
\hline $\begin{array}{l}\text { f ea } \\
\text { the }\end{array}$ & 0.0 & 0.0 & 0.0 & 0.0 & 0.0 \\
\hline $\begin{array}{l}\text { e second day } \\
\text { was. }\end{array}$ & 4.09 & 4.43 & 6.47 & 3.45 & 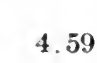 \\
\hline day of the & & & & & \\
\hline th day of & & & & & \\
\hline & 17.54 & 16.66 & 15.81 & 10.85 & 16. \\
\hline & 14.75 & 13.04 & 27.47 & 13.08 & 14 \\
\hline 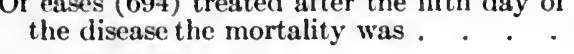 & 14.87 & 18.40 & 15.00 & 10.85 & 14. \\
\hline 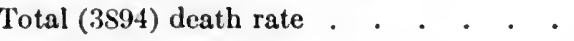 & & & & & \\
\hline
\end{tabular}

The Department of Health of the Commonwealth of Pennsyliania has been giving antitoxin free, for a period of four years, to those who are unable to pay for it. 'The accompanying graphic diagram (p. \$16) shows its efficacy. At the end of $1909,20,794$ cases had been treated with serum furnished by the Iepartment of Health, with a death rate of $8.4 S$ per cent.

Site of Infection. - I prefer to inject antitoxin subcutaneously over the abdomen, although in giving immunizing doses I frequently inject along the thigh or in the back at the angle of the scapula. 'The skin in each instance should be cleansed as carefully as though an operation were to be performed. In profoundly toxic cases with much cervical cellulitis, "bull necks," I have occasionally had splendid results by giving the first doses intravenously. 'The difficulty of plunging the needle into a vein in young children, in the absence of trained attendants in homes, practically limits this method of administration to hospital practice. 
Immunizing.- It is my custom to advise that all children who have been exposed in the household and the nurse, if she happens to be the mother or a near relative, be given immunizing doses of antitoxin-1000 units given to each individual is usually sufficient to protect for a period of three or four weeks. I have seen diphtheria occur nineteen days after a child had been immunized, but I believe this to be an exceptional experience.

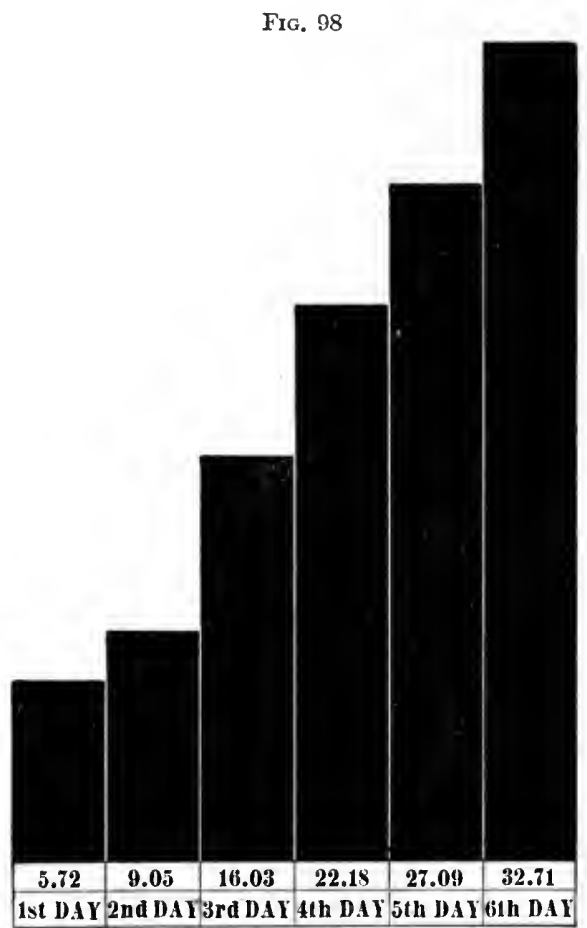

Diagram showing percentage of deaths in 20,794 cases treated according to duration of disease.

Dosage Used by Others.-(1) McCollom begins with a slightly larger dose than I have recommended, and repeats it in from four to six hours. (2) Baginsky gives a much smaller dose, and does not repeat it frequently. (3) Northrup's treatment is well shown by the following paragraph:

"From 2000 to 3000 units to a child over a year in an ordinary case of diphtheria. From 3000 to 5000 units for severe cases and all laryngeal cases of any age. For a child under one year, in an ordinary case, 1500 to 2000 units. 'These doses are to be repeated in twelve hours, or even less if the symptoms are increasing, and in eighteen to twenty-four hours if there is not decided improvement. A third dose, or even more, may be given, if necessary, in another twenty-four hours."

(4) Ker, of Edinburgh, uses a large dose frequently repeated. The following quotation from his Text-book on Infectious Diseases shows his method: 
"As to the total amount of serum to be administered, continued injections at short intervals are required until improvement has taken place. So long as there is membrane on the throat it is probably worth while to push the treatment, unless, of conrse, it is obviously and rapidly disintegrating. In severe nasopharyngeal and laryngeal cases it may be necessary to give in all 40,000 inits or even more. My own largest total dose has never exceeded 64,000 units, a comparatively low figure when compareal with the enormous totals reported by MeCollom and others. In not a few cases there is no visible improvement until the patient bas received over 20,000 units, and there is no doubt that when a diphtheritic bronchopneumonia is supervening in a laryngeal case much is to be gained by pushing the serum until the respirations and temperature fall. Except in this class of cases a fall of temperature is not to be regarded as a sign to stop the treatment, unless, indeed, the patient is much improvel in other respects. 'The worst instances of diphtheria of ten run their course with the temperature normal or subnormal."

(5) Welch, of Philadelphia, is inclined to give small doses at twelve and twenty-four hours' intervals, with a total dosage less than is usually recommended.

Other Methods of Treatment.-Perhaps no other remedy is as helpful in supplementing antitoxin treatment of diphtheria as alcohol. It is indicated in all types of the disease and may best be administered in the form of good whisky or brandy. 'Those who are more fastidiously inclined may be given champagne or sherry. 'The next most useful drug is strychnine; occasionally the citrate of caffeine may be given with great advantage to the patient. Whisky in half-dram or dram dosesin adults still larger quantities-at intervals of three hours, invariably improves the profoundly septic case, steadies the heart, and insures rest. When the heart is weak and the pulse intermittent, strychnine in full dose serves well when used with moderate amounts of alcohol. In the septic case with marked degenerative changes in the heart musculature, when the area of cardiac dulness is gradually increasing and when the liver shows prominently below the rib, caffeine citrate given hypodermically in large doses often gives satisfactory results. Opiates are alwars contraindicated. I have never seen patients improved by giving them iron, bichloride of mercury solution, or chlorate of potash internally, and I believe that these drugs should be withheld. Iron not only upsets digestion, but by its action on the psendo-membrane fills the mouth with ropy material, which, besides being hard to dislodge, is disgusting to the patient.

Local Treatment.-Tonsillar diphtheria with great inflummation of the soft parts or with swollen cervical lymph glands is greatly relieved by the local use of eold-best applied dry by using ice collars or ice bags. If these are not available I'do not hesitate to use thick cloths wrung from ice water at intervals of a minute.

After a long trial of various types of antiseptics, a trial including the use of bichloride solution, hydrogen peroxide, liquor antisepticus, boricacid solution, chlorate of potash, and solutions of potassium permangavol. 1. -52 
nate, I gradually eliminated one after the other, in the form of even sprays, and at last came to depend on copious irrigations with normal salt solution.

Irrigations are best carried out by keeping the very sick in a recumbent position, bringing the patient to the edge of the bed, or by laying them on a table with the head extending beyond. An ordinary fountain syringe or a metal irrigating can with a four or five inch nozzle, with large enough caliber to throw a good stream, is the most satisfactory instrument for this purpose. For hospital use I found it best to have nickel-plated tubes six inches in length with a $15 \mathrm{~mm}$. bore for use in irrigating the mouth, and a blunt glass nozzle most convenient for irrigating the nostrils. A foul mouth should be irrigated every second or third hour. The patient with clean membrane and without ropy deposits will do well when irrigated three times each day. I have practised irrigating the nose with a large series of cases having nasal involvement, and believe this to be good practice, although many physicians with large experience do not approve of this method of treatment. McCollom is among those who have discontinued this nasal irrigation. His great experience leads him to believe that middle-ear infections are more common in cases so treated. My experience would not seem to bear out his conclusions. I have found it useful to follow nasal irrigations with local applications of oil; liquid cosmoline, or the more refined product albolene, serves well. Irrigations are to be discontinued, of course, if hemorrhages supervene.

In the type of nasal diphtheria, characterized by serious bleeding, the physician is of ten puzzled as to the proper treatment. In these cases a careful packing of each nostril, using long strips of gauze and long-bladed application forceps, beginning the packing high in the posterior nares and alternately packing in the superior space, and then far back along the floor of the nose until the entire cavity is filled and a uniform pressure is secured, has often enabled me to control bleeding. When this type of dry packing fails I treat the hemorrhages by wetting the gauze packing with 1 to 3000 adrenalin solution. Occasionally it becomes necessary to pack the posterior nares through the mouth, reaching this cavity by carrier forceps and a thread or by passing a carrier through a catheter. 'The anterior nares should then be packed in the usual way and the entire plug tied in so as to be held firmly in place. After packing, pledgets of gauze wet with ice water should be frequently applied over the bridge of the nose.

I have tried calcium chloride and gelatin internally without great encouragcment.

'The diphtheria patient showing areas of purpura occasionally recovers. Usually, however, drug therapy is of little value. I have found nothing that aids antitoxin in this type of the disease.

Paralyses are perhaps more frequently seen today than before the antitoxin era. 'This is no doubt due to the fact that many patients are saved that in pre-antitoxin days would have died before paralysis developed. In treating these paralyses simple tonics, small doses of strychnine or iron, gentle massage, soothing electric currents, and nourishing food is all that 


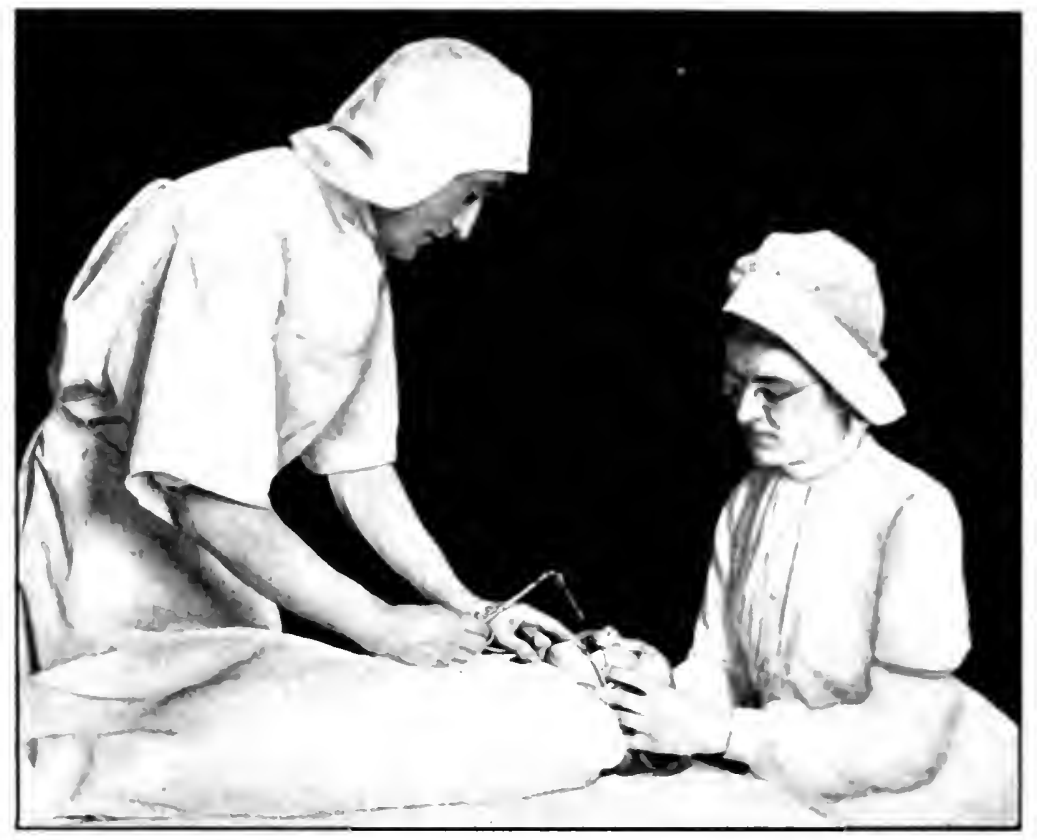

Fig. 1. Showing the first steps of intubation in the dorsal position. Photographed by Dr. B. Franklin Royer.)

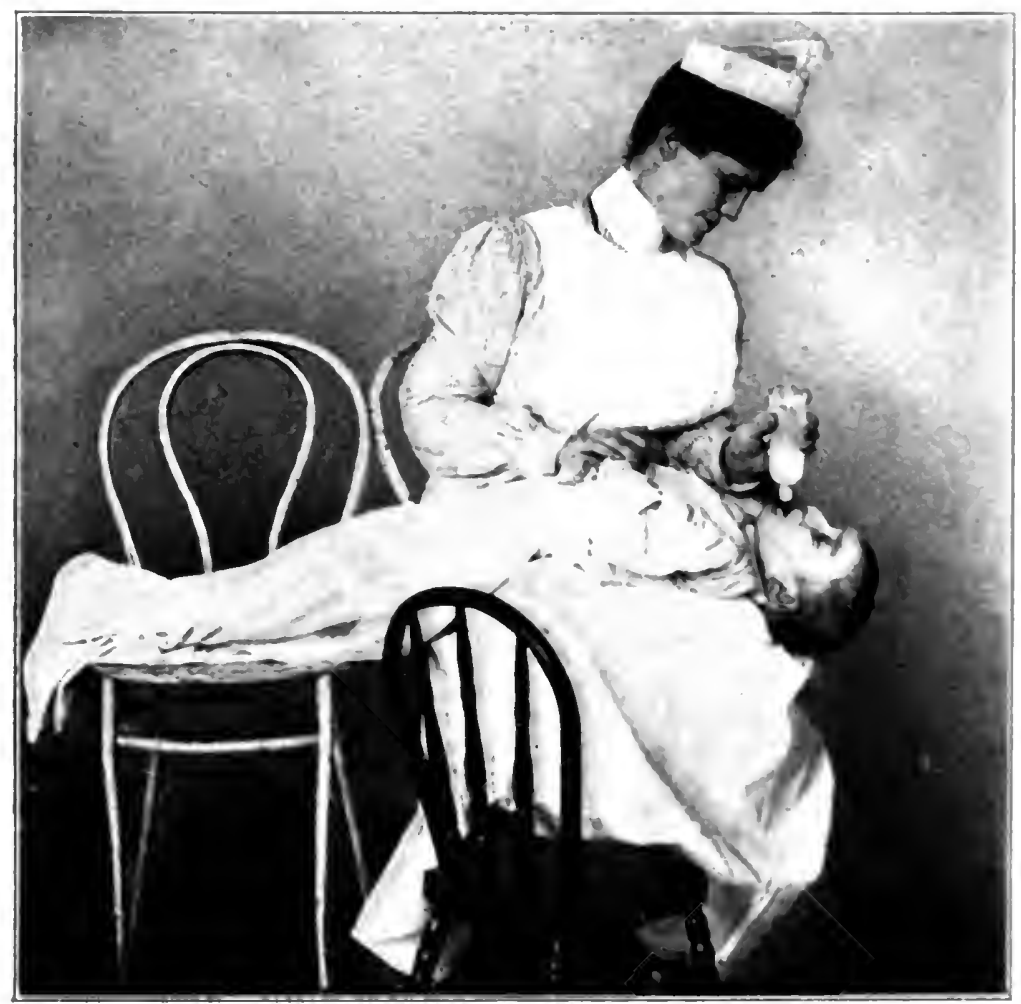

Fig. 2.-Casselberry's position for feeding intubated cases. Alter Nathrug 

is required. 'The paralyses tend to get well without treatment and rarely" present great difficulty. Occasionally, however, patients are unable to swallow, then it is necessary to practise gavage.

Operative Treatment. - Laryngeal diphtheria of ten requires operative interference. 'The young doctor is apt to operate too early in the disease, older physicians of ten delay interference until the patient is exhausted in his fight for air. A good rule to follow is to avoid interference so long as the patient's color remains good and rest is secured. When extreme restlessness supervenes, or on the appearance of cyanosis and retraction of the xiphoid cartilage, one of two things should be done: intubation-the operation of choice in cities and hospitals, or tracheotomy-the operation of choice in adults and in children in the country far removed from the trained surgeon.

Intubation is a simple procedure, but requires a good deal of skill. 'Tracheotomy is a more formidable operation, easier to perform

Having determined that operative interference is demanded, the patient should be placed upon his back on a low table, a hard bed, or on the floor of the room. He should be wrapped in a sheet with the arms firmly fastened to the side, a hard pillow should be placed under the neck, and, if intubation is to be the operation of choice, an assistantpreferably a trained nurse or doctor, or if such persons are not at hand any available person who will do as they are told-should hold the head firmly in a natural position with the chin a little elevated and should grasp the mouth gag so as to keep the jaws widely separated. The operator takes his place on the patient's right side, with the forefinger holds the epiglottis against the base of the tongue, with his right hand grasps the introducer and passes the intubation tube along his left forefinger until it reaches the top of the larynx, and almost enters it, when by elevating the right hand to right angles with the neck the tube enters the larynx and descends. With his left forefinger he then holds the tube in place while with the tip he dislodges the obturator and waits for the patient to breathe comfortably before determining what shall be done with the thread that is always to be drawn through the eye of the tube.

It has been my custom to remove this thread. Some operators prefer to keep it in place, carrying it in a loop about the patient's ear or strapping it to the cheek. In young children without molars this may work wellolder children soon cut this thread with their teeth and the end either drags into the top of the esophagus, annoying the patient, or is swallowed.

Extubation is practised with the patient in the same position, prepared in the same way, the operator reversing his manipulation.

Caution.-In order to successfully perform intubation the plivsician must have a "light hand," follow the course of least resistance, and must remember to elevate the introducer to a right angle with the neck at the same time the tube enters the larynx. Descent into the larymx and trachea should take place almost by the weight of the tube. O'Dwrer's set of tubes, made of vulcanite, with a light metal lining, as manufactured by Ermold, are the most satisfactory instruments in practice.

Laryngeal cases requiring intubation are best treated in hospitals, and 
if the family cannot afford to have a competent physician remain in the house the patient should, if possible, be removed to a diphtheria hospital.

When tracheotomy is to be the operation of choice the technique to be followed is that described in all books of surgery. In practice the site of selection is the "middle of the neck." In other words, select a point just above the isthmus of the thyroid. The operation is usually done in haste, and there is but scant opportunity for elaborate technique. The secrets of success lie in rapid work, avoiding large bloodvessels, exposing the trachea before cutting into it, and securing a dry field of operation before making the final incision. Having opened the trachea, be prepared to retract its walls at once and insert the cannula.

In my experience adults do better if tracheotomy is performed as soon as interference is indicated. In children with suitable surroundings better results will be obtained by performing intubation, and it is always easier to secure a parent's consent for the latter operation. The chances of accident and the difficulty of securing trained attendants in country practice often forces physicians to choose the more formidable operation in order to secure greater safety for the patient. Intubation tubes are sometimes dislodged by patients, and a life is lost before the physician can reach the bedside, when great distances separate the doctor from the patient. Any handy mother can be taught to cleanse a tracheotomy cannula, and the physician will be spared great anxiety if he chooses the cutting operation.

I cannot explain why adults apparently do better with tracheotomy. It has been my experience, however, and I believe it is that of most others who have treated large numbers of diphtheria patients, that better results are secured by doing tracheotomy at once in grown men and women.

The after-care of operative cases counts for a great deal, and serious lung infections may be avoided by properly guarding the patient.

Intubation cases in children old enough to take table food do better when semisolids are given-young babies may be fed in Casselberry's position. Older children quickly learn to take a liberal soft diet. Tracheotomy cases may be fed in the same manner as any other sick patient, but great care should be practised to prevent the inhalation of dust particles or foreign bodies, and the cannula should be kept strictly clean and ought to be removed at intervals of two days. The intubation tube may be removed at intervals of two days, but I believe better results are obtained by keeping it in place from three to four days and then removing it at intervals of three or four days until the patient breathes well without it.

Bronchopneumonia.--After each operation the patient should be kept in the open air, and if pneumonia develops he should certainly be treated in the open air. Drugs are indicated as in any other bronchopneumonia patient. When the pseudomembrane has largely disappeared but little further good is accomplished by giving antitoxin, although MeCollom believes that many of these cases are improved by continuing its administration, and Ker, already quoted, continues giving serum during the febrile stage. 


\title{
ACUTE AR'TICULAR RHEUMATISM
}

\author{
BY THOMAS A. CIAY'TOR, M.I.
}

Historical.-Not until Sydenham in the seventeenth century differentiated rheumatism from gout did there exist a definite treatment for the former disease. He based his treatment on what he regarded as the striking similarity that rheumatism bore to pleurisy. It was a time when venesection was at its zenith, bleeding being the treatment for all acute febrile diseases. It was, therefore, natural that it should have been the main feature of Sydenham's treatment of rheumatism. He took 10 ounces of blood from the vein of the arm on the side of the affected joint. 'This was repeated on the following day, and again on alternate days, till four bleedings had been made. Rarely did he find it necessary to bleed a fifth time. Sydenham recognized limitations to the employment of this measure, as in the case of oid persons and very weak patients. His successors were not so conservative: Bouillaud bled far more frequently, copiously, and persistently than did Sydenham. 'The latter (Sydenham) prescribed drinks flavored with water-lilies, lemon, and violets. He forebade meat and meat broths. He took the patient out of bed a few hours every day, because he believed the heat of continued confinement promoted and aggravated the disease. He regarded opiates as contraindicated. Under this treatment "the pains would be lessened, but would not wholly disappear until the season changed, this change producing a physical condition contrary to that which had induced the disease. 'Then, and after the patient had recovered from his loss of blood, all symptoms would disappear, and the convalescent grow strong as a wrestler." Laennec advocated tartar emetic in full and repeated doses, vomiting and purging being the objects to be obtained.

During the nimeteenth century a variety of methods were recommended, and were successively in fashion for longer or shorter periods. Casenave, of Pau, introduced large and repeated doses of opium. 'This practice was introduced into England by Corrigan, and was ardently advocated by him. 'To Corrigan more than to any one else belongs the credit of forcing the abandonment of the extreme depleting measures that prevailed up to his time.

Colchicum was at one time in vogue, its advocates employing the drug to the production of vomiting, purging, and excessive diuresis, much in the same manner that tartar ensetic was used at an earlier period. 
Antiphlogistic treatment, with large and repeated doses of mercury, was much employed, and was regarded as being especially efficacious in those cases associated with pericarditis.

Guaiac and einchona were advocated, the first by Seymour, and the latter by Fothergill, Haygarth, and Farquhar. 'They are still used by some as adjuvants, especially in England.

Potassium nitrate in large dosage was introduced in the latter half of the eighteenth century by Brocklesby, and its use was revived in 1844 by Basham.

About this time Rees introduced the lemon-juice treatment. As much as 6 to $S$ ounces of lemon-juice were given daily by the disciples of this school. Rees was perhaps the first to conceive that this or any other acute disease might be dealt with by other means than by the administration of powerful remedies.

Todd (1843) evolved the eliminative treatment, by which he endeavored to remove the materia morbi from the body. To this end he called on all the emunctories in the vigorous fashion of his time. Calomel, jalap, and magnesium sulphate kept the bowels moving four or five times daily. Dover's powders, potassium nitrate, tartar emetic, and opium were used to induce sweating, and the latter also to relieve pain. Salines, colchicum, and potassium tartrate were also used as purgatives. Todd states that he did not use active diuretics, but maintained a copious flow of urine by giving large quantities of liquids. Locally, he used leeches, warm fomentations, and poultices. He gave a more liberal diet than Sydenham, beginning with meat and animal broths on the third day of the disease. He recommended tonics, especially iron, and a liberal diet during convalescence.

In 1852 Fuller published the results of his treatment of rheumatism by the alkaline method. This was based on the theory that the disease was associated with excessive acidity of the blood, and that this acidity accounted for the symptoms. He first employed the alkalies, but soon abandoned them for their organic salts. He claimed to shorten the duration of the attack by ten days to several weeks in the prolonged cases. He used citrate of potassium or ammonium and potassium or sodium bicarbonate in large and frequent doses; also potassium acetate. In connection with these, Rochelle salts was employed as a laxative. Fuller's explanation of the rationale of the alkaline treatment was as follows:

1. It restored the alkaline condition of the system, which had become acid under the influence of the disease.

2. It maintained the solubility of fibrin, thereby preventing deposition on the valvular apparatus of the heart.

3. It exerted a powerful sedative effect, calming the action of the heart and arteries.

4. It increased the metamorphosis of tissue, proving an active provocative of the secretion of urine, increasing both the fluid and solid elements, whereby the elimination of the materia morbi was assisted.

The history of the development of the use of salieyl preparations in 
rheumatism forms an interesting chapter in medical annals, chicfly owing to the fact that the substance was in use for more than a century before its most importint field of usefulness was discovered. 'The first mention of the use of willow-bark as medicine in modern times is found in a paper by Speckbuck (1769), in which he diseusses it chiefly from a botanical standpoint, and recommends it in intermittent fevers in conjunction with Peruvian bark. Günz (1772) compares willow-bark with Peruvian bark. His paper is interesting chicfly because of the references it contains to the use of willow anong the ancients. His references to the writings of Galen, Avicenna. Serenus, Dodona, Pliny, Theophrastus, Fallopius, and others show that this plant was in common use among them. 'They employed it in the form of powder, poultice, and a vinous decoction. 'The latter was used in nervous diseases-the powdered root or ash was mixed with water or vinegar, and used as an astringent or drying agent in gout and earache; the various preparations were used to remove callosities, warts, and blemishes of the face; to cleanse ulcers; to make wounds unite; to stop hemorrhage, especially from the lungs; in impaired vision and in swellings of the eyes; in obstruction of the liver; to promote excretion; to check the menstrual flow; in baths to strengthen the weak legs of infants; an oily solution of the bark was used to dye the beard black.

James (1792) credits the discovery of the beneficial therapeutic effect of willow-bark to an English elergyman, Mr. E. Stone, who communicated it to the Royal Society in 1763. Wilkinson (1803) compares willow with Peruvian bark, to the great advantage of the former, which he claimed to be better as an antiperiodic, antipyretic, analgesic, and antiseptic. He praises it for its cheapness and because his countrymen were not dependent on the Spanish for the supply.

Interest in the drug seems to have subsided until it was revived in 1830 by the discovery of salicin by Leroux. Blaincourt first used salicin in intermittent fevers. For the next ten years it was extensively used, generally as a substitute for quinine. It was also recommended in neuralgia, hyperaesthesia, irritable states, shuggish digestion, cachexias, phthisis, muscular weakness, and convulsive congh.

Salicylic acid was discovered in 1837 by Piria and Ettling, but was for a long time used only as an antiseptic. In 1875 Riess reported on its use in typhus and pneumonia as an antipyretic. It will be seen from the foregoing that the development of the use of the salicyl preparations in medicine was brought about by the search for a substitute for cinchona, a fact of pharmacological history that was duplicated a century later in the discovery of the efficacy of the coal-tar derivatives. Quinolin, a member of the benzene series, was found to be a decomposition product of quinine, and this fact stimulated the effort to produce a substitute for quinine by working on the benzene series by analytic and synthetic methods, an effort which has resulted in furnishing us with a large number of more or less valuable medicinal and commercial substances known as the coal-tar derivatives.

There is some conflict of opinion as to who deserves the credit of first 
using salicyl in the treatment of acute articular rheumatism. The first publication on this subject is that of Buss, of Basle, who, in 1875, gave the results of his investigations on salicylic acid as an antipyretic. Among the cases he réported were 4 of acute articular rheumatism. He began his investigation in December, 1874, and the drug was first employed by him in a case of rheumatism on the 28th of that month. In addition to the antipyretic effect, he also noted the marked effect in relieving pain, and declared that salicylic acid had a specific favorable influence on acute articular rheumatism. Stricker, January, 1876, published a series of cases treated with the acid in Traube's clinic, where it was first used in October, 1875. In June, 1876, von Graeffner published the results in 21 cases, acknowledging the precedence of Stricker's work. Maclagan, who has been generally credited with initiating the treatment of this disease with salicyl-containing drugs, did not publish the results of his work until March 4, 1876. He states in his original communication that he first used salicin November 26, 1874, which antedated Buss' use of salicylic acid by one month.

Germain-Sée in 1877, introduced salicylate of soda in place of salicylic acid, and its advantages over the latter were so obvious that it soon supplanted the acid, and its use became almost universal.

In order to realize the results that have been wrought in the treatment of this disease by the introduction of salicyl treatment, it is of interest to note the statistics of earlier authors as to its duration. Warren's classical observation that the only cure for rheumatism was six weeks reflects very accurately the experience of others.

Sir Charles Scudamore stated it at three weeks to two months; Chomel (1834) gave four weeks as the duration of the average case, and claimed that none were less than twenty days; Macleod (1842) gave five to six wecks as the a verage duration; Todd said "a mild case is over in a fortnight, an ordinary one in three to four weeks, and a severe one lasts fully six weeks." 'Todd's statistics included children. Fuller states that under the ordinary methods of treatment, convalescence began in four to five weeks, and that the patient never left the hospital before the end of the fifth week. Some cases lasted much longer-as much as fifteen to seventeen weeks. Under judicious (alkaline) treatment this period was shortened by ten days to five weeks.

Compare these with the following quotation from a modern work on therapeutics: Referring to the effects of the salicylate treatment"Lsually in twenty-four hours considerable relief is experienced, and both pain and swelling will often completely disappear within three to five days. At the same time there is generally a steady and rapid fall of temperature, and the patient may be quite free from fever in from three to six days" (Yeo).

Treatment.-In the treatment of this disease I follow, as a rule, the following plan:

The patient is put immediately to bed, and is not allowed to get up for any purpose during the acute stage of the disease. The conditions that ordinarily obtain in the modern well-equipped hospital 
meet satisfactorily the hygienic needs of the case. In the home, however, there are likely to be several elements lacking whose alsence materially affects the comfort and welfare of the sufferer, and for this reason it is well to enumerate them.

'The siek-room should be large, dry, well lighted, and well ventilated. 'The bed should be so placed that proper ventilation may' be obtained without exposing the patient to draughts. 'The bed itself is important. It should be of suflicient height and of such width as to permit the nurse to npproach and manage the patient from either side. 'The mattress should he firm and smooth, without being too hard, and the bed-spring should be rigid, so as not to sag under the weight of the patient. One has only to treat a single case of acute articular rheunatism in the low double bed commonly found in the homes of the poorer and middle classes, or in one with flaccid worn-ont springs, to appreciate the importance of these factors. 'The temperature of the room should be kept uniform, and not too high, $65^{\circ}$ to $70^{\circ} \mathrm{F}$. being sufficient under ordinary conditions. Where dampness is excessive, and in elderly and anemic patients, it may be necessary to have a slightly higher temperature than this. 'The night-dress should contain some wool, so as to absorb the perspiration and protect against chilling. Outing flannel is a suitable material. I have not found it necessary to dispense with sheets and keep blankets next to the patient, as is recommended by some.

The only drugs that have any influence on the disease are those containing the radicle of salicylic acid. 'The preparation that gives the greatest satisfaction in the largest number of cases is salicylate of soda. 'This is given every two hours in doses of from 10 to $20 \mathrm{gr}$., with an equal quantity of sodium bicarbonate. In order to avoid the unpleasant taste which is objectionable to many patients, the drug is given in capsules. An abundance of water is given at the same time, thus avoiding irritation of the stomach. The sodium bicarbonate is incorporated in a portion of this water, to which some flavoring substance may be added if desired. I have observed that salicylates are better borne and act more promptly when given on an empty stomach, provided that a large quantity of water be given at the same time. I, therefore, give the medicine between feedings rather than immediately after, as is often advised. It is well to give a larger dose just before the patient goes to sleep for the night than has been the rule through the day. Experience has taught me that the drug is most eflicient administered at this time, and in cases where the symptoms are very mild, a single daily dose may suffice to control them.

In the case of children and adults who cannot swallow capsules, it will be necessary to give the drug in solution. Here we combine the alkali directly with the salicylate, and disguise the taste by some aromatic flavoring substance, as ginger.

This treatment is kept up until the acute symptoms are markedly ameliorated, a result that is usually obtained in from twelve to fortyeight hours. 'The fever is reduced, the pain disappears, the swelling 
of the joints subsides, and the pulse returns to its normal rate and volume. As the acute stage subsides, the dose of the above is gradually reduced, and the interval lengthened, until the patient is taking 20 to $30 \mathrm{gr}$. of each drug daily. 'This reduced quantity should be continued as long as he is under observation, that is, until all the local and general manifestations of the disease have disappeared. The purpose of this is to prevent relapses or extensions to other joints.

Theoretically, it might be assumed that this continued administration of the salicylate has some effect in preventing the occurrence of those complications that so frequently follow articular rheumatism, endocarditis, pericarditis, etc., but satisfactory evidence that such is the case is lacking.

As has been said, the large majority of patients are able to take and respond to this treatment satisfactorily. Compared with the other salicylate preparations, it seems to combine the greatest efficiency with the fewest objections. Salicylic acid is decidedly more irritant, and there is no reason to think that its effects on the disease are any better than those obtained by the sodium salt. Salicin, the original preparation of willow-bark used by Maclagan, has no advantages except that it is not liable to the impurities and defects of manufacture that may occur in the preparations that are made synthetically. It has been claimed that it is less depressant than the acid or the sodium salt, but it is also less effective, and must be given in larger doses. Pure and reliable preparations of the salicylate are to be had, and defects of manufacture can be avoided if this fact is remembered.

Such preparations as salophẹn, salipyrin, and salol have no advantage over sodium salicylate, and as they contain the radicles of the more toxic members of the coal-tar series, antipyrin, and phenol, they cannot be given with safety in sufficient doses to obtain the full effect of the salicyl radicle. If it should be desired to get the antipyretic and analgesic effect of these drugs, they should be administered separately, though I have not found it necessary to employ them. Occasionally we meet with patients who, by reason of an excessively irritable condition of their stomachs, or because of idiosyncrasy, cannot take sodium salicylate. For these we have a valuable and efficient substitute in acetyl salicylate (aspirin). This is given in doses of $7 \frac{1}{2}$ grains with the same frequency. Some who cannot take either of these will tolerate the oil of gaultheria. 'The important point to be remembered is that the active agent in combatting this disease is the salicyl radicle, which is contained in all these preparations, and our object should be to introduce a sufficient quantity of this radicle in the form that is most easily absorbed, and at the same time least irritating and toxic.

It is my experience, and this accords with that of other clinicians, that the results of salicyl administration are most brilliant in direct proportion to the acuteness of the attack of articular rheumatism; that it has a far greater effect on the arthritic type of rheumatic invasion than on the other forms; and that its effect is more satisfactory in adults than in children. The exact manner of its action is not yet under- 
stood, nor can we expect it to be clear until the etiology of the disease is satisfactorily established. While it may not be a true specific in the sense that quinine is in malaria, or mercury in syphilis, its effect in rheumatism is so pronounced, that from a clinical standpoint, it may well be considered as such. Strong efforts have been made in recent years to overthrow the established conviction that salicyl has any action in acute rheumatic arthritis that can be described as specific. 'The brilliant results obtained by the use of the drug in this discase, its utter failure to produce anything like parallel results in other forms of arthritis, together with the fact that other substances, which have ordinarily greater antipyretic, analgesic, and antiseptic action, fo not approach it in its influence on rheumatic arthritis, offer a clinical bulwark against such attacks that has not as yet been seriously disturbed.

I have not found it necessary to give the enormous quantities of salicylates that some have recommended, and consider that such a procedure is contraindicated. Most patients who take salicylates in the quantities above prescribed have ringing in the ears, but this is not sufficient reason for stopping the drug or lessening the dosage. I have had one unfortunate experience in the sudden death from cardiac failure of an elderly patient, to whose importunities for larger doses of salicylate I once yielded. Others have had similar experiences, and in the light of such occurrences, the practice of massive dosage is unjustifiable.

Salicylic acid ( $\left.\mathrm{C}_{6} \mathrm{H}_{4} \mathrm{OH} . \mathrm{COOH}\right)$ is a benzol derivative, and partakes to a greater or less degree in the physiological actions that are common to all the members of this group. 'They are depressant to the entire central nervous system; they are antipyretic and analgesic; they are locally toxic to protoplasm, and they cause the formation of methemoglobin. 'The differences in the medicinal action of the different members of the group are due to their possessing these qualities in different degrees. Salicyl partakes of them all, and while the toxic effects are rarely produced by the doses ordinarily employed, it is wise to remember that, theoretically, it may produce them, and that they are to be expected in greater or less severity when excessive doses are given, when the patient is debilitated or the subject of chrcnic disease, or in cases of idiosyncrasy. Furthermore, it is very decidedly irritant to the kidney, and is, therefore, contraindicated, or to be used cantionsly, in cases having nephritis. It is to be remembered, however, that rheumatism, as does any other acute febrile disease, often causes a transient albuminuria with the presence of casts, and we are not to regard this as a contraindication to the use of salicyl unless the diagnosis of nephritis has been made previous to the onset of the rheumatic attack, or there are other evidences of renal defect.

What is true of the dosage is also true of the duration of the active treatment. 'The good effects of salicyl medication in articular rheumatism are manifested promptly, and if amelionation of the symptoms does not begin in forty-eight hours, it will probably not he produced hy this treatment, and we have presented to us the consideration of some other method, or the reconsideration of our diagnosis. 
Rationale of the Use of Alkalies.-It has been noted that previous to the introduction of salicyl the accepted treatment of rheumatism was the alkaline method of Fuller. There seems to be no reasonable doubt that this treatment was a distinct improvement on any method practised up to that time; that by it the duration and severity of the disease was lessened. While the large quantities of alkali recommended by Fuller are unnecessary and harmful, and while we cannot accept in its entirety his theory of its manner of action, the weight of opinion among clinicians is that the use of moderate quantities of alkali is advantageous, and our knowledge of the action of these agents on the human economy would seem to place their use on a sound rational as well as empirical basis.

Alkalies increase both the fluid and solid elements of urine; they diminish the acidity of the urine, or make it alkaline; they promote oxidation; urea nitrogen is increased, and ammonia nitrogen is diminished. It is also claimed that the bactericidal power of the body tissues is heightened by increasing the alkalinity of the body fluids. It is obvious that it is desirable to attain these ends in a disease characterized by the presence of irritating toxins, as is the case in acute rheumatism. 'The addition of an alkali to the salicyl preparation has a corrective, as well as an adjuvant, function. In cases where it is necessary to give the salicylate in a liquid form, the alkali has a good effect in disguising the unpleasant taste of the former. Also salicylates are better borne by the stomach when given with an alkali. More important than these is the fact that the irritant effect of the salicyl on the kidney is lessened by making the urine alkaline. Frey has shown by experiments on rabbits, dogs, and in his own person that salicylates in sufficient doses produce albuminuria when the urine is acid, but fail to do so in the same dosage when the urine is previously rendered alkaline.

Local Treatment.-During the acute stage of the disease the pain in the affected joints is excruciating, and the slightest movement or pressure is intolerable. Careful attention to the position of the involved members and skill and patience in handling the patient saves much severe suffering. In no disease does conscientious and intelligent nursing add more to the comfort of the sufferer. The leg or arm should be evenly supported throughout its entire length in a position of slight flexion of all the joints, especially the one involved. The rheumatic knee causes far more pain when the limb is fully extended, and suspended between the heel and the tuberosity of the ischium, than when it is slightly flexed and evenly supported throughout its length. Much can be accomplished by the intelligent use of pillows. Mechanical devices or sand-bags may be of use. I have not found it necessary to encase the member in a plaster cast, as is recommended by some, except in cases where it is necessary to transport the patient from one place to another.

I do not practise the administration of salicylates locally, although they are readily absorbed through the skin, especially the volatile salicylic esters, such as methyl salicylate. This absorption, however, does not take place in large enough quantity, or with sufficient certainty, 
to meet the needs of the case of acute rheumatism, and cannot be compared in efficiency with administration by mouth.

A great many remedies have been recommended and used with benefit by local application. Analysis of their effects would seem to indicate that the beneficial effects of them all result from the production of a greater or less degree of active hyperemia of the part. I have found nothing of more service than the ordinary 10 per cent. ichthyol ointment. It is soothing to the patient, and can be applied continuously and indefinitely without destroying the skin or producing vesication.

General Measures. - The bowels should be kept freely open throughout the attack, the patient using the bedpan and urinal in responding to nature's calls during the active stage. 'The milder saline laxatives, citrate of magnesia and Rochelle salts, are the most suitable agents for regulating the bowels. If the patient is constipated when first seen, the question will be presented of giving a cathartic before beginning the salicyl treatment. 'There is no doubt that constipation increases the constitutional disturbance, and that the salicylates are better absorbed and act more quickly and efficiently if the bowels are emptied beforehand. On the other hand, the disturbance of the patient incident to the repeated use of the bedpan causes much additional suffering, which can be avoided if we can get him under the influence of the salicylates beforehand. 'The relief caused by this drug is generally so prompt, and the transition from exquisite suffering to relative comfort so speedy, that the postponement of catharsis for a few hours, till a time when it does not cause such distress, seems justifiable. Each case must be weighed on its merits, and a decision reached after consideration of both sides of the question.

It is rare that I find it necessary to use any other remedies than those already alluded to. Where pain is intense, we should not hesitate to use morphine to control it until the effect of the salicylates can be established. A single hypodermic injection of $\frac{1}{8}$ to $\frac{1}{4} \mathrm{gr}$. is generally all that will be required. Rheumatism presents in itself no contraindication to the use of morphine, and the same principles should govern its use here as in other conditions associated with pain and great restlessness.

'The patient should receive a sponge-bath in bed at least once a day, and oftener, if the temperature is very high or the sweating is profuse.

Diet.-During the acute stage of the disease I give a liquid diet, preferably milk. I find that this is usually all the patient will take, and it furnishes all the nutriment necessary, and at the same time an abundance of fluid which is required in large quantities. I do not consider that any especial diet is indicated in rheumatism, nor do I believe that any one food has any specific influence in causing recurrence or complications. 'The same principles that govern the dietary of any other acute febrile condition are to be observed here. 'The quantity of food should be limited to the digestive capacity of the patient. Here, as elsewhere, dietary errors are more often quantitative than qualitative. I do not keep up the liquid diet for a prolonged period, but begin to increase the quantity and variety of the food as soon as the acute symp- 
toms subside, using the patient's appetite as an index. For years clinicians have advocated a very meagre diet in rheumatism. A review of the older, and of even some of the modern dietaries, reveals a startling deficiency in caloric value, especially in the proteid content, this element having been regarded as being especially active in the production of the disease and in causing relapse. When we consider that the disease is more prevalent among the poor, whose diet, as well as their other hygienic requirements, is defective; and that rheumatism, when unchecked, produces a very rapid impoverishment of the blood, we have a rational basis for placing these patients on a mixed diet of substantial caloric value as soon as the acute symptoms have subsided.

Water should be given abundantly, especially during the febrile period. 'The thirst of the patient is generally a good guide to the quantity needed. Water may be rendered grateful and refreshing by being acidulated with lemon-juice or some other acid. Thirst is greater, and the quantity of water needed is larger when the sweating is profuse.

Prevention of Complications, Extension, and Recurrence.-The cardiac complications of rheumatism are so frequent, and the results of cardiac involvement so grave, that its prevention constitutes the most important feature in the management of the case. Valvular endocarditis is the most frequent, with a smaller, though considerable, proportion of cases of pericarditis. It is probable that the myocardium is affected to a greater or less degree whenever the other two occur. The younger the patient, the greater the liability to cardiac involvement, and the graver its results. The arthritis in children is slight in comparison with that of adults. Cardiac involvement is so much more serious, and its occurrence so constant, appearing sometimes before, sometimes without joint involvement, that it would seem almost justifiable to consider the rheumatic invasion in children as a separate disease from the rheumatic arthritis of adults, and the joint lesions as incidental rather than the dominant feature.

The heart of the rheumatic patient should be examined carefully every day that he is under observation. With reference to the cardiac condition we may observe the following classification: (1) Those in whom the organ is unaffected; (2) those in whom there is a fresh active involvement; (3) those who have no active involvement, but who have mechanical defects of valves or pericardium resulting from previous attacks; (4) those who have a fresh involvement engrafted on an old mechanical defect. In all four groups the principles of treatment are the same, and vary only in degree and in the duration of their application.

It is as well to admit that the salicylates have no specific influence on the heart lesions of rheumatism, either in preventing their occurrence or in promoting their removal. Realization of this will spare us much error in therapy and disappointment in prognosis. It is the chief reason why we are not able to assign to the drug a position as a specific in rheumatism parallel to that of quinine in malaria. It is useless to speculate on the explanation of clinical facts whose settlement must rest on the unknown factors of etiology. Experience has shown us that salicylates 
do not exert an effect on the other manifestations of the rheumatic invasion that are in any way comparable to that exhibited in the arthritic type. 'The only effect they have in preventing complications is by shortening the duration of the attack of arthritis, and thus lessening the time during which vulnerable organs are exposed to the etiological factors of the disease. 'The statement made by some that salicylates increase the liability to cardiac involvement has no substantial foundation.

To shorten the duration of the attack of arthritis, the institution of salicylate treatment should be prompt and vigorous, full doses being used from the beginning. 'The prophylactic doses of smaller size should be kept up at least two weeks after the fever has disappeared or even longer. The only other factor that influences the occurrence of complications or recrudescences is rest. 'The length of time that we should insist on rest in bed varies greatly with different cases. As has been said, it is exceedingly difficult to keep patients in bed, or even in the hospital, for a sufficient length of time to protect against further trouble. 'The conditions that make a prolonged stay in bed necessary are as follows:

(1) Childhood. If we should be so fortunate as to have a child under fifteen years pass through an attack of acute rheumatism without any cardiac lesion developing, we should spare no pains or patience to maintain this condition.

(2) When, after the subsidence of the fever and joint symptoms, even though auscultation show's no valvular defect, there is an exaggerated muscular action of the heart, or an increase in its rate either at rest or on slight exertion.

(3) Whenever there is the evidence of an old cardiac inflammation, since these cases are more liable to recurrence.

(4) When there is a history of one or more previous attacks of rheumatic arthritis.

(5) When resolution of the joint lesions is not complete and satisfactory.

With these considerations in mind, we must form our opinion in each case, weighing the character of the patient, his environment at home, and his capacity for and intelligence in following instructions as to taking care of himself.

In those instances where a cardiac complication arises, the management of the case becomes that of the heart disease, and is discussed under that title.

Hyperpyrexia.- This complication does not often occur, and the factors that produce it are not understood. Without any apparent reference to the severity of the case, certain patients, without warning, suddenly develop excessively high temperature, with accompanving cerebral symptoms, such as convulsions and delirium, followor by stupor and coma. 'There is at the same time profound general depression. The condition is a very grave one, and its rarity accounts chicfly for the small mortality that arises immediately from this disease. 'The best results are obtained by external application of cold, a fact that owes 
its recognition to Wilson Fox. Fox placed the patient in a tub-bath at $90^{\circ}$ to $100^{\circ} \mathrm{F}$., and gradually reduced the temperature to $75^{\circ}$ or $70^{\circ} \mathrm{F}$. He was removed when the rectal temperature was reduced to $102^{\circ} \mathrm{F}$. He found that it was not necessary to go below this, as the temperature continued to fall after the patient was removed from the bath. After removal from the bath the patient was wrapped in blankets and, if necessary, hot bottles were applied to the feet and body. Fox stimulated freely with whisky and brandy where it seemed to be required. In case the above method did not succeed in reducing the temperature, he added applications of ice to the abdomen, chest, and spine, and affusions of ice-water poured from pails.

The similarity of the clinical picture of hyperpyrexia in rheumatism to that presented by insolation (sunstroke) is very striking. It was noted before the time of Fox, and was alluded to by him. The important difference is that in rheumatic hyperpyrexia the patient is already depressed by having been exposed for a time to the toxins of disease, and there does not occur the sthenic type, as we find in insolation. For this reason depleting measures, such as bleeding, which is often beneficial in the latter condition, is contraindicated in rheumatic hyperpyrexia. The older authors recognized that not only did it weaken the patient, but it had no influence on the fever. With this exception, the treatment of the two conditions is identical. Baruch collected the statistics of the cases of insolation treated in the New York hospitals in 1896 by various hydrotherapeutic methods. They show that much the lowest mortality was obtained by wrapping the patient in a sheet and applying cold sprays or affusions with force, stopping the treatment when the temperature had fallen to $104^{\circ}$ or $103^{\circ} \mathrm{F}$.

Pneumonia and pleurisy are not infrequent complications of acute articular rheumatism, and occur generally in those cases in which heart involvement has already arisen. Pneumonia is apt to be a serious matter, and greatly prolong the illness and convalescence. Its treatment does not differ from that of other secondary pneumonias. Pleurisy is not often of serious import, and is easily controlled by the measures ordinarily employed in this condition.

Convalescence.-As has been said, the resolution of the inflammation of the joints is generally complete, and there is no resulting disability. Occasionally we meet with subacute cases in whom convalescence is tedious. Sometimes, after the acute attack is recovered from, there persists a certain degree of pain and stiffness, which is aggravated by damp weather and exposure to chilling. This is due to incomplete absorption of the periarticular infiltration, and is apt to be present in elderly patients, or in those who have had repeated attacks. In such instances absorption should be promoted during convalescence by passive movements and massage of the affected joints alone, or combined with some of the stimulating hydrotherapeutic and thermic measures, such as local hot-packs and hot-air baths, douches, etc. 'This treatment is best carried out at thermal resorts or institutes, where its administration is in trained hands. 
In former times a much-dreaded sequel of rheumatism was anemia. All the early authors testify to the severe and rapid anemia that the disease produced, and this was evidently independent of the depleting treatment that characterized its early therapy. Germain-See (1S77) first noted that under salicylate treatment anemia did not occur. In my experience it has been very unusual in uncomplicated rheumatic arthritis, and is limited to those cases that have by reason of complication had their illness prolonged, or is caused by subsequent heart disease. Its practical disalppearance as a sequel to the arthritis is undoubtedly due to the shortening of the period of activity of the toxins of the disease by salicylates. In event of its occurrence it should be treated according to the principles that apply in secondary anemias in general, that is, by improved hygienic environment, hypernutrition, and tonics. Iron, arsenic, and quinine are useful, and some cases, especially children, respond admirably to cod-liver oil.

Local Use of Salicyl Preparations.-As I have already said, I do not advocate the practice of trying to neutralize the toxic material of rheumatic arthritis by the application of salicyl preparations to the involved joints. My experience with the internal administration has been such that I am convinced that it is the most satisfactory method of using the drug. There is no question that salicylic acid and the organic salicylates are absorbed by the skin quite readily, as may be demonstrated by testing the urine, but in how great quantity it is difficult to say. It is not very probable that absorption through the skin takes place as rapidly over an inflamed surface as over healthy tissue. The only reason, therefore, for making applications of salicyl to the inflamed joints must be based on the assumption that it has some specific local effects on the inflammatory products, which is not exerted to the same extent when it is introduced through the circulation. Some have reported favorable results from this method, but the reports are not so numerous or convincing as to lead one to think that it will give results comparable to the more usual manner of administration. It may be tried in cases that for any reason cannot take salicylates by mouth, as in cases of excessive gastric irritability and idiosyncrasy. I have, however, learned by experience that in case of idiosyncrasy, toxic effects, such as nausea, may result from applying the drug by inunction. The preparation generally used for local application is salicylic acid incorporated in olive oil, lanolin, or some other bland diluent, or else one of the preparations containing methyl salicylate, as oil of gaultheria, or some of the numerous synthetic products.

Hydrotherapy. - Various local procedures, such as hot bathing of the joints, hot-packs, and cold compresses, have from time to time been recommended by various authors for the purpose of relieving the pain and reducing the inflammation of the joints. Their beneficial action is due to the local hyperemia they produce, and they may be used for this purpose by those who prefer them.

A few have undertaken, however, to treat acute articular rheumatism by hydrotherapy alone. Annong these is Hauffe, who reported 233

VoL. I-53 
cases of articular rheumatism, including 101 acute cases treated by this method. Hauffe's method consists in placing the patient at the begin. ning in a hot full bath at $100.4^{\circ} \mathrm{F}$, and gradually raising the temperature by the addition of hot water, to $113^{\circ} \mathrm{F}$, or higher. The patient is kept in the bath until he begins to sweat, which is generally in fifteen to twenty minutes. After the bath he is placed in an ordinary full wetpack, where he remains one hour. Then he is put naked to bed and lightly covered, where he remains for some time. The inflamed joints are now dressed with a mixture of turpentine and oil, covered with gutta-percha tissue. The hyperemic effect produced by the latter may be heightened, if desired, by the addition of hot-water bottles. The above procedure is repeated as indicated. Passive movements of the joints are begun as soon as they cause no discomfort to the patient, and he is allowed to get out of bed early. Hauffe claims admirable results by this method. His report is reviewed by Plehn, who reports at the same time 343 cases of acute articular rheumatism, taken without selection from his hospital records, treated with salicylic acid. The comparison shows for the salicyl treatment a shorter duration of the febrile period, a shorter stay in the hospital, and a smaller percentage of recurrences and complications. He draws special attention to the relatively large number of cases of pleurisy that developed under Hauffe's treatment.

Hyperemia (Bier).-While the application of passive hyperemia has generally been limited to those affections of the joints that are septic or chronic in their nature, a few have recommended it in acute articular rheumatism. Klemperer and Steinitz laud its superiority over salicyl medication in this disease, and each reports a series of cases to support his contention. Plehn disposes of these claims at the same time that he deals with those of Hauffe, and reasserts the generally accepted facts as to the curative effects of salicyl, and shows that the statements as to its toxicity have been greatly exaggerated.

The value to be attached to the employment of hydrotherapy and other physical methods of treating acute articular rheumatism is well expressed in the words in which Plehn closes his discussion of the subject in the paper previously referred to:

"The so-called physical therapy (passive hyperemia, baths, packs. massage, etc.) may assist the effect of medicinal treatment, but can never entirely replace it."

I would only consider their employment as adjuvants to salicyl therapy, or else in those cases of acute rheumatism which have demonstrated beyond question their inability to take any form of salicylate, or who, by reason of advanced heart or kidney disease, cannot take it in sufficient quantity to be effective, and finally, in those cases in which salicylates have, after a fair trial, failed to produce favorable results. 


\section{CHOLERA}

BY LEONARD ROGERS, M.D., F.R.C.P.

Tив sudden and rapid onset of very serious symptoms makes Cholera Asiatica one of the most terrifying of human diseases; yet there is probably no known specific disease in which a greater proportion of valuable lives can be saved by prompt and efficient treatment, and certainly none in which recovery from an almost moribund condition can so frequently be brought about. 'The necessary measures require care and experience to obtain the best results, but the principles on which they are based are simple, and afford a sure guide in the rapidly varying phases of the disease.

The Nature of the Pathological Process. - The essential features of cholera to bear in mind from the practical point of view is that the causative comma bacillus of Koch is almost limited to the intestinal canal, and does not penetrate the tissues to any marked extent. 'The toxins which produce the symptoms are, therefore, formed in the bowel and absorbed thence into the circulation. 'This results in a rapid outpouring of fluid through the mucous membrane of the stomach and intestine, so that several pints, or even quarts, of fluid escape from the body within a few hours. If the loss of fluid is very great, collapse ensues, with little or no pulse at the wrist, and death may soon take place from circulatory failure. Should the patient survive the collapse stage, and the pulse pick up again, the nearly equally dangerous reaction stage is entered on, with increased absorption of toxins from the bowel producing a rise of temperature, which may occasionally become hyperpyrexial or typhoid-like. During this period, also, the deadly combined toxemia and uremia, resulting from failure of the kidneys to resume their excretory functions, occurs.

The principles of treatment of cholera consist in: (1) Checking the loss of fluid if possible; (2) limiting the absorption of toxins from the bowel; (3) restoring the lost fluid so as to maintain the circulation; (4) reëstablishing the action of the kidneys, and (5) supporting the strength of the patient through an extremely exhausting disease.

Stage of Premonitory Diarrhœa.-During the prevalence of cholera severe diarrhœe of an ordinary type, which yields to astringent remedies, frequently occurs. Some of these cases are doubtless very mild choleraic infections which do not go on to the fully developed disease, and others of a similar nature pass into true cholera in spite of the same treatment. The passage of such loose fecal stools is described as the premonitory stige of cholera, and acids and opium, the latter commonly in the form of chlorodyne, are generally recommended. Proof is, however, wanting that attacks apparently cut short by these remedies would have 
gone to true cholera if they had not been treated by astringents. The unsuccessful experimental attempts to produce cholera in the human subject by Koch's bacillus given by the mouth show the great resisting power of the system against this infection. Metchnikoff, in 1893, was able to produce well-marked diarrhœa in men at the Pasteur Institute, but in only one instance did typical rice-water stools ensue without active treatment. For these reasons, I am somewhat skeptical regarding the power of astringent remedies to cut short a true cholera infection, which would otherwise have gone on to a typical manifestation of the disease. As, however, it is impossible to say in this stage whether the attack is the premonitory diarrhœa of cholera or not, I consider it should be treated, on ordinary principles, with astringent remedies, such as dilute sulphuric acid. 'The use of opium requires greater care and consideration, for it is an exceedingly dangerous drug in the fully developed stages of cholera. Nevertheless, as it frequently appears to cut short severe diarrhœa in the tropics, a single dose of 20 minims of the tincture of opium, or 15 minims of chlorodyne, may be given if the stools are still fecal; but I never use opium, once rice-water evacuations have commenced. The dose should not be repeated, for if collapse quickly ensues, the drug may not be absorbed until the circulation revives in the stage of reaction, when it is most actively harmful. If cholera is suspected, purgatives should not on any account be given. C. Macnamara thoroughly tried Johnstone's castor oil treatment in India, but abandoned it, as the mortality was "fearful." Nature's eliminative methods are more than efficient in cholera, and do not require any artificial aid.

Stage of Copious Evacuations and Collapse.-The great majority of cases of cholera only come under the observation of the physician after very copious diarrhœa and vomiting has occurred, and the patient has already become more or less collapsed. If the pulse has markedly failed, drugs can be of little or no use, as they will not be absorbed. On the other hand, if the circulation is fully restored and maintained, the toxins are quickly excreted through the kidneys, and rapid recovery commonly takes place without the aid of any drugs whatever. I shall, therefore, first deal with measures to restore the lost fluid, which is the primary consideration in the treatment of cholera.

The Necessity of Replacing the Fluids and Salts Lost from the Blood in Cholera. - The rapid loss of several pints of fluid from the body in cholera, leaving little or no pulse at the wrist, at once suggests the advisability of replacing some of the deficiency. Yet Dr. George Johnson, in his strenuous advocacy of a purgative, or, as he called it, "eliminative" treatment of cholera, denied that there is any relationship between the quantity of the evacuations and the severity of the disease. It is thus of vital importance to determine the true relationship between the loss of fluid from the blood and the degree of collapse and danger. I have, therefore, estimated the amount of fluid lost from the blood by measuring with the hematocrite the volume of serum and corpuscles respectively in a consecutive series of cases of cholera in Calcutta. In a number of them estimations were also made of the percentage of chlorides 
in the serum. 'The results are summarized in 'Table I, which includes the normal figures for natives of India.

Table I.-Blood Chanoes in Cholem and the Efyect of Hypertosic Saline INJECTIONS.

\begin{tabular}{|c|c|c|c|c|}
\hline . & Normal. & $\begin{array}{l}\text { Avernge of } 7 \\
\text { fatal cases. }\end{array}$ & $\begin{array}{l}\text { Average of } 12 \\
\text { cases recover- } \\
\text { ing after trans- } \\
\text { fusion. }\end{array}$ & $\begin{array}{l}\text { Average of } 5 \\
\text { mild ceses not } \\
\text { transfused. }\end{array}$ \\
\hline 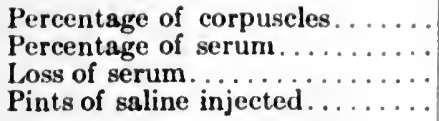 & $\begin{array}{l}45 \\
55 \\
\cdots\end{array}$ & \begin{tabular}{|c|}
71 \\
29 \\
64 per cent. \\
3.7
\end{tabular} & \begin{tabular}{|c|}
63 \\
37 \\
52 per cent. \\
3.6
\end{tabular} & $\begin{array}{c}60 \\
40 \\
35 \text { per cent. }\end{array}$ \\
\hline Blood-pressure before injection. . & $\left\{\begin{array}{l}100-110 \\
\text { mm.Hg. }\end{array}\right.$ & $\left\{\begin{array}{c}50 \mathrm{~mm} . \\
\mathrm{Hg} .\end{array}\right\}$ & $51 \mathrm{~mm} . \mathrm{Hg}$. & $71 \mathrm{~mm} . \mathrm{Hg}$. \\
\hline $\begin{array}{l}\text { Blood-pressure after injection... } \\
\text { Chlorides before injection ...... } \\
\text { Chlorides after injection. ...... }\end{array}$ & $\ddot{0 . \dot{s}}$ & $\begin{array}{c}97 \mathrm{~mm} . \mathrm{Hg} . \\
0.79 \\
0.95\end{array}$ & \begin{tabular}{|}
$107 \mathrm{~mm} . \mathrm{Hg}$. \\
0.90 \\
1.07
\end{tabular} & 0.92 \\
\hline
\end{tabular}

In the mild cases, recovering without intravenous saline injections having been necessary, the average loss of fluid from the serum of the blood amounted to 35 per cont. In typically severe cases, requiring transfusion, but which ultimately recovered, the average loss amounted to 52 per cent., while in 7 very severe cases, which proved fatal in spite of repeated intravenous injections, the average loss of fluid from the serum was no less than 64 per cent. of the whole. There is thus a very definite relationship between the amount of fluid lost from the blood and the severity of the disease. Moreover, in a large majority of the cases the loss of fluid is so great, and the consequent increased viscosity of the blood so marked, as at once to indicate the necessity of replacing it if the circulation is to be adequately maintained.

For clinical work, a simpler guide to the concentration of the blood in cholera is the specific gravity. For its estimation, I prefer to use the method in which a small drop of blood from the finger is gently blown by a capillary pipette, into the middle of a mixture of glycerine and water of known specific gravity, and watching to see if it rises or falls; this process is repeated until the solution, in which it just floats for a second or two, is found, which gives the desired result. By having the series of small glass-stoppered bottles in a box, containing solutions of every alternate degree from 1040 to 1076 , the specific gravity can readily be determined at the bedside in two or three minutes. In the tropies it is well to make up the solutions at about the mean temperature, and they should he renewed from time to time from stock bottles. In the collapse stage of cholera the specific gravity of the blood rises from a normal of 1056 to about 1065 in a case of moderate severity, while it may rarely reach over 1070 in very marked collapse, the viscosity of the bloorl being seen to be greatly increased on opening a vein in the latter cases.

Of equally great significance is the effect of the evacuations on the salts of the blood. It might naturally be expected that, with the concentration of the blood, the percentage of its salts would be considerably 
increased. Such is not the case, for I have confirmed Edmund Parke's observations of about fifty years ago, that the rice-water stools of cholera contain a large amount of chlorides, 0.5 per cent. and more, although the watery vomit contains very little. In order to estimate the effect of this loss on the blood in cholera, I have estimated the percentage of chlorides in the serum separated from the clotted blood, and thus obtained the figures given in Table $I$. The results are most instructive, for in the worst cases of cholera, in which an average of two-thirds of the fluid of the serum was lost, the chlorides were slightly lower than normal, and in somecases considerably lower. As little as 0.6 per cent. has been met with, the serum exuding from the clot sometimes showing distinct hemolysis, which disappeared when the chlorides had been raised to 0.9 per cent. or over by transfusion with a hypertonic salt solution. Further, the lowest percentage of chlorides was usually found in the worst cases of cholera, and if it was raised to 1 per cent. or over, recovery usually took place. There is thus evidence of a distinct relationship between the loss of chlorides from the blood and the severity of the disease, and a clear indication is afforded for replacing the lost salts, as well as the lost fluid, in cholera.

Methods of Replacing the Lost Fluids and Salts in Cholera.-Having now cleared the ground, I may next consider the methods of restoring, as far as possible, the circulation of a patient coming under observation in the collapse stage of cholera, and the indications for each, beginning with the simplest measures.

Rectal Injections of Salt Solutions.-Althongh the stools are very copious and frequent at the onset of cholera, once collapse has set in they become much less so. Rectal injections of salt solutions are then commonly retained long enough for much of them to be absorbed, for the mucous membrane of the lower part of the large bowel is not affected by the disease. The difficulty is to know when it is safe to rely on this simple measure. This can be decided by estimating the blood-pressure at the wrist, preferably by one of the many modifications of the Riva-Rocci mercury sphygmometers, of which the most convenient is perhaps that recently described by Leonard Hill. As a result of several years' observations, I have for some time begun the treatment with rectal saline injections if the blood-pressure is 70 millimeters or over, unless other strong indications for intravenous injections were present. A large majority of such cases respond well to this treatment, the blood-pressure rising and the specific gravity of the blood falling. A most careful watch must be kept for any further failure of the pulse, as a single copious stool or vomit may suddenly lower the blood-pressure to a dangerous extent, necessitating immediate active measures. Half to one pint of normal saline may be injected every two hours at a slow rate, with the vessel only elevated one or two feet above the buttocks. If it is not retained when given in such quantities, the plan may be tried of continued very slow injection, as in Murphy's method of treating peritonitis. A stop-cock in the course of the tubing allows of easy regulation of the flow. Watch must be kept that the tube is not expelled with a passage of a stool into the bed. I have several times seen the pulse pick up, and 
the specific gravity of the blood fall, as a result of slow, continued rectal injections in patients with a blood-pressure of only (i5 to 75 ) millimeters, and intravenous injections were thus rendered unnecessary where I scarcely expected to be able to do without them. With presisures below (b) to 70 millimeters this methor fails, as the fluid is not absorbed. For rectal injection I use 90 grains solium chloride to a pint of water at blood heat. It is most important that rectal injections should be continued until free action of the kidneys is obtained; this is seldom less than two days, although the quantity and frequency of the rectal saline injections may be reduced as soon as a fair amount of urine is passed.

Subcutaneous Injections of Salt Solutions.-A more certain and rapid method of getting the salt solution into the circulation in cholera is by injecting it into the loose subeutaneous tissues of the chest-wall, axilla, or thighs, as is often done in the treatment of surgical shock. 'This plan was, I believe, first largely used in cholera by Wall, loth in India and in Europe. It is of considerable value, especially when the circumstances do not allow of the more efficient intravenous injections. 'The objections to the method are that it is difficult to introduce subcutaneously the large quantities of fluid necessary in the severe collapse of cholera, and in addition, if the pulse at the wrist has completely failed, the fluid may not be absorbed in time to restore the rapidly ebbing circulation. Indeed, I have seen a patient die of collapse with a large amount of fluid in his subcutaneous tissues, which had been injected by the slow, continued method, but had not been absorbed. Another drawback is that, owing to the low vitality of the tissues in cholera and the frequent absence of perfect aseptic conditions, very extensive abscess formation is unavoidable in a certain number of cases, which will greatly retard convalescence, even if it does not turn the scale against the patient. For these reasons, I very seldom use the subcutaneous method in the collapse stage in hospital practice, where facilities for the more satisfactory intravenous injections are always ready at hand. Nevertheless, in the absence of the latter, repeated subcutaneous salines are of great value, and hypertonic solutions should be used in all cases in which rectal injections are not likely to be of service, the strictest antiseptic and aseptic precautions being taken.

Intraperitoneal Injections of Salt Solutions.-In order to overcome the drawbacks to the subcutaneous injections, and to furnish a more simple and rapid method of replacing the lost fluids than the intravenous one, I have devised the instrument shown in Fig. 99, to allow of intraperitoneal injections. It consists of a steel cannula, the distal end of which is sharpened like a cork borer, while a flange near the other end prevents it slipping in too far. A blunt stilette is provided for cleaning and oiling the interior. After carefully cleansing the skin, as for a surgical operation, an incision about half an inch in length is made with a narrow-bladed knife, just below the navel (as the perioneum is adherent here, and will not strip in front of the canmula), and carried through the subcutaneous tissues as deeply as is safe. The cannula (without the stilette) is now inserted to the bottom of the wound, and, 
with a finger placed near the point to prevent it suddenly slipping forcibly into the peritoneal eavity, it is made to penetrate the remaining tissues by a steady, boring motion, during which the abdominal wall is held up by an assistant. As mueh as three pints of hypertonie salt solution can be easily run in within about ten minutes in an adult, this being the maximum advisable at one time. After withdrawing the cannula, a stitch is inserted through the skin and-fascia and collodion applied. 'The injection can readily be repeated through the same wound

Fig. 99.

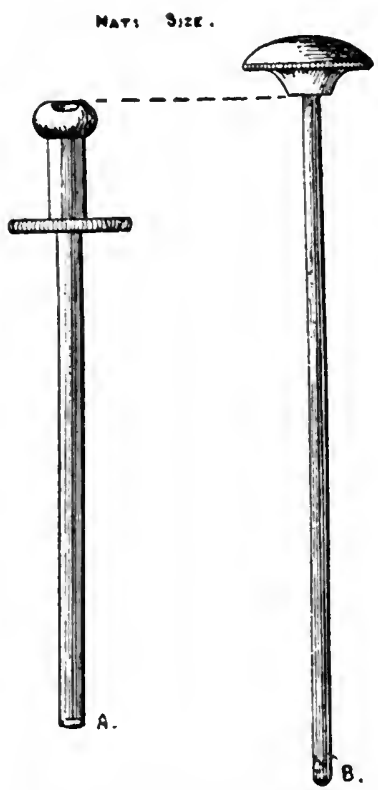

Cannula for intraperitoneal transfusion.

if necessary. Care must be taken not to run in sufficient fluid to embarrass the action of the diaphragm. 'The abdomen should never be distended so that the fluid is under marked pressure. If any pulse remains at the wrist the fluid is rapidly absorbed, with an accompanying rise of blood-pressure and a decrease in the concentration of the blood. I have also seen early reëstablishment of the renal secretion ensue. Favorable results following the use of this method have been reported to me from two of the Calcutta hospitals. I have also used it with suceess in several cases, chiefly in children, when veins are very small. I much prefer the intravenous method when feasible, but consider the intraperitoneal injections to be a useful substitute when eircumstances do not allow of the former, as it is simpler and quieker. In moribund patients the fluid will not be absorbed quickly enough from the abdominal cavity, and intravenous injections are essential. With this exception the indieations are the same as for the later method. Some further rise of blood-pressure can be obtained by pressure on the dilated portal system, by means of an abdominal binder applied after the intraperitoneal injection; the effect on the respirations should be carefully watched.

Intravenous Injections of Salt Solution.-The history of the use of intravenous saline injections in cholera is a long and checkered one, which may be only briefly referred to here. They were first used by Drs. Latta and Mackintosh, in Edinburgh in 1831 in 166 eases, with a mortality of 84 per cent. From that time on they have been repeatedly tried and as often abandoned. The immediate effeet in reviving apparently moribund patients is remarkable, but of very brief duration. Wall, in 1893, with the advantage of Lord Lister's antiseptie methods, enthusiastically advoeated intravenous injection in eholera, and recorded a success after six injections, yet his mortality in 193 cases was a little over 70 per cent. Goodeve, of the Calcutta Medical College Hospital, writing in 1870, was 
very doubtful whether the mortality of eholera was reduced by this measure. The method largely went out of use in India in recent years up to 1906 , when it was again tried by myself, with the help of Captain J. W. D. Megaw, I. M. S. I ike our predecessors, we used normal salt solution, but only obtained very fleeting improvement, while the reduction in the mortality was so slight (51 per cent. against 59 previously) that the method was once more given up, during 1907 , as of very little value.

It then occurred to me that if the salts of the blood become concentrated as a result of the great loss of fluid in cholera, then the injection of normal salt solution would once more reduce the proportion of salts in it, and so might aid the outflow through the damaged intestinal mucous membrane. I, therefore, determined to try the effect of injections of stronger saline solutions, to maintain a high salt content, which would tend to eause the osmotic currents to carry fluid into the blool rather than to allow it to escape from it. At the same time, I carried out an investigation of the amount of chlorides in the serum in cholera, which furnished an additional reason for using strong saline solutions to replace the large amount of salts lost in the rice-water stools of cholera. I am greatly indebted to Captain Maxwell Mackenzie, I. M. S., for carrying out the earlier, very successful hypertonic transfusions for me.

Table II.-Results of Different Forms of Treatment of Cholera.

\begin{tabular}{|c|c|c|c|c|c|}
\hline \multirow{2}{*}{ Period. } & \multirow{2}{*}{ Treatment. } & \multirow{2}{*}{ Cases. } & \multirow{2}{*}{ Deaths. } & \multicolumn{2}{|c|}{ Percentage. } \\
\hline & & & & Deaths. & Recoveries. \\
\hline 1895-1905. & Reetal and subeutaneous salines & 1243 & 783 & 59.0 & 41.0 \\
\hline $1906 \ldots$ & Normal saline intravenously.... & 112 & 57 & 51.9 & 49.1 \\
\hline 1907. & Reetal and subeutaneous salines. . & 158 & 94 & 59.5 & 40.5 \\
\hline $1908-1909$ & Hypertonic intravenous salines..... & 294 & 96 & 32.6 & 67.4 \\
\hline $\left.\begin{array}{l}\text { Aug., } 1909 \\
\text { May, } 1910\end{array}\right\}$ & 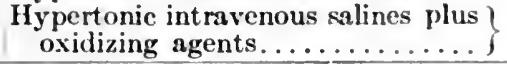 & 91 & 22 & 24.2 & 75.8 \\
\hline
\end{tabular}

Calcutta is an ideal place for testing the value of the hypertonic saline transfusions, as records exist of the results of former treatment at the Medical College Hospital for eleven years before the revival of intravenous injections in 1906 . The death-rate differed little from year to year, although each quarter has its own mortality, which is highest in the first three months, and lowest in the third or rainy quarter. 'Table II gives the data illustrating the results of different forms of treatment, and clearly shows that the death-rate has been reduced to but little more than half of the former figure by the introduction of hypertonic transfusions. During the last ten months, by the addition of treatment directerl toward destroying the toxins within the howel, the death-rate has fallen still further to 24 per cent., or a two and a balf fold reduction on the original mortality for the same months of the eleven years, from 1595 to $1905 \mathrm{in}-$ clusive. As my observations of the hypertonic injections now extend over two and a half years, during which upward of 350 cases have been admitted, no possible doubt can remain that a great advance has been made in the treatment of this terrible disease. All deaths in patients admitted 
in a moribund state, as well as those suffering from complications, are included. Moreover, for some time past the diagnosis has been confirmed bacteriologically, and as only cases with a characteristic history and typical symptoms are included, the figures may safely be taken as reliable.

Indications for Intravenous Saline Injections in Cholera.-I regard a blood-pressure below 70 millimeters of $\mathrm{Hg}$. at the wrist as the most essential indication for the injection of saline solution into a vein. This means a very feeble and soft pulse. It should be accompanied by an increase in the specific gravity of the blood to 1060 or over, for in the later reaction stage of cholera low blood-pressure may be combined with a normal or even a subnormal specific gravity, in which case copious

Fig. 100.
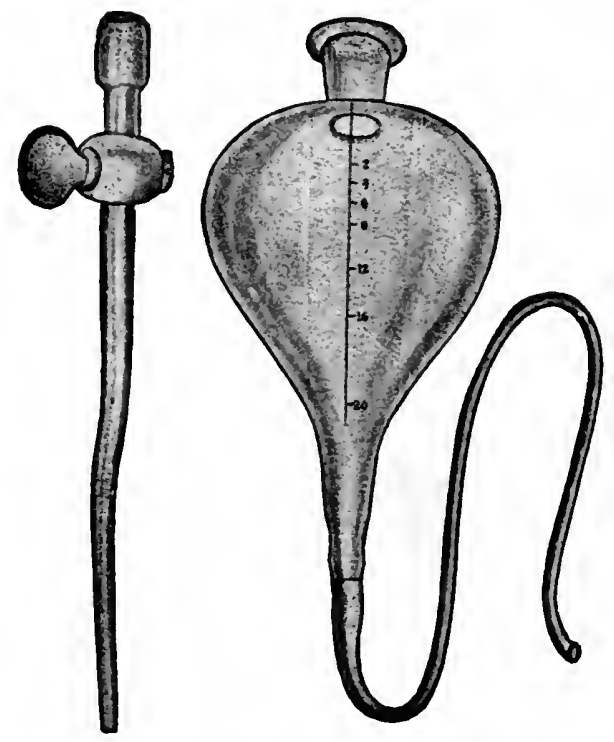

Stopcock cannula and graduated bulb for transfusion at regulated rates.

saline intravenous injections are dangerous, on account of their tendency to produce cedema of the lungs. In addition to these primary indications there are others depending on the condition of the patient; namely, marked restlessness, severe cramps, or cyanosis. These symptoms are practically alwavs associated with very marked failure of the pulse and concentration of the blood, due to great loss of fluid. When the bloodpressure is on the borderland, between 65 and 75 millimeters, if restlessness and cramps are present, or the specific gravity of the blood is above 1065, no time should be lost in giving an intravenous injection, but if the patient is quiet, and the general condition fairly good, it may be advisable to first try rectal injections. Restlessness, cramps, and cyanosis are at once relieved by an intravenous injection, the patient 
not infrequently falling asleep before the injection is completed. One of the veins at the bend of the elbow is most convenient, especially the median basilic. A large vein is also available in front of the inner malleolus, and is well marked in children, but the fluid will run in more slowly here. In children I have also used the internal saphenous in the thigh, on account of its great size, but this is seldom necessary in those over six years of age if the cannula shown in Fig. 100 is at hand. 'The end tapers slightly, so that it readily enters quite a small vessel, and, being pushed well in, can be securely tied. It has been made for me by Messrs. Down Bros. of London. As the veins are collapsed in cholera, it is advisable to tie a bandage round the limb above the site of injection to distend the vessel. 'The vein may be exposed by a small incision, no anesthetic being necessary. After tying the lower end, an oblique cut is made with scissors through about half the circumference, just distal to a point held by a forceps gripping only the superficial wall, thus forming a small flap, beneath which the cannula can be readily passed into the vessel. All air having been previously expelled from the tubing, the bandage is loosened, and the fluid allowed to run in by gravitation, the vessel being placed on a stand two or three feet above the level of the bed. A piece of sterile gauze should be laid on the wound, to protect it during the injection, and the vessel of fluid also covered.

Strength and Composition of the Solution.-I have used with success from 120 to 150 grains of sodium chloride to the pint, but, in view of the large amounts commonly necessary, I advise the following:

$$
\begin{aligned}
& \text { Sodium chloride, } \\
& \text { Calcium chloride, } \\
& \text { Potassium chloride, }
\end{aligned}
$$

$120 \mathrm{gr}$.

4 "

The addition of the last two salts furnishes the three chlorides in the same proportion as Ringer's fluid, with which a mammalian heart can be kept beating for many hours, although with sodium chloride alone it will soon cease to act. I have arranged with both Messrs. Burroughs and Wellcome and Messrs. Parke, Davis and Co. to put this combination in tablet form, so that four of them dissolved in one pint of sterile water (any pure water which has been filtered through cotton-wool and boiled for fifteen minutes suffices in an emergency) will make a solution of the above strength. If three of them are dissolved in the same quantity of water, the solution will be suitable for rectal injections or for late injections in the reaction stage after collapse is over, and the urinary secretion is deficient. 'Two drachms to the pint of sodium chloride alone (1.25 per cent.) is also quite satisfactory, and has, indeed, been used in the majority of my successful transfusions.

The Temperature of the Fluid.-Although in cholera collapse the surface temperature is much helow normal, yet I find the rectal reading is as often above as below $98.4^{\circ} \mathrm{F}$. Experience also shows that saline injections into the veins are almost invariably followed by a rigor, with a rapid rise of the axillary temperature to $100^{\circ}$ to $104^{\circ} \mathrm{F}$, and very occasionally ending in hyperpyrexia. 'The deficiency of the surface heat is 
thus clearly due to the great diminution of the blood circulating through the superficial parts and extremities, the internal temperature being commonly high. I, therefore, inject the fluid at as nearly as possible the normal body temperature whenever the rectal reading is $99^{\circ} \mathrm{F}$. or over. To allow for cooling during passage through the tubing, the fluid should be at $100^{\circ} \mathrm{F}$, in the flask. If, however, the rectal temperature is below normal, the fluid in the vessel should be at a temperature of from $102^{\circ}$ to $104^{\circ} \mathrm{F}$. at the commencement of the injection, being lowered later if the surface heat returns. Cases with a very low rectal temperature, such as below $97^{\circ} \mathrm{F}$., are extremely critical, and nearly always terminate fatally. Nichols and Andrews, in the Philippine Islands, advise a temperature of $43^{\circ} \mathrm{C}$. $\left(109.5^{\circ} \mathrm{F}\right.$.) for the fluid in the flask.

The Quantity of Saline to be Injected.-By measuring the volume of corpuscles and serum before and after rapid intravenous transfusions, I found that in an adult patient with marked collapse, and little or no pulse at the wrist, four pints of fluid were usually required to dilute the blood to about the normal consistency and specific gravity. In some very severe cases even this quantity was not sufficient for the purpose. In the Philippines 1500 cubic centimeters, or about three pints, was the average amount used, although larger quantities were occasionally employed. Here again the specific gravity of the blood is a valuable clinical guide. If it is found to be raised to over 1064, four pints may be injected in an adult male in the collapse stage, while the effect can be rapidly gauged by taking the specific gravity again before concluding the injection. The blood may be safely diluted to several degrees below normal, so as to allow for some further loss of fluid taking place without collapse being at once reproduced. I have frequently reduced the specific gravity to 1050 , or even less, with good results, while, if the last pint or two is given slowly, it will have time to pass into the tissues and does not further materially dilute the blood.

The effect on the blood-pressure is also of great importance. It is not sufficient to restore the pulse to a state in which it is just felt at the wrist. An apparently very fair pulse-beat will often be found with a blood-pressure of only 70 to 80 millimeters. This is sufficient to maintain the circulation for the time being, but not high enough to insure renewed secretion of urine, which is so much to be desired to remove from the blood the toxins already absorbed from the bowel. Further, a moderate loss of fluid from the gastro-intestinal tract will rapidly reproduce dangerous collapse if the pressure has only been raised to about 80 millimeters. A normal pressure of about 110 millimeters or more should be aimed at, which will insure immediate action of the kidneys and also allow of some fall due to further diarrhœa without collapse being at once induced. In females about $3 \frac{1}{2}$ pints will suffice, and in children from ten to fifteen years of age, abont 2 pints, the specific gravity and blood-pressure serving as guides to the precise amounts required.

Rate of Transfusion and Method of Regulating It.-When marked collapse is present, the saline should be first run in quickly to restore the 
pulse. It may, as a rule, be given at the rate of about 1 pint in five minutes, or 4 ounces per minute, but a careful watch must be kept for any signs of distress, especially in elderly persons. If severe headache or oppression of the chest is produced, or the breathing becomes quickener, the rate should be at once slowed. If the stop-cock cannula is not available, this may be done by lowering the height of the vessel containing the fluid, or by means of a screw clamp on the rubber tubing. In order to be able to run in fluid at any required rate, I have devised the simple apparatus shown in Fig. 100. 'The cannula has a stop-cock, by means of which the flow through it can be easily regulated. 'The glass bulb for holding the saline is graduated from above down, to show every 2 to 4 ounces. 'T'o regulate the flow the bulb is filled to the neck, and the quantity which enters the vein in one minute is timed. If it is faster than is desired, the stop-cock is turned partly off and the rate again timed, and so on, until the desired flow is obtained, which can usually be done by the time about 6 ounces have rum in. The speed can be readily slowed down to under 1 ounce a minute. In cases in which severe diarrhoa continues and the specific gravity of the blood is very high, it is sometimes advisable to give more than 4 pints at a single injection. In such patients 3 or 4 pints are first given quickly, and subsequently 1 or 2 more may be run in at the rate of 1 ounce per minute, so that it will pass into the tissues without materially further diluting the blood. The sunken appearance of the eyes may then largely disappear, the voice once more regain its strength, and the patient be afforded a reserve of fluid which will lessen the liability to a rapid recurrence of collapse. I adopted this plan, as I have occasionally met with cases in which, after 4 pints had been given with immediate good effect, a few hours later the passage of a very copious stool of 2 pints or more was accompanied by extreme collapse, proving fatal before a second transfusion could be performed. Slow, continued transfusion can also be carried out in the same manner, and I have kept it up for several hours in desperate cases, although without much success.

Dr. Cox, in Shanghai, has used continued intravenous transfusion of normal saline by means of an ingenious self-regulating apparatus. He has thus given as much as 17 pints at one time, and in one instance $2 S$ pints, with ultimate recovery, the fluid passing out by the bowel as fast as it ran into the veins. He has reported favorable results in Chinese patients. With hypertonic solutions I have not found it necessary to give anything like such heroic quantities, the administration of which requires more continuous skilled attendance and elaborate apparatus than is usually likely to be available in cholera outbreaks in tropical countries. Dr. Cox ruus in the fluid at a temperature of $103^{\circ}$ to $104^{\circ} \mathrm{F}$., and also applies warmth externally.

Nichols and Andrews, in the Philippine Islands, have used intravenous injections of normal saline solutions (0.5) per cent. sodium chloride), with a total mortality of 43.6 per cent., which was 25 per cent. lower than with subcutaneous injections. The average number of intrivenous injections in cases recorering was 1.S, and in fatal ones 2.6. 
This is much higher than with my hypertonic solutions, with which second injections were comparatively seldom required. Nearly half of those cases which survived collapse developed uremic symptoms, and of these, two-thirds proved fatal.

M. A. Vassilieva, working in Russia during 1909, used intravenous injections of normal saline solution in 129 cholera cases, of which only 50 recovered, or 38.7 per cent. With hypertonic salines during the last ten months I have had 68 per cent. of recoveries among 62 cases.

Repetition of Intravenous Injections.-In a great majority of cases a single hypertonic transfusion suffices to tide over the collapse stage, although such a favorable result was rare with normal salines. A repetition of the hypertonic saline was only given in 28.82 per cent. of my cases. Should collapse recur after the former, the gravity of the prognosis is greatly increased. Such cases usually terminated fatally up to the time I conmenced the plan, to be presently described, of destroying the toxins in the bowel. Since then a much larger proportion of double and even triple intravenous injections have been successful in saving life, while in one recovery followed a fourth transfusion. The indications for a repetition of the process are the same as for the first injection, the hypertonic solution being again used if the watery diarrhœa continues. If the rectal temperature is above normal, the saline may safely be given at even a slightly subnormal heat, such as $96^{\circ} \mathrm{F}$.

Fluid by the Mouth.-Extreme thirst is one of the most distressing symptoms of cholera. Drinking fluid often excites vomiting, but the sickness of cholera is mainly due to the evacuation of watery fluid excreted by the gastric mucous membrane, and probably removes some toxins from the system. As there is seldom much retching, any exhaustion resulting is due rather to the amount of fluid lost than to the efforts of vomiting. It is useless to give a large amount of fluid by the mouth at one time, as it is sure to be rapidly expelled; but by administering an ounce or two at short intervals, it is surprising how much will be retained and absorbed-much to the bencfit of the patient. If collapse is marked, and especially if the rectal temperature is low, it is not advisable to give ice to suck, but in the contrary conditions it is often of value in allaying the irritability of the stomach. I have tried weak saline and acid solutions as drinks, but have not seen any definite benefit from them, while they have the disadvantage of reducing the amount of fluid taken, owing to the patient objecting to their taste. I, therefore, prefer plain water, with the addition of toxin-destroying substances, to be mentioned later.

\section{Medicinal Treatment in the Stage of Copious Evacuations and} Collapse.-So various are the remedies that have been extolled as of great value in the acute stages of cholera, that it is safe to conclude that few or none of them have any specific action in the disease. Their sponsors have too often been misled by the apparent success of a new drug in the later mild terminal cases of an epidemic, forgetting that at the end of an outbreak the mortality will often be but about half of that of the earlier cases, even when all have been treated in the same manner. To obtain reliable data regarding any remedy in cholera, either every other case must be 
treated by it through a fairly long series, or it must be used for many months in an endenic area, where the ordinary mortality of each season is known. Moreover, the drug treatment must be combined with saline injections, preferably of hypertonic solutions, if marked collapse is present, for unless the circulation is first restored, the drug will have little or no chance of exerting any beneficial action. As earlier recommendations have largely lacked the desired scientific control, I shall first give the conelusions I have arrived at as a result of several years' careful observation under very favorable conditions in Calcutta hospitals.

Should Opium or Morphine Be Given in Fully Developed Cholera?-'The belief of the older Anglo-Indian writers in the value of opium in cholera is striking. As late as 1893 Wall advised, in the stage of evacuations, before complete collapse had set in, that 12 minims of the tincture of opium and 10 minims of dilute sulphurie acid should be given and repeated if necessiry after two hours. If vomiting was excessive, he gave the opium subcutaneously. All writers are agreed that during collapse opium should not be given. If the drug is of value in the stage of evacuations, it should also do good in checking the diarrhoea and tendeney to recurrence of collapse once that condition has been removed by copious saline injections. In order to test this, I gave a hypodermic injeetion of morphine or opium, either before collapse had set in or after it had been relieved by intravenous salines, to every other patient of two series, at different seasons of the year, amounting to 56 cases. The mortality among those who received the narcotic drug was 39.3 per eent., while among an equal number treated without any opium or morphine the death-rate was only 2i) per cent. Moreover, no less than one-fourth of the total cases treated with this drug died with uremic symptoms, against 3.57 , or one-seventh of the former number, among the non-opium cases. Nor did the drug appear to have any effect in checking the evaeuations and tendency to the recurrence of collapse. I am, therefore, convinced that opium in all its forms is absolutely injurious in cholera once typical colorless evacuations have set in, and I never allow it to be given in my wards.

Acids.-Dilute hydrochloric acid in the form of a weak drink was freely given to every other patient in a series of cases, but was abandoned, as the results were distinctly unfavorable. This treatment also failed in the Hamburg outbreak in 1892. Dilute acetic acid has been strongl! recommended by Davidson and others, but I have very little experience with it, and am not disposed to recommend it after the failure in my hands of dilute mineral acids.

Intestinal Antiseptics.-From a very early date large doses of calomel were greatly used in cholera in India, while, since the discovery of the comma bacillus by Koch in 1SS3, inmmerable chemicals have been recommended to destroy or limit the growth of the organism in the intestine. None of them have come into general use, nor do any appear to be an improvement on calomel itself. Goodeve considered calomel to be at least harmless, but Wall condemned it, together with castor oil, as positively dangerous. 'These writers referred to large doses (20 to 
30 grains) usually given in their day. Calomel was given in $\frac{1}{2}$-grain doses, with an equal quantity of camphor, every quarter of an hour for from four to eight doses. This was the routine treatment both before and during 1908 in Calcutta, when the mortality was so greatly reduced by hypertonic salines. It is well known that the appearance of bile in the stools is a favorable sign in cholera; so that small doses of calomel are theoretically correct, and, I believe, are of some service, although large amounts are dangerous. Salol, in 5-grain doses, appears to me to have been of use, although I am now using bismuth salicylate in 10-grain doses as a more active antiseptic of the same class. During the early part of 1909 eucalyptus oil was tried, but the results were not quite so favorable as with small doses of calomel. Perchloride of mercury has also been recommended, but its irritant qualities are disadvantageous in cholera. In short, I do not think there is any clear evidence in favor of the curative properties of any of the antiseptic drugs hitherto used in cholera.

The Destruction of Toxins in the Bowel as a Curative Treatment of Cholera.-The failure of intestinal antiseptics to reduce the mortality of cholera may be partly explained by the following considerations. We know that the toxins of cholera are mainly, if not entirely, of an intracellular nature. Any antiseptic drug which kills the comma bacilli within the bowel may cause them to break up and dissolve in the alkaline contents, and so lead to rapid absorption of the toxins with aggravation of the symptoms. For this reason, a more rational treatment for cholera would be to attempt to destroy the toxins, rather than the bacilli, in the intestinal canal. A little reflection will show that this may not prove as difficult a task as might at first sight appear. In cholera the bowel is so thoroughly cleared out of fecal matter that any substance having the power of destroying the toxins may readily come into intimate contact with the poisons formed there, as the comma bacillus penetrates but little into the mucous membrane, and the toxins must be absorbed mainly from the interior of the digestive tube. Again, bacterial poisons belong, for the most part, to the class of albumoses and other unstable forms of albumin, while the quantity absorbed from the intestine must be very small in actual weight, on account of its extreme toxicity. As I showed some years ago, the exceedingly toxic albumoses which constitute the active principles of snake venoms are all readily rendered inert by about their own weight of such an oxidizing agent as permanganate of potash. This drug can be readily given in large amounts in such a form as to dissolve only on reaching the small intestine. It can also be given as a weak solution by mouth, which will destroy any toxin excreted in the watery evacuations from the mucous membrane of the stcmach, much in the same way as it acts as an antidote in morphine poisoning, while some of it will doubtless pass on into the small intestine. Since I have adopted this plan of treatment, in addition to the hypertonic saline injections, I have obtained markedly better results than in previous years, as shown in the last line of 'Table II. Thus, during the ten months, from August, 1909, to May, 1910, inclusive, there have been only 22 deaths 
among 91 cases treated, or a mortality of 24.2 per cent. During the same months of eleven years, 1895-190;), before hypertonic transfusions were used, the mortality was just 60 per cent., giving a two and a half fold reduction in the death-rate by the present treatment. A still better test of the value of the administration of permanganates hy the mouth in cholera is afforded by comparing the mortality of the severe cases requirung intravenous transfusion before and after the use of this remerly. 'Thus, with hypertonic salines alone, out of 170 cases treated by intravenous injections the death-rate was 80 , or 47 per cent. Since the addition of oxidizing agents by the mouth, 62 hypertonic transfusions have been done, with only 20 deaths, or 32 per cent., leaving 68 per cent. of recoveries. When it is remembered that extremely few of these patients could have recovered without transfusion, these results are remarkably good. The severity of the disease may be gathered from the fact that in 37 per cent. of the total admissions during the ten months of the new treatment there was either no pulse to be felt at the wrist, or too small a one to allow the blood-pressure to be estimated; that is, these patients were almost moribund. Yet no less than 56 per cent. of these extreme cases were saved. Again, the recent further improvement in the results is certainly not due to the disease being of a milder type, for no less than 68 per cent. of the admissions required transfusion-a higher proportion than in any previous period.

Another marked feature of the permanganate treatment is the rapidity with which the character of the stools completely changes from very copious rice-water ones to small green-colored, but still fluid, evacuations. At the same time the number of comma bacilli in them enormously decreases. These changes have frequently occurred within twelve hours of admission, and almost always, within twenty-four hours, the general condition and blood-pressure improving markedly at the same time. In short, after very careful and prolonged trial of the permanganates, I am convinced that they do destroy a large amount of the toxins within the bowel in cholera, and have a definite specific effect in reducing the severity of the attacks.

The simplest way of giving permanganates is by mouth, in solution. Owing to the intense thirst in cholera, it is frequently possible to get patients to drink as much as 4 or more grains to the pint, which makes a very dark solution. I have known as much as 12 grains to the pint to be taken hy a European, suffering from a very severe attack, and rapid recovery ensue. As a rule, it has to be diluted much further before it will readily be swallowed. The potassium salt may be used for this purpose, but I much prefer calcium permanganate, as it is less astringent to the taste, while it is divalent, and so gives off more oxygen. The patient is encouraged to drink as much as he can of the solution, and it may be given in the place of small quantities of water to lessen the tendency to sickness. In addition, the permanganate salt is given in pill form as follows:

Potassium permanganate, हr. 2:

Kaolin and vaseline, q. 8 .

VoL. I -54 
Make a pill and coat with melted salol or with keratin, so that it will not dissolve until it reaches the intestine, where its action is desired. The pills are made up as small as possible, so as to readily pass through the pylorus. They are rarely vomited. One pill is given every quarter of an hour for the first hour, and then every half-hour for three hours more, making a total of 20 grains of potassium permanganate in the first four hours, in addition to the quantity given in drinks. The pills are then omitted for four hours, and barley water given, if thought necessary, to keep up the patient's strength. I have lately also given 20 grains of bismuth salicylate as an antiseptic in 5-grain eachets during this second four-hourly period. From the ninth to the twelfth hour one permanganate pill is again given every half-hour, making 16 grains of the salt in the four hours, after which another pause of the same time is allowed. This procedure is continued until the stools have changed color and become much less in quantity, which is usually the case within twenty-four hours. At the beginning of the second day, 16 grains more of the permanganate salt is administered to guard against a relapse. When the acute stage is over, the blood-pressure improves and the thirst is greatly diminished. If the patient objects to the permanganate drinks, it is advisable to substitute water, as it is important to get as much fluid absorbed by the stomach as possible in this stage. Calcium and sodium permanganate are so extremely deliquescent that it is difficult to make them up in pill form in the tropics. I have also tried another oxidizing agent in similar doses, namely, sodium persulphate, both in solution and pill form, but it is also difficult to make up in the latter form in the hot season, while it is more irritating to the stomach than permanganates. Moreover, the persulphate has much less effect in destroying cholera toxins than permanganates. For example, three-fourths of a culture-tube of dead cholera bacilli mixed with 3 milligrams of calcium permanganate could be safely injected intravenously into a pigeon, although one-third of that amount without the salt proved fatal in a few hours, showing that the intracellular toxins are rendered inert by permanganate salts, and furnishing a scientifie basis for my plan of treatment. These tests, taken with the results already mentioned, justify me in recommending this method of dealing with cholera in addition to the hypertonic transfusions, especially as good reports of the combination have reached me from several medical men in India who have recently tried it.

The success of such an oxidizing agent, given by the mouth in cholera, suggests a trial of oxygen inhalations in severe cases, with a view to destroying the toxins already absorbed into the blood. Owing to the unfortunate miscarriage of an order for the necessary apparatus from England, I have not yet been able to put this idea to the test, but it is worthy of remembrance.

Stage of Reaction.-When the copious colorless evacuations are replaced by smaller bile-containing stools, the pulse begins to slowly regain its strength and the surface warmth reappears: the stage of reaction is entered on. This was regarded by the older Anglo-Indian writers as being quite as dangerous as the collapse stage. Apart from the less 
frequent complications, there are two great causes of fatalities at this period; namely, excessive febrile reaction and continued suppression of urine, and later uremia. The latter is the more frequent of the two, and will be dealt with later.

Excessive Febrile Reaction.-As Norman Chevers pointed out, cholera is really a fever in which the rise of temperature is masked by the deficiency of surface circulation. 'The frequency of a high rectal temperature, even in collapse, and the febrile rise when the circulation revives, bears out his statement. 'This reaction is not rarely excessive, producing either a typhoid-like state, or only too frequently actual hyperpyrexia, especially in the hot season. 'This may occur quite apart from the use of any saline injections. 'Thus, at the Calcutta European Hospital, fatal hyperpyrexia caused 10 per cent. of the deaths during recent years when saline injections of any kind were rarely given. Indeed, every patient whose temperature rose to $103^{\circ} \mathrm{F}$. or over, apart from any transfusion, died in the reaction stage. 'This very serious reactive fever is doubtless due to absorption of the cholera toxins from the bowel with the revival of the circulation. In this connection it is worthy of note that deaths from this cause have become less frequent since my plan of using permanganates to render the toxins in the bowel inert has been usecl. Opium appears to predispose to dangerous reaction, for in the European hospital the drug had been given twice as frequently to those dying in this stage as it had to those who recovered from the disease.

Copious intravenous salines at body heat are almost invariably followed by a marked rise of temperature, commonly accompanied by a rigor, and reaching from $100^{\circ}$ to $103^{\circ} \mathrm{F}$. Such reactions are of a favorable nature, while a rise of the axillary temperature to even $104^{\circ}$ or $105^{\circ}$ F., if of short duration, and not accompanied by delirium or loss of consciousness, does no harm, although alarming at the time. On the other hand, the absence of any reaction at all is of grave import, being most common in elderly patients with low vitality. Hyperpyrexia may rarely occur, and, if unconsciousness ensues, it will terminate fatally in spite of the fever being rapidly reduced by ice to the head, cold pack, and iced-water enemata. It is more frequent in European than in native patients, being evidently due to an overwhelming dose of toxins being absorbed from the bowel. I have met with several such cases in a single outbreak of very severe cholera, in which the stools presented a pure culture of Koch's bacillus. The occasional occurrence of this serious complication in such severe attacks, in which recovery could not have taken place without transfusion, is no valid argument against the routine use of this life-saving measure, which has actually reduced the number of deaths in the reaction stage.

The pructical indication is to avoid the use of hot-water bottles and other methods of combating the low surface temperature when transfusion is done and in the reaction stage. I have seen hot-water bottles still surrounding a patient at the time his temperature was rapidly becoming hyperpyrexial during an intravenous injection of saline fluid. Personally, I never allow hot-water bottles to be used in the collapse stage, 
as the rectal temperature is very rarely below normal, except in patients requiring immediate transfusion, which in itself will raise it. 'The deficiency of circulation through the surface and extremities is a conservative process to retain the small amount of blood for the vital centres. To drain it into less important parts is only to increase the collapse and thus to harm the patient.

Do Not Check the Diarrhœa in the Stage of Reaction.-Once the collapse stage is over and the pulse has returned, the natural inclination is to again resort to astringent remedies to finally stop the now greatly diminished diarrhœa. Experience has convinced me that this is absolutely wrong, owing to its leading to a greater absorption of toxins through the damaged intestinal mucous membrane. I, therefore, use no astringent remedies whatever in this stage, but leave the reparatory processes in the bowel entirely to nature. Opium and acetate of lead at this period are absolutely deadly as actively predisposing to fatal suppression of urine. Acids are not so positively injurious, but I have seen fatal, late reactive fever follow their use, as if toxic absorption had been increased. The diarrhœa will cease by itself in good time if the diet is properly regulated. As long as rectal saline injections are continued, fairly frequent but colored watery motions will be passed, but this is of no consequence, and stops when the salt solutions can be safely omitted.

Diet.-I have already mentioned that only barley-water is given in the earlier stages of cholera. This should be continued more freely during reaction. The utmost caution is necessary in adding to the diet. The too early administration of milk, soup, or jellies which contain animal albumins is particularly liable to bring on a relapse, with copious watery stools, and may thus be far more dangerous than a couple of days' starvation. In severe cases they should be entirely withheld for two or three days, and farinaceous foods only given, thin arrowroot being first added. When milk is given it should be in the form of whey, so as to avoid the irritant action of casein curds. - When the diarrhœea has stopped for two or three days, custards and other light solid food may be commenced, and the diet gradually increased with the improvement of the general condition and appetite of the patient. As long as the kidneys are not acting freely, especial caution in giving soups rich in nitrogenous extractives must be exercised. It is surprising how rapidly the digestive tract usually resumes its functions after the terrible upset caused by the cholera. With the above precautions the diet rarely gives much trouble in the after-treatment of the disease.

Medicinal Agents.-Alcohol was nearly universally condemned by the older Anglo-Indian writers as absolutely injurious in cholera. Macnamara earnestly protested against the use of any alcohol in the disease, which he believed, "both theoretically and practically, to be the cause of unnitigated evil." Wall, on the other hand, considered that dilute alcohol in small quantities was beneficial, but in large ones highly injurious. 'The most serious objection to this drug is that its powerful effect in dilating the surface vesscls only increases the collapse, while it is useless to stimulate the heart when the circulatory failure is due 
to deficient fluid in the vessels. I hold strongly that it should not be given as a routine measure, and never in the acite stages. During convalescence it may oceasionally be of service in those who have been accustomed to its regular use.

Ammonia is recommended as a stimulant by many of the older writers. I have found it of great value in the reaction stage, especially if there is any tendency to congestion of the lungs. It mav be given in the form of ammonium carbonate with sal volatile every four hours, five minims of tincture of digitalis, and 10 misins of tincture nucis vomica being added if the blood-pressure is deficient and urinary secretion not free.

Chloroform and amyl mitrite have been recommended for the relief of cramps in cholera, but when this symptom is severc, it is an indication for immediate transfusion, which stops the pain at once by removing the deficient cireulation and oxygenation of the muscles, so I have never had occasion to use these powerful drugs in cholera.

Atropine has been suggested in the stage of collapse by Sir Iauder Brunton, but I have not yet been able to adequately test it. In one patient, admitted with threatened uremia, who had taken an excess of opiates, hypodermics of atropine certainly did much good.

Serum Therapy.-A great deal of research work has been done in European laboratories in the preparation of anticholeraic serums, and these have been shown experimentally to have distinct anti-endotoxic action. In practice they have not yet been proved to be very efficacious, although some apparent reduction in the mortality of cholera has been reported in a few small series of cases. 'Thus Berdnikoff, using Schurupow's serum, had a death-rate of 59 per cent. of 44 cases treated with the serum, against 72 per cent. of deaths in 25 controls. From 30 to 50 cubic centimeters of serum were injected, either intravenously or subcutaneously. It is hardly to be expected that this treatment by itself conid be of very much value in the severer forms of cholera with marked collapse, but the serum treatment is well worthy of further trial, in combination with hypertonic saline injections, to determine if a still further reduction of the mortality over that obtained by hypertonic salines alone can be obtained. It should be used in every other ('ase in which transfusion is necessary, so that an equal number of control cases will be available to allow of correct deductions as to its value being drawn.

Measures to Insure Free Action of the Kidneys After Cholera.By far the most important part of the treatment of the later stages of cholera is concerned with the reëstablishment of the renal secretion, which is entirely in abeyance during collapse of any severity. Unless this takes place within a very few days, death is inevitable from a combination of toxemia and uremia. There are two classes of cases in which this serious complication is most commonly met with. First, very severe attacks, which have with difficulty been tided over the collapse stage by copious transfusions, and, second, much milder ones, in patients who are usually only brought to the hospital with commencing uremia about the third day after the onset of the disease. It has long been held that the 
continued suppression of urine after cholera is due to the secreting epithelium having been irretrievably damaged by the toxins of the disease. $\mathrm{My}$ observations, however, indicate that this is rarely the case, the trouble being nearly always solely dependent on deficient blood-pressure. This will be clear from the simple statement that during 1909 no patient whose blood-pressure was raised to over 105 died with uremic symptoms, while every patient surviving collapse, but whose blood-pressure remained permanently below 95 to 100 millimeters of $\mathrm{Hg}$., did succumb to this complication. Recently cases of fatal uremia have very occasionally been met with a higher blood-pressure, but when a postmortem was obtained, either old-standing organic disease of the kidney, suclı as a greater or less degree of granular change or tight stricture of the urethra with back-pressure, was almost always found. Moreover, I have carried out a number of experiments to ascertain what pressure was necessary to obtain free perfusion of normal saline solution through the renal circulation postmortem. They showed that in fatal post-choleraic uremia a pressure of 80 to 100 millimeters of $\mathrm{Hg}$. pressure was required for this purpose, against one of 20 to 30 millimeters in the kidneys of subjects dying of such diseases as pneumonia, etc. This márked obstruction to the renal circulation after cholera appeared to be usually due to extreme congestion and often hemorrhages into the substance of the organ, and I have observed that uremia is most frequent in cases in which the stools had been hemorrhagic in character in the acute stage. The pressure required for perfusion postmortem could usually be reduced by 10 to 20 millimeters by peeling off the tense capsule. Enough has been said to show the imperative necessity of daily estimations of the blood-pressure in the after-treatment of cholera, and the adoption of every possible means to raise it to the point necessary to insure the free action of the kidneys. Since this has been done, the mortality from uremia has been greatly reduced, and a number of cases with prolonged, complete or nearly complete, suppession of urine have been saved. These measures were adopted in 1909, during which the proportion of deaths from uremia to total admissions fell from 13 to 7 per cent., in spite of many severe cases of cholera being tided over collapse by hypertonic transfusions to face the dangers of continued suppression of urine. The following are the measures which have been successfully used for preventing and treating post-choleraic uremia. In the Philippine Islands 28 per cent. of the patients who survived collapse died of uremia. My corresponding figure is 16 per cent., so it is clear that hypertonic transfusion does not predispose to uremia.

Flushing the System with Fluid.-As already mentioned, copious intravenous saline injections are often followed by the immediate secretion of considerable quantities of urine-clear proof that damage to the renal epithelium is not the cause of absence of secretion during collapse. If the blood-pressure remains at about 100 millimeters after the copious evacuations have ceased, there is usually no difficulty in getting sufficient fluid into the system to flush the kidneys and rapidly remove all signs of toxemia. For this purpose, drinks of small quantities of fluid at a time 
should assiduously be pressed on the patient. In addition, from half to one pint of normal saline solution should be given by the rectum every two to four hours until some 40 ounces of urine have been passed within twenty-four hours. If the urinary secretion remains deficient, the bloodpressure and the specific gravity of the blood should be estimated. If the blood-pressure is low, such as from 80 to 90 millimeters, and the specific gravity be above the normal, such as 1060 or over, it is clear that there is still a dangerous deficiency of fluid in the circulation. One or two pints of normal saline solution should, therefore, be given, either subcutaneously or by the slow intravenous method, in accordance with the degree of deficiency. Several critical cases have recently been saved by these measures, the renal secretion having been restored within a few hours after having been in nearly or complete abeyance for two or more days. If, however, the specific gravity of the blood is already below the normal, it will not be safe to inject saline solutions for fear of inducing œdema of the lungs. Reliance must now be placed on the following two classes of remedies to raise the blood-pressure to the necessary point.

Cardiac tonics should be given to increase the foree of the heart. For this purpose I first give digitalin in $\frac{1}{10}$-grain doses morning and evening, the tincture being omitted from the ammonia mixture if that is also being taken. Caffeine may be given hypodermically instead of

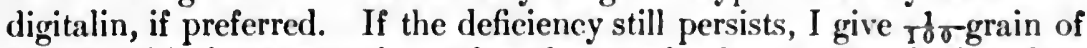
strophanthin intravenously not less than twelve hours after the last dose of digitalin, and have seen it followed by freer action of the kidneys and ultimate recovery in several serious cases. It has the advantage of dilating the renal vessels in addition to stimulating the heart.

$V$ asoconstrictor drugs, such as adrenalin and pituitary extract, are also of great value. The former may be given in the rectal injections and hypodermically in 10- to 20-minim doses of the 1 to 1000 solution, every four to six hours. Intravenously in very dilute form (1 to $100,000)$ it may also be useful in flushing the kidney, but the danger of the sudden high pressure thus induced on the greatly enfeebled heart necessitates great caution in this mode of administration. By the use of these various drugs I was able to raise the blood-pressure of a patient, admitted very late with extremely labored breathing of advanced postcholeraic uremia, from 84 to 105 , when urine once more appeared, after three days' complete suppression. On the following day the pressure reached 115 , and 40 ounces of urine were passed, followed by 150 in the succeeding twenty-four hours, with complete recovery. In short, I regard the discovery of the relationship between deficient blood-pressure and post-choleraic uremia as only second in importance to the use of hypertonic salines in the earlier stages of the disease.

Dry-cupping over the kidneys is also carried out twice daily to relieve the congestion, three cups being placed over each. I have also tried wetcupping for uremia, but without success, and consider that the disadvantage of the wounds outweighs any possible benefit derived from this severer measure.

Hot-air baths have not proved of much value in this condition in my 
experience. Where the blood-pressure is already too low, they may do harm by decreasing it further. I now only use them in the exceptional cases in which uremic symptoms persist with a high blood-pressure, when they may help to prolong life and so give time for other remedies to act.

In one case of continued suppression, with a blood-pressure of 105 millimeters, in a woman, I got a surgeon to separate the capsule of one kidney and incise the cortex. 'The patient stood the operation well, but it failed to cause the organ to resume its functions. On examining the kidneys postmortem they were found to present early granular changes microseopically, which accounted for the failure of the operation, which may be worthy of further consideration in this condition.

I have also punctured the kidney through the lumbar region with a large hypodermic syringe to relieve the congestion, but without nuch apparent benefit.

Complications.-Low Inflammatory Sequels.-The severest forms of cholera are liable to be followed during the stage of reaction by congestion or inflammation going on to sloughing. 'These affect especially the cornea, the parotid gland, producing abscess, and, more rarely, gangrene of the scrotum. The eyes require careful cleansing and protection if asthenia is prolonged after cholera, while the other complications mentioned must be treated on ordinary surgical principles, powerful antiseptic applications being avoided.

Pneumonia and bronchitis are also frequent and dangerous, complications, especially in the colder seasons and climates. This complication was met with in no less than 41 per cent. of cases in the Hamburg outbreak in 1892. Good ventilation, combined with protection from cold draughts, form the most important preventive measures, while full doses of ammonium carbonate have been most useful in my hands in its treatment. In these late complications alcohol is also permissible.

Dysentery occasionally follows cholera in tropical countries, and, although a grave complication, I have found it to commonly yield to ordinary antidysenteric treatment, which was also Wall's experience.

Asthenia may be grave and prolonged in old people, and requires a simple nourishing diet and stimulants for ịts treatment.

Convalescence.-In all but the most severe forms of cholera recovery is usually remarkably rapid in fairly young and otherwise healthy subjects. Complete rest in bed is, however, necessary for some days, owing to the strain the disease puts on the heart. Once the diarrhœa has completely ceased, the bowels often remain unopened for two or three days, but commence to act naturally again as the diet is gradually increased, so that it is rarely necessary to interfere with them. A simple enema or a dose of castor oil may occasionally be required. The patient's stools should continue to be disinfected as long as he remains under medical care, as the comma bacillus has not infrequently been found for some days, or more rarely for-some weeks, after recovery from the disease. Although rapid recovery is the rule, still occasionally debility and lassitude may remain for a long time, necessitating a 
prolonged change to a cooler climate if the patient has been attacked in the tropics.

Summary.-In view of the length of this article, it may be well to recapitulate the main points in the treatment of a cholera patient from the time of his admission. In the first place, the blood-pressure and specific gravity of the blood should be estimated and the axillary and rectal temperature taken. If the blood-pressure is 70 millimeters of $\mathrm{Hg}$. or over, the patient quiet, and the general condition good, rectal saline injections may be tried, a careful watch being kept on the pulse, so that any further fall may at once be detected and more active measures adopted. If the specific gravity is very high, such as about 1070 , transfusion should not be delayed. If the blool-pressure on admission or later falls below 70 , and especially if the patient is restless, cranoserl, and suffering from severe cramps, no time should be lost in giving an intravenous injection, the hypertonic Ringer solution being used. Sufficient should be injected to obtain not only a fair, but a strong full, pressure-pulse, so as to at once restore the urinary secretion. Four pints are commonly necessary in a severe case in an adult male, and proportionally less in women and children. It should be given at body heat unless the rectal temperature is below $99^{\circ} \mathrm{F}$., when the temperature of the solution should be a few degrees higher until the surface temperature is restored. A careful watch should be kept on the temperature reaction which always follows intravenous salines, and the necessary measures taken to prevent an excessive rise occurring. In the acute stage nothing should be given by the mouth except the permanganate, drinks and pills, and some antiseptic, such as bismuth salicrlate. If the acute stage is prolonged, barley-water may also be necessary. If collapse recurs, the hypertonic injection must be repeated, being given at a slower rate if the specific gravity of the blood is not much raised, and at a subnormal temperature if there is fever present. If a second hypertonic injection is required, the prognosis becomes much graver, although a majority of even such cases may be saved.

Once the stage of copious evacuations and collapse is past, the allimportant indication is to dilute the blood to at least the normal point, and to raise the blood-pressure high enough to restore the secretory activity of the kidneys, and so insure the excretion of the toxins and the prevention of uremic complications. If the specific gravity of the blood remains above normal, and is not rapidly reduced by continuing the salines by the bowel and water by the mouth, a further smaller subcutaneous or slow intravenous injection of normal saline solution is indicated. In addition, cardiac tonics and vasoconstricting drugs are of great service.

Great caution is necessary in adding to the diet during early convalescence, animal albumins being especially liable to induce a relapse, while starvation for two or three days is less injurious in otherwise healthy subjects whose age is not at either of the extremes of the scale. 



\section{PLAGUE}

\section{By THOMAS WRIGHT JACKSON, M.D.}

Prophylaxis.-At present the most promising and the most rationally based phase of treatment is that of prevention. 'The reason for this is plainly apparent. If the conclusions of the Plague Commissioners and students of epidemiology the world over are correct, to eradicate plague we need only to control its carriers. 'To exterminate the rat (and perhaps the marmot and ground-squirrel), to prevent the transportation of rats or of infected rat-fleas in ships, trains, elothing, merchandise, and upon the bodies of man and animals, from the numerous foci or plague-centres of the world to non-infected localities, is a beautiful plan indeed.

Restricted to single communities, even where the intelligence, patriotism, effort, and wealth of the whole people are enlisted, the undertaking is formidable with obstacles to its execution, and discouragement must often be expected. Extended in its application to the whole plagueinfected world, it becomes an undertaking seemingly impossible of accomplishment.

Yet we are encouraged to face the situation by a glance at what has been accomplished. 'The United States, perhaps, presents the highest example of achievement in the case of San Francisco. The work is too recent and has been too well published to require detailed review here. A successful campaign against rats in 1907 practically terminated an epidemic of considerable proportions well within a year. Behind this movement, however, were the powerful machinery of the Federal Government, money in generous amount, and a considerably aroused public, resentful of the gross mismanagement of the 1903 epidemic by the California state government, whereby, through pure fear of financial loss to commercial interests and by a disgraceful suppression of the truth, California was made, permanently perhaps, one of the world's plague centres.

It has been estimated that the rat population of the world is equal to the human population, and this estimate does not appear to be unreasonable when one considers as indices the destruction of the rodents in cities by the hundreds of thousands, upon single farms by the thousand, and the wonderful procreative powers of the rat.

It is certain that the economic importance of rat destruction upon grounds other than those purely sanitary must be impressed upon the public wherever a rat campaign is to be carried on. 'The absolute inutility of the rat, its enormous destructiveness to crops, to merchandise 
in warehouses and in transit, to poultry, eggs, fruits, and vegetables, to buildings and furniture, and its incendiary habits, causing annual fire losses of considerable magnitude, must be emphasized in season and out of season. Such items as the value of the grain consumed by a single rat per year, as estimated by the experts of the United States Agricultural Department, are convincing arguments in the case. At a daily consumption of 2 ounces, the ration for a full-grown rat, this grain value varies from $\$ .60$ per year, for wheat, to $\$ 2.00$ per year, for oatmeal, for each rat subsisted. Similar data in great variety relating to direct and indirect losses are available for the purpose of making impressive the economic need for rat destruction.

Accumulated experience from various countries and cities shows plainly that there is no single method of rat destruction to be depended upon to the exclusion of all others, and it also shows that without governmental direction and supervision, backed by ample authority and the ability and willingness to expend considerable money, neither single nor combined methods will be successful. Moreover, in the countries where special effort is most needed, there is often distrust on the part of the natives, religious prejudice against the destruction of animal life, and frequently open opposition to the authorities in their efforts to destroy rats. The same superstitions and religious beliefs which prevent the killing of venomous snakes in India, at the annual cost of thousands of human lives, operates against most measures of rat destruction proposed by the government.

The plans and weapons of warfare against rats include the use of poisons, traps, starvation, rat-proof construction of buildings, wharves, bakeries, stables, granaries, etc., the introduction of diseases among the rat population by bacterial viruses, and the conservation of the natural enemies of the rat, such as the cat, the dog, the ferret, the mongoose, and certain wild animals and birds of the woods and fields.

Among the most widely used and most effective poisons is arsenious acid, in the form of paste (mixed with cheese or cornmeal), or placed upon sweets and fruits. Crude phosphorus is largely used in similar pastes. When mixed with glucose, its inflammable properties are said to be lost. Its inflammability is, of course, a serious obstacle to its general use. Strychnine, owing to its bitter taste, is of little value in poisoning rats, and when used is best combined with glucose and 1 per cent. of cyanide of potassium. Soaked wheat, bread, or similar food is then treated with this mixture and placed where rats may eat it. It is said to be eaten readily by ground squirrels witl fatal effect. It is expensive, however, and apt to be taken by domestic fowls. Most rat poisons have the disadvantage of being dangerous to human life, and must be used with caution wherever children and ignorant persons are about.

Trapping has been found to be a very effective method of rat destruction in cities. 'Traps are of several varieties, and are constructed upon various principles. It is desirable to catch the rats alive and uninjured, and for this purpose barrel traps, wire cage traps, and similar devices are placed in the rat highways. 'These highways are readily discovered in 
the cities. Considerable care must be taken to overcome the natural caution of the rat, and this includes judgment in the use of attractive bait, the concealing and smoking of traps after handling, and perhaps the use of some scent, such as the oil of anise, of which rats seem to be fond. As a general rule, bait should differ from the food naturally supplied by the locality. For example, about granaries and stables fresh animal food should be used for bait, while about slaughter houses, meat-markets, fish-markets, and similar places, where animal offal is abundant, the rat should be tempted with vegetable bait.

Where the circumstances will permit, and this is apt to be so for ground-squirrel destruction, the burrows may be filled with some asphyxiating or poisonous gas. In this manner whole families of rodents, and their fleas as well, are destroyed. 'The system is not often applicable in houses, but aboard ships it is found most effective, the holds of ships being flooded with sulphur dioxide, developed by burning sulphur in a special furnace provided with a pumping and piping system for delivering the gas at distant parts of the ship. In empty ships' holds and elsewhere the simple burning of sulphur in open vessels effects the same results, provided sufficient sulphur and a sufficient number of vessels be used, and further provided that the generation and confining of gas be sufficiently prolonged. In San Francisco harbor, where for more than a year nine vessels were disinfected per day, this method was adopted as more effective, speedy, and economical than any other system. It has the disadvantage, in the case of laden ships, of affording some danger of fire.

Carbon bisulphide has recently heen extensively used in California in the burrows of ground-squirrels. Its fumes, being heavier than air, penetrate the burrows and promptly poison or asphyxiate all living animals and fleas. Absorbent material of some kind is saturated with the liquid and placed in the entrance of the burrow, which is then quickly sealed to confine the gas.

It will be seen that, in common with other methods of rat destruction, fumigation has a limited application and a number of serious objections. It is particularly useful aboard ships. The method should never be employed by unskilled persons or those unacquainted with the dangers to human life from noxious or asphyxiating gases.

'The subjects of the starvation of rats and rat-proof construction may be considered together.

Just as the pig in the Philippine Islands and elsewhere in the Orient must give place as a scavenger of human excreta to modern and decent methods of waste disposal, so must the rat, a garbage seavenger the world over, give place to systematic garbage collection and removal, with temporary storage of garbage in covered metal cans (rat proof). Incidentally, it may be mentioned that the effect of such measures upon the prevalence of flies and the transmission of disease by these insects will be very great and very beneficial to the public health.

Food must be kept from rats, and rats must be kept from the food. Perhaps the greatest resorts of rats are the places where cattle are fed, where grain is stored, and where animals are killed. Slaughter houses, 
markets, grocery stores, restaurants, bakeries, wharves, and warehouses must be regulated by ordinances duly enforced. Much can be done with screens of heavy iron wire with a mesh of less than 1 inch. When concrete and metal have displaced wood and plaster as construction materials; when plank sidewalks and refuse piles are no more, and when the catch basins of sewers have been made rat-proof, the subsistence problem for the rat will be greatly increased in difficulty and starvation should then begin to lessen the rat population, at least in the cities. Municipal authorities should take up the matter of rat-proof construction for new buildings and the rat-proofing of old ones by approved alterations. In Manila, Hong Kong, and elsewhere these methods are receiving attention and encouraging reports are recorded, more particularly with regard to the disappearance of plague in districts so treated rather than in the disappearance of rats. This is most important, for if the rat and his fleas are excluded from houses, and, therefore, from intimate association with man (an apparently feasible matter through the ratproof construction of buildings), protection against human plague is in great measure accomplished. In Manila the disappearance and continued absence of human plague seem to date from the introduction of systematic rat-proofing in sections where cases of human plague occurred. It is rather more than a coincidence that these measures were instituted in 1906, and that plague disappeared from Manila in the same year and has not since reappeared. Particularly is this so in view of that city's experience with rat destruction. From 1900 to 1905 , \$15,000 were paid in rat bounties and $\$ 325,000$ were paid for salaries, wages, and expenses in rat-catching, with little appreciable effect upon the number of rats, and without causing the plague entirely to disappear. It must be admitted, however, that practical control of the disease was attained during this period. Rat-proofing of dwelling-houses is less expensive than wholesale rat destruction, and, judging from Manila's experience, is a more effective measure against human plague. In the suppression of the San Francisco epidemic in 1907 rat-proofing was also extensively resorted to.

The expense of rat-proofing has been generally considered as prohibitive, but if the work be confined at first to the vicinity of infected centers, and if it be carried on subsequent to rat destruction in corresponding areas, the expense need not always be prohibitive, at least in American-governed cities. The Manila plan of plotting the city into "plague-infected" areas, corresponding with the capture of plaguediseased rats, and systematically working within geographic boundaries in which rat-plague exists or is likely to spread, as determined by rat captures and examinations of the rats for signs of plague, has proved itself to be a good plan.

To prevent the transportation of rats in ships, trains, and merchandise is an undertaking of difficulty as well as importance.

In the case of vessels it involves an understanding of the manner by which rats gain ingress to the ship and the ways of preventing them from entering. Few facts are better known, perhaps, than the fact that 
all ships harbor rats, but except to the initiated, the extent to which some ships are infested is by no means understood. I have made voyages upon steamships, which upon alternate trips carried forage for animals in the holds, when the conditions were, to say the least, uncomfortable. 'To have one's stateroom taken possession of by rats, his clothing carried away, or to awake with a rat in one's berth are unpleasant experiences, but not uncommon ones. I personally know of the case of a woman prostrated with sea-sickness who was obliged to remain in her berth and see four large rats disport themselves about her room. In another case known to me a rat jumped from the washstand into the berth of a sleeping woman, running across her exposed face and arm. In traveling upon small dirty steamers in the Orient I have often slept on deck, quite as much to avoid the rats and vermin in the staterooms as for better ventilation. In a certain ship in which I traveled some of the ship's officers amused themselves by shooting rats with an air-rifle in the lower decks, quietly hiding themselves in dimly lighted places and shooting the rats as they erossed the lighter spaces. In many ships the rat population far exceeds the human population. In San Francisco 310 rats were destroyed by a single fumigation on a vessel of only 260 tons burden. In Bombay 1300 rats were destroyed at one time upon a single ship, and in London 1700 were secured at one fumigation. 'The ease with which rats adapt themselves to new environment is shown by the fact that they live, when permitted to do so, in cold-storage and refrigerating rooms, where they grow heavy coats of fur for protection against the cold.

They gain ingress to ships in three principal ways: (1) By coming overside upon gang-planks, wharf-stringers, etc.; (2) by passing along the lines by which the ship is made fast to the dock, through hawse-holes, the rat being an expert rope walker; (3) by coming aboard in the cargo. By the latter method rats are often brought aboard by whole families, their fleas included. Many styles of packages, such as barrels, bales, crated goods, grain in sacks, and matting in rolls present the rat with abundant opportunity to take passage, and it is probably thus, as stowaways, that rats go to sen in the largest number. Plainly then the placing of rat-funnels upon all lines from ship to wharf, the use of special fenders, the raising of gang-planks, and even anchorage in the stream will not prevent rats from getting aboard ships unless cargo disinfection be practised before loading the vessel. 'The ship itself should be fumigated every three months if possible.

Rats are doubtless carried in considerable numbers upon railway cars, both freight and passenger cars. While riding in a crowded street-car in Minila I saw a good-sized rat come out of the space between the walls of the car, into which the windows are dropped, run along the window-sill within a foot of a passenger, and disappear in another window space. The same principles which obtain in the ease of ships apply to cars and trains as well. Grain cars in particular should receive special attention.

The proposal to destroy rats by wholesale, by spreading epizootic diseases among them through feeding them bacterial virus, has received 
much attention in the last ten years. In 1900 Danysz isolated a bacillus from field-mice suffering from an epidemic disease communicable to rats, and great hopes were entertained that by means of this method decided reductions in the rat population would result. Indeed, the results in Cape Town, South Africa, in 1901, and in Odessa, Russia, in 1902, seemed to justify the hope to some extent, and certain observers still believe the method to be effective. Experience with the Danysz and other organisms has shown, however, that introduced epidemic diseases do not destroy rats in sufficient number to do much good, and that nearly all the viruses experimented with are more or less unreliable. Most of the organisms are apparently related to the colon, typhoid, or hog-cholera groups. The mouse-typhoid bacillus (Bacillus typhi murium) was originally isolated by Löffler in 1899. The paratyphoid bacillus and Gärtner's Bacillus enteriditidis correspond closely with the Danysz organism, and can scarcely be separated culturally. In rodents they produce enteritis, sometimes hemorrhagic in character, and they are by no means to be regarded as harmless for man, as originally supposed. In Japan, in particular, serious and fatal cases of diarrheal diseases have followed the accidental eating by man of food treated with these bacterial poisons.

On account of the natural resistance of rats to diseases of bacterial causation-plague being the most notable exception to this rule-and the clinical fact that no sufficient death-rate among rodents is produced by feeding them upon bacterial viruses, as well as on account of the dangers to man just mentioned, this method of rat destruction is not in favor at present. Poisoning rats and ground-squirrels by chemical poisons seems to be a preferable method, at least equally effective and without most of the disadvantages of uncertainty and danger which attach to the bacterial viruses.

Concerning the utility of such domestic animals as are natural enemies of the rat, in the warfare against the offending rodents, there is considerable difference of opinion, based upon varying experiences. I leave out of consideration all but the cat and the dog. It will be found that wherever cats and dogs are wcll housed (indoors) and well fed they are apt to be fat, lazy, and inefficient. House cats of this class will catch mice, but will often leave rats alone, but half-wild cats, obliged to forage for their own subsistence, are often excellent rat-catchers. Small, active dogs, particularly of the terrier breeds, will often keep houses practically free from rats, and upon farms they are especially valuable, particularly if the construction of buildings is such as to permit them to get beneath the floors. The employment of these animals will necessarily be confined to individuals for the freeing of individual premises from rats. A fact to be borne in mind is one already cited, viz.: that cats and dogs sometimes harbor the same fleas as the rat. Infected rat-fleas often leave dead rats for other animals, and, all things considered, there are many other objections to the intimate house-dog and house-cat, which find comfortable resting-places impartially upon the beds of adults or the cribs of babies and children. 
Before passing to the treatment of the individual, both protective and active, I would sum up the measures of preventive treatment for the community. There must be-(1) active warfare against rats and other plague-affected rodents and their fleas; (2) quarantine applied to persons, goods, and animals; (3) disinfection of cargoes shipped from infected ports; (4) isolation of the sick and proper disposal of the dead; (5) international notification between governments of the occurrence of plague within their respective territories; (6) lastly, but perhaps first in importance, the early recognition of the presence of plague and rapid diagnosis in individual cases, both dependent upon laboratory workers.

All these measures must be fostered, directed, and aided in every possible way by competent authority (national, if possible), whose officers must be men of great moral courage and of unselfish purpose. Behind all this imust be generous financial support.

Protective Treatment.-A detailed recital of the efforts made to secure a safe, effective, and reliable prophylactic treatment for the non-immune individual would require more space than is here available. Moreover, as these efforts have not yet been crowned with unqualified success, it is not necessary to enter into minute details. I will, therefore, give the briefest possible synoptic review of this work. These efforts date from the proposal to make use of killed cultures of Bacillus pestis by hypodermatic injection in the hope of creating immunity against plague, Haffkine's prophylactic injection being first proposed and practised in India in 1897 . He offered as a prophylactic injection killed bouillon cultures of pest bacilli. As used by him at present, the preparation is practically as originally proposed. The bouillon cultures are so treated with phenol as to represent a 0.5 per cent. carbolic solution. The dose injected varies from 0.5 to $1 \mathrm{cc}$., and in special cases as much as $20 \mathrm{cc}$, and one or two repetitions of the injection are advised. 'This prophylactic received the approval of the India Plague Commission.

Gaffky, Pfeiffer, and others varied the method by using killed agar cultures of Bacillus pestis, the dose being one agar culture of forty-eight hours' growth. Virulent pest bacilli are grown on agar slants, the growth being then suspended in saline solution. The death of the bacilli is accomplished by heat, and similar carbolization to that used in the Haffkine prophylactic is practised to preserve the product. 'This preparation was adopted by the German Plague Commission in 1599 .

In 1897 Galeotti and Lustig, of Florence, prepared a chemically extracted nucleoproteid from agar cultures of Bacillus pestis. It was in the form of a powder, and was administered hypodermatically in a 0.5 per cent. solution of sodium carbonate, the dose of the nucleoproteid being 3 milligrams.

Terni and Bandi (1900) utilized the peritoneal exudate from guineapigs injected intra-abdominally with Bacillus pestis. Just before death from plague the animal is killed, and the exudate so sterilized that the serum-albumins are not coagulated. It is preserved by a mixture of carbolic acid, sodium chloride, and sodium carbonate, the dose of the injection being 1.5 to $2.5 \mathrm{cc}$.

VoL. I -55 
Shiga's prophylactic, proposed in 1900, consisted of an agar culture of Bacillus pestis suspended in a saline solution. The cultures used were of three days' growth and 3 oesen of organisms were thoroughly mixed with 3 ec. of salt solution. Heat was applied to destroy the pest bacilli, and preservation was effected by making a 0.5 per cent. carbolic solution. Equal parts of this preparation and of pest-immune serum were used for injection. Two injections were advised, the first to consist of 0.6 to $1 \mathrm{cc}$. of the mixture. 'The second, to be given after reaction lad disappeared, was to be administered without the serum, the saline suspension of bacilli being given alone in the same dose.

Besredka's preparation consisted of an agglutination of killed pest bacilli precipitated from a saline solution by the addition of a plague-immune serum. The precipitated bacteria were washed free from serum and were then used for injection.

Gosio (1905) proposed a variation of this method, whereby large amounts of plague prophylactic could be obtained.

Klein (1906) prepared a dried extract of the organs of plague-affected guinea-pigs dead of subacute plague. The organs were cut into minute parts and dried in layers on glass over sulphuric acid at $46^{\circ} \mathrm{C}$. After three days the dried particles were powdered and bottled. From 5 to 7 milligrams of the powder are injected in emulsion in warm distilled water. This virus for inoculation certainly does not answer to the necessary condition prescribed for a prophylactic, viz., that it shall be harmless. On the contrary, while it apparently produced decided immunity in a certain percentage of animals injected, it also produced death in a large percentage, especially when the extract was derived from acute cases of guinea-pig plague.

Thus it will be seen that most of the proposed prophylactics for human plague are derived from cultures of Bacillus pestis grown either in bouillon or upon agar, killed by heat, and injected into the human body in suspensions in various solutions and in some cases in mixture with the serum of a plague-immune animal. In a few cases the preparations are extracts from the organs of plague-diseased animals and from peritoneal exudates caused by the intra-abdominal injection of pest bacilli into guinea-pigs. These preparations manifestly contain, besides the bodies and toxins of pest bacilli, other complex substances.

It is proper to state that not all of the proposed prophylactics of this class have actually been used on man, most immunity experiments having been made with animals susceptible to plague.

Applying as a test of usefulness the conditions that a virus for inoculation must be harmless, reasonably certain in action, and of definite protective value, it is doubtful whether any of the preparations described above will survive. Indeed, most of them have already been passed upon unfavorably. It is true that the Haffkine prophylactic is credited with the endorsement of the British Plague Commission, which finds(1) That inoculation with this preparation lessens the incidence of plague in the inoculated population, but that the protection is not absolute; (2) that it greatly diminishes the death-rate among the inoculated popula- 
tion, both from lessened rate of attack and from lessened fatality of attacks; (3) that no great degree of protection is conferred by incculation within the first few days after the operation. Nevertheless, satisfactory statistical confirmation of the effects of Haffkine's prophylactic is lacking, or at least but half-convincing. 'Tables usually apply to limited districts, and do not indicate the fact that on account of the unwillingness of the population the inoculation is rarely general. It is not to be disputed, however, that when given in large doses and frequently repeated, Haffkine's prophylactic does protect certain individuals from plague, at least temporarily.

On account of a most unfortunate accident in India in 1902, when the population of an entire province was about to receive inoculation (over $5,000,000$ persons), 18 inoculated persons died from tetanus, infection occurring through impure plague prophylactic, accidentally contaminated with the tetanus bacillus. 'This caused the abandonment of the plan which, if carried out, might have definitely decided the value or worthlessness of the method. It also caused the method and its originator to lose prestige seriously in the eyes of the people who were already apathetic toward the movement, and even actively antagonistic to it in certain cases.

A similar fatal accident occurred in Manila, in November, 1906, when 24 persons who were to be inoculated with a prophylactic against cholera accidentally received cultures of Bacillus pestis instead. Of the whole number, 14 died. As the injections were made by a prominent worker at the Bureau of Science, where immunity experiments were being carried on, it caused pronounced public opposition to further experimentation of this kind, not to mention threats of prosecution for criminal carelessness, averted only by a report from an official investigation commission.

These two most unfortunate occurrences, both of which took place during investigations conducted under the auspices of the British and American governments, respectively, practically put an end, for the time being, to human immunity experiments with killed cultures and with attenuated living cultures of Bacillus pestis.

Of the latter method of producing immunity, viz., with attenuated living cultures of Bacillus pestis, Strong, who has made a most thorough study of plague immunity in man and animals, including monkers, has a very high opinion. He has recommended its use in the Philippines, but largely on account of the unfortunate loss of 14 lives in Manila, a result attributable to pure accident and in no sense connected with plague immunity work, little has been done or is at present likely to be done publicly along this line of work, which is perhaps that of greatest promise in the search for a true prophylactic. Fortunately, plague has disappeared from Manila.

Strong, in 1905, was the first to "vaccinate" man with attenuated live pest bacilli. In this connection he uses the term raccination as indicating immunization with living attenuated organisms, and not with dead bacteria or their extracts. 
As a result of extended study of the subject and experiments upon both animals and man, Strong concludes that, while the method is not infallible, and while very brilliant results are not always to be expected, a certain proportion of the population may be immunized and an appreciable degree of immunity may be retained for at least nine months. $\mathrm{He}$ also believes that in plague-stricken districts the whole population may be gradually immunized by this "vaccination," which, he maintains, is entirely safe.

In the matter of antipest sera, $i$. e., the sera of animals rendered immune to plague by killed or living cultures of pest bacilli administered in ascending doses, there has also been much experimentation, and it has been possible to develop sera (horse sera in particular) with pronounced protective properties, as indicated by their ability to preserve the lives of plague-affected animals, including mice, rats, guinea-pigs, and monkeys. In some of the series of cases as high as 90 per cent. of the animals have survived, but all observers agree that the duration of the infection before antipest serum is administered is of the greatest importance in determining the curability of the disease by serum. In the case of animals, and in the case of man as well (Choksy), it has been found that twenty-four hours is the longest period which may elapse, generally speaking, between the onset of symptoms and the administration of the serum. The hopefulness of successful treatment with serum after the lapse of a day is enormously lessened. The serum should be administered by intravenous injection or by intraperitoneal injection, absorption from the tissues being entirely too uncertain. Manifestly, the potency of the serum and the dosage are of great importance.

Those who have had actual experience in treatment agree that early injection, within a few hours after the onset of symptoms if possible, repetition within a few hours, and a dosage of $100 \mathrm{cc}$. per injection give the greatest promise of success. It is denied by some that the curative properties of antipest serum are due to antitoxins, or, at least, that marked antitoxic properties are developed. They believe, rather, that its properties are "anti-infectious" and opsonic. At any rate, the prophylactic properties of antipest serum are esteemed higher than its curative properties by some workers. Manson speaks of the practical employment of antipest serum in India as disappointing. Choksy's statistics of 1408 cases treated in Bombay in 1907, however, show a a mortality of 28.9 per cent. of cases treated with serum upon the first day, and of 53.3 per cent. of all cases treated with serum. In view of the normal death-rate of plague, at times reaching 85 or 90 per cent. of all cases, these figures must be considered as favorable to the treatment, and the outlook for serum therapy must still be accounted promising.

The theory of neutralization of plague toxins in the human body by means of the antidotal principles present in the serum of immune animals may have to be abandoned or modified in the light of slight agglutinin and bacteriolysin formation in immune serum, the opsonic action of the serum and, perhaps, a better understanding of anaphylaxis and its influence. 
It is clear, at least, that in our analyses we need to take into account circumstances which at first we entirely overlooked.

Experimentation in the laboratory must continue, for, as Simpson pointed out several years ago, "The serum which shall possess evident and indisputable specific or antidotal powers against plague has still to be discovered."

Supportant and Symptomatic Treatment.-In 1901, in Hong Kong, and again in 1903, the internal administration of large doses of carbolic acid was practised with a mortality of 76.5 per cent. in 1901, and of 34.6 per cent. in 1903 (J. C. 'Thomson). 'The carbolic acid was given in a mixture of orange syrup and peppermint water in doses of 12 grains ( 0.8 gram) every two hours. In one case 2500 grains of carbolic acid were given, with recovery of the patient. 'The drug did not produce poisoning and only oceasionally was carboluria observed. 'The omission of a few doses of carbolic acid caused the urine to become clear, after which the treatment was resumed. In India Moorhead used the drug in similar doses with apparent benefit, his mortality being 65 per cent.

Other treatment of plague cases is comprehended under the terms "supportant" and "symptomatic."

Supportant treatment includes the nursing of the patient, a most important part of the conduct of a case of plague. 'The occupation of a plague nurse is difficult and dangerous, and calls for the exercise of fearlessness and an intelligent appreciation of the danger of infection, the manner of conveyance of the disease, and the need of unceasing watchfulness. The patient must be confined to bed, carefully fed, restrained if necessary, and prevented from expending his strength uselessly. His excreta must be disinfected and disposed of, his buboes must be dressed, and emergeney treatment for collapse or hemorrhage must be applied if called for. 'The isolation and quarantine of the patient should be carefully secured and maintained, and naturally the place for treatment should be within a hospital. The sick-room should be rat-proof and insect-proof. In addition to suitable insect netting a barrier of sticky fly-paper may be used to intercept fleas. The maximum amount of sunlight and fresh air possible to be secured are necessary.

In the light of our present knowledge the dangers of personal contagion may be considered slight to the nurse or medical attendant (assuming that insects are completely exchuded from the sick-room), except in cases of pneumonic plague, in which the sputum is highly infectious. In Bombay a nurse was fatally infected by a particle of plague sputum conghed into her eye by a patient suffering with pneumonic plague. Gotschlich ${ }^{1}$ has shown that pest bacilli capable of killing guinea-pigs can be recovered from the sputum of cases of pneumonic plague as long as forty-eight days after a normal temperature is attained. 'The importance of continued disinfection of the sputum is, therefore, apparent, and in the treatment of plague pneumonia the wearing of

'Medical Diseases of Egypt, Sandwith, 1905, p. 169. 
suitable cheese-cloth masks, kept moist with an antiseptic solution, is desirable for nurse and physician. Slight abrasions of the mucous membrane anywhere about the face invite infection. In the dressing of buboes or other suppurating surfaces the discharges must, of course, be assumed to contain plague bacilli and contact with the skin must be avoided by the use of rubber gloves. Abrasions, "hang-nails," cuts, or other lesions of the skin of the attendant should be sealed up with collodion or other occlusive dressings, and suitable overgarments should be worn. The employment of barefooted native nurses or dressers should, of course, be forbidden.

Nurses and physicians engaged in the care of patients suffering with plague should undergo prophylactic inoculation with Haffkine's or Shiga's prophylactic, or with some other well-tested preparation. It seems probable that in Europeans and Americans, who are less susceptible to plague than the natives of India, the protection afforded will be more pronounced than in susceptible Orientals. Inoculation or "vaccination" with killed or attenuated pest bacilli should be subcutaneous and never directly into the blood current.

It is probable that the practice of "evacuation" in communities where plague is present should be limited to premises where plague cases actually occur, and rat destruction and thorough sulphur fumigation, using 3 pounds of sulphur to 1000 cubic feet of air-space, with rat-proofing of the building, should be practised before reoccupation.

If called upon to administer to plague cases, I should pursue as nearly as possible the following plan of treatment. Assuming that a diagnosis of plague has been made, no time should be lost in administering serum, regardless of the type of plague, a matter often indeterminable in the earliest hours. In view of the distribution of plague throughout the world to-day, the disease is always to be feared and suspected in seaboard towns, where direct communication with infected ports may arise.

It is also constantly to be sought for and suspected in sections where rodent plague infections have been shown to exist, as, for example, in California. An attitude of suspicion toward all cases of fever with adenitis and all atypical fevers resembling typhoid and pneumonia should be preserved, and recourse to laboratory diagnosis should be had in the earliest possible hours of suspicion. Fortunately, direct microscopic examination of aspirated matter from swollen glands, of sputum, direct blood examination in a small percentage of cases, and the cultivation of Bacillus pestis from the diseased tissues or from blood give us early and definite information of plague infection; supplemented by the production of plague in laboratory animals by injected material from the case under suspicion. With the technical laboratory details, however, we have nothing to do in this discussion. The diagnostic procedures should be turned over to the bacteriologist. Laboratories should be prepared, however, to conduct these investigations upon the instant, and with the exercise of due care cases should not be overlooked, except perhaps the very earliest ones occurring in a community. 
The choice of a serum is a matter often beyond the control of the physician. He is obliged to use that which is at hand. In Hong Kong and Manila sera made in Paris were found upon trial to be of little value. Yersin, Calmette, Roux, Borrel, Lustig, Kitasato, and others have elaborated sera, but it is unlikely that all the sera commercially obtainable are standardized and of identical dosage. Other things being equal, the serum of most recent preparation (prepared, if possible, in the United States, if for use here) should be used when required. We have no reason to expect anything but a superior product from the American manufacturers of serum of established reputations. In Manila, at the end of the year 1909, there were on hand, ready for instant use, 2:30 bottles of antipest serum and 1024 of plague prophylactic, although no cases of plague had occurred within two years. It would be interesting to know how well other eities are prepared for an outbreak of plague. State and municipal health departments should constantly have on hand adequate supplies, preferably prepared and tested in their own laboratories.

At the Bombay Medical Congress of 1909 Choksy again reported upon the use of antipest serum, having secured a mortality of 25.3 per cent. for cases treated upon the first day of the discase, the mortality rising with each day of delay in beginning treatment. He recommends subeutaneous injections of $100 \mathrm{cc}$. of serum, three doses to be given within forty-eight hours, the further use of serum depending upon the patient's condition. 'The originators of the serum treatment, however, insist, with reasons that seem to be sound, that the injections should be intravenous.

The serum treatment need not and should not interfere with the symptomatic treatment. At the outset I would administer 5 grains $(0.324$ gm.) of calomel, followed in six hours with a saline aperient. Aside from this initial purgation, I would avoid the use of laxatives. Indeed, it may be necessary to combat diarrhœa, in which case salol, opium, and bismuth, either separately or in combination, should be given. So long as the disease is acute, the diet should be liquid or limited to soft foods requiring little or no digestion. Peptonized milk, egg-albumen, barleywater, and junket may be combined with stimulants according to the special needs of the case. The feeding should be performed every three hours, day and night. Thirst will perhaps be distressing, and pellets of ice should be frequently administered, as well as frequent small amounts of iced Vichy or other carbonated water. With the development of toxemic or septicemic symptoms, alcohol is called for and should be administered cautiously, but in rather liberal amounts, whisky or brandy, well diluted, champagne, or sherry being given. Morphine will be needed in most of the bubonic cases for the control of pain. It will also serve to produce sleep and to allay restlessness better than any other drug. It should be given by needle in average doses of $\frac{1}{4}$ grain, the intervals being determined by the effect of the drug, which should be carefully watched.

In the event of persistent vomiting, feeding per orem will have to be 
discontinued, and nutrient enemata may be substituted for direct feeding if emesis is long continued.

Efforts to reduce pyrexia should generally be confined to cold sponging. Antipyretic drugs are contraindicated, and should not be given. The icc-bag applied to the head is most useful to control headache and to subdue delirium. It probably has a definite value as a temperature depressant also. Ice-bags locally applied are also useful to lessen the pain of buboes, although, as a rule, the buboes should be treated with hot fomentations to favor suppuration and discharge, or to hasten the time for incision. The ice-pack should be employed rarely, if at all, for the control of fever. Cold water, iced, if ice can possibly be secured, should be used for sponging, alcohol being added to the water to increase its evaporation; or plain alcohol sponging may be substituted. In the absence of ice, tepid sponging may be found useful.

The foregoing treatment is applicable to all forms of plague, and, indeed, there is no definite or separate symptomatic treatment for the various clinical types of plague, except the employment of surgical measures in the bubonic and cellulocutaneous types, which I will outline later. In all types of plague except the pneumonic type, in which symptomatic treatment can hardly be expected to do more than give comfort, certain critical stages appear in the course of the disease. These critical stages are accompanied by two definite dangers- $-(a)$ the danger of cardiovascular paresis, dependent upon toxemia; and $(b)$ the danger of septicemia or generalized infection of the blood-current (Choksy).

Progressive toxemia is indicated by a gradual enfeeblement of the circulation, and may induce cardiac syncope and slowly developing oedema of the lungs. The symptoms include air-hunger and delirium. The special therapeutic indication is for alcohol, oxygen, cardiac stimulants, and perhaps the incision of buboes.

In the septicemic cases the therapeutic indication is for more antipest serum. Septicemia is to be recognized by an increasing number of colonies of Bacillus pestis, grown from the blood upon agar slants, as shown by frequent cultures $(0.1 \mathrm{cc}$. of blood to the agar slant). This index is an important one, and as the septicemia becomes more intense, the number of pest bacilli per cubic centimeter of blood may rise as high as $1,000,000$, just before death.

The symptoms are not unlike the symptoms of toxemia, but include hemorrhagic developments and often culminate in a terminal pneumonia. In our use of therapeutic weapons against sudden cardiac failure or threatened collapse we have the choice of camphor, digitalis, and strophanthus, while strychnine may be given with advantage through the course of the disease. Whenever possible, these drugs should be given hypodermatically and in good dosage. In the stage of slow convalescence following the subsidence of acute symptoms, or the mild septicopvemia cases of chronic tendency, or when extreme sloughing or suppuration supervene, we must supplement the surgical treatment with constructive tonics, iron, stimulants, liberal feeding, and the best of nursing. 
Surgical treatment in plagne is, of course, limited to bubonic cases and to cases developing the so-called "plague carbuncles." Choksy has termed this latter type of cases the "cellulocutaneous" type, thus indicating a localized pest bacillus infection of the skin and subcutineous cellular tissue, usually accompanied by very limited lymph-node involvement. Owing to frequent extensive necroses, these cases are of ten prolonged, but clinically the type is the least fatal of the several varieties of well-developed plague. 'The "carbuncle" areas develop rapidly from coalescing small vesicles, appearing over a discolored spot in the skin. The resulting larger vesicle often contains a pure culture of Bacillus pestis, and results in an ulcer or local slough, with indurated, sharply defined edges, surrounded by extensive oedema.

Without discussing the pathology of buboes, which are variously designated as primary buboes of the first and second order, and as secondary buboes, let us understand that primary buboes are those which occur in the lymph-glands nearest to the points of entrance of the pest bacillus, and that secondary buboes are those secondarily infected through the blood-current. 'The primary buboes are most likely to suppurate.

Early and complete extirpation of the buboes prior to suppuration has been seriously proposed, but the present-day weight of opinion is against such surgical interference, for the reason that other glands than those giving rise to the primary first order bubo are invariably invaded by pest bacilli before there is sufficient evidence of bubo formation to suggest extirpation. I would freely incise the suppurating bubo as soon as evidence of suppuration is present, drain with tubes or gauze wicks, and keep antiseptic dressings constantly applied and frequently changed. Antiseptic poulticing may sometimes be used with advantage to hasten the suppuration of buboes before incision.

The surgical treatment of the so-called "carbuncles," the local sloughing areas of the cellulocutaneous type of plague, is somewhat different. In some cases the infected skin area and the underlying cellular tissue may be excised, all incisions being through healthy surrounding tissues. In these cases I recommend the use of the actual cautery after extirpation, or extirpation with the cautery knife, which seals divided lymph-vessels and blood-vessels and thus lessens the chances of further systemic infection.

Usually, however, the surgical treatment of these cases is limited to the application of antiseptic absorbent dressings, moist and hot, with the object of hastening the separation and removal of the slough. When a definite line of demarcation occurs, the necrotic tissue may often be removed from its base, thus hastening repair by granulation. If, as sometimes occurs, there is very extensive destruction of soft tissues, other subsequent surgical procedures may be necessary to cover exposed bone, cartilage, or other of the deep tissues.

In this discussion of the treatment of plague I have not attempted to present in detail the symptomatology, clinical classification as to varieties, or physical diagnosis of the disease, but I trust that sufficient 
reference has been made to the various forms of plague to make clear the indications for treatment. I have consciously omitted from separate consideration the mild types of infection known as ambulant or larval plague, or pestis minor, and will simply remind the reader that in plague, as in many other infectious diseases, we will occasionally encounter cases in which clinical expression is incomplete when compared with the classical symptomatology of the disease. These cases are principally dangerous if they occur, as often happens, at the beginning of an epidemic, on account of the probability that they will escape attention and so spread the aisease in its more serious forms by distributing pest bacilli in the various ways possible. They are, therefore, of the greatest importance from the viewpoint of diagnosis.

In spite of our unsatisfactory position in the matter of agencies for the curative treatment of plague, the situation is not one for despair. On the contrary, we have great reason for hope that our pursuit of a dependable method of curative treatment will soon be successful, while in our extensive knowledge of the causes and means of prevention of plague we are, in theory at least, already in control of the disease. 


\section{INFLUENZA}

BY O. H. WILSON, M.D.

The term "influenza" has been loosely applied to a host of conditions, some of which justified its employment, others did not. The recent investigations of ${ }^{3}$ Holt and Wollstein have shed considerable light upon this subject. In their researches they considered as influenza only those cases in which the bacillus of Pfeifier could be demonstrated." This organism was discovered by cultures in 85 persons, of whom 42 were suspected of having influenza and 43 were not, 1 is of the latter being nurses or physicians who were in intimate contact with patients. In cases of acute nasopharyngeal catarrh and its complications, such as otitis media and adenitis, the Bacillus influenza was conspicuous by its absence. Cultures were made from 29 cases of otitis media after fresh paracentesis, none showed influenza bacilli; likewise 5 cases of acute nasopharyngeal catarrh gave negative results. From an analysis of the results obtained by the above-mentioned investigators it is evident that the term "influenza" must be more restricted in its application. It is the epidemic form of the disease that will receive especial consideration in this article.

The successful management of a case of influenza will often tax to the utmost the resources of the medical attendant. When we consider that the disease is one which clinically has protean aspects, it at once becomes apparent that our principal rule for treatment should be to individualize as much as possible, and treat the patient rather than the disease. During epidemics and endemics mild cases are frequently encountered, and as these are often accompanied or followed by serious complications and sequels, they should merit as much care and attention as those characterized by severe clinical manifestations. Influenza per se is a comparatively benign affection; this is especially true when the disease occurs in young and healthy individuals. It is the complications and sequels that are most dreaded - the latter frequently cast the victim a physical wreck upon the shores of health.

As yet no specific has been discovered for this prostrating malady. The pathological changes produced by the bacillus of I'feiffer do not appear to be distinctive; therefore, our drug treatment is largely empirieal and symptomatic. 'The therapeutist will best serve the interests of his patient by not employing any remedial agent unless the indications for its use are clearly defined, never forgetting, however, the possible contraindications. With our increasing knowledge of the etiology, pathology,

${ }^{1}$ The Arehives of Internal Medieine, May, 1910. 
and modes of transmission of infectious diseases it becomes of paramount importance for the medical attendant to institute measures to prevent their dissemination. 'The prevention or prophylaxis of influenza, therefore, first demands our attention.

Prophylaxis.- That influenza is a highly infectious disease seems to be definitely settled. The infection spreads with marked rapidity-not faster, however, than the modes of travel, which fact illustrates what an important factor personal contact is in spreading the infection. Isolation should be practised whenever possible, only those attending the patient being admitted. As the bacillus of Pfeiffer shows a special predilection for the respiratory system, the secretions from this tract must be considered as important agents in spreading the infection. The sputum should be expectorated into a special receptacle, containing a germicidal solution, such as 5 per cent. phenol, and afterward cremated. A piece of gauze should be employed to catch particles expelled during the act of coughing or sneezing; these should subsequently be burned. Patients should be warned against moistening the fingers in the mouth for any purpose. Bed-clothes and soiled linen of any kind should be thoroughly boiled before sending to the laundry. Thermometers should be sterilized and table utensils boiled. Cleaning the bed-room should be done in such a manner as will prevent dissemination of dust.

In inter-epidemic periods individuals are found who suffer with chronic cough and expectoration, their sputum being laden with influenza bacilli, by reason of which they become important sources of infection. 'These disease carriers should be warned of the danger of infecting others. and proper instructions given for disposal of their sputum. As much care should be exercised as is usual with the sputum of tuberculous patients.

Holt and Wollstein report the finding of influenza bacilli in the secretions of 16 apparently healthy persons. Further investigations may show that such individuals may be capable of spreading infection. If future reports prove our supposition to be correct, the isolation and control of this class of persons would be a valuable but difficult prophylactic measure.

In the presence of an epidemic all should strive to maintain their general health at the highest possible level. This precaution is most essential in persons suffering with general debility and catarrhal conditions of the mucous membranes, on account of their marked predisposition to infection. Tuberculous patients, infants, and the aged must be carefully protected from all possible sources of contagion. If the disease is contracted by this group of individuals, it is apt to be characterized by symptoms of marked severity; and on account of their general feebleness they are little able to withstand the onslaughts of the disease. It is doubtful if any drugs possess distinct prophylactic properties, but such action has been attributed to quinine, salicin, and eucalyptus.

General Treatment.-Of all the measures employed in the treatment of influenza, rest in bed is the most important. 'This should be insisted upon, no matter how mild the case. 'There is a great tendency upon the part of many patients to fight the onslaughts of the disease by remaining 
on their feet and attending to their usual occupation. This indiscretion often prolongs the course of the disease, and renders the sufferer more susceptible to the development of complications and sequels. Such a needless expenditure of energy and exposure should not receive the sanction of the medical attendant. 'The sick-chamber should be well ventilated, all dranghts being a voided - an even temperature of about $68^{\circ} \mathrm{F}$. is probably best. In most cases the use of the bed-pan is not necessary, but it is advisable for the patient to attend to such calls within the room.

If the patient is seen shortly after the onset, perspiration should be encouraged by wrapping the body in blankets and by administering ten grains of Dover's powder with a hot drink containing a little brandy or whisky. 'This procedure is frequently effective in relieving pain and producing nervous sedation. It is important to keep the skin warm at all times, for by so doing internal congestions are relieved, toxic matter is eliminated, and the burden of the kidneys materially lessened. 'The bowels should be freely opened by giving a grain or two of calomel in divided doses, followed in four or five hours by a saline purge. Some writers believe that such purgation may excite an obstinate diarrloea, and that drugs of this class should be used with caution. I invariably employ them, believing that their good results far outweigh any possible deleterious effect. 'The dose of the saline, however, should be carefully regulated to suit the individual case.

One of the most prominent and distressing manifestations for which the patient demands relief is the "aching pain" in the muscles and bones. Locally, for this condition, I employ heat in the form of hot-water bottles, hot sand or salt bags. Applications of heat seem to be more efficacious than cold, and are always more grateful to the patient. Cold, locally applied, has a limited range of usefulness in this disease-rarely is it necessary to resort to it for its antipyretic influence. Liniments of various kinds have been emploved for the relief of the general muscular pain and tenderness. Mueh friction cannot be used on account of the extreme sensitiveness of the tissues. I never employ them, as other remedies have proved more efficacious in my hands. The salicylates and the coal-tar derivatives give the most prompt and satisfactory results. The former drugs seem at times to influence favorably the course of the malady. The following combination has vielded excellent results in the practice of the writer:

R. Codeinæ sulphatis, Quinina hydrochloridi, Acetphenetidini, Aspirin,

Misce et pone in capsulas No. xii.

gr. iij:

gr. xij;

gr. $x i j$ :

gr. slviij.

Sig.- One capsule every two hours.

The codeine in this prescription not only enhances the analgesic action of the acetphenetidin and aspirin, but also serves to quiet the nervous excitation frequently present in the initial period. Salol, salicin, salicylic acid, strontium, or sodium salicylate may be employed in place of aspirin; 
my preference is for the latter drug, as it is better tolerated by the stomach than are most of the other salicylic compounds. The other coal-tar derivatives, such as acetanilide, antipyrine, and salipyrine, may occasionally be used to advantage. The employment of this class of remedies in the treatment of influenza has frequently been condemned on account of their depressing action. The question as to whether or not they should be administered can only be decided by a careful consideration of the individual case. In the presence of profound prostration, great cardiac weakness, or serere gastric symptoms, they must be withheld. In no case should they be employed in large doses, as good results can usually be obtained by smaller ones, without the dangers attending the administration of larger quantities. Discontinue their administration as soon as the distressing pains are controlled. That they increase susceptibility to infection, especially when the patient is exposed to cold, should always be borne in mind. When the coal-tar products are contra-indicated, morphine may be given, hypodermically or by the mouth, to relieve pain. Insomnia is usually due to pain; with the relief of this symptom sleep is generally secured. The salicylates are decidedly depressing and must be used with care.

Quinine is a drug which has unquestionably been abused in the treatment of la grippe, especially by the laity. It appears to be useful, but I do not believe there is any advantage to be gained by the administration of large doses. Such quantities frequently produce an annoying tinnitus and congestion of the tympanum, which is distinctly disadvantageous when we consider how frequently otitis media occurs as a complication. Small doses are of service and possess valuable tonic properties. Administered in this way it is never depressing, and may be continued with advantage throughout the disease and during convalescence.

The employment of stimulants is a valuable and rational procedure. Their administration should never be made a routine practice, being given only to meet special indications. Many cases will be seen in which, owing to the mildness of the infection, or the resisting powers of the individual, the use of this class of remedies would be a needless drugging of the patient. Others are encountered in which the prostration is profound from the onset, so that stimulation in some form may be required intermittently or continuously throughout the course of the malady. This profound asthenia or adynamia is most apt to occur in the aged, and in those suffering from pre-existing general debility. The deleterious influence of the toxins of the bacillus of Pfeiffer upon a heart already afflicted with myocardial degeneration, or the seat of a valvular defect, are too well known to necessitate elaboration at this time. In this class of cases the foregoing remedies frequently find their greatest field of usefulness. During the febrile period, stimulants, as a rule, should be withheld, unless clear indications exist for their administration. At this time they frequently increase the nervous excitation and suffering. When prostration is a prominent feature, alcohol often yields excellent results. This remedy has been much abused in the treatment of influenza, its administration in large quantities for many days being frequently 
resorted to. I cannot sanction its employment in this way, as I have observed cases in which the depressing ifter-effects of large doses of alcohol were greater than the depression of the disease itself. Small doses give good results without the possible deleterious influence of large ones. When ordering alcoliol, the tendency to form a habit should never be forgotten. It is usually administered in the form of brandy or whisky; if for any reason these are not well tolerated, or if special indications are to be met, a good wine or dry champagne may be substituted to advantage. When a rapid effect is desired, the brandy or whisky should be given hot and concentrated; if a more prolonged action is desirable, it can best be attained by giving the stimulant cold and well diluted, preferably with food. 'The use of all forms of alcohol should be discontinued as soon as possible.

One of the most valuable agents to rally the vital forces and tide the patient over critical periods is strychnine. It may be ingested or given hypodermically, according to the urgency of the symptoms. If cardiac failure is threatened, rapidly acting diffusible stimulants, such as aromatic spirits of ammonia or Hoffman's anodyne, should be administered hypodermically. These remedies, like strychnine, must be reserved for emergencies; to utilize them continuously often causes a needless expenditure of reserve force, so that when the decisive struggle ensues, there are no reinforcements available. During the febrile period, cardiac tonics, like digitalis, strophanthus, and cactus grandiflorus, have a very limited range of usefulness. In the presence of marked vascular relaxation atropine hypodermically is indicated.

Symptoms referable to the respiratory system are undoubtedly the most common manifestations of influenza. The infection of this anatomical tract leads to the development of rhinitis, rhinopharyngitis, tracheitis, or bronchitis; one or all of these conditions may be present in a given case. The inflammation of the nasal mucous membrane is usually severe, the inflammatory process displaying a marked tendency to spread and involve the accessory sinuses. It is essential that the nasal chambers and the nasopharynx should be frequently and thoroughly cleansed by some alkaline antiseptic fluid; for this purpose Dobell's solution, used warm in a Birmingham douche, answers admirably. By securing good drainage for the nasal secretions the liability of involvement of the accessory sinuses is reduced to a minimum. Applications of cocaine solution (4 per cent.) or adrenalin chloride (1 to 1000), by contracting the swollen and hyperemic mucosa, aid very materially in maintaining patulous the avenues of exit of the mucopurulent secretions. The shrinking of the mucous membranes not only favors drainage, but permits more thorough cleansing of the deeper portions of the nasal chambers. Adrenalin does not produce secondary congestion of the mucous membrane; this undesirable result is common after cocaine applications. After a thorough cleansing of the nasal chambers with the alkaline aqueous solution I apply to the mucous membrane a solution containing chloretone, camphor, menthol, and oil of cinnamon in liquid albolene. Following the subsidence of the acute manifestations, there is often a 
tendency for the Schneiderian mucous membrane to remain thickened and irritated. This condition is best treated by the administration of alteratives, especially potassium iodide. If the tracheitis and laryngitis are sufficiently serere and annoying to demand treatment, considerable relief may be obtained by inhaling steam laden with compound tincture of benzoin, one or two drams of this preparation being added to a pint of boiling water.

Bronchitis has been considered by some writers as a complication; when confined to the larger tubes, I believe it best to regard it as one of the essential manifestations of the malady. 'The treatment of this condition differs little from that usually employed in bronchial inflammations arising under other conditions. In the early stages, when secretion is scanty and the patient is harassed by a troublesome and unproductive cough, codeine is exceedingly useful. I prefer this drug to heroin, as I have often observed considerable depression following the administration of this new morphine derivative. Codeine may advantageously be combined with sedative expectorants, as in the following prescription:

R. Codeinæ sulphatis, Potassii citratis,

Liquoris ammonii acetatis,

Sig.-Teaspoonful in water every two hours

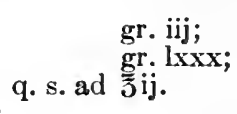

gr. Lxxx;

Depressing drugs, like antimony, aconite, and veratrum viride, should be avoided. Counterirritation of the chest at this time is a valuable adjunct. For this purpose we may employ a mustard plaster or a turpentine stupe. I prefer the latter applied to the anterior, lateral, and posterior aspects of the chest. When numerous moist rales become audible, indicating the period of active secretion, stimulating expectorants are the remedies of choice. The most useful drugs of this class are ammonium chloride and carbonate, terpin hydrate, terebene, oleoresin of cubebs, and oil of sandalwood. The tendency of these expectorants to disorder digestion should not be lost sight of, as an unimpaired alimentary tract is one of the patient's most valuable assets. Ammonium chloride seems to be the least offensive in this connection, and is the remedy of choice in most cases. Should the cough be excessive, codeine, heroin, or hyoscyamus can be given simultaneously. Some such combination as the following may of ten be used to advantage:

R. Codeinæ sulphatis,

Ammonii chloridi,

Tinct. hyoscyami,

Fluidextracti glycyrrhizæ,

Misce. Aquæ destillatæ,

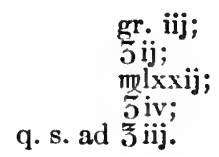

Sig.- Teaspoonful in water every three hours.

It will frequently be beneficial to vary from time to time the expectorant employed.

Diet.-The importance of selecting a proper diet cannot be overestimated. Of all factors aiding recovery, the maintenance of nutrition is the most potent. While the fever lasts, the stomach is usually irritable, 
so the diet must be fluid and restricted. Food should be given in small quantities and at frequent intervals. For two or three days an exclusive milk diet is often best; if well tolerated, at least two quarts should be drunk. In some cases, with a foul tongue and marked gastro-intestinal disturbance, milk is not well borne; under these circumstances we may resort to concentrated brotlss of beef, mutton, and chicken. If vomiting is a troublesome symptom, it is best to withhold food for a time, giving nothing but small quantities of albumin water. 'This procedure usually yields better results than can be obtained by administering drugs. The quantity of fool given at this time should be governed entirely by the patient's ability to digest and assimilate. Often the stomach is not in a receptive mood; to encourage the taking of large quantities of food under such circumstances can only be productive of harm. As the fever subsides and the condition of the gastro-intestinal mucosa improves, raw eggs, milk toast, eggnog, milk-punch, and custards may be added to the dietary.

When solid food can be eaten, scraped beef sand wiches, eggs, poached, soft-boiled, or scrambled, oysters, tender breast of chicken, sweetbreads, and light farinaceous articles with which cream is taken are appropriate.

During convalescence the food should be highly mutritious and served in the most palatable manner, and as much as the condition of the stomach will allow. At this time abundant solid nitrogenous food, such as roast-beef, beefsteak, chicken, eggs, etc., should be given; in addition, the patient should receive a quart of milk daily, provided it is well tolerated. If the appetite is poor and the digestive functions are tardy in regaining their full physiological activity, alcohol in some form is a valuable aid. For this purpose we may utilize whisky or brandy well diluted, good Burgundy, champagne, or some of the malt liquors. 'Their best effects are seen when administered with food. As adjunets in overcoming the anorexia of convalescence, the bitter tonics, like gentian and calumbo, are often useful.

Complications.-Respiratory.-The severity of the attack does not scem to bear any distant relation to the development of complications. They are frequently due to negligence or indiscretion upon the part of the patient. 'Too early resumption of their customary duties and undue exposure are often important factors in their causation. 'The most frequent and important complications are those involving the respiratory tract. Catarrhal or croupous pneumonia may be encountered, the former much more frequently than the latter. 'The existence of a bronchitis should make us apprehensive, as the inflammatory process often extends rapidly and involves the bronchioles and alveoli. Bronchopneumonia, due to the bacillus of Pfeiffer, differs little from that due to other causes, except that the associated asthenia or adynamia is usually more profound. Cardiac embarrassment is especially prone to occur during the course of influenzal pneumonia, on account of the well-known deleterious influence of the toxines of this disease upon the myocardium. With signs of circulatory failure active stimulation is often imperative, and in a crisis of this kind the remedies must be given hypodermically and for effect. 'The most use-

VoL. I -56 
ful drugs under these circumstances are strychnine, atropine, aromatic spirits of ammonia, Hoffman's anodyne, camphor, and caffeine; the last-mentioned drug should be given hypodermically as caffeine sodiobenzoate. Ammonium carbonate is a remedy which is often useful as a cardiac stimulant and expectorant; it may be administered by the mouth in small doses for some time. If gastric disturbances follow its administration, it should be discontinued. In children an emetic is occasionally useful, to aid in the expulsion of mucus; for this purpose we may employ the wine or syrup of ipecac. Tartar emetic is contraindicated on account of its depressing properties; even the former drug must be used cautiously or withheld in the presence of severe prostration.

Counterirritation of the chest is a valuable and rational procedure. For this purpose we may employ blisters, turpentine stupes, or dry cups, according to the effect desired. In children I prefer to use turpentine stupes; in older patients I employ dry cups. An ice poultice applied to the chest is often efficacious in relieving pain. For combating the toxemia, reducing the temperature, and producing nervous sedation, nothing is so serviceable as the cold sponge. The temperature of the sponge should be regulated by the ability of the patient to react. If the cerebral symptoms are pronounced and cough is excessive, morphine or codeine may be required. Morphine must be employed with great care or withheld, especially when secretion is profuse and cyanosis is present. With increasing cyanosis and cardiac weakness, ominous rales in the throat, and marked stupor, every effort must be made to arouse the patient. Alternate douches of hot and cold water and electricity may be tried: they often yield excellent results.

The food should be liquid and concentrated, milk, broths, and eggalbumin forming the basis of the diet. Upon the subsidence of the acute manifestations, stimulating expectorants are indicated to aid in the expulsion of mucus and to favor the restoration to normal of the mucous membranes. Delayed resolution is to be treated by the administration of iodides and by counterirritation to the affected area. When the pulmonary manifestations are unduly prolonged, other complications should be suspected, especially abscess, gangrene, or empyema. These conditions should be promptly recognized and appropriate treatment instituted.

Cardiac Disorders.-In many cases of influenza the heart appears to bear the brunt of the attack. Such selective action of the toxines, as previously pointed out, becomes especially serious in the presence of pre-existing myocardial degeneration or valvular defects. In this class of cases the question of cardiac efficiency should demand a large share of our attention. By promptly recognizing impending danger and instituting appropriate treatment, much can be done to avert disaster. That the bacillus of influenza is capable of producing acute endocarditis, myocarditis, and pericarditis seems to be definitely settled. 'The manifestations of cardiac involvement are variable both in degree and kind; they may be organic or functional in origin. Tachycardia, bradycardia, arrhythmia, and dicrotism are frequently observed; in the presence of 
much myocardial degeneration embryocardia is usually pronounced. Palpitation is often an annoying symptom, and syncopal attacks are common, the latter frequently follows the slightest exertion, such as raising the head from the pillow.

The treatment of acute cardiac insufficiency occurring during the active stage of the malatly has been considered. 'T'o conserve cardiac energy and prevent subsequent accidents rest in bed is one of the most important therapeutic measures at our disposal. 'The resumption of activities must be gradual, being controlled entirely by the ability of the heart to meet the demands for increased work. In ardition to rest, cardiac tonics are valuable adjuncts in favoring restoration of normal heartaction. The remedy of choice and the dose can be determined only by a careful study of the individual case. Strychnine often yields splendid results, and is especially valuable on account of its general tonic properties. When feeble heart action is associated with palpitation, arrhythmia, and tachycardia, the bromides combined with digitalis often act promptly and efficiently. In these cases, with little or no impairment of the first sound of the heart, but with tachycardia and arrhythmia, the following combination, suggested by Dr. H. A. Hare, has yielded excellent results in my hands.

17. Tinct. aconiti, Tinct. belladonnæ, Tinet. digitalis, Tinct. gentian. comp.,

Sig. - A teaspoonful three or four times a day.

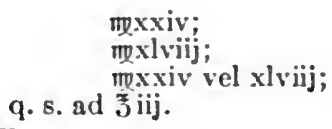

mxxiv; mexlviij; mpxiv vel xlviij; q. s. ad 节ij.

If the cardiac asthenia is associated with high arterial tension, nitroglycerin may be combined with digitalis, or the latter drug may be replaced to advantage by strophanthus. If the heart is rapid and irregular and palpitation is an annoying symptom, an ice-bag applied to the precordium gives great relief; it may be used continuously or intermittently; I prefer the latter method. Obstinate cases of cardiac disorders only improve with the restoration of the general health and nutrition, consequently all measures tending to promote the early establishment of these conditions, such as rest, should be instituted.

Gastro-intestinal Complications.-There seems to be considerable difference of opinion regarding the existence of a distinct gastro-intestinal type of influenza. Mild gastric and intestinal disorders are so common that they cannot be considered as complications. Undoubtedly cases occur in which symptoms referable to the alimentary tract dominate the clinical picture. It is quite possible that these marked disturbances are purely toxic in origin, and result from some other form of disease. It seems best not to consider this form as a clinical entity until more definite information is available.

Nausea and vomiting, when slight, usually yield to ordinary methods of treatment. If the tongue is heavily coated, calomel in minute doses is usually followed by good results. Other remedies commonly employed, especially if the tongue is clean, are pellets of ice, small doses of 
brandy, cocaine, bismuth subnitrate, cerium oxalate, chloretone, lime water, and acetanilide. The last-mentioned drug should be employed with care or withheld if much depression exists. A favorite combination of the writer's for this condition is the following:

R. Sodii bromidi,

Sodii bicarbonatis,

Spiritus ammoniæ aromatici, Misce. Aquæ menthæ piperitæ,

\author{
āā gr. lxxx; \\ q. s. ad. $5 \mathrm{ij}$.
}

Sig.-Teaspoonful in a little ice water every hour until relieved.

If the vomiting is intense and uncontrollable, all medicines and food should be temporarily discontinued; the head should be kept low and absolute quiet must be insisted upon. Counterirritation to the epigastrium is useful, preferably in the form of a mustard plaster. As the vomiting subsides and the stomach becomes more tolerant, milk and albumin water may be allowed in teaspoonful doses at frequent intervals, the quantity being gradually increased as the condition improves. The restoration of a liberal diet should be made as soon as possible, so as to maintain the nutrition. If diarrhœa is a troublesome symptom, it is to be treated by the usual remedies employed for that condition, care being exercised not to select a remedial agent capable of influencing adversely the general condition of the patient. While the diarrhoea persists, the diet should be fluid and restricted, if necessary. Alcoholic stimulation may be necessary, especially if the diarrhoea is an indication of the existence of a severe enteritis or enterocolitis.

Nephritis.-The renal disorders accompanying influenza are usually trivial, and resemble those found in any acute infectious disease. True renal inflammation is occasionally encountered, but it must be regarded as an infrequent complication. If mild nephritis exists, no special treatment is necessary, but strict prophylactic measures must be maintained, especially the avoidance of exposure to cold. When the symptoms and signs indicate a more severe grade of kidney involvement, an exclusive milk diet is best; it should be continued until decided improvement occurs. The alkaline diuretics, such as potassium citrate, acetate, and bicarbonate, are often useful. If there is associated cardiac feebleness, digitalis often gives excellent results; the tincture is the preparation of choice, especially if gastric irritability is present. In the presence of much renal irritation or inflammation it is best not to employ turpentine locally, for fear it may be absorbed and aggravate the existing inflammation.

Aural Complications.-Otitis media is a frequent and dangerous complication of influenza. The inflammatory process may be catarrhal or suppurative- too often the latter condition is present. By thoroughly cleansing the nose and nasopharynx much may be done to avert this dangerous complication. As a nasal wash any bland alkaline and antiseptic solution is serviceable; I invariably employ Dobell's solution. Recent investigations have shown that urotropin is eliminated in the secretions from the tympanum. 'This discovery would suggest the advisability of administering this drug as a prophylactic and curative agent. 
Further experience will be necessary to determine its value in this connection.

In the early stage, when there is great hyperemia, the real or artificial leech should be applied in front of and close to the tragus. When the mastoid is simultaneously involved, the leceh should also be placed behind the auriele, just over the antrum or mastoid tip. 'This treatment seems to mitigate and shorten the course of many cuses of otitis media. For the relief of pain instillations of hot water into the external anditory canal are exceedingly useful; a hot-water bottle or bag of hot salt may be employed for the same purpose. Cocaine solntion (4 per cent.) may be warmed and instilled with benefit; also the following:
R. Atropina sulphatis, Acidi borici,
Glycerini, Misce.
Aqux destillatx,

$$
\begin{aligned}
& \text { gr. ij; } \\
& \text { gr. } \mathrm{x} \text {; }
\end{aligned}
$$

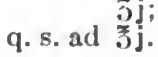

'The tympanic membrane should be ineised freely as soon as bulging of this structure becomes apparent, as well as in those cases in which pain persists in spite of all antiphlogistic treatment. 'The value of early incisions cannot be overestimated, for it minimizes the danger of the development of serious complications, such as mastoiditis, meningitis, and cerebral abscess.

Nervous and Mental Complications and Sequels.-Nervous phenomena in greater or less degree appear to be present in nearly all cases of influenza, but there is a certain group of patients in whom this anatomical system is especially implicated. 'I'his selective action may result from some pre-existing weakness, either congenital or acquired. The treatment of the initial nervous symptoms has been considered. Of the later manifestations, persistent nerve pains are common; the nerves most frequently involved are the supra-orbital, intercostal, and sciatic. The iodides and sulicylates are the most useful remedies for this persistent neuritis. Counter-irritation is a valuable adjunct, applied in that form best suited to the region affected. One of the most potent factors in establishing a cure of this distressing condition is the early restoration of the general health and mutrition.

Neurasthenia may be initiated; especially is this apt to occur if any predisposition exists. If mild, it usually yields to general hygienic measures, but if severe and occurring in an individual with a neuropathic disposition, a formal rest cure may be required. (See Rest Cure.)

Psychoses of various kinds have been deseribed in connection with influenza. In some cases they appear to be primarily due to the infection; in the greater number, however, a psychopathic disposition can be demonstrated; and it would seem as if the infection had kindled into activity some latent process. 'Io prevent and cure these mental disturbances every effort should be made to overcome the profound depression. Prolonged rest in bed, nourishing food, and tonics are essential. If much nervous excitation is present, the bromides often give considerable relief. Insomnia, which is of ten a troublesone symptom, may be banished 
by the administration of trional, sulphonal, veronal, hyoscine, or chloral hydrate. 'The last-named drug is contraindicated if much cardiac weakness exists. When the mental aberration is not marked and the patient can readily be controlled, home treatment is generally satisfactory. It is best to reserve sanatorium and asylum treatment for the more severe cases that are difficult to manage.

Convalescence.-When the storm has abated, we must not delude ourselves into believing that all danger is past, for it is just at this time that the utmost vigilance is necessary to save our patient from the many pitfalls which beset his path. Prostration frequently far outlasts the symptoms of the disease. After the active symptoms subside, rest in bed should be insisted upon, no matter how mild the attack has been. If the infection has been characterized by marked symptoms of severity, or if complications were present, this period of rest must be more prolonged. Physical or mental exertion when the patient first gets out of bed must be undertaken gradually, and it should never be carried to the point of fatigue. Avoidance of all nervous and mental strain and prolonged abstinence from business worries and cares aid very materially in hastening recovery. The diet should receive a large share of our attention; this important matter has been previously considered in a former chapter. At this time tonics are a valuable aid; I employ the following combination with excellent results:

\section{R. Strychninæ sulphatis, \\ Arsenii trioxidi, \\ Pulv. digitalis, \\ Quininæ hydrochloridi, \\ Ferri reducti, \\ Extracti gentianæ,}

Misce et pone in capsulas No. $\mathrm{xxx}$.

Sig.-One capsule three times a day after food.

$$
\begin{aligned}
& \text { āā gr. ss; } \\
& \text { gr. viij; } \\
& \text { gr. xxx; } \\
& \text { gr. vj; } \\
& \text { gr. xxx. }
\end{aligned}
$$

In this prescription the strychnine is valuable in overcoming the physical and mental depression, and in conjunction with the digitalis it renders signal service by stimulating the enfeebled heart. The iron and arsenic are employed to combat anemia, which is common during convalescence; the arsenic also cxercises a valuable alterative influence upon the depraved mucous membranes. The quinine is useful for its general tonic properties, and when combined with the gentian and strychnine, is valuable for correcting persistent anorexia. The above tonic prescription may be administered for several weeks; after a short time the digitalis should be omitted. Obstinate cases are encountered, in which the physical and mental depression persist in spite of all treatment, full restoration of health under these circumstances only being secured by a change of climate, an ocean vovage, or a visit to a health resort.

With persons predisposed to tuberculosis especial care must be exercised, as the influenzal infection appears at times to establish a point of least resistance, which permits ready infection with the tubercle bacillus. A pre-existing and unsuspected tuberculosis may be present, the latent foci being stimulated into activity with the onset of the acute in- 
fection. The debilitated condition of the patient at this time may permit the tuberculous process to make rapid strides, consecquently it is of great importance to recognize promptly the existence of this condition, and to institute appropriate treatment. Chronic cough and expectoration, independent of any tuberculous process, are common and persistent symptoms during convalescence. 'The importance of this group of cases as carriers of infection has already been considered. 'This chronic bronchial inflammation or irritation is best treated with stimulating expectorants, preferably creosote, terpin hydrate, terebene, or oil of sandalwood. Codeine is valuable if the eough is excessive and unproductive.

Holt observed that positive influenza eultures were frequently obtained during the spring months, and that with the advent of warm weather the organism disappeared entirely from the secretions. 'This observation suggests to us the advisability of sending our cases with persistent symptoms, whenever possible, to a warm, equable climate. 



\title{
YELLOW FEVER
}

\author{
BY ARÍSTIDES AGRAMONTE, M.D.
}

General Remarks.-It is the physician's strictest duty not only to cure the sick whenever called upon to attend them, but also to prevent the occurrence of disease, particularly when such might be spread by the cases under his care; hence, before entering at length upon the method to be followed in treating yellow fever, I may be permitted to note here the most salient etiologic factors, as well as those pertaining to the diagnosis of the disease, which ought to be borne in mind when assuming charge of a case.

An important feature, and one that the modern practitioner must defend against the antiquated notions of former days, in reference to the means of propagation, is the fact that "fomites" play no part in the spread of epidemics; no part of the patient, his clothing or surroundings, can in any way be considered as directly infective: there is no need, as formerly recommended, to disinfect the urine, feces, and romitus, nor to employ any unusual method in treating the bed-clothing or garments of the patient. 'The troublesome and often unsatisfactory measures necessary in dealing with other infectious diseases are entirely uncalled for in yellow fever. Sufficient evidence in support of this doctrine has been furnished by the exhaustive experiments carried out by the U.S. Army Yellow-fever Commission, corroborated by the various authorities who have since investigated the matter, to say nothing of the foul and nauseating tests undertaken by previous investigators without success as far as obtaining an artificially infected case of yellow fever is concerned.

The only thing one must guard against is the access of mosquitoes to our patients. Inmediately upon coming across a suspicious case of yellow fever the physician must see that it is covered by a mosquito-bar that will effectually prevent the sting of these insects, and this precaution is of the utmost importance during the first three days of the disease, for at this period the infective agent is circulating in the blood of the patient and can then be taken up into the stomach of the attacking mosquito.

When, as it often happens, the case is not seen before the second or third day or even after, or the diagnosis, which is at times very difficult, is not made before, then all care should be taken so as to prevent the escape of mosquitoes which may have already bitten the patient, and which experience has demonstrated seldom travel far away from the case, and 
less so after having partaken of a full meal. For this purpose the least disturbance should be made about the room; if not screened, the windows should be made insect proof by covering or stretching mosquito-netting upon the frame and the doors kept closed. After the fourth day there is no need to protect the patient; he can then be removed from the room and the latter subjected to thorough fumigation with sulphur. This practice will very likely cause the destruction of any mosquitoes which may have stung the patient during the infective stage of the first three days.

Aside from these precautions, the question of prophylaxis is beyond the sphere of the attending physician, and the matter comes under the domain of the sanitary authorities.

A few words must be said regarding the diagnosis of yellow fever. It is very unfortunate that men who have been considered as authorities in this specific disease have gone so far as to convey the idea that the diagnosis of yellow fever is comparatively easy and that it can be made with some degree of certainty during the early stages of the disease. The fact is that perhaps no other of the prevailing tropical diseases is more difficult to diagnose at a period when it is of the greatest importance that the actual conditions be recognized. Of course, when the physician comes upon a case of icterus with fever, vomiting, and albuminuria, at the fourth day of his illness, the diagnosis presents no difficulties; it is the cases of comparative mildness, those in which we probably find nothing bevond a suffused face with injected eyes and backache, which puzzle and confound the most expert. Yet one case is just as dangerous as the other, so far as causing the spread of the infection and producing other cases of greater or lesser intensity is concerned.

I think that physicians practising in tropical countries should always be on the lookout for cases of yellow fever, for, although the spontaneous generation of cases is not to be thought of for a moment, in such countries there will always be the possibility of importation, so long as yellow fever remains endemic in any of the African or American ports, and the requirements of commerce between civilized nations demand the curtailing of quarantine restrictions. Another point to be kept in mind is the fact that native children often serve as the means of keeping up the sources of infection in countries where the disease is not endemic by virtue of the mildness of their attacks, which may repeatedly pass unrecognized as such.

It is but seldom that we see a case of yellow fever upon the first day of the disease; usually on the second or third day, and it is not an uncommon thing for individuals to seek medical aid only after black vomit or a typhoid state has been developed. The history is generally alike in the majority of cases: the onset is more or less sudden, with a chill or sensations of cold and slight shivers, accompanied or immediately followed by severe frontal headache and lumbar pains of varying degrees of severity: at this time the face is flushed, the eyes injected, and there may be slight epigastric tenderness on pressure, also fever and a pulse increased both in strength and frequency. On the third day, or there- 
abouts, albumin appears in the urine, a slight jeteric tint may be ofserved in the conjunctiva, while the nausea and the fever may decrease. In the mild cases defervescence eontinues and paticnts do not suffer from the secondary rise and the exacerbation of all the symptoms, which is the case in most instances. After this "period of calm," as it has been called, which is evident on the third or fourth day of the disease, patients usually have a second access, another rise of temperature if it had remitted, gastric pain, nausea, or even vomiting, bleeding from various mucous membranes (gums, mouth, or stomach), increased albuminuria, often with bile-stained renal casts in the urine, and all the signs of an intense intoxication. At this stage the disease is readily recognized and is not dangerous from the epideniologic point of view.

From the fact that yellow fever has generally been a disease of the tropies, it is not strange to find this infection co-existing with malarial infection in the same patient. I have personally observed a number of cases in which the malarial parasite was demonstrated in the blood, while they suffered, some of them recovering, from well-marked and typical atticks of yellow fever.

We must also remember that all the salient symptoms of this disease are to be found in other conditions-malarial, typhoid or other fevers, dengue, hepatic disturbances, etc. 'The flushed face and injected eves of the first two days of yellow fever are frequently seen at the onset of other febrile diseases, but here they are generally accompanied by intense rachialgia. The fever may well be confounded with that of dengue, but we can usually see in yellow fever the tendency for the temperature to diverge from the pulse-rate, that is, the fever rising or keeping high, while the pulse gradually subsides. 'This discrepancy between pulse and temperature is otherwise only seen in cases of intense and prolonged icterus or intoxications of various kinds. 'The icterus in yellow fever, appearing on the third or fourth day, is never so intense as in the acute obstructive type or in infectious jaundice. As a rule, the feces are of their natural color, there is no acholia, except in prolonged cases in which the icterus really becomes intense and secondary conditions supervene after the original attack of yellow fever. Vomiting can only be considered as pathognomonic when it becomes bloody, showing the characteristic appearance of "coffee-grounds" as a result of the action of the gastric juice upon the blood.

This brief outline will show that although none of the symptoms can be considered as pathognomonic in yellow fever, still there is a group which, concurring at a given period in the course of the disease, may well serve as a basis for a positive diagnosis in most cases.

It is beyond the scope of this article to treat at any greater length of the symptomatology of yellow fever; in going over the methods of treatment, some of the symptoms will necessarily be referred to again.

A few temperature charts are herein inserted, showing the course of common types of the disease: the note at the foot of each gives the principal data in connection with the case. 
FIG. 101.

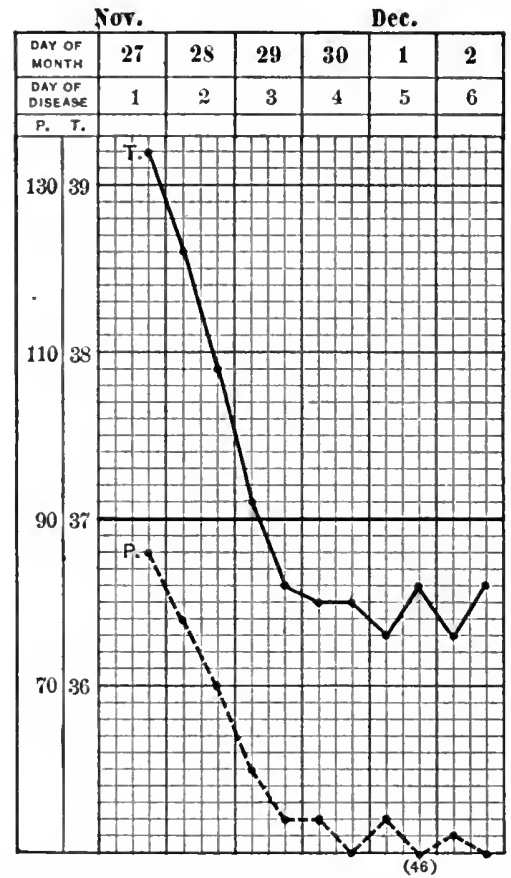

Case No. 1.-Mild case. Slight bleeding of gums. No internal medication. Very rapid recovery. $\mathrm{T} .=$ temperature; $\mathrm{P} .=$ pulse-rate.

FIG. 102.

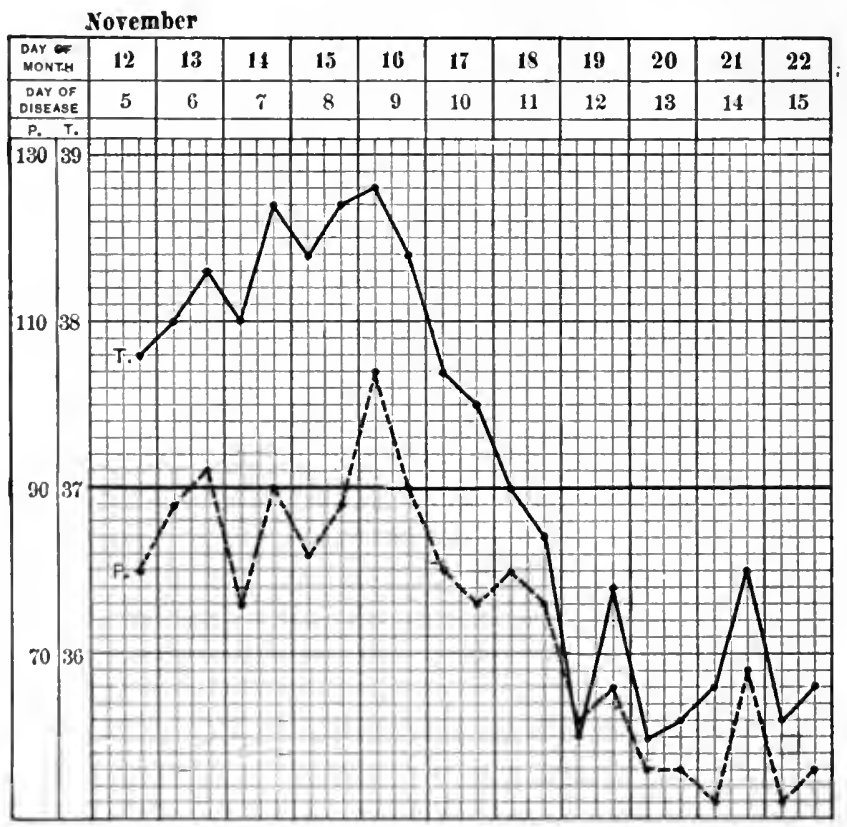

Case No. 2.-Quite typical of the secondary rise. Mainly symptomatic treatment. Rapid recovery. 'T.=temperature; $\mathrm{P}$.= pulse-rate. 


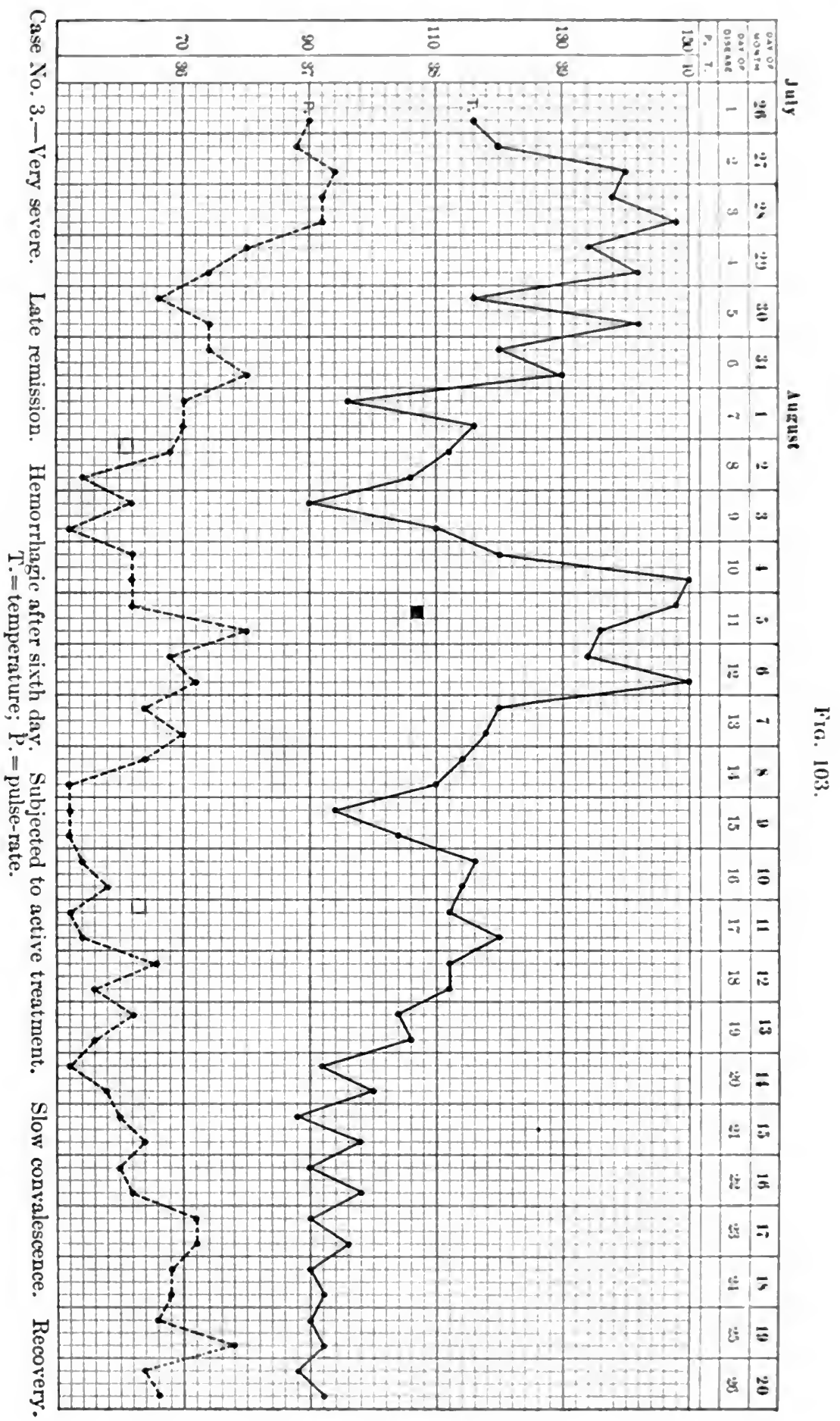




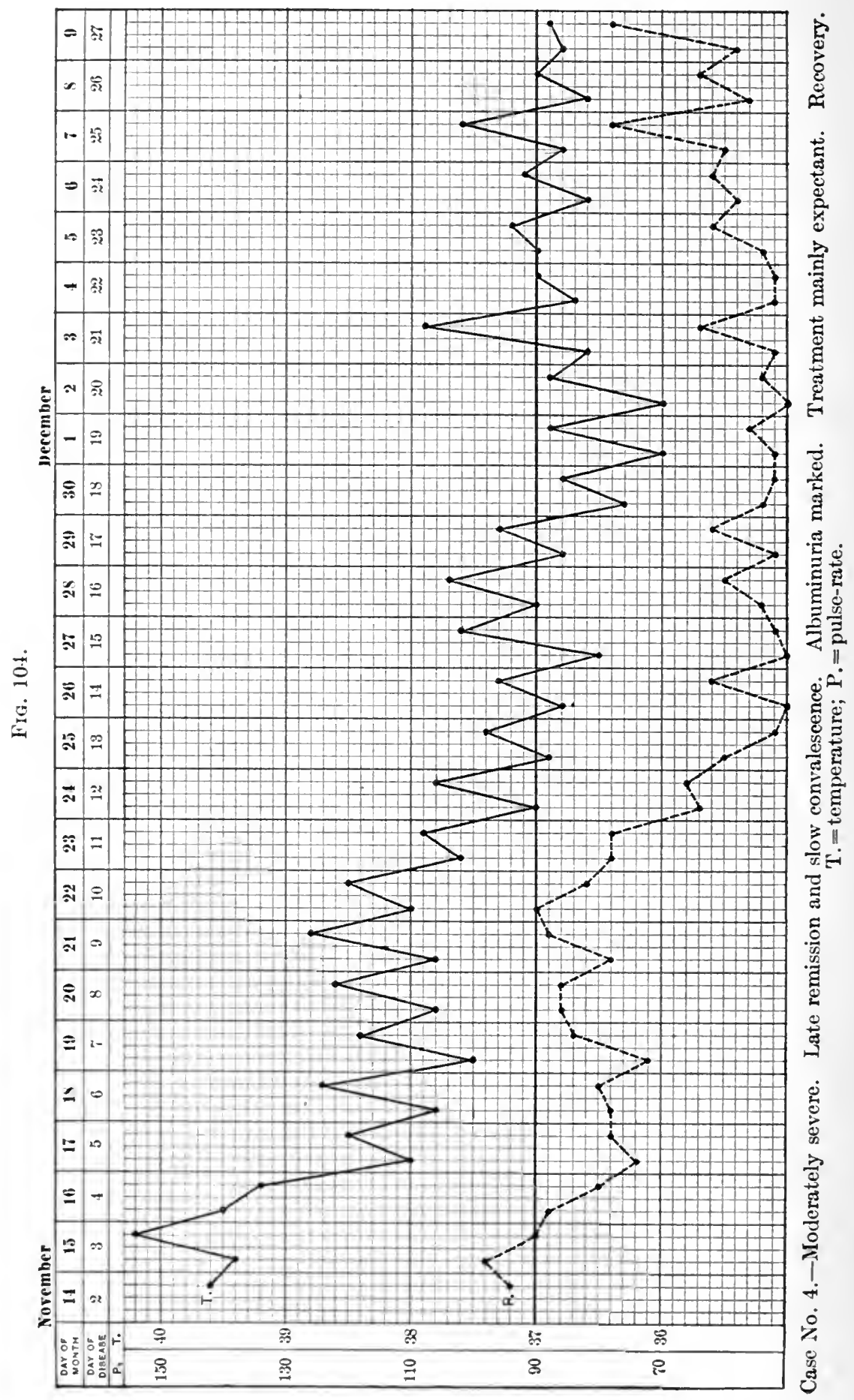


Fig. 105.

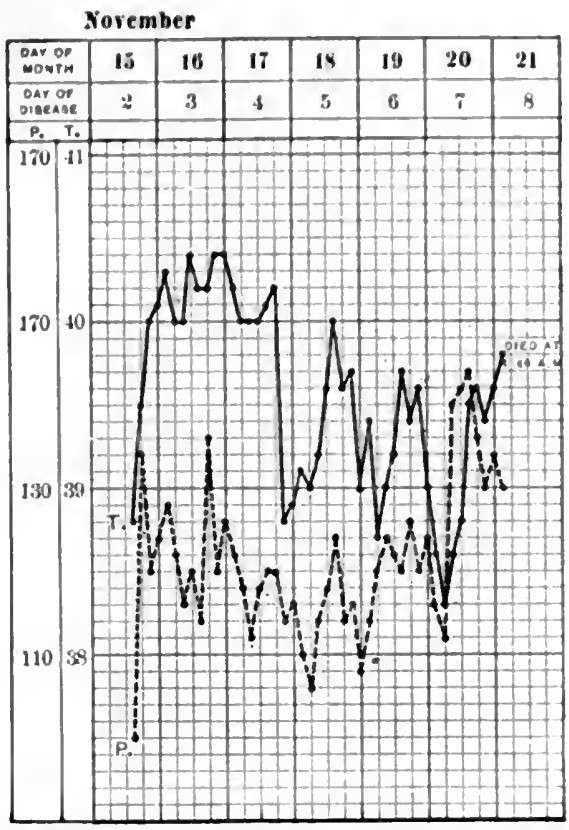

Case No. 5.-Hemorrhagic from the beginning. Albuminuria slight. Very high tem. perature and pulse-rate. Died on the eighth day. $T .=$ temperature; $P .=$ pulse-rate.

Treatment.- The treatment of yellow fever has been materially changed from the moment that scientific research demonstrated beyond the shadow of a doubt that we have to deal with a blood disease and not with a gastric or gastro-intestinal infection. All attempts to disinfect the digestive tract or in any way to affect the course of the disease by the administration of drugs that will act upon these channels, or their contents, must be abandoned or practised only with the purpose of meeting certain symptoms which shall be considered later.

Although the treatment of yellow fever may be considered in its greater part as expectant, there is no doubt that much good can be accomplished when cases are seen during the first three days, and in many cases the prognosis depends mainly upon whether patients come under the physician's eare within that time. Cases of continued fever, in which there is no distinct remission at the third or fourth day, always do badly and not rarely terminate fatally in spite of treatment. Besides these, there are a few cases which hardly give us time for action and from the very beginning show very pointedly their fatal termination. I do not remember to have seen any of these cases in which treatment has seemed to make any impression. It is evident that the anatomical lesions, the result of an unusually intense intoxication, are of such a degree of severity in these patients as to soon be beyond repair; thus we have seen at the autopsy table cases which have succumbed within a very short period, in which the hepatic degeneration was certainly as intense and 
general as in cases of acute yellow atrophy or phosphorus-poisoning. The kidners may also be the seat of a marked parenchymatous nephritis, instead of the usual congestion and cloudy swelling found in the ordinary cases of yellow fever.

Looking upon the subject from a general standpoint, it might be said that, in dealing with yellow-fever infection, our efforts should be directed mainly in two directions: in the first place, to sustain the normal vital forces of our patients, and, secondly, to assist in the elimination of the yellow fever toxin, whichever that may be.

The successful treatment of this disease depends largely upon the manner in which the symptoms are met or their occurrence prevented: hence, it is of the greatest importance that a careful clinical history of each case be made by a painstaking and observing nurse; unfortunatcly, a trained assistant cannot always be obtained in private practice, and less so in times of epidemic, so that it then becomes the duty of the physician to instruct those who take care of the cases upon the special dutics and the particular observations which should be recorded. If a temperature curve cannot be traced, at least the thermometer should be used every three hours and the temperature noted down as well as the frequency and character of the pulse. Every new symptom should be recorded and the hour of its appearance, its increasing or diminishing intensity, etc. The amount of urine should be measured, and it should be tested for albumin at every visit.

The removal of the patient from home to the hospital, whenever practicable, should be done at the very earliest moment and under the most careful instruction, so as to cause the least disturbance, both mentally and physically, not forgetting the simple measures to avoid infecting mosquitoes during the transfer.

If the case is to be treated at home, the patient must be removed to a distant room, where the greatest degree of quiet may be obtained: plenty of ventilation and sunlight should be provided, but the windows and doors must be kept screened until after the fourth day. The room in which the patient took sick and the other rooms in the building can then be fumigated without disturbing our patient.

Experience has shown that cases of yellow fever do very well in open tents, where free circulation of air is had by simply raising the side walls; whenever treated under these conditions, the danger of spreading infection is also minimized from the fact that inspection of the quarters can be more readily made and mosquitoes more effectively excluded by means of individual mosquito-bars.

As to the actual handling of the case, my plan has been to do nothing in the way of active medication during the first three days of the disease. It is rarely that any call arises for the administration of drugs at this stage, and I have seen cases where evident harm has resulted from needless therapeutics. 'The same method practically has been followed by the best authorities, although most of these recommend an initial cathartic. I could never see the reason for such practice, except it be to follow the routine of ancient times; it is not advised or done in the case of 
other infectious diseases, and no one has yet demonstrated that in yellow fever the disease follows a more favorable course, or in any wa becomes ameliorated by virtue of the preliminary purgative. ( ) f course, if the patient suffer from habitual constipation, or nomovement from the bowels has taken place during the previous two or three days, the indication in such a case is evident, almost regardless of the nature of the illness which called for our interference. I think that the routinc practice of administering a cathartic in all cases of fever is very properly being abandoned by modern clinicians.

I have always insisted upon absolute rest after giving the patient a cleansing bath. It is not sufficient for the pationt to take to his bed; he must be kept there, flat on his back, and on no account should he be permitted to leave it; a bed-pan must be provided, which should be used with the least disturbance to the patient, and it is better to use a feeding-cup or glass tube than to allow the patient to sit up for the purpose of drinking.

'The value of absolute rest during the first days of yellow fever has been recognized by all who have had wide experience in the treatment of this disease. Besides this, no food is allowed until the remission of the fever, on the third or fouth day, if this takes place; if the high temperature is continuous, - an unfarorable sign,- the nourishment must be still longer withheld. Some good results have been obtained from rectal feeding instituted at the outset, contimued during three or four days, and then suspended for two days, to be renewed again as before: since it is evident that our main defense consists in reinforcing the natural body powers of resistance, this method is worthy of more extended trial whenever practicable. The object in suppressing all food is principally to relieve the stomach from performing its functions, thus diminishing, in a marked degree, the intense irritability with which this organ is affected in yellow fever.

A certain amount of liquid, preferably iced, in the shape of weak bicarbonate of sodium solution (one drachm to the quart), or alkaline mineral waters of the Vichy type, or even mild lemonade, may be allowed to relieve the thirst caused by the fever, and in an indirect manner assist the kidneys in performing their function.

It must be understood that, while recommending in a general way that treatment be directed, hesides sustaining the patient's vital forces, also toward contributing to the elimination of the yellow fever toxin, I do not intend to advise that active diuretics, diaphoretics, etc., be employed, as I believe these are counterindicnted: their use would not be really aiding in the toxin elimination, but a forcing process instituted upon the functional activities of organs which it would be unwise to irritate; further, it would not be in accord with our present knowlelge of the final destiny of toxins in the infectious diseases.

Dr. John Guitéras, without the mention of whose name any writing upon this subject seems incomplete, holds that eliminative trentment is absolutely unnecessary in yellow fever, and to a certain extent we must rely for its cure upon neutralizing the toxins in the body of our patients,

Vol. I -57 
as we know not how or when they are eliminated. However, he recommends the administration of large quantities of water, "even up to eighty ounces in twenty-four hours," as well as calomel and jalap or castor oil at the beginning of the treatment.

To relieve subjective symptoms at this early period of the disease external applications are of the greatest benefit. 'The intense headache can be lessened by the use of an ice-cap, either upon the head or placed on the back of the neck; ice-water compresses to the forehead are often satisfactory.

The marked rachialgia may be treated by sinapisms upon the lumbar region, or, instead, massage of the muscles, or rubbing with alcohol or some aromatic lotion.

The fever demands our attention. Whenever the temperature reaches above $103^{\circ} \mathrm{F}$, an attempt should be made to lower it, preferably without the use of antipyretics. I believe the advisability of using the coal-tar derivatives in yellow fever quite questionable. Whenever they are used, it should be done with the greatest caution, and one should always be on the lookout for the first sign of their well-known depressing effects. Antipyrine and phenacetine are, perhaps, more to be recommended for their analgesic than their antithermic properties.

To lower the fever, cold water should be our standby: it relieves congestion, gives a sense of well-being after its use, promotes leucocytosis, and very effectively brings down the temperature. It is best applied in the form of sponge baths, repeated at four-hour intervals if the temperature rises above $103^{\circ} \mathrm{F}$. Tubbing or the Brand method is not applicable in vellow fever on account of the handling necessary; it certainly has never been tried as extensively as in typhoid fever, while the cold sponging may be readily carried out by a single attendant, without much disturbance to the patient. The temperature of the water should, as a rule, be not lower than $70^{\circ} \mathrm{F}$., but if little impression is thus made upon the fever, the water may be made still colder, even to $50^{\circ} \mathrm{F}$., by the addition of ice. Some observers recommend the use of alcohol in the water, others a certain proportion of common salt, etc. But there is no doubt that the benefit is derived mainly from the cold application itself.

As mentioned above, mild cases do well after the third or fourth day, and no further treatment than the one so far outlined is necessary; when, however, the fever rises again or does not remit at all, and vomiting and increased albuminuria set in, we are compelled to do more if we hope to save our patient.

Nausea and romiting are to a certain extent controlled by the absolute suppression of all feeding or medication by the mouth, aided by the application of cold (ice-cap) to the epigastrium or to the root of the neck in front; a mustard plaster to the epigastrium may be of use where the ice-cap fails to relicve the nausea.

For the nausea alone, effervescing drinks in small quantities often suffice; teaspoonful doses of the separate solutions of Seidlitz powders, or small amounts of ginger-ale; iced dry champagne, about half an ounce, 
repeated not too often (every three or four hours), besides relieving the nausea, contributes a stimulating effect, very desirable in some cases. It must be remembered that overloading the stomach with any liquid will promote vomiting, hence care and good judgment must be exercised in allowing the use of all drinks. In administering eracked ice, very useful sometimes, the actual amount must be measured, else $t(x)$ much liquid may thus be given unawares.

So soon as the presence of exuded blood is evident in the vomitus, the best practice is that followed by Guiteras, and one that I have no doubt has prevented in many cases the.injurious effects of profuse gastric hemorrbages. It consists in administering 5 drops of the tincture of the chloride of iron every two or three hours; this may be done in a small quantity of lime-juice and glycerine. I believe the iron chloride acts directly upon the mucous membrane, at the site where the hemorrhage would likely take place; hence it is best to stop its administration when the blood specks no longer appear in the vomitus, or whenever signs of increased gastric irritability manifest themselves: it is a fact that by this method vomiting is often decreased markedly.

Experience has shown the uselessness of the usual stypties in controlling the gastric hemorrhage, and adrenal extract, styptol, calcium salts, etc., have proved unaviling. For the bleeding of the gums, however, local applications of the common astringents are often satisfactory.

The problem of nourishment at this period of the disease is one that must be solved with the greatest care. 'The obvious necessity of administering but the scantiest amount of fluid by mouth, while the gastric irritability continues or is increased, as is often the case at the fourth or fifth day, compels us to resort to rectal feeding. This is best carried out on alternate days, so as to allow the mucous membrane a certain amount of rest that will prevent or at least defer rectal irritation. The best method is perhaps the injection of a mixture composed of-

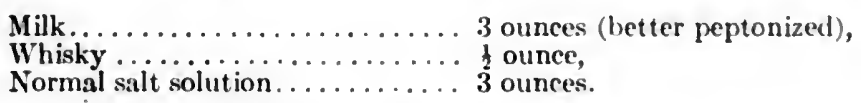

The enema should be administered very slowly, that it may be better retained, and repeated every four hours. Rectal feeding must be preceded by a cleansing enema of plain water.

If the vomiting has not been severe and other conditions of the patient permit it, at the sixth or seventh day a certain amount of milk may be allowed to be taken in small quantities, either pure or mixed with limewater or Seltzer water, as the case may be. 'This should never be done if the temperature remains high-above $102^{\circ} \mathrm{F}$.

The exact relation that the urinary secretion bears to the prognosis in yellow fever may be summed up in few words: the more urine is voided, the more favorable the prognosis. Anuria is uniformly fatal, and a defective kidney action at the beginning is, indeed, a most infavorable sign. The amount of albumin, on the other hand, is of less moment, and is not looked upon as an index worthy of much reliance; cases with great 
amounts of albumin and with abundant casts in their urine very often recover if the permeability of the kidneys is retained, while others prove fatal when only traces of albumin can be detected in the urine. For this reason the exact amount passed by the patient during the twentyfour hours should be measured, and, if possible, each emission kept separately for its analysis.

In this connection, as medicines by mouth must be aroided, enemas of normal salt solution are most useful, and if administered cold, they serve the double purpose of increasing urinary secretion and at the same time lowering the fever.. 'The amount given each time should be about one pint, by means of a long rectal tube, allowing the liquid to flow very slowly, so as to secure its retention. Of course, when rectal feeding is being resorted to, the enemas are prepared with an excess of salt solution, if it is desired to obtain the action upon the kidneys that I refer to here.

Besides the conditions so far presented here, the treatment of yellow fever consists mainly in supporting the patient's strength and meeting the various accidents which may arise from the defective action of important organs, some of which may well have been affected before the yellow fever infection took place. 'Thus we often have to deal with cases where the heart requires stimulation, leading us to the use of strychnine or digitalin ( $\frac{1}{60}$ or $-\frac{1}{12} 0$ grain, t. i. d.), which drugs should be used hypodermically. Digitalin may be particularly useful because it increases the urine at the same time it stimulates the heart; for its action upon the kidneys alone, however, it should never be employed; for this purpose Guiteras advocates the use of calomel in doses of 2 or 3 grains every four hours. Alcohol is a useful drug to support the heart, of special benefit in persons addicted to its use; it must be given very cantiously and mainly in enemas, except in case of great emergency, when, to relieve impending syncope, it may be used hypodermically, as well as ether, caffeine, or camphorated oil. When possible to administer it by mouth, champagne should be preferred, the next choice being whisky and soda.

'The serum treatment of yellow fever has not been attempted beyond the limits of the experimental field, by the use of human sera obtained from convalescents. In a series of 9 cases treated exclusively by these means I had only 1 death: there were no hemorrhages and the cases convalesced with marked rapidity. Other observers, however, have not followed out this line of investigation; I recognize the slight value of its practical utility.

The attempts at treatment with artificial sera have, of course, ended disastrously and need not be recorded here.

In these pages I have endeavored to give the main lines upon which the treatment of yellow fever should be carried ont, based principally upon personal experience. I have purposely left out all methods dependent on the idea that this infection is a gastro-intestinal one, as not being in accord with the modern conception of the etiology of the disease, and, therefore, misleading if not absolntely useless. I have also omitted to note special drugs advocated by different men and which have not 
received the sanction of other authorities upon the subject, or which have given the result claimed for them only in isolated cases.

'The names of 'Touatre, Sternberg, Chaille, Anderson, Finlay, and others perhaps ought to appear here; I set them down with pleasure: their methods of treatment do not differ very widely from that advocated by me, as in a general way they have been my teachers, except that their practice was obtained at a period when the disease was being studied from a different point of view.

Convalescence.-Cases of yellow fever which have gone through the so-called typhoid state recover very slowly, while the ordinary cases have a remarkably short convalescence; in fact, it is not unusual to see men about their daily work twelve or fifteen days after their initial chill.

The feeding of these convalescents demands a certain amount of care, and serious consequences have supervened from too early an allowance of solid or mixed food. 'The condition of the kidneys should serve to indicate when a more varied diet than milk and gruel should be allowed. If albumin is diminishing rapidly from the urine and the condition of the digestive tract permit it, meat-broth or mutton-broth gelatines may be given, and the quantity and variety of the food increased very gradually.

'The prolonged cases, and particularly those patients who have been profoundly debilitated by profuse hemorrhages and adynamic conditions, must be kept upon liquid diet for a longer period. Fach case must be considered on its own merits and treated accordingly: in some it may be even necessary to continue the administration of tonics for a time, while in others the simple regulation of the diet may suffice for a rapid and complete recovery. 



\section{INDE.X OF VOLUME I}

A

Abdominal compress, 235 therapeutic application of, $23 \mathrm{~S}$ neuroses, bydrotherapy for, $\mathbf{2 4 5}$

Ablution, 254 for typhoid fever, 254

Abnormal electrical reactions, $29 \mathrm{~S}$

Abscess complicating smallpox, 652 of tonsil complicating smallpox, 683

Accessory sinuses, carcinoma of, $x$-rays for, 345

Acetanilid for fever in tuberculosis, 785 for pleurisy, 732 untoward effects of, 115, 116

Acetic acid, dilute, in cholera, $8+7$

Acetphenetidine, untoward effects of, 116

Acids in cholera, 847

Acne, electricity for, 325 rosacea, $x$-rays for, 335 vulgaris, $x$-rays for, 335

Aconite group, 45 untoward effects of, 115

Aconitine, untoward effects of, 115

$\Lambda$ ddison's disease, adrenal extracts in, $56 \mathrm{~S}$ exercise in, 185

Adenitis, tuberculous, $x$-rays for, 350

Administration of exercise, 176

Adnexa, uterine, disease of, hydrotherapy for, 250

Adonidine, 44

Adrenal extract, 566

in Addison's disease, $56 \mathrm{~S}$

in asthma, $56 \mathrm{~S}$

in Graves' disease, 569

in lemorrhages of mucous membrane, 567

therapeutics of, 567 gland, 566

in whooping-cough, 569

Adrenaline, 48

Affusions, 254

for typhoid fever, 254

Agglutinins in serum therapy, 4S5

Air, fresh, for anemia in tulserculosis, 795 for night sweats in tuberculosis, 791

in tuberculosis, 745

Alcohol, 46

action of, on heart, 46

in cholera, 852

in diphtheria, $\mathrm{S} 17$

as food, 376
Acohol in influenza, sis

in plague, $87 \mathrm{I}$

in sinallpox, 676

in tuberculosis, $393,787,810$

Alkalies in acute articular rheumatism, $82 S$

Alkaline waters, 202

Alopecia complicating smallpox, 683

electricity for, 325

$x$-ray's for, 333

Altitude, high, 153

contraindications for, 153

diseases of nervous system, 154

heart disease, 154

nephritis, 154

tuberculosis, advanced, 1.54

indications for, 153

asthma, 153

convalescents, 1.53

tubereulosis, 15.3

in ifland climates, 137,146

low, 154

indications for, 154

in inland climates, 137,141

medium, 154

indications for, 154

in inland climates, 137,144

American waters, $21 \mathrm{~s}$

Amebic dysentery, hydrotherapy for, 249

Ammonia, 47

in cholera. 553

Amyl nitrite, 50

in cholera, 553

for hemoptysis in tubereulosis, 790

Anabolism, influence of exercise upon, 165

Analgesics, spinal, 38

Anaphylaxis, 114

Anemia, effect of high freguency currents on, 289

exercise in, 189

pernicious, diet in, 396

hydrotherapy for, 249

secondary, diet in, 396

in tuberculosis, 795

in yellow fever, $\$ 99$

Anesthesia, choice of, in tuberculosis, $\mathrm{SO}$

Aneurysn, diet in, 395

electricity for, 317

quiescent, exercise in, 1ss

Angina pectoris, diet in, 396 
Ankyloses, exercise in, 192

Anodynes, central, 38

$$
\text { coal-tar, } 39
$$

peripheral, 37

Anorexia in tuberculosis, 799

Anosmia, electricity for, 324 .

Anthraquinone series of cathartics, 52

Anthrax, serum therapy of, 512

Antidiphtheritic serum, 813

Antimeningitic serum, 688

Antimeningococcus serum, 504

Antimony, 49

Antipest sera, 868,871

Antipyretics in typhoid fever, 626 untoward effects of, 115

Antipyrinc for fever in tuberculosis, 785 for pleurisy, $7: 32$ untoward effects of, 115

Antiseptics, intestinal, in cholera, $\$ 47$ in typhoid fever, 627

Antitoxin, diphtheria, 490, 814,816 in cerebrospinal meningitis, 686 in scarlet fever, 649 in serum therapy, 485

Antityphoid inoculations, 539 vaccines, 540

Anus, fissure of, clectricity for, 321

Apilating agent, $x$-rays as an, 333

Appendicitis, diet in, 419 hydrotherapy for, 238, 239 in typhoid fever, 633

Appetite, 371

Arsenic for anemia in tuberculosis, 79.5 in tuberculosis, 810 untoward effects of, 116 waters, 211

Arteriosclerosis, climatic treatment of, 159

electricity for, 318

exercise in, 1 sis thyroid gland in, $5.5 \mathrm{~s}$

Arthritis, chronic, exercise in, 192 complicating smallpox, 683

deformans, climatic treatment of, 160 electricity for, 322

hydrotherapy for, 246

in scarlet fever, 651

Asiatic cholera, 835

Aspirin for pleuritic pain in tubcrculosis, 795

Asthenia in cholera, 850

Asthma, adrenal extract in, 568

bronchial, $x$-rays for, 353

climatic treatment of, 157

diet in, 394

electricity for, 324

exercise in, 189

Asthmatic attacks in tuberculosis, 79:3

Atony of stomach, diet in, 410

Atrophy, idiopathic muscular, electricity for, 317

progressive muscular, exercise in, 191

Atropine, 48, 49

sulphate in cholera, 853

for hemoptysis in tuberculosis, 792
Atropine sulphate for night sweats in tuberculosis, 791

Attitudes, faulty, exercise in, 192

Aural complications in influenza, 884

Autointoxication, hydrotherapy for, 243

\section{B}

BACILLEN emulsion tuberculin, 580

filtrate, tuberculin, 579

Bacilluria in typhoid fever, 634

Bacillus coli vaccine, 547

paratyphosus vaccine, $\mathbf{5 4 8}$

typhosus vaccine, 548

Bacterial immunity, 515 cellular theory of, 515

humoral theory of, $\mathbf{5 1 5}$

Bacterination in typhoid fever, 614

Bacteriolysins in serum therapy, 486

Bath, bed, 239

Brand, 250

carbon dioxide, 254

in cerebrospinal meningitis, 705

cold friction, 239

drip, 232

therapeutics of, 240

electrostatic, 281,282

hammock, 240,254

hip, 244

hot pack, 242

hot-air, for cholera, 855

hydro-electric, 292

sheet, $231,233,253$

Bathing in individual hygiene, 436

for night sweats in tuberculosis, 792

Battery, faradic, 282

galvanic, 269

Bedding, disinfection of, 473

in typlioid fever, 622

Belladonna, untoward effects of, 117

Beraneck tuberculin, 579

Beta-naphthol for diarrhea in tuberculosis, $\mathrm{SO} 2$

Beverages, composition of, 404

Bicarbonated alkaline waters, 202

Bier's hyperemia in acute articular rheumatism, 834

Bilious attacks in tuberculosis, 800

Biological effects, relations of chemical structure to, 20

of $x$-rays, 327

products, supervision of, in national liygiene, 456

Bismuth for diarrhea in tuberculosis, $\mathbf{8 0 2}$ untoward effects of, 117

Bladder, irrigation of, 249

Blaud's pills for anemia in tuberculosis, 795

Blisters for pleurisy, 731

Blood cells, effect of hydrotherapy on, 227

changes in cholera, 837

diseases of, diet in, 396

effect of high-frequency currents on, 289 
Blood pressure after administration of nitrites, 50

in cholera, $837,842,854,85.5$

drugs used to raise, 46 Calcareous alkaline mineral waters, 204

Carpase, 47, is

effect of high-frequency currents Calcium in food, 375 on, 289

of hydrotherapy oll, 2a-

high, exereise in, 189

serum for hemophilia, 513

for prevention of hemorrhage, $51: 3$

specific gravity of, in cholera, $8: 3$

tetanus antitoxin in, persistence of, 497

toxin in, 496

Boils in typlooid fever, 633

Bone cofiditions, exercise in, 192

lesions in typhoid fever, 634

Borax, untoward effects of, 118

Boric acid, untoward effects of, 118

Bowels, care of, in pneumonia, 714 in typhoid fever, 621

Brachial neuralgia, electricity for, 309

Bradycardia, exercise in, 188

Brain, diseases of, electricity for, 312 electro-diagnosis of, 312 excrcise in, 191

effect of galvanic electricity on, 277

Brand bath, 250

method in typhoid fever, 625

Bread, substitutes for, 429

Breathing exercises after thoracentesis in pleurisy; 737

Bromides, 38, 41

in cerebrospinal meningitis, 70.5 .

untoward effects of, 119

Bronchial asthma, $x$-rays for, 353

secretion, drugs used to affect, 62

Bronchitis, acute, diet in, 394 hydrotherapy for, 237

capillary, hydrotherapy for, 231

in cholera, 856

chronic, climatic treatment of, 156 exercise in, 189

indications for coast climate, 153 $x$-rays for, 353

liydrotherapy for, 238

in influenza, 880

in measles, 664

in typhoid fever, 633

use of water in, 201

Bronchopncumonia in diphtheria, $\$ 20$

in measles, 258,665

hydrotherapy in, $25 \mathrm{~s}$

Buboes in plaguc, 873

Bubonocele, exercise in, 190

Bubonic plague. See Plague.

Buccal discharges, disinfection of, 472

Buri's method of treating pockmarking

in smallpox, 683 3

Burns from $x$-rays, first degree, 328

second degree, 329

third degree, 329

Buttermilk as food, 373

in tubereulosis, 772,802

lactate, 562

for hemoptysis in tuberculosis, 791

metalolism, $: 375$

Californin, Southern, winter months in, 140

Calisthenic exercises, 179

Calomel in cholera, 845

Camploor, 47

Camphoric acid for night sweats in tuherculosis, 792

Cancer, thymus gland in, $5 \pi 0$

of urogenital tract, hydrotherapy for, 241

Cantharides, untoward effects of, 119

Carbohydrate food, 368

Carbohydrates in tuberculosis, 774

Carbolic acid. Sce also Phenol.

for plague, 869

untoward effects of, 119

Carbon dioxide bath, 254 for typhoid fever, 254

Carbonated alkaline waters, 202

Carbuncles in plague, 873

Carcinoma of accessory sinuses, $x$-rays for, 345

of breast, $x$-rays for, 341

of heart, $x$-rays for, 342

of larynx, $x$-rays for, 345

of stomach, diet in, 414

$x$-rays for, 341

Cardiac disorders in influenza, $8 \$ 2$ failure, lydrotherapy for, 237

stimulants, 46

tonics in cholera, 855

weakness in tuberculosis, 794

Cardiants, 32

Cardiovascular disense, diet in, 395 system, diseases of, electricity for, 317

in pneumonia, 720

in typhoid fever, 629

(ars, disinfection of, 480

Cascara sagrada for constipation in tuber culosis, 803

Castor oil in cholera, 847

Cataphoresis in galvanic electricity, 276

Catarrh, intestinal, ncute, diet in, 41 s

chronic, diet in, 418

hydrotherapy for, 24.5

uncomembranous, of intestine, diet in, 420

nusal, electricity for, 323

Cutarrhal conditions of mucous membranes, use of water in. 202

diseases, acute, hydrotherapy for, 244

hrdrotherapy for, 233

jaundice, diet in, 423

Cathartic oils, 52

Cathartics, 51 
Cathartics, anthraquinone series, 52 cathartic oils, 52 mercury, 54 resinous group, 53 salines, 51 therapeutic uses of, $54^{\circ}$

Cellular theory of bacterial inmunity,515 Central anodynes, 38

Cerates, 84

Cereals, composition of, 404

Cerebral excitement, hydrotherapy for, 241

galvanization, 313

Cercbrospinal meningitis, 655, 704 serum therapy of, 502

Chalybeate waters, 210

Chemical structure, relations of, to biological effects, 20

Chest compresses, 236 therapeutic application of, 237 strapping of, in pleurisy, 228 weights, exercises for, 181

Chickenpox, 670

Children, constipation in, 384

dose of drugs in, 30

feeding of, 380

food intoxications in, 381

gastritis in, 382

jliocolitis in, 383

intestinal jndigestion in, 383

rickets in, 384

typhoid psychosis in, 635

Chloral hydrate, untoward effeets of, 120

Chloroform, 42

as anesthetic for tuberculosis, 807 in cholera, 853

Chlorosis, diet in, 396 exercise in, 189

Cholecystitis in typhoid fever, 633 use of water in, 214

Cholelithiasis, diet in, 424

Cholera, 390, 835

acids in, 847

alcohol in, 752

ammonia in, 853

amyl nitrite in, 853

Asiatic, 835

asthenia in, 856

atropine sulphate in, 853

blood changes in, 837

pressure in, $837,842,854,855$

bronchitis in, 856

calomel in, 848

cardiac tonics in, 855

castor oil in, $\$ \$ 7$

chloroform in, 853

complications of, 856

convalescence in, 856

diarrhea in, premonitory, 835

in stage of reaction of, 852

diet in, 390,851

dry cupping for, 855

dysentery in, 856

fluid loss in, 836

method of replacing, 836

by mouth in, 846,854
Cholera, heart in, 855

hot-air baths for, 855

hyperpyrexia in, 851

inflammatory sequels in, 856

intestinal antiseptics in, 847

kidneys in, 853

medicinal agents in, 852

treatment of, 846

mercury perchloride in, 848

morphine in, 847

nephritis in, 854

opium in, 847

pathology of, $\$ 35$

permanganate of potassium in, 848

pneumonia in, 856

saline injections in, 837

intraperitoneal, 839 caunula for, 840

intravenous, 840

composition of, 843

indications for, 842

quantity of, 844

repetition of, 846

temperature of, 843

transfusion of, rate of, 844

rectal, 838

results of, 841

subcutaneous, 839

salve in, 848

serum therapy in, 508, 853

specific gravity of blood in, 837

stage of collapse in, 836,846

of copious evacuations in, 836 , $\$ 46$

of premonitory diarrhea in, 835

of reaction in, 850

summary of treatment of, 857

toxins in bowel, destruction of, 848 .

transfusion for, $\$ 40-\$ 46$

uremia in, 854

vasoconstrictor drugs in, 855

Chordee, hydrotherapy for, 245

Chorea, exercise in, 191

Sydenham's, electricity for, 313

Circulatory diseases, climatic treatment of, 158

stimulants, 55

Cirrhosis of liver, diet in, 424

Climate, coast, 136, 153

resorts, 138

desert, 138154

indications for, 154

inland, 134, 136, 137, 141, 153

altitude, high, 137,146

effect of, 137

low, 137, 141

medium, 137, 144

indications for, 153

ocean, 134,135

for tuberculosis, 745

types of, in United States, 134

for winter months, 141

Climatic considerations in tuberculosis, 745

fact ors, 133 
Climatic factors, financial resources, 134 homesickness, 134 individual peculiarities, 134 mental attitude of patient, 134 stage of disease, 134

therapeutics, definition of, 133 treatment, advantages of, 150 of arteriosclerosis, 159 of arthritis deformans, 160 of asthma, 157

of bronchitis, chronic, 156 of circulatory diseases, 158 contraindications for, 152 of convalescents, 157 of cough, chronic winter, 156 of diabetes, 160 of digestive disturbances, 160 disadvantages of, 150 diseases benefited by, 155 of emphysema, 157 of gout, 159 of heart disease, 158 indications for, 152 of kidney disease, 157 of nephritis, 157

of nervous disease, 161 of rheumatic conditions, 159 of tuberculosis, 155

Clothing, disinfection of, 473

in individual hygiene, 437

in tuberculosis, 780

Coal-t ar anodyues, 39 drugs for pleurisy, 732

Coast climate, 136,153 contraindications for, 153 tuberculosis, pulmonary, 153

indieations for, 153 bronchitis, chronic, 153 emphysena, 153 heart disease, 153 nephritis, 153 tuberculosis, 153 resorts, 138

resorts, summer, 139 winter, 139

Cocaine series, 37 untoward effects of, 120

Cocon as food, 376

Codeine, 38

Coffee as food, 763

Cold applications, continuous, 238 therapeutic application of, 239

for pleurisy, 729

friction batl, 239

physiological action of, in hydrotherapy, 223

Colds, acute, hydrotherapy for, 233 in tuberculosis, 796

Coley's toxins for sarcoma, 346

Collapse in croupous pneumonia, 724

Colic, chronic, hydrotherapy for, 2.35 gallstone, hydrotherapy for, 243 lead, hydrotherapy for, 249 nephritic, hydrotherapy for, 243
Collyrium, prescription for, 89

Communicable discase in communal hygiene, 443

in national hygiene, $45-4$

Compress, abdominal, 238

chest, 236

hot, for pleurisy, 730

local, 238

wet, $2: 36$

Congestions, internal, hydrotherapy for, 243

Conjunctival discharges, disinfection of, 473

tuberculin test, 599

Conjunctivitis in varicella, 671

Constipation in children, $3 \$ 4$

chronic, diet in, 421 use of water in, 215

electricity for, 320

hydrotherapy for, 245

spasmodic, electricity for, 320

in tuberculosis, $\mathrm{S03}$

Constitutional diseases, electrieity for, 322

Continuous cold applications, 235

Contractions, muscular, mechanical effects of, 164

Contraindieations for exercise, 176

Convalescence, exereise for, 185

Convalescents, climatic treatment of, 157

Convulsions, drugs used to check, 40

Cord, spinal, diseases of, electricity for, 310

Corneal ulcers complieating smallpox, 653

Cory'za in tuberculosis, 796

Cough, chronic winter, climatic treatment of, 156

in pneumonia, 718

in tuberculosis, 770,781

Counter-irritants for pleurisy, 730

Counter-irritation for pleuritic pain in tuberculosis, 794

Cretinism, thy roid gland in, 555

Creosote for cough in tuberculosis, $7 \mathrm{~S} 3$ and derivatives in tuberculosis, 809 for diarrhea in tuberculosis, 802

Cupping for pleurisy, 731

Currents, high-frequency, therapeutic action of, 290

Cutaneous horns, $x$-rays for, 338 tuberculin test, $59 \mathrm{~S}$

Cystitis, hydrotherapy for, $2+1$

\section{D}

D.AIRY products, composition of, 403

Day camps in dispensary treatment of tuberculosis, 756

Death, electricity as test for, 294 regist ration of, in communal iygiene, 442

Debility, general, exercise in, 191

Degeneration, reaction of. 300

Delirium in pneumonia, 719 
Delirium tremens, hydrotherapy for, Diet in glycosuria, moderately severe $234,235,241$

in typhoid fever, 630

Dermatitis, Röntgen, 329

Dermatologic paste, $\$ 3$

Dermatoses, pruriginous, $x$-rays for, 335

Desert elimate, 138

Detre's differential tuberculin test, 601

Diabetes, climatic treatment of, 160

diet in, 425 cures for, 428

electricity for, 323

exercise in, 191

insipidus, use of water in, 215

mellitus, tuberculosis and, 804

use of water in, 202, 204

Diarrhea, chronic, diet in, 4:1

hydrotherapy for, 241,245

infantile, dict in, 382

hydrotherapy for, 249

in measles, 667

premonitory, in cholera, 835

in scarlet fever, 657

in stage of reaction in cholera, 852

in tuberculosis, 801

in typhoid fever, 630

use of water in, 217

Diastasis recti, exereise in, 190

Diet in acute articular rheumatism, $\$ 28$ in anemia, 396

in aneurysms, 395

in angina peetoris, 396

in appendieitis, 419

in arterioselerosis, 395

in asthma, 394

in bronchitis, acute, 394

in cardiovascular disease, 395

in cerebrospinal meningitis, 704

in chlorosis, 396

in cholelithiasis, 421

jn cholera, 851

Asiatic, 390

in constipation, chronic, 421

cures for diabetes, 428

for obesity, 4:32

in diabetes, 425

in diabetic coma, 428

in diarrhea, chronic, 421

of infants, 382

in tuberculosis, 801

is diphtheria, 390

in diseases of blood, 396

of lings, 393

of stomach, $39 \mathrm{~s}$

in dysentery, 419

in dyspepsia, nervous, 415

in enteroptosis, 415

in fevers, 385

in gastric disorders, 406

disturbances, 407

irritability, 393

in gastritis, acute, 408

chronie, 408

in gastroptosis, 415

in glycosuria, in elderly persons, 427 mild forms of, 427

forms of, 428

severe forms of, 428

in young persons, 427

in gout, 396

in hemoptysis, 790

in hemorrhage from lungs, 393

in hemorrhoids, 421

in hyperchlorhydria, 415

in hypersecretion, 415

in influenza, 880,852

in intestinal catarrh, 418

diseases, 417

obstruction, 419

in intestines, mucomembranous eatarrh of, 420

nervous affections of, 420

in jaundice, eatarrhal, 423

list, Penzoldt's, 401

in liver, cirrhosis of, 424 diseases of, 423

in measles, 660

in nephritis, 397

for the nursing mother, 385

in obesity, 429

in peritonitis, 422

in pertussis, 389

in plague, 871

in pleurisy, 394,727

in pneumonia, 712

lobar, 394

in pregnancy, 384

in puerperium, 385

in rabies, 391

in rest cure, 359

salt-free, 433

scale, Leube's, 399

in searlet fever, 389,646

in scurvy, 425

infantile, 425

in smallpox, 389,675

in stomach, atony of, 410 careinoma of, 414

dilatation of, 409

ulcer of, 411

in tetanus, 391

in tuberculosis, 391, 767 of intestines, 393

in typhoid fever, $388 \quad 622$

in ulecrs, duodenal, 419 of intestine, 419

in whooping cough, 389

in yellow fever, $\$ 99,901$

Dietetics, 371

Digestion, hydrotherapy for, $24 \mathrm{~S}$

Digestive disturbances, climatic treatment of, 160

tract, diseases of, electricity for, 318

Digitalin, untoward effects of, 121

Digitalis, 48, 56

group, 43

for hemoptysis in tubereulosis, 791

untoward effects of, 121

Digitoxin, 45

Dilatation of stomach, diet iu, 409

Diphtheria, 813 
Diphtheria, alcolool in, 817

antitoxin, 490

accidents from, 495

administration by mouth, 40,

in cerebrospinal meningitis, $6 \times 6$

dosage in, 814, 816

early use of, 815

immunizing dose of, 816

nuture of, 490

as preventive, 493

production of, 491

rashes from, 494

results from, 494

in scarlet fever, 649

site of injeetion of, $\$ 15$

unit of, 491

use of, 492

bronchopneumonia in, $\$ 20$

cold applications in, 817

diet in, 390

intubation in, 819

irrigations of throat in, 818

laryngeal, 810

paralyses in, 818

serum therapy of, 489

strychnine in, 817

tracheotomy in, $\$ 20$

treatment of, local, 817 operative, 819

Direct elect ricity, 266

Disinfection, 469

of hedding, 473

of buccal discharges, 472

of ears, 480

of clothing, 473

of conjunetival discharges, 473

of feces, 471

formaldehyde for, 476

of instruments, 475

of nasal diseharges, 472

of rooms, 475

of ships, 481

of skin, 473

sulphur for, 47t

dioxide for, 4S0

in typhoid fever, 6:2

of urine, 472

of utensils, 475

Dislocations, exercise in, 192

Dispensary treatment of tubereulosis, 754

Distention, abdominal, in pneumonia,715

Distilled water, 200

Diuretics for pleurisy, 733

in pneumonia, 719

saline, 57

stimulating, 57

Dosage forms, extemporaneous, it

of galvanic electricity, 278

Doses of drugs, 98-10s

in children, 30

measures for, 85

Wright's, for vaccines, 534

Douche, 245,262

electrostatic, 2\$1, $2 \$ 2$

for neurasthenia, 262

prescription for, $\$ 9$
Douche, therapentices of, 246, 263

Doumer's reaction, :301

Dover's powder for pleuritic pain in euberculisis, 795

"Draughts," f('ar of, 751

Drinking water, 197

hot, 198

as source of infection in typhoid fever, 609

Drinks in pneumonia, 71.4

in tuberculosis, itit

Drugs, best method of dispensing, 98-105 circumstances modifying effects of, 2.5 combination of, 6.5

control of, in national hygiene, 4:5t

doses of, $95-108$

in children, 30

in fever in tubereulosis, 787

individual idiosynerasies to, 111

physiological action of, 32

relation of physiological action to

therapeutic uses of, 32

treatment of tuberculosis by, $\mathbf{8 0 9}$

in typhoid fever, 626

untoward effects of, 109

used to affect bronchial secretion, 62 to check convulsions, 40

to inercase quantity of urine, 5.5 circulatory stimulants, 55

saline diureties, $5 j$

stimulating diu-

respiration, 60 retics, 57

sweat, 59

to lower blood pressure, 49

to raise blood pressure, 46 cardiac stimulants, 46

vasomotor stimulants, 47

to relieve pain, 36

to slow the pulse, 4?

to soothe the respiratory centre, 61

Dry cupping for cholera, 8.55

bysentery, amebic, hydrotherapy for, 249

in cholera, 8.56

dict in, 410

Shiga's, hydrotherapy for, 249

serum therapy of, 50.5

Dysmenorrhea, neuralgie, hydrotherapy for, $24 j$

Dyspepsia, catarrhal, hydnotherapy for, 245

hydrotherapy for, 245

nervous, diet in, 415

hydrotherapy for, 245

Dyspnea in pneumonia, 721

in tuberculosis, 792

\section{$\mathbf{E}$}

Linn, diseases of, electricity for, 324

electrodes, 273 
Ear in scarlet fever, 652

Earthy alkaline mineral waters, 204

Eclampsia, hydrotherapy for, 235

Eczema, electricity for, 325 seborrheic, $x$-ray's for, 335

\section{Edema, 5.4}

of glottis complicating smallpox, 683 pulmonary, in pneumonia, 725

Eggs, 406

diet of, in tuberculosis, 772 as food, 373

Electric excitation, $17 \mathrm{~s}$ injection for pncumonia, 715 sleep, 326

vibratory massage, 301

physiological action of, 303

Electrical currents for industrial purposes, 294

friction, 281

reactions, 294,298

abnormal, 298

faradic current in, 298

galvanic current in, 298

sparking, 281

Electricity, 265

for acne, 325

for alopecia, 325

for aneurysms, 317

for anosmia, 324

for anterior poliomyelitis, 310

for arteriosclerosis, 318

for arthritis, 322

for astlınia, 324

for constipation, 320

for constitutional diseases, 322

for diabetes, 323

for diffuse myclitis, 312

for dilatation of stomach, 319

direct, 266

for diseases of brain, 312

of cardiovascular system, 317

of digestive tract, $31 \mathrm{~s}$

of ear, 324

of muscles, 317

of nerves, 306

of respiratory tract, $32: 3$

of skin, 325

of spinal cord, 310

of urinary tract, 321

for eczema, 325

for endocarditis, 317

for esophageal stricture, 318

for exophthalmic goitre, 316

laradic, 266

for fissure of anus, 321

Franklinic, 266

frictional, 266

for functional nervous disorders, 313 galvanic, 266

determination of polarity in, 269

Fleming cell in, 268

Grenet cell in, 267

Grove cell in, 268

McIntosh cell in, 268

negative pole, 269

Smee cell in, 267
Electricity, galvanic, varieties of cells in, 267

for gout, 323

for hemorrhoids, 274,321

for hypertrichosis, 326

for hysteria, 313

for idiopathic muscular atrophy, 317 indirect, 266

interrupted, 266

for intestinal occlusion, 320

for locomotor ataxia, 311

for lupus, 325

for nasal catarrh, 323

for nephritis, 322

for neuralgia, 308

brachial, 309

paresthetica, 310

sciatic, 310

trifacial, 309

for neurasthenia, 314

for neuritis, 306

facial, 306

of lower extremity, 307

median, 307

multiple, 306

musculospiral, 307

sciatic, 308

ulnar, 307

for obesity, 323

for otitis media, 324

for ozena, 323

for paralysis agitans, 316

for primary lateral sclerosis, 312

myopathy, 317

for pruritus, 325

ani, 321

for pulmonary tuberculosis, 324

for pyorrhea alveolaris, 319

for reflex vomiting, 319

for Rigg's disease, 318

for sciatica, 310

static, 266,278

accessories, 280

condensers, 280

conduction, 278

conductors, 280

electrodes for, 280

forms of, 281

induction, 278

insulating platform, 280

machines for producing, 279

Holtz, 279

Toepler, 279

Wimshurst, 279

methods of applying, 281

motor reaction with, 281

physiological effects of, 281

polarity of, 281

sensory reaction witl, 281

vasomotor effect of, 281

for Sydenham's chorea, 313

for syringomyelitis, 312

as a test for death, 294

therapeutic uses of, 306

for tinnitus aurium, 324

for urinary incontinence, 321 
Electricity for urticaria, 325; varieties of, 266 voltaic, 266

Electrification, Einhorn's intragastric, of stomach, 319

Electrodes, 271

car, 273

nasal, 273

pad, 272

rectal, 274

for static electricity, 280

stomach, 274

tongue, 273

vaginal, 274

Electro-diagnosis, 294

faradie reactions in, 295

galvanic reactions in, 296

Electrolysis in galvanic electricity, 275, 276

Electrolyte, 275

Electromotive force, 274

Electro-pathology, 292

galvanic current for, 293

high-frequency current for, 294

static machines for, 293

Elcetrostatic aigrette, 281,282

bath, 281, 282

douche, 281, 282

effleuve, 281,282

Electrotherapeuties, 265

Fliminauts, 532

Emphysema, climatic treatment of, 157 exercise in, 189 indications for coast climate, 153 in tuberculosis, 797

Employment after arrest of disease in tuberculosis, 763

Endoeardial troubles, hydrotherapy for, 239

Endocarditis, chronic, exereise in, $15 \mathrm{~s}$ electricity for, 317

Enteroclysis, hydrotherapy for, 248, 249

Enterocolitis, hydrotherapy for, $23 \mathrm{~S}$

Enteroptosis, diet in, $\mathbf{4 1 5}$

Environment in tuberculosis, 745

Ephemeral fevers, hydrotherapy for, 240

Epidemic cercbrospinal meningitis. Sce Meningitis.

Epidemics, management of, $45 \mathrm{~S}$ coöperation of public in, $\mathbf{4 6 1}$ depopulation of infected areas in, 466 destruction of insects in, $46 \mathrm{~S}$ of rodents in, 468

detection of infected food in, 461

educational measures in, 459

isolation of sick in, 463

protective inoculations in, 462

sanitary organization in, 460 survey of, 463

supervision of traffic in, 467

surveillance of contracts in, 465

Epilepsy, exercises in, 191 thyroid gland in, $55 \mathrm{~S}$

Fpistaxis in typhoid fever, 633

Epithelioma of lip, $x$-rays for, 340

of mouth, $x$-rays for, 340
Epithelioma of mucuus meontiranes, $x$-rays for, 339

Epitheliomata, $x$-rays for, 338

Eirgot, 48, 49

untoward effects of, 122

Erythrocytes, diminished, exercise in, 189

Eirythrol tetranitrite, 50

Esophageal stricture, elcetricity for, 318

Esophagus, carcinona of, $x$-rays for, 345

Exanthemata, 258

hydrotherapy in, 258

lixcretion, influence of exercise upon, 165

Exercise for abnormal cases, 186

in Addison' disense, 168

administration of, 176

in anemia, 189

in ancurysm, quiescent, 188

in ankyloses, 192

application of, 186

in arteriosclerosis, 188

in arthritis, chronic, 192

in asthma, 189

in bone conditions, 192

in bradycardia, 188

in bronchitis, chronic, 189

in bubonocele, 190

calisthenic, 179

execution of, 179

form of, 179

movements in, 179

for chest weights, 181 movements in, $1 \mathrm{Sl}$

in chlorosis, 189

in chorea, 191

in conditions with increased abdominal pressure, 190

intrathoracic pressure, 190

contraindications for, $176,18 \mathrm{~s}$

for convalescence, 188

in debility, general, 191

in cliabetes, 191

in diastasis recti, 190

in diminished erythrocytes, 159

hemoglobin, 189

in diseases of brain, 191

in dislocations, 192

in emphysema, 189

in endocarditis chronic, $18 \mathrm{~S}$

in epilepsy, 191

in faulty attitudes, 19:2

in flat foot, 19:

in fractures, 192

in functional diseases, 191

games for, 179

general, $16: 3$

in gout. 191

in hemi:o, 190

in high blood pressure, 1sS

in hysteria, 191

indientions for, 175

influence of, upon anabolism, lfis upon exeretion, las upon function, $16-1$ upon katabolism. 165 upon structure, lfs

in joint conditions, 192 
Exercise, katabolic products of, hormone action of, 165

in locomotor ataxia, 191

in mental diseases, 191

in myocarditis, chronic, 188

in nephritis, $1 S S$ chronic, 190

in nervous fatigue, 191

in neurasthenia, 191

in neuritis, 191

for normal individual, 186

in paralysis, 191

agitans, 191 spastic, 191

in paresis, 191

in pericarditis, chronic, $1 S 8$

physiological effects of, 172

physiology of, 16:3

in pneumonia, chronic, 159

in poliomyelitis, 191

in progressive muscular atrophy, 191 proplyylactic use of, 170

in rheumatism, 191

in scar weakness in abdominal wall, 190

in scoljosis, 192

for sedentary habits, 186

sports for, 179

in subluxations, 192

in tachycardia, 188

therapeutic use of, 170

in tuberculosis, 190,757

untoward effects of, 173

in varices, 188

varieties of, 177

eleetric excitation, $17 \mathrm{~s}$

games, 179

gymnasium apparatus, 781

massage, 177

mechanical percussion, $17 i$

passive motion, $17 \mathrm{~S}$

sports, 179

Zander machines, $17 \mathrm{~s}$

Exercising devices. See after page 193

Exophthalmic goitre, electricity for, 316 thymus gland in, 570 thyroid gland in, 556 $x$-ravs for, 352

Extremities, bandaging of, for hemoptysis in tuberculosis, 791

Extremity, lower, neuritis of, electricity for, 307

Eye complieations in measles, 669

Eyes, care of, in pneumonia, 712

\section{F}

FACE, pockmarking of, in smallpox, 68 . Facial neuritis, eleetricity for, 306

Faradic hattery, 282

eurrent in ahnormal electrieal reactions, 298

for hydro-electric haths, 292

electricity, 266, 2\$2

cffect of, on nerves, 283,284
Faradic electricity, electrodes in, 284

methods of applying, 284

physiological action of, 284

short circuiting in, 282

reactions in electrodiagnosis, 295

Faradism for neurasthenia, 315

Fatigue, nervous, exercise in, 191

Fats, 406

as food, 368,374

in tuberculosis, 773,800

Favus, $x$-rays for, 334

lebrile affections, hydrotherapy for, 238 diseases, hydrotherapy for, 233, 240

Feces, disinfection of, 471

leeding of children, 380

in fever's, 385

forced, in gastric disturbances, 407

of infants, 380

in measles, 661

in plague, 872

in pneumonia, 717

and pregnancy in typhoid, 635

rectal, 377

in scarlet fever, 650

in smallpox, 678

suppurative, in smallpox, 681

in tuberculosis, 785

in yellow fever, 898

Fermented milk for tuberculosis, 772,802

Fevers, diet in, 385

ephemeral, hydrotherapy for, 240

"Fireless cooker," 770

Fish, 406

composition of, 404

as food, 373

Fissure of anus, electricity for, 321

Fistula, hydrotherapy for, 249

Fistula-in-ano in tuberculosis, 803

Flat foot, exercise in, 192

Fleischer's table, 416

Fleming cell in galwanic electricity, 268

Flies, as source of infection in typhoid fever, 613

Florida, winter months in, 140

Fluid by mouth in cholera, 846

Fly hlister for cough in tuberculosis, 783

Folliculitis of beard, $x$-rays for, 334

Fomentations, hot, 244

for pleurisy, 730

Food, absorbability of, $36 \mathrm{~s}$

alcohol, 376

buttermilk, 373

calcium, 375

carbohydrate. 368

classes of, 372

cocoa, 376

coffee, 376

composition of most common, 403

control of, in national hygiene, 457

eggs, 37.3

fat, 368,374

fish, 374

fuel value of, $36 \mathrm{~S}$

relatinous forms of, 406

infected, detection of, in epidemies, 461 
Food intoxications in eluldren, 381 iron, 375

liquid, 40:5

meats, 373

milk, 372

nutrition and, 367

phosphorus, 375

protein, 367

regulation of, in communal hygin.ne, 445

salts, 375

as source of iufection in typlosicl fever, 612

sugar, 374

tea, 375

vegetalsles, 37.4

water, 375

Foreign waters, 218

Formaldehyde for disinfection, 476

Fractures, exercise in, 192

Franklin electricity, 260

liresh-air treatment in pneumonia, 709

Frictional elect ricity, 266

Fruits, composition of, 104

Function, influence of exercise upom, 165

Functional diseases, exercise in, 191

nervous disorders, electricity for, $31: 3$

Furunculosis complicating smallpox, 6S2

\section{G}

Gallstove colic, hydrotherapy for, 243

Gallstones, use of water in, 214

Galvanic battery, 269

current in abnormal eleetrical reactions, 298

for electropathology, 293

for hydro-electric haths, 292

electricity, 266, 275

applieation of, 278

cataphoresis in, 270

dosage of, 278

effect of, on brain, 27 motor, 277 on musele, non-strialed, 277 physiological, 277 sensory, 277 variations of current, 277

electrolysis in, 275, 276

method of application of, $27 \%$

physiological action of, 275

polarity in, effects of, 276

therapeutics of. 278

reactions in electrodiagnosis, 296

Galvanism for neurasthenia, 315

Galvanization, cerebral, 313

Galvanofaradic currents, 25.5

for hydro-elect ric baths, 292

physiologieal action of, 285

Game, composition of. 403

Games for exercise, 179

Gangrene of skin in varicella, 673

Gargle, prescription for, 90

Gastric disorders, diet in, 40\%

disturbances, diet in, 407
Gastric dinturhanceon, furcerd fordling in, $410 \%$

kavage in, toi

grape cure it, 4117

milk curve in, $40 \%$

rest cure ind, fils

in tulsereulusis, 795

irritalbility, dlict in, 3983

neurosis, hyelrotherapy for, 24s

Gastritis, acutc, diet in, diss

in children, :25:3

chronic, diet in, fos

hydrutherapy for, $2: 25$

11:' of water in, :20:3

(iastro-intestinnl complications in influ'nuza, sis?

trace in mensles, 667

in searlet fiver, 6.57

(iastroptosis, diet in. 415

Gavare in gastric disturbaners, flo

(ierman measle:s, 669

Gianders, visceines for, 549

Gilandular colargement in scarlet fever, 6ii2

therapy, 5.51

general consideration of, 551

Glottis, edema of, complicating smallpxix, 6S3

Gilycerogelatin as ointment bave, s:3

Glyeosuria, inild forms of, diet in, 427

Givitre, exophthulmic, $x$-rays for, 352

Gonococcus infections, serum therapy of, 511

vaccine, $5+16$

Gout, 264

climatic treatinent of, 159

diet in, 396;

electricity for, 323

exercise in, 191

hydrotherapy for, 243, 246, 264

use of water in, 202, 212

Graduated labor in tubereulosis, 760

Grape cure in gastrie disturbances, 407

Graves' disease, adrenal extract in, 569 thyroid gland in, 556

Grenet cell in galvanie electricity, 26i

Grove cell in galvanic electricity, 265

Guaiacol earbonate for cough in tulerculosis, 7S4

for cough in tuberculosis, 784

Gymnasium apparatus, $17 \mathrm{~S}$

Gymnastics, pulmonary, in tuberculosis, 765

\section{H}

H.AFrktNE's pmphylactic for plague, \$6.5

Hair, fulling out of, from $x$-rays, 329

Hanmock inth, 2.10, 254 therapeut ic applieation of, 241

for typhoil fever, 251

IIeadaches, hydmotherapy for, 233

in smallpox, 675

in typhoid forver, 629

Ifealth nesorts, types of, in Cnited states, 134,135

vol. I. $-5 S$ 
Heart, action of alcohol on, 46 in acute articular rheumatism, 830 carcinoma of, $x$-rays for, 342 in cholera, 855

disease, climatic treatment of, 158 indieations for coast climate in, 153

failure, hydrotherapy for, 237 in typhoid fever, 629

in influenza, 884

in measles, 666

in pneumonia, 720

in searlet fever, 656

in smallpox, 678

in yellow fever, 900

Heat, physiological action of, in hydrotherapy, 223

Hemoglobin, diminished, exercise in, 189

Hemophilia, blood serum for, 513

Hemoptysis, diet in, 790

in tubereulosis, 787

Hemorrhage, blood serum for prevention of, 513

from lungs, diet in, 393

of mucous membrane, adrenal extract in, 567

in typhoid fever, 630, 635

Hemorrhagic searlet fever, 657

Hemorrhoids, diet in, 421

electricity for, 274,321

hydrotherapy for, 245

Hernix, exercise in, 190

Heroin, 38,62

for pleuritic pain in tubereulosis; 795

High-frequency current, 265, 287

- accessories for, 288

application of, 290

effect of, on anemia, 289

on blood, 289

pressure, 289

on cxcitability, 289

local, 289

on respiration, 289

on urine, 289

for neurasthenia, 315

physiological action of, 289

Hip bath, 244 therapentic action of, 290

Hoarseness in tuberculosis, 797

Hodgkin's disease, $x$-rays for, 351

Holtz machine for producing static electricity, 279

Home, sanitation of, in individual hygiene, 440

treatment of tuberculosis, 748

Homesickness as a elimatic factor, 134

Hormone action of katabolie products of physical exercise, 165

Hospitals, isolation, for scarlet fever, 642

Hot-air baths for cholera, 855

Hot bath pack, 242

therapentic application of, 243

fomentations, 244

therapeutic application of, 244
Housing, regulation of, in communal hygiene, 448

Humoral theory of bacterial immunity, 515

Hydrochloric acid, dilute, in cholera, 847

Hydro-electric baths, 291

faradic current for, 292

galvanic current for, 292

galvano-faradic current for, 292

physiological effect of, 292

sinusoidal current for, 292

Hydrophobia, serum therapy of, 512

Hydrotherapeutic law of Baruch, 222

procedures, ablution, 228

affusion, 230

dosage of, 222

technique of, 228

Hydrotherapy, 221

for abdominal neuroses, 245

in acute articular rleumatism, 833

catarrhal diseases, 244

colds, 233

nephritis, 241

for appendicitis, 238,239

for arthritis, 246

for auto-intoxication, 243

for bronchitis, 237, 238

in bronchopneumonia in measles, 258

for eancer of urinary tract, 241

for capillary bronchitis, 231

for cardiac failure, 237

for catarrhal diseases, 233

jaundice, 249

for cerebral excitement, 241

for chordee, 245

for colic, chronic, 238

for constipation, 245

for cystitis, 241

for delirium tremens, 234, 235, 241

for diarrhea, 241, 245

for digestion, 248

for disease of uterine adnexa, 250 of uterus, 250

for dysentery, 249

amebic, 249

Shiga's, 249

for dyspepsia, 248

catarrhal, 248

nervous, 248

for eclampsia, 235

effect of, on blood cells, 227

pressure, 227

general, 224

local, 223

metabolic, 225

on muscle power, 227

on nervous system, 228

respiratory, 225

temperature, 226

for endocardial troubles, 239

for enteroclysis, 248,249

for ephemeral fevers, 240

in exanthemata, 258

for febrile affections, 238

diseases, 233, 240

for fever in tuberculosis, $7 \& 7$ 
Hydrotherapy for fistulas, 249

for gallstone colie, $24: 3$

for gastric neurosis, 248

for gastritis, 23s

for gout, 243, 246, 264

for headaclic:s, 2:33

for heart failure, $2: 37$

for hemorrhojds, 245

for hyperemia, 243

for hyperemesis gruvidarmm, 2:w

for hysteria, $\mathbf{2 4 6}$

for infantile dinrrhen, 249

for influenza, $23: 3$

for insanity, 236

for insomnia, 262

for internal congestions, $24: 3$

for intestinal catarrh, 245

for lead colic, 249

for locomotor ataxia, 241

for lumbago, $243,244,246$

for mania, 236, 241

in measles, 258,662

for melancholia, 236

for meningitis, 241

for metabolic activity, 2.35

for muscular rleumatism, 24;

for myelitis, 241

for myocarditis, 239

for nepliritic colic, 243

for nephritis, $233,235,243$

for neuralgia, 233, 243

for neuralgic dysmenorrhea, 245

for neurasthenia, 246,260

for neuritis, 243

for neuroses, 239

ncurovascular training $\mathrm{l} y, 230$

for obesity, 243

for paresis, 236

for paroxysmal tacliyeardia, 2:39

for pericarditis, 239

for peritonitis, 239

for pernicious anemia, 249

for phthisis, 237, 260

physiological action of cold in, 223 of heat in, 223

for pneumonia, 233, 237, 238, 259, 716

for prolonged menstruation, 245

for prostatic disease, 249

for renal insufficiency, 249

for rheumatism, 246, 264

for scarlatina, 231,235

in scarlet fever, 253

for sciatica, 243, 244, 246

for stiffened muscles, 244

for sunstroke, 234,259

for synovitis, 246

for tencsmus, 245

for tonsillitis, 233, 23i

for tuberculosis, $238,239,260$, $7 \mathrm{~A}()$

for tympanitis, $2: 38$

for typhoid fever, 241, 250, 25.5, 62.4

for ulcer of stomach, 233

for uremia, 249

for urethritis, 245

for vaginal injections, 249
Hydrotherapy for vasomoter relaxation, 23.5

Hydrons wool fat as ointment Lase, 83

Hygienc, conmunal, 441

communical, le diseases in, 443

dixpossal of dead in, 449

prevention of nuivances in, 4.49

registrution of desthe in, $\$ 42$ of sickness in, 4.2

regulation of dangerous trades in, $\cdot 1-17$

of frods in, 4.45

of lousing in, 44s

of offensive trades in, $4 \mathbf{4}$

relation of state and local ans-

thorities in, 450)

removal of refuse in, 446

of sewage in, 146

supervision of water supplies in, 444

individual, 435

hathing in, 436

hodily cleanliness in, 436

clothing in, 437

pure air in, 43S

rest in, 439

sanitation in the home in, 440

sleep in, 439

sunshine in, 435

national, 450

communicable diseases in, 454

control of drugs in, 4S7 of foods in, 457

educational measures in, 456

foreign quarantine in, 451

insular quarantine in, $\mathbf{4 5 1}$

interstate quarantine in, 45.3

medical inspection of inmigrants in, 452

prevention of pollution of waters in, 455

relation to local authorities in, $45 \mathrm{~s}$

to State authorities in, 455

sanitary agreements in, $45^{\circ} 2$ reports in, 4.5 .5

statistics in, 455

supervision of biological produets in, 456

Hygicnic measures, 435, 441

Hyperacidity of stomach and turinary

tract, use of water in, $20-4$

Hyperemia, hydrotherapy for, $24: 3$

Hyperehlorhydria, diet in, 415

use of water in, 202

Hyperemesis gravidarum, hydrotherapy for, 238

Hyperpyrexia in acute articular rheumatism, $\mathbf{S 3 1}$

in cholern, 851

in scurlet fever, 650

IIypersecretion, diet in, 115

Ilypersensibility to tuberculin, 55s

Hypertrichosis, electricity for, 326

$x$-rais for, 333

Hypnotics, 36 
Hypophosphates in tuberculosis, 810

Hysteria, electricity for, 313

exercise in, 191

hydrotherapy for, 246

\section{I}

ILEOCOLITIS in children, 383

Immigrants, medical inspection of, in national hygiene, 452

Immune bodies, Spengler serum, 602

Immunity, bacterial, 515

Immunization, serum therapy and, 488 in typhoid fever, 614

Immunizing dose of diphtheria antitoxin, 816

Incontinence, urinary, electricity for, 321 Indigestion, intestinal, in children, 383

Indirect eleetricity, 266

Infantile diarrhea, hydrotherapy for, 249

Infants, diet in diarrhea of, 382 feeding of, 380

Infections, paratyphoid, 637

Infectious diseases, 607

Influenza, 875

alcohol in, 878

bronchitis in, 880

cardiac disorders in, 882

coal-tar drugs. in, 877

complications in, 881

aural, 884

gastro-intestinal, 883

mental, 885

nervous, 885

respiratory, 881

convalescence in, 886

diet in, 880,882

heart in, 884

hydrotherapy for, 233

kidneys in, 884

nephritis in, 884

pain in, 877

prophylaxis in, 876

psychoses in, 885

quinine in, 878

respiratory symptoms in, 879

rest in, 876

stimulants in, 878

strychnine in, 879,886

treatment in, 876

Inhalations in tuberculosis, 810

Injections, vaginal, hydrotherapy for, 249

Inland climates, 134, 136, 137

Inoculation, protective, in epidemies, 462

Insanity in children in typhoid fever, 635 hydrotherapy for, 236 post-typhoid, in typhoid fever, 635

Insects, destruction of, in epidemies, 468 as source of infection in typhoid fever, 613

Insomnia, 35, 262, 365

hydrotherapy for, 262

in tuberculosis, 804

in typhoid fever, 630

Interrupted elcctricity, 266
Intestinal antiseptics in cholera, 847

in typhoid fever, 627

catarrh, diet in, 418

diseases, diet in, 417

indigestion in children, 383

occlusion, electricity for, 320

Intestines, carcinoma of, $x$-rays for, 345

Intoxications, food, in children, 381

Intraperitoneal saline injections in cholera, 839

Intravenous saline injections in cholera, 840

Iodide of potassium in tuberculosis, 804

Iodides, untoward effects of, 122

Iodine, tincture of, for pleurisy, 731 in tuberculosis, 810 untoward effects of, 122

Iodobromic waters, 212

Iodoform, untoward effects of, 123

Iroll as food, 375

in tubereulosis, 810

untoward effects of, 124

waters, 210

Irrigations, 247

therapeutic application of, 248

Isolation hospitals for scarlet fever 642 of sick in epidemies, 463

JAundice, catarrhal, diet in, 423

hydrotherapy for, 249

Joint conditions, exereise in, 192

Joints in scarlet fever, 654

\section{$\mathbf{K}$}

Katabolic products of exercise, hormone action of, 165

Iatabolism, influence of exercise upon, 164

Kefir for tuberculosis, 772, 802

Keloid, $x$-rays for, 337

Kidney in eholera, 853 disease, climatic treatment of, 157

Kidneys in influenza, 884

in measles, 669

in scarlet fever, 655

in yellow fever, 899

Iienbock quantimeter, 328,348

Koumiss for tuberculosis, 772,802

$\mathbf{L}$

IABOR, graduated, in tuberculosis, 760

Iactic acid bacteria for diarrhea in tuberculosis, 802

Iaryngeal diphtheria, 819 spasm in measles, 663

Larynx, carcinoma of, $x$-rays for, 345 in measles, 662

Lead colic, hydrotherapy for, 249 poisoning, 124 
Lead, untoward effects of, 124

Leube's diet scale, 399

Jeukocyte extract in cerebrospinul mesingitis, 656

Ieukocytic extract in serum therapy, \&:7

Leucorrhca in tubereulosis, $\mathrm{SO}$ )

I.eukemia, $x$-rays for, 349

Lichen cordeus, $x$-ray's for, 336 ; ruber planus, $x$-rays for, $3: 36$

I iniment, alcoholic, prescription for, 90 oily, prescription for, 91 soupy, prescription for, 91

I.ithia water, 203

Liver, cirrhosis of, dict in, 424 disease of, dict in, 423

Iocal compresses, $2: 38$

Locomotor ataxia, electricity for, 311 exercise in, 191 hydrotherapy for, 241

I.otion, prescription for, 89

I.tumbago, liydrotherapy for, $243,244,246$

Lumbar puncture in ccrebrospinal meningitis, 686

Ilungs, diseases of, dict in, $39: 3$

I.upus, elcetricity for, 325 ery thematosus, $x$-rays for, 337 vulgaris, $x$-rays for, 336

I.ymphadenoma, $x$-rays for, 351

lymphatic glands, $x$-rays for, 350 leukemia, $x$-rays for, 350

\section{$\mathbf{M}$}

McIxtosu cell in galvanic electricity, 268 rheotome, 284

Magnetism, 303

Malignant growths, thymus gland in, 570 thyroid gland in, 559

Mania, hydrotherapy for, 236, 241

Maragliano's serum, 601

Marmorck's antituberculin serum, 60)'

Massage, 177, 285

for obesity, $4: 31$

vibratory, clectric, 301

Measles, 258, 658

arrangement of room, 6.59

hronchitis in, 664

bronehopneumonia in, $25 S, 659$

diarrhen in, 667

diet in, 660

eye complications in, 669

fever in, 661

gastro-jntestinal tract in, 667

German, 669

heart in, $666^{\circ}$

hydrotherapy in, 258, 662

kidneys in, 669

laryngeal spasm in, 66:3

larynx in, 602

pharyngeal irritability in, 663

pharynx in, 662

prophylaxis in, 658

skin in, 668

stimulants in, 660

stomatitis in, 667
Meat, 406

composition of, 403

diet in tulsereulosis, 753

as food, 373

Median neuritis, electricity for, 307

Medical inspection of iminigrants in national hygiene, 452

Melancholia, hydrotherapy for, 2:;6

Meningitis, 685

cerebrospinal, 685, 704

baths in, 705

bromides in, 705

charts showing dose of serum for, $695,697,699$

cold applications in, 705

diet in, 704

diphtheria antitoxin, 686

forms of, 685

lenkocyte extraet, 656

lumbar puructure in, 686

pain in, 705

rare forms of, 704

rigidity of limbs in, 705

serum treatment of, 502 , 686 , $6 \mathrm{~S} 6$

administration of, 690

frequency of, 696

indications for, 693

technique for, 690

in chronic cases, 700

dose of, size of, 694

in fulminating cases, 697

nature of, 658

low obtained, 689

in prolonged cases, 698

in relapses, 699

in resistant cases, 700

summary of, 701

value of, $6 \mathrm{~s} S$

stimulants in, 705

symptomatic treatment of, 704

urotropin in, 706

vaccine therapy in, $6 S f$;

chronic, hydrotherapy for, 241

pneumococcus, $70: 3$

staphylococcus, 703

streptococcus, 703

syphilitic, 704

tuberculous, 702

in typhoid fever, 634

vaccines for, 547,703

Meningococcus vaccine, 547

Menstrual disturbances in tuberculosis, 805

Menstruation, prolonged, hydrotherapy for, 245

Mental complications in influenza, SS5 diseases, exercise in, 191 occupation in tulereulosis. 766

Menus for tuberculosis, $775,776,777,775$, 779,780

Mercury as cathartic, 54 perchloride in cholern, 845 in tuberculosis, 804,810 untoward effects of, 125

Metabolic activity, hydrotherapy for, $\mathbf{2 3 5}$ 
Metabolic disturbances, thyroid gland in, 556

effect of hydrotherapy, 225

Metabolism, calcium, 375

Meteorism in pneumonia, 715

Metric system, 87

Micrococcus catarrhalis vaccine, 547 neoformans vaccine, 547

Mikulicz's disease, $x$-ray's for, 352

Milk cure in gastric disturbances, 407 for obesity, 432

diet in tuberculosis, 771

as a food, 372

peptonized, in gastric disturbances in tuberculosis, 800

as a source of infection in typlooid fever, 612

Milliamperemeter, 271

Mineral springs, 195

waters, radio-activity of, 196

Morphia, untoward effects of, 127

Morphine, 38, 62

in cholera, $8+7$

for dyspnea in tuberculosis, 793 in pneumonia, 720

Morro reaction of tuberculin, 600

Mosquitoes in yellow fever, danger from, 889

Motor effect of galvanic electricity, 277 reaction with static electricity, 281

Mouth, care of, 380

in pneumonia, 711

wash in pneumonia, 712

prescription for, 89

Multiple neuritis, electricity for, 306

Murphy treatment in typhoid fever, 626

Muscle, non-striated, effect of galvanic electricity on, 277

power, effect of hydrotherapy on, 277

Muscles, diseases of, electricity for, 317 stiffened, hydrotherapy for, 244

Muscular atrophy, idiopathic, electricity for, 317

electrodiagnosis of, 317

electrotherapeutics of, 317

contractions, mechanical effects of, $16 t$

Musculospiral neuritis, electricity for, 307

fermented, for tuberculosis, 772 , 802

Mustard plaster for pleurisy, 731

Myasthenic reaction, 301

Mycosis fungoides, $x$-rays for, 337

Myelitis, diffuse, electricity for, 312

hydrotherapy for, 241

Myclogenous leukemia, $x$-rays for, 349

Myc arditis, chronic, exercise in, 188 nydrotherapy for, 239

Myopathy, primary, electricity for, 317 electrodiagnosis of, 317 electrotherapeutics of, 317

Myotonic reaction, 301

Myxedema, thyroid gland in, 555
$\mathbf{N}$

Nevus, $x$-rays for, 338

Nasal catarrh, electricity for, 323 discharges, disinfection of, 472 electrodes, 273 spray, prescription for, 90

Nastin, Tb.-A. tuberculin, 580 Tb.-L. tuberculin, 580

Nausea in typhoid fever, 630 in ycllow fever, 898

Nephritic colic, hydrotherapy for, 243

Nephritis, acute, hydrotherapy for, 241 in cholera, 854 chronic, exercise in, 190

climatic treatment of, 157

diet in, 397

electricity for, 322

exercise in, $18 \delta$

hydrotherapy for, $233,235,243$

indications for coast climate, 153

in influenza, 884

in scarlet fever, 655

use of water in, 201, 213

Nephtria, 366

Nerves, diseases of, electricity for, 306

Nervines, 32

Nervous affections of intestines, diet in, 420

disease, climatic treatment of, 161

complications in influenza, 885

disorders, functional electricity for, 313

excitement in typhoid fever, 630

manifestations in smallpox, 678

system, effect of hydrotherapy on, 228

Neuralgia, brachial, electricity for, 309

electricity for, 308

hydrotherapy for, 233,243

paresthetica, electricity for, 310

sciatic, electricity for, 310

trifacial, electricity for, 309

Neuralgic dysmenorrhea, hydrotherapy for, 245

Neurasthenia, 260, 355

douche for, 262

electricity for, 314

exercise in, 191

faradism for, 315

galvanism for, 315

high-frequency currents for, 315

hydrotherapy for, 246,260

Ncuritis, electricity for, 306

exercise in, 191

facial, electricity for, 306

hydrotherapy for, 243

of lower extremity, electricity for, 307

median, electricity for, 307

multiple, electricity for, 306

musculospiral, electricity for, 307

sciatic, electricity for, 308

in typhoid fever, 635

ulnar, electricity for, 307

Neuroses, abdominal, hydrotherapy for, 
Neurosis, gastric, hydrotherapy for, 245 hydrotherapy for, 239

Neurovascular training by hydrotherapy, 230

Night camps in dispensary treatment of tuberculosis, 755 sweats in tuherculosis, 791

Nitrite, amyl, in cholera, 853 sodium, 50

Nitrites, 50

administration of, blood pressure after, 50

effect of, 50

for hemoptysis in tuberculosis, 790 untoward effects of, 126

Nitroglycerin, 50

for hemoptysis in tubereulosis, 790

Nitrous oxide gas as anesthetic for tuberculosis, 807

Noma in scarlet fever, 652

Nose, care of, in pneumonia, 712 in scarlet fever, 6.51

Nursing mother, diet for, 385

Nutrition and food, 367

Nux vomica in tuberculosis, 810

Obesity, diet in, 429

cures for, 432

electricity for, 323

hydrotherapy for, 243

massage for, 431

milk cure for, 432

thyroid gland in, 559

use of water in, 200

Obstruction, intestinal, diet in, 419

Occupation, mental, in tuberculosis, 766

Ocean climates, 134, 135

Ohm's law, 275

Ointment, 84

bases, 83

glycerogelatin, 83

hydrous wool fat, 83

saponated petrolatum, solid, 83

preparation of, 84

Open-air treatment in pneumonia, 709

Opium in cholera, 817

for cough in tuberculosis, 803

for diarrhea in tuberculosis, 803

untoward effects of, 127

Opsonic index, 515

method of determining, 520

significance of, 522

technique for, 520,522

vaccines and, 535

value of, 522

Opsonins, 515

history of, 516

nature of, 516

in serum therapy, $4 S 7$

Otitis media, electricity for, 324

Oxaluria, use of water in, 212

Oxygen for dyspnea in tuberculosis, 793
Oysters as source of infection in typhoid fever, 612

Ozena, electricity for, 323

\section{$\mathbf{P}$}

PACk, wet, 234

Pad clectrodes, $2 \pi 2$

l'ain in ecrehrospinal meningitis, $7(25)$ drugs used to relieve, 36

in influenza, 877

in pneumonia, 719

$x$-rays for, 330

Pancreas, 570

Paralysis agitans, electricity for, 316 exercise in, 191 parathyroid gland in, $5 f \circ 3$

exereise in, 191

spastic, exercise in, 191

I'arathyroid gland, 560

extract of, 561

in paralysis agitans, 563

products of, therapeutic use of, 562

properties of, 562

in tetany, 562

Parathyroidectomy, 560

Paratyphoid infections, 637

Paresis, exercise in, 191

hydrotherapy for, 236

Parotitis, chronic, $x$-rays for, 352

in typhoid fever, 633

Paroxysmal tachycardia, hydrotherapy for, 239

Passive motion, 17s

Paste, dermatologic, 83 pencils, 84

Pectorants, 32

Pencils, paste, 84

Penzoldt's diet lists, 401

Peptonized milk in gastric disturbances in tuberculosis, 800

Percussion, mechanical, a form of exercise, 178

Peri-anal abseess in tuberculosis, $\mathrm{S03}$

Pericardial effusions complicating smallpox, 6S3

Pericarditis, chronic, exercise in, 1SS hydrotherapy for, 239

Peripheral anodines, 37

Pcritonitis, diet in, 421 hydrotherapy for, 239

Perlsucht emulsion tuberculin, 550 tuberculin, 579

Pernicious anemia, hydrotherapy for, 249

Pertussis, diet in, 359

Pestis minor, 874

Petrolatum, solid, saponated, as ointment base, $\$ 3$

Phagocytosis, 515 in serum therapy, 4 s

Pharmacodynamics, 17 limitations of, 17 value of, 17

Pharyngeal irritability in measles, 663 
Pharyux in measles, 662

Phenacetin for fever in tuberenlosis, 785 for pleurisy, 732 untoward effeets of, 116

Phenol series, 37 for plague, 869 untoward effects of, 119

Phlebitis in typhoid fever, 634

Phosphaturia, use of water in, 215

Phosphorus as food, 375 untoward effects of, 128

Phototherapy, 304

blue light in, 305

red light in, 305

Phthisis, 260

hydrotherapy for, 237,260

Physiological aetion of cold in hydrotherapy, 223

of drugs, 32

of faradic electricity, 282

of galvanic eleetricity, 275

of heat in hydrotherapy, 223

effects of excrcise, 172

of galvanic electricity, 277

of hydro-electric baths, 292

Pilocarpine, 59 of static electricity, 281

untoward effects of, 128

Pilo-sebaceous diseases, $x$-rays for, 333

Pituitary gland, 564 extract, 565

Pityriasis versicolor in tubereulosis, 804 Plague, 859

alcohol for, 871

ambulant form of, 874

buboes in, 873

carbolic acid for, 809

carbuncles, 873

diet in, 871

fever in, 872

Haffkine's prophylaetic for, 865

larval form of, 874

nurses for, 869

phenol for, 869

pneumonic type of, 872

prophylaxis in, 859

protective treatment, 865

rats in spread of, 859

septicemic cases of, 872

sera for, 768,871

serum therapy of, 512,871

Shiga's prophylaetic for, 866

squirrels in spread of, 861

supportant treatment in, 869

surgical treatment in, 873

symptomatic treatment, 869

toxemia in, 872

vaccination against, 867

Pleural effusions complicating small-pox, 683

Pleurisy, 726

in tuberculosis, 797

acute articular rheumatism and, 832

after-treatment of, 737

coal-tar drugs for, 732

acetanilid, 732
Pleurisy, coal-tar drugs, antipyrine, 732 phenacetin, 732

cold applications for, 729

counterirritants in, 730

blisters, 731

cupping, 731

iodine tincture, 731

mustard plaster, 731

diet in, 324, 727

diureties for, 733

hot compresses for, 730

fomentations for, 730

poultice for, 730

salicylates for, 728

strapping of chest in, 728

thoracentesis in, 733

accidents from, 736

apparatus for, 734

breathing exercises after, 737

teehnique of, 735

tuberculous nature of, 737

in typhoid fever, 633

Pleuritic pain in tuberculosis, 724

Pneumococcus infections, serum therapy of, 511

meningitis, 703

vaccine, 544

Pneumonia, 259

abdominal distention in, 715

acute articular rheumatism and, 832

bed, arrangement of, 709

bowels in, care of, 714

cardiovascular system in, 720

care of body in, 711

in eholera, 856

chronic, exercise in, 189

convalescence in, 725

cough in, 718

croupous, 707 collapse in, 724

delirium in, 714

diet in, 712

diuretin in, 719

drinks in, 714

dyspnea in, 724

edema in, 725

eyes in, care of, 712

fever in, 717

fresh-air treatment in, 709

heart in, 720 stimulants for, 721

hydrotherapy for, $233,237,238,259$, 716

injection for, clectrie, 715 saline, by bowel, 715, 719

lobar, diet in, 394

meteorism in, 715

morphine in, 720

mouth, eare of, 711 wash, 712

nose in, care of, 712

open-air treatment in, 709

pain in, 719

prophylaxis for, 726

rest in, 708

specific treatment in, 725 
Pneumonia, symptomatic treatment of, 717

toxemia in, 719

in typhoid fever, 6.33

vaccines for, 544

water by mouth in, 714, 719

Pneumonic type of plague, 872

Pneumothorax in tuberculosis, 797

Pockmarking of face in smallpox, 6s:3

Poisoning, lead, 125

snake, serum therapy of, 512

Polarity, determination of, in electricity, 269

of static electricity, 2S1

Poliomyelitis, anterior, electricity for, $: 310$ exereise in, 191

Post-typhoid insanity, 635

in typhoid fever, 635

Potassium ehlorate, untoward effects of 125

iodide of, in tuberculosis, $\mathrm{SOH}$

permanganate of, in cholera, $84 \mathrm{~S}$

Poultice for pleurisy, 730

Precipitins in serum therany, 485

Pregnancy, diet in, 384

in tuberculosis, 805

Prescription blank, 66 character of, 66

directions on, 67

in capsule form, 91

for eollyrium, $\$ 9$

construction of, 71, 87-98

for external use, $\$ 9$

for internal use, 88,92

for douche, 89

for gargle, 90

for liniment, alcoholic, 90 oily, 90

soapy, 91

for local application, $91,94,96$

for lotion, 89

in lozenge form, 94

for mouth wash, 89

for nasal spray, 90

in pill form, 93

in powder form, 91,96

for reetal injection, 91

renewal of, 69

in tablet form, 94

for throat spray, 90

types, 87-98

for uretliral in jection, 91

writing, 65,70

Progressive muscular atroplyy, exercise in, 191

Prophylactic use of exercise, 170 of vaccines, 539

Prophylaxis against measles, 658 scarlet fever, 639

in influenza, 876

in plague, 859

for pneumonia, 726

Prostatic disease, hydrotherapy for, 219 hypertmphy, $x$-rays for, 353

Protein food, 367

Prurigo, $x$-rays for, 336

Quarastive, foreign, in national hygiene, 451

insular, in national hygiene, 451

interstate, in national hygiene, 453

Quinine in influenza, 878

untoward effects of, 129

\section{R}

RABIES, diet in, 391

serum therapy of, 512

Rachialgia in yellow fever, $\$ 98$

Radio-active substances, $32 \%, 333$

Radio-activity of mineral waters, 196

Radiodermatitis, 329

Rashes from diphtheria antitoxin, 494

Rats, ships infested with, 862 in spread of plague, 859 traps for, 860

Reactions of regencration, 300

Rectal electrodes, 274

feeding, 377

injection, prescription for, 91

saline injections in cholera, 83S

Remak's reaction, 301

Renal insufficieney, hydrotherapy for, 249

Resinous group of eathartics, 53

Resorts, coast, climate, $13 \mathrm{~S}$

summer, 139

winter, 139

Respiration, drugs used to increase, (i) effect of higls-frequency currents on, 289

Respiratory centre, drugs used to soothe, 61

complications in influenzs, SS1

effect of hydmilherapy, 225

symptoms in influenza, 879

system in typhoid fever, 633

tract, diserses of, electricity for, 323

Rest in acute articular rheumatism, $\$ 31$ cure, $355-366$

diet in, 359

in gast ric disturbances, 407

for diarrhea in tuherenlosis, so1

for fever in tuberculosis, 75s, 7S5 
Rest in influenza, 876

in pneumonia, 708

in tuberculosis, 757

Rheophores, 274

Rheostat, 270

Rheotome, McIntosh, 254

Rheumatic conditions, climatic treatment of, 159

Rheumatism, 264

acute articular, $\$ 21$

alkalies in, $\$ 2 S$

Bier's hyperemia for, 834

complications of, 830

convalescence in, 832

diet in, $\$ 29$

extension of, 830

general measures in, $\$ 29$

heart in, 830

historical, 821

hydrotherapy in, 833

hyperpyrexia in, 831

local treatment of, 828

management of, 824

pleurisy and, 832

pneumonia and, 832

recurrences in, 830

rest in, 831

salicylate of sodium in, 825

salicylic acid in, 823, 825, $\$ 27,833$

chronic, use of water in, 212

exercise in, 191

hydrotherapy for, 246,264

muscular, hydrotherapy for, 243

use of water in, 202

Rich's reaction, 301

Rickets in children, 384

Rigg's disease, electricity for, 318

Rodent ulcer, $x$-rays for, 340

Rodents, destruction of, in epidemics, 468

Röntgen dermatitis, 329

Rooms, disinfection of, 475

Rubella, 669

Rubner's table, 417

s

Salicylate of sodium in acute articular rheumatism, 825

Salicylates for pleurisy, 728

for pleuritic pain in tuberculosis, 795

untoward effects of, 130

Salicylie acid in acute articular rheumatism, $823,825,827,833$

Saline eathartics, 51 untoward effects of, 130

diuretics, 57

injection by bowel for pneumonia, 715,719

in cholera, 837

waters, 206

Salivary glands, $x$-rays for, 350

Salol in cholera, 848

for diarrhea in tuberculosis, 802

Salt as food, 375
Salt-free diet, 433

solutions in eholera, 837

Salve mulls, 84

Sanitary organization in epidemics, 460 reports in national hygiene, 455 statisties in national hygiene, 455

Sanitation of home in individual hygiene, 440

Santonin, untoward effects of, 130

Saponated solid petrolatum as ointment base, 83

Saratoga Springs, 208

Sarcoma, Coley's toxins for, 346 $x$-rays for, 346

Scar weakness in abdominal wall, exercise in, 190

Scarlatina. See Scarlet fever, Scarlet fever, 25\$, 639

arrangement of sickroom in, 644 arthritis in, 654

desquamation in, 643

diarrhea in, 657

diet in, 389,646

diphtheria antitoxin in, 649

ears in, 652

fever in, 650

gastro-intestinal tract in, 657

general care of, 647

glandular enlargements in, 652

heart in, 656

hemorrhagic, 657

hydrotherapy in, 231, 235, 258

hyperpyrexia in, 650

irrigation of throat, 643

isolation hospitals for, 642 of patient in, 639

kidneys in, 655

medical precautions in, 645 supervision in, 645

medication in, 648

nephritis in, 655

noma in, 652

nose in, 651

placarding house in, 639

prophylaxis against, 639

public schools and, 640

relapses in, 642

throat in, 650

irrigation of, 643

vaccines for, 541

Schools, closing of, in scarlet fever, 642

public, scarlet fever and, 640

Sciatic neuralgia, electricity for, 310

neuritis, electricity for, 308

Sciatica, electricity for, 310

hydrotherapy for, $243,244,246$

Scleroderma, $x$-rays for, 337

Sclerosis, primary lateral, electricity for, 312

Scoliosis, exercise in, 192

Scurvy, diet in, 425

infantile, diet in, 425

Sea voyages, 136

Seborrhea, diseases associated with, $x$-rays for, 335

Sedentary habits, exereise for, 186 
Sensory: effect of galvanic eleetricity, 277 reaction with static elect ricity, $2 \$ 1$

Septicemic cases of plague, 872

Serum, 601

antidiphtberitic, 813

antimeningococeus, 504

antimeningitic, 658

blood, for prevention of hemorrhage, 513

for hemophilia, 513

immune bodies, Spengler, 602

Maragliano's, 601

Marmorek's antitubercular, 602

for plague, 868,871

therapy, 483

agglutinius in, 485

of anthrax, 512

autitoxins in, 485

baeteriolysins in, 486

of eerebrospinal meningitis, 502 , 686

of eholera, 50S, 553

of diphtheria, 489

of dysentery, 505

of gonococcus infections, 511

history of, 483

of hydrophobia, 512

immunization and, 488

leukoeytic extraet in, 487

opsonins in, 487

phagocytosis in, 487

of plague, 512,871

bubonic, 512

of pneumococeus infections, 511

precipitins in, 485

prineiples of, 484

of rabies, 512

of snake poisoning, 512

of staphylococeus infeetions, 511

of streptococeus infections, 509

of tetanus, 495

of tubereulosis, 511

of typhoid fever, 507, 627

treatment of yellow fever, 900

Sewage, removal of, in communal hygiene, 446

Sexual glands, 571

Shect bath, 231, 233, 253

drip, 232

for typhoid fever, 253

therapeutic application of, 233

Shellfish as source of infection in typhoid fever, 612

Shiga's dysentery, hydrotherapy for, 249

prophylaetic for plague, 866

Ships, disinfection of, 481

infested with rats, 862

Silver, untoward effeets of, 131

Sinusoidal current, 286

application of, 287

for hydro-elect rie baths, 292

physiological action of, 256

Skin, diseases of, electrieity for, 325

thyroid gland in, $55 \mathrm{~S}$

disinfection of 473

distance of $x$-rays from, 331
Skin, gangrene of, in varicella, 67.3

itching of, in varicella, 671

lotions in varicella, 671

in measles, 658

protection of, from $x$-ray's, 331, 34s

in smallpox, 679

sleep, electric, 326

simallpox, 673

alcohol for, 676

cumplications of, 65:2

alscesses, 6x:2

aloprecia, 65i3

arthritis in, 653

corneal ulcers, 653

edema of glottis, 653

furuneulosis, 652

pericardial effusions, 653

pleural effusions, 683

tonsil, alsceess of, 653

convalescence, 683

diet in, 359, 675

fever in, 678

suppurative, 681

general care of, 673

headache in, 677

heart in, 678

medieinal treatment of, 677

nervous manifestations in, 678

pockmarking of face in, 683

Buri's method of treating, 653

purpuric, 652

pustules in, 679

skin in, 679

temperature of room, 67.4

vaceination against, 674

ventilation of room, 674

smec cell in galvanie electricity, 267

Snake poisoning, serum therapy of, 512

Social service in tubereulosis, 754

Sodic-potassic-magnesic muriated saline waters, 208

Sodium chloride solutions in cholera, $\$ 37$ nitrite, 50

Somnifacients, 33

Soups, composition of, 404

Southern California, winter resorts in, 140

Spengler's tuberculin, 5SO

Spinal analgesies, 38

anesthesia in tuberculosis, $\mathrm{SO}^{-}$

cord, diseases of, electricity for, 310

Sports for exercise, 179

Springs, mineral, 195

Saratoga, 208

thermat, 195

Squirrels, earbon hisulphide for, 861

in spread of plague, $\$ 61$

Staphylococeus infections, serum therapy of, 511

meningitis, 7033 vaccines for, $: 03$

vaceine, 543

Static electricity, 266, 279

machines, 279

Steel waters, 210

Stimulating diuretics, 57 
Stimulants, cardiae, 46 eirculatory, $\overline{5} 5$ in influenza, 878 vasomotor, 47

stomaeh, atony of, diet in, 410 carcinoma of, diet in, 414 . $x$-rays for, 345

dilatation of, diet in, 409 electricity for, 319

diseases of, diet in, $39 \mathrm{~S}$

Finhorn's intragastrie electrifieation of, 319

electrodes, 274

ulcer of, diet in, 411 hydrotherapy for, 238

urinary tract and, hyperacidity of, use of water in, 204

Stomatitis in measles, 667

Strapping of chest in pleurisy, 728

Streptococcus infections, serum therapy of, 509

meningitis, 703

vaccine, 541,544

Stricture, esophageal, electricity for, 318

Strophanthus, 44

Strychnine, 48,49

in diphtheria, 817

in influenza, 879,886

untoward effeets of, 131

Subcutaneous saline injections in cholera, 839

tuberculin test, 596

Subluxations, exercise in, 192

Sugar as food, 374

substitutes for, 429

Sulphonal, 34 untoward effeets of, 131

Sulphones, untoward effects of, 131

Sulphur dioxide for disinfection, 480 for disinfection, 477

Sulphuric acid for night sweats in tuberculosis, 792

Summer coast resolts, 139

Sunstroke, 259

hydrotherapy for, 234,259

Suppositories, 81,95 preparation of, 81

Sweat, drugs used to increase. 59

Sycosis of beard, $x$-ray's for, 334

Sydenham's chorea, electricity for, 313 electrodiagnosis of, 313

Synovitis, hydrotherapy for, 246

syphilis, tuberculosis and, 804

Syphilitic meningitis, 704

Syringomyelitis, electricity for, 312

\section{$\mathbf{T}$}

Tachycardia, exercise in, 188 paroxysmal, bydrotherapy for, 239 in tuberculosis, 793

Tea as food, 375

Temperature charts of yellow fever, 892 effect of hydrotherapy on, 226 observations of yellow fever, 896
Tenesmus, hydrotherapy for, 245

Tetanus antitoxin, 496

absorption of, 499

immunization by, 501

large doses of, 502

methods of administering, 501

persistence of, in blood, 497

results of, 500

toxin and, 498

unit of, 497

diet in, 391

serum therapy of, 495

toxin, 496, 489

action of, 496

in blood, 496

Tetany, parathyroid glands in, 562

Theobromine, 58

Theocine, 58

Thermal springs, 198

Thermogenesis, 290

Thoracentesis in pleurisy, 733

Throat in scarlet fever, 650

spray, prescription for, 90

Thymus gland, 569

in cancer, 570

in exophthalmic goitre, $\mathbf{5 7 0}$

in malignant growths, 570

Thyroid gland, 552

in arteriosclerosis, 558

in cretinism, 555

in epilepsy, 558

in exophthalmic goitre, 556

in Graves' disease, 556

in malignant growths, 559

in metabolic disturbances, 556

in myxedema, 555

in obesity, 559

in skin diseases, 558

Tinnitus aurium, electricity for, 324

Tobacco, use of, in tuberculosis, 808

Toepler machine for produeing static electricity, 279

Toes, tender, in typhoid fever, 633

Tongue electrodes, 273

Tonsil, abscess of, complicating smallpox, 683

Tonsillitis, hydrotherapy for, 233, 235

Toxemia in plague, 872

in pneumonia, 719

in typhoid fever, 628

Toxin in bowel, destruction of, in eholera, 848

tetanus, 496,498

antitoxin and, $49 \mathrm{~S}$

Trades, dangerous, regulation of, in communal hygiene, 447

offensive, regulations of, in communal hygiene, 447

Transfusion in cholera, 840-846

intravenous, 840-846

Trichophytes, $x$-rays for, 334

'Trifacial neuraligia, electricity for, 309

Trional, 34

untoward effects of, 131

Tub bath in typhoid fever, 625

Tuberculin, 573 
Tuberculin, action of, 575

administration, method of, 580

bacillen cmulsion, 580

$$
\text { filtrate, } 579
$$

Beraneck, 579

clinical advantages of, 576

curative action of, 575

as a diagnostic agent, 573,593

diluting, method of, 5S1

discovery of, 574

doses of, large and small, 586

effects upon symptoms when administered, 592

guide to dosage of, 548

hypersensibility to, 588

inijection of, $58^{\circ} 2$

muscular, deep, 583

site of, 583

subcutancous, $5 \$ 3$

time of day, 582

Morro reaction of, 600

nature of, 574

nastin, Tb.- $A ., 580$

old, 578

Tb.-I., 580

P. V., 580

perlsucht, 579

emulsion, 580

preparations of, 579

bacillen emulsion, 580

baeillus filtrate, 579

nastin, Tb.-A., Deycke of Mueh, 580

Tb.-I.., Deycke \& Much, 580

old tuberculin. 579

P. V., 580

perlsucht emulsion, 580 tuberculin, 579

T. B. V., 5 SO

tuberculin R., 580

Beraneck, 579

watery extract, 580

R., 580

reaction, 583

is it a safe remedy for general use, 592

schemata for dosage of, 585

Spengler's, 580

T. B. V., 580

for tachycardia in tuberculosis, 591

test, conjunctival, 599

cutaneous, 598

Detre's differential, 601

importance of, in diagnosis, 59.4

interpretation of, 594

intracutaneous, 601

sisbcutaneous, 586

as a therapeutic agent. 573

trentment, choice of patients for, $5 \mathrm{~s} 9$ duration of, 592

varieties of, 577

watery extract, 580

Tuberculosis, 739

air in, 745

alcohol in, 393, 810 'luberculosis, anemia in, 795

arsenic for, 795

Blaud's pill for, 795

freah air for, 795

anesthesia, choice of, in, sut

mesthetic for, 807

chloroform. 807

nitrous oxide gas, sot

spinal anesthesia, 807

anorexia in, 799

arsenic in, 810

asthmatic attacks in, 793

bilious attacks in, 800

cardiac weakness in, 794

climate for, 745

selection of, $7 \notin 7$

climatic considerations in, 74.5

treatment of, 15.5

clothing in, 780

colds in, 796

constipation in, 803

cascara sagrada for, 803

coryza in, 796

cough in, 781

control of, 782

creosote for, $7 \mathrm{~K}_{3}$

fly blister for, 783

guaiacol for, 784

carbonate for, 784

local measures for, 783

opium for, 784

creosote and its derivatives in, 809

diabetes mellitus and, 804

diarrhea in, 801

beta-naphthol for, 802

bismuth for, 802

creosote for, 802

diet for, 801

lactic acid bacilli for, sor

opium for, 803

rest for, 801

salol for, 802

diet in, 391, 767

carbohydrates, iit

drinks, 7 it

eggs, 772

fats, 773

hemorrhage, 790

meat, 773

menus for, $735,76 t, 777,7 i s$, 779,780

milk, 771

vegetables, $7 \mathrm{TH}$

directory, 743

dispensary treatment of, 754

day camps in. 756

night camps in, 756

tuberculosis class in, 755

dyspnea in, 792

morphine for, 793

oxygen for, 793

employment after arrest of cliscase in, 763

empyems in, 797

environment in, 745

exercise in, 757,755 
Tuberculosis, exercise in, contraindieations for, 758 method of, 759

fat intolerance in, 800

fever in, 758, 785

acetanilid for, 785

alcohol for, $7 S T$

antipyrine for, 785

drugs in, 787

hydrotherapy for, 787

phenacetin for, $7 \$ 5$

pyramidon for, 785

rest for, 758,785

tuberculin for, 591

finaneial resources in, 742

fistula in ano in, 803

gastric disturbances in, 798

Iydrochloric acid, dilute, in, 800

peptonized milk in, 800

graduated labor in, 760

liemoptysis in, 787

amyl nitrite for, 790

atropine sulphate for, 791

bandaging extremities for, 791

calcium lactate for, 791

digitalis for, 791

isolation for, $78 \mathrm{~S}$

nitrites for, 790

nitroglyeerin for, 790

turpentine for, 791

veratrum viride for, 791

hoarseness in, 797

home treatment of, 748

accessory details of, 752

arrangement of bed in, 752

home in, 750

supervision of patient in, 750

weather, eold, sit ting out in, 752

hot, arrangements in, 753

hydrotherapy for, $237,238,780$

hypophosphites in, 810

indieations for eoast climate, 153

individual prophylaxis for, 739

inhalations in, 810

insomnia in, S04

of intestines, diet in, 393

iodide of potassium in, 804

iodine in, 810

iron in, 810

kefir for, 772,802

koumiss for, 772,802

leucorrhea in, 805

management of a hopeless case of, 808

marriage in, 806

medical supervision in, 744

menstrual disturbances in, 805

mental occupation in, 766

mercury in, 804,810

milk, fermented, for, 772, 802

mixed infections in, 603

night sweats in, 791
Tuberculosis, night sweats in, atropine sulphate for, 792

bathing for, 792 camploric acid for, 792

fresh air for, 791

sulphuric acid for, 792 vinegar for, 792

nux vomica in, 810

pcri-anal abscess in, 803

physical status of patient in, 743

pityriasis versicolor in, 804

pleural effusion in, 797

pleuritic pain in, 794 aspirin for, 795

counterirritation for, 794

Dover's powder for, 795

heroin for, 795

salicylates for, 795

strapping chest for, 795

pneumothorax in, 797

pregnancy in, 805

pulmonary, 260

contraindieations for coast climate in, 153

electricity for, 324

exercise in, 190

gymnastics in, 765

hydrotherapy for, 260

rest in, 757

serum therapy of, 511

social service in, 754

symptoms in, 781

syphilis and, 804

tachycardia in, 793

tuberculin for, 591

tinca versicolor in, 804

tobacco, use of, in, 808

treatment by drugs in, 809

tuberculin for, 573

Tuberculous adenitis, $x$-rays for, 350

meningitis, 702

nature of pleurisy, 737

Turpentine for hemoptysis in tuberculosis, 791

untoward effects of, 132

Tympanitis, hydrotherapy for, $\mathbf{2 3 8}$

in typhoid fever, 630

Typhoid carriers, eontrol of, 636

fever, 607

ablution for, 254

affusions for, 254

appendicitis in, 633

bacilluria in, 634

bacterination in, 614

boils in, 633

bone lesions in, 634

Brand treatment of, 250

bronchitis in, 633

carbon dioxide bath for, 254

cardiovascular system in, 629

care of bowels in, 621

cholecystitis in, 633

convalescence, 635

delirium in, 630

diarrhea in, 630

diet in, 388, 622 
Typhoid fever, disinfection in, 622 drugs in, 626

antipyretics, 626

intestinal antiseptics, $62 \bar{\tau}$

dutics of nurse in, 620

epistaxis in, 633

general care of, 619

lammock bath for, 254

headache in, 629

leart failure in, 629

lemorrhage in, 6:30, 6:31

hydrotherapy for, $241,250,2.5$, 624

Brand incthod, 625

Murphy treatment, 626

packs, 625

sponging, 624

tub bath, 625

immunization in, 614

inoculation for, 618

insanity in, post-typhoid, 63.5 in children, 635

insomnia in, 630

medical supervision of, 620

meningit is in, 634

methods of prevention of, 614

nausea in, 630

nervous excitement in, 630

neuritis in, $6: 35$

parotitis in, 633

perforation in, 632

phlebitis in, 634

pleurisy in, 633

pneumonia in, 633

pregnancy and, 635

psyclioses in children, 635

relapses in, 634

respiratory system in, 633

room for patient with, 618

serum therapy of, 507,627

shect bath for, 253

sources of infection in, 609 contact with patients, 614

drinking water, 609

flies, 613

foods, 612

insects, 613

milk, 612

oysters, 612

shellfish, 612

vegetables, 612

symptoms and complications of, 628

tender toes in, 633

toxemia in, $62 \mathrm{~s}$

tympanitis in, 630

urine in, retention of, 634 seanty, 634

use of water in, 201

vaceination in, 614,617

raccines for, $539,540,5-18,627$

vomiting in, 630

water drinking in, 624

spine, 635

vaccine, 616
Typhoid vacciue, dosage of, 616 effect of, 617

preparation of, 616

\section{U}

Lickn, coneal, complicating smallpox, 653

duodenal, diet in, 419

intestinal, other furns of, diret in, $\mathbf{4 1 9}$

of intestine, diet in, 419

of stomach, diet in, 411

hydrotherapy for, 238

rodent, $x$-rays for, 340

Unar neuritis, eleetricity for, 307

United States, types of climates in, 134 health resorts in, 134, 125

Lintoward effects of acephenetidine, I16 of acetanilid, 115, 116

of aconite, 115

of aconitine, 115

of antipyretics, 115

of antipyrine, 115

of arsenic, 116

of belladonna, 117

of bismuth, 117

of borax, $11 \mathrm{~s}$

of boric acid, 118

of bromides, 119

of cantharides, 117

of carbolic acid, 119

of chloral hydrate, 119

of cocaine, 120

of digitaline, 121

of digitalis, 121

of drugs, 109

of ergot, 122

of exercise, 173

of iodides, 122

of jodine, 122

of iodoform, 123

of iron, 124

of lead, 124

of mercury, 125

of morphia, 127

of nitrites, 126

of opium, 127

of phenacetin, 116

of phenol, 119

of phosphorus, 12S

of pilocarpine, $12 \mathrm{~S}$

of potassium chlorate, 125

of quinine, 129

of salicylates, 130

of salicylic acid, 130

of santonin, 130

of silver, 131

of strychinine, 131

of sulphonal, 131

of sulphones, 131

of trional, 131

of turpentine, 132

Uremia in cholera, $\mathbf{5 5 4}$

hydrotherapy for, $\mathbf{2 4 9}$

Urethral infection, prescription for, 91 
Urethritis, hydrotherapy for, 245

Urinary gravel, use of water in, 215

incontinence, electricity for, 321

tract, diseases of, electricity for, 321

Urine, disinfeetion of, 472

drugs used to increase quantity of, 55

effect of high-frequency currents on, 289

retention of, in typhoid fever, 634

scanty, in typhoid fever, 634

Urotropin in cerebrospinal meningitis, 706

Urticaria, electricity for, 325

Utensils, disinfection of, 475

Uterus, disease of, hydrotherapy for, 250

\section{V}

VAccisatox against plague, 867 smallpox, 673

in typhoid fever, 614,617

Vaccines, 524

administration of, 532

antityphoid, 539

Bacillus coli, 547

Friedländer, 549

mallei, 549

paratyphosus, 5.48

pueumoniae, 549

pyocyaneus, 549

typhosus, 548

bacterial emulsion, 529

clinical application of, 542

containers, 531

dispensing of, 531

dosage of, 532,537

for glanders, $\mathbf{5 4 9}$

gonococcus, 546

for gonorrhea, 546

inoculation of, 532

for meningitis, 547

meningococens, 547

Micrococcus catarrhalis, 547 neoformans, 547

opsonic index and, 535

phenomena following, 535

pneumococcus, 544

for pnenmonia, 544

preparation of, 529

propnylactic use of, 539

in scarlet fever, $5+1$

in typhoid fever, 539,540

reaction from, 538

focal, 538

general, 538

local, 538

for scarlet fever, 541

standardization of, $\mathbf{5 3 0}$

staphylococcus, 543

meningitis, 703

sterilization of, 531

streptococcus, 541,544

theoretical considerations of, 53.4

therapy, 515, 524

in cerebrospinal meningitis, 686 typhoid, 539, 540, 548, 616, 627
Vaccines, Wright's doses for, 534

Vagina, irrigation of, 249

Vaginal eleetrodes, 274 infections, hydrotherapy for, 249 Varicella, 670

conjunctivit is in, 671

constitutional measures in, 671

isolation, 670

itehing of skin in, 671

local measures in, 671

skin in, gangrene of, 673

lotions for, 671

Varices, exereises in, 188

Variola. See Smallpox.

Vasoconstrictor drugs in cholera, 855

Vasomotor relaxation, hydrotherapy for, 235

stimulants, 47

Vegetables, 406

composition of, 404

as food, 374

as source of infeetion in typhoid fever, 612

in tuberculosis, 774

Vegetarianism, 371

Veratrum viride, 45

for hemoptysis in tuberculosis, 791

Verruca, $x$-rays for, 338

Vibratory massage, electric, 301

Vinegar for night sweats in tuberculosis, 792

Voltaic electricity, 266

Vomiting, reflex, electricity for, 319

in typhoid fever, 630

in yellow fever, 898

Vomitus, bloody, in yellow fever, 899

\section{$\mathbf{W}$}

Water, distilled, 200

draughts of cold, 199

drinking, 199

hot, 198

source of infection in typhoid fever, 609

as food, 375

internal use of, 250

by mouth in pneumonia, 714, 719

supplies, supervision of, 444

use of, in bronchitis, 201

in catarrhal conditions of mucous membranes, 202

in cholecystitis, 214

in chronic constipation, 215

-rheumatism, 212

in diabetes, 202,214

insipidus, 215

in diarrhea, 217

in gallstones, 214

in gastritis, 203

in gout, 202, 212

in hyperacidity of stomach, and urinary tract, 204

in hyperchlorhydria, 202 
Water, use of, in nephritis, 201, 213 in obesity, 200 in oxaluria, 215 in phosplasturia, 215 in rheumatism, 202 in typhoid fever, 201 in urinary gravel, 215

Waters, alkaline, $20^{2}$ bicarbonated, 202 carbonated, 202

American, 218

arsenic, 211 springs of, 211

chalybeate, 210

foreign, 218 springs of, 211

iodobromic, 212

iron, 210

lithia, 203

mincral, alkaline, 204

artificial, 197 radio-activity of, 196

prevention of pollution of, in national hygienc, 455

purgative, 207

saline, 206

sodic-potassic-magnesic muriated, 206, 203

steel, 210 springs of, 208

sulphuretted, 209

Watery extract tuberculin, 580

Wet compresses, 236 pack, 234

modus operandi of, 235

therapeutic application of, 23.5

Whooping cough, adrenal gland in, 569 diet in, 389

Wimshurst machine for produeing static electricity, 279

Winter coast resorts, 139 months, climate for, 141 in Southern California, 140

Wool fat, hydrous, as ointment base, $\$ 3$

\section{$\mathbf{x}$}

X-nars for acne rosacea, 335 vulgaris, 335

for alopecia, 333

for asthma, bronchial, 353

as an apilating agent, 333

biological effects of, 327 on disensed tissue, $3: 30$

for bronclitis, chronic, 353

hurn from, first degree, 326 second degree, $3: 9$ third degree, 329

for carcinoma, 341

of accessory sinuses, 34.5

of lireast, 341

of esophagus, 345

of henrt, 342

ante-operative treatment in. 343

VOI. I. -59
X-rays for carcinoma of heart, frefueuey of tresetine:nts, 34:3

inoperable, ist2

metsestatic growths in, 3H

postoperative treatmopst in, 314

quality of rays, 342

recurrent growths $\mathrm{jn}$, int

results, 343

trochinique of 312

intestines, 345

of laryux 345

of stonuch, 345

for cutaneniss horms, 3.3.

for deep-seated diseases, $3: 31$

flermatitis from, $3: 9$

for dermatoses, pruriginous, 33.5

distance of, from skin, 331, 342

duration of treatment, $3: 32$

for cczema, selorrheic, 3.35)

for epithelioma of lip, 340

of mouth, 340

of mucous membrane, 339

for epitheliomata, 333

for ophthalmic guitre, 3.52

falling out of hair from, 329

for favus, 3:44

filters for, 332

for folliculitis of head, 331

frequeney of treatment, 3.32

for Hodgkin's dicease, 351

for hypertrichosis, 33;

for keloid, 337

for leukemia, 349

lymphatic, 350

myelogenous, 349

for lichen corneus, 336; ruber planus, 336

for lupus erythematosus, 3:37 vulgaris, 336

for lynphadenoma, 351

for lymplazt ic glands, 350)

for Slikulicz's disease, 3.52

for mycosis fungoides, 337

for nevus, 335

for pain, 3:30

for parotitis, cluronic, 352

for pilo-sebsecons diveases, 333 postoperative treatment. 349

for prostatic hypert rophy. 33:3

protection of operat or from, 3:3:2 of patient from, 333

for prurigo, 336

for pruritus, anal, 335 vulvar, 33í

for pseudoleukemia, 351

reaction, i:2s

for rodent uleer, 340

for salivary glands, 3.50)

for sarcomia, 346

compared to other methods, $3+6$ recurreuces, 347

technique of, 345 amount of eurrent, 345 distance from skin, 345 duration of treatment, 348 
X-rays for sarcoma, teehnique of profor scleroderma, 327 teetion of skin, 348

for seborrhea, diseases - associated with, 335

strength of current for, 331

for superfieial application, 331

for sycosis of beard, 334

technique of, 330

for trichophytes, 334

tube, 330

and its vacuum, 330

for tuberculous adenitis, 350

use of anode in, 331

for verruca, 338

\section{$\mathbf{Y}$}

YeLI.ow fever, SS9

anemia in, 899

bloody vomitus in, 899
Yellow fever, convalescence in, 901

diagnosis of, 890

diet in, 899, 901

eliminative treatment of, 897

fever in, 898

heart in, 900

kidneys in, 899

mosquitoes in, danger from, $8 \$ 9$

nausea in, 898

rachialgia in, 898

rest in, 897

serum treatment of, 900

symptomatology of, 890,891

temperature charts of, 892

observations in, $\$ 96$

treatment of, 895

vomiting in, 898

$\mathbf{Z}$

ZANDER machines, 178 




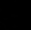

.

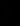

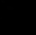


University of Toronto Library

DO NOT REMOVE THE CARD FROM THIS POCKET Acme Library Card Pocket Under Pat "Ref. Loder FUle" Made by LIBRARY bUReau 
